
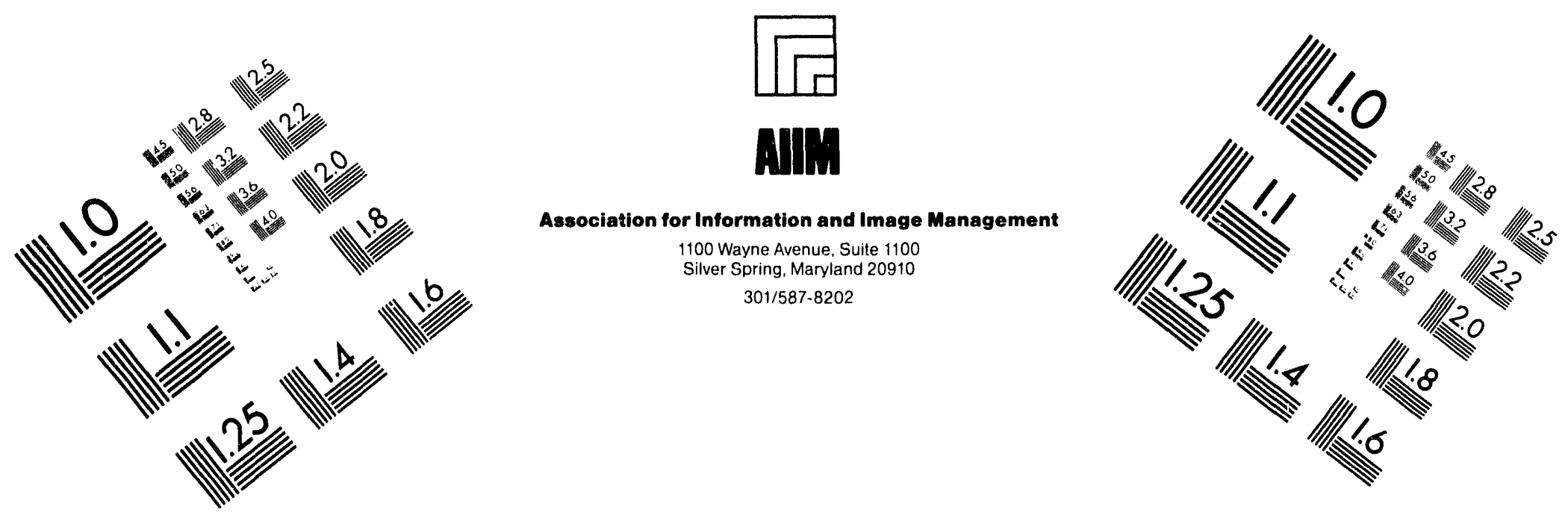

\title{
Centimeter
}

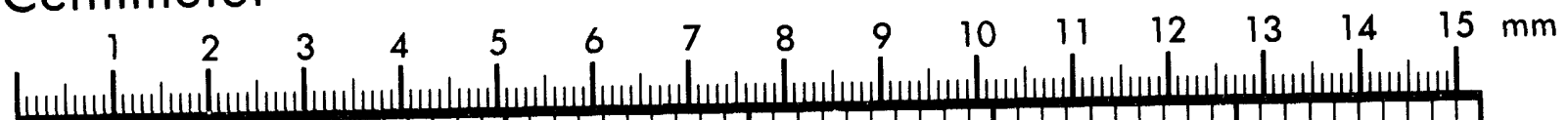

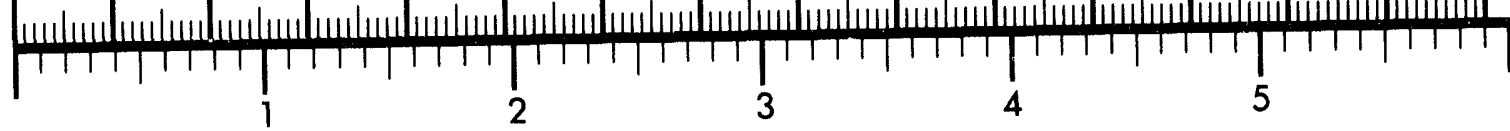
Inches
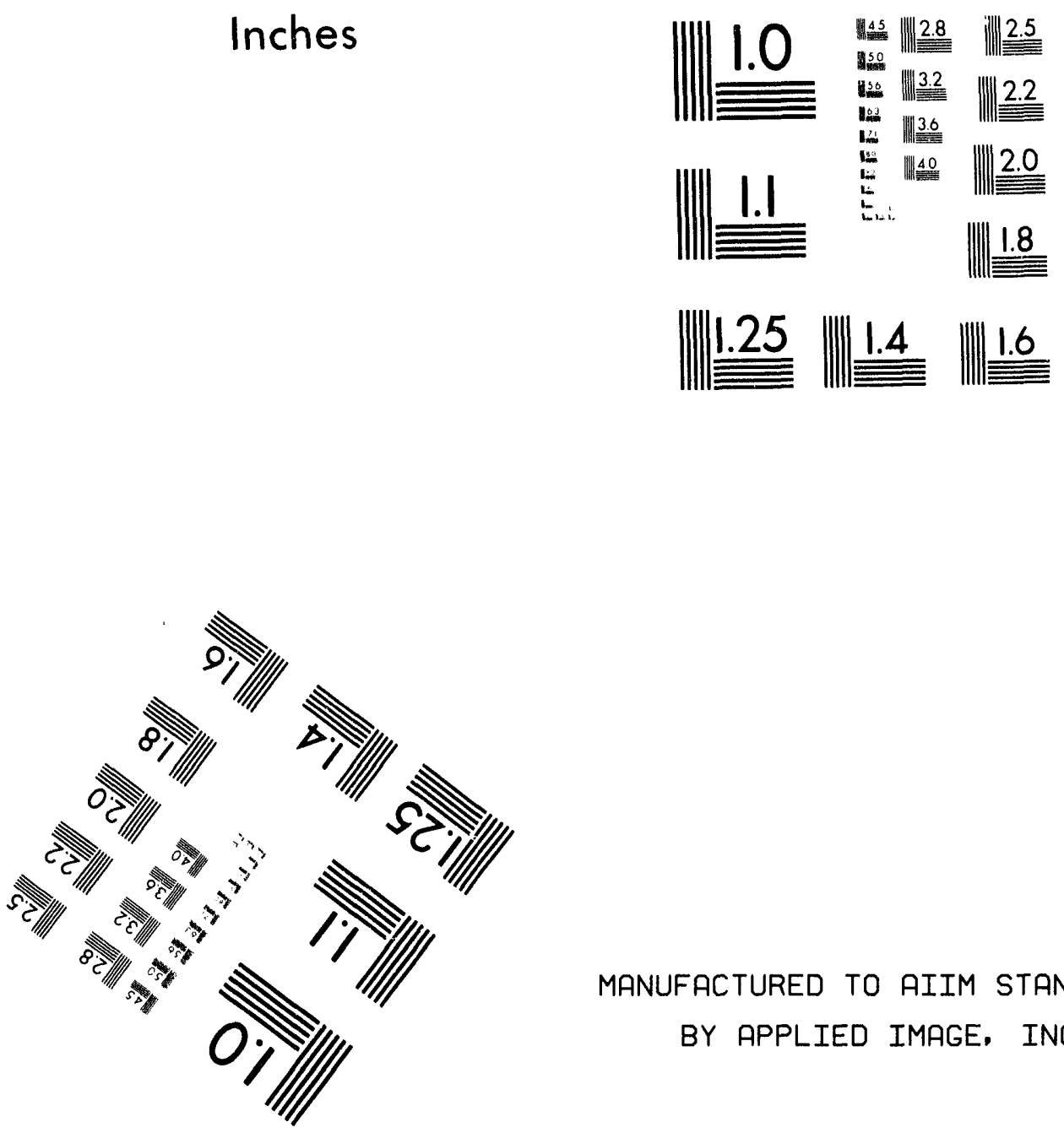

MANUFACTURED TO AIIM STANDARDS

$$
\text { BY APPLIED IMAGE, INC. }
$$

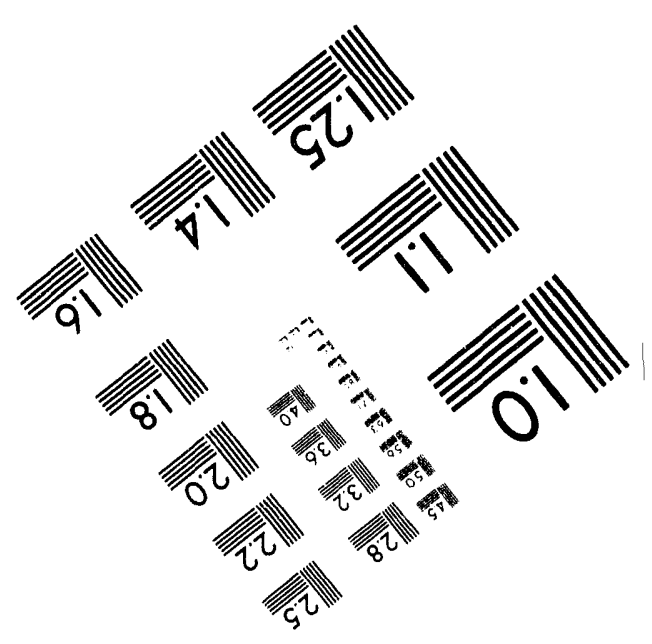



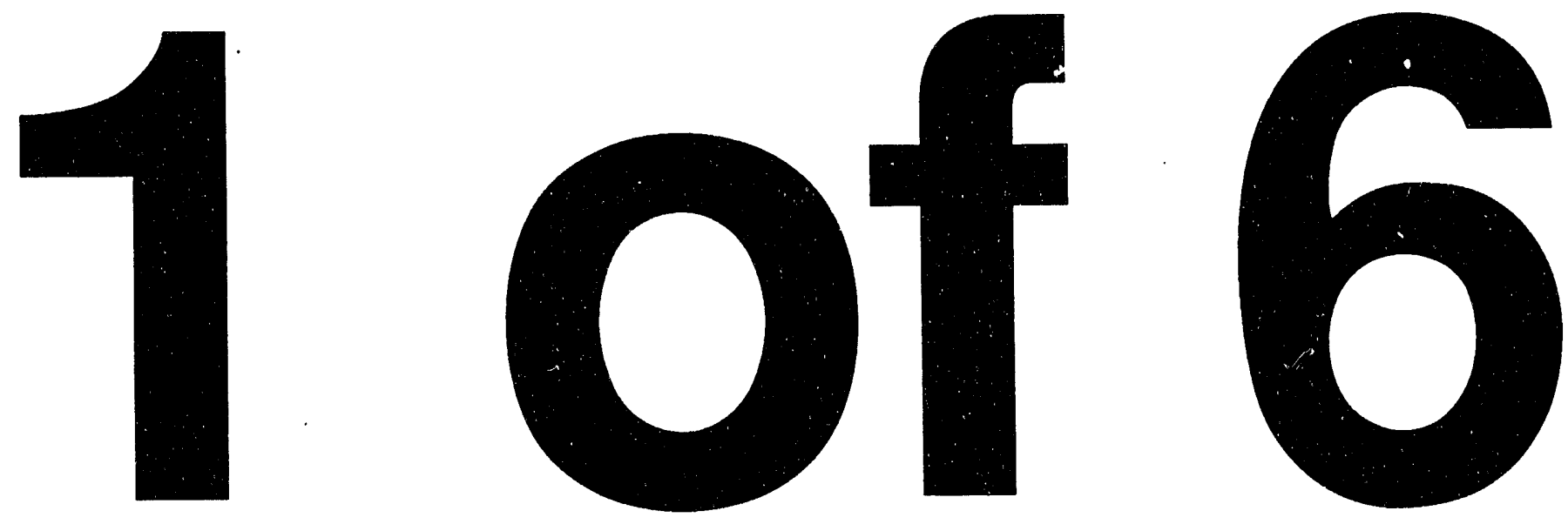
WiHC-EP-0791

UC-2030

\section{Extensive Separations (CLEAN) Processing Strategy Compared to TRUEX Strategy and Sludge Wash lon Exchange}

B. J. Knutson

G. Jansen

B. D. Zimmerman

S. E. Seeman

Westinghouse Hanford Company

L. Lauerhass

M. Hoza

Pacific Northwest Laboratory

Date Published

August 1994

Prepared for the U.S. Department of Energy Office of Environmental Management

Office of Technology Development

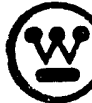

Westinghouse

Hanford Company

P.O. Box 1970

Richland, Washington 99352

Hanford Operations and Engineering Contractor for the

U.S. Department of Energy under Contract DE-AC06-87RL10930

Approved for Public Release 
LECAL DISCLAIMER

This report was prepared as an account of work sponsored by en agency of the United States Government. Neither the United States Government nor any agency thereof, nor any of their employees, nor any of their contractors, subcontractors or their omployees, makes any warranty, express or implied, or assumes any legal liability or responsibility for the accuracy, completeness, or any third party's use or the results of such use of any information, apparatus, product, or process disclosed, or represents that its use would not infringe privately owned rights. Reference herein to any specific commercial product, process, or service by trade name, tradomark, manufacturer, or otherwise, does not necessarily constitute or imply its endorsement, recommendation, or favoring by the United States Government or any agency thereof or its contractors or subcontractors. The views and opinions of authors expressed herein do not necessarily state or reflect those of the United States Government or any egency thereof.

This report has been reproduced from the best available copy. Available in peper copy and microfiche.

Aveilable to the U.S. Department of Energy and its contractors from

Office of Scientific and Technical Information

P.O. Box 62

Oek Ridge, TN 37831

(615) 576-8401

Available to the public from the U.S. Department of Commerce National Technical Information Service

5285 Port Royal Road

Springfield, VA 22161

(703) $487-4650$

Printed in the United Staten of Americe

DISCLM-1.CHP (1-91) 


\section{RELEASE AUTHORIZATION}

\section{Document Number: WHC-EP-0791}

Extensive Separations (CLEAN) Processing Strategy

Document Title: $\quad$ Compared to TRUEX Strategy and Sludge Wash lon

Exchange

Release Date:

August 22, 1994

This document was reviewed following the procedures described in WHC-CM-3-4 and is:

APPROVED FOR PUBLIC RELEASE

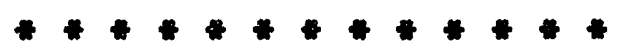

WHC Information Release Administration Specialist:

$$
\text { M. Bostan M. N. Boston }
$$


WHC-EP-0791

Document Title: Extensive Separations (CLEAN) Processing Strategy Compared to TRUEX Strategy and Sludge Wash Ion Exchange

Prepared by:

Bundt Hutton

B. J. Knutson, Principal Engineer

Westinghouse Hanford Company

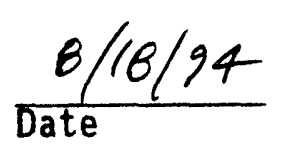

$\frac{8 / 18 / 94}{\text { Date }}$

$\frac{8 / 18 / 94}{\text { Date }}$

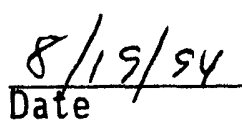

Approved by:

G.W. Jackson, Director Systems Engineering

iv 


\section{ACKNOWLEDGENENT}

The work of students K. C. Larson, B. M. Grace, W. J. Young, and $M$. E. Johnston of the Westinghouse Hanford Company in writing various spreadsheet scripts to process the model input and output data was invaluable in accomplishing this work. Funding for this project was provided by the U.S. Department of Energy, Office of Technology Development (EM-50), through the Efficient Separations and Processing Integrated Program. 
WHC-EP-0791

This page intentionally left blank. 


\section{EXTENSIVE SEPARATIONS (CLEAN) PROCESSING STRATEGY COMPARED TO TRUEX STRATEGY AND \\ SLUDGE WASH ION EXCHANGE}

\section{EXECUTIVE SUMMARY}

Numerous pretreatment flowsheets have been proposed for processing the radioactive wastes in Hanford's 177 underground storage tanks. On one extreme are minimal treatment flowsheets containing a few separations that moderately concentrate radionuclides into a high-level waste (HLW) glass product. To the other extreme are complex flowsheets with dozens of separation processes intended to minimize the HLW volume and radiotoxicity of the low-level waste (LLW) glass or grout product. The CLEAN Option is an example of this latter type of pretreatment flowsheet whose analys is is the subject of this report. The CLEAN Option is examined along with two other flowsheet alternatives to quantify the trade-off of greater capital equipment and operating costs for aggressive separations with the reduced waste disposal costs and decreased environmental/health risks.

The effect on the volume or HLW glass product and radiotoxicity of the LLW giass or grout product is predicted with current assumptions about waste characteristics and separations processes using a mass balance model. The prediction is made on three principal processing options:

- Washing of tank wastes with removal of cesium and technetium from the supernatant, with washed solids routed directly to the glass (referred to as the Sludge Wash ( processing strategy)

- The previous steps plus dissolution of the solids and removal of transuranic (TRU) elements, uranium, and strontium using solvent extraction processes (referred to as the Transuranic Extraction Option C (TRUEX-C) processing strategy)

- An aggressive yet feasible processing strategy for separating the waste components to meet several main goals or objectives (referred to as the CLEAN Option processing strategy), such as the LLW is required to meet the U.S. Nuclear Regulatory Commission Class A limits; concentrations of 
technetium, fodine, and uranium are reduced as low as reasonably achievable; and HLW will be contained within 1,000 borosilicate glass canisters $\left(0.625 \mathrm{~m}^{3}\right.$ /canister) that meet current Hanford Waste Vitrification Plant (HWVP) glass specifications.

Key characteristics of the different processing strategies, which could be compared quantitatively, are summarized in the results summary table. Costs are provided for the CLEAN option with and without compact processing units (CPUS).

Results Summary Table.

\begin{tabular}{|c|c|c|c|c|}
\hline \multirow[b]{2}{*}{ COMPARISON } & \multirow{2}{*}{$\begin{array}{l}\text { WTHCPUS } \\
\text { CEAN }\end{array}$} & \multicolumn{3}{|c|}{ WITHOUT CPUS $\cdots$} \\
\hline & & CEAN & TRUEXC & SLUDGE WASHC \\
\hline UTLIZES COMPACT PROCESSING UNTS (CPUS)? & YES & NO & NO & NO \\
\hline TOTAL COST (\$BILLLON) & $\$ 31.1 \mathrm{~B}$ & $\$ 41.2 \mathrm{~B}$ & $\$ 32.5 \mathrm{~B}$ & $\$ 36.4 B$ \\
\hline $\begin{array}{l}\text { PROCESSING OOST } \\
\text { HLW TREATMENT \& DISPOSAL COST } \\
\text { LWW TREATMENT \& DISPOSAL COST } \\
\text { TERTIARY WASTE TREATMENT \& DISPOSAL COST } \\
\text { TANK WASTE RETRIEVAL COST } \\
\text { OTHEA }\end{array}$ & $\begin{array}{l}\$ 7.6 \mathrm{~B} \\
2.5 \\
2.4 \\
9.5 \\
8.1 \\
0.4\end{array}$ & $\begin{array}{l}\$ 14.3 B \\
2.5 \\
2.4 \\
12.8 \\
8.1 \\
0.4\end{array}$ & $\begin{array}{l}\$ 5.2 B \\
6.0 \\
3.5 \\
8.9 \\
8.1 \\
0.4\end{array}$ & $\begin{array}{l}\$ 3.78 \\
15.3 \\
2.7 \\
5.9 \\
8.1 \\
0.4\end{array}$ \\
\hline IMPACT OF 1 WT\% P2O5 GLASS LIMIT ON TOTAL COST & \$0.1B & \$0.1B & $\$ 1.6 \mathrm{~B}$ & $\$ 17.58$ \\
\hline TERTIAAY WASTE CONTRIBUTION TO TOTAL COST & $30 \%$ & $31 \%$ & $27 \%$ & $16 \%$ \\
\hline $\begin{array}{l}\text { WITH P2O5 GLASS LIMT RELAXED: } \\
\text { HLW GLASS CANISTERS (0.625 M3/CANISTER) } \\
\text { LLW GLASS-IN.SULFUA VAULTS }\end{array}$ & $\begin{array}{c}750 \\
65\end{array}$ & $\begin{array}{c}750 \\
65\end{array}$ & $\begin{array}{c}8880 \\
76\end{array}$ & $\begin{array}{c}41450 \\
60\end{array}$ \\
\hline TERTTARY WASTE SALT GROUT VAULTS & 169 & 237 & 155 & 98 \\
\hline LW CLASSIFICATION & CLASSA & CLASSA & CLASSB & CLASSB \\
\hline $\begin{array}{l}\text { UW ONSITE \& OFFSITE SHORT TERM RELATIVE } \\
\text { HEALTH RISK INDEX }\end{array}$ & 1.0 & 1.0 & 49.5 & 71.2 \\
\hline $\begin{array}{l}\text { RELATIVE OCCUPATIONAL HEALTH RISK INDEX: } \\
\text { BETA RADIATION } \\
\text { GAMMA RADIATION }\end{array}$ & $\begin{array}{l}19.2 \\
1.24\end{array}$ & $\begin{array}{l}19.2 \\
1.24\end{array}$ & $\begin{array}{l}8.7 \\
1.20\end{array}$ & $\begin{array}{l}1.0 \\
1.00\end{array}$ \\
\hline NE2O CONTENT OF LOWLEVEL WASTE & $74 \mathrm{WT} \%$ & 74 WT\% & 85 WT\% & $89 \mathrm{WT} \%$ \\
\hline RELATTVE PAOCESS STRATEGY TECHNICAL RISKINDEX & $>9.8$ & 9.8 & 5.0 & 1.0 \\
\hline
\end{tabular}


The following conclusions are supported by the study results.

- The reduction in the HLW glass canister count achieved by the CLEAN Option sharply decreases the associated HLW treatment and disposal cost relative to the TRUEX $-C$ and Sludge Wash $C$ flowsheets.

- The greater number of separation processes in the CLEAN flowsheet is reflected in higher separation costs when compared to the alternative options.

- Of the three options examined (excluding CLEAN Option with CPUS), TRUEX-C has the lowest total cost. TRUEX- $C$ demonstrates the most economic trade-off of separation complexity with HLW disposal cost reduction.

- A $24 \%$ reduction in the total cost of the CLEAN Option is achieved if CPUs (for alkaline ion exchange processing of supernate and salt cake to remove cesium, strontium, and technetium) are used instead of a Central Processing Facility. A similar total cost impact is expected if CPUs are used in the TRUEX-C and Sludge Wash $C$ flowsheets.

- The contribution of tertiary waste to total cost is substantial for all flowsheets. Tertiary costs varied from $16 \%$ (for Sludge Wash C) to $31 \%$ (for CLEAN Option) of the total cost.

- With regard to health risk posed by LLW, the CLEAN Option is substantially superior to the other options. The CLEAN Option slowsheet is the only option in this study capable of producing Class A LLW.

- The CLEAN Option poses greater occupational health risk because more extensive separation operations increase worker exposure to radioactivity. 
The total costs cited for the different flowsheets do not take into consideration a number of intangible factors important in flowsheet selection.

- The CLEAN Option carries more technical risk than the alternative flowsheets because it relies more heavily on emerging technologies still undergoing development. For example, there is considerable uncertainty in the optimistic acid side dissolution assumptions so critical to CLEAN Option's dramatic HLW volume reduction relative to alternative pretreatment strategies.

- The more than an-order-of-magnitude improvement in short-term onsite and offsite health risk achieved by the CLEAN Option could make this option preferable to lower cost alternatives.

- Credit is not taken in the CLEAN Option for reducing HLW volume to the point that a separate vitrification facility may not be needed. If vitrification can be incorporated directly into the separations plant as an acid process, the cost of permitting and approval efforts for an additional stand-alone facility could be avoided, and costs associated with neutralization, material transport, and storage of waste at a separate facility would be eliminated.

Additional conclusions resulting from this study include the following.

- LLW processing and disposal costs are comparable for LLW glass-insulfur and grout products.

- Residual waste (or hee1) remaining in the tanks aftrr sludge retrieval represents the dominant onsite and offsite health hazard.

Numerous assumptions were made for each of the three processing strategies. Four assumptions, which strongly affect total cost and merit further technical effort to substantiate, are as follows:

- The 1 wt\% $\mathrm{P}_{2} \mathrm{O}_{5}$ maximum limit for HWVP borosilicate glass

- The $20 \mathrm{wt} \% \mathrm{Na}_{2} \mathrm{O}$ maximum limit for LLW borosilicate glass 
- The effectiveness of the organic destruction step in destroying metal complexants in the supernatant

- The extent of sludge dissolution for siudge treatments proposed in the three processing strategies.

The results of this study suggest investigating the following issues in future work under this project.

- Determine the cost impact of including a sodium salt removal process in the pretreatment flowsheet to substantially decrease the volume of LLW that must be processed.

- Quantify the effect on costs of substituting developing ion-exchange technologies (cesium and strontium) for baseline technologies.

- Consider a greater range of disposal alternatives by not recombining the waste fractions produced by a processing strategy, such as the CLEAN Option.

- Reevaluate the process strategies using different tank group feedstreams (perhaps 10 to 12 for single-shell tanks) versus an all tank (single- and double-shell tank) mixed waste feedstream currently used in the analyses. Sludge dissolution factors included in the process models would change for each specific tank sludge waste group based on experimental/analytical data. This course of analysis could be phased in as appropriate. 
WHC-EP-0791

This page intentionally left blank. 


\section{CONTENTS}

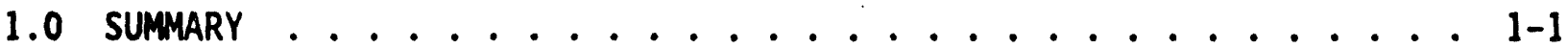

1.1 BACKGROUND ....................... 1-8

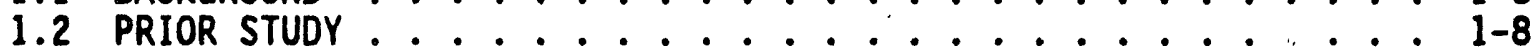

1.3 WASTE MINIMIZATION PHILOSOPHY ............... 1-9

2.0 TECHNICAL ISSUES ....................... 2-1

2.1 WASTE STREAM .................. 2-1

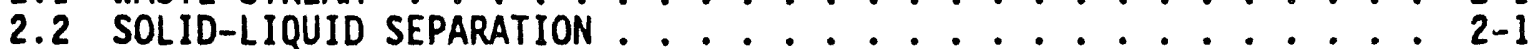

2.3 SOLIDS DISSOLUTION .................. 2-2

2.4 HIGH- AND LOW-LEVEL WASTE FORM MINIMIZATION . . . . . . . 2-2

2.5 GENERATION OF SECONDARY AND TERTIARY WASTE . . . . . . . 2-3

3.0 PROCESS DESCRIPTION AND IMPORTANT ASSUMPTIONS ..........

3.1 WASTE STREAM .................... 3-1

3.1.1 Supernatant ................. 3-1

3.1.2 STudge .. . . . . . . . . . . . . . . 3-2

3.2 HIGH- AND LOW-LEVEL WASTE STREAMS .............. 3-3

3.3 SOLVENT EXTRACTION ................. 3-5

3.4 SOLVENT WASTE MINIMIZATION .............. . . 3-5

3.5 SODIUM HYDROXIDE AND NITRIC ACID RECYCLE . . . . . . . . 3-6

4.0 RESULTS ........................ 4-1

4.1 TERTIARY WASTE GENERATION ................. . 4-1

4.2 COSTS . . . . . . . . . . . . . . . . 4-1

4.3 GLASS COMPOSITION OPTIMIZATION .............. $4-4$

4.4 WASTE PRODUCT MASS BALANCES ................. . 4-6

4.5 RELATIVE HEALTH HAZARD ESTIMATES ............ 4-7

4.5.1 Long-Term Offsite Health Hazard . . . . . . . . . . . . 4-7

4.5.2 Long-Term Onsite Health Hazard . . . . . . . . . . . . 4-7

4.5.3 Short-Term Onsite And Offsite Health Hazard . . . . . 4 4-8

4.5.4 Occupational Exposure ............... 4-9

4.5.5 Waste Classification and Radionuciide Concentrations . . 4-10

4.6 PROCESS STRATEGY TECHNICAL RISK . . . . . . . . . . . 4-10

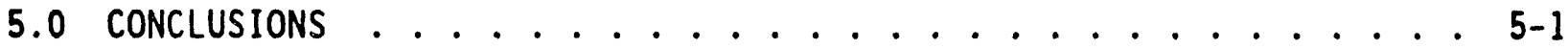

6.0 REFERENCES ........................ 6-1

\section{APPENDICES}

A GENERAL DESCRIPTION OF ASPEN PLUS . . . . . . . . . . . . A-1

B DETAILED CLEAN OPTION MODEL: ASPEN PLUS IMPLEMENTATIION $\cdot \cdots \cdot \cdots$

C HANFORD STRATEGIC ANALYSIS MODEL: ASPEN PLUS IMPLEMENTATION . . . . C-1

D GLOBAL LEVEL MODEL: ASPEN PLUS IMPLEMENTATION . . . . . . . . . . D-1

E TERTIARY NONPROCESS WASTE GENERATION ................ E-1

F COST ESTIMATION MODELS . . . . . . . . . . . . . . . . . . F-1

G GLASS COMPOSITION OPTIMIZATION . . . . . . . . . . . . . . . . G-1

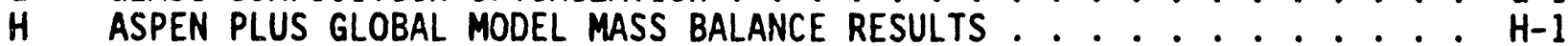

I RELATIVE HEALTH RISK ESTIMATES . . . . . . . . . . . . . . . I-1

$\mathrm{J}$ TANK WASTE INVENTORIES . . . . . . . . . . . . . . . . . J-1

$K$ PROCESS STRATEGY TECHNICAL RISK . . . . . . . . . . . . . . . . K-1 


\section{LIST OF FIGURES}

1-1 Process Strategy Flowsheet Comparison . . . . . . . . . 1-10

3-1 High-Level Waste Oxide Stream Content for Flowsheet Options . . . 3-7

3-2 Radionuctide Fraction to Low-Level Waste . . . . . . . . . . . 3-7

4-1 Tertiary Waste Generation . . . . . . . . . . . . . 4-12

4-2 Cost Summary for Primary and Tertiary Waste Processing . . . . . . 4-13

4-3 High-Level Waste Glass Canister Summary . . . . . . . . 4-14

4-4 Low-Level Waste Glass and Grout Vault Summary . . . . . . . . . 4-14

4-5 Normalized Long and Short Term Onsite Health Hazard . . . . . . 4-15

4-6 Occupational Health Hazard Summary . . . . . . . . . . 4-15

4-7 Total Technical Risk Index by Process Strategy . . . . . . . . 4-16

4-8 Average Technical Risk Index by Process Strategy . . . . . . . . 4-16 
WHC-EP-0791

\section{LIST OF TABLES}

3-1 High-Level Waste Oxide Stream Content for Flowsheet Options . . . 3-8

3-2 Radionuclide Fraction to Low-Level Waste . . . . . . . . . . 3-8

4-1 Total Cost Summary for Process Strategies . . . . . . . . . 4-17

4-2 Summary of Glass Optimization Results . . . . . . . . . . 4-18

4-3 Summary of Waste Product Generation ............. 4-19 


\section{LIST OF TERMS}

$\begin{array}{ll}\text { CE } & \text { crown ether } \\ \text { CMPO } & \text { N-diisobutylcarbamoylmethylphosphine oxide } \\ \text { CPU } & \text { compact processing unit } \\ \text { DF } & \text { decontamination factor } \\ \text { DOE } & \text { U.S. Department of Energy } \\ \text { DST } & \text { double-shell tank } \\ \text { EDTA } & \text { ethylenediaminetetraacetic acid } \\ \text { ESPIP } & \text { Efficient Separations and Process Integrated Program } \\ \text { GTCC } & \text { Greater than Class C } \\ \text { HHI } & \text { Health Hazard Index } \\ \text { HLW } & \text { high level waste } \\ \text { HWVP } & \text { Hanford Waste Vitrification Plant } \\ \text { IX } & \text { ion exchange } \\ \text { LLW } & \text { low-level waste } \\ \text { NRC } & \text { U.S. Nuclear Regulatory Commission } \\ \text { OTHHI } & \text { Other upper limit } \\ \text { SCWO } & \text { supercritical water oxidation } \\ \text { SE } & \text { solvent extraction } \\ \text { SLS } & \text { solid/liquid separation } \\ \text { SOLEX } & \text { Solvent Extraction Option } \\ \text { SREX } & \text { strontium extraction } \\ \text { SST } & \text { single-shell tank } \\ \text { TBP } & \text { tributyl phosphate } \\ \text { TCIX } & \text { technetium ion exchange } \\ \text { TOC } & \text { total organic carbon } \\ \text { TPA } & \text { Tri-Party Agreement } \\ \text { TRU } & \text { transuranic } \\ \text { TRUEX } & \text { transuranic extraction } \\ \text { TRUEX-C } & \text { Transuranic Extraction Option C } \\ \text { WHC } & \text { Westinghouse Hanford Company } \\ & \end{array}$




\section{EXTENSIVE SEPARATIONS (CLEAN) PROCESSING STRATEGY \\ COMPARED TO TRUEX STRATEGY AND \\ SLUDGE HASH ION EXCHANGE}

\subsection{SUMMARY}

The effect on the volume of high-level waste (HLW) glass and radiotoxicity of the low-level waste (LLW) grout or glass is predicted with current assumptions about waste characteristics and separations processes using a mass balance model. The prediction is made on three principal processing options: (1) washing of tank wastes with removal of cesium and technetium from the supernatant, with washed solids routed directly to the glass (referred to as the Sludge Wash C processing strategy); (2) the previous steps plus dissolution of the solids and removal of transuranic (TRU) elements, uranium, and strontium using solvent extraction processes (referred to as the Transuranic Extraction Option C [TRUEX-C] processing strategy); and (3) an aggressive yet feasible processing strategy for separating the waste components to meet several main goals or objectives (referred to as the CLEAN Option processing strategy). Among the goals or objectives of the CLEAN Option strategy are the following: LLW meets U.S. Nuclear Regulatory Commission (NRC) Class A limits, concentrations of technetium and iodine in the $L L W$ fraction are reduced as low as reasonably achievable, and HLW will be contained within 1,000 borosilicate glass canisters that meet current Hanford Waste Vitrification Plant (HWVP) canister size and glass composition specifications. (Each canister is asşumed to be $2 \mathrm{ft}$ in diameter by $10 \mathrm{ft}$ in length with a glass volume of $0.625 \mathrm{~m}^{3}$ ).

Recent Tri-Party Agreement (TPA) changes have adopted an Enhanced In-Tank Sludge Wash process strategy as the technical baseline. Figure 1-1 shows generally how the current baseline process strategy relates to the three process strategies evaluated in this report. The Enhanced In-Tank Sludge Wash process strategy will be evaluated in a future report.

The predicted HLW volumes and radiotoxicity of the LLW depend on the following assumptions:

- Assumed composition of the waste stream

- Assumed distribution of various components in the waste between the sludge and supernatant phases

- Assumed fraction of various components in the sludge that dissolve in the assumed acid dissolution $\operatorname{step}(s)$

- Assumed maximum allowable mass fraction of phosphate in the HLW or LLW glass

- Assumed relationship of certain glass properties to glass composition

- Assumed use of a stripping agent for the transuranic extraction (TRUEX) step that is not an organophosphate 
- Assumed fraction of TRU elements complexed in the supernatant and the extent of destruction of complexants in a supercritical water oxidation step

- Assumed ability of a sodium titanate sorption step to remove the remaining, presumably complexed, dissolved species

- Assumed extent of extraction and stripping of various components in the TRUEX and strontium extraction (SREX) solvent extraction processes

- Assumed ion exchange (IX) removal efficiency for radionuclides (i.e., americium, strontium, cesium, and technetium).

The amounts of actinide elements in the waste supernatant that are routed to the LLW form are consequences of the following key assumptions:

(1) their assumed concentrations in the supernatant, (2) the assumption that these concentrations are the result of complexation, and (3) that $99 \%$ of the complexants are destroyed in an oxidation step.

The amount of HLW glass produced may be controlled by the masses of waste components in the sludge that remain undissolved (especially for the TRUEX-C and Sludge Wash ( process strategies). The CLEAN Option processing strategy contains three acid dissolution steps to overcome these dissolution problems. In the flowsheets considered for this report, undissolved solids are routed directly to HLW glass. Based on the assumed waste composition, these solids may include substantial mass fractions of phosphate (especially for the TRUEX-C and Sludge Wash $C$ process strategies). The assumed allowable mass fraction of phosphate as $\mathrm{P}_{2} \mathrm{O}_{5}$ in the glass is 1 wt\%; based on this 1 imit the HLW loading in the glass must be constrained to accommodate the phosphate in the undissolved solids. The amount of HLW glass that would be required if the maximum phosphate mass fraction constraint is relaxed was al so calculated for comparison and also depends mainly on the extent to which sludge remains undissolved.

Based on a draft ietter report issued in July 1993 (Knutson et al. 1993), the HLW stream, if calcined, would be nearly the same in composition as the calcined undissolved solids stream (for the TRUEX $-C$ and Sludge Wash $C$ process strategies) because on average $90 \%$ of the HLW equivalent oxides are contributed by the undissolved solids stream. Therefore, the reduction in waste mass, on a calcined basis, from the feed to the HLW of a factor of 22 is 1 imited by the assumed $90 \%$ dissolution of species in the solids. Therefore, EM-30/50 should focus on the solids dissolution pretreatment step because it has the largest impact on the glass waste product. Certainly, the CLEAN Option processing strategy focuses on this aspect along with additional element separations to meet its low HLW glass canister count and LLW toxicity goals.

Recent TPA changes have also designated glass as the final waste product form for the LLW stream. The LLW glass is expected to be stored as gravelsized glass chunks (cullet) within a sulfur cement matrix in underground vaults. However, no extensive analyses or experimental glass formulation data relative to expected LLW stream compositions are available. Therefore, the analyses in this report used the HLW glass composition specifications and 
associated optimization models for the LLW glass streams also. It is expected that the LLW glass will have different glass comoosition and property limits that will be defined in future work.

Significant reductions in the HLW glass canister and LLW glass-in-sulfur vault counts were achieved when the $\mathrm{P}_{2} \mathrm{O}_{5}$ high constraint limit was relaxed in the glass optimization calculations. With these aggressive pretreatment strategies it is clear that more glass property data and glass melting data in HLW and LLW waste composition ranges could possibly allow relaxing current composition constraints. If such limits were relaxed, higher waste oxide loadings in the glass could be achieved (and hence, reduced glass canister and vault counts, reduced cost.s, etc.) and other pretreatment process choices would be seen. Indeed, the critical component constraints have not been explored as a function of the mass fraction of major alass components, such as $\mathrm{SiO}_{2}$ or $\mathrm{Na}_{2} \mathrm{O}$, and, therefore, should be taken as current estimates and not as scientifically established values that cannot be challenged if they represent severe cunstraints on the rest of a tank waste remediation system. Therefore, there should be additional focus on HLW and LLW immobilization activities with emphasis on currentiy constraining limits, such as $\mathrm{P}_{2} \mathrm{O}_{5}, \mathrm{Na}_{2} \mathrm{O}$, etc.

The majority of LLW glass mass inventory (kilograms) is due primarily to the large amount of $\mathrm{Na}_{2} \mathrm{O}$ in the LLW oxide streams. Indeed, $\mathrm{Na}_{2} \mathrm{O}$ constitutes 74, 85, and $89 \%$ of the LLW oxide stream for the CLEAN Option, TRUEX-C, and Sludge Wash $C$ process strategies, respectively. Removing this sodium would substantially reduce the LLW glass mass inventory. One alternative for reducing $\mathrm{Na}_{2} \mathrm{O}$ in the LLW streams is a "Clean" Salt process (or Fractional Crystalization of $\mathrm{NaNO}_{3}$ ) (Herting 1992), where separation and production of "Clean" sodium salts (such as $\mathrm{NaNO}_{3}$ ) is achieved. If these "Clean" sodium salts are fed to an electrodialysis salt splitting process, $\mathrm{NaOH}$ and $\mathrm{HNO}_{3}$ could be produced for use in other processing. Alternatively, the "Clean" sodium salts could possibly be used in industrial applications or perhaps find disposal in lower cost drum storage instead of glass. This process will be evaluated in a later report.

The three process strategy cost estimates were based on the use of a central processing facility. Included in this report for one of the three process strategies, namely, the CLEAN Option, were cost estimates for the use of a combination of compact processing units (CPUS) for processing supernate and salt cake, along with a central facility for processing sludge. This report also considers both the primary LLW to salt grout and to glass-insulfur product options as part of the analyses along with their cost impact comparisons.

Costs associated with processing the primary waste stream and those associated with processing the tertiary nonprocess waste streams (i.e., wastes that did not result directly from splitting or partitioning of the main process streams) are calculated separately. In a previous report (Knutson et al. 1993) the costs associated with tertiary waste processing and disposal for the TRUEX-C and Sludge Wash $C$ process strategies were overestimated. This report includes better estimates of the tertiary waste processing costs for each of these process strategies. 
Summarizing the cost estimate results, the CLEAN Option without CPUs had total costs of $\$ 41.28$, which were approximately $27 \%$ greater than those for the TRUEX-C process strategy (\$32.5B), approximately $13 \%$ greater than those for the Sludge Wash C process strategy (\$36.4B), and approximately $32 \%$ greater than the CLEAN Option with CPUS (\$31.1B). Cost comparison ratios for the primary LLW to grout option were comparable to those for the primary LLW to glass option stated above.

Using CPUs reduced the total CLEAN Option process strategy cost by approximately $24 \%$. Similar cost reductions could potentially apply to the TRUEX-C and Sludge Wash C process strategies if similar CPU use is included. Comparing the primary LLW to glass option to the LLW to salt grout option shows that total primary LLW processing and disposal costs were essentially the same for both options. However, the costs associated with both the HLW and LLW glass products can vary dramatically, depending on which glass composition optimization models and associated composition constraints are used. For example, the $\mathrm{P}_{2} \mathrm{O}_{5}$ constraint was relaxed for all process strategy glass product results. If this constraint was included, cost increases of $\$ 1.6 \mathrm{~B}, \$ 17.5 \mathrm{~B}$, and \$0.085B would be seen for the TRUEX-C, Sludge Wash C, and CLEAN Option process strategies, respectively.

The tertiary waste processing costs were approximately $31,27,16$, and $30 \%$ of the total costs for the CLEAN Option without CPUs, TRUEX-C, siudge Wash C, and CLEAN Option with CPUs process strategies, respectively. These percentages essentially apply to either the primary LLW to grout or glass product options. The ratios of tertiary to primary costs were approximately $45,37,19$, and $44 \%$ for these same process strategies, respectively.

Note that all process strategy costs assume that a $0 \%$ heel (i.e., the unretrieved waste) was left in the waste tanks. Generally, if a $5 \%$ heel was assumed, the process strategy costs would be reduced by approximately 5 to $6 \%$ because of the reduced process stream flows (Knutson et al. 1993).

For the primary LLW glass product option, the CLEAN Option process strategy without CPUs had primary waste processing costs approximately $20 \%$ greater than those for the TRUEX-C process strategy, approximately $7 \%$ less than those for Sludge Wash $C$, and approximately $31 \%$ greater than those for the CLEAN Option with CPUs. However, the tertiary waste process costs for the CLEAN Option without CPUs were approximately $45 \%$ greater than those for the TRUEX-C process strategy, approximately $117 \%$ greater than those for the Sludge Wash C process strategy, and approximately $35 \%$ greater than for the CLEAN Option with CPUs.

To further show the basis of the cost differences between the process strategies the costs were combined into major process categories. The costs associated with tank waste retrieval and liquid waste partitioning are substantial (approximately \$8.1B) and are assumed to be the same for each process strategy. Costs associated with waste tank immobilization were estimated to be $50.43 \mathrm{~B}$, again, assumed to be the same for each process strategy. Again, the primary LLW treatment and disposal costs for the salt grout and glass-in-sulfur product options were essentially the same. 
The costs for separation of solids and liquids along with solids dissolution for the CLEAN Option process strategy without CPUs required approximately 1.3 and $\$ 0.99 \mathrm{~B}$ more than the TRUEX $-C$ and Sludge Wash $C$ process strategles, respectively. The costs for nuclide removal from alkal ine liquids for the CLEAN Option process strategy without CPUs required approximately 1.0 and $\$ 1.2 B$ more than the TRUEX-C and STudge Wash $C$ process strategies, respectively. The costs for nuclide removal from acidic liquids for the CLEAN Option process strategy without CPUs required approximately 2.6 and $\$ 4.2 B$ more than the TRUEX-C and Sludge Wash $C$ process strategies, respectively. The costs for removal and purification of bulk chemicals for the CLEAN Option process strategy without CPUs required approximately \$4.2B more than the TRUEX-C and Sludge Wash $C$ process strategies.

Combining the above four processing categories, the CLEAN Option process strategy without CPUs required approximately 9.1, 10.6, and \$6.7B more than the TRUEX-C, Sludge Wash C, and CLEAN Option with CPUs process strategies, respectively. The trade-off for these higher processing costs for the CLEAN Option process strategy is seen in the reduced $H L W$ and LLW processing and disposal costs as compared to the TRUEX-C and Sludge Wash $C$ process strategies. When the HLW and LLW processing and disposal costs are included with the four processing categories, the CLEAN Option process strategy without CPUs required approximately 4.6 and $\$ 6.7 B$ more than the TRUEX-C and CLEAN Option with CPUs process strategies, respectively, but $\$ 2.4 B$ less than the siudge Wash C process strategy for the primary LLW to glass option.

However, the increased processing in the CLEAN Option process strategy generated more tertiary waste than the other process strategies. Thus, the tertiary waste treatment and disposal costs for the CLEAN Option process strategy without CPUS required approximately $4.0,6.9$, and $\$ 3.3 \mathrm{~B}$ more than the TRUEX-C, Sludge Wash C, and CLEAN Option with CPUs process strategies, respectively. Consequently, reductions in the HLW and LLW treatment and disposal costs for the CLEAN Option process strategy compared to the other process strategies were offset by the increased cost of tertiary waste treatment and disposal.

In summary, the cost savings associated with CPU use was significant and should be incorporated into any process strategy. The large tertiary waste costs could be decreased significantly if process design is also focused on reducing the amount of tertiary waste produced. More glass composition constraint evaluation and experimentation on key waste oxide stream components could allow relaxing the current constraints, resulting in significantly reduced HLW glass canister and LLW glass vauit counts (i.e., higher waste oxide loadings in glass) and their associated costs. The trade-off for the higher processing costs for the CLEAN Option process strategy without CPUs is seen in the reduced $H L W$ and $L L W$ processing and disposal costs as compared to the TRUEX-C and Sludge Wash C process strategies. However, the increased processing in the CLEAN Option process strategy generated more tertiary waste and higher associated treatment and disposal costs, offsetting the reductions in the HLW and LLW treatment and disposal costs. 
The toxicity of the primary LLW stream is significantly affected by complexation in alkaline supernatant, especially after the organic destruction step. Therefore, greater understanding of this complexation is needed to assure successfui process definition and development.

The relative long-term offsite health hazard estimates suggest that this health hazard is comparable for all of the process strategies. The greatest long- and short-term onsite health hazard (controlled by excavation rather than by a drilling scenario) would be in the HLW glass product if the environment were not protected from it by geologic and geochemical barriers in and around the repository. The CLEAN Option long- and short-term onsite health hazards were more than an order of magnitude greater for the HLW glass product than the TRUEX-C and Sludge Wash C process strategies because of the greater HLW concentration. However, it is highly unlikely that any excavation would uncover a deep repository. The drilling scenario with different controlling components would probably produce more short-term hazard from the repository than the excavation scenario during the first 500 years.

Excluding the HLW glass product, the tank heel (i.e., the unretrieved tank waste) stream dominates both long- and short-term onsite health hazards. Otherwise, the only significant variations in the long- and short-term onsite health hazards between the pretreatment process strategies occurs for the primary LLW grout product and the uranium product. Assuming that the uranium product does not remain onsite but is added to the U.S. national stockpile, only the primary LLW grout product health hazards are compared. For the primary LLW grout product long-term onsite health hazard, the TRUEX-C process strategy was a factor of approximately 5 and 7 higher than for the CLEAN Option and Sludge Wash C process strategies, respectively. For the primary LLW grout product short-term onsite heaith hazard, the CLEAN Option process strategy was more than an order of magnitude lower than for the TRUEX-C and Sludge Wash C process strategies, respectively. While no estimate of the primary LLW glass product onsite health hazard is included, it is expected to be lower than that resulting from primary LLW grout because the glass represents a more stable waste form than grout.

Considering occupational exposure, the assumed plant designs for the CLEAN Option flowsheets result in about 3 and $24 \%$ more occupational gamma exposure, a factor of 2.3 and 12.1 more occupational alpha exposure, and a factor of 2.2 and 19.2 more occupational beta exposure, than the TRUEX-C and Sludge Wash C plant designs, respectively. These larger health hazards for the CLEAN Option result from its greater processing requirements.

Generally, more efficient removal of major exposure-causing components in the first process elements to contact the tank waste would lower the occupational exposure in all process strategies. For example, the CLEAN Option process strategy has alkaline-side cesium removal before strontium and technetium removal, thereby reducing gamma exposure in these subsequent removal operations. However, cesium removal follows after other removal operations in the acid-side processing. If the acid-side cesium removal could also be placed before the other acid-side removal operations, it would reduce the gamma exposure in those subsequent removal operations. 
Considering waste product classification, the tank waste feedstreams, the HLW glass stream destined for a HLA repository, and the tank heel stream are classified as Greater than Class C (GTCC). The primary LLW grout streams are LLW Class A for the CLEAN Option process strategy, while the TRUEX-C and Sludge Wash C process strategies were LLW Class B because of the strontium Iimits. Using uranium LLW classification Iimits proposed by Kocher a d Croff (Kocher and Croff 1988), the uranium product stream was al so GTCC for the CLEAN and Sludge Wash C process strategies, while the TRUEX-C process strategy uranium stream was LLW Class C. Higher decontamination factors (DFs) may be needed for the atmospheric releuse of the vitrification offgas where different limits apply than for solid LLW.

Finally, an assessment of the technical risk associated with each process strategy was made by developing a technical risk estimation method based on tabulated data on alternative process technology development levels and extents (Sutherland et al. 1993). These process development data were tabulated using the following categories:

- Maturity of particular process technologies--extent and scale of lesting with simple or realistic simulants (e.g., laboratory, bench, pilot-plant, full-scale, operating full-scale facility)

- Potential benefit of particular process technologies--activity level and timeliness of testing and development

- Current U.S. Department of Energy (DOE) funding level of particular process technologies--extent of funding and the associated advance of maturity.

To allow an assessment of the technical risk associated with each of the process strategies, weighting factors were assigned to the above process technology development levels and extents in each category. Using these weights, numerical estimates of technical risk factor indexes were calculated for particular process technologies in each development category. These process technology technical risk factor indexes were combined for processes in each process strategy to assess the technical risk of each strategy.

Summarizing the technical risk assessment results, the DOE current level of effort contributes considerably to the technical risk of many process technologies, representing the slow advance of the process maturity. These processes (e.g., solids dissolution, SREX, $\mathrm{NaOH} / \mathrm{HNO}_{3}$ recycle, technetium ion exchange [TCIX], and uranium separation associated with the TRUEX process) may require renewed or increased levels of effort to provide successful application of a tank waste pretreatment process strategy. The CLEAN Option process strategy without CPUs had a total combined category technical risk index that was a factor of approximately 2 and 10 times higher than the TRUEX-C and Sludge Wash C process strategies, respectively. The process average combined category technical risk index for the CLEAN Option process strategy without CPUs was nearly the same as the TRUEX-C process strategy and a factor of approximately 3 higher than for the Sludge Wash C process strategy. The CLEAN Option using the new and developing CPU concept should be considered to have more technical risk than the CLEAN Option based only on a central processing facility concept. 


\subsection{BACKGROUND}

This report describes work done in accordance with the fiscal year 1993/1994 milestone to provide an evaluation of the CLEAN Option processing strategy, for the combined tasks "Technology Evaluation and Process Definition --Unit Process Level" (RL332008) and "Technology Evaluation and Process Definition--Global Level" (RL411205). The objectives of the combined tasks are as follows:

- Determine and compare incentives for including pretreatment technologies in an integrated tank waste remediation system

- Determine the incentive in terms of the overall cost and operating schedule of the integrated system

- Consider the costs of retrieval and disposal

- Link technologies into an integrated system by modeling process flowsheet mass balances in a common modeling format.

Work done in the combined tasks is first reviewed with developers of the technologies being evaluated and second with a peer review group defined by Efficient Separations and Process Integrated Program (ESPIP) management. The results are provided to the ESPIP to help direct the program and communicate the benefits of the program to those who will use the separations technologies that are developed.

\subsection{PRIOR STUDY}

A prior study of pretreatment options conducted by EM-30 was issued by the Westinghouse Hanford Company (WHC) as the rank Waste Technical Options Report (Boomer et al. 1993). That report included a number of options, each defined in terms of a set of separations technologies integrated into a pretreatment system. The "SLUDGE WASH-C," "TRUEX-A" (later named Solvent Extraction Option [SOLEX] A or SOLEX-A), "TRUEX-C" (1 ater named SOLEX-C), and "CLEAN" options of that report have been considered in this task and are described below.

The so-called TRUEX or SOLEX options include the TRUEX technology as part of a subsystem used to separate radionuclides from dissolved solids. It is important to understand that the designation "TRUEX" for a process strategy involving many steps and processes does not imply that all consequences of the strategy result from inclusion of the TRUEX process per se, as is discussed further below.

In a previous letter report, Advanced Chemical Separations Study: TRUEX-A Versus Sludge Wash C (Knutson et al. 1992), the consequences of including solvent extraction (SE) and other separation technologies to separate cesium, technetium, TRUs, etc., from solids and supernatant in Hanford waste tanks were described. Two pretreatment system options, one including sludge dissolution ("TRUEX-A") and one for which solids are not treated other than by water washing ("SLUDGE WASH-C"), which had been proposed as part of the Technical Options Report, were assumed for our previous report. 
The predicted benefits of including the TRUEX technology depend on other technologies included in the flow sheet and on how they were integrated into the pretreatment system and on assumptions made about the performance of the TRUEX technology.

An alternate pretreatment option, "TRUEX-C," which includes complexant destruction, was a better choice for comparison to the "SLUDGE WASH-C" option, because both include complexant destruction and TCIX. This option and the SLUDGE WASH-C option were compared and the evaluation results were described in a letter report Advanced Chemical Separations Study: TRUEX Strategy Compared To sludge Wash Ion Exchange (Knutson et a1. 1993). This report included the development of an expanded set of performance measures to use in the comparison calculations, including unit process cost formulations, $\mathrm{HLW}$ glass composition optimization, revised tertiary waste estimate formulations, and several short-and long-term relative health risk indexes.

This report compares the "CLEAN" option processing strategy to the "TRUEX-C" and "SLUDGE WASH-C" processing strategies. A summary of these process strategies is given briefly below with more detailed discussion given in Appendix B.

\subsection{WASTE MINIMIZATION PHILOSOPHY}

In pretreatment approaches designed to greatly minimize the volume of $H L W$ glass, the sludge portion of the waste must be dissolved so that the radionuclide components (e.g., TRUs and ${ }^{90} \mathrm{Sr}$ ) can be separated from the inert components (e.g., iron, chromium, aluminum, and bismuth). The separations processes then give two acidic streams: (1) the radionuclides stream that is routed to $\mathrm{HLW}$ vitrification and (2) the inert component stream that is routed to $L L W$.

In the TRUEX-C and CLEAN Option flowsheets, it was assumed that both of these acidic streams were neutralized with sodium hydroxide: the radionuclide stream for interim storage in carbon steel tanks before vitrification and the inert component stream in preparation for salt grouting or vitrification. These neutralizations form sodium nitrate, which may lead to increased volumes of both the HLW and LLW forms. However, these volume increases result from the assumed course of action and are not a necessary consequence of treating the dissolved sludge solutions. An alternate approach of providing direct calcination of the HLW acidic waste stream was evaluated in the previous report (Knutson et al. 1993).

The CLEAN Option processing strategy focuses on achieving a high degree of dissolution of the sludge solids with further acid-stream and alkalinestream separatior. of key elements having large contributions to HLW glass to reduce the glass canister counts below a specified goal ( 1,000 canisters). Another goal is to produce a LLW stream that can be classified as NRC Class $A$. 


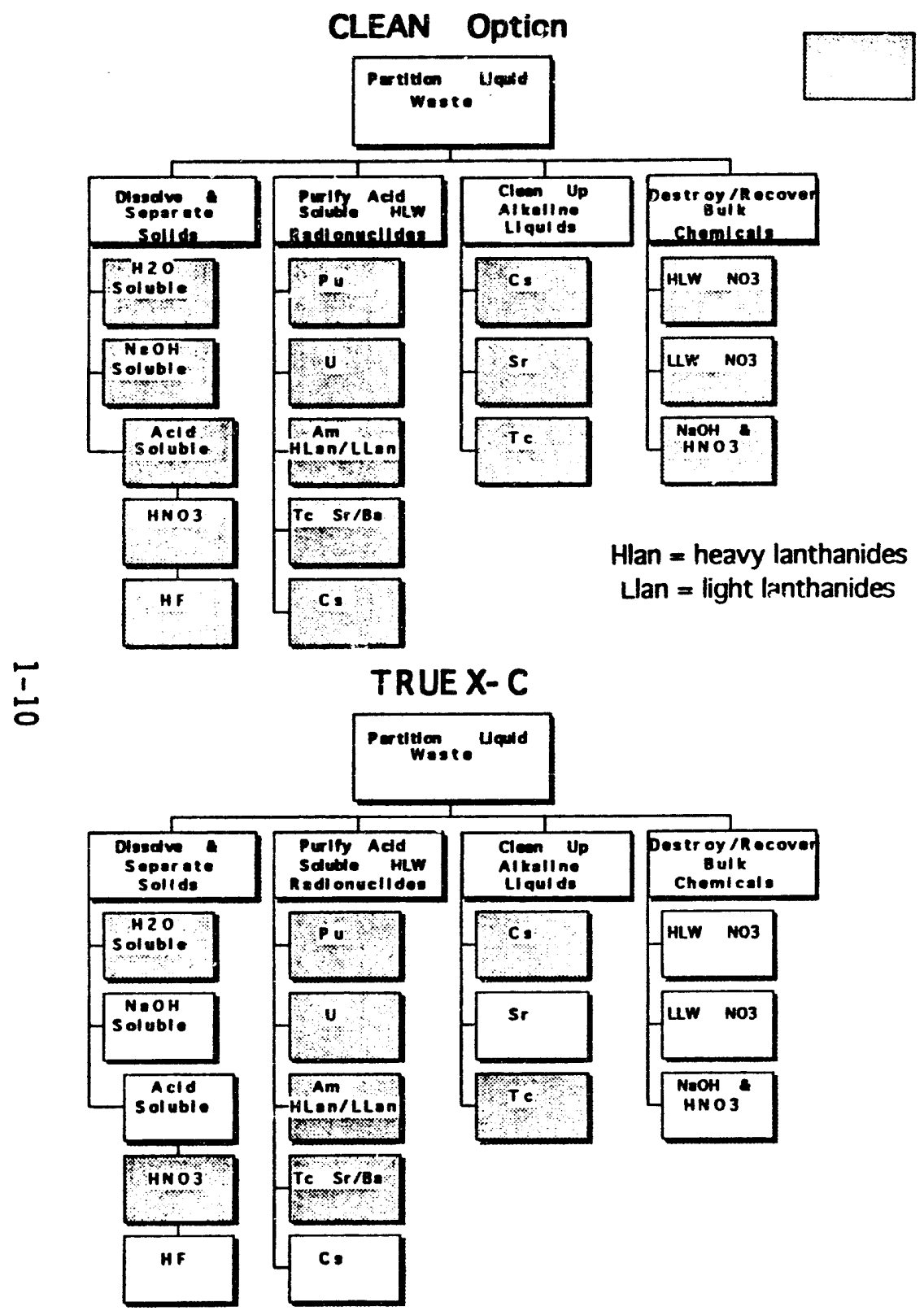

Process Included

In Strategy
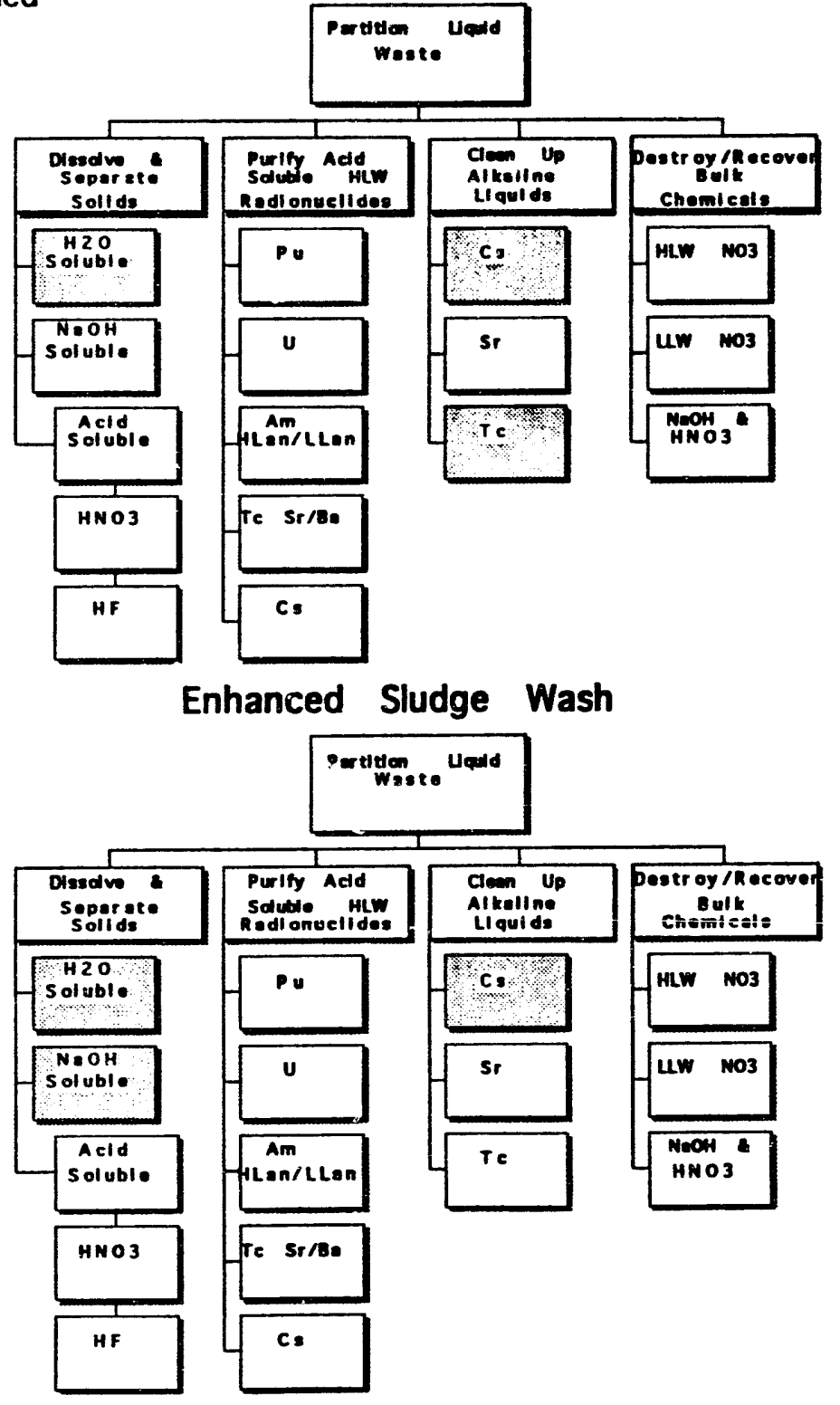
WHC-EP-D791

\subsection{TECHNICAL ISSUES}

\subsection{MASTE STREAM}

The contents of the waste tanks are known only approximately. The assumed composition of the waste stream to be treated can affect conclusions drawn about the relative worth of different pretreatment options. The Hanford tank waste stream radioactive and mass inventories assumed for this report were based on a review of available data as discussed in Appendix $J$. These tank waste streams are defined as though all waste (sludge, supernatant, and salt cake) from all tanks (single- and double-she11) were blended together and processed in a single campaign. This assumption allows the effects of pretreatment options and the various included technologies to be evaluated without forcing discussions about the large number of permutations among retrieval and blending options. Also, it is consistent with assuming that pretreatment will be done in a single, central processing facility. As EM-30 focuses on other specific approaches, such as retrieval and pretreatment of a portion of the waste in a tank or from some subset of the tanks, those approaches can be incorporated in the global systems model to evaluate separations technology in the particular context of each approach. Indeed, the CLEAN Option processing strategy considers the use of a combination of CPUs for processing tank supernate and salt cake and a central facility for processing tank sludge. Cost savings resulting from this approach are discussed in this report.

\subsection{SOLID-LIQUID SEPARATION}

In the sludge wash and SE models for solid/liquid separation (SLS) that had been assumed so far in work done as part of Hanford EM-30 activities (Boomer et a 1. 1993), a centrifugation step is included to provide a gross solid-liquid separation before filtration. A subsequent filtration step is currently modeled as a perfect solid-liquid separation. Although a perfect separation is not possible, we retained this model because it is simple. If a process were constructed that provided a very efficient separation of strontium from dissolved sludge, then most of the strontium passing to the LLW form could be the result of an imperfect SLS failing to prevent strontiumladen solids from escaping into the alkal ine liquid stream. Whenever efficient separation of strontium from dissolved sludge is proposed, the actual performance of solid-liquid separation steps will need to be considered.

The CLEAN Option flowsheet employs centrifuges for SLS in six different portions of the flowsheet: initial treatment of feed from waste tank retrieval, after the Organic Destruction processing, after caustic dissolution, after both acid dissolution steps, and after the chromium reduction step before caustic production and recycling. Each of these centrifuge operations and downstream filters is assumed to achieve $0 \%$ solid in clarified supernate. Appendix $B$ includes more detailed discussion regarding the SLS model assumptions. 


\subsection{SOLIDS DISSOLUTION}

The components present in undissolved solids can define a substantial fraction of the glass volume when advanced separations are used to treat the dissolved waste. Therefore, the actual solids phases in the sludge and their behavior during acid dissolution need to be understood to better determine the benefit of integrating an advanced separations technology into a pretreatment system. For example, the solids currently assumed to appear in a blended Hanford waste tank stream include an aluminosilicate mineral, termed "cancrinite," with the nominal composition $2 \mathrm{NaAlSiO}_{4}^{\circ} 0.52 \mathrm{NaNO}_{3} \cdot 0.68 \mathrm{H}_{2} \mathrm{O}$. For our current TRUEX model, this species is assumed to be insoluble in the siudge dissolution step, and, consequently, all of this material is routed directly to the HLW form. Similarly, the fate of sodium titanate, which is added as part of a sorption step and which is routed to the sludge dissolution step in the TRUEX and Sludge Wash C models, is not known, and it is assumed to be insoluble. These assumptions are discussed further below. Such assumed behavior during sludge dissolution leads to a prediction that part of the waste feed will be sent to the HLW form irrespective of subsequent processing steps, such as TRUEX SOLEX.

The CLEAN Option flowsheet contains three dissolution steps along with undissolved sludge recycle to minimize the amount of sludge routed to HLW. Caustic leaching, the first operation in the dissolution cycle, is critical for solubilizing cancrinite (50\% is assumed to dissolve with each pass) and nickel ferrocyanide $\left(\mathrm{Ni}_{2} \mathrm{Fe}(\mathrm{CN})_{6}\right)$ ( $90 \%$ is assumed to dissolve with each pass).

One CLEAN Option dissolution cycle involves sequential sludge processing through the following steps: (1) caustic leach, (2) nitric acid/oxalic acid dissolution, and (3) nitric acid/hydrofluoric acid dissolution, where SLS occurs after each of these steps. Solids remaining after the third step are returned to the caustic leach step as part of a 9-cycle batch processing scheme that achieves approximately $96 \%$ dissolution of cancrinite. Appendix $B$ includes more detailed discussion regarding the solids dissolution model assumptions.

\subsection{HIGH- AND LOW-LEVEL WASTE FORM MINIMIZATION}

A facility, such as the HWVP, will mix waste with glass formers whose composition is selected based on the composition of the waste to be vitrified. In this context it is assumed that large volumes of waste of nearly constant composition will be processed in a series of campaigns. An appropriate glass former composition will be selected to maximize the waste loading in the resulting glass; substantially different waste loadings can result. The three general types of constraints on waste loading are: (1) constraints on the properties of the homogeneous glass when in the melter (e.g., viscosity) or on the properties of the glass after cooling (e.g., durability); (2) constraints on heat loading (or possibly radiation levels); and (3) constraints on the solubility in molten glass of species, such as $\mathrm{SO}_{3}, \mathrm{Cr}_{2} \mathrm{O}_{3}$, and noble metal oxides that can render the molten glass inhomogeneous, or constraints on the loading of species, such as $\mathrm{P}_{2} \mathrm{O}_{5}$ that can inhibit the vitrification rate in some melter designs. 
Allowable mass fractions in glass of certain critical components have been estimated for the HWVP project as follows: $\mathrm{Cr}_{2} \mathrm{O}_{3}<0.005 ; \mathrm{F}-<0.017 ; \mathrm{P}_{2} \mathrm{O}_{5}$ $<0.01 ; \mathrm{SO}_{3}<0.05 ; \mathrm{Rh}_{2} \mathrm{O}_{3}, \mathrm{PdO}$, and $\mathrm{Ru}_{2} \mathrm{O}_{3}<0.025$. Of these, the constraint on phosphate is probably the most disadvantageous, because phosphate is abundant in the waste. The critical component constraints have not been explored as a function of the mass fraction of major glass components, such as $\mathrm{SiO}_{2}$ or $\mathrm{Na}_{2} \mathrm{O}$, and, therefore, should be taken as current estimates and not as scientificaliy established vaiues that cannot be challenged if they represent severe constraints on the rest of a tank waste remediation system.

The current glass optimization models used in this report are based on analyses and experimental data relative to the anticipated HLW stream compositions as described in Appendix G. Recent TPA changes have also designated glass as the final waste product form for the LLW stream. The LLW glass is expected to be stored as gravel-sized glass chunks (cullet) within a sulfur cement matrix in underground vaults. However, no extensive analyses or experimental glass formulation data relative to expected LLW stream compositions are available. Therefore, this report uses the HLW glass composition specifications and associated optimization models for the LLW glass streams also. It is expected that the LLW glass will have different glass composition and property limits that will be defined in future work.

The majority of LLW glass mass inventory (kilograms) is due primarily to the large amount of $\mathrm{Na}_{2} \mathrm{O}$ in the LLW oxide streams. Indeed, $\mathrm{Na}_{2} \mathrm{O}$ constitutes 74,85 , and 89 wt\% of the LLW oxide stream for the CLEAN Option, TRUEX-C, and Sludge Wash C process strategies, respectively. Removing this sodium would substantially reduce the LLW glass mass inventory. One alternative for reducing $\mathrm{Na}_{2} \mathrm{O}$ in the $L L W$ streams is a "Clean" Salt process (or Fractional Crystallization of $\mathrm{NaNO}_{3}$ ) (Herting 1992), where separation and production of "Clean" sodium salts (such as $\mathrm{NaNO}_{3}$ ), is achieved. If these "Clean" sodium salts are fed to an electrodialysis salt splitting process, $\mathrm{NaOH}$ and $\mathrm{HNO}_{3}$ could be produced for use in other processing. Alternatively, the "Clean" sodium salts could possibly be used in industrial applications or perhaps find disposal in lower cost drum storage instead of glass. This process will be evaluated in a later report.

\subsection{GENERATION OF SECONDARY AND TERTIARY MASTE}

Whenever separation processes are added to any given separations scheme, further addition of process chemicals and associated generation of waste will occur. We use the term "secondary waste" to describe waste resulting directly from the routing of process chemicals to an effluent stream, to distinguish it from "primary waste," which is the waste retrieved from HLW tanks. Secondary waste can be minimized by recycle and careful selection and integration of the separation processes. The predicted generation of secondary waste is computed as part of the mass balance model approach reported here.

We use the term "tertiary waste" to describe various nonprocess waste streams or wastes that did not result directly from splitting or partitioning of the main process streams (described in Appendix E). This waste includes trash and incidental capture of liquid and solid emissions. For example, included as noncombustible materials (e.g., glass, metal, and soil) are items, 
such as contaminated or irradiated equipment, waste and spill site soil, silver-çated packing material, smoke detectors, and basin sediment sources (e.g., $\left.{ }^{57} \mathrm{Co},{ }^{139} \mathrm{Cs},{ }^{239} \mathrm{Pu},{ }^{235} \mathrm{U}\right)$. Included as combust ible materials (e.g., absorbents, cellulose, plastic, rubber, liquids) are items such as toxic cleanup material, laboratory waste, filter cartridges, deionizer resins, contaminated clothing, lubricants, solvent waste. Included as aqueous wastes are items such as scintillation solution, high- and low-activity waste, decontamination solutions. Because data for cell air and offgas rates were not available, the volume of filters (e.g., high-efficiency particulate air filters and in-tank precipitation filters) resulting from offgas treatment was substituted.

The generation of tertiary waste is estimated and included in the mass balances. However, these estimates are necessarily rough, being based on the generation of similar wastes during operation of large chemical plants at Javannah River, Hanford, and other DOE sites as described in Appendix E. The estimates are probably conservative relative to operation of future facilities, assuming waste minimization will be a design and operating objective. The mass balances also include estimates of the chemical adds for tertiary waste treatment. 
WHC-EP-0791

\subsection{PROCESS DESCRIPTION AND IMPORTANT ASSUMPTIONS}

\subsection{WASTE STREAM}

The masses, concentrations, and compositions of certain process streams are described in Appendix B. The component names have been adopted from the Technical Options Report and often do not describe the expected chemical form of the elements or compounds. For example, silicon will exist as $\mathrm{SiO}_{2}$ or silicate minerals in the solid phase or as the silicate anion or silicic acid in solution but is described in the data set as "Si+4," which does not exist in the materials dealt with during tank waste processing. Similarly, "TOC" represents total organic carbon and is arbitrarily assigned a molecular weight equal to the atomic weight of carbon. Such component names describe the occurrence of an element where often the actual chemical and physical form are unknown. All that is required to perform the calculations supporting this report is to maintain a mass balance, which is often achieved by using "stand in" component formulae, such as "Si+4" and "TOC."

Table Bl of Appendix B lists the thirty most abundant species, by mass, of the species defined for the waste feed streams representing the Hanford single- and double-shell tanks (SSTs and DSTs), respectively. The waste is primarily a $\mathrm{NaNO}_{3}-\mathrm{NaOH}-\mathrm{NaNO}_{2}-\mathrm{Na}_{3} \mathrm{PO}_{4}-\mathrm{Na}_{2} \mathrm{CO}_{3}-\mathrm{Na}_{2} \mathrm{SO}_{4}$ mixture with substantial aluminum in both the supernatant (presumably as the aluminate anion) and precipitated (presumably as an aluminum hydroxide or an aluminosilicate). In the supernatant, sodium is the dominant cation by far, with a mole concentration more than 50 times greater than the next most abundant cation, potassium. The assumed solid phase includes primarily the sodium aluminosilicate "cancrinite," nickel ferrocyanide, and an aluminum-uraniumzirconium-sodium-iron-cesium cation mixture balanced by the principal anions phosphate, hydroxide, nitrate, and carbonate.

\subsubsection{Supernatant}

Table B2 of Appendix B describes important characteristics and assumptions pertaining to the supernatant. This stream is assumed to be subjected to supercritical water oxidation (SCWO) to destroy most of the organic complexants that are assumed to hold some otherwise insoluble species in solution. The SCWO step is assumed to destroy $99 \%$ of the complexant and to, thereby, cause $99 \%$ of certain species in the supernatant to precipitate to form corresponding solid species. Those species assumed to be affected are apparent in Table B2. Some aluminum is also assumed to be precipitated (assumed 30\%).

Because it is assumed that $1 \%$ of complexants are not destroyed by SCWO, it would be consistent to propose that the remaining species of precipitated elements are in a complexed form and possibly not available for chemical separations, depending on the power of the separating agent. This calls into question the efficacy of the sodium titanate sorption step in the TRUEX-C and Sludge Wash C processing strategies. 


\subsubsection{Sludge}

Tables B3 and B13 of Appendix B describe characteristics and assumptions pertaining to the solid portion of the waste stream (sludge), including acid dissolution of the solids for the CLEAN Option and TRUEX-C process strategies, respectively. The assumed solids in the waste feed are essentially an al uminum-sodium-iron-zirconium-uranium mixture of hydroxides, phosphates, carbonates, and nitrates.

The CLEAN Option process strategy uses three dissolution steps along with undissolved sludge recycle to minimize the amount of sludge routed to $\mathrm{HLW}$. One dissolution cycle involves sequential sludge processing involving caustic leach, nitric acid/oxalic acid dissolution, and nitric acid/hydrofluoric acid dissolution, where SLS occurs after each of these steps. Table B3 shows the extents of dissolution assumed by component for each dissolution process. Note that the caustic leach step is assumed to significantly solubilize cancrinite (50\% dissolution with each pass) and nickel ferrocyanide $\left(\mathrm{Ni}_{2} \mathrm{Fe}(\mathrm{CN})_{6}\right)$ (90\% dissolution with each pass).

In the CLEAN Option ASPEN PLUS ${ }^{1}$ model, 95\% of the undissolved solids from the second acid dissolution step are returned for caustic leaching to begin another dissolution cycle, with the remaining $5 \%$ solids diverted to HLW treatment. Recycling 95\% of the undissolved solids is necessary to achieve the same total dissolution of cancrinite $(96 \%)$ as is attained in the proposed 9-cycle batch processing scheme, which cannot be modeled in ASPEN PLUS. Although recycling $95 \%$ of the undissolved solids slightly overpredicts (relative to the 9-cycle batch scheme) the amount of solid components that accompany cancrinite to HLW treatment, the impact of this overprediction on total HLW volume is not significant because cancrinite is the dominant constituent of the undissoived solids.

For the TRUEX-C process strategy the fraction of these solids assumed to dissolve is adopted from the Technical Options Report (Boomer et al. 1993) and is shown in Table B13 as taken from the draft letter report (Knutson et a1. 1993). Even though a slightly different feedstream was used in the previous report the results shown in Table B13 are still indicative of the dissolution performance in the TRUEX-C process strategy. None of the cancrinite ("CANCR") or nickel ferrocyanide $\left(\mathrm{Ni}_{2} \mathrm{Fe}(\mathrm{CN})_{6}\right)$ is assumed to dissolve. The fraction assumed to be dissolved for alf other species is close to $90 \%$ and could, as well, be taken as exactly $90 \%$ for the purposes of this report. The undissolved solids that would result are relatively enhanced in cancrinite and nickel ferrocyanide because of the assumed dissolution characteristics, as shown in the last columns in Table B13. The actual undissolved solids also contain contributions from process recycle streams; in particular, sodium titanate is routed from alkaline stream processing and is assumed, for the purpose of this study, not to dissolve in the acid dissolution step.

${ }^{1}$ ASPEN PLUS is a trademark of Aspen Technology, Inc. 


\subsection{HIEH- ND LOW-LEVEL MASTE STREAMS}

All three of the process strategies produce waste fractions that are assumed to be recombined into HLW and LLW mixtures. Only the CLEAN Option and TRUEX-C process strategies produce a purified uranium stream for shipment to national stockpiles. Also, any bulk chemical recovery is used mostly for recycle within the process flowsheet. The following discussion considers the HLW and LLW streams and their composition related to the objectives of each process strategy. An alternative to mixing the waste fractions is considered following that discussion.

Tables 3-1 and 3-2 provide the HLW oxide stream content and radionuclide fraction reporting to LLW for each of the process strategies, respectively. Figures 3-1 and 3-2 show this same data. Note that the CLEAN Option process strategy has a concentrated HLW oxide stream that is high in strontium, aluminum, silicon, sodium, barium, and thorium oxides. The TRUEX-C process strategy has a HLG oxide stream dominated by aluminum, silicon, sodium, iron, uranium, nickel, cerium, and phosphorus. The Sludge Wash C process strategy has a HLW oxide stream dominated by aluminum, silicon, sodium, iron, uranium, zirconium, and phosphorus. If the phosphorus upper constraint limit of 1 wt\% for the HLW glass composition cannot be relaxed, as assumed in these analyses, the phosphorus would have a major impact on the magnitude of the final HLW glass product for the TRUEX-C and Sludge Wash $C$ process strategies (Section 4.3).

Considering the radionuclide fraction sent to the LLW stream, the assumed CLEAN Option process strategy was effective at reducing the ${ }^{90} \mathrm{Sr}$ reporting to LLW to a level that allows the LLW to achieve an NRC Class A classification. The amount of $\mathrm{LLW}{ }^{90} \mathrm{Sr}$ assumed in the other process strategies only allowed them to attain an NRC Class $B$ classification. The other major radionuclides that required significant removal to meet $N$ RC Class $A$ limits were ${ }^{137} \mathrm{Cs},{ }^{239} \mathrm{Pu}$, and ${ }^{241} \mathrm{Am}$. Nickel-63 removal was not added to the CLEAN Option flowsheet but may be needed for processing specific tanks without sufficient blending with other waste streams.

The ${ }^{99} \mathrm{Tc},{ }^{129} \mathrm{I}$, and ${ }^{238} \mathrm{U}$ can be prime contributors to the long-term radioactivity-related risk even at concentrations allowed by the LLW Class $A$ limits. Thus, the CLEAN Option flowsheet included steps to reduce these radionuclide concentrations in the LLW fraction. As shown in Table 3-2 and Figure $3-2$, the CLEAN Option has reduced the amount of ${ }^{99} \mathrm{TC}$ and ${ }^{238} \mathrm{U}$ reporting to the LLW. Only the ${ }^{238} \mathrm{U}$ in the Sludge Wash C process strategy has a lower value. The separated uranium undergoes purification in the CLEAN Option and TRUEX-C process strategies so that it can be added to the existing uranium stockpiles.

The CLEAN Option flowsheet also includes separations to send the ${ }^{232} \mathrm{Th}$ and ${ }^{159} \mathrm{Sm}$ to the HLW fraction. Thorium separation is included because it is likely to occur with plutonium while the samarium separation can occur with the americium separation from the bulk chemical lanthanides. 
Although not evaluated in this report, the following observations provide possible disposal alternatives to mixing separated waste fractions into HLW and LLW mixtures. If the waste fractions produced by a processing strategy, such as the CLEAN Option, are not recombined into the two HLW and LLW mixtures, HLW repository may not be technically necessary for anything but spent fuel.

- The concentrated high radiation, high-heat ${ }^{137} \mathrm{Cs}$ fraction can be placed in underground storage sites stable enough for the ${ }^{137} \mathrm{Cs}$ to decay to LLW in 300 to 500 years or in safe surface storage if temporary uses, such as food irradiation, are attractive enough to promote temporary withdrawal from storage.

- The concentrated high-heat ${ }^{90} \mathrm{Sr}$ fraction can be stored for 300 to 500 years similarly to the ${ }^{137}$ Cs but without gamma shielding, and some parts of it may still find temporary use in radioisotopic power generators.

- The thousands of tons of separated and purified uranium can be added to the nation's stockpile with little penalty (already assumed in the report).

- The vast bulk of the tank waste (mainly $\mathrm{NaNO}_{3}$ or $\mathrm{NaOH}$ if denitrated) can be decontaminated from ${ }^{3}$ Cs to drinking water levels of radioactivity and reintroduced as a chemical feedstock into chemical plants producing nonfood products.

- Other groundwater insoluble, long-lived radioactivities from fission products $\left(e . g .,{ }^{93} \mathrm{Zr}\right.$ and $\left.{ }^{159} \mathrm{Sm}\right)$ and neutron activation products (e.g., ${ }^{63} \mathrm{Ni}$ ) should also be acceptable to an alpha waste repository because they have little radiation field and little heat generation.

- Each of the soluble long-lived fractions $\left({ }^{14} \mathrm{C}\right.$ with $a 5,000$-year half-life, ${ }^{90}$ Tc with a 243,000-year half-life, and ${ }^{129} I$ with a 16-million-year half-life) have their own peculiar disposal problems, which require further study. They are not well suited to disposal even in a HLW repository, and they will eventually return to the environment before they have decayed much. They have such low specific activities even when concentrated into small volumes that the level of danger to mankind and the environment is questionable. Transmutation, isotopic dilution, dispersal in the ocean, and even containment on the earth's moon where there can be no water or air pollution have been suggested.

If a CLEAN Option solution to the Hanford tank waste problem proves workable and the above alternatives are included in the processing strategy, its technology and disposal options could be attached to nuclear fuel reprocessing plants worldwide. This could cancel the need for HLW repositories in countries that do not choose spent fuel as their nuclear waste. 
WHC-EP-0791

\subsection{SOLVENT EXTRACTION}

The CLEAN Option processing strategy makes use of three SE processes: (1) a tributyl phosphate (TBP) SE step to remove tetravalent and hexavalent actinides (pentavalent neptunium is converted to the hexavalent state for removal also), (2) a $\mathrm{N}$-difsobutylcarbamoylmethylphosphine oxide (CMPO) SE process for removal of trivalent actinides (trivalent 1 anthanides are also removed), and (3) a crown ether (CE) SE process for removal of strontium and technetium. These SE processes are discussed briefly below and in more detail in Appendix $B$.

The solvent composition for the first SE process is the same, $30 \%$ TBP in hydrocarbon diluent, as is usually used in the well-known plutonium-uranium extraction process. In the first extraction contactor of this TBP cycle, uranium, plutonium, neptunium, and thorium are extracted into the TBP organic phase. The extracted plutonium, neptunium, and thorium are selectively stripped back into an aqueous phase in a second contactor (to be routed to HLW glass for disposal), and the uranium is stripped in a third contactor.

The raffinate from the first TBP cycle is routed to a CMPO SE process to remove trivalent lanthanides, americium, and bismuth. The solvent used is $0.2 M$ CMPO + 1.4M TBF in hydrocarbon diluent, a typical solvent composition proposed for the TRUEX process. In this extraction step, the lanthanides, americium, and bismuth in the dissolved sludge solution are extracted by the solvent. The raffinate from this extraction retains essentially all the cesium, strontium, and technetium and undergoes further processing for removal of these components as described in Appendix B. Americium and trivalent actinides are stripped from the CMPO extract into dilute nitric acid in the second contactor. Bismuth is retained in the extract and is removed from the solvent in a sodium carbonate wash step with added EDTA complexant to prevent bismuth precipitation.

The raffinate from the first CMPO cycle, which contains all of the components of the dissolved sludge solution, except the actinides, the lanthanides, and the bismuth, is fed to a CE SE step in which strontium and technetium are removed.

The assumed performance of these various SE processes is described in Appendix B.

\subsection{SOLVENT MASTE MINIMIZATION}

Inevitably, some fraction of solvent used in extraction steps will dissoive and entrain in the aqueous phase. Aqueous streams exiting the overall extraction process (i.e., extraction, stripping, and washing) are evaporated to concentrate the streams. However, evaporation al so serves to distill the solvent, which then condenses in high enough concentration to be immiscible in the condensate.

In the CLEAN Option flowsineet the TBP and CMPO solvent losses are assumed to be minimal (0.001\%) because downstream evaporators recover and recycle any solvent carried away in the aqueous outlet streams of the extraction,

stripping, and solvent wash steps. 
In the assumed TRUEX-C flowsheet, adopted from the Technical Options Report (Boomer et al. 1993), the solvent is separated by decanting and then recycled to the extraction process to minimize the generation of tertiary waste.

\subsection{SODIUN HYDROXIDE ND NITRIC ACID RECYCLE}

A major feature of the CLEAN Option process strategy is the recycle of process chemicals (especially sodium hydroxide) to minimize the "growth" of waste during processing. The CLEAN Option flowsheet includes a calcination process, based on work under way in EM-55's Underground Storage Tank Integrated Demonstration, primarily as a means of destroying nitrates, nitrites, and organic complexants so that the LLW waste form will be less toxic and will have greater stability. Calcination also allows the recovery of sodium hydroxide. Sodium hydroxide results from the calcination step because of the presence of sodium in the waste being treated; and, consequently, when the calcine product is redissolved, it is alkaine and suitable for tank storage if desired. All of the sodium hydroxide will ultimately be disposed of in the LLW disposal form, but, during process operations, a portion can be reused.

Because the tank wastes contain large quantities of sodium and recycled sodium hydroxide is used to neutralize excess acids, recycling of nitric acid is not needed to minimize the increase in LLW disposal volume. However, the CLEAN Option flowsheet also includes nitric acid recovery primarily to minimize the release of nitrate destruction products to the atmosphere, to decrease the processing load on calcination and NOx destruction operations, and to provide flowsheet flexibility for other low-sodium waste streams. The bulk of the nitric acid recovery occurs from recovery of the NO and NOx that are evolved when the concentrated acidic LLW and HLW waste solutions are denitrated by reaction with sucrose. Other nitric acid recovery occurs during evaporation steps.

The TRUEX-C process strategy assumes that nitric acid added to dissolve solids and to keep various species in solution during separation steps is destroyed rather than neutralized by the addition of sodium hydroxide. The impact of adding a HLW calcination step to avoid sodium hydroxide addition for neutralizing acidic HLW was evaluated in the previous report

(Knutson et al. 1993). 
Figure 3-1. High-Level Waste Oxide Stream Content for Flowsheet Options.

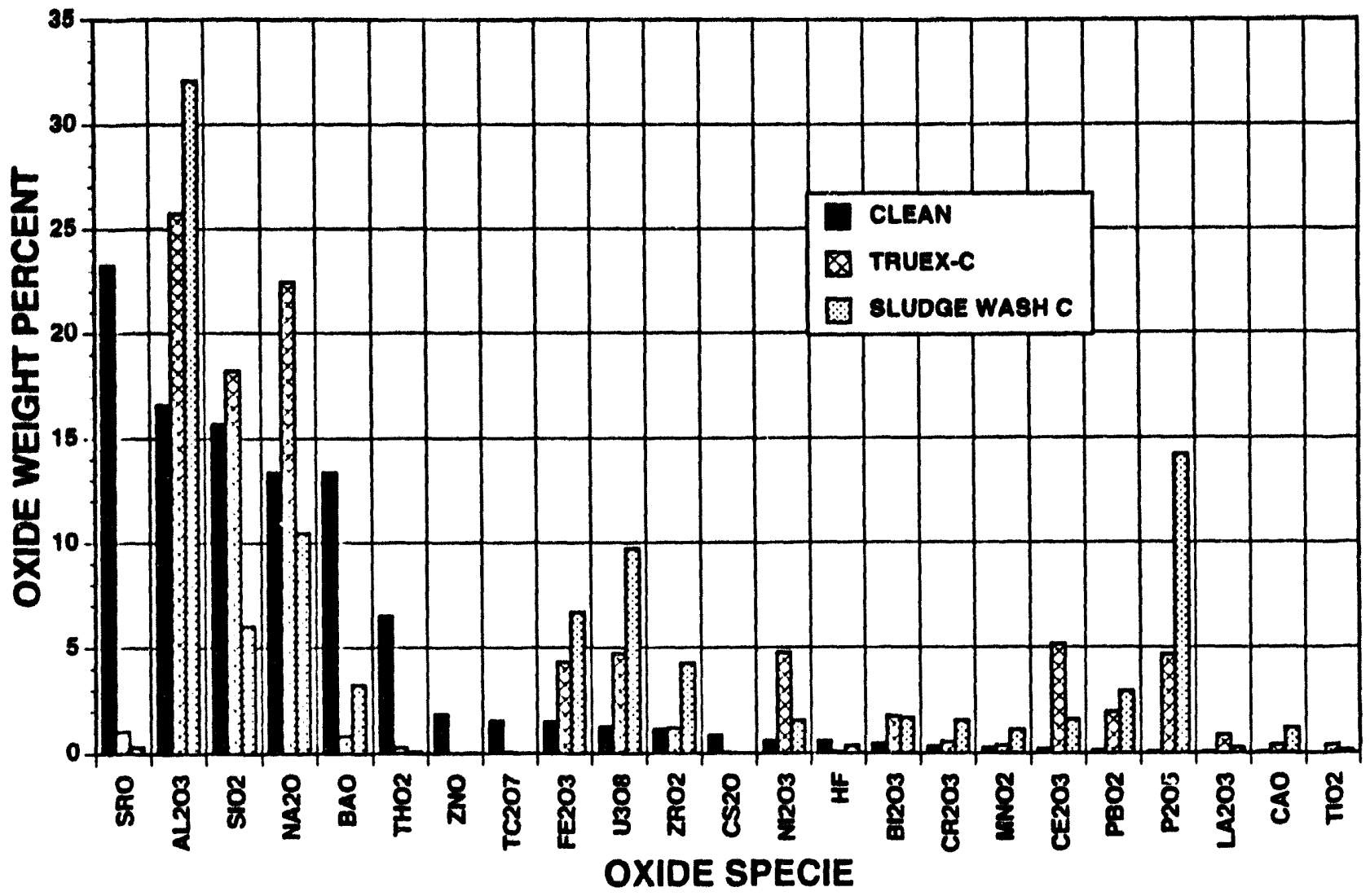

Figure 3-2. Radionuclide Fraction to Low-Level Waste.

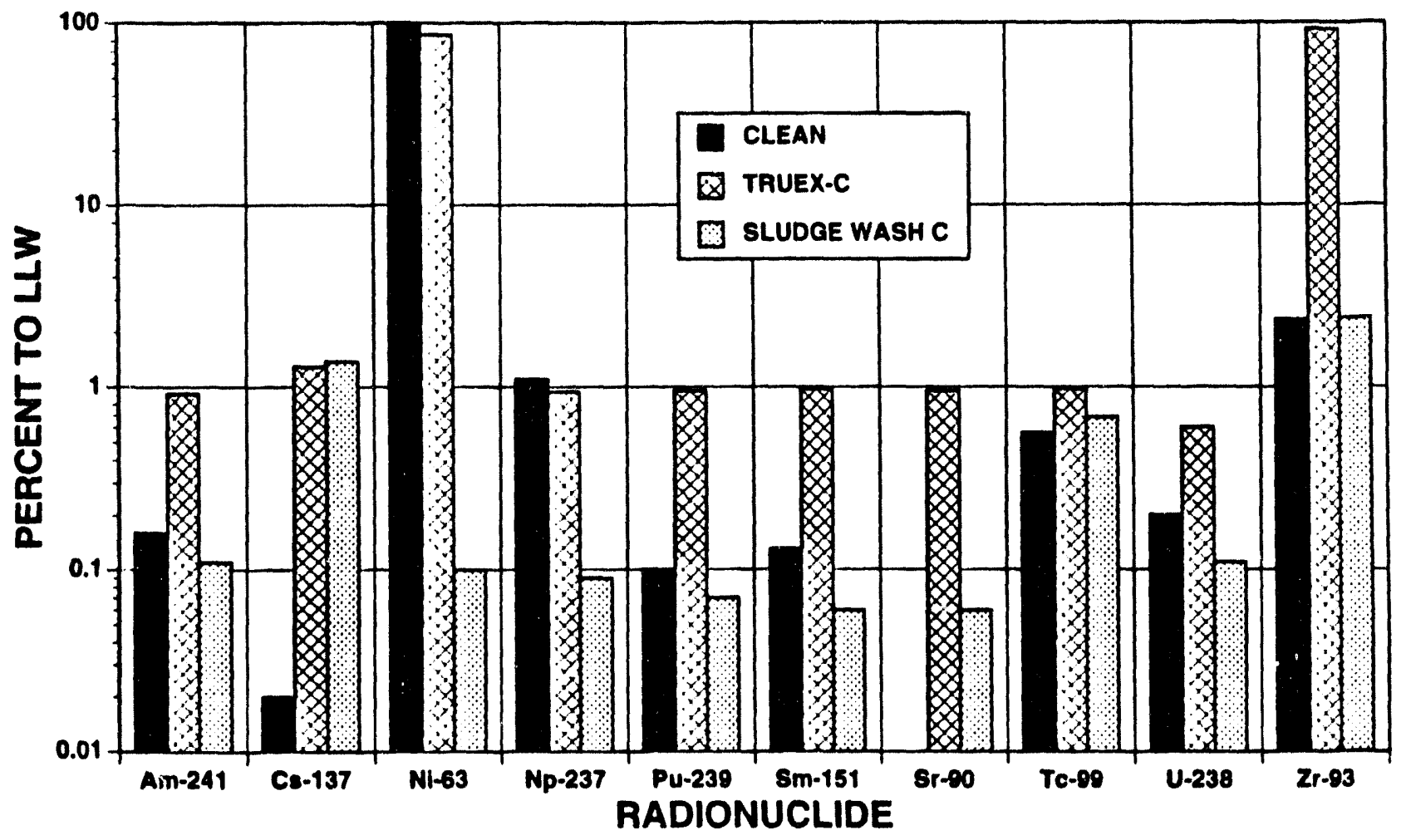


Table 3-1. High-Level Waste Oxide Stream Content for Flowsheet Options.

\begin{tabular}{|l|c|c|c|}
\hline Oxide & $\begin{array}{c}\text { CLEAN } \\
\text { (wt\%) }\end{array}$ & $\begin{array}{c}\text { TRUEX } \\
\text { (wt\%) }\end{array}$ & $\begin{array}{c}\text { S1udge Wash C } \\
\text { (wt\%) }\end{array}$ \\
\hline SRO & 23.28 & 1.04 & 0.32 \\
AL203 & 16.61 & 25.72 & 32.08 \\
S102 & 15.68 & 18.24 & 6.03 \\
NA20 & 13.35 & 22.43 & 10.47 \\
BA0 & 13.35 & 0.82 & 3.21 \\
THO2 & 6.50 & 0.29 & 0.09 \\
ZNO & 1.83 & 0.01 & 0.02 \\
TC207 & 1.53 & 0.07 & 0.02 \\
FE203 & 1.48 & 4.32 & 6.68 \\
U308 & 1.23 & 4.72 & 9.71 \\
ZR02 & 1.13 & 1.18 & 4.25 \\
CS20 & 0.83 & 0.04 & 0.01 \\
NI203 & 0.58 & 4.76 & 1.52 \\
HF & 0.58 & 0.08 & 0.35 \\
BI203 & 0.44 & 1.73 & 1.66 \\
CR203 & 0.30 & 0.52 & 1.53 \\
MNO2 & 0.23 & 0.37 & 1.09 \\
CE203 & 0.19 & 5.17 & 1.58 \\
PBO2 & 0.12 & 1.93 & 2.89 \\
P205 & 0.06 & 4.67 & 14.19 \\
LA203 & 0.05 & 0.85 & 0.26 \\
CAO & 0.05 & 0.39 & 1.17 \\
T102 & 0.00 & 0.38 & 0.12 \\
\hline Tota1 & 99.41 & 99.74 & 99.25 \\
\hline TOt & & & \\
\hline
\end{tabular}

TRUEX = transuranic extraction.

Table 3-2. Radionuclide Fraction to Low-Level Waste.

\begin{tabular}{|c|c|c|c|}
\hline Radionuclide & $\begin{array}{c}\text { CLEAN } \\
\text { (\% to LLW) }\end{array}$ & $\begin{array}{c}\text { TRUEX } \\
(\% \text { to LLW) }\end{array}$ & $\begin{array}{c}\text { Siudge Wash C } \\
\text { (\% to LLW) }\end{array}$ \\
\hline${ }^{249} \mathrm{Am}$ & 0.16 & 0.92 & 0.11 \\
${ }^{137} \mathrm{Cs}$ & 0.02 & 1.29 & 1.38 \\
${ }^{63} \mathrm{Ni}$ & 100.00 & 86.29 & 0.10 \\
${ }^{237} \mathrm{~Np}$ & 1.10 & 0.94 & 0.09 \\
${ }^{239} \mathrm{Pu}$ & 0.10 & 0.95 & 0.07 \\
${ }^{151} \mathrm{Sm}$ & 0.13 & 0.97 & 0.06 \\
${ }^{90} \mathrm{Sr}$ & 0.01 & 0.95 & 0.06 \\
${ }^{90} \mathrm{TC}$ & 0.56 & 0.97 & 0.68 \\
${ }^{238} \mathrm{O}$ & 0.20 & 0.60 & 0.11 \\
${ }^{93} \mathrm{Zr}$ & 2.35 & 91.74 & 2.40 \\
\hline
\end{tabular}

$L L W$ - low-level waste.

TRUEX = transuranic extraction. 


\subsection{RESULTS}

The sections below present summaries of the analysis results. More detailed discussion of these results along with summary figures and tables will be found in the appropriate appendices. The ASPEN PLUS software is described in Appendix A. Appendices B, C, and D give broader descriptions of the detailed, higher, and global-level ASPEN PLUS models, respectively. Appendix $E$ describes the tertiary nonprocess waste generation models. Appendix $F$ describes the cost estimation models. Appendix $G$ describes the glass composition optimization models. Appendix $H$ describes the global model mass balance results with summary tables showing the waste product inventories. Appendix I describes the relative health hazard estimates. Finally, Appendix $J$ describes the tank waste radiological and mass inventory estimates.

\subsection{TERTIARY WASTE GENERATION}

Figure 4-1 shows the tertiary waste generation and treatment inventory for each process strategy in terms of its main components (noncombustible materials, solids treatment adds, combustible materials, aqueous wastes, evaporator and IX adds, and process offgas representing filter volume) as compared to the primary waste feedstream. This data is summarized from results described in Appendix $E$.

As shown in Figure 4-1, the tertiary waste flows were larger than the primary waste feedstream flow; the total tertiary to primary waste flow ratios were $4.4,2.9,1.8$, and 3.1 for the CLEAN Option without CPUS, TRUEX-C, sludge Wash $C$, and CLEAN Option with CPUs process strategies, respectively. In terms of individual processes, the cesium, strontium, and TCIX processes produced the most tertiary waste because of their high throughput and high SE cycle equivalency assignment (Appendix $E$ ).

It should be clear from the data that the tertiary waste generation is substantial and cannot be ignored when evaluating process technologies. The CLEAN Option with and without CPUs generates the largest amount of tertiary waste owing to its intensive chemical processing strategy. Indeed, the cost for processing this tertiary waste is very substantial. The large tertiary waste costs could be decreased significantly if process design is also focused on reducing the amount of tertiary waste produced.

\subsection{CoSTS}

Table 4-1 shows the cost estimates for each processing strategy as summarized from results described in Appendix F. Appendix F discusses the development of the cost estimation models and has tables and figures summarizing the detailed capital and expense costs by process. Figure 4-2 shows some of the cost results. 
As shown in Table 4-1 and Figure 4-2, using CPUs reduced the total CLEAN Option process strategy cost by approximately $24 \%$. Thus, the cost savings associated with CPU use was significant and should be incorporated into any process strategy. Similar cost reductions could potentially apply to the TRUEX-C and Sludge Wash C process strategies if similar CPU use is included. Comparing the primary LLW to glass option to the LLW to salt grout option shows that total primary LLW processing and disposal costs were essentially the same for both options.

The costs associated with the HLW and LLW glass products can vary cramatically, depending on which glass optimization models and associated constraints are used (Appendix G). For example, the $\mathrm{P}_{2} \mathrm{O}_{5}$ constraint was relaxed for all process strategy glass product results. If this constraint was included, cost increases of $\$ 1.6 \mathrm{~B}, \$ 17.5 \mathrm{~B}$, and $\$ 0.085 \mathrm{~B}$ would be seen for the TRUEX-C, STudge Wash C, and CLEAN Option process strategies, respectively. Thus, more glass composition constraint evaluation and experimentation on key waste oxide stream components could allow relaxing the current constraints, resulting in significantly reduced HLW glass

canister and LLW glass vault counts (i.e., higher waste oxide loadings in glass [Appendix G]) and their associated costs.

As shown in Table 4-1 and Figure 4-2, the tertiary waste processing costs were approximately $31,27,16$, and $30 \%$ of the total costs for the CLEAN Option without CPUS, TRUEX-C, Sludge Wash C, and CLEAN Option with CPUs process strategies, respectively. These percentages essentially apply to either the primary LLW to grout or glass product options. The ratios of tertiary to primary costs were approximately $45,37,19$, and $44 \%$ for these same process strategies, respectively. The large tertiary waste costs could be decreased significantly if process design is also focused on reducing the amount of tertiary waste produced.

Note that all process strategy costs assume that a $0 \%$ heel was left in the waste tanks. Generally, if a $5 \%$ heel was assumed, the process strategy costs would be reduced by approximately 5 to $6 \%$ because of the reduced process stream flows (Knutson et a1. 1993).

For the primary LLW glass product option, the CLEAN Option process strategy without CPUs had primary waste processing costs approximately $20 \%$ greater than those for the TRUEX-C process strategy, approximately $7 \%$ less than those for Sludge Wash $C$, and approximately $31 \%$ greater than those for the CLEAN Option with CPUs. However, the tertiary waste process costs for the CLEAN Option without CPUS were approximately $45 \%$ greater than those for the TRUEX-C process strategy, approximately $117 \%$ greater than those for the Sludge Wash C process strategy, and approximately $35 \%$ greater than for the CLEAN Option with CPUs.

To further show the basis of the cost differences between the process strategies, the costs were combined into major process categories. The costs assnciated with tank waste retrieval and liquid waste partitioning are substantial (approximately \$8.1B) and are assumed to be the same for each process strategy. Costs associated with waste tank immobilization were estimated to be $50.43 \mathrm{~B}$, again, assumed to be the same for each process strategy. Again, the primary LLW treatment and disposal costs for the salt grout and glass-in-sulfur product options were essentially the same. 
The costs for separation of solids and liquids along with solids dissolution for the CLEAN Option process strategy without CPUs required approximately 1.3 and $50.99 B$ more than the TRUEX $-C$ and Sludge Wash $C$ process strategies, respectively. The costs for nuclide removal from alkal ine liquids for the CLEAN Option process strategy without CPUs required approximately 1.0 and $\$ 1.2 B$ more than the TRUEX-C and Sludge Wash $C$ process strategies, respectively. The costs for nuclide removal from acidic liquids for the CLEAN Option process strategy without CPUs required approximately 2.6 and \$4.2B more than the TRUEX-C and Siudge Wash $C$ process strategies, respectively. The costs for removal and purification of bulk chemicals for the CLEAN Option process strategy without CPUs required approximately $\$ 4.2 B$ more than the TRUEX-C and Sludge Wash C process strategies.

Combining the above four processing categories together the CLEAN Option process strategy without CPUs required approximately $9.1,10.6$, and $\$ 6.7 \mathrm{~B}$ more than the TRUEX-C, Sludge Wash C, and CLEAN Option with CPUs process strategies, respectively. The trade-off for these higher processing costs for the CLEAN Option process strategy is seen in the reduced HLW and LLW processing and disposal costs as compared to the TRUEX-C and sludge Wash $C$ process strategies. When the HLW and LLW processing and disposal costs are now included with the four processing categories, the CLEAN Option process strategy without CPUs required approximately 4.6 and $\$ 6.7 \mathrm{~B}$ more than the TRUEX-C and CLEAN Option with CPUS process strategies, respectively, but $\$ 2.4 B$ less than the Sludge Wash C process strategy for the primary LLW to glass option.

However, the increased processing in the CLEAN Option process strategy generated more tertiary waste than the other process strategies. Thus, the tertiary waste treatment and disposal costs for the CLEAN Option process strategy without CPUs required approximately $4.0,6.9$, and $\$ 3.3 \mathrm{~B}$ more than the TRUEX-C, Sludge Wash C, and CLEAN Option with CPUS process strategies, respectively. Consequently, reductions in the HLW and LLW treatment and disposal costs for the CLEAN Option process strategy compaced to the other process strategies were offset by the increased cost of tertiary waste treatment and disposal.

In summary, the cost savings associated with CPU use was significant and should be incorporated into any process strategy. The large tertiary waste costs could be decreased significantly if process design is also focused on reducing the amount of tertiary waste produced. More glass composition constraint evaluation and experimentation on key waste oxide stream components could allow relaxing the current constraints, resulting in significantly reduced HLW glass canister and $L L W$ glass vauit counts (i.e., higher waste oxide loadings in glass) and their associated costs. The trade-off for the higher processing costs for the CLEAN Option process strategy without CPUs is seen in the reduced HLW and LLW processing and disposal costs as compared to the TRUEX-C and Sludge Wash $C$ process strategies. However, the increased processing in the CLEAN Option process strategy generated more tertiary waste and higher associated treatment and disposal costs, offsetting the reductions in the HLW and LLW treatment and disposal costs. 
WHC-EP-0791

\subsection{GLASS COMPOSITION OPTIMIZATION}

The current glass optimization models are based on analyses and experimental glass formulation data relative to expected HLW stream compositions. Recent TPA changes have also designated glass as the final waste product form for the LLW stream. However, because no extensive analyses or experimental glass formulation data relative to expected LLW stream compositions are available, the HLW glass composition specifications and associated optimization models were used for the LLW glass streams also. is expected that the LLW glass will have different glass composition and property limits that will be defined in future work.

To obtain feasible solutions when optimizing the glass composition the $\mathrm{Fe}_{2} \mathrm{O}_{3}$ lower 1 imit of 2 wt\% was removed in all HLW and LLW glass calculations. This was because the waste oxide streams resulting from processing all SST and DST wastes together ( $i . e .$, as a blended waste stream) contained $\mathrm{Fe}_{2} \mathrm{O}_{3}$ in quantities of small overail mass fraction, requiring unattainable high waste loadings to achieve the lower constraint 1 imit. The $\mathrm{Fe}_{2} \mathrm{O}_{3}$ lower 1 imit was originally based on maintaining sufficient $\mathrm{Fe}_{2} \mathrm{O}_{3}$ in the glass to act as a redox buffer to protect against uranium+5 reduction to uranium+4. This lower limit should be reexamined to determine if it is necessary.

The limiting constraint in most models was the $\mathrm{P}_{2} \mathrm{O}_{5}$ upper limit of 1 wt\%. When this constraint was removed the HLW glass streams were limited by the other upper limit (OTHHI) (this limit represents everything other than the nine main components considered in the Composition Variability Study discussed in Appendix G) of 10 wt\% while the LLW glass streams were limited by the $\mathrm{Na}_{2} \mathrm{O}$ upper limit of 20 wt\%.

Relaxing the OTHHI was definitely considered to be extrapolating outside the range of applicability of the glass property models and constraints. However, relaxing the $\mathrm{P}_{2} \mathrm{O}_{5}$ upper limit was not considered to represent a large extrapolation outside the range of model applicability. Although the same reasoning could be applied to the $\mathrm{Na}_{2} \mathrm{O}$ upper limit, only the $\mathrm{P}_{2} \mathrm{O}_{5}$ upper limit was relaxed for the process strategy comparisons. Thus, for the LLW glass compositions the limiting constraint was the $\mathrm{Na}_{2} \mathrm{O}$ upper 1 imit of 20 wt\% and for the HLW glass compositions the limiting constraint was the OTHHI of 10 wt\%.

Table 4-2 shows the HLW and LLW glass production results for each processing strategy as summarized from Tables 65 through 67 of Appendix $G$. Appendix $G$ discusses the development of the glass composition optimization models and has tables and figures summarizing the glass composition calculations. Figures 4-3 and 4-4 show the HLW and LLW glass production data in terms of canisters and vaults, respectively.

The impact of the glass composition constraints on waste loading in the glass is shown in Table 4-2. Waste loadings of approximately 19 wt\%, 36 wt\%, and 26 wt\% were achieved in the CLEAN Option, TRUEX-C, and Sludge Wash C HLW glass products, respectively, without the $\mathrm{P}_{2} \mathrm{O}_{5}$ upper limit of 1 wt\% in place. The resulting $\mathrm{P}_{2} \mathrm{O}_{5}$ weight fraction in glass was 1.7 and $3.6 \%$ for the TRUEX-C and Sludge Wash $C$ HLW glass products, respectively. However, with the $\mathrm{P}_{2} \mathrm{O}_{5}$ upper limit, the HLW waste loadings were significantly lower, approximately 21 and 7 wt\% for the TRUEX-C and Sludge Wash $C$ models, respectively. No impact 
on the CLEAN Option $\mathrm{HLH}$ glass resulted from relaxing the $\mathrm{P}_{2} \mathrm{O}_{5}$ limit because it was not the limiting constraint. Increasing the OTHHI of 10 wt\% will also have a significant impact on the HLW glass production as shown by the comparison of the CLEAN Option HLW glass stream results in Table 4-2. When the OTHHI was relaxed, the CLEAN Option HLW glass stream showed a $39 \%$ reduction of canisters ( 750 down to 457 ), with a substantial increase in waste loading from 19 to 32 wt\%.

The LLW glass had waste loadings from 22 to 24 wt\% with the $\mathrm{P}_{2} \mathrm{O}_{5}$ upper limit in place. When the $\mathrm{P}_{2} \mathrm{O}_{5}$ upper limit was relaxed and the $\mathrm{Na}_{2} \mathrm{O}$ upper limit became binding, only the CLEAN Option LLW glass waste loading increased significantly from 22 to $27 \mathrm{wt} \%$. Increasing the $\mathrm{Na}_{2} \mathrm{O}$ upper 1 imit will also have a significant impact on the $L L W$ glass production as shown by the comparison of the Sludge Wash C LLW glass stream results with and without the $\mathrm{Na}_{2} \mathrm{O}$ upper 1 imit of 20 wt\% relaxed, where an $8 \%$ reduction of vaults ( 60 down to ${ }^{25}$ ) was seen. The resulting $\mathrm{Na}_{2} \mathrm{O}$ weight fraction in glass was $21.5 \%$. The Tank Waste Technical Options Report (Boomer et al. 1993) suggests that a higher $\mathrm{Na}_{2} \mathrm{O}$ limit of $25 \mathrm{wt} \%$ is possible because of the ability to recycle out of specification glass cullet. It also claims that higher $\mathrm{Na}_{2} \mathrm{O}$ limits in borosilicate glass compositions are reported in other iiterature. Increasing this limit will have a significant impact on the LLW glass production as shown by the comparison of the Sludge Wash C results.

Note that the CLEAN Option HLW glass canister count was dramatically lower than the other process strategies, with only approximately 5 and $1 \%$ of the TRUEX-C and Sludge Wash $C$ process strategy glass product magnitudes with the $\mathrm{P}_{2} \mathrm{O}_{5}$ upper limit, respectively, and only approximately 8 and $2 \%$ without the $\mathrm{P}_{2}^{2} \mathrm{O}_{5}$ upper limit, respectively. Relaxing the $\mathrm{P}_{2} \mathrm{O}_{5}$ upper 1 imit reduced the TRUEX $-C$ and Sludge Wash C HLW canister counts by $4 f$ and $72 \%$, respectively, and the LLW vault counts by 18 and $0.4 \%$ for the CLEAN Option and TRUEX-C process strategies, respectively.

The CLEAN Option LLW glass-in-sulfur vault total was highest for the $\mathrm{P}_{2} \mathrm{O}_{5}$ limiting case but ranged between the TRUEX-C and Sludge Wash $C$ results without the $\mathrm{P}_{2} \mathrm{O}_{5}$ upper limit, showing vault counts of 86 and $109 \%$ of the TRUEX-C and Sludge Wash $C$ results, respectively. Note the large amount of glass mass inventory (kilograms) associated with the LLW glass. This is due to the large amount of $\mathrm{Na}_{2} \mathrm{O}$ in the $\mathrm{LLW}$ oxide streams. Indeed $\mathrm{Na}_{2} \mathrm{O}$ constitutes 74, 85, and 89 wt\% of the LLW oxide stream for the CLEAN Option, TRUEX-C, and Sludge Wash $C$ process strategies, respectively. Removing this sodium would dramatically reduce the LLW giass mass inventory. One alternative for reducing $\mathrm{Na}_{2} \mathrm{O}$ in the LLW streams is a "Clean" Salt process (or Fractional Crystallization of $\mathrm{NaNO}_{3}$ ) (Herting 1992), where separation and production of "Clean" sodium salts (e.g., $\mathrm{NaNO}_{3}$ ) is achieved. If these "Clean" sodium salts are fed to an electrodialysis salt splitting process, $\mathrm{NaOH}$ and $\mathrm{HNO}_{3}$ could be produced for use in other processing. Alternatively, the "Clean" sodium salts could possibly be used in industrial applications or perhaps find disposal in lower cost drum storage instead of glass. This process will be evaluated in a later report.

As discussed in the previous report on the evaluation of the TRUEX-C and Sludge Wash C process strategies (Knutson et al. 1993), over $90 \%$ of the HLW glass oxides in those two models come from the undissolved solids stream that 
is sent to glass before TRUEX processing and eventual neutralization. Therefore, significant glass oxide reduction for those two models must come in the acid dissolution process step.

Based on previous work (Knutson et al. 1993), a 5\% heel left in the tanks during retrieval would result in an approximately $5 \%$ reduction in the HLW glass canister counts (Knutson et al. 1993).

With these aggressive pretreatment strategies it is clear that more glass property data and glass melting data in HLW and LLW waste composition ranges could possibly allow relaxing current composition constraints. If such limits were relaxed, higher waste oxide loadings in the glass could be achieved (and hence, reduced glass canister and vault counts, costs, etc.) and other pretreatment process choices would be seen. Indeed, the critical component constraints have not been explored as a function of the mass fraction of major glass components (e.g., $\mathrm{SiO}_{2}$ or $\mathrm{Na}_{2} \mathrm{O}$ ) and, therefore, should be taken as current estimates and not as scientifically established values that cannot be challenged if they represent severe constraints on the rest of a tank waste remediation system. Therefore, there should be additional focus on HLW and LLW immobilization activities with emphasis on currently constraining limits (e.g., $\mathrm{P}_{2} \mathrm{O}_{5}$, Other, and $\mathrm{Na}_{2} \mathrm{O}$ ).

\subsection{WASTE PRODUCT MASS BALANCES}

Table 4-3 contains a brief summary of the waste product generation for the CLEAN Option, TRUEX-C, and Sludge Wash C process strategies extracted from Table $\mathrm{H} 2$ of Appendix $H$. Appendix $H$ contains the process stream mass balance results for the global CLEAN Option, TRUEX-C, and Sludge Wash C process models, along with tables summarizing the waste product results. Figures 4-3 and 4-4 show the HLW and LLW glass/grout production data in terms of canisters and vaults, respectively.

As discussed previously, a large amount of tertiary waste is generated for each of the process strategies. With tertiary waste treatment, approximately $38 \%$ of the tertiary waste is destined for grout, approximately $26 \%$ of the tertiary waste is destined for water treatment, and $36 \%$ is destined for landfill burial. Note that the CLEAN Option process strategy produces a factor of 1.5 and 2.4 more tertiary waste than the TRUEX-C and Siudge Wash $C$ process strategies, respectively, because of its more process-intensive strategy.

Using CPUs to process the supernate and salt cake reduced the CLEAN Option tertiary waste generation by approximately $29 \%$. This reduction was estimated by processing only the sludge portion of the tank waste (i.e., approximately 36 and $4 \%$ of the SST and DST waste, respectively) through a central processing facility.

Figure 4-4 shows the LLW vault requirements for both primary and tertiary waste. Note that the primary LLW to glass option required only $30 \%$ of the vaults as the LLW salt grout option required. Also, note that the CLEAN Option tertiary LLW waste required nearly the same number of salt grout vaults as the primary LLW. Using CPUS, the CLEAN Option tertiary LLW waste required only $73 \%$ of the salt grout vaults as the primary LLW. The TRUEX-C and Sludge 
Wash $C$ process strategies had tertiary grout vault requirements that were approximately 57 and 50\% of those associated with primary LLW, respectively.

Based on primary LLW to glass option production ratios the CLEAN Option process strategy produces $14 \%$ less primary LLW glass than the TRUEX-C process strategy and $9 \%$ more than the Sludge Wash C process strategy.

Based on the primary LLW to salt grout option production ratios the CLEAN Option process strategy produces $15 \%$ less primary $L L W$ salt grout than the TRUEX-C process strategy and $19 \%$ more than the Siudge Wash C process strategy.

Based on HLW glass production ratios the CLEAN Option process strategy produces only 8 and $2 \%$ of the HLW glass than the TRUEX-C and Sludge Wash C process strategies produce, respectively.

Again, the LLW and HLW glass production results are based on the glass composition optimization results discussed in Appendix G. Relaxation of key glass component constraints can have a significant impact on the glass production results shown above.

Note that all process strategies assumed that a $0 \%$ heel was left in the waste tanks. Generally, if a $5 \%$ heel was assumed, the process strategy would decrease the HLW glass product by 4 to $5 \%$ as compared to the $0 \%$ heel case, while decreasing the LLW product by 2 to $3 \%$ (Knutson et al. 1993).

\subsection{RELATIVE HEALTH HAZARD ESTIMATES}

\subsubsection{Long-Term Offsite Health Hazard}

The comparison results show that the relative long-term health hazard for the CLEAN Option process strategy is within $1 \%$ of the health hazard for the TRUEX-C and Sludge Wash C process strategies for the $0 \%$ tank waste heel case. Based on previous analyses of the TRUEX-C and Sludge Wash $C$ process strategies (Knutson et al. 1993), these relative health hazards will increase by a factor of approximately 2.4 if the heel percentage increases from 0 to $5 \%$. This health hazard increase is associated with more radioactive waste products left behind in the tanks that will be immobilized in place. Thus, the data suggest that the relative long-term health hazard is comparable for all process strategies.

\subsubsection{Long-Term Onsite Heaith Hazard}

Figure 4-5 gives a graphical summary of the long- and short-term onsite health hazards normalized to the initial feedstream values as taken from data shown in Appendix I. Appendix I describes the relative health hazard estimates for the various process strategies and has tables and figures summarizing those estimates. 
As shown in Figure 4-5, the greatest long-term onsite waste health hazard would be in the HLW glass product if the environment were not protected from it by formidable barriers around the repository. The uranium product has the highest Health Hazard Index (HHI) for the Sludge Wash C process because it is highly concentrated. However, it is assumed that the uranium product does not remain onsite but is added to the U.S. national stockpile. Thus, the tank heel (requiring dome fill) stream dominates the onsite health hazard. otherwise, the primary LLW grout stream contains the only significant variation between pretreatment process strategies, where the TRUEX-C process strategy value was a factor of approximately 5 and 7 higher than for the CLEAN Option and Siudge Wash C process strategies, respectively. Note that while no estimate of the primary LLW glass HHI is included, it is expected to be lower than that resulting from primary LLW grout because the glass represents a more stable long-term waste form than grout.

The contributions of each component to the normalized long-term onsite HHI for the HLW glass (Appendix I) showed that the CLEAN Option HLW glass has substantially more contribution to the long-term onsite HHI (compared to the original total feedstream value) from plutonium, technetium, americium, thorium, neptunium, and heavy lanthanides, because of the more extensive separation processing performed for these elements. This greater processing in the CLEAN Option al so produced less contribution to HLW glass from uranium, fission products (FP2), and ${ }^{63} \mathrm{Ni}$ (OTH3) compared to the other process strategies.

Overall, the CLEAN Option long-term onsite HHI was more than an order of magnitude greater for the HLW glass product than the TRUEX-C and Sludge Wash C process strategies because of the greater $H L W$ concentration. However, it is highly unlikely that any excavation would uncover a deep repository. The drilling scenario with different controlling components would produce more health hazard from the repository than the excavation scenario.

The contributions of each component to the normalized long-term onsite HHI for primary LLW grout show that the CLEAN Option had larger contributions from the iodine, fission products (FP2), ${ }^{63} \mathrm{Ni}$ (OTH3), thorium, and neptunium than the TRUEX-C and Sludge Wash C process strategies because of the greater primary LLW concentration. However, the overall long-term onsite HHI for primary LLW grout is substantially lower when compared to the initial feedstream.

\subsubsection{Short-Term Onsite And Offsite Health Hazard}

Figure 4-5 gives a summary of the long- and short-term onsite health hazards normalized to the initial feedstream values as taken from data shown in Appendix I. Appendix I describes the relative health hazard estimates for the various process strategies and has tables and figures summarizing those estimates.

As shown in Figure 4-5, the greatest short-term onsite waste health hazard would be in the HLW glass stream if the environment were not protected from it by formidable barriers around the repository. Excluding the HLW glass product, the tank heel (requiring dome fill) stream dominates the short-term onsite health hazards. Otherwise, the only significant variations in the 
short-term onsite health hazards between the pretreatment process strategies occurs for the primary LLW grout product stream and the uranium product stream. Assuming that the uranium product does not remain onsite but is added to the U.S. national stockpile, only the primary LLW grout product health hazards are compared. The CLEAN Option process strategy has a primary LLW grout product health hazard value that is more than an order of magnitude less than the TRUEX-C and Sludge Wash C process strategy values. Note that while no estimate of the primary LLW glass HHI is included, it is expected to be lower than that resulting from primary LLW grout because the glass represents a more stable short-term (up to 500 years) waste form than grout.

The contributions of each component to the normalized short-term onsite HHI for the HLW glass (Appendix I) showed that the CLEAN Option HLW glass has substantially more contribution to the short-term onsite HHI (compared to the original total feedstream value) from plutonium, cesium, strontium, technetium, americium, thorium, neptunium, and heavy lanthanides resulting from the more extensive separation prc essing performed for these elements. This greater processing in the CLEAN Option also produced less contribution to HLW giass from uranium, fission products (FP2), and ${ }^{63} \mathrm{Ni}$ (OTH3) compared to the other process strategies.

Overal1, the CLEAN Option short-term onsite HHI was more than an order of magnitude greater for the HLW glass product than the TRUEX-C and Sludge Wash C process strategies because of the greater $H L W$ concentration. However, it is highly unlikely that any excavation would uncover a deep repository in the first 500 years after closure. The drilling scenario with different controlling components would probably produce more health hazard from the repository than the excavation scenario during the first 500 years.

The contributions of each component to the normalized short-term onsite HHI for primary LLW grout show that the CLEAN Option had larger contributions from the iodine, fission products (FP2), ${ }^{63} \mathrm{Ni}(0 \mathrm{TH} 3)$, thorium, and neptunium than the TRUEX-C and Sludge Wash C process strategies because of the greater primary LLW concentration. However, the CLEAN Option had substantialiy smaller contributions from cesium and strontium because of the more extensive separation processing performed for these elements. Overall, the short-term onsite HHI for primary LLW grout is substantially lower when compared to the initial feedstream.

\subsubsection{Occupational Exposure}

The previous report (Knutson et al. 1993) considered occupational exposure resulting from ${ }^{13}{ }^{C S}$ weighted by the feedstream and tertiary waste flows to particular processes, along with the relative alpha contamination from ${ }^{238} \mathrm{U}$ and ${ }^{239} \mathrm{Pu}$ and beta contamination from ${ }^{90} \mathrm{Sr}$. To increase the accuracy and detail of the occupational exposure calculations more components were evaluated in this report.

Figure 4-6 gives a summary of the occupational exposure health hazard estimates as taken from data shown in Appendix I. Appendix I describes the relative health hazard estimates for the various process strategies and has tables and figures summarizing those estimates. 
As shown in Figure 4-6, the plant designs for the CLEAN Option flowsheets result in about 3 and $24 \%$ more occupational gamma exposure, a factor of 2.3 and 12.1 more occupational alpha exposure, and a factor of 2.2 and 19.2 more occupational beta exposure, than the TRUEX-C and Siudge Wash $C$ plant designs, respectively. These larger indexes for the CLEAN Option result from its greater processing requirements.

Considering the contributions of various processes in each process strategy to the occupational exposure (Appendix I), the IX processes were weighted more heavily in terms of exposure than the solid separation steps because they were assumed to have the same amount of tertiary waste as a single cycle of SE. The solid separation steps are only assumed to generate $0.25 \%$ as much tertiary waste as one SE cycle judged from equipment fioor space in the Savannah River Purex $F$ and $H$ canyons (Appendix $E$ ).

Generally, more efficient removal of major exposure causing components in the first process elements to contact the tank waste would lower the occupational exposure in all process strategies. For example, the CLEAN Option process strategy has alkaline-side cesium removal before strontium and technetium removal, thereby reducing gamma exposure in these subsequent removal operations. However, cesium removal follows after other removal operations in the acid-side processing. If the acid-side cesium removal could also be placed before the other acid-side removal operations, it would reduce the gamma exposure in those subsequent removal operations.

\subsubsection{Waste Classification and Radionuclide Concentrations}

The tank waste feedstreams, the HLW glass stream destined for a HLW repository, and the tank heel stream are classified as either TRU or GTCC. The primary LLW to grout option streams are LLW Class A for the CLEAN Option process strategy, while the TRUEX-C and Sludge Wash $C$ process strategies were LLW Class B because of the strontium limits. Higher DFs may be needed for the atmospheric release of the vitrification offgas where different limits apply than for solid LLW. Using uranium LLW classification limits proposed by Kocher and Croff (Kocher and Croff 1988), the uranium product stream was also GTCC for the CLEAN and Sludge Wash C process strategies, while the TRUEX-C process strategy uranium stream was LLW Class $C$.

\subsection{PROCESS STRATEGY TECHNICAL RISK}

Figures 4-7 and 4-8 give a summary of the process strategy technical risk estimates as taken from data shown in Appendix $K$. Appendix $K$ describes the process strategy technical risk estimation method and has tables and figures summarizing those estimates.

The assessment of the technical risk associated with each process strategy was made by developing a technical risk estimation method based on 
tabulated data on alternative process technology development levels and extents (Sutherland et al. 1993). These process development data were tabulated using the following categories:

- Maturity of particular process technologies--extent and scale of testing with simple or realistic simulants (e.g., laboratory, bench, pllot plant, full-scale, operating full-scale facility)

- Potential benefit of particular process technologies--activity level and timeliness of testing and development

- Current DOE funding level of particular process technologies--extent of funding and the associated advance of maturity.

To allow an assessment of the technical risk associated with each of the process strategies, weighting factors were assigned to the above process technology development levels and extents in each category. Using these weights, numerical estimates of technical risk factor indexes were calculated for particular process technologies in each development category. These process technology technical risk factor indexes were combined for processes in each process strategy to assess the technical risk of each strategy.

Summarizing the technical risk assessment results, the DOE current level of effort contributes considerably to the technical risk of many process technologies, representing the slow advance of the process maturity. These processes (e.g., solids dissolution, SREX, $\mathrm{NaOH} / \mathrm{HNO}_{3}$ recycle, TCIX, and uranium separation associated with the TRUEX process) may require renewed or increased levels of effort to provide successful application of a tank waste pretreatment process strategy. The CLEAN Option process strategy without CPUs had a total combined category technical risk index that was a factor of approximately 2 and 10 times higher than the TRUEX-C and Sludge Wash $C$ process strategies, respectively. The process average combined category technical risk index for the CLEAN Option process strategy without CPUs was nearly the same as the TRUEX-C process strategy and a factor of approximately 3 higher than for the Sludge Wash C process strategy. The CLEAN Option using the new and developing CPU concept should be considered to have more technical risk than the CLEAN Option based only on a central processing facility concept. 
Figure 4-1. Tertiary Waste Generation.

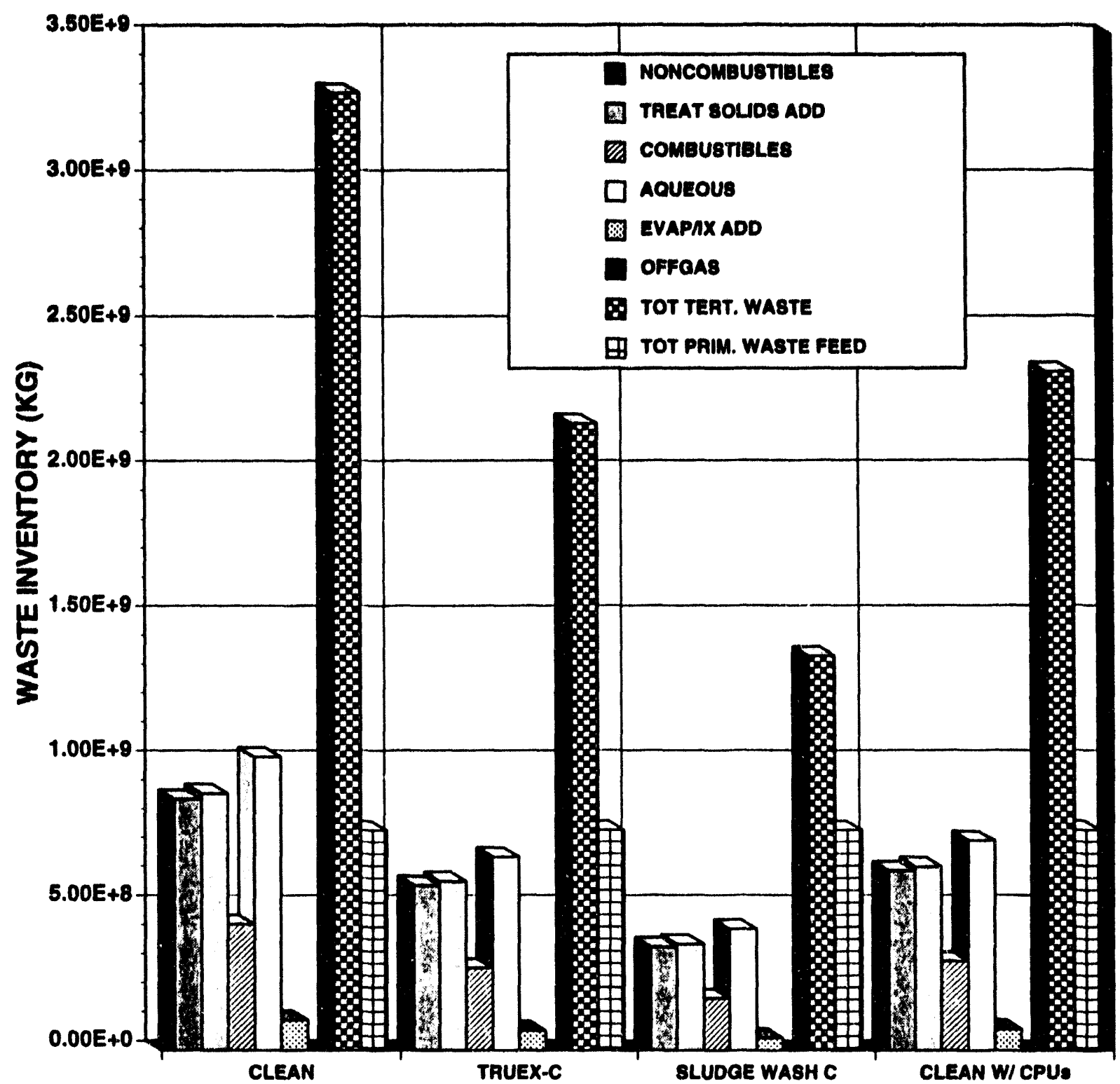

\begin{tabular}{|c|c|c|c|c|c|c|c|c|}
\hline$\therefore$ & NoN(I)U & $|K| 1 \mid$ & I $(3) 1131$ & A(p) I: & $111 / 11$ & (1)1.1:15 & $10111 \mathrm{KI}$ & IOII IKIII. \\
\hline CLEAN & $8.66 E+8$ & $8.86 E+8$ & $4.33 E+8$ & $1.01 \mathrm{E}+9$ & $1.04 E+8$ & $7.12 \mathrm{E}+5$ & $3.30 \mathrm{E}+9$ & $7.57 E+8$ \\
\hline TRUEX-C & $5.66 \mathrm{E}+8$ & $5.80 E+8$ & $2.83 E+8$ & $6.63 E+8$ & $6.78 E+7$ & $4.66 \mathrm{E}+5$ & $2.16 \mathrm{E}+9$ & $7.57 \mathrm{E}+8$ \\
\hline SLUDGE WASH C & $3.56 \mathrm{E}+8$ & $3.65 E+8$ & $1.78 E+8$ & 4.17E+8 & $4.26 E+7$ & $2.93 E+5$ & $1.36 E+9$ & $7.57 \mathrm{E}+8$ \\
\hline CLEAN W/ CPUs & $6.15 E+8$ & $6.29 E+8$ & $3.07 E+8$ & $7.19 E+8$ & $7.36 E+7$ & $5.05 E+5$ & $2.34 E+9$ & $7.57 E+8$ \\
\hline
\end{tabular}


Figure 4-2. Cost Summary for Primary and Tertiary Waste Processing (amounts given in S Million).

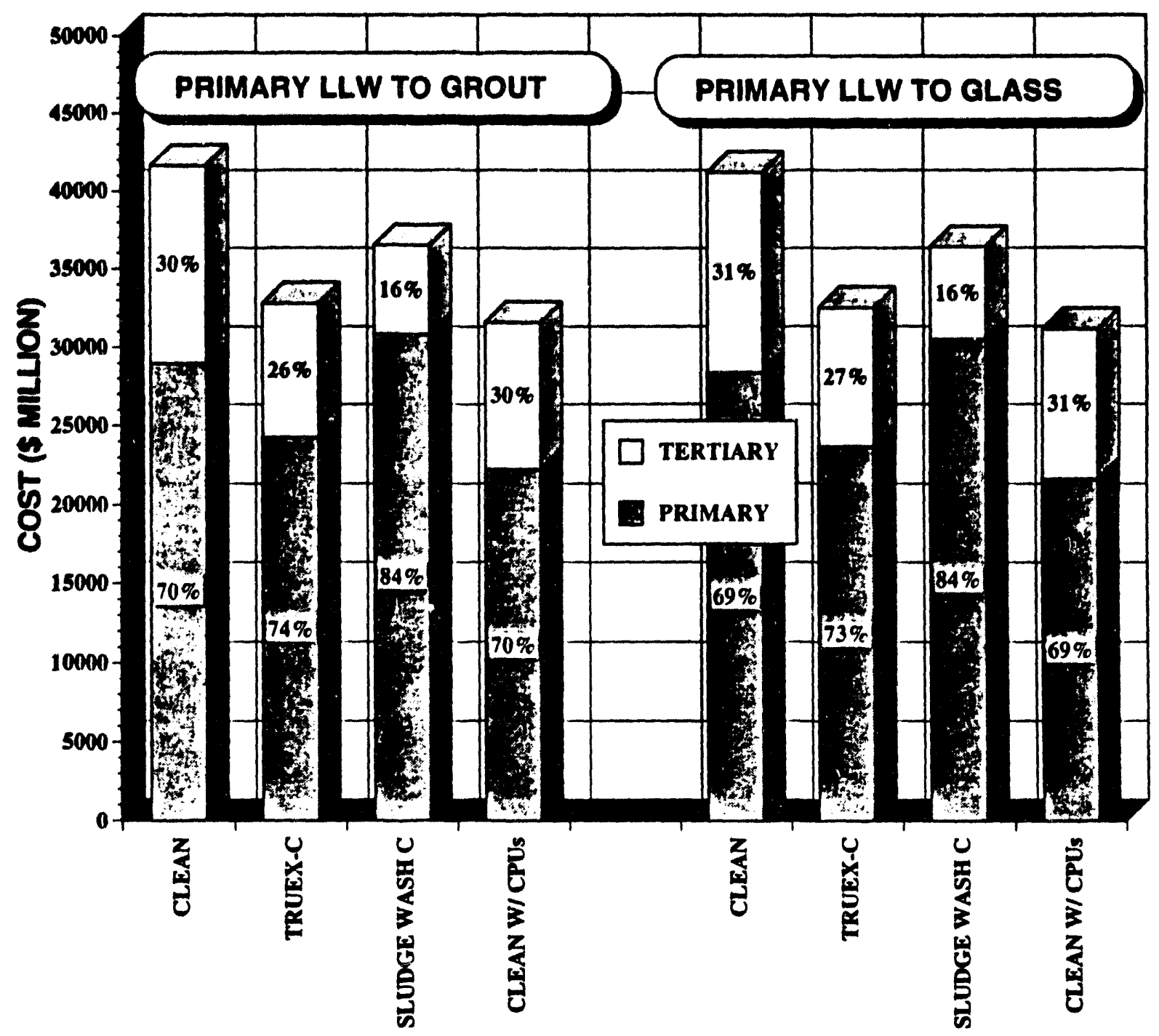

\begin{tabular}{|c|c|c|c|}
\hline IRIMARY LLWY TOCROUT & PRIVIARY & TLKTIAKY & TOTAI. \\
\hline CLEAN & 28970 & 12660 & 41630 \\
\hline TRUEX-C & 24250 & 8557 & 32807 \\
\hline SLUDGE WASH C & 30840 & 5683 & 36523 \\
\hline CLEAN W/ CPUs & 22240 & 9352 & 31592 \\
\hline \multicolumn{4}{|l|}{ PRIMARY LLW TOCILASS } \\
\hline CLEAN & 28373 & 12840 & 41213 \\
\hline TRUEX-C & 23665 & 8856 & 32521 \\
\hline SLUDGE WASH C & 30499 & 5919 & 36418 \\
\hline CLEAN W/ CPUs & 21641 & 9506 & 31147 \\
\hline
\end{tabular}


Figure 4-3. High-Level Waste Glass Canister Summary.

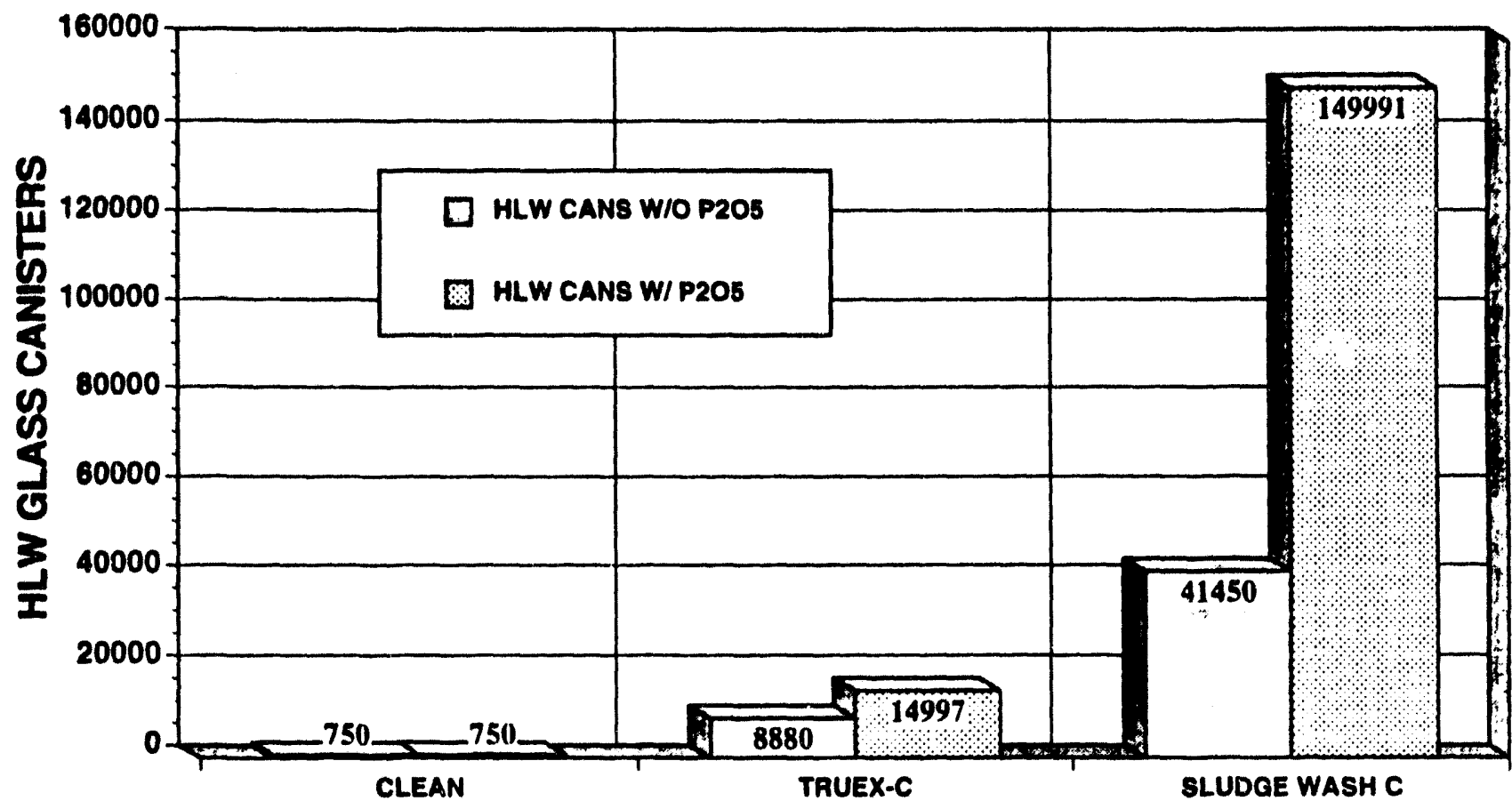

Figure 4-4. Low-Level Waste Glass and Grout Vault Summary.

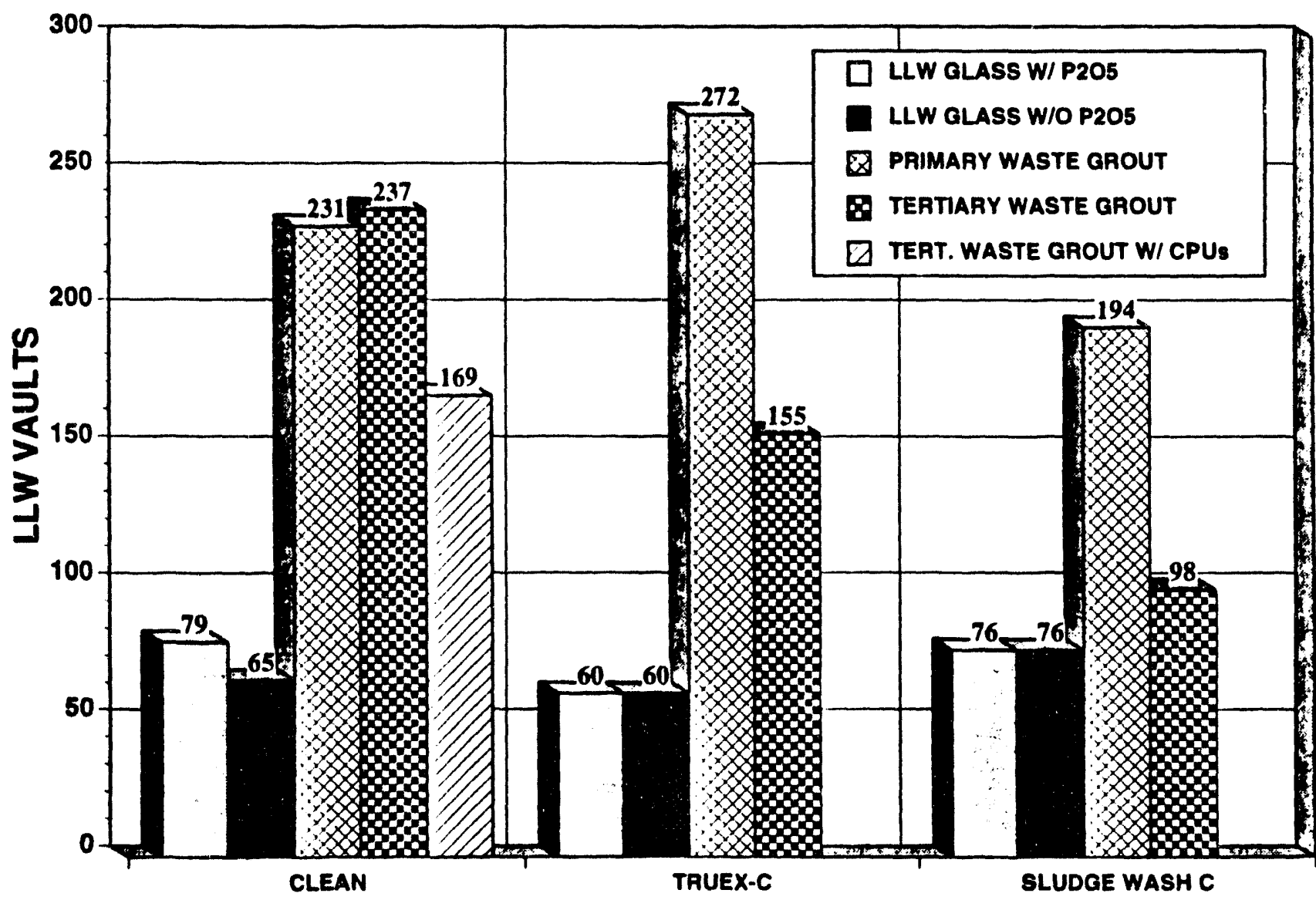


Figure 4-5. Normalized Long- and Short-Term Onsite Health Hazard.

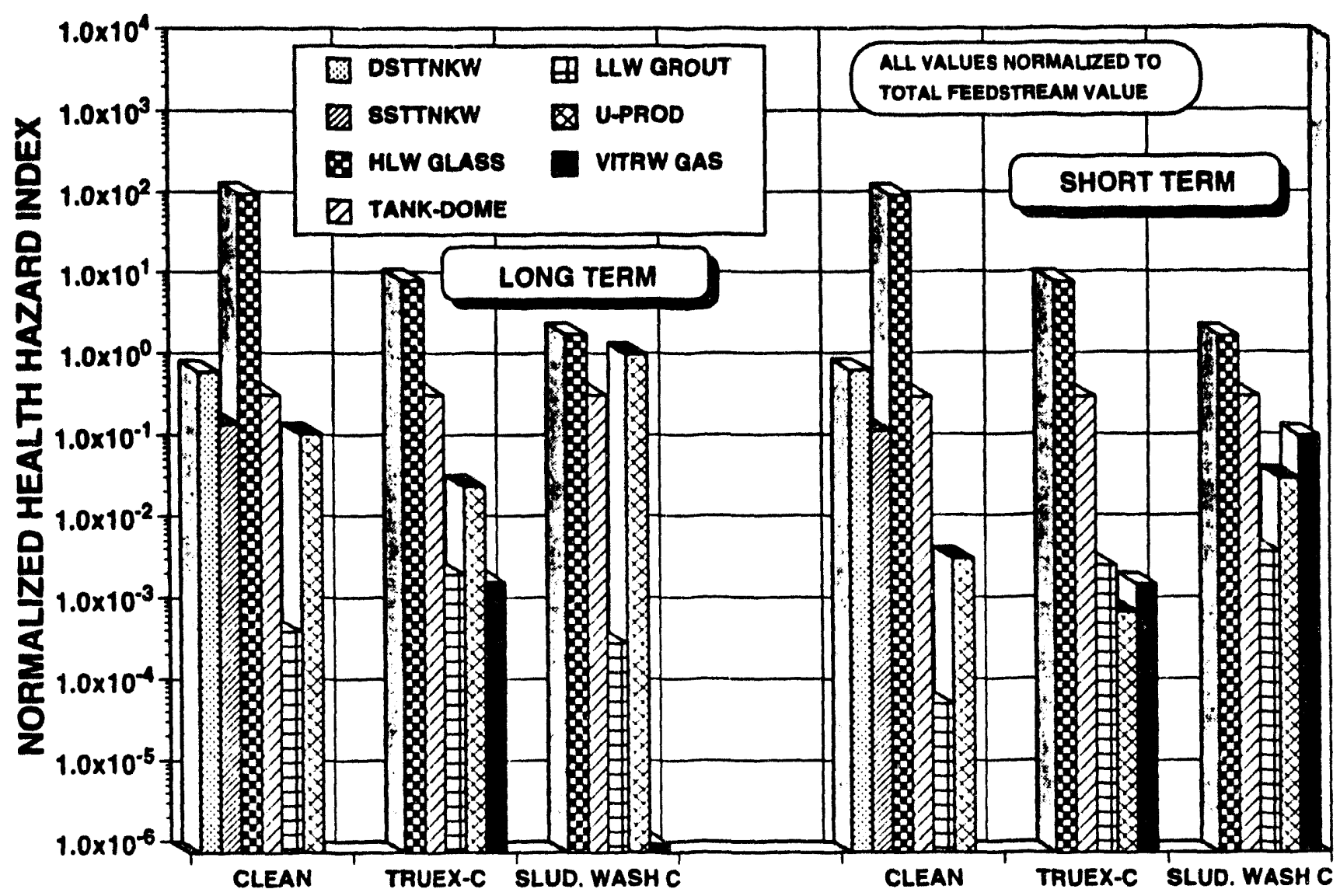

Figure 4-6. Occupational Health Hazard Summary.

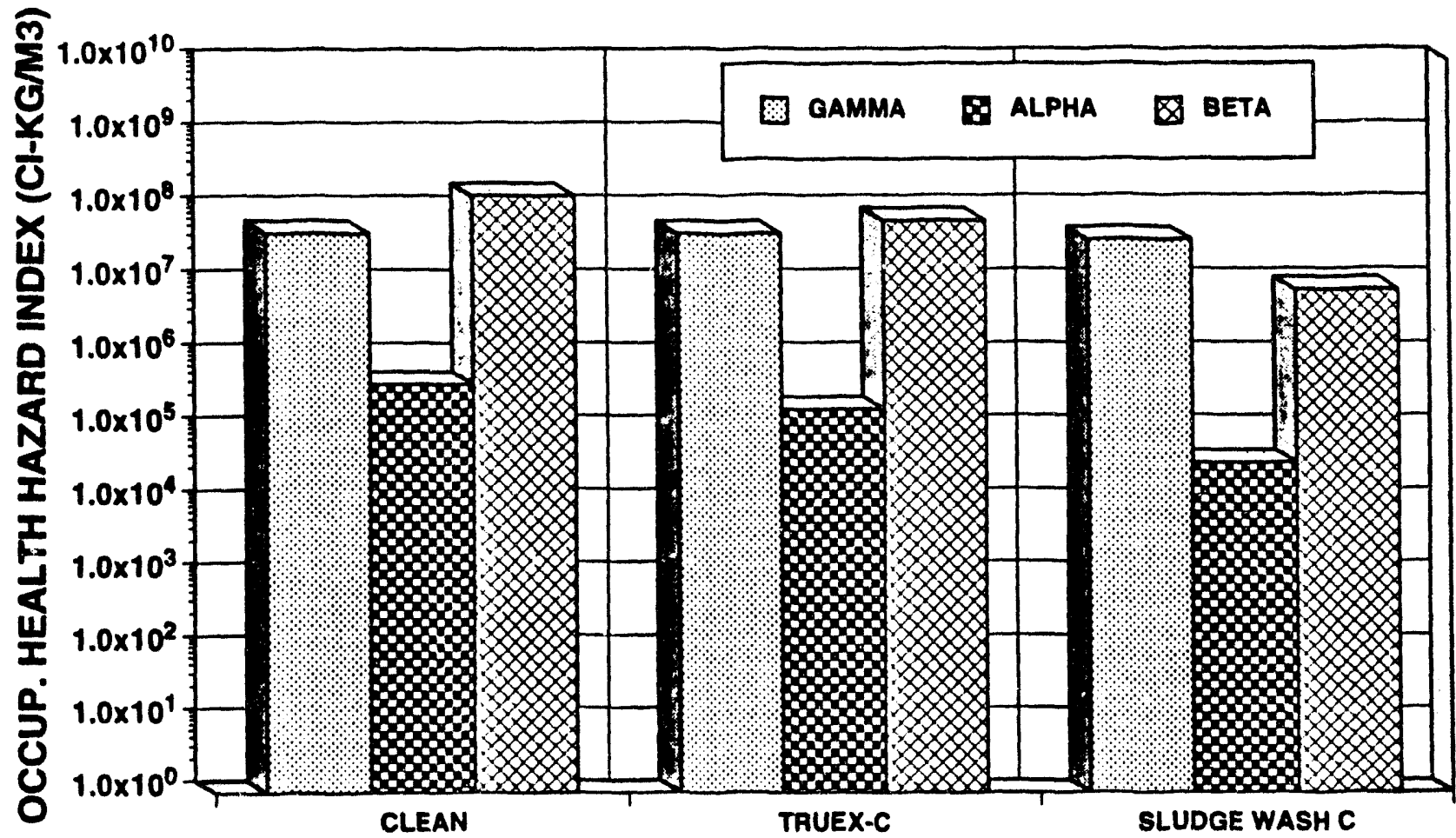


Figure 4-7. Total Technical Risk Index by Process Strategy.

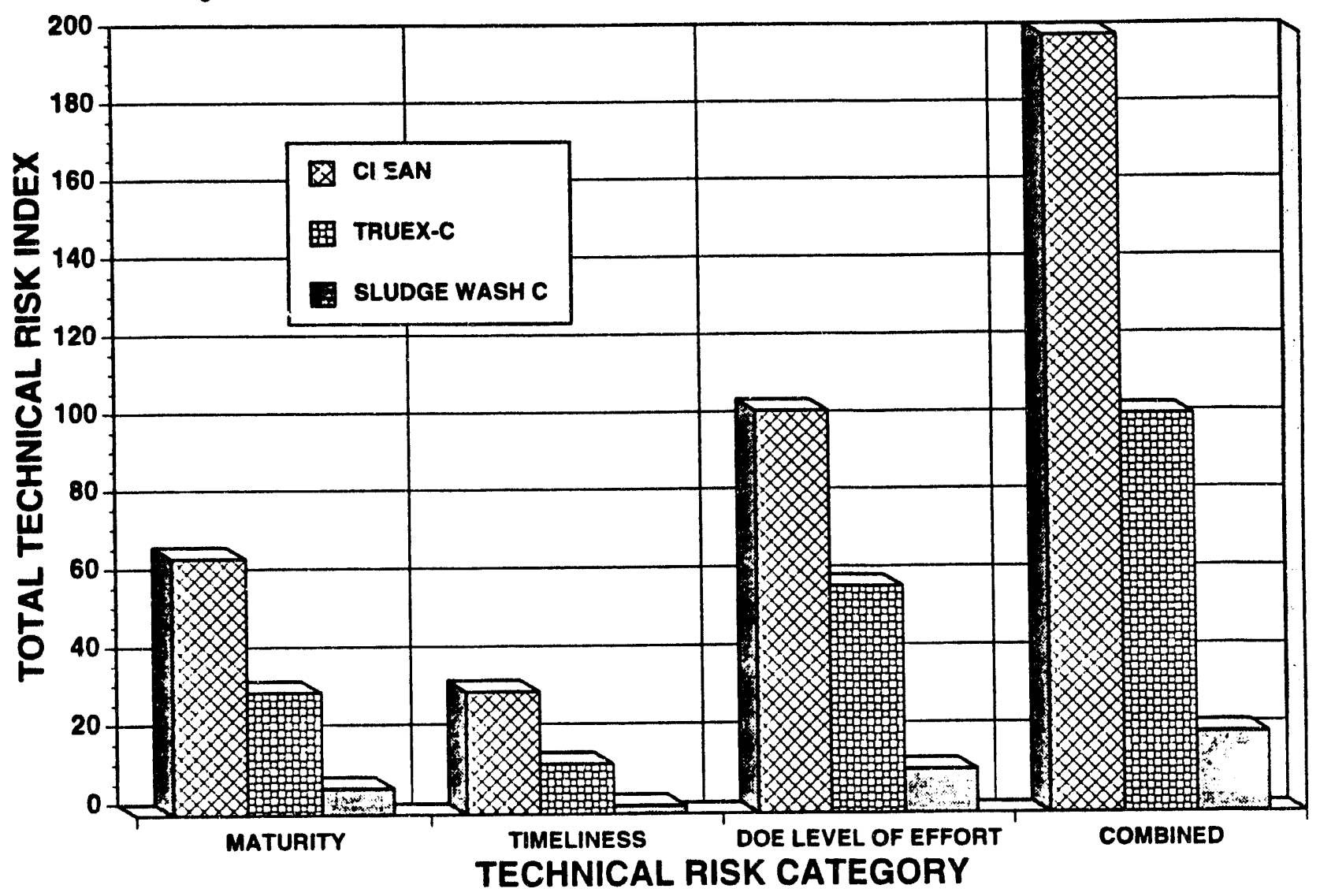

Figure 4-8. Average Technical Risk Index by Process Strategy.

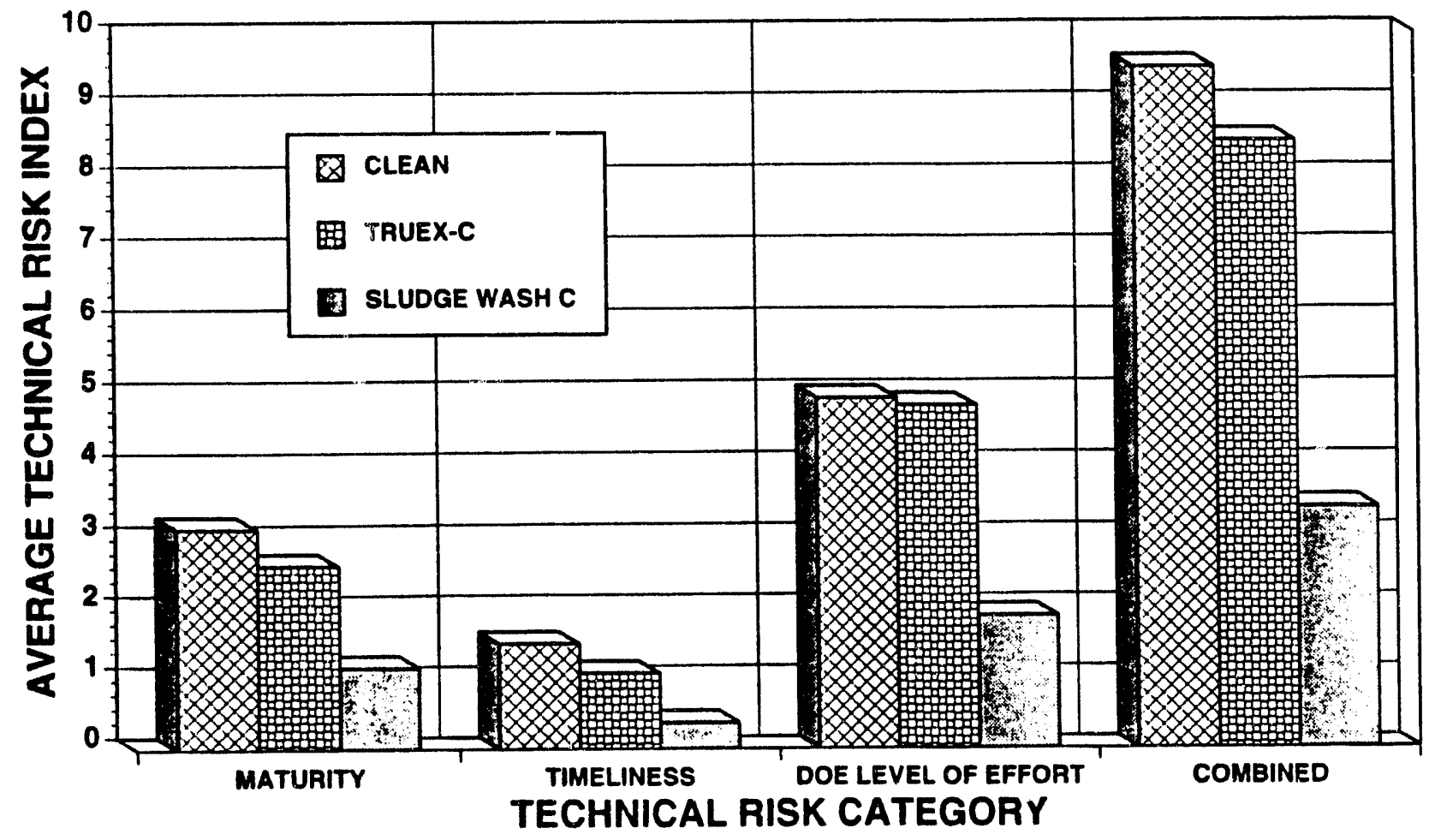


Table 4-1. Total Cost Summary for Process Strategies.

\begin{tabular}{|l|c|r|r|r|r|r|r|r|}
\hline \multirow{2}{*}{ Mode 1: } & \multicolumn{9}{|c|}{ Primary LLW to grout } & \multicolumn{4}{c|}{ Primary LLW to glass } \\
\cline { 2 - 9 } & CLEAN & TRUEX-C & SLUDW-C & $\begin{array}{r}\text { CLEAN } \\
+ \text { +CPUS }\end{array}$ & CLEAN & TRUEX-C & SLUDW-C & $\begin{array}{r}\text { CLEAN } \\
+ \text { CPUs }\end{array}$ \\
\hline \multicolumn{8}{|c|}{ Waste stream } \\
\hline $\begin{array}{l}\text { Primary } \\
\text { Tertiary }\end{array}$ & $\begin{array}{r}28,970 \\
12,660\end{array}$ & $\begin{array}{r}24,250 \\
8,557\end{array}$ & $\begin{array}{r}30,840 \\
5,683\end{array}$ & $\begin{array}{r}22,240 \\
9,352\end{array}$ & $\begin{array}{r}28,373 \\
12,840\end{array}$ & $\begin{array}{r}23,665 \\
8,856\end{array}$ & $\begin{array}{r}30,499 \\
5,919\end{array}$ & $\begin{array}{r}21,641 \\
9,506\end{array}$ \\
\hline Total & 41,630 & 32,807 & 36,523 & 31,592 & 41,213 & 32,521 & 36,418 & 31,147 \\
\hline $\begin{array}{l}\text { Tertiary/ } \\
\text { Primary }\end{array}$ & 0.44 & 0.35 & 0.18 & 0.42 & 0.45 & 0.37 & 0.19 & 0.44 \\
\hline
\end{tabular}

Percentage of cost:

\begin{tabular}{|l|l|l|l|l|l|l|l|l|}
\hline Primary & 69.6 & 73.9 & 84.4 & 70.4 & 68.8 & 72.8 & 83.7 & 69.5 \\
Tertiary & 30.4 & 26.1 & 15.6 & 29.6 & 31.2 & 27.2 & 16.3 & 30.5 \\
\hline
\end{tabular}

CLEAN/"MODEL" cost:

\begin{tabular}{|l|c|l|l|l|l|l|l|l|}
\hline Primary & -- & 1.19 & 0.94 & 1.30 & -- & 1.20 & 0.93 & 1.31 \\
Tertiary & -- & 1.48 & 2.23 & 1.35 & -- & 1.45 & 2.17 & 1.35 \\
\hline Total & -- & 1.27 & 1.14 & 1.32 & -- & 1.27 & 1.13 & 1.32 \\
\hline
\end{tabular}

CLEAN +C.PUS/"MODEL" cost:

\begin{tabular}{|l|l|l|l|l|l|l|l|l|}
\hline Primary & 0.77 & 0.92 & 0.72 & -- & 0.76 & 0.91 & 0.71 & -- \\
Tertiary & 0.74 & 1.09 & 1.65 & -- & 0.74 & 1.07 & 1.61 & -- \\
\hline Total & 0.76 & 0.96 & 0.86 & -- & 0.76 & 0.96 & 0.86 & -- \\
\hline
\end{tabular}

LLW grout versus glass product cost:

\begin{tabular}{|l|c|c|c|c|c|c|c|c|}
\hline Primary & -- & -- & -- & -- & 0.98 & 0.98 & 0.99 & 0.97 \\
Tertiary & -- & -- & -- & -- & 1.01 & 1.03 & 1.04 & 1.02 \\
\hline Total & -- & -- & -- & -- & 0.99 & 0.99 & 1.00 & 0.99 \\
\hline
\end{tabular}

NOTE: Dollar amounts are given in millions. $C P U=$ compact processing unit.

$L L W=$ low-level waste.

SLUDW-C = Sludge Wash $C$.

TRUEX-C = Transuranic Extraction Option C. 
Table 4-2. Summary of Glass Optimization Results.

\begin{tabular}{|c|c|c|c|c|c|c|}
\hline \multirow[b]{2}{*}{ Model: } & \multicolumn{3}{|c|}{ High-level waste glass } & \multicolumn{3}{|c|}{ Low-level waste glass } \\
\hline & CLEAN & TRUEX-C & SLUDW-C & CLEAN & TRUEX-C & SLUDW-C \\
\hline \multicolumn{7}{|c|}{ With the $\mathrm{P}_{2} \mathrm{O}_{5}$ high constraint } \\
\hline $\begin{array}{l}\text { Glass (kg) } \\
\text { Canisters } \\
\text { Vaults } \\
\text { Load (wt\%) }\end{array}$ & $\begin{array}{c}1.24 E+06 \\
750 \\
-- \\
19.3 \\
\end{array}$ & $\begin{array}{c}2.47 \mathrm{E}+07 \\
14,997 \\
-- \\
21.4\end{array}$ & $\begin{array}{c}2.47 \mathrm{E}+08 \\
149,991 \\
- \\
7.0\end{array}$ & $\begin{array}{c}6.74 E+08 \\
\overline{79} \\
22.3\end{array}$ & $\begin{array}{c}6.49 E+08 \\
-\overline{76} \\
23.5\end{array}$ & $\begin{array}{c}5.08 E+08 \\
-- \\
60 \\
22.5\end{array}$ \\
\hline \multicolumn{7}{|c|}{ Without the $\mathrm{P}_{2} \mathrm{O}_{5}$ high constraint } \\
\hline $\begin{array}{l}\text { Glass (kg) } \\
\text { Canisters } \\
\text { Vaults } \\
\text { Load (wt\%) }\end{array}$ & $\begin{array}{c}1.24 E+06 \\
750 \\
-- \\
19.3\end{array}$ & $\begin{array}{c}1.47 E+07 \\
8,880 \\
-- \\
36.1\end{array}$ & $\begin{array}{c}6.84 E+07 \\
41,450 \\
-- \\
25.5\end{array}$ & $\begin{array}{c}5.53 \mathrm{E}+08 \\
-\overline{65} \\
27.2\end{array}$ & $\begin{array}{c}6.46 \mathrm{E}+08 \\
-\overline{76} \\
23.6\end{array}$ & $\begin{array}{c}5.08 E+08 \\
-- \\
60 \\
22.5\end{array}$ \\
\hline \multicolumn{7}{|c|}{ Without the $\mathrm{NA}_{2} \mathrm{O}$ high constraint } \\
\hline $\begin{array}{l}\text { Glass (kg) } \\
\text { Vaults } \\
\text { Load (wt\%) }\end{array}$ & $\begin{array}{l}-- \\
--\end{array}$ & $\begin{array}{l}-- \\
-- \\
--\end{array}$ & $\begin{array}{l}-- \\
-- \\
--\end{array}$ & $\begin{array}{l}-- \\
-- \\
--\end{array}$ & $\begin{array}{l}-- \\
--\end{array}$ & $\begin{array}{c}4.71 E+08 \\
55 \\
24.3\end{array}$ \\
\hline \multicolumn{7}{|c|}{ Without the OTHHI high constraint } \\
\hline $\begin{array}{l}\text { Glass (kg) } \\
\text { Canisters } \\
\text { Load (wt\%) }\end{array}$ & \begin{tabular}{|c|}
$7.55 E+05$ \\
457 \\
31.7
\end{tabular} & $\begin{array}{l}-- \\
-- \\
--\end{array}$ & $\begin{array}{l}-- \\
-- \\
--\end{array}$ & $\begin{array}{l}-- \\
-- \\
--\end{array}$ & $\begin{array}{l}-- \\
-- \\
--\end{array}$ & $\begin{array}{l}-- \\
-- \\
--\end{array}$ \\
\hline \multicolumn{7}{|c|}{ Without $\mathrm{P}_{2} \mathrm{O}_{5} /$ with $\mathrm{P}_{2} \mathrm{O}_{5}$} \\
\hline Reduct (\%) & $\begin{array}{l}1.00 \\
0.0\end{array}$ & $\begin{array}{c}0.59 \\
40.8\end{array}$ & $\begin{array}{c}0.28 \\
72.4\end{array}$ & $\begin{array}{c}0.82 \\
17.9\end{array}$ & $\begin{array}{l}1.00 \\
0.4\end{array}$ & $\begin{array}{l}1.00 \\
0.0\end{array}$ \\
\hline
\end{tabular}

CLEAN/ "MODEL" :

\begin{tabular}{|l|l|l|l|l|l|l|}
\hline With $\mathrm{P}_{2} \mathrm{O}_{5}$ & -- & 0.05 & 0.01 & -- & 1.04 & 1.33 \\
Without $\mathrm{P}_{2} \mathrm{O}_{5}$ & -- & 0.08 & 0.02 & -- & 0.86 & 1.09 \\
\hline
\end{tabular}

Low-level waste glass $(\mathrm{kg}) / \mathrm{high}-$ level waste glass $(\mathrm{kg})$ :

\begin{tabular}{|l|l|l|l|l|l|l|}
\hline With $\mathrm{P}_{2} \mathrm{O}_{5}$ & -- & -- & -- & 544.39 & 26.23 & 2.05 \\
Without $\mathrm{P}_{2} \mathrm{O}_{5}$ & -- & -- & -- & 446.91 & 44.11 & 7.43 \\
\hline
\end{tabular}

OTHHI = Other upper limit.

SLUDW-C = Sludge Wash $C$.

TRUEX-C = Transuranic Extraction Option C. 
Table 4-3. Summary of Waste Product Generation.

\begin{tabular}{|c|c|c|c|c|}
\hline Model: & CLEAN & TRUEX-C & Sludge Wash C & CLEAN + CPUS \\
\hline \multicolumn{5}{|c|}{$\begin{array}{c}\text { Waste production ratio: } \mathrm{kg} \text { of waste product produced } \\
\text { per } \mathrm{kg} \text { of initial primary waste. }\end{array}$} \\
\hline $\begin{array}{l}\text { Total TERW } \\
\text { HLW glass } \\
\text { LLW glass } \\
\text { Salt grout } \\
\text { TERW grout } \\
\text { All grout }\end{array}$ & $\begin{array}{l}4.36 \\
0.002 \\
0.73 \\
2.58 \\
2.66 \\
5.24\end{array}$ & $\begin{array}{l}2.85 \\
0.019 \\
0.85 \\
3.04 \\
1.74 \\
4.78\end{array}$ & $\begin{array}{l}1.79 \\
0.090 \\
0.67 \\
2.17 \\
1.09 \\
3.27\end{array}$ & $\begin{array}{l}3.10 \\
-- \\
-- \\
-- \\
1.89 \\
4.47\end{array}$ \\
\hline \multicolumn{5}{|c|}{ Ratio of CLEAN results to other models } \\
\hline $\begin{array}{l}\text { HLW glass } \\
\text { LLW glass } \\
\text { Salt grout } \\
\text { TERW grout } \\
\text { All grout }\end{array}$ & $\begin{array}{l}-- \\
-- \\
-- \\
-- \\
--\end{array}$ & $\begin{array}{l}0.08 \\
0.86 \\
0.85 \\
1.53 \\
1.10\end{array}$ & $\begin{array}{l}0.02 \\
1.09 \\
1.19 \\
2.43 \\
1.60\end{array}$ & $\begin{array}{l}-- \\
-- \\
-- \\
1.41 \\
1.17\end{array}$ \\
\hline
\end{tabular}

$C P U=$ compact processing unit.

$H L W=$ high-level waste.

$L L W=$ low-level waste.

TERW = tertiary waste.

TRUEX-C = Transuranic EXtraction Option C. 
WHC-EP-0791

This page intentionally left blank. 


\subsection{CONCLUSIONS}

The cost savings associated with CPU use was significant (approximately 24\% for the CLEAN Option process strategy) and should be incorporated into any process strategy. The large tertiary waste costs (ranging from approximately 16\% [for Sludge Wash C] to 31\% [for CLEAN Option] of the total process strategy cost) could be decreased significantly if process design is also focused on reducing the amount of tertiary waste produced. Overall, the CLEAN Option without CPUs had a total cost of \$41.2B, which was approximately 27\% greater than that for the TRUEX-C process strategy (\$32.5B), approximately 13\% greater than that for the Sludge Wash C process strategy (\$36.4B), and approximately $32 \%$ greater than the CLEAN Option with CPUs (\$31.1B).

Comparing the primary $L L W$ to glass option to the $L L W$ to salt grout option shows that total primary $L L W$ processing and disposal costs were essentialiy the same for both options. Therefore, the choice of a LLW glass-in-sulfur product appears very advantageous compared to grout from a waste form stability aspect and also for its potential for future retrievability and processing.

The costs associated with the HLW and LLW glass products can vary dramatically, depending on which glass optimization models and associated composition constraints are used. For example, the $\mathrm{P}_{2} \mathrm{O}_{5}$ constraint was relaxed for all process strategy glass product results. If this constraint was included, cost increases of $\$ 1.6 \mathrm{~B}, \$ 17.5 \mathrm{~B}$, and $\$ 0.085 \mathrm{~B}$ would be seen for the TRUEX-C, Sludge Wash C, and CLEAN Option process strategies, respectively. Therefore, more glass composition constraint evaluation and experimentation on key waste oxide stream components could allow relaxing the current constraints, resulting in significantly reduced $H L W$ glass canister and LLW glass vault counts (i.e., higher waste oxide loadings in glass) and their associated costs.

The large amount of glass mass inventory (kilograms) associated with the LLW glass was due primarily to the large amount of $\mathrm{Na}_{2} \mathrm{O}$ in the LLW oxide streams. Indeed, $\mathrm{Na}_{2} \mathrm{O}$ constitutes 74,85 , and 89 wt\% of the LLW oxide stream for the CLEAN Option, TRUEX-C, and Sludge Wash C process strategies, respectively. Removing this sodium would substantially reduce the LLW glass mass inventory. One alternative for reducing $\mathrm{Na}_{2} \mathrm{O}$ in the $\mathrm{LLW}$ streams is a "Clean" Salt process (or Fractional Crystallization of $\mathrm{NaNO}_{3}$ ) (Herting 1992), where separation and production of "Clean" sodium salts (such as $\mathrm{NaNO}_{3}$ ) is achieved. If these "Clean" sodium salts are fed to an electrodialysis salt splitting process, $\mathrm{NaOH}$ and $\mathrm{HNO}_{3}$ could be produced for use in other processing. Alternatively, the "Clean" sodium salts could possibly be used in industrial applications or perhaps find disposal in lower cost drum storage instead of glass. This process will be evaluated in a later report.

The aggressive pretreatment process flowsheet that represented the CLEAN Option process strategy was successful in meeting the major goals of producing less than 1,000 HLW canisters along with a LLW Class A waste stream. Again, this success depends on the previously discussed process modeling assumptions. 
Credit is not taken in the CLEAN Option for reducing HLW volume to the point that a separate vitrification facility may not be needed. If vitrification can be incorporated directly into the separations plant as an acid process, the cost of permitting and approval efforts for an additional stand-alone facility could be avoided, and costs associated with neutralization, material transport, and storage of waste at a separate facility would be eliminated.

The toxicity of the LLW stream is significantly affected by complexation in alkaline supernatant, especially after the organic destruction step. Therefore, greater understanding of this complexation is needed to ensure successful process definition and development.

The relative long-term offsite health hazard estimates suggest that this health hazard is comparable for all of the process strategies. The greatest long- and short-term onsite health hazard (controlled by excavation rather than by a drilling scenario) would be in the HLW glass product if the environment were not protected from it by geologic and geochemical barriers in and around the repository. The CLEAN Option long- and short-term onsite health hazards were more than an order of magnitude greater for the HLW glass product than the TRUEX-C and Sludge Wash C process strategies because of the greater HLW concentration. However, it is highly unlikely that any excavation would uncover a deep repository. The drilling scenario with different controlling components would probably produce more short-term hazard from the repository than the excavation scenario during the first 500 years.

Thus, excluding the HLW glass product, the tank heel stream dominates both long- and short-term onsite health hazards. Otherwise, the only significant variations in the long- and short-term onsite health hazards between the pretreatment process strategies occurs for the primary LLW grout product and the uranium product. Assuming that the uranium product does not remain onsite but is added to the U.S. national stockpile, only the primary LLW grout product health hazards are compared. For the primary LLW grout product long-term onsite health hazard, the TRUEX-C process strategy was a factor of approximately 5 and 7 higher than for the CLEAN Option and Siudge Wash C process strategies, respectively. For the primary LLW grout product short-term onsite health hazard, the CLEAN Option process strategy was more than an order of magnitude lower than for the TRUEX-C and Sludge Wash $C$ process strategies, respectively. While no estimate of the primary LLW glass product onsite heaith hazard is included, it is expected to be lower than that resulting from primary LLW grout because the glass represents a more stable waste form than grout.

Considering occupational exposure, the plant designs for the CLEAN Option flowsheets resuit in about 3 and $24 \%$ more occupational gamma exposure, a factor of 2.3 and 12.1 more occupational alpha exposure, and a factor of 2.2 and 19.2 more occupational beta exposure, than the TRUEX-C and Sludge Wash $C$ plant designs, respectively. These larger health hazards for the CLEAN Option result from its greater processing requirements.

Generally, more efficient removal of major exposure-causing components in the first process elements to contact the tank waste would lower the occupational exposure in all process strategies. For example, the CLEAN Option process strategy has alkaline-side cesium removal before strontium and 
technetium removal, thereby reducing gamma exposure in these subsequent removal operations. However, cesium removal follows after other removal operations in the acid-side processing. If the acid-side cesium removal could also be placed before the other acid-side removal operations, it would reduce the gamma exposure in those subsequent removal operations.

Considering waste product classification, the tank waste feedstreams, the HLW glass stream destined for a HLW repository, and the tank heel stream are classified as GTCC. The primary LLW grout streams are LLW Class A for the CLEAN Option process strategy, while the TRUEX-C and Sludge Wash $C$ process strategies were LLW Class B because of the strontium limits. Higher DFs may be needed for the atmospheric release of the vitrification offgas where different limits apply than for solid LLW. Using uranium LLW classification limits proposed by Kocher and Croff (Kocher and Croff 1988), the uranium product stream was also GTCC for the CLEAN and Sludge Wash C process strategies, while the TRUEX-C process strategy uranium stream was LLW Class $C$.

The DOE current level of effort contributes considerably to the technical risk of many process technologies, representing the slow advance of the process maturity. These processes (e.g., solids dissolution, SREX, $\mathrm{NaOH} / \mathrm{HNO}_{3}$ recycle, TCIX, and uranium separation associated with the TRUEX process) may require renewed or increased levels of effort to provide successful application of a tank waste pretreatment process strategy. The CLEAN Option process strategy without CPUs had a total combined category technical risk index that was a factor of approximately 2 and 10 times higher than the TRUEX $-C$ and Sludge Wash C process strategies, respectively. The process average combined category technical risk index for the CLEAN Option process strategy without CPUs was nearly the same as the TRUEX-C process strategy and a factor of approximately 3 higher than for the Sludge Wash C process strategy. The CLEAN Option using the new and developing CPU concept should be considered to have more technical risk than the CLEAN Option based only on a central processing facility concept.

Future work could consider possible disposal alternatives to mixing separated waste fractions into HLW and LLW mixtures. If the waste fractions produced by a processing strategy, such as the CLEAN Option, are not recombined into the two HLW and LLW mixtures, a HLW repository may not be technically necessary for anything but spent fuel.

Also, the process strategies could be reevaluated using different tank group feedstreams (perhaps 10 to 12 for SSTs) versus an all-tank (SST and DST) mixed waste feedstream currently used in the analyses. Sludge dissolution factors included in the process models would change for each specific tank sludge waste group based on experimental/analytical data. This course of analysis could be phased in as appropriate. 
WHC-EP-0791

This page intentionally left blank. 


\subsection{REFERENCES}

Boomer, K. D., et al., 1993, Tank Waste Technical Options Report, WHC-EP-0616, Westinghouse Hanford Company, Richland, Washington.

Herting, D. L., 1992, "Clean Salt Process End-0f-Fiscal-Year Report," Internal Memo, 12110-PCL92-078, October 1, 1992, Westinghouse Hanford Company, Richi and, Washington.

Knutson, B. J., et a1., 1992, "Advanced Chemical Separation Study: TRUEX-A Yersus Sludge Wash C," DRAFT letter report, August 1992, Westinghouse Hanford Company, Richland, Washington.

Knutson, B. J., et al., 1993, "Advanced Chemical Separation Study: TRUEX Strategy Compared To Sludge Wash Ion Exchange," DRAFT letter report, July 1993, Westinghouse Hanford Company, Richiand, Washington.

Kocher, D. C., and A. G. Croff, 1988, A Proposed Classification System For High-Level And Other Radioactive Wastes, Research Sponsored by DOE Contract DE-AC05-840R21400, Radioactive Waste Management and the Nuclear Fuel Cycle, Vol. LL(3), Pp. 227-268, Oak Ridge National Laboratory, Oak Ridge, Tennessee.

Sutherland, D. G., et al., 1993, Pretreatment Status Report On The Identification And Evaluation of Alternative Processes, WHC-SD-WM-PRS-012, Rev. O, Westinghouse Hanford Company, Richland, Washington. 
WHC-EP-0791

This page intentionally left blank. 
WHC-EP-0791

\section{APPENDIX A}

GENERAL DESCRIPTION OF ASPEN PLUS

A-1 
WHC-EP-0791

This page intentionally left blank.

A-2 
ASPEN PLUS' (Advanced System for Process Engineering) is a software system for computer aided process design. The code was designed for performing rigorous steady state material and energy balances for plants in the process industries. It is a tool that can be used by engineers to model any type of process for which there is a continuous flow of materials and energy from one processing unit to the next. Input to the model consists of information normaliy contained in process flowsheets. The program input files contain keywords that define the flowsheet connectivity, the chemical components with in the flow streams, the methods for calculating thermodynamic properties (1.e., equations of state), and unit operation information, such as type of equipment, operation sequence, and performance specifications. The code will perform the necessary calculations, including equilibrium calculations, to produce a material and energy balance that describes the flowsheet. The output includes flow rates and compositions of feed, product, internal, and recycle streams. Key equipment specifications and energy consumption are also determined, and capital and operating costs of the modeled process can be estimated. ASPEN PLUS also includes capabilities for running sensitivity analysis case studies, optimizing the process for a specified parameter (i.e., cost), and data regression for estimating various equilibrium and physical property parameters. User-defined FORTRAN blocks can be used to supplement or supersede the calculations performed by ASPEN PLUS to control chemical makeup calculations or change process unit specifications based on stream characteristics.

Material balances are rigorously calculated using the internal physical and thermodynamic property models selected by the user. ASPEN PLUS contains several property models for this purpose, including models for equations of state, enthalpy, activity coefficients, transport properties, and others. Some of the physical property parameters required to use these property models are contained in ASPEN PLUS data banks for common chemical components. However, binary and ternary ion interaction parameters and chemical equilibrium data for solid dissolution and precipitation for most of the Hanford tank waste species are not available in the data banks. Special data libraries were developed to represent the ionic species and chemical compounds unique to the Hanford waste streams. These user-defined data libraries include information related to specie radioactivity and toxicity.

An expert system interface to ASPEN PLUS, called ModelManager ${ }^{2}$, provides the user with a graphical tool to facilitate model development, evaluation, and representation. The expert system includes built-in checks to prevent over- and under-specification of model parameters during model development, allows interactive control and display of the ASPEN PLUS model run data, and provides data spreadsheet and table development.

The ASPEN PLUS computational model consists of process blocks and streams. Each block represents a process or group of processes which make up a function. Streams represent the flow of materials by appropriate means into

'ASPEN PLUS is a trademark of Aspen Technology, Inc.
2ModelManager is a trademark of Aspen Technology, Inc. 
and out of the processes. Each block can have one or more input streams and one or more output streams, depending on which particular process is represented by that block. Each input and output stream flow is divided into components. or groups of materials, based on their chemical and radiological properties.

For mass flow calculations, four different types of process blocks are used. The first process type is a "mixer" (MIXER), which consists of one or more input streams and one output stream. The mass flow in the output stream is just the sum (or mix) of the mass flows of the input streams. The second process type is a "splitter" (FSPLIT), which separates (or splits the flow) of one or more input streams into two or more output streams. For each of the chemical components in a stream, the mass flow of each output stream is some fraction of the sum of the mass flow input into the process. This fraction is referred to as the separation factor. Because of the conservation of mass, the separation factors for each component for all of the streams must sum to unity. For a splitter, the separations for each component of a particular output stream are equal. In other words, all outlet streams have the same composition and properties.

The third process type modeled is a "separator" (SEP, SEP2). A separator is a type of splitting process which has one or more input streams and two or more output streams. For a separator, the component separation values within a particular stream may be different, which distinguishes a separator from a splitter. Again, the mass flow for the output stream is defined by the sum of the input streams multiplied by a separation factor between zero and one, and the sum of the separation factors for each component over all the output streams must sum to unity. This representation results in an algebraic computational model. The separation factor in a separator varies inversely with the commonly used decontamination factor, which is the ratio of the concentration of a component in an exit stream to its concentration in the feed stream. This definition differs significantly from the classical separation factor, which is the ratio of two components in one exit stream divided by the ratio of the same two components in another exit stream.

The last process type modeled is a "reactor" (RSTOIC, RYIELD). The reactor is used to simulate chemical reactions. Ali reactors may have any number of input or feed streams, which are mixed internally. The RYIELD reactor process simulates a reacter with specified reaction yields of each component. The RSTOIC reactor process simulates a reactor with specified reaction stoichiometry and reaction extents. In both reactor simulations, no reaction kinetics parameters are required.

In addition to the above process blocks, user-defined FORTRAN blocks were created to automate (i.e., provide run-time calculations of) many of the process-dependent calculations. These include feed flow dependent chemical makeup calculations, tertiary nonprocess waste generation calculations, process cost calculations, and glass composition optimization calculations. 
WHC-EP-0791

\section{APPENDIX B}

DETAILED CLEAN OPTION MODEL: ASPEN PLUS' IMPLEMENTATION

\footnotetext{
'ASPEN PLUS is a trademark of Aspen Technology, Inc.
} 


\section{LIST OF TERMS}

APM
CE
CMPO
CSIX
DCH 18-Cr-6
DTPA
EDTA
HLW
IDEF
LLW
NOX
NPH
PFD
PUREX
SCWO
SE
SLS
SOLEX
SREX
TBP
TCIX
THFTCA
TOC
TRU
TRUEX
TRUEX-C
UREX

ammonium phosphomolybdate

crown ether

N-di isobutylcarbamoylmethylphosphine oxide

cesium ion exchange

dicyclohexano 19-crown-6 ether

diethylene-triamine-penta-acetic acid

ethylenediaminetetraacetic acid

high-level waste

Integrated Computer-Aided Manufacturing (ICAM) Definition

(Language)

low-level waste

oxides of nitrogen

normal paraffin hydrocarbon

process flow diagram

plutonium-uranium extraction

supercritical water oxidation

solvent extraction

solid/liquid separation

Solvent Extraction Option

strontium extraction

tributyl phosphate

technetium ion exchange

tetrahydrofurantetracarboxylic acid

total organic content

transuranic

transuranic extraction

Transuranic Extraction Option C

uranium extraction 
WHC-EP-0791

\section{DETAILED CLEAN OPTION MODEL: ASPEN PLUS IMPLEMENTATION}

\section{B1.0 INTRODUCTION}

The ASPEN PLUS unit process model developed for this report is a streamlined model of the CLEAN process flow diagram (PFD) presented in Tank Waste Technical Options Report, WHC-EP-0616, (Boomer et al. 1993). Figure Bl shows the ASPEN PLUS block diagram representing the CLEAN Option flowsheet model. The stream numbers mentioned in the CLEAN Option model description refer to this block diagram. Figure B2 shows the Sludge Wash C, Transuranic Extraction Option C (TRUEX-C), and CLEAN Option process strategies as they relate to the waste separations technology options defined in the Tank Waste Technical Options Report (Boomer et al. 1993). Following the CLEAN Option model description are descriptions of the Sludge Wash $C$ and TRUEX-C models (Knutson et al. 1993) that are added for completeness.

Following the TRUEX $-C$ and Sludge Wash $C$ model descriptions is a CLEAN Option flowsheet representation using the ICAM (Integrated Computer-Aided Manufacturing) Definition (IDEF) Language software tool (Pajunen 1992). The IDEF tool provided integration of the CLEAN Option process strategy within the total Hanford cleanup system (Appendix C). It also provided a flowsheet description at increasingly decomposed states (i.e., a hierarchical representation) or levels of detail, from which an appropriate detail level could be selected for implementation using ASPEN PLUS. Figure B5 shows the IDEF hierarchy of functions with the global level representation highlighted.

The streamlined CLEAN Uption model described in this appendix is based upon an example flowsheet proposed by J. L. Swanson (Swanson 1993) and is the same, except for removal of the following processing steps:

1. Evaporative removal of water from feed to basic-side separations

2. Precipitation of ferric hydroxide before basic-side separations

3. Caustic fusion of solids after the second acid dissolution step.

Sufficient detail is contained in the model for performing a material balance calculation. Process equipment shown in the PFD in WHC-EP-0616 (Boomer et al. 1993) is not included in the model if it does not impact the distribution of components between the waste streams produced by the flowsheet. For example, identical process trains, which run in parallel, are modeled as a single train; a mixing tank followed by a unit operation (e.g., reactor or extraction step) is modeled as an appropriate unit operation block with multiple feed streams.

The amount of process unit detail contained in the material balance model closely follows the level of process unit information presented in Clean Option: An Alternative Strategy for Hanford Tank Waste Remediation, Volume 2, Detailed Description of First Example Flowsheet (Swanson 1993). In fact, this reference is the source for most of the design specifications and bases for chemical additions. In addition, it contains the rationale for the selection and sequencing of the various process steps comprising the CLEAN flowsheet option. 
The process descriptions included in this appendix are only intended as summaries of information presented in WHC-EP-0616 (Boomer et al. 1993) and PNL-8388 (Swanson 1993). For further details, the reader is referred to these sources.

\section{B2.0 SOLID-LIQUID SEPARATION TREATMENT/ASSUMPTIONS}

The CLEAN flowsheet employs centrifuges for solid/liquid separation (SLS) in six different portions of the flowsheet: initial treatment of feed from waste tank retrieval, after the organic destruction processing, after caustic dissolution, after both acid dissolution steps, and after the chromium reduction step before caustic production and recycling. The common assumptions regarding separation efficiency and operation for all of these SLS steps are as follows.

a. Centrifuge operation and downstream filters achieve $0 \%$ solid in clarified supernate.

b. Polyelectrolyte solution is blended in line with the slurry feed to the centrifuge to coagulate colloidal solids and, thereby, improve solids recovery. This solution has a weight equivalent to $19 \%$ of the solids in the centrifuge feed and has a composition of 1 wt\% polyelectrolyte and 99 wt\% water.

c. Supernate entrainment in the solids from the centrifuge is $12 \%$ of the solid weight in the centrifuge feed.

d. Centrifuge wash water amount is 4 times the total solids weight. of this $400 \%$ solid weight equivalent wash water, 88 solid wt\% accompanies the solid to the centrifuge catch tank; the remainder accompanies the clarified supernate.

e. Dilution water equivalent in weight to $200 \%$ solids is added to the catch tank to produce a pumpable slurry, which is $25 \mathrm{wt} \%$ solid.

\section{B3.0 INITIAL SOLID-LIQUID SEPARATION}

Sludge and dissolved salt cake from waste storage tanks undergo SLS as the first processing step. The simplified slate of components and their distribution between liquid and solid phases is provided in Table Bl. The solid slurry from the centrifuge is fed to the caustic dissolution section. The clarified liquid is routed to the organic destruction step. 


\section{B4.0 ORGANIC DESTRUCTION}

Supercritical water oxidation (SCWO) destroys organics complexed with metal ions and precipitates the multivalent metals out of solution as their hydroxides. Process conditions for SCWO are $500{ }^{\circ} \mathrm{C}$ and $3,000 \mathrm{psi}$. For simplicity, organic destruction is modeled with the following reactions in which only the total organic content (TOC) of organic complexes is considered (except for ferrocyanide):

$$
\begin{aligned}
& \mathrm{Fe}[\mathrm{CN}]_{6}^{-3}(\mathrm{aq})+6 \mathrm{NO}_{3}^{-}(\mathrm{aq})=\mathrm{Fe}^{+3}(\mathrm{aq})+6 \mathrm{CO}_{3}^{-2}(\mathrm{aq})+6 \mathrm{~N}_{2}(\mathrm{~g}) \\
& 5 \mathrm{TOC}(\mathrm{aq})+4 \mathrm{NO}_{3}^{-}(\mathrm{aq})+2 \mathrm{H} 2 \mathrm{O}(\mathrm{aq})=5 \mathrm{CO}_{2}(\mathrm{~g})+4 \mathrm{OH}^{-}(\mathrm{aq})+2 \mathrm{~N}_{2}(\mathrm{~g})
\end{aligned}
$$

Table B2 contains the fractional component conversions for organic and ferrocyanide destruction, as well as metal hydroxide precipitation.

With cooling and pressure reduction of the outlet stream from the reactor, evolved $\mathrm{CO}_{2}$ and $\mathrm{N}_{2}$ gases are readily separated and sent to offgas treatment. Subsequent SLS results in a solid slurry containing metal hydroxides that are routed to caustic dissolution; liquid from the centrifuge is one of several feeds to the basic-side separations.

\section{B5.0 SOLID DISSOLUTION}

Three dissolution steps along with undissolved sludge recycle are included in the flowsheet to minimize the amount of sludge routed to highlevel waste $(H L W)$. One dissolution cycle involves sequential sludge processing through the following steps: (1) caustic leach, (2) nitric acid/oxalic acid dissolution, and (3) nitric acid/hydrofluoric acid dissolution, where SLS occurs after each of these steps. Several hours of digestion at approximately $90^{\circ} \mathrm{C}$ is anticipated for all these processes, under the following base/acid conditions:

$$
\begin{aligned}
& \text { Caustic Leach: } \quad\left[\mathrm{OH}^{-}\right]=4.0 \mathrm{M} \\
& \begin{array}{l}
\text { Nitric/0xalic } \\
\text { Acid Dissolution: } \quad\left[\mathrm{C}_{2} \mathrm{O}_{4}{ }^{-2}\right]=0.3 \mathrm{M} \text { (approx. } 1 \text { mole/mole }
\end{array}
\end{aligned}
$$

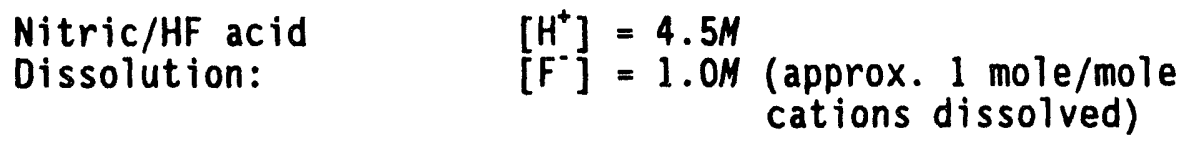

The extents of dissolution assumed by component are shown in Table B3 for each dissolution process.

In the ASPEN PLUS model, 95\% of the undissolved solids from the second acid dissolution step are returned for caustic leaching to begin another dissolution cycle, with the remaining $5 \%$ solids diverted to HLW treatment. Recycling 95\% of the undissolved solids is necessary to achieve the same total 
dissolution of cancrinite (96\%) as is attained by J. Swanson's proposed 9-cycle batch processing scheme (Swanson 1993), which cannot be modeled in ASPEN PLUS. Although recycling 95\% of the undissolved solids slightly overpredicts (relative to the 9-cycle batch scheme) the amount of solid components that accompany cancrinite to HLW treatment, the impact of this overprediction on total HLW volume is not significant because cancrinite is the dominant constituent of the undissolved solids.

Offgas from both acid dissolution processes contains $\mathrm{NO}_{2}$ formed by nitrite ion acidification. The offgas is contacted with water and oxygen to convert $90 \%$ of the $\mathrm{NO}_{2}$ to nitric acid. This regenerated nitric acid is recycled to the dissolvers to reduce nitric acid feed requirements.

\section{B6.0 TRIBUTYL PHOSPHATE SOLVENT EXTRACTION OF TRANSURANIC COMPONENTS}

The tributyl phosphate (TBP) solvent extraction (SE) process is designed for the removal of uranium, plutonium, neptunium, and thorium. The combined feed to this process consists of the dissolved sludge stream from the acid dissolution portion of the flowsheet and a waste solution from the subsequent uranium purification cycle. The solvent composition is the same, 30\% TBP in hydrocarbon diluent, as is usually used in the well-known plutonium-uranium extraction (PUREX) process.

In the first extraction contactor of this TBP cycle, uranium, plutonium, neptunium, and thorium are extracted into the TBP organic phase, which is then scrubbed with nitric acid to increase the separation from the bulk metal ions, some of which have a slight extractability. The raffinate from this contactor contains lanthanides and the remaining components of the dissolved sludge solution and is routed to $\mathrm{N}$-diisobutylcarbamoylmethylphosphine oxide (CMPO) SE of lanthanides. The extracted plutonium, neptunium, and thorium are selectively stripped back into an aqueous phase in the second contactor (to be routed to HLW glass for disposal), and the uranium is stripped in the third contactor. The uranium is purified further in another TBP cycle, described in Section B7.0, so that it can be added to the existing uranium stockpile. The solvent is then washed with sodium carbonate solution to prepare it for reuse.

Table B4 presents the percentage split of feed components among the various product streams (see Figure B1) from this process. The dissolved sludge stream is assumed to be decontaminated from uranium and plutonium by factors of $10^{4}$ and from thorium and neptunium by factors of $10^{2}$. Cesium, strontium, americium, and trivalent lanthanides are virtually inextractable by TBP, and technetium extracts very little at the high acidity used here $\left(\sim 4 \mathrm{HNO}_{3}\right)$. Thus, these elements will remain in the dissolved sludge stream in this step, along with the nonradioactive elements present in the stream. The TBP solvent loss is assumed to be minimal $(0.001 \%)$ because downstream evaporators recover and recycle any solvent carried away in the aqueous streams. 


\section{B7.0 URANIUM PURIFICATION}

Final uranium purification is accomplished in a second TBP cycle that is based on the 2D Cycle of the Hanford PUREX Plant. In this cycle, the uranium stream is first concentrated by evaporation and is then sent to a TBP extraction contactor; this contactor is run at low acidity and at a high degree of saturation of the TBP by uranium; hydroxylamine reductant is employed in the scrub stream to enhance removal of fission product and transuranic (TRU) elements. These conditions lead to a significant uranium "loss" from the contactor, but the "loss" is recovered by backcycling this stream (after evaporation) to the start of the first TBP cycle. Finally, the purified uranium is again stripped into dilute acid; this stream is concentrated by evaporation and then calcined to convert the uranium to $\mathrm{UO}_{3}$ for transfer to the stockpile.

The distribution of key components among the outlet streams from this operation is shown in Table B5. The solvent from the second TBP cycle is also washed with carbonate to prepare it for reuse. The TBP solvent losses are assumed to be $0.001 \%$ because of downstream evaporators that will recover TBP from the aqueous streams.

\section{B8.0 CMPO SOLVENT EXTRACTION FOR LANTHANIDE REMOVAL}

The raffinate from the first TBP cycle is routed to a CMPO SE process to remove trivalent 1 anthanides, americium, and bismuth. This extraction process contains extraction, stripping, and solvent wash steps. The solvent used is $0.2 M$ CMPO + 1.4M TBP in hydrocarbon diluent.

In the extraction step, $99.99 \%$ of the trivalent lanthanides and americium, and $99.5 \%$ of the bismuth in the dissolved sludge solution are extracted by the solvent. The raffinate from this extraction retains essentially all the cesium, strontium, and technetium and undergoes further processing for removal of these components as described in later sections.

Americium and trivalent actinides are stripped from the CMPO extract into dilute nitric acid in the second contactor. Bismuth is retained in the extract under these stripping conditions and is removed from the solvent in a subsequent sodium carbonate wash step. EDTA complexant is added in this wash step to prevent bismuth precipitation. The resulting carbonate solution containing bismuth is sent to low-level waste (LLW) treatment; the washed solvent is recycled to the CMPO extraction step.

Table B6 shows the distribution of key components among the outlet streams from this process. Solvent losses are assumed to be $0.001 \%$ because downstream evaporators will recover solvent from the aqueous outlet streams of the extraction, stripping, and solvent wash steps. 


\section{B9.0 AMERICIUM/LNTTHANIDE SEPARATION BY BAND DISPLACEMENT CATION EXCHANGE}

Two cation exchange resins loaded in sequential columns are used to separate americium and curium from lanthanides in the strip solution from the first CMPO cycle. Component distributions among the outlet streams from this operation are provided in Table B7.

In the first cation exchange column, americium, curium, and trivalent lanthanides are selectively sorbed onto the resin. Following a water wash of the resin, the trivalent ions are eluted with $0.05 \mathrm{~N} \mathrm{Na}$ diethylene-triaminepenta-acetic acid (DTPA) solution onto a zinc-loaded cation exchange resin in the second column. Continued elution through a series of columns establishes discrete bands of metal ion-DTPA complexes, which allow americium and curium to be isolated as a middle cut. After elution, the first and second cation exchange resin columns are regenerated with $\mathrm{HNO}_{3}$ and $\mathrm{Zn}\left(\mathrm{NO}_{3}\right)_{2}$ solutions, respectively. Subsequent water washes prepare the resin beds for the next cycle of operation.

\section{B10.0 CROWN ETHER SOLVENT EXTRACTION FOR STRONTIUM AND TECHNETIUM REMOVAL}

The raffinate from the first CMPO cycle, which contains all of the components of the dissolved sludge solution except the actinides, the lanthanides, and possibly the bismuth, is then fed to a crown ether (CE) SE step in which strontium and technetium are removed.

In this CE extraction cycle, the strontium and technetium are extracted from the dissolved sludge solution aqueous phase into the CE organic phase, which is then scrubbed with $\sim 1 \mathrm{M} \mathrm{HNO}_{3}$ to remove less strongly extracted species. The strontium and technetium are then stripped from the organic phase into a dilute acid aqueous phase. As shown in Table B8, this aqueous dilute acid stream contains $99.99 \%$ of the strontium and barium and $99 \%$ of the technetium in the feed to this operation.

\section{B11.0 STRONTIUM/BARIUM/TECHNETIUM SEPARATION BY BAND DISPLACEMENT CATION EXCHANGE}

Two cation exchange resins loaded in sequential columns are used to separate strontium, barium and technetium in the dilute acid stream from the CE extraction step described in the previous section.

In the first cation exchange column, strontium and barium are selectively sorbed onto the resin. Following a water wash of the resin, these ions are eluted with $0.05 \mathrm{M} \mathrm{Na}$ EDTA solution onto a zinc-loaded cation exchange resin in the second column. Continued elution through a series of columns establishes discrete bands of metal ion-EDTA complexes, which allows $99.99 \%$ of 
the strontium to be isolated as a middle cut. This strontium cut is handled as HLW and is assumed to include residual zinc and barlum as detalled in Table B9. The first cut containing zinc and the last cut containing barium are routed to LLW treatment.

After elution, the first and second cation exchange resin columns are regenerated with $\mathrm{HNO}_{3}$ and $\mathrm{Zn}\left(\mathrm{NO}_{3}\right)_{2}$ solutions, respectively. Subsequent water washes prepare the resin beds for the next cycle of operation.

\subsection{AMHONIUN PHOSPHOMOLYBDATE ION EXCHANGE FOR CESIUM REMOVAL}

The final acid-side processing step uses ammonium phosphomolybdate (APM) to remove $99.99 \%$ of the cesium from the residual sludge solution produced by the CE extraction operation described in Section 10.0. It is assumed that the resin used is APM on an alumina substrate (10 wt\% APM) to meet the mass and heat transfer requirements for this application. Because cesium cannot be readily eluted from APM, the loaded sorbent ( $50 \mathrm{~g}$ cesium $/ \mathrm{kg} \mathrm{APM}$ ) is routed to the caustic leach step of the sludge dissolution process (Section 5.0). The APM and its associated cesium will dissolve under caustic conditions and become part of the feed to the basic-side ion exchange processes.

\section{B13.0 RESORCINOL-FORMALDEHYDE ION EXCHANGE FOR CESIUM REMOVAL}

The combined basic-side feed undergoes $99.99 \%$ cesium removal via ion exchange with a resorcinol-formaldehyde resin. Table B10 provides volumetric information on the regeneration/wash streams required for this ion exchange process. Once the column is loaded with cesium, sodium hydroxide and water washes flush feed from the column and prepare it for elution with formic acid. The acid solution containing cesium stripped from the resin is routed to HLW processing. A subsequent eluant flush, using water, is followed by regeneration with sodium hydroxide to prepare the column for the next cycle.

\section{B14.0 CHELATING ION EXCHANGE FOR STRONTIUM REMOVAL}

The basic stream remaining after cesium ion exchange (CSIX) is sent to a chelating cation exchange column containing resin, which removes $99.99 \%$ of the strontium, barium, and calcium. Volumetric information on regeneration streams is provided in Table Bll.

After the resin has been loaded with strontium, barium, and calcium, it is flushed with sodium hydroxide and then water before eluting with $1 \mathrm{M} \mathrm{HNO}_{3}$. This acid stream containing strontium is concenivated by an evaporator to $\left[\mathrm{H}^{+}\right]=4.5 \mathrm{M}$ before being routing to the first acid dissolution step. 
An eluant water wash followed by sodium hydroxide regeneration prepares the fon exchange column for the next cycle.

\section{B15.0 NNION EXCHANGE REMOVAL OF TECHNETIUM}

The basic stream remaining after strontium ion exchange undergoes anion exchange to achieve $99.5 \%$ removal of technetium. Table B12 contains regeneration stream volumetric data assumed for this operation.

In this process, the pertechnetate ion is removed from the basic-side solution by sorption on a strong-base anion exchange resin. After the resin bed is loaded, it is washed with sodium hydroxide to remove residual feed solution and then with dilute nitric acid to ensure conversion of the resin to the nitrate form before the elution step is complete. The technetium is eluted with $6 \mathrm{M}$ nitric acid; the bed is prepared for the next loading cycle by displacing the acid first with water and then with sodium hydroxide.

Using acid distillation, the technetium product is concentrated to $12 M$ nitric acid and routed to HLW processing. The dilute acid stream resulting from the distillation is used in the next ion exchange cycle to reduce nitric acid consumption by this ion exchange process.

\section{B16.0 INITIAL TREATMENT FOR ACIDIC LOW-LEVEL WASTE TREATMENT}

The first treatment for the combined acidic LLW stream (No. 503 in Figure Bl) is evaporation to reduce its volume. Vapor from this evaporation results in a dilute nitric acid stream, which is sent to acid recovery. The concentrated liquid from evaporation $\left(5 \mathrm{M} \mathrm{HNO}_{3}\right)$ then undergoes denitration by reaction with sucrose according to the following overall stoichiometry:

$$
24 \mathrm{H}^{+}+24 \mathrm{NO}_{3}^{-}+\mathrm{C}_{12} \mathrm{H}_{22} \mathrm{O}_{11}=12 \mathrm{CO}_{2}+12 \mathrm{NO}+12 \mathrm{NO}_{2}+23 \mathrm{H}_{2} \mathrm{O}
$$

Sufficient sucrose is supplied to this denitration step to achieve $0.5 \mathrm{M}$ $\mathrm{HNO}_{3}$ in the liquid after complete sucrose conversion. This liquid is next fed to a calcination step as discussed in the next section. The oxides of nitrogen $\left(\mathrm{NO}_{\mathrm{x}}\right.$ ) produced by denitration is sent to the acid recovery portion of the flowsheet for conversion back to $\mathrm{HNO}_{3}$.

\section{B17.0 LOW-LEVEL WASTE CALCINATION}

The combined basic LLW stream (No. 500 in Figure B1) is concentrated by evaporation of water to achieve $7 \mathrm{M} \mathrm{NaOH}$ in the condensate (stream No. 517 in Figure B1) from the calcination step about to be described. This concentrated LLW stream from evaporation (stream No. 502 in Figure B1) is mixed with the 
dilute acid stream (No. 512 in Figure B1) obtained from the initial acidic LLW treatment and fed to a calcination process for destruction of organic complexants and conversion of nitrates and nitrites to hydroxide. The reaction stoichiometry for conversion to caustic is described by the following reactions, where $90 \%$ conversion of nitrate and nitrite is assumed.

$$
\begin{aligned}
\mathrm{NaNO}_{3} & =\mathrm{NaNO}_{2}+0.5 \mathrm{O}_{2} \\
2 \mathrm{NaNO}_{2} & =\mathrm{Na}_{2} \mathrm{O}^{2}+\mathrm{N}_{2}+1.5 \mathrm{O}_{2} . \\
\mathrm{Na}_{2} \mathrm{O}+\mathrm{H}_{2} \mathrm{O} & =2 \mathrm{NaOH} .
\end{aligned}
$$

The 10\% remaining sodium nitrate and sodium nitrite decompose to NOx, which is destroyed by addition of ammonia. The overall calcination/NOx destruction stoichiometry is as follows.

$$
\begin{aligned}
& 3 \mathrm{NaNO}_{3}+5 \mathrm{NH}_{3}=4 \mathrm{~N}_{2}+6 \mathrm{H}_{2} \mathrm{O}+3 \mathrm{NaOH} \text {. } \\
& \mathrm{NaNO}_{2}+\mathrm{NH}_{3}=\mathrm{N}_{2}+\mathrm{H}_{2}^{2} \mathrm{O}+\mathrm{NaOH} \text {. }
\end{aligned}
$$

It is assumed that $100 \%$ excess $\mathrm{NH}_{3}$ is supplied, where unreacted $\mathrm{NH}_{3}$ is oxidized to nitrogen and water.

After condensing the reactions products, nitrogen gas is separated from the caustic slurry $(7 \mathrm{M} \mathrm{NaOH})$, which undergoes treatment as described in the next section.

\section{B18.0 CAUSTIC TREATMENT FOR SODIUM HYDROXIDE REUSE}

The caustic slurry produced in the calcination operation contains $7 M$ sodium hydroxide, which can be recovered for process uses to minimize LLW disposal volume. To el iminate chromium from recycled sodium hydroxide, soluble $\mathrm{Cr}$ (VI) is reduced to insoluble $\mathrm{Cr}$ (III) with hydroxylamine. After solid-liquid separation, approximately $30 \%$ of this caustic stream must be recycled to meet flowsheet requirements for sodium hydroxide (e.g., the caustic leach step of the solid dissolution operation). The remaining portion of the caustic slurry is incorporated into a LLW form for disposal.

\section{B19.0 HIGH-LEVEL HASTE STREAM TREATMENT}

The HLW streams, from the separation steps described in the previous sections, are combined and concentrated by evaporation to a $3 \mathrm{M} \mathrm{HNO}$ solution. Dilute acid vapor from this operation is sent to acid recovery. The $3 \mathrm{M} \mathrm{HNO}_{3}$ solution and formic acid eluant containing cesium from resorcinol-formaldehyde ion exchange (Section 13.0) are mixed and undergo denitration by reaction with sucrose and formic acid destruction.

$$
\begin{aligned}
& 24 \mathrm{H}^{+}+24 \mathrm{NO}_{3}^{-}+\mathrm{C}_{12} \mathrm{H}_{22} \mathrm{O}_{11}=12 \mathrm{CO}_{2}+12 \mathrm{NO}+12 \mathrm{NO}_{2}+23 \mathrm{H}_{2} \mathrm{O} \\
& \mathrm{HCOOH}+\mathrm{HNO}_{3}=2 \mathrm{NO}_{2}+\mathrm{CO}_{2}+2 \mathrm{H}_{2} \mathrm{O} .
\end{aligned}
$$


Sufficient sucrose is supplied to this denitration step to achieve $0.5 \mathrm{M}$ $\mathrm{HNO}_{3}$ in the liquid after complete sucrose conversion. This liquid is next fed to the HLW glass vitrification process along with undissolved solids from the acid dissolution operation. The $\mathrm{NO}_{x}$ produced by denitration are sent to the acid recovery portion of the flowsheet for conversion back to $\mathrm{HNO}_{3}$.

\section{B20.0 NITRIC ACID RECYCLING}

Acidic evaporator overheads (from HLW treatment, strontium ion exchange, and acidic LLW treatment) are contacted with $\mathrm{NO}_{\mathrm{x}}$ streams (from denitration and calcination steps) with the addition of hydrogen peroxide and air to absorb and convert $\mathrm{NO}_{x}$ to nitric acid. Conversion of $80 \% \mathrm{NO}_{x}$ to acid is accomplished by oxidation by oxygen.

$$
\begin{aligned}
& 2 \mathrm{NO}+1.5 \mathrm{O}_{2}=2 \mathrm{HNO}_{3}+\mathrm{H}_{2} \mathrm{O} . \\
& 2 \mathrm{NO}_{2}+.5 \mathrm{O}_{2}=2 \mathrm{HNO}_{3}+\mathrm{H}_{2} \mathrm{O} .
\end{aligned}
$$

The remaining $20 \%$ of the $\mathrm{NO}_{x}$ is oxidized by hydrogen peroxide.

$$
\begin{aligned}
& \mathrm{H} 2 \mathrm{O}_{2}+\mathrm{NO}=\mathrm{NO}_{2}+\mathrm{H}_{2} \mathrm{O} . \\
& \mathrm{H}_{2} \mathrm{O}_{2}+2 \mathrm{NO}_{2}=2 \mathrm{HNO}_{3} .
\end{aligned}
$$

The resulting dilute acid liquid is concentrated by acid fractionation to recover $99.5 \%$ of the nitric acid as $12 \mathrm{M} \mathrm{HNO}_{3}$. The weak acid overhead is used as water recycle.

\section{B21.0 SLUDGE WASH C AND TRUEX-C MODEL DESCRIPTIONS}

The following model descriptions find reference in the systems Engineering Study for the Closure of Single-Shell Tanks (Boomer et al. 1991) and Tank Waste Technical Options Report (Boomer et al. 1993). In the latter document, the TRUEX $C$ process strategy is referred to as the Solex Extraction (SOLEX) C Option. Figure B2 shows the Sludge Wash C and TRUEX-C process strategies as they relate to the waste separations technology options defined in the Tank Waste Technical Options Report (Boomer et a1. 1993).

\section{B21.1 SLUDGE WASH C}

The following recoveries for radionuclides from combined sludge and salt to the fraction being disposed of offsite represent the principal process criteria for the Sludge Wash $C$ process strategy:

$$
\text { Sr } 99.9 \% \text { TC } 99 \% \text { Cs } 99 \% \text { Pu } 99.9 \% \text { Am } 99.9 \% \text {. }
$$


The Sludge Wash $C$ process strategy is made up of the following process steps: (a) Siudge Washing, (b) Organic Destruction using SCWO, (c) Sodium Titanate Sorption, (d) CSIX and Waste Concentration, and (e) Technetium Ion Exhange (TCIX) and Waste Concentration. A flowsheet of the Sludge Wash C process strategy is shown in Figure B3.

Tank waste is fed from retrieval to the sludge washing step, where a supernatant-solids separation on the retrieved waste is performed with continuous centrifuges as the solids are washed with water. The solid fraction is then neutralized before being transferred to HLW/TRU treatment, while the centrate is filtered. The filtrate is then sent to CSIX, and the supernate is further processed with organic destruction.

To destroy organic complexants, which solubilize significant multivalent TRU, fission products, and cations in the alkaline supernatant from sludge washing, a scwo process is used. In this process, complexants are oxidized, allowing multivalent cations to precipitate out in their hydroxide forms and be separated out later in the Sludge Wash $C$ process, resulting in a clarified supernatant with lower concentrations of TRU and strontium. To facilitate oxidation, high pressure oxygen in excess quantities is injected into a heated supernatant and fed to a high temperature vessel where organic destruction occurs. The temperature and pressure of the treated stream are reduced and the liquid stream is further treated with a sludge washing operation to remove the precipitated salts resulting from the destruction process. The solids are sent on to HLW treatment, while the supernatant is further processed with sodium titanate sorption.

The liquid stream from organic destruction is then treated to remove residual strontium and transuranics that remained in solution during this process. The adsorbent used is $37 \% \mathrm{NaTi}_{2} \mathrm{O}_{5} \mathrm{H}$ loaded on a macroreticular resin. The adsorbent is sent to HLW/TRU treatment after each loading cycle.

Clarified supernatant from sodium titanate adsorption is further treated with a cation ion exchange process, where cesium is removed in a series of columns loaded with a strong base organic anion exchange resin, Duolite ${ }^{1}$ CS-100. The cesium product solution is then neutralized with $\mathrm{NaOH}$ before being sent to HLW/TRU treatment, while the effluent regeneration streams are further processed using an anion exchange process.

To remove technetiul. from the CSIX effluent regeneration stream, a series/parallel configuration of ion exchange columns is used. Strong base resin is used for this process. The technetium product stream is neutralized before transfer to Tank Farm Storage/HLW treatment, while the supernatant is adjusted for hydroxide and nitrite content before being sent to LLW storage.

The process facility is sized to process all tank waste in 13 years of operation with a $60 \%$ online efficiency $(2,850$ operating days).

'Duolite is a trademark of Rohm and Haas. 


\section{B21.1.1 Sludge Wash C Chemical Makeups}

Fewer reagents are needed for chemical processing in Sludge Wash $C$ than in the TRUEX-C process because of the complexity of the TRUEX-C design. Wash and dilution water is used in the sludge washing, SCWO, and SCWO Effiuent clarification process steps. Oxygen gas is pressurized before utilization in the SCWO process. Nitric acid is used in the cesium and technetium ion exchange processes during column regeneration. Sodium hydroxide is used in these latter process steps and also added to neutralize various product streams before HLW/TRU treatment. Sodium titanate is the adsorbent used to remove residual strontium and TRUs from the SCWO effluent clarification section and is flushed out and replaced after loading has been accomplished.

\section{B21.2 TRUEX-C}

The TRUEX-C pretreatment option represents an additional degree of partitioning, which reduces the radionuclide content and increases the amount of inert solids going to onsite disposal, while increasing the radionuclide content and reducing the amount of inert solids destined for offsite disposal. The following recoveries for radionuclides from combined sludge and salt to the fraction being disposed of offsite represent the principal process criteria for the TRUEX-C process strategy:

$$
\text { Sr } 99 \% \text { TC } 99 \% \text { Cs } 99 \% \text { Pu } 99 \% \text { Am } 99 \% \text {. U } 99 \% \text {. }
$$

The TRUEX-C process strategy is made up of the following process steps: (a) Sludge Washing, (b) Organic Destruction using SCWO, (c) Sodium Titanate Sorption, (d) CSIX and Waste Concentration, (e) TCIX and Waste Concentration, (f) Dissolution, (g) Transuranic Extraction (TRUEX), (h) Technetium Removal, (i) Uranium Recovery, and $(j)$ Strontium Extraction (SREX). A flowsheet of the TRUEX-C process strategy is shown in Figure B4.

Solvent Extraction Feed Clarification is included in the dissolution step, TRUEX is split into extraction/scrubbing and the stripping process blocks, and SREX raffinate and CSIX feed clarification are included in the CSIX step.

Tank waste is pumped as a slurry from retrieval and is first separated into supernatant and solid fractions with continuous centrifuges and filters in a sludge washing process. The solids are sent to dissolution, and the supernatant is routed to the CSIX and Waste Concentration process, where the cesium is removed with a strong base organic anion exchange resin. Table B13 describes characteristics and assumptions pertaining to the solid portion of the waste stream ("sludge"), including acid dissolution of the solids for the TRUEX-C process strategy, respectively. The assumed solids in the waste feed are essentially an aluminum-sodium-iron-zirconium-uranium mixture of hydroxides, phosphates, and nitrates.

The Solids Dissolution process uses dissolvers to decrease the amount of solids sent to offsite waste treatment and dispocal. The slurry stream, made up of slurries from the sludge washing step, the Organic Destruction step, and the Sodium Titanate Sorption step, is split and pumped into large batch 
dissolvers where the solids are first reacted with nitric acid and then with sodium oxalate solution to facilitate dissolution, each batch cycle taking 48 hours total, including recharging of dissolvers. Ninety percent dissolution of all solids is assumed except for cancrinite and nickel (0\%).

Clarified dissolver solution is processed in the TRUEX process, a liquidliquid extraction process employing two banks of centrifugal contactors. The process serves to selectively remove the actinides and lanthanides from the feed, and then separate uranium, technetium, and thorium from the actinides and lanthanides. A commercially available bifunctional organophosphorus reagent, octyl(phenyl)-N, CMPO with TBP in a normal paraffin hydrocarbon (NPH) diluent, is used as the extractant in the TRUEX process. Process products include a raffinate stream, a TRU rich stream, and a technetium and uranium stream (nearly 100\% recovery here). The technetium-/uranium-rich stream from the TRUEX process is routed to a separation system where $99 \%$ of the technetium is removed by absorption on an amine-loaded substrate. The stream containing technetium is sent to Waste Concentration, the product of which is transferred to interim lag storage. Liquid-liquid extraction is again used to selectively extract and purify $84 \%$ of the uranium fed to TRUEX-C for resale. The raffinate stream from the TRUEX process is transferred to the SREX section where it is processed through centrifugal contactors for SREX, scrubbing, and stripping. The strontium-rich stream is sent to HLW/TRU lag storage.

The partitioning facility is sized to process all Tank waste in 13 year of operation with a $60 \%$ on ine efficiency $(2,850$ operating days). The process equipment includes evaporator capacity and tanks to replace existing tank farm evaporators.

\section{B21.2.1 Modifications Made to Existing TRUEX-C Model}

Flow split blocks were added to reroute the $\mathrm{HLW}$ around neutralization steps to provide an option for direct calcination of acidic HLW before neutralization. Also, reactor blocks were added to represent the oxidation of the HLW stream during either the calcination or vitrification process steps.

\section{B21.2.2 TRUEX-C Chemical Makeup}

Numerous chemical compounds are used throughout the TRUEX-C process, including nitric acid, polyelectrolyte, sodium hydroxide, sodium oxalate, octyl(phenyl)-N, CMPO with TBP in a NPH, oxalic acid, tetrahydrofurantetracarboxylic acid (THFTCA), ascorbic acid, sodium carbonate and bicarbonate, ferrous sulfamate, and dicyclohexano 19-crown-6 ether (DCH 18-Cr-6) in an n-octanol diluent. Large volumes of water are also used in the TRUEX-C process. Ion exchange resin is also used and regenerated. However, it will prubably require periodic changeout.

Nitric acid is lised in the TRUEX-C for several process steps with varied reasons. It is added to the dissolution step to dissolve the solids and to create a $3 \mathrm{MHNO}_{3}$ dissolver product, to the extraction, scrub, and strip 
contactors of TRUEX, uranium extraction (UREX), and SREX to aid in the solvent-solvent extraction, and to the CSIX columns to remove the captured cesium and regenerate the column.

Sodium hydroxide is added to TRUEX-C to regenerate the CSIX columns and to neutralize excess $\mathrm{H}^{+}$to meet tank farm specifications.

Sodium oxalate is used in the dissolution step subsequent to nitric acid digestion to promote further dissolution of the solids. Enough is added so that the exiting dissolver solution is $0.2 \mathrm{M} \mathrm{C}_{2} \mathrm{O}_{4}^{-2}$.

Oxalic acid is added to TRUEX-C to increase the amount of iron and zirconium extracted.

A CMPO/TBP/NPH mixture is used to extract the actinides, lanthanides, thorium, uranium, and technetium from the feed to the TRUEX step. The TBP/NPH is the organic extractant used in the UREX process step and serves to selectively extract in excess of $99 \%$ of the uranium from the feed.

A THFTCA/ascorbic acid/nitric acid solution is added to TRUEX to strip the uranium and technetium from the CMPO/TBP/NPH extractant.

Sodium carbonate is added to the TRUEX wash contactors to remove virtually all of the uranium, technetium, and thorium from the organic (THFTCA $/ \mathrm{C}_{6} \mathrm{H}_{6} \mathrm{O}_{8} / \mathrm{HNO}_{3}$ ) solution and is added to the technetium absorption column downstream of the contactors to remove the captured technetium.

Sodium bicarbonate is used to wash organic degradation products and impurities from the recycled organic routed to the solvent feed tanks for the TRUEX, SREX, and UREX process steps.

Ferrous sulfamate is added to the UREX feed to change the valence of plutonium from 4 to 3 . This increases the amount of plutonium that will be scrubbed out and, therefore, increases the $U 2^{+2}$ purity. process.

DCH 18-Cr-6 in an n-octanol diluent is the extractant used on the SREX

Air is fed to the dissolver offgas equipment and used in the conversion of $\mathrm{NO}_{x}$ to $\mathrm{HNO}_{3}$. A jet steam stream is used to propel the tank contents from one process to another. It is assumed that this adds 3 wt\% of water to the solution being jetted.

\section{B22.0 REFERENCES}

Boomer, K. D., et al., 1991, Systems Engineering Study for the Closure of Single-Shell Tanks, WHC-EP-0405, Rev. 0, Westinghouse Hanford Company, Richland, Washington.

Boomer, K. D., et al., 1993, Tank Waste Technical Options Report, WHC-EP-0616, Rev. 0, Westinghouse Hanford Company, Richland, Washington. 
Knutson, B. J., et al., 1993, "Advanced Chemical Separation Study: TRUEX Strategy Compared To Sludge Wash Ion Exchange," Letter report, Westinghouse Hanford Company, Richland, Washington.

Pajunen, A. L., et al., 1992, Hanford Strategic Analysis Study, WHC-EP-0549, Westinghouse Hanford Company, Richland, Washington.

Swanson, J. L., 1993, Clean Option: An Alternative Strategy for Hanford Tank Waste Remediation, Volume 2, Detailed Description of First Example

Flowsheet, PNL-8388, Pacific Northwest Laboratory, Rich1and, Washington. 
WHC-EP-0791

This page intentionally left blank.

B-18 

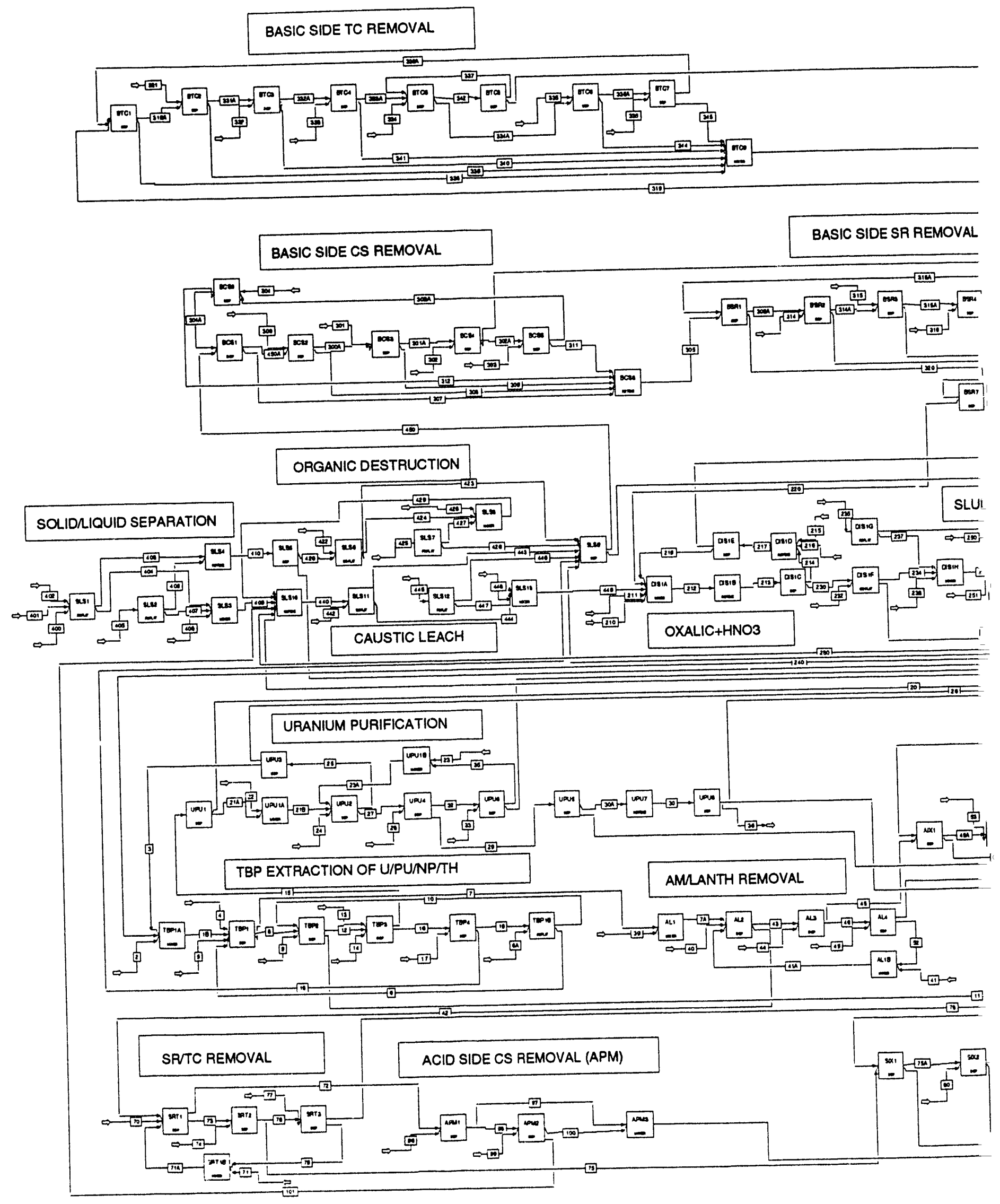
WHC-EP-0791

Figure B1. CLEAN Option Flowsheet.




Figure B2. Waste Separations Technology Options

as Defined in WHC-EP-0405, Rev. 0 .

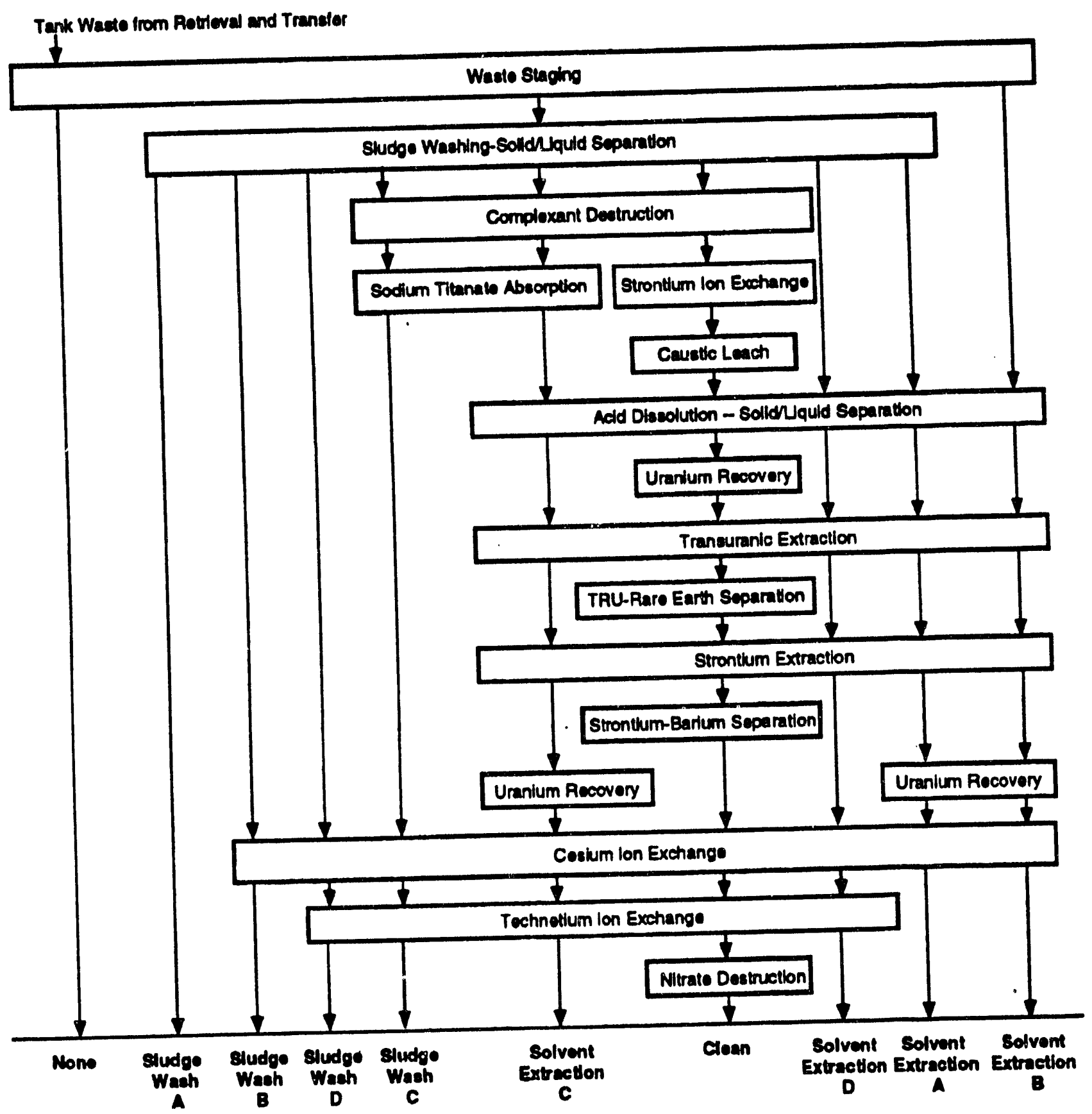


Figure B3. Sludge Wash C.

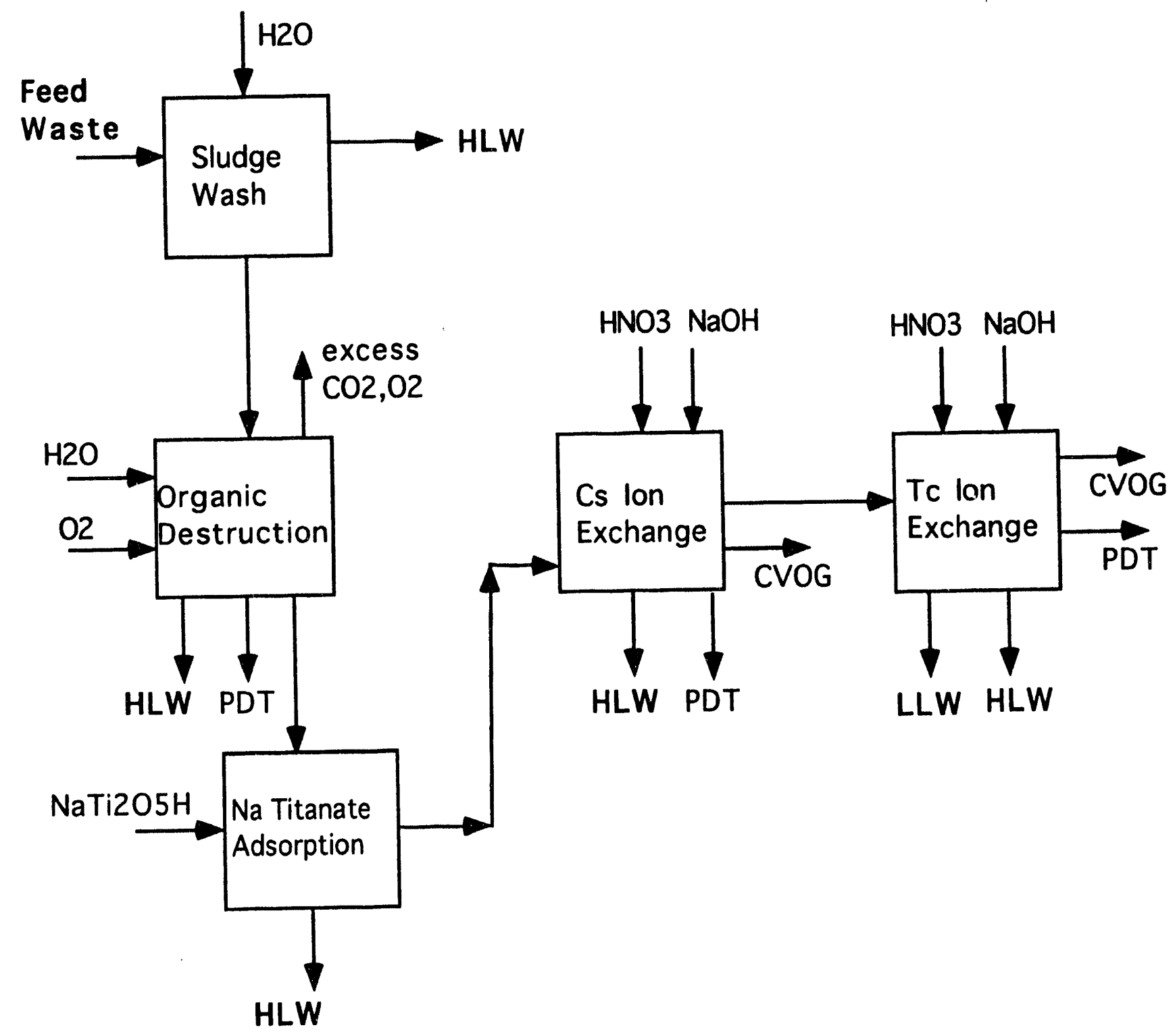


Figure B4. TRUEX-C.
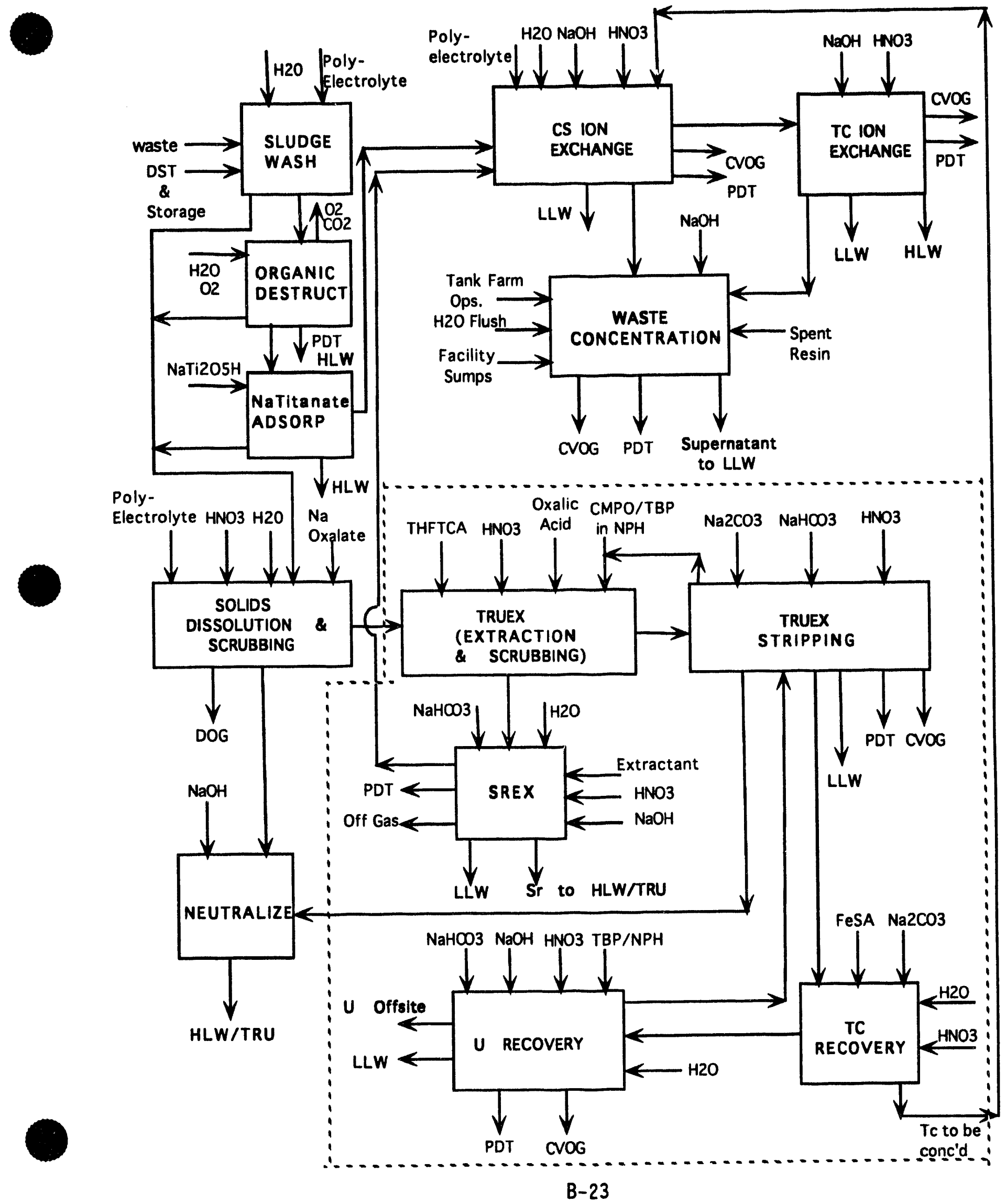
Figure B5. CLEAN Option IDEF Flowsheet Structure.

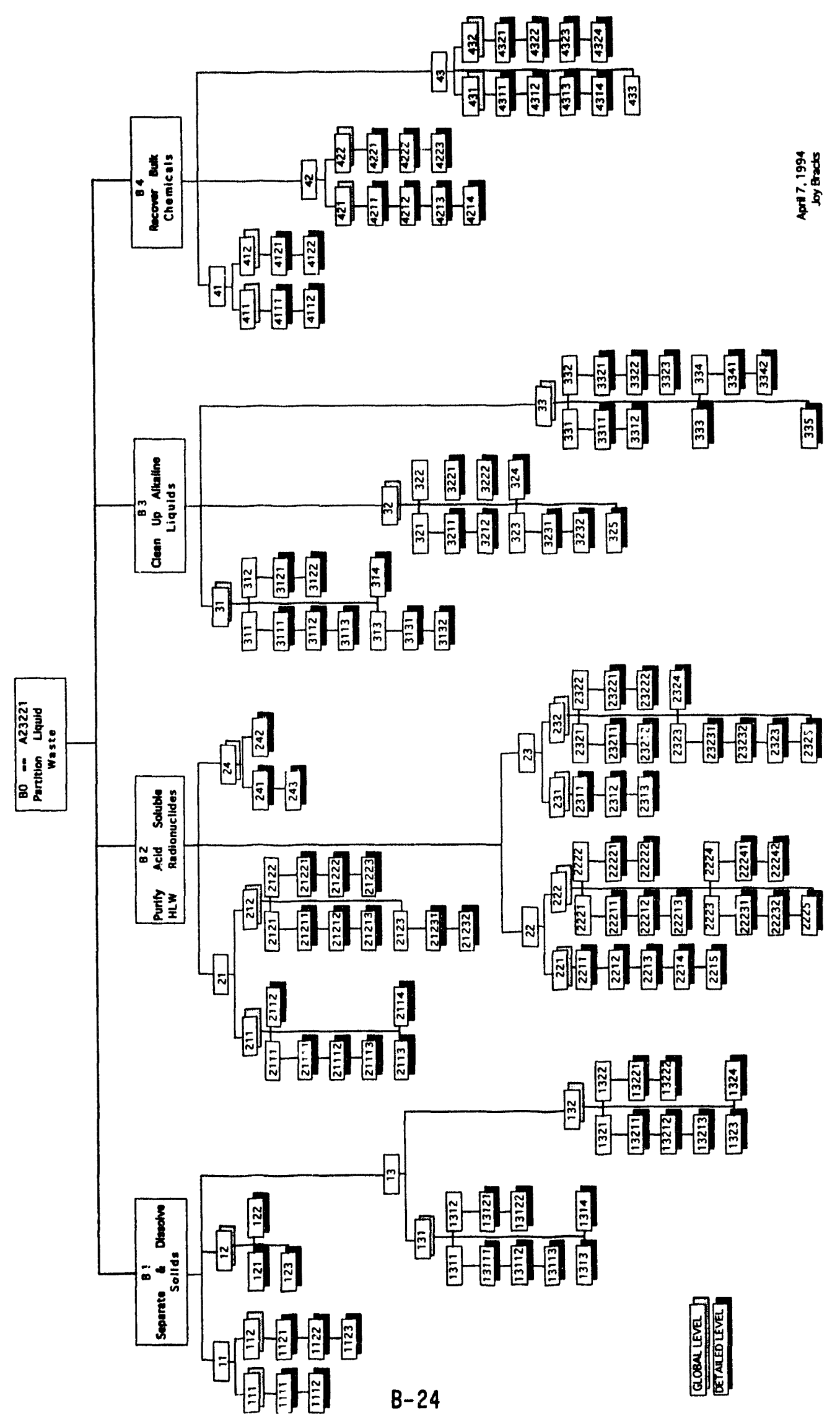









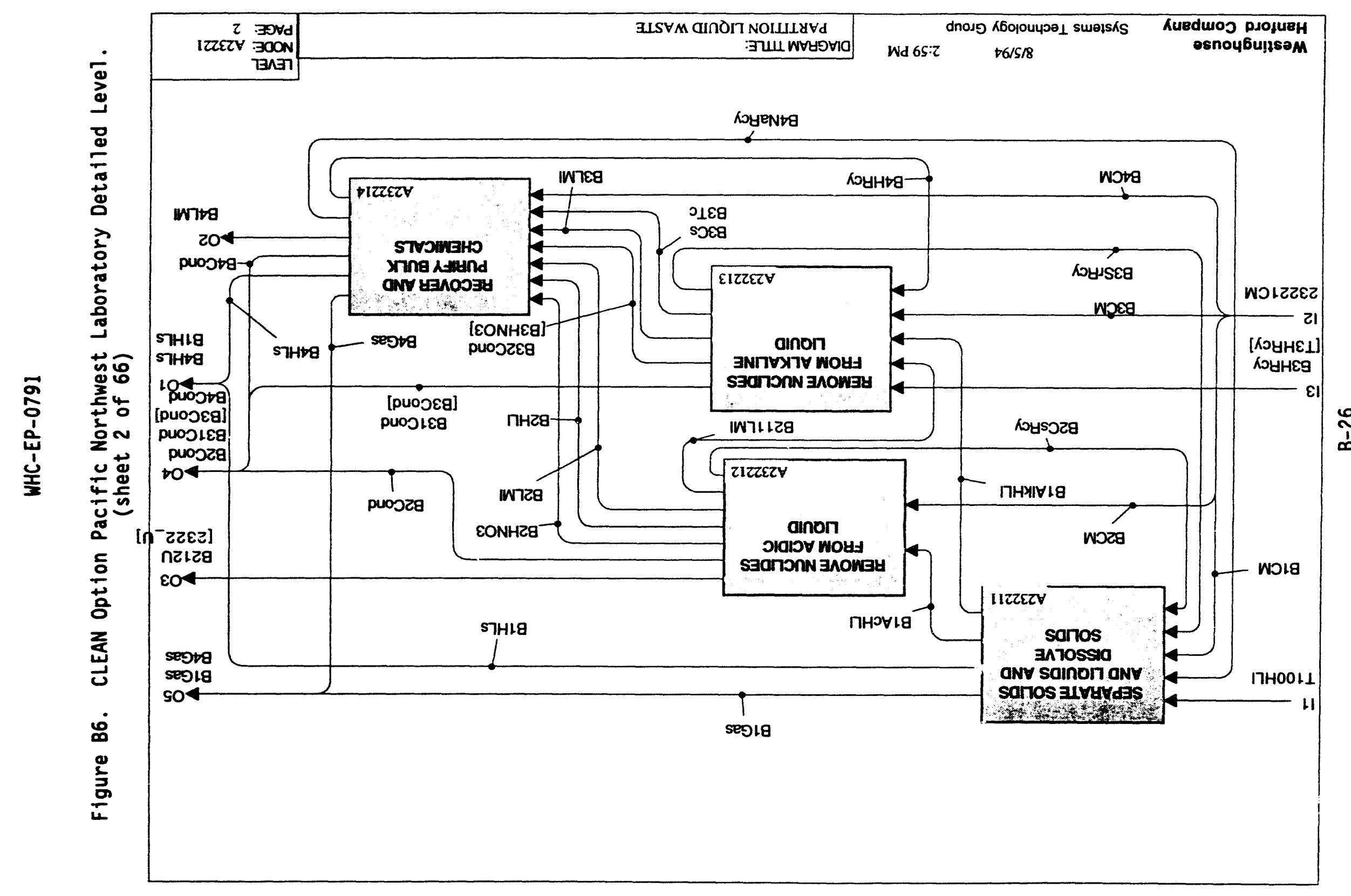


WHC-EP-0791

Figure B6. CLEAN Option Pacific Northwest Laboratory Detailed Level. (sheet 3 of 66)

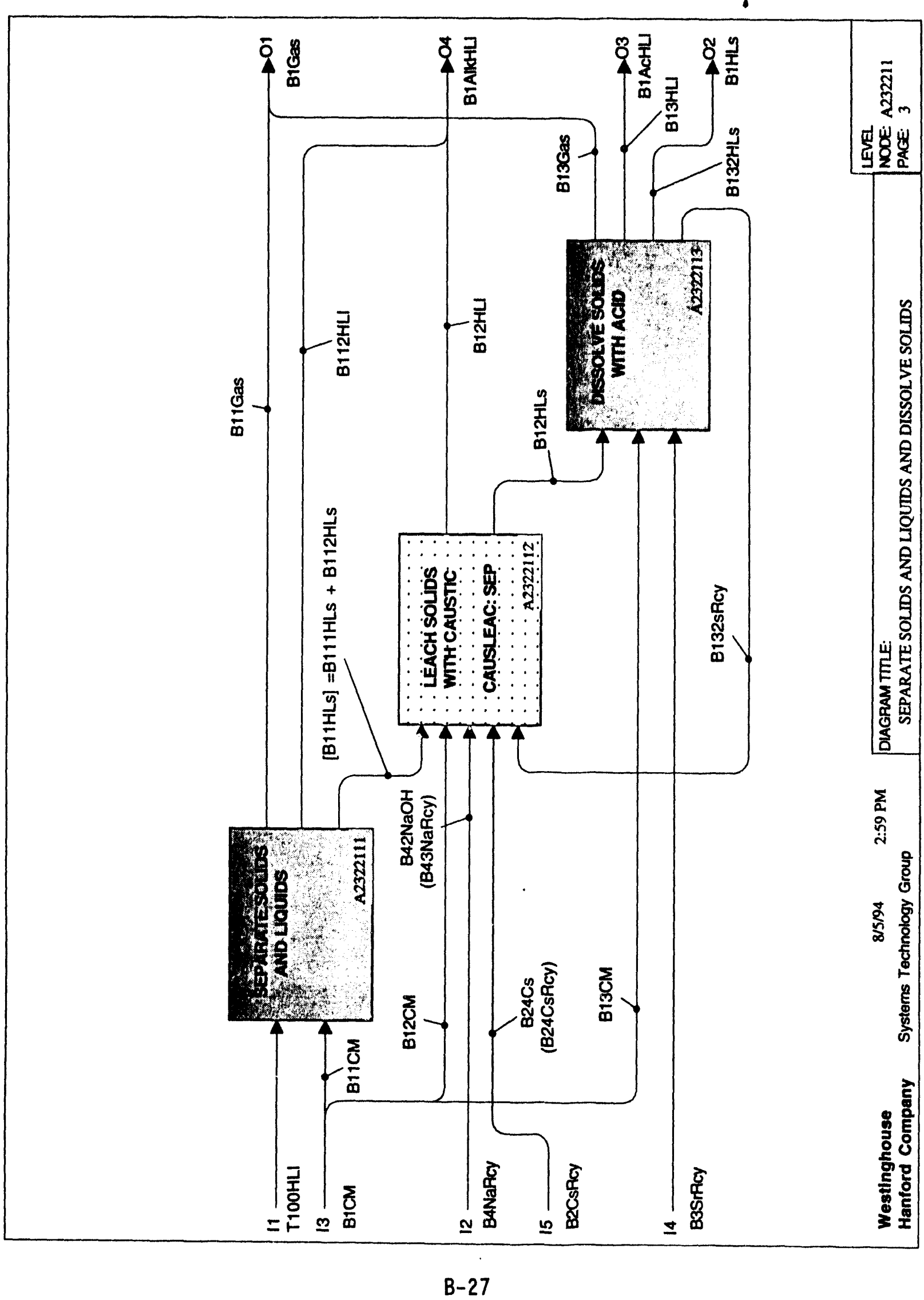



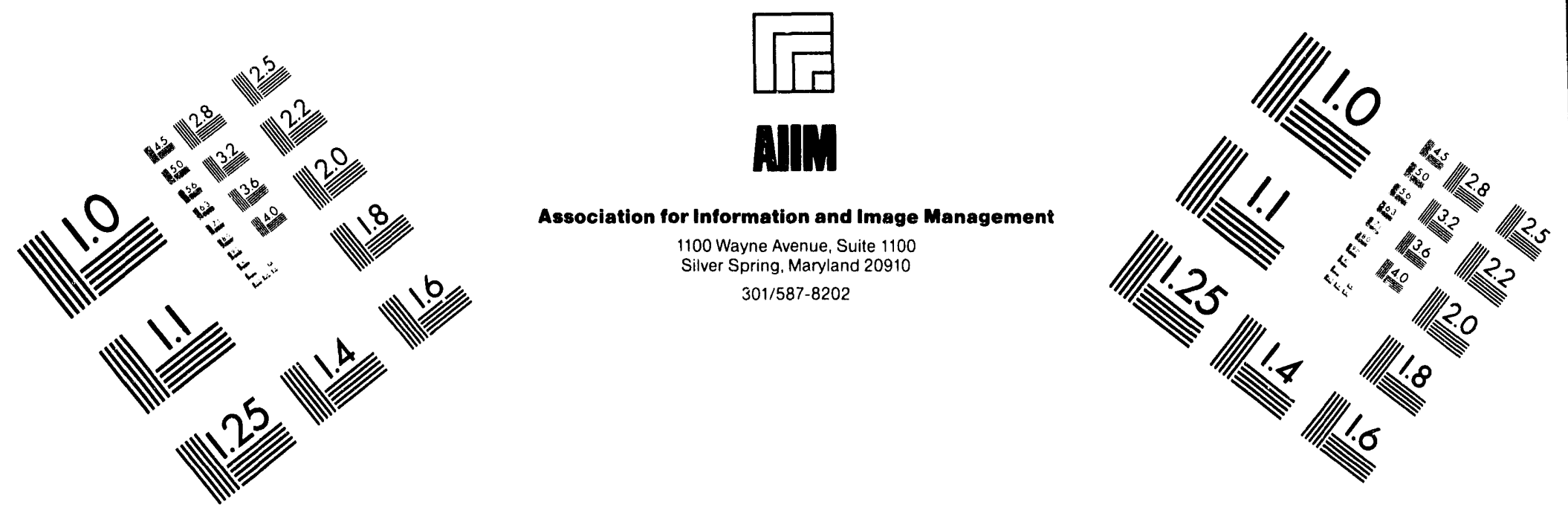

\section{Centimeter}

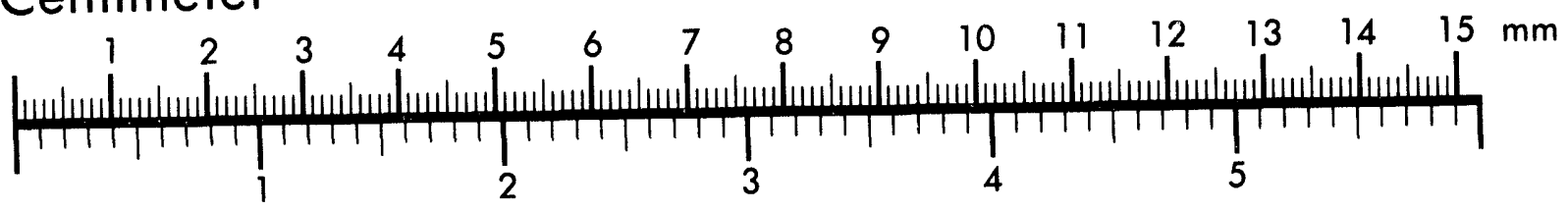
Inches
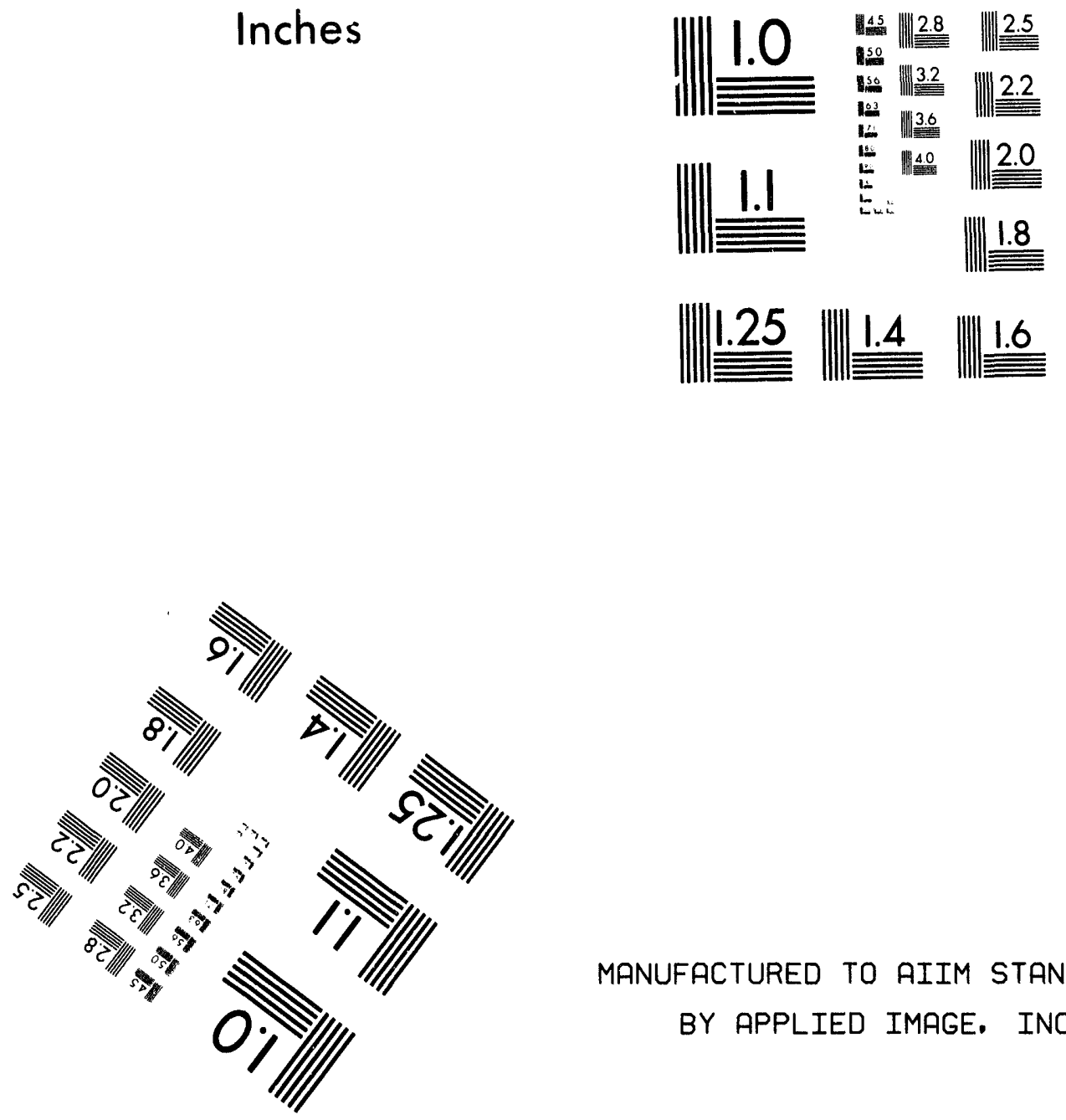

MANUFACTURED TO AIIM STANDARDS BY APPLIED IMAGE. INC.

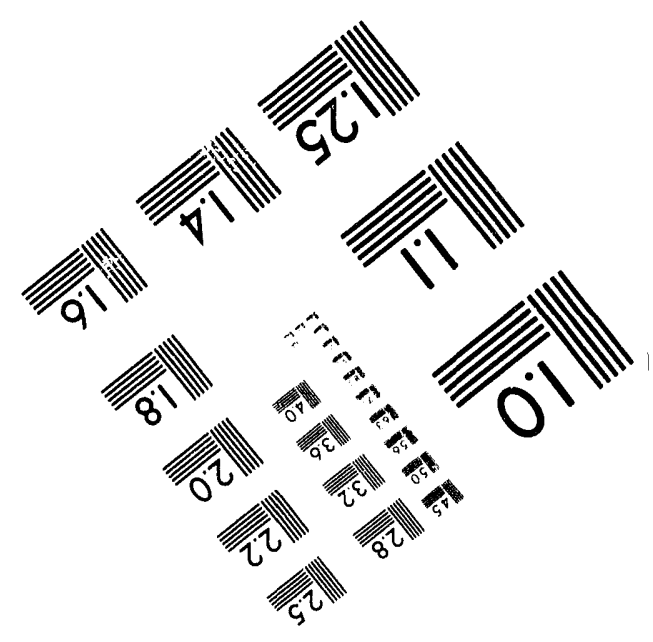



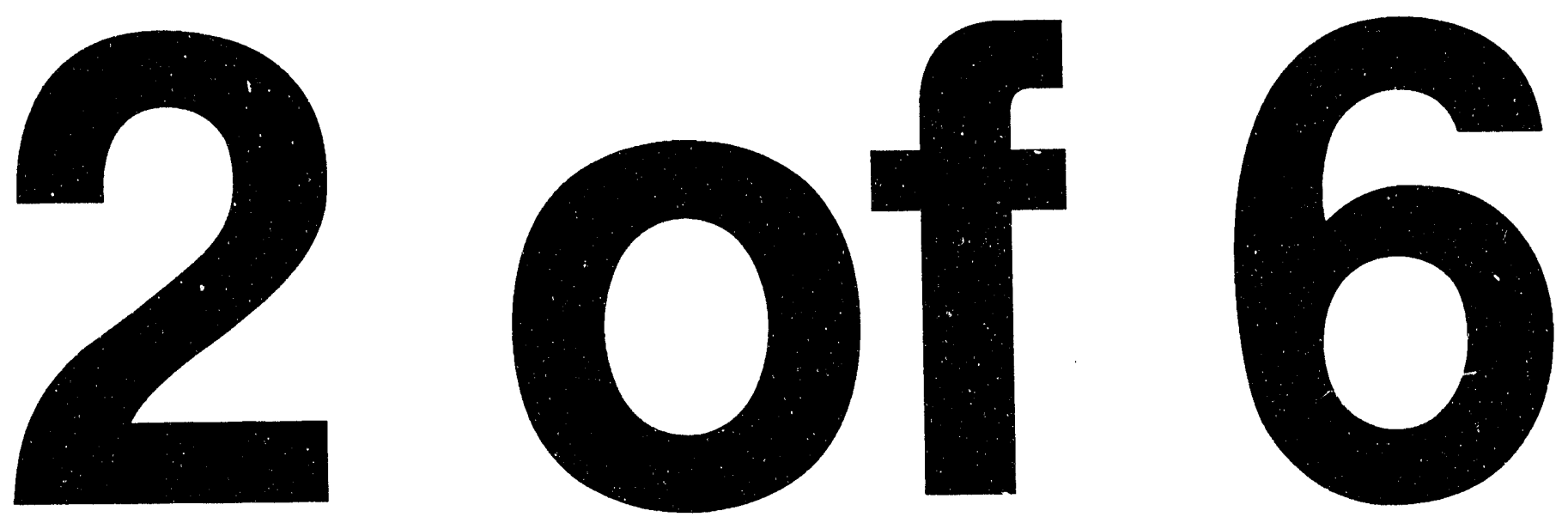


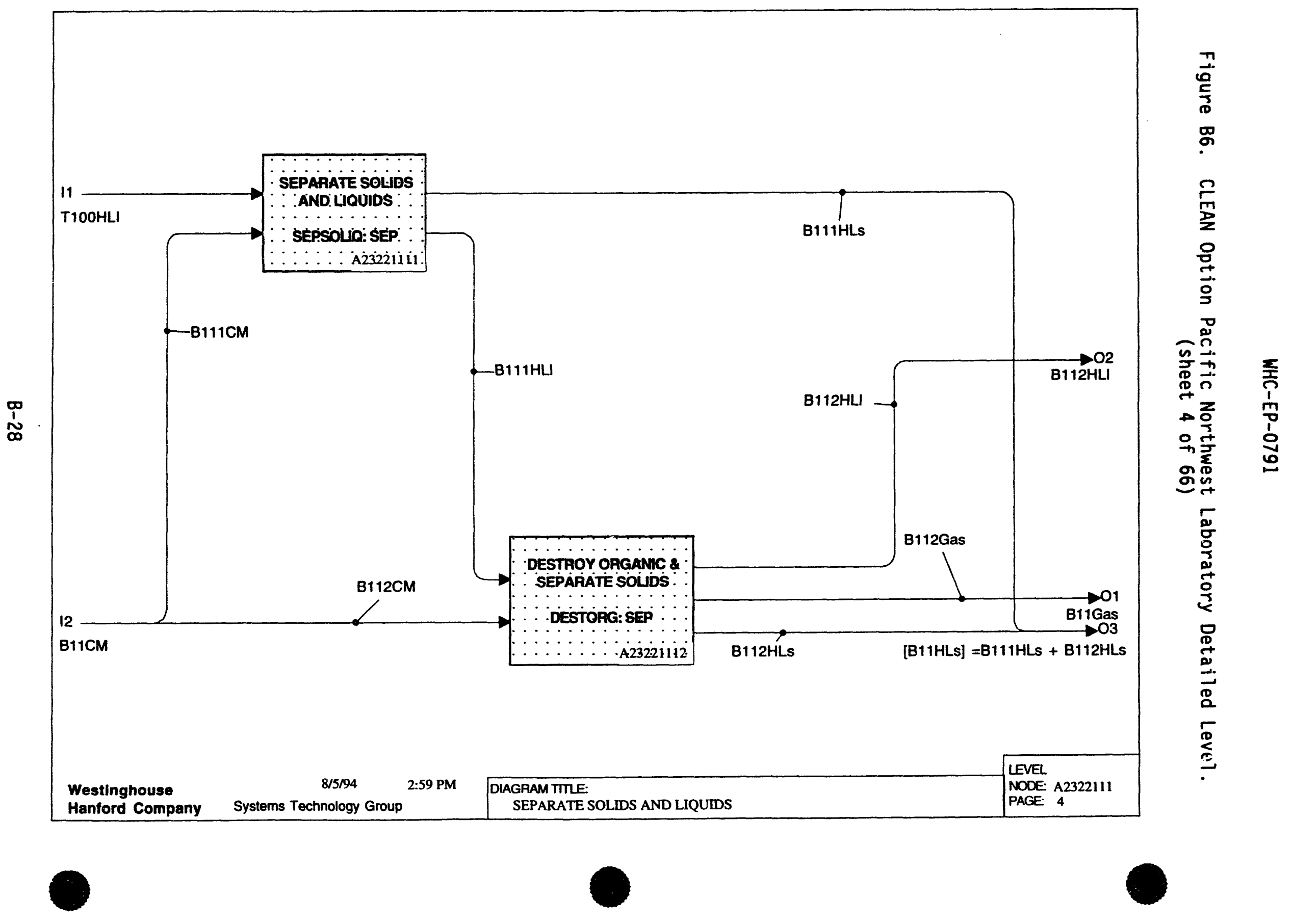




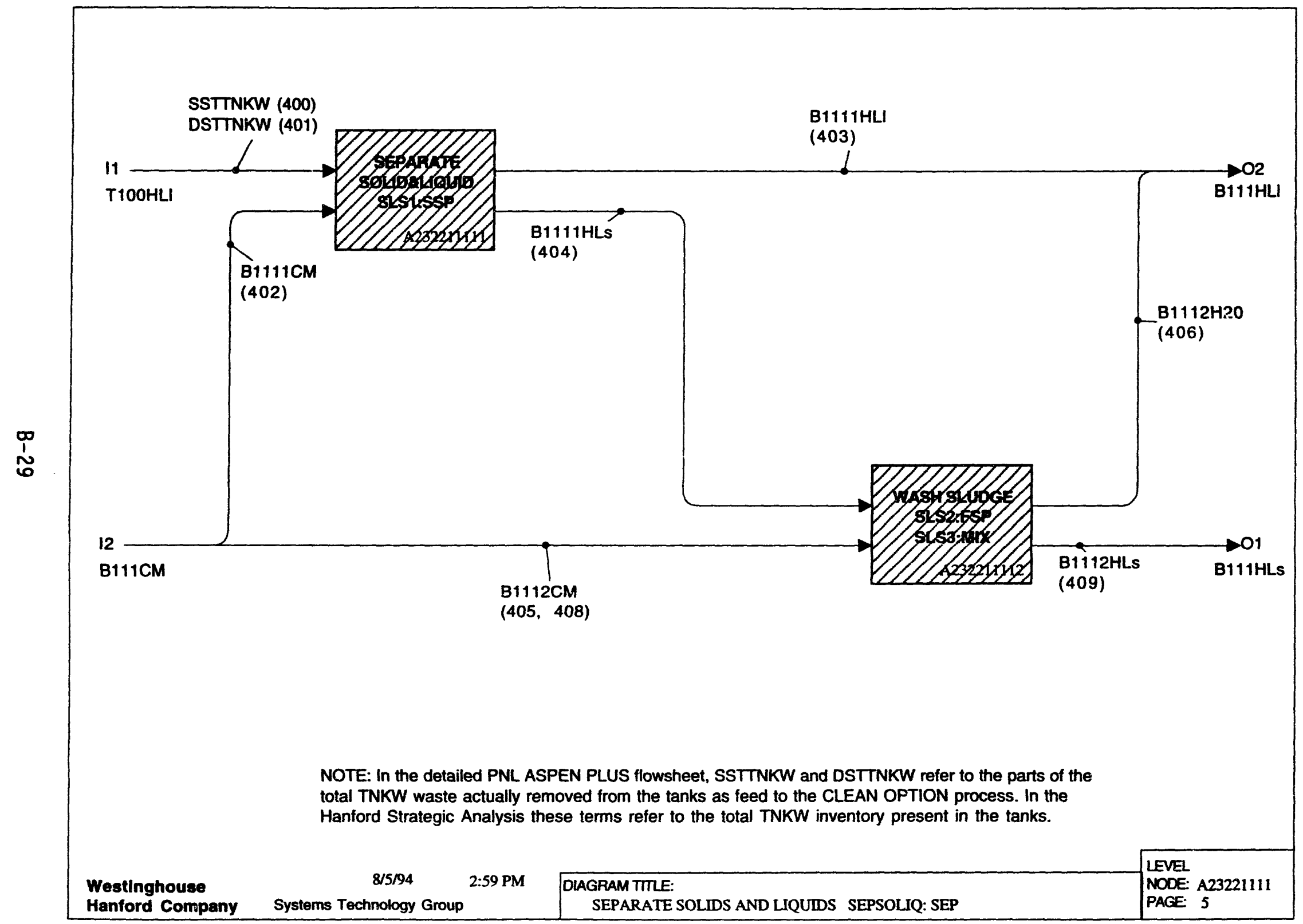




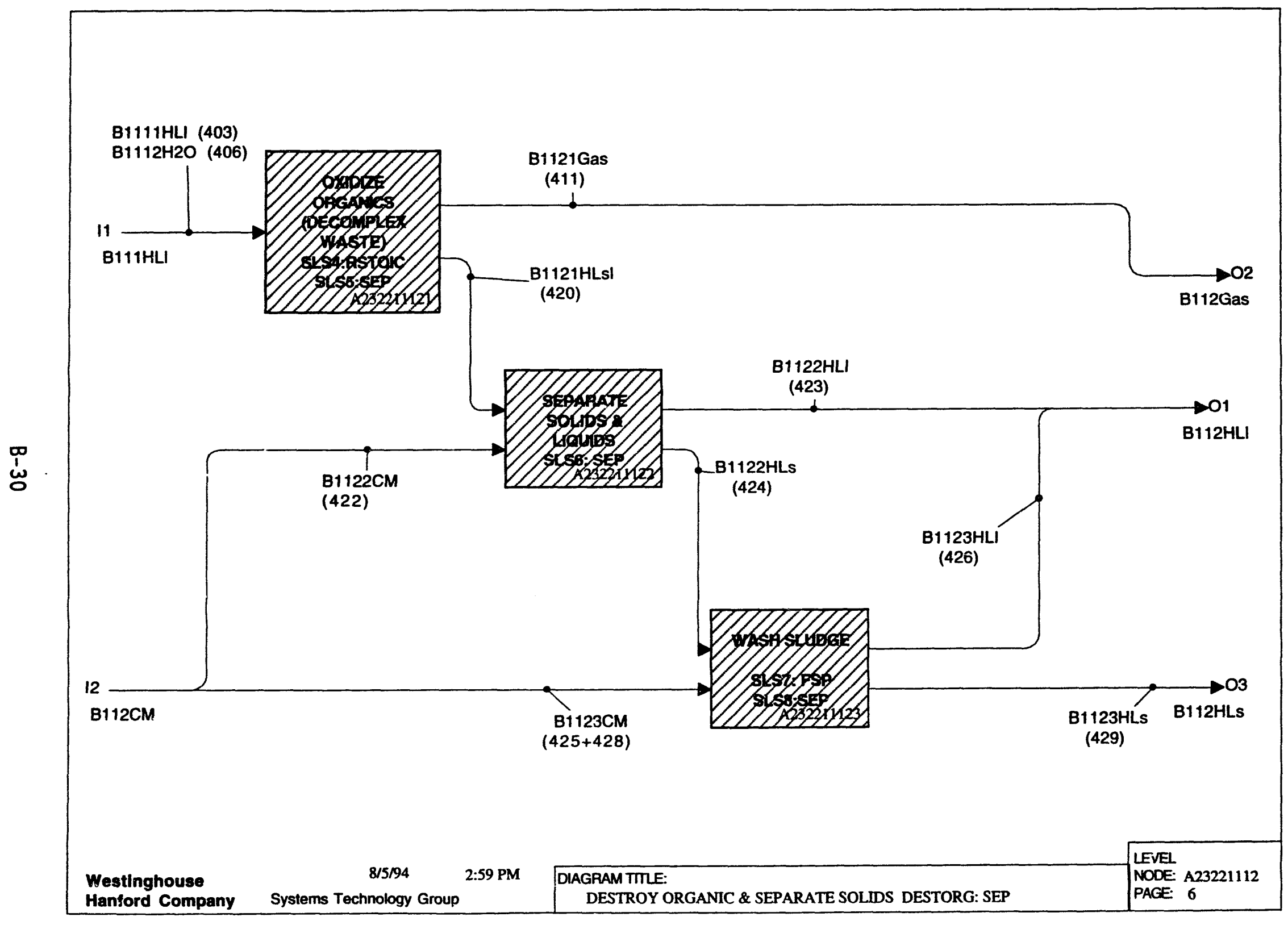

ำ

品

定

罗

뭄.

苟

势声

$+2$

욱

ตัฒ

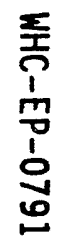

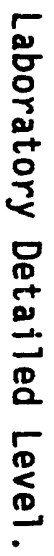




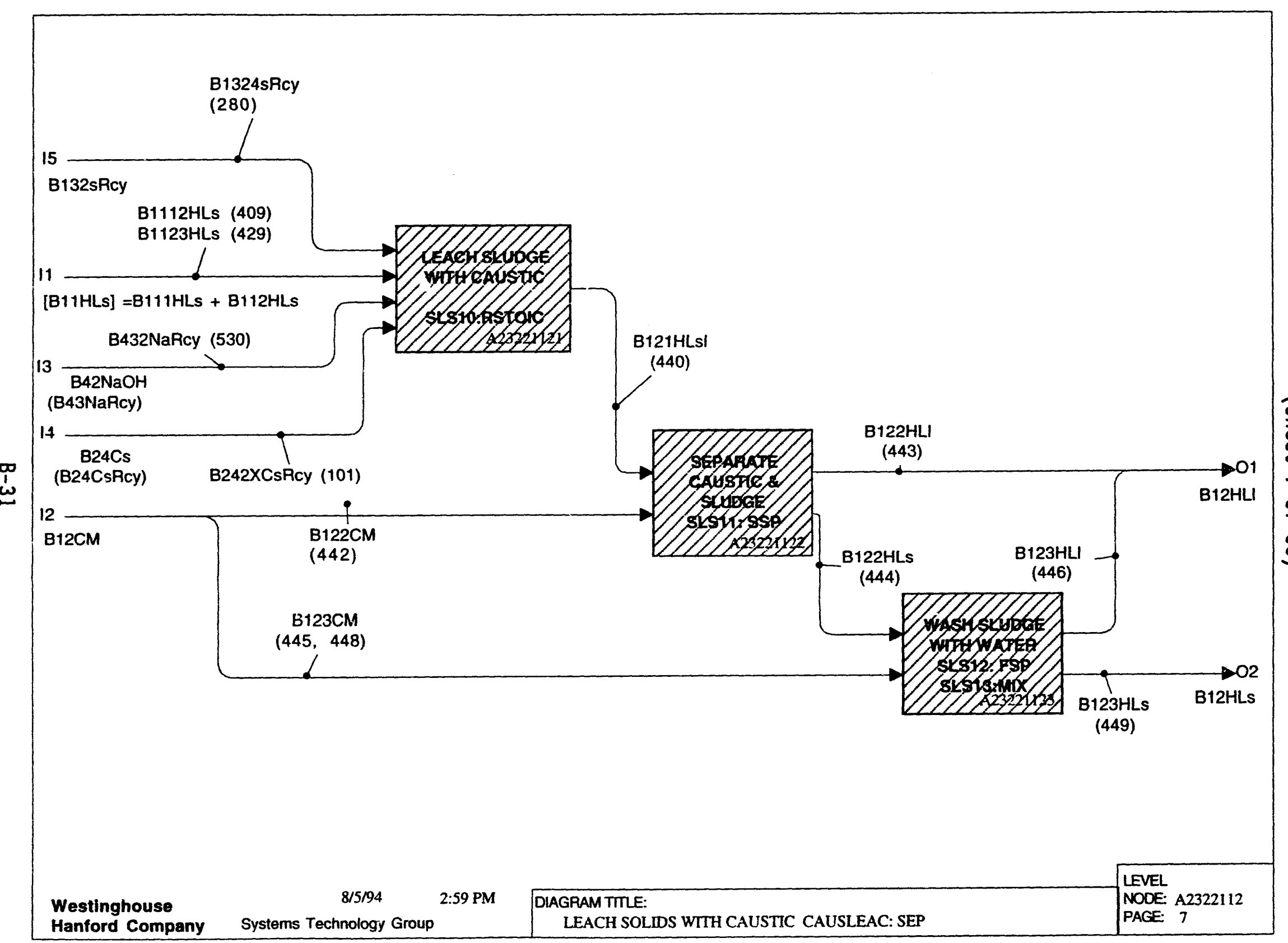

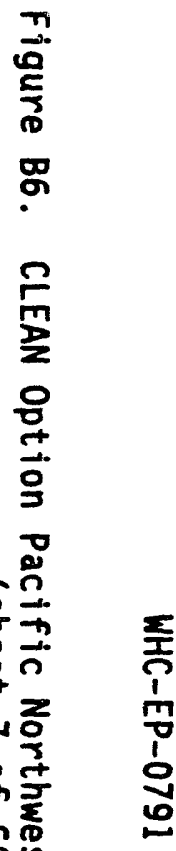




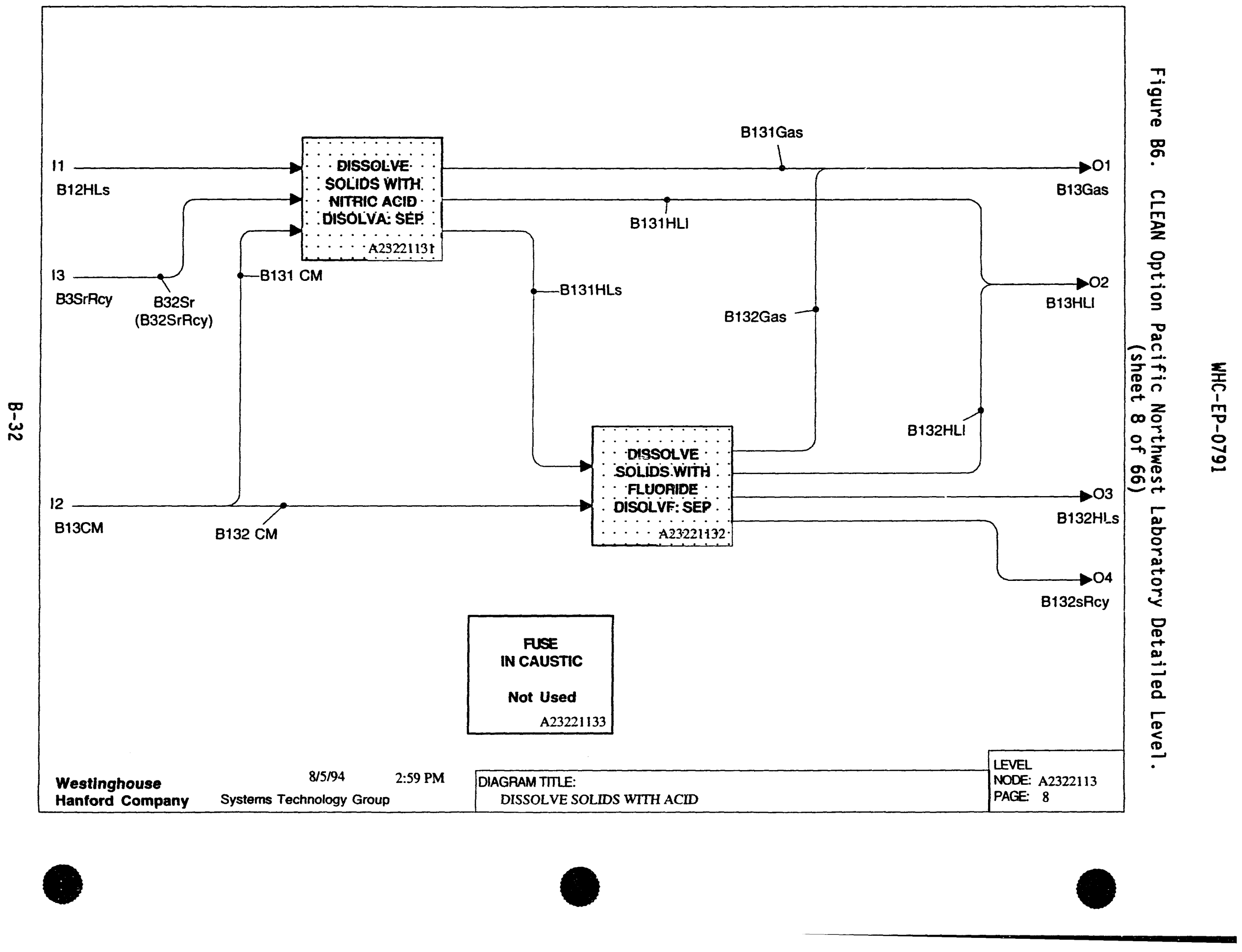




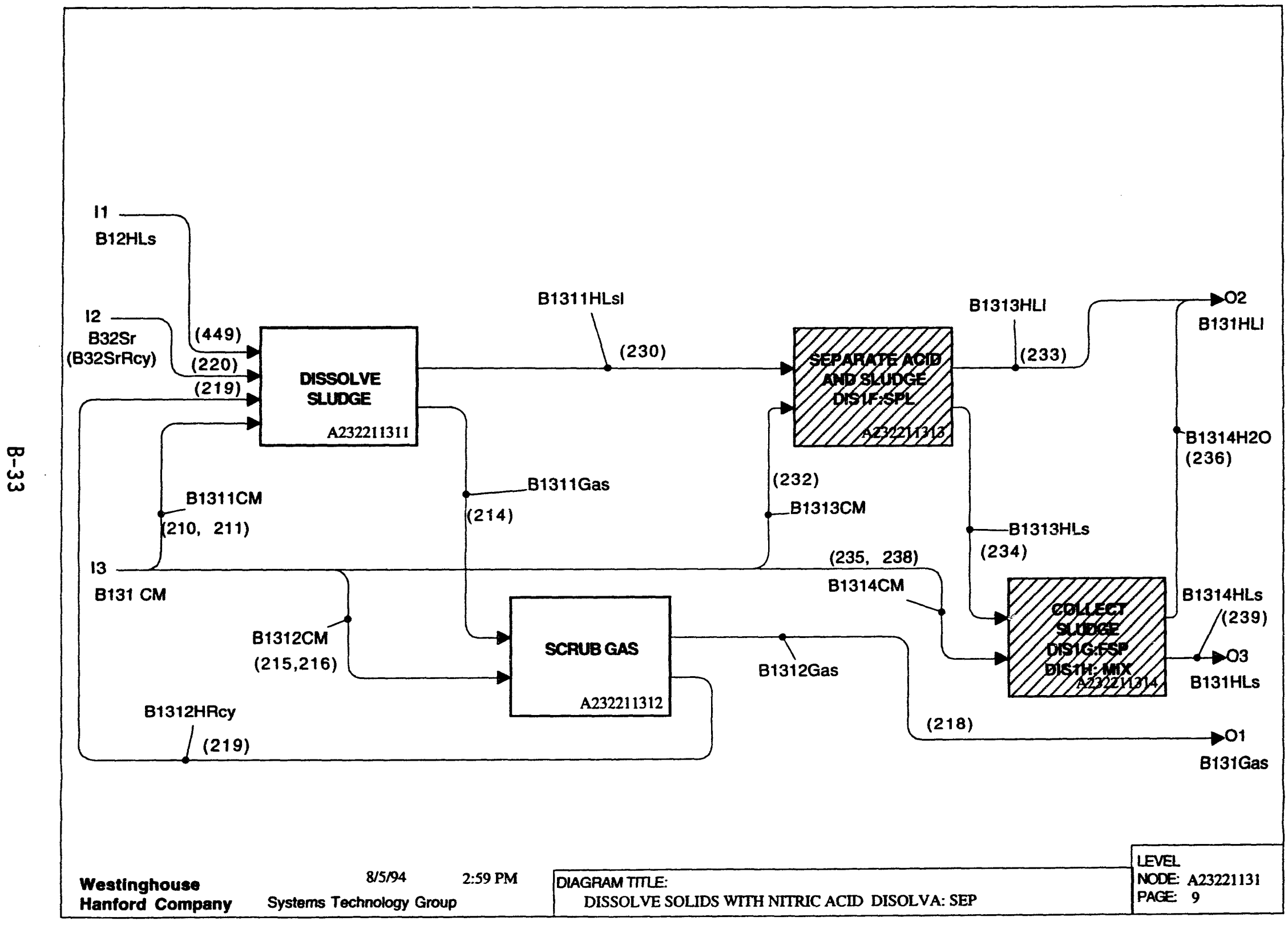

年

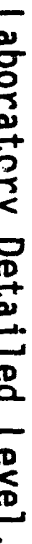




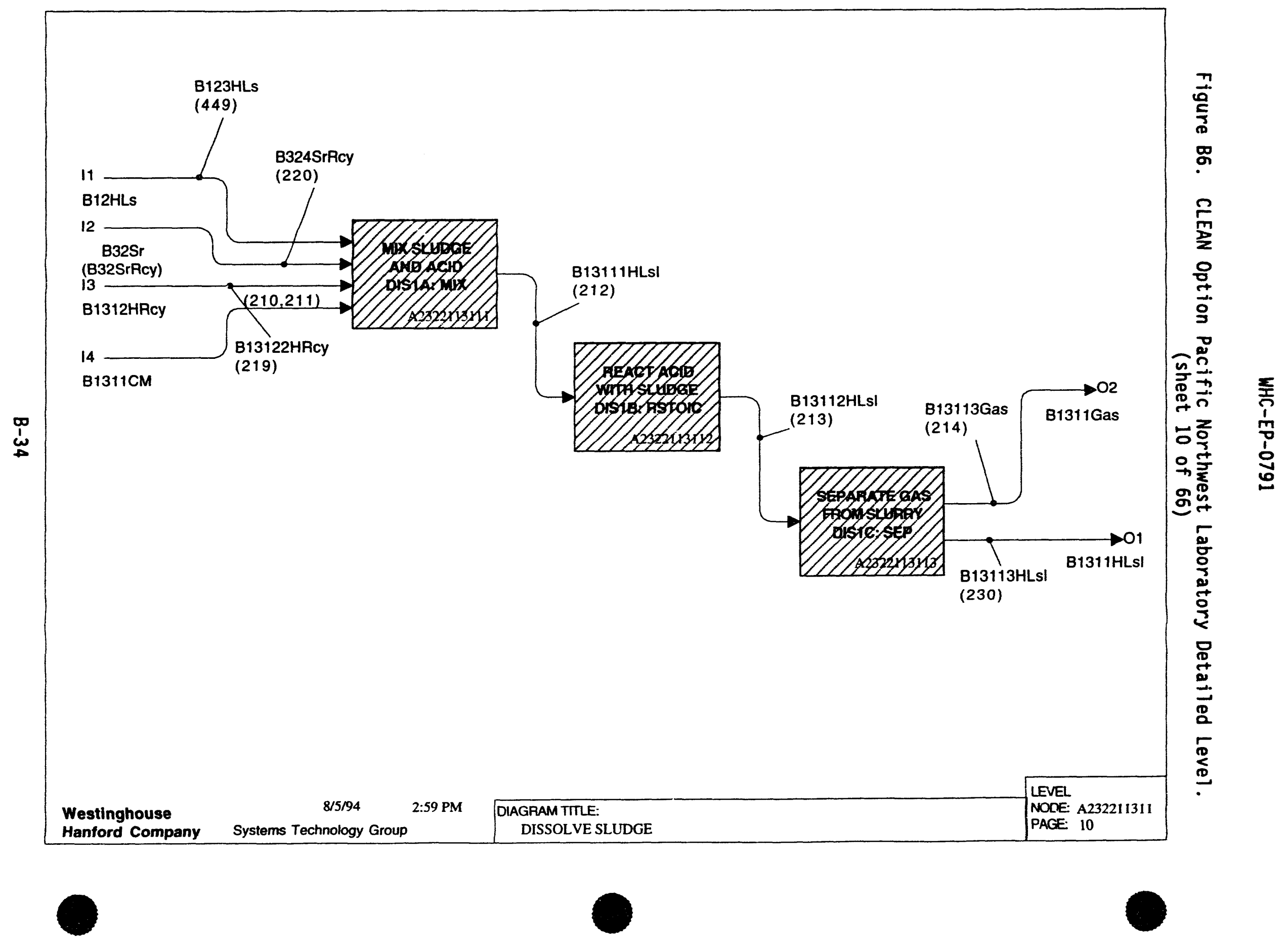




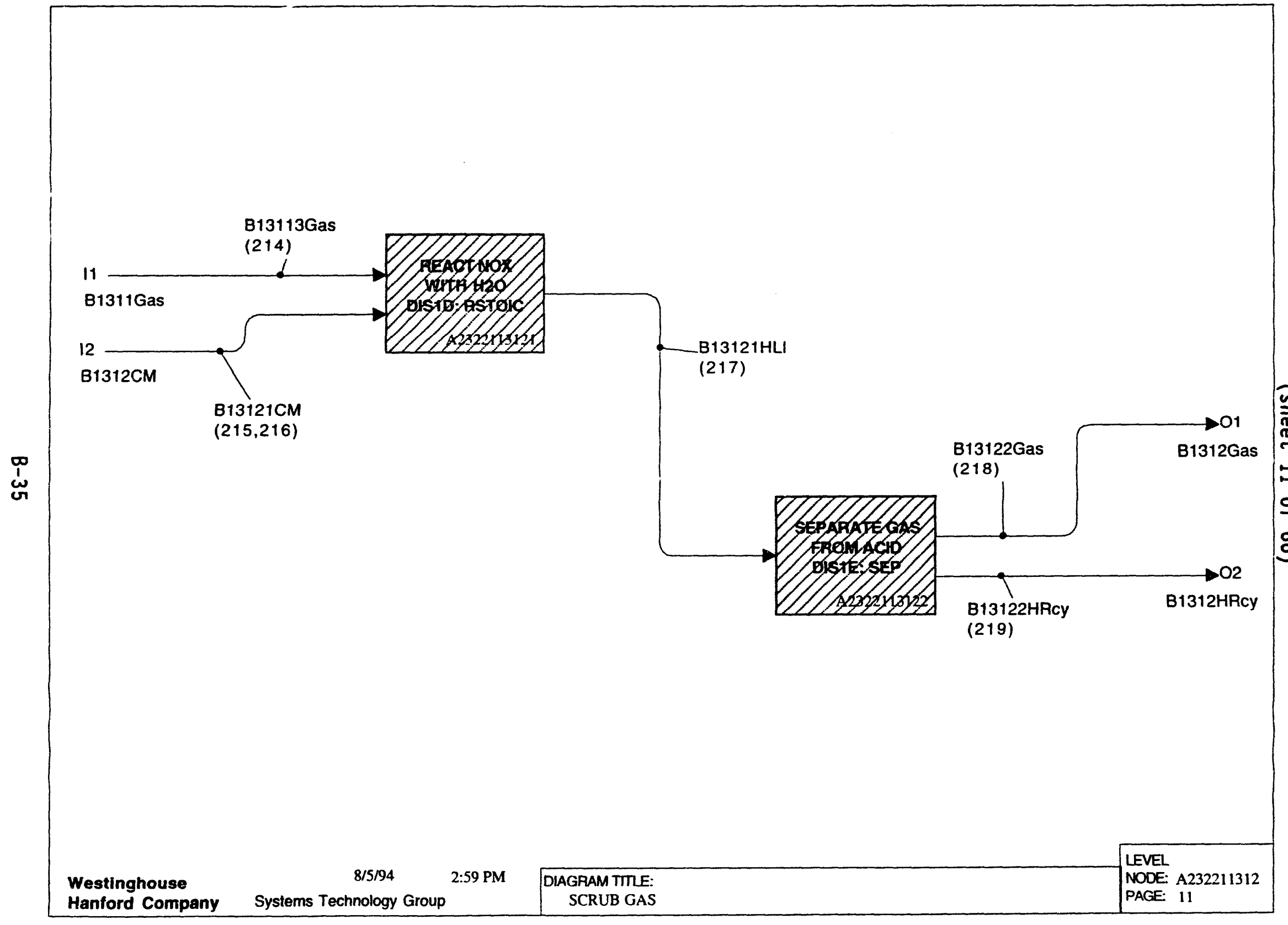




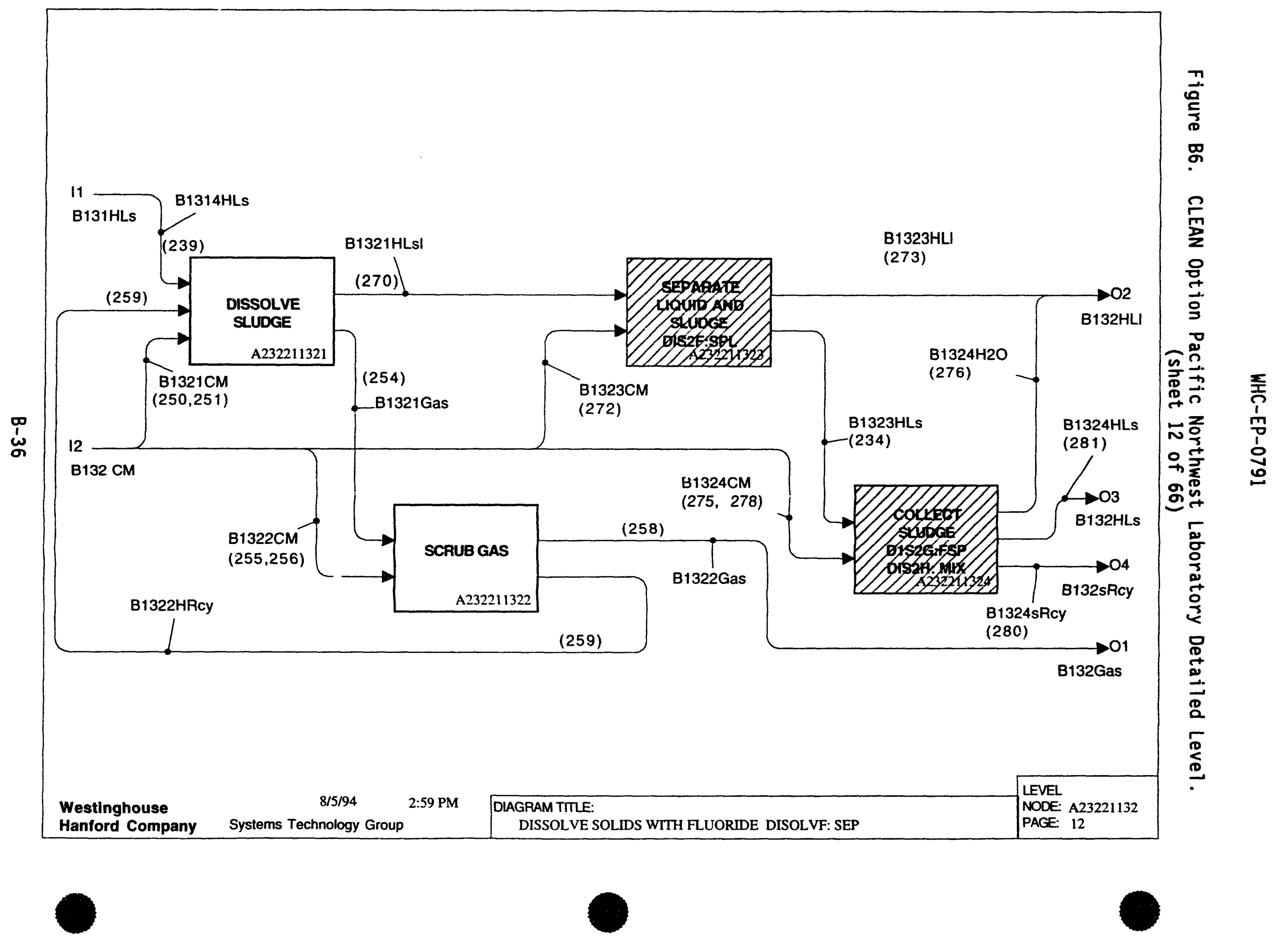




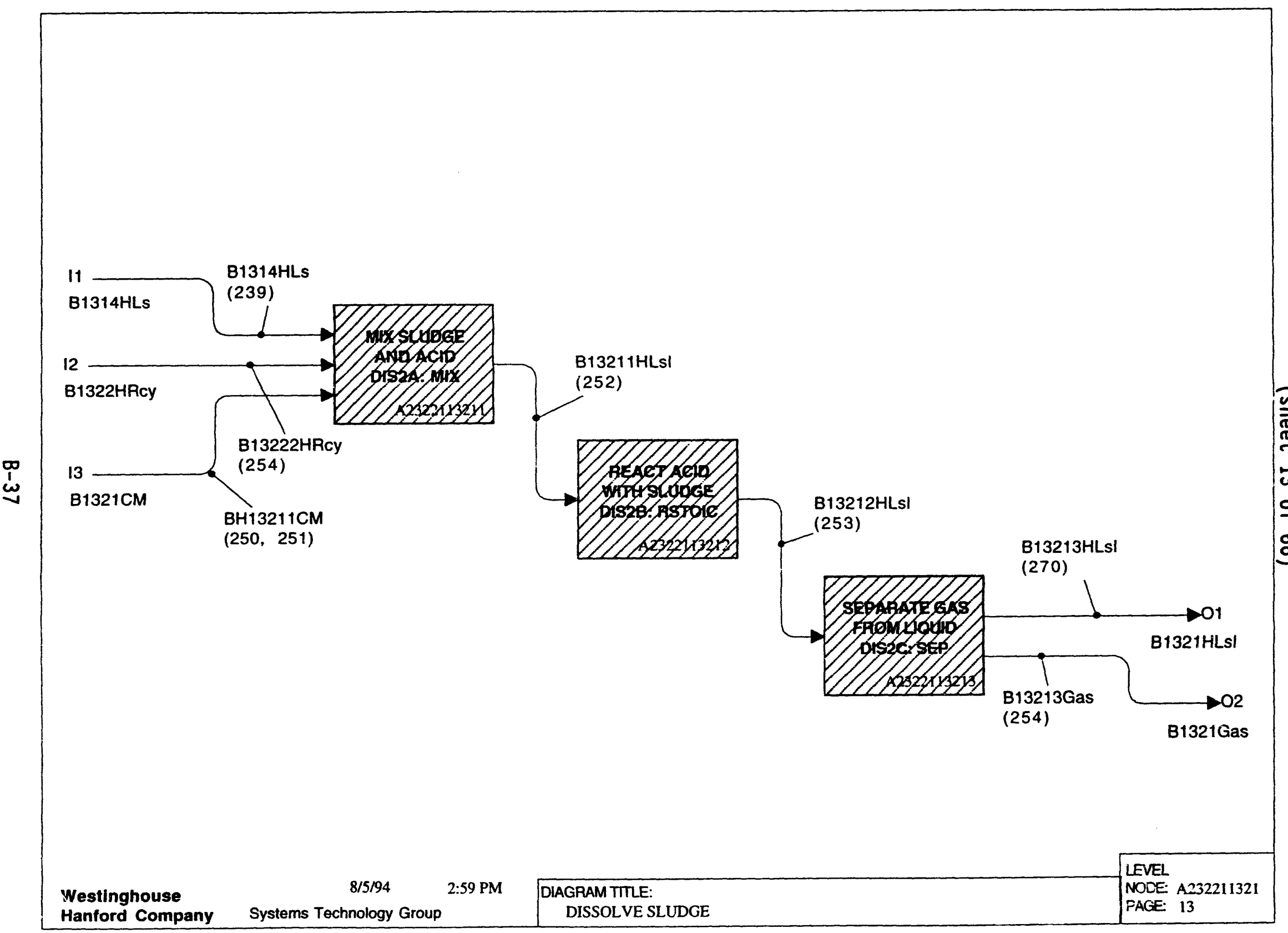




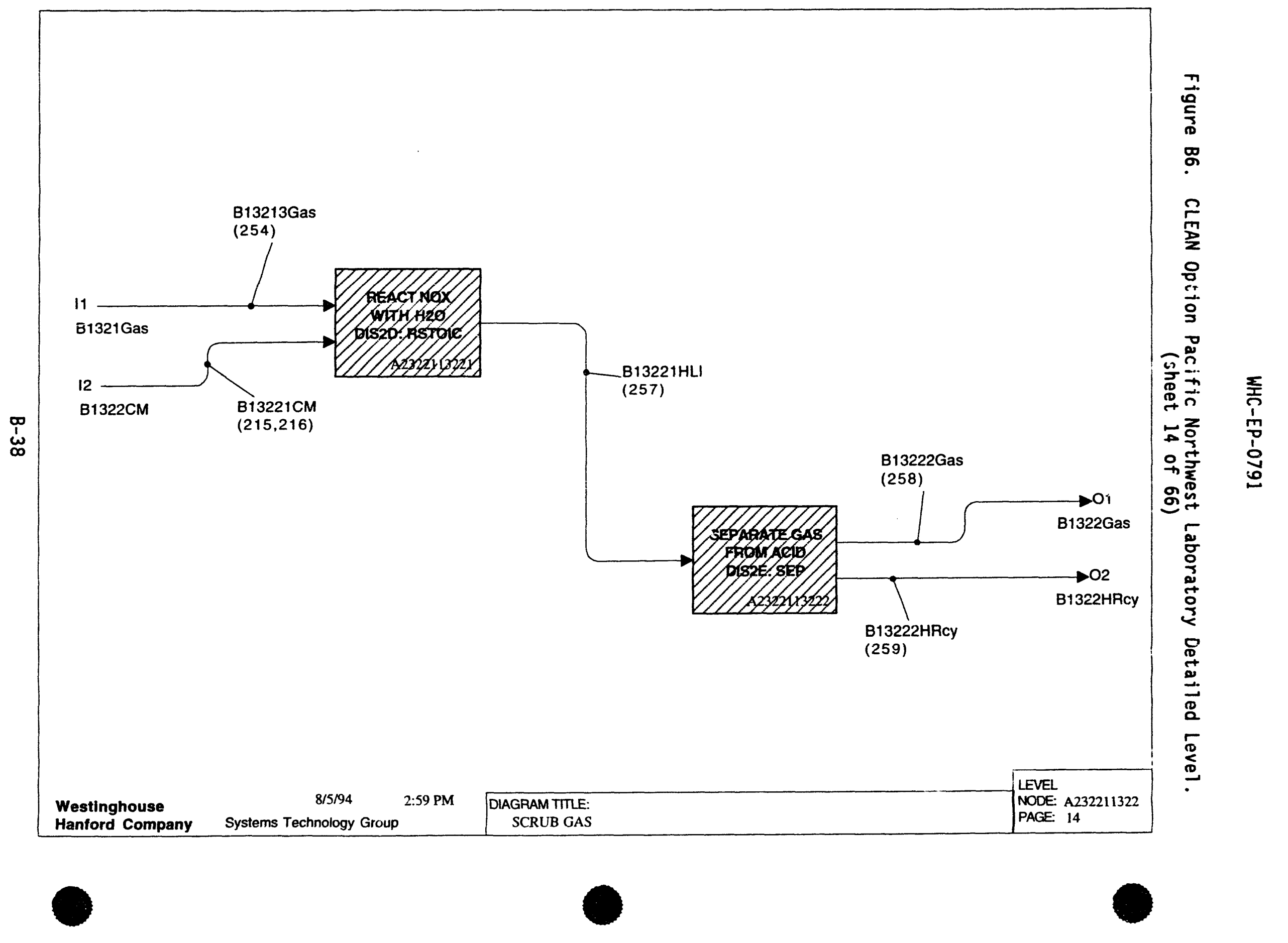




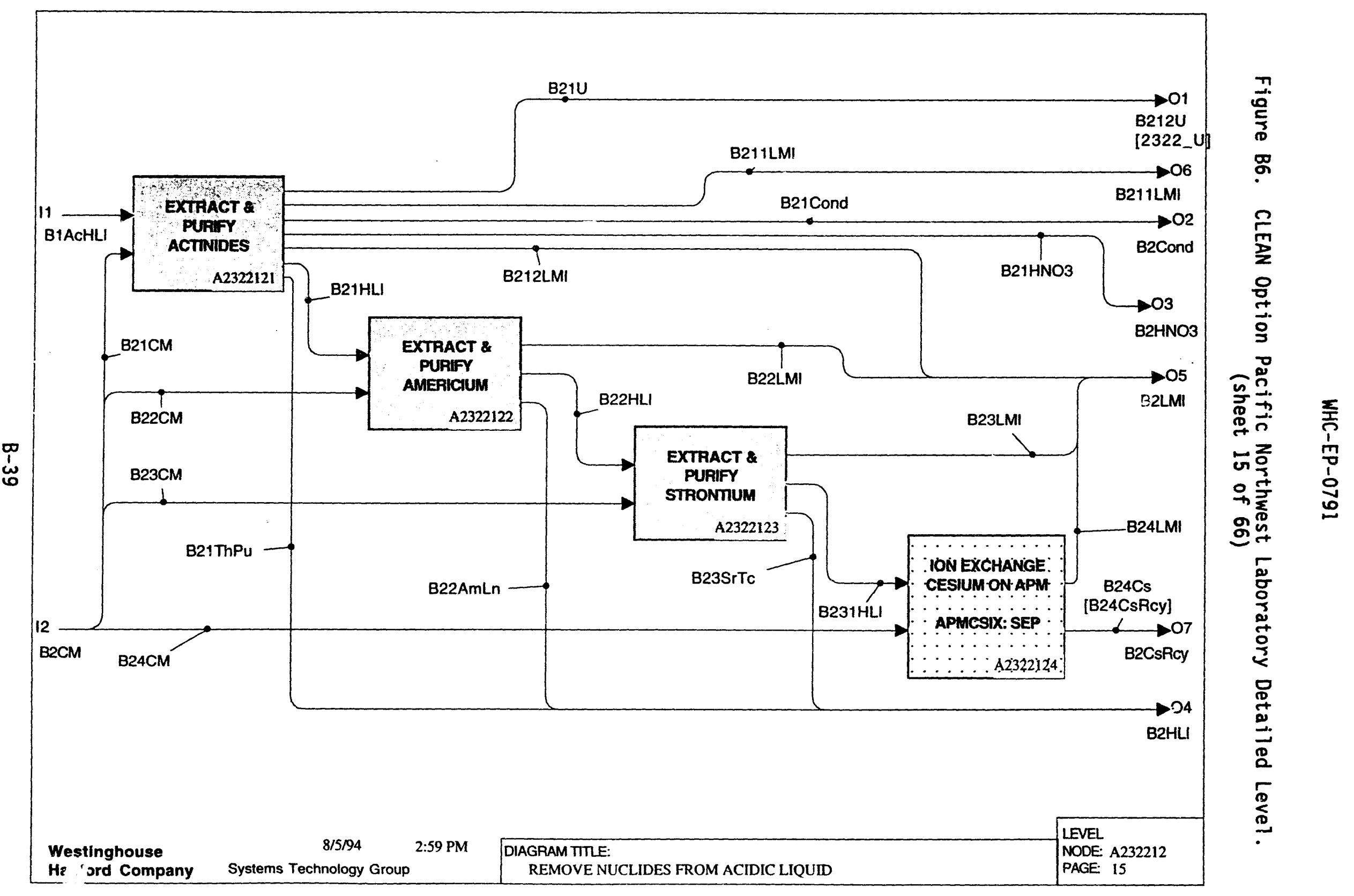




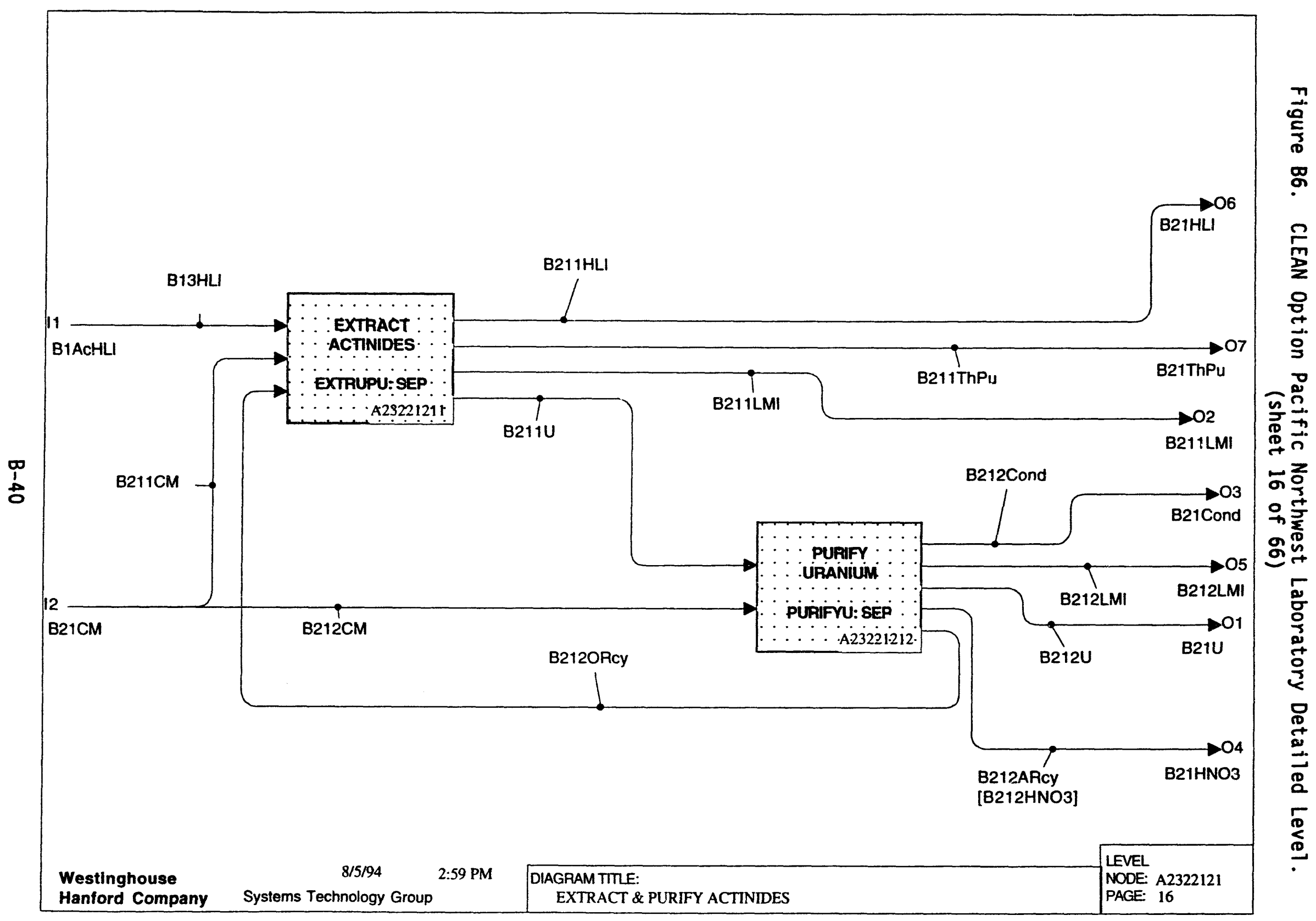




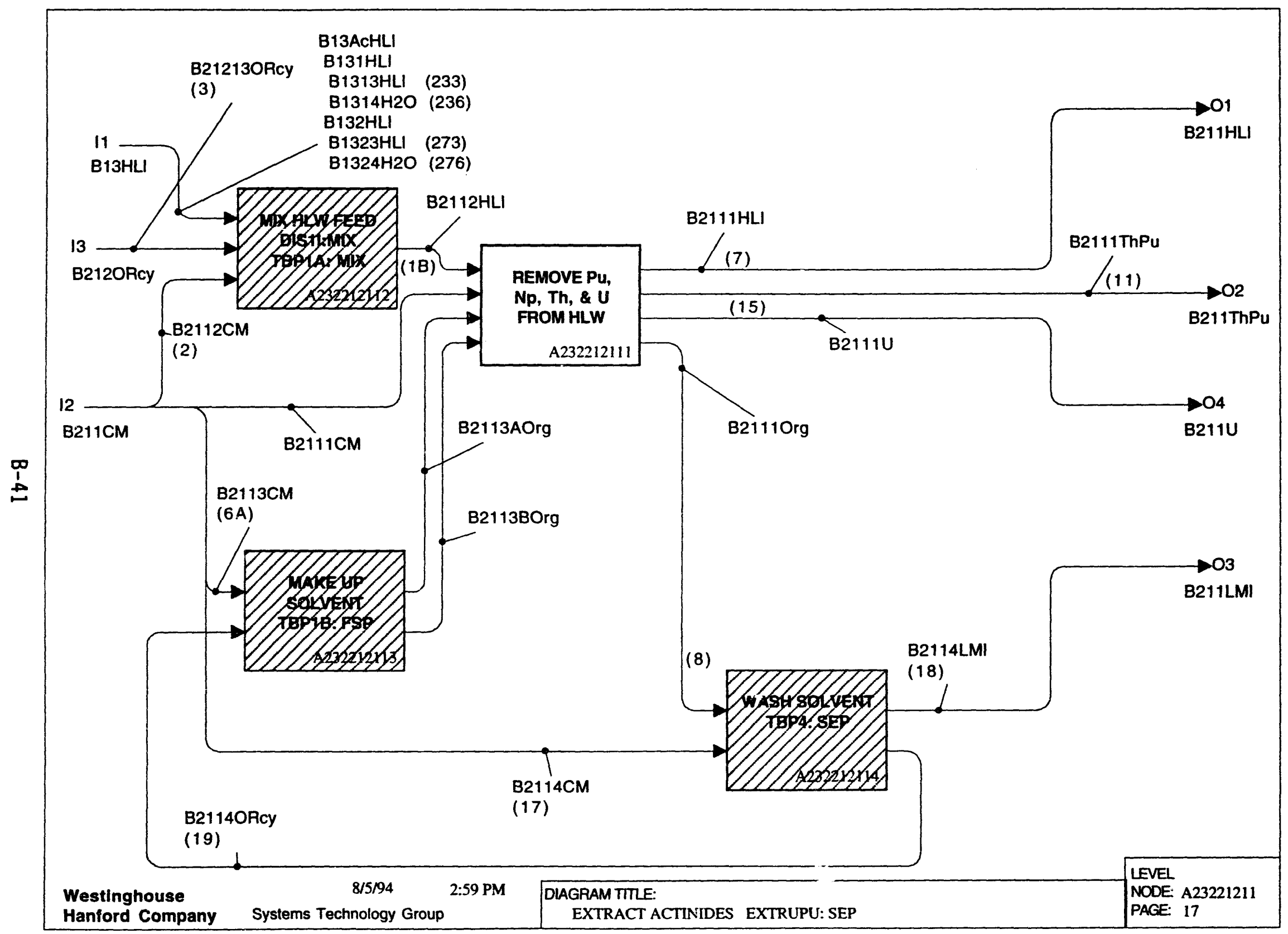

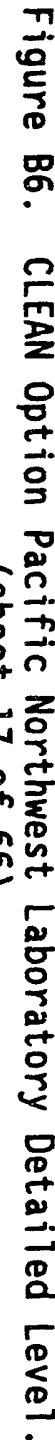




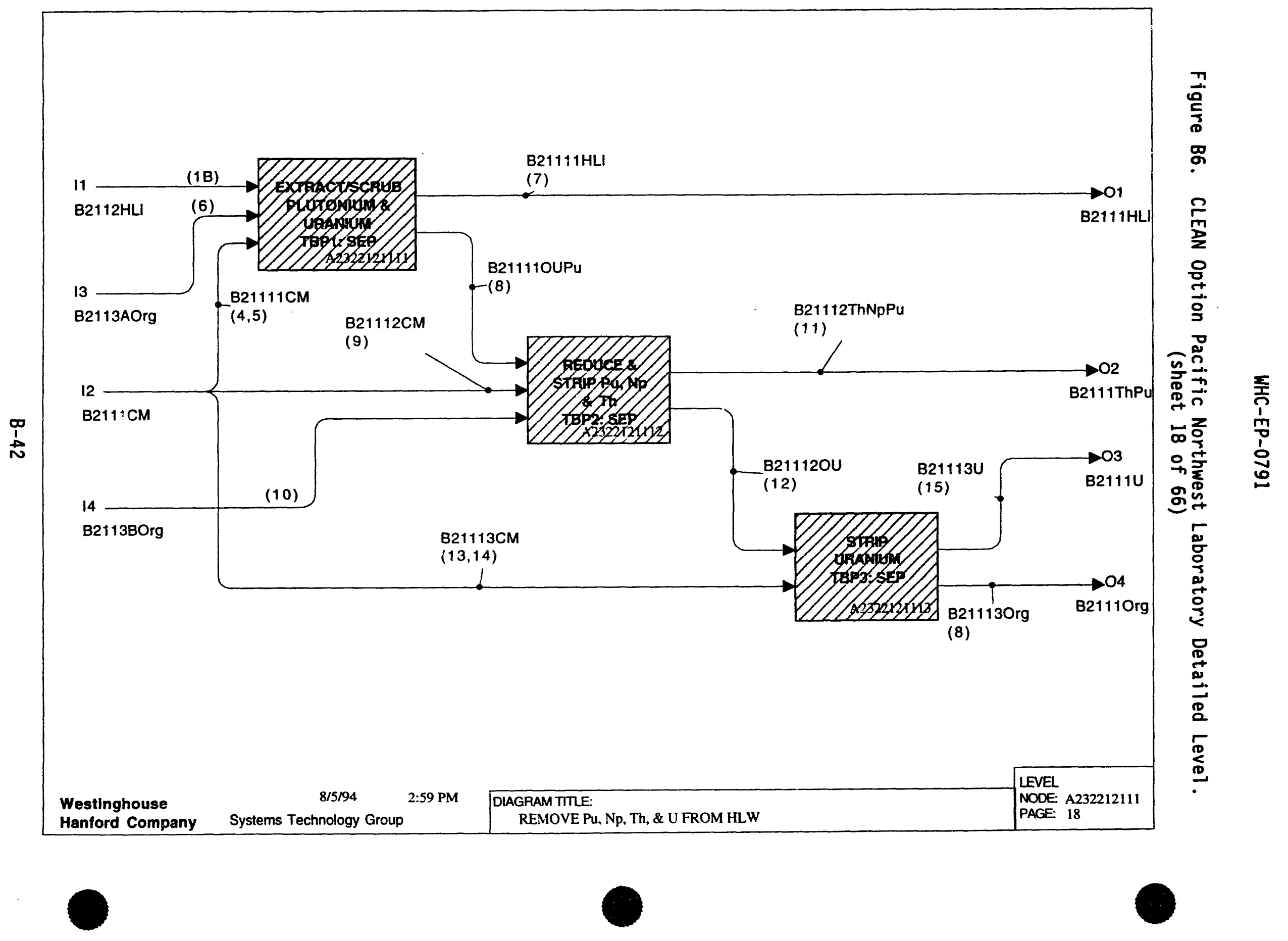




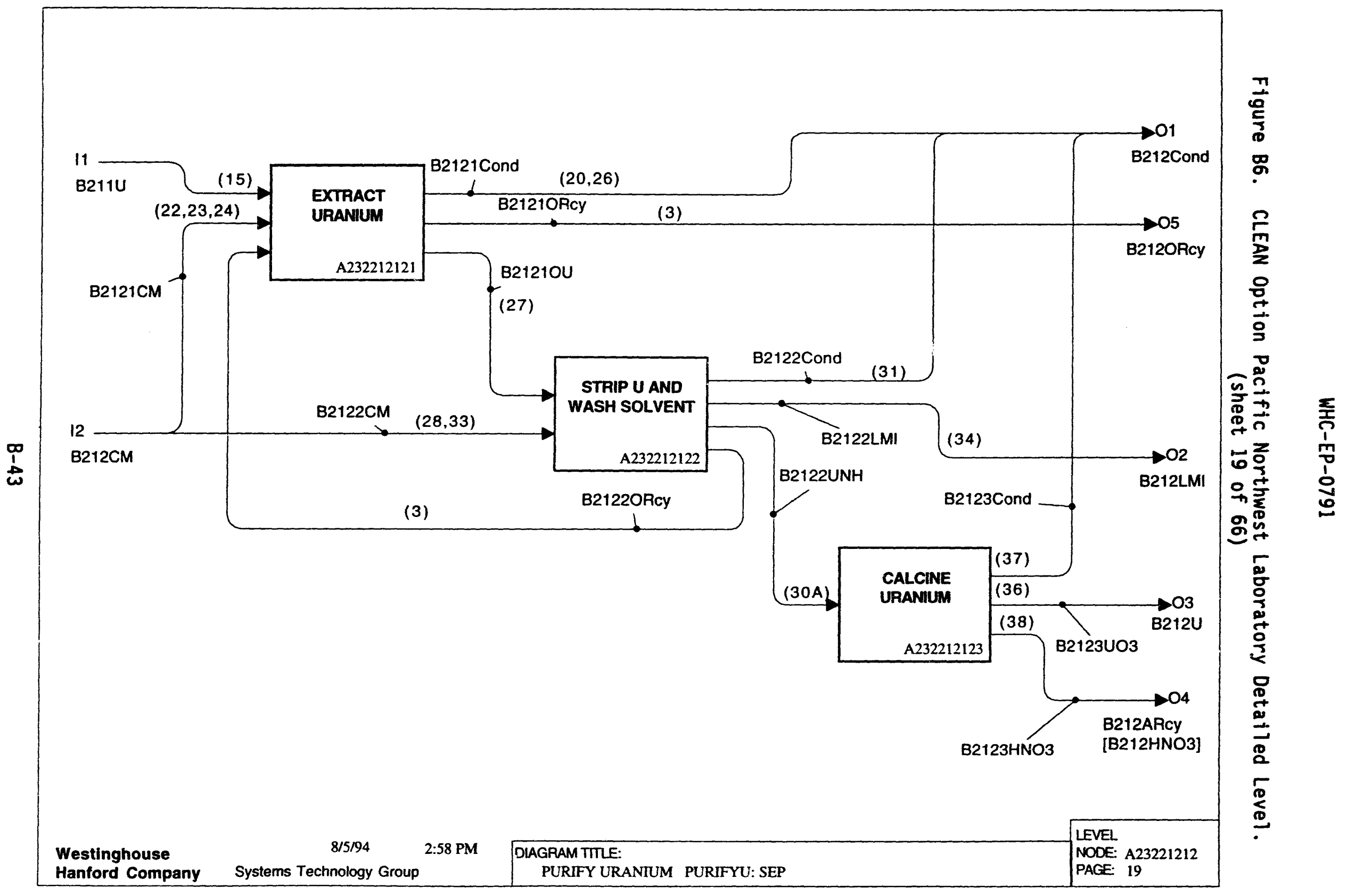




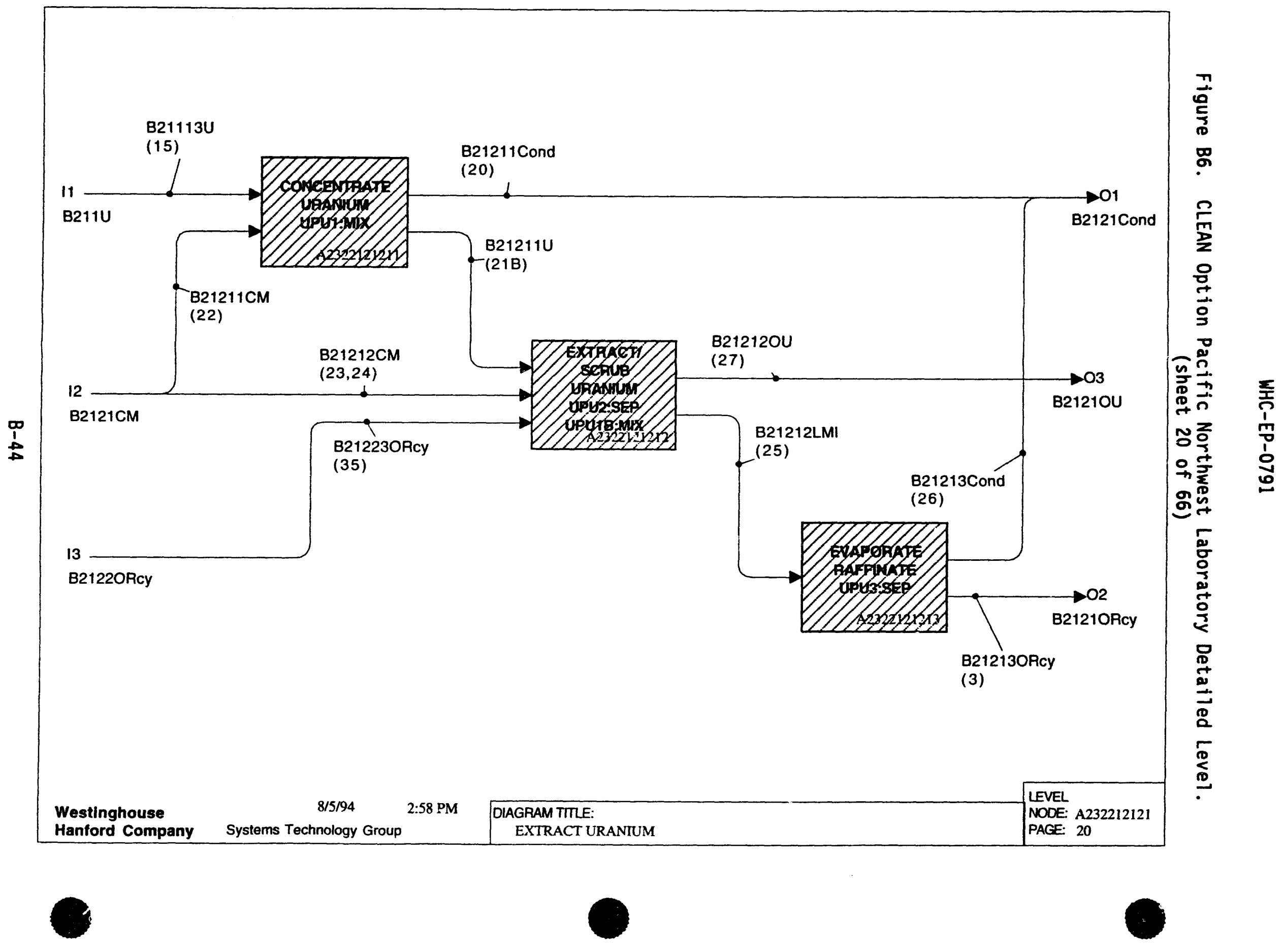




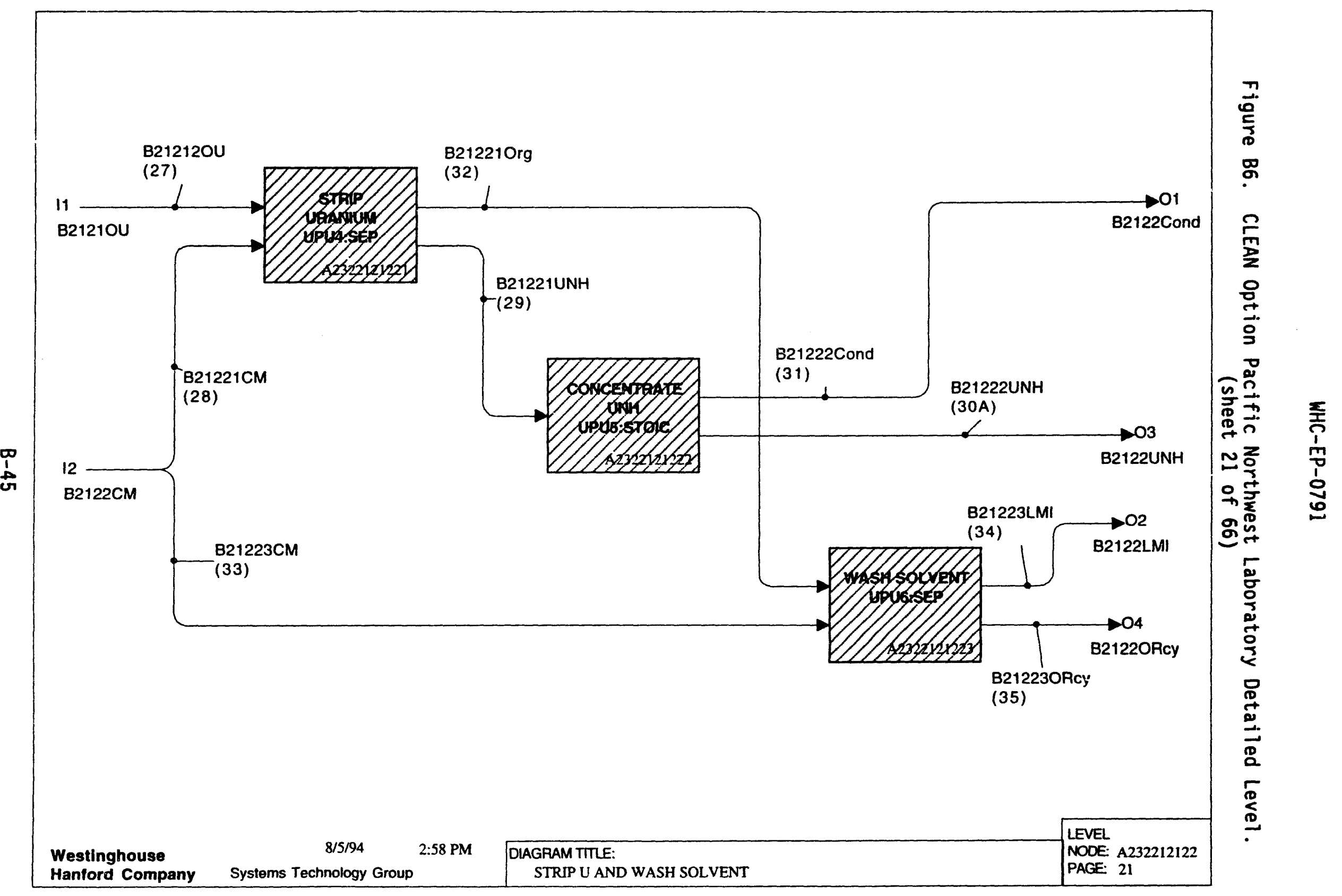




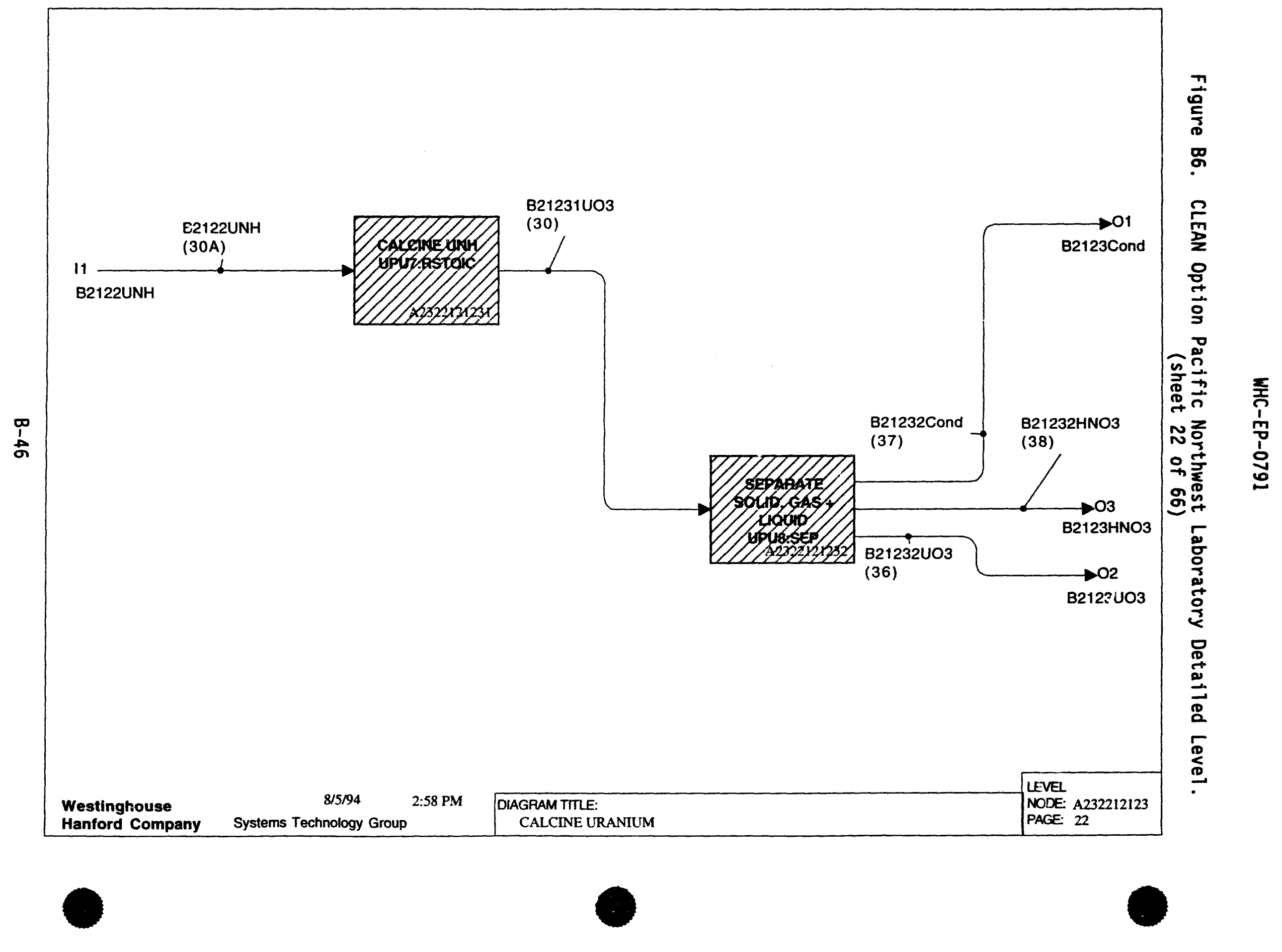




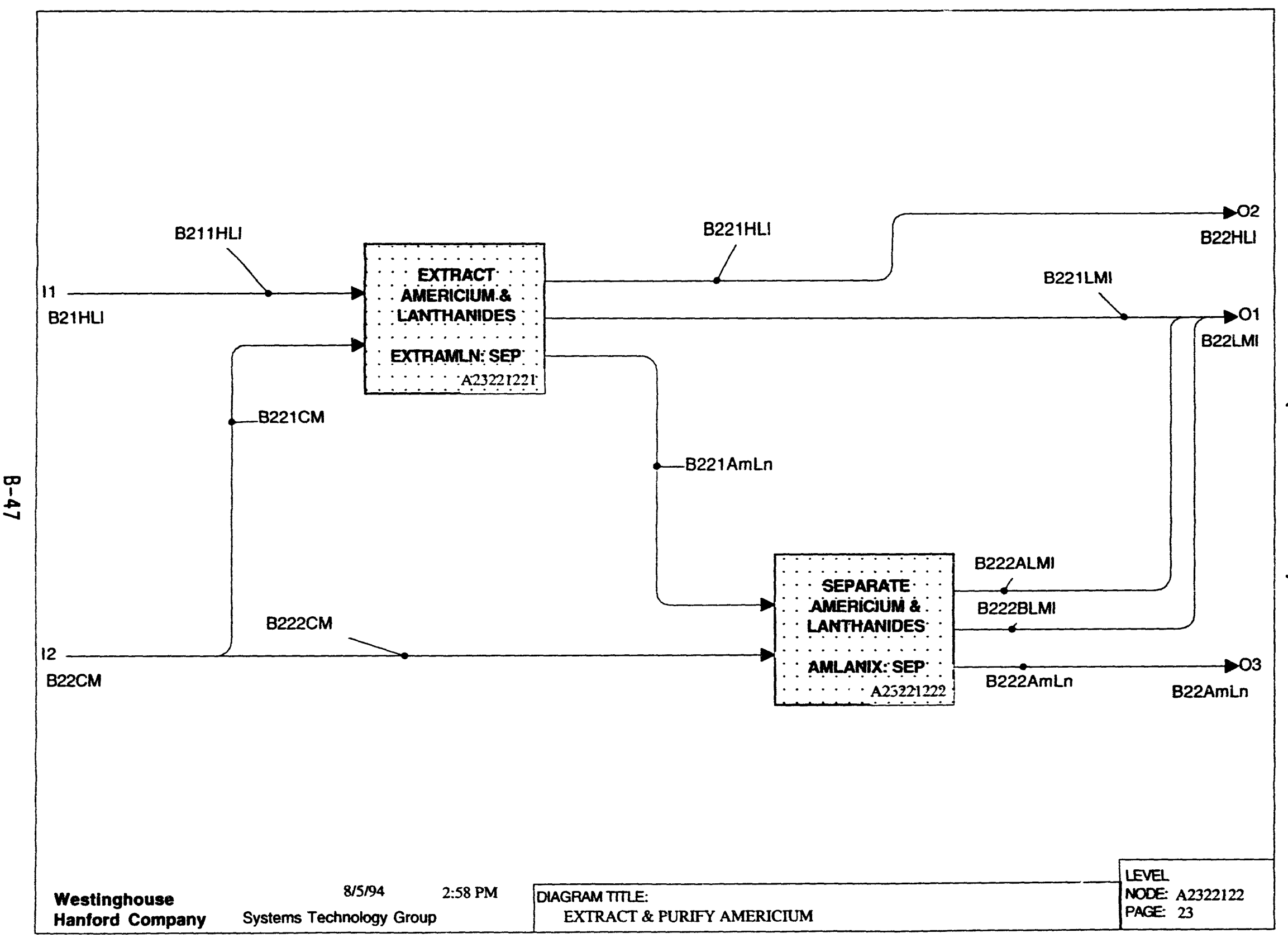

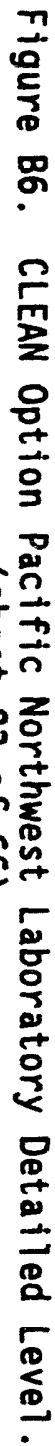




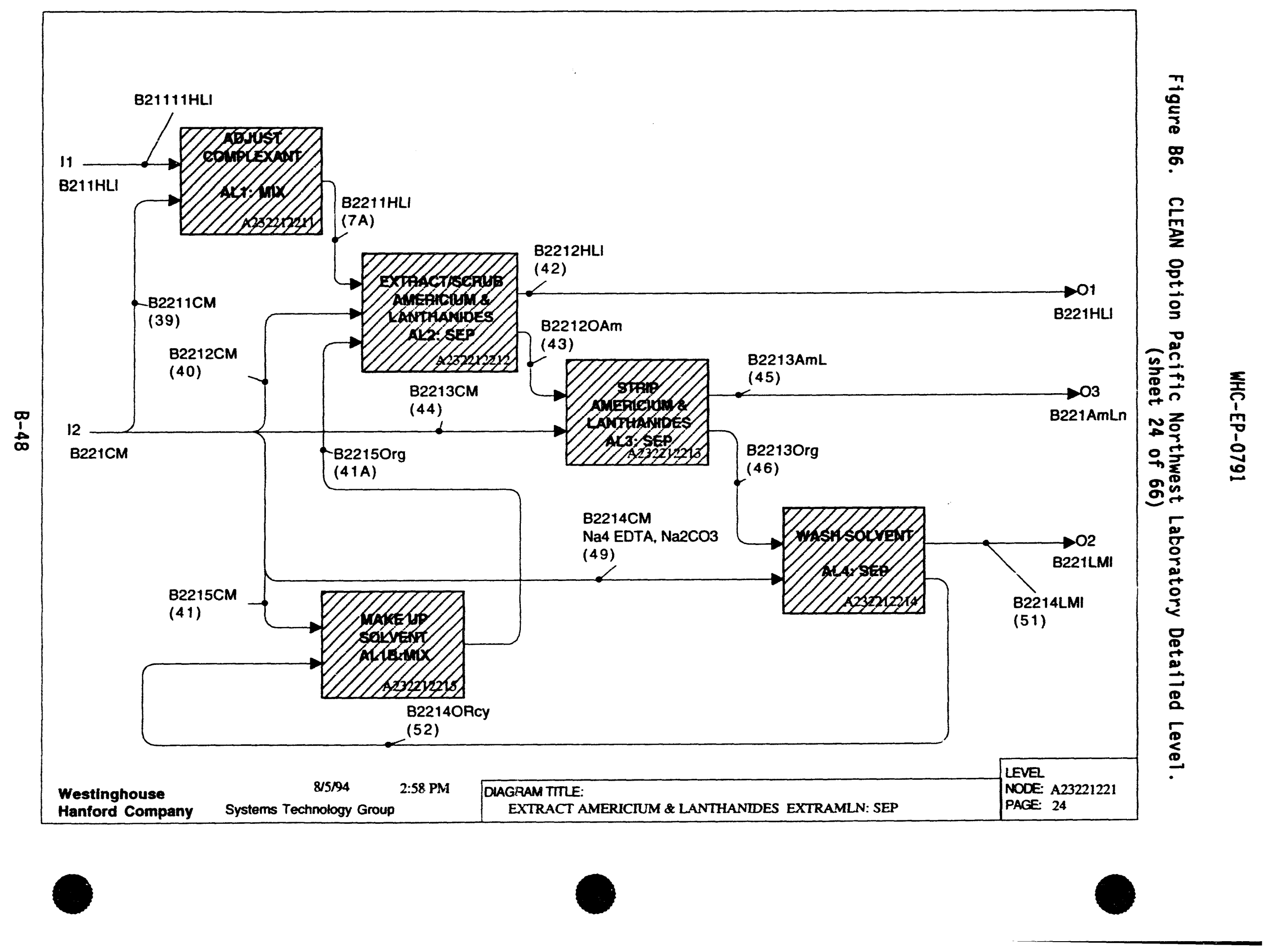




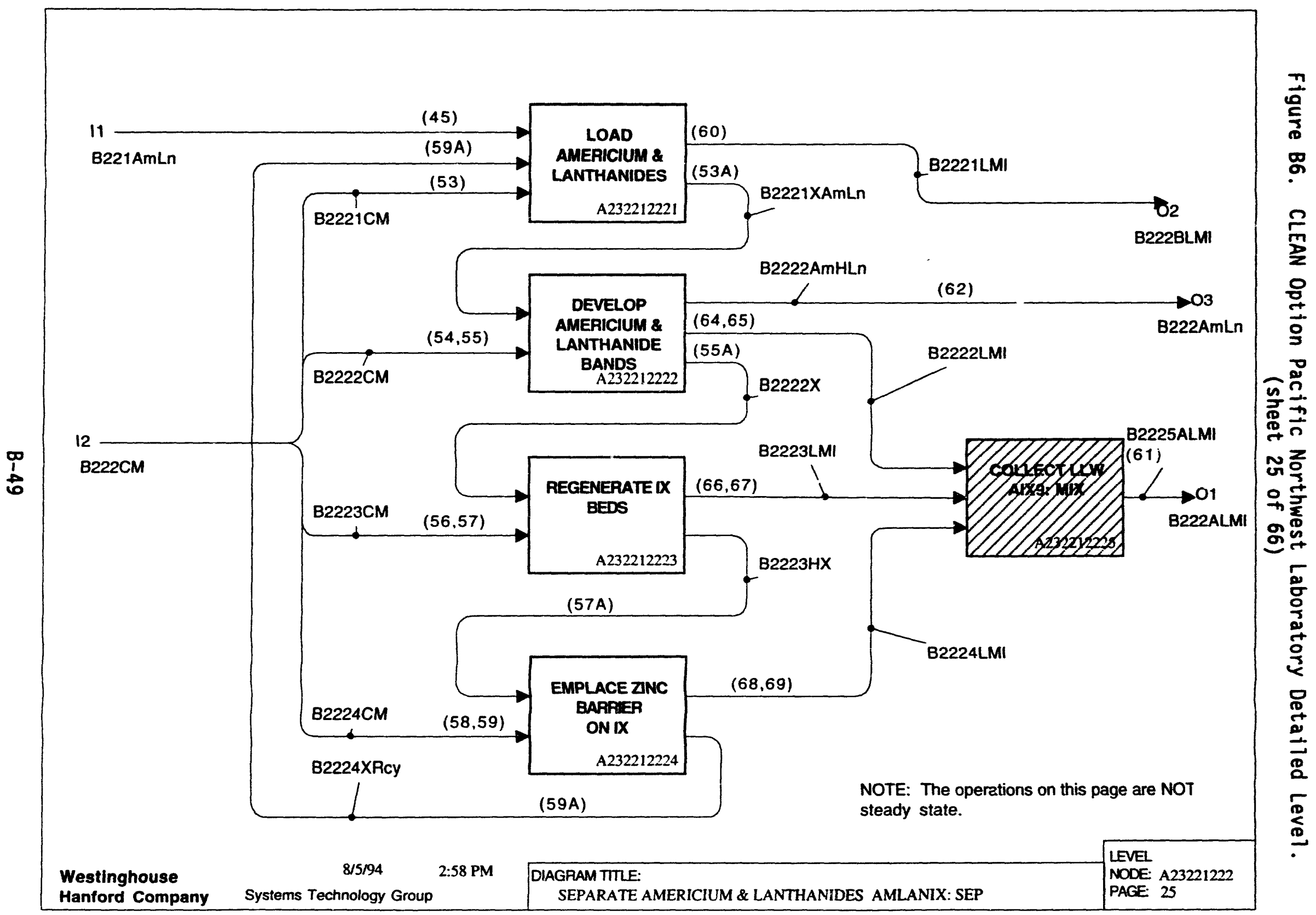




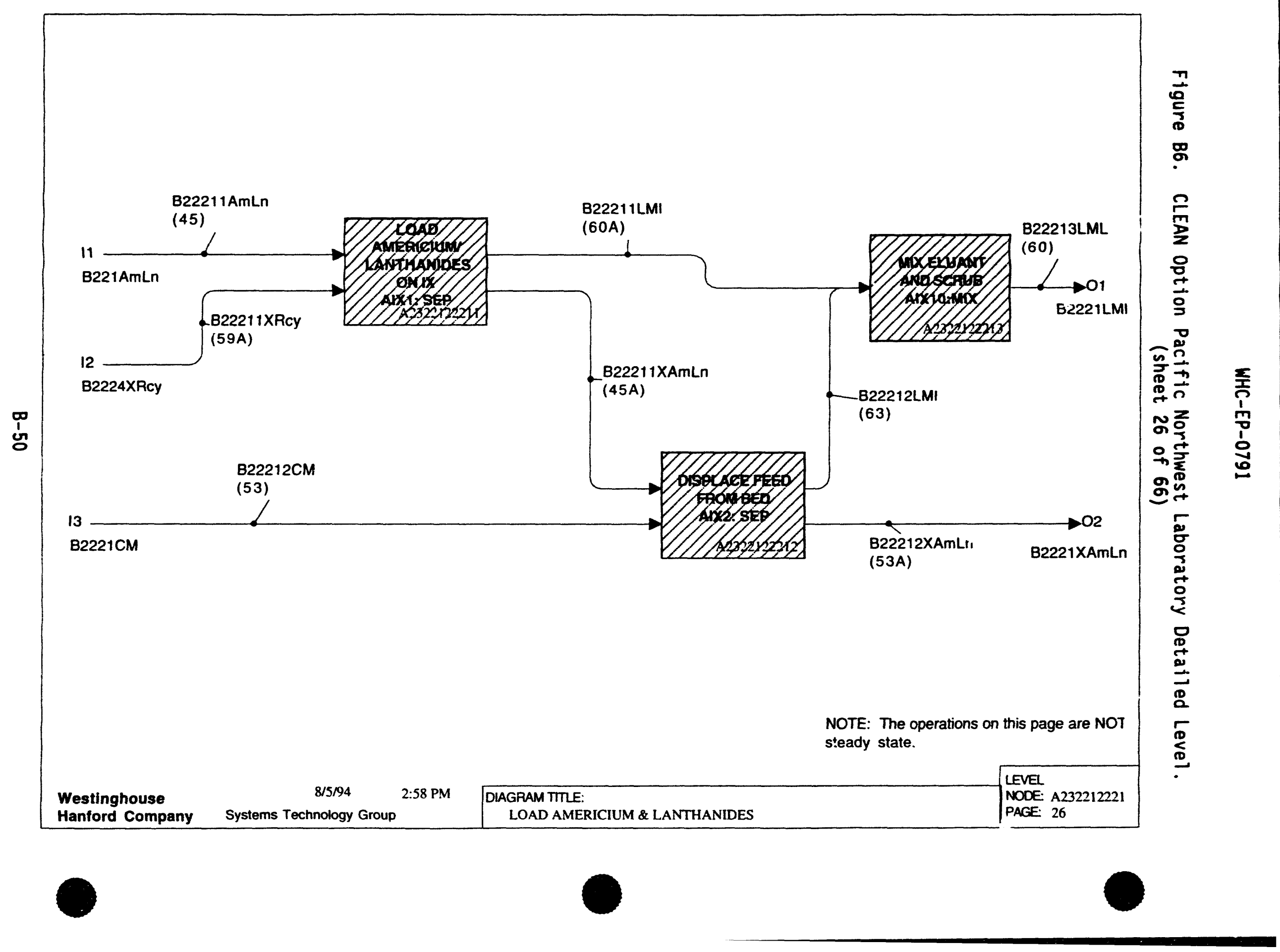




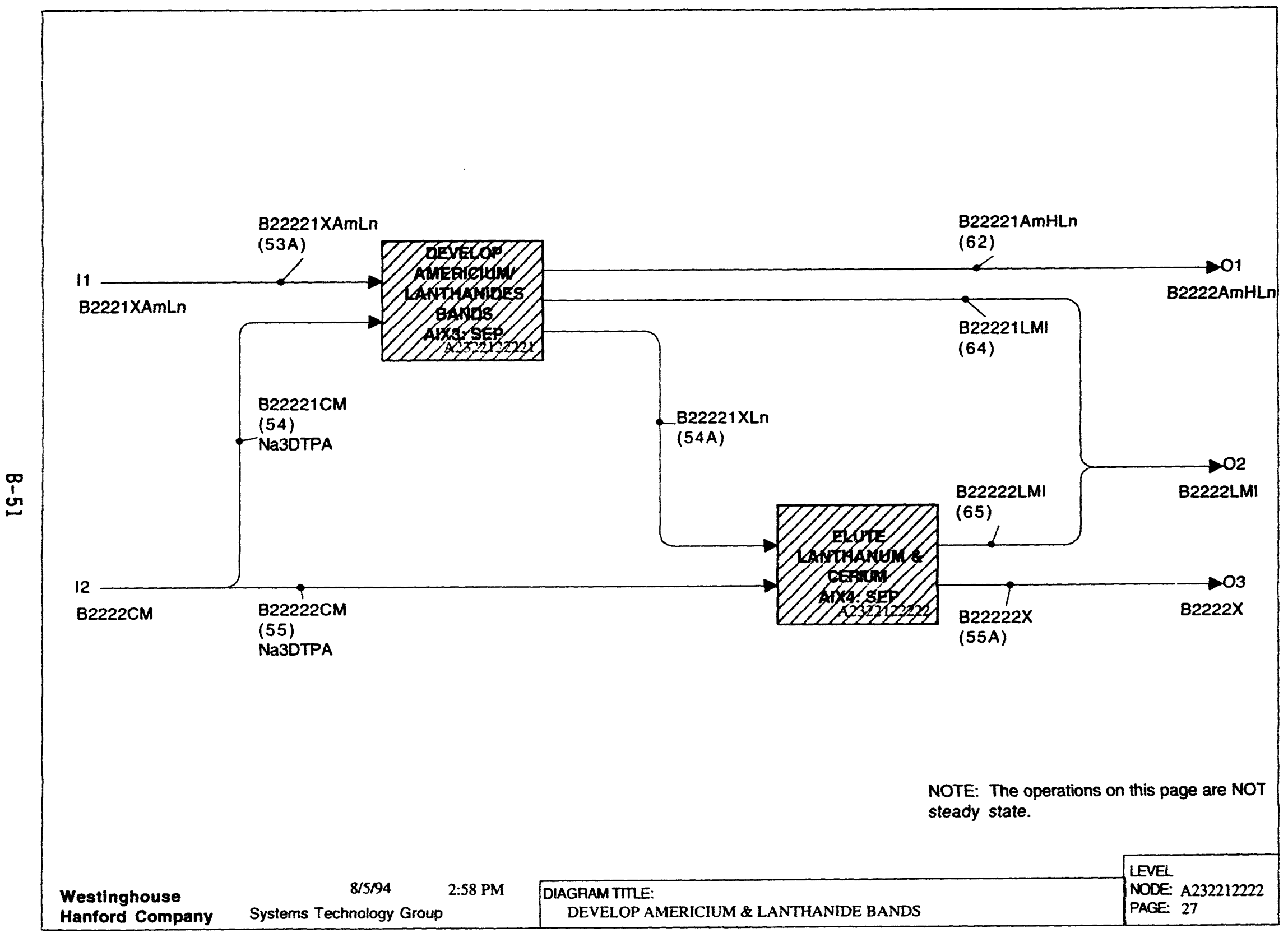




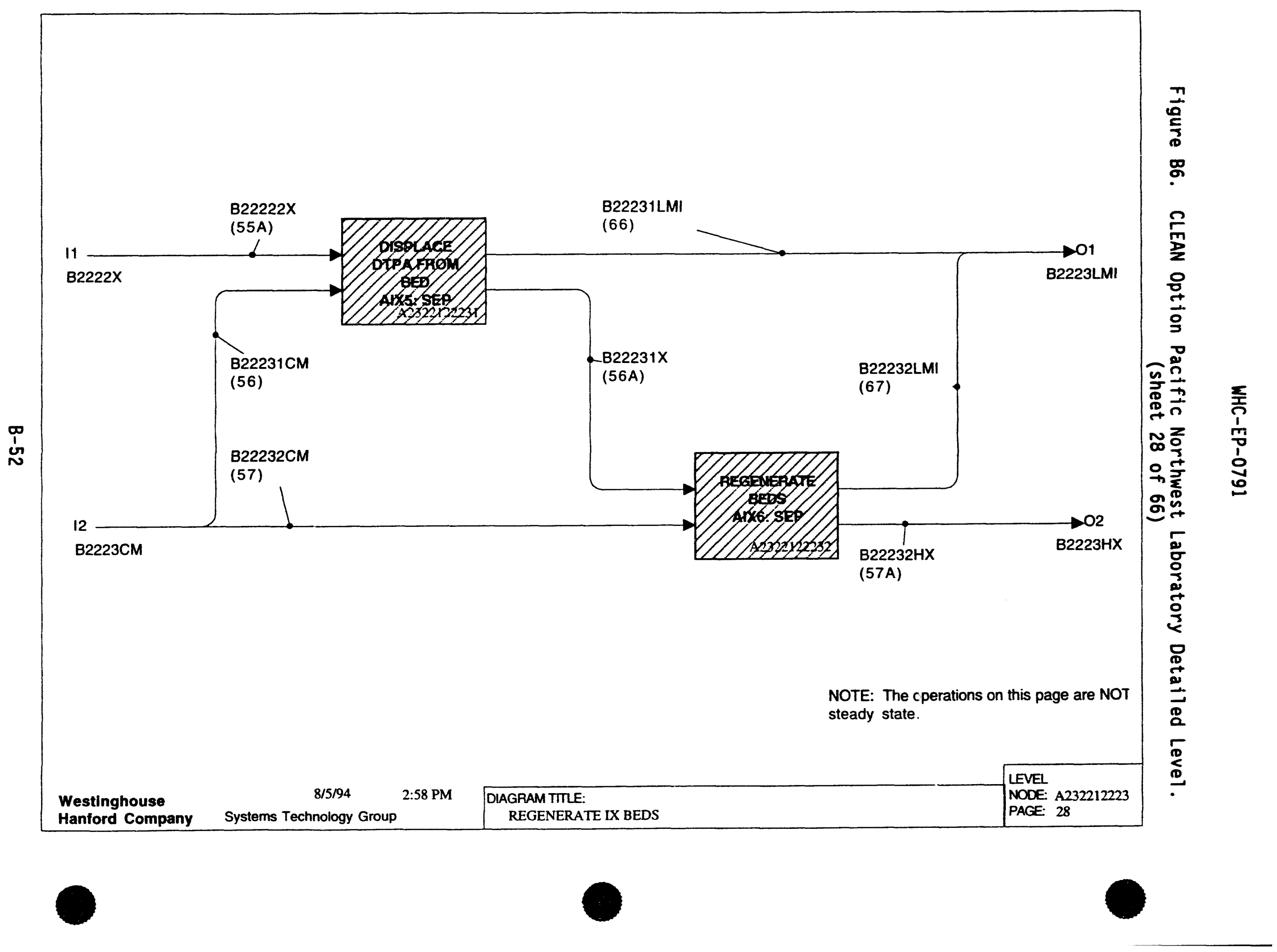




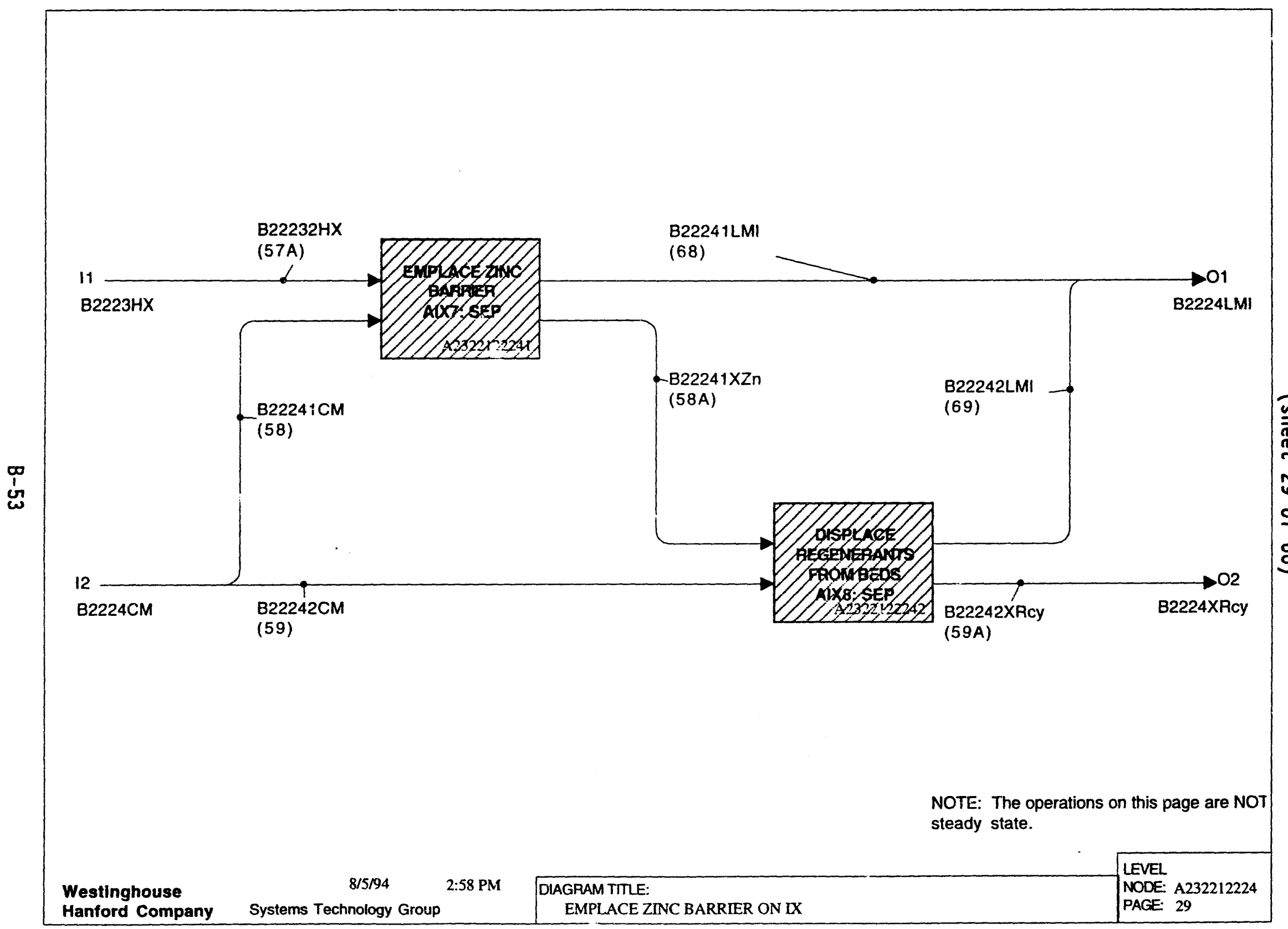




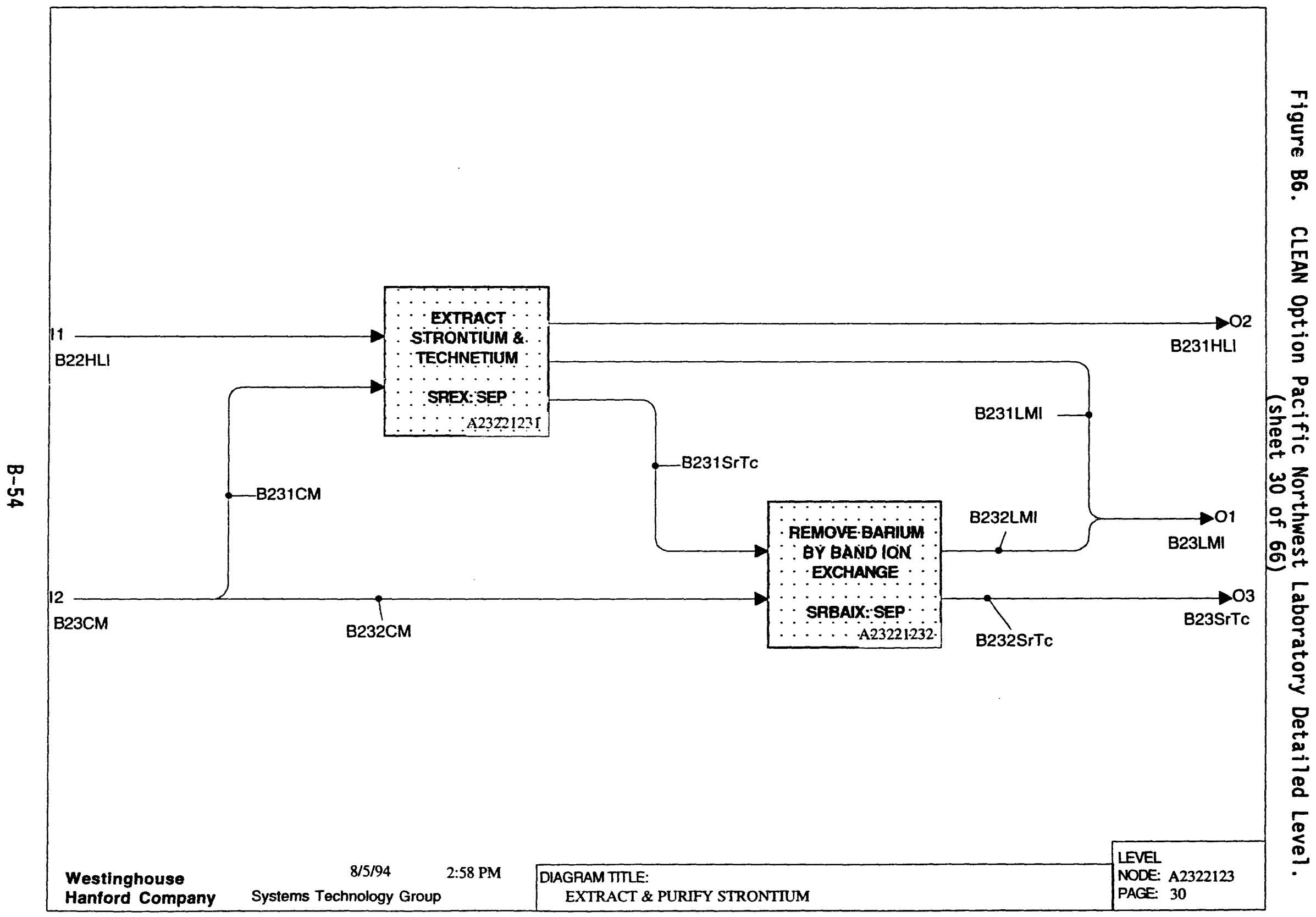




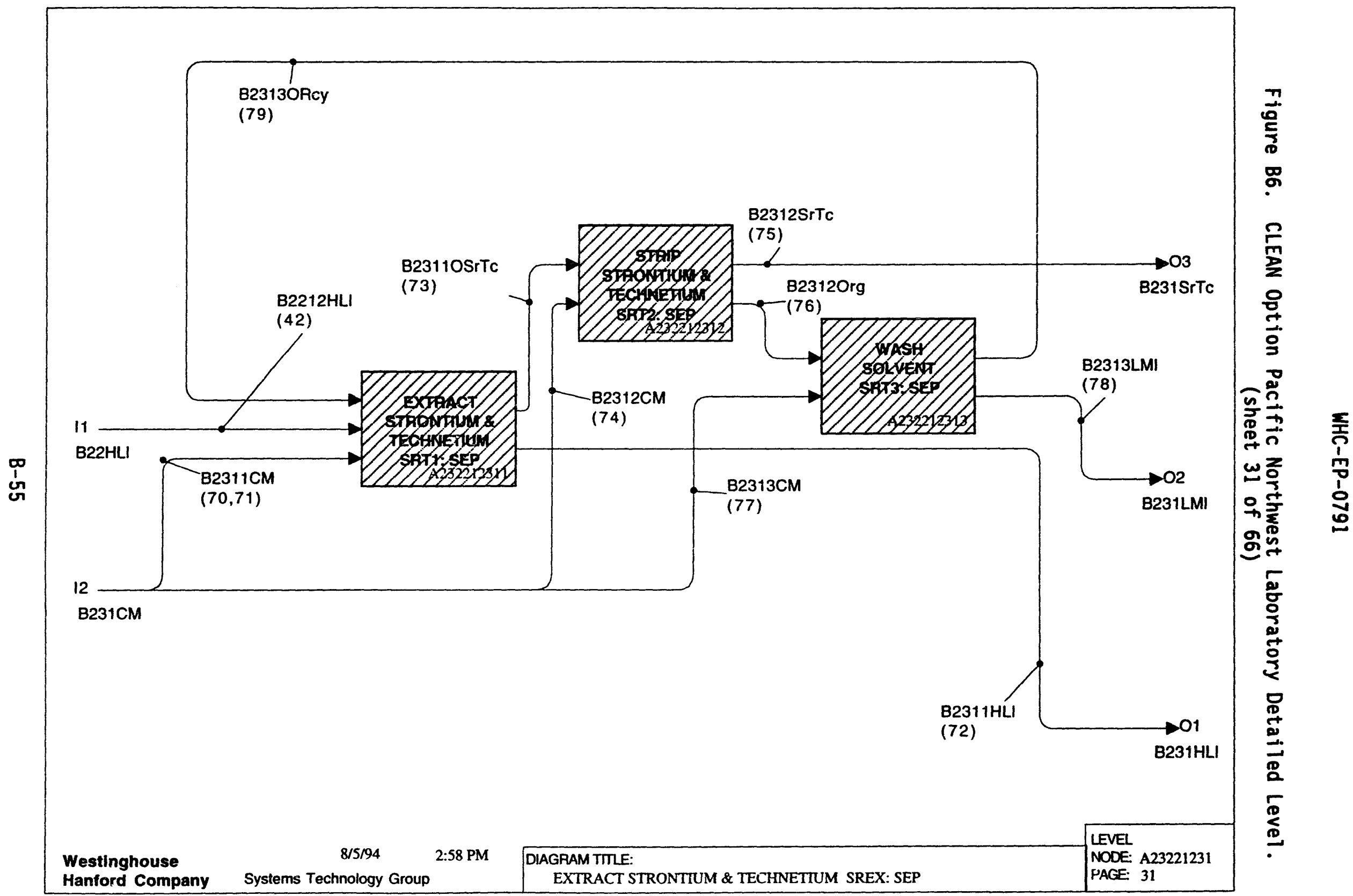




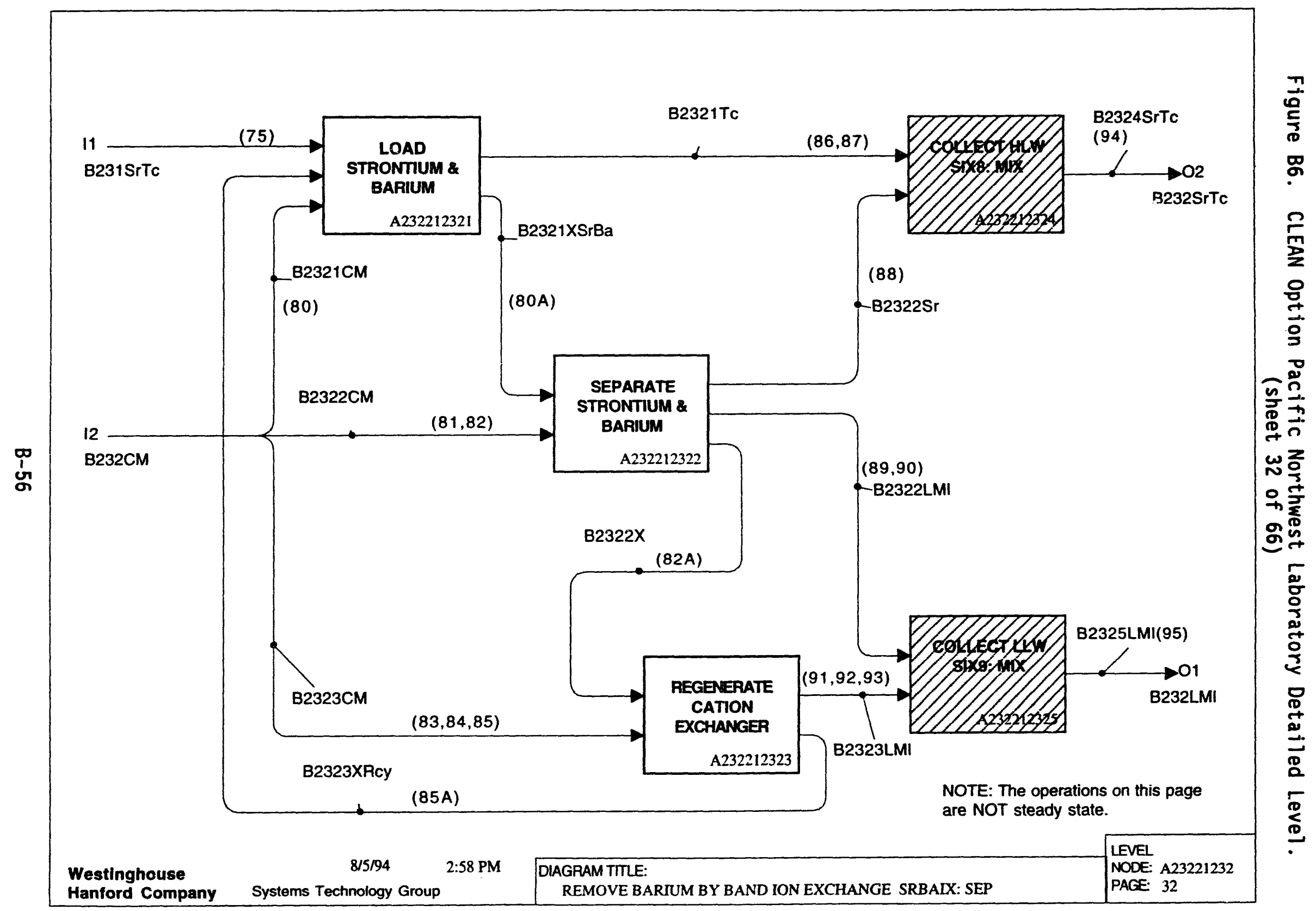



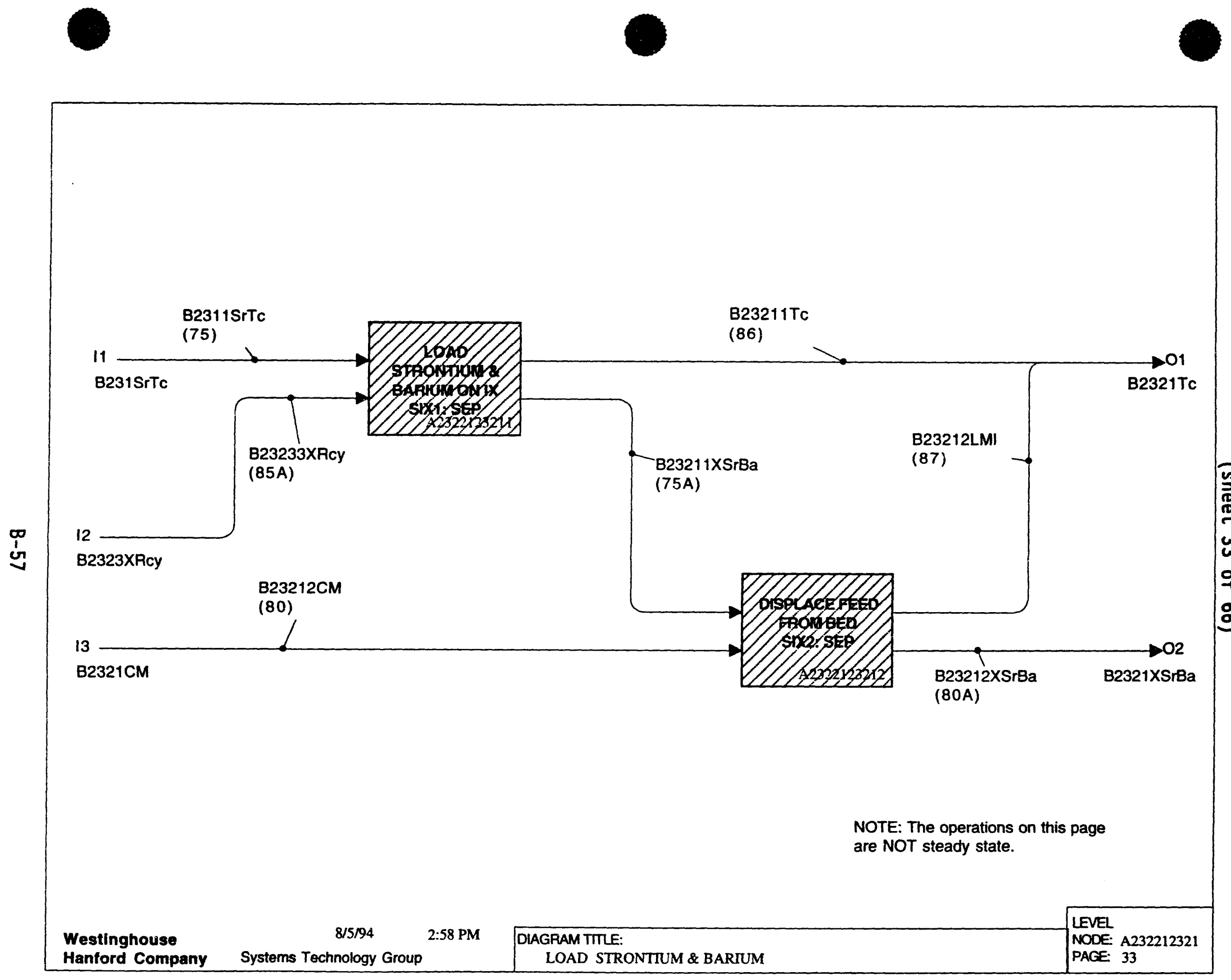

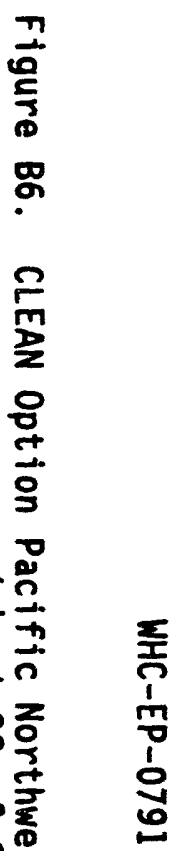




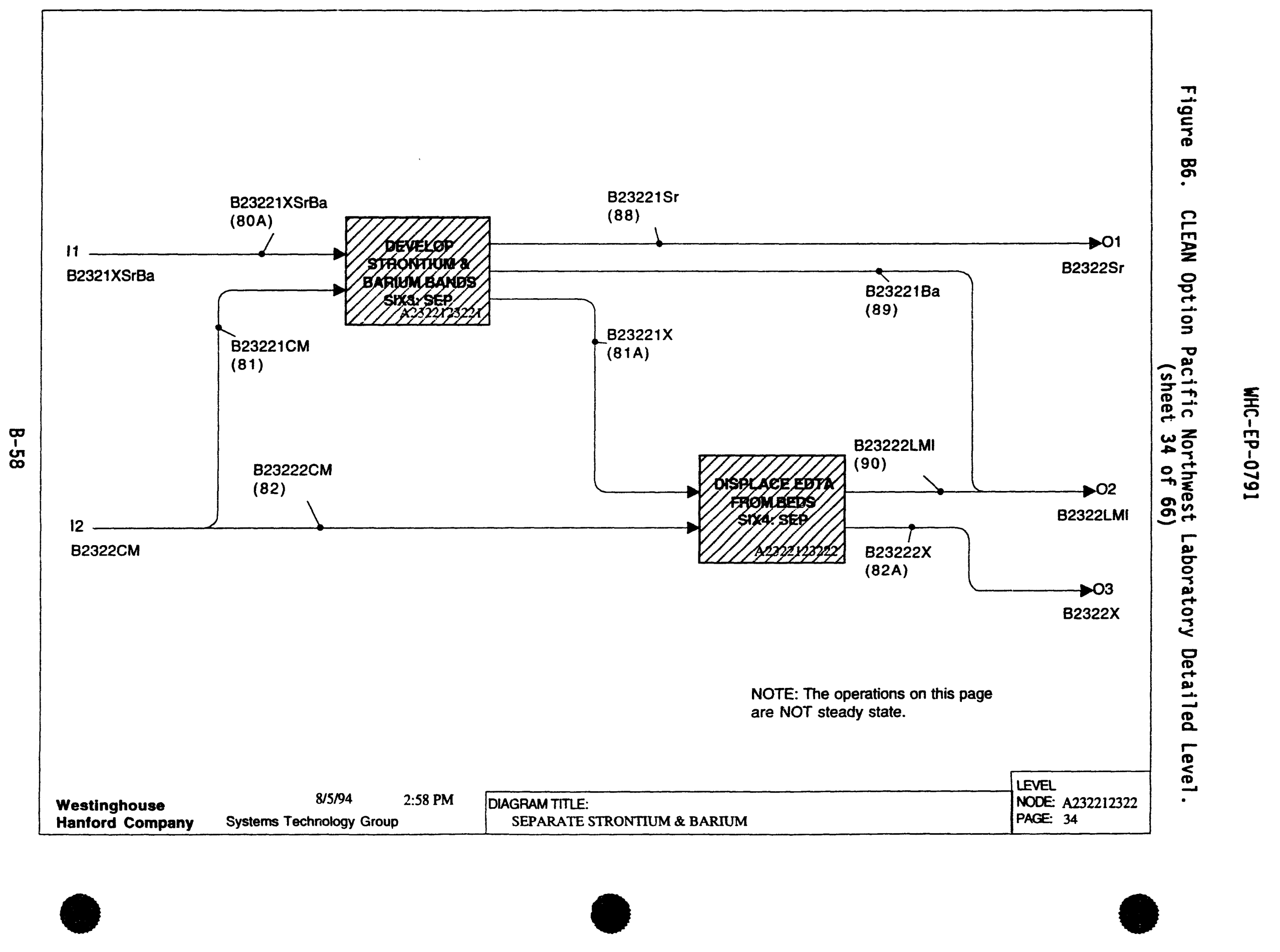




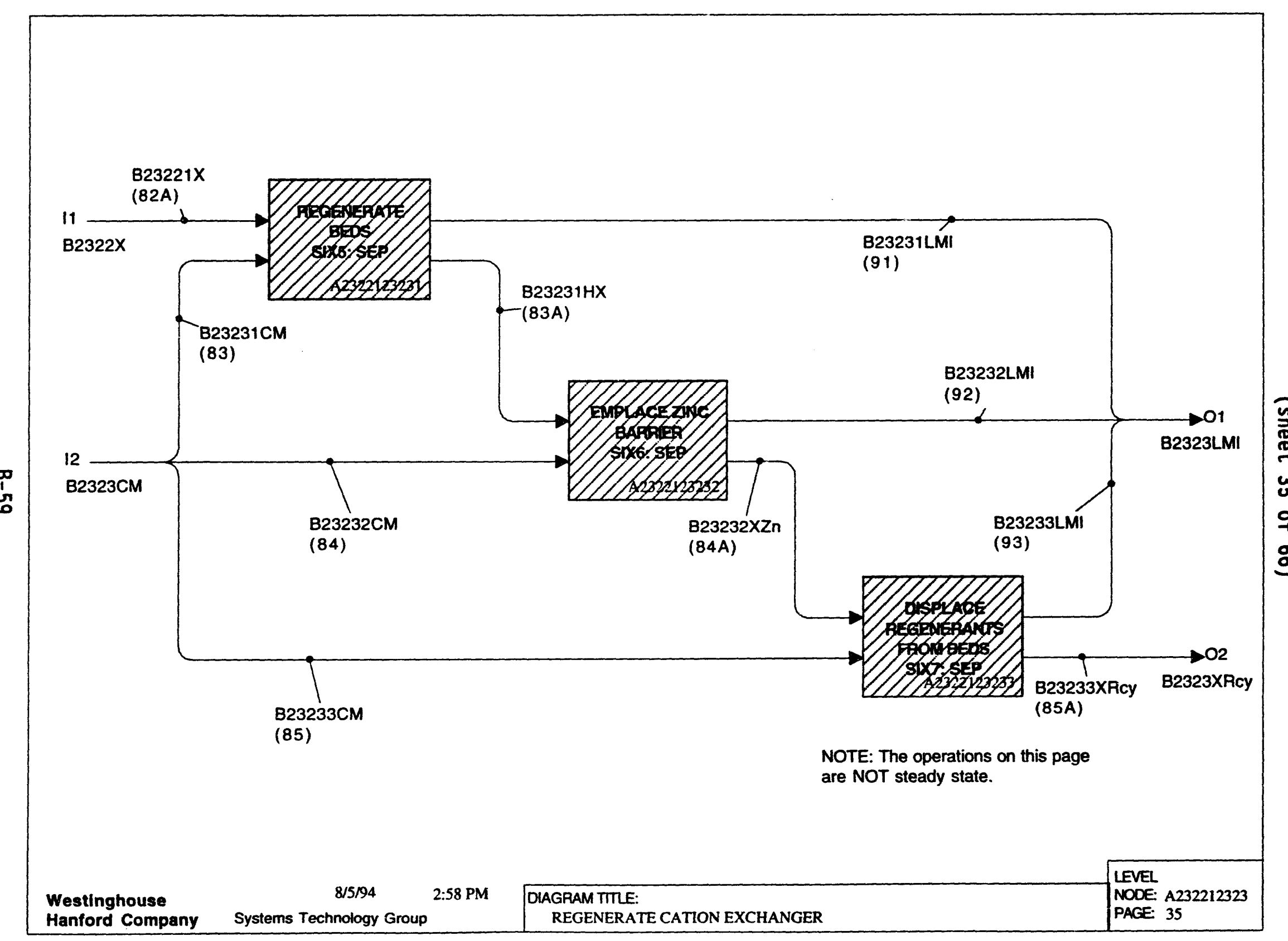

要

$\stackrel{0}{+}$

जi

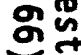

: 


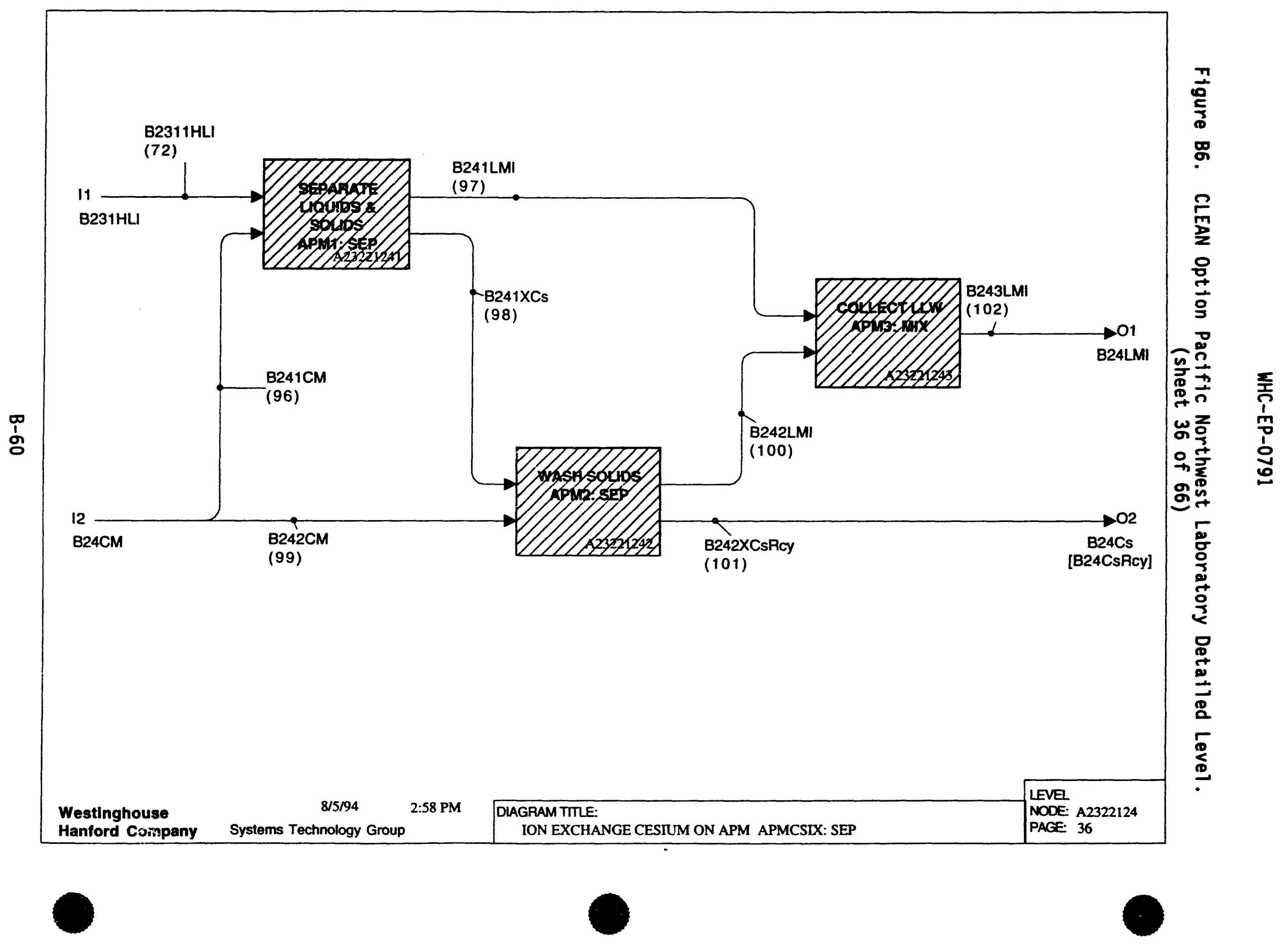




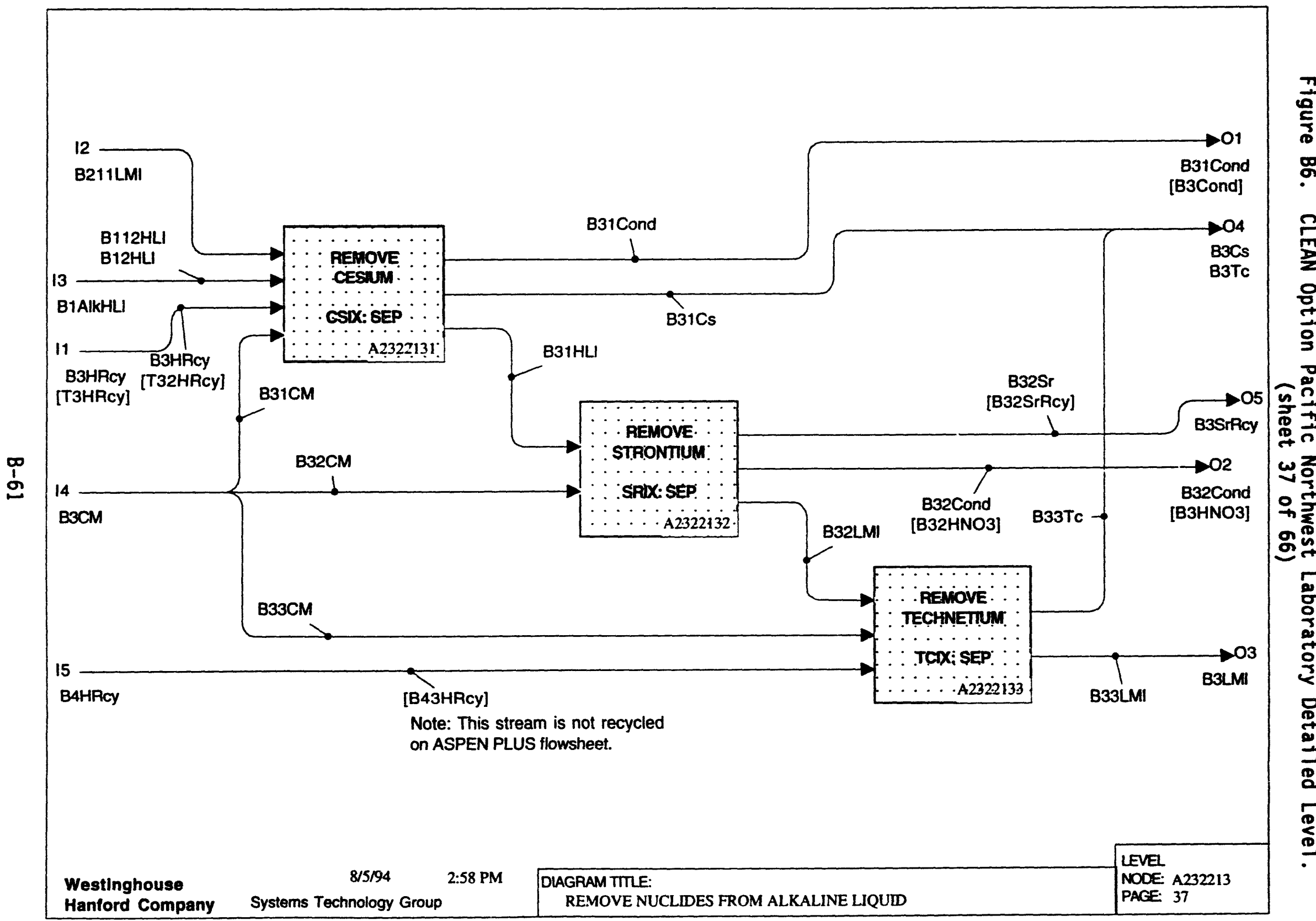




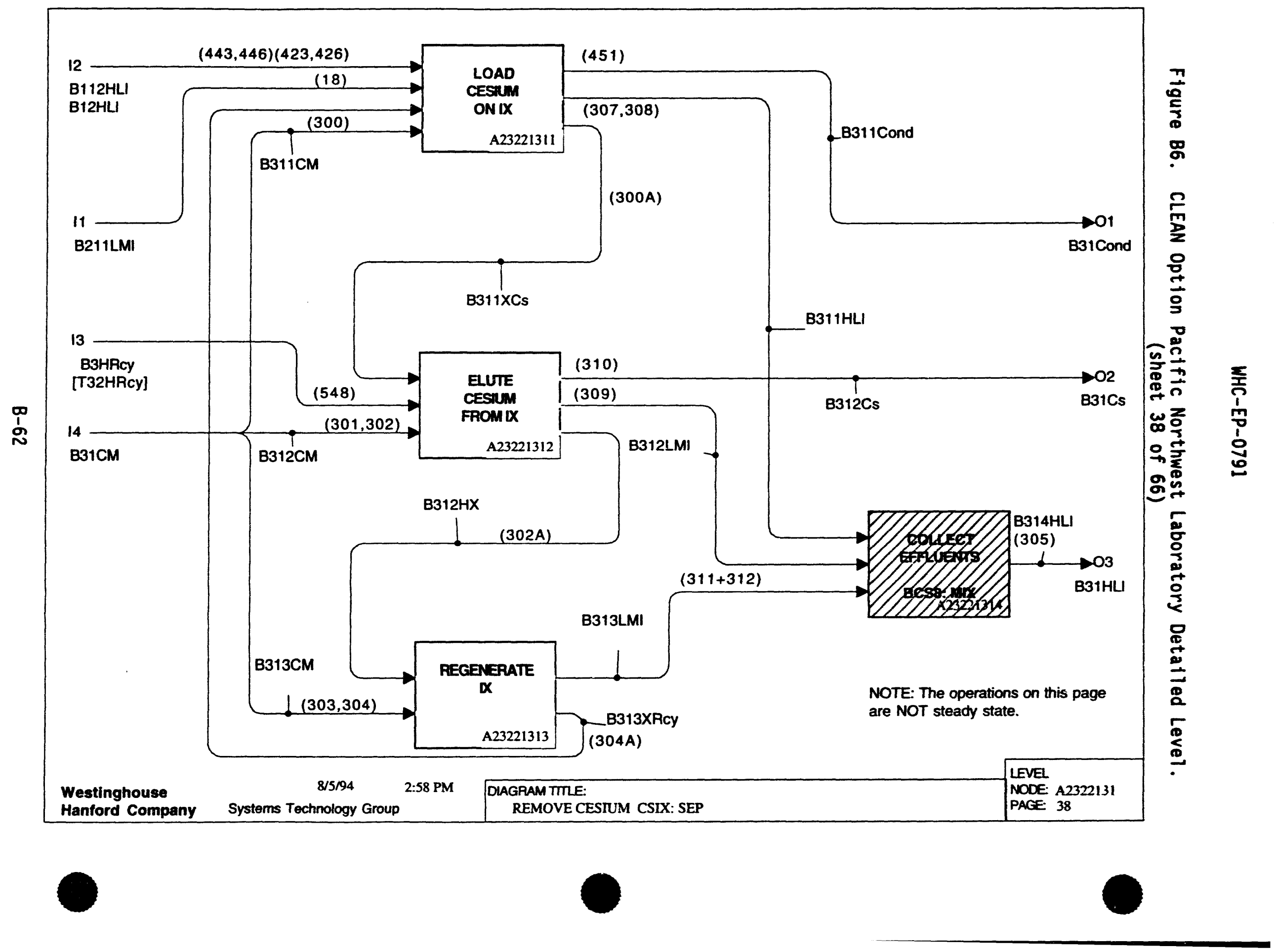




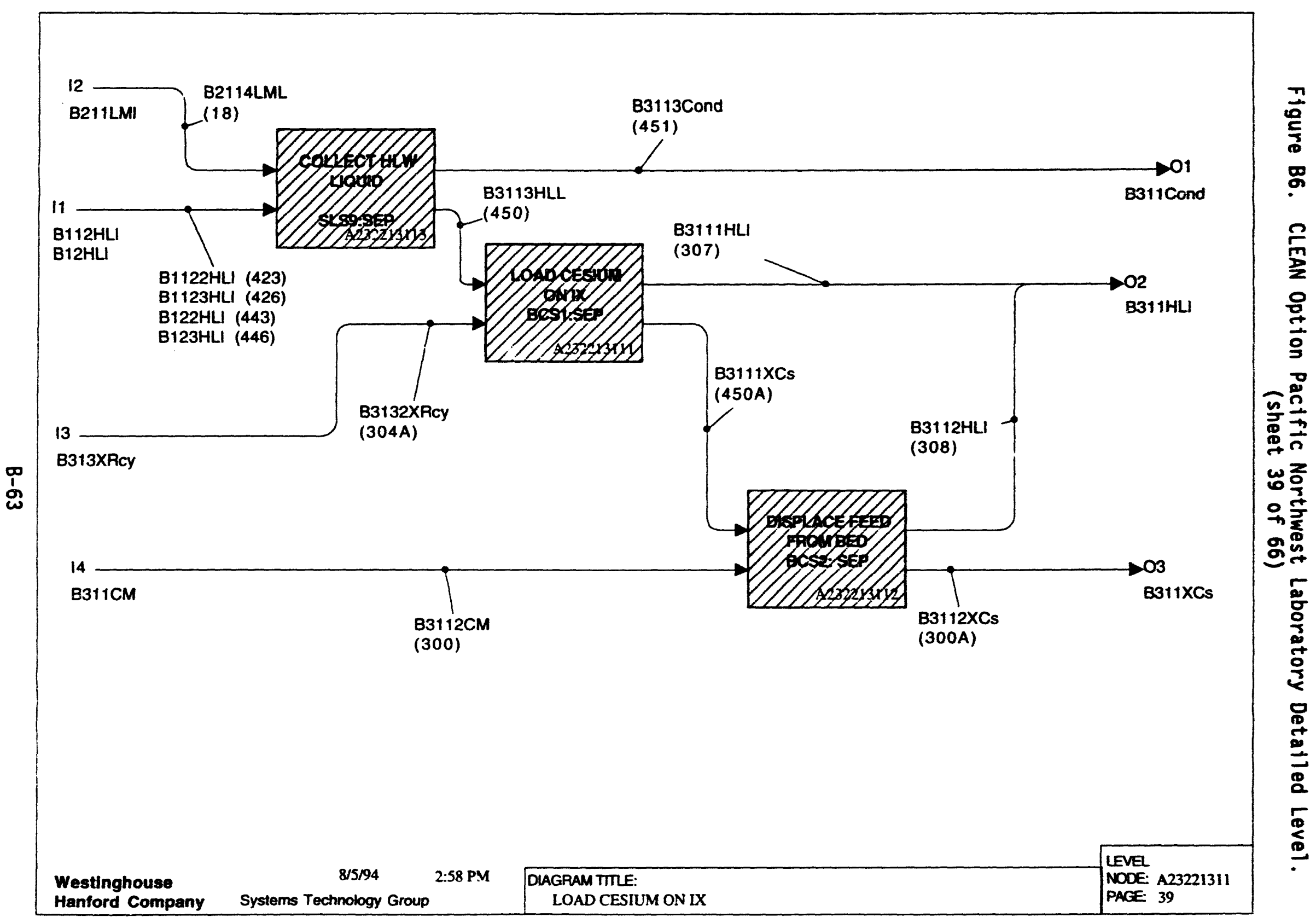




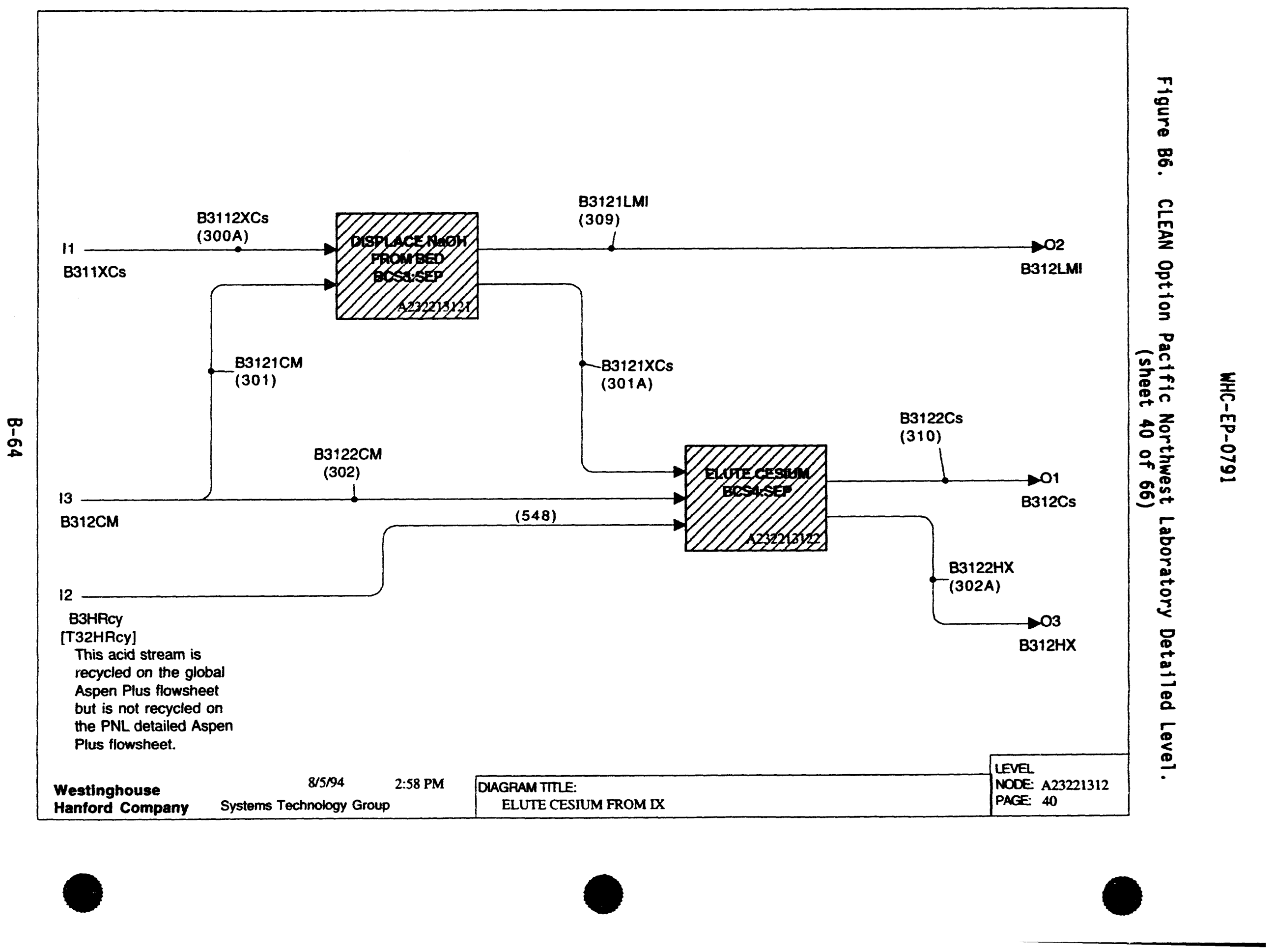




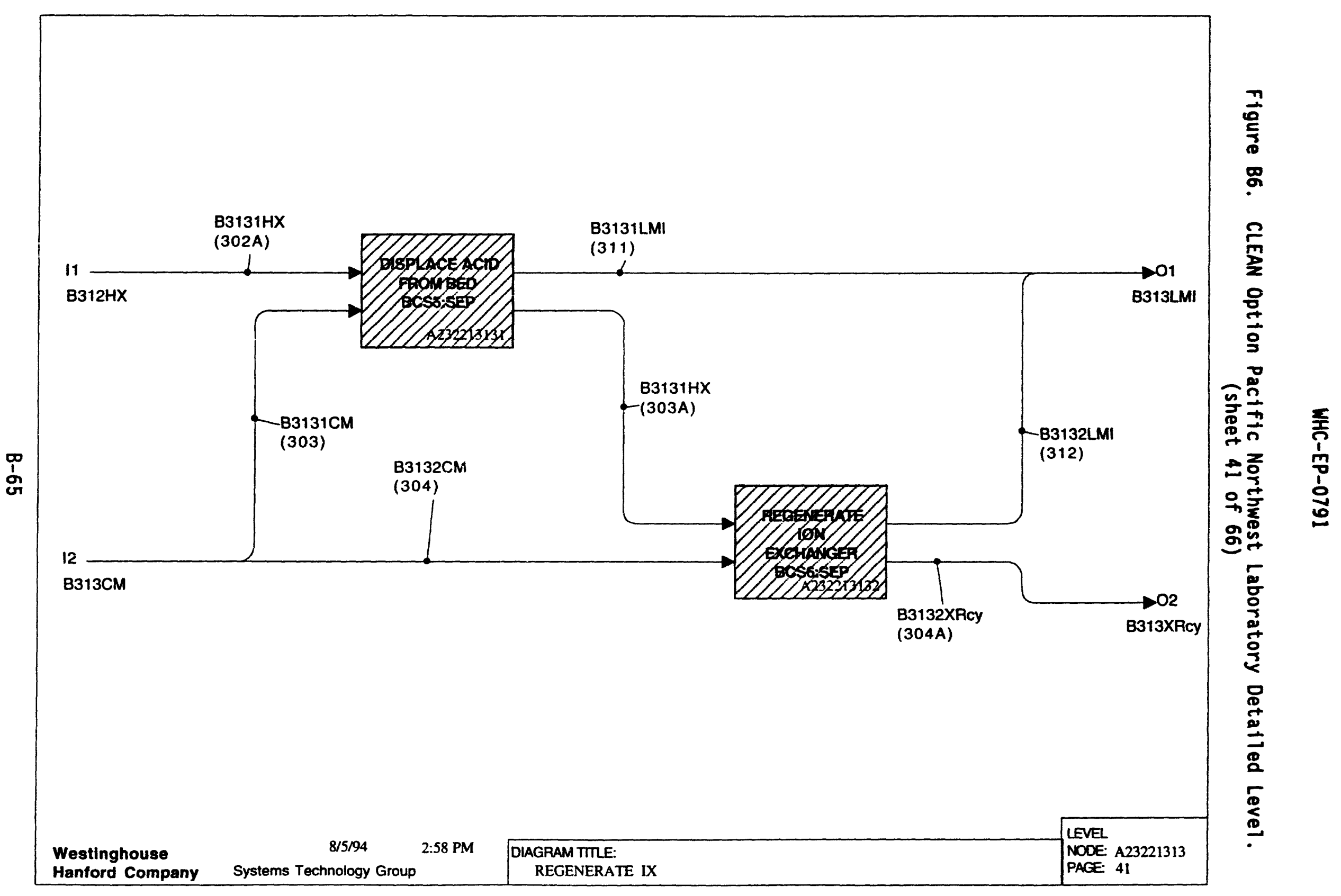




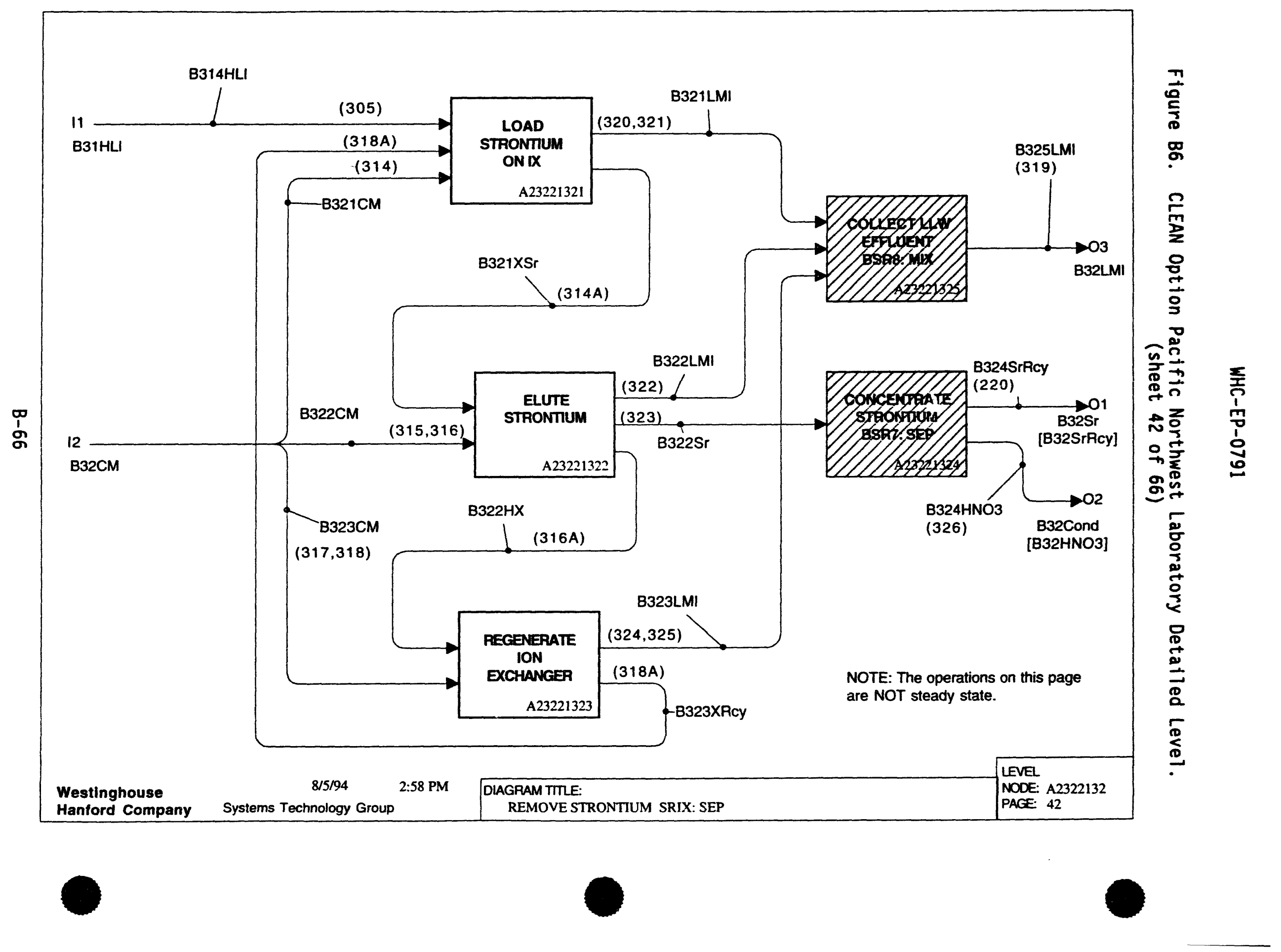


1 1

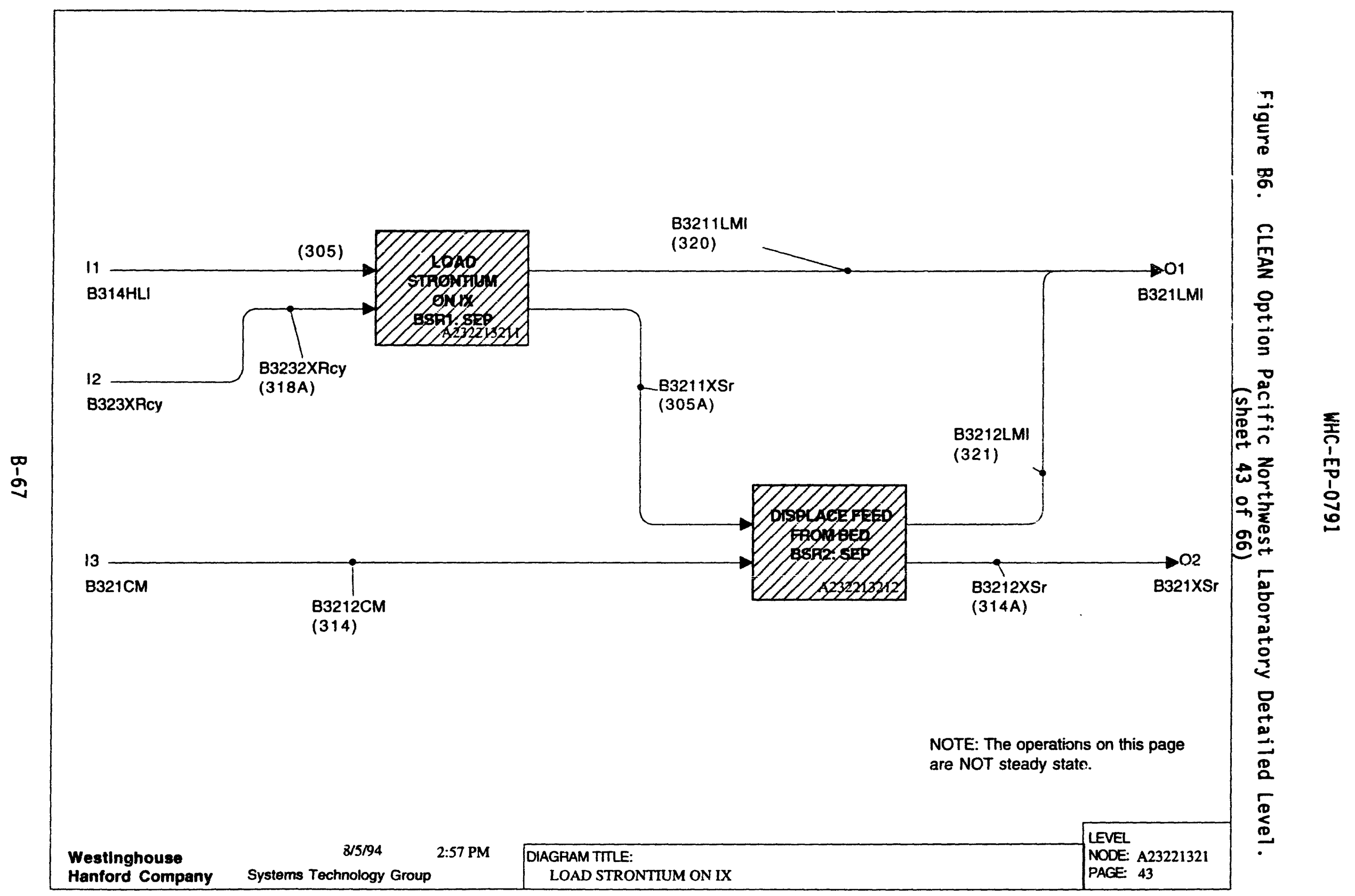




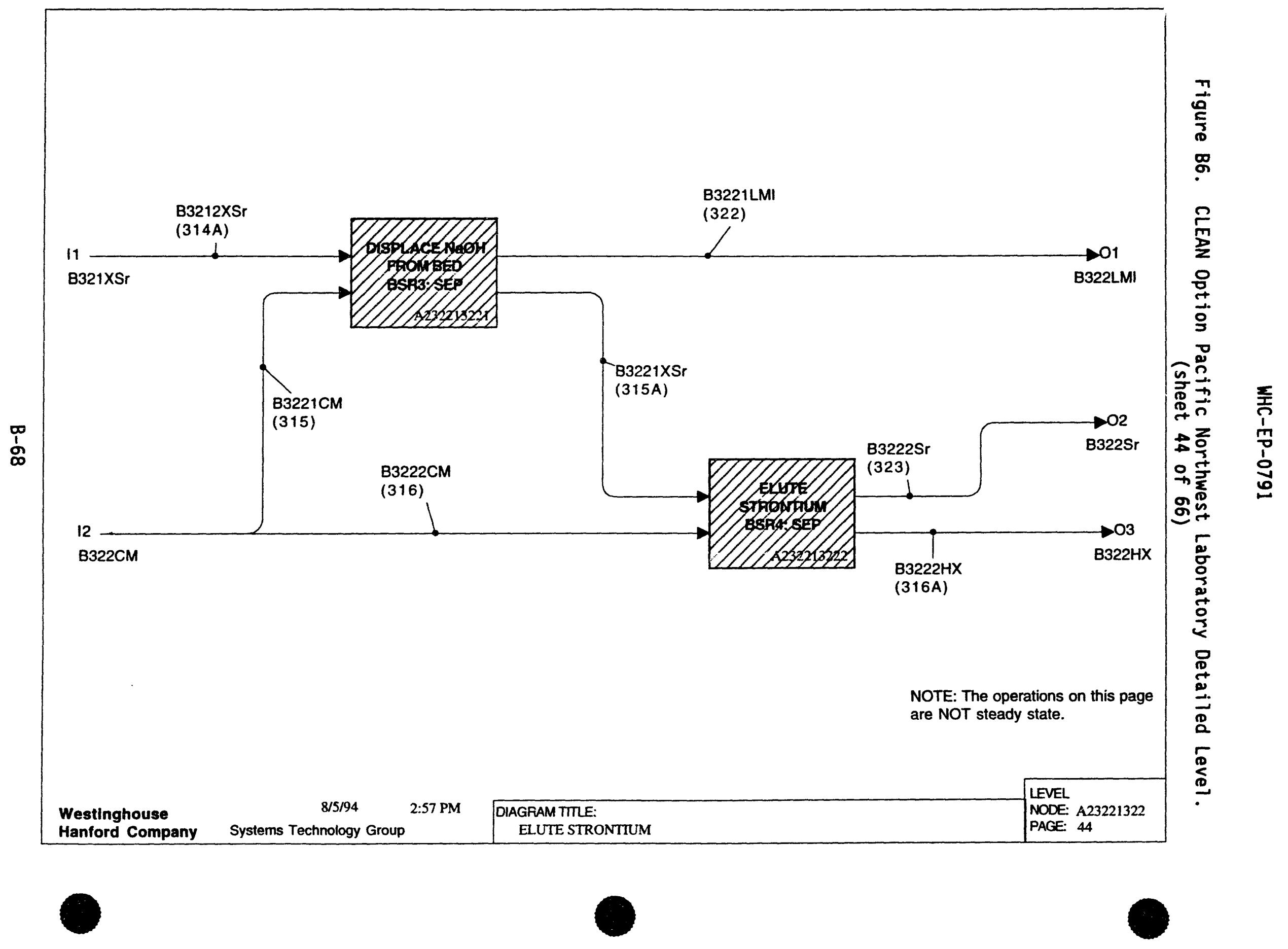




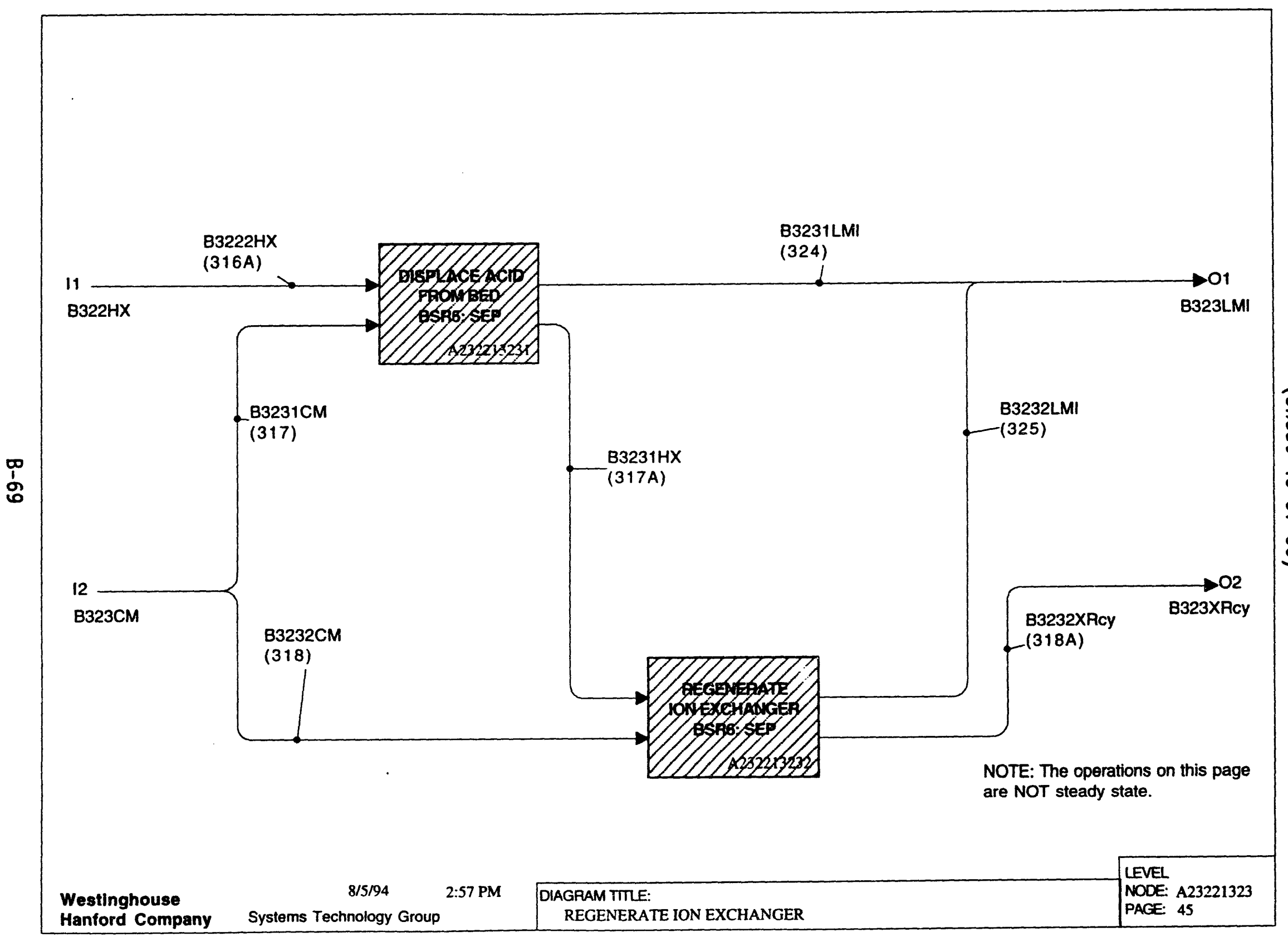

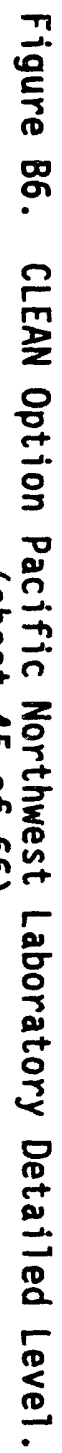




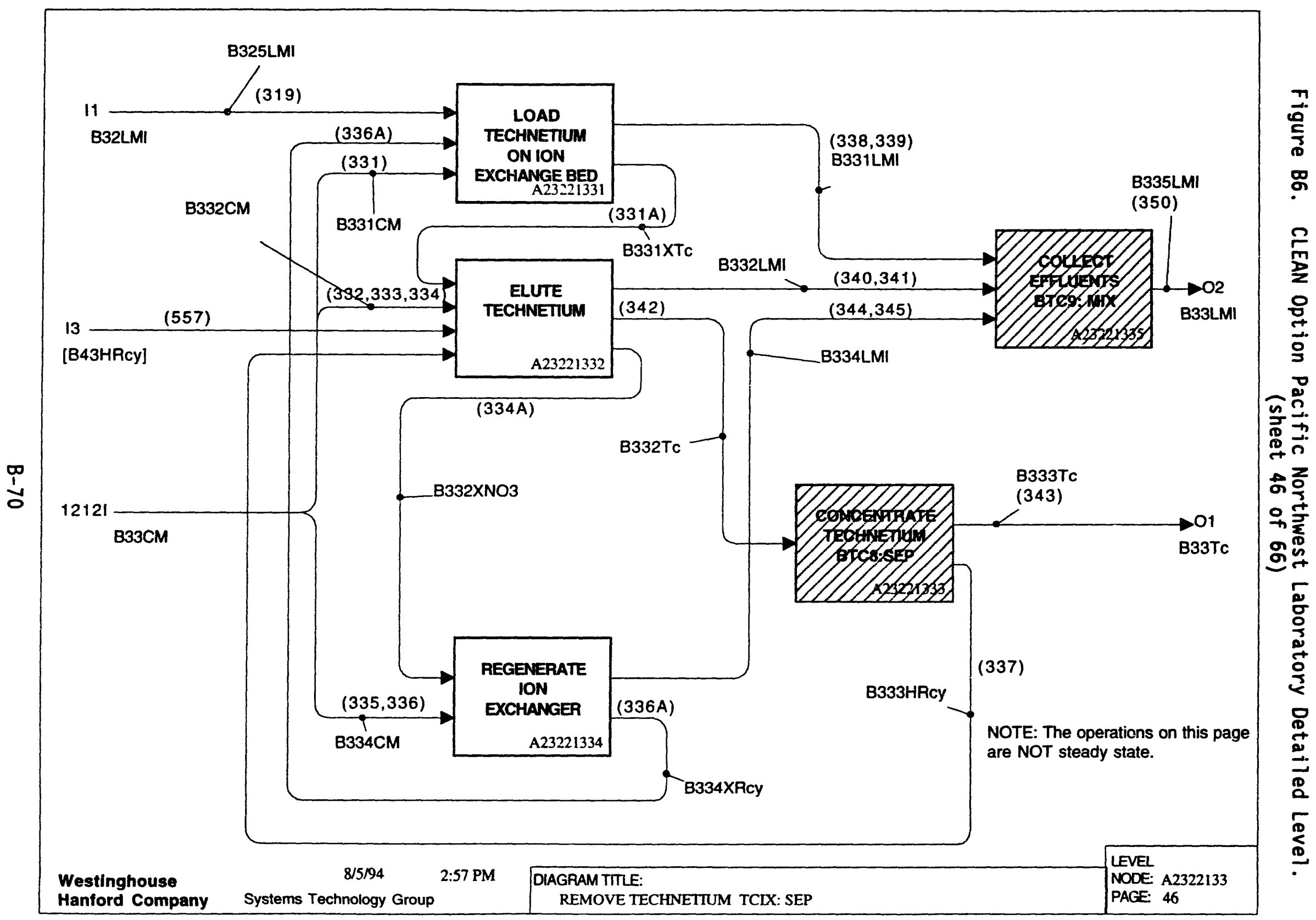




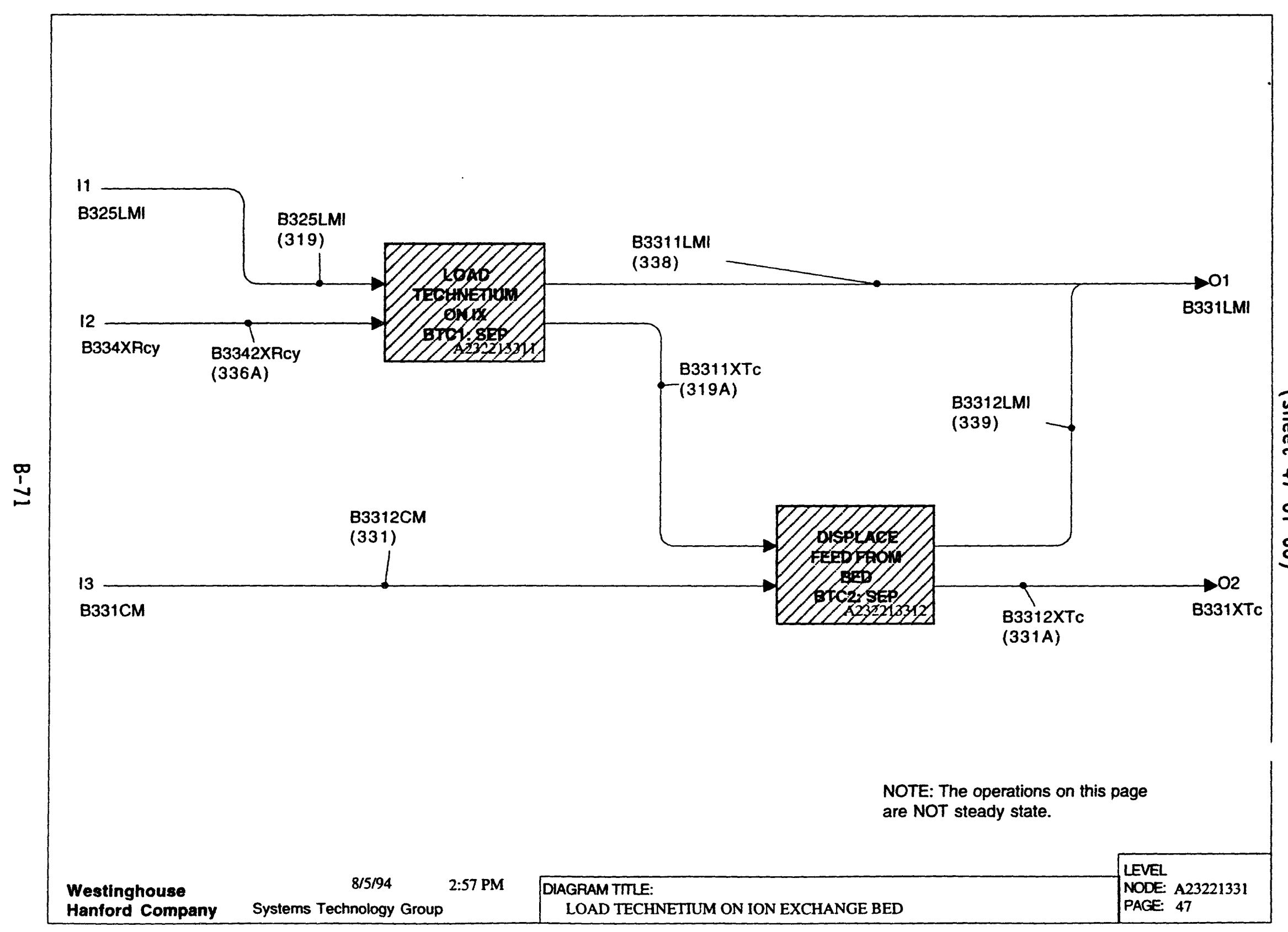




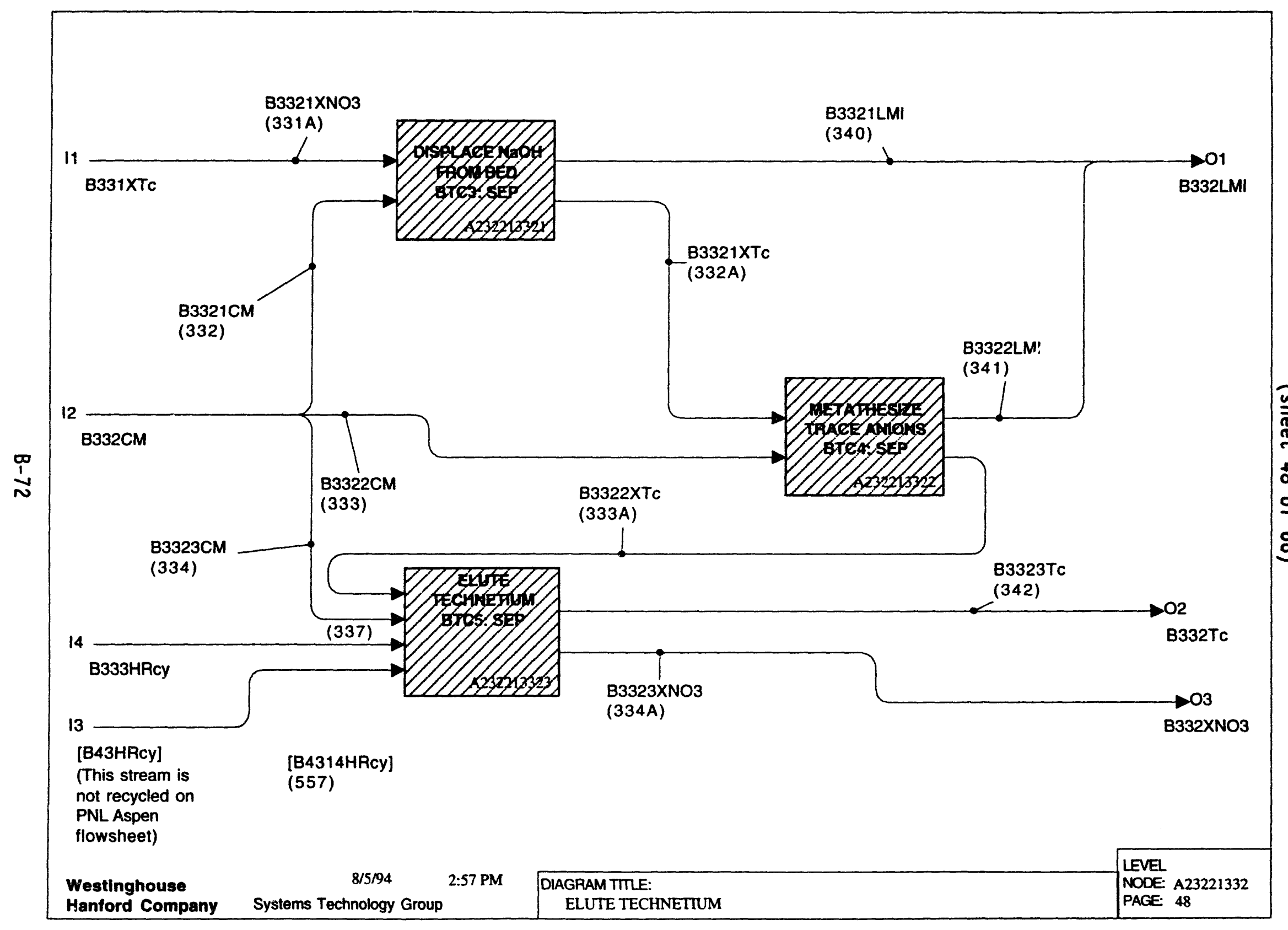

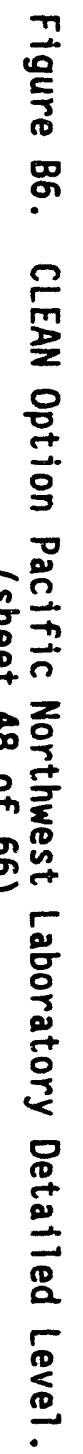

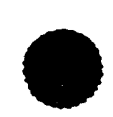




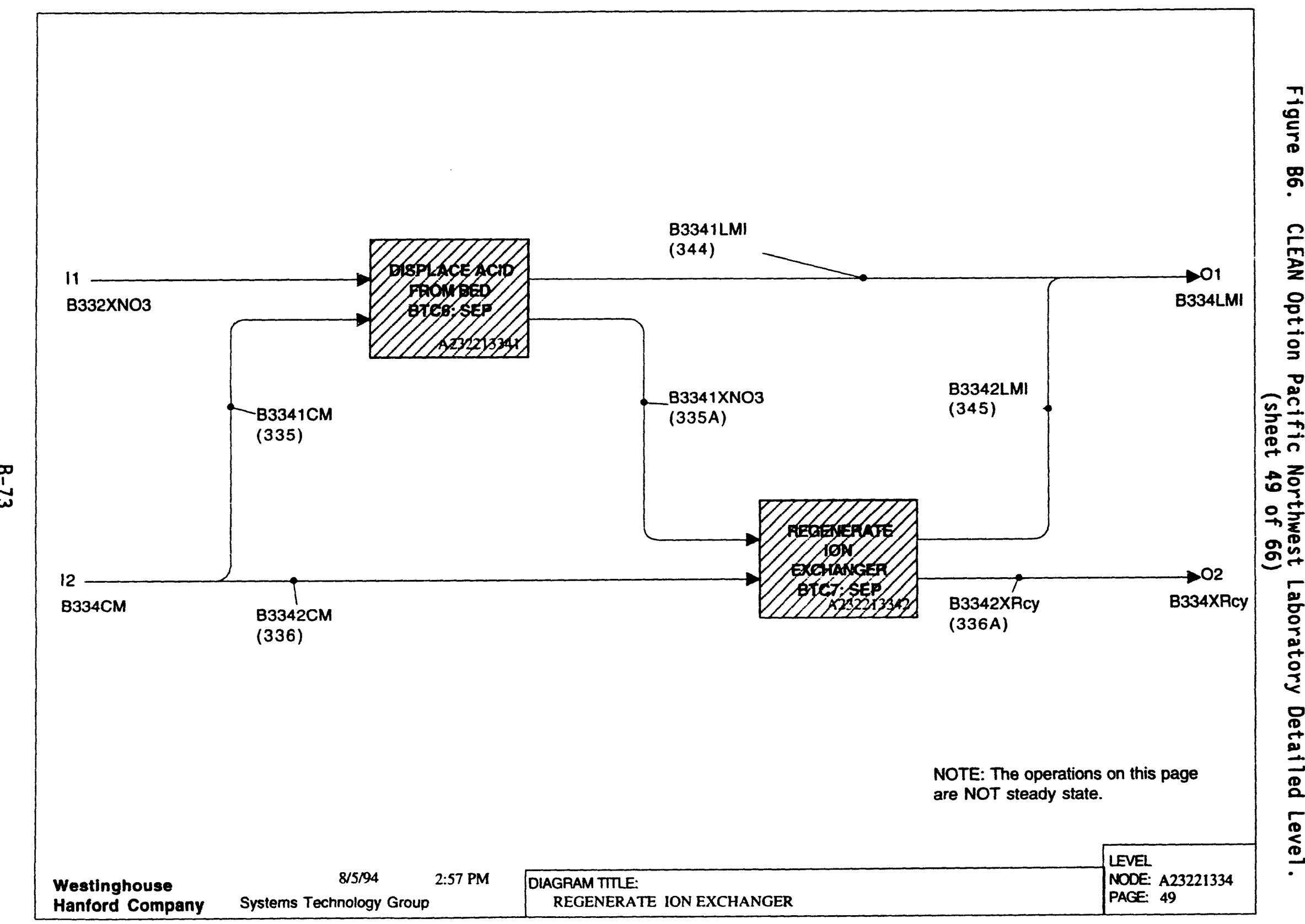




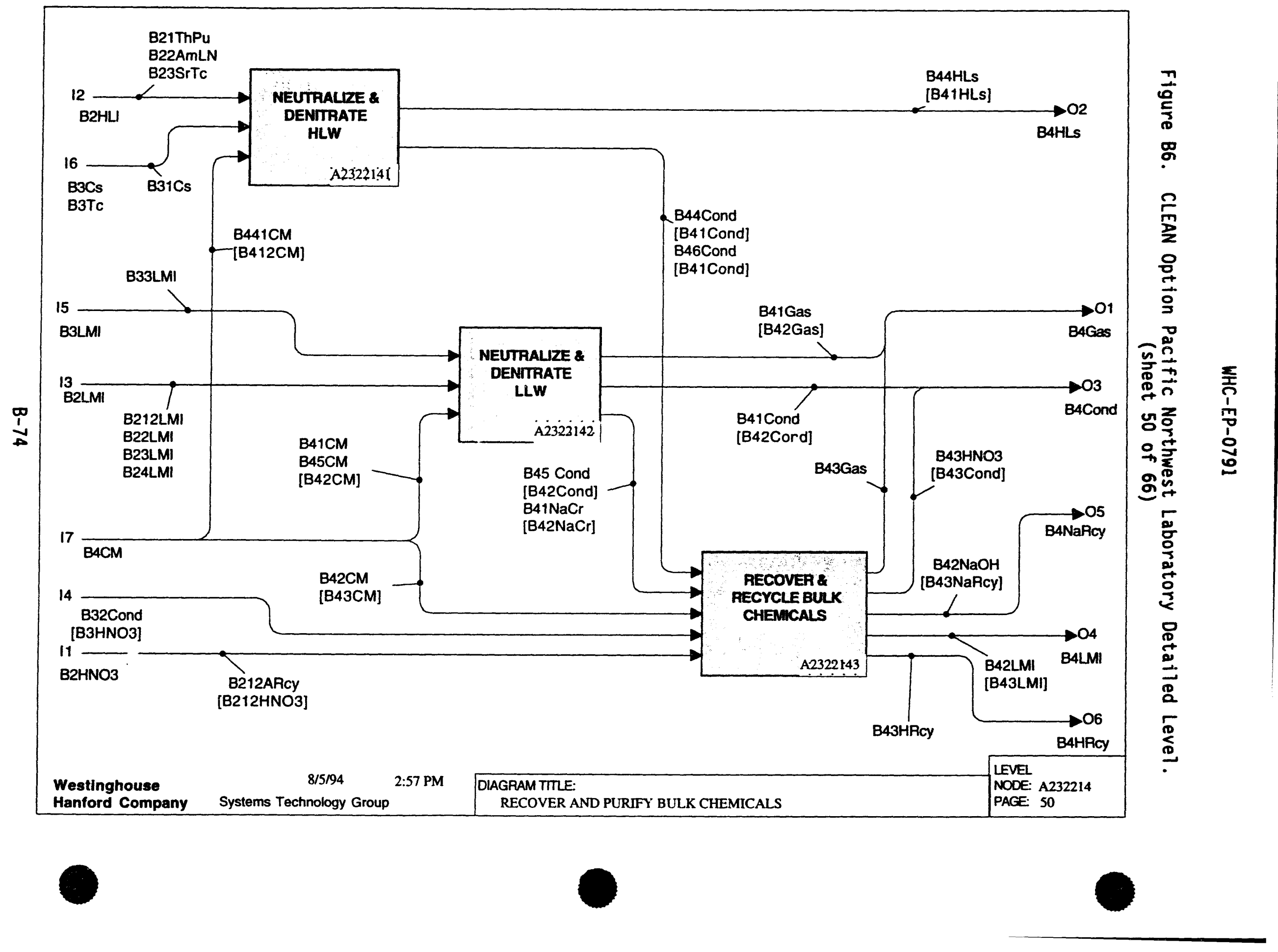




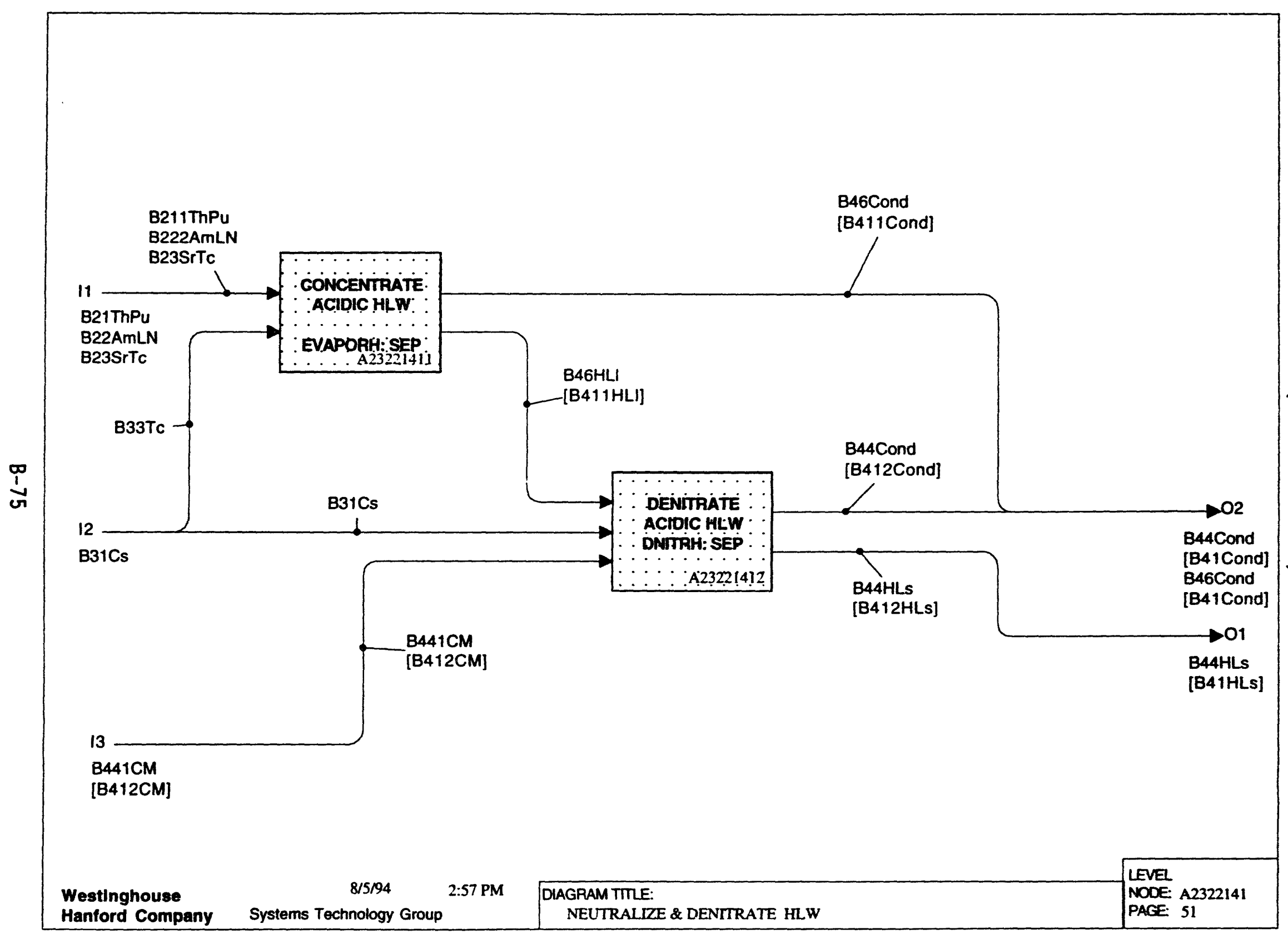




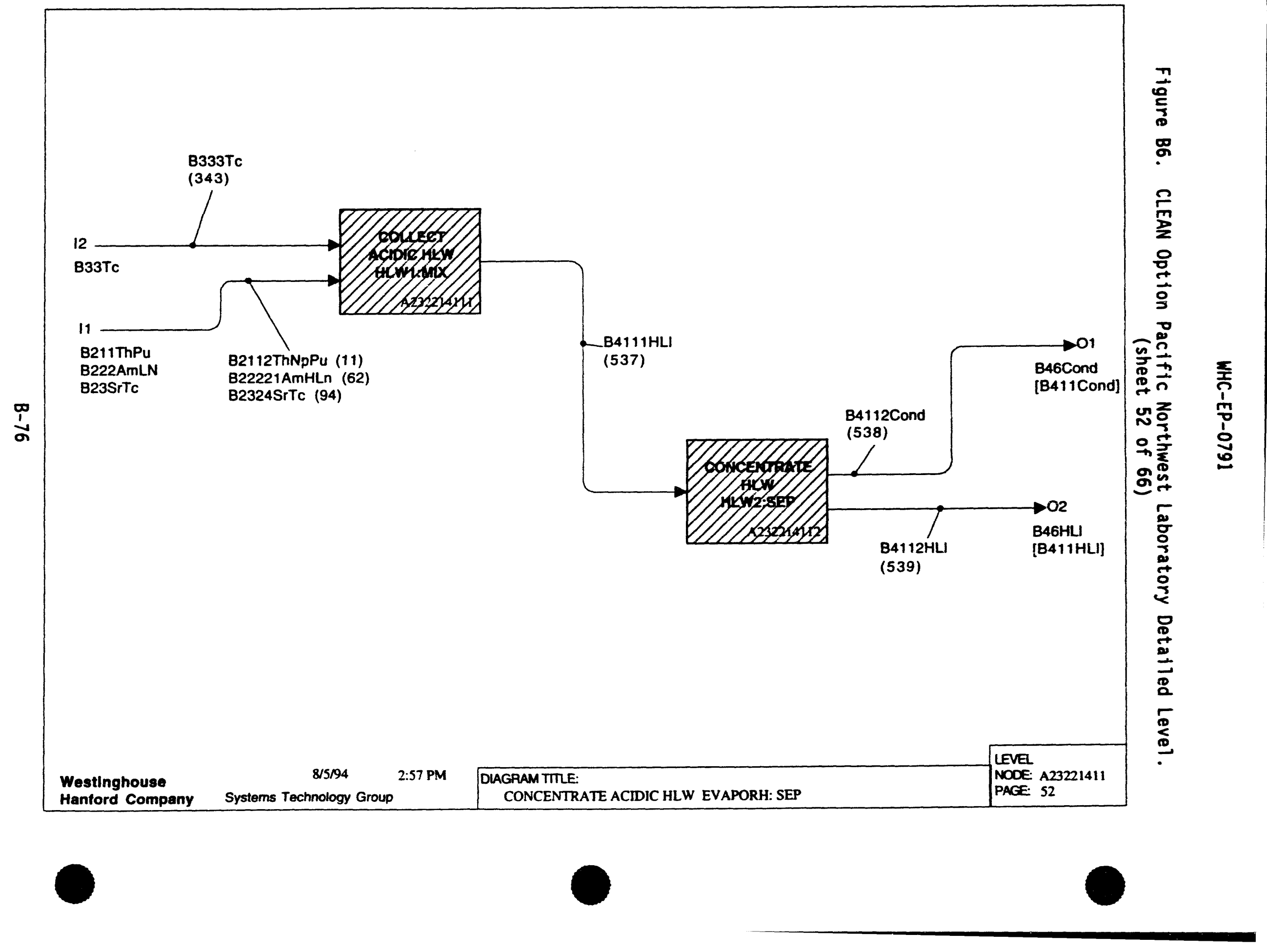




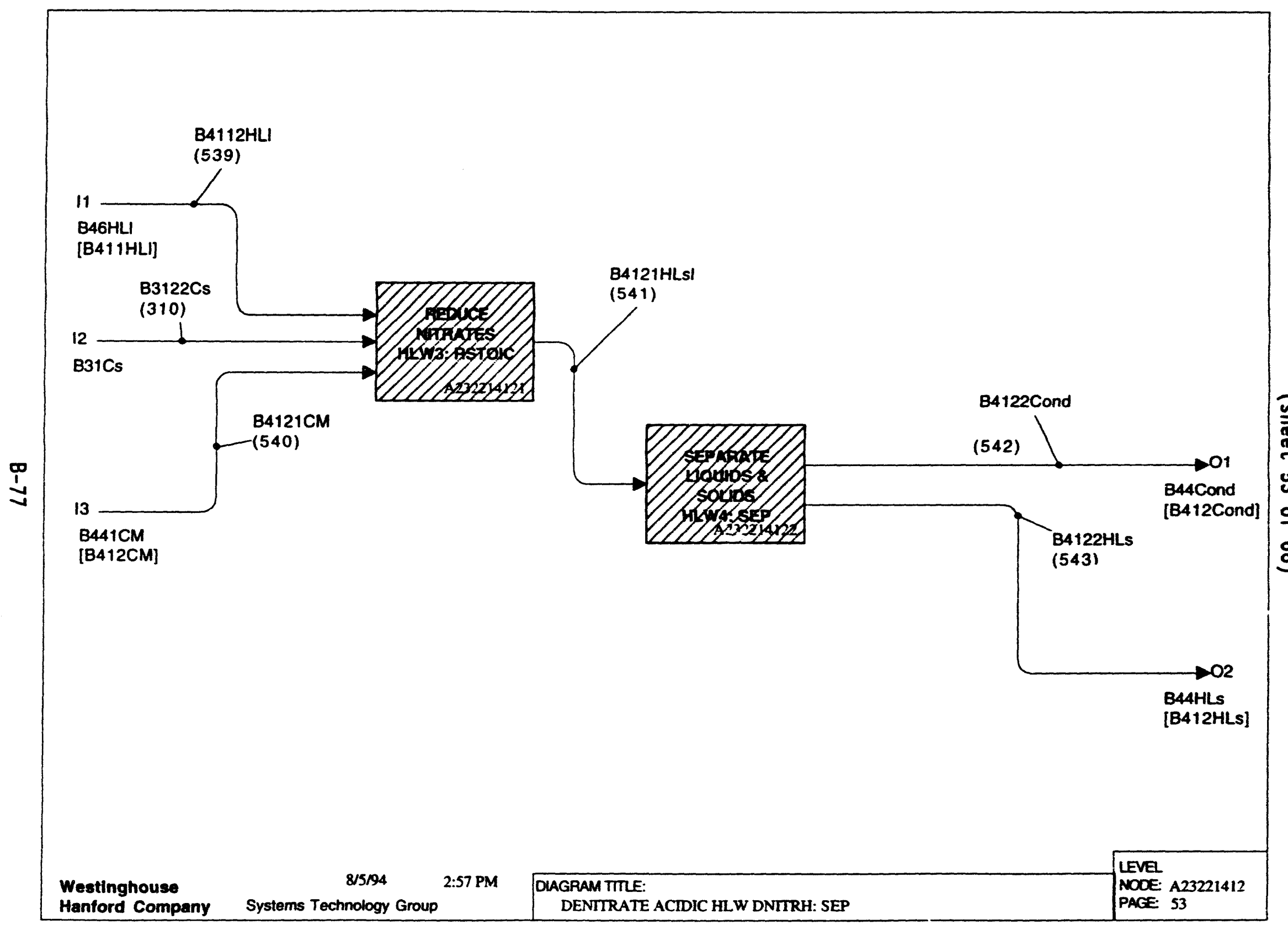

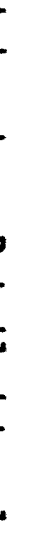




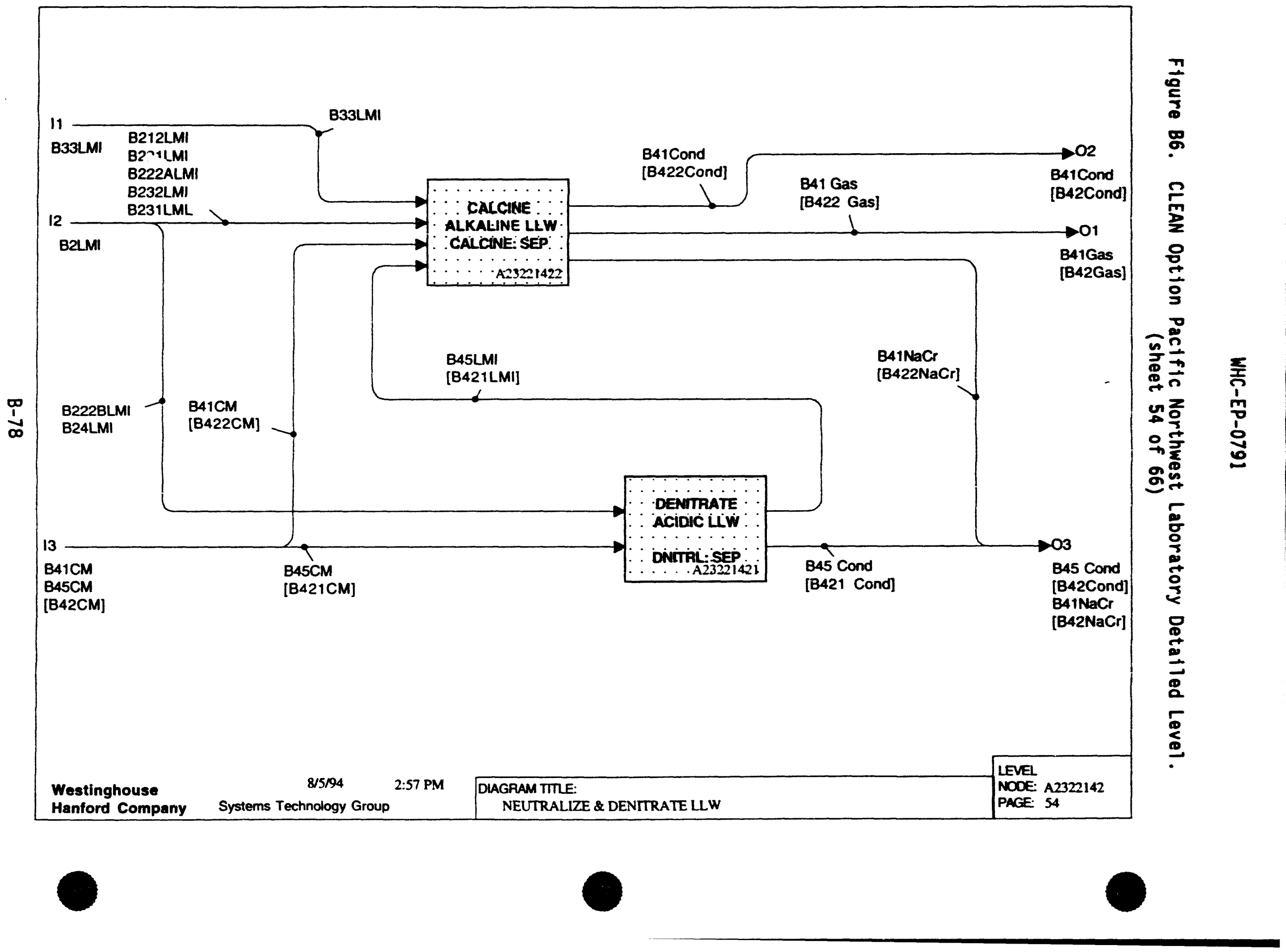




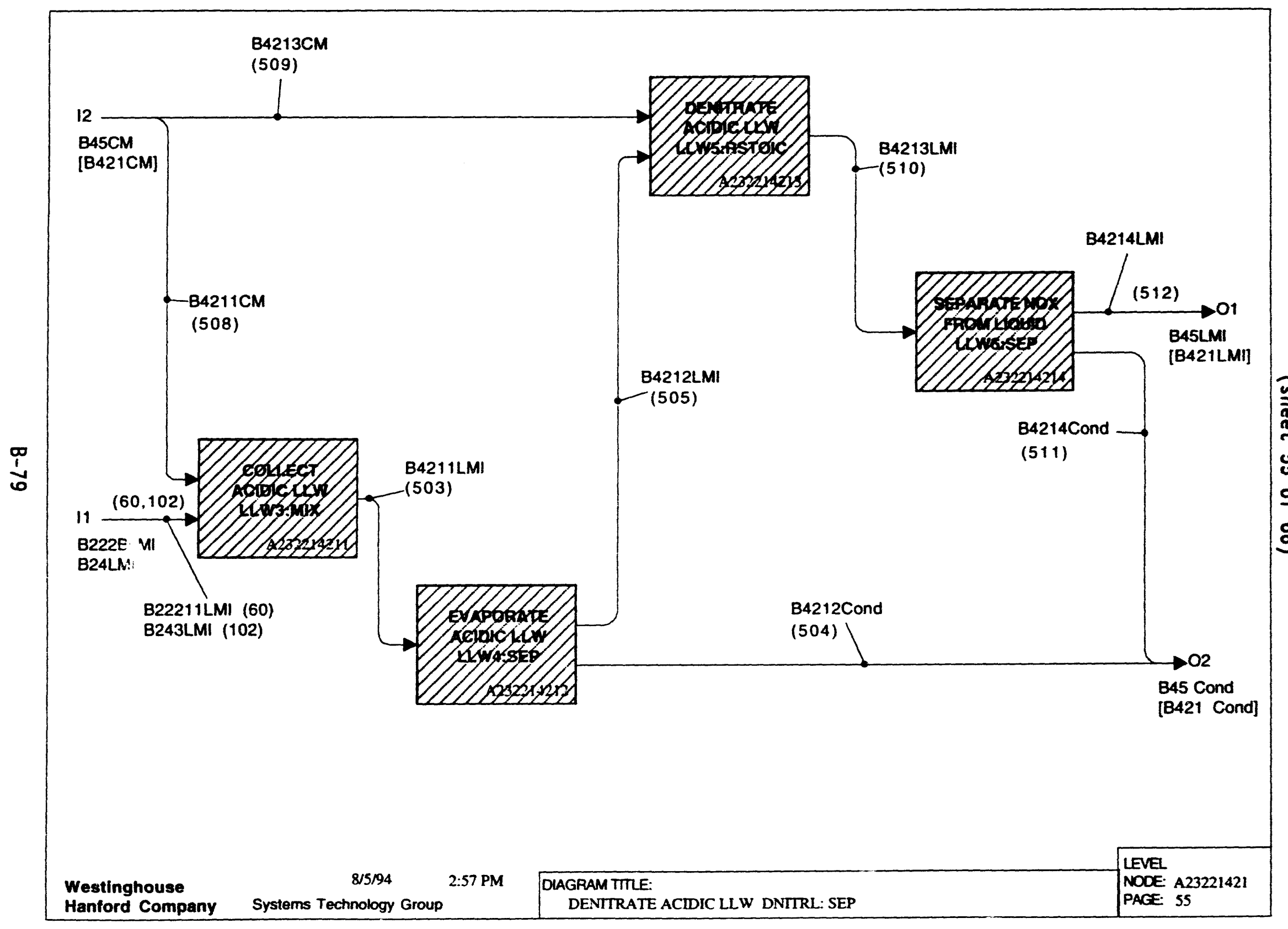

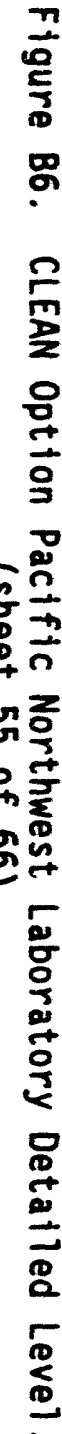




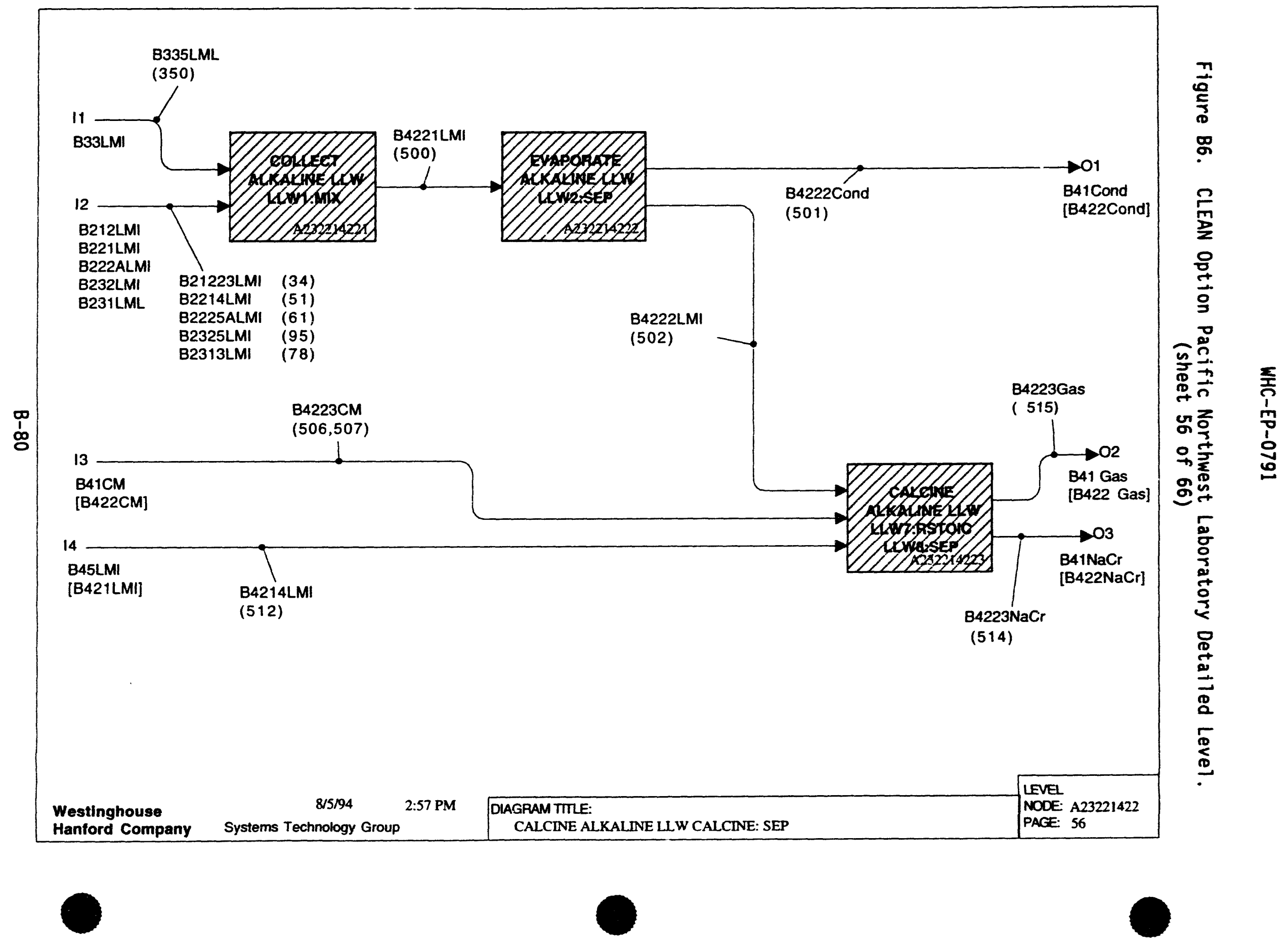




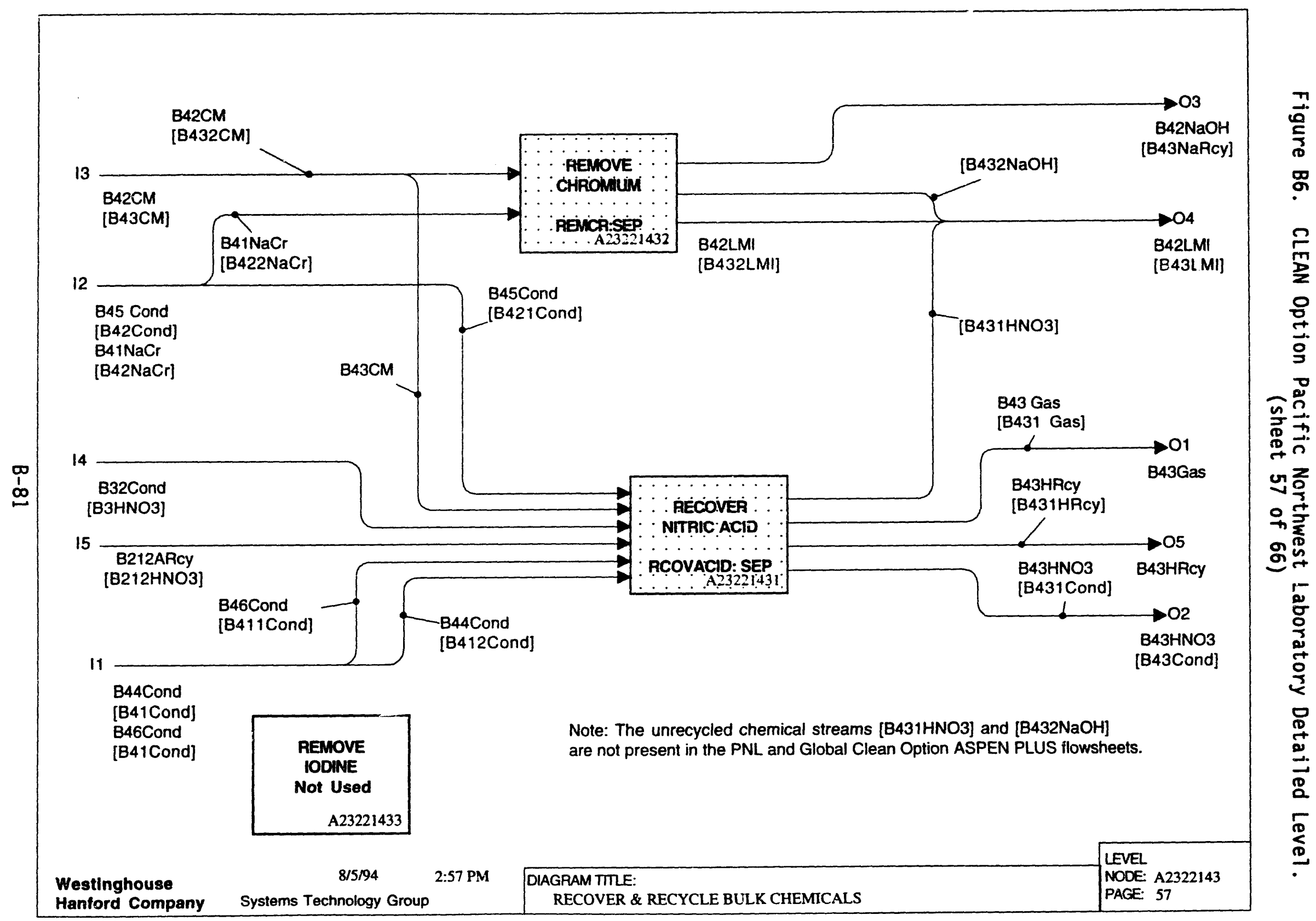




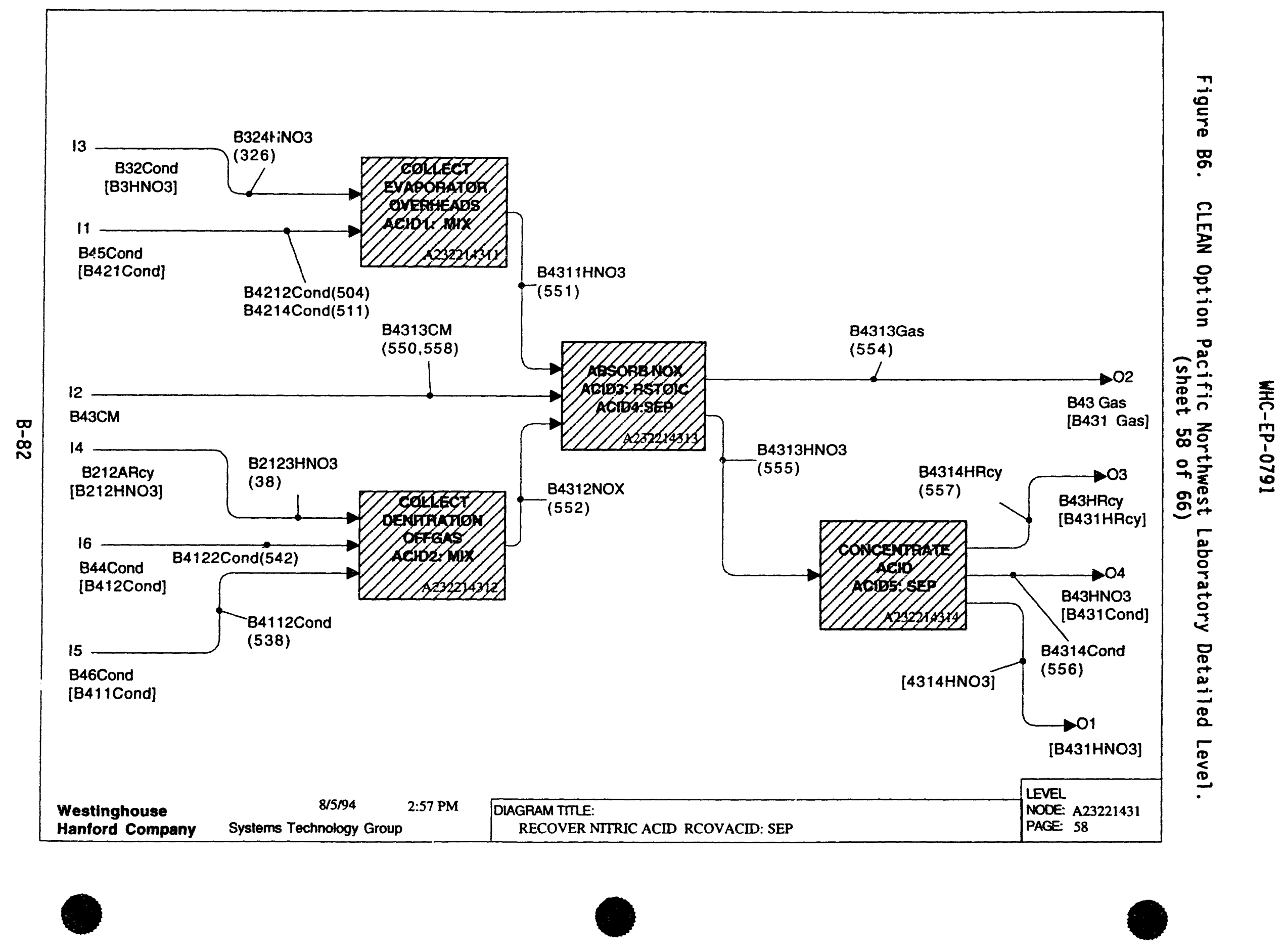




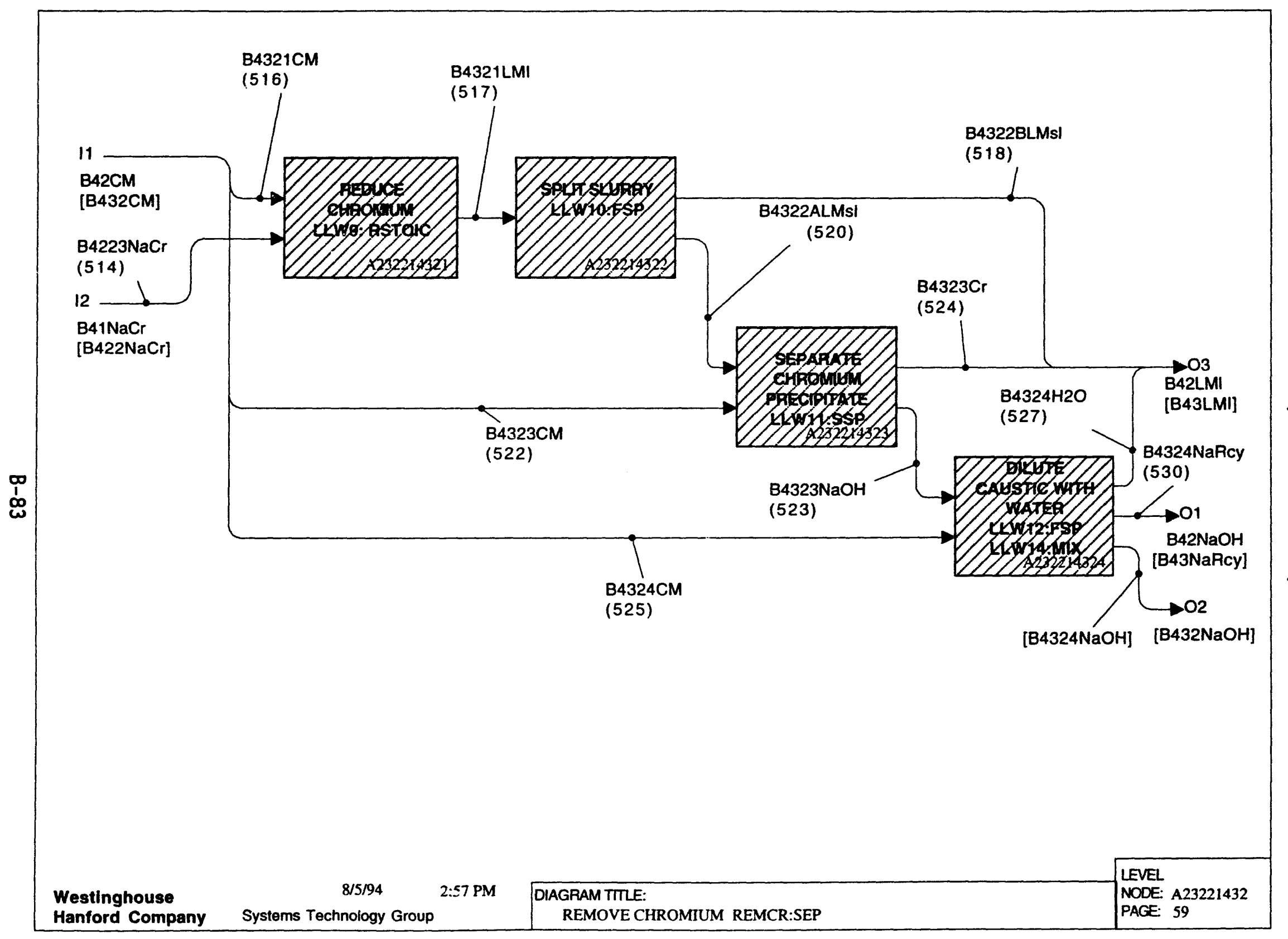

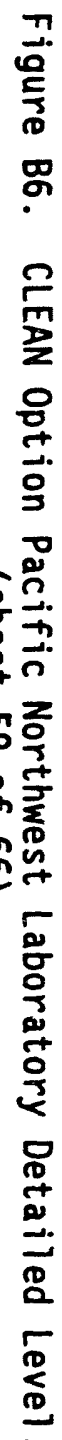




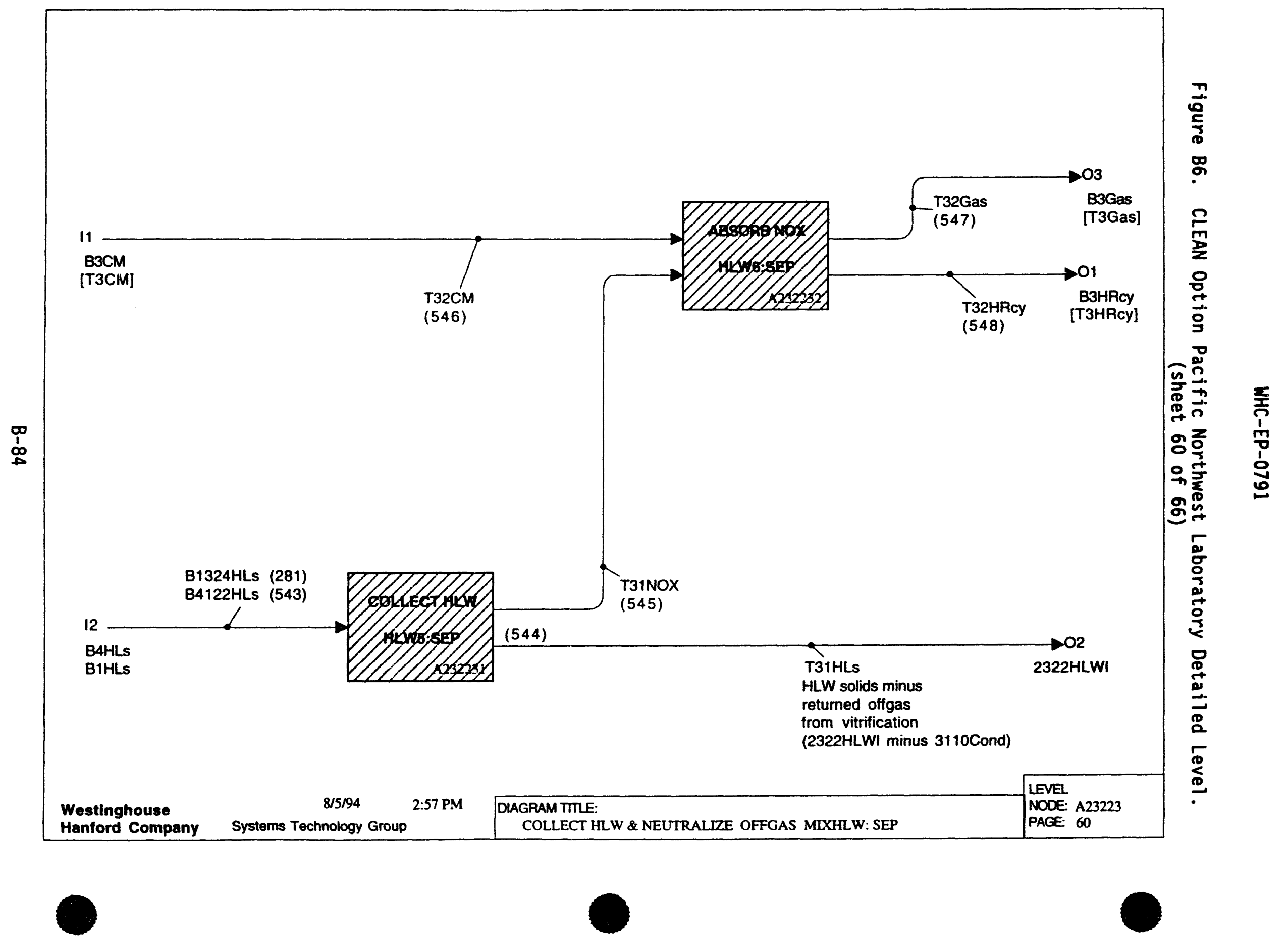




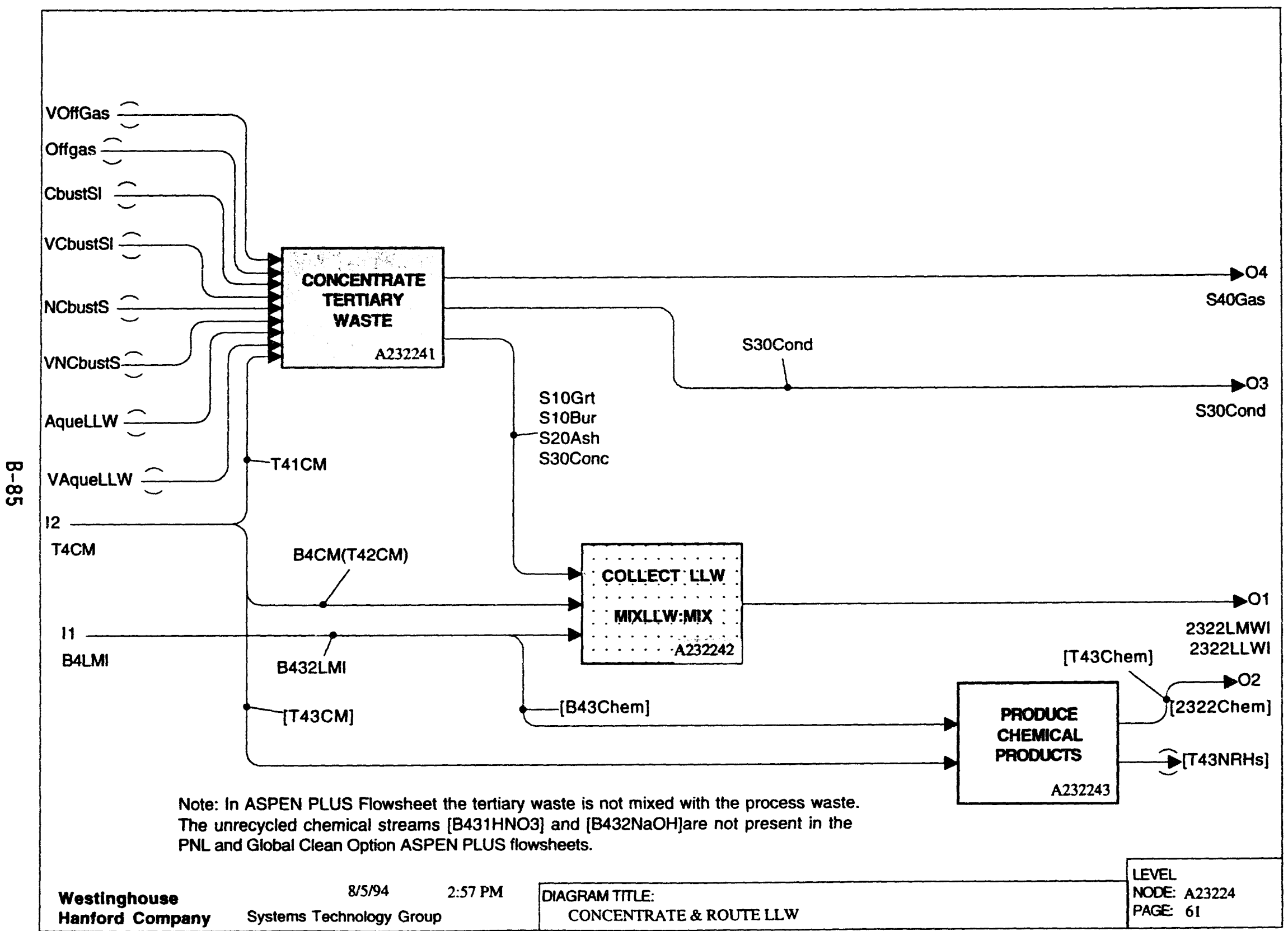




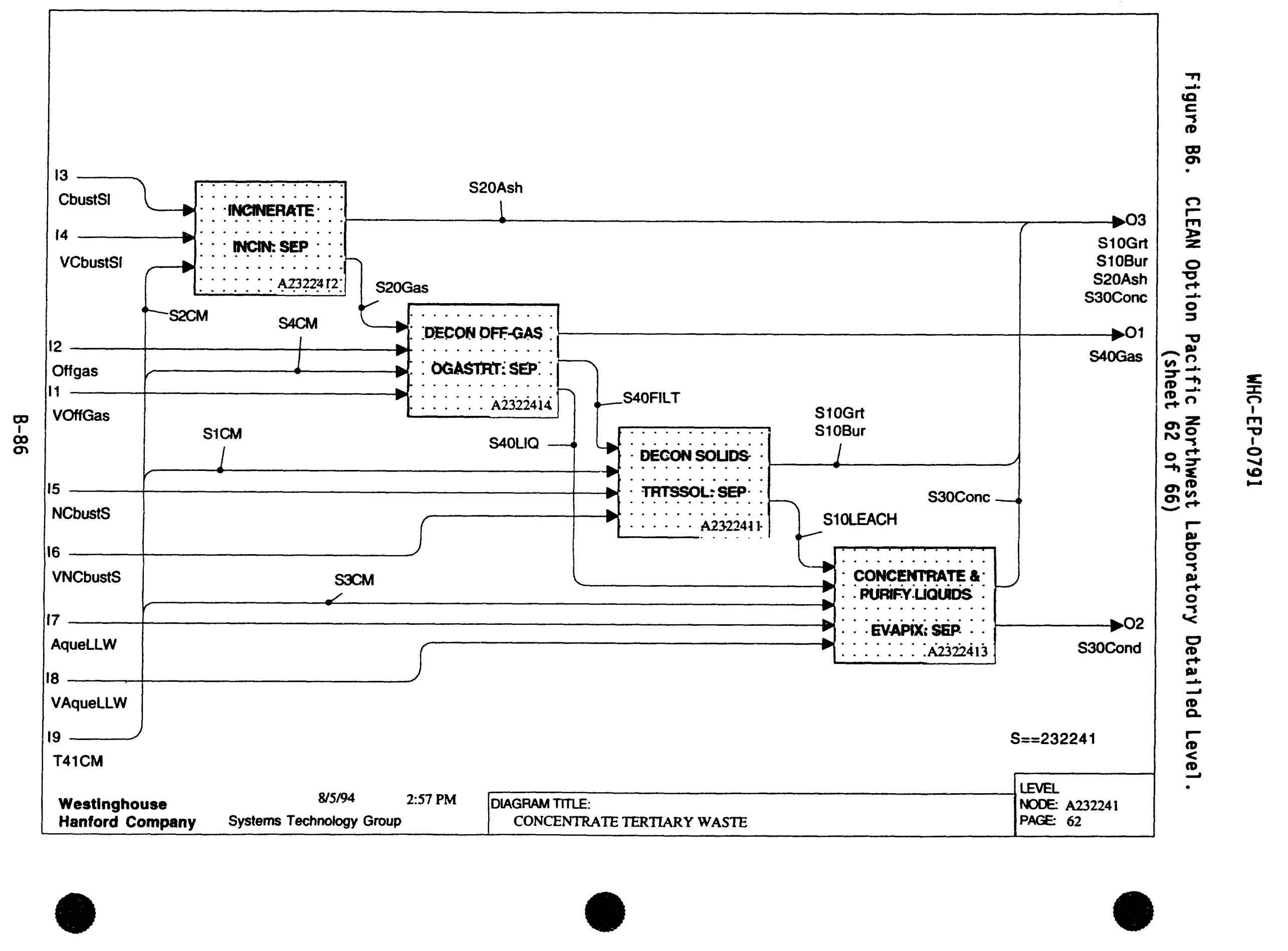



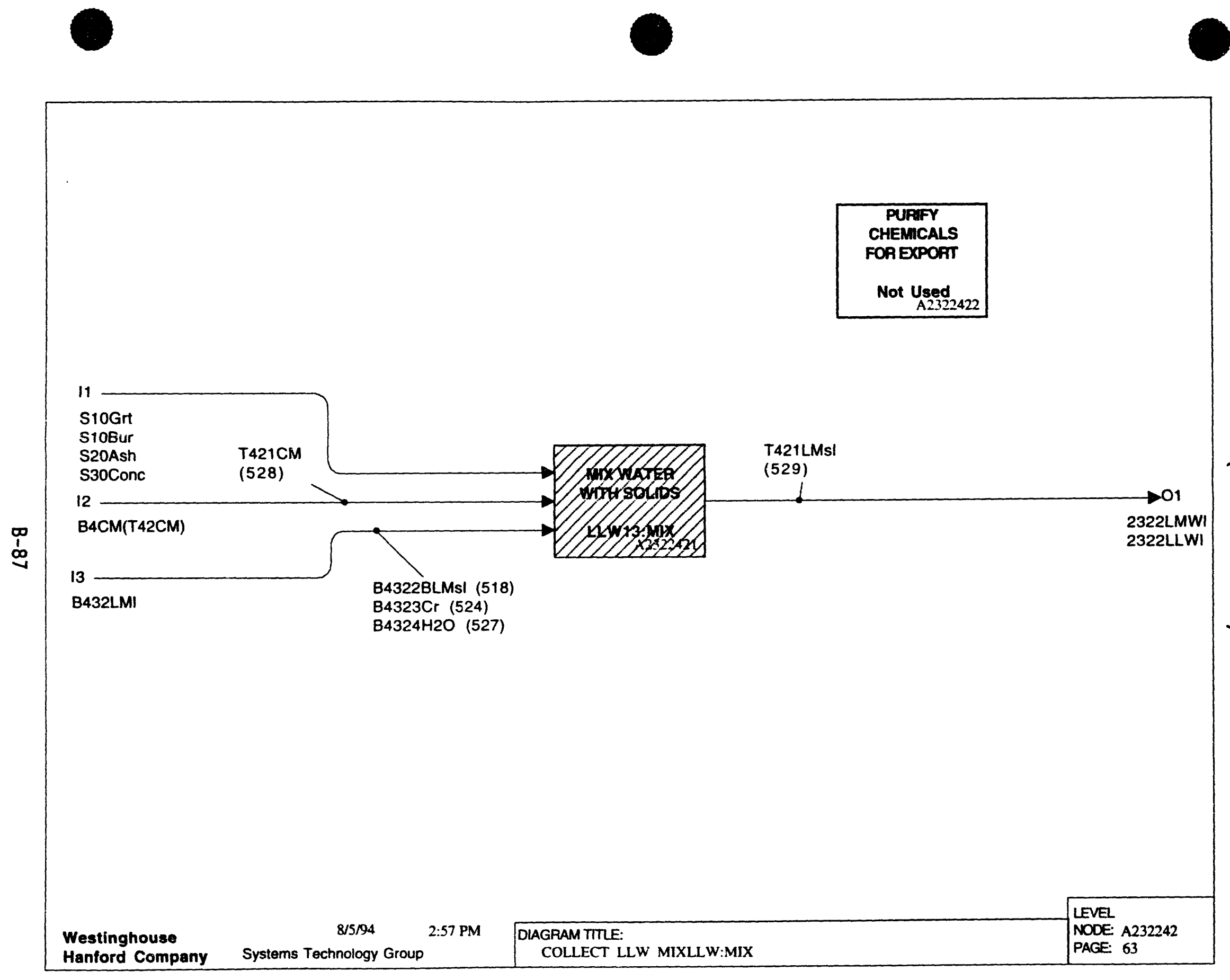

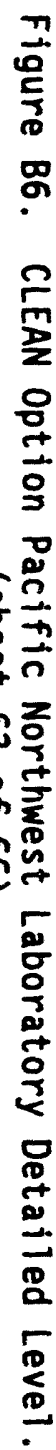




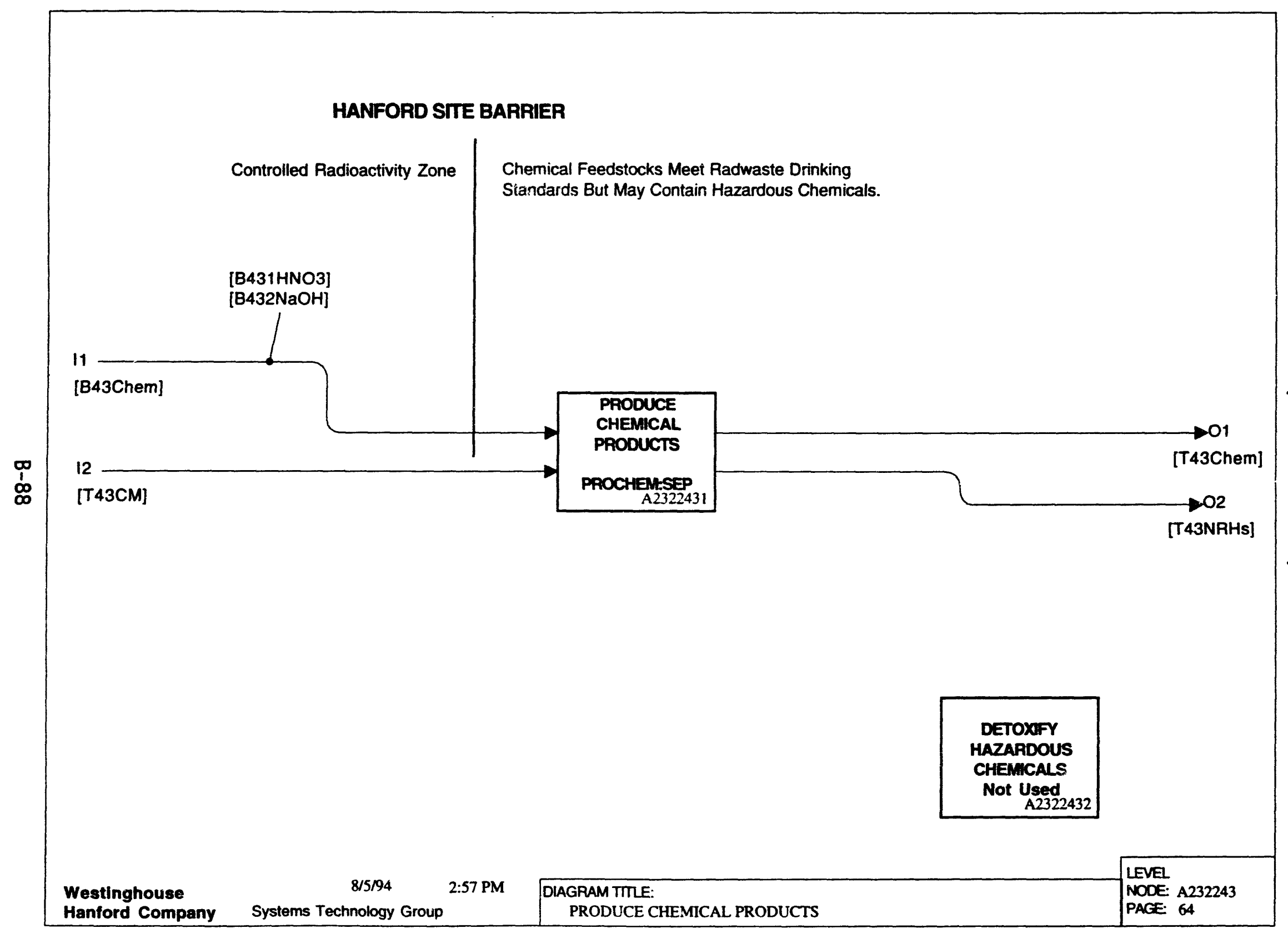




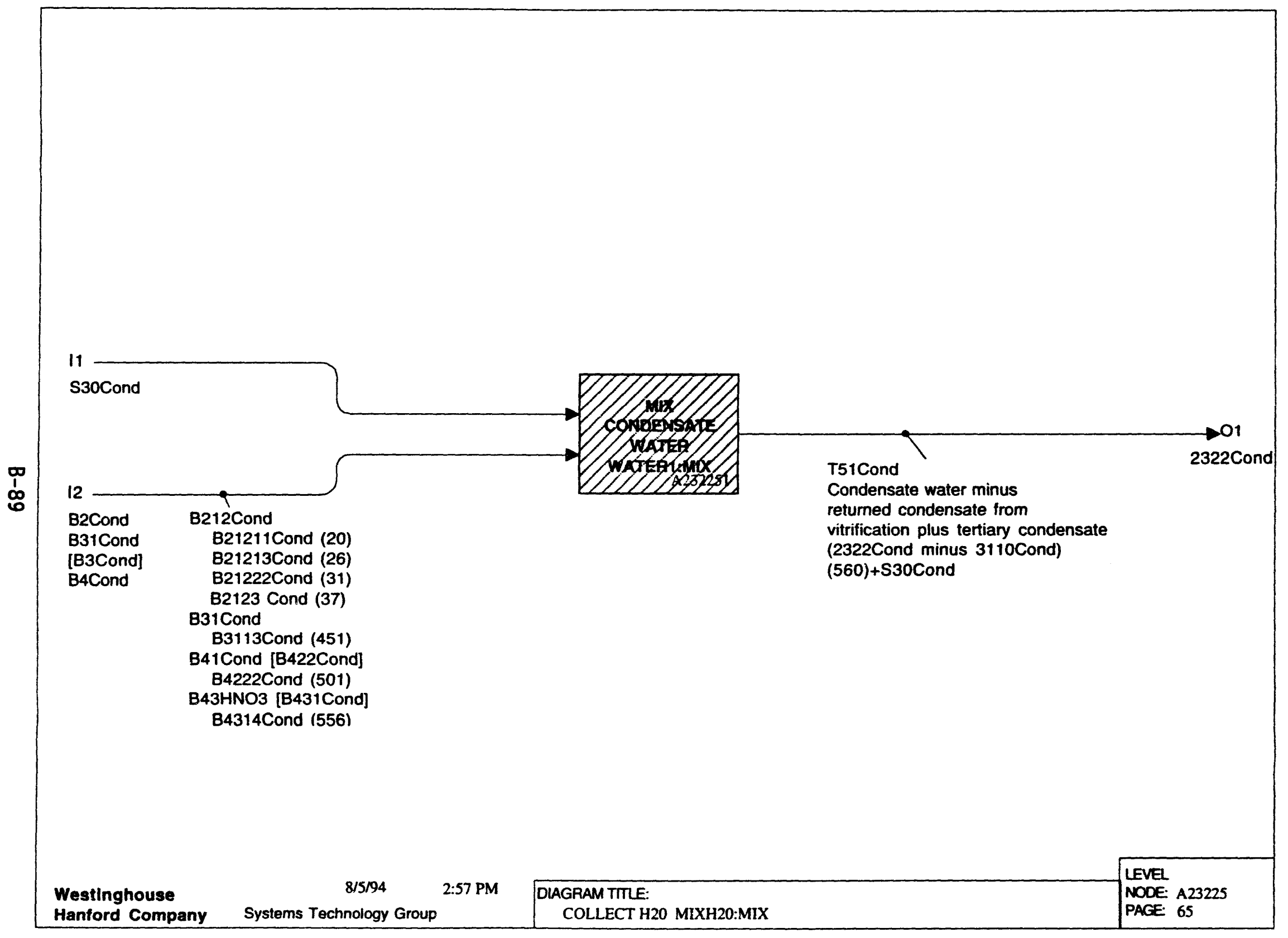

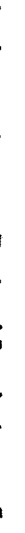




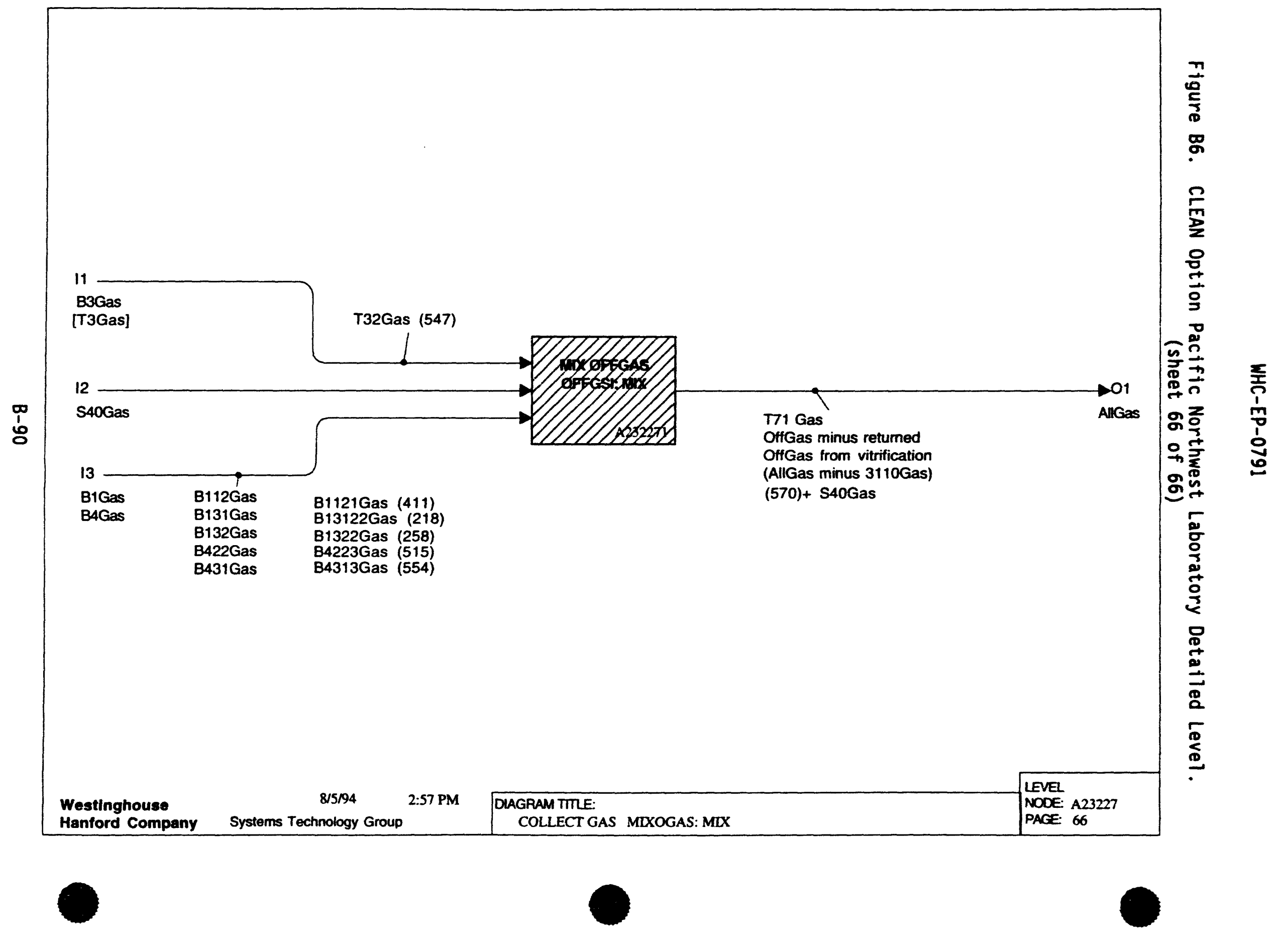


Table B1. Thirty Most Abundant Species by Mass in Waste Feed Streams.

\begin{tabular}{|c|c|c|c|c|c|c|c|c|}
\hline & \multicolumn{2}{|c|}{ SST MASS $(\mathrm{Kg})$} & \multicolumn{2}{|c|}{ DST MASS $(\mathrm{Kg})$} & \multicolumn{2}{|c|}{ COMBINED PHASES } & \multirow{2}{*}{$\begin{array}{l}\text { LIOUID } \\
\text { PHASE } \\
\text { CONC. } \\
\text { (molai) }\end{array}$} & \multirow{2}{*}{$\begin{array}{l}\text { SOLID } \\
\text { PHASE } \\
(w t \%)\end{array}$} \\
\hline & 110010 & $50 L 10$ & LIOUID & SOLID & $(\mathrm{Kg})$ & (gMole) & & \\
\hline TOTAL & $5.56 E+08$ & $1.64 E+07$ & $1.83 E+08$ & $1.88 E+06$ & $7.57 E+08$ & $3.54 E+10$ & & \\
\hline $\mathrm{H} 2 \mathrm{O}$ & $3.86 E+08$ & $0.00 E+00$ & $1.31 E+08$ & $0.00 E+00$ & $5.17 E+08$ & $2.87 E+10$ & & \\
\hline NO3. & $9.60 E+07$ & $9.74 E+05$ & $1.39 E+07$ & $5.81 E+04$ & $1.11 \mathrm{E}+08$ & $1.79 E+09$ & 3.431 & $5.64 \%$ \\
\hline NAt & $5.12 E+07$ & $5.12 E+05$ & $1.67 E+07$ & $2.03 E+05$ & $6.86 E+07$ & $2.98 E+09$ & 5.713 & $3.91 \%$ \\
\hline $\mathrm{OH}$. & $7.10 E+06$ & $2.05 E+06$ & $9.72 E+06$ & $5.83 E+05$ & $1.94 \mathrm{E}+07$ & $1.14 E+09$ & 1.913 & $14.36 \%$ \\
\hline NO2. & $4.75 E+06$ & $4.86 E+04$ & $5.04 E+06$ & $1.46 E+04$ & $9.85 E+06$ & $2.14 E+08$ & 0.412 & $0.35 \%$ \\
\hline PO4 - 3 & $5.50 E+06$ & $3.30 E+66$ & $2.01 E+05$ & $1.61 E+04$ & $9.02 E+06$ & $9.49 E+07$ & 0.116 & $18.11 \%$ \\
\hline$A L+3$ & $5.53 E+05$ & $1.65 E+06$ & $2.11 E+06$ & $9.42 E+04$ & $4.40 E+06$ & $1.63 E+08$ & 0.191 & $9.51 \%$ \\
\hline $\mathrm{CO} \cdot 2$ & $1.53 E+06$ & $1.67 E+05$ & $1.10 E+06$ & $1.69 E+04$ & $2.82 E+06$ & $4.70 E+07$ & 0.085 & $1.01 \%$ \\
\hline CANCRINITE & $0.00 E+00$ & $2.70 E+06$ & $0.00 E+00$ & $0.00 E+00$ & $2.70 E+06$ & $7.93 E+06$ & 0.000 & $14.76 \%$ \\
\hline $\mathrm{SO} 4 \cdot 2$ & $1.62 E+06$ & $3.36 E+04$ & $3.74 E+05$ & $5.67 E+03$ & $2.03 E+06$ & $2.11 E+07$ & 0.040 & $0.21 \%$ \\
\hline$k+$ & $3.95 E+05$ & $4.69 E+03$ & $1.42 \mathrm{E}+06$ & $1.69 E+04$ & $1.84 E+06$ & $4.71 E+07$ & 0.090 & $0.12 \%$ \\
\hline $402+2$ & $8.16 E+04$ & $1.51 E+06$ & $1.34 E+04$ & $3.01 E+04$ & $1.63 E+06$ & $6.04 E+06$ & 0.001 & $8.40 \%$ \\
\hline$F$ & $7.69 E+05$ & $4.09 E+04$ & $3.65 E+05$ & $1.68 E+04$ & $1.19 E+06$ & $6.27 E+07$ & 0.116 & $0.32 \%$ \\
\hline $2 \mathrm{RO} 2: 2 \mathrm{H} 2$ & $2.14 E+04$ & $4.15 \mathrm{E}+05$ & $1.72 E+03$ & $5.45 E+05$ & $9.83 E+05$ & $6.17 E+06$ & 0.000 & $5.24 \%$ \\
\hline $10 C$ & $1.80 t+05$ & $2.01 E+04$ & $6.02 E+05$ & $1.32 E+04$ & $8.15 E+05$ & $6.79 E+07$ & 0.126 & $0.18 \%$ \\
\hline $\mathrm{FE}+3$ & $6.24 E+03$ & $6.24 E+05$ & $1.23 E+03$ & $1.01 E+05$ & $7.32 E+05$ & $1.31 \mathrm{E}+07$ & 0.000 & $3.96 \%$ \\
\hline$B A+2$ & $0.00 E+00$ & $5.00 E+05$ & $3.09 E+02$ & $1.45 E+03$ & $5.02 E+05$ & $3.65 E+06$ & 0.000 & $2.74 \%$ \\
\hline NI2FECNG & $0.00 E+00$ & $4.98 E+05$ & $0.00 E+00$ & $0.00 E+00$ & $4.98 E+05$ & $1.51 E+06$ & 0.000 & 2.728 \\
\hline $\mathrm{CL}$. & $3.80 E+04$ & $2.01 E+03$ & $4.46 E+05$ & $5.10 E+02$ & $4.87 E+05$ & $1.37 E+07$ & 0.026 & $0.01 \%$ \\
\hline$P B+4$ & $0.00 E+00$ & $4.36 E+05$ & $2.69 E+03$ & $9.96 E+02$ & $4.40 E+05$ & $2.12 E+06$ & 0.000 & $2.39 \%$ \\
\hline $81+3$ & $6.41 E+04$ & $1.97 E+05$ & $0.00 E+00$ & $0.00 E+00$ & $2.61 E+05$ & $1.25 E+06$ & 0.001 & $1.08 \%$ \\
\hline$C E+3$ & $2.33 E+03$ & $2.33 E+05$ & $2.45 E+01$ & $8.20 E+02$ & $2.36 E+05$ & $1.68 E+06$ & 0.000 & $1.28 \%$ \\
\hline MNO2 & $9.50 E+03$ & $1.80 \mathrm{E}+05$ & $1.19 E+04$ & $1.04 E+04$ & $2.12 \mathrm{E}+05$ & $2.44 E+06$ & 0.000 & $1.04 \%$ \\
\hline$C R+3$ & 1. $14 E+04$ & $1.04 E+05$ & $3.20 E+04$ & $3.63 E+04$ & $1.83 E+05$ & $3.52 E+06$ & 0.002 & $0.76 \%$ \\
\hline$C A+2$ & $6.42 E+03$ & $1.24 E+05$ & $3.69 E+03$ & $1.30 E+04$ & $1.47 E+05$ & $3.66 E+06$ & 0.000 & $0.75 \%$ \\
\hline$S I+4$ & $0.00 E+00$ & $0.00 E+00$ & $1.34 E+04$ & $4.66 E+04$ & $6.00 E+04$ & $2.14 E+06$ & 0.001 & $0.25 \%$ \\
\hline$S R+2$ & $4.67 E+02$ & $4.64 E+04$ & $3.23 E+00$ & $2.44 E+02$ & $4.71 E+04$ & $5.38 E+05$ & 0.000 & $0.26 \%$ \\
\hline$L A+3$ & $2.81 E+02$ & $2.76 E+04$ & $4.54 E+01$ & $1.07 E+04$ & $3.87 E+04$ & $2.78 E+05$ & 0.000 & $0.21 \%$ \\
\hline$P 205: 24 W$ & $0.00 E+00$ & $2.01 \mathrm{E}+04$ & $0.00 E+00$ & $0.00 E+00$ & $2.01 E+04$ & $3.29 E+03$ & 0.000 & $0.11 \%$ \\
\hline $\mathrm{TH}+4$ & $1.34 E+02$ & $1.29 E+04$ & $2.91 \mathrm{E}+01$ & $7.97 \mathrm{E}+02$ & $1.38 E+04$ & $5.96 E+04$ & 0.000 & $0.07 \%$ \\
\hline
\end{tabular}


Table B2. Key Species in the Supernatant and Supercritical Water Oxidation Impact.

\begin{tabular}{|c|c|c|c|c|}
\hline Species & $\begin{array}{c}\text { Starting Liquid } \\
\text { Phase Mass }(\mathrm{kg})\end{array}$ & $\begin{array}{c}\text { Precipitation/ } \\
\text { Destruction } \\
\text { by SCWO }\end{array}$ & $\begin{array}{c}\text { Liquid Phase } \\
\text { Mass }(\mathrm{kg})\end{array}$ & $\begin{array}{c}\text { SCWO } . . . . . . . \\
\text { Liquid Phase } \\
\text { Conc. (molal) }\end{array}$ \\
\hline $\begin{array}{l}\text { TOTAL } \\
\mathrm{H} 20 \\
\mathrm{NA}+ \\
\mathrm{NO} 3- \\
\mathrm{OH}- \\
\end{array}$ & $\begin{array}{l}7.39 E+08 \\
5.17 E+08 \\
6.79 E+07 \\
1.10 E+08 \\
1.68 E+07 \\
\end{array}$ & & $\begin{array}{l}6.79 E+07 \\
1.10 E+08 \\
1.68 E+07 \\
\end{array}$ & $\begin{array}{l}5.71 E+00 \\
3.43 E+00 \\
1.91 E+00 \\
\end{array}$ \\
\hline $\begin{array}{l}\mathrm{NO2}- \\
\mathrm{Al}+3 \\
\mathrm{PO} 4-3 \\
\mathrm{~F}- \\
\mathrm{K}+\end{array}$ & $\begin{array}{l}9.79 E+06 \\
2.66 E+06 \\
5.70 E+06 \\
1.13 E+06 \\
1.82 E+06\end{array}$ & $30 \%$ & $\begin{array}{l}9.79 E+06 \\
1.86 E+06 \\
5.70 E+06 \\
1.13 E+06 \\
1.82 E+06\end{array}$ & $\begin{array}{l}4.12 E-01 \\
1.33 E-01 \\
1.16 E-01 \\
1.16 E-01 \\
9.00 E-02\end{array}$ \\
\hline $\begin{array}{l}\mathrm{CO}-2 \\
\mathrm{SO}-2 \\
\mathrm{CL}- \\
\mathrm{TOC} \\
\mathrm{S} \mathrm{I}+4\end{array}$ & $\begin{array}{l}2.64 E+06 \\
1.99 E+06 \\
4.84 E+05 \\
7.82 E+05 \\
1.34 E+04 \\
\end{array}$ & $99 \%$ & $\begin{array}{l}2.64 E+06 \\
1.99 E+06 \\
4.84 E+05 \\
7.82 E+03 \\
1.34 E+04 \\
\end{array}$ & $\begin{array}{l}8.50 E-02 \\
4.01 E-02 \\
2.64 E-02 \\
1.26 E-03 \\
9.25 E-04\end{array}$ \\
\hline $\begin{array}{l}2 \mathrm{RO2}: 2 \mathrm{H2} \\
\mathrm{MO}+6 \\
\mathrm{MG}+2 \\
\mathrm{~W}+6 \\
\mathrm{TCO} 4 .\end{array}$ & $\begin{array}{l}2.32 E+04 \\
3.27 E+03 \\
5.35 E+02 \\
3.87 E+03 \\
2.98 E+03\end{array}$ & & $\begin{array}{l}2.32 E+04 \\
3.27 E+03 \\
5.35 E+02 \\
3.87 E+03 \\
2.98 E+03\end{array}$ & $\begin{array}{l}2.81 E-04 \\
6.59 E-05 \\
4.26 E-05 \\
4.07 E-05 \\
3.56 E-05\end{array}$ \\
\hline $\begin{array}{l}\mathrm{PB}+4 \\
\mathrm{CS}+ \\
\mathrm{CR}+3 \\
\mathrm{U} 02+2 \\
\mathrm{BI}+3\end{array}$ & $\begin{array}{l}2.69 E+03 \\
1.67 E+03 \\
4.34 E+04 \\
9.49 E+04 \\
6.41 E+04\end{array}$ & $\begin{array}{l}99 \% \\
99 \% \\
99 \%\end{array}$ & $\begin{array}{l}2.69 E+03 \\
1.67 E+03 \\
4.34 E+02 \\
9.49 E+02 \\
6.41 E+02\end{array}$ & $\begin{array}{l}2.52 E-05 \\
2.43 E-05 \\
1.61 E-05 \\
6.80 E-06 \\
5.94 E-06\end{array}$ \\
\hline $\begin{array}{l}C A+2 \\
M N O 2 \\
F E+3 \\
C D+2 \\
C E+3\end{array}$ & $\begin{array}{l}1.01 E+04 \\
2.14 E+04 \\
7.46 E+03 \\
2.30 E+03 \\
2.35 E+03\end{array}$ & $\begin{array}{l}99 \% \\
99 \% \\
99 \% \\
99 \% \\
99 \%\end{array}$ & $\begin{array}{l}1.01 \mathrm{E}+02 \\
2.14 \mathrm{E}+02 \\
7.46 \mathrm{E}+01 \\
2.30 \mathrm{E}+01 \\
2.35 \mathrm{E}+01\end{array}$ & $\begin{array}{l}4.88 E-06 \\
4.77 E-06 \\
2.59 E-06 \\
3.96 E-07 \\
3.25 E-07\end{array}$ \\
\hline $\begin{array}{l}\text { FECN6-3 } \\
2 N+2 \\
N I+3 \\
S R+2 \\
L A+3\end{array}$ & $\begin{array}{l}3.54 E+03 \\
8.83 E+02 \\
5.78 E+02 \\
4.70 E+02 \\
3.26 E+02\end{array}$ & $\begin{array}{l}99 \% \\
99 \% \\
99 \% \\
99 \% \\
99 \%\end{array}$ & $\begin{array}{l}3.54 E+01 \\
8.83 E+00 \\
5.78 E+00 \\
4.70 E+00 \\
3.26 E+00\end{array}$ & $\begin{array}{l}3.23 \mathrm{E}-07 \\
2.61 \mathrm{E}-07 \\
1.91 \mathrm{E}-07 \\
1.04 \mathrm{E}-07 \\
4.54 \mathrm{E}-08\end{array}$ \\
\hline $\begin{array}{l}\mathrm{BA+2} \\
\mathrm{TH}+4 \\
\mathrm{SN}+4 \\
\mathrm{PU}+4 \\
\mathrm{SM}+3 \\
\mathrm{NP}+4 \\
\mathrm{AM}+3 \\
\mathrm{CM}+3\end{array}$ & $\begin{array}{l}3.09 E+02 \\
1.63 E+02 \\
1.65 E+01 \\
1.54 E+01 \\
4.87 E+00 \\
4.53 E+00 \\
1.99 E+00 \\
3.84 E-04\end{array}$ & $\begin{array}{l}99 \% \\
99 \% \\
99 \% \\
99 \% \\
99 \% \\
99 \% \\
99 \% \\
99 \%\end{array}$ & $\begin{array}{l}3.09 E+00 \\
1.63 E+00 \\
1.65 E-01 \\
1.54 E-01 \\
4.87 E-02 \\
4.53 E-02 \\
1.99 E-02 \\
3.84 E-06\end{array}$ & $\begin{array}{l}4.35 E-08 \\
1.36 E-08 \\
2.68 E-09 \\
1.24 E-09 \\
6.27 E-10 \\
3.70 E-10 \\
1.58 E-10 \\
3.00 E-14\end{array}$ \\
\hline
\end{tabular}


WHC-EP-0791

Table B3. Component Dissolution by Base/Acid Process.

\begin{tabular}{|c|c|c|c|}
\hline \multirow{2}{*}{$\begin{array}{l}\text { SOUNO PHASE } \\
\text { SPECES }\end{array}$} & \multicolumn{3}{|c|}{$<\ldots \ldots$ DISSOLUTION $\ldots . . . . . .>$} \\
\hline & CAUSTIC & NITAIC IOXALIC & NITRIC /HF \\
\hline $\mathrm{AQ}+$ & & $80 \%$ & $90 \%$ \\
\hline$\overline{A L+3}$ & $90 \%$ & $50 \%$ & $50 \%$ \\
\hline AL2O3 & $50 \%$ & & \\
\hline$A M+3$ & & $90 \%$ & $50 \%$ \\
\hline APM. & $90 \%$ & & \\
\hline AS+5 & & $90 \%$ & $80 \%$ \\
\hline $\mathrm{B}+3$ & & $90 \%$ & $90 \%$ \\
\hline$B A+2$ & & $90 \%$ & $90 \%$ \\
\hline$B E+2$ & & $90 \%$ & $90 \%$ \\
\hline $\mathrm{Bl}+3$ & & $90 \%$ & $50 \%$ \\
\hline C14 & & $90 \%$ & $90 \%$ \\
\hline $\mathrm{CA}+2$ & & $90 \%$ & $90 \%$ \\
\hline CANCAINITE & $50 \%$ & & $10 \%$ \\
\hline $\mathrm{CD}+2$ & & $90 \%$ & $90 \%$ \\
\hline $\mathrm{CE}+3$ & & $90 \%$ & $90 \%$ \\
\hline $\mathrm{CL}$ - & & $90 \%$ & $80 \%$ \\
\hline $\mathrm{CM}+3$ & & $90 \%$ & $90 \%$ \\
\hline $\mathrm{CO}+3$ & & $90 \%$ & $90 \%$ \\
\hline $\mathrm{CO} 3.2$ & & $80 \%$ & $80 \%$ \\
\hline $\mathrm{CR}+3$ & & $90 \%$ & $50 \%$ \\
\hline CSt & & $80 \%$ & $90 \%$ \\
\hline $\mathrm{Cu}+2$ & & $90 \%$ & $90 \%$ \\
\hline F. & & $90 \%$ & $90 \%$ \\
\hline$F E+3$ & & $90 \%$ & $50 \%$ \\
\hline FECN6-3 & & $90 \%$ & $90 \%$ \\
\hline $\mathrm{HG}+2$ & & $90 \%$ & $90 \%$ \\
\hline 1. & & $90 \%$ & $90 \%$ \\
\hline $\mathrm{K}+$ & & $90 \%$ & $90 \%$ \\
\hline $\mathrm{LA}+3$ & & $90 \%$ & $50 \%$ \\
\hline $\mathrm{LIt}$ & & $90 \%$ & $90 \%$ \\
\hline$M G+2$ & & $90 \%$ & $80 \%$ \\
\hline$M N+2$ & & $90 \%$ & $90 \%$ \\
\hline MNO2 & & $90 \%$ & $50 \%$ \\
\hline $\mathrm{MO}+6$ & & $90 \%$ & $80 \%$ \\
\hline NAt & & $90 \%$ & $90 \%$ \\
\hline $\mathrm{NB}+5$ & & $90 \%$ & $90 \%$ \\
\hline $\mathrm{NI}+3$ & & $90 \%$ & $90 \%$ \\
\hline NI2FECN6 & $90 \%$ & & \\
\hline NO2. & & $90 \%$ & $90 \%$ \\
\hline NO3. & & $90 \%$ & $90 \%$ \\
\hline $\mathrm{NP}+4$ & & $90 \%$ & $50 \%$ \\
\hline $\mathrm{OH}$ & & $90 \%$ & $90 \%$ \\
\hline P205:24W & & $90 \%$ & $90 \%$ \\
\hline $\mathrm{PB}+4$ & & $90 \%$ & $80 \%$ \\
\hline PO4-3 & $90 \%$ & $90 \%$ & $90 \%$ \\
\hline $\mathrm{PU}+4$ & & $90 \%$ & $50 \%$ \\
\hline $\mathrm{RB}+$ & & $90 \%$ & $90 \%$ \\
\hline $\mathrm{RE}+7$ & & $90 \%$ & $90 \%$ \\
\hline $\mathrm{RH}+3$ & & $90 \%$ & $90 \%$ \\
\hline$R U+3$ & & $80 \%$ & $90 \%$ \\
\hline $\mathrm{SB}+5$ & & $90 \%$ & $90 \%$ \\
\hline$S E+6$ & & $90 \%$ & $80 \%$ \\
\hline$S 1+4$ & & $90 \%$ & $90 \%$ \\
\hline$S M+3$ & & $90 \%$ & $90 \%$ \\
\hline $\mathrm{SN}+4$ & & $90 \%$ & $90 \%$ \\
\hline $504-2$ & $90 \%$ & $90 \%$ & $90 \%$ \\
\hline $\mathrm{SP}+2$ & & $90 \%$ & $80 \%$ \\
\hline TCO4. & & $80 \%$ & $90 \%$ \\
\hline$T E+6$ & & $90 \%$ & $90 \%$ \\
\hline $\mathrm{TH}+4$ & & $80 \%$ & $50 \%$ \\
\hline $\mathrm{TI}+4$ & & $90 \%$ & $90 \%$ \\
\hline$T L+3$ & & $00 \%$ & $90 \%$ \\
\hline TOC & & $90 \%$ & $90 \%$ \\
\hline $402+2$ & & $90 \%$ & $90 \%$ \\
\hline$V+5$ & & $90 \%$ & $90 \%$ \\
\hline$w+6$ & & $80 \%$ & $90 \%$ \\
\hline $2 \mathrm{~N}+2$ & & $90 \%$ & $90 \%$ \\
\hline $2 R+4$ & & $90 \%$ & $90 \%$ \\
\hline $\mathrm{ZRO} 2: 2 \mathrm{H} 2$ & & $90 \%$ & $50 \%$ \\
\hline
\end{tabular}


Table B4. Tributyl Phosphate Extraction of Transuranics Section: Component Distribution.

(--- Component distribution among outlet streams -..--->

Stream (St)* St No. 7 St No. 11 St No. 15 St No. 18 St No. 19

Species To Am/La To To Uran. To Basic Solvent

- Separation HLW Purif. Side Feed Recycle

$\begin{array}{lrrrr}\text { Am+3 } & 99.99 \% & .01 \% & & \\ B i+3 & 99.90 \% & .10 \% & & \\ \text { Ce+3 } & 99.99 \% & .01 \% & & \\ \text { Cm+3 } & 99.99 \% & .01 \% & & \\ \text { Fe+3 } & 99.99 \% & .01 \% & & \\ \text { La+3 } & 99.99 \% & .01 \% & .01 \% & \\ \text { Np+4 } & 1.00 \% & 98.99 \% & .01 \% & \\ \text { Pu+4 } & .01 \% & 99.98 \% & .01 \% & \\ \text { Sm+4 } & 99.99 \% & .01 \% & .05 \% & \\ \text { TC04- } & 99.90 \% & .05 \% & .01 \% & \\ \text { Th+4 } & 1.00 \% & 98.99 \% & .01 \% & \\ \text { U02+2 } & .01 \% & .10 \% & 99.88 \% & .01 \% \\ \text { Zr+4 } & 99.90 \% & .10 \% & & \\ \text { TBP Solvent } & & & .001 \% & \end{array}$

Table B5. Uranium Purification Section: Component Distribution. (---- Component distribution among outlet streams ----->

Stream (St)* St No. 3 St No. 36 St No. 34 St No.31 St No. 35

Species To Extract. Uran. To To To Water Solvent Iransuranic Stockpile LLW Recycle Recycle

$\begin{array}{llllll}{[\mathrm{H}+]} & 2.4 \mathrm{M} & & & \text { remainder } & \\ \mathrm{Np}+4 & 100 \% & & & \\ \mathrm{Pu}+4 & 100 \% & & & & \\ \mathrm{Tc04}- & 100 \% & & & & \\ \text { Th+4 } & 100 \% & & & & \\ \text { [U02+2] } & 0.18 \mathrm{M} & \text { remainder } & .01 \% & .001 \% & 99.999 \% \\ \text { Solvent } & & & & .009\end{array}$

*Stream identification numbers from Figure BI CLEAN Option Flowsheet. 
Table B6. CMPO Extraction Americium/Lanthanum Section: Component Distribution.

--- Component distribution among outlet streams --->

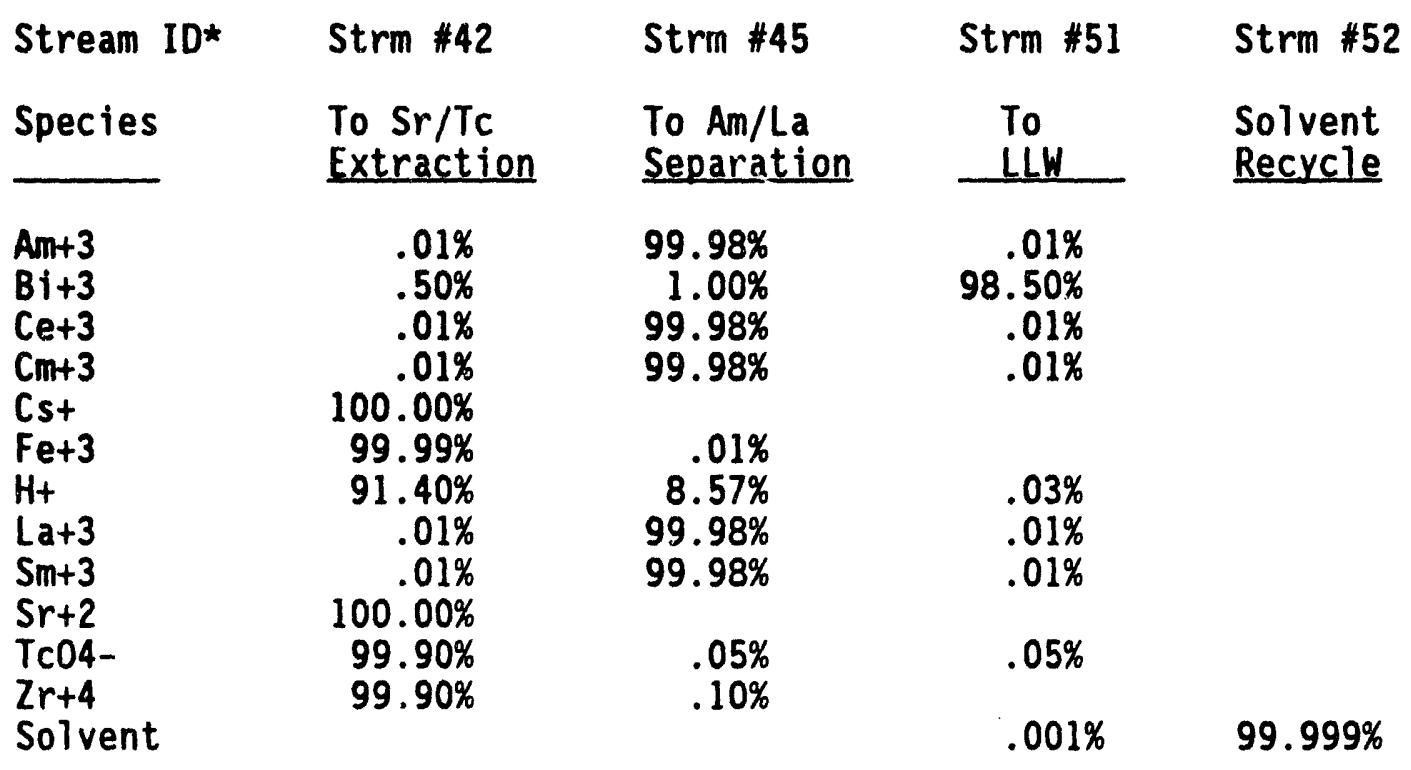

Table B7. Americium/Lanthanum Band Displacement Section: Component Distribution.

<-- Component distribution among outlet streams -- $\rightarrow$

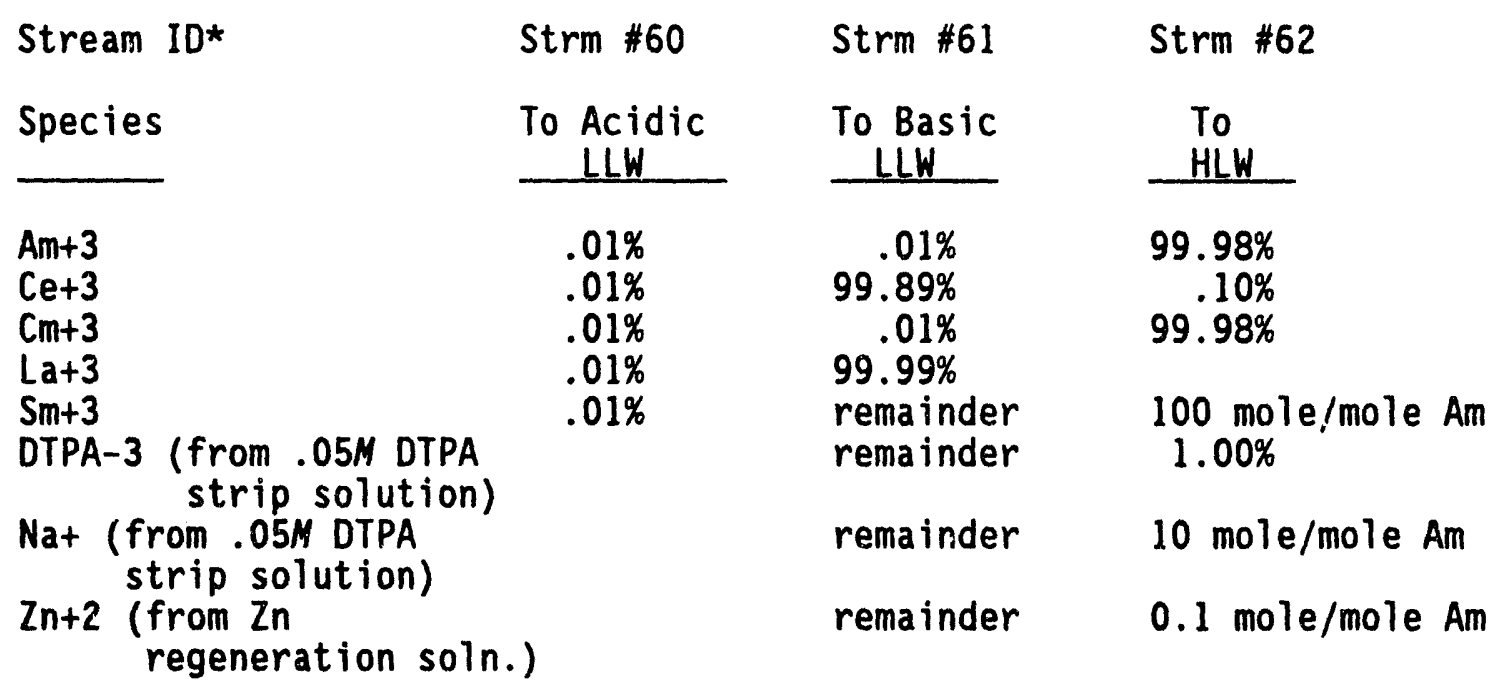

*Stream identification numbers from Figure B1 CLEAN Option Flowsheet. 
Table B8. Crown Ether Extraction Strontium/Technetium Section: Component Distribution.

--- Component distribution among outlet streams --->

\begin{tabular}{|c|c|c|c|c|}
\hline Stream ID* & Strm $\# 75$ & Strm \#72 & Strm \#78 & Strm $\# 79$ \\
\hline Species & $\begin{array}{l}\text { To } \mathrm{Sr} / \mathrm{Ba} / \mathrm{TC} \\
\text { Separation }\end{array}$ & $\begin{array}{l}\text { To Cs APM } \\
\text { Extraction }\end{array}$ & $\begin{array}{c}\text { Basic } \\
\text { LLW }\end{array}$ & $\begin{array}{l}\text { Solvent } \\
\text { Recycle }\end{array}$ \\
\hline $\begin{array}{l}\text { Ba+2 } \\
\text { Sr+2 } \\
\text { Tc04- } \\
\text { HNO3 } \\
\text { Solvent }\end{array}$ & $\begin{array}{l}99.99 \% \\
99.99 \% \\
99.00 \% \\
2.6 \mathrm{M}\end{array}$ & $\begin{array}{l}.01 \% \\
.01 \% \\
1.00 \% \\
\text { remainder }\end{array}$ & $.001 \%$ & $99.999 \%$ \\
\hline
\end{tabular}

Table B9. Strontium/Barium Band Displacement Section: Component Distribution.

--- Component distribution ---->

\begin{tabular}{|c|c|c|}
\hline Stream ID* & Strm \#95 & Strm \#94 \\
\hline Species & $\begin{array}{c}\text { To Basic } \\
\text { LLW }\end{array}$ & $\begin{array}{l}\text { To } \\
\text { HLW } \\
\end{array}$ \\
\hline $\begin{array}{l}\mathrm{Ba}+2 \\
\text { Sr+2 } \\
\text { Tc04- } \\
\text { EDTA-3 (from .05M EDTA } \\
\text { strip solution) }\end{array}$ & $\begin{array}{l}\text { remainder } \\
.01 \% \\
0.00 \% \\
\text { remainder }\end{array}$ & $\begin{array}{l}0.3 \mathrm{~mole} / \mathrm{mole} \mathrm{Sr}+2 \\
99.99 \% \\
100 \% \\
7.20 \%\end{array}$ \\
\hline $\begin{array}{l}\text { Nat (from .05M EDTA } \\
\text { strip solution) } \\
\text { Zn+2 (from } Z n \\
\text { regeneration soln.) }\end{array}$ & $\begin{array}{l}\text { remainder } \\
\text { remainder }\end{array}$ & $\begin{array}{l}0.13 \mathrm{~mole} / \mathrm{mole} S r+2 \\
0.1 \mathrm{~mole} / \mathrm{mole} S r+2\end{array}$ \\
\hline
\end{tabular}

Table B10. Basic Cesium Ion Exchange: Relative Flow Volumes of Streams.

\begin{tabular}{|c|c|c|c|}
\hline Stream Name & $\begin{array}{l}\text { Stream } \\
\text { Number* }\end{array}$ & $\begin{array}{l}\text { Column } \\
\text { Volumes }\end{array}$ & Composition \\
\hline $\begin{array}{l}\text { Column Feed } \\
\text { Regeneration streams }\end{array}$ & 450 & 2000 & --- \\
\hline $\begin{array}{l}\text { Feed flush } \\
\text { Feed flush } \\
\text { Cesium Eluant } \\
\text { Eluant Flush } \\
\text { Regeneration }\end{array}$ & $\begin{array}{l}300 \\
301 \\
302 \\
303 \\
304\end{array}$ & $\begin{array}{l}3 \\
6 \\
20 \\
3 \\
3\end{array}$ & $\begin{array}{l}2 M \mathrm{NaOH} \\
\text { Water } \\
\text { Formic acid } \\
\text { Water } \\
2 M \mathrm{NaOH}\end{array}$ \\
\hline
\end{tabular}

*Stream identification numbers from Figure B1 CLEAN Option Flowsheet. 
Table B11. Basic Strontium Ion Exchange: Relative Flow Volumes of Streams.

$\begin{array}{llll}\text { Stream Name } & \begin{array}{l}\text { Stream } \\ \text { Number* }\end{array} & \begin{array}{l}\text { Column } \\ \text { Volumes }\end{array} & \text { Composition } \\ \text { Column Feed } & 305 & 2000 & -- \\ \text { Regeneration streams } & & & \\ \text { Feed flush } & 314 & 3 & 2 \mathrm{~N} \mathrm{NaOH} \\ \text { Feed flush } & 315 & 6 & \text { Water } \\ \text { Strontium Eluant } & 316 & 20 & 1 \mathrm{MNO} \\ \text { Eluant Flush } & 317 & 3 & \text { Water } \\ \text { Regeneration } & 318 & 3 & 2 \mathrm{NaOH}\end{array}$

Table B12. Basic Technetium Ion Exchange: Relative Flow Volumes of Streams.

\begin{tabular}{llll} 
Stream Name & $\begin{array}{l}\text { Stream } \\
\text { Number* }\end{array}$ & $\begin{array}{l}\text { Column } \\
\text { Volumes }\end{array}$ & Composition \\
\cline { 2 - 4 } Column Feed & 319 & 100 & -- \\
Regeneration streams & & & \\
Feed flush & 331 & 8 & $2 \mathrm{~N} \mathrm{NaOH}$ \\
Feed flush & 332 & 12 & Water \\
Nat displacement & 333 & 6 & $0.25 \mathrm{HNO}$ \\
Technetium Eluant & 337 & 20 & $6 \mathrm{~N} \mathrm{HNO3}$ \\
Eluant Flush & 335 & 6 & Water \\
Regeneration & 336 & 8 & $2 M \mathrm{NaOH}$
\end{tabular}

*Stream identification numbers from Figure B1 CLEAN Option Flowsheet. 
Table B13. Characteristics of Solid Portion of Waste Stream ("Sludge").

\begin{tabular}{llllllll} 
& \multicolumn{2}{c}{ Waste Feed Solids } & \multicolumn{2}{c}{ Dissolution } & \multicolumn{2}{c}{ Undissolved Solids } \\
Species & Kg & Moles & Mole\% & Species & \%Diss & Moles & Mole\% \\
Tota1 & $1.79 e+07$ & $3.53 e+05$ & & & & $4.17 e+04$ & \\
OH- & $2.33 e+06$ & $1.37 e+05$ & $38.90 \%$ & OH- & $91.58 \%$ & $1.16 e+04$ & $27.71 \%$ \\
AL+3 & $1.75 e+06$ & $6.49 e+04$ & $18.39 \%$ & CANCR & $0.00 \%$ & $7.94 e+03$ & $19.03 \%$ \\
P04-3 & $4.39 e+06$ & $4.62 e+04$ & $13.10 \%$ & AL+3 & $88.77 \%$ & $7.28 e+03$ & $17.47 \%$ \\
NAt & $7.07 e+05$ & $3.07 e+04$ & $8.72 \%$ & PO4-3 & $90.03 \%$ & $4.61 e+03$ & $11.05 \%$ \\
NO3- & $1.02 e+06$ & $1.65 e+04$ & $4.68 \%$ & NAt & $92.10 \%$ & $2.43 e+03$ & $5.83 \%$ \\
FE+3 & $6.97 e+05$ & $1.25 e+04$ & $3.54 \%$ & NO3- & $88.34 \%$ & $1.92 e+03$ & $4.61 \%$ \\
CANCR & $2.70 e+06$ & $7.94 e+03$ & $2.25 \%$ & NI2FECN6 & $0.00 \%$ & $1.51 e+03$ & $3.63 \%$ \\
ZRO2 & $9.63 e+05$ & $6.05 e+03$ & $1.71 \%$ & FE+3 & $90.47 \%$ & $1.19 e+03$ & $2.85 \%$ \\
UO2+2 & $1.45 e+06$ & $5.37 e+03$ & $1.52 \%$ & UO2+2 & $90.16 \%$ & $5.29 e+02$ & $1.27 \%$ \\
CA+2 & $1.47 e+05$ & $3.66 e+03$ & $1.04 \%$ & ZRO2 & $92.39 \%$ & $4.60 e+02$ & $1.10 \%$ \\
F- & $5.65 e+04$ & $2.97 e+03$ & $0.84 \%$ & CA+2 & $90.20 \%$ & $3.59 e+02$ & $0.86 \%$ \\
C03-2 & $1.75 e+05$ & $2.91 e+03$ & $0.83 \%$ & TOC & $90.00 \%$ & $2.77 e+02$ & $0.66 \%$ \\
TOC & $3.32 e+04$ & $2.77 e+03$ & $0.78 \%$ & CO3-2 & $90.83 \%$ & $2.67 e+02$ & $0.64 \%$ \\
MNO2 & $1.91 e+05$ & $2.20 e+03$ & $0.62 \%$ & SI+4 & $86.59 \%$ & $2.22 e+02$ & $0.53 \%$ \\
CR+3 & $9.89 e+04$ & $1.90 e+03$ & $0.54 \%$ & MN02 & $90.20 \%$ & $2.16 e+02$ & $0.52 \%$ \\
CE+3 & $2.34 e+05$ & $1.67 e+03$ & $0.47 \%$ & F- & $92.87 \%$ & $2.12 e+02$ & $0.51 \%$ \\
SI+4 & $4.66 e+04$ & $1.66 e+03$ & $0.47 \%$ & CR+3 & $90.05 \%$ & $1.89 e+02$ & $0.45 \%$ \\
NI2FECN6 & $4.98 e+05$ & $1.51 e+03$ & $0.43 \%$ & CE+3 & $89.96 \%$ & $1.68 e+02$ & $0.40 \%$
\end{tabular}


WHC-EP-0791

\section{APPENDIX C}

HANFORD STRATEGIC ANALYSIS MODEL:

ASPEN PLUS' ${ }^{\prime}$ IMPLEMENTATION

'ASPEN PLUS is a trademark of Aspen Technology, Inc.

C-1 
WHC-EP-0791

\section{LIST OF TERMS}

HSA Hanford Strategic Analysis

IDEF Integrated Computer-Aided Manufacturing (ICAM) Definition (Language)

C-2 
HANFORD STRATEGIC AMALYSIS MODEL: ASPEN PLUS IMPLEMENTATION

Several process simulation models were developed for the Hanford Strategic Analysis (HSA) Study (Pajunen et a1. 1992). The steady-state process simulation program ASPEN PLUS was selected for the modeling of the HSA models to provide mass or material balance calculations for each process flowsheet design. Special material data libraries were created for use with the ASPEN PLUS program to allow modeling the various material waste stream flows. These libraries allowed using radioactive and hazardous materials as components of each stream. However, global level model runs subsequent to those analyses have incorporated component radioactivity data by making a separate model run with curie inventories substituted in the feedstream.

To control the model complexity or abstraction level, waste stream component lumping (i.e., detailed to more general) was used extensively to allow modeling the entire waste processing system, from cradle to grave, with reasonable detail to provide a good assessment of various waste cleanup strategies and options, including sensitivity and parametric studies.

The ASPEN PLUS model implementation started with process flowsheets developed for the various waste cleanup strategies and their respective options. The process flowsheet for the representative Strategy IX, Option 2, is shown in Figure $\mathrm{Cl}$. The process flowsheets were subsequently modeled using the Integrated Computer-Aided Manufacturing (ICAM) Definition (Language) (IDEF) software tool (Pajunen et al. 1992) at a higher abstraction or less detailed level showing all major feed and product streams and the main process blocks. The IDEF tool allowed showing the flowsheet description at increasingly decomposed states (i.e., a hierarchical representation). The higher level model allowed good understanding of the overall waste flow in each strategy/option and facilitated the development of the detailed process models. Each main process and stream in the higher level model was further broken down or decomposed to an appropriate detail level for implementation using ASPEN PLUS. Thus, the IDEF representations became the ASPEN PLUS flowsheet specifications. The IDEF flowsheet representation for Strategy IX, Option 2, is shown in Appendix D with the CLEAN Option process strategy appropriately integrated. Figure C2 shows the final ASPEN PLUS implementation model for Strategy IX, Option 2.

The IDEF model flowsheet specifications were used to build the ASPEN PLUS flowsheet model connectivity with all the specified feed and product streams. In place of rigorous chemical and physical process models, the HSA ASPEN PLUS models used simpler mixers and splitter models, referred to as MIXER, FSPLIT, and SEP process unit blocks (Appendix A). The MIXER block combines material streams into one stream. The FSPLIT block combines material streams and divides the resulting stream into two or more streams, with all outlets having the same composition and properties. The SEP block combines streams and separates the resulting stream into two or more streams according to splits or separation factors specified for each component. 
The ASPEN PLUS HSA model detail consisted of between 30 and 40 process blocks for each strategy/option, with between 20 and 30 feedstreams and 20 to 30 product streams. Including internal connectivity streams, the ASPEN PLUS models contained between 140 and 170 streams. Each stream contained 14 pseudo (lumped) components as shown below. Figure C3 shows the lumped component attribute tree structure. A more detailed breakdown of each of these components into more specific chemical species is described in Appendix $D$.

\begin{tabular}{|c|c|}
\hline $\begin{array}{l}\text { Pseudo- } \\
\text { Component } \\
\text { Name }\end{array}$ & Long name \\
\hline $\begin{array}{l}\text { H2O } \\
\text { TRU2 } \\
\text { ACT2 } \\
\text { LAN } \\
\text { CS } \\
\text { SR } \\
\text { TC } \\
\text { I } \\
\text { FP2 } \\
\text { AP1 } \\
\text { NaNO3 } \\
\text { OHAZ1 } \\
\text { SOI } \\
\text { OTH2 }\end{array}$ & $\begin{array}{l}\text { WATER } \\
\text { TRANSU } \\
\text { ACTINIDE } \\
\text { LANTH } \\
\text { CESIUM } \\
\text { STRONTIUM } \\
\text { TECHNETIUM } \\
\text { IODINE } \\
\text { FISPROD2 } \\
\text { ACTPROD1 } \\
\text { NaNO3 } \\
\text { OHAZ1 } \\
\text { SOIL } \\
\text { OTHER2 }\end{array}$ \\
\hline
\end{tabular}

Definition

Water, including all 1 iquids

All elements with atomic number above uranium (92) Uranium and thorium and daughters

All lanthanides

All cesium

All strontium

Technetium, ${ }^{90} \mathrm{TC}$

All iodine

All remaining fission products

All Activation Products

Sodium nitrate including $\mathrm{NaNO}_{2}$ and $\mathrm{NaNO}_{3}$

Hazardous chemicals

All normal soil (dirt) constituents, excluding water

All other solids, except macroscopic dirt constituents

To provide data to run the ASPEN PLUS models, separate spreadsheet tables were created with all the required feedstream mass ( $\mathrm{kg}$ or curie) inventories. These spreadsheet files are referred to as the Inventory Specifications. These inventory data are shown on the output results for the Strategy IX, Option 2 model (Table Cl). Similarly, the separation or stream component split factors needed for the FSPLIT and SEP process blocks were tabulated in spreadsheet files for each process block and its product streams. Thus, there were 14 separation factors per product stream except for the single split factor required by the FSPLIT blocks. These split factors are referred to as the Separation Factor Specifications and are shown in Table C2.

Initially the interactive/graphics model management tool, ModelManager', a program provided with ASPEN PLUS, was used to create the ASPEN PLUS models. The graphical user interface within ModelManager allowed relatively fast creation of the flowsheet connectivity while its built-in expert system guided the model development. Menu driven data sheets al so facilitated data entry with checking for model completeness and correctness. As the HSA analyses progressed, the model data entry was automated using special spreadsheet script programs. These scripts output the specification data to files in the correct ASPEN PLUS format and, thus, avoided the table entry requirements within ModelManager. A word processor or editor was then used to incorporate each section into a complete ASPEN PLUS input file.

'ModelManager is a trademark of Aspen Technology, Inc. 
The chemical makeup mass inventories were also specified for each base strategy (option 2) model. Spectal FORTRAN blocks were written using in-line FORTRAN statements to reproduce these base strategy makeup inventories and provide automatic run-time chemical or physical makeup addition calculations for use in the other strategy/option models. These FORTRAN blocks adjusted the makeup stream component mass flows before they were used by the individual process blocks. The makeup mass calculations consisted of appiying a makeupto-input mass ratio for each process block, based on either the total amount of solids flow (defined as the mass flow of all components less $\mathrm{H}_{2} \mathrm{O}$ ) or the total mass flow of all components, into each process block. These ratios represented the actual makeup inventory modeled in the base strategy runs. When the makeup was determined, three additional ratios split the makeup inventory into three components, $\mathrm{H}_{2} \mathrm{O}, \mathrm{OHAZ1}$, and OTH2. A few special cases included the addition of soll through the makeup calculations. These chemical makeup mass ratios are shown for Strategy IX, Option 2, in Table C3.

\section{REFERENCES}

Pajunen, A. L., et al., 1992, Hanford Strategic Analysis Study, WHC-EP-0549, Westinghouse Hanford Company, Richland, Washington. 
Figure C1. Hanford Strategic Analysis Study - Strategy IX, Option 2.

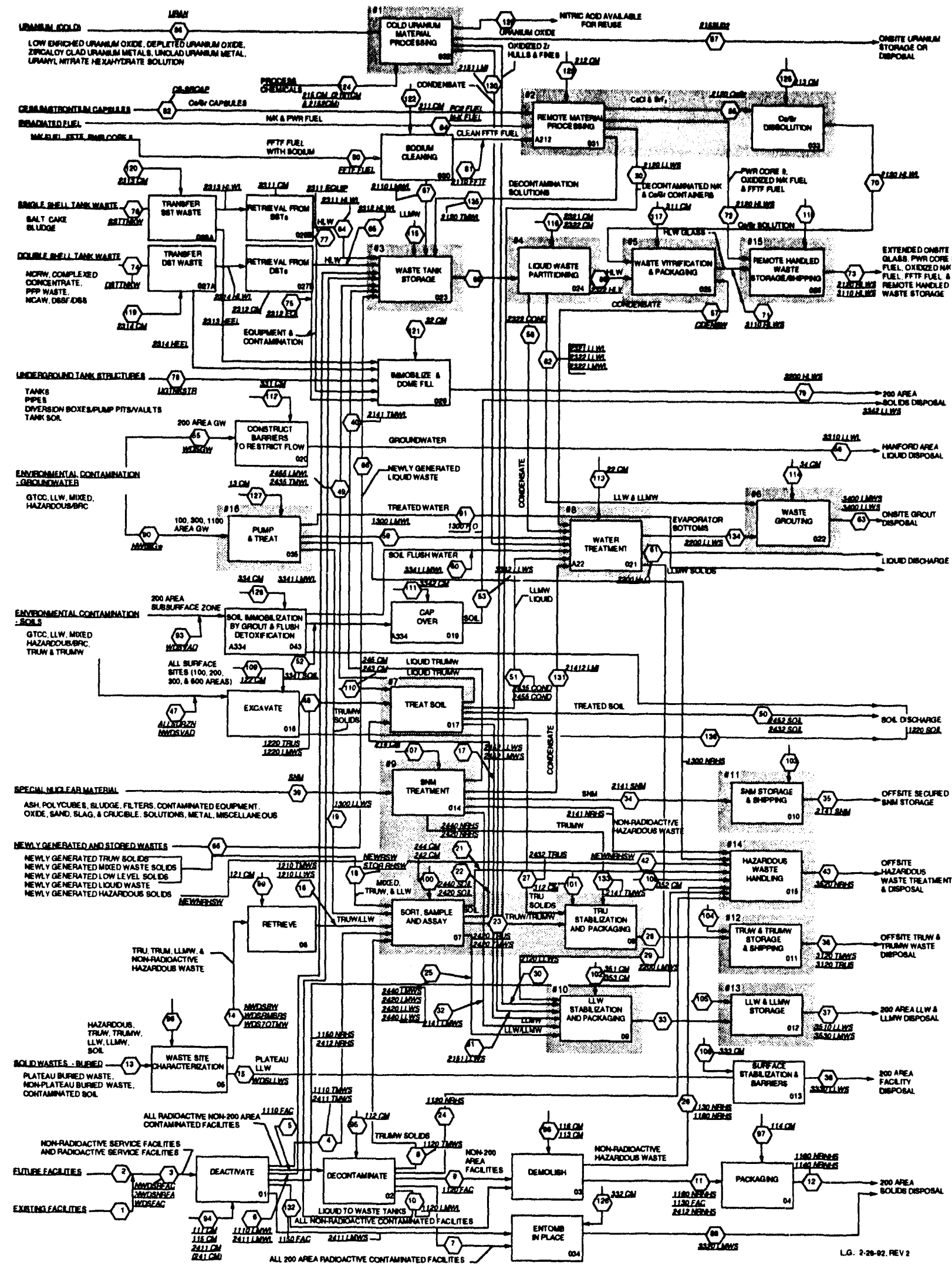


Figure C2. Hanford Strategic Analysis Model - Strategy IX, Option 2.

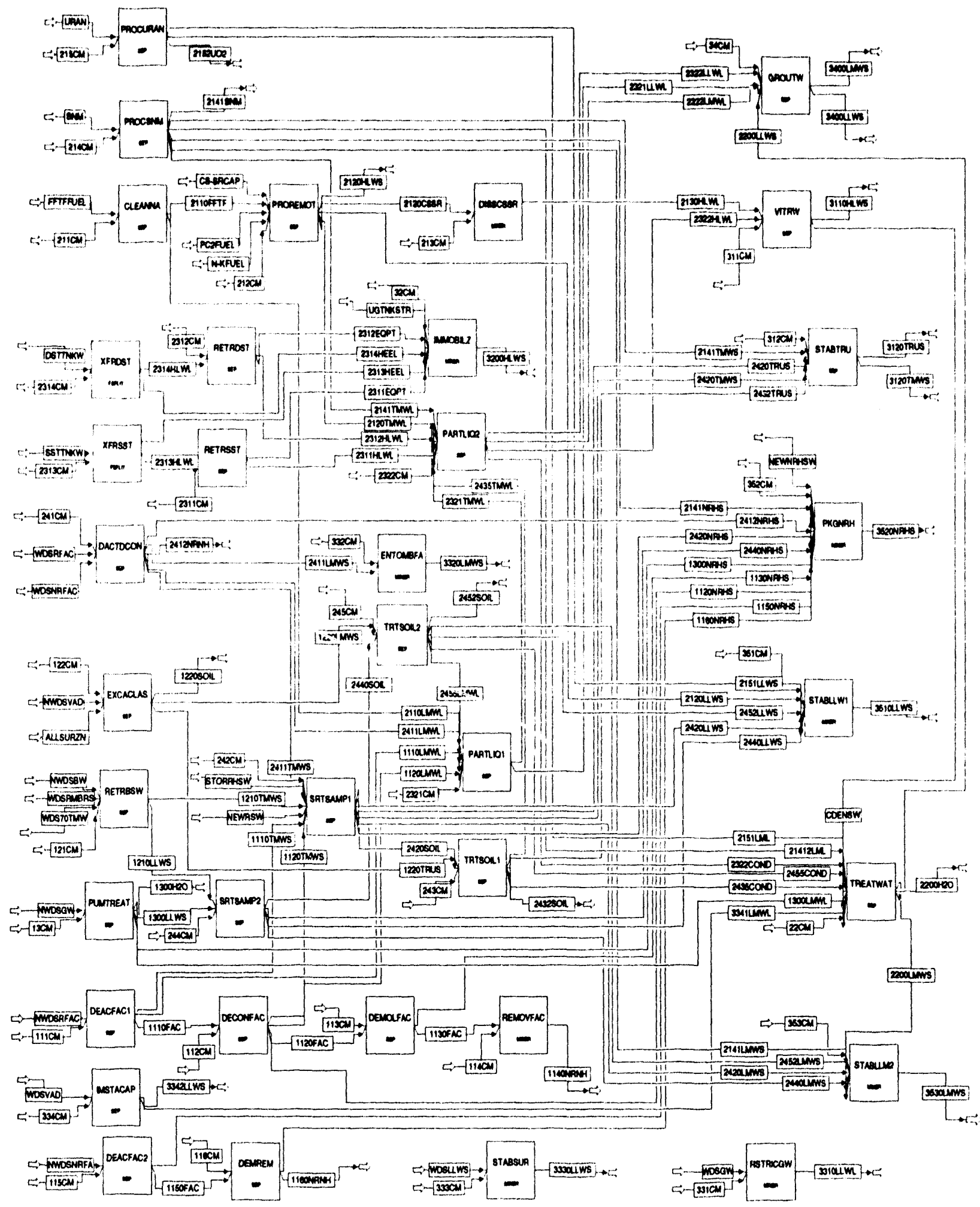

C-7 
WHC-EP-0791

Figure (3. Pseudo Component Attribute Tree.

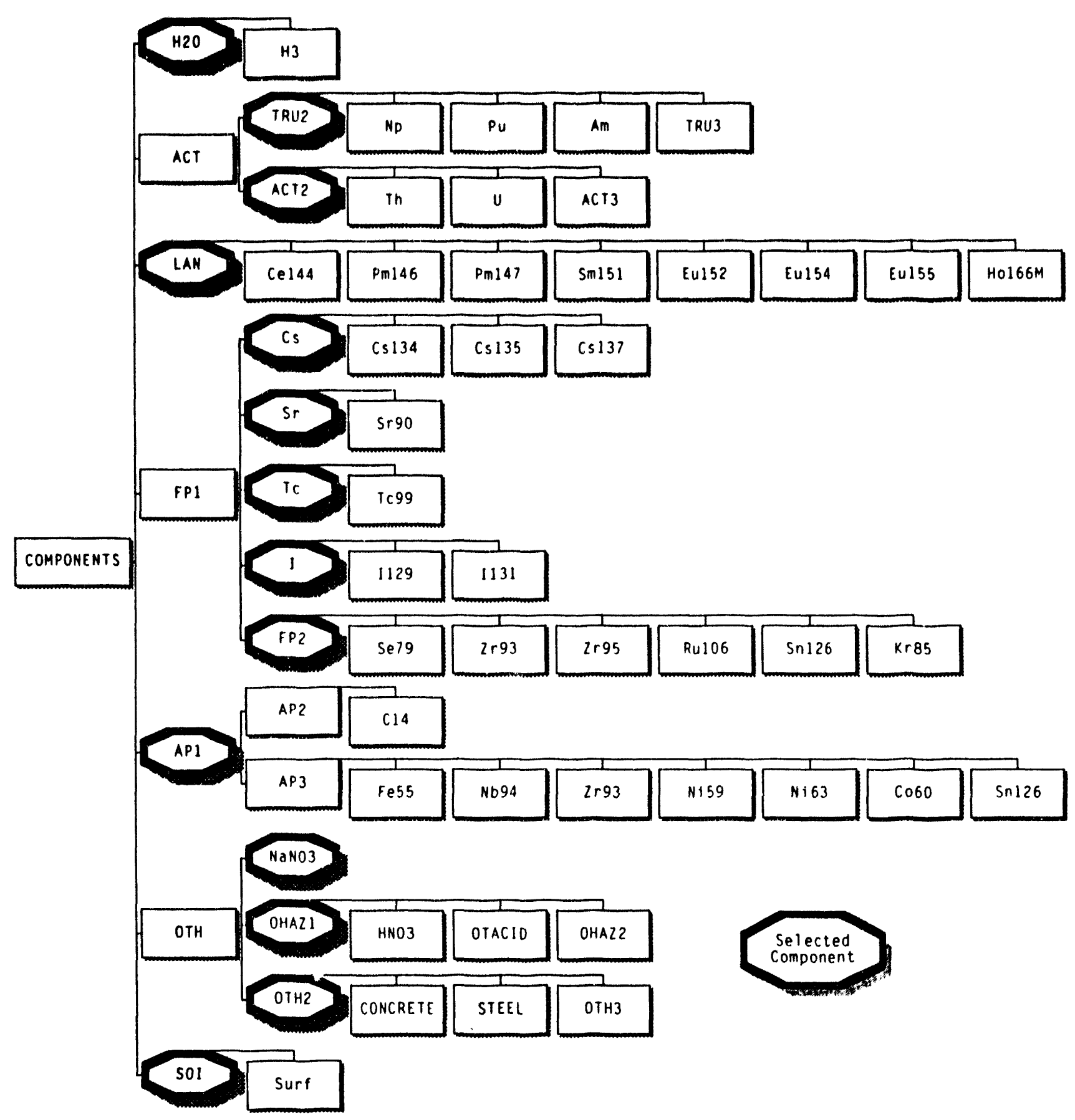


Table C1. Hanford Strategic Analysis Mass Balance Output Results (kilogram). (sheet 1 of 5) FHo: C.MSPRUNSLSO837142.NP MSA BASE RUN, STRATEGY 09. 37 BLOCKS. 14 COMPONENTS. OPTION 2)

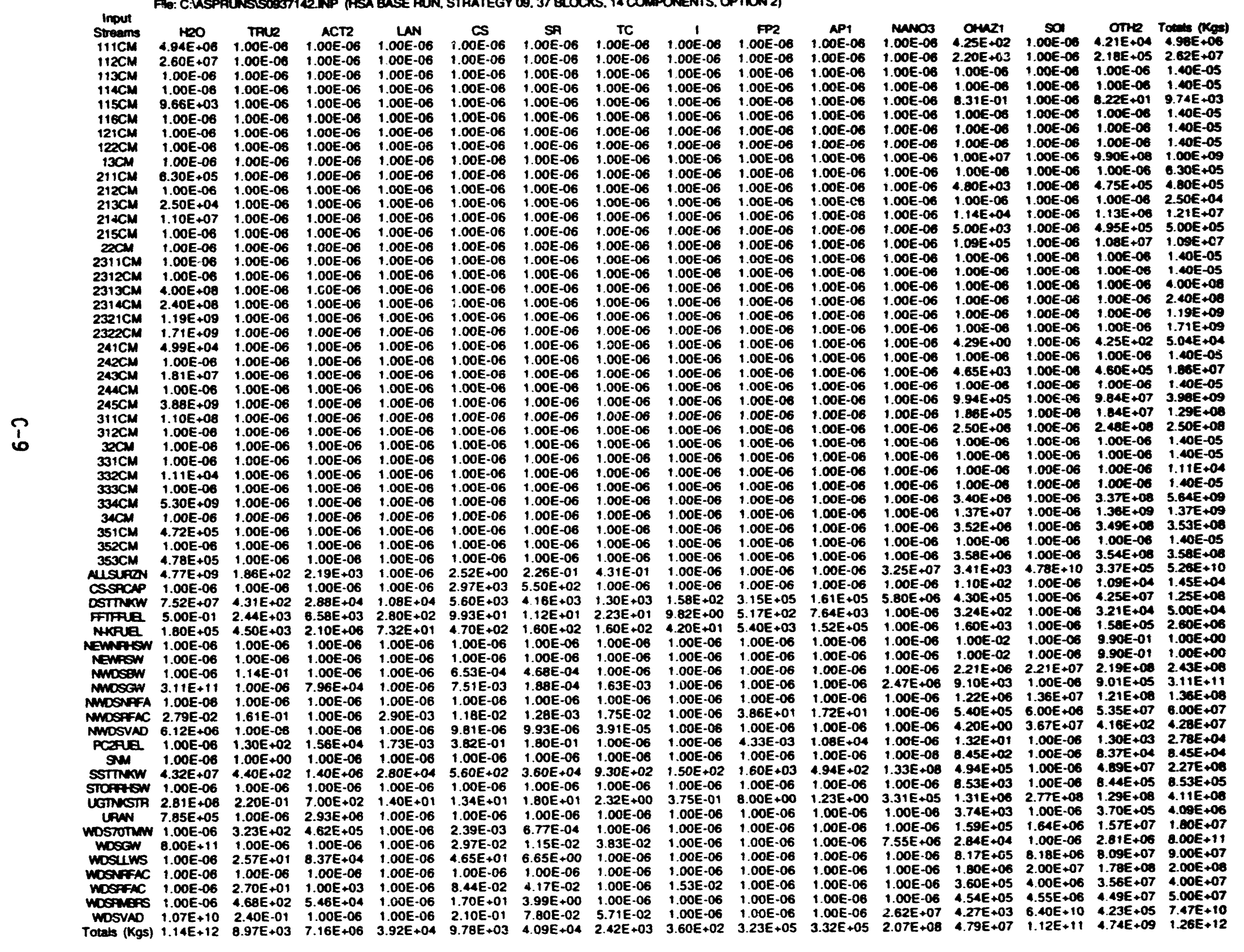


Table C1. Hanford Strategic Analysis Mass Balance Output Results (kilogram). (sheet 2 of 5)

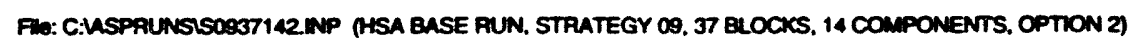

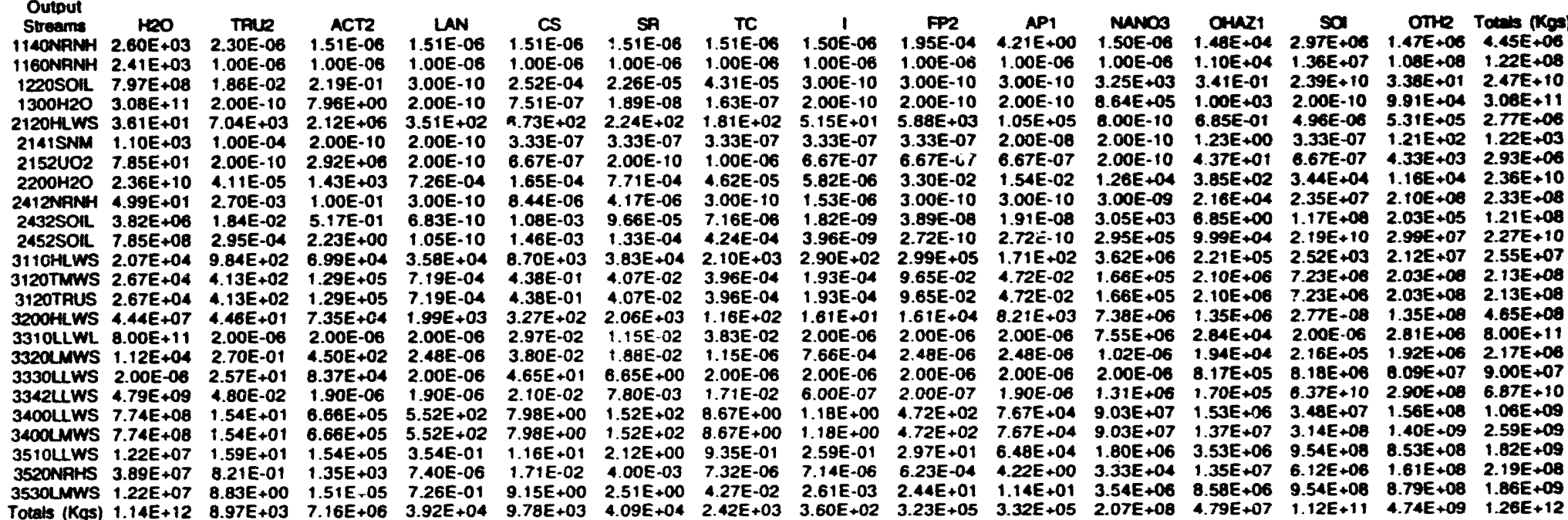


Table C1. Hanford Strategic Analysis Mass Balance Output Results (kilogram). (sheet 3 of 5) CISPRNSISO937142.INP (HSA BASE RUN. STRATEGY 09. 37 BLOCKS, 14 COMPONENTS. OPTION 2)

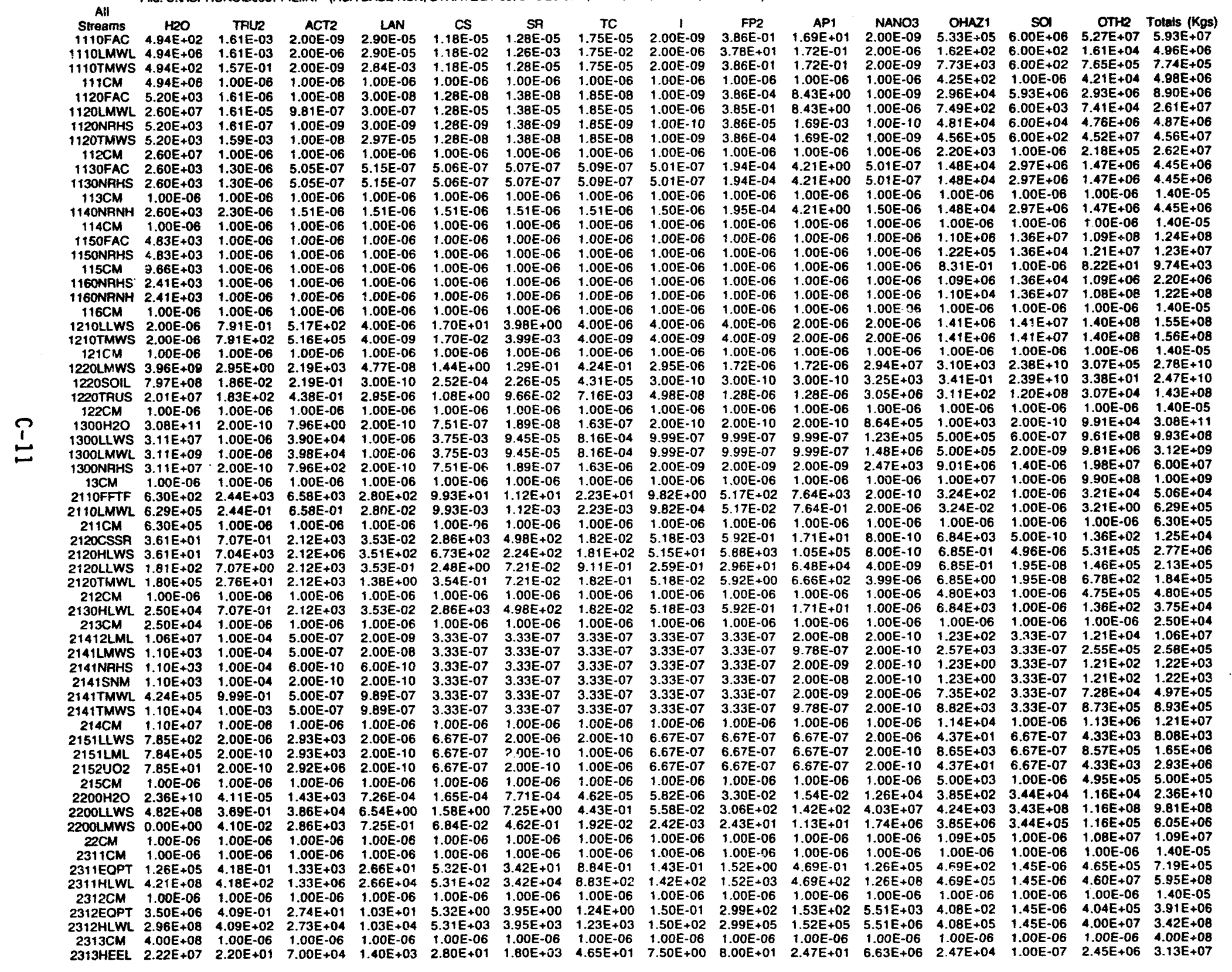


Table C1. Hanford Strategic Analysis Mass Balance Output Results (kilogram). (sheet 4 of 5)

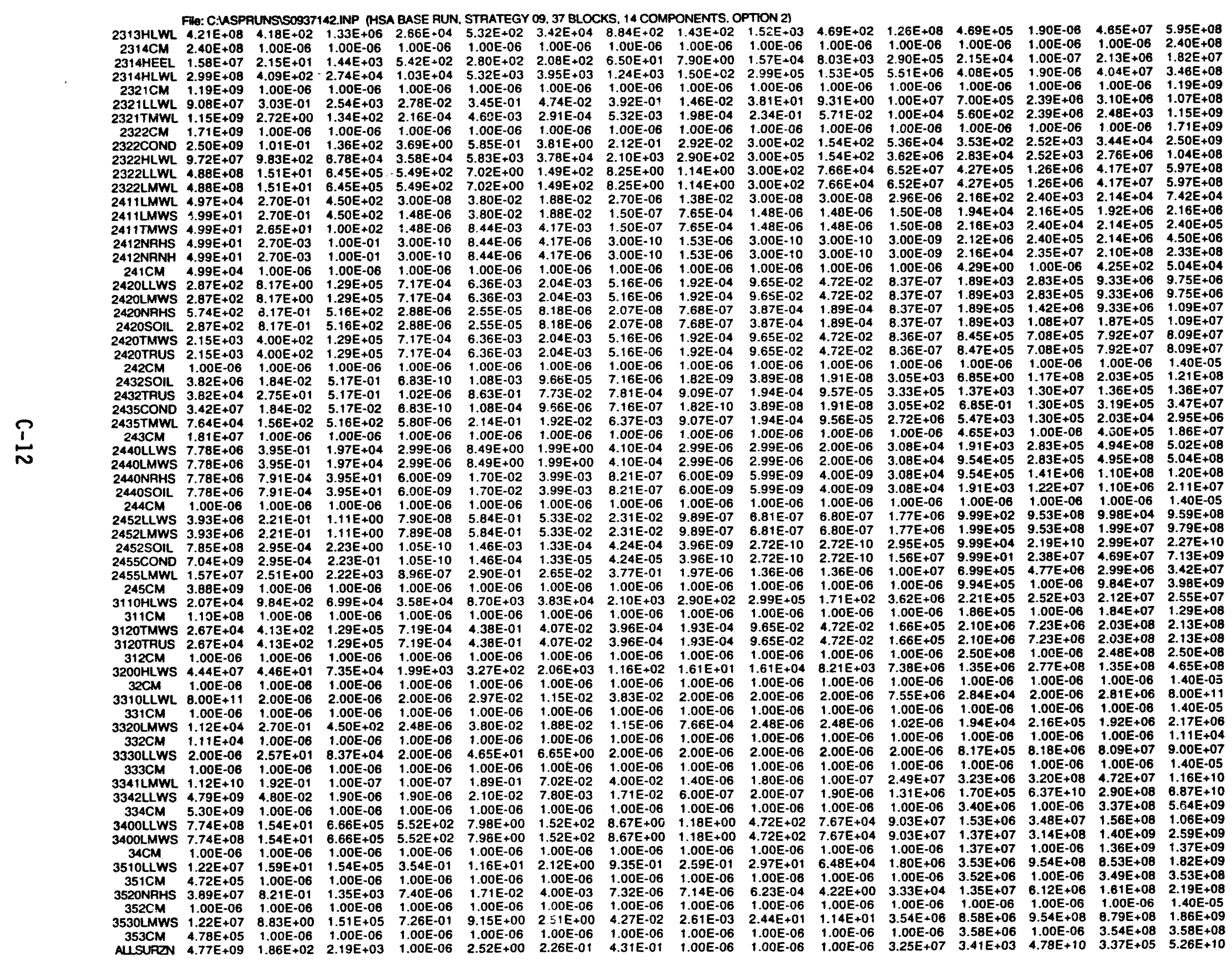


Table C1. Hanford Strategic Analysis Mass Balance Output Results (kilogram). (sheet 5 of 5)

\begin{tabular}{|c|c|c|c|c|c|c|c|c|c|c|c|c|c|c|c|}
\hline \multirow{5}{*}{$\begin{array}{l}\text { COENSW } \\
\text { CSSRCAP } \\
\text { DSTINWW } \\
\text { FTIFUE }\end{array}$} & & & & & & & & & & & & & & & \\
\hline & & & 6.99E+00 & & 8.70E-01 & & & & & & & & & & \\
\hline & & $\begin{array}{l}1.00 E-06 \\
\end{array}$ & $1.00 \mathrm{E}-06$ & $1.00 E-06$ & $2.97 E+03$ & & & & & & & & & & \\
\hline & & & $58 E+03$ & & & & & & & & $\begin{array}{l}=+06 \\
E-06\end{array}$ & $\begin{array}{l}05 \\
02\end{array}$ & $\begin{array}{l}1.00 E-06 \\
1.06\end{array}$ & & \\
\hline & & & 2. & 1 & 4.70 & & 1.60 & & & & & & & & \\
\hline & & & & & & & & & & & & & & & \\
\hline & & & & & & & & & & & & & & & \\
\hline & & & & & & & & & & & & & & & \\
\hline & & & & & & & & & & & & & & & \\
\hline & & & & & & & & & & & & & & & \\
\hline & & & & & & & & & & & & & & & \\
\hline & & & & & & & & & & & & & & & \\
\hline & & & & & & & & & & & & & & & \\
\hline & & & & & & & & & & & & & & & \\
\hline & $4.32 \mathrm{E}$ & 4.40 & & & & & & & & & & & & & \\
\hline & & & & & & & & & & & & & & & \\
\hline & 2.81 & 2.20 & & & & & & & & & & & & & \\
\hline & & & & & & & & & & & & & & & \\
\hline & & & & & & & & & & & & & & & \\
\hline & & & & & & & & & & & & & & & \\
\hline$A C$ & $\begin{array}{l}1.0 \\
1.0\end{array}$ & $\begin{array}{l}2.57 \mathrm{E} \\
1.00\end{array}$ & & & & & & & & & & & & & \\
\hline & & & & & & & & & & & & & & & \\
\hline & & & & & & & & & & & & & & & \\
\hline & & & & & & & & $.00 E-06$ & & & & & & & \\
\hline
\end{tabular}


Table C2. Hanford Strategic Analysis Separation Specifications. (sheet 1 of 2) TEDOC1. SEPAPATION SPECIFCATIONS FLE: SEPS_IX_OP2_PB REV. 18 P.HAPMSEN 14 FEB 92

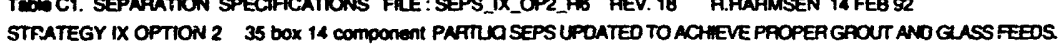

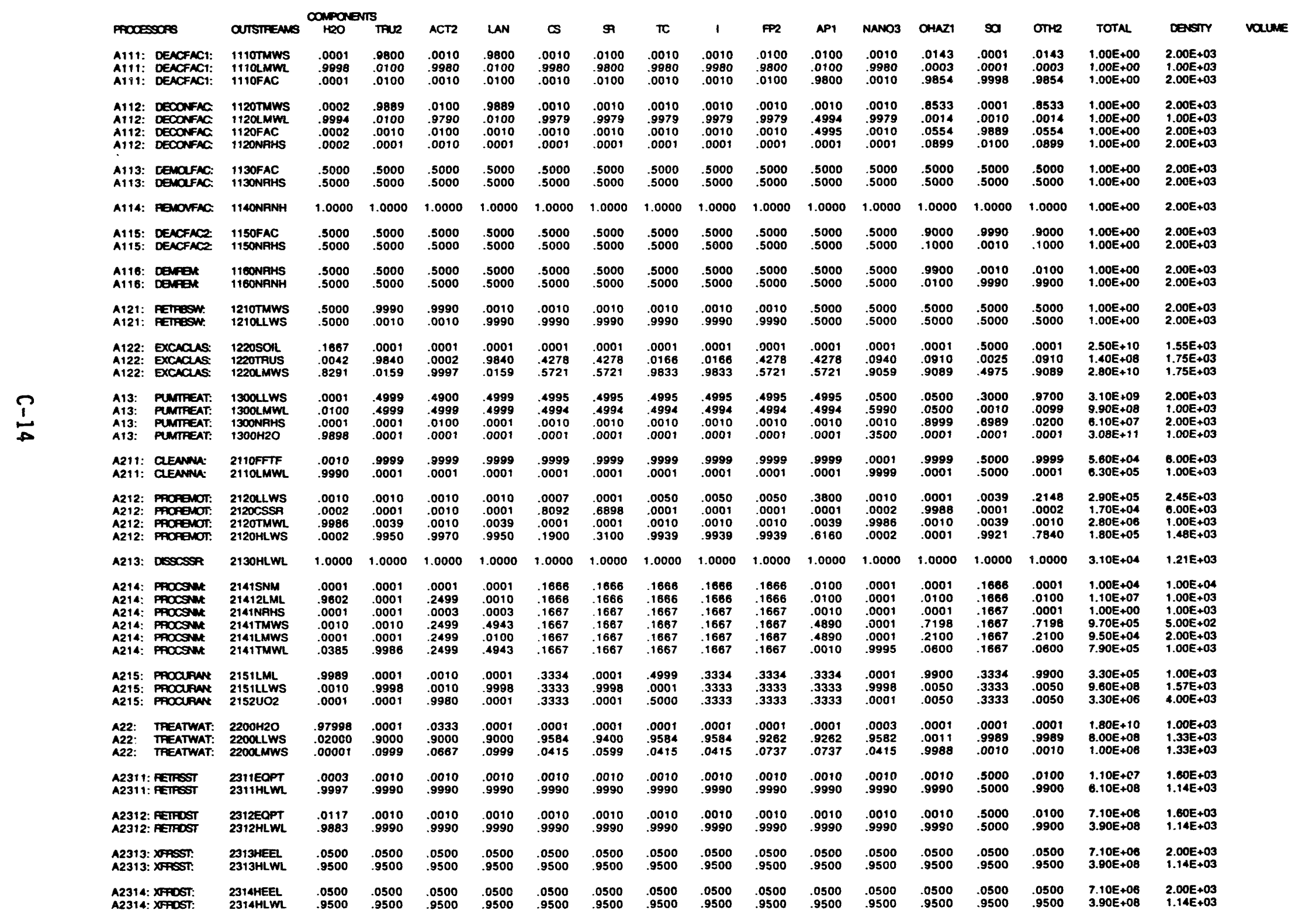


Table C2. Hanford Strategic Analysis Separation Specifications. (sheet 2 of 2)

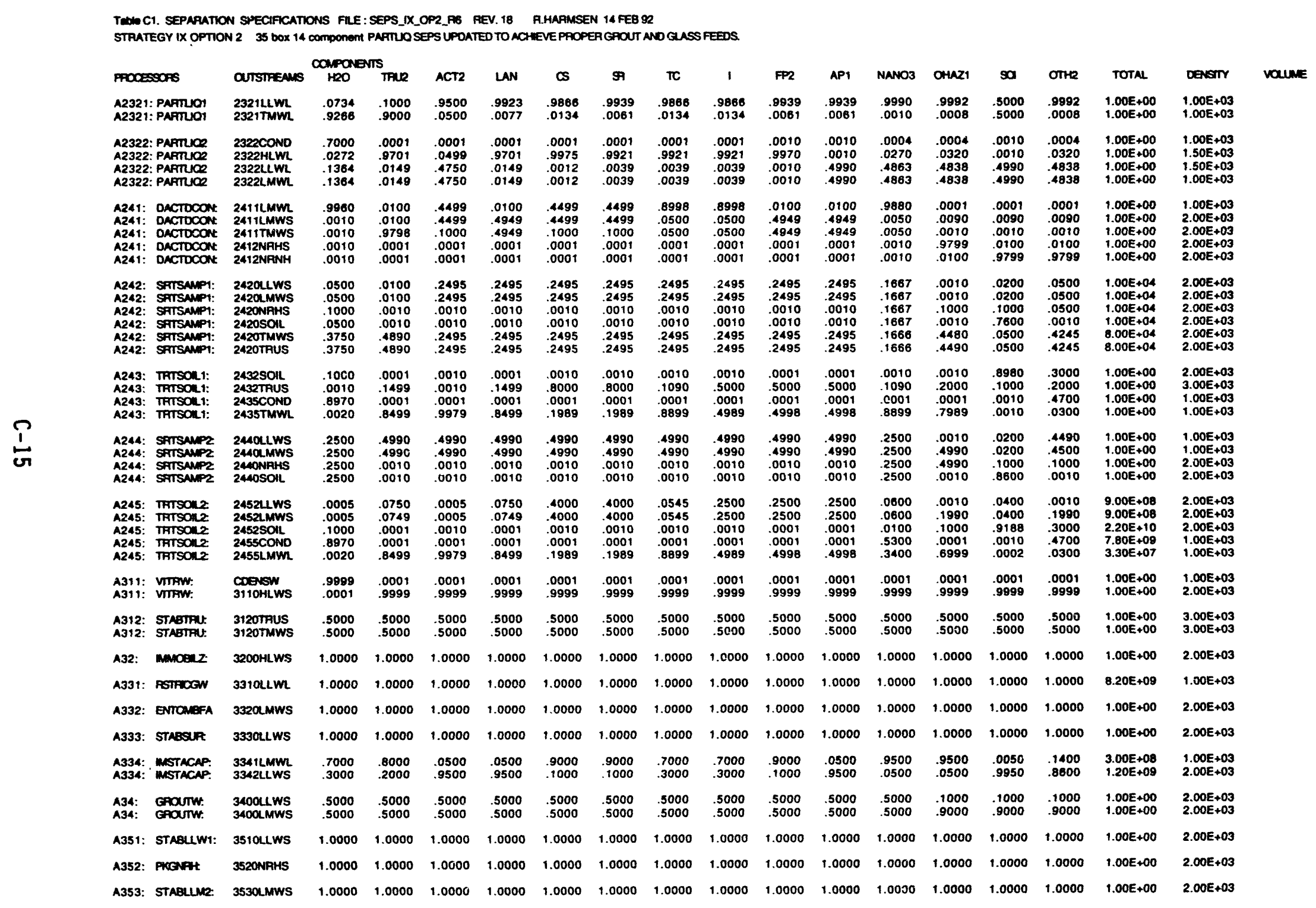


Table C3. Ratios of Chemical Makeup Mass to Process Block Input Mass Along With Makeup Component Ratios.

\author{
Strategy IX Option 2
} (S0937142)

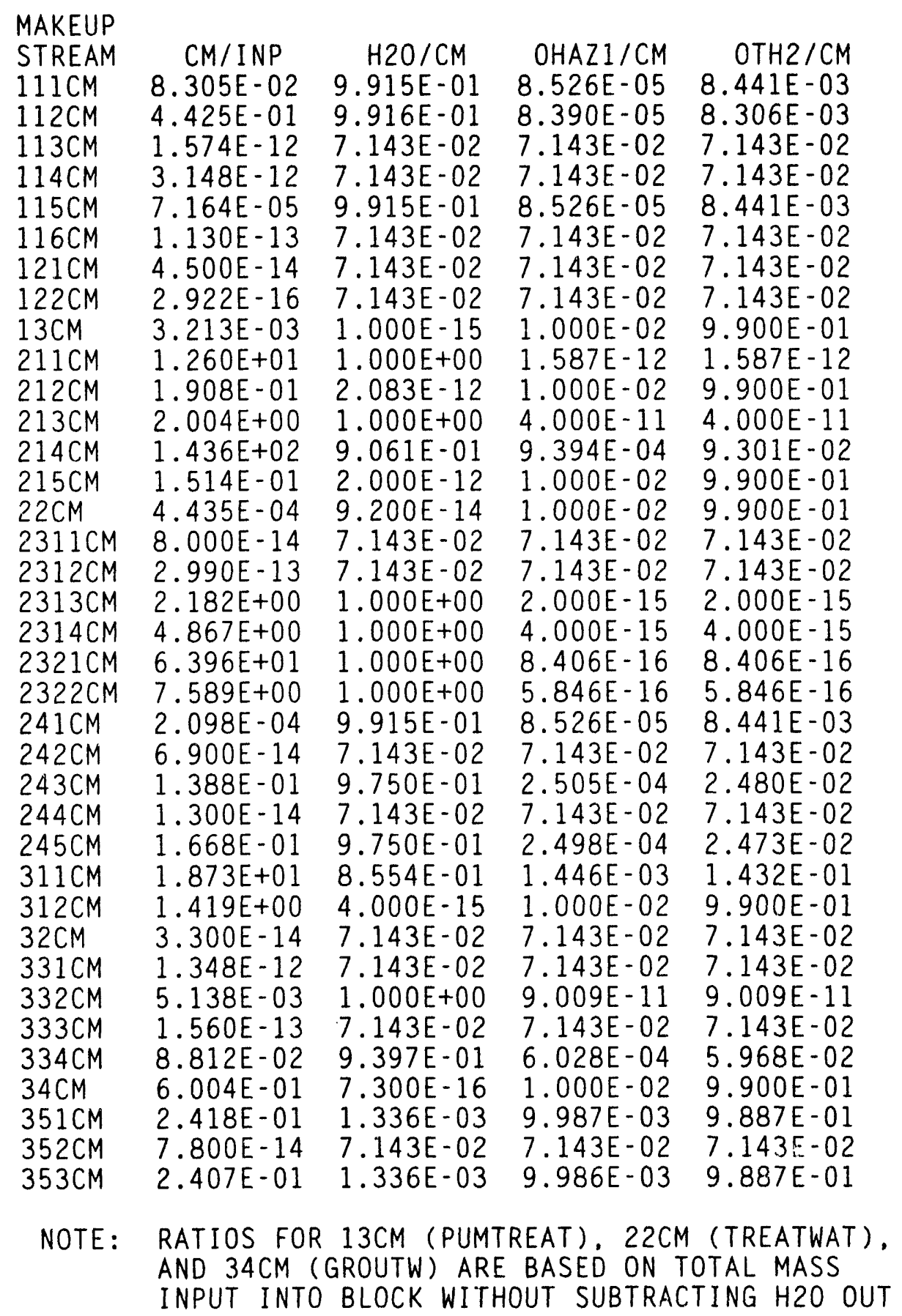


WHC-EP-0791

\section{APPENDIX D}

GLOBAL LEVEL MODEL: ASPEN PLUS ${ }^{1}$ IMPLEMENTATION

${ }^{1}$ ASPEN PLUS is a trademark of Aspen Technology, Inc.

D-1 
WHC-EP-0791

\section{LIST OF TERMS}

IDEF Integrated Computer-Aided Manufacturing (ICAM) Definition (Language)

TRUEX-C Transuranic Extraction Option C 
WHC-EP-0791

\section{GLOBAL LEVEL MODEL: ASPEN PLUS IMPLEMENTATION}

The ASPEN PLUS global model development began with a process flowsheet modeled using the Integrated Computer-Aided Manufacturing (ICAM) Definition (Language) (IDEF) software tool (Pajunen et a1. 1992) at a higher abstraction or less detailed level showing all major feed and product streams and the main process blocks. The IDEF tool allowed showing the flowsheet description at increasingly decomposed states (i.e., a hierarchical representation) as shown in Figure D1. The higher level model allowed good understanding of the overall waste flow in each process and facilitated the development of a more detailed process model. Each main process and stream in the higher level model was further broken down or decomposed to an appropriate detail level for implementation using ASPEN PLUS. Thus, the IDEF representations became the ASPEN PLUS flowsheet specifications. The IDEF flowsheet representation for the CLEAN Option process strategies is shown in Figure D2.

The IDEF model flowsheet specifications were used to build the ASPEN PLUS flowsheet model connectivity with all the specified feed and product streams. In place of rigorous chemical and physical process models, the ASPEN PLUS models used simpler mixers and splitter models, referred to as MIXER, FSPLIT, and SEP process unit blocks (see Appendix A). The MIXER block combines material streams into one stream. The FSPLIT block combines material streams and divides the resulting stream into two or more streams, with all outlets having the same composition and properties. The SEP block combines streams and separates the resulting stream into two or more streams according to splits or separation factors specified for each component. The last process type modeled is a "reactor" (RYIELD). The reactor is used to simulate chemical reactions. The RYIELD reactor process simulates a reactor with specified reaction yields of each component.

A global (or intermediate) level model was developed to represent the CLEAN Option (CLEAN) process strategy described in Appendix B. This global model represented these process strategies with 63 blocks (23 reactors, 33 seps, 4 mixers, and 3 fsplits) and was self-contained in modeling the tank waste retrieval, treatment, and disposal processes, along with the tertiary nonprocess waste generation. The global model was created to run with either 25 pseudo (or lumped) or 168 specie components. Calculations of stream component masses, stream separation factors, and reactor yield factors using the detailed model data, were automated using spreadsheet script programs. This automation allows using any component lumping scheme desired in processing the detailed model results for implementation in the global model.

A detailed oxidation model was included in the detailed CLEAN Option model to accurately account for oxide creation during the high-level waste vitrification process. The resulting oxide product stream was used in a glass composition optimization routine, described in Appendix G, to find the maximum waste oxide loading in the glass along with the glass former composition. This optimization routine accounts for high-level waste glass property constraints (viscosity, electrical conductivity, durability-boron release), heat loading constraints, critical solubility constraints, and single- and multiple-component constraints (Appendix G). Because specifications for low-level waste glass have not yet been determined, the low-level waste mass was converted to glass using the same high-level waste constraints. However, 
for further model comparison purposes, a previously developed grout calculation module used in the Transuranic Extraction Option C (TRUEX-C) and Sludge Wash $C$ models was also added to the detailed CLEAN Option model to provide a similar grout mass calculation. FORTRAN blocks were developed and used in the global ASPEN PLUS models to account for chemical makeup additions, tertiary nonprocess waste generation (Appendix E), process block cost calculations (Appendix F), and output stream file creation.

The actual global ASPEN PLUS flowsheet model for the CLEAN Option process strategy is shown in Figure D3. Because the CLEAN Option model will be compared to the previously developed TRUEX-C and Sludge Wash C process strategies (Knutson et al. 1993), their global flowsheet model is shown in Figure D4. In Figure D4, the FSPLIT blocks allowed routing the waste streams to the appropriate processes to represent a particular process strategy (e.g., TRUEX-C and Sludge Wash C).

Table D1 describes stream-naming conventions and provides a brief description of the CLEAN Option global model blocks. Table D2 describes stream-naming conventions and provides a brief description of the TRUEX-C and Sludge Wash C global model blocks. Table D3 shows the relationship between the CLEAN Option global model streams and the detailed model streams. Table D4 provides the component lumping scheme used for the CLEAN Option global model streams. This table shows the global model stream components and the detailed model stream species that are included in those global components.

Table D5 provides the separation factor and reactor component yield data used in the CLEAN Option global model blocks. Table D6 provides the separation factor and reactor component yield data used in the TRUEX-C global model blocks. Table 07 provides the separation factor and reactor component yield data used in the Sludge Wash C global model blocks. The separation factors were based on rolling up (or lumping) the detailed model stream and component data for individual output streams and comparing the component distributions between streams to the total output stream. The reactor yield factors were based on comparing the distribution of component masses in the total output stream compared to the total input stream for a particular process block. Note that accurate representation of the detailed models was achieved in the global model by representing a group of detailed process blocks with a reactor and a separation block together. The reactor blocks accounted for component mass changes resulting from chemical reactions within the group of detailed model process blocks while the separation blocks separated those components into their appropriate output streams.

\section{REFERENCES}

Knutson, B. J., et al., 1993, "Advanced Chemical Separation Study: TRUEX Strategy Compared To Sludge Wash Ion Exchange," Letter report, Westinghouse Hanford Company, Richland, Washington.

Pajunen, A. L., et al., 1992, Hanford Strategic Analysis Study, WHC-EP-0549, Westinghouse Hanford Company, Richland, Washington. 
WHC-EP-0791

Figure DI. IDEF Structured Flowsheet.

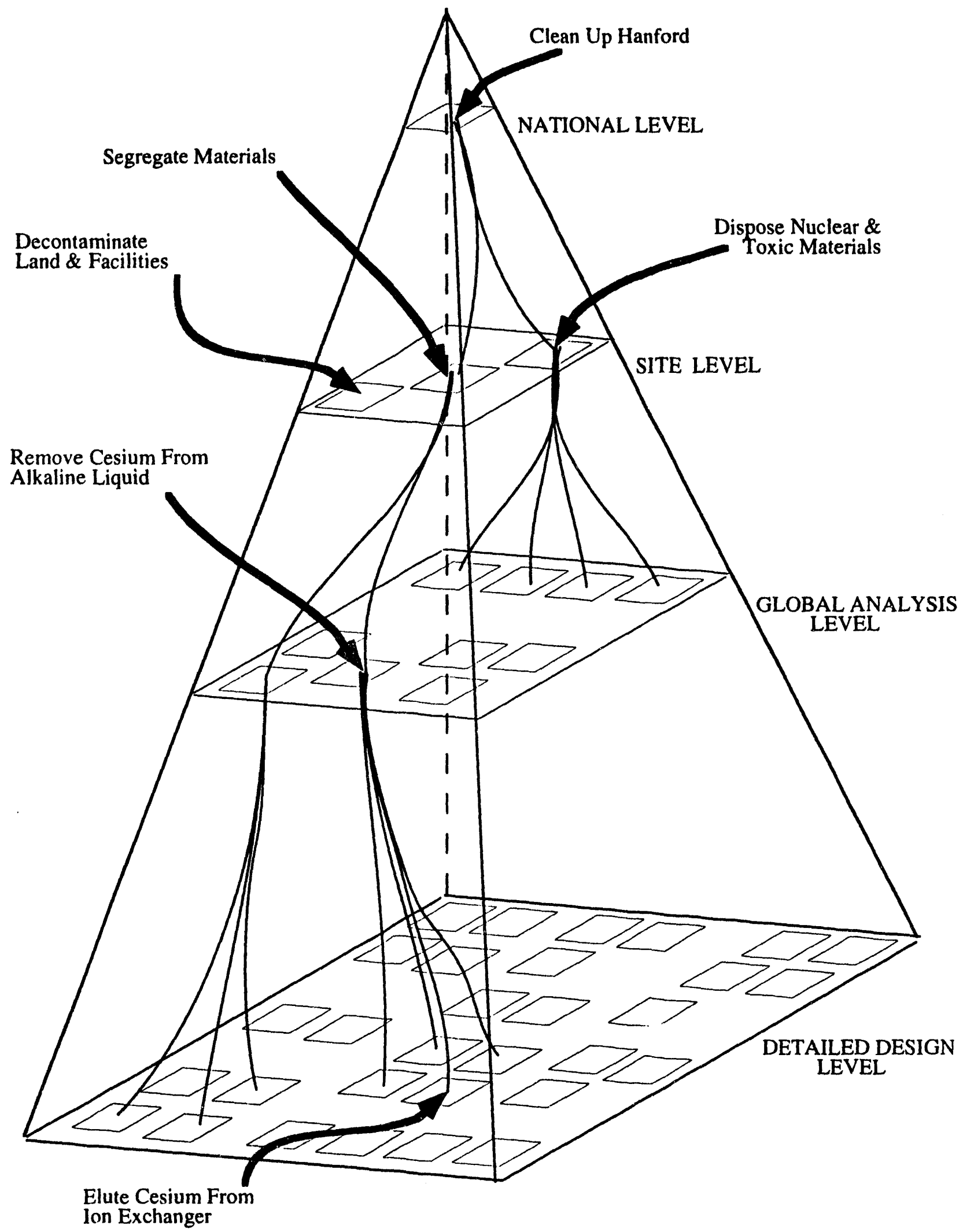




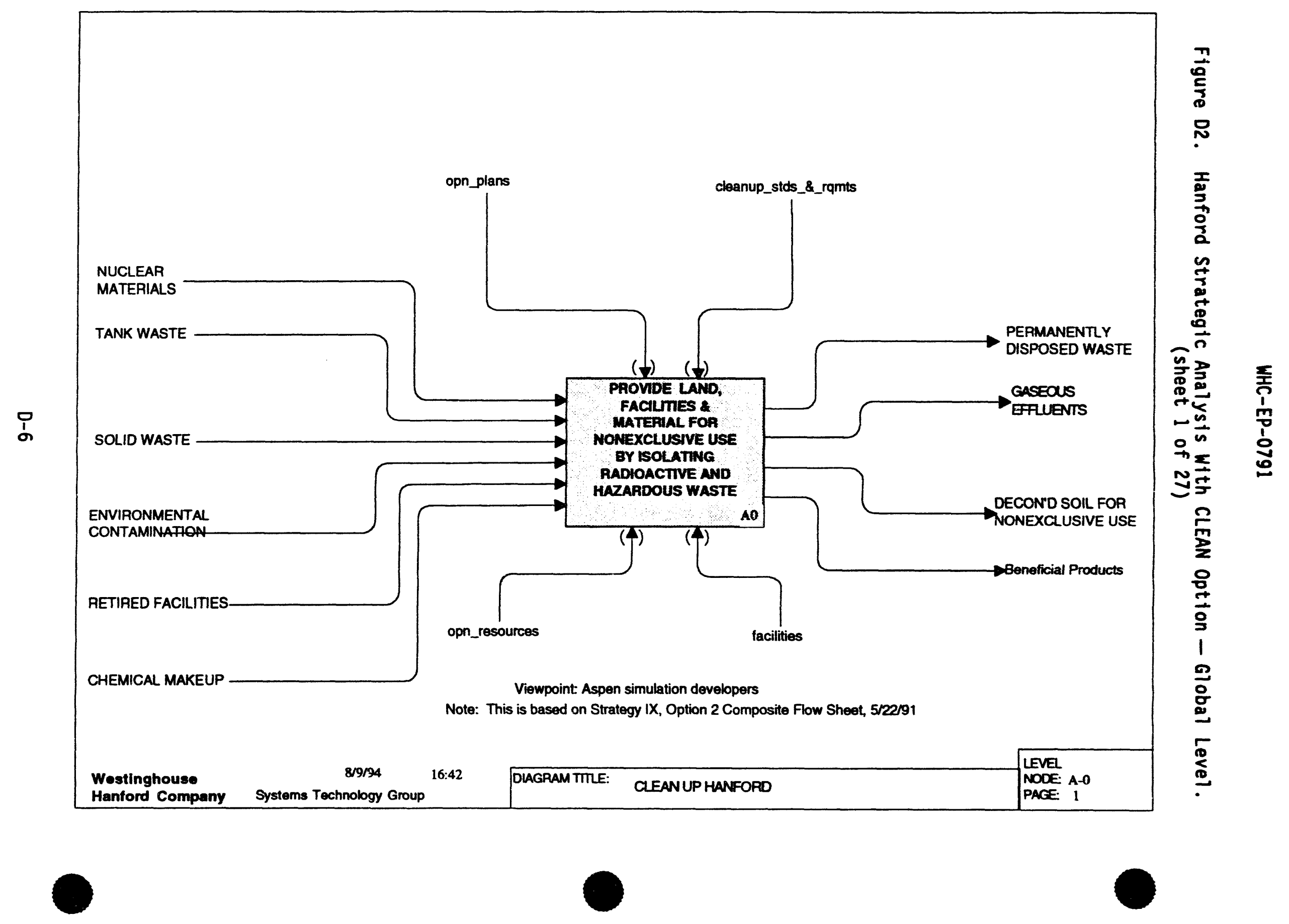




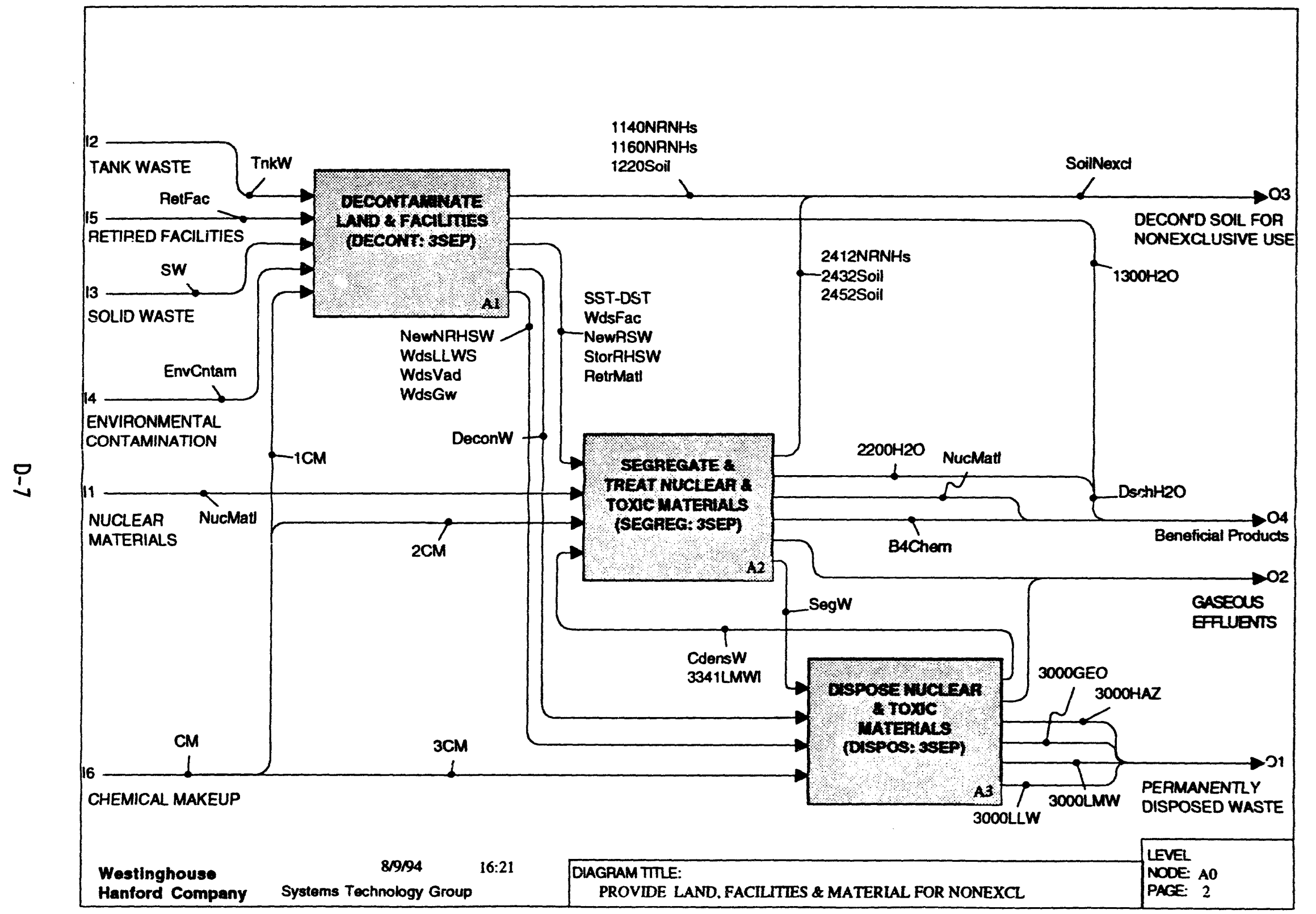




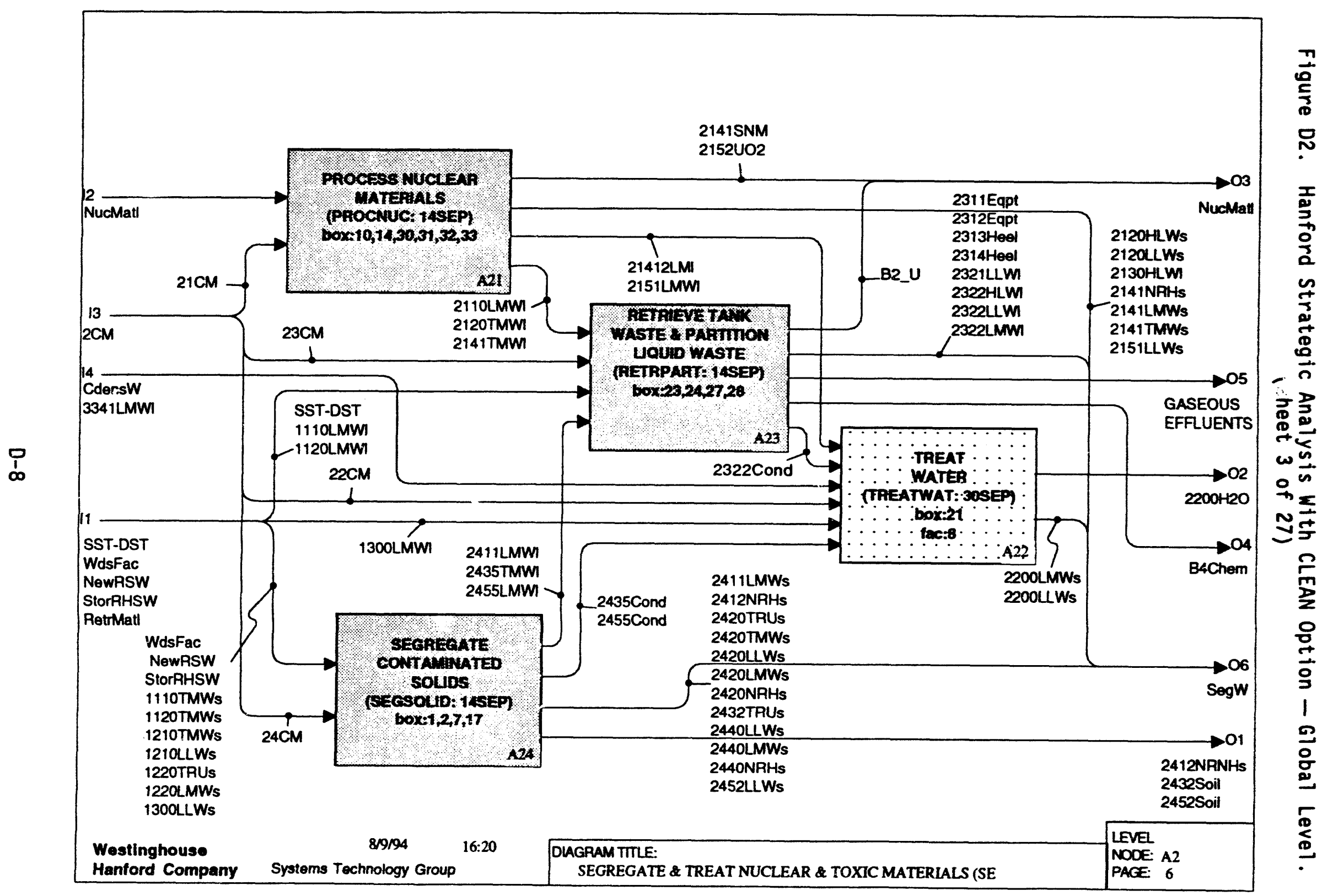



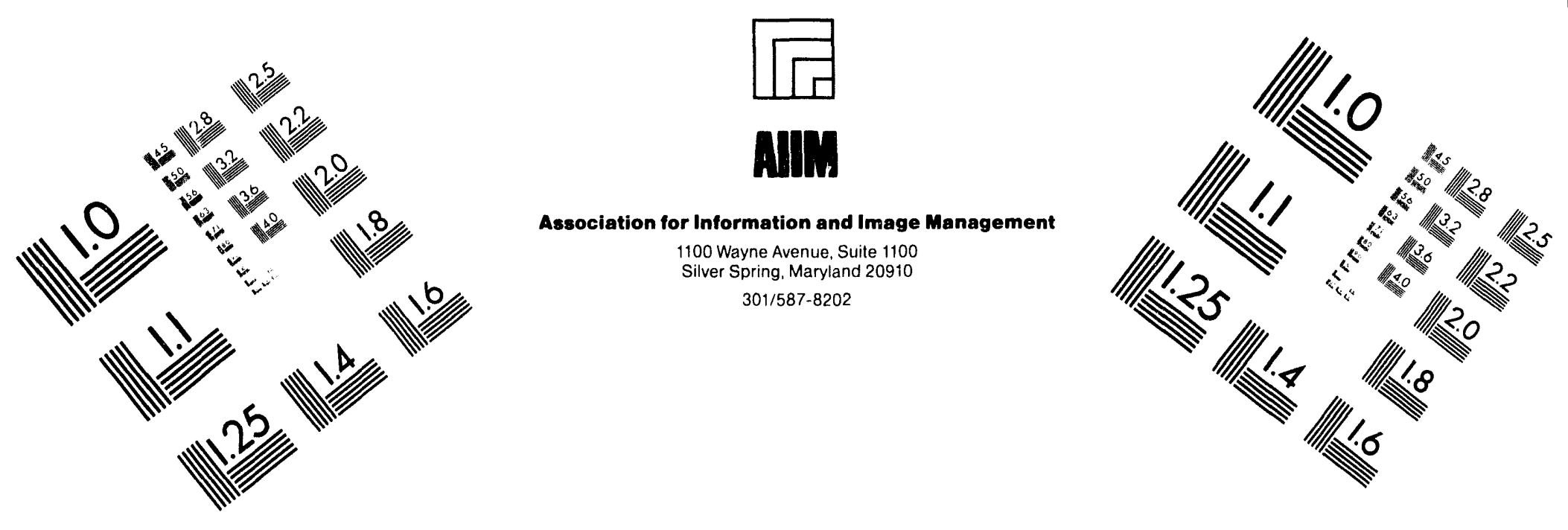

\section{Centimeter}

$\begin{array}{llllllllllllllll}1 & 2 & 3 & 4 & 5 & 6 & 7 & 8 & 9 & 10 & 11 & 12 & 13 & 14 & 15 & \mathrm{~mm}\end{array}$

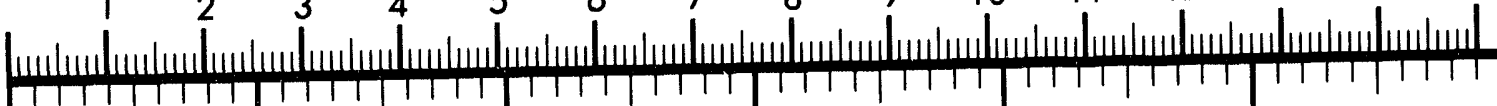

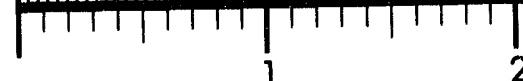
Inches

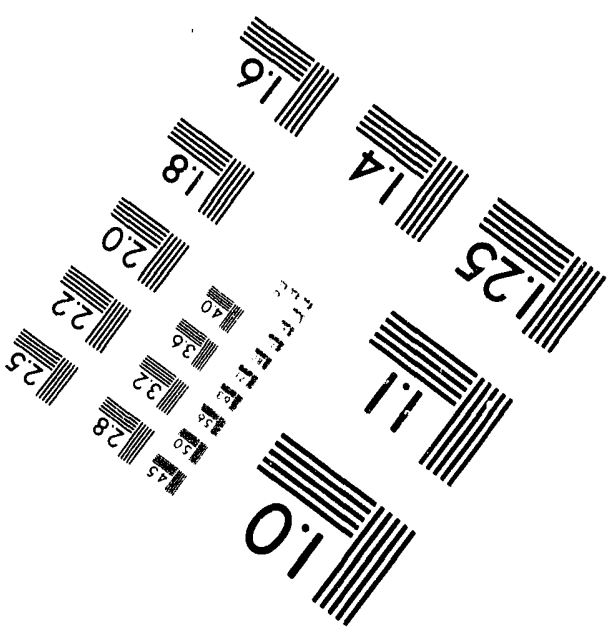

MANUFACTURED TO AIIM STANDARDS

BY APPLIED IMAGE, INC.

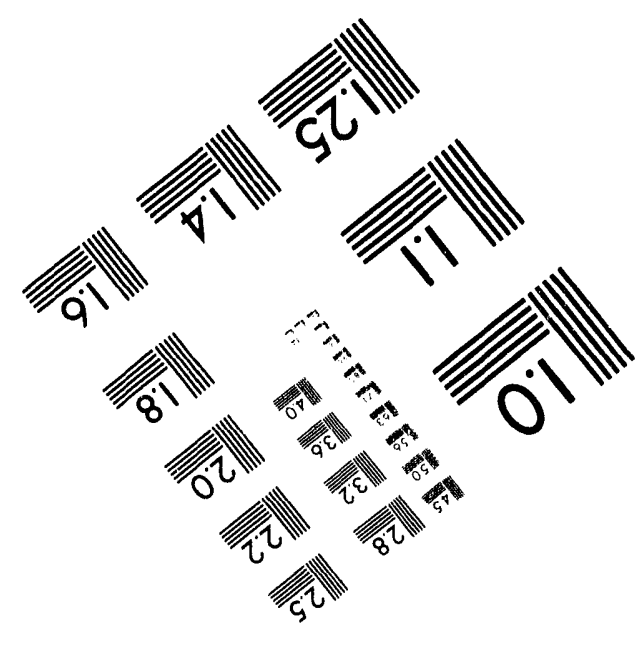



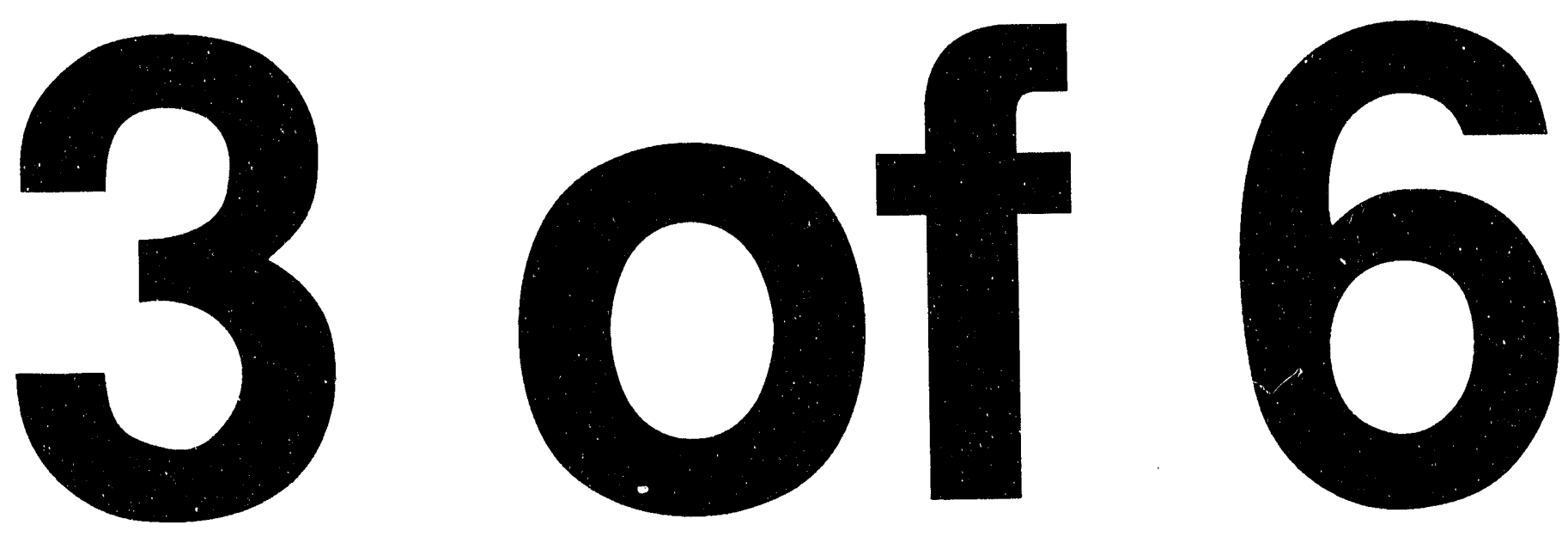


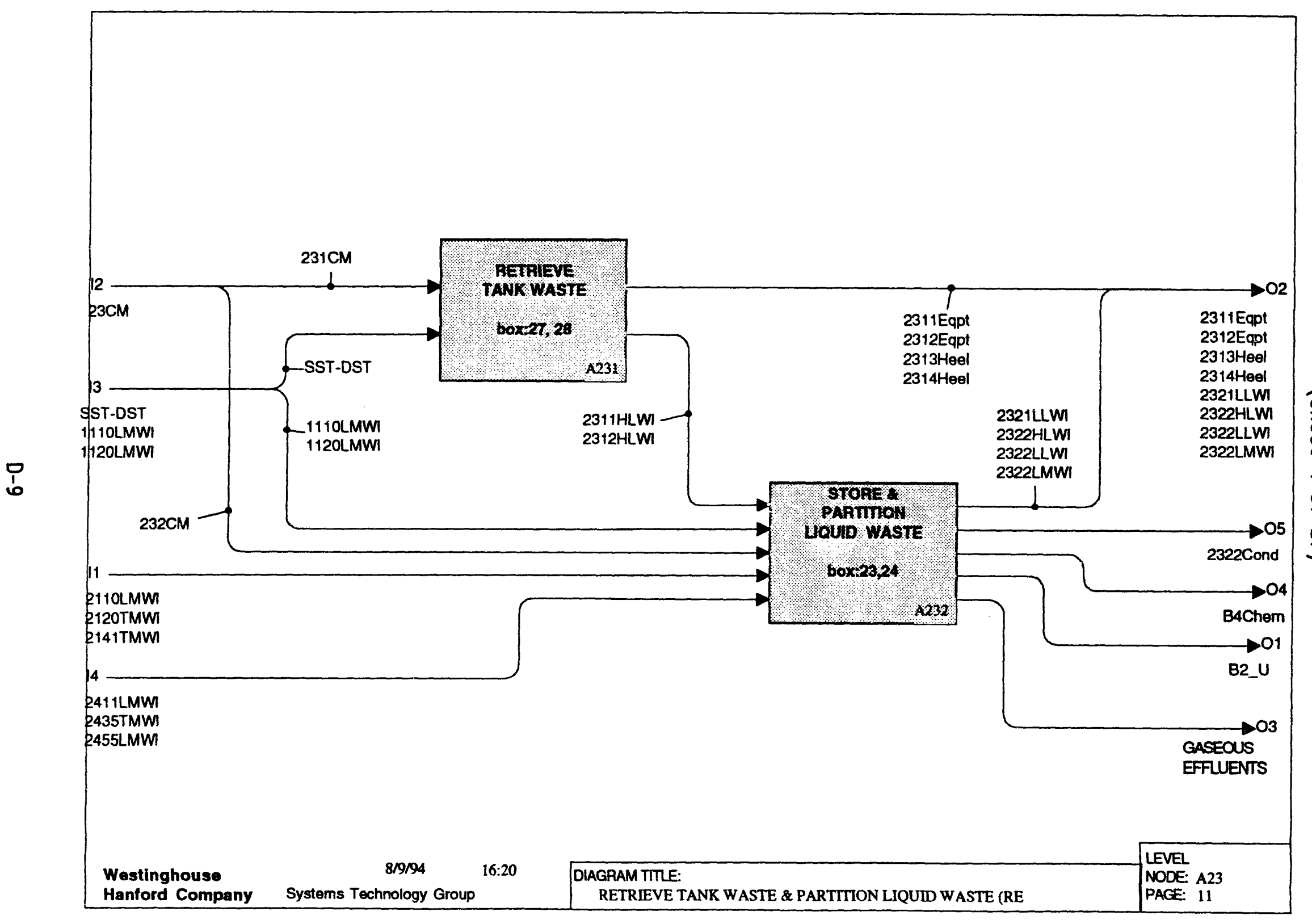

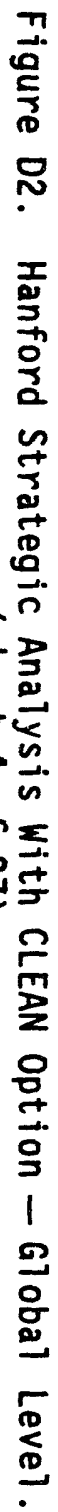




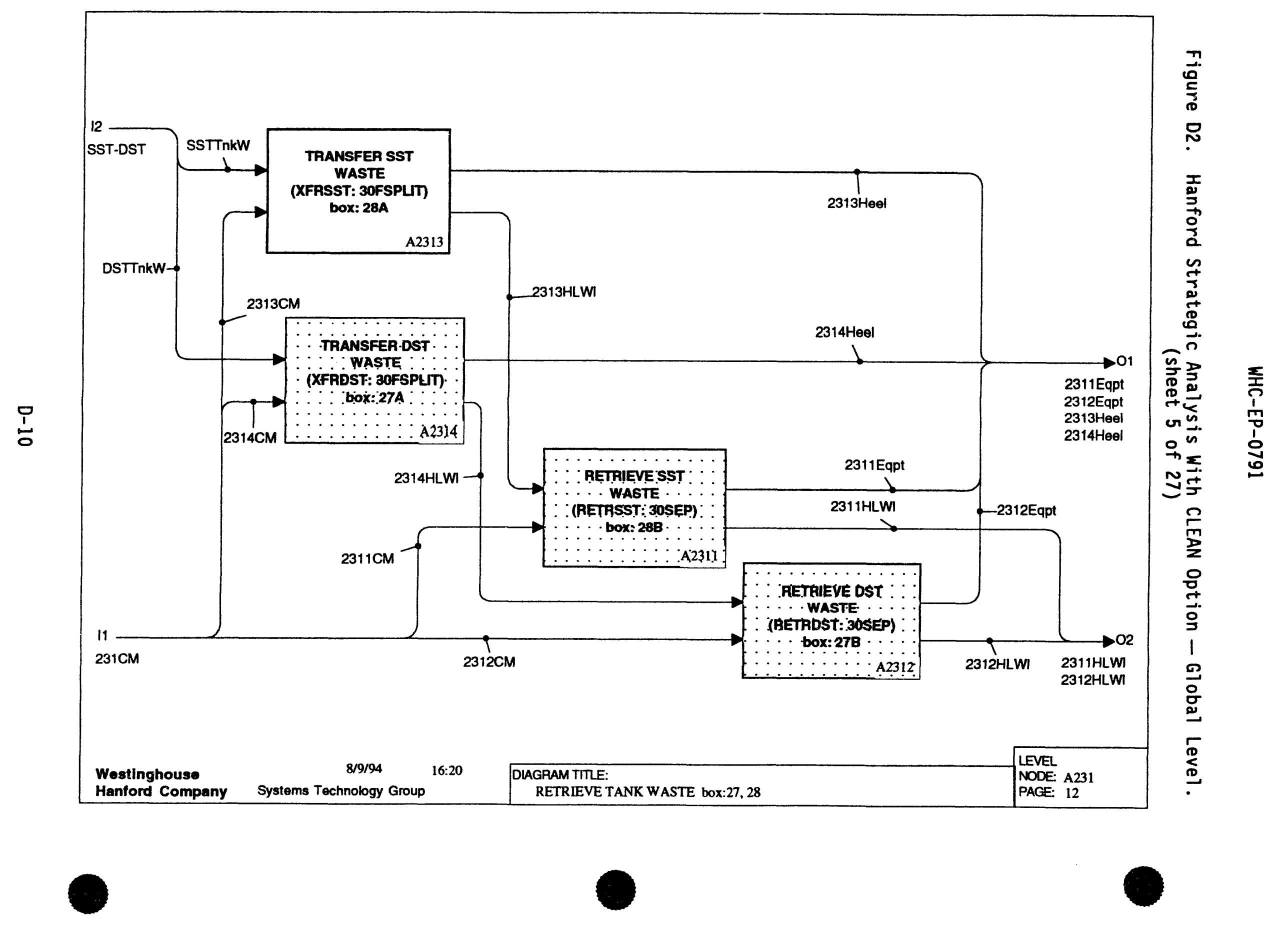




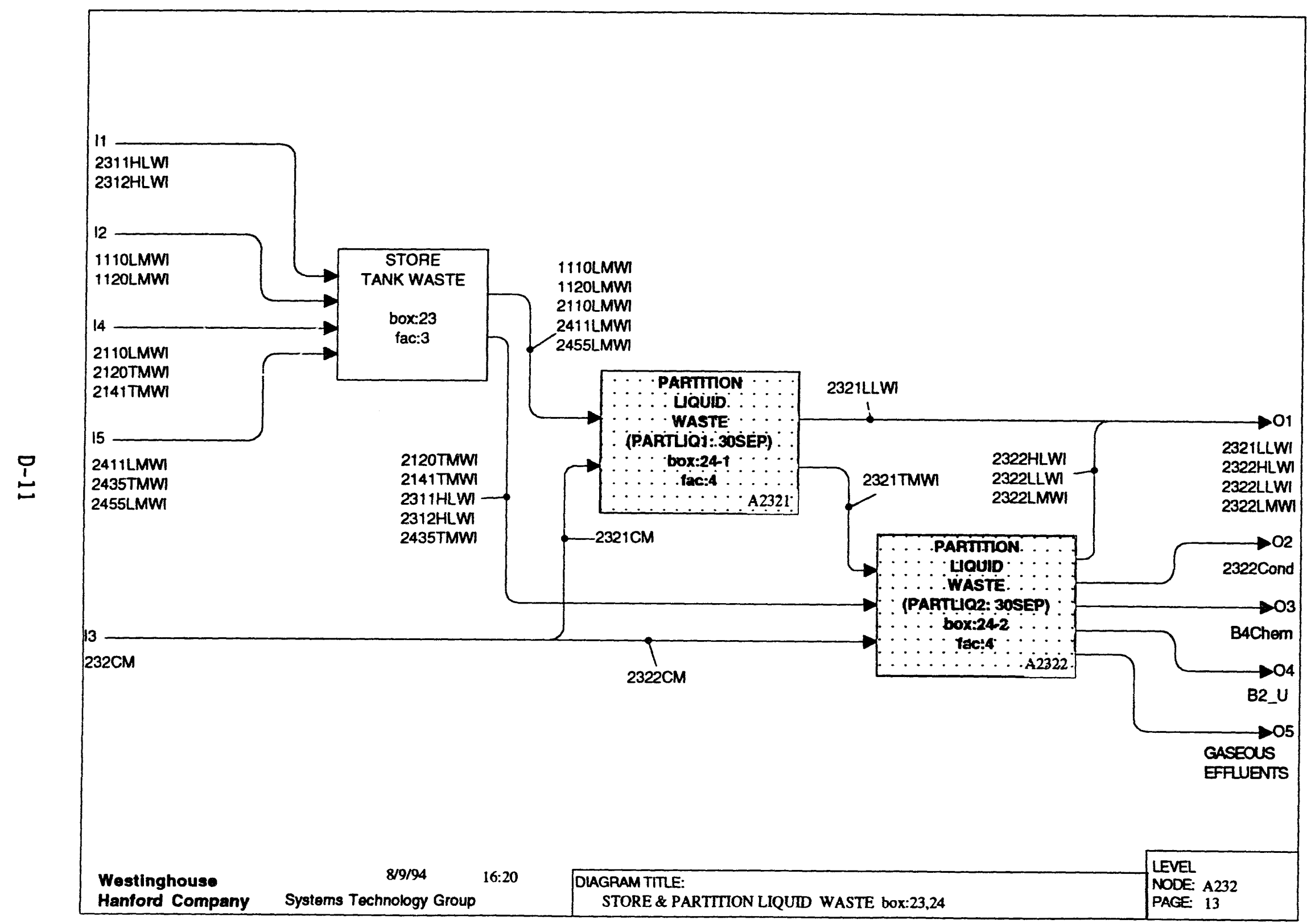




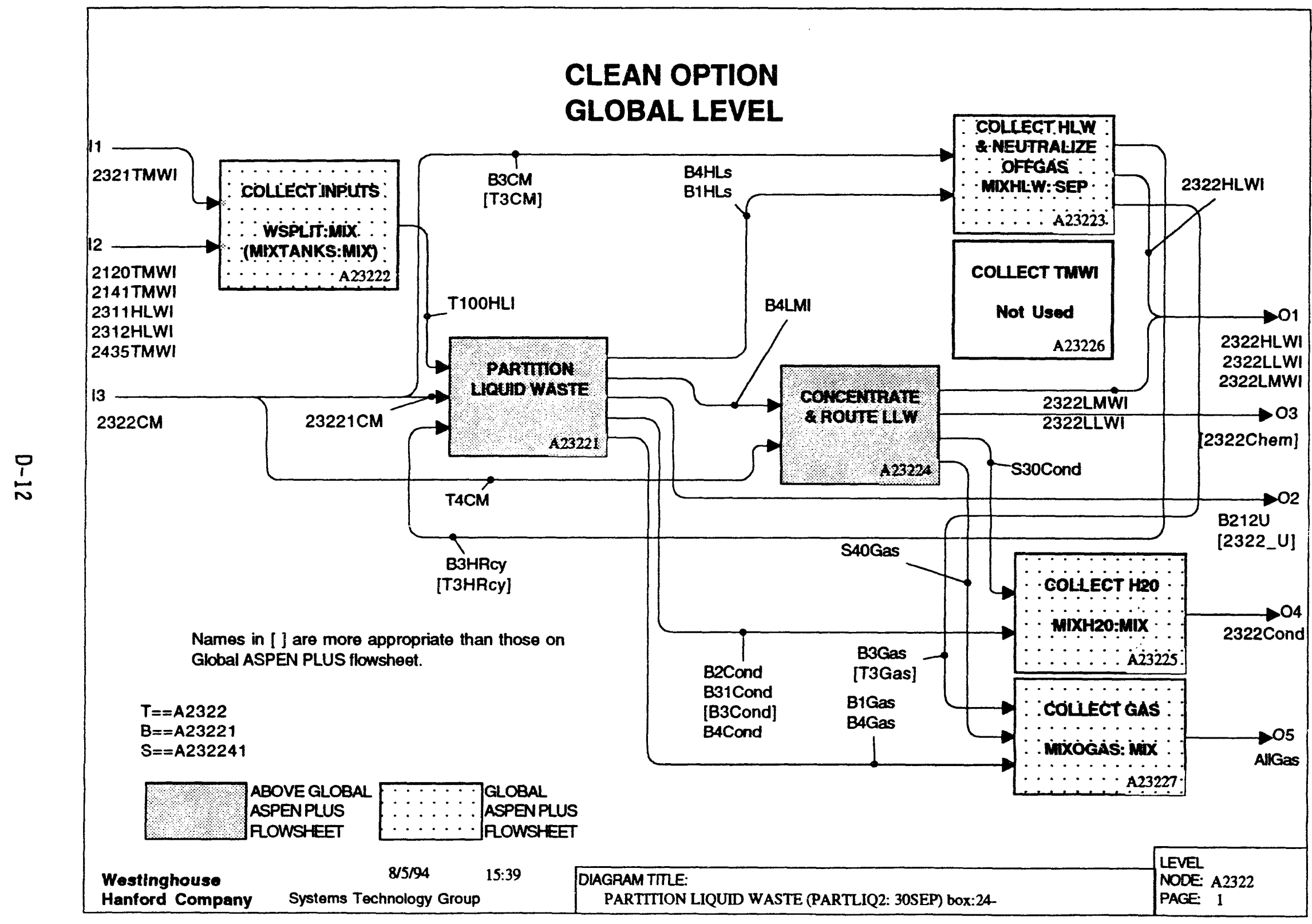

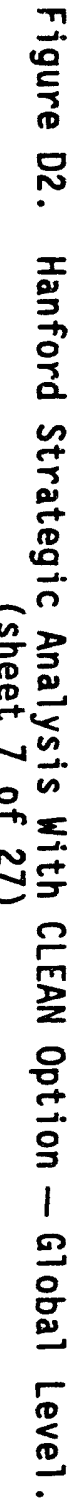




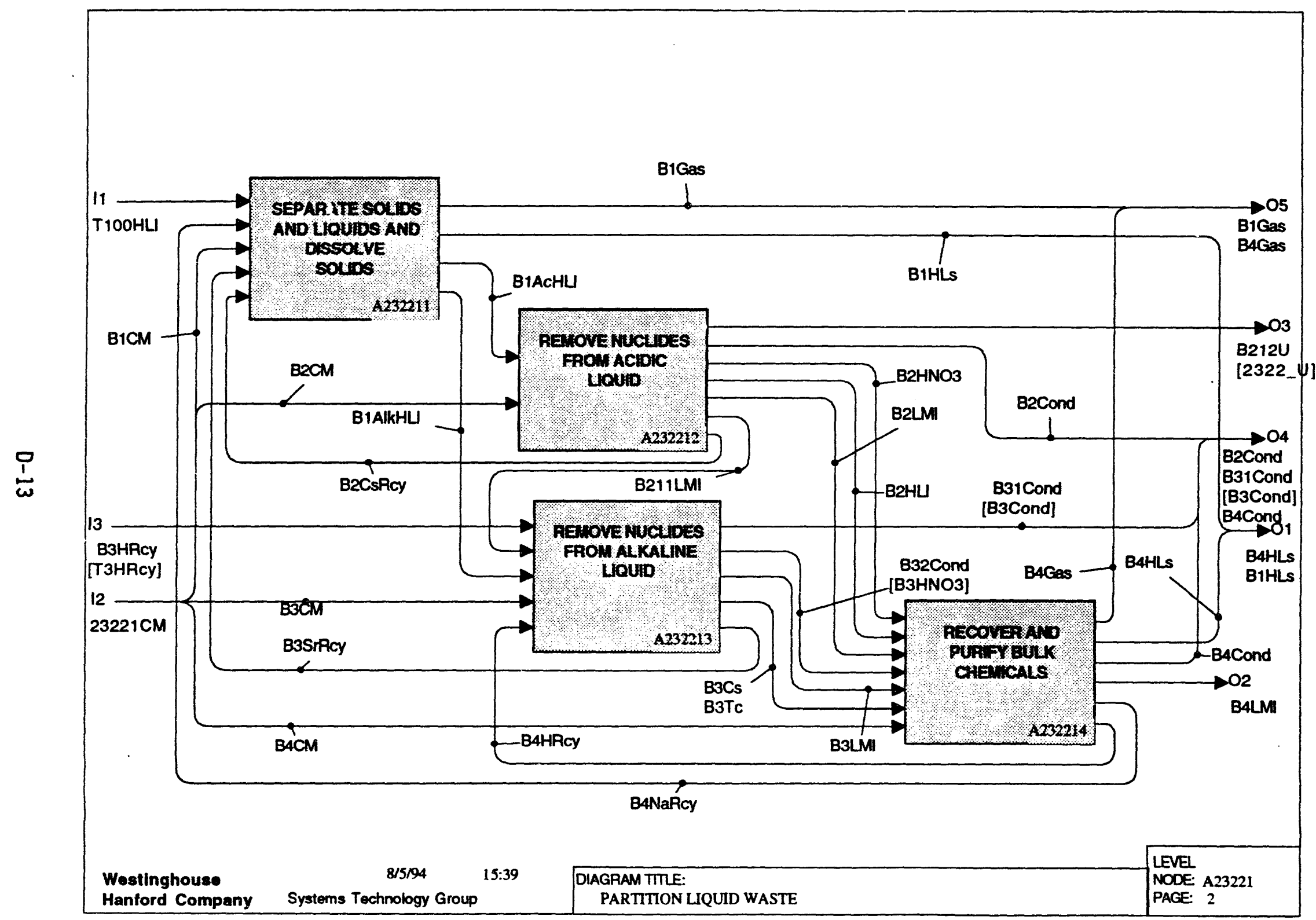




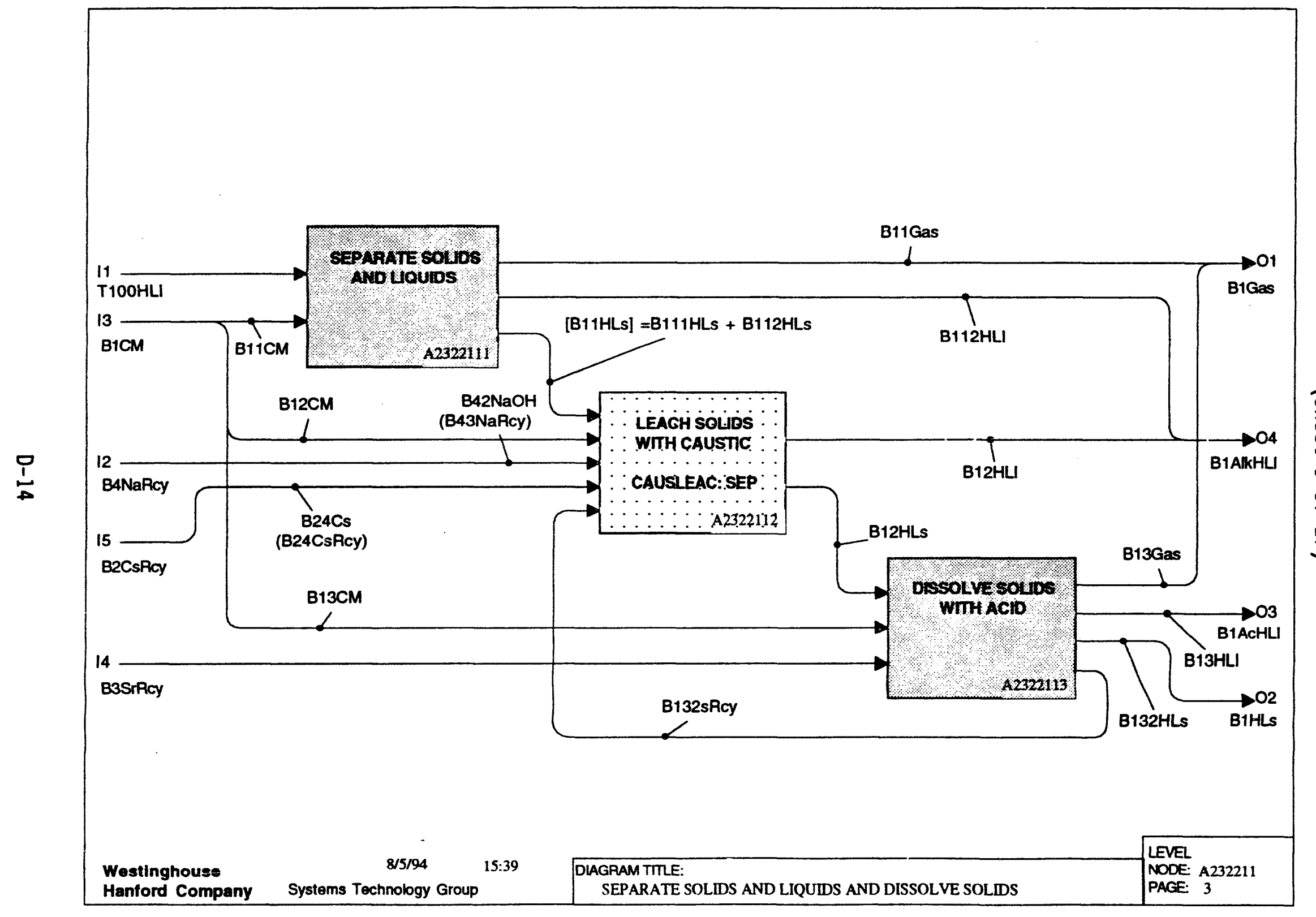

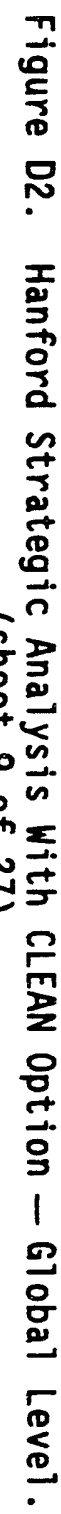




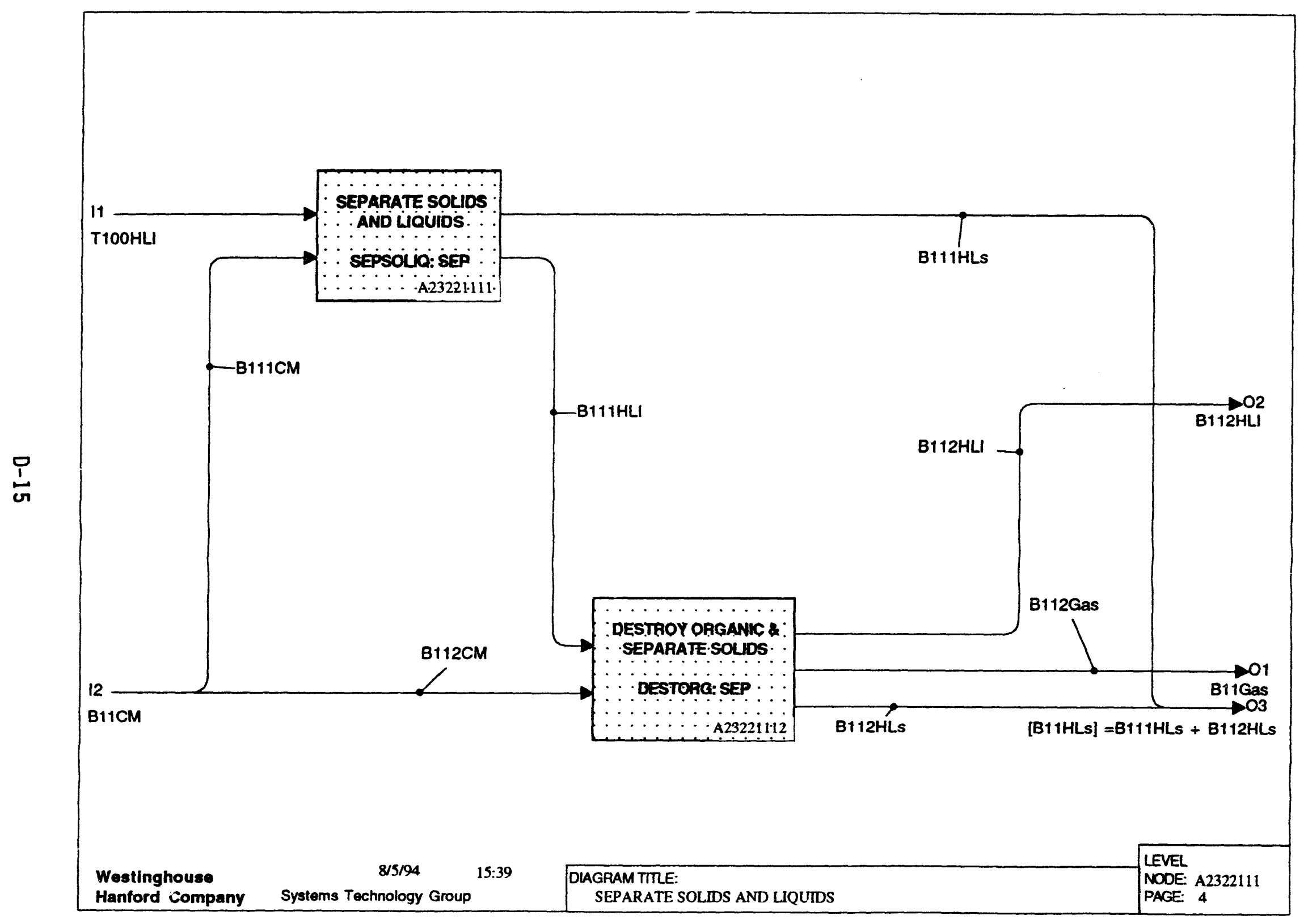

苟 


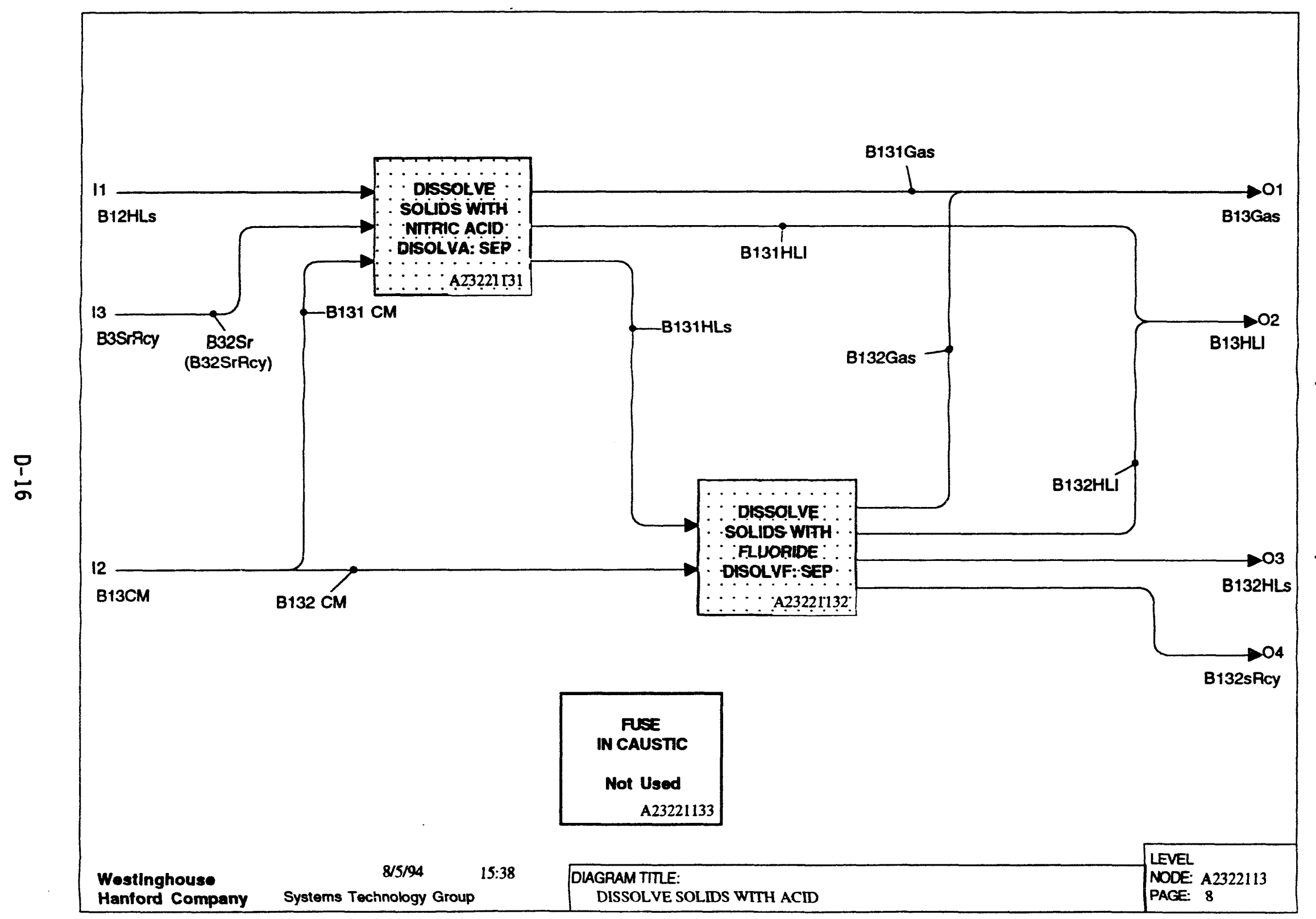

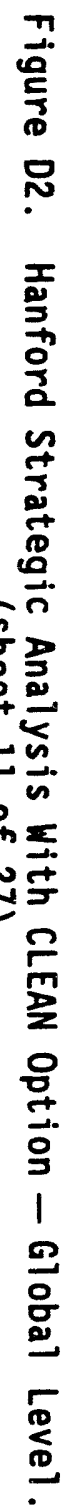



Figure D2. Hanford Strategic Analysis With CLEAN Option - Global Leve1.
(sheet 12 of 27)

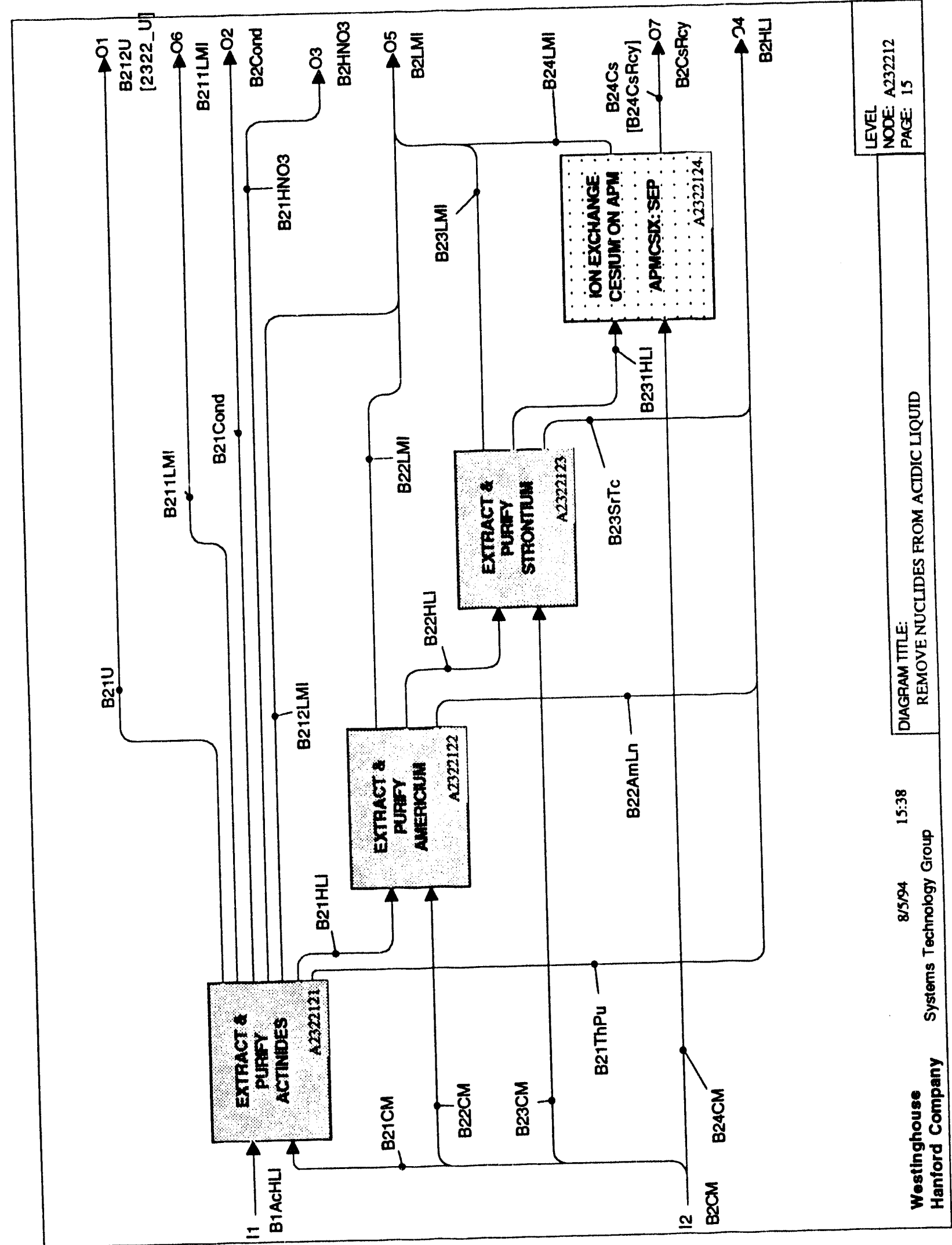




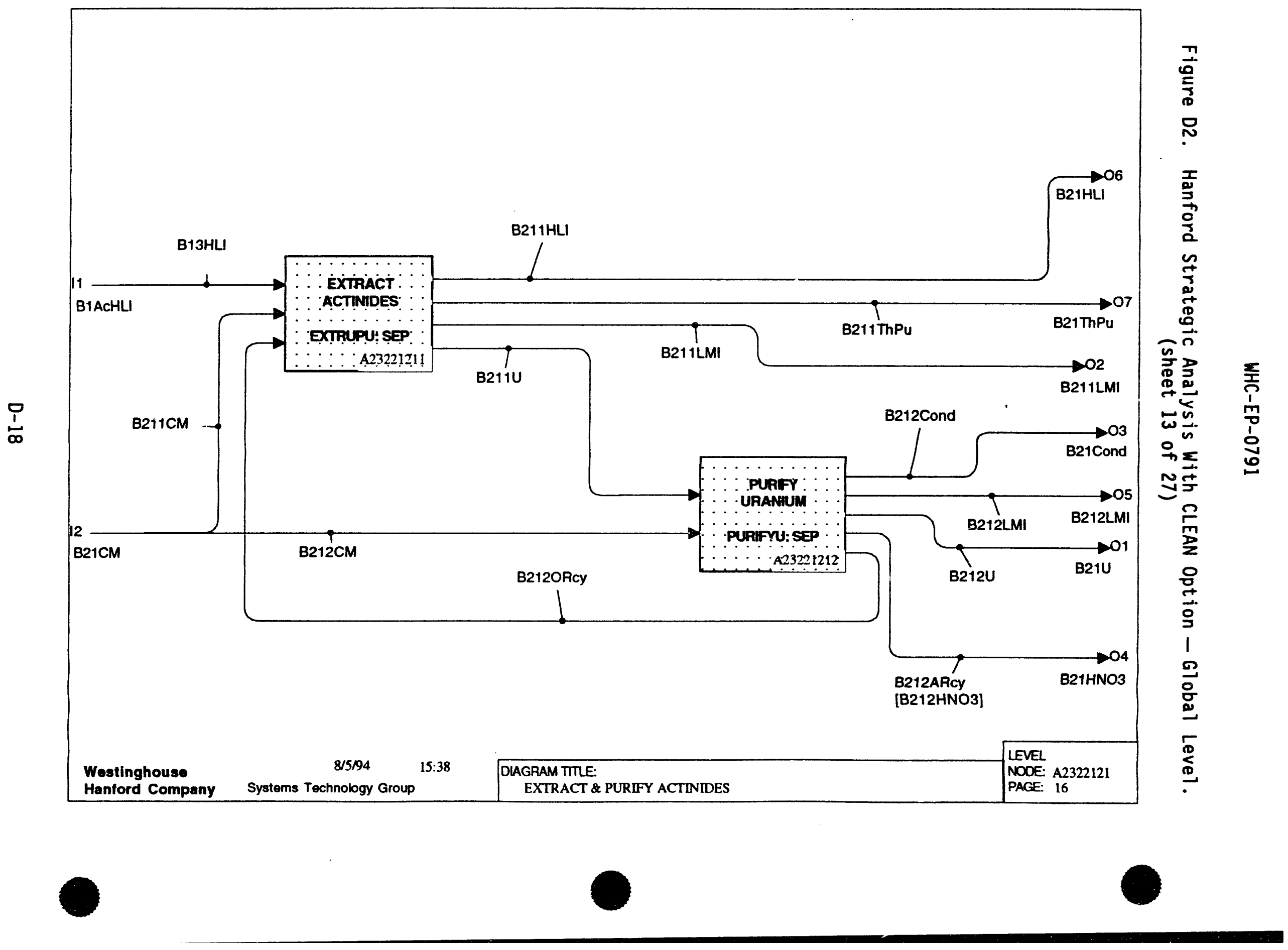




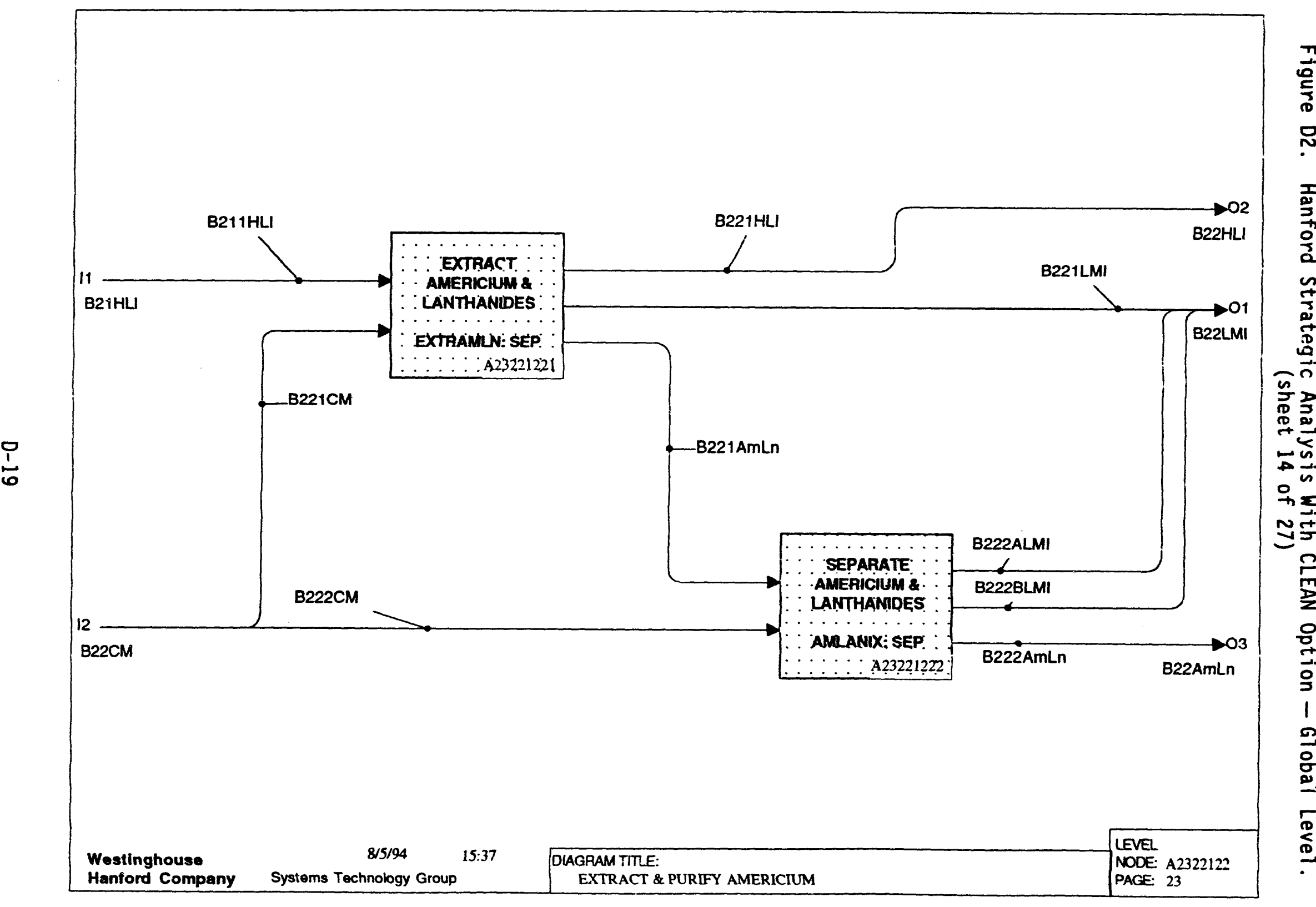




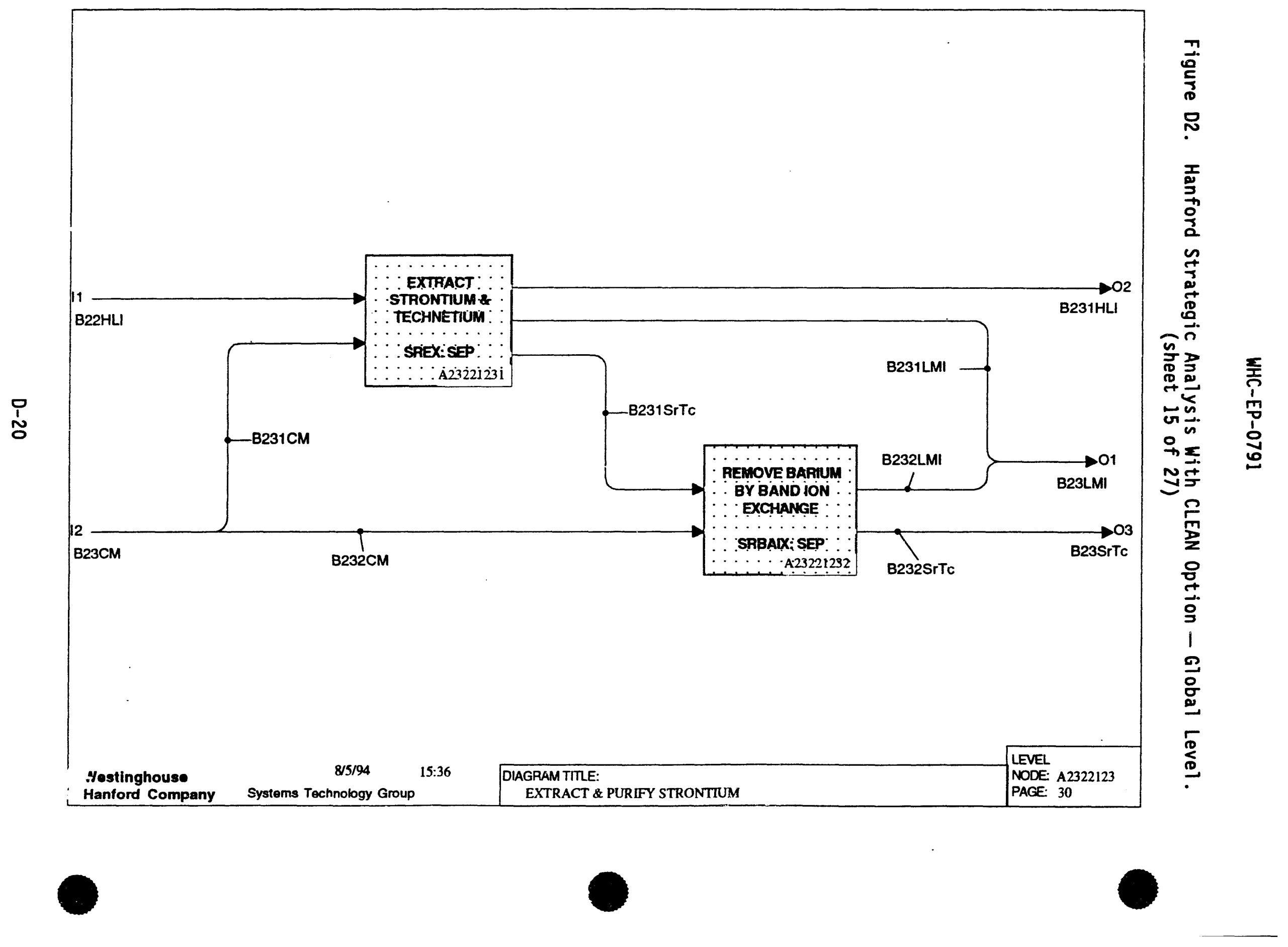




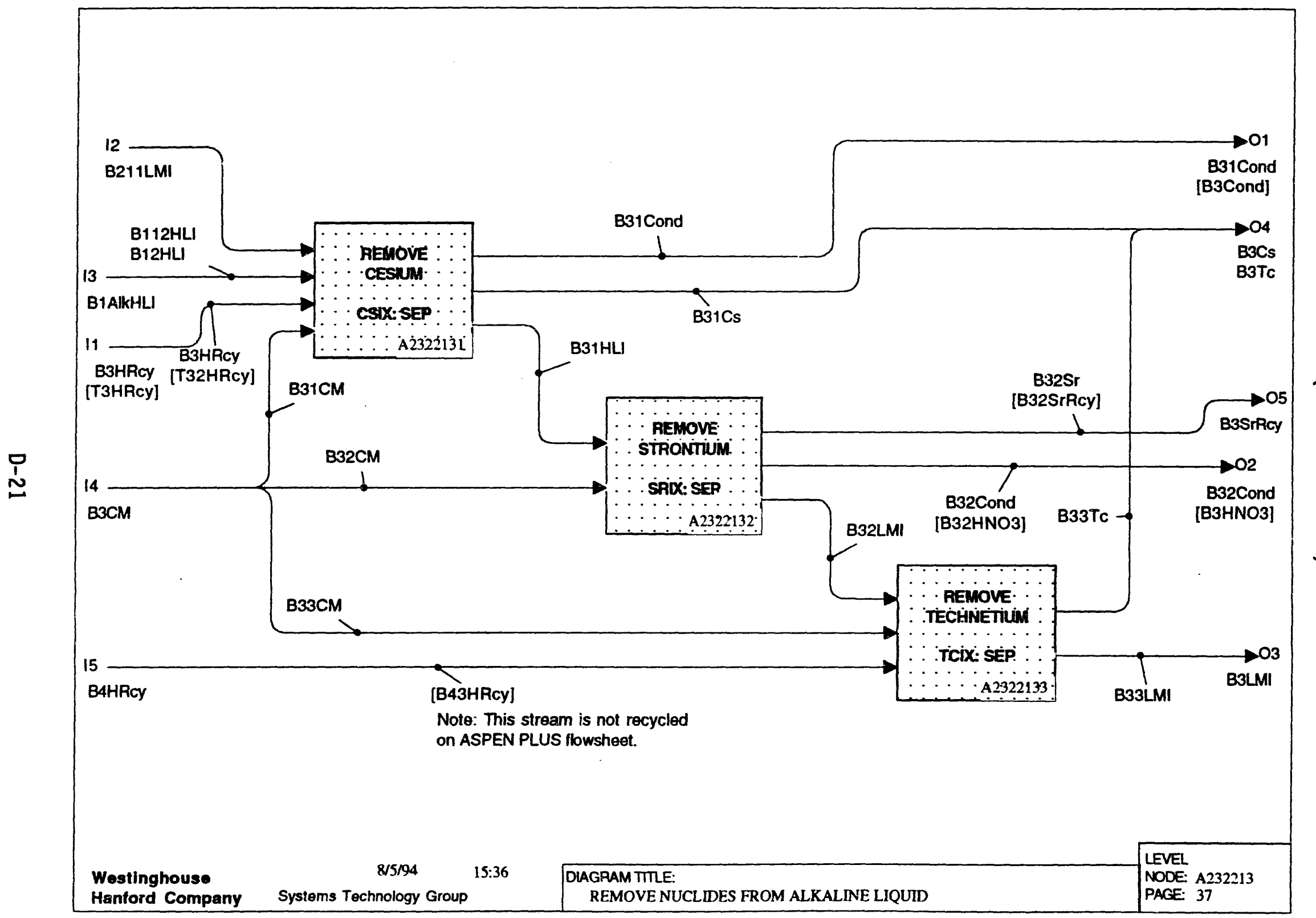




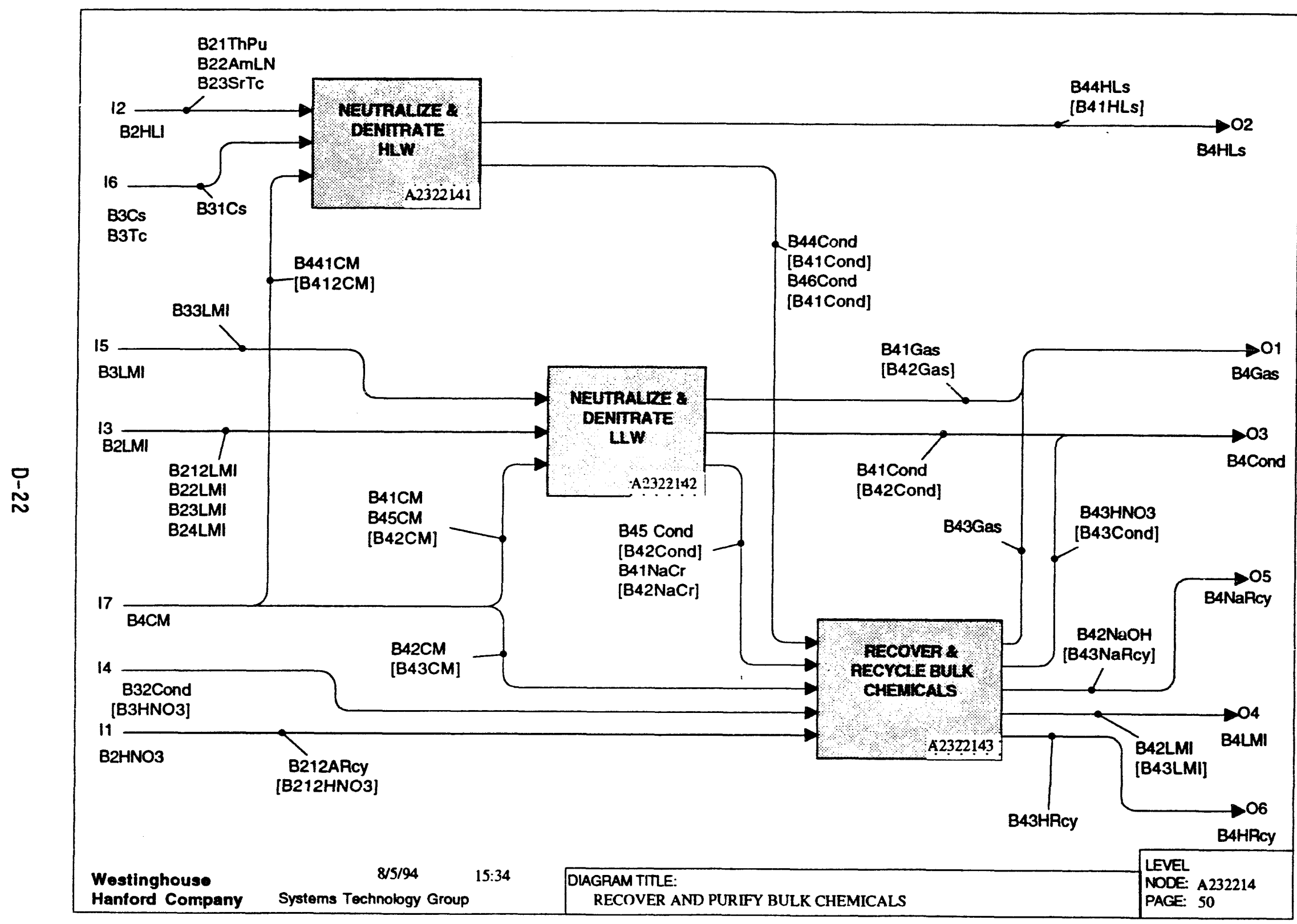

告 


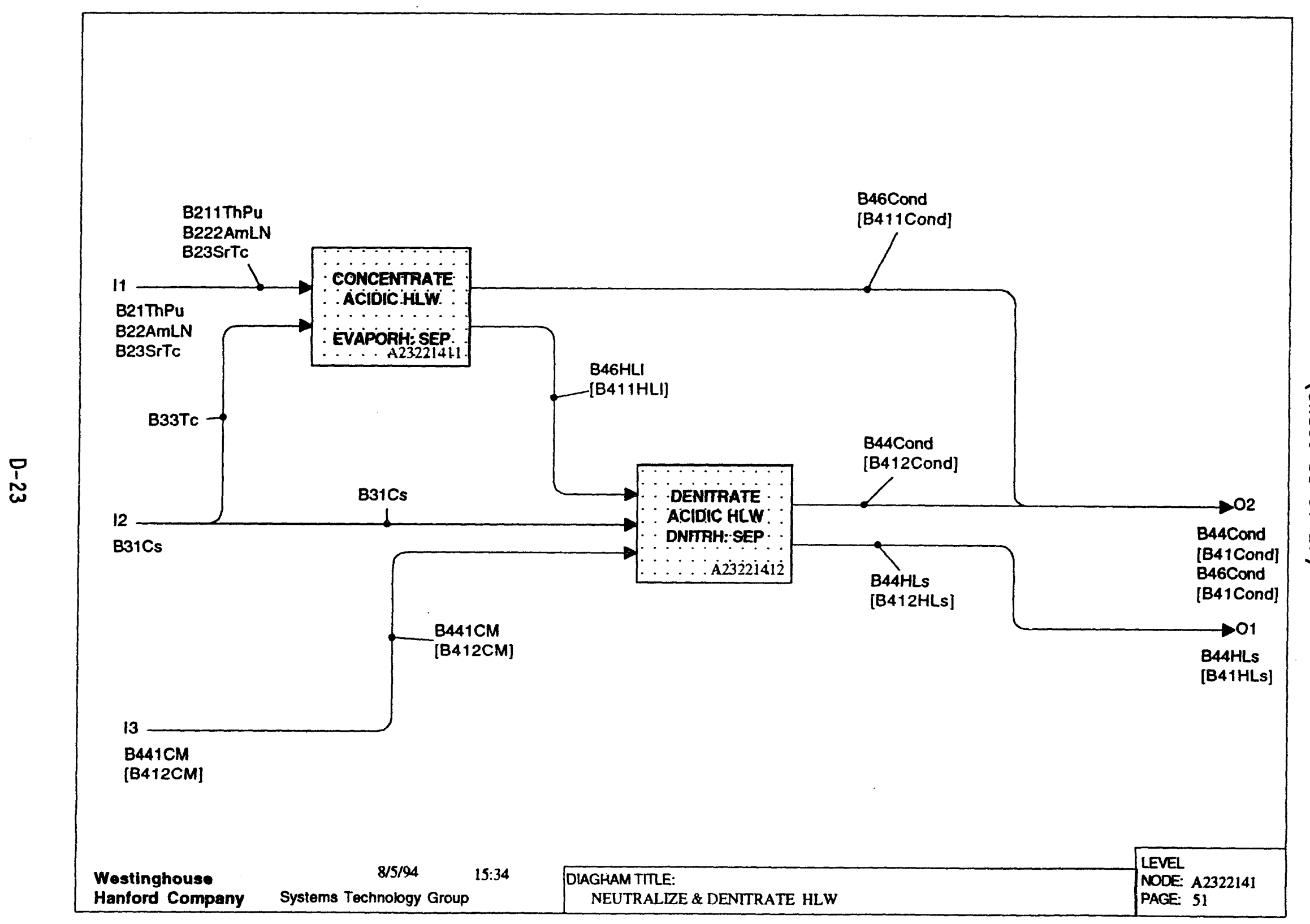

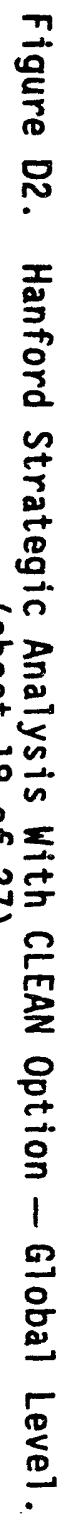




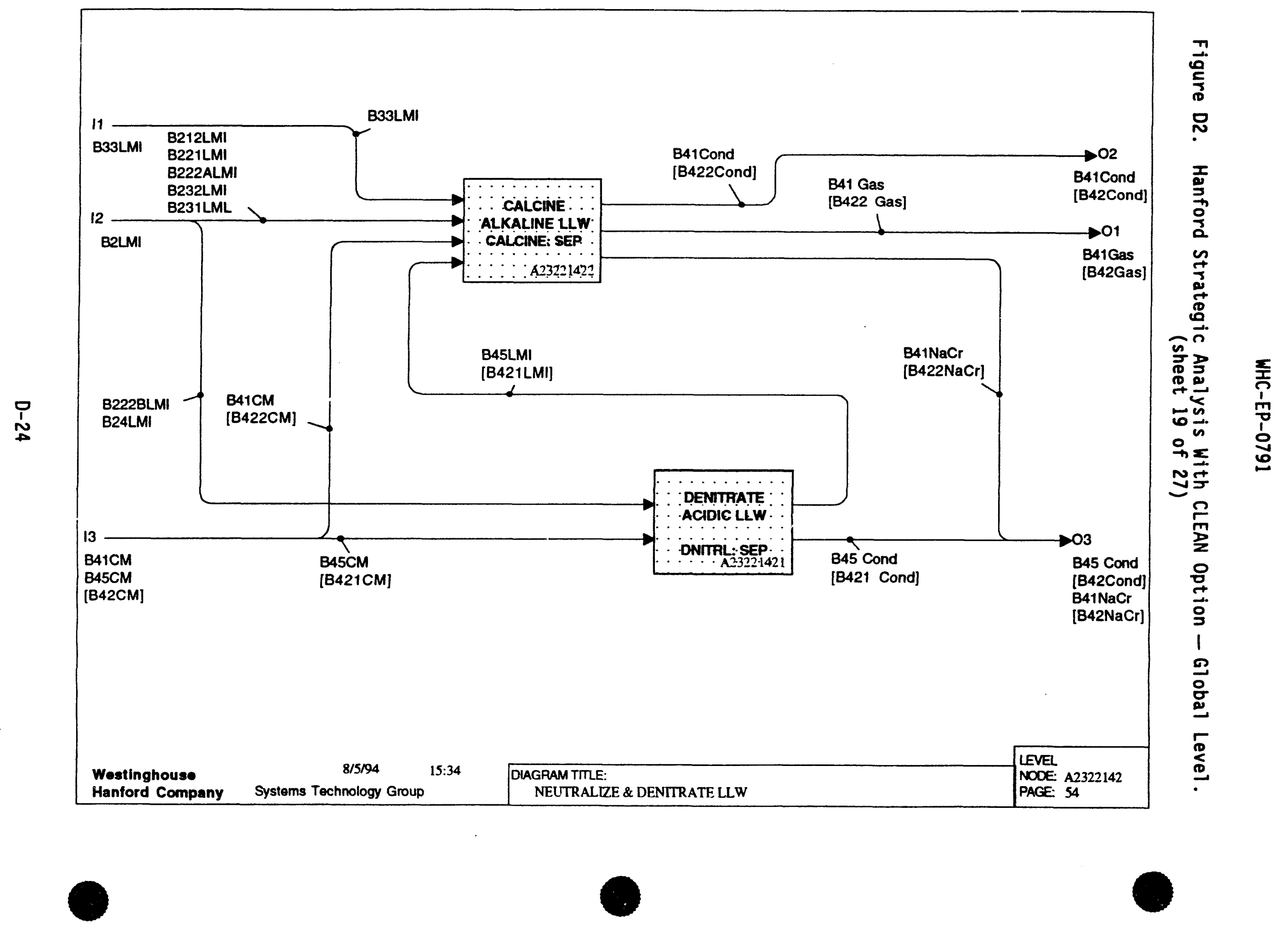




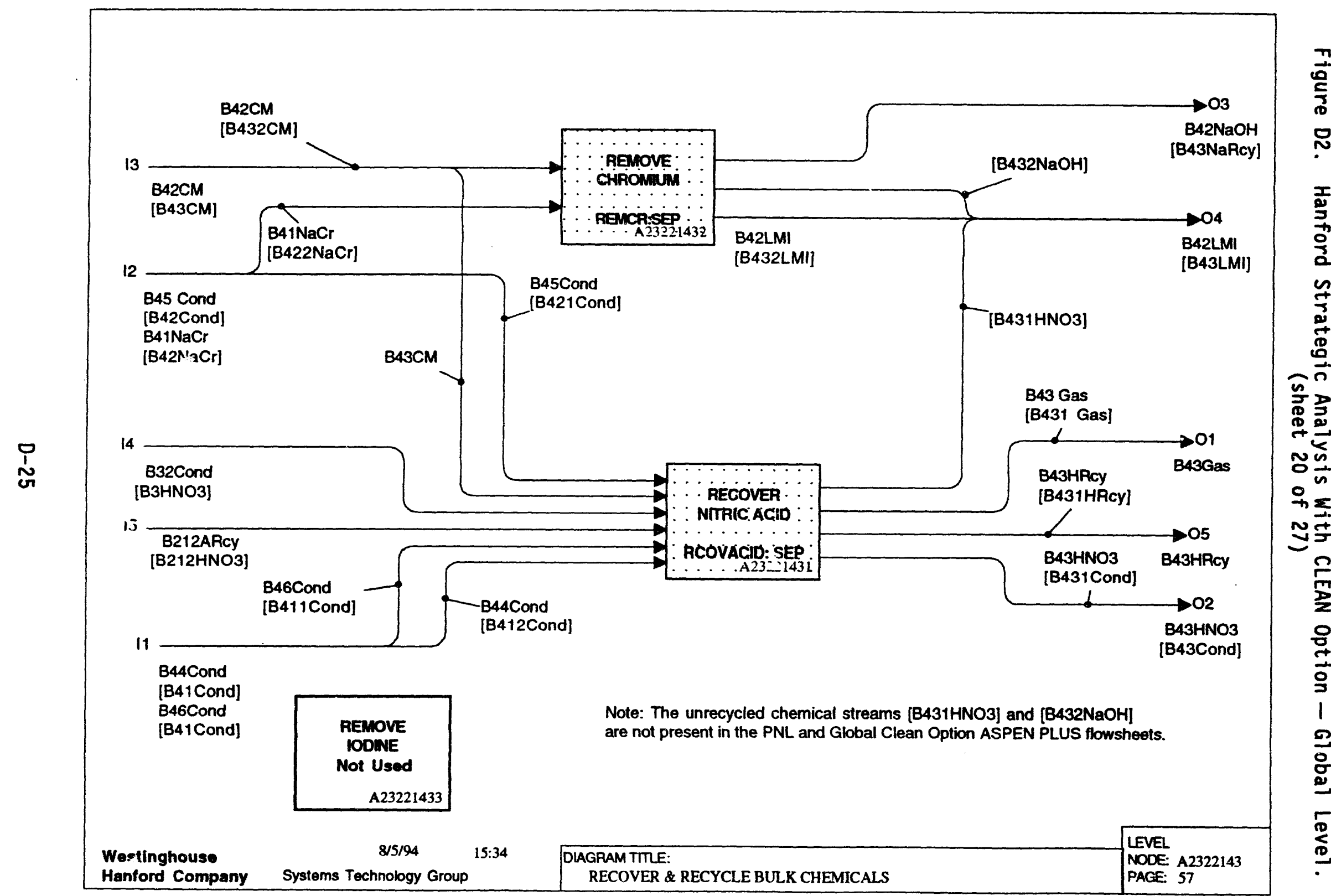




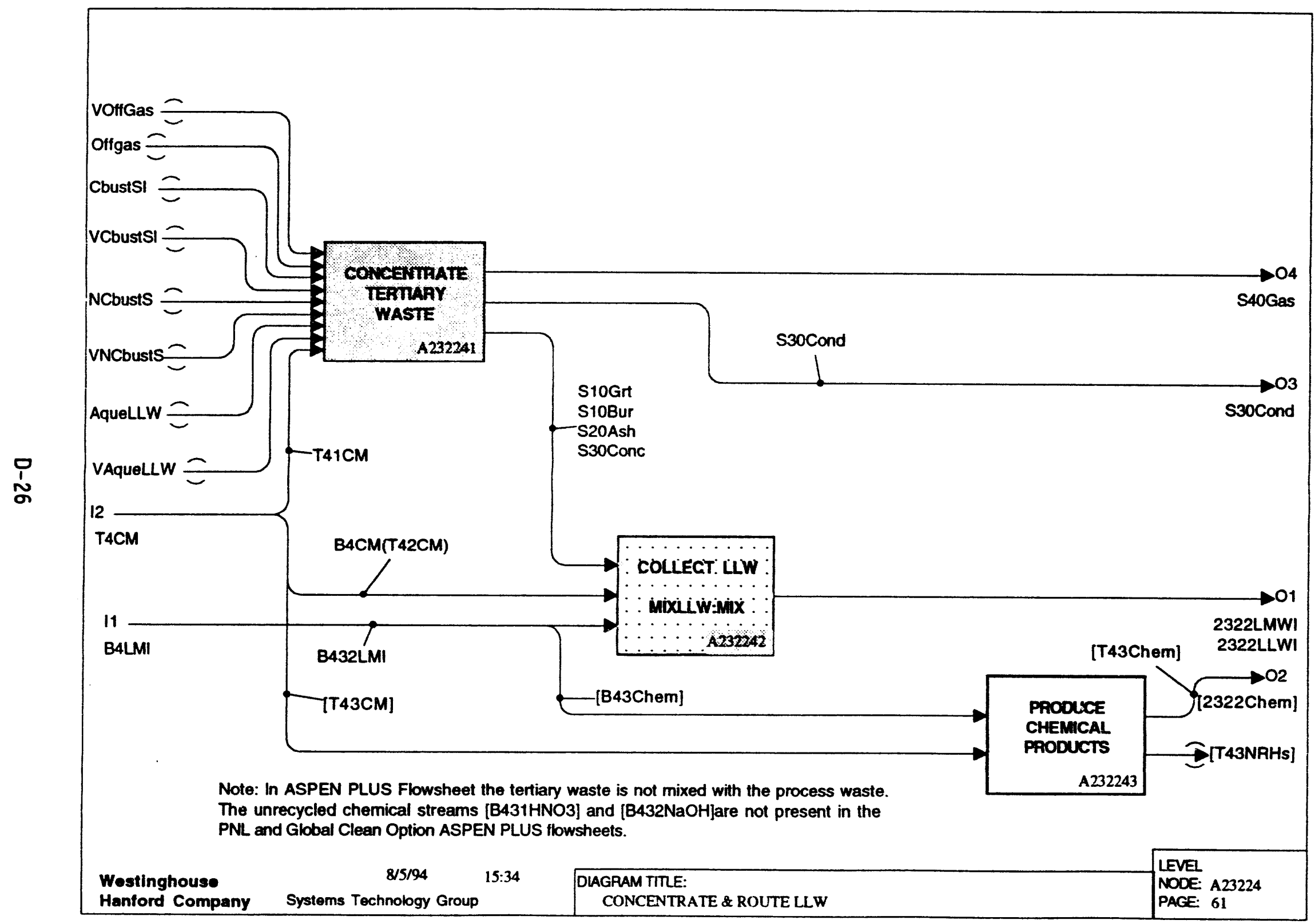

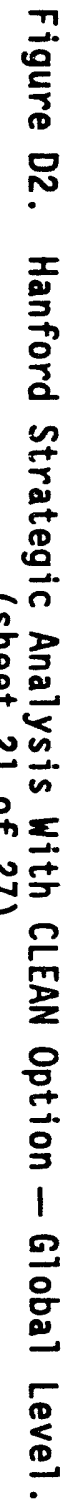




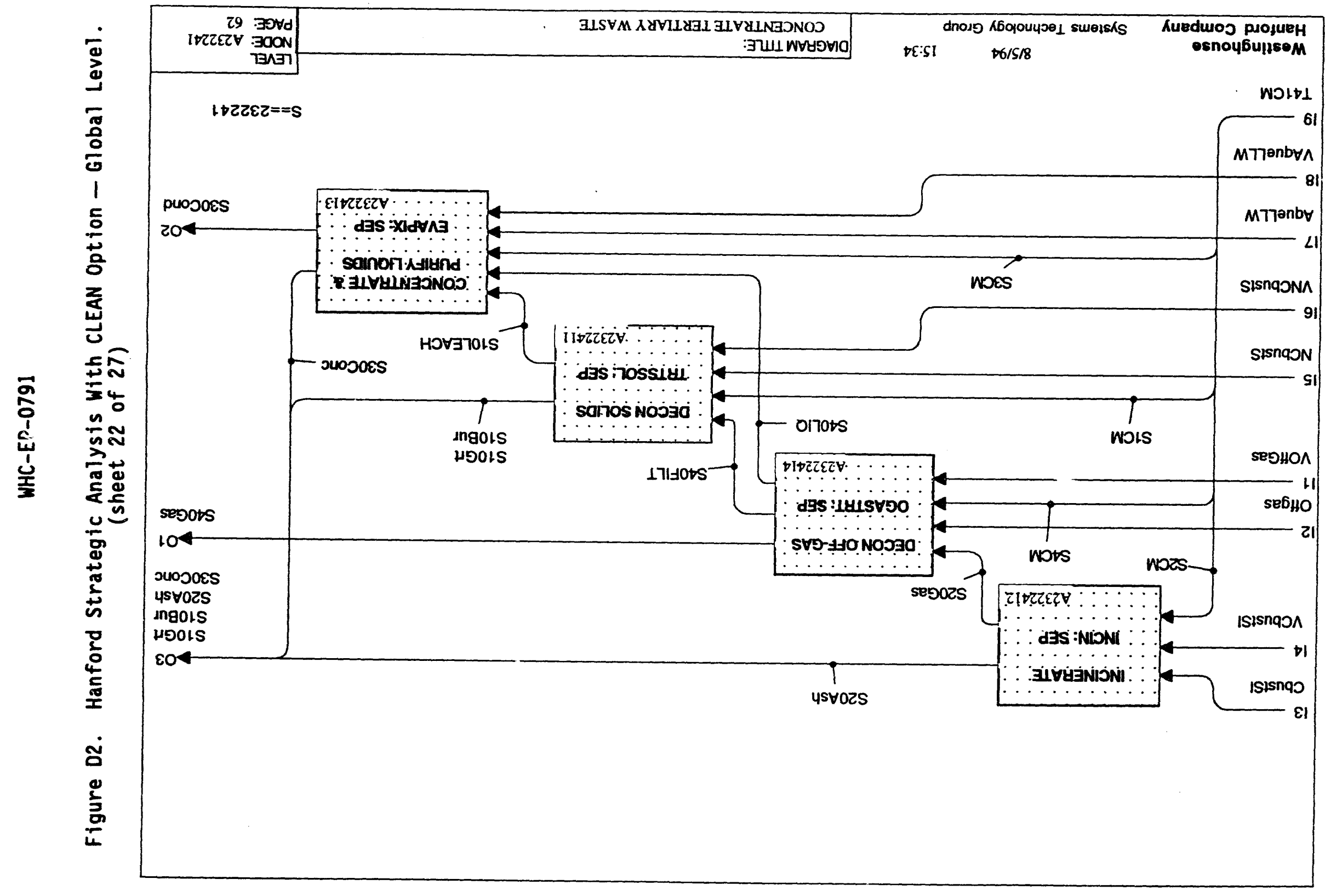




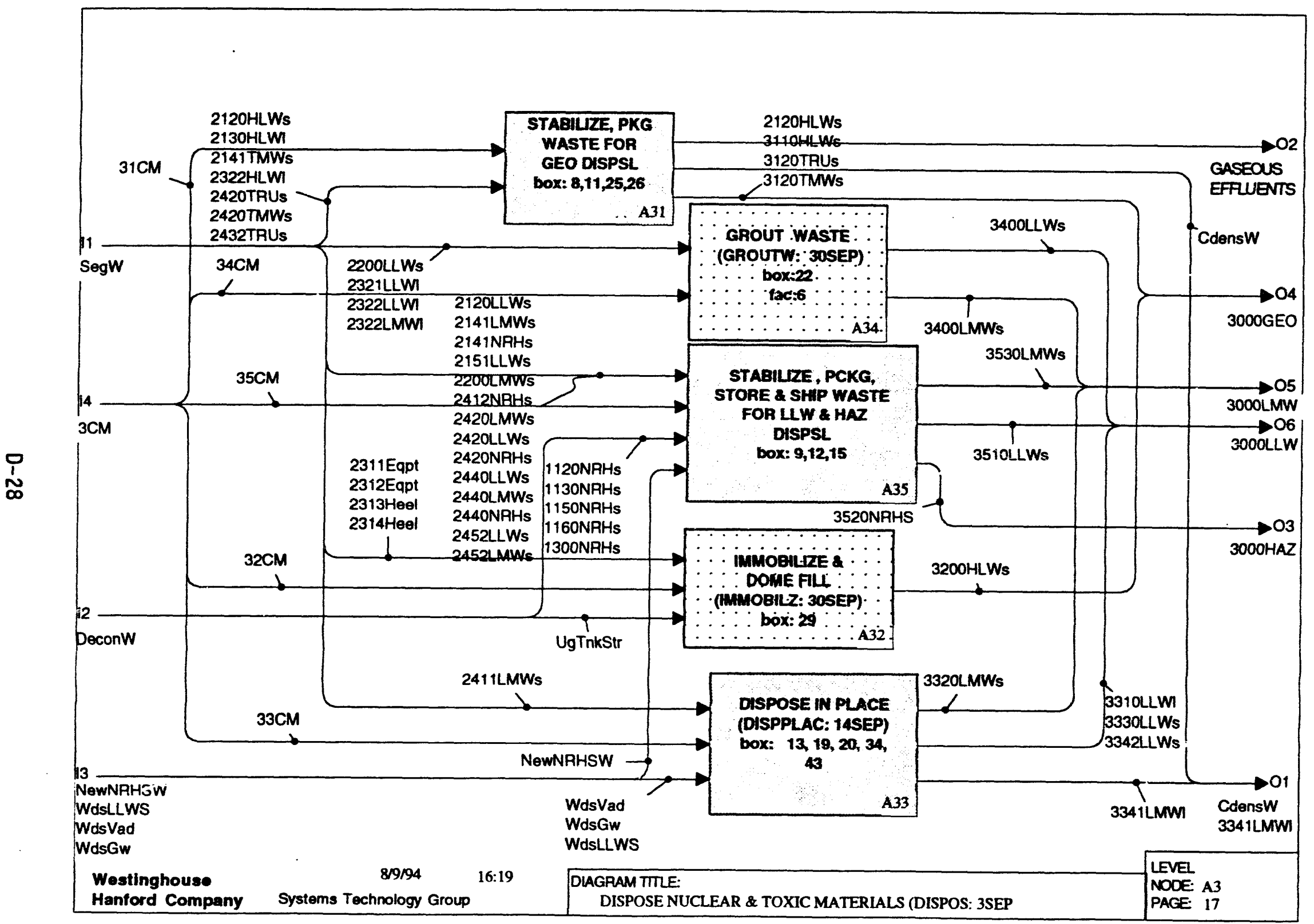




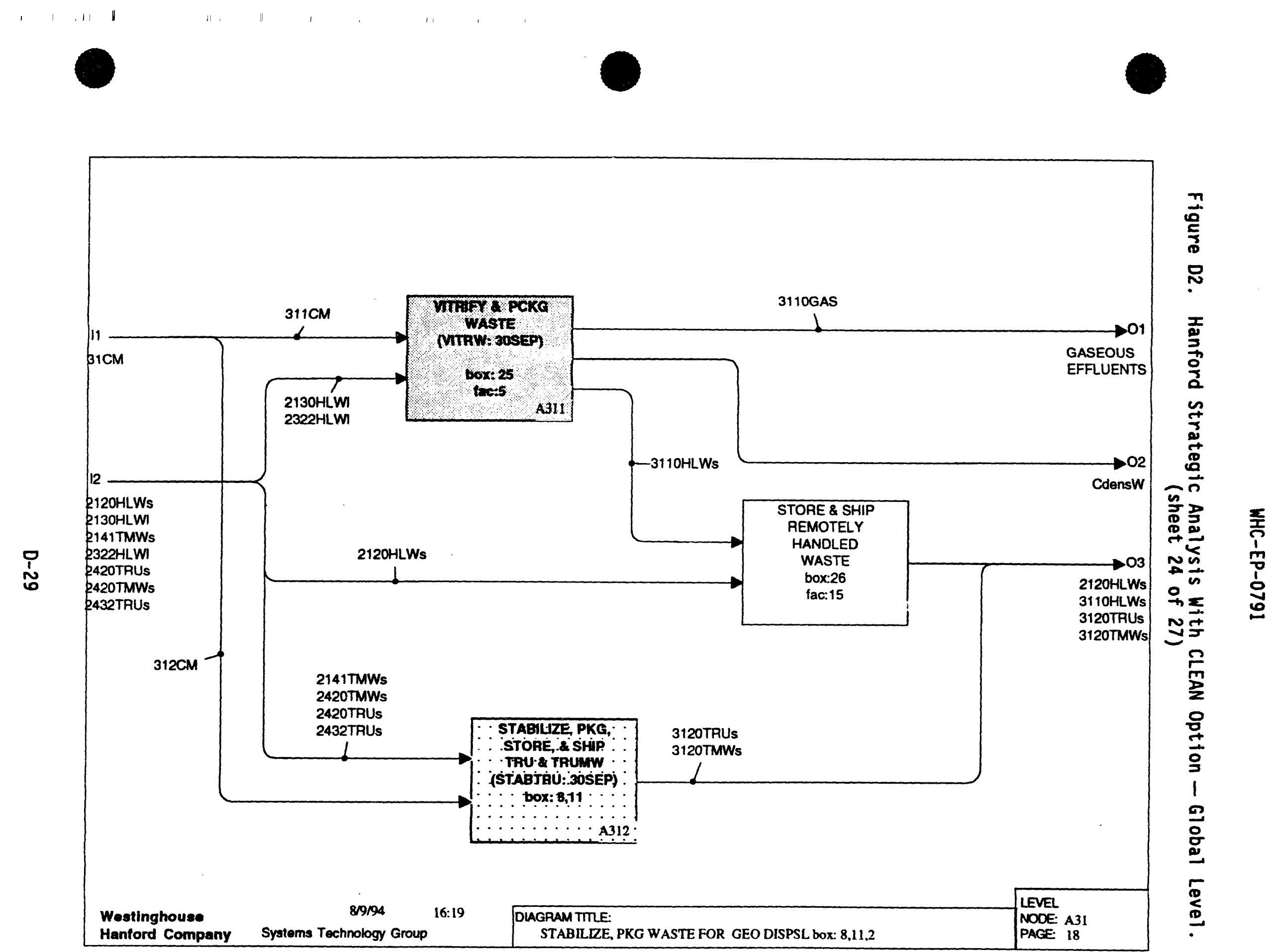




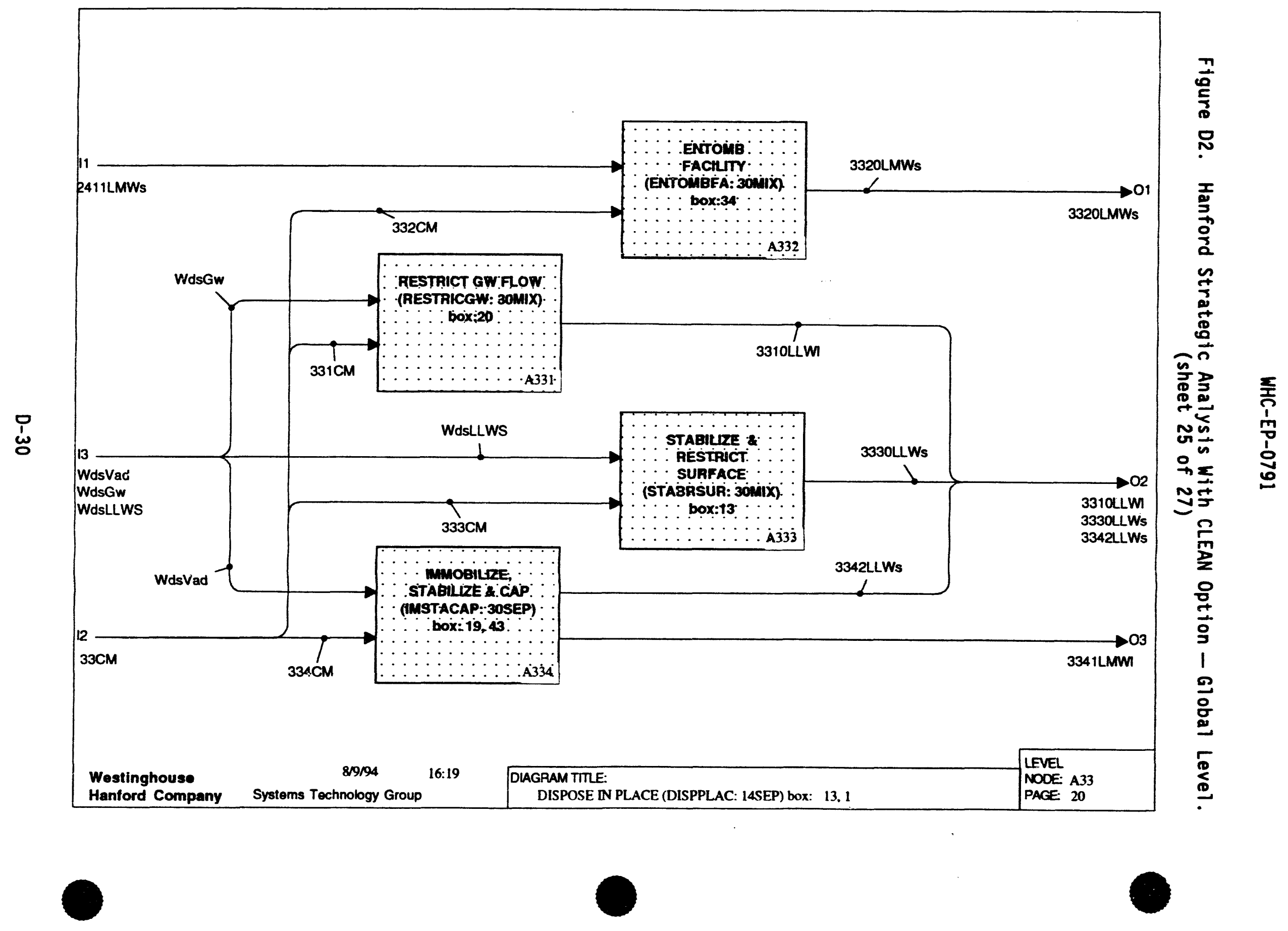




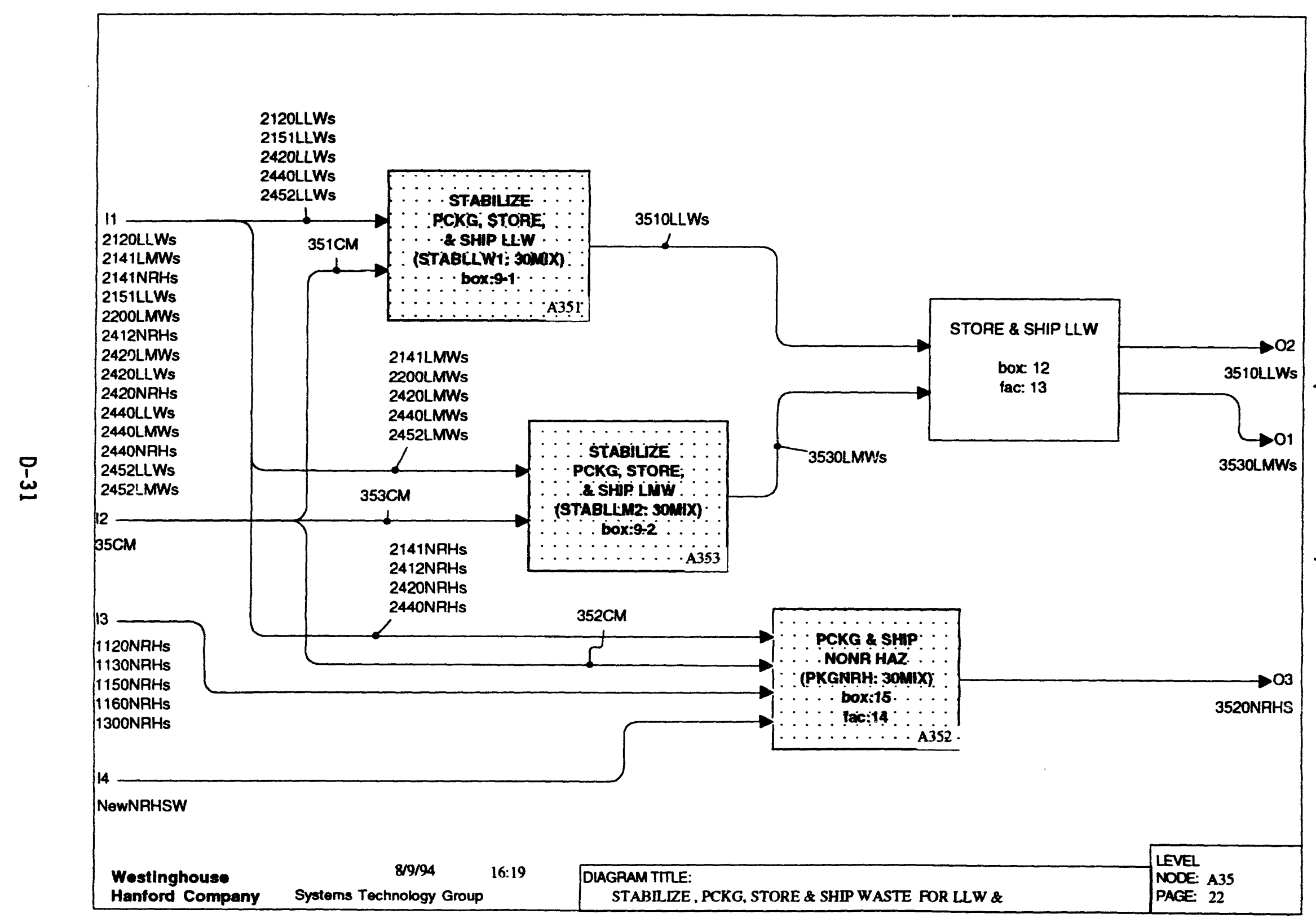




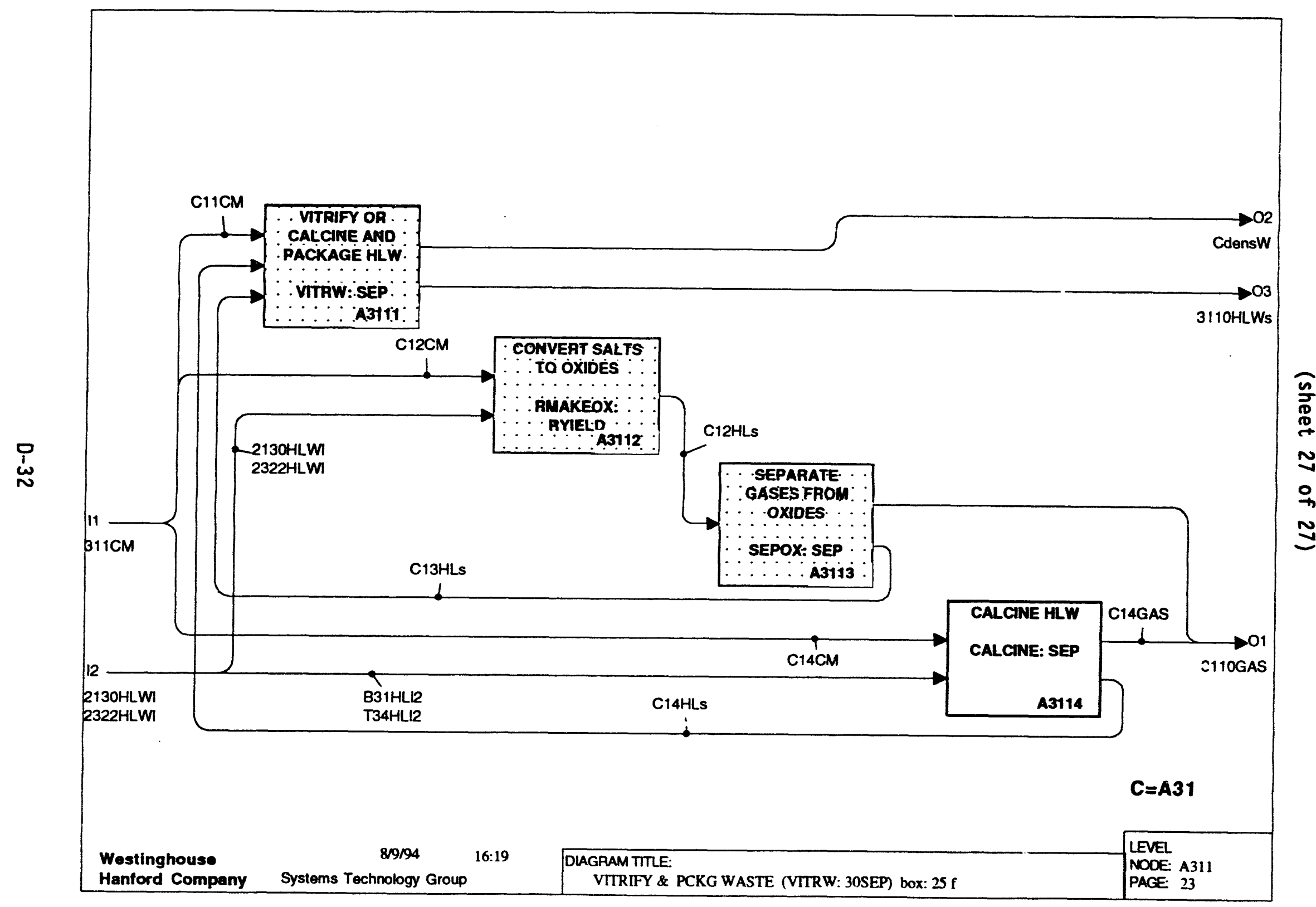

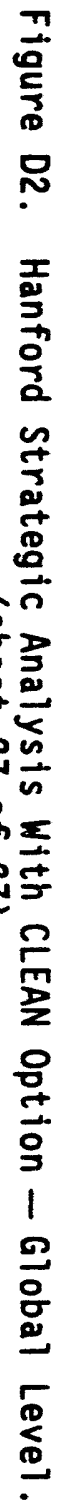




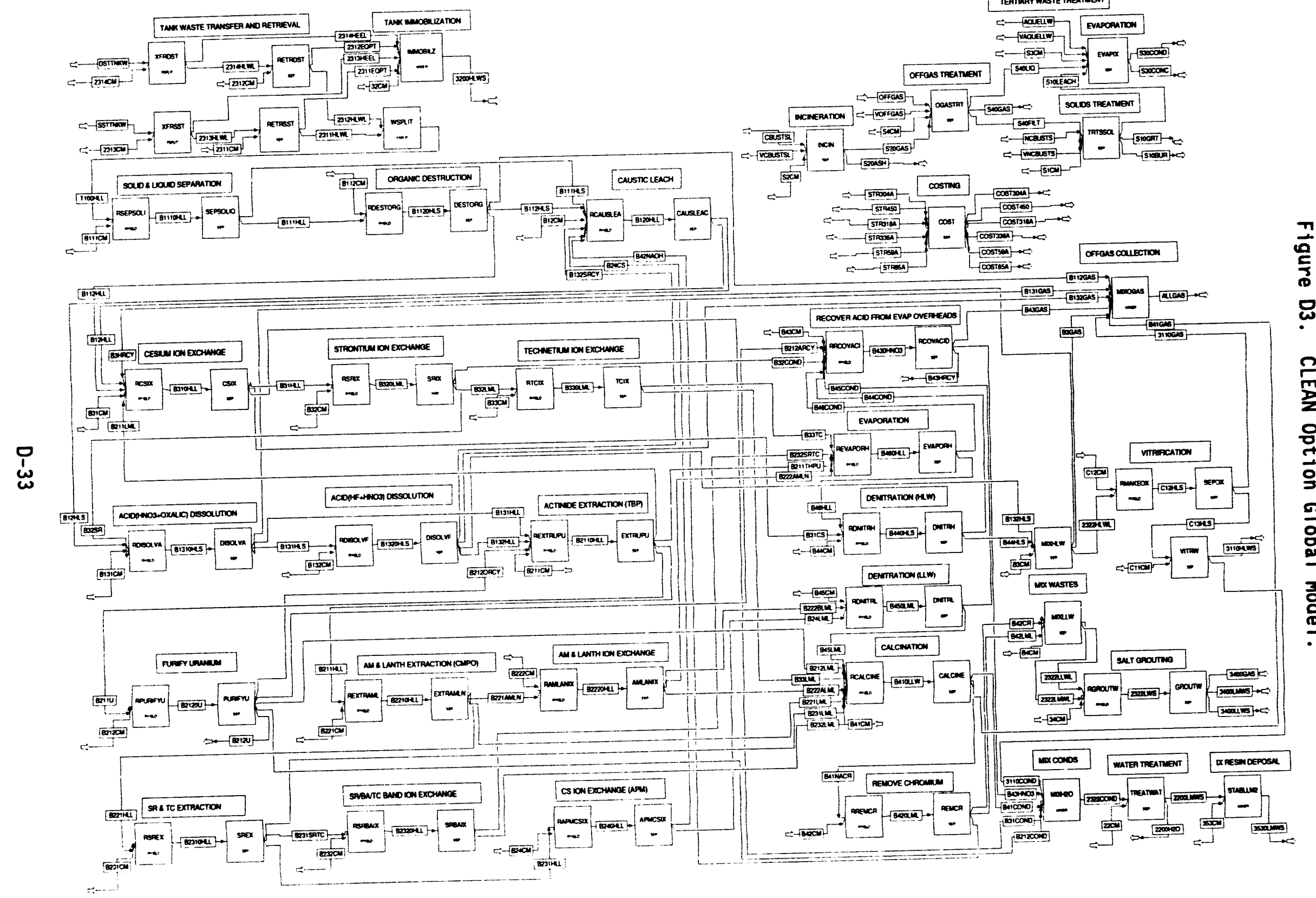


Figure D4. Transuranic Extraction (TRUEX)/Sludge Wash Global Model.

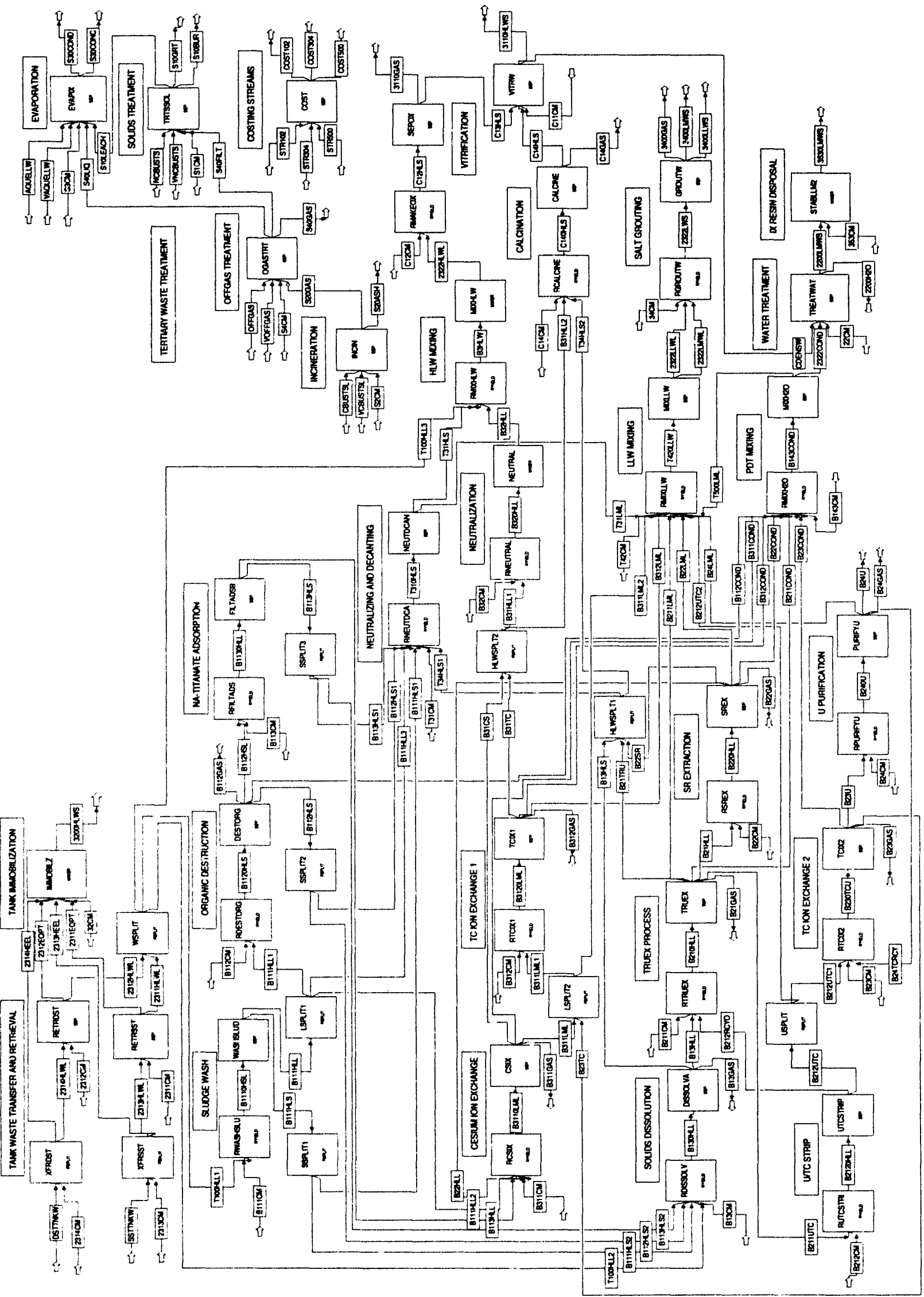


Table D1. ASPEN PLUS Intermediate CLEAN Option Model Stream Naming and Process Block Descriptions. (sheet 1 of 8 )

\section{CLEAN OPTION STREAM NAMING CONVENTIONS:}

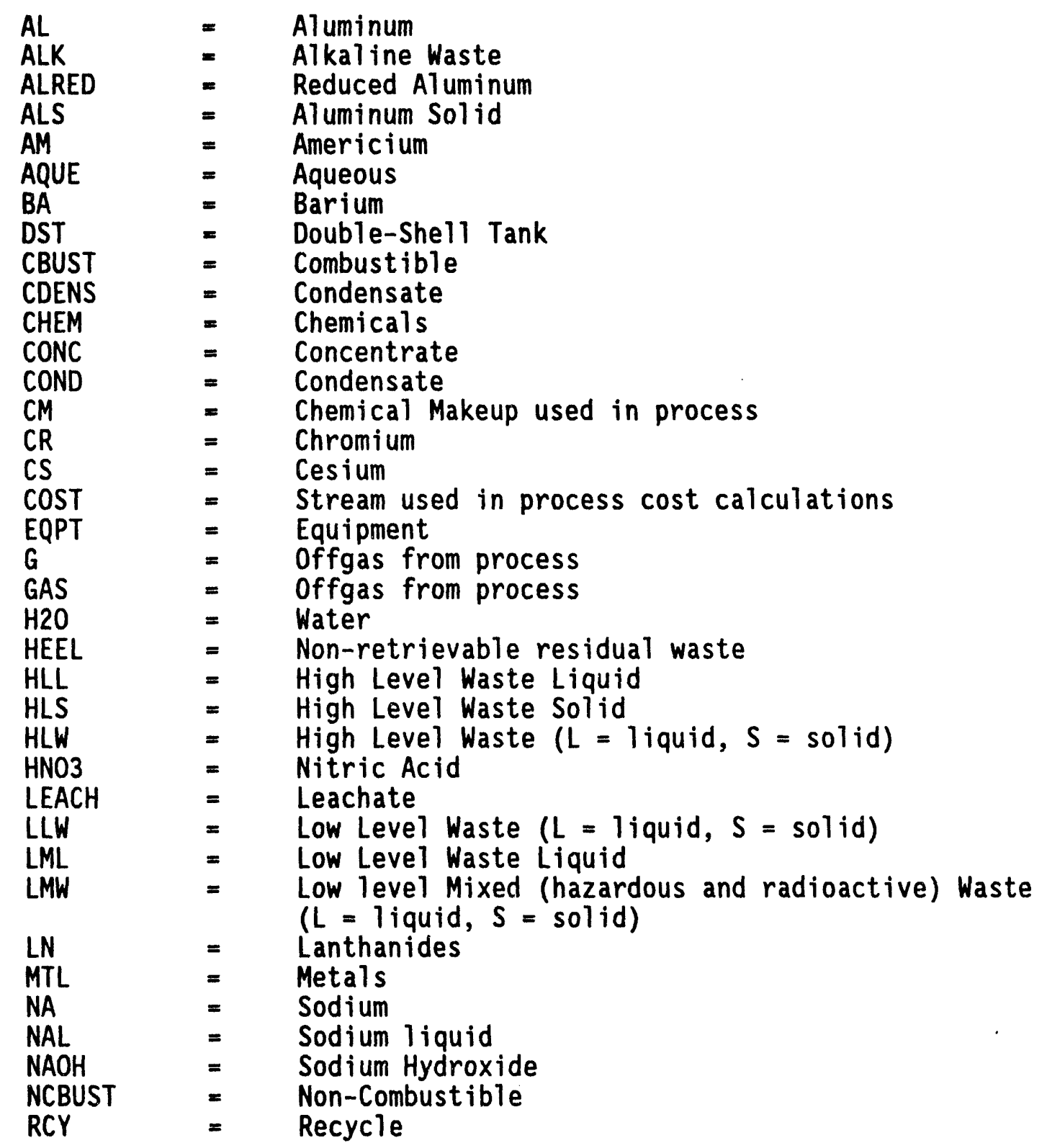


Table D1. ASPEN PLUS Intermediate CLEAN Option Model Stream Naming and Process Block Descriptions. (sheet 2 of 8 )

\section{CLEAN OPTION STREAN MAMING CONVENTIONS (continued):}

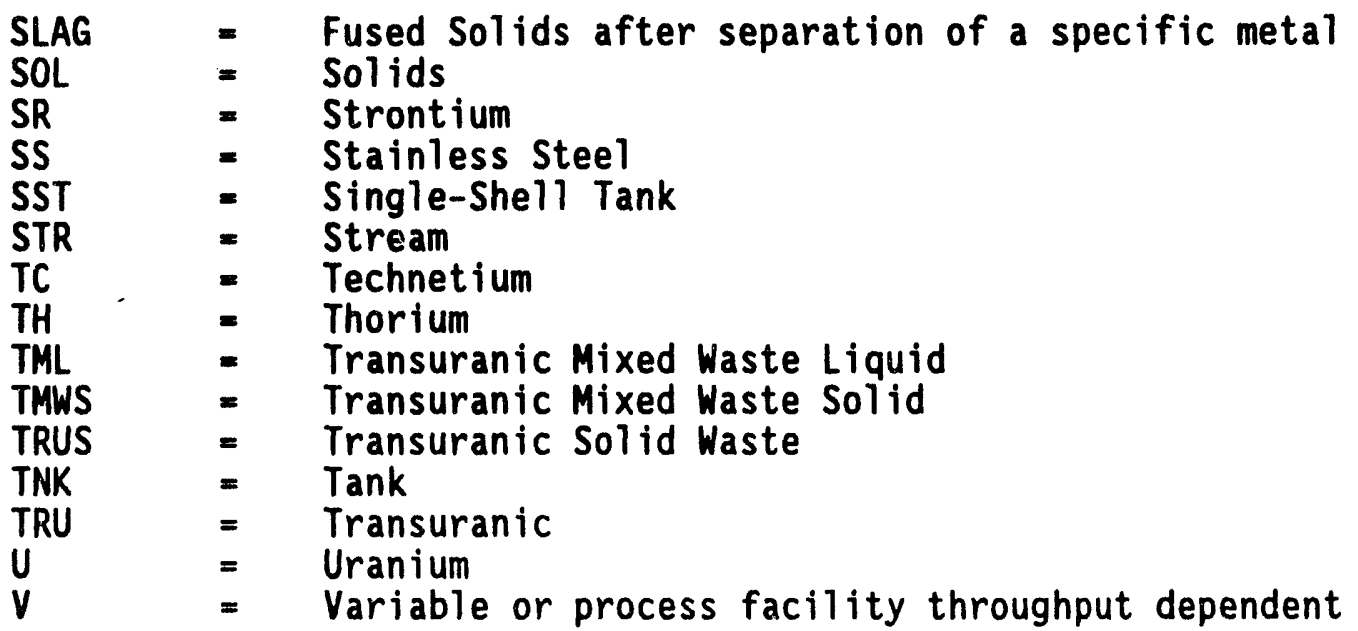


Table D1. ASPEN PLUS Intermediate CLEAN Option Model Stream Naming and Process BTock Descriptions. (sheet 3 of 8 )

\section{CLEAN OPTION BLOCK DESCRIPTIONS:}

XFRSST = Transfer SST waste from tanks using additional H2O, producing a HLW 1 iquid stream and a residual or heel HLW stream.

XFRDST = Transfer DST waste from tanks using additional $\mathrm{H} 20$ producing a HLW 1 iquid stream and a residual or heel HLW stream.

RETRSST = Retrieve SST waste from interim storage tanks producing a HLW liquid stream and a residual contaminated equipment HLW waste stream.

RETRDST = Retrieve DST waste from interim storage tanks producing a HLW liquid stream and a residual contaminated equipment $H L W$ waste stream.

WSPLIT = A stream splitter for routing the primary waste streams along optional processing steps (if needed).

SEPSOLIQ = Wash the SST and DST waste sludge streams with $\mathrm{H} 2 \mathrm{O}$ and Polyelectrolyte and separate the solid and liquid using either filtration or centrifugation to produce a $H(W$ liquid (HLL) supernate stream and a HLW solids (HLS) stream.

DESTORG = Perform organic complexant destruction using Super Critical Water Oxidation (SCWO) to precipitate TRU, fission products, and cations in the alkaline solution.

CAUSLEAC $=$ Dissolve selected solids by caustic leaching and separate the solid and liquid using either filtration or centrifugation to produce HLL and HLS waste streams.

CSIX $=$ Perform CS Ion Exchange and evaporation of the HLW Tiquid supernate to produce a LML concentrated supernate stream, a HLW stream containing mostly $C S$ and a condensate stream.

SRIX $=$ Perform Sr Ion Exchange and evaporation of the HLW Iiquid supernate to produce a LML concentrated supernate stream, a HLW stream containing mostly $\mathrm{Sr}$ and a condensate stream.

TCIX $=$ Perform TC Ion Exchange of the LML stream from the SRIX process to produce a LML stream and a concentrated HLW stream containing mostly Tc. 
Table D1. ASPEN PLUS Intermediate CLEAN Option Mode1 Stream Naming and Process Block Descriptions. (sheet 4 of 8 )

\section{CLEAN OPTION BLOCK DESCRIPTIONS Continued:}

DISOLVA = Dissolve the HLW solids using mixed nitric-oxalic acids to produce a dissolved HLW solids stream, an undissolved $H L W$ solids stream, and an offgas stream.

DISOLVF = Dissolve the HLW solids using mixed nitric-hydrofluoric acids to produce a dissolved HLW solids stream, an undissolved HLW solids stream, an offgas stream, and a sludge waste recycle stream returning to CAUSLEAC.

EXTRUPU $=$ Perform Actinide Extraction using TBP to extract tetravalent and hexavalent actinide and lanthanide elements from acidic waste streams to produce an actinide stream, an offgas stream, a $U$ rich stream, and a LML stream.

PURIFYU $=$ Perform concentration of $U$ rich stream from EXTRUPU to produce a $U$ product stream, a LML stream, an acid recycle stream, an organic recycle stream, and a condensate stream.

EXTRAMLN = Perform Actinide Extraction using CMPO to extract trivalent actinide and lanthanide elements and separate them from Bismuth in the acidic waste streams to produce an actinide/lanthanide stream, a HLW stream, and a LML stream.

AMLANIX = Perform Americium/Lanthanide Ion Exchange on the respective EXTRAMLN actinide/lanthanide stream to produce two LML streams, one directly calcinable and the other requiring denitration, and a concentrated $\mathrm{HLW}$ actinide/lanthanide stream.

SREX = Perform Strontium Extraction using crown ether solvent to extract $\mathrm{Sr}$ and $\mathrm{Tc}$ from acidic waste streams to produce a concentrated Sr/Tc stream, a HLW stream, and a LML siream.

SRBAIX = Perform band displacement cation exchange to extract the Sr and $\mathrm{Ba}$ to produce a concentrated $\mathrm{Sr} / \mathrm{Ba}$ and Tc stream and a LML stream.

APMCSIX = Perform CS Ion Exchange of the concentrated Sr/Tc stream from the SREX process to produce a CS rich stream and a LML stream. 
Table 01. ASPEN PLUS Intermediate CLEAN Option Model Stream Naming and Process Block Descriptions. (sheet 5 of 8 )

\section{CLEAN OPTION BLOCK DESCRIPTIONS Continued:}

RCOVACID $=$ Recover nitric acid from evaporator overheads and process gaseous NOX streams from denitration to produce a $\mathrm{HNO}$ product stream, an offgas stream, and water recycle stream.

EVAPORH - Evaporation of various HLW streams produced from acidic and alkaline side processing to produce a HLW Iiquid stream and an $\mathrm{HNO}_{3}$ stream.

DNITRH = Perform denitration of HLW waste streams to produce a HLW solid stream and a gaseous NOx stream.

DNITRL = Perform denitration of LML waste streams to produce a LML stream and a gaseous NOx stream.

CALCINE = Perform calcination of LML waste streams to destroy nitrates and nitrites and produce a caustic slurry stream, an offgas stream, and a water recycle stream.

REMCR = Remove $\mathrm{Cr}$ from LML waste streams to produce a $\mathrm{NaOH}$ recycle stream, a LML stream, and a Cr rich LML stream.

MIXLLW = Separate LLW streams into HSA higher level model streams.

MIXHLW = Combine HLW streams into HSA higher level model stream.

MIXH2O = Combine condensate streams into HSA higher level model stream.

MIXOGAS = Combine offgas streams into HSA higher level model stream.

IMMOBILZ = Immobilize the HLW contaminated tank and equipment using a dome filling technique.

STABTRU = Stabilize the TMW and TRU solids in waste packages for shipment to a TRU waste isolation repository.

STABLLM2 = Stabilize the LMW solids using an in-situ grouting technique.

TREATWAT = Treat condensate waste liquid to produce a recycle $\mathrm{H} 2 \mathrm{O}$ stream and LLW and LMW streams for grouting.

GROUTW = Combine LLW solids in a salt grout for vault storage.

VITRW = Combine HLW solids with glass formers and vitrify mixture into glass logs for storage in an offsite HLW repository. 
Table D1. ASPEN PLUS Intermediate CLEAN Option Model Stream Naming and Process Block Descriptions. (sheet 6 of 8 )

\section{CLEAN OPTION BLOCK DESCRIPTIONS Continued:}

INCIN = Incinerate combustible secondary wastes produced from SST waste treatment processes to produce a waste ash stream and an offgas stream requiring scrub treatment.

TRTSSOL = Treat noncombustible secondary waste solids to produce a groutable waste stream, a landfill qualified solid waste stream for burial, and a leachate stream.

OGASTRT = Treat waste-processing and cell-air offgasses producing contaminated filters, releasable offgases, and a condensate stream.

EVAPIX = Use evaporation and ion exchange processes on secondary waste aqueous streams to remove excess liquid and selected waste elements, respectively.

RSEPSOLI = Account for waste stream component reactions or mass transfer between components within the SEPSOLIQ process.

RDESTORG = Account for waste stream component reactions or mass transfer between components within the DESTORG process.

RCAUSLEA = Account for waste stream component reactions or mass transfer between components within the CAUSLEAC process.

RCSIX $=$ Account for waste stream component reactions or mass transfer between components within the CSIX process.

RSRIX = Account for waste stream component reactions or mass transfer between components within the SRIX process.

RTCIX $=$ Account for waste stream component reactions or mass transfer between components within the TCIX process.

RDISOLVA = Account for waste stream component reactions or mass transfer between components within the DISOLVA process.

RDISOLVF = Account for waste stream component reactions or mass transfer between components within the DISOLVF process.

REXTRUPU = Account for waste stream component reactions or mass transfer between components within the EXTRUPU process. 
Table D1. ASPEN PLUS Intermediate CLEAN Option Model Stream Naming and Process Block Descriptions. (sheet 7 of 8 )

\section{CLEAN OPTION BLOCK DESCRIPTIONS Continued:}

\begin{tabular}{|c|c|c|}
\hline RPURIFYU & $=$ & $\begin{array}{l}\text { Account for waste stream component reactions or mass } \\
\text { transfer between components within the PURIFYU process. }\end{array}$ \\
\hline REXTRAML & $=$ & $\begin{array}{l}\text { Account for waste stream component reactions or mass } \\
\text { transfer between components within the EXTRAMLN process. }\end{array}$ \\
\hline RAMLANIX & $=$ & $\begin{array}{l}\text { Account for waste stream component reactions or mass } \\
\text { transfer between components within the AMLANIX process. }\end{array}$ \\
\hline RSREX & $=$ & $\begin{array}{l}\text { Account for waste stream component reactions or mass } \\
\text { transfer between components within the SREX process. }\end{array}$ \\
\hline RSRBAIX & $=$ & $\begin{array}{l}\text { Account for waste stream component reactions or mass } \\
\text { transfer between components within the SRBAIX process. }\end{array}$ \\
\hline RAPMCSIX & $=$ & $\begin{array}{l}\text { Account for waste stream component reactions or mass } \\
\text { transfer between components within the APMCSIX process. }\end{array}$ \\
\hline RRCOVACID & $=$ & $\begin{array}{l}\text { Account for waste stream component reactions or mass } \\
\text { transfer between components within the RCOVACID process. }\end{array}$ \\
\hline RNEUTRAL & $=$ & $\begin{array}{l}\text { Account for waste stream component reactions or mass } \\
\text { transfer between components within the NEUTRAL process. }\end{array}$ \\
\hline RDNITRH & $=$ & $\begin{array}{l}\text { Account for waste stream component reactions or mass } \\
\text { transfer between components within the DNITRH process. }\end{array}$ \\
\hline RDNITRL & $=$ & $\begin{array}{l}\text { Account for waste stream component reactions or mass } \\
\text { transfer between components within the DNITRL process. }\end{array}$ \\
\hline RCALCINE & $=$ & $\begin{array}{l}\text { Account for waste stream component reactions or mass } \\
\text { transfer between components within the CALCINE process. }\end{array}$ \\
\hline RREMCR & $=$ & $\begin{array}{l}\text { Account for waste stream component reactions or mass } \\
\text { transfer between components within the REMCR process. }\end{array}$ \\
\hline RMIXLLW & $=$ & $\begin{array}{l}\text { Account for waste stream component reactions or mass } \\
\text { transfer between components within the LLW stream mixing } \\
\text { process. }\end{array}$ \\
\hline RMIXHLW & $=$ & $\begin{array}{l}\text { Account for waste stream component reactions or mass } \\
\text { transfer between components within the HLW stream mixing } \\
\text { process. }\end{array}$ \\
\hline
\end{tabular}


Table D1. ASPEN PLUS Intermediate CLEAN Option Model Stream Naming and Process Block Descriptions. (sheet 8 of 8 )

CLEAN OPTION BLOCK DESCRIPTIONS Continued:

RMIXH2O = Account for waste stream component reactions or mass transfer between components within the $\mathrm{H} 2 \mathrm{O}$ stream mixing process.

RGROUTW = Account for waste stream component reactions or mass transfer between components within the GROUTW process.

RMAKEOX = Account for waste stream component reactions or mass transfer between components within the HLW vitrification process.

SEPOX $=$ Separate the oxide stream from the offgas stream. 
Table D2. ASPEN PLUS Intermediate TRUEX-C/Sludge Wash C Model Stream Naming and Process Block Descriptions. (sheet 1 of 6 )

TRUEX-C/SLUDGE MASH C STREAN NAMING CONVENTIONS:

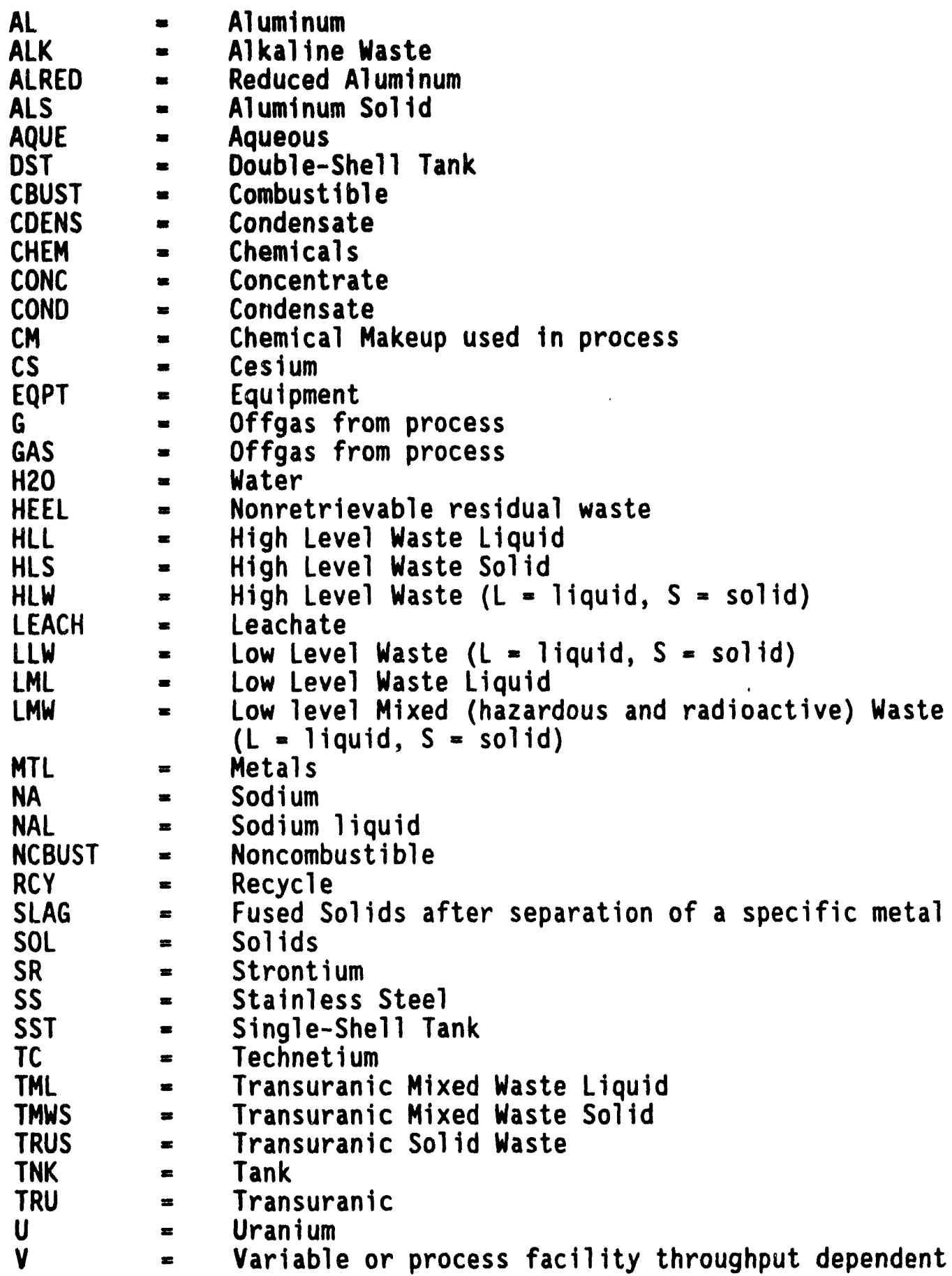


Table D2. ASPEN PLUS Intermediate TRUEX-C/S1udge Wash C Model Stream Naming and Process Block Descriptions. (sheet 2 of 6 )

TRUEX-C/SLUDGE WASH C BLOCK DESCRIPTIONS:

XFRSST $\quad=\quad$ Transfer SST waste from tanks using additional H2O, producing a HLW liquid stream and a residual or heel HLW stream.

XFRDST = Transfer DST waste from tanks using additional $\mathrm{H} 20$ producing producing a HLW 1 iquid stream and a residual or heel HLW stream.

RETRSST - Retrieve SST waste from interim storage tanks producing a HLW liquid stream and a residual contaminated equipment HLW waste stream.

RETRDST - Retrieve DST waste from interim storage tanks producing a HLW I lquid stream and a residual contaminated equipment HLW waste stream.

WASHSLUD = Wash the SST waste sludge stream with $\mathrm{H} 2 \mathrm{O}$ and Polyelectrolyte to produce a HLW liquid supernate stream and a HLW solids stream.

DESTORG - Perform organic complexant destruction using Super Critical Water oxidation (SCWO) to precipitate TRU, fission products, and cations in the alkaline solution.

FILTADSB - Perform sodium titanate sorption to adsorb residual Sr and TRU from the SCWO effluent.

CSIX $=$ Perform CS Ion Exchange and evaporation of the HLW liquid supernate from the FILTADSB process to produce a LML concentrated supernate stream, a HLW stream containing mostly $\mathrm{Cs}$, an offgas stream, and a condensate stream.

TCIXI = Perform Tc Ion Exchange of the LML stream from the CSIX process to produce a LML stream, a condensate stream, and a concentrated HLW stream containing mostly TC.

DISSOLVA = Qissolve the HLW solids from the WASHSLUD process using additional $\mathrm{H} 2 \mathrm{O}, \mathrm{HNO3}$, and Polyelectrolyte, to produce a dissolved HLW solids stream, an undissolved HLW solids stream, and an offgas stream.

TRUEX $\quad=$ Perform Transuranic Extraction to extract actinide and lanthanide elements from acidic waste streams to produce a U/TC concentrated stream, a TRU stream, an offgas stream, and a condensate stream. 
Table D2. ASPEN PLUS Intermediate TRUEX-C/STudge Wash C Model Stream Naming and Process Block Descriptions. (sheet 3 of 6 )

\section{TRUEX-C/SLUDGE MASH C BLOCK DESCRIPTIONS Continued:}

SREX $\quad$ - Perform Strontium Extraction to extract Sr from acidic waste streams to produce a concentrated Sr stream, a recycle HLW stream, an LML stream, an offgas stream, and a condensate stream.

UTCSTRIP = Perform strip of U/TC from HLW stream to produce a concentrated U/TC stream and an organic solvent recycle stream.

TCIX2 = Perform TC Ion Exchange of the U/TC rich stream from the UTCSTRIP process to produce concentrated $U$ and TC streams, a condensate stream, and an offgas stream.

PURIFYU - Perform concentration of $U$ rich stream from TCIX2 to produce a $U$ product stream, a LML stream, a Tc recycle stream, and an offgas stream.

NEUTRAL = Neutralize the concentrated CS and TC HLW streams produced from alkaline side processing to produce a HLW liquid stream.

NEUTDCAN = Neutralize and Decant the HLW streams resulting from acid side processing to produce a HLW Iiquid stream.

CALCINE = Perform calcination of acidic HLW waste streams to produce a HLW solid (oxide) stream and an offgas stream.

SSPLIT\# = A stream splitter for routing the solid waste streams along optional processing steps.

LSPLIT\# = A stream splitter for routing the liquid waste streams along optional processing steps.

WSPLIT = A stream splitter for routing the primary waste streams along optional processing steps.

USPLIT = A stream splitter for routing the U/TC waste stream from process UTCSTRIP along optional processing steps.

HLWSPLT1 = A stream splitter for routing the HLW streams from acid side processing to neutralization or calcination process steps.

HLWSPLT2 = A stream splitter for routing the HLW streams from alkaline side processing to neutralization or calcination process steps. 
Table D2. ASPEN PLUS Intermediate TRUEX-C/Sludge Wash C Model Stream Naming and Process Block Descriptions. (sheet 4 of 6 )

\section{TRUEX-C/SLUDGE WASH C BLOCK DESCRIPTIONS ContinUed:}

MIXLLW - Separate LLW streams into HSA higher level model streams.

MIXHLW = Combine HLW streams into HSA higher level model stream.

MIXH2O = Combine condensate streams into HSA higher level model stream.

IMMOBILZ - Immobilize the HLW contaminated tank and equipment using a dome filling technique.

STABTRU = Stabilize the TMW and TRU solids in waste packages for shipment to a TRU waste isolation repository.

STABLLM2 = Stabilize the LMW solids using an in-situ grouting technique.

TREATWAT = Treat condensate waste liquid to produce a recycle $\mathrm{H} 20$ stream and LLW and LMW streams for grouting.

GROUTW = Combine LLW solids in a salt grout for vault storage.

VITRW = Combine HLW solids with glass formers and vitrify mixture into glass logs for storage in an offsite $H L W$ repository.

INCIN = Incinerate combustible secondary wastes produced from SST waste treatment processes to produce a waste ash stream and an offgas stream requiring scrub treatment.

TRTSSOL = Treat noncombustible secondary waste solids to produce a groutable waste stream, a landfill qualified solid waste stream for burial, and a leachate stream.

OGASTRT = Treat waste-processing and cell-air offgasses producing contaminated filters, releasable offgases, and a condensate stream.

EVAPIX = Use evaporation and ion exchange processes on secondary waste aqueous streams to remove excess liquid and selected waste elements, respectively.

RWASHSLU $=$ Account for waste stream component reactions or mass transfer between components within the WASHSLUD process. 
Table D2. ASPEN PLUS Intermediate TRUEX-C/Sludge Wash C Model Stream Naming and Process Block Descriptions. (sheet 5 of 6 )

\section{TRUEX-C/SLUDGE MASH C BLOCK DESCRIPTIONS Continued:}

\begin{tabular}{|c|c|c|}
\hline RDESTORG & $=$ & $\begin{array}{l}\text { Account for waste stream component reactions or mass } \\
\text { transfer between components within the DESTORG process. }\end{array}$ \\
\hline RFILTADS & $=$ & $\begin{array}{l}\text { Account for waste stream component reactions or mass } \\
\text { transfer between components within the FILTADSB process. }\end{array}$ \\
\hline RCSIX & $=$ & $\begin{array}{l}\text { Account for waste stream component reactions or mass } \\
\text { transfer between components within the CSIX process. }\end{array}$ \\
\hline RTCIXI & $=$ & $\begin{array}{l}\text { Account for waste stream component reactions or mass } \\
\text { transfer between components within the TCIXI process. }\end{array}$ \\
\hline RDISSOLV & $=$ & $\begin{array}{l}\text { Account for waste stream component reactions or mass } \\
\text { transfer between components within the DISSOLVA process. }\end{array}$ \\
\hline RTRUEX & $=$ & $\begin{array}{l}\text { Account for waste stream component reactions or mass } \\
\text { transfer between components within the TRUEX process. }\end{array}$ \\
\hline RSREX & $=$ & $\begin{array}{l}\text { Account for waste stream component reactions or mass } \\
\text { transfer between components within the SREX process. }\end{array}$ \\
\hline RUTCSTRI & $=$ & $\begin{array}{l}\text { Account for waste stream component react } \\
\text { transfer between components within the } U\end{array}$ \\
\hline RTCIX2 & $=$ & $\begin{array}{l}\text { Account for waste stream component reactions or mass } \\
\text { transfer between components within the TCIX2 process. }\end{array}$ \\
\hline RPURIFYU & $=$ & $\begin{array}{l}\text { Account for waste stream component reactions or mass } \\
\text { transfer between components within the PURIFYU process. }\end{array}$ \\
\hline RNEUTRAL & $=$ & $\begin{array}{l}\text { Account for waste stream component reactions or mass } \\
\text { transfer between components within the NEUTRAL process. }\end{array}$ \\
\hline RNEUTDCAN & $=$ & $\begin{array}{l}\text { Account for waste stream component reactions or mass } \\
\text { transfer between components within the NEUTDCAN process. }\end{array}$ \\
\hline RPURIFYU & $=$ & $\begin{array}{l}\text { Account for waste stream component reactions or mass } \\
\text { transfer between components within the PURIFYU process. }\end{array}$ \\
\hline RMIXLLW & $=$ & $\begin{array}{l}\text { Account for waste stream component reactions or mass } \\
\text { transfer between components within the LLW stream mixing } \\
\text { process. }\end{array}$ \\
\hline
\end{tabular}

RMIXHLW = Account for waste stream component reactions or mass transfer between components within the HLW stream mixing process. 
Table D2. ASPEN PLUS Intermediate TRUEX-C/Sludge Wash C Mode1 Stream Naming and Process Block Descriptions. (sheet 6 of 6 )

TRUEX-C/SLUDGE HASH C BLOCK DESCRIPTIONS Continued:

RMIXH20 = Account for waste stream component reactions or mass transfer between components within the Hấ stream mixing process.

RGROUTW = Account for waste stream component reactions or mass transfer between components within the GROUTW process.

RCALCINE = Account for waste stream component reactions or mass transfer between components within the CALCINE process.

RMAKEOX = Account for waste stream component reactions or mass transfer between components within the HLW vitrification process.

SEPOX = Separate the oxide stream from the offgas stream. 
Table D3. Stream Relationships: Global Versus Detailed Clean Option Models. (sheet 1 of 6)

GLOBAL MODEL

RSEPSOLI/SEPSOLIQ: (SLUDGE NASH AND SOLIDS AND LIQUID SEPARATION)

FEEDS: SSTTNKW 400

EEEDS: DSTTNKW 401

FEEDS: B111CM 402

PRODUCTS: B111HLL 403

PRODUCTS: BIIIHLS 409
DETAILED MODEL

405408

406

RDESTORG/DESTORG: (ORGANIC DESTRUCTION WITH SCWO AND SOLIDS SEPARATION)

$\begin{array}{lllll}\text { FEEDS: } & \text { B111HLL } & 403 & 406 & \\ \text { FEEDS: } & \text { B112CM } & 422 & 425 & 428 \\ \text { PRODUCTS: } & \text { B112HLL } & 423 & 426 & \\ \text { PRODUCTS: } & \text { B112HLS } & 429 & & \\ \text { PRODUCTS: } & \text { B112GAS } & 411 & & \\ \text { RCAUSLEA/CAUSLEAC: } & \text { (CAUSTIC LEACH) } \\ \text { FEEDS: } & \text { B132SRCY } & 280 & & \\ \text { FEEDS: } & \text { B24CS } & 101 & & \\ \text { FEEDS: } & \text { B42NAOH } & 530 & & \\ \text { FEEDS: } & \text { B111HLS } & 409 & & \\ \text { FEEDS: } & \text { B112HLS } & 429 & & \\ \text { FEEDS: } & \text { B12CM } & 442 & 445 & 448 \\ \text { PRODUCTS: } & \text { B12HLL } & 443 & 446 & \\ \text { PRODUCTS: } & \text { B12HLS } & 449 & & \end{array}$

RCSIX/CSIX: (CESIUM ION EXCHANGE)

\begin{tabular}{lllllll} 
FEEDS: & B3HRCY & 548 & & & & \\
\hline FEEDS: & B112HLL & 423 & 426 & & & \\
FEEDS: & B12HLL & 443 & 446 & & & \\
FEEDS: & B211LML & 18 & & & & \\
FEEDS: & B31CM & 300 & 301 & 302 & 303 & 304 \\
PRODUCTS: B3ICS & 310 & & & & \\
PRODUCTS: & B3IHLL & 305 & & & & \\
PRODUCTS: & B31COND & 451 & & & &
\end{tabular}

RSRIX/SRIX: (STRONTIUM ION EXCHANGE)

FEEDS: B3IHLL 305

$\begin{array}{lllllll}\text { FEEDS: } & \text { B32CM } & 314 & 315 & 316 & 317 & 318\end{array}$

PRODUCTS: B32SR 220

PRODUCTS: B32LML 319

PRODUCTS: B32COND 326 
Table D3. Stream Relationships: Global Versus Detailed Clean Option Models. (sheet 2 of 6 )

STREAN RELATIONSHIPS: GLOBAL VS. DETAILED CLEAN OPTION MODELS CONTINUED

RTCIX/TCIX: (TECHNETIUM ION EXCHANGE)

\begin{tabular}{|c|c|c|c|c|c|c|c|c|c|}
\hline$\frac{\frac{\text { EEEDS: }}{\text { EEEDS: }}}{\text { PRODUCTS; }}$ & $\begin{array}{l}\text { B32LML } \\
\text { B33CM } \\
\text { B33TC } \\
\text { B33LML }\end{array}$ & $\begin{array}{l}319 \\
331 \\
343 \\
350\end{array}$ & 332 & 333 & 334 & 335 & \multicolumn{3}{|l|}{336} \\
\hline \multicolumn{2}{|c|}{ RDISOLVA/DISOLVA: } & (ACID & \multicolumn{3}{|c|}{ (OXALIC+HNO3) } & \multicolumn{2}{|c|}{ DISSOLUTION } & OF & SOLIDS) \\
\hline $\begin{array}{l}\frac{F E E D S:}{\text { FEEDS: }} \\
\frac{\text { FEEDS: }}{\text { PRODUCTS: }} \\
\text { PRODUCTS: } \\
\text { PRODUCTS: }\end{array}$ & $\begin{array}{l}\text { B32SR } \\
\text { B12HLS } \\
\text { B131CM } \\
\text { B131HLL } \\
\text { B131HLS } \\
\text { B131GAS }\end{array}$ & $\begin{array}{l}220 \\
449 \\
210 \\
233 \\
239 \\
218\end{array}$ & $\begin{array}{l}211 \\
236\end{array}$ & 215 & 216 & 232 & 235 & 238 & \\
\hline \multicolumn{2}{|c|}{ RDISOLVF/DISOLVF : } & (ACID & \multicolumn{2}{|c|}{ (HF+HN03) } & \multicolumn{3}{|c|}{ DISSOLUTION OF } & \multicolumn{2}{|c|}{ SOLIDS) } \\
\hline $\begin{array}{l}\text { FEEDS: } \\
\text { FEEDS: } \\
\text { PRODUCTS: } \\
\text { PRODUCTS: } \\
\text { PRODUCTS: } \\
\text { PRODUCTS: }\end{array}$ & $\begin{array}{l}\text { B131HLS } \\
\text { B132CM } \\
\text { B132SRCY } \\
\text { B132HLL } \\
\text { B132HLS } \\
\text { B132GAS }\end{array}$ & $\begin{array}{l}239 \\
250 \\
280 \\
273 \\
281 \\
258\end{array}$ & $\begin{array}{l}251 \\
276\end{array}$ & 255 & 256 & 272 & 275 & 278 & \\
\hline \multicolumn{2}{|c|}{ REXTRUPU/EXTRUPU: } & \multicolumn{2}{|c|}{ (ACTINIDE } & EXTRA & CTION & I USIN & G TBP & & \\
\hline $\begin{array}{l}\frac{\text { FEEDS: }}{\text { FEEDS: }} \\
\frac{\text { FEEDS: }}{\text { FEEDS: }} \\
\text { PRODUCTS: } \\
\text { PRODUCTS: } \\
\text { PRODUCTS: } \\
\text { PRODUCTS: }\end{array}$ & $\begin{array}{l}\text { B131HLL } \\
\text { B132HLL } \\
\text { B2120RCY } \\
\text { B211CM } \\
\text { B211THPU } \\
\text { B211U } \\
\text { B21:1ML } \\
\text { B211HLL }\end{array}$ & $\begin{array}{l}233 \\
273 \\
3 \\
2 \\
11 \\
15 \\
18 \\
7\end{array}$ & $\begin{array}{l}236 \\
276\end{array}$ & 5 & 9 & 13 & 14 & 17 & $6 A$ \\
\hline \multicolumn{2}{|c|}{ RPURIFYU/PURIFYU: } & \multicolumn{5}{|c|}{ (URANIUM PURIFICATION) } & & & \\
\hline $\begin{array}{l}\frac{\text { EEEDS: }}{\text { FEEDS: }} \\
\text { PRODUCTS: } \\
\text { PRODUCTS: } \\
\text { PRODUCTS: } \\
\text { PRODUCTS: } \\
\text { PRODUCTS: }\end{array}$ & $\begin{array}{l}\text { B211U } \\
\text { B212CM } \\
\text { B212LML } \\
\text { B2120RCY } \\
\text { B212ARCY } \\
\text { B212COND } \\
\text { B212U }\end{array}$ & $\begin{array}{l}15 \\
22 \\
34 \\
3 \\
38 \\
37 \\
36\end{array}$ & 23 & 24 & 28 & 33 & & & \\
\hline
\end{tabular}




$$
\text { WHC-EP-0791 }
$$

Table D3. Stream Relationships: Global Versus Detailed Clean Option Models. (sheet 3 of 6 )

STREAN RELATIONSHIPS: GLOBAL VS. DETAILED CLEAN OPTION MODELS CONTINUED

GLOBAL MODEL DETAILED MODEL

REXTRAML/EXTRANLN: (AMERICIUN AND LANTHANIDES EXTRACTION USING CMPO)

EEEDS: B21IHLL 7

EEED: $\quad$ B221CM $\quad 39 \quad 40 \quad 41 \quad 44 \quad 49$

PRODUCTS: B22IAMLN 45

PRODUCTS: B221HLL 42

PRODUCTS: B221LML 51

RAMLANIX/AMLANIX: (AMERICIUM AND LANTHANIDES ION EXCHANGE)

FEEDS: B22IAMLN 45

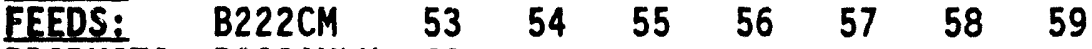

PRODUCTS: B222AMLN 62

PRODUCTS: B222ALML 61

PRODUCTS: B222BLML 60

RSREX/SREX: (STRONTIUM AND TECHNETIUM EXTRACTION)

FEEDS: B221HLL 42

$\begin{array}{llllll}\text { FEEDS: } & \text { B231CM } & 70 & 71 & 74 & 77\end{array}$

PRODUCTS: B231SRTC 75

PRODUCTS: B231HLL 72

PRODUCTS: B23ILML 78

RSRBAIX/SRBAIX: (STRONTIUN, BARIUM, AND TECHNETIUN ION EXCHANGE)

FEEDS: B231SRTC 75

FEEDS: B232CM 80

PRODUCTS: B232SRTC 94

PRODUCTS: B232LML 95

$\begin{array}{lllll}81 & 82 & 83 & 84 & 85\end{array}$

RAPMCSIX/APMCSIX: (CESIUM ION EXCHANGE USING APM)

FEEDS: B231HLL 72

FEEDS: B24CM 9699

PRODUCTS: B24CS 101

PRODUCTS: B24LML 102 
Table D3. Stream Relationships: Global Versus Detailed Clean Option Models. (sheet 4 of 6)

STREAM RELATIONSHIPS: GLOBAL VS. DETAILED CLEAN OPTION MODELS CONTINUED

GLOBAL HODEL DETAILED MODEL

RCALCINE/CALCINE: (WASTE CALCINING)

EEEDS: B33LML 350

FEEDS: B212LML 34

FEEDS: B221LML 51

FEEDS: B222ALML 61

FEEDS: B231LML 78

EEEDS: B232LML 95

EEEDS: B45LML 512

FEEDS: B41CM $506 \quad 507$

PRODUCTS: B4ICOND 501

PRODUCTS: B4INACR 514

PRODUCTS: B41GAS 515

RREMCR/REMCR: (CHROMIUM REMOVAL)

$\begin{array}{lllll}\text { EEEDS: } & \text { B41NACR } & 514 & & \\ \text { FEEDS: } & \text { B42CM } & 516 & 522 & 525 \\ \text { PRODUCTS: } & \text { B42NAOH } & 530 & & \\ \text { PRODUCTS: } & \text { B42LML } & 518 & 524 & 527\end{array}$

RDNITRL/DNITRL: (LLW DENITRATION)

FEEDS: B222BLML 60

FEEDS: B24LML 102

FEEDS: $\quad$ B45CM 508509

PRODUCTS: B45LML 512

PRODUCTS: B45COND 504511

REVAPORH/EVAPORH: (HLW EVAPORATION)

FEEDS: B211THPU 11

FEEDS: B33TC 343

FEEDS: B232SRTC 94

FEEDS: B222AMLN 62

PRODUCTS: B46HLL 539

PRODUCTS: B46COND 538 
Table D3. Stream Relationships: Global Versus Detailed Clean Option Models. (sheet 5 of 6 )

STREAM RELATIONSHIPS: GLOBAL VS. DETAILED CLEAN OPTION MODELS CONTINUED

GLOBAL MODEL DETAILED MODEL

RRCOVACI/RCOVACID: (HNO3 ACID RECOVERY)

$\begin{array}{llll}\text { EEEDS: } & \text { B45COND } & 504 & 511 \\ \text { FEEDS: } & \text { B32COND } & 326 & \\ \text { FEEDS: } & \text { B212ARCY } & 38 & \\ \text { FEEDS: } & \text { B44COND } & 542 & \\ \text { FEEDS: } & \text { B46COND } & 538 & \\ \text { FEEDS: } & \text { B43CM } & 550 & 558 \\ \text { PRODUCTS: } & \text { B43HRCY } & 557 & \\ \text { PRODUCTS: } & \text { B43HNO3 } & 556 & \\ \text { PRODUCTS: B43GAS } & 554 & \end{array}$

RDNITRH/DNITRH: (HLW DENITRATION)

EEEDS: B31CS 310

FEEDS: B46HLL 539

EEEDS: B44CM 540

PRODUCTS: B44HLS 543

PRODUCTS: B44COND 542

MIXHLW: (COMBINE HLW WASTE STREAMS)

FEEDS: B44HLS 543

EEEDS: B132HLS 281

FEEDS: B3CM 546

PRODUCTS: 2322HLWL 544

PRODUCTS: B3HRCY 548

PRODUCTS: B3GAS 547

MIXLLH: (COMBINE LLW MASTE STREAMS)

EEEDS: B42LML $518 \quad 524 \quad 527$

FEEDS: B4CM 528

PRODUCTS: 2322LLWL 529

MIXOGAS: (COMBINE OFFGAS MASTE STREAMS)

FEEDS: B111GAS 411

FEEDS: B13IGAS 218

EEEDS: B132GAS 258

FEEDS: B4IGAS 515

FEEDS: B43GAS 554

FEEDS: B3GAS 547

PRODUCTS: ALLGAS 570 
Table 03. Stream Relationships: Global Versus Detailed Clean Option Models. (sheet 6 of 6 )

STREAM RELATIONSHIPS: GLOBAL VS. DETAILED CLEAN OPTION MODELS CONTINUED GLOBAL MODEL DETAILED MODEL

MIXH20: (CONBINE PDT MATER STREAMS)

$\begin{array}{llllll}\text { EEEDS: } & \text { B31COND } & 451 & & & \\ \text { FEEDS: } & \text { B212COND } & 37 & 26 & 20 & 31 \\ \text { FEEDS: } & \text { B43HNO3 } & 556 & & & \\ \text { FEEDS: } & \text { B41COND } & 501 & & & \\ \text { PRODUCTS: } & \text { 2322COND } & 560 & & & \end{array}$

RMAKEOX: (MAKE OXIDES FROM NEUTRALIZED HLW)

FEEDS: $2322 \mathrm{HLWL} 544$

FEEDS: CI2CM HYDROX

PRODUCTS: C12HLS TCOX

SEPOX: (OXIDE STREAM SEPARATION)

$\begin{array}{lll}\text { FEEDS: } & \text { C12HLS } & \text { TCOX } \\ \text { PRODUCTS: } & \text { C13HLS } & \text { HLWOXS } \\ \text { PRODUCTS: } & \text { 3110GAS } & \text { REST }\end{array}$

VITRW: (VITRIFICATION)

$\begin{array}{lll}\text { FEEDS: } & \text { C13HLS } & \text { HLWOXS } \\ \text { FEEDS: } & \text { C11CM } & \text { FRIT } \\ \text { PRODUCTS: } & \text { 3110HLWS } & \text { GLASS }\end{array}$ (GLASS FORMERS)

RGROUTW/GROUTW: (GROUTING OF LLW)

\begin{tabular}{|c|c|c|c|c|c|}
\hline $\begin{array}{l}\frac{\text { FEEDS: }}{\text { FEEDS: }} \\
\text { PRODUCTS: } \\
\text { PRODUCTS: } \\
\text { PRODUCTS: }\end{array}$ & $\begin{array}{l}2322 \text { LLWL } \\
34 \text { CM } \\
3400 \text { LMWS } \\
3400 \text { LWS } \\
\text { 3400GAS }\end{array}$ & $\begin{array}{l}529 \\
\text { H2OF IX } \\
\text { GRLMWS } \\
\text { GRLLWS } \\
\text { GRGAS }\end{array}$ & DRYMIX & AIRIN & AIRLK \\
\hline COST: (CC & OSTING) & & & & \\
\hline $\begin{array}{l}\text { FEEDS: } \\
\text { FEEDS: } \\
\text { FEEDS: } \\
\text { FEEDS: } \\
\text { FEEDS: } \\
\text { FEEDS: } \\
\text { PRODUCTS: } \\
\text { PRODUCTS: } \\
\text { PRODUCTS: } \\
\text { PRODUCTS } \\
\text { PRODUCTS: } \\
\text { PRODUCTS: } \\
\text { PRODUCTS: }\end{array}$ & $\begin{array}{l}\text { STR304A } \\
\text { STR450 } \\
\text { STR318A } \\
\text { STR336A } \\
\text { STR59A } \\
\text { STR85A } \\
\text { COST304A } \\
\text { COST450 } \\
\text { COST318A } \\
\text { COST336A } \\
\text { COST59A } \\
\text { COST85A }\end{array}$ & $\begin{array}{l}304 A \\
450 \\
318 A \\
336 A \\
59 A \\
85 A \\
304 A \\
450 \\
318 A \\
336 A \\
59 A \\
85 A\end{array}$ & & & \\
\hline
\end{tabular}


Table D4. Component Lumping Scheme Used for CLEAN Option Global Models.

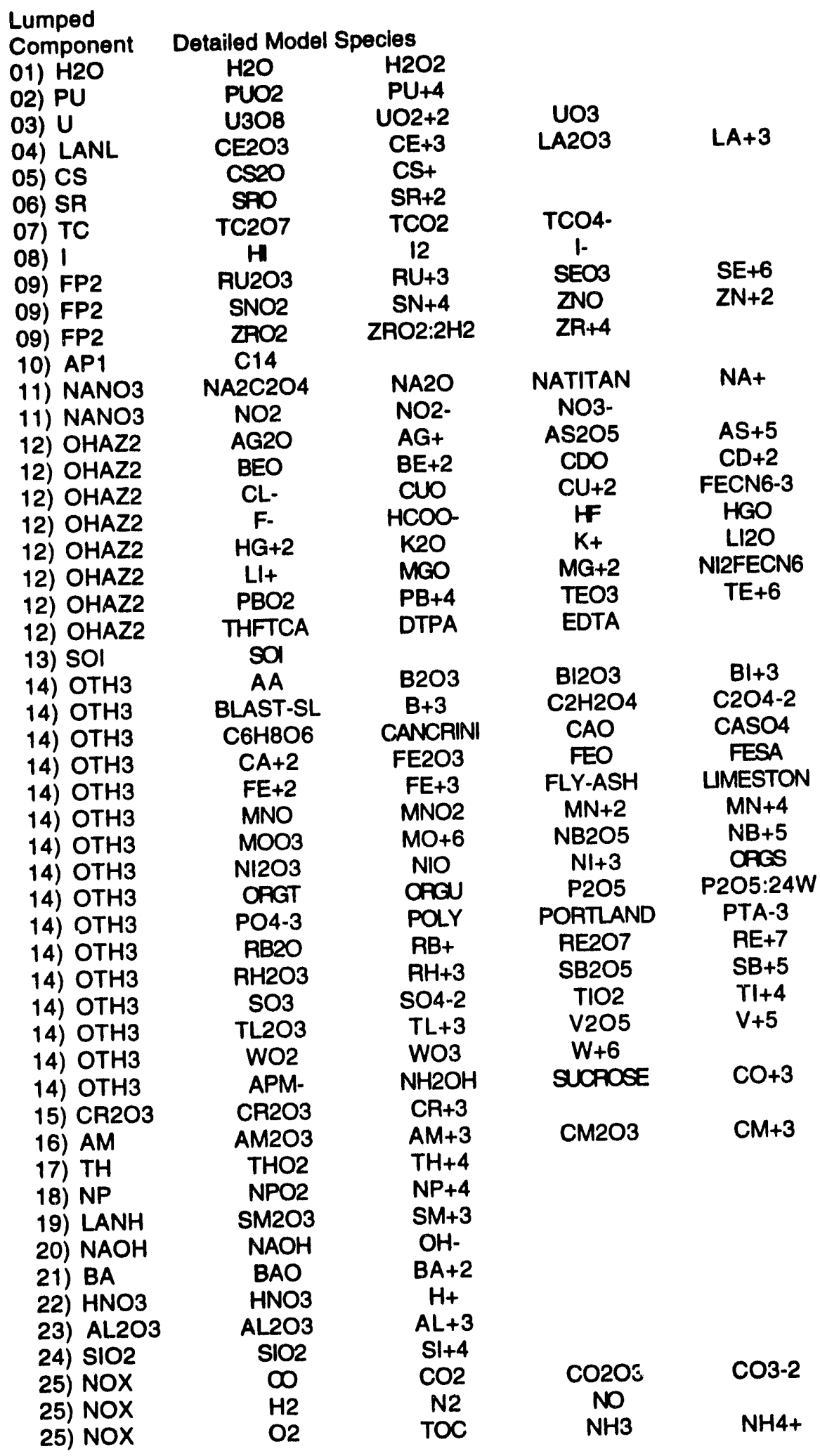


Table 05. Separation Factors and Reactor Component Yield Data for Model CLEAN31. (sheet 1 of 4)

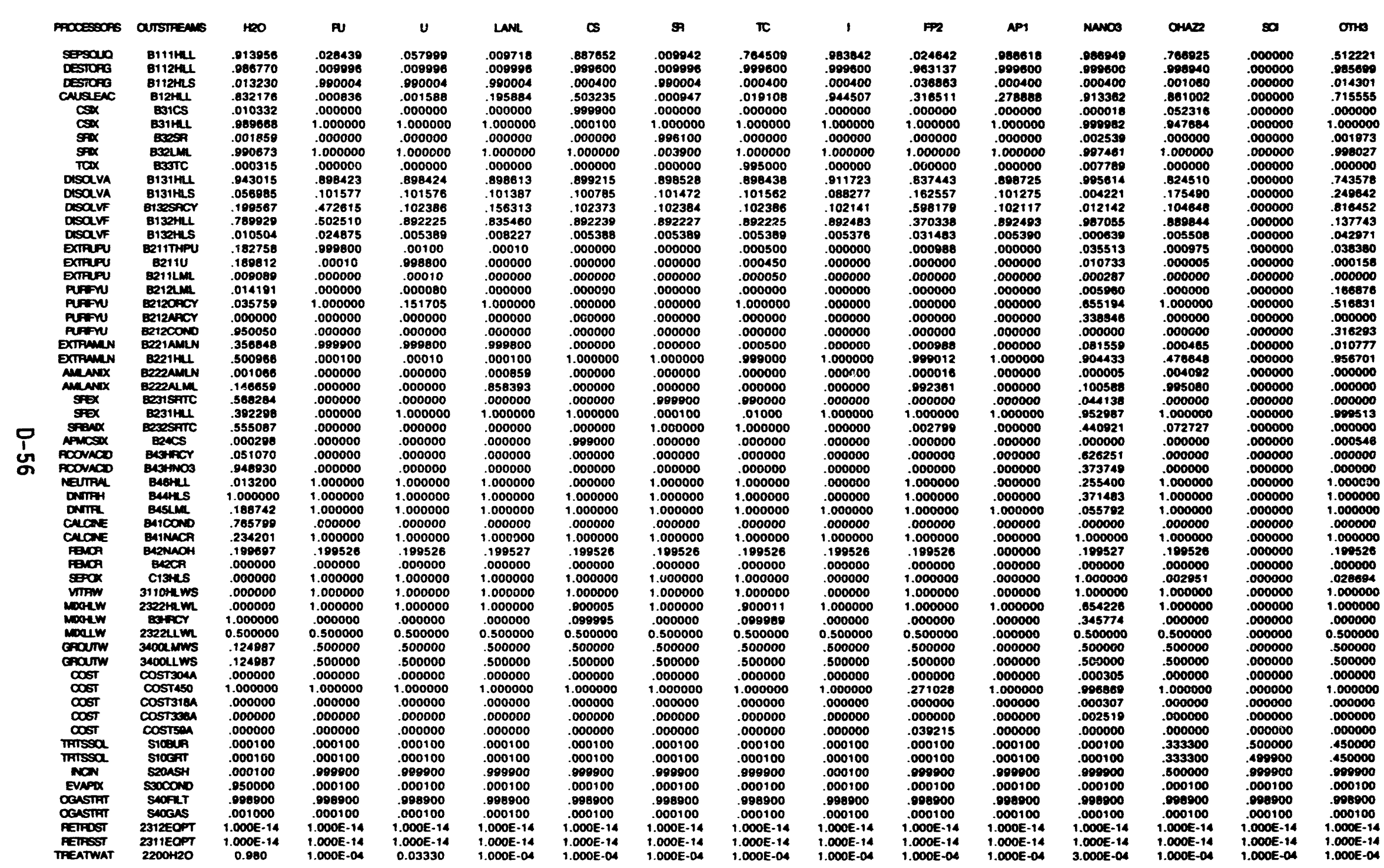


WHC-EP-0791

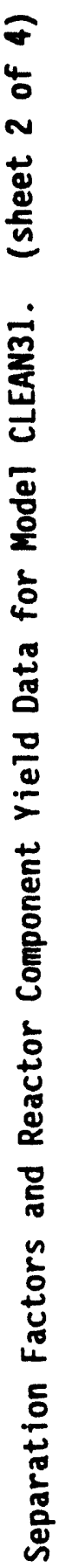

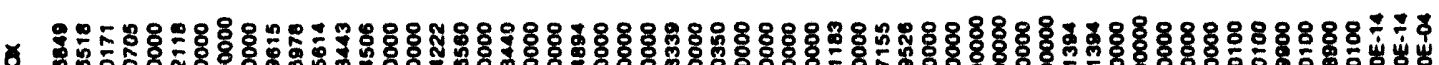
2.

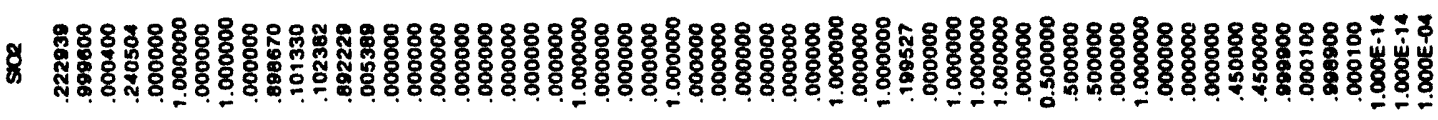
ร)

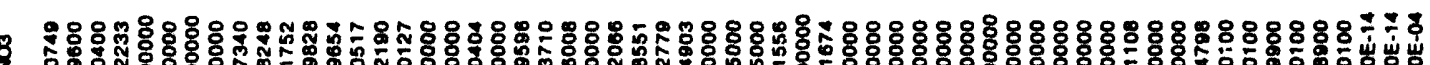

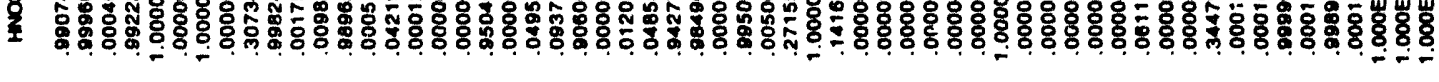

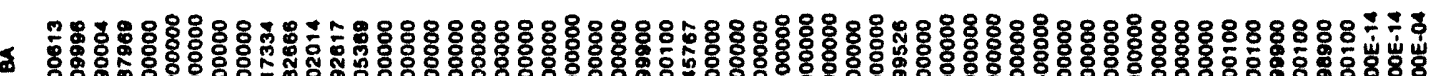

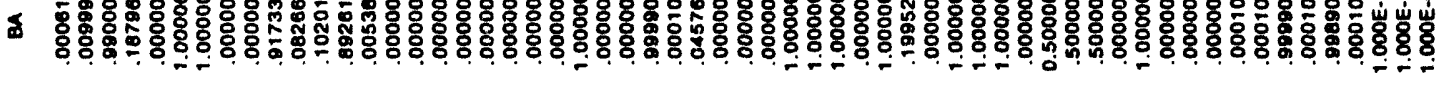

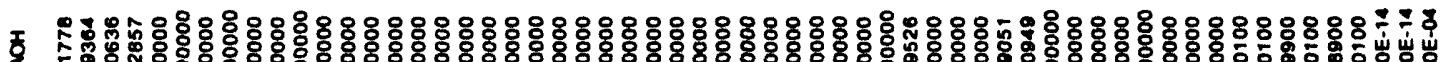

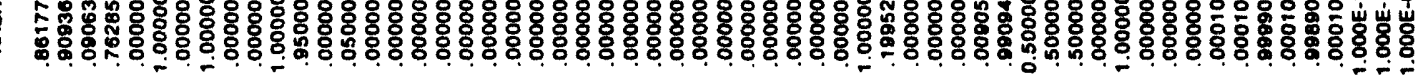

3

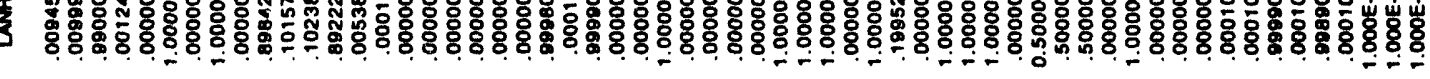

2

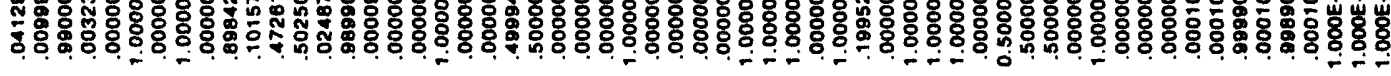

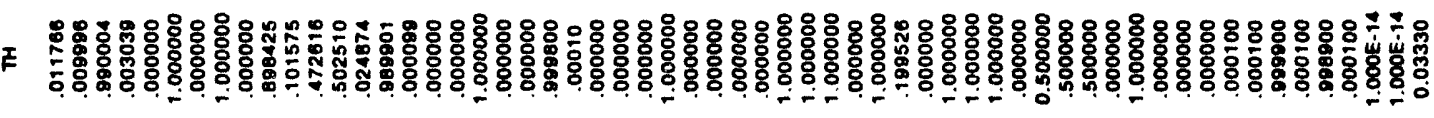

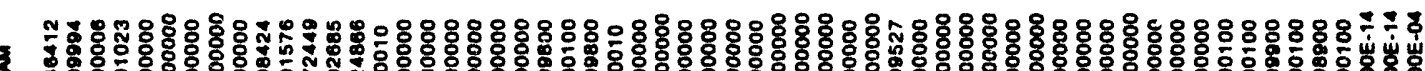

₹

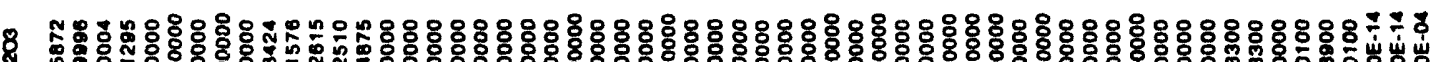
药

至 万 [1ำ D-57 
Table D5. Separation Factors and Reactor Component Yield Data for Model CLEAN31. (sheet 3 of 4 )

\begin{tabular}{|c|c|c|c|c|c|c|c|c|c|c|c|c|c|c|c|}
\hline & & & & & & & & & & & & & & OTHo & recos \\
\hline 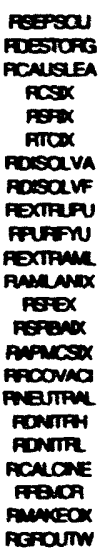 & 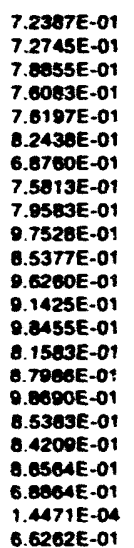 & 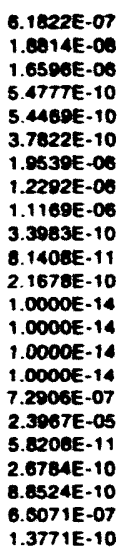 & 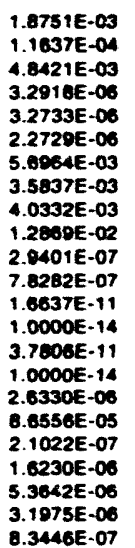 & 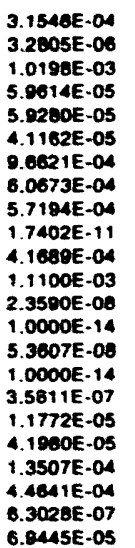 & 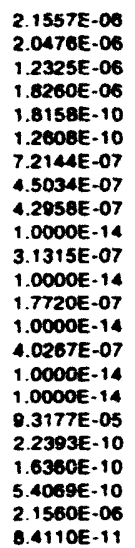 & 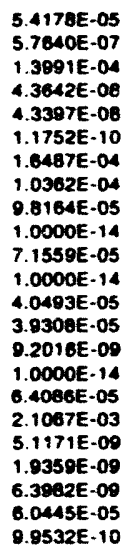 & 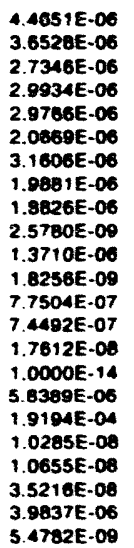 & 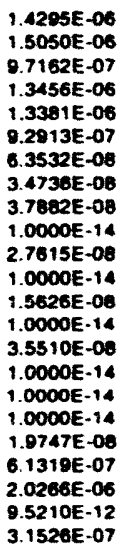 & 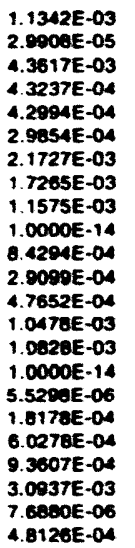 & 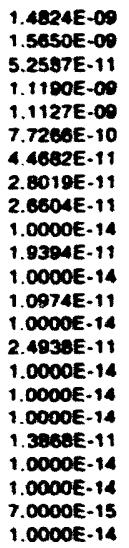 & 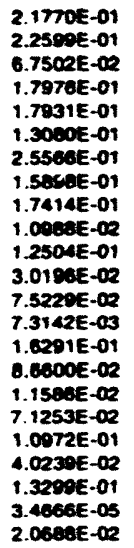 & 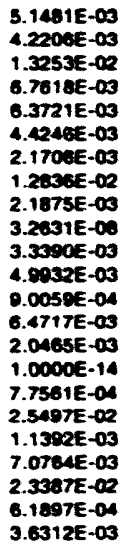 & 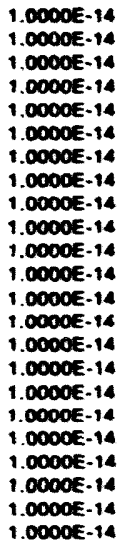 & 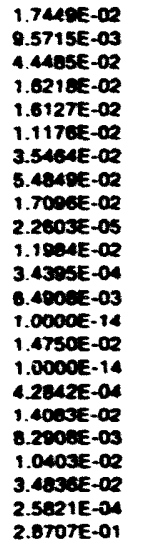 & \\
\hline
\end{tabular}


Table D5. Separation Factors and Reactor Component Yield Data for Model CLEAN31. (sheet 4 of 4)

\begin{tabular}{|c|c|c|c|c|c|c|c|c|c|c|}
\hline & 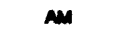 & n & & LaNH & $\mathrm{MOH}$ & Ba & Hacos & NL2O3 & 500 & Nox \\
\hline 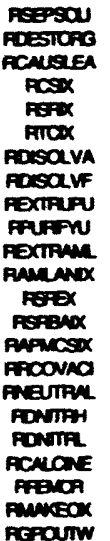 & 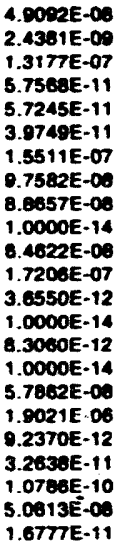 & 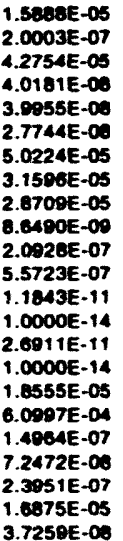 & 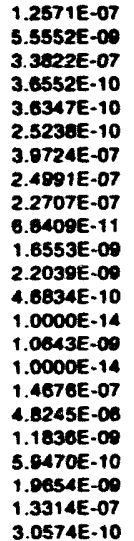 & 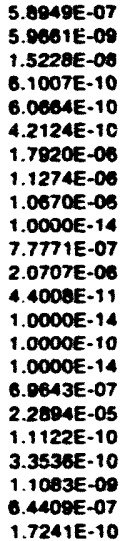 & 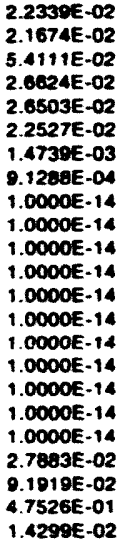 & 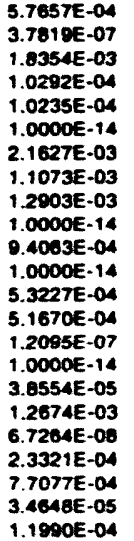 & 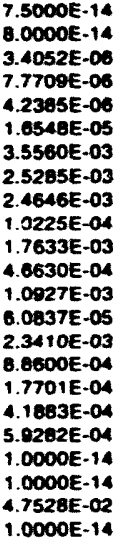 & 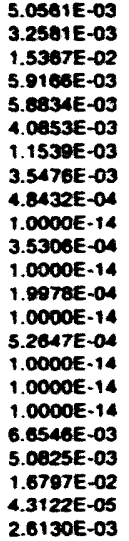 & 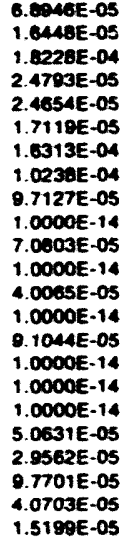 & 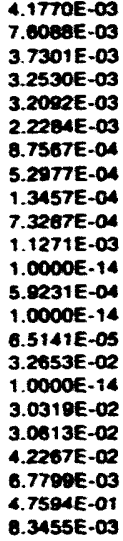 \\
\hline
\end{tabular}




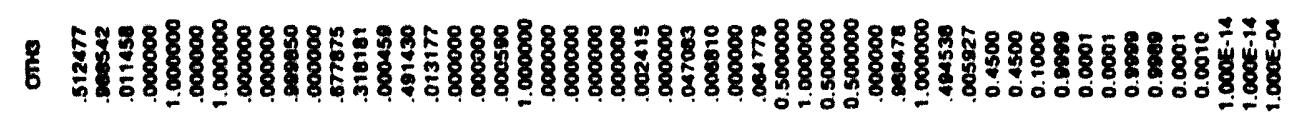

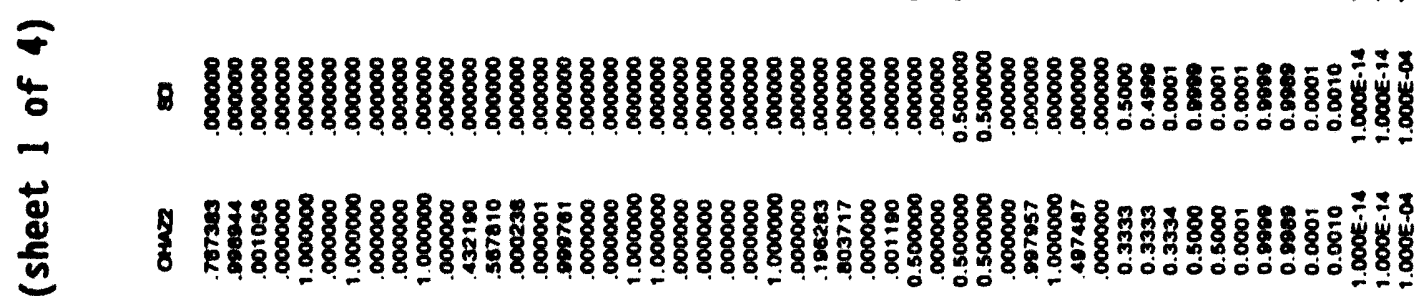

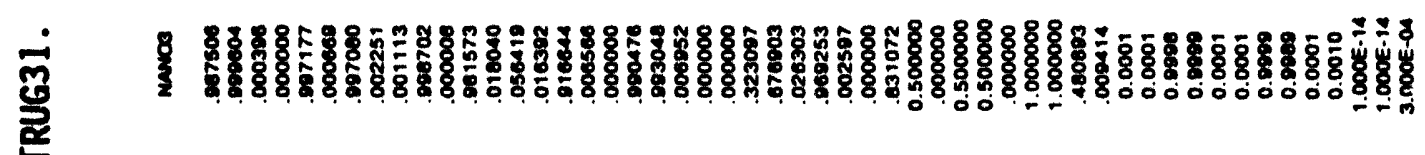

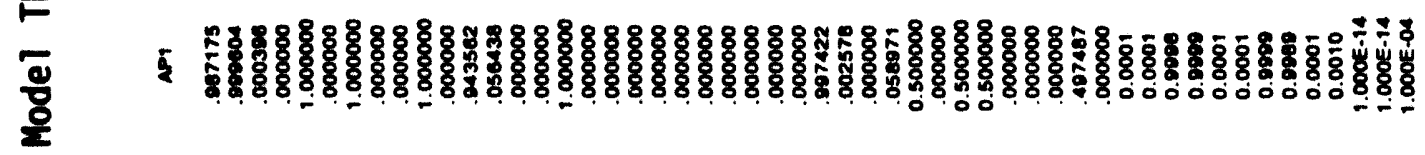
\&

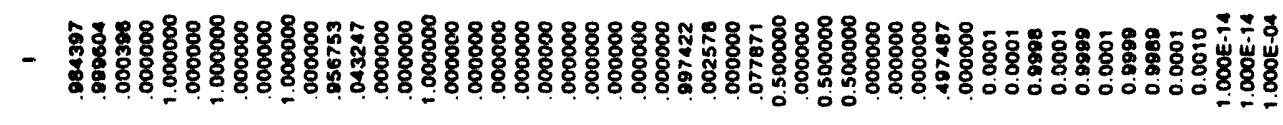

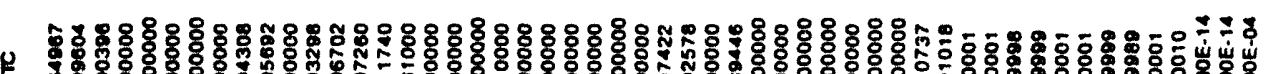

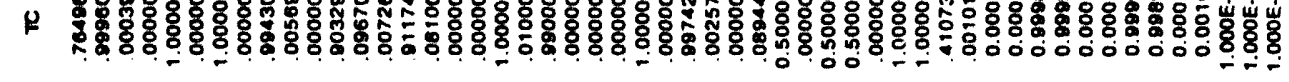

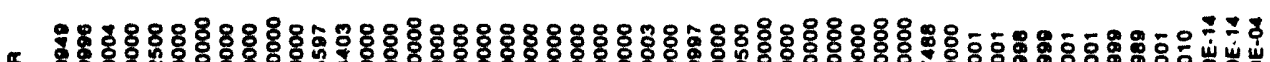

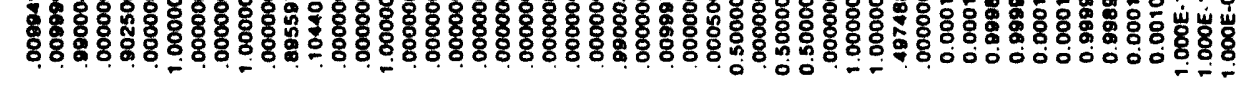

5

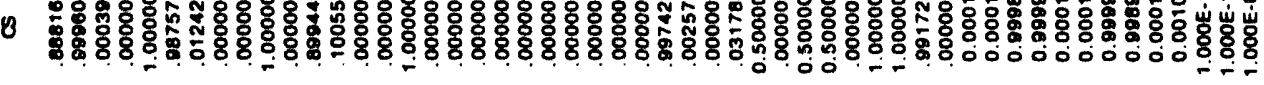

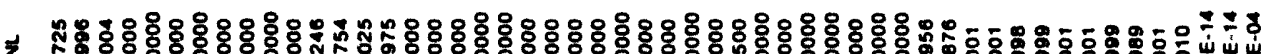

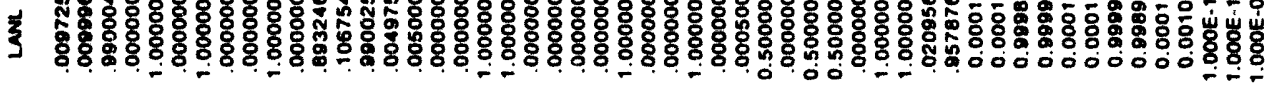

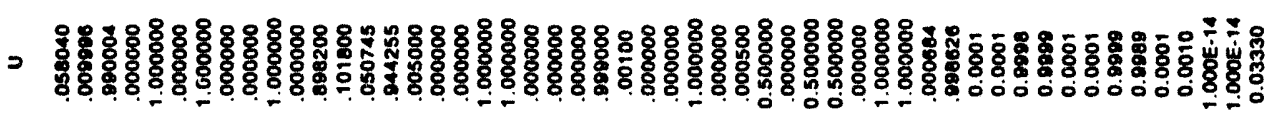

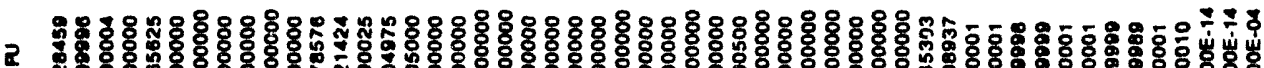

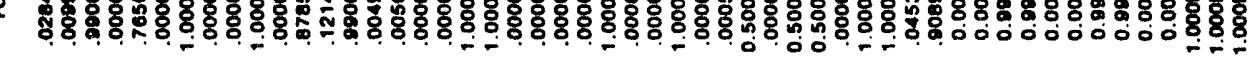

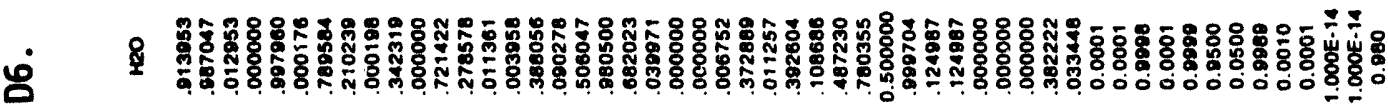

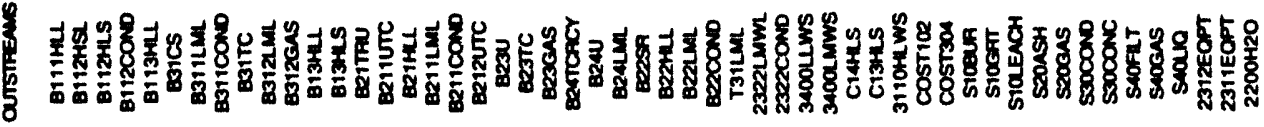

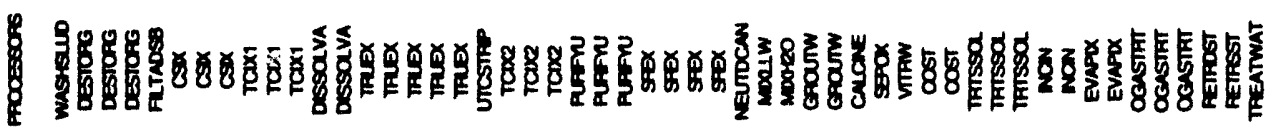
D-60 
WHC-EP-0791

$\sigma$
4
$\sim$
$\sim$
$\frac{g}{\tilde{E}}$

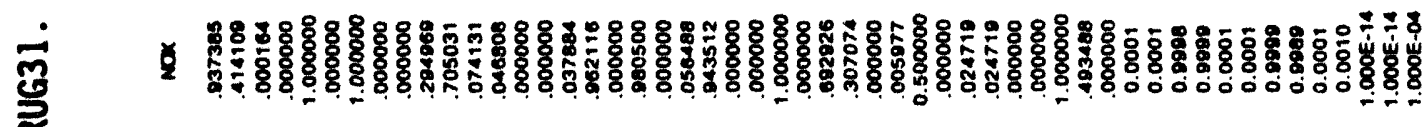

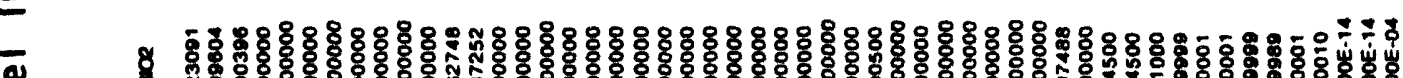

吾 \%

它 不

苟

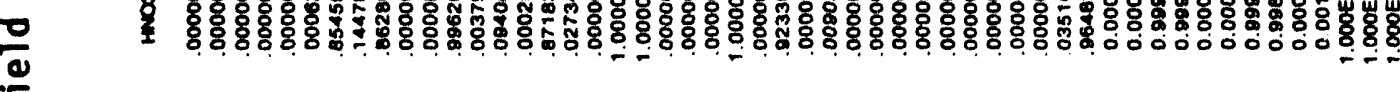

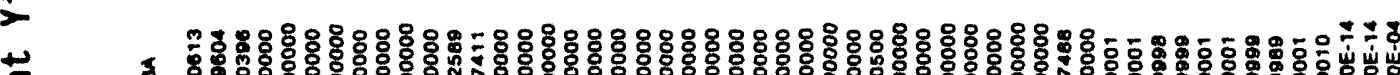

\

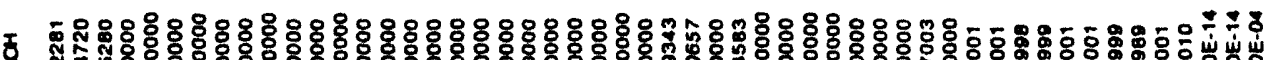

Z

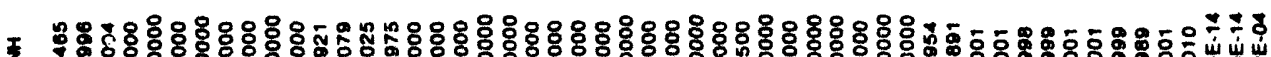

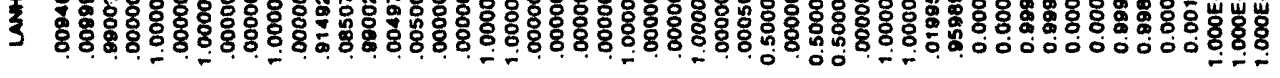

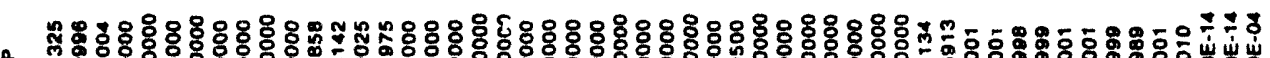

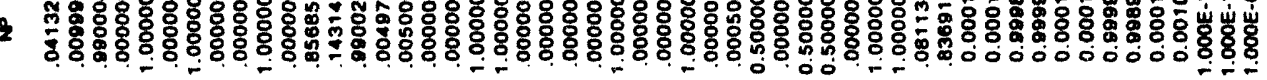

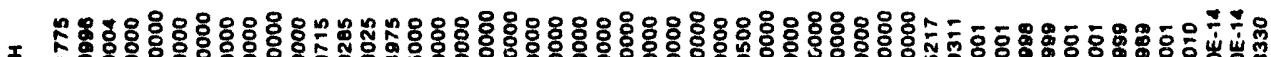

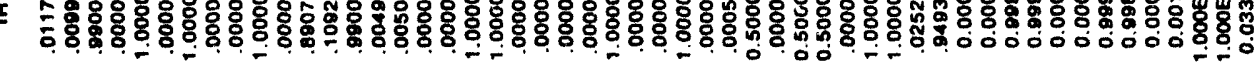

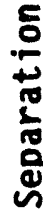

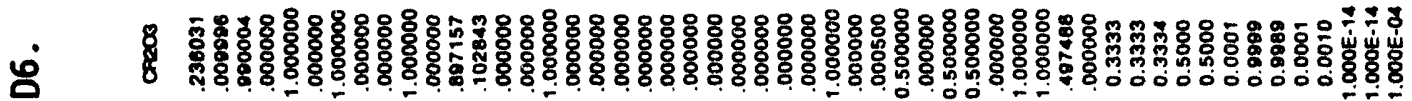

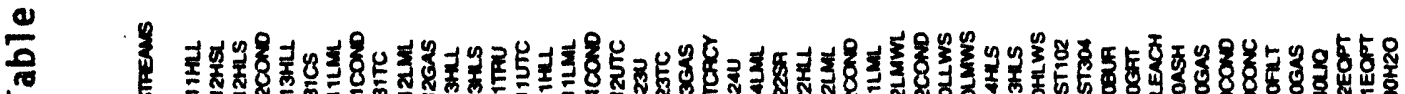

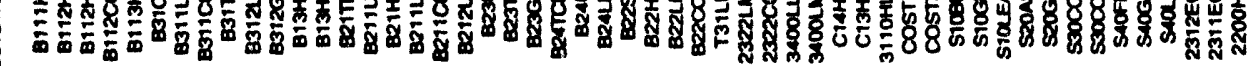

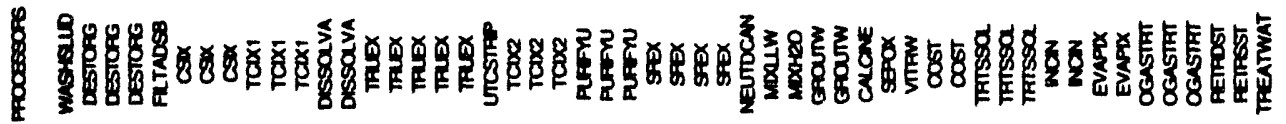

D-61 
Table D6. Separation Factors and Reactor Component Yield Data for Model TRUG31. (sheet 3 of 4)

$\operatorname{acc}$

nеO

PU

LANL

$\infty$

9

$\pi$

FP

AP1

NANOS

Orenes

sa

OTH

Creos

FWAStou $\begin{array}{lllllll}7.2433 E-01 & 6.1710 E-07 & 1.8717 E-03 & 3.1480 E-04 & 2.1521 E-06 & 5.4079 E-05 \\ 7.3121 E-01 & 1.8370 E-00 & 1.1554 E-04 & 3.2571 E-06 & 2.6330 E-08 & 5.7227 E-07\end{array}$ $7.3438 E-01 \quad 1.0980 E-10 \quad 1.172$

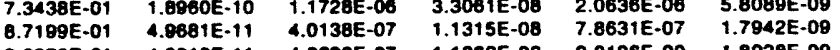

4.4575E-06 $1.4271 E-06$

$\begin{array}{ll}1.1322 E-03 & 1.47 \\ 2.9695 E-05 & 1.55 \\ 2.9042 E-05 & 1.57\end{array}$

$\begin{array}{lll}1.4789 E-09 & 2.173 \\ 1.5539 E-09 & 2.234\end{array}$

$2.1734 E-01 \quad 5.13$

\begin{tabular}{ll|l}
$5.1393 E-03$ & $1.0000 E-14$ \\
$4.1948 E-03$ & $1.0000 E-14$ \\
$1.2550 E$ & 1.7
\end{tabular}

$\begin{array}{rr}1.7425 E-02 & 2.1026 E-04 \\ 0.5063 E-03 & 5.2783 E-05\end{array}$

Forssav

$7.4095 \mathrm{E}-01$

$1.1369 \mathrm{E}-08 \quad 9.8198 \mathrm{E}-09$

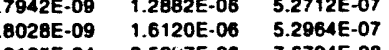

$9.9392 E-06$
$8.986 E-06$

$\begin{array}{ll}2.272 E-161 \\ .4659 E-10 & 1.0921 \mathrm{E}-01\end{array}$

$\begin{array}{ll}4.2550 E-03 & 1.0000 E-14 \\ 1.4938 E-03 & 1.0000 E-14 \\ 1.5010 & \end{array}$

$\begin{array}{ll}0.5405 E-03 & 5.3579 E-07 \\ 0.2772 E & 1.0397-07\end{array}$

\begin{tabular}{lllll}
$.2940 E-03$ & $1.0594 E-03$ & $8.1236 E-07$ & $1.8195 E-04$ \\
\hline $.2569 E-03$ & $2.1040 E-04$ & $1.6245 E-07$ & $3.6229 E-05$
\end{tabular}

$\begin{array}{ccccc}1.612 E E-06 & 5.2964 E-07 & 9.9866 E-06 & 5.4920 E-10 \\ .5267 E-06 & 7.6794 E-08 & 2.2576 E-03 & 6.5808 E-11 \\ 7.067\end{array}$

$1.1093 E-01$
$1.8805 E-01$

$\begin{array}{ll}1.5010 E-03 & 1.0000 E-14 \\ 4.0365 E-03 & 1.0000 E-14\end{array}$

6.3071E-03 $1.8424 E-07$

$\begin{array}{ll}1.5135 E-01 & 1.5189 E-02 \\ 5.7533 E-01 & 3.4605 E-00\end{array}$

7.8807E-06 1.0000E-14

\begin{tabular}{ll}
$1.0000 E-14$ & $2.0279 E-06$ \\
\hline & $4.8660 E-06$ \\
\hline
\end{tabular}

\begin{tabular}{ll|}
$.0000 E-14$ & $1.0000 E-14$ \\
\hline $.0000 E-14$ & $1.0000 E-14$
\end{tabular}

\begin{tabular}{ll}
$1.0000 E-14$ & $3.0668 \mathrm{E}-02$ \\
\hline &
\end{tabular}

. $4550 E-09 \quad 1.0000 E-14$

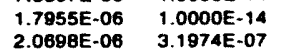

$.0000 E-14$
$7.1305 E-05$
.1 .1

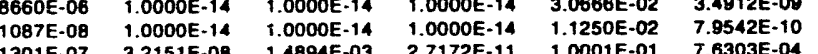

$\begin{array}{ccc}\text { OOOOOE-14 } & 8.0946 E-01 & 1.0000 E-14 \\ .0000 E-14 & 3.9987 E-03 & 1.0000-14 \\ 1.0000 E-14\end{array}$

(6.350.01

$3.9891 E-09$

. 2365E-05

1.0698E-06 $3.1974 E-07$

$4.9952 E-04$
$1.0000 E-14$

\begin{tabular}{lllll}
$3.2151 E-08$ & $1.4894 E-03$ & $1.7172 E-11$ & $1.0001 E-01$ \\
\hline $.2004 E-09$ & $8.7055 E-04$ & $1.0289 E-11$ & $7.7949 E-02$ \\
\hline
\end{tabular}

$1.0000 E-14$
$1.0000 E-14$

$\begin{array}{llllll}2.1590 E-03 & 1.0000 E-14 & 1.0000 E-14 & 1.0000 E-14 & 3.4830 E-01\end{array}$

\begin{tabular}{ll}
$0.0454 E-08$ & $3.0000 E-14$ \\
\hline & $3.1234 E-03$ \\
\hline
\end{tabular}

3.4755E-11 $\quad 7.0562 E-02$

.0000E-14

.0000E-14

$\begin{array}{ll}2.7700 E-04 \\ .7274 E-02 & 2.0108 E-04\end{array}$

$\begin{array}{llllllllll}7.5505 E-01 & 3.8711 E-09 & 7.4218 E-06 & 1.0809 E-06 & 1.8389 E-08 & 3.4154 E-07 & 2.8627 E-08\end{array}$

8. $9962 E-01 \quad 1.0000 E-14$

\begin{tabular}{|l|l|l|}
\hline $.0000 E-14$ & $1.0000 E-14$ & $1.80000 E-14$ \\
\hline
\end{tabular}

1.0000E-14 $1.0000 E-14$

\begin{tabular}{llllll}
$.14439 E-07$ & $6.8767 E-04$ & $9.7925 E-10$ & $2.0706 E-01$ \\
$.0000 E-14$ & $1.0000 E-14$ & $1.0000 E-14$ & $3.8087 E-04$ \\
\hline
\end{tabular}

2.2768E-02

1.0000E-14

$1.0000 E-14$

PMNOEOS

PCNONE

\begin{tabular}{llllll}
\hline $.3300 E-13$ & $1.0000 E-14$ & $1.0000 E-14$ & $1.0000 E-14$ & $1.0000 E-14$ \\
$2.0916 E-02$ & $6.3849 E-07$ & $2.6389 E-04$ & $3.3660 E-04$ & $2.0748 E-06$
\end{tabular}

$\begin{array}{llllllll}1.0000 E-14 & 1.0000 E-14 & 1.000 E E-14 & 1.000 E-14 & 1.0000 E-14 & 1.000 \\ 3 & 8.4513 E-10 & 6.6943 E-05 & 9.5700 E-13 & 1.2552 E-03 & 1.1\end{array}$

1.2513E-01 7.2143E-04

$\begin{array}{ccc}1.0000 E-14 & 1.0000 E-14 & 1.0000 E-14 \\ & 9.4940 E-04 & 2.9031 E-05\end{array}$

号 
Table D6. Separation Factors and Reactor Component Yield Data for Model TRUG31. (sheet 4 of 4)

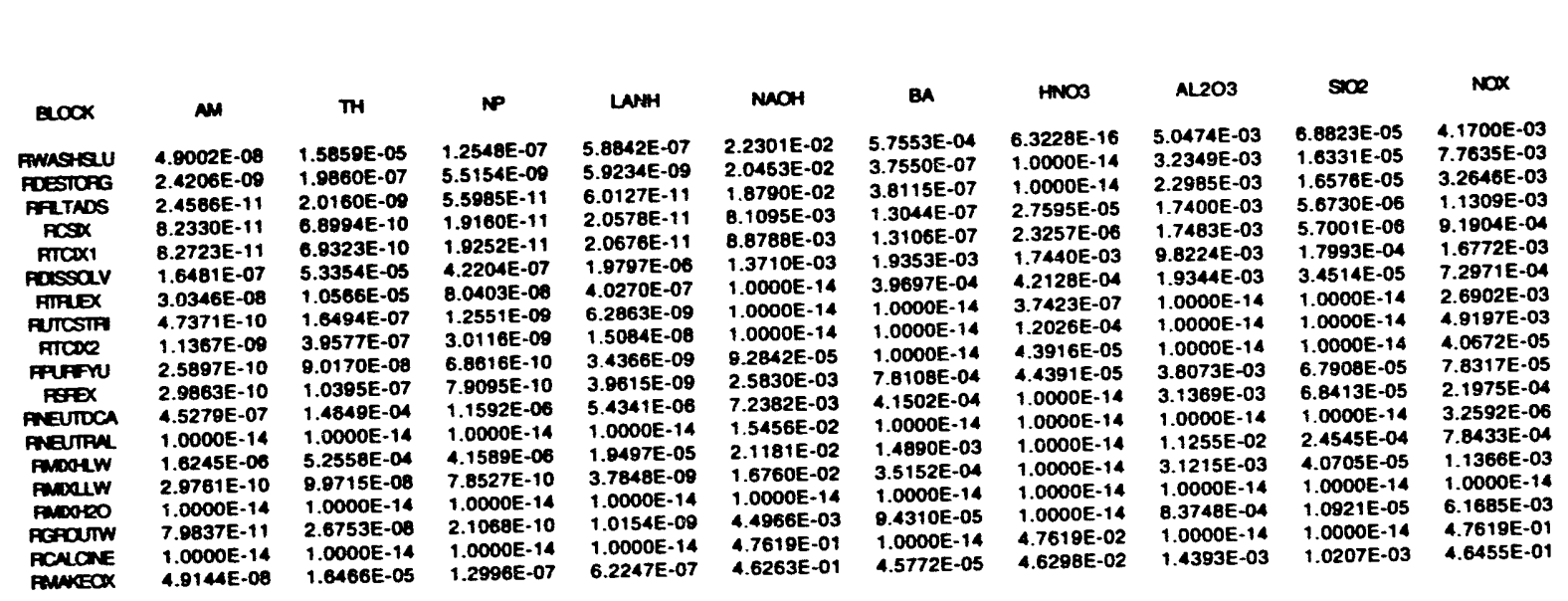


$69-0$

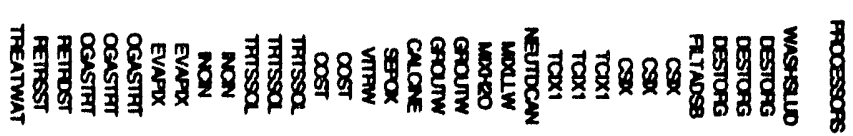

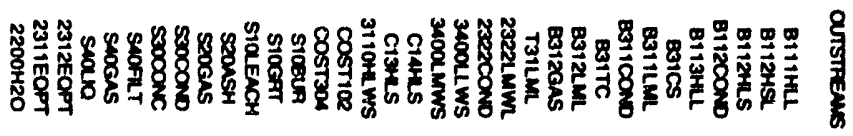

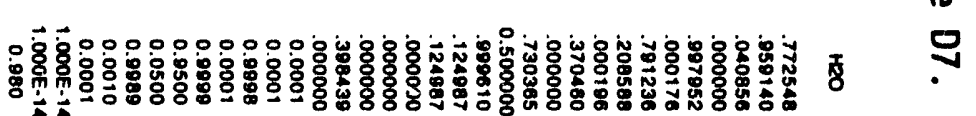

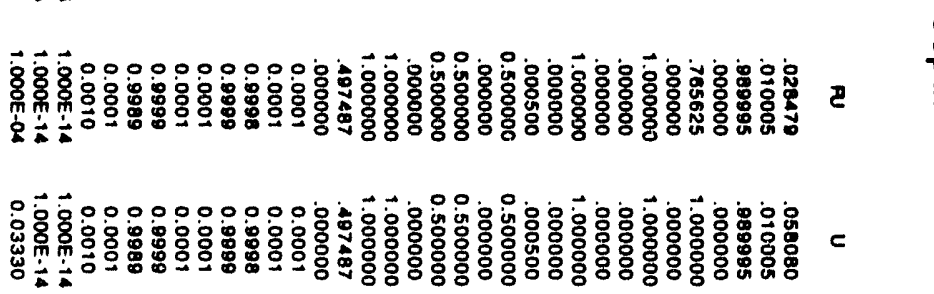

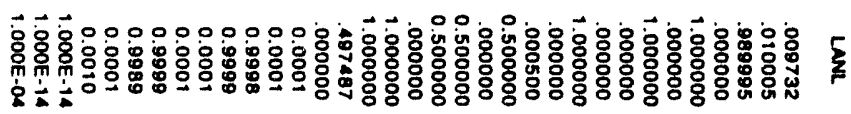

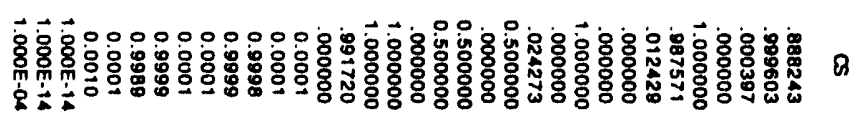

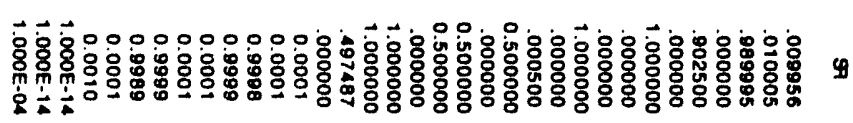

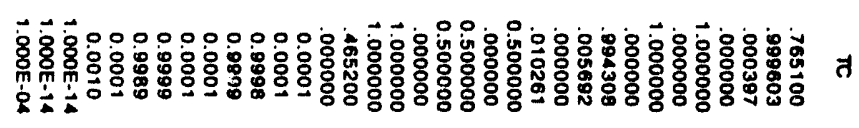

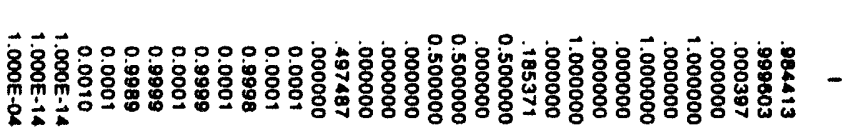

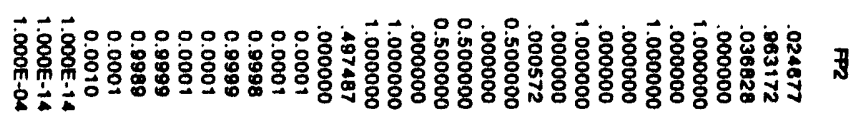

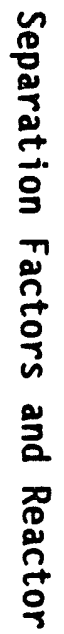

을

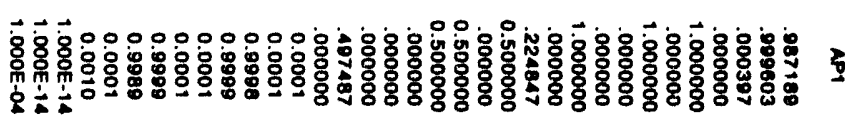

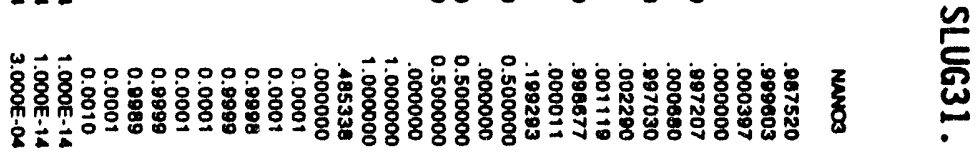

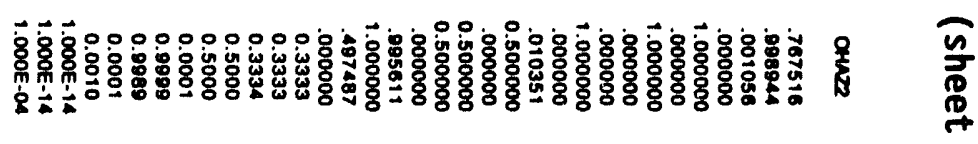

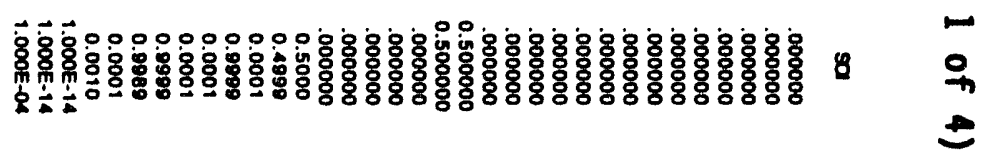

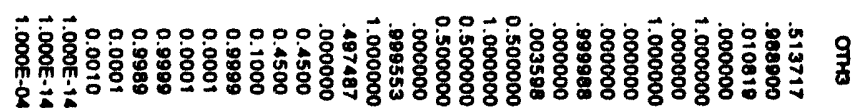


WHC-EP-0791

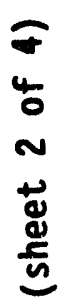

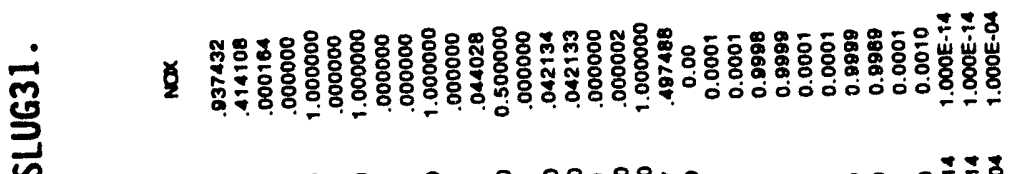

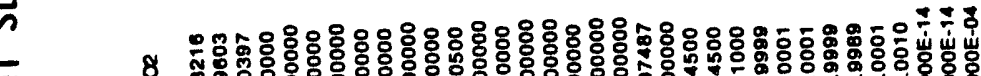

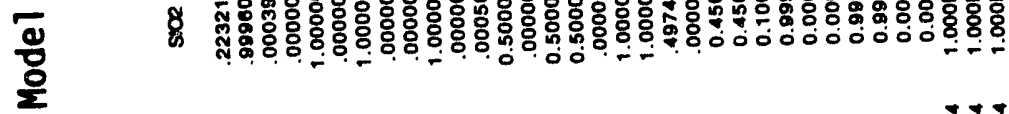

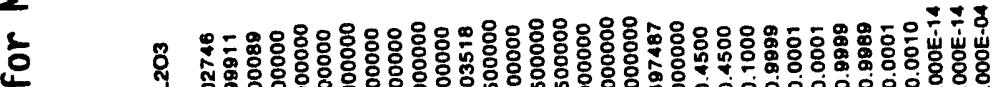

₹

焉

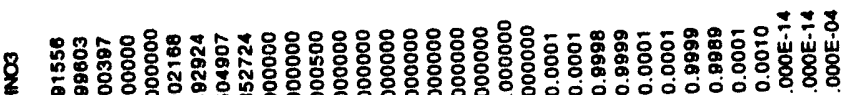

—

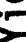

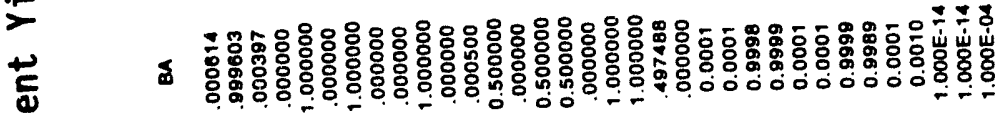

言

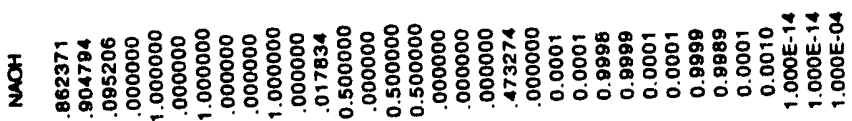

bे

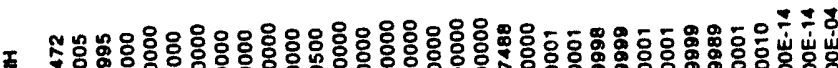

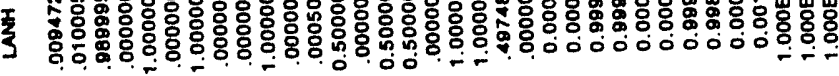

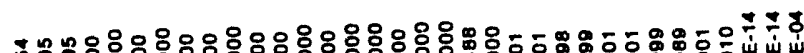

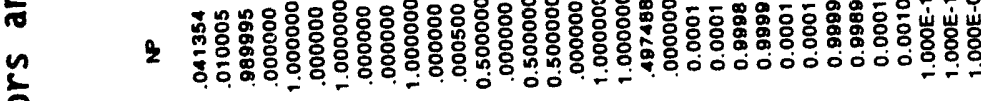

蒈

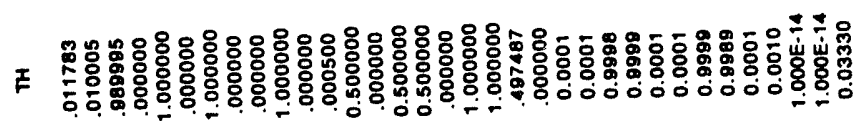

둥

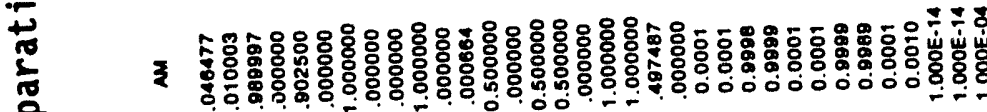

ஸे

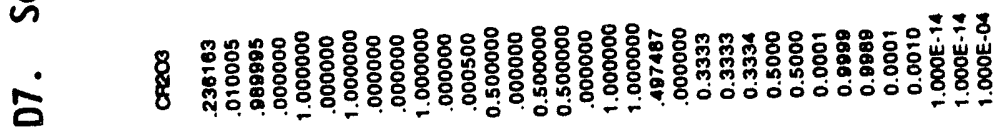

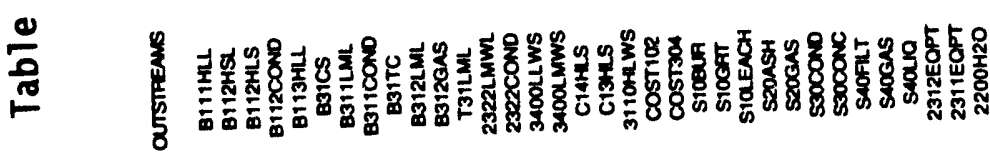

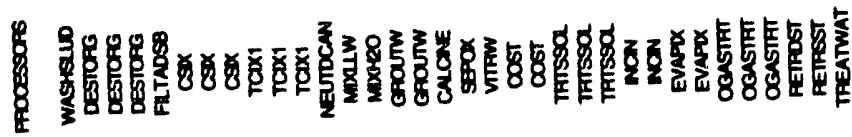


Table D7. Separation Factors and Reactor Component Yield Data for Model SLUG31. (sheet 3 of 4)

acox

Amarean

Fostoris

$\operatorname{pcox}$

Prcoxic

Avarima

ancotw

andored

Faroum

FCNCONE

7.5398E-01 $\quad \mathbf{1 . 0 4 7 7 7 - 0 0}$

$7470 E-01 \quad 6.4011 E-11$

B.7195E-01

O.

.0676E-08 $\quad 6.2688 E-03$

(6.1799E-14

.0000E-14 $1.0000 E-14$

$\begin{array}{cc}1.0933 E-10 & 4.0907 E-07 \\ 1.0000 E-14 & 1.0000 E-14\end{array}$

$\begin{array}{ccc}1.0000 E-14 & 1.0000 E-14 & 1.0000 E-14 \\ 0.0858 E-02 & 5.0476 E-07 & 1.6811 E-03\end{array}$
LANL CS

$2.7916 E-04 \quad 1.9092 E-06 \quad 0.78$

\begin{tabular}{lll}
$1.4578 E-08$ & $2.0864 E-06$ \\
\hline $1.0995 E-07$
\end{tabular}

.4699E-08 $1.1404 \mathrm{E}-08$ - $2.3117 \mathrm{E}-09$

$\begin{array}{lll}1.0552 E-03 & 8.0910 E-07 & 1.8122 E-04\end{array}$

1538E-03

1.0000E-14

2.5959E-08

\begin{tabular}{ll}
$1.0000 E-14$ & $1.0000 E-14$ \\
\hline $.6418 E-08$ & $7.3123 E-09$
\end{tabular}

$1.3123 E-149$
$1.0000 E-14$

$1.0000 E-14$
$7.8725 E-09$

5.52B2E-05 1.0000E-14

\section{$\pi$}

.0540E-06 1.2681E-06 $\begin{array}{ll}1.4794 E-08 \\ 3.7221 E-06 & 1.5335 E-06\end{array}$

6.6879E-07

$7.6470 E-08$
$1.0000 E-14$

$1.8628 E-07$
$1.2351 E-06$

$1.0000 E-14$
$3.4791 E-07$
$3.000 E-14$

1.0000E-14
$7.4164 E-04$

\begin{tabular}{|c|c|c|c|c|c|}
\hline AP1 & NANDS & ortuz2 & sa & onts & \\
\hline $\begin{array}{l}5947 \\
9548 \\
0126 \\
5529 \\
0000 \\
5190 \\
2844 \\
00000 \\
6180\end{array}$ & $\begin{array}{l}2119 E-01 \\
2093 E-01 \\
.0723 E-01 \\
.0928 E-01 \\
.1495 E-02 \\
.4771 E-01 \\
.2894 E-02 \\
.0055 E-01 \\
.0215 E-04 \\
.6493 E-02 \\
.0000 E-14 \\
.8122 E-03\end{array}$ & & & $\begin{array}{l}1.5419 E-02 \\
9.4042 E-03 \\
9.6439 E-03 \\
4.2059 E-03 \\
4.2409 E-03 \\
2.8668 E-02 \\
1.0000 E-14 \\
8.5418 E-02 \\
7.7710 E-03 \\
7.0313 E-08 \\
2.2447 E-01 \\
1.0000 E-14 \\
4.6546 E-03\end{array}$ & $\begin{array}{l}1.8643 E-04 \\
5.2213 E-05 \\
5.4171 E-07 \\
2.3625 E-07 \\
2.3822 E-07 \\
7.0294 E-04 \\
1.0000 E-14 \\
2.1010 E-03 \\
5.2690 E-07 \\
1.0000 E-14 \\
1.4842 E-07 \\
1.0000 E-14 \\
2.6502 E-04\end{array}$ \\
\hline
\end{tabular}

禺

잉 
Table D7. Separation Factors and Reactor Component Yield Data for Model SLUG31. (sheet 4 of 4)

soox

Amasew

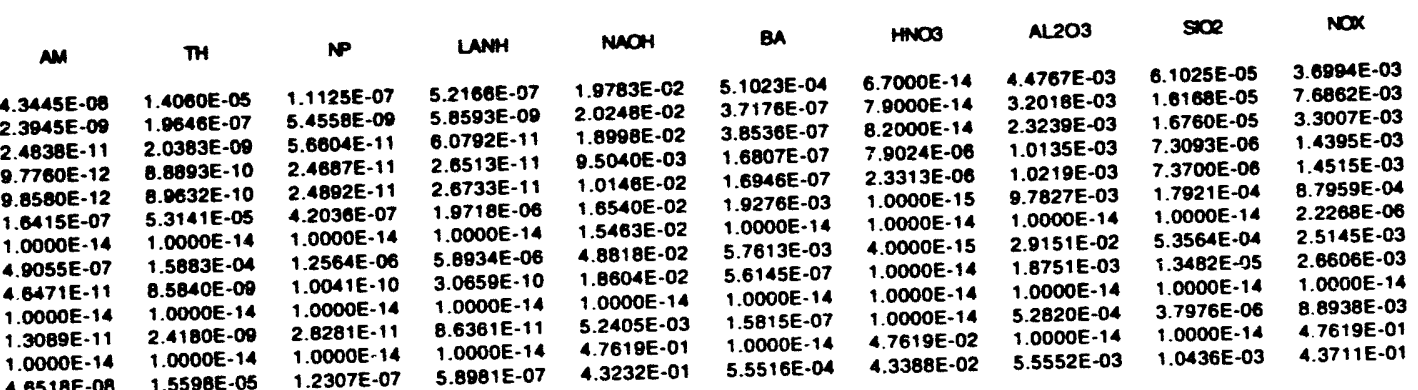


WHC-EP-0791

This page intentionaliy left blank. 
WHC-EP-0791

APPENDIX $\mathbf{F}$

COST ESTIMATION MODELS

F-1 


\section{LIST OF TERMS}

CMPO

CPU

D\&D

DST

HLW

HSA

HWVP

ICF

ISV

LLW

SCWO

SST

SWA

SWB

SWC

TBP

TRU

TRUEX-C

TWRS

WIPP
$\mathrm{N}$-di isobutylcarbamoylmethlphosphine oxide compact processing unit decontamination and decomissioning double-shell tank

high-level waste

Hanford Strategic Analys is

Hanford Waste Vitrification Plant

Consolidated Incinerator Facility

in situ vitrification

low-level waste

supercritical water oxidation

single-shell tank

Sludge Wash A

Sludge Wash B

Sludge Wash D

tributyl phosphate

transuranic

Transuranic Extraction Option C

Tank Waste Remediation System

Waste Isolation Pilot Plant 


\section{COST ESTIMATION MODELS}

This appendix discusses the development of the cost estimation models and the results from using these models. The cost models are based on cost data taken from a library of cost data compiled to support Hanford Site tank waste retrieval systems engineering efforts. In most cases, the data exist in the form of cost functions, though some data may be in the form of point estimates.

\section{F1.0 DEVELOPMENT OF THE COST ESTIMATION MODELS}

\section{FI.1 COST FUNCTIONS}

Cost functions predict the cost of some facility or activity based on input parameters, such as design throughput capacity, degree of chemical separation, amount of material treated or disposed, or other parameters as needed. Consequently, cost functions predict costs over a wide range of possible system configurations, rather than for just one specific configuration.

\section{F1.1.1 Throughput Capacity}

Cost function terms depending on design throughput capacity are based on the well known exponent scaling method. That is, terms such as the following:

$$
\text { (actual capacity/reference capacity) } \star \star E x p
$$

are used, where the ratio of the desired capacity to a reference capacity is raised to an exponent between 0.0 and 1.0. One of these terms is multiplied times a reference capital cost to obtain a capacity-scaled capital cost. A similar term (generally with a different exponent) is multiplied times a reference operating cost to obtain a capacity-scaled operating cost. Many published tables of suggested exponents exist for specific chemical processes and processing facilities. Where no process-specific exponent can be found, a generic value of 0.7 is often used for the capital cost component. A value of 0.75 is usually used for the operating cost component.

\section{F1.1.2 Chemical Separation}

Cost function terms, depending on the degree of chemical separation, are based on the estimation that precipitation processes tend to have costs that increase linearly with increasing separation, solvent extraction processes tend to have costs that increase as the logarithm of separation, and ion exchange processes tend to have costs that increase as the logarithm of separation raised to the 0.55 power. These functional dependencies are semiquantitative estimates based on first principles considerations. 
WHC-EP-0791

\section{F1.1.3 Other}

Other cost function terms have been derived from speciffc avallable data. For example, the cost function describing the cost for high-level waste disposal in the Nevada Repository is a curve fit based on cost estimates for spectfic volumes of material disposed.

\section{F2.1 FUNCTION CREATION}

In fully general form, a cost function should be independent of a particular process or particular facility. This is accomplished, in principle, in the following manner.

1. Cost functions are developed for several processes or factilities that perform a similar waste processing operation. Often these process/facility functions have different volume or separation performance ranges of applicability, but not always.

2. Overall process/facility-independent cost functions are developed by combining the individual functions in a way that seems most cost-advantageous or reasonable.

In practice, funding has not been available to develop and cost a wide enough range of process and facility alternatives to produce more than a few process/facility-independent cost functions using the two-step process described above. More typically, only Step l above is performed. That is, only a single process or facility is assumed available. The way in which this single process/facility cost varies with capacity or separation (or other appropriate parameter) is then estimated to produce a cost function based on that one process/facility.

Typically, individual cost functions are developed by multiplying together terms that correct for throughput capacity and terms that account for desired separation (if applicable). In instances where no separation is involved, an appropriate cost term is often simply multiplied times the total volume of waste involved in the process or facility. In other, unusual cases, some other scheme for developing the final cost function may be employed, but in all cases the end result is some combination of curve fitting, exponent scaling, and assumed functional forms for the process or activity under consideration.

Typically, capital and operating cost terms are represented separately in the cost functions.

\section{F3.1 POINT ESTIMATES}

In a few instances, information has not been located to allow development of even a "one process" cost function (using only Step 1 above). Where functions are not available, "point estimates" have been made for the cost of processes or facilities of a specific size and with specific, fixed parameters. 


\section{F4.1 COST FUNCTIONS AND POINT ESTIMATES USED}

The previous report on the Transuranic Extraction Option C (TRUEX-C) and sludge Wash C evaluation (Knutson et al. 1993) contained process cost function derivations based largely on the detalled cost estimates contained in the Tank Waste Technical Options Report (Boomer et al. 1993) work. These cost est imates were based on using a central processing facllity. However, the CLEAN Option work did not include such detailed process cost estimations. Therefore, to provide a simflar process cost breakdown for the CLEAN Option strategy based on the central facility concept, process cost function estimates were made based on matching the CLEAN Option processes with similar type processes in the TRUEX-C and Sludge Wash $C$ process strategles for which cost functions were available. Because most of the available cost functions were related to process flows and separation factor performance, these function matches were considered adequate to provide reasonable cost estimates for the anticipated CLEAN Option processes. These CLEAN Option process matching assumptions are shown below.

Other CLEAN Option cost estimates have been made based on using some combination of compact processing units (CPUS) for processing supernate and salt cake and a central facility for processing siudge. A discussion of cost estimates for this concept is given in an individual writeup for CLEAN that follows.

CLEAN Option process matching assumptions for central facility concept:

1. Cestum Ion Exchange--same as before

$$
\text { CSIX = CSIX (TRUEX-C). }
$$

2. Strontium Ion Exchange--same as technetium ion exchange

$$
\text { SRIX }=\text { TCIXI (TRUEX-C). }
$$

3. Technetium Ion Exchange--same as before

$$
\text { TCIX }=\text { TCIXI (TRUEX-C). }
$$

4. Acid ( $\mathrm{HNO}_{3}+0 \times a \mathrm{ic}$ ) Dissolution--set to $15 \%$ of previous TRUEX/SREX/technetium recovery cost

$$
\text { DISOLVA }=0.15^{\star}(\text { TRUEX+SREX+DISSOLVA+..) }(\text { TRUEX-C }) \text {. }
$$

5. Acid (HF+HNO ${ }_{3}$ ) Dissolution--same as 4; Acid Dissolution

$$
\text { DISOLVF = DISOLVA. }
$$

6. Actinide Extraction (TBP)--set to 1.33 times the purify uranium cost below

$$
\text { EXTRUPU }=1.33 *(\text { PURIFYU }) \quad(\text { TRUEX }-C)
$$


7. Purify Urantum--same as previous UREX process

$$
\text { PURIFYU = PURIFYU (TRUEX-C). }
$$

8. $A$ and Lanth Extraction (CMPO)--set to $50 \%$ of previous TRUEX/SREX/technetium recovery cost

$$
\text { EXTRAMLN }=0.50 *(\text { TRUEX+SREX+DISSOLVA+...) (TRUEX-C). }
$$

9. Amoricium and Lanth Ion Exchange--use general IX module

$$
\text { AMLANIX = TCIXI (TRUEX-C). }
$$

10. Strontium and Technetium Extraction--set to 35\% of previous TRUEX/SREX/technetium recovery cost

$$
\text { SREX }=0.35 *(\text { TRUEX+SREX+DISSOLVA+...) (TRUEX-C). }
$$

11. Strontium/Barium/Technetium Band Ion Exchange--use general IX module

$$
\text { SRBAIX = TCIXI (TRUEX-C). }
$$

12. Cesium Ion Exchange (APM)--use general IX module

$$
\text { APMCSIX - TCIXI (TRUEX-C). }
$$

13. Recover Acid From Evap Overheads--assume 50\% dissolver (evaporation+distillation)

$$
\text { RCOVACID }=0.50 * \text { DISOLVA. }
$$

14. Evaporation (HLW)--assumed to be small compared to other processes

$$
\text { EVAPORH = none. }
$$

15. Denitration (HLW)--(sugar denitration) dissolver in reverse

$$
\text { DNITRH = DISOLVA. }
$$

16. Denitration (LLW)--(sugar denitration) dissolver in reverse

$$
\text { DNITRL - DNITRH. }
$$

17. Calcination--calcining of LLW

$$
\text { CALCINE = CALCINE (TRUEX-C). }
$$

18. Remove Chromium--(probably a precipitation and centrifuge process)

$$
\text { REMCR = SEPSOLIQ. }
$$


19. Separate Solid/Liquid--water wash and centrifuge SEPSOLIQ - Sludge Wash A strategy.

20. Caustic Leaching--caustic wash and centrifuge

$$
\text { CAUSLEAC = SEPSOLIQ. }
$$

\section{Organic Destruction with SCHO}

$$
\begin{aligned}
& \text { DESTORG }=\text { (DESTORG+FILTADSB) (TRUEX-C) } \\
& \text { (Assume FILTADSB contribution small compared to DESTORG). }
\end{aligned}
$$

Following the summary results section and its associated figures and tables are individual writeups for the functions and point estimates used to develop costs for this report. The functions/estimates may be sorted into the following categories:

- Retrieval

- Retrieval (10-27-92)

- Pretreatment

- Sludge Wash A (3-27-93)

- Uranium Recovery (3-29-93)

- Cesium Separation To HLW (3-26-93)

- Plasma Torch Calcine (6-14-93)

- Strontium Cost Equation For Use With TRUEX and Sludge Wash Type Processes (3-27-93)

- TRUEX, SREX, Technetium Recovery (3-30-93)

- Technetium Ion Exchange (6-14-93)

- Low-Level Waste (LLW) Disposal

- Salt Grout Plant (4-5-94)

- Transportation and Disposal Cost For Class A LLW (1-4-93)

- Mixed Waste Incineration (4-6-93)

- Glass-in-Sulfur Plant (3-3-94)

- Waste Water Treatment

- Water Treatment Plant - Facility No. 8 (3-17-93)

- High-Level Waste Disposal

- HLW Disposal To Glass (5-11-93)

- Volume Scaleup For HLW Glass Production Plant (1-24-93)

- Transuranic Disposal

- WIPP Disposal (1-27-93) 
WHC-EP-0791

- In Situ Disposal

- In Situ Grout (3-23-93)

- Empty Tank and Past-Practice Unit Disposal

- Immobilization and Dome Filling (3-24-93)

- clean Option

- Clean (9-1-93)

- CPU COST

- Generic CPU Cost (8-30-93).

It may be noted that throughput capacity is often quoted in terms of "dry" throughput. This refers to flow with the free water subtracted out.

\section{F2.0 SUMMARY OF RESULTS}

Figures F1 and F2 show cost summary plots for the CLEAN Option and TRUEX $-C$ and Sludge Wash $C$ process strategies, respectively, with the primary LLW going to a salt grout product. Figures F3 and F4 show similar cost summary plots with the primary LLW going to a glass-in-sulfur product. These same cost data are given in a more detailed breakdown between capital and expense costs in Tables Fl through F4. The cost estimates in Tables Fl through $\mathrm{F} 3$ reflect the use of a central processing facility that processes all single- and double-shell tank waste with a 13-year campaign. Table F4 shows a cost estimate for the CLEAN Option process strategy based on using a combination of CPUs for processing supernate and salt cake and a central facility for processing sludge. Table $F 5$ gives a summary of the total primary waste and tertiary waste (i.e., on process waste streams or wastes that did not result directly from splitting or partitioning of the main process streams; see Appendix $E$ for a more detailed description) processing costs associated with each process strategy along with comparison ratios.

To further show the basis of the cost differences between the process strategies, the costs were combined into major process categories. These process categories are listed in Table $\mathrm{F} 6$ with their corresponding process block distribution (from Tables Fl through F4) for each process strategy. Table F7 presents a summary of the cost data for these major process categories by process strategy and primary LLW disposal option (i.e., salt grout or glass-in-sulfur products). Figures $F 5$ and $F 6$ show these same process category cost data for the primary LLW disposal to glass-in-sulfur and salt grout products, respectively. Table F8 presents differential process category costs compared to the CLEANG31 model (i.e., CLEAN Option without CPUs in Table F7). 
Note from Table F5 that the CLEAN Option total primary and tertiary waste processing costs and, hence, the overall cost are reduced by approximately 24\% when the CLEAN Option uses CPUs along with a central processing facility. Similar cost reductions could potentially apply to the TRUEX-C and sludge Wash C process strategies if similar CPU use is included.

Note that costs for both primary LLW salt grout and glass-in-sulfur product options are included for each process strategy. Because no LLW glass composition specifications have yet been formulated (Appendix G), high-level waste (HLW) glass composition specifications were al so applied to primary LLW glass. Note from Tables F1 through F5 that total LLW processing and disposal costs for the primary LLW to glass and to grout product options were essentially the same. Small increases in the tertiary salt grout costs occur for the primary LLW to glass product option because previousiy shared primary and tertiary LLW grout plant costs are now borne only by the tertiary LLW for this option.

Note that the costs associated with the HLW and LLW glass products can vary dramatically, depending on which glass optimization models and associated composition constraints are used (Appendix G). For example, the $\mathrm{P}_{2} \mathrm{O}_{5}$ constraint was relaxed for all process strategy glass product results. If this constraint were included, the HLW glass canister count would increase by factors of 1.7 and 3.6 (Appendix G, Tabie G7) for the TRUEX-C and Sludge Wash C process strategies, respectively, while the LLW glass vault count would increase by a factor of 1.2 for the CLEAN Option process strategy. The corresponding cost increases would be $\$ 1.6 \mathrm{~B}, \$ 17.5 \mathrm{~B}$, and $\$ 0.085 \mathrm{~B}$ for the TRUEX-C, STudge Wash C, and CLEAN Option process strategies, respectively.

From Table $F 5$ note the magnitudes of the costs associated with processing the primary waste stream and those associated with processing the tertiary nonprocess waste streams. These "primary" and "tertiary" costs are calculated separately. The tertiary waste processing costs were approximately $31,27,16$, and $30 \%$ of the total costs for the CLEAN Option without CPUS, TRUEX-C, sludge Wash C, and CLEAN Option with CPUs process strategies, respectively. These percentages essentially apply to either the primary LLW to grout or glass product options. The ratios of tertiary to primary costs were approximately $45,37,19$, and $44 \%$ for these same process strategies, respectively.

Because the CLEAN Option and TRUEX-C process strategies produce less HLW than the Sludge Wash C process strategy, the associated HLW glass-related costs are lower, especially for the CLEAN Option process strategy. The LLW grout-related costs are lowest for the CLEAN Option strategy because less LLW reports to grout than either the TRUEX-C strategy or Sludge Wash C process strategies. However, when the LLW is converted to oxides during vitrification, the CLEAN Option and TRUEX-C process strategies have nearly similar oxide stream mass inventories, while the Sludge Wash $C$ oxide stream is 25 and $22 \%$ 1ess in inventory than the CLEAN Option and TRUEX-C process strategies, respectively (Appendix G).

For the primary LLW glass product option, results in Table F5 show the CLEAN Option process strategy without CPUs had primary waste processing costs approximately $20 \%$ greater than those for the TRUEX-C process strategy, approximately $7 \%$ less than those for Sludge Wash $C$, and approximately $31 \%$ greater than those for the CLEAN Option with CPUS. However, the tertiary 
waste process costs for the CLEAN Option without CPUs were approximately $45 \%$ greater than those for the TRUEX-C process strategy, approximately $117 \%$ greater than those for the Sludge Wash C process strategy, and approximately 35\% greater than for the CLEAN Option with CPUs.

Overal1, the CLEAN Option without CPUs had total costs of \$41.2B, which were approximately $27 \%$ greater than those for the TRUEX-C process strategy (\$32.5B), approximately 13\% greater than those for the Sludge Wash C process strategy (\$36.4B), and approximately 32\% greater than the CLEAN Option with CPUs (\$31.1B). Cost comparison ratios for the primary LLW to grout option were comparable to those for the primary LLW to glass option stated above.

Note that al1 process strategy costs assume that a $0 \%$ heel was left in the waste tanks. Generally, if a $5 \%$ heel was assumed, the process strategy costs would be reduced by approximately 5 to $6 \%$ because of the reduced process stream flows (Knutson et a1. 1993).

From Table F7 note that the costs associated with tank waste retrieval and liquid waste partitioning are substantial (approximately \$8.1B) and are assumed to be the same for each process strategy. Costs associated with waste tank immobilization were estimated to be $\$ 0.43 \mathrm{~B}$, again, assumed to be the same for each process strategy. Note that the LLW treatment and disposal costs for the salt grout and glass-in-sulfur products were essentially the same.

Therefore, the following discussion will relate the costs associated with the primary LLW to glass product option because the results for the primary LLW to grout option were comparable.

Table $F 8$ shows that the costs for separation of solids and liquids along with solids dissolution for the CLEAN Option process strategy without CPUs required approximately 1.3 and 50.99B more than the TRUEX-C and S1udge Wash $C$ process strategies, respectively. The costs for nuclide removal from alkaline Tiquids for the CLEAN Option process strategy without CPUs required approximately 1.0 and $\$ 1.2 B$ more than the TRUEX $C$ and $S 7$ udge Wash $C$ process strategies, respectively. The costs for nuclide removal from acidic liquids for the CLEAN Option process strategy without CPUs required approximately 2.6 and \$4.2B more than the TRUEX-C and Sludge Wash C process strategies, respectively. The costs for removal and purification of bulk chemicals for the CLEAN Option process strategy without CPUs required approximately $\$ 4.2 B$ more than the TRUEX $C$ and Sludge Wash $C$ process strategies.

Combining these four processing categories together (item $\mathrm{Cl}$ in Tables $\mathrm{F7}$ and $F 8$ and Figures $F 5$ and $F 6$ ) showed that the CLEAN Option process strategy without CPUs required approximately 9.1, 10.6, and \$6.7B more than the TRUEX-C, Sludge Wash C, and CLEAN Option with CPUs process strategies, respectively. The trade-off for these higher processing costs for the CLEAN Option process strategy is seen in the reduced HLW and LLW processing and disposal costs as compared to the TRUEX-C and Sludge Wash $C$ process strategies. Items $\mathrm{C2}$ and $\mathrm{C} 3$ in Tables $\mathrm{F} 7$ and 58 show how the combined processing differential cost $\mathrm{Cl}$ is impacted by including the HLW and LLW processing and disposal costs. As shown in Table F8, the CLEAN Option process strategy without CPUs required approximately 4.6 and \$6.7B more than the TRUEX-C and CLEAN Option with CPUS pruiess strategies, respectively, but $\$ 2.4 B$ less than the Sludge Wash C process strategy for the primary LLW-to-glass option. 
However, the increased processing in the CLEAN Option process strategy generated more tertiary waste than the other process strategies. Thus, the tertiary waste treatment and disposal costs for the CLEAN Option process strategy without CPUs required approximately $4.0,6.9$, and $\$ 3.38$ more than the TRUEX-C, STudge Wash C, and CLEAN Option with CPUs process strategies, respectively (see item $C_{4}$ in Table F8). Consequently, reductions in the HLW and LLW treatment and disposal costs for the CLEAN Option process strategy compared to the other process strategies were offset by the increased cost of tertiary waste treatment and disposai.

In summary, the cost savings associated with CPU use was significant and should be incorporated into any process strategy. The large tertiary waste costs could be decreased significantly if process design is also focused on reducing the amount of tertiary waste produced. More glass composition constraint evaluation and experimentation on key waste oxide stream components could allow relaxing the current constraints, resulting in significantly reduced $H L W$ glass canister and $L L W$ glass vault counts (i.e., higher waste oxide loadings in glass) and their associated costs. The trade-off for the higher processing costs for the CLEAN Option process strategy without CPUS is seen in the reduced $H L W$ and $L L W$ processing and disposal costs as compared to the TRUEX-C and Sludge Wash $C$ process strategies. However, the increased processing in the CLEAN Option process strategy generated more tertiary waste and higher associated treatment and disposal costs, offsetting the reductions in the HLW and LLW treatment and disposal costs.

\section{F3.0 REFERENCES}

Boomer, K. D., et a T., 1993, Tank Waste Technical Options Report, WHC-EP-0616, pp. 7-51, Westinghouse Hanford Company, Richland, Washington.

Knutson, B. J., et a1., 1993, "Advanced Chemical Separation Study: TRUEX Strategy Compared to Sludge Wash Ion Exchange," DRAFT letter report, July 1993, Westinghouse Hanford Company, Richland, Washington. 


\section{RETRIEVAL}

10-27-92

Estimates of this cost can be obtained from both the Hanford Strategic Analysis (HSA) project work ${ }^{(1)}$ and from data contained in the decision analysis computer model developed for the Tank Waste Remediation System (TWRS) program.

\section{HANFORD STRATEGIC NMALYSIS}

The equation developed to support HSA ${ }^{(1)}$ is as follows:

$$
\text { Total cost }=\$ 5.7 e 9\left(1-S F \_H E E L_{-} T C\right)-\$ 3.47 e 8 \mathrm{ln}\left(\mathrm{SF} \_\right. \text {HEEL_TC), }
$$

where SF HEEL TC is the separation factor (fraction) of technetium left in the "heel" of waste remaining in the tank after retrieval. This equation was originally based on information from Bob Parazin (2) and included cost for both single-shell tanks (SSTs) and double-shell tanks (DSTs).

This equation produces the following values:

\begin{tabular}{cc} 
Separation factor & Cost \\
\hline 1.0 & $\$ 0$ \\
0.01 & $\$ 7.3 \mathrm{~B}$ \\
0.001 & $\$ 8.2 \mathrm{~B}$
\end{tabular}

There is no data to support use of this equation outside these ranges.

\section{THRS DECISION ANALYSIS SPREADSHEET MODEL}

The TWRS spreadsheet includes the following cost data for tank retrieval. (The spreadsheet provides best estimate values, pessimistic values, and optimistic values.)

\begin{tabular}{|c|c|c|c|c|}
\hline $\begin{array}{l}\text { Pessimistic } \\
\text { Best estimate } \\
\text { Optimistic }\end{array}$ & $\begin{array}{l}\text { RT1 } \\
15 \% \\
10 \% \\
5 \%\end{array}$ & $\begin{array}{l}\text { Estima } \\
\text { RT2 } \\
5 \% \\
1 \% \\
0.1 \%\end{array}$ & $\begin{array}{r}\text { ted HEEL } \\
\text { RT3 } \\
1 \% \\
0.1 \% \\
0.05 \%\end{array}$ & $\begin{array}{r}\text { RT4 } \\
1 \% \\
0.1 \% \\
0.05 \%\end{array}$ \\
\hline $\begin{array}{l}\text { Pessimistic } \\
\text { Best estimate } \\
\text { Optimistic }\end{array}$ & $\begin{array}{l}\text { RT1 } \\
\text { \$4OM } \\
\$ 20 M \\
\text { \$10M }\end{array}$ & $\begin{array}{c}\text { Estimated DST } \\
\text { RT2 } \\
- \\
- \\
-\end{array}$ & $\begin{array}{c}\text { Per Tank Cost }{ }^{(3)} \\
\text { RT3 } \\
\$ 250 M \\
\$ 100 M \\
\$ 50 M\end{array}$ & $\begin{array}{c}\text { RT4 } \\
- \\
- \\
-\end{array}$ \\
\hline $\begin{array}{l}\text { Pessimistic } \\
\text { Best estimate } \\
\text { Optimistic }\end{array}$ & $\begin{array}{c}\text { RTI } \\
- \\
- \\
-\end{array}$ & $\begin{array}{c}\text { Estimated SST } \\
\text { RT2 } \\
\$ 35 M \\
\$ 30 M \\
\$ 25 M\end{array}$ & $\begin{array}{c}\text { Per Tank Cost } \\
\text { RT3 } \\
\$ 15 M \\
\$ 10 M \\
\$ 5 M\end{array}$ & $\begin{array}{l}\text { RT4 } \\
\text { S25M } \\
\text { S20M } \\
\$ 15 M\end{array}$ \\
\hline
\end{tabular}


where: $R T 1$ - Mixer pump retrieval system.

RT2 = Mechanical retrieval system.

RT3 = High-volume sluice retrieval system.

RT4 = Hydraulic or pneumatic retrieval system.

SST = Single-shell tank.

DST = Double-shell tank.

Because high-volume sluice is the only system currently considered feasible for both SSTs and DSTs ("-" indicates not currently considered feasible), that option is focused on, and best estimate separation factors are used.

Assuming 28 DSTs and 149 SSTs are retrieved:

$$
\begin{aligned}
& \text { Cost (pessimistic) }=\$ 250 M(28)+\$ 15 M(149)=\$ 9.2 B(\text { dtTc }=0.001) \\
& \text { Cost (best estimate) }=\$ 100 M(28)+\$ 10 M(149)=\$ 4.3 B(n) \\
& \text { Cost (optimistic) }=\$ 50 M(28)+\$ 5 M(149)=\$ 2.1 B(")
\end{aligned}
$$

Note that the spreadsheet recognizes the existence of aluminum, chromium, sodium, nicke1, $\mathrm{NO}_{3}$, cesium, strontium, technetium, and transuranic in the tanks. However, the retrieval separation factors are treated as the same for all isotopes.

\section{AMALYSIS}

The range of costs for SF HEEL TC derived from the spreadsheet data (\$2.1B to \$9.2B) is consistent with ${ }^{-}$the HSA equation estimate of $\$ 8.2 \mathrm{~B}$ for this separation factor. Therefore, there is no reason not to continue using this equation.

Consistent with the spreadsheet assumption, the SF Heel separation factor value is considered to be the same for all isotopes.

\section{REFERENCES}

1. Tank Retrieval, Bruce Zimmerman, personal notes for HSA cost functions, February 26, 1992, Westinghouse Hanford Company, Richland, Washington.

2. "SST Closure Estimate for Sensitivity Study," Bob Parazin, personal communication, January 24, 1992, Stabilization and Retrieval Engineering, Westinghouse Hanford Company, Richland, Washington.

3. Reick, C. A., WHC-SD-W211-CDR-011, Rev. 0, Conceptual Design Report, Initial Tank Retrieval System, Project $W-211$, Westinghouse Hanford Company, Richland, Washington. 


\section{SLUDGE MASH A}

$$
\text { 3-27-93 }
$$

The relevant data are as follows:
Capital
Expense
Dry throughout
S0.96B(1)
$\$ 1.13 B^{(1)}$
$5.4 \mathrm{E}+4 \mathrm{~kg} / \mathrm{day} *$

*The total flow into Sludge Wash A (SWA) per operating day is $2.7 \mathrm{E}+5 \mathrm{~kg} / O D$ (kilograms per operating day), the water portion is $1.8 \mathrm{E}+5 \mathrm{~kg} / 0 \mathrm{D}$, so the "dry" flow is $9 \mathrm{E}+4 \mathrm{~kg} / 0 \mathrm{D}$. Because the operating efficiency is assumed to be 60\%, the dry flow per 24-hour day is $5.4 \mathrm{E}+4 \mathrm{~kg} / \mathrm{day}$. Note that these flows correspond to processing a homogeneous mixture of single- and double-shell tank waste in 13 years ${ }^{(2)}$.

These cost values correspond to the following separations:

$$
\begin{array}{ll}
\text { SF_HLW_TC } & 0.23^{(3)} \\
\text { SF_HLW_CS } & 0.11^{(3)} \\
\text { SF_HLW_Sr } & 0.99^{(3)} \\
\text { SF_HLW_Na } & 0.02^{(4)} \\
\text { SF_HLW_AT } & 0.47^{(4)}
\end{array}
$$

where, for example, SF HLW tc refers to the separation factor (fraction) of technetium into the high-lēvel waste stream.

The capacity scaling is done using a standard 0.7 exponent for capital equipment ${ }^{(5)}$ and 0.75 for expense costs ${ }^{(6)}$.

$\$ c a p(B)=\$ 0.96 B($ actual daily dry throughput $/ 5.4 E+4 \mathrm{~kg} /$ day $) * * 0.7$

$\operatorname{Sexp}(B)=\$ 1.13 B$ (actual daily dry throughput $/ 5.4 \mathrm{E}+4 \mathrm{~kg} / \mathrm{day}) * * 0.75$

\section{REFERENCES}

1. Boomer, et al., 1993, Tank Waste Technical Options Report, WHC-EP-0616, p. 7-51, Westinghouse Hanford Company, Richland, Washington.

2. "Separations Data," personal communication (cc:mail) from Eric Slaathaug to Bruce Zimmerman, dated March 30, 1993, Westinghouse Hanford Company, Richland, Washington.

3. Ibid. 1, p. 7-11.

4. Ibid. 1, p. 7-12. 
5. Remer, D. S., and L. H. Chai, August 1990, "Design Cost Factors For Scaling-Up Engineering Equipment," Chemical Engineering Progress, American Institute of Chemical Engineers, New York, New York, p. 82.

6. Black, J. H., "Scaling Operating Costs By Cost-Capacity Factors," 1982 Transactions of the American Association of Cost Engineers 26th Annual Meeting, June 27 - June 30, 1982, Houston, Texas, H.3.1. 


\section{URANIUH RECOVERY}

\section{3-29-93}

The TRUEX-C and TRUEX-D chemical separation processes have previously been costed in detall. TRUEX-C is approximately the same as TRUEX-D, except that TRUEX-C contains a uranium recovery module and an organic destruction/ titinate (SCWO) module that TRUEX-D does not. Consequentiy, the cost of the uranium recovery module (UREX) can be estimated as:

$$
\text { UREX }=(\text { TRUEX-C) - (SCWO) - (TRUEX-D). }
$$

The relevant data are ${ }^{(1)}$ as follows:

\begin{tabular}{cccr} 
& IRUEX-C & SCWO & IRUEX-D \\
Capital cost & $\$ 2.69 \mathrm{~B}$ & $\$ 0.44 \mathrm{~B}$ & $\$ 2.10 \mathrm{~B}$ \\
Expense cost & $\$ 2.66 \mathrm{~B}$ & $\$ 0.13 \mathrm{~B}$ & $\$ 2.48 \mathrm{~B}$ \\
\multicolumn{4}{c}{$($ SCWO $=$ supercritical water oxidation) }
\end{tabular}

So, for UREX:

$$
\begin{array}{ll}
\text { Capital cost } & \$ 0.15 B \\
\text { Expense cost } & \$ 0.05 B
\end{array}
$$
TRUEX-C ${ }^{(2)}$.

It was assumed that the uranium separation fraction to $L L W$ is $1 \%$ for

The total flow rate into the UREX module of TRUEX $-C$ is $2.73 \mathrm{E}+4 \mathrm{~kg} / O D$ (kilograms per operating day), the water flow is $2.51 \mathrm{E}+4 \mathrm{~kg} / 0 \mathrm{D}^{(3)}$, so the "dry" flow is $2.2 \mathrm{E}+3 \mathrm{~kg} / 0 \mathrm{D}$. Because the assumed operating efficiency is $60 \%$, the dry flow per 24-hour day is $1.32 \mathrm{E}+3 \mathrm{~kg} / \mathrm{day}$. This flow assumes that the homogenized single- and double-shell tank waste is processed in 13 years. These flows are expected to be approximately the same as the flows for processing only single-shell tank waste in 10 years.

The capacity scaling is done using the standard 0.7 exponent for capital equipment ${ }^{(4)}$ and 0.75 for expense costs ${ }^{(5)}$ :

$$
\begin{gathered}
\$ c a p(B)=\$ 0.15 \text { (actual daily dry feed into the UREX module/ } \\
1.32 \mathrm{E}+3 \mathrm{~kg} / \text { day) } \star \star 0.7
\end{gathered}
$$

\section{REFERENCES}

1. Boomer, et al., 1993, Tank Waste Technical Report Options, WHC-EP-0616, p. 7-51, Westinghouse Hanford Company, Richland, Washington.

2. Ibid., 1 p. 7-11. 
3. "Separations Data," personal communication (cc:mail) from Eric Slaathaug to Bruce Zimmerman, dated March 30, 1993, Westinghouse Hanford Company, Richland, Washington.

4. Remer, D. S., and L. H. Cha1, August 1990, "Destgn Cost Factors For Scaling-Up Engineering Equipment," Chemical Engineering Progress, American Institute of Chemical Engineers, New York, New York, p. 82.

5. Black, J. H., "Scaling Operating Costs By Cost-Capacity Factors," 1982 Transactions of the American Association of Cost Engineers 26th Annual Meeting, June 27 - June 30, 1982, Houston, Texas, H.3.1. 


\section{CS SEPARATION TO HLH 3-26-93}

The Sludge Wash A (SWA) and Sludge Wash B (SWB) processes have previously been costed in detail. Sludge Wash $A$ and Sludge Wash $B$ are the same, except that Sludge Wash B includes a cesium ion exchanger. Consequently, the difference in these costs represents the cost of the ion exchanger.

\section{BASIC DATA}

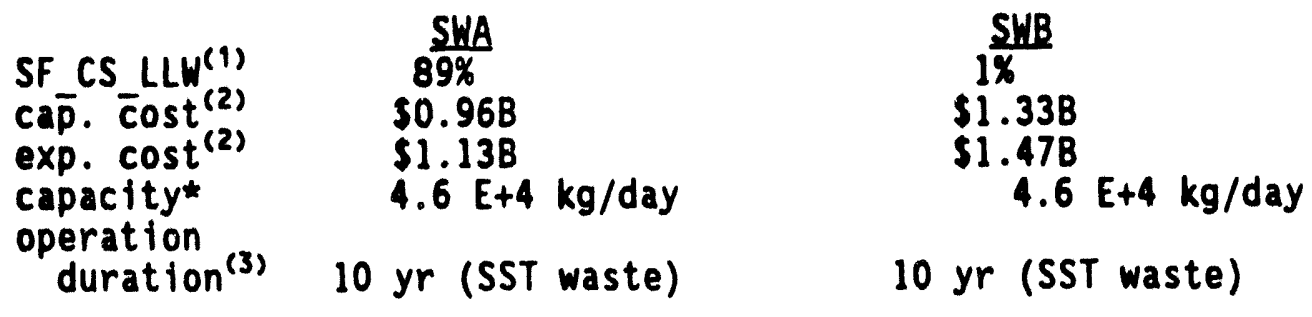

*Assumed capacity for this stream is $2.89 \mathrm{E}+5 \mathrm{~kg} / 00$ (kilograms per operation day), $2.13 \mathrm{E}+5 \mathrm{~kg} / 0 \mathrm{D}$ is water, so the dry design capacity is $7.6 \mathrm{E}+4$ $\mathrm{kg} / 0 D^{(4)}$. However, at $60 \%$ assumed efficiency, the actual dry throughput per day, is $4.6 \mathrm{E}+4 \mathrm{~kg} / \mathrm{day}$. The process is sized to treat all single-shell tank waste in 10 years $(3)(4)$, which is approximately the same as processing all single- and double-shell tank waste in 13 years.

SF CS LLW = the separation factor (fraction) of cesium that goes to the low-levēl waste stream.

The costs to be assigned to the cesium ion exchanger are then:

$$
\begin{aligned}
& \text { Capital cost }=\$ 0.37 \mathrm{~B} \\
& \text { Expense cost }=\$ 0.34 \mathrm{~B}
\end{aligned}
$$

\section{ION EXCHANGER FUNCTIONAL FORM}

Based on the derivation in Reference 5 , the generic functional form for ion exchanger cost versus separation factor is as follows:

$$
S=A *(1-\ln (S F)) \star \star 0.55-A
$$

where: $S F=0$ means perfect separation. In the context here, SF is SF_CS_LLW.

Normally, $S F=1$ (no separation) would correspond to so. In the present instance, however, SF=0.89 corresponds to 50 ion exchanger cost, because no ion exchanger is needed to get $S F=0.89$. The equation's offset must be something different than "A":

$$
S=A^{*}(1-\ln (S F)) \star \star 0.55+B .
$$


Determining the constants " $A$ " and " $B$ " for both capital cost and expense cost (assuming that capital and expense costs would scale similarly with separation factor), gives the following:

$$
\begin{aligned}
& \text { Capital cost(SB): } \quad S=\$ 0.244\left(1-\ln \left(S S_{-} C_{-} S_{L} L W\right)\right) * * 0.55-\$ 0.259 \\
& \text { Expense } \operatorname{cost}(\$ B): \quad S=\$ 0.224\left(1-\ln \left(S F \_C S \_L L W\right)\right)^{\star * 0.55}-\$ 0.238
\end{aligned}
$$

Including a capacity scaling term of 0.55 for the capital cost (assumes throughput is proportional to resin bed size ${ }^{(5)}$ ) and 0.75 for the expense cost ${ }^{(8)}$ :

$$
\begin{aligned}
& \text { Capital cost: } \quad \$=\{\$ 0.244(1-\ln (S F \text { CS LLW }) * \star 0.55-\$ 0.259\} * \\
& \text { \{actual daily dry throughput } / 4.6 \mathrm{E}+4 \mathrm{~kg} / \text { day } * \star 0.55 \\
& \text { Expense cost: } \quad S=\left\{\$ 0.224(1-\ln (S F \text { CS_LLW }))^{\star *} 0.55-\$ 0.238\right\}^{*} \\
& \text { \{actual datly dry throughput } / 4.6 \mathrm{E}+4 \mathrm{~kg} / \text { day }\} * * 0.75
\end{aligned}
$$

\section{REFERENCES}

1. Boomer, K. D., et a1., 1993, Tank Waste Engineering Study, WHC-EP-0616, P. 7-11, Westinghouse Hanford Company, Richland, Washington.

2. Ibid. ref.1, p. 7-51.

3. Ibid. ref.1, p, 7-22, 7-24.

4. "Sludge Wash B," personal communication (cc:mail) from Eric Slaathaug to Bruce Zimmerman, dated March 26, 1993, Westinghouse Hanford Company, Richland, Washington.

5. "Ion Exchanger Generic Function Derivation," B. Zimmerman personal notes, December 29, 1992, Westinghouse Hanford Company, Richland, Washington.

6. Black, J. H., "Scaling Operating Costs By Cost-Capacity Factors", 1982 Transactions of the American Association of Cost Engineers 26th Annual Meeting, June 27 - June 30, 1982, Houston, Texas, H.3.1. 


\section{PLASMA TORCH CALCINE \\ 6-14-93}

As suggested in references 1 and 2 , this process can be thought of as decomposable into the following separate steps: calcine, offgas treatment, dissolution, technetium ion exchange, cesium ion exchange.

Recognizing that the dissolution, technetium ion exchange, and cestum ion exchange steps are collectively similar to the previously costed Siudge Wash C process ${ }^{(2)}$, and that the overall plasma torch calcine facility may be comparable to the previousiy costed TRUEX-D facility(2), the following rough cost estimates can be made.

\begin{tabular}{|c|c|c|c|c|}
\hline & calcine/offoas" & dissolution* & ICIX $x^{\star \star}$ & $\operatorname{csi}\left(x^{\star \star * 1}\right.$ \\
\hline Capital & $50.78^{(2)}$ & $\$ 1.4 B$ & \$0.07B & S0.37B \\
\hline \multicolumn{3}{|c|}{ (Including allowance for D\&D) } & $\$ 0.34 B$ & $\$ 0.34 B$ \\
\hline \multicolumn{5}{|c|}{$\begin{array}{l}0.54 \text { value determined as } 2.48-1.26-0.34-0.34^{(3)} \text {; the } 2.48 \\
\text { number is for TRUEX-D } \\
\text { values determined as (Sludge Wash C) - (TcIX) - (CSIX)(2)(3) } \\
\text { values determined as (Sludge Wash D) } \text { ( Sludge Wash B)(3) } \\
\text { values determined as (Sludge Wash B) - (Sludge Wash A)(3) }\end{array}$} \\
\hline
\end{tabular}

Based on inspection of ASPEN PLUS flow sheet results ${ }^{(4)}$, it appears that essentially all the sodium and aluminum separation occurs in the dissolution step. For sodium and aluminum, the separation is estimated to be about $5 \%$ to high-level waste (HLW) and $95 \%$ to the low-level waste (LLW) stream, while the technetium and cesium are mostly passed through to the ion exchangers ${ }^{(4)(5)}$.

\section{DISSOLUTION}

Developing a functional form for wash dissolution as a function of sodium separation gives:

Input data: So capital, so expense implies 1.0 of $\mathrm{Na}$ to $\mathrm{HLW}$ $\$ 1.4 \mathrm{~B}$ capital, \$1.26B expense implies 0.05 of $\mathrm{Na}$ to HLW (actually applies to both aluminum and sodium) 


\section{Sodium}

Resulting equations:

$$
\begin{aligned}
& \text { Capita) \$B }=-\$ 1.47 B(S F \text { CALC HLWNA }+\$ 1.47 B \\
& \text { Expense \$B }=-\$ 1.33 B(S F \text { CALC_HLWNA }+\$ 1.33 B
\end{aligned}
$$

The 1 inear form was suggested by George Jansen ${ }^{(6)}$, along with the restriction that SF_CALC_HLW_NA should be restricted to the range 0.01 to 0.1 .

Note that the expense costs include an allowance for $D \& D^{(2)}$.

\section{Ion Exchange}

For technetium and cesium ion exchange the previousiy derived equations $^{(7)(8)}$ were used:

IC

$$
\begin{aligned}
& \text { capital SB }=\left[0 . 0 4 8 B \left(1-\ln (1-S F \text { HLW TC) } * \star 0.55-\$ 0.055]^{\star}\right.\right. \\
& \text { \{actual daily dry throughput } / 0.486 \mathrm{E}+5 \mathrm{~kg} / \text { day } * * 0.55
\end{aligned}
$$

$\underline{\text { Cs }}$

$$
\begin{aligned}
& \text { Capital SB }=\left\{\$ 0.244(1-\ln (1-S F \text { HLW CS }))^{\star \star 0} 0.55-\$ 0.259\right\} \\
& \text { (actual daily dry throughput } / 4.6 \mathrm{E}+4 \mathrm{~kg} / \text { day\} } \star \star 0.55 \\
& \text { Expense SB }=\left\{\$ 0.224(1-\ln (1-S F \text { HLW CS }))^{\star * 0.55-\$ 0.238}\right\}^{*} \\
& \text { \{actual daily dry throughput } / 4.6 \mathrm{E}+4 \mathrm{~kg} / \text { day } * \star 0.75
\end{aligned}
$$

\section{VOLUME CORRECTION TERMS}

It is assumed that the reference case throughput for the ion exchange terms of $4.6 \mathrm{E}+4 \mathrm{~kg} /$ day dry feed $=1.68 \mathrm{E}+7 \mathrm{dry} \mathrm{kg} / \mathrm{yr}$, as specified above. For the caicine, offgas, and dissolution steps, the throughput for the reference case was calculated as follows:

233 Mkg of original total "dry" waste (salt cake and sludge) ${ }^{(9)}$ divided by 13 years assumed total processing time ${ }^{(2)}$

\section{$1.79 \mathrm{E}+7 \mathrm{~kg} / \mathrm{yr}$}

The following scaling exponents were used to scale for throughput(10)(11):
All expense terms
Calcine step capital term
Dissolution step capital term
0.75 (personnel)
0.8 (furnaces)
Ion exchange capital terms
0.8 (centrifuges)
0.55 (ion exchangers) 
Putting all the above components together produces the final equation:

$S B=\$ 0.7 B^{*}$ (actual annual dry throughput/1.79 E+7 kg/yr)**0.8

$+\$ 0.54 B^{\star}$ (actual annual dry throughput/1.79 E+7 kg/yr)**0.75

$+\left[-\$ 1.47 B^{\star}(\right.$ SF CALC HLW_NA) $\$ 1.47 B]$ (actual annual dry throughput $\left./ \mathrm{T} .79^{-} \mathrm{E}+7 \mathrm{~kg} / \mathrm{yr}\right) \approx * 0.8$

$+\left[-\$ 1.26 B *\left(S F \_C A L C \_H L W \_N A\right)+\$ 1.26 B\right]$ (actual annual dry throughput $/ 1.79 \mathrm{E}+7 \mathrm{~kg} / \mathrm{yr}) * * 0.75$

$+\{0.0488(1-\ln (1-S F$ HLW_TC)**0.55 - \$0.055 \}*

\{actual daily dry throughput/0.486 E+5 kg/day\}**0.55

$\left.+\left\{\$ 0.235(1-\ln (1-S F \text { HLW_TC }))^{\star * 0.55-\$ 0.267}\right\}\right\}^{*}$

\{actual dail $\bar{y}$ dry throughput $/ 0.486 \mathrm{E}+5 \mathrm{~kg} /$ day $* * 0.75$

$+\{\$ 0.244(1-\ln (1-S F$ HLW_CS $)) * * 0.55-\$ 0.259\}$

\{actual daily dry throughput/4.6 E+4 kg/day\} $\star \star 0.55$

$+\{\$ 0.224(1-\ln (1-S F$ HLW CS $)) * * 0.55-\$ 0.238\} *$

\{actual dail $\bar{y}$ dry throughput $/ 4.6 \mathrm{E}+4 \mathrm{~kg} /$ day $* \star 0.75$

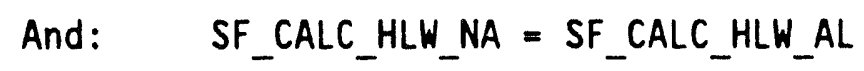

where: SF_CALC_HLW_NA should be restricted to the range 0.01 to 0.1 .

It is assumed that annual dry throughput = daily dry throughput $X 365$.

\section{REFERENCES}

1. Evaluation of Hanford Tank Waste Treatment Alternatives:

Calcination/Dissolution Compared To TRUEX-A and sludge Wash $C$,

Figure Bl, September 1992, Systems Technology, Westinghouse Hanford Company, Richland, Washington.

2. "Cost Estimate For Calcine Facility," meeting notes, December 11-15, 1992, Zimmerman, B. D., Westinghouse Hanford Company, Richland, Washington.

3. Boomer, K. D., et al., 1993, Tank Waste Technical Options Report, WHC-EP-0616, p. 7-51, Westinghouse Hanford Company, Rich 1and, Washington.

4. Ibid ref. 1., Table B2. 
5. George Jansen, personal computer database printout for ASPEN PLUS blocks A23221111 and A23221112, Dec. 16, 1992, Westinghouse Hanford Company, Richland, Washington.

6. Personal communication, G. Jansen to B. Zimmerman, Dec. 15, 1992, Westinghouse Hanford Company, Richland, Washington.

7. "Tc Ion Exchange," personal notes, B. Zimmerman, June 14, 1993, Westinghouse Hanford Company, Richland, Washington.

8. "Cs Separation To HLW," personal notes, B. Zimmerman, March 26, 1993, Westinghouse Hanford Company, Richland, Washington.

9. "Table 1. Tank Waste Inventory For TWRS Parametrics," personal communication, R. Harmsen to B. Zimmerman, June 1993, Westinghouse Hanford Company, Richland, Washington.

10. Remer, D. S., and L. H. Chai, August 1990, "Design Cost Factors For Scaling-Up Engineering Equipment," Chemical Engineering Progress, American Institute of Chemical Engineers, New York, New York, pp. 77-82.

11. Black, J. H., "Scaling Operating Costs By Cost-Capacity Factors," 1982 Transactions of the American Association of Cost Engineers 26th Annual Meeting, June 27 - June 30, 1982, Houston, Texas, H.3.1. 


\section{STRONTIUN COST EQUATION FOR USE HITH SLUDGE MASH AND TRUEX TYPE PROCESSES 3-27-93}

In the typical Sludge Wash and transuranic solvent extraction process (TRUEX) separation processing trains, the first process is a sludge wash (followed by ion exchanges, solvent extractions for specific isotopes, etc.). A Sludge Wash alone will typically produce a strontium separation to the lowlevel waste (LLW) steam (SF SR LLW) of approximately $1 \%(1)$. When the TRUEX step is added into the high-level waste (HLW) stream to separate the transuranics (TRUS) from the HLW, a so-called strontium extraction (SREX) (a solvent extraction) is also added into the HLW stream to prevent the transuranic extraction from returning large amounts of strontium to the LLW stream.

For the analysis here, it is assumed that an SREX module is always bundled with the transuranic extraction process in the HLW stream and is costed as part of the transuranic extraction. Variations in the overall strontium separation (of which TRUEX-SREX are one part) are, therefore, achieved by adding strontium separation modules to the LLW stream. These strontium separation modules are expected to be of either the supercritical water oxidation (plus titinate bed) type or the ion exchange type.

Supercritical water oxidation (SCWO) is primarily a precipitation process. Precipitation processes tend to exhibit a linear relationship between cost and separation factor, at least over a limited range ${ }^{(2)}$.

Because the sludge wash step alone produces a strontium separation to LLW (SF SR LLW) of $1 \%$, the $1 \%$ is considered to be obtained "for free." The cost function derived below deals with separations beyond this $1 \%$.

Assume, as is typical of the processes described in reference 1, that SCWO would be employed to reduce SF SR LLW from $1 \%$ to approximately $0.1 \%$. The cost for an SCWO module can be estimated as follows.

SWC SWD

$\begin{array}{lll}\text { components: } & \text { Sludge Wash } & \text { Sludge Wash } \\ \text { CSIX } & \text { CSIX } \\ & \text { SCWO } & \\ & \text { TCIX } & \text { TXIX }\end{array}$

$\begin{array}{lll}\text { Capital cost } & \$ 1.84 B & \$ 1.40 B \\ \text { Expense cost } & \$ 1.94 B & \$ 1.81 B\end{array}$

All of these cost figures are from reference 3 .

Then, the cost of an SCwo module must be about:

$\begin{array}{ll}\text { Capital cost } & \$ 0.44 B \\ \text { Expense cost } & \$ 0.13 B\end{array}$


Using the linear form for SCWO with the values:
separation factor
SF_SR_LLW
0.01
0.001
Capital cost
\$o
Expense cost
\$O
\$0.44B
\$0.13B

produces the following two equations:

Capital cost $(\$ B)=-\$ 48.9 B * S F S R L L W+\$ 0.489 B$

Expense cost $(S B)=-\$ 14.4 B * S F_{-}^{-} S R_{-}^{-} L L W+\$ 0.144 B$

These equations are good for SF_SR_LLW in the range 0.01 to 0.001 .

It is reasonable to assume that separation better than 0.001 would achieved by adding a Sr ion exchanger into the LLW stream ${ }^{(4)}$. This possibility is not considered here at this time.

\section{CAPACITY}

The design capacity for the SCWO system is $2.78 \mathrm{E}+5 \mathrm{~kg} / 0 \mathrm{D}$ (kilograms per operating day) (5). $2.02 \mathrm{E}+5 \mathrm{~kg}$ of this is water, so the "dry" flow rate is $7.6 \mathrm{E}+4 \mathrm{~kg} / 0 \mathrm{D}$. Because the plant is only expected to have an operating efficiency of $60 \%$, the actual average throughput in a 24-hour period would be $7.6 \mathrm{E}+4 \mathrm{~kg} * 0.6=4.6 \mathrm{E}+4 \mathrm{dry} \mathrm{kg} /$ day.

For capital cost scaling a capacity scaling factor of 0.7 is appropriate ${ }^{(6)}$. This is a typical value because a more specific value for this process is not ayailable. For expense cost scaling a scaling factor of 0.75 will be assumed $^{(7)}$.

Employing these factors gives:

$$
\begin{aligned}
& \text { Total cost }(S B)=\{-\$ 48.9 * S F \text { SR } L L W+\$ 0.489\} \text { actual daily dry } \\
& +\{-\$ 14.4 * S F S R L L W+\$ 0.144\} \text { actual daily dry } \\
& \text { throughput } / 4 . \overline{6} \mathrm{E}+4 \text { dry } \mathrm{kg} / \text { day }\} * 0.75
\end{aligned}
$$

where: SF SR LLW is restricted to be $<=0.01$, and where there is no design dat $\bar{a}$ t $\overline{0}$ support SF_SR_LLW $<0.001$.

Note that these flow values are based on the assumption that homogeneousiy mixed single-shell and double-shell tank waste is processed in 13 years $(5)$. This is expected to be approximately the same flow as required to process only single-shell tank waste in 10 years. 


\section{REFERENCES}

1. Boomer, K. D., et a1., 1993, Tank Waste Technical Options Report, WHC-EP-0616, p. 7-11, Westinghouse Hanford Company, Richland, Washington.

2. Personal communication, G. Jansen to B. Zimmerman, Jan. 15, 1993, Westinghouse Hanford Company, Richland, Washington.

3. Ibid. ref.1, p. 7-51.

4. How Would One Extend The Strontium Separation For Sludge Wash Type Processes?," meeting notes, Jan. 18, 1993, B. D. Zimmerman, Westinghouse Hanford Company, Richland, Washington.

5. "Separations Data," personal communication (cc:mail) from Eric S1aathaug to Bruce Zimmerman, dated March 30, 1993, Westinghouse Hanford Company, Richland, Washington.

6. Remer, D. S., and L. H. Chai, August 1990, "Design Cost Factors For Scaling-Up Engineering Equipment," Chemical Engineering Progress, American Institute of Chemical Engineers, New York, New York, p. 82.

7. Black, J. H., "Scaling Operating Costs By Cost-Capacity Factors," 1982 Transactions of the American Association of Cost Engineers 26th Annual Meeting, June 27 - June 30, 1982, Houston, Texas, H.3.1. 


\section{TRUEX, SREX, TECHNETIUA RECOVERY \\ 3-30-93}

The primary difference between TRUEX-D and Sludge Wash $D$ is the TRUEX, SREX, and technetium recovery modules. Consequently, these two previously costed waste processing configurations can be used to estimate the cost of these modules.

The relevant data are is follows:

TRUEX-D
STudge wash
Cesium ion exchange
Technetium Ion exchange
TRUEX
SREX
Technetium Recovery

capital cost ${ }^{(1)}$ expense cost ${ }^{(1)}$

Sr sep. to on-site(2) $U$ sep. to on-site $(2)$ Pu sep. to on-site ${ }^{(2)}$
$\$ 2.18$ $\$ 2.48 \mathrm{~B}$

\section{Sludge Wash D \\ STudge wash} Cesium ion exchange Technetium Ion Exchange

"Sep. to onsite" refers to the fraction of the isotope that remains in the low-level waste stream that is currently assumed to be disposed of on site.

From reference 3 , the total flow rate into the TRUEX module of TRUEX-D is $5.83 \mathrm{E}+4 \mathrm{~kg} / 0 \mathrm{D}$ (kilograms per operating day), the water flow is $4.15 \mathrm{E}+4 \mathrm{~kg} / 0 \mathrm{D}$. Therefore, the "dry" flow is $1.68 \mathrm{E}+4 \mathrm{~kg} / 0 \mathrm{D}$. Because the assumed operating efficiency is $60 \%$, the dry flow per 24-hour day is

$1.01 \mathrm{E}+4 \mathrm{~kg} / \mathrm{day}$. These flows are based on the assumption that all single- and double-shel1 tank waste is processed in 13 years. These flows are expected to be approximately the same as the flows for processing single-shell tank waste only in 10 years. then:

The cost to be assigned to the TRUEX/SREX/Technetium recovery modules is

$$
\begin{aligned}
& \text { Capital cost }=\$ 0.7 \mathrm{~B} \\
& \text { Expense cost }=\$ 0.67 \mathrm{~B}
\end{aligned}
$$


The capacity scaling is obtained using a standard 0.7 exponent for capital equipment ${ }^{(4)}$ and 0.75 for expense costs ${ }^{(5)}$ :

\$Capital(B) = \$0.7B (actual daily dry feed into TRUEX module/1.01 E+4 $\mathrm{kg} /$ day) $* \star 0.7$

SExpense $(B)=\$ 0.67 B$ (actual daily dry feed into TRUEX module/1.01 E+4 $\mathrm{kg} / \mathrm{day}) \star \star 0.75$

\section{REFERENCES}

1. Boomer, K. D., et al., 1993, Tank Waste Technical Options Report, WHC-EP-0616, p. 7-51, Westinghouse Hanford Company, Richland, Washington.

2. Ibid., p. 7-11.

3. "Separations Data," personal communication (cc:mai1) from Eric Slaathaug to Bruce Zimmerman, dated March 30, 1993, Westinghouse Hanford Company, Richland, Washington.

4. Remer, D. S., and L. H. Chai, August 1990, "Design Cost Factors For Scaling-Up Engineering Equipment," Chemical Engineering Progress, American Institute of Chemical Engineers, New York, New York, p. 82.

5. Black, J. H., "Scaling Operating Costs By Cost-Capacity Factors," 1982 Transactions of the American Association of Cost Engineers 26th Annual Meeting, June 27 - June 30, 1982, Houston, Texas, H.3.1. 


\section{WHC-EP-0791}

\section{TECHNETIUN ION EXCHANGE}

$$
\text { 6-14-93 }
$$

Sludge Wash D (SWD) and Sludge Wash B (SWB) are the same, except that Sludge Wash $D$ includes a technetium ion exchanger. Consequentiy, the difference in these previously costed configurations represents the cost of the ion exchanger.

\begin{tabular}{|c|c|c|}
\hline Basic Data & SWD & SWB \\
\hline $\begin{array}{l}\text { SF HLW TC }{ }^{(1)} \\
\text { CapitaT cost(2) } \\
\text { Expense cost } \\
\text { Capacity* } \\
\text { oper. duration } \\
\text { (3) }\end{array}$ & $\begin{array}{c}99 \% \\
\$ 1.40 \mathrm{~B} \\
\$ 1.81 \mathrm{~B} \\
0.486 \mathrm{E}+5 \mathrm{~kg} / \mathrm{day} \\
10 \text { years }\end{array}$ & $\begin{array}{c}23 \% \\
\$ 1.33 \mathrm{~B} \\
\$ 1.47 \mathrm{~B} \\
\text { for TC ion exchanger } \\
10 \text { years }\end{array}$ \\
\hline
\end{tabular}

*Assumed operating capacity for this stream is $4.03 \mathrm{E}+5 \mathrm{~kg} / 0 \mathrm{D}$ (kilograms per operating day) total, of which $3.22 \mathrm{E}+5 \mathrm{~kg} / 0 \mathrm{D}$ is water and $0.81 \mathrm{E}+5 \mathrm{~kg} / 0 \mathrm{D}$ is "dry." Because the assumed operating efficiency is $60 \%$, the dry flow in this stream per 24-hour day is $0.81 E+5 * 0.6=0.486 \mathrm{E}+5 \mathrm{~kg} / \mathrm{day}$. This flow rate is designed to handle all single-shell tank waste in 10 years $^{(4)}$.

SF HLW TC = separation factor (fraction) of technetium to the high-level waste stream.

The costs to be assigned to the technetium ion exchanger are then:

$$
\begin{aligned}
& \text { Capital cost }=\$ 0.07 \mathrm{~B} \\
& \text { Expense cost }=\$ 0.34 \mathrm{~B}
\end{aligned}
$$

\section{ION EXCHANGER FUNCTIONAL FORM}

Based on internal analysis, the generic functional form for ion exchanger cost versus separation factor has been estimated to be:

$$
\$=A *\left(1-\ln \left(1-S F_{-} H L W_{-} T C\right)\right) * * 0.55+B .
$$

where: $S F=1$ means perfect separation for SF_HLW_TC. In the present instance, SF HLW TC $=0.23$ corresponds to $\$ 0$, because $\bar{a}$ technetium exchanger is not needed to obtain this separation value. (Sludge wash alone produces 0.23. )

Fitting this functional form to the capital and expense cost data produces the following equations:

$$
\begin{aligned}
& \text { Capital cost (SB) }=\$ 0.048\left(1-\ln \left(1-S F_{-} H_{L} W_{-} T C\right)\right)^{\star * 0} 0.55-\$ 0.055 \text {. } \\
& \text { Expense } \operatorname{cost}(S B)=\$ 0.235\left(1-\ln \left(1-S F_{-} H_{L} W_{-} T C\right)\right)^{\star \star 0} 0.55-\$ 0.267 \text {. }
\end{aligned}
$$


Including a capacity scaling term of 0.55 for the capital cost (assunies throughput is proportional to resin bed size) and 0.75 for the expense cost ${ }^{(5)}$ :

Capital cost(SB) $=\left\{\$ 0.048(1-\ln (1-S F \text { HLW TC }))^{\star * 0.55-\$ 0.055}\right\} *$ \{actual daily dry throughpuE $/ 0.486 \mathrm{E}+5 \mathrm{~kg} /$ day\} $* * 0.55$

Expense $\operatorname{cost}(\$ B)=\left\{\$ 0.235(1-\ln (1-S F H L W T C))^{\star \star 0} 0.55-\$ 0.267\right\} *$ \{actual daily dry through

\section{REFERENCES}

1. Boomer, K. D., et al., 1993, Tank Waste Technical Options Report, WHC-EP-0616, p. 7-11, Westinghouse Hanford Company, Richland, Washington.

2. Ibid. p. 7-51.

3. Ibid. p. 7-29.

4. Ibid. p. G7-74.

5. Black, J. H., "Scaling Operating Costs By Cost-Capacity Factors", 1982 Transactions of the American Association of Cost Engineers 26th Annual Meeting, June 27 - June 30, 1982, Houston, Texas, H.3.1. 


\section{SALT GROUT PLANT \\ 4-5-94}

The following data are applicable for a salt grout plant:

Capital Operating Assumed cavacity Dry feed capacity

$\$ 0.605 B^{(1)} \quad \$ 3.29 B^{(1)} \quad 3.83 E+5 \mathrm{~kg} / O D^{(2)} \quad 1.04 E+5 \mathrm{~kg} / 0 D^{(2)}$

$B=$ SBillions; $O D=$ operating day $=2,847$ days (see calculation below)

The operating cost includes an allowance for decontamination and decommissioning (D\&D) and the capital cost includes required double-shell tanks (DSTs) to support the facility. The plant operating costs are based on a 13-year operating period with a $50 \%$ plant efficiency. The capacity numbers are for waste flowing into the salt grout plant and do not include added grout cement binding material. Using the assumed $50 \%$ plant efficiency, this implies a 24-hour day capacity of $5.21 \mathrm{E}+4 \mathrm{dry} \mathrm{kg} / \mathrm{day}$.

The $\$ 3.29 B$ breaks down as follows ${ }^{(3)}$ :

Staff cost: $\quad \$ 49.9 \mathrm{M} / \mathrm{yr}$ for 15 years $=\$ 749 \mathrm{M}$

Essential mat'ls: $\$ 16.9 \mathrm{M} / \mathrm{yr}$ for 13 years $=\$ 220 \mathrm{M}$

Vaults:

\$7.65M per vault (with diffusion barriers and liners) +

$\$ 2 M$ per acre for closure barriers $=\$ 2074 M$

[ Two vaults require approximately 1 acre of 1 and. (3)

Each vault holds $1.4 \mathrm{E}+6$ gallons or $5.3 \mathrm{E}+3 \mathrm{~m}^{3}$ of waste. (4)

The salt grout product flow $=7.18 \mathrm{E}+5 \mathrm{~kg} / 0 \mathrm{D}$, yielding a total of 241 vaults $^{(2)}$ \}

Relating the vault cost to actual $\mathrm{kg}$ grout deposited, the final salt grout vault cost equation becomes:

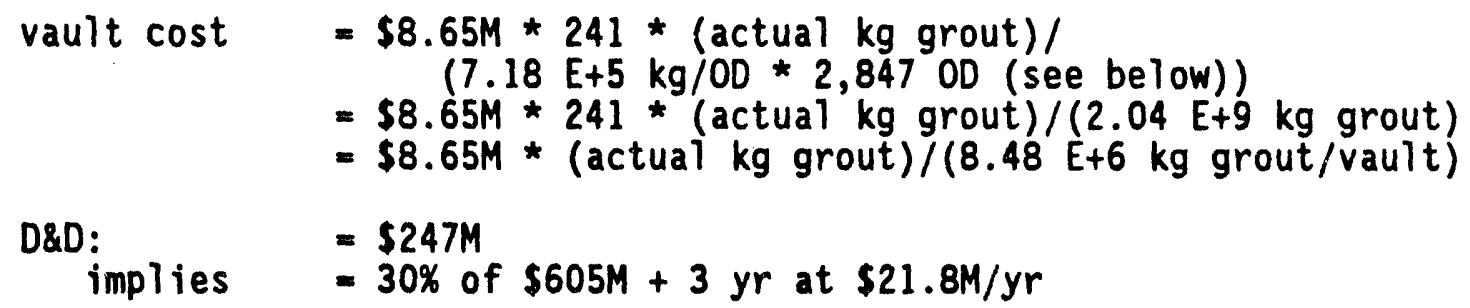


These data imply the following equation:

dryfeed = actual dry feed capacity per day (kg/day)

lifefeed = actual total lifetime grout dry feed $(\mathrm{kg})$

[Total $\mathrm{kg}$ dry grout feed estimated as $1.04 \mathrm{E}+5 \mathrm{~kg} / O D * 2,847 \mathrm{OD}=2.96 \mathrm{E}+8 \mathrm{~kg}$ dry feed $]$

$(2,8470 D=13 y * 365 * 60 \%$ oper. effic. actually used in flowsheet model

$\$($ total $)=\$ 605 M($ dryfeed $/ 5.21 \mathrm{E}+4 \mathrm{~kg} /$ day $) * 0.78$

$+\$ 749 \mathrm{M}$ (dryfeed $/ 5.21 \mathrm{E}+4 \mathrm{~kg} /$ day) $* * 0.75$

$+\$ 220 M(1$ ifefeed $/ 2.96 \mathrm{E}+8 \mathrm{~kg}$ )

$+\$ 8.65 M$ * (actual $\mathrm{kg}$ grout) $/(8.48 \mathrm{E}+6 \mathrm{~kg}$ grout/vault)

$+(0.3)(\$ 605 \mathrm{M})$ (dryfeed $/ 5.21 \mathrm{E}+4 \mathrm{~kg} /$ day) $* \approx 0.78$

$+(3)(\$ 21.8 M)($ dryfeed $/ 5.21 \mathrm{E}+4 \mathrm{~kg} /$ day $) \star \star 0.78$

Capital equipment exponent $(0.78)^{(5)}$, operating cost exponent $(0.75)^{(6)}$.

\section{REFERENCES}

1. Boomer, K. D., et al., 1993, Tank Waste Technical Options Report, WHC-EP-0616, pp. 8-33 and 8-34, Westinghouse Hanford Company, Richland, Washington.

2. Ibid. pp. H3-34 and H3-35.

3. Ibid. p. H3-30.

4. Ibid. Pp. 8-6 through 8-10.

5. Remer, D. S., and L. H. Chai, August 1990, "Design Cost Factors For Scaling-Up Engineering Equipment," Chemical Engineering Progress, American Institute of Chemical Engineers, New York, New York, p. 78 (blenders).

6. Black, J. H., "Scaling Operating Costs By Cost-Capacity Factors," 1982 Transactions of the American Association of Cost Engineers 26th Annual Meeting, June 27 - June 30 1982, Houston, Texas, H.3.1. 
WHC-EP-0791

\section{TRANSPORTATION AND DISPOSAL COST FOR CLASS A \\ LOW-LEVEL WASTE \\ (IN LLH LANDFILL) \\ $1-4-93$}

The following values are applicable $e^{(1)}$.

TRANSPORTATION COSTS
30-miles
300 -miles
500 -miles
$\$ 0.0172 / \mathrm{kg}$
\$0.0295/kg
$\$ 0.0389 / \mathrm{kg}$

(based on current commercial transportation rates).

DISPOSAL (LANDFILL) CHARGES

$\frac{\text { Current US Ecology Washington }}{\text { Nuclear Center Charge }} \frac{\text { Median Value From }}{\text { Study }}$

$\$ 1.18 / \mathrm{kg}$

$\$ 2.57 / \mathrm{kg}$

For the time being, the $\$ 2.57$ disposal value may be used. One of the transportation charges could be added, but they are negligible. The assumed density of low-level waste is $128 \mathrm{lb} / \mathrm{ft} \star \star 3=2,050 \mathrm{~kg} / \mathrm{m}^{\star \star 3}{ }^{(2)}$.

\section{REFERENCES}

1. Schlueter, R., J. J. Schafer, 1992, "Low-Level and Transuranic Waste Transportation, Disposal, and Facility Decommissioning Cost Sensitivity Analysis," EGG-WTD-10092, INEL, Idaho Falls, Idaho.

2. Personal communication, R. W. Harmsen to B. D. Zimmerman, Jan. 4, 1993, Westinghouse Hanford Company, Richland, Washington. 


\section{MIXEO WASTE INCINERATION}

$$
\text { 4-6-93 }
$$

For the Savannah River Laboratory (SRL) Consolidated Incinerator Facility (ICF) design, the expected capital cost is approximately \$150M, with an expected throughput of $5.6 \mathrm{M} \mathrm{ib} /$ year of solids and $860,000 \mathrm{Ib} /$ year of liquids(1).

The Oak Ridge mixed waste incinerator has approximately the same capacity as the ICF ${ }^{(1)(2)}$ but is already in operation. The annual operating cost for the Oak Ridge facility is approximately $\$ 26 \mathrm{M} /$ year.

Using standard scaling exponents, and basing the throughput scaling on the total pounds-per-year capacity ${ }^{(3)(4)}$ : $\begin{aligned} \text { Capital } \operatorname{cost}(\$) & =\$ 150 M(\text { actual solid }+1 \text { lquid throughput } / 6.46 \mathrm{M} \\ & 1 \mathrm{~b} / \text { year }) \star * 0.8\end{aligned}$

Expense cost $(\$)=\$ 26 M$ (actual solid+liquid throughput/6.46M lb/ year) $* \star 0.75$

\section{REFERENCES}

1. Personal communication, H. Pope to B. Zimmerman, April 5, 1993, U.S. Department of Energy, Savannah River Operations Office, Aiken, South Carolina.

2. Personal communication, V. Adams (ORNL) to B. Zimmerman, April 6, 1993, Oak Ridge National Laboratory, Oak Ridge, Tennessee.

3. Remer, D. S., and L. H. Chai, August 1990, "Design Cost Factors For Scaling-Up Engineering Equipment," Chemical Engineering Progress, American Institute of Chemical Engineers, New York, New York, p. 79 (furnaces).

4. Black, J. H., "Scaling Operating Costs By Cost Capacity Factors," 1982 Transactions of the American Association of Cost Engineers

26th Annual Meeting, June 27 - June 30, 1982, Houston, Texas, H.3.1. 
WHC-EP-0791

\section{GLASS-IN-SULFUR PLANT}

3-3-94

The following data are applicable for a glass in sulfur plant:

\section{Capital Operating Assumed capacity Dry feed capacity}

$\$ 1.26 B^{(1)} \quad \$ 2.24 B^{(1)} \quad 3.10 E+5 \mathrm{~kg} / O D^{(2)} \quad 1.25 E+5 \mathrm{~kg} / O D^{(2)}$

B = \$Billions; $O D=$ operating day $=2,420$ days (see calculation below)

The operating cost includes an allowance for D\&D and the capital cost includes any required double-shell tanks to support the facility. The plant operating costs are based on a 13-year operating period with a $60 \%$ plant efficiency. The capacity numbers are for waste flowing into the glass-insulfur plant and do not include added sulfur cement binding material. Using the assumed $60 \%$ plant efficiency, this implies a 24-hour day capacity of $7.47 \mathrm{E}+4 \mathrm{dry} \mathrm{kg} / \mathrm{day}$.

The \$2.24B operating cost breakdown is as follows:

Staff cost: $\quad \$ 62.5 \mathrm{M} / \mathrm{yr}$ for 15 years $=\$ 937 \mathrm{M}$

Essential materials: $\$ 39.8 \mathrm{M} / \mathrm{yr}$ for 13 years $=\$ 517 \mathrm{M}$

Vaults: $\$ 4.95 M$ per vault (without liners) + $\$ 2 M$ per acre for closure barriers $=\$ 331 \mathrm{M}$

[ Two vaults require approximately 1 acre of 1 and ${ }^{(3)}$.

Each vault holds $1.4 \mathrm{E}+6$ gal or $5.3 \mathrm{E}+3 \mathrm{~m}^{3}$ of waste ${ }^{(1)}$.

The $L L W$ glass density is assumed to be $2.67 \mathrm{E}+3 \mathrm{~kg} / \mathrm{m}^{3}$.

The glass cullet aggregate makes up $67.1 \mathrm{wt} \%$ of the final glass-in-sulfur product ${ }^{(2)}$.

The glass-in-sulfur product flow $=2.94 \mathrm{E}+5 \mathrm{~kg} / 0 \mathrm{D}$, yielding a total of 56 vaults $^{(2)}$. ]

Relating the vault cost to actual kilograms of glass deposited, the final glass-in-sulfur vault cost equation becomes:

vault cost $=\$ 5.95 \mathrm{M} * 56$ * (actual $\mathrm{kg} \mathrm{glass}) / 2.94 \mathrm{E}+5 \mathrm{~kg} / 0 \mathrm{D} * 0.671$ * 2,420 OD (see below))

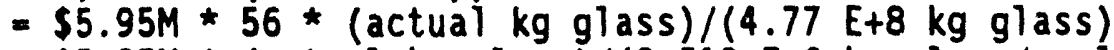

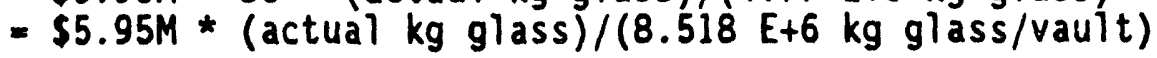

D\&D: $\quad=\$ 455 \mathrm{M}$

implies $=30 \%$ of $\$ 1260 M+3$ years at $\$ 25.7 M / y r$ 

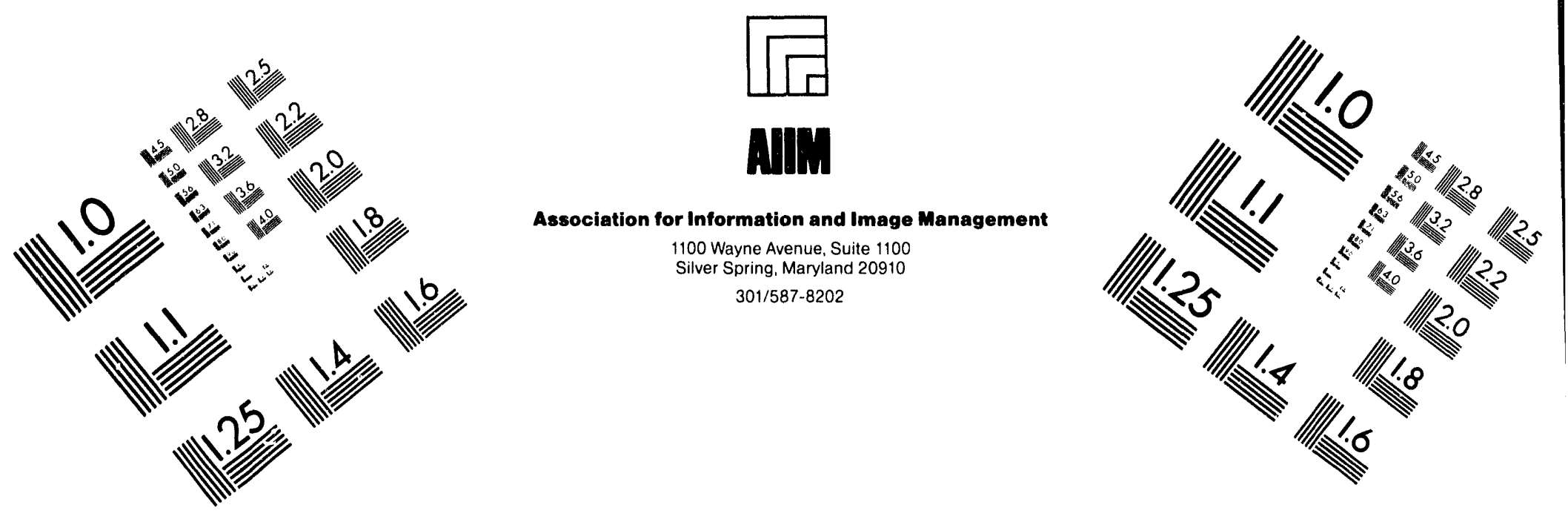

\section{Centimeter}

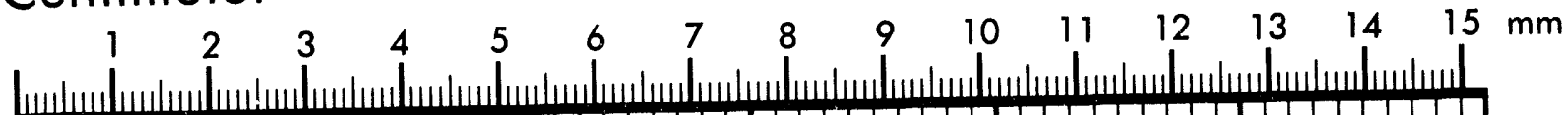

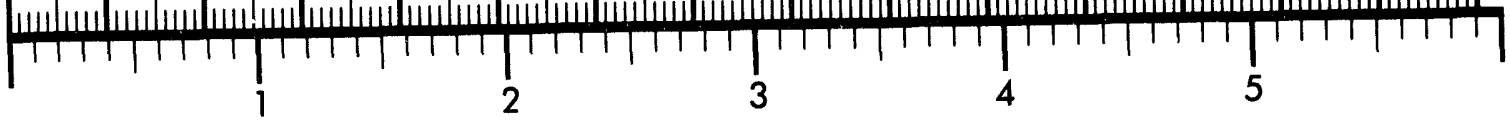
Inches
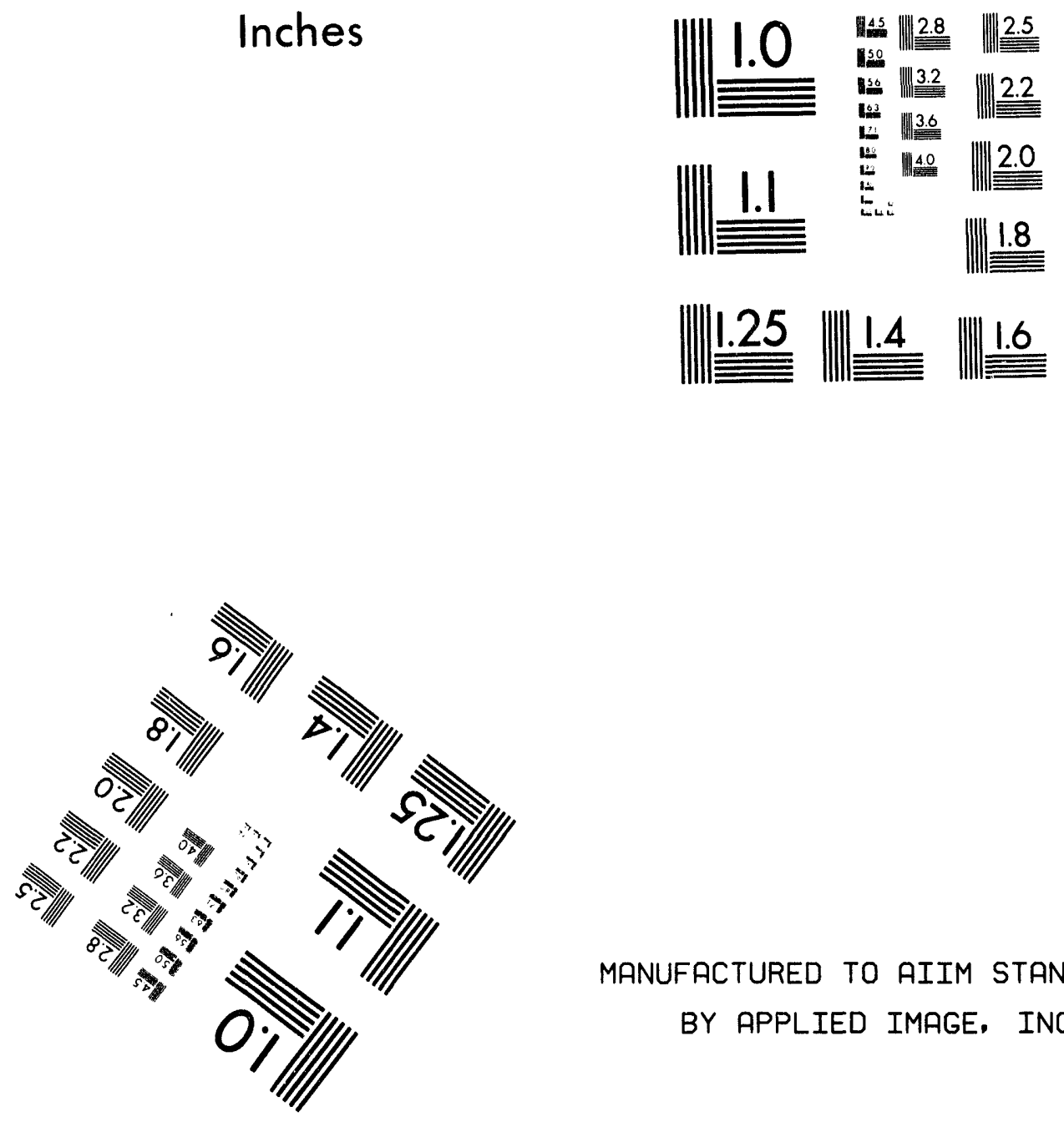

MANUFACTURED TO AIIM STANDARDS BY APPLIED IMAGE, INC.

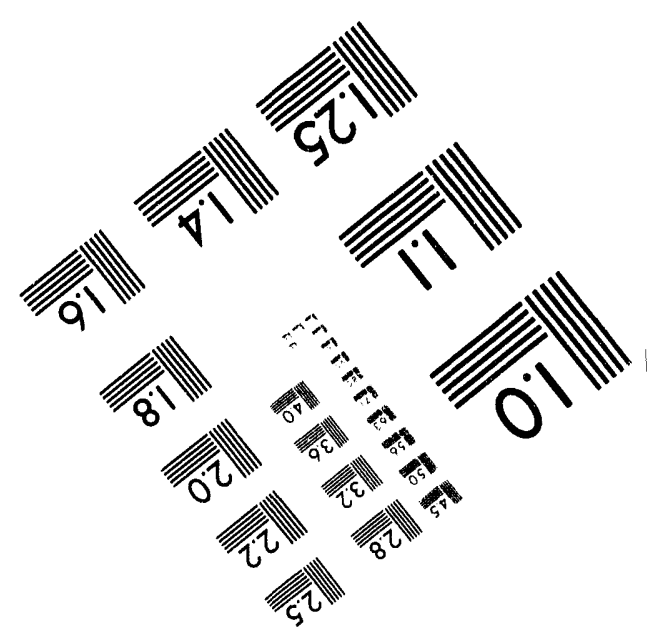



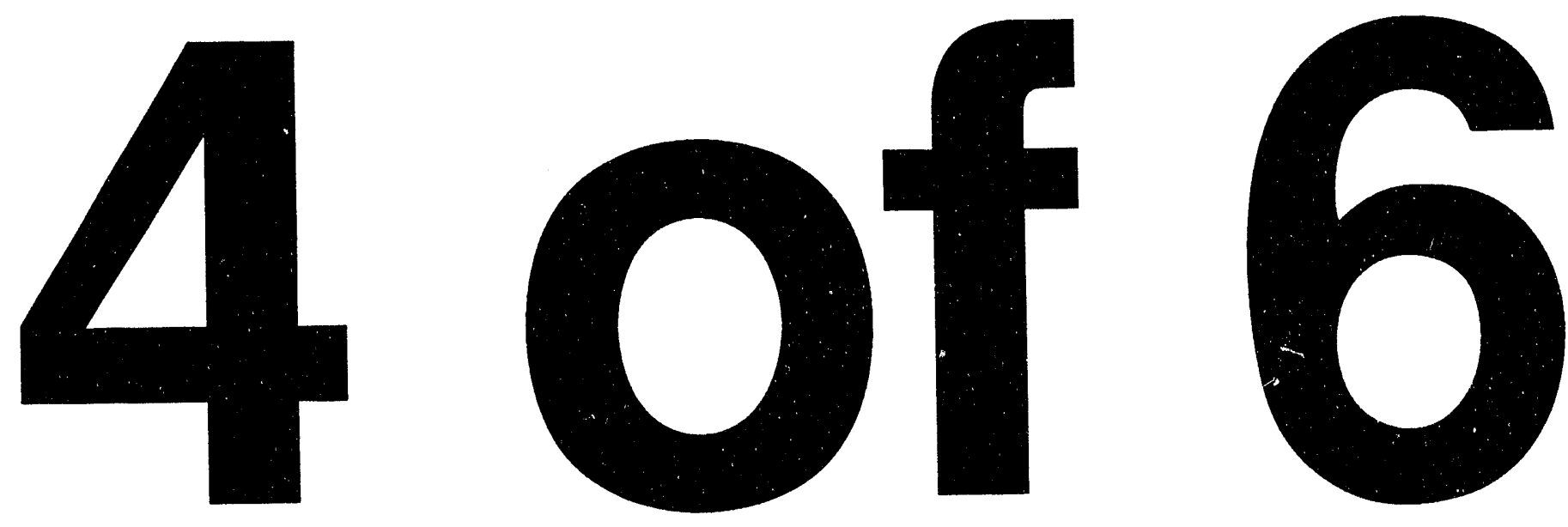
These data imply the following equation:

dryfeed = actual dry feed capacity per day (kg/day)

Lifefeed - actual total lifetime grout dry feed (kg)

[Total $\mathrm{kg}$ dry grout feed estimated as

$1.25 \mathrm{E}+5 \mathrm{~kg} / 0 \mathrm{0D} 2,4200 \mathrm{OD}=3.02 \mathrm{E}+8 \mathrm{~kg}$ dry feed

$(2,420$ OD $=13 y * 365 * 60 \%$ oper. effic. * 0.85 actually used in flowsheet model

$\$($ total) $=\$ 1260 M($ dryfeed $/ 7.47 \mathrm{E}+4 \mathrm{~kg} /$ day) $\star \star \star 0.78$

$+\$ 937 M($ dryfeed $/ 7.47 \mathrm{E}+4 \mathrm{~kg} /$ day) $\star * 0.75$

$+\$ 517 M(1$ ifefeed/3.02 E+8 kg)

$+\$ 5.95 \mathrm{M}$ * (actual $\mathrm{kg}$ glass) $/(8.518 \mathrm{E}+6 \mathrm{~kg}$ glass/vault)

$+(0.3)(\$ 1260 \mathrm{M})$ (dryfeed $/ 7.47 \mathrm{E}+4 \mathrm{~kg} /$ day) $\star \star 0.78$

$+(3)(\$ 25.7 M)($ dryfeed $/ 7.47 \mathrm{E}+4 \mathrm{~kg} /$ day $) \star \star 0.78$

Capital equipment exponent $(0.78)^{(5)}$, operating cost exponent $(0.75)^{(6)}$.

\section{REFERENCES}

1. Boomer, K. D., et al., 1993, Tank Waste Technical Options Report, WHC-EP-0616, Pp. 8-33 and 8-34, Westinghouse Hanford Company, Richland, Washington.

2. Ibid. Pp. H3-44 through H3-51.

3. Ibid. p. H3-30.

4. Ibid. pp. 8-21 through 8-24.

5. Remer, D. S., and L. H. Chai, August 1990, "Design Cost Factors For Scaling-Up Engineering Equipment," Chemical Engineering Progress, American Institute of Chemical Engineers, New York, New York, p. 78 (blenders).

6. Black, J. H., "Scaling Operating Costs By Cost-Capacity Factors," 1982 Transactions of the American Association of Cost Engineers 26th Annual Meeting, June 27 - June 30 1982, Houston, Texas, H.3.1. 


\section{MATER TREATMENT PLANT - FACILITY NO. 8

$$
\text { 3-17-93 }
$$

The ROM Cost Estimate document ${ }^{(1)}$, suggests a water treatment facility cost estimate of $\$ 3.43 \mathrm{~B}$ ( $\$ 1.31 \mathrm{~B}$ capital and $\$ 2.12 \mathrm{~B}$ operations, for the life of the plant) for an actual lifetime feed into the plant of $34.3 \mathrm{M} \mathrm{m}$ $(34.3 \mathrm{E}+9 \mathrm{~kg}$ ) over a 30 -year operating life. Using a general 0.7 scaling exponent, this leads to the formula:

Water treatment cost $=\$ 3.43 B($ actual lifetime feed $/ 34.3 \mathrm{E}+9 \mathrm{~kg}) \star \approx 0.7$

This presumably includes tanks and decontamination and decommissioning (D\&D). Decontamination and decommissioning is generally assumed to be $30 \%$ of capital cost +3 years of operating cost, which would $=\$ 0.56 \mathrm{~B}$.

Decontamination and decommissioning is always included as part of operating cost. The number of tanks assumed in the estimate, if any, is unknown.

\section{REFERENCES}

1. "ROM Cost Estimate For Hanford Strategic Analys is Study Strategy IX. Option 2," Facility Specification Sheet for :acility No. 8, January 29, 1992, R. C. Hoyt, Westinghouse Hanford Company, Richland, Washington. 


\section{HLH DISPOSAL TO GLASS}

$$
\text { 5-11-93 }
$$

\section{GLASS PLANT}

The term for the glass plant includes capital, expense, tanks, and D\&D. The plant probably operates for about 35 years. It is derived in detail in reference 1 .

\section{INTERIM STORAGE}

The second term is for interim storage of glass at Hanford. Relevant data are:

$$
\text { Cap. cost }{ }^{(2)} \mathrm{m}^{\star \star 3} \text { of glass(3) } \mathrm{kg} \text { of glass } \$ / \mathrm{kg}
$$

$\begin{array}{lrrrr}\text { HWV option } & \$ 0.56 \mathrm{~B} & 7,000 & 1.87 \mathrm{e} 7^{*} & \$ 29.95 \\ \text { Large melter option } & \$ 1.22 \mathrm{~B} & 23,400 & 6.25 \mathrm{e}^{\star} & \$ 19.52\end{array}$

*based on $2.67 \mathrm{E}+3 \mathrm{~kg} / \mathrm{m} \star \star 3$ for $\mathrm{glass}$

Based on these values, $\$ 25 / \mathrm{kg}$ for interim storage seems reasonable.

\section{TRANSPORTATION}

\begin{tabular}{|c|c|c|c|}
\hline \multirow[b]{2}{*}{$\begin{array}{l}\text { Option } 1 \\
\text { Option } 2 \\
\text { Option } 3 \\
\text { Option } 4\end{array}$} & Iransportation cost ${ }^{(4)}$ & Canisters $^{(5)}$ & $\$ /$ canisters \\
\hline & $\begin{array}{r}\$ 290 M \\
\$ 66 M \\
\$ 8 M \\
\$ 1,712 M\end{array}$ & $\begin{array}{r}16,800 \\
4,900 \\
890 \\
96,400\end{array}$ & $\begin{array}{r}\$ 17,262 \\
\$ 13,469 \\
\$ 8,989 \\
\$ 17,759\end{array}$ \\
\hline & $\$ 2,076 M$ & $1.1899 E+5$ & avg. $\$ 17,447$ \\
\hline
\end{tabular}

Relevant data are:

Assuming $3,738 \mathrm{~kg}$ per $\left(1.4 \mathrm{~m}^{\star \star 3}\right)$ canister: $\$ 17,447 / 3,738 \mathrm{~kg}=\$ 4.67 / \mathrm{kg}$.

\section{REPOSITORY CHARGE}

A curve fit of the following data was made to estimate repository charge as a function of total kilograms of glass disposed ${ }^{(6)}$ :

\begin{tabular}{crrr} 
Total canisters & \multicolumn{1}{c}{ Total kg* } & Total $\$$ & \multicolumn{1}{l}{$\mathbf{k g}$} \\
890 & $3.33 \mathrm{E}+6$ & $\$ 0.49 \mathrm{~B}$ & $\$ 112.63$ \\
4,900 & $18.32 \mathrm{E}+6$ & $\$ 2.56 \mathrm{~B}$ & $\$ 139.91$ \\
16,800 & $62.8 \mathrm{E}+6$ & $\$ 7.07 \mathrm{~B}$ & $\$ 148.21$ \\
96,400 & $360.34 \mathrm{E}+6$ & $\$ 24.73 \mathrm{~B}$ & $\$ 68.75$
\end{tabular}


The curve fit produced the following equation:

Repository charge $=\$ 0.342 \mathrm{E}-15^{*}(\mathrm{~kg}$ of glass $) * * 3-0.3244 \mathrm{E}-6 *(\mathrm{~kg}$ of glass $) * 2+\$ 142.4 *(\mathrm{~kg}$ of glass $)$

The functional form used for the fit was a quadratic. The resulting equation was multiplied by $\mathrm{kg}$ of glass to produce the total repository charge.

The data used to produce the equation were for the Hest Valley canister, assuming two repositories are required. Note that if BR-100 canisters are assumed, the cost would be lowered by $\$ 1 B$ or less for $<=63 \mathrm{M} \mathrm{kg}$ of glass. $(63 \mathrm{M} \mathrm{kg}$ of glass corresponds to the case where only sludge wash is performed for separation.) The cost would be lowered by about \$4B (\$24.7B versus $\$ 20.9 B$ ) in the case of $360 \mathrm{Mg}$ of glass, where $360 \mathrm{M} \mathrm{kg}$ of $\mathrm{glass}$ corresponds to no pretreatment.

\section{TOTAL}

The resulting total cost equation is:

$\$($ total cost) $=$

$\$ 3.74 \mathrm{E}+9$ (actual $\mathrm{kg}$ per day dry feed $/ 0.683 \mathrm{E}+3 \mathrm{~kg} /$ day) $\star * 0.29$

$+\$ 25 *(\mathrm{~kg}$ of glass)

$+\$ 4.67 *(\mathrm{~kg}$ of glass $)$

$+\$ 0.342 E-15 *(\mathrm{~kg}$ of glass $) \star \star 3-0.3244 E-6 *(\mathrm{~kg}$ of glass $) \star \star 2+$ $\$ 142.4^{*}(\mathrm{~kg}$ of $\mathrm{glass})$

\section{REFERENCES}

1. Zimmerman, B. D., "Volume Scale-Up For HLW Glass Production Plant," personal notes dated Jan. 22, 1993, Westinghouse Hanford Company, Richland, Washington.

2. Boomer, K. D., et al., 1993 Tank Waste Technical Options Report, WHC-EP-0616, p. 9-32, Westinghouse Hanford Company, Richland, Washington.

3. Ibid., p. 9-6.

4. "Total System Life Cycle Cost Estimates, Preliminary Draft," Table 4 of estimated repository costs from Weston, Feb. 20, 1993, Washington, D.C.

5. Ibid., Table 1.

6. "Summary of Preliminary Repository Disposal Fee Estimates For Hanford Tank Waste Remediation Study Cases (2/25/93), "from P. LaMont, U.S. Department of Energy, Richland Operations Office, Richland, Washington. 


\section{VOLUME SCALE-UP FOR HLH GLASS PRODUCTION PLANT \\ 1-24-93}

There are at least three concept for HLW glass plants of differing capacities: HWVP, large melter plant, and multi-melter plant. References 1 and 2 provide the following data:

$\begin{array}{ccc}\text { HWVP } & \text { Large Melter } & \text { Multi-Melter } \\ 25 \text { wt\% waste } & 25 \text { wt\% waste } & 15 \text { wt\% Na20 in glass } \\ 2.6 \text { MT/OD glass } & 9 \text { MT/OD glass } & 220 \mathrm{MT} / 00 \mathrm{glass} \\ 40 \text { years op. } & 33 \text { years op. } & 30 / 13 \text { years op. } \\ \$ 1.06 B & \$ 2.0 B & \$ 6.4 B \\ \$ 2.68 B & \$ 2.53 B & \$ 3.5 B\end{array}$

NOTE: For the multi-melter, it appears that the WHC-EP-0405, Rev. A, flowsheet values are based on a 30 -year assumed operating period, whereas the Rev. 0 values and figures derived from them (i.e., 220 MT/OD) are based on a 13-year assumed operating period.

The following data is also relevant:

dry feed $\quad 1.14 \mathrm{E}+3 \mathrm{~kg} / O \mathrm{D}^{(3)} \quad 3.2 \mathrm{E}+3^{\prime} \mathrm{kg} / O D^{(4)} \quad 2.77 \mathrm{E}+4 \mathrm{~kg} / O \mathrm{D}^{(5)}$

24-hr day

at $60 \% \mathrm{eff} .0 .68 \mathrm{E}+3 \mathrm{~kg} /$ day $\quad 1.9 \mathrm{E}+3 \mathrm{~kg} /$ day $\quad 1.66 \mathrm{E}+4 \mathrm{~kg} / \mathrm{day}$

Graphing these data suggests that it makes the most sense to correlate total cost with volume of dry feed (rather than expense and capital costs individually). The result is:

$\$($ captexp) $=\$ 3.74 \mathrm{E}+9$ (actual $\mathrm{kg}$ per day dry feed capacity) $0.683 \mathrm{E}+3 \mathrm{~kg} / \mathrm{day}) \star \star 0.29$

\section{REFERENCES}

1. Boomer, K. D., et a1., 1991, Tank Waste Systems Engineering Study, WHC-EP-0405, Rev. A, p. H-56, Westinghouse Hanford Company, Richland, Washington.

2. Boomer, K. D., et al., 1992, Tank Waste Systems Engineering Study, WHC-EP-0405, Rev. 0, p. 9-31, Westinghouse Hanford Company, Richland, Washington.

3. Ibid. P. H-5.

4. Ibid. p. H-36.

5. Ibid. P. H-60. 


\section{MIPP DISPOSAL}

$$
1 / 27 / 93
$$

Tang Chou (1) has uncovered the following numbers for disposal of transuranic material at the Waste Isolation Pilot Plant (WIPP) repository (processed into glass):

preparation for shipment:

transportation to WIPP:

WIPP charge:
$\$ 9,175 / m \star \star 3$

$\$ 750 / m^{* \star 3}$

$\$ 13,520 / m * * 3$

Assuming a glass density of $2,650 \mathrm{~kg} / \mathrm{m}^{* \star 3}$ :

preparation for shipment:

transportation to WIPP:

WIPP charge:
$\$ 3.46 / \mathrm{kg}$

$\$ 0.28 / \mathrm{kg}$

$\$ 5.10 / \mathrm{kg}$

$\$ 8.84 / \mathrm{kg}$

\section{REFERENCES}

1. Personal communication, R. Karnesky to B. Zimmerman, January 27, 1993, Westinghouse Hanford Company, Richland, Washington. 


\section{IN SITU GROUT}

3-23-93

This activity consists of mixing in-tank waste with a grout mixture, possibly including selected chemicals, completely filling the tank with grout. An offgas system is required, and engineered intrusion and infiltration barriers are installed.

For treatment of single-shell tanks, the estimated costs are:

capital operating waste volume des \& const time oper.time

$\$ 1.9 B^{(1)} \quad \$ 2.1 B^{(1)} \quad 37 \mathrm{Mgal}^{(2)} \quad 9$ years $^{(3)} \quad \begin{gathered}8 \text { years } \\ \text { effic. }\end{gathered}$

Reference 5 suggests that the construction cost for one chemical stabilization unit is $\$ 162 M$, which implies that increases in required processing capacity would be obtained by building more units. In this situation, capital and operating cost would likely vary directly with required capacity.

$S\left(\right.$ total cost) $=\$ 4.0 B^{*}$ (actual volume of waste to be retrieved/37 $\left.E+6 \mathrm{gal}\right)$

It may be noted that this option involves grouting all high-level waste in place. This option has been dropped from recent documents and seems very unlikely to ever be feasible from a regulatory stand point.

\section{REFERENCES}

1. Boomer, K. D., et al., 1992, Tank Waste Systems Engineering Study, WHC-EP-0405, Rev. 0, p. 12-17, West inghouse Hanford Company, Richland, Washington.

2. Ibid, 1. P. 4-5.

3. Ibid, 1. P. 12-9.

4. Boomer, K. D., et al., 1991, Tank Waste Systems Engineering Study, WHC-EP-0405, Rev. A, P. K-19, Westinghouse Hanford Company, Richland, Washington.

5. Ibid. 4. P. K-27. 
WHC-EP-0791

\section{IMMOBILIZATION AND DOME FILLING}

$$
\text { 3-24-93 }
$$
heading:

There are several possible activities that might be included under this

- Filling tanks for which retrieval activities are complete with gravel or grout ("Dome Filling")

- Filling past-practice unit facilities, such as trenches and pits, with gravel or grout ("Past-Practice Unit Stabilization")

- Installing intrusion barriers above emptied tanks ("Intrusion Barriers")

- In situ vitrification (ISV) of emptied tanks rather than grout filling.

The following costs are applicable for the 149 single-shell tanks:

\section{Qperating}

$\$ 0.27 B^{(1)}$

$\$ 0.27 B^{(1)}$

$\$ 0^{(1)}$

total of $50.55 B$ more than

simple grout dome filling(2)

To include both single-shell and double-shell tanks, the above numbers were multiplied by $177 / 149$ (the ratio of single-shell + double-shell tanks to single-shell tanks).

Note that the dome filling or ISV costs assume that these activities are taking place in conjunction with retrieval, so that the tank confinement system is already in place. The tank confinement system cost is not included in the numbers given here; it is assumed to be included as part of retrieval cost.

\section{REFERENCES}

1. "SST Closure Estimate For Sensitivity Study," personal communication R. Parazin to B. Zimmerman, January 24, 1992, Westinghouse Hanford Company, Richland, Washington.

2. "Question On Heel Vitrification Cost," personal communication by CC:Mai1, R. Parazin to B. Zimmerman, Feb. 5, 1992, Westinghouse Hanford Company, Richland, Washington. 


\section{CLEAN \\ 9-1-93}

The assumption here is that CLEAN will be accomplished by some combination of CPUs (compact processing units) for supernate and salt cake and a central facility for sludge.

\section{CENTRAL FACILITY}

This sludge processing facility is assumed to contain unit operations to perform the following processing steps ${ }^{(5)}$ :

- Sludge washing/clarification

- Organic destruction by supercritical water oxidation with precipitation/separation of transuranic (TRU)/actinides

- Cesium ion exchange and waste concentration

- Strontium ion exchange and concentration

- Technetium ion exchange and concentration

- Calcination of LLW fractions and dissolution of calcine and reductive precipitation of chromium from calcine solution

- Recycle of caustic from calcine solution

- Sequential dissolution of solids by caustic, nitric/oxalic acid, and nitric/hydrofluoric acid

- Tributyl phosphate (TBP) extraction from dissolver solution and stripping of plutonium, neptunium, and thorium followed by stripping of uranium

- Purification of uranium for recycle

- CMPO extraction from the TBP raffinate and stripping of americium and lanthanum followed by bismuth stripping

- Crown ether extraction of strontium, barium, and technetium from CMPO raffinate

- Ammonium phosphomolybdate ion exchange of cesium from Crown ether raffinate

- Band ion exchange separation of americium from lanthanides in the CMPO25 strip solution

- Band ion exchange separation of strontium and technetium from barium in the Crown Ether strip solution. 
The total sludge to be processed in SSTs and DSTs is $5.1 \mathrm{E}+4 \mathrm{~m}^{3}$ (1).

It is assumed that the central facility will process this sludge in 13 years at an operating availability of $60 \%^{(2)}$. Densities from sludge, salt cake, and supernate are ${ }^{(6)}$ :

sludge: $1.7 \mathrm{E}+3 \mathrm{~kg} / \mathrm{m}^{* * 3}$ salt cake: $1.5 \mathrm{E}+3 \mathrm{~kg} / \mathrm{m} * \star 3$

supernate (interstitial liquor): $1.6 \mathrm{E}+3 \mathrm{~kg} / \mathrm{m}^{* \star 3}$

The costs associated with the central sludge processing facility are estimated to be ${ }^{(3)(4)}$ :

$$
\text { Capital: \$2.68B Expense: \$3.98B (includes staff, }
$$
materials, and D\&D)

Because there is a variety of chemical processing equipment in the CLEAN facility, a capacity scaling factor of 0.7 for capital equipment scaling will be used $(7)$. Also, a fairly standard expense scaling factor of 0.75 will be used $^{(8)}$.

The average daily sludge processing capacity for the facility is:

$(5.1 \mathrm{E}+4 \mathrm{~m} * \star 3)\left(1.7 \mathrm{E}+3 \mathrm{~kg} / \mathrm{m}^{\star \star 3}\right) /((13 \mathrm{years})(365 \mathrm{days} / \mathrm{yr}))=1.83 \mathrm{E}+4 \mathrm{~kg} / \mathrm{day}$ facility:

The above information leads to the following equations for the central

Capital:

$S=\$ 2.68 \mathrm{E}+9$ (avg. daily capacity at $60 \%$ avail. $/ 1.83 \mathrm{E}+4 \mathrm{~kg} /$ day) $* * 0.7$

Operating:

$S=\$ 3.98 E+9$ (avg. daily capacity at $60 \%$ avail./1.83 E+4 kg/day) $\star \star 0.75$

\section{COMPACT PROCESSING UNITS}

Cost equations for CPUs are derived in detail in reference 9 . To summarize, the equations are:

Capital $\$=\$ 9.9 \mathrm{M}$ (actual throughput per day $/ 2.1 \mathrm{E}+4 \mathrm{~kg} /$ day) $* \star 0.7$ per CPU

Expense $\$=\$ 4 M$ (actual throughput per day $/ 2.1 \mathrm{E}+4 \mathrm{~kg} /$ day) $* * 0.75$ per CPU per year

Capital $\$=\$ 7.4 M$ per tank farm, per CPU operating in parallel

Capital $\$=\$ 2.2 M$ per CPU type

Capital $\$=\$ 0.96 \mathrm{M}$ one time charge 
There are various ideas about how CPUs would be employed as part of CLEAN. For example, one concept ${ }^{(10)}$ is that three different types of CPUs would be created: cesium removal, technetium removal, and nitrate/nitrite processing. One of each of these three types would be operating at a time (i.e., in parallel). If CPU processing extends over a period of 17 years ${ }^{(11)}$ and CPUS last an average of 3 years, then:

- Integer $(17 / 3) \star 3=18$ CPUs total would be required

- (18 tank farms) * (3 sets of infrastructure per farm) $=54$ sets of tank farm infrastructure would be required if tanks in all farms are to be processed.

The total CPU cost, for the nominal throughput of 2.5 gal/minute, would then be:
Cap. $\$=\$ 9.9 M * 18=$
$\$ 178 M$
Exp. $S=\$ 4 M * 3$ CPUS $* 17$ years $=$
$\$ 204 M$
Cap. S(infrastructure) $=\$ 7.4 \mathrm{M} * 54$ sets $=\$ 400 \mathrm{M}$
Cap. $\$($ per CPU type) $=\$ 2.2 * 3=$
$\$ 7 M$
Cap. \$(enclosure design) =
$\$$ ! in

$\$ 790 M$

Note, however, that a 2.5 gal/minute CPU operating for 17 years will process approximately $22 \mathrm{Mgal}$ of material. The SST and DST inventories are as follows ${ }^{(1)}$ :

$\begin{array}{ll}\text { SST salt: } & 8.77 \mathrm{E}+4 \mathrm{~m}^{* * 3}=23.2 \mathrm{Mgal} \\ \text { SST sludge: } & 4.72 \mathrm{E}+4 \mathrm{~m}^{* * 3}=12.5 \mathrm{Mgal} \\ \text { SST supernate: } 2.29 \mathrm{E}+3 \mathrm{~m}^{* * 3}= & 0.6 \mathrm{Mgal} \\ \text { DST sludge: } & 3.83 \mathrm{E}+3 \mathrm{~m}^{* * 3}=1.0 \mathrm{Mgal} \\ \text { DST supernate: } 7.43 \mathrm{E}+4 \mathrm{~m}^{* * 3}=19.6 \mathrm{Mgal}\end{array}$

Consequently, one 2.5 gal/minute CPU operating for 17 years can approximately process either the SST salt and supernate, or the DST supernate (no sait inventory given for DSTs), but not both. If both SST and DST salt and supernate are required to be processed, the capacity and/or the number of CPUs must be increased.

Another different approach to the CPU reusability issue assumes that CPUS can be repaired in a maintenance facility, so that they nominally last for the entire cleanup campaign rather than being discarded when they fail. It has been estimated that such a maintenance facility might have a capital cost of $\$ 100 M$ to $\$ 200 M^{(12)}$ and an annual expense cost of approximately $\$ 20 M^{(13)}$. 
Because the current CLEAN concept (centrai facility/CPUs) is $i 11$ defined, the above data are given so that a cost appropriate to a particular concept may be created as needed.

\section{REFERENCES}

1. Boomer, K. D., et a1., 1993, Tank Waste Technical Options Report, WHC-EP-0616, Rev. 0, Appendix D, "Tank Waste Radionuclide and Chemical Compositions," Table D-3 and Table D-8, Westinghouse Hanford Company, Richiand, Washington.

2. Ibid., Appendix G, "Waste Receipt, Storage, and Separations," April 1993, Pp. G13-3 and G13-9.

3. Ibid. 2., 613-10.

4. Shen, P. K., 1993, Internal Memo, Systems Engineering, to M. L. Grygiel, Westinghouse Hanford Company, Richland, Washington, "Transmittal of Report 'TWRS System Engineering Analys is Costing Däta, Estimated Cost Validation'", March 29, 1993, pp. A-2 and C-3.

5. Ibid. 2., Pp. G13-3 and G13-4.

6. O'Toole, S. M., Internal Memo, "Tank Waste Inventory Revision," to J. N. Appel et al., May 24, 1993, Table B.2.2, Westinghouse Hanford Company, Richland, Washington,.

7. Remer, D. S., and L. H. Chai, August 1990, "Design Cost Factors For Scaling-Up Engineering Equipment," Chemical Engineering Progress, American Institute of Chemical Engineers, New York, New York, p. 82.

8. Black, J. H., "Scaling Operating Costs By Cost-Capacity Factors", 1982 Transactions of the American Association of Cost Engineers 26th Annual Meeting, June 27 - June 30, 1982, Houston, Texas, H.3.6.

9. B.D. Zimmerman, "GENERIC CPU COST", August 30, 1993, personal notes, Westinghouse Hanford Company, Richland, Washington.

10. Ibid. 4., p. 9.

11. Ibid. $4 .$, p. 4.

12. Tauscher, H. T., "CleanS- .XLS", Feb. 18, 1993, personal spreadsheet notes, Westinghouse Hanford Company, Richland, Washington.

13. Tauscher, H. T., to B. D. Zimmerman, "CPU Cost Estimate Spreadsheet Assumptions," August 11, 1993, personal communication. Telecon states that a staff of 200 is reyuired. Ref. 12 states that $\$ 100 \mathrm{~K}$ per person is assumed. 


\section{GENERIC CPU COST}

8-30-93

The CPU concept really consists of the following pieces:

- Mobile CPU chemical processing unit (ion exchange equipment and enclosure)

- Mobile instrumentation and control unit

- Mobile power module

- Assorted piping, offgas facilities, and diversion boxes (infrastructure).

A different CPU chemical processing unit is assumed required for each chemical processing unit operation.

\section{CAPITAL COSTS}

The following data are applicable. These data are actually based on a cesium separation CPU:

\section{CPU chemical processing unit}

Ion exchange equipment fabrication $\$ 2,484,000^{(1)(9)}$

Enclosure fabrication

$$
\$ 6,072,000^{(1)(9)}
$$

Initial unit disposal cost is

Expected lifetime is 3 years $(1)$

Expected flow rate is $2.5 \mathrm{gal} / \mathrm{minute}$

( $5 \mathrm{gal} / \mathrm{minute} 050 \%$ availability)

$$
\$ 9,920,000
$$

\section{Per CPU type}

Ion exchanger design

Ion exchanger test and installation

Instrumentation \& control design

S $\left.497,000^{(9)(9)}(1)(6) .4\right)$

Instrument. \& control test/install

$\$ 146,000^{(1)(9)}$

$\$ 1,525,000^{(1)(9)}$

$\$ 2,168,000$

\section{Per-tank-farm per parallel CPU}

Instrumentation \& control fabrication \$730,000(1)(9)

Tank farm infrastructure design $\$ 1,017,000(1)(9) \quad(2.6)$

Tank farm infrastructure fabrication $\$ 5,681,000$ (1)( $(9)$

$\$ 7,428,000$ 
("Parallel CPUs" refers to CPUs that would require duplicate sets of infrastructure and instrumentation and control modules at each tank farm).

\section{One-time Charges}

\section{Enclosure design}

$$
5960,000^{(1)(9)}
$$

Note that the numbers in () and [] near the right margin are index numbers from reference 1 .

\section{EXPENSE COSTS}

Reference 3 estimates the total cost of CPUs for 15 years of operation to be bet:jeen (10W) $\$ 2.25 \mathrm{~B}$ and (high) \$3.0B. These estimates are for a total of 50 CPUs, operating for 15 years each ${ }^{(4)}$, which implies a per CPU, per year cost of:

$$
\$ 2.25 B /(50 * 15)=\$ 3 M
$$

or

$$
\$ 3 B /(50 \star 15)=\$ 4 M \quad \text { (high) }
$$

Both of these figures include an allowance of approximately $10 \%$ for operation of a maintenance facility. The $54 M$ figure is intended to al so include an allowance for miscellaneous expenses, such as chemical makeups.

Another estimate of operating cost was made in reference 5, where direct operating labor only was estimated to be $\$ 8.2 M$.

Based on review of the estimating effort involved in producing these various estimates, it appears the $\$ 4 M$ per CPU per year figure is most likely to be accurate.

\section{CAPACITY SCALING}

Because there is a variety of equipment involved in the CPUS, a capital equipment scaling factor of 0.7 is appropriate ${ }^{(6)}$. A standard 0.75 scaling factor will be used for operating costs $(?)$.

Assuming that an individual CPU has a processing capacity of $2.5 \mathrm{gal} / \mathrm{minute}$ ( $5 \mathrm{gal} / \mathrm{minute}$ with $50 \%$ availability) ${ }^{(2)}$, and the following densities ${ }^{(8)}$ :

Salt cake: $1.5 \mathrm{E}+3 \mathrm{~kg} / \mathrm{m}^{\star \star} 3$

Supernate (interstitial liquor): $1.6 \mathrm{E}+3 \mathrm{~kg} / \mathrm{m}^{* * 3}$

then:

(2.5 gal $/$ minute) $\left(1.55 \mathrm{E}+3 \mathrm{~kg} / \mathrm{m}^{\star \star * 3}\right)(60 \mathrm{minute} / \mathrm{h})(24 \mathrm{~h} /$ day $) /$

(264.2 gal $/$ minute $\star * 3)=2.1 \mathrm{E}+4 \mathrm{~kg} /$ day 
WHC-EP-079]

The final equations then become:

Capital \$ = \$9.9M (actual throughput per day/2.1 E+4 kg/day)**0.7 per CPU

Expense $\$=\$ 4 M$ (actual throughput per day $/ 2.1 \mathrm{E}+4 \mathrm{~kg} /$ day) $* * 0.75$ per CPU per year

Capital $\$=\$ 7.4 M$ per tank farm, per CPU operating in parallel

Capital $\$=\$ 2.2 M$ per CPU type (i.e., CS, TC, nitrate, etc.)

Capital $\$=\$ 0.96 \mathrm{M}$ one time charge

\section{REFERENCES}

1. Richmond, W. G., "Compact Processing Unit, Cost Estimate Review," personal correspondence, Pacific Northwest Laboratory, Richland, Washington.

2. Richmond, W. G., "Compact Processing Unit Assumptions/Bas is for Systems Study Input," personal correspondence, Pacific Northwest Laboratory, Richland, Washington.

3. Tauscher, H. T., "CLEANS- .XLS," Feb. 18, 1993, personal spreadsheet notes, Westinghouse Hanford Company, Richland, Washington.

4. Tauscher, H. T., to B. D. Zimmerman, "CPU Cost Estimate Spreadsheet Assumptions," August 11, 1993, personal communication, Westinghouse Hanford Company, Richland, Washington.

5. Richmond, W. B., note to cost estimator at Kaiser Engineers Hanford, ("My Dearest Friends at KEH, ..."), Pacific Northwest Laboratory, Richland, Washington.

6. Remer, D. S., and L. H. Chai, August 1990, "Design Cost Factors For Scaling-Up Engineering Equipment," Chemical Engineering Progress, American Institute of Chemical Engineers, New York, New York, p. 82.

7. Black, J. H., "Scaling Operating Costs By Cost-Capacity Factors," 1982 Transactions of the American Association of Cost Engineers 26th Annual Meeting, June 27 - June 30, 1982, Houston, Texas, H.3.1.

8. O'Toole, S. M., Internal Memo, "Tank Waste Inventory Revision," to J. N. Appe1, et a1., May 24, 1993, Table B.2.2, Westinghouse Hanford Company, Richland, Washington.

9. Richmond, W. B., "CPU COSTS - II", CC:Mail message to B. Zimmerman, August 26, 1993, Westinghouse Hanford Company, Richland, Washington. 


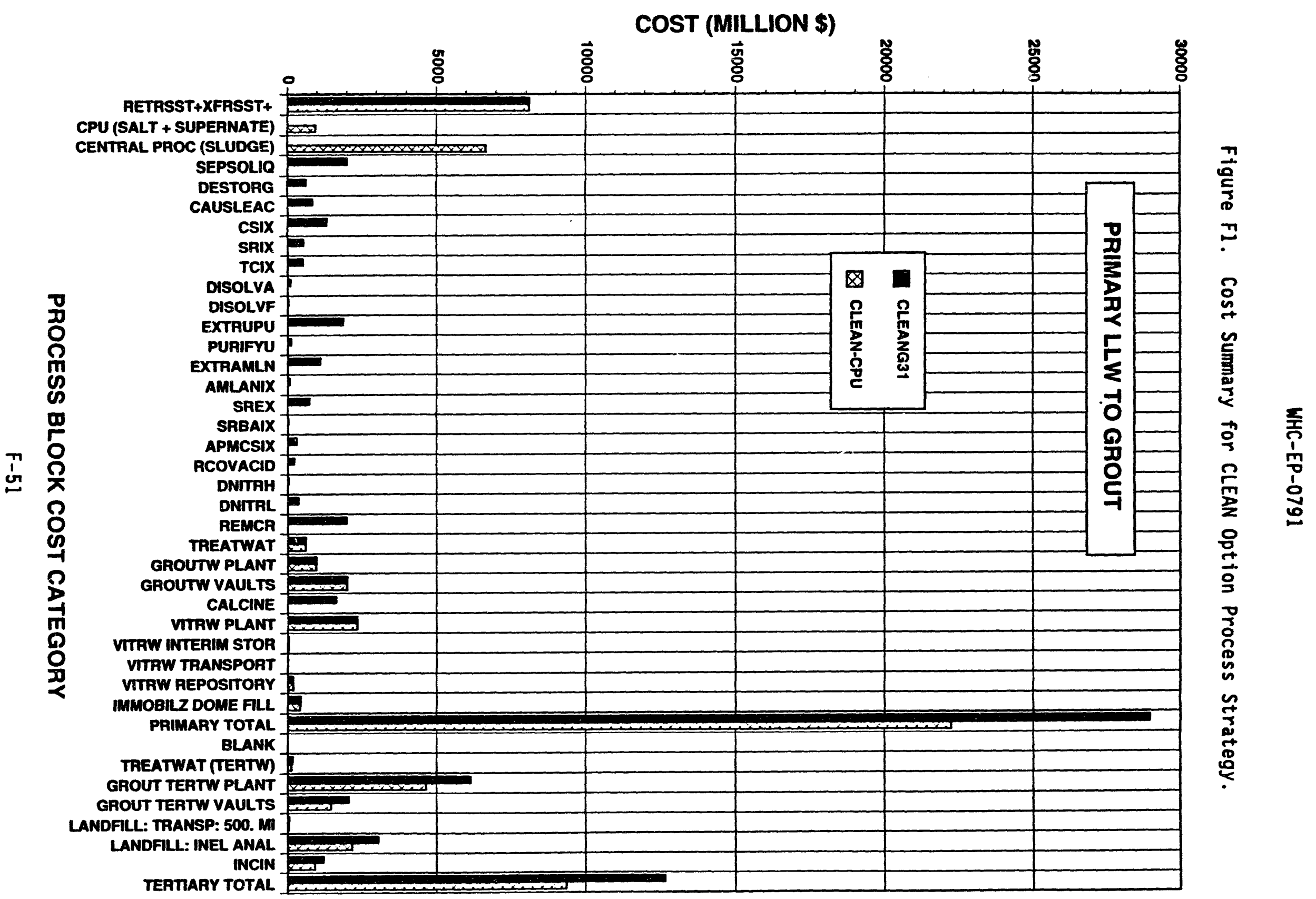




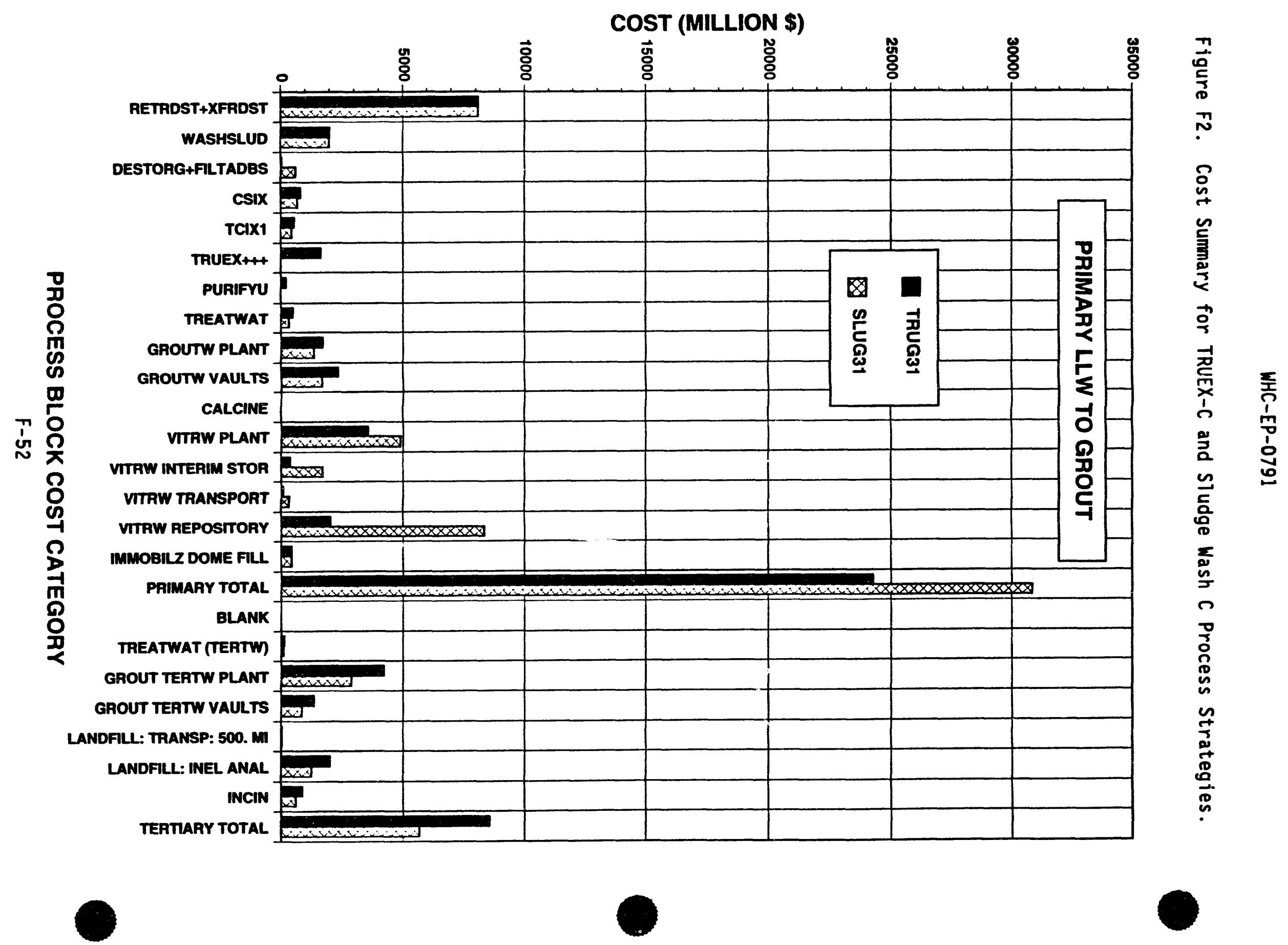




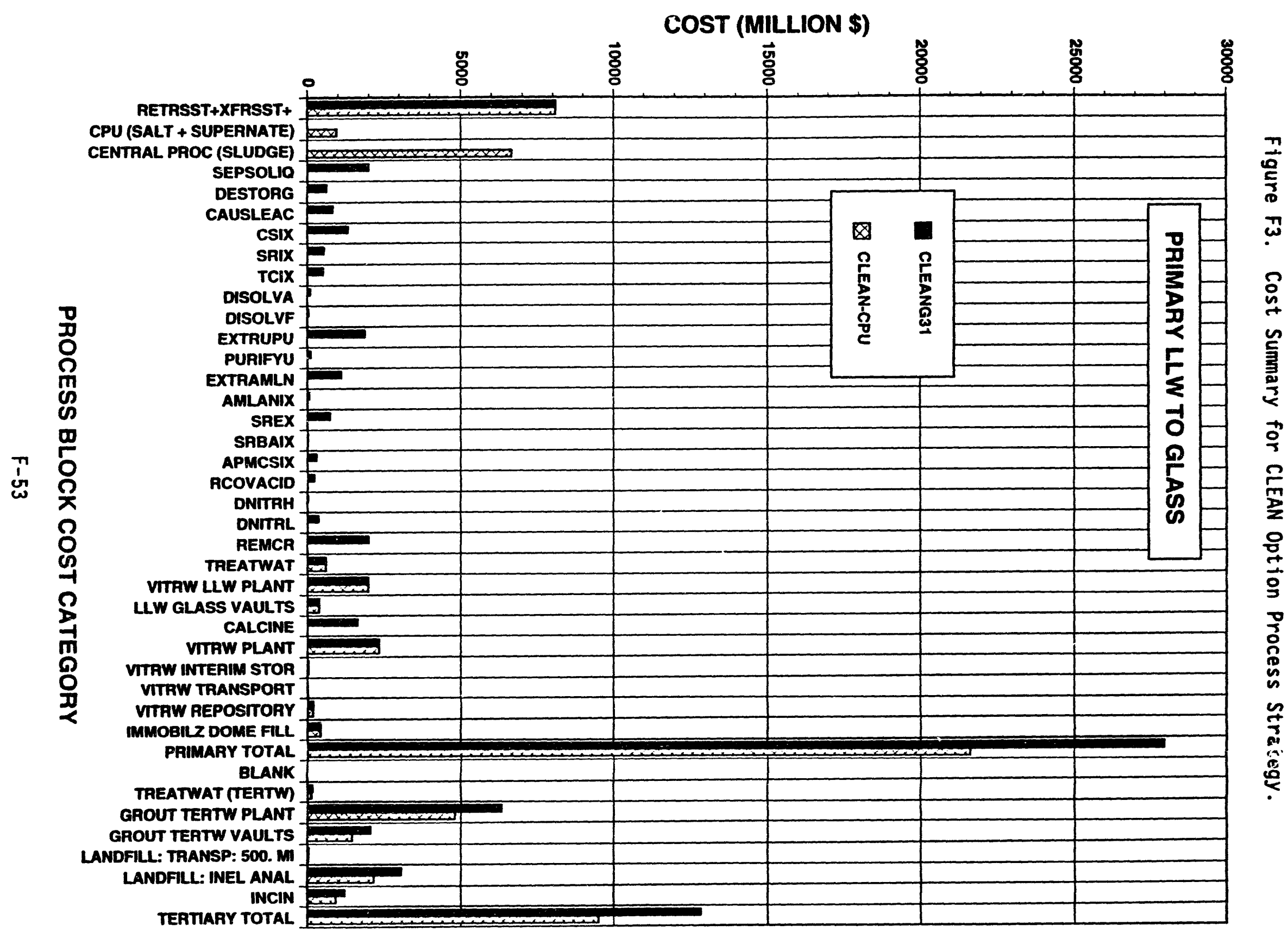


Figure F4. Cost Summary for TRUEX-C and Sludge Wash C Process Strategies.

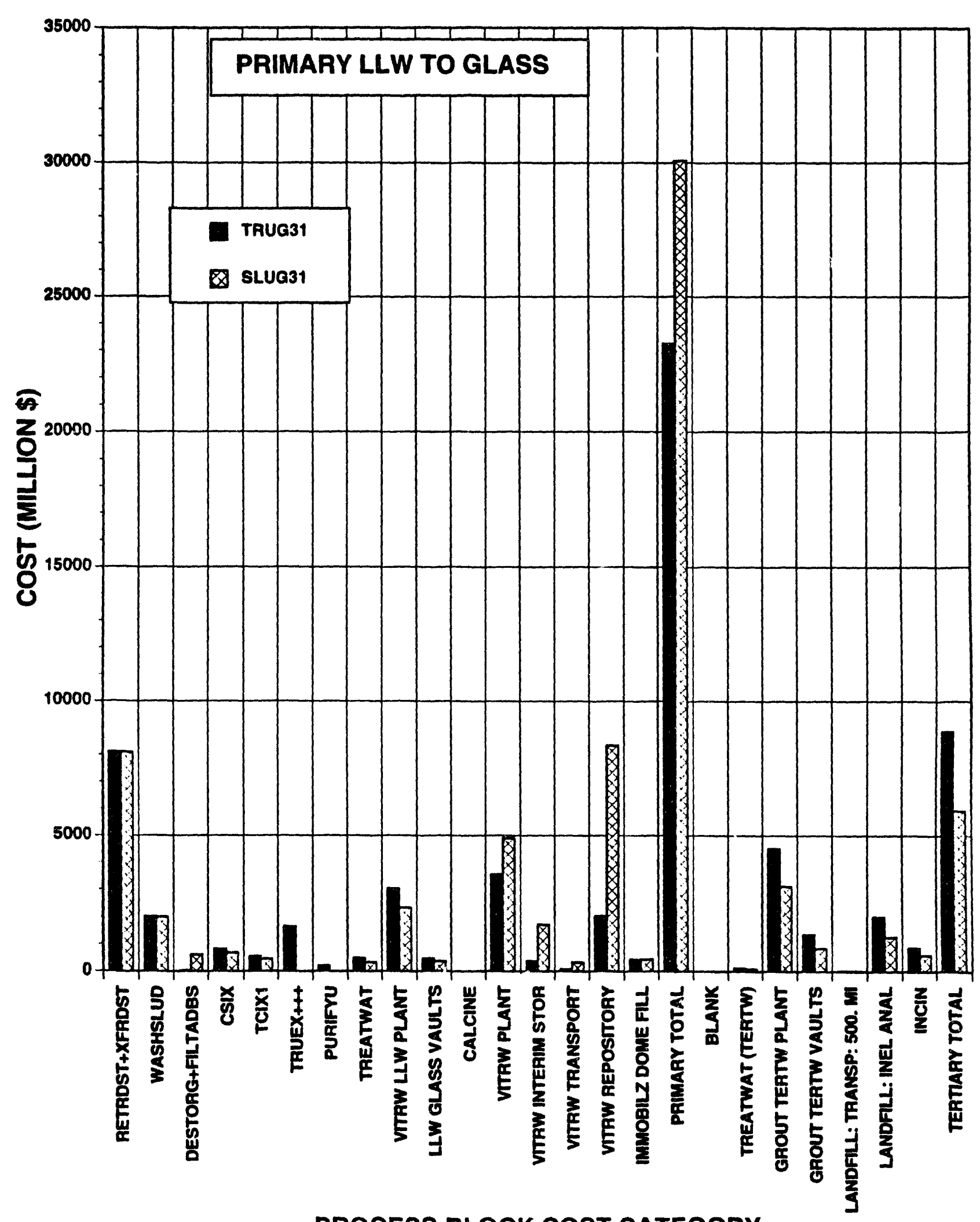

PROCESS BLOCK COST CATEGORY 
Figure F5. Cost Summary of Major Process Categories With Primary Low-Level Waste Glass Product.

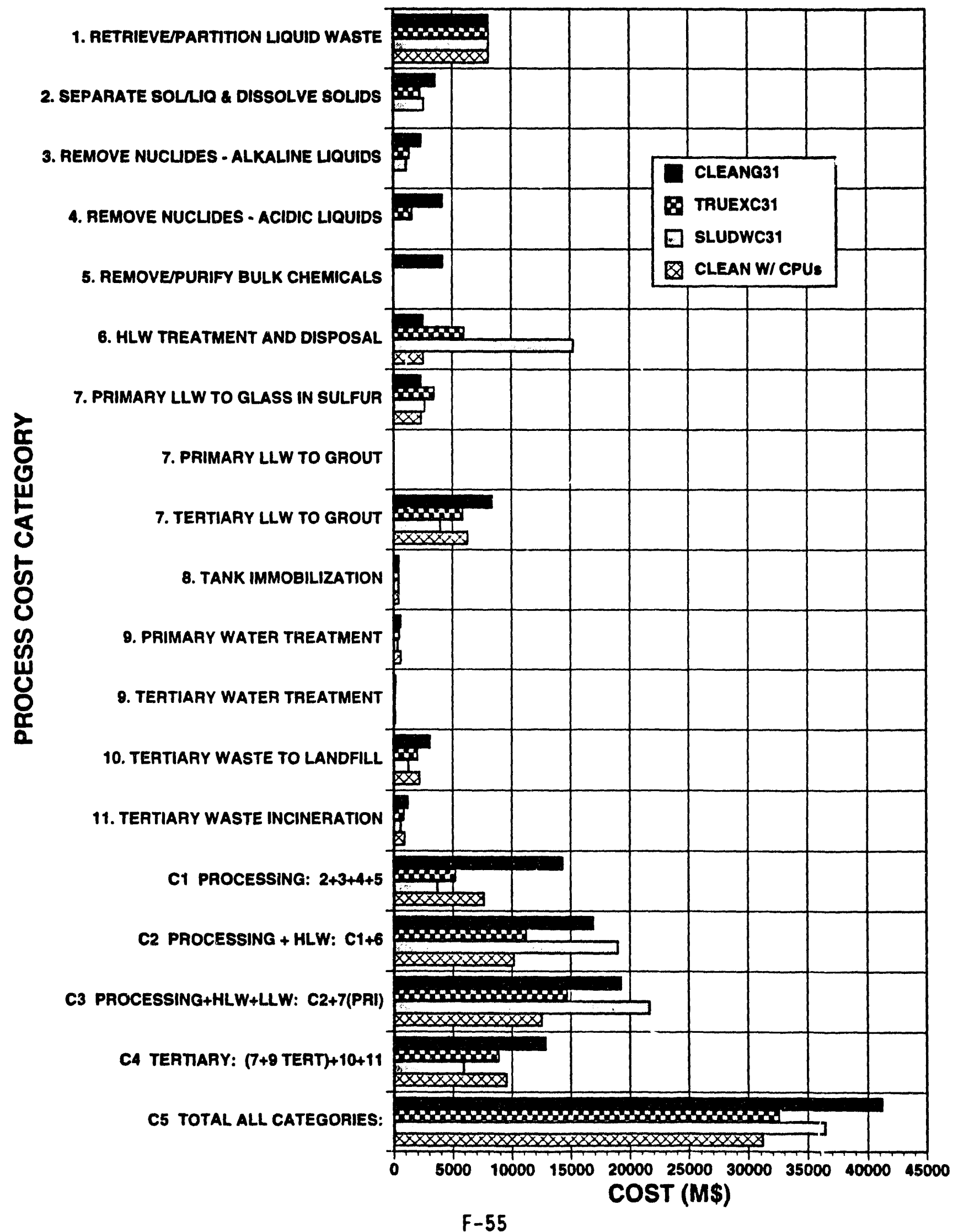


Figure F6. Cost Summary of Major Process Categories With Primary Low-Level Waste Grout Product.

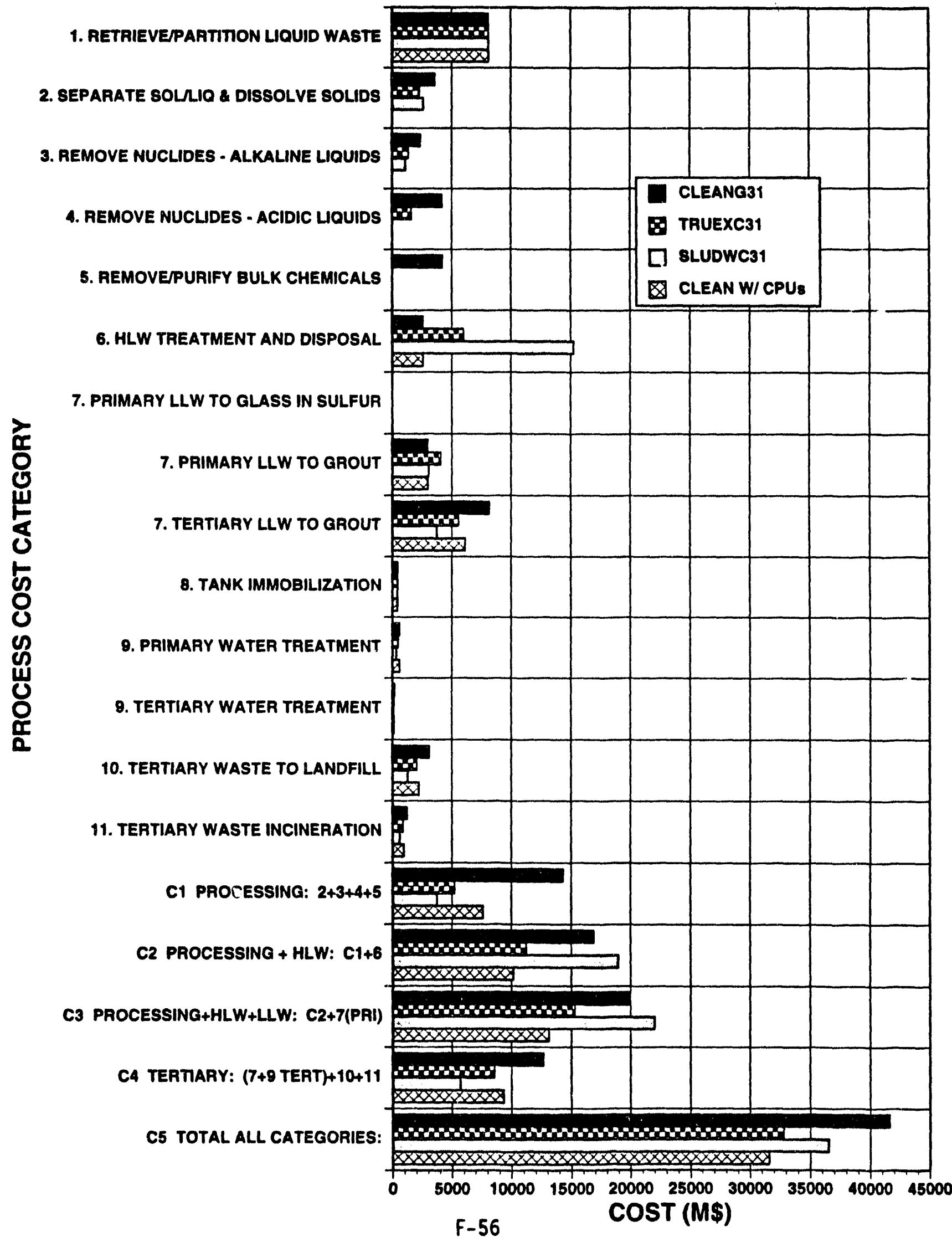


Table F1. Cost Summary for Model CLEANG31.

PAIMARY LIWTOGROUT COST (M\$) SUMMMAY (PRMAARY WASTE)

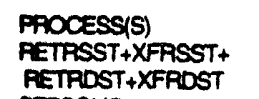
RETRDST+XFPDST EePsovo

DESTOPG

CAUSLEAC

$\cos x$

$\sin$

TaX

DISOLVA

DISOLVF

EXTFUPU

PURTN

EXTRAMLN

AMLANIX

SFOX

SPBAIX

APMCSIX

ACOVACID

DNITPH

DNITAL

FAMR

TAEATWAT

GROUTW(LIW)

PLANT

VAULTS

CALCINE

VITRW(HLW)

PLANT

WIEAM STOA

TRANSPORT

PEPOSTORY

DOME FILLNG

DOME FILUNG

NTRUSN BARRIER
INSITU VITRIF.

$$
017.70
$$

DPARE

8091.00
$1077.00 \quad 1095.00$

$\begin{array}{lll}484.90 & 142.80 & 627.70\end{array}$

$\begin{array}{llr}394.60 & 435.90 & 830.50\end{array}$

$\begin{array}{llr}671.60 & 644.20 & 1316.00\end{array}$

$\begin{array}{lll}87.61 & 446.10 & 533.70\end{array}$

$\begin{array}{lll}84.58 & 430.90 & 515.50\end{array}$

$\begin{array}{lll}49.35 & 44.74 & 94.07\end{array}$

$48.35 \quad 44.74$

1350.00

86.07

551.50

15.53

515.70
27.57

27.57

545.30

40.71

363.10

15.44

238.20

107.90

14.47

176.10

1071.00

177.20
912.90

319.20

1995.00

647.50

926.70

647.50
702.40

$2335.00 \quad \ldots$

$\begin{array}{ll}\ldots & 30.94 \\ \ldots & 5.78\end{array}$

175.80

106.90

178.20

320.70

0.00

TOTAL

...
TOTAL.

84.07
33.92

33.82
1865.00

113.60

1097.00

57.24

731.50

21.95

296.00

215.10

31.67

353.30

1984.00

602.30

966.70

1995.00

2335.00

30.94
5.78

175.80

427.70

PERCENT

27.0

6.9
2.2

2.9

4.5
1.8

1.8

0.3

6.4

0.4
3.8

0.2

2.5
0.1

0.1
1.0
0.7

0.1

1.2
6.8

2.1

VITRW(LLW):

3.3

6.9

\section{1}

0.1

0.6

1.5

33.20

... $\quad 28970.00$

100.0

PAMMAY UW TO CUASS N SULFUR

COST (MA) SUMMARY (PAMARY WASTE)

CAPTTAL DFENE TOTAL PECENT

$\begin{array}{rrrr}\ldots 1.0 & \cdots & 6091.00 & 28.5 \\ 917.70 & 1077.00 & 1095.00 & 7.0 \\ 484.90 & 142.80 & 627.70 & 2.2 \\ 384.60 & 435.90 & 830.50 & 2.9 \\ 671.60 & 644.20 & 1316.00 & 4.6 \\ 87.61 & 446.10 & 533.70 & 1.9 \\ 84.58 & 130.90 & 515.50 & 1.8 \\ 49.33 & 44.74 & 94.07 & 0.3 \\ 18.38 & 15.53 & 33.92 & 0.1 \\ 1350.00 & 515.70 & 1865.00 & 6.6 \\ 86.07 & 27.57 & 113.60 & 0.4 \\ 551.50 & 545.30 & 1097.00 & 3.8 \\ 16.53 & 40.71 & 57.24 & 0.2 \\ 368.40 & 363.10 & 731.50 & 2.6 \\ 6.51 & 15.44 & 21.95 & 0.10 \\ 57.89 & 238.20 & 296.00 & 1.0 \\ 107.20 & 107.90 & 215.10 & 0.8 \\ 17.20 & 14.47 & 31.67 & 0.1 \\ 177.20 & 176.10 & 353.30 & 1.2 \\ 912.90 & 1071.00 & 1984.00 & 7.0 \\ . \cdots & \ldots 1.0 & 602.30 & 2.1\end{array}$

$\begin{array}{llll}778.40 & 1188.00 & 1976.40 & 7.0\end{array}$

$\begin{array}{rrrr}386.30 & \cdots & 386.30 & 1.4 \\ 826.70 & 702.40 & 1629.00 & 5.7\end{array}$

$\begin{array}{llll}2335.00 & \cdots & 2335.00 & 8.2\end{array}$

8.2
0.1
0.0

0.0

1.5

106.90
178.20

244.40

$\begin{array}{rr}320.70 & 427.70 \\ 0.00 & \ldots\end{array}$

*. 28372.97

100.0

$\cdots$ " TABLE PLACE HOLDER

COST (MS) SUMMAPY (TERTIARY WASTE)

PAOCESS(S)

TREATWAT

GROUTW:

PLANT

VAULTS

TRANSP: $30 . M$

TRANSP: $300 . \mathrm{MI}$

TRANSP: 500 . MI

USECOLOGY

INEL ANALYSIS

NCEN

$$
\text { CAPITAL }
$$

CAPITAL EPENE TOTAL

$2026.00 \quad 4110.00 \quad 6137.00$

$\begin{array}{rrr}2026.00 & 4110.00 & 6137.00\end{array}$

$\ldots \quad 20.40$

$\begin{array}{rr}\ldots & 20.40 \\ \ldots & 35.00 \\ \ldots & 46.15 \\ \ldots & 1400.00 \\ \ldots & 3049.00\end{array}$

1048.00

160.90

-...

TOTAL

•..

$\cdots$ = TABLE PLACE HOLDER

TERTAPYPAMMARY

0.44

2961.70

(PLANT + VAULTS

...

COST (M\$) SUMMARY (TERTIARY WASTE)

PETCENT

48.5

16.2

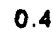

24.1

100.0

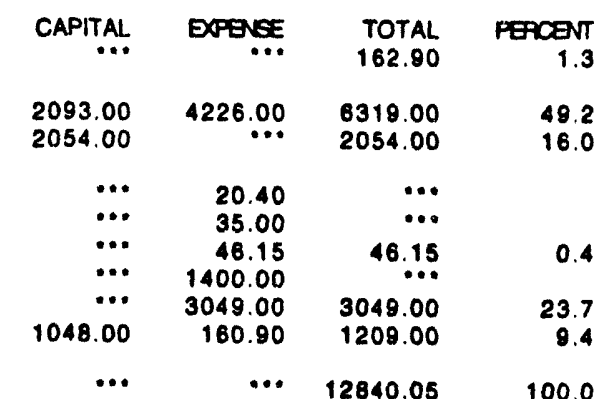

0.45

2362.70

0.80

GLASS / GROUT 
Table F2. Cost Summary for Model TRUEXC31.

PRMAPY LWT TO GROUT

COST (MS) SUMM TY (PAIMARY WASTE)

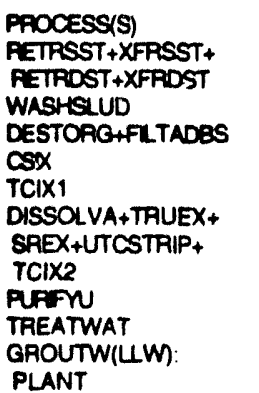

PLANT

VAULTS

VITRW(HLW):

PLANT

WTERM STOR

TRANSPORT

REPOSTTOFY

MMOBILZ

DOME FILUNG

NTRUSN BARRIEA

INSITU VITRIF.

CAPITAL EPENEE TOTAL

... $\quad \cdots \quad 8091.00$

$017.70 \quad 1077.00 \quad 1895.00$

22.72

410.60

6.69

396.30

451.40

29.41

806.90

538.50

$828.00 \quad 802.10 \quad 1630.00$

150.50

50.

200.70

$\begin{array}{lll}566.30 & 1141.00 \quad 1707.00\end{array}$

2349.00

1141.00

1707.00
2349.00

$\begin{array}{rrr}0.00 & 0.00 & 0.00\end{array}$

3548.00

8.00
$\ldots$
$\ldots$

... 3548.00

$\begin{array}{rr}366.40 & 366.40\end{array}$

$2018.00 \quad 2018.00$

106.90

178.20

320.70

427.70

244.40

0.00
733.20

427.70
$\ldots$
..

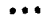

... 24250.00

TOTAL

$\cdots=$ TABLE PLACE HOLDER

COST (M\$) SUMMARY (TERTLARY WASTE)

PROCESS(S)
TREATWAT
GROUTW.
PLANT
VAULTS
LANDFILL:
TRANSP: $30 . \mathrm{MI}$
TRANSP: $300 \mathrm{MI}$
TRANSP: $500 \mathrm{MI}$
USECOLOGY
INEL ANALYSIS
NCIN

$\begin{array}{rr}\text { CAPITAL } & \text { EPEESE } \\ 1395.00 & 2810.00 \\ 1344.00 & \ldots \\ \ldots & 13.35 \\ \ldots & 22.90 \\ \ldots & 30.20 \\ \ldots & 916.10 \\ \ldots & 1995.00 \\ 746.60 & 117.10\end{array}$

TOTAL

PERCENT

EOENT

33.4

8.2
0.1

3.3
2.2

6.7
0.8

2.0

VITAW(LLW)

7.0
9.7

0.0

14.6

1.5
0.3

8.3

1.8

100.0

4205.00

1344.00

49.1

15.7

...

30.20

1995.00

863.70

8557.00

TOTAL

...

.. = TABLE PLACE HOLDER

TERTLAYPPAMAPY

0.35

0.4

23.3

100.0
PAMMAY LW TO CLASS IN SULFUA

COST (MA) SUMMAPY (PAMMRY WASTE)

\begin{tabular}{|c|c|c|c|}
\hline CAPTTAL & DPENEE & TOTAL & FERCENT \\
\hline $\begin{array}{r}\cdots \\
917.70 \\
22.72 \\
410.80 \\
87.05\end{array}$ & $\begin{array}{r}\cdots \\
1077.00 \\
6.68 \\
396.30 \\
451.40\end{array}$ & $\begin{array}{r}8091.00 \\
1995.00 \\
28.41 \\
808.90 \\
538.50\end{array}$ & $\begin{array}{r}34.2 \\
8.4 \\
0.1 \\
3.4 \\
2.3\end{array}$ \\
\hline $\begin{array}{r}828.00 \\
150.50 \\
\ldots\end{array}$ & $\begin{array}{r}802.10 \\
50.18 \\
\ldots\end{array}$ & $\begin{array}{r}1630.00 \\
200.70 \\
476.60\end{array}$ & $\begin{array}{l}6.0 \\
0.8 \\
2.0\end{array}$ \\
\hline $\begin{array}{r}1170.00 \\
451.30 \\
0.00\end{array}$ & $\begin{array}{r}1847.00 \\
0.00\end{array}$ & $\begin{array}{r}3017.00 \\
451.30 \\
0.00\end{array}$ & $\begin{array}{r}12.7 \\
1.8 \\
0.0\end{array}$ \\
\hline $\begin{array}{r}3548.00 \\
\ldots \\
\ldots\end{array}$ & $\begin{array}{r}\ldots \\
366.40 \\
68.44 \\
2018.00\end{array}$ & $\begin{array}{r}3548.00 \\
366.40 \\
68.44 \\
2018.00\end{array}$ & $\begin{array}{r}15.0 \\
1.5 \\
0.3 \\
8.5\end{array}$ \\
\hline $\begin{array}{l}106.90 \\
178.20 \\
244.40\end{array}$ & $\begin{array}{r}320.70 \\
0.00 \\
733.20\end{array}$ & $\begin{array}{r}427.70 \\
\ldots\end{array}$ & 1.8 \\
\hline$\cdots$ & ... & 23664.95 & 100.0 \\
\hline
\end{tabular}

COST (M\$) SUMMARY (TERTIARY WASTE)

\begin{tabular}{|c|c|c|c|}
\hline CAPITAL & EPFESE & $\begin{array}{r}\text { TOTAL } \\
118.70\end{array}$ & $\begin{array}{r}\text { PEROENT } \\
1.3\end{array}$ \\
\hline $\begin{array}{l}1503.00 \\
1344.00\end{array}$ & $\begin{array}{r}3001.00 \\
\ldots\end{array}$ & $\begin{array}{l}4504.00 \\
1344.00\end{array}$ & $\begin{array}{l}50.9 \\
15.2\end{array}$ \\
\hline$\ddot{\cdots}$ & $\begin{array}{l}13.35 \\
22.90\end{array}$ & $\ddot{\ldots}$ & \\
\hline$\ddot{\cdots}$ & $\begin{array}{r}30.20 \\
916.10\end{array}$ & $\begin{array}{r}30.20 \\
\ldots\end{array}$ & 0.3 \\
\hline 746.60 & $\begin{array}{r}1985.00 \\
117.10\end{array}$ & $\begin{array}{r}1995.00 \\
863.70\end{array}$ & $\begin{array}{r}22.5 \\
9.8\end{array}$ \\
\hline$\cdots$ & $\cdots$ & 8855.60 & 100.0 \\
\hline
\end{tabular}

0.37

3488.30

0.86

(PLANT + VAULTS)

4056.00 
WHC-EP-0791

Table F3. Cost Summary for Model SLUG31.

PRMARY UWTO GROUT

COST (MS) SUMMARY (PRIMARY WASTE)

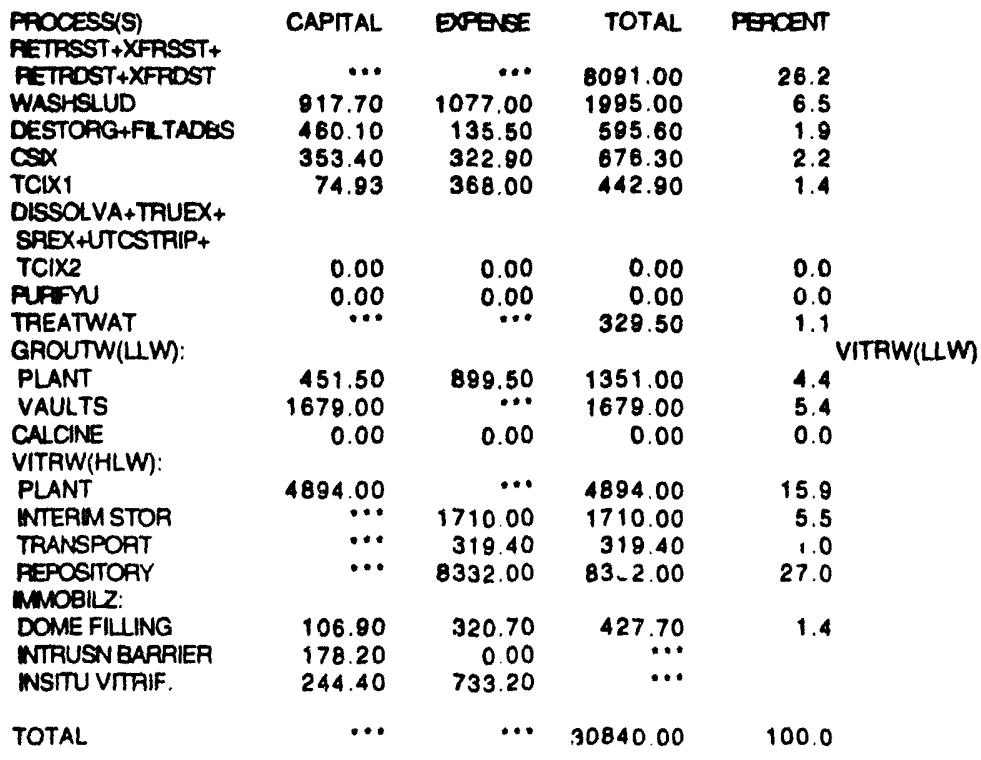

.. - TABLE PLACE HOLDER

COST (MS) SUMMARY (TERTIAAY WASTE)

PROCESSIS)
TREATWAT
GROUTW:
PLANT
VAULTS
LANDFILL:
TRANSP: $30 . \mathrm{MI}$
TRANSP: 300. MI
TRANSP: 500. MI
USECOLDGY
INEL ANALYSIS
WCIN

$\begin{array}{rrrr}\text { CAPITAL } & \text { EXENEE } & \begin{array}{r}\text { TOTAL } \\ 86.98\end{array} & \text { PERCENT } \\ 062.00 & 1917.00 & 2879.00 & 1.5 \\ 845.40 & \ldots & 845.40 & 50.7 \\ \ldots & & 14.9 \\ \ldots & 14.40 & \ldots & \\ \ldots & 18.99 & 18.99 & 0.3 \\ \ldots & 576.10 & \ldots & \\ \ldots 15.20 & 1255.00 & 1255.00 & 22.1 \\ \ldots & 82.67 & 597.80 & 10.5 \\ \ldots & \ldots & 5683.00 & 100.0\end{array}$

.. = TABLE PLACE hOLDER

TERTLARYPPIMAAYY

PRMARY UW PROCESSNG COSTS:

(PLANT + VAULTS)

GLASS I GROUT
0.18

0.18

PAMARY LWW TO GLASS W SULFUR

COST (MA) SUMMARY (PRIMAPY WASTE)

CAPITAL EPEESE TOTAL

$\begin{array}{rrr}\ldots 1 . & \ldots & 8091.00 \\ 917.70 & 1077.00 & 1995.00 \\ 460.10 & 135.50 & 595.60 \\ 353.40 & 322.90 & 676.30 \\ 74.93 & 368.00 & 442.90\end{array}$

PERCENT$$
74.90
$$

$\begin{array}{rrrr}0.00 & 0.00 & 0.00 & 0.0 \\ 0.00 & 0.00 & 0.00 & 0.0 \\ \cdots & \cdots & 328.50 & 1.1\end{array}$

$\begin{array}{rrrr}912.60 & 1418.00 & 2330.60 & 7.6 \\ 354.90 & \ldots & 354.90 & 1.2 \\ 0.00 & 0.00 & 0.00 & 0.0\end{array}$

$\begin{array}{llll}4894.00 & \ldots & 4894.00 & 16.0\end{array}$

$\begin{array}{llll}\ldots & 1710.00 & 1710.00 & 5.6\end{array}$

$\begin{array}{rrrr}\ldots & 318.40 & 319.40 & 1.0 \\ \ldots & 8332.00 & 8332.00 & 27.3\end{array}$

$\begin{array}{lrrr}106.90 & 320.70 & 427.70 & 1.4\end{array}$

$\begin{array}{rrr}178.20 & 0.00 & \ldots \\ 244.40 & 733.20 & \ldots\end{array}$

... $\quad$.. 30498.80

100.0

COST (MS) SUMMARY (TERTIARY WASTE)

\begin{tabular}{|c|c|c|c|}
\hline CAPITAL. & EXPESE & $\begin{array}{l}\text { TOTAL. } \\
86.98\end{array}$ & $\begin{array}{r}\text { PETCENT } \\
1.5\end{array}$ \\
\hline $\begin{array}{r}1047.00 \\
645.40\end{array}$ & $\begin{array}{r}2068.00 \\
\ldots\end{array}$ & $\begin{array}{r}3115.00 \\
845.40\end{array}$ & $\begin{array}{l}52.6 \\
14.3\end{array}$ \\
\hline$\ddot{*}$ & $\begin{array}{r}8.40 \\
14.40\end{array}$ & $\ldots$ & \\
\hline$\ddot{\ldots}$ & $\begin{array}{r}18.99 \\
576.10\end{array}$ & $\begin{array}{r}18.99 \\
. . .\end{array}$ & 0.3 \\
\hline $\begin{array}{r}\ldots 1 . \\
515.20\end{array}$ & $\begin{array}{r}1255.00 \\
82.67\end{array}$ & $\begin{array}{r}1255.00 \\
597.80\end{array}$ & $\begin{array}{l}21.2 \\
10.1\end{array}$ \\
\hline$\cdots$ & ... & 5919.17 & 100.0 \\
\hline
\end{tabular}

3030.00

2685.50

0.89 
Table F4. Cost Summary for Model CLEANG31 Incorporating Compact Processing Units.

PRIMARY UIW TOGROUT COST (M\$) SUMMAAYY (PRIMARY WASTE)

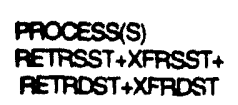

CENTRALPAOC

(SLUDGE)

CPU ISALT +

SUPERNATE)

TREATWAT

GROUTW(UW):

PLANT

VAULTS

VITRW(HLW):

PLANT

NTERM STOR

TRANSPORT

PEPOSTIOFY

MMOBIL:

DOME FILUNG

NIRUSN BARRIER

NSTU VITRIF.

CAPITAL

ERENEE

....

TOTAL

PECENT

0000.00

2600.00

745.8

3980.00

8091.00

36.4

TOTAL

$\cdots$ = TABLE PLACE HOLDER

COST (M\$) SUMMARY (TERTIARY WASTE)

PAOCESSSS
TAEATWAT
GROUTW:
PLANT
VAULTS
LANDFILL:
TRANSP: 30. MI
TRANSP: 300. MI
TRANSP: 500. MI
USECOIOGY
INEL ANALYSIS
NCIN

$\begin{array}{rrrr}\text { CAPTAL. } & \text { EPPESE } & \text { TOTAL } & \text { PERCENT } \\ 121.70 & 1.3 \\ 1547.00 & 3107.00 & 4654.00 & 49.8 \\ 1458.00 & \ldots & 1458.00 & 15.6 \\ \ldots & 14.49 & \ldots & \\ \ldots & 24.85 & \ldots . . & \\ \ldots & 32.76 & 32.76 & 0.4 \\ \ldots & 993.90 & \ldots 1.0 & \\ 796.90 & 2165.00 & 2165.00 & 23.1 \\ \ldots & 124.40 & 921.40 & 8.9 \\ \ldots & \ldots & 9352.00 & 100.0\end{array}$

$\cdots=$ TABLE PLACE HOLDER

TERTIARYPPIMARY

2961.70

(PLANT + VAULTS)
PAIMARY LWTO GLASS IN SULFUR

COST (MS) SUMMARY (PAIMAAY WASTE)

\begin{tabular}{|c|c|c|c|}
\hline CAPITAL & DPESE & TOTAL & PEACENT \\
\hline$\cdots$ & $\cdots$ & 8091.00 & 37.4 \\
\hline 2680.00 & 3980.00 & 6660.00 & 30.8 \\
\hline 745.86 & 204.00 & 948.86 & 4.4 \\
\hline$\cdots$ & $\cdots$ & 602.30 & 2.8 \\
\hline $\begin{array}{l}778.40 \\
386.30\end{array}$ & 1198.00 & $\begin{array}{r}1976.40 \\
386.30\end{array}$ & $\begin{array}{l}0.1 \\
1.8\end{array}$ \\
\hline $\begin{array}{r}2335.00 \\
\ldots \\
\ldots \\
\ldots\end{array}$ & $\begin{array}{r}30.94 \\
5.78 \\
175.80\end{array}$ & $\begin{array}{r}2335.00 \\
30.94 \\
5.78 \\
175.80\end{array}$ & $\begin{array}{l}10.8 \\
0.1 \\
0.0 \\
0.8\end{array}$ \\
\hline $\begin{array}{l}106.90 \\
178.20 \\
244.40\end{array}$ & $\begin{array}{r}320.70 \\
0.00 \\
733.20\end{array}$ & $\begin{array}{r}427.70 \\
\ldots \\
\ldots\end{array}$ & 2.0 \\
\hline & $\cdots$ & 21641.08 & 00.0 \\
\hline
\end{tabular}

COST (M\$) SUMMAAY (TERTIARY WASTE)

\begin{tabular}{|c|c|c|c|}
\hline $\begin{array}{r}\text { CAPITAL } \\
\ldots\end{array}$ & EPENEE & $\begin{array}{r}\text { TOTAL } \\
121.70\end{array}$ & $\begin{array}{r}\text { PERCENT } \\
1.3\end{array}$ \\
\hline $\begin{array}{l}1602.00 \\
1458.00\end{array}$ & $\begin{array}{r}3205.00 \\
\ldots\end{array}$ & $\begin{array}{l}4807.00 \\
1458.00\end{array}$ & $\begin{array}{l}50.6 \\
15.3\end{array}$ \\
\hline ... & $\begin{array}{r}14.49 \\
24.85\end{array}$ & $\ldots$ & \\
\hline$\ldots$ & $\begin{array}{r}32.76 \\
\end{array}$ & 32.76 & 0.3 \\
\hline$\because$. & $\begin{array}{r}993.90 \\
\end{array}$ & & \\
\hline 796.90 & $\begin{array}{r}2165.00 \\
124.40\end{array}$ & $\begin{array}{r}2165.00 \\
821.40\end{array}$ & $\begin{array}{r}22.8 \\
9.7\end{array}$ \\
\hline$\cdots$ & .• & 9505.86 & 100.0 \\
\hline
\end{tabular}

0.44

2362.70 0.80

GLASS / GROUT 
Table F5. Total Cost (Million \$) Summary for Process Strategies.

\begin{tabular}{|c|c|c|c|c|c|c|c|c|}
\hline \multirow{2}{*}{ moore. } & \multirow{2}{*}{$\begin{array}{l}\text { CLENGS1 } \\
\text { CEEN OPTION } \\
\text { (OO\% HEEL) }\end{array}$} & \multicolumn{2}{|c|}{ PRAMARY LW TO GROYT } & \multirow[b]{2}{*}{$\begin{array}{l}\text { CLEAN W/ CPUS } \\
\text { CLENOPTON } \\
\text { (O\% HEEL) }\end{array}$} & \multirow[b]{2}{*}{$\begin{array}{l}\text { CEENG31 } \\
\text { CEN OPTON } \\
\text { (O\% HEEL) }\end{array}$} & \multicolumn{3}{|c|}{ PRMUAY UWTO QLASS W SULFUA } \\
\hline & & $\begin{array}{l}\text { TRUEXCO1 } \\
\text { TREXXC } \\
\text { (O\% HEEL) }\end{array}$ & $\begin{array}{l}\text { SUUDWC31 } \\
\text { SUDGE WASHC } \\
\text { (O\% HEEL) }\end{array}$ & & & $\begin{array}{l}\text { TRUEXCO1 } \\
\text { MIEXCC } \\
(0 \% \text { HEEL) }\end{array}$ & $\begin{array}{l}\text { SUDWC31 } \\
\text { SUOGE WASHC } \\
\text { (0\% HEEL) }\end{array}$ & $\begin{array}{l}\text { CLEAN W/ CPU } \\
\text { CEAN DPTON } \\
\text { (O\% HEEL) }\end{array}$ \\
\hline \multicolumn{9}{|l|}{ WASTE STREAM } \\
\hline $\begin{array}{l}\text { PAMAN } \\
\text { TERTWFY }\end{array}$ & $\begin{array}{l}28970 \\
12660\end{array}$ & $\begin{array}{l}24250 \\
8557\end{array}$ & $\begin{array}{l}30840 \\
5683\end{array}$ & $\begin{array}{c}22240 \\
8352\end{array}$ & $\begin{array}{l}28373 \\
12840\end{array}$ & $\begin{array}{c}23665 \\
8856\end{array}$ & $\begin{array}{c}30408 \\
5810\end{array}$ & $\begin{array}{l}21641 \\
0506\end{array}$ \\
\hline TOTAL & 41630 & 32807 & 36523 & 31582 & 41213 & 32521 & 36418 & 31147 \\
\hline TERTAPYPPAMARYY & 0.44 & 0.35 & 0.18 & 0.42 & 0.45 & 0.37 & 0.18 & 0.44 \\
\hline $\begin{array}{l}\text { PECENT OF COST: } \\
\text { PFOWAYY } \\
\text { TEMTHYY }\end{array}$ & $\begin{array}{l}68.6 \\
30.4\end{array}$ & $\begin{array}{l}73.8 \\
26.1\end{array}$ & $\begin{array}{l}84.4 \\
15.6\end{array}$ & $\begin{array}{l}70.4 \\
28.6\end{array}$ & $\begin{array}{l}68.8 \\
31.2\end{array}$ & $\begin{array}{l}72.8 \\
27.2\end{array}$ & $\begin{array}{l}83.7 \\
16.3\end{array}$ & $\begin{array}{l}60.5 \\
30.5\end{array}$ \\
\hline \multicolumn{9}{|l|}{ CLEANG31/MODEL" COST: } \\
\hline $\begin{array}{l}\text { PFAMAPY } \\
\text { TEITUPY }\end{array}$ & & $\begin{array}{l}1.18 \\
1.48\end{array}$ & $\begin{array}{l}0.94 \\
2.23\end{array}$ & $\begin{array}{l}1.30 \\
1.35\end{array}$ & & $\begin{array}{l}1.20 \\
1.45\end{array}$ & $\begin{array}{l}0.93 \\
2.17\end{array}$ & $\begin{array}{l}1.31 \\
1.35\end{array}$ \\
\hline TSTAL & & 1.27 & 1.14 & 1.32 & & 1.27 & 1.13 & 1.32 \\
\hline \multicolumn{9}{|l|}{ TRUEX-CrMODEL" COST: } \\
\hline $\begin{array}{l}\text { PFAMAYY } \\
\text { TERTAAYY }\end{array}$ & $\begin{array}{l}0.84 \\
i .68\end{array}$ & & $\begin{array}{l}0.79 \\
1.51\end{array}$ & $\begin{array}{l}1.09 \\
0.91\end{array}$ & $\begin{array}{l}0.83 \\
0.69\end{array}$ & & $\begin{array}{l}0.78 \\
1.50\end{array}$ & $\begin{array}{l}1.09 \\
0.83\end{array}$ \\
\hline TOTAL & 0.79 & & 0.80 & 1.04 & 0.79 & & 0.80 & 1.04 \\
\hline \multicolumn{9}{|c|}{ SLUDGE WASH C/MODEL" COST: } \\
\hline $\begin{array}{l}\text { PAMAPY } \\
\text { TERTARY }\end{array}$ & $\begin{array}{l}1.06 \\
0.45\end{array}$ & $\begin{array}{l}1.27 \\
0.56\end{array}$ & & $\begin{array}{l}1.39 \\
0.61\end{array}$ & $\begin{array}{l}1.07 \\
0.46\end{array}$ & $\begin{array}{l}1.29 \\
0.67\end{array}$ & & $\begin{array}{l}1.41 \\
0.62\end{array}$ \\
\hline TOTAL & 0.88 & 1.11 & & 1.16 & 0.88 & 1.12 & & 1.17 \\
\hline \multicolumn{9}{|c|}{ CLEAN W/ CPU\&FMODEL- COST: } \\
\hline $\begin{array}{l}\text { PRAMAPY } \\
\text { TERTLAY }\end{array}$ & $\begin{array}{l}0.77 \\
0.74\end{array}$ & $\begin{array}{l}0.92 \\
1.09\end{array}$ & $\begin{array}{l}0.72 \\
1.65\end{array}$ & & $\begin{array}{l}0.76 \\
0.74\end{array}$ & $\begin{array}{l}0.91 \\
1.07\end{array}$ & $\begin{array}{l}0.71 \\
1.61\end{array}$ & \\
\hline TOTAL & 0.76 & 0.86 & 0.86 & & 0.76 & 0.96 & 0.86 & \\
\hline \multicolumn{9}{|c|}{ LW GROUT VE. GLASS PRODUCT COSTS: } \\
\hline $\begin{array}{l}\text { PAIMADY } \\
\text { TERTLAY }\end{array}$ & & & & & $\begin{array}{l}0.98 \\
1.01\end{array}$ & $\begin{array}{l}0.98 \\
1.03\end{array}$ & $\begin{array}{l}0.89 \\
1.04\end{array}$ & $\begin{array}{l}0.8 \% \\
1.02\end{array}$ \\
\hline TOTAL & & & & & 0.89 & 0.99 & 1.00 & 0.89 \\
\hline
\end{tabular}


WHC-EP-0791

Table F6. Summary of Process Distribution Across Major Process Categories.

\begin{tabular}{|c|c|c|c|c|}
\hline MAOAPROCESS CATESOFY & $\begin{array}{l}\text { CLEANG31 } \\
\text { CEAN OPTION }\end{array}$ & $\begin{array}{l}\text { Thuexca1 } \\
\text { ThUEXC }\end{array}$ & $\begin{array}{l}\text { SUDWC31 } \\
\text { SUDEE WASHC }\end{array}$ & $\begin{array}{l}\text { CLEAN W/CPUS } \\
\text { CENOPTION }\end{array}$ \\
\hline $\begin{array}{l}\text { RETPIEVE TANK WASTE } \\
\text { AND PARTTION LOUID WASTE: }\end{array}$ & 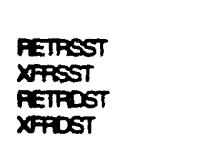 & $\begin{array}{l}\text { PETREST } \\
\text { XFPST } \\
\text { RETPST } \\
\text { XFTOST }\end{array}$ & $\begin{array}{l}\text { PETRST } \\
\text { XFAST } \\
\text { FETRST } \\
\text { XFODST }\end{array}$ & $\begin{array}{l}\text { RETRST } \\
\text { XPFST } \\
\text { RETPST } \\
\text { XFPDST }\end{array}$ \\
\hline $\begin{array}{l}\text { SEPAPATE SOUDS AND LOUIDS } \\
\text { AND DISSOLVE SOUDS: }\end{array}$ & $\begin{array}{l}\text { SEPSOAO } \\
\text { DESTOPG } \\
\text { CAUSEAC } \\
\text { DISOLVA } \\
\text { DISOLVF }\end{array}$ & $\begin{array}{l}\text { WASTHSUD } \\
\text { DESTOAG } \\
\text { FLTADES } \\
\text { DISSOLVA. }\end{array}$ & $\begin{array}{l}\text { WASHSLUD } \\
\text { DESTORG } \\
\text { FITADES }\end{array}$ & : \\
\hline $\begin{array}{l}\text { REVOVE NUOA DES FFOM } \\
\text { ALKALINE LUUIDS: }\end{array}$ & $\begin{array}{l}\operatorname{cs} \alpha \\
\operatorname{sp\alpha } \\
\operatorname{Tax}\end{array}$ & $\cos _{\text {TCIX1 }}$ & $\cos _{\operatorname{Tc} x}$ & $\begin{array}{l}\text { CENTRA PAOC } \\
\text { (SLUDGE) } \\
\text { CPU (SALT + } \\
\text { SUPERNATE) }\end{array}$ \\
\hline ACIDIC LIOUIDS: & $\begin{array}{l}\text { EXTAUPU } \\
\text { PUPFN } \\
\text { EXTRAMIN } \\
\text { AMLANIX } \\
\text { SFEX } \\
\text { SPBAXX } \\
\text { APMCSIX }\end{array}$ & $\begin{array}{l}\text { TREX } \\
\text { PUPFN } \\
\text { UTCSTRIP } \\
\text { TCIX2 } \\
\text { SFEX }\end{array}$ & & $\vdots$ \\
\hline $\begin{array}{l}\text { RENONE AND PUAIFY } \\
\text { BULK CHEMICALS: }\end{array}$ & $\begin{array}{l}\text { RCONACID } \\
\text { DNTAH } \\
\text { DNITRL } \\
\text { PEMOR } \\
\text { CALCINE }\end{array}$ & & & $:$ \\
\hline HLW TREATMENT AND DISPOSAL: & $\begin{array}{l}\text { VITRWWHLW): } \\
\text { PLANT } \\
\text { NTERMSTOP STO } \\
\text { TRANSPORT } \\
\text { REPOSTOFY }\end{array}$ & $\begin{array}{l}\text { VITRW(HLW): } \\
\text { PLANT } \\
\text { NTERIM STOR } \\
\text { TRANSPORT } \\
\text { REPOSTTORY }\end{array}$ & $\begin{array}{l}\text { VITRW(HLW): } \\
\text { PLANT } \\
\text { WTERIMSTOR } \\
\text { TRANSPOAT } \\
\text { REPOSTOFY }\end{array}$ & $\begin{array}{l}\text { VITRW(HLW): } \\
\text { PLANT } \\
\text { WTERM STOR } \\
\text { TRANSPOAT } \\
\text { REPOSTOFY }\end{array}$ \\
\hline \multicolumn{5}{|l|}{ UW TREATMENT AND DISPOSAL: } \\
\hline PRIMARY WASTE: & $\begin{array}{l}\text { VITAW(LLW): } \\
\text { PLANT } \\
\text { VAULTS }\end{array}$ & $\begin{array}{l}\text { VITRW(LLW): } \\
\text { PLANT } \\
\text { VAULTS }\end{array}$ & $\begin{array}{l}\text { VITAW(LLLW): } \\
\text { PLANT } \\
\text { VAULTS }\end{array}$ & $\begin{array}{l}\text { VITRW(LLW): } \\
\text { PLANT } \\
\text { VAULTS }\end{array}$ \\
\hline \multicolumn{5}{|l|}{ OPTION 2: GROUT } \\
\hline PAIMARY WASTE: & $\begin{array}{l}\text { GROUTW(LW): } \\
\text { PLANT } \\
\text { VAULTS }\end{array}$ & $\begin{array}{l}\text { GROUTW(LLM): } \\
\text { PLANT } \\
\text { VAULTS }\end{array}$ & $\begin{array}{l}\text { GROUTW(LLM): } \\
\text { PLANT } \\
\text { VAULTS }\end{array}$ & $\begin{array}{l}\text { GROUTW(LI): } \\
\text { PLANT } \\
\text { VAULTS }\end{array}$ \\
\hline TERTIARY WASTE: & $\begin{array}{l}\text { GROUTW: } \\
\text { PLANT } \\
\text { VAULTS }\end{array}$ & $\begin{array}{l}\text { CROUTW: } \\
\text { PLANT } \\
\text { VAULTS }\end{array}$ & $\begin{array}{l}\text { GAOUTW: } \\
\text { PLANT } \\
\text { VAULTS }\end{array}$ & $\begin{array}{l}\text { GAOUTW: } \\
\text { PLANT } \\
\text { VAULTS }\end{array}$ \\
\hline TANK IMUOBILEATION: & $\begin{array}{l}\text { MMOOHZ: } \\
\text { DOME FILING } \\
\text { NTRUSNBARRIER } \\
\text { INSTU VITRIF. }\end{array}$ & $\begin{array}{l}\text { MMOBHLZ } \\
\text { DOME FILNG } \\
\text { NITUSN BARALER } \\
\text { WSTUU VITAIF. }\end{array}$ & 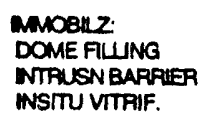 & $\begin{array}{l}\text { MMOBHZ: } \\
\text { DOME RUUNG } \\
\text { NIRUSN BARAIE } \\
\text { NSTTU VITAIF. }\end{array}$ \\
\hline \multicolumn{5}{|l|}{ WATER TREATMENT: } \\
\hline PRIMARY WASTE: & TREATWAT & TREATWAT & TREATWAT & TAEATWAT \\
\hline TERTIARY WASTE: & TREATWAT & TREATWAT & TREATWAT & TREATWAT \\
\hline TERTIARY WASTE TOLANDFIL: & $\begin{array}{l}\text { LANDFIL: } \\
\text { TRANSP: } 30 . M I \\
\text { TRANSP: } 300 . M 1 \\
\text { TRANSP: } 500 . \text { MI } \\
\text { USECOLOGY } \\
\text { INEL ANALYSIS }\end{array}$ & $\begin{array}{l}\text { LANDFILL: } \\
\text { TRANSP: } 30 . \mathrm{MI} \\
\text { TRANSP: } 300 . \text { MI } \\
\text { TRANSP: } 500 . \mathrm{MI} \\
\text { USECOOOGY } \\
\text { INEL ANALYSIS }\end{array}$ & $\begin{array}{l}\text { LANDFILL: } \\
\text { TRANSP: } 30 . M I \\
\text { TRANSP: } 300 . M I \\
\text { TRANSP: } 500 . M I \\
\text { USECOOGY } \\
\text { INEL ANALYSIS }\end{array}$ & $\begin{array}{l}\text { LANDFILL: } \\
\text { TRANSP: } 30 . \text { MI } \\
\text { TRANSP: } 300 . \text { MI } \\
\text { TRANSP: } 500 . \text { MI } \\
\text { USECOOOGY } \\
\text { INEL ANALYSIS }\end{array}$ \\
\hline TERTAPYY WASTE INCINERATION: & MaN & NaN & MCN & NCIN \\
\hline
\end{tabular}

- DISSOLVA $=0.15^{\circ}$ (TRUEX + SAEX+DISSOLVA+TCIX2+UTCSTRIP) 
Table F7. Cost (Million \$) Summary of Major Process Categories.

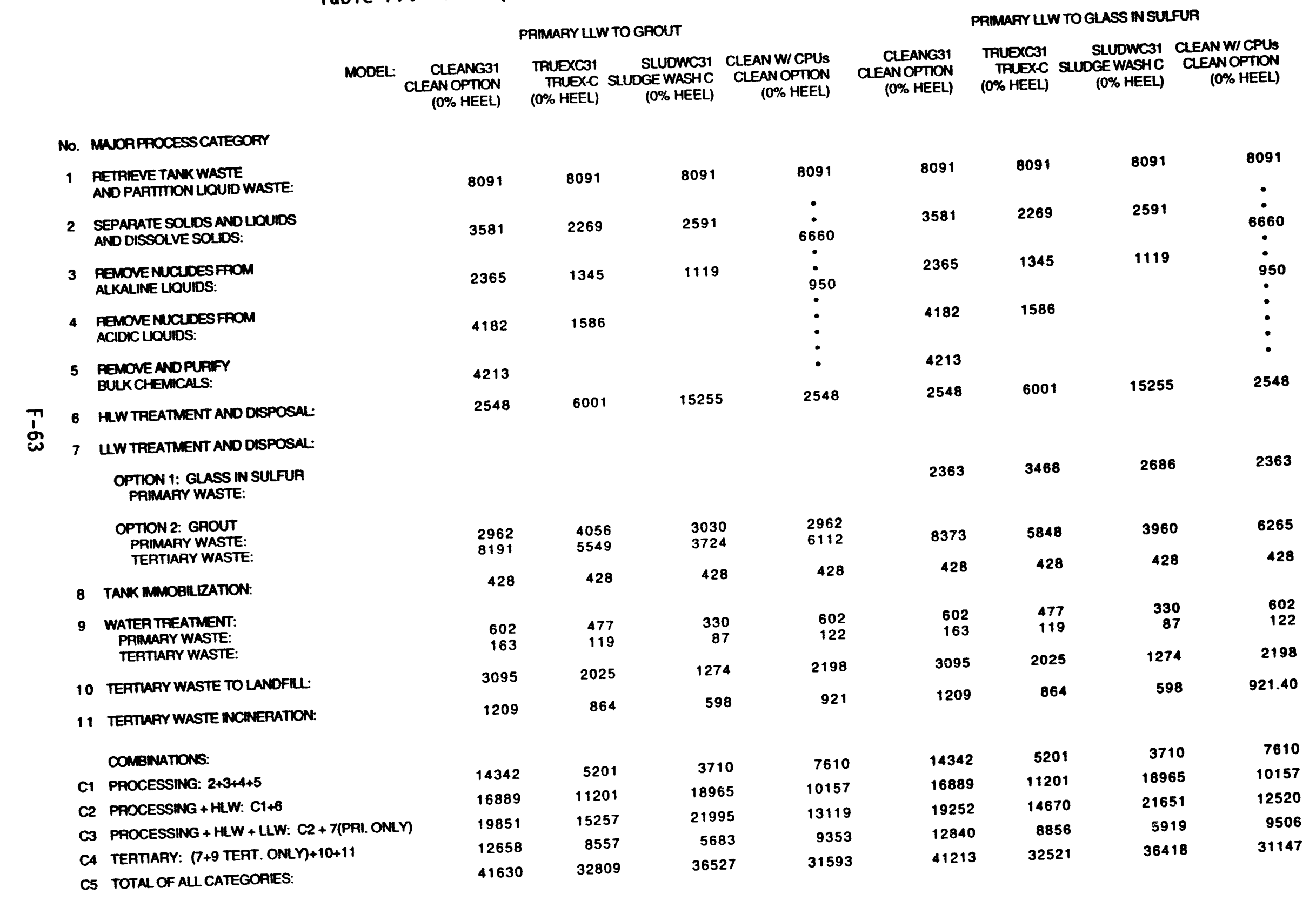


Table F8. Differential Cost (Million \$) Compared to Model CLEANG31 for Major Process Categories.

PRIMARY ULW TO GROUT

\begin{tabular}{|c|c|c|c|c|}
\hline MOD & $\begin{array}{r}\text { CLEANG31 } \\
\text { CLEANOPTON }\end{array}$ & $\begin{array}{r}\text { TRUEXC31 } \\
\text { TRUEX-C } \\
\text { (0\% HEEL) }\end{array}$ & $\begin{array}{r}\text { SLUDWC31 } \\
\text { SLUDGE WASHC } \\
\text { (0\% HEEL) }\end{array}$ & $\begin{array}{r}\text { CLEAN W/ CPUS } \\
\text { CLEAN OPTON } \\
\text { (O\% HEEL) }\end{array}$ \\
\hline
\end{tabular}

PAMAFY LLW TO GLASS N SULFUR CLEANG31 (O) HEEL)
TRUEXC31

CLEAN WICPUS

IRUEXC SLUDGE WASHC CLEANOPTION

(0\% HEEL) (0\% HESL) (O\% HEEL)

No. MAOAPAOCESSCATEGOFN

RETRIEVE TANK WASTE AND PARTIION LQUID WASTE:

2 SEPARATE SOUDS ANDLIOUIDS AND DISSOLVE SOLDS:

3 FEMOVENUCLOESFAOM ALKALINE LOUIDS:

4 PEMOVENUCLDESFAOM ACIDIC LOULS:

5 REMOVEANDPURFY BUIKCHEMACALS

T1 6 HWW TREATMENT AND DISPOSAL:

o 7 UW TREATMENT AND DISPOSAL.

OPTION 1: GLASS IN SULFUR

PAIMARY WASTE:

OPTION 2: GROUT

PRIMARY WASTE:

TERTIARY WASTE.

- TANK MMMOBLIRATION:

9 WATER TREATMENT:

PRIMARY WASTE:

TERTARY WASTE:

10 TERTIAPY WASTE TO LANDFILL:

11 TERTLAYY WASTE NCINERATION:

COMBNATIONS:

C1 PAOCESSING: $2+3+4+5$

C2 PROCESSIMG + HLW: C1+6

C3 PROCESSING + HLW + ULW: C2 + 7PPRI. ONLY

C4 TERTIARY: $(7+9$ TERT. ONLY $)+10+11$

C5 TOTAL OF ALL CATEGORIES

0

$\begin{array}{rr}0 & 0 \\ -1312 & -991 \\ -1020 & -1246 \\ -2596 & -4182 \\ -4213 & -4213 \\ 3453 & 12708\end{array}$

12708

0

1094
-264
2

68
-4467

0
-2079

0

-126
-44

$-1070$

$-345$

$-9141$

.5688

$-4594$

$-4101$

$-8821$

0

$-273$

-76
-1821

$-611$

$-10632$

2076

2144

.6975

.5103
$-991$

$-1312 \quad-991$

$-2596$

$-4213$

3453

$-4182$

$-4213$

12708

1106

323

$-2525$

$-4413$

0

$\begin{array}{rrr}0 & 0 & 0 \\ -126 & -273 & 0 \\ -44 & -76 & -41 \\ -1070 & -1821 & -897 \\ -345 & -611 & -288 \\ & & \\ -.9141 & -10632 & -6732 \\ -5688 & 2076 & -6732 \\ -.4582 & 2399 & -6732 \\ -3984 & -6921 & -3334 \\ -8692 & -4795 & -10066\end{array}$


WHC-EP-0791

\section{APPENDIX E}

TERTIARY NONPROCESS WASTE GENERATION

E-1 


\section{LIST OF TERMS}

AQUELLW
CBUSTL
CPU
DISS
DOE
DST
HLW
INEL
OFFGAS
NCBUSTS
SE
SRS
SST
TRU
VAQUELLW
VCBUSTL
VOFFGAS
VNCBUSTS
WMIS
WVDP

aqueous liquids combustible solids and liquids compact processing unit dissolver U.S. Department of Energy doubie-shell tank high-level waste Idaho National Engineering Laboratory cell air and offgas noncombustible solids solvent extraction Savannah River Site single-shell tank transuranic varied aqueous liquids varied combustible solids and liquids varied cell air and offgas varied noncombustible solids Waste Management Information System West Valley Demonstration Project 


\section{TERTIARY NONPROCESS WASTE GENERATION}

This appendix discusses the development of the tertiary waste generation estimation models and the results from using these models. The tertiary waste models are based on data collected from various U.S. Department of Energy (DOE) sites. These models were implemented in the global process models using FORTRAN calculation blocks.

\section{E1.0 DEVELOPMENT OF THE TERTIARY WASTE GENERATION MODEL}

In previous work the wastes that did not result directly from splitting or partitioning of the main process streams were termed secondary waste. To avoid confusion with the smaller process streams these wastes are renamed here as tertiary nonprocess waste or simply tertiary waste.

For this study, the tertiary waste has been divided into combustible solids and liquids (CBUSTL), noncombustible solids (NCBUSTS), aqueous liquids (AQUELLW), and cell air and offgas (OFFGAS). The portions of these wastes that vary when the primary process flow rate is varied, after the plant design has been fixed, are called VCBUSTL, VNCBUSTS, VAQUELLW, and VOFFGAS.

To estimate the tertiary waste feed rates, printouts were requested from L. J. Wachter, manager of the Waste Management Information System (WMIS) database at Hazardous Waste Remedial Actions Program in Oak Ridge, Tennessee, for the major DOE integrated processing sites: Hanford, Idaho National Engineering Laboratory (INEL), Rocky Flats, and West Valley Demonstration Project (WVDP). This gave values of inventories and rates for 563 DOE waste streams. The streams were then classified into the four desired tertiary waste categories (CBUSTL, NCBUSTS, AQUELLW, and OFFGAS) plus the primary aqueous waste streams. Because data for cell air and offgas rates were not available, the volume of filters resulting from offgas treatment was substituted. Aqueous solutions used for offgas scrubbing were assumed to be already included in the primary flowsheets.

For each DOE site the ratio of the volume of each tertiary waste category to the volume of the primary stream was estimated. Whenever possible, the current rates were used, but for plants that are no longer operating it was necessary to use data from accumulated inventories from unknown past practices. For sites that had sufficient information, the ratio of transuranic (TRU) waste to nONTRU waste was estimated for each waste category.

The aggregated waste rates, shown in Table El, gave ratios that were often inconsistent. Each site seemed to have a different ratio of streams, perhaps because each DOE site was a special case: Rocky Flats had no primary aqueous waste stream; INEL included much stored waste from Rocky $\mathrm{Fl}$ ats in its solid wastes; and the Savannah River Site (SRS) included tritiated wastes from its reactors and tritium processing plants. No processes are currently operating at the Hanford Site and its only nonhigh-level liquid waste was an 
enormous volume of very dilute process condensate. There is a great deal of information relevant to tertiary wastes contained in the WMIS reports. However, in the future it is recommended that access be gained to the detailed tertiary process waste rates from individual facilities with currently operating processes before the wastes are reprocessed and aggregated into DOE site integrated totals.

After examining wastes from the major DOE sites (Hanford, INEL, Rocky Flats, SRS, and WVDP) it was concluded that only the Savannah River $F$ and $H$ canyon data in Tables E2 and E3 covered a long enough time period of startups, shutdowns, and idle periods to provide substantive data for establishing credibie tertiary waste generation rates as they relate to specific pretreatment processes. Nevertheless, the data from other sites provided more complete breakdowns of the tertiary waste that could be classified into four main categories for which typical treatment processes could be defined. The flow diagram for treatment of these tertiary waste categories is shown in Figure EI as implemented in the ASPEN PLUS ${ }^{1}$ global models.

Although the SRS operations include wastes from several plants, the 98 waste streams gave the best opportunity for estimating tertiary process waste rates for operating solvent extraction (SE) plants. The flowsheet in Figure E2 was devised to represent waste flows from Savannah River SE operations. It is a composite flowsheet, which combines major stream flow data for a typical period of startups, shutdowns, and idle periods in Table E2 from 1987 to 1992 with the more detailed solid waste data from Table E3 for the 1992 calendar year predicted as of August 29, 1991. The major difficulties are that the bulk of the combustible and noncombustible wastes are mixed in two categories, compactible and job control wastes, and that the ratio of tertiary-to-primary wastes from $F$ canyon is six times higher than from $\mathrm{H}$ canyon. An $\mathrm{F}-\mathrm{H}$ canyon composite volume ratio of tertiary-to-primary of 2:1 was used for the study. When the Savannah River waste stream categories, classifications, and compositions were inadequate, estimates of ratios were taken first from the Hanford Site and then from INEL and Rocky Flats.

The four waste types and treatment processes are as follows.

1. Treat noncombustible solids by processes, such as compaction, leaching, and decontamination. (TRTSSOL)

2. Incinerate combustible solids and liquids. (INCIN)

3. Ion exchange and concentrate aqueous wastes. (EVAPIX)

4. Treat cell air and offgas with high-efficiency particulate air filters. (OGASTRT)

The net result of combining all of the site information is shown in Figure El for the SRS $F$ and $H$ canyons.

'ASPEN PLUS is a trademark of Aspen Technology, Inc. 
Nearly all of the tertiary wastes were found to be only a function of the size of the process and plant design. Only a small fraction, mainly TRU wastes, varied with the plant throughput after the plant had been operating. The variable tertiary waste fraction was designated VCBUSTL, VNCBUSTS, VAQUELLW, and VOFFGAS. Early plant data suggested that a satisfactory description of treatment of tertiary waste would require a matrix of the constant plant design throughput portion and the variable throughput portion of the four waste types for all of the processes in the plant. However, there was insufficient information available on the layouts of the $F$ and $H$ canyon processes to ascribe the tertiary wastes to individual processes. Thus, the matrix approach was temporarily abandoned, and, instead, the composition and ratio of the total $F$ and $H$ canyon tertiary waste flow to the total high-level waste (HLW) flow rate in our plant design was applied to give tertiary waste flows and compositions.

Additional information has now been obtained on the SRS plant layouts and on the proposed Hanford TRUEX-D space allocations (Figure E3) that has allowed improving the estimation of the tertiary wastes from the individual processes in our plant flowsheets. Therefore, we can return to the matrix approach.

To facilitate the waste allocations to individual process the following assumptions were made:

- The variable throughput coefficients (VCBUSTL, VNCBUSTS, VAQUELLW, and VOFFGAS) were not used (i.e., they were temporarily set to zero and included in the design stream) because the current analyses are only concerned with plant design and not with variable rate of operation of a plant after it has been designed.

- The tertiary waste volume was proportional to space allocation for each process in the SRS $F$ or $H$ canyon.

- The SRS F and $H$ canyons were operated at the same waste flow rate (i.e., the flow rates were averaged).

- It was assumed that an SRS dissolver (DISS) is equivalent to 0.25 SE cycles for the purposes of tertiary waste generation estimation.

- It was assumed that the high activity waste throughput rate is the same as the feed rate to the $F$ or $H$ canyon. The average 1987 to 1990 rate from the data given in Table E2 was $182,000 \mathrm{gal} / \mathrm{yr}$ HLW or $91,000 \mathrm{gal} / \mathrm{yr}$ for one canyon. Converting units gives $344.5 \mathrm{~m}^{3} / \mathrm{yr}$ $\left(3.786 \mathrm{~m}^{3} / \mathrm{gal}\right.$ ) or $344,500 \mathrm{~kg} / \mathrm{yr} \mathrm{HLW}$ (assuming a liquid density of $\left.1,000 \mathrm{~kg} / \mathrm{m}^{3}\right)$.

- It was assumed that the flow rates through all process equipment in the SRS $F$ and $H$ canyons were equal because the actual relative flow rates through the $F$ or $H$ canyon equipment or any other part of the main SRS processing plants were not available for use.

From SRS canyon layout data the total tertiary waste generation volume was assigned to the various canyon processes based on the number of pieces of major equipment involved in each of the specific processes relative to the total. This assignment method is based on the fact that tertiary waste 
generation seems to depend most on the number of staff operating the plant processes. This number was assumed to be proportional to the number of tanks and major sets of equipment involved in the plant processes because such entities tend to require observation, sampling, control, and maintenance, which requires staff attention and particulariy staff ingress into radistion zones. These assignments are shown in Table E4 in the form of equipment fractions or volume ratios for each process.

From the equipment ratio data the four-cycle SE process accounts for $44 \%$ of the total tertiary waste generation or $11 \%$ for each SE cycle $(9 * 11 \% \approx 100 \%)$. Therefore, the total tertiary waste generation from $F$ or $H$ canyon is equivalent to approximately nine SE cycles. For nine SE cycles (in series), Table $E 5$ shows the mass ratios of tertiary wastes to primary waste liquid HLW along with the mass ratios per SE equivalent. To apply this waste generation data, the CLEAN Option and TRUEX-C/STudge Wash C suite of processes were first represented by 21 and 13 main processes, respectively, each of which was categorized by SE equivalents as shown in Table E6. These SE equivalents were chosen, based on estimates of major pieces of equipment needed for each specific process. The tertiary waste generation rate from each of these processes is found using the following equation:

$$
\text { TertWaste }_{j}=\text { ProcFlow * Equivs * MassRatio }_{j}
$$

where:

$$
\begin{aligned}
\text { Tertwaste }_{j}= & \text { Tertiary waste } \mathrm{j} \text { generation }(\mathrm{kg}) . \\
\text { Procflow }= & \begin{aligned}
\text { Primary waste flow }(\mathrm{kg}) \text { through the equipment in a } \\
\text { particular process. }
\end{aligned} \\
\text { Equivs }= & \text { SE equivalents ( } 1 \text { DISS }=0.25 \mathrm{SE} \text { ) for a particular } \\
\text { process. } & \\
\text { MassRatio }= & \begin{array}{l}
\text { Mass ratio of tertiary waste } \mathrm{j} \text { to the primary waste per } \\
\text { SE cycle. }
\end{array}
\end{aligned}
$$

For example, if the design mass flow rate through the WASHSLUD process was $100,000 \mathrm{~kg} / \mathrm{yr}$, then NCBUSTS (noncombustibles) tertiary waste is calculated as follows:

$$
\text { NCBUSTS(WASHSLUD) }=100,000 *(0.5 * 0.25) * 0.12=1,500 \mathrm{~kg} \mathrm{NCBUSTS} / \mathrm{yr}
$$

The calculations of tertiary waste generation were implemented in FORTRAN subroutines that compiled directiy with each global model ASPEN PLUS run. These FORTRAN subroutines automatically retrieve the specific process flow rate and use the above equation to calculate the 12 elements comprising the tertiary waste (i.e., NCBUSTS, CBUSTSL, etc.) for each main process. 
The waste generation for each process is then summed to provide the input streams for the tertiary process waste treatment portion of the model. The tertiary waste was assigned to four components as follows:

\section{AQUELLW}

$\begin{array}{ll}\mathrm{H}_{2} \mathrm{O} & (89 \%) \\ \mathrm{NaNO}^{2} & (10 \%) \\ \text { OHAZI } & (1 \%) \\ \text { OTH2 } & (<1 \%)\end{array}$

CBUSTL, NCBUSTS, OFFGAS

$\begin{array}{ll}\mathrm{H}_{2} \mathrm{O} & (\mathrm{O}) \\ \mathrm{NaNO}_{3} & (0 \%) \\ \text { OHAZ1 } & (10 \%) \\ \text { OTH2 } & (90 \%)\end{array}$

\section{E2.0 SUMMARY OF RESULTS}

Figure $E 4$ shows the calculated tertiary waste generation by process for the CLEAN Option (with and without compact processing units [CPUs]) and TRUEX-C/Sludge Wash C processes with $0 \%$ heel to show maximum process throughput. For the CLEAN Option using CPUs, the tertiary waste stream flows were estimated by using single-shell tank (SST) and double-shell tank (DST) feedstreams with only 36 and $4 \%$ of the SST and DST tank waste feedstream mass flows, respectively. These percentages represent only the sludge portion of the respective feedstreams that would be processed in a central processing facility when CPUs are also used.

As shown in Figure E4, the cesium ion exchange, strontium ion exchange, and technetium ion exchange processes produced the most tertiary waste because of their high throughput and high SE cycle equivalency assignment (see Table E6). Figure E5 shows the tertiary waste stream flows for each of the process strategies. Note how large the tertiary waste flows are compared to the primary waste feedstream flow of approximately $7.6 \mathrm{E}+08 \mathrm{~kg}$. As discussed in Appendix $\mathrm{H}$, the total tertiary to primary waste flow ratios were $4.4,2.9$, 1.8 , and 3.1 (calculated based on results in Table ElO) for the CLEAN Option without CPUs, TRUEX-C, Sludge Wash C, and CLEAN Option with CPUs, respectively.

Tables E7 through ElO show the detailed tertiary waste generation data for each global model from which Figures E4 and E5 were created. The tert tary waste table data are shown for each of the main global model processes with a breakdown into each tertiary waste stream. The process flow rates that determined the quantity of tertiary waste generated are also shown.

It should be clear from the data that the tertiary waste generation is substantial and cannot be ignored when evaluating process technologies. As shown by Figure E5, the CLEAN Option with and without CPUs generates the largest amount of tertiary waste owing to its intensive chemical processing strategy. Indeed, the cost for processing this tertiary waste is very substantial as discussed in Appendix $F$. The large tertiary waste costs could be decreased significantly if process design is also focused on reducing the amount of tertiary waste produced. 


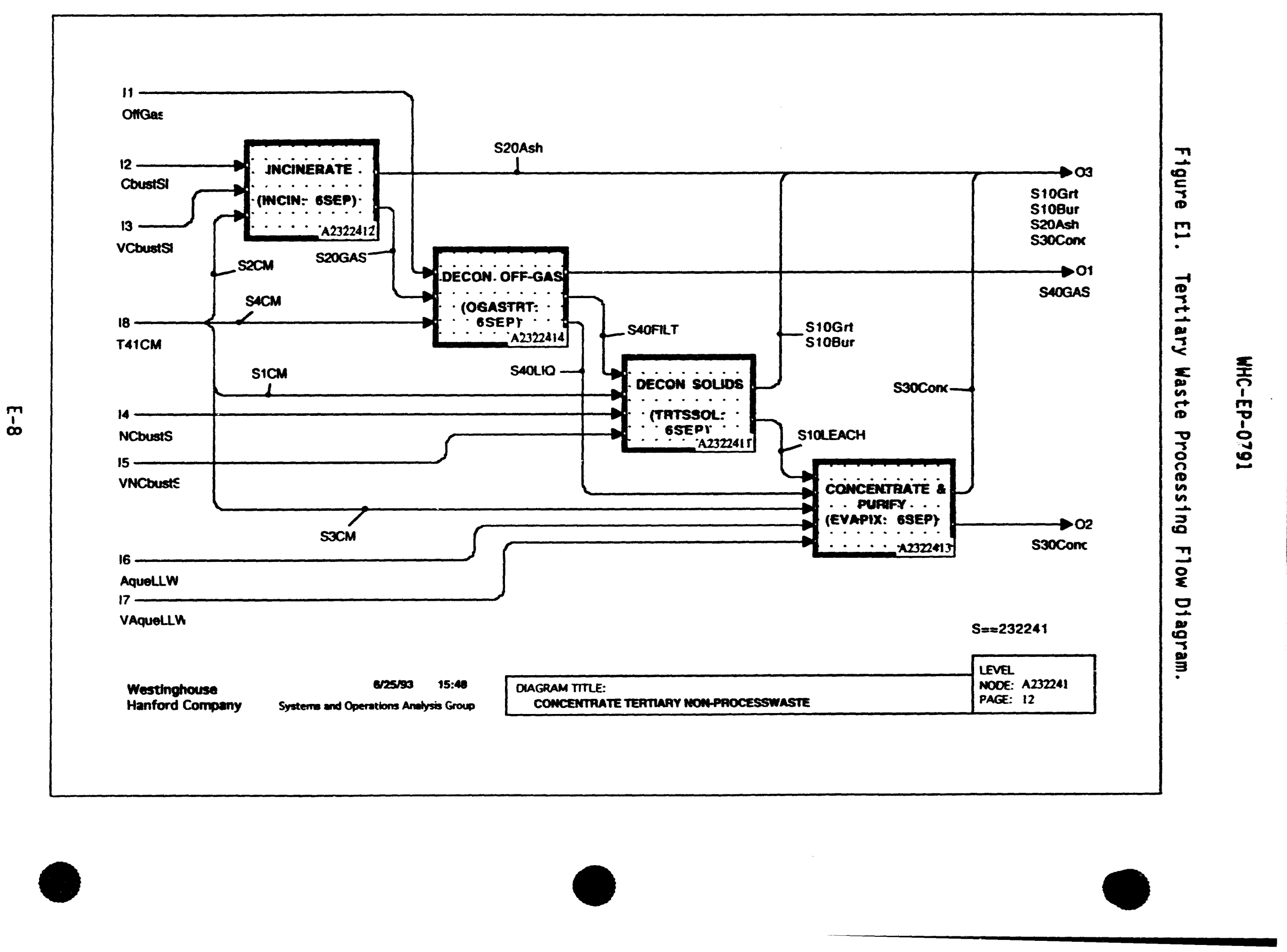


Figure E2. Savannah River Site Waste Management Information System Waste Streams (1987 - 1991 Average).

(all flows expressed as volume ratio to HLW flow rate)

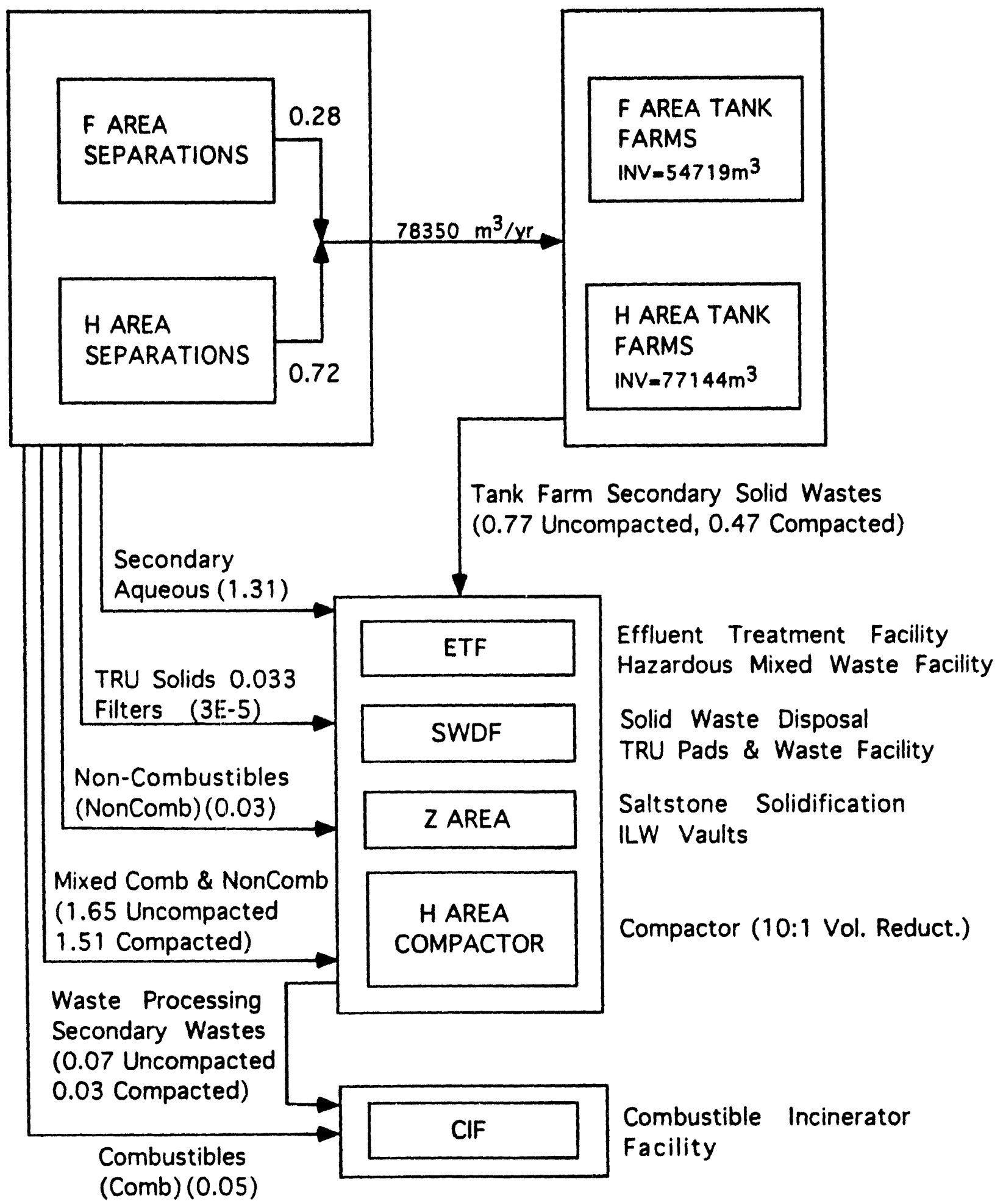




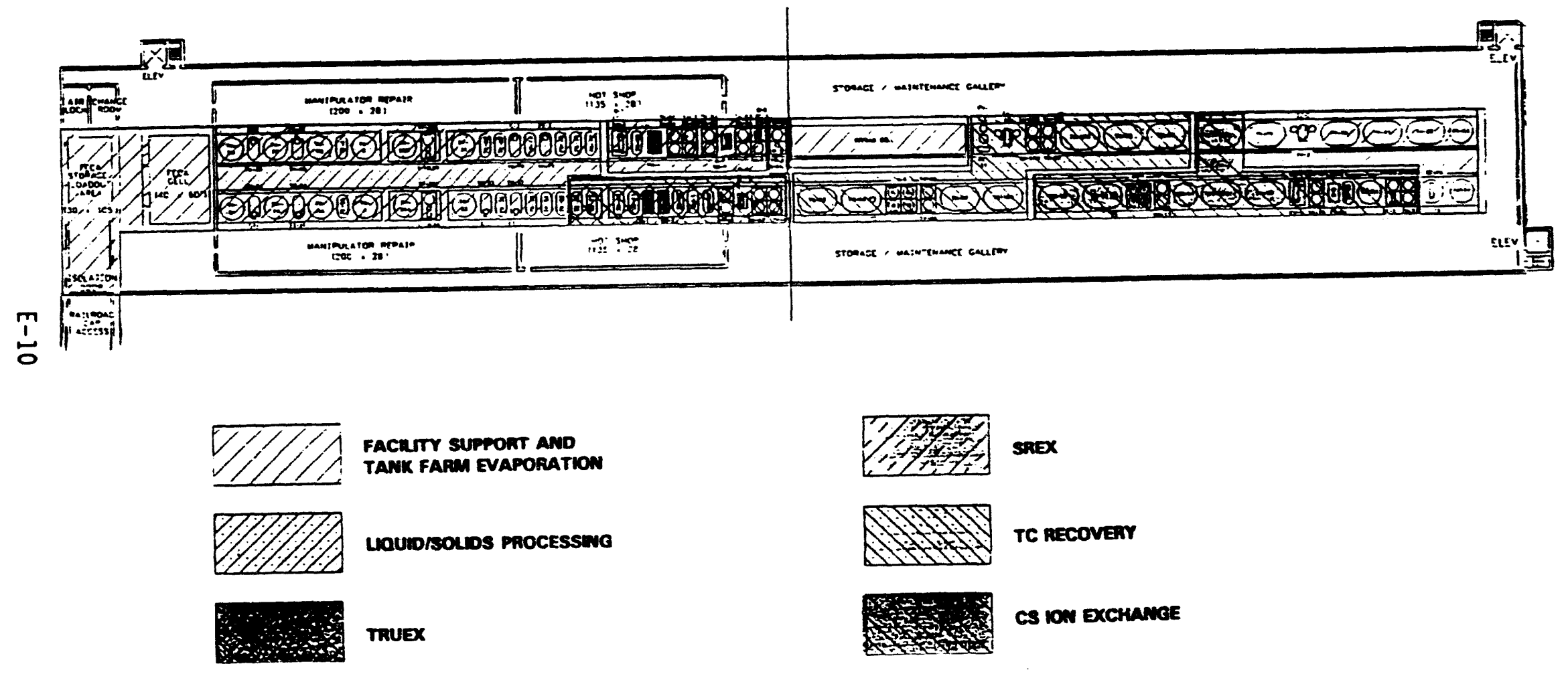

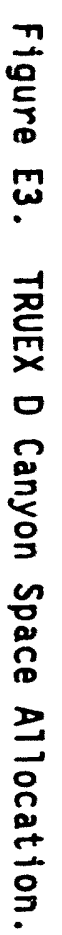

폼 
Figure E4. Tertiary Waste Generation by Process.
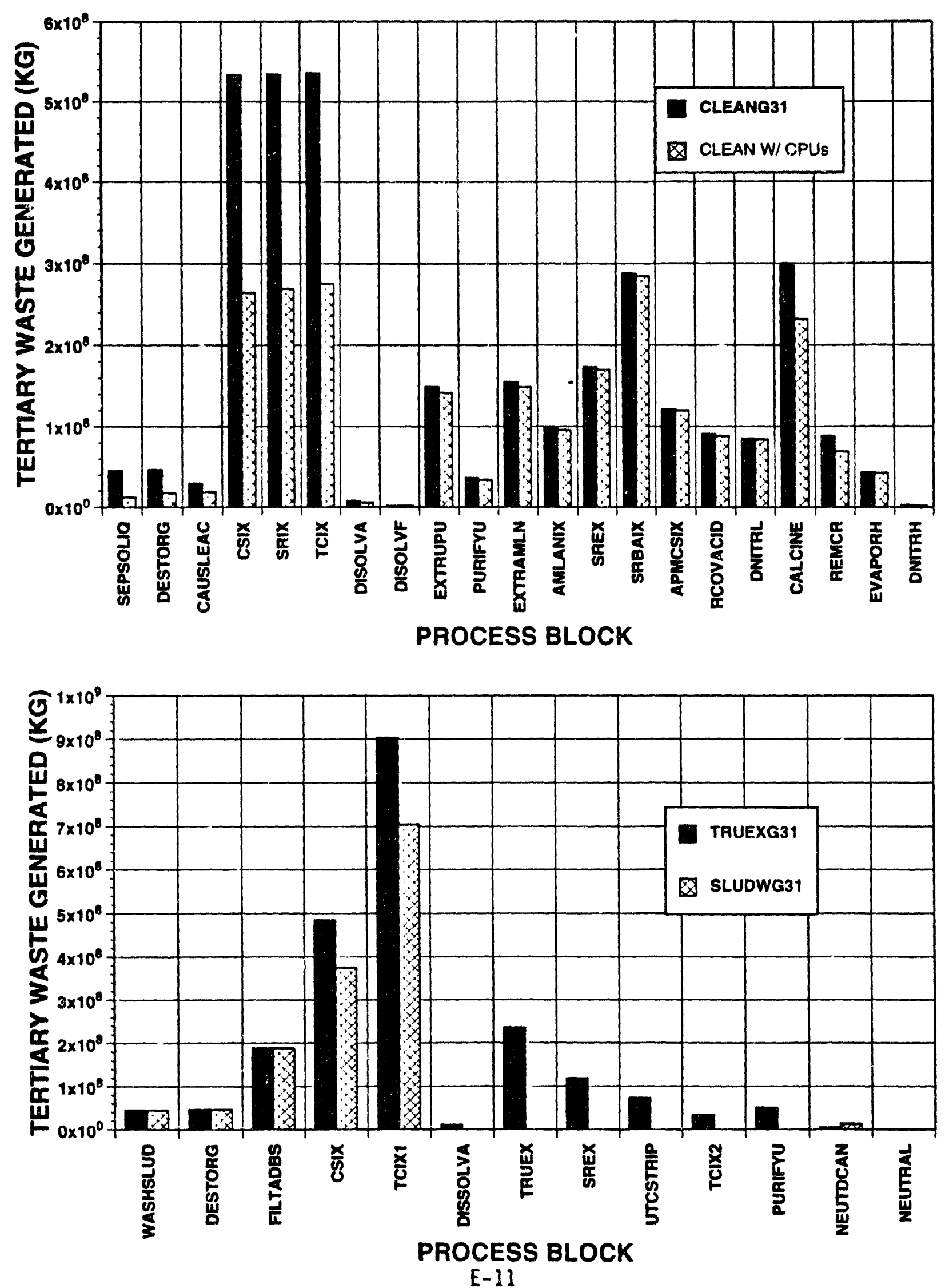
WHC-EP-0791

Figure E5. Tertiary Waste Stream Mass Flows.

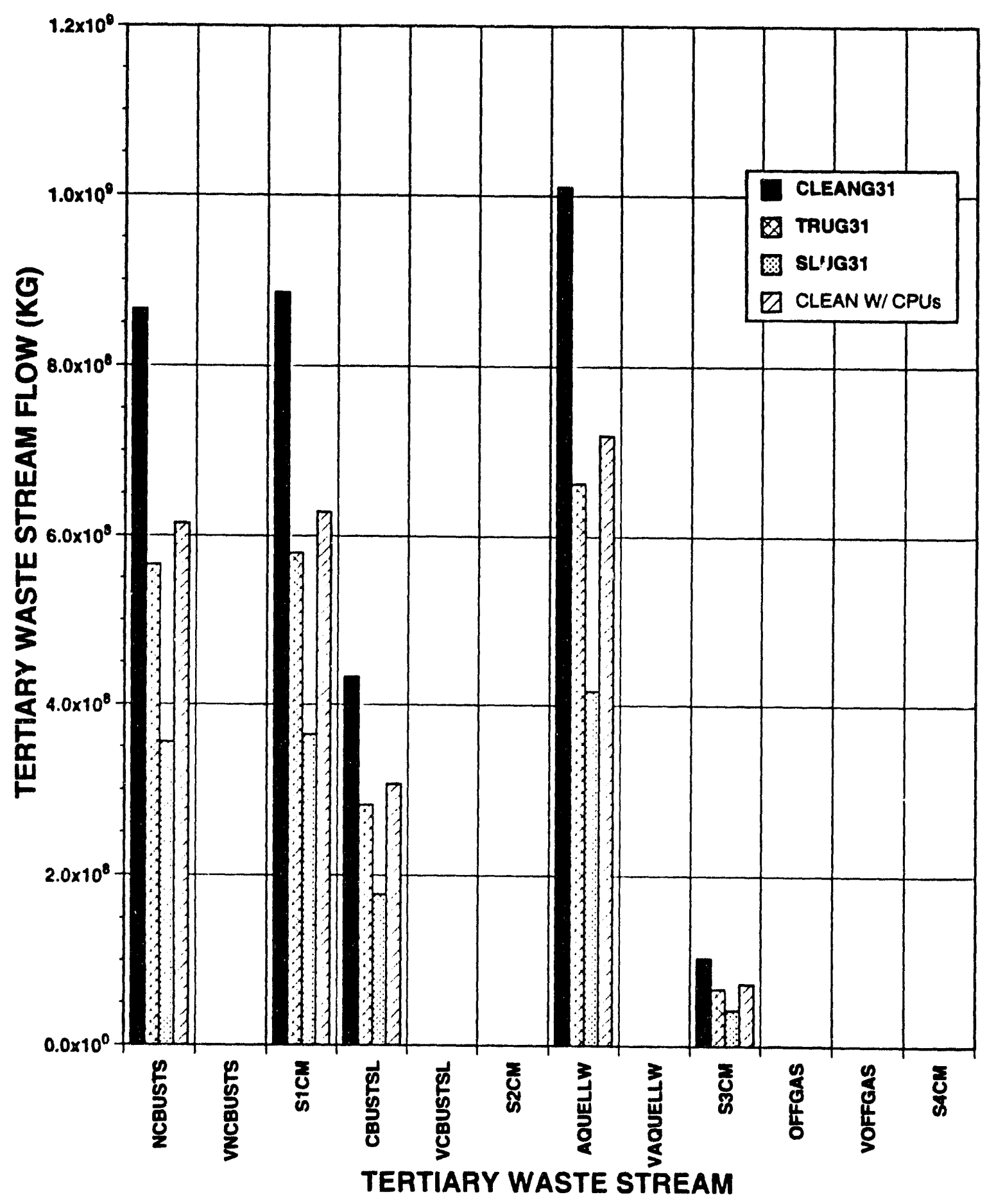


Table El. Aggregated Rates and Inventories for U.S. Department of Energy Sites (Projected for 1992 as of August 29, 1991).

\begin{tabular}{|c|c|c|c|c|c|}
\hline $\begin{array}{l}\text { HLW Liquid, } \mathrm{m}^{3} / \mathrm{yr} \\
\text { Inventory, } \mathrm{m}^{3}\end{array}$ & $\begin{array}{c}\text { HANFORD } \\
464 \\
45190\end{array}$ & $\begin{array}{l}\text { INEL } \\
2500 \\
11000\end{array}$ & $\begin{array}{c}\text { ROCKY F } \\
0 \\
0\end{array}$ & $\begin{array}{l}\text { SAV RIV } \\
8539 \\
131853\end{array}$ & $\begin{array}{r}\text { WVDP } \\
0 \\
5360\end{array}$ \\
\hline $\begin{array}{c}\text { Secondary Aqueous, } \mathrm{m}^{3} / \mathrm{yr} \\
\text { Inventory, } \mathrm{m}^{3} \\
\text { TRU Inv, } \mathrm{m}^{3}\end{array}$ & - & $\frac{2243}{538}$ & $\begin{array}{c}3 \\
-\cdots-- \\
-\cdots--\end{array}$ & $\frac{4285}{210}$ & $\begin{array}{l}2 \\
2\end{array}$ \\
\hline $\begin{array}{c}\text { Combustibles, } \mathrm{m}^{3} / \mathrm{yr} \\
\text { TRU, } \mathrm{m}^{3} / \mathrm{yr} \\
\text { Inventory, } \mathrm{m}^{3} \\
\text { TRU Inv, } \mathrm{m}^{3}\end{array}$ & $\begin{array}{c}464 \\
100 \\
0.13\end{array}$ & 30287 & 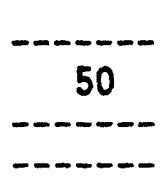 & 7122 & $\begin{array}{l}-- \\
4\end{array}$ \\
\hline $\begin{array}{l}\text { Mixed Comb.\& Nonc, } \mathrm{m}^{3} / \mathrm{yr} \\
\text { Inventory, } \mathrm{m}^{3} \\
\text { TRU Inv, } \mathrm{m}^{3}\end{array}$ & - & - & - & $\frac{28764}{435}$ & - \\
\hline $\begin{array}{c}\text { Noncombustibles, } \mathrm{m}^{3} / \mathrm{yr} \\
\text { TRU, } \mathrm{m}^{3} / \mathrm{yr} \\
\text { Inventory, } \mathrm{m}^{3} \\
\text { TRU Inv, } \mathrm{m}^{3}\end{array}$ & $\begin{array}{c}325 \\
607 \\
14\end{array}$ & $\begin{array}{l}2985 \\
240196 \\
24095\end{array}$ & $\begin{array}{r}753 \\
17 \\
8000 \\
\end{array}$ & \begin{tabular}{c}
3450 \\
\hdashline 12600 \\
$-\cdots$
\end{tabular} & 8 \\
\hline $\begin{array}{l}\text { Filters, } \mathrm{m}^{3} / \mathrm{yr} \\
\text { Inventory, } \mathrm{m}^{3} \\
\text { Tru Inventory, } \mathrm{m}^{3}\end{array}$ & - & $\begin{array}{r}4 \\
2089 \\
328\end{array}$ & $\begin{array}{l}70 \\
64 \\
49\end{array}$ & $\begin{array}{l}11 \\
40 \\
---\end{array}$ & -n-n \\
\hline
\end{tabular}


Table E2. Savannah River Waste Management Information System Waste Stream Data (Operation During 1987 through 1991).

Low Level Job

Control Waste

1987

F Separations

H Separations

\section{8}

F Separations

H Separations

\section{9}

F Separations

H Separations

\section{0}

F Separations

H Separations

\section{1}

F Separations

H Separations

1992 (up to 4/92)

F Separations

H Separations
285,300

124,200

322,700

134,300

237,400

161,400

208,900

159,100

124,500

107,300

20,100

21,400
Low Level

Compactor Waste
TRU

Waste
12,200

1,200

26,100

8,060

5,950

920

31,860

17,900

5,211

610

13,300

29

5,950

1,323

NOTES:

All values are in cubic feet per year.

TRU values for 1989 are estimates. 
Table E3. Savannah River Waste Management Information System List of 98 Waste Streams

(Projected for 1992 as of August 29, 1991). (sheet 1 of 3)

C=COMBUSTIBLE F=FILTERS M=MIXED COMBUSTIBLE-NONCOMBUSTIBLE N=NONCOMBUSTIBLE SOLIDS $A=A Q U E O U S$ C=COMBUSTIBLE $F=F$ ILTERS $M=M I X E D$ TANK FARM OPERATIONS $W=$ FROM WASTE TREATMENT

\section{SITE MIS MASTE STREAM MAME}

A $221-$ F CAMYON HIGH LEVEL LIOUID MASTE

HLW RH A SAVR 221-H CANYOA HIGH LEVEL LICUID WASTE

HLW RH A SAVR 244-H RBOF HIGH ACTIVIT

LLU CH A SAVR SCINTILLA ACTIVITY MASTE

LLW CH A SAVR SRL MIGA ACTIVITY MASTE

LLH CH A SAYR SRL LOW ACTMTAMIMATED APPLICATORS (LLW)

LLW CH C SAVR SOLVENT TRU UASTE

TRU CH C SAVR SOLVENT TRU WASTE HCI/G TRU RAD

LLW CH C SAVR SOLVENT MASTE MTERIAL

LLW CH C SAVR TOXIC CLEAMP CATED HEPA FILTERS

LLW CH F SAVR CAOMIUH - COAT

LLY RH F SAVR ITP FTITERS UASTE - TT2F

LLW CH M SAVR COMPACTIBLE UASTE - TT2F SEPARATIONS

LLW CH SAVR COMPACTIBLE WASTE - MREA SEPARATIONS

LLW CH M SAVR COMPACT

LLW CH M SAVR COMPACTIBLE WASTE SERVICES LLN SOLID MASTE

LLW CH M SAVR FACILITIES AMD SERVICES LCNTROL - SRL

$\underset{1}{m}$ $m^{3} / \mathrm{rR}$

1700.0000 4000.0000 2838.7500 200.0000 970.0000
0.1000

0.2000

8.5000

0.7100

85.0000
3400.0000

312.0000

45.0000
21.0000

61.0000

9268.0000

2280.0000

1800.0000

540.0000

200.0000

12.5000

198.0000

24.5000
8.1000

623.0000

623.0000
31.0000

31.0000
91.0000

127.0000

6.0000
40.0000

40.0000

1.0000
$<0.0200$

$<0.0200$

$<0.0200$

$<0.0200$

$<0.0200$

$<0.0200$
2.0000

1360.0000

1564.0000

29.0000
368.0000
MB INV REMARKS

54719.0000 IUY IS F-AREA HLY TANK 27 ONLY

THV IS F-AREA HLW TAMK 27 GM ONLY

64825.0000 INV IS H-AREA MLU TAMK 21 OLLY

作

71.1000 ANALYZED

ANALYZED FISSIOA PROOUCT VALUES

3853.0000 ISOTOPES IDEWTIFIED

3269.0000 ISOTOPES IDENTIFIED

38.7000 O908 0000 KG

AIXED FISSION PRODUCT VALUES

MIXED FISSION PRODUCT VALUES

MIXED FISSION PROOUCT VALUES

MIXED FISSION PROOUCT VALUES

MIXED FISSIOM PROOUCT VALUES

MIXED FISSIOA PRODUCT VALUES

MIXED FISSION PRCOUCT VALUES

MIXED FISSION PRCOUCT VALUES

MIXED FISSION PRODUCT VALUES

MIXED FISSION PRODUCT VALUES

PU COAPOSITIOU VALUES

PU COMPOSITION VALUES

PU COAPOSITION VALUES

ISOTOPES IDENTIFIED

ISOTOP IDETIFIED

PLUTOUI COMOSITIO

PLUTONIUA CONPOSIO PRODUCT VALUES

MIXED FISSION PRODCT VALUES

MIXED FISSION PRCDLCT VALLES

$202.000012470 \mathrm{KG} / \mathrm{YR}$

MIXED FISSION PRCOUCT VALUES

MIXED FISSIOU PRCOUCT

AM241

COBALT -57

16.0000 ISOTOPES IDENTIFIED

MIXED FISSIOU PROOUCT VALUES MIXED FISSION PROOUCT VALUES MIXED FISSION PRODUCT VALUES MIXED FISSION PRODUCT VALUES 
Table E3. Savannah River Waste Management Information System List of 98 Waste Streams

(Projected for 1992 as of August 29, 1991). (sheet 2 of 3)

\section{A=AQUEOUS C=COMBUSTIBLE F=FILTERS O=OTHER UNRELATED ACTIVITIES}

\section{SITE MIS MUSTE STREAM MAME}

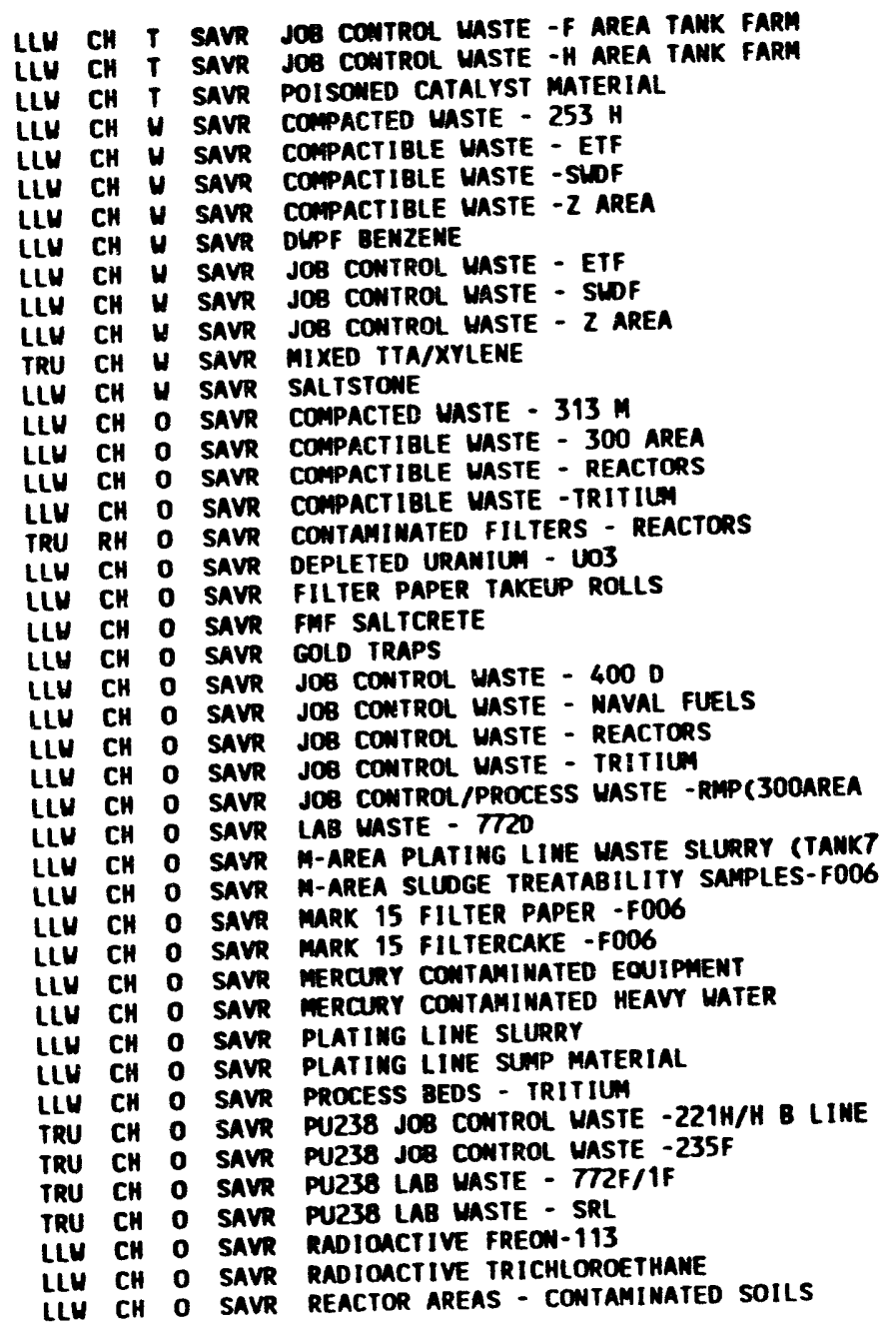

$M=M I X E D$ COMBUSTIBLE-NONCONBUSTIBLE N=NONCOMBUSTIBLE SOLIDS $T=$ FROM TANK FARM OPERATIONS $W=$ FROM WASTE TREATMENT

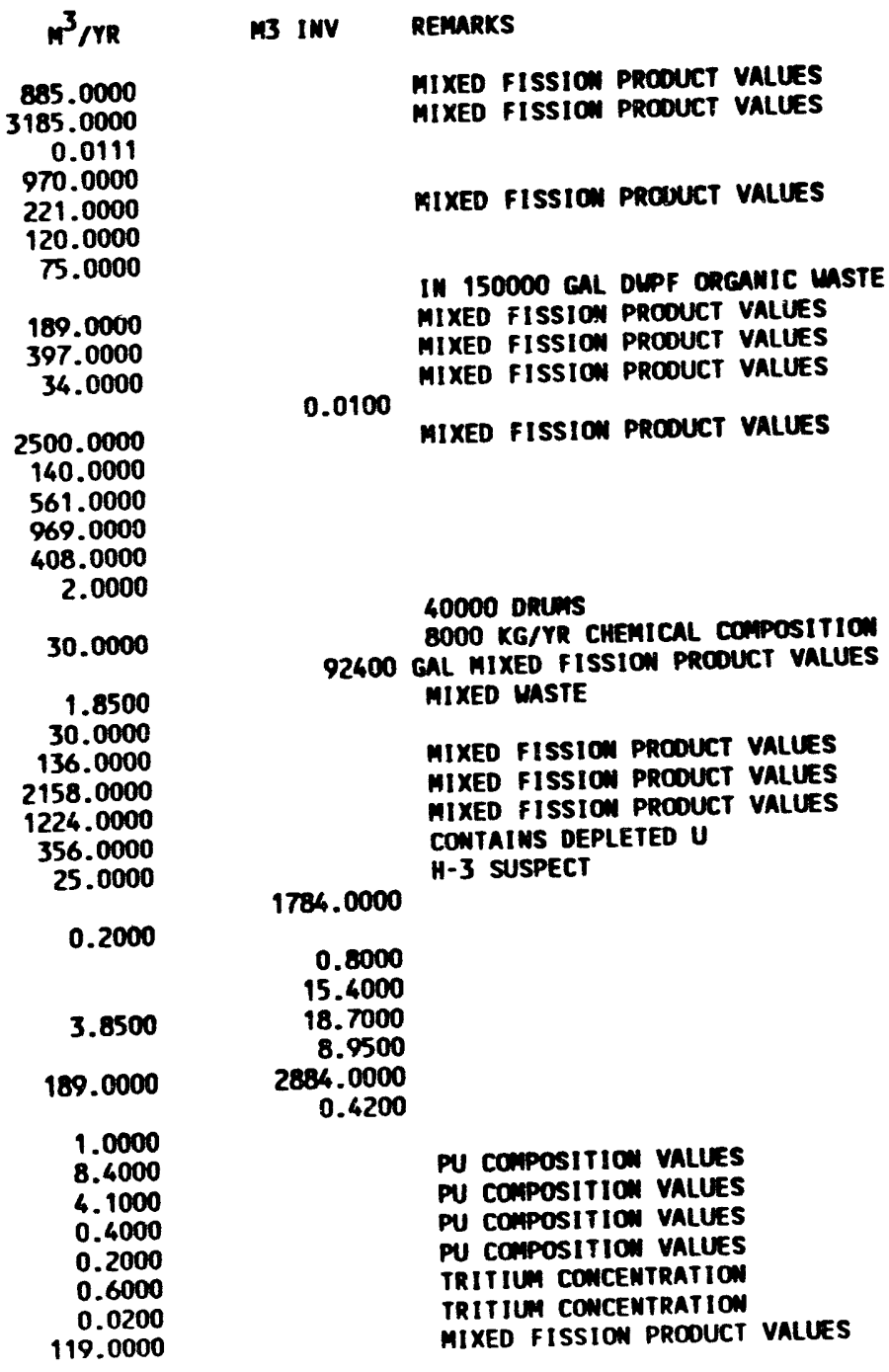



Table E3. Savannah River Waste Management Information System List of 98 Waste Streams
(Projected for 1992 as of August 29, 1991). (sheet 3 of 3)

$A=$ AQUEOUS $C=$ COMBUSTIBLE F=FILTERS O=0THER UNRELATED ACTIVITIES

SITE WIS MASTE STREAM MANE

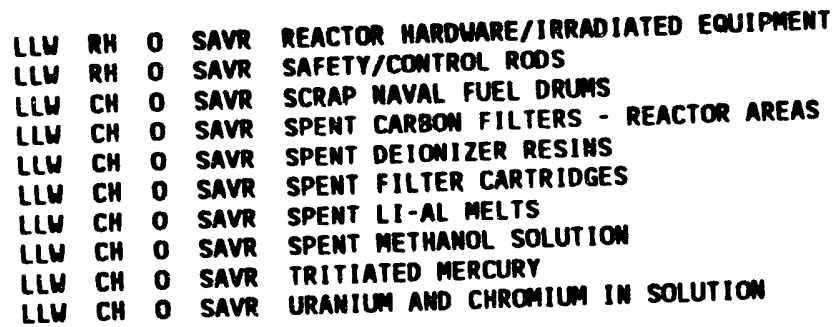

LLW CH O SAVR

LLW CH O
$M=M I X E D$ COMBUSTIBLE-NONCOMBUSTIBLE N=NONCOMBUSTIBLE SOLIDS FROM TANK FARM OPERATIONS $H=F R O M$ WASTE TREATMENT

$m^{3} / \mathrm{rR}$

130.0000

0.0200

70.0000

35.0000
M3 INV

\section{REMARKS}

MIXED FISSIOU PROOUCT VALUES

SOME INVEWTORIES

10,008 DRUNS/YR FILLED UITH EROUT

MIXED FISSIOA PROOUCT VALUES

0.8400 MIXED FISSION PRODUCT VALLES

TRITIUN \& $C O-60$

0.1400 AMALYZED

0.1820 
Table E4. Equipment Ratios for Processes Involved in Tertiary Waste Generation.

\begin{tabular}{|l|c|}
\hline \multicolumn{1}{|c|}{ Process type } & Equipment ratio \\
\hline Overhead Processes & 0.16 \\
Facility support & 0.12 \\
Rerun and frame waste & 0.12 \\
Solvent recovery & 0.40 \\
\hline Subtotal & \\
\hline Waste Processes & 0.04 \\
Dissolvers & 0.08 \\
First cycle solvent extraction & 0.36 \\
Third cycle solvent extraction & 0.48 \\
\hline Subtotal & 0.12 \\
\hline High-Activity Waste and Head End & 1.00 \\
\hline Total F or H canyon & \\
\hline
\end{tabular}


Table E5. Ratios of Tertiary Wastes to Primary Liquid High-Level Waste (Revised to Savannah River Site 1987 through 1991 Basis).

$\begin{array}{lll}\text { MASS } & \text { DENSITY MASS RATIO/ } \\ \text { STREAM RATIO } & \mathrm{kg} / \mathrm{m}^{3} & \text { SE CYCLE }\end{array}$

TREAT NONCOMBUSTIBLE SOLIDS (TRTSSOL)

$\begin{array}{llll}\text { NCBUSTS } & 1.0942 & 2.00 E+3 & 0.12 \\ \text { VNCBUSTS } & 0.0257 * & 2.00 E+3 & 0.0029 \\ \text { SICM } & 1.1199 & 1.00 E+3 & 0.12\end{array}$

INCINERATE COMBUSTIBLE SOLIDS AND LIQUIDS (INCIN)

$\begin{array}{llll}\text { CBUSTSL } & 0.5471 & 1.00 E+3 & 0.061 \\ \text { VCBUSTSL } & 0.0129 * & 1.00 E+3 & 0.0014 \\ \text { S2CM } & 0.0000 & 1.00 E+3 & 0.0\end{array}$

ION EXCHANGE AND EVAPORATE TERTIARY AQUEOUS WASTES (EVAPIX)

$\begin{array}{llll}\text { AQUELLW } & 1.2801 & 1.00 E+3 & 0.14 \\ \text { VAQUELLW } & 0.0299 * & 1.00 E+3 & 0.0033 \\ \text { S3CM } & 0.1310 & 1.00 E+3 & 0.015\end{array}$

TREAT CELL AIR AND OFFGAS WITH HEPA FILTERS (OGASTRT)

$\begin{array}{llll}\text { OFFGAS } & 0.0009 & 1.00 E+0 & 0.0001 \\ \text { VOFFGAS } & 0.0001 * & 1.00 E+0 & 0.00001 \\ \text { S4CM } & 0.0000 & 1.00 E+3 & 0.0\end{array}$

* Assumed zero but included in design stream 
Table E6. Solvent Extraction Equivalent Assignments.

Main CLEAN Option Processes

Main Process Equivalents

1.) SEPSOLIQ

2.) DESTORG

3.) CAUSLEAC

4.) CSIX

5.) SRIX

6.) TCIX

7.) DISOLVA

8.) DISOLVF

9.) EXTRUPU

10.) PURIFYU

11.) EXTRAMLN

12.) AMLANIX

13.) SREX

14.) SRBAIX

15.) APMCSIX*

16.) RCOVACID

17.) DNITRL

18.) CALCINE

19.) REMCR

20.) EVAPORH

21.) DNITRH

TOTALS
0.5 DISS

0.5 DISS

1.0 DISS

$1.0 \mathrm{SE}$

$1.0 \mathrm{SE}$

$1.0 \mathrm{SE}$

$1.0 \mathrm{SE}$

$1.0 \mathrm{SE}$

$1.0 \mathrm{SE}$

$1.0 \mathrm{SE}$

$1.0 \mathrm{SE}$

$1.0 \mathrm{SE}$

$0.5 \mathrm{SE}$

1.0 DISS

1.0 DISS

1.0 DISS

1.0 DISS

0.5 DISS

1.0 DISS

9.5 DISS

(2.38 SE)

$9.5 \mathrm{SE}$
1.0 DISS

1.0 DISS
Main TRUEX-C/SLUDGE WASH C Processes Main Process Equivalents
1.) WASHSLUD
2.) DESTORG
3.) FILTABS
4.) $\operatorname{csI} x$
5.) TCIXI
6.) DISSOLVA
7.) TRUEX
8.) SREX
9.) UTCSTRIP
10.) TCIXZ
11.) PURIFYU
12.) NEUTDCAN
13.) NEUTRAL

3.0 DISS

$(0.75 \mathrm{SE})$

$7.0 \mathrm{SE}$

Equivalent Waste from DISSOLVER (DISS) $=0.25$ SOLVENT EXTRACTION (SE)

* The APM is not eluted but is redissolved in an existing dissolver CAUSLEAC. 
Table E7. Tertiary Waste Generation Data for Model CLEANG31.

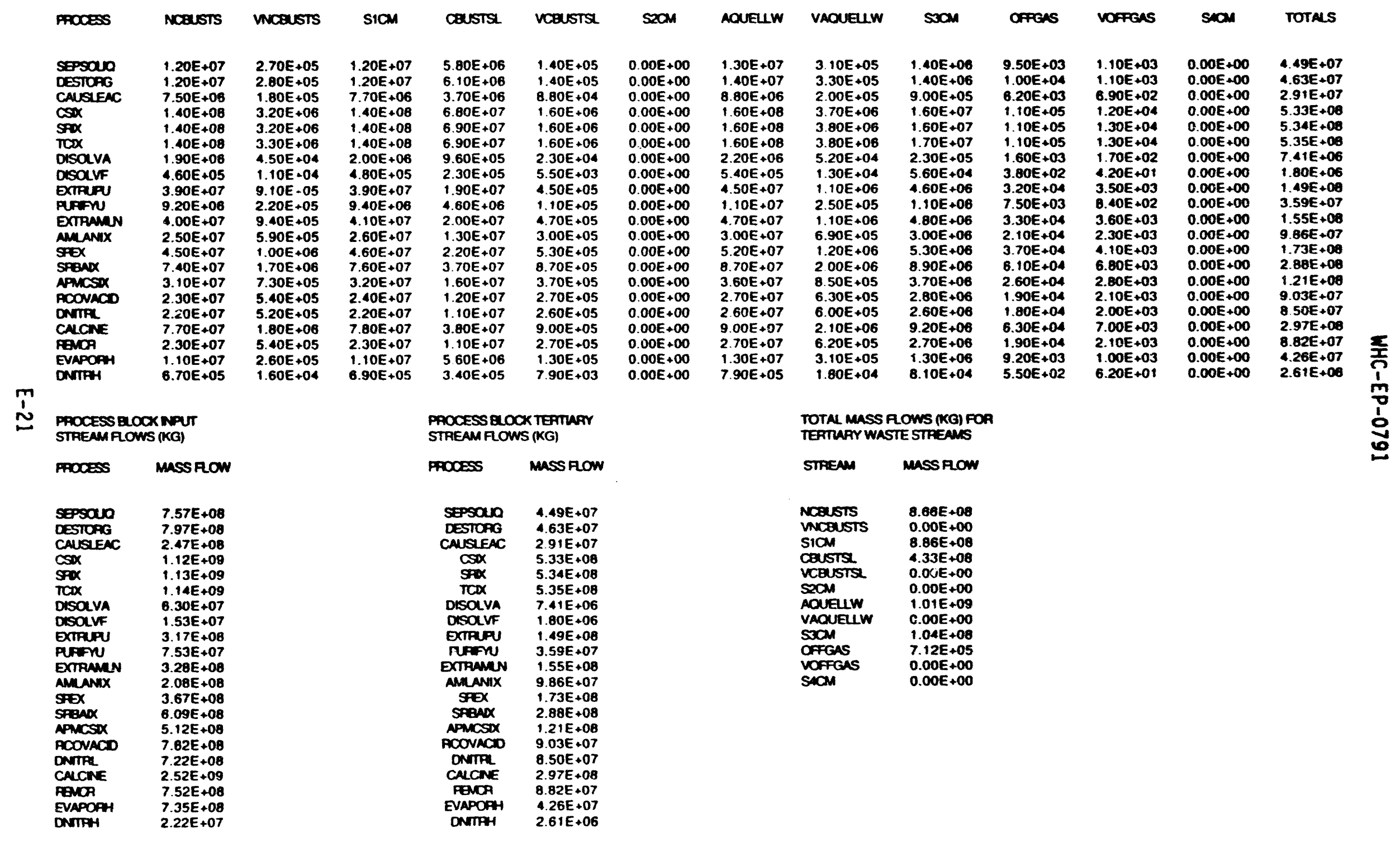


Table E8. Tertiary Waste Generation Data for Model TRUG3l.

TOTAL MASS FLOWS (KG):

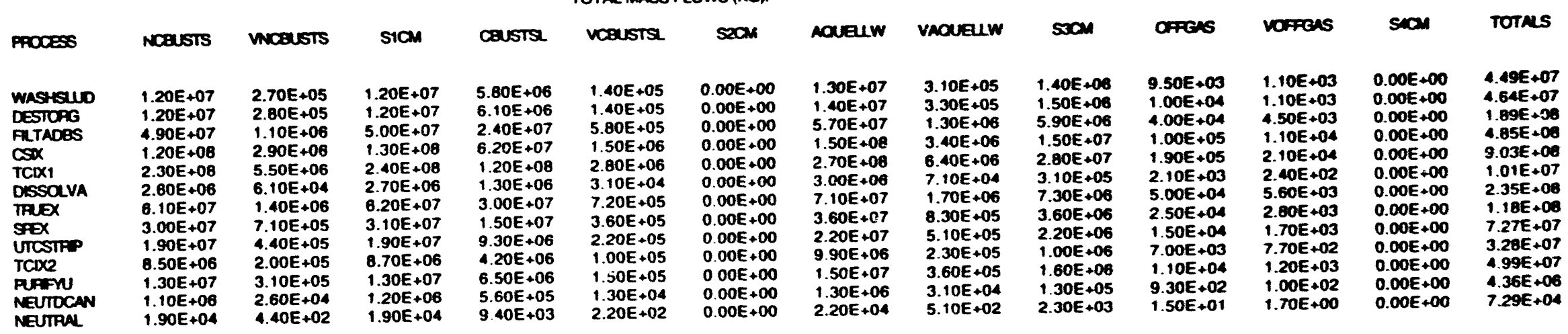

PAOCESS QLOCKNPUT

STREAMROWS (KG)

PROCESS Massfow

WASHSWD $7.57 E+08$

IN FLTADQS $8.05 E+08$

$\cos \quad 1.02 E+09$

TCIX1 $1.93 E+09$

OISSOLVA 6.55E+07

TREX 5.01E+08

FEX 2.50E+08

UTCSTPP $\quad 3.05 E+00$

TCIX2 $2.96 E+07$

PUFFU 1.07E+08

NEUTDCAN $7.40 E+07$
PAOCESS BLOCK TERMAPY

STREAM FLOWS (KG)

PROCESS MASSROW

$\begin{array}{ll}\text { WASHSWD } & 4.49 E+07 \\ \text { DESTOFG } & 4.64 E+07 \\ \text { FLTADBS } & 1.89 E+08 \\ \text { CSX } & 4.85 E+08 \\ \text { TCIX1 } & 9.03 E+08 \\ \text { OISSOLVA } & 1.01 E+07 \\ \text { IFUEX } & 2.35 E+08 \\ \text { FEX } & 1.18 E+08 \\ \text { UTCSTRP } & 7.27 E+07 \\ \text { TCIX2 } & 3.28 E+07 \\ \text { PUFY } & 4.99 E+07 \\ \text { NEUTOCAN } & 4.36 E+06 \\ \text { NEUTHAL } & 7.29 E+04\end{array}$

TOTAL MASS F OWS (KG) FOA TERTARY WASTE STREAMS

STREMM MUSSROW

NCBUSTS $\quad 5.66 E+08$

WICBUSTS $0.00 E+00$

5.80E+09

CaUSTS 2.83E+0

sacm

AOVZUW $6.63 E+08$

VAOUEUW $0.00 E+00$

$53 \mathrm{CM} \quad 6.78 E+07$

OFGes $4.66 E+05$

VOFGAS $0.00 E+00$

$\begin{array}{ll}\text { TOFGAS } & 0.00 E+00 \\ \text { SAO } & 0.00 E+00\end{array}$ 
Table E9. Tertiary Waste Generation Data for Model SLUG31.

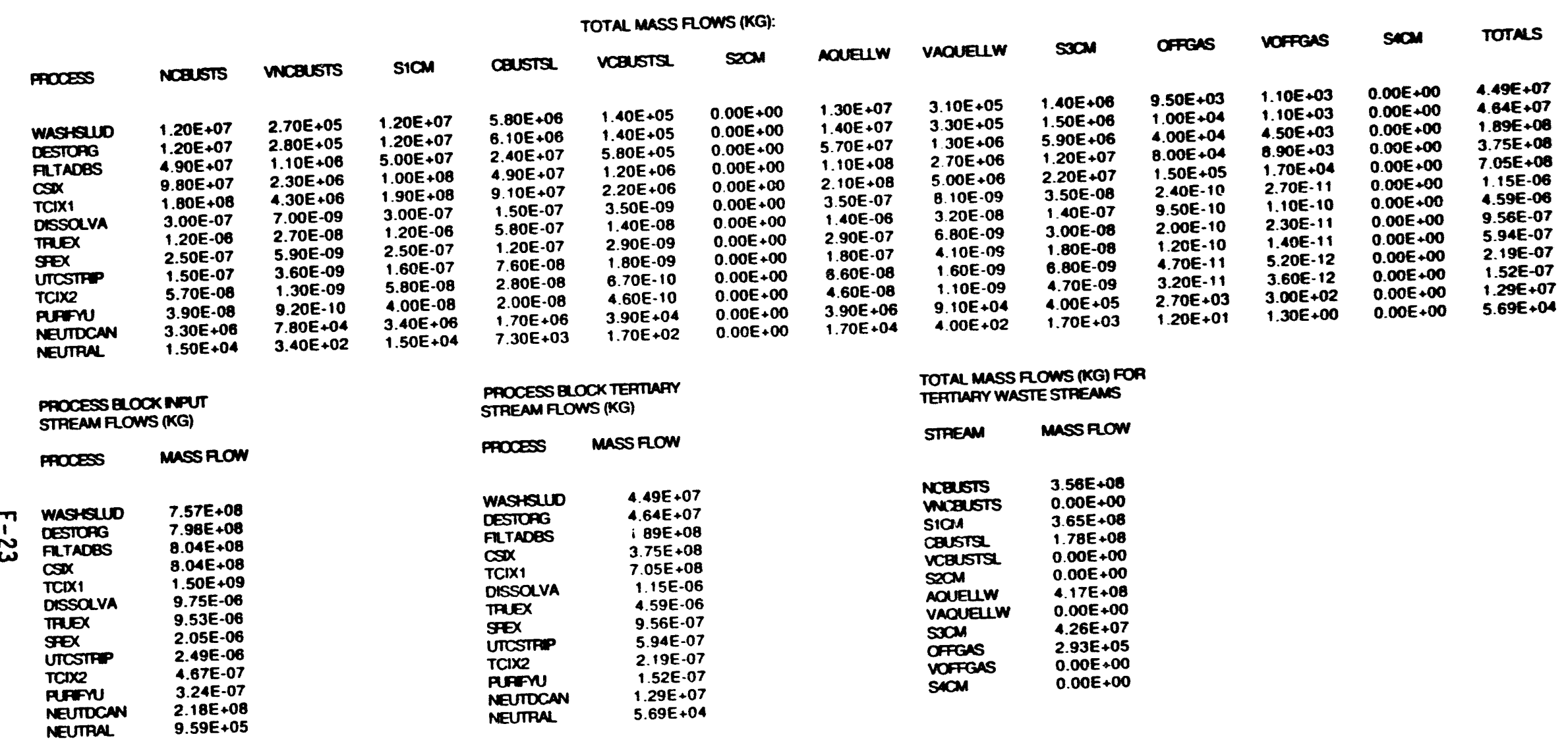


Table E10. Tertiary Waste Generation Data for Model CLEANG31

With Compact Processing Units.

TOTAL MASS FLOWS (KG):

\begin{tabular}{|c|c|c|c|}
\hline PPOCESS & NCaUSTS & MCBusts & SicM \\
\hline 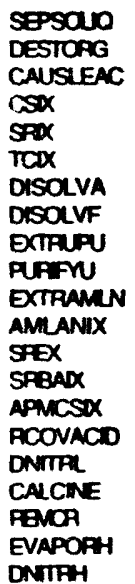 & $\begin{array}{l}3.20 E+06 \\
4.00 E+06 \\
4.90 E+06 \\
6.80 E+07 \\
7.00 E+07 \\
7.10 E+07 \\
1.40 E+06 \\
1.40 E+05 \\
3.70 E+07 \\
8.80 E+06 \\
3.90 E+07 \\
2.50 E+07 \\
4.40 E+07 \\
7.40 E+07 \\
3.10 E+07 \\
2.30 E+07 \\
2.20 E+07 \\
6.00 E+07 \\
1.00 E+07 \\
1.10 E+07 \\
5.30 E+05\end{array}$ & $\begin{array}{l}7.60 E+04 \\
1.10 E+05 \\
1.10 E+05 \\
1.60 E+06 \\
1.60 E+06 \\
1.70 E+06 \\
3.30 E+04 \\
1.00 E+04 \\
8.60 E+05 \\
2.10 E+05 \\
9.00 E+05 \\
5.80 E+05 \\
1.00 E+06 \\
1.70 E+06 \\
7.30 E+05 \\
5.40 E+05 \\
5.10 E+05 \\
1.0 E E+06 \\
4.20 E+05 \\
2.60 E+05 \\
1.20 E+04\end{array}$ & $\begin{array}{l}3.30 E+06 \\
4.60 E+06 \\
5.00 E+06 \\
7.00 E+07 \\
7.10 E+07 \\
7.30 E+07 \\
1.40 E+06 \\
4.50 E+05 \\
3.70 E+07 \\
9.10 E+06 \\
3.90 E+07 \\
2.50 E+07 \\
4.50 E+07 \\
7.50 E+07 \\
3.20 E+07 \\
2.30 E+07 \\
2.20 E+07 \\
6.10 E+07 \\
1.80 E+07 \\
1.10 E+07 \\
5.40 E+05\end{array}$ \\
\hline
\end{tabular}

CBUSTSL
$1.60 E+06$
$2.30 E+06$
$2.40 E+06$
$3.40 E+07$
$3.50 E+07$
$3.60 E+07$
$7.10 E+05$
$2.20 E+05$
$1.80 E+07$
$4.40 E+06$
$1.90 E+07$
$1.20 E+07$
$2.20 E+07$
$3.70 E+07$
$1.50 E+07$
$1.10 E+07$
$1.10 E+07$
$3.00 E+07$
$8.90 E+06$
$5.50 E+06$
$2.70 E+05$

vCEusTS
$3.80 E+04$
$5.40 E+04$
$5.80 E+04$
$6.00 E+05$
$8.20 E+05$
$8.40 E+05$
$1.70 E+04$
$5.20 E+03$
$4.30 E+05$
$1.00 E+05$
$4.50 E+05$
$2.90 E+05$
$5.20 E+05$
$8.70 E+05$
$3.60 E+05$
$2.70 E+05$
$2.60 E+05$
$7.00 E+05$
$2.10 E+05$
$1.30 E+05$
$6.20 E+03$

$\begin{array}{cc}\text { SOCM } & \text { AOUEWW } \\ & \\ 0.00 E+00 & 3.80 E+06 \\ 0.00 E+00 & 5.30 E+06 \\ 0.00 E+00 & 5.70 E+06 \\ 0.00 E+00 & 8.00 E+07 \\ 0.00 E+00 & 8.10 E+07 \\ 0.00 E+00 & 8.30 E+07 \\ 0.00 E+00 & 1.70 E+06 \\ 0.00 E+00 & 5.10 E+05 \\ 0.00 E+00 & 4.30 E+07 \\ 0.00 E+00 & 1.00 E+07 \\ 0.00 E+00 & 1.50 E+07 \\ 0.00 E+00 & 2.90 E+07 \\ 0.00 E+00 & 5.10 E+07 \\ 0.00 E+00 & 8.60 E+07 \\ 0.00 E+00 & 3.60 E+07 \\ 0.00 E+00 & 2.70 E+07 \\ 0.00 E+00 & 2.50 E+07 \\ 0.0 E E+00 & 7.00 E+07 \\ 0.00 E+00 & 2.10 E+07 \\ 0.00 E+00 & 1.30 E+07 \\ 0.00 E+00 & 6.20 E+05\end{array}$

VACUELIW

$6.90 E+04$
$1.20 E+05$
$1.30 E+05$
$1.90 E+06$
$1.90 E+06$
$1.90 E+06$
$3.90 E+04$
$1.20 E+04$
$1.00 E+06$
$2.40 E+05$
$1.10 E+06$
$6.80 E+05$
$1.20 E+06$
$2.00 E+06$
$8.50 E+05$
$6.30 E+05$
$5.90 E+05$
$1.60 E+06$
$4.90 E+05$
$3.00 E+05$
$1.40 E+04$

$\operatorname{sran}$

afres

3.90E+05

$5.40 E+05$
$5.90 E+05$

B. $20 \mathrm{E}+06$

8. $30 E+06$

$8.50 E+06$
$1.70 E+05$

$1.70 E+05$
$5.30 E+04$

5.
$4.40 E+06$

$1.10 E+06$
$4.60 E+05$

$4.60 E+06$
$3.00 E+06$

5.30E +06

8.80E+06

3.70E+06

$2.70 E+06$
$2.60 E+06$

2. $60 E+06$
$7.10 E+06$

2.10E+06

1.30E+06

$2.70 E+03$
$3.70 E+03$

$4.00 E+03$

$5.60 E+04$

$5.70 E+04$
$5.80 E+04$

.20E+03

$3.60 E+02$

$7.30 E+03$

$3.20 E+04$

$2.00 E+04$

$3.60 E+04$

6. $10 E+04$

$1.90 E+04$

1. BOE +04

$4.90 E+04$

$1.50 E+04$

$9.10 E+03$
$4.40 E+02$

VOFGas

$3.00 E+02$
$4.20 E+02$
$4.50 E+02$
$6.20 E+03$
$6.40 E+03$
$6.50 E+03$
$1.30 E+02$
$4.00 E+01$
$3.30 E+03$
$8.10 E+02$
$3.50 E+03$
$2.30 E+03$
$4.00 E+03$
$6.70 E+03$
$2.80 E+03$
$2.10 E+03$
$2.00 E+03$
$5.50 E+03$
$1.60 E+03$
$1.00 E+03$
$4.80 E+01$

sean

Totals

PAOCESS BLOCK TERTURY

TOTAL MASS FOOWS (KG) FOR

PAOCESSEOOKNPU
STREAM FLOWS (KG)

PROCESS MUSSFLOW

STPEAM FOWS (KG)

PFOCESS MUSSFIOW TETAL MASS FLW STRE)

STREAM MASSRLOW

$0.00 E+00$

$0.00 E+00 \quad 1.09 E+07$

$0.00 E+00 \quad 2.65 E+08$

$0.00 E+00 \quad 2.70 E+00$

$0.00 E+00$ S $5.47 E+00$

$0.00 E+00 \quad 1.70 E+06$

$0.00 E+00,1.42 E+08$

$0.00 E+00 \quad 3.40 E+07$

$0.00 E+00 \quad 1.49 E+08$

$0.00 E+00 \quad 9.56 E+07$

$0.00 E+00 \quad 1.70 E+00$

$0.00 E+00 \quad 2.85 E+08$

$0.00 E+00 \quad 1.20 E+08$

$0.00 E+00 \quad 8.82 E+07$

$0.00 E+00 \quad 8.40 E+07$

$0.00 E+00 \quad 2.32 E+06$

$\begin{array}{ll}0.00 E+00 & 6.91 E+07 \\ 0.00 E+00 & 4.25 E+07\end{array}$

$0.00 E+00$
$0.00 E+00$

\begin{tabular}{|c|c|}
\hline $\begin{array}{l}\text { SEPSOWO } \\
\text { DESTOAG }\end{array}$ & $\begin{array}{l}1.25 E+07 \\
1.75 E+07\end{array}$ \\
\hline CAUSLEAC & $1.89 E+07$ \\
\hline $\operatorname{css} x$ & $2.65 E+08$ \\
\hline SFX & $2.70 E+08$ \\
\hline $\operatorname{TCD}$ & $2.76 \mathrm{E}+08$ \\
\hline Disolva & $5.47 E+06$ \\
\hline DẼZVF & $1.70 E+06$ \\
\hline EXIRTPY & $1.42 E+08$ \\
\hline PUAFNU & $3.40 E+07$ \\
\hline EXTRAMEN & $1.49 E+08$ \\
\hline AMLANIX & $9.56 E+07$ \\
\hline $\operatorname{FEx}$ & $1.70 E+08$ \\
\hline SPReAx & $2.85 E+08$ \\
\hline APMicsix & $1.20 E+08$ \\
\hline PCOVACID & 8. $82 E+07$ \\
\hline DNNTRL. & $8.40 E+07$ \\
\hline CALCNE & $2.32 E+08$ \\
\hline PEMCR & $6.91 E+07$ \\
\hline EVAPOPH & $4.25 E+07$ \\
\hline
\end{tabular}

$\begin{array}{ll}\text { NCBUSTS } & 6.15 E+08 \\ \text { WVCBUSTS } & 0.00 E+00 \\ \text { SICM } & 6.29 E+08 \\ \text { CBUSTS } & 3.07 E+08 \\ \text { VCBUSTS } & 0.00 E+00 \\ \text { SECM } & 0.00 E+00 \\ \text { AOUEUW } & 7.19 E+08 \\ \text { VAOUEUW } & 0.00 E+00 \\ \text { SOCM } & 7.36 E+07 \\ \text { OFFGAS } & 5.05 E+05 \\ \text { VOFGGA } & 0.00 E+00 \\ \text { SAM } & 0.00 E+00\end{array}$

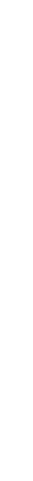


WHC-EP-0791

APPENDIX 6

GLASS COMPOSITION OPTIMIZATION

G-1 


\section{LIST OF TERMS}

$\begin{array}{ll}\text { CLEAN31 } & \text { CLEAN Option HLW stream } \\ \text { CLELLW } & \text { CLEAN Option LLW stream } \\ \text { CVS } & \text { Composition Variability Study } \\ \text { HLW } & \text { high-level waste } \\ \text { LLW } & \text { low-leve1 waste } \\ \text { OTHHI } & \text { Other upper limit } \\ \text { SLUD31 } & \text { Sludge Wash C HLW stream } \\ \text { SLULLW } & \text { Sludge Wash C LLW stream } \\ \text { TPA } & \text { Tri-Party Agreement } \\ \text { TRUEX } & \text { transuranic extraction } \\ \text { TRUEX-C } & \text { Transuranic Extraction Option C } \\ \text { TRULLW } & \text { TRUEX-C LLW stream } \\ \text { TRUX31 } & \text { TRUEX-C HLW stream }\end{array}$




\section{GLASS COMPOSITION OPTIMIZATION}

The nonlinear optimization methods used in determining the glass composition are described in this section. The discussion first considers a general nonlinear optimization problem and proceeds to describe the method of finding a global optimum to the problem. These nonlinear optimization methods are then applied to the optimal glass waste loading problem with development of a glass composition optimization model. This model was developed by Mark Hoza of Pacific Northwest Laboratory. He also provided the glass optimization calculations along with tha model description text that follows. The final section describes the glass optimization results for the different process model runs.

\subsection{THE GENERAL CONSTRAINED OPTIMIZATION PROBLEM}

This section describes a general optimization problem. In optimization problems, one wants to find the point $x$ that produces the maximum (or minimum) value of some function and that meets a set of criteria called constraints. The problem would be stated as:

$$
\begin{gathered}
\text { maximize } \quad f\left(x_{1}, x_{2}, \ldots, x_{n}\right) \text { (objective function) } \\
\text { subject to } \quad h_{1}\left(x_{1}, x_{2}, \ldots, x_{n}\right)=0 \quad \text { (equality constraints) } \\
h_{2}\left(x_{1}, x_{2}, \ldots, x_{n}\right)=0 \\
\vdots \\
h_{2}\left(x_{1}, x_{2}, \ldots, x_{n}\right)=0 \\
g_{1}\left(x_{1}, x_{2}, \ldots, x_{n}\right) \leq 0 \text { (inequality constraints) } \\
g_{2}\left(x_{1}, x_{2}, \ldots, x_{n}\right) \leq 0 \\
\vdots \\
g_{g}\left(x_{1}, x_{2}, \ldots, x_{n}\right) \leq 0
\end{gathered}
$$

The objective function is a single (scalar) function of the unknown variables $\left(x_{1}, x_{2}, \ldots, x_{n}\right)$, which one wants to maximize or minimize. Each of the equality and inequality constraints is also a function of the unknowns. In general, the objective function and any or all constraints may be nonitinear.

The above problem can te stated more compactly in vector notation, in which a vector is represented in bold type.

$$
\begin{array}{ll}
\max & f(x) \\
\text { subject to } & h(x)=0 \\
& g(x) \leq 0
\end{array}
$$




\subsection{UNDERSTANDING THE CONSTRAINED OPTIMIZATION PROBLEM}

It is useful to visualize a few simple optimization problems. Consider first a simple unconstrained optimization problem, as represented by the surface in Figure Gl. One can easily identify the minimum of that function. For an unconstrained problem, the gradient of the function at the optimum must equal zero. This indicates there is no direction one can go from the optimum to produce a better (lower) value.

The situation is analogous for constrained optimization. Figure Gl shows the same simple function but with two linear constraints, one an equality and the other an inequality. The minimum must lie on the function surface, within the equality constraint $p l a n e$, and to the left of the inequality (vertical) plane. The feasible region is the arc resulting from the intersection of the equality plane and the function surface which lies to the left of the inequality constraint. The optimum is identified on the figure. For a constrained optimum, the Karusch-Kuhn-Tucker conditions must be satisfied. These essentially state that the constraints must be satisfied and that the gradient of the Lagrangian (a function made up of the objective function and the constraints) be equal to zero. Much more detail can be found in any optimization book, such as Optimization of Chemical Processes (Edgar and Himmelblau 1988).

\subsection{FINDING THE GLOBAL OPTIMUM}

In any moderately complex system, there will likely be more than one point that satisfies the optimality conditions. These points are called local optima. Only one of them, however, is the true (global) optimum. The following discussion will try to provide an intuitive understanding of why this is so.

Consider a simple function of one variable as shown in Figure Gl.

This function has two local minima, A and B. A is the global minimum. If the starting point for the calculation is $C$ or $D$, the optimization will return $B$ as the optimum. (At either of those points, the gradient points toward B.) If the starting point is either $E$ or $F$, the correct global optimum is found.

This does not mean that the optimization algorithm does not work. An optimization algorithm can be proven to find the global optimum only for a convex function (which will, by definition, only have one optimum). Because many functions of interest are nonconvex, the best one can do directly is to find a local optimum. By providing a number of different starting points over a fairly wide range; however, one can select the best local optimum and be reasonably sure it is the global optimum. 


\subsection{THE OPTIMAL WASTE LOADING PROBLEH}

This section discusses the simple waste loading problem around which most of the waste optimization calculations are based. In this problem, glass formers are added to a single waste composition, and the mass fraction of waste in the glass is maximized such that all the constraints are satisfied. The glass former composition is varied as part of the optimization. The general formulation of the problem is given below, while the objective function and the constraints are described in the following subsections. Specialized variations of this general problem are described later in this report.

For the single mixture or single tank case, the optimization problem can be generally stated as follows.

\section{Single Mixture Waste Loading Optimization Problem:}

$\begin{array}{ll}\text { or } \min & \text { number of canisters } \\ \max & \text { waste loading per canister } \\ \text { s.t. } & \text { mass balance constraints } \\ & \text { single component constraints } \\ & \text { critiple component constraints } \\ & \text { glass property constraints }\end{array}$

The possible objective functions and each category of constraints will be discussed in turn in the next few sections.

\subsection{OBJECTIVE FUNCTION}

For the process model evaluations, it is desired to minimize the number of canisters of glass produced. The number of canisters can be expressed as total mass of waste oxides divided by the total mass of waste in the glass per canister. In the notation used throughout the optimal waste loading (OWL) models, our objective is to

$$
\text { minimize } \frac{\text { OxideMass }}{\text { TMWG }}
$$


Because the numerator is constant for a given problem, one can equivalently maximize the amount of waste in each canister or the mass fraction of waste in each canister.

TMWG maximize

or

TMWG

MaxLogMass

For the single mixture calculations one can use the latter form for the objective function.

\subsection{MASS BALANCE CONSTRAINTS}

The mass balance constraints are equalities. A list was developed to include the mass balance constraints for the waste + glass formers $=$ glass (i.e., no recycle) case. The extensions for the recycle case are straight forward.

Mass of glass in a canister = Total Mass of Waste in the Glass + Total Mass of Glass Formers in the Glass

$$
\text { MaxLogMass }=\text { TMWG + TMFG. }
$$

Define the mass fraction of each component in the glass (FG) in terms of its mass fractions in the waste (FW) and the glass formers (FF), and the masses of waste and glass formers in the glass

$$
F G_{i}=\frac{F W_{i} * T M W G+F F_{i} * T M F G}{\text { MaxLogMass }}
$$

And finally, require that the mass fractions of all ten CVS components sum to one in the glass former stream and in the glass product.

$$
\sum_{i=1}^{10} F F_{i}=1.0 \quad \sum_{i=1}^{10} F G_{i}=1.0
$$




\subsection{SINGLE COMPONENT CONSTRAINTS}

These constraints limit the range of the mass fraction values each component can have in the glass. They reflect the composition region over which the Composition Variability Study (CVS) was performed. The upper and lower limits used in the calculations are the bounds from phase $2 a$ of CVS-II (Hrma and Piepel 1992). For each of the ten cys components, these constraints would be expressed as:

$$
F G_{i, L L} \leq F G_{i} \leq F G_{i, U L}
$$

The limits for each component are given in Table Gl.

\subsection{MULTIPLE CONPONENT CONSTRAINTS}

These constraints (also known as crystallinity constraints) limit the range of values some combination of components can have. The constraints and the names by which they will be referred are given below:

$$
\begin{aligned}
& \frac{F G_{\mathrm{SiO}_{2}}}{F G_{\mathrm{AL}_{2} \mathrm{O}_{3}}} \geq 3.0 \quad \text { Crystall } \\
& F G_{190}=F G_{\text {co0 }} \leq 0.08 \quad \text { Crysta12 } \\
& \mathrm{FG}_{\mathrm{FE}_{2} \mathrm{O}_{3}}+\mathrm{FG}_{\mathrm{AL}_{2} \mathrm{O}_{3}}+\mathrm{FG}_{2 \mathrm{rO}_{2}}+\mathrm{FG}_{\text {other }} \leq 0.225 \quad \text { Crystal3 } \\
& F G_{\mathrm{Al}_{2} \mathrm{O}_{3}}+F G_{2 \mathrm{rO}_{2}} \leq 0.18 \quad \text { Crystal4 } \\
& F G_{\text {MgO }}+F G_{\mathrm{CsO}}+F G_{2 \mathrm{rO}_{2}} \leq 0.18 \quad \text { Crystal5 }
\end{aligned}
$$




\subsection{CRITICAL SOLUBILITY CONSTRAINTS}

These constraints limit the maximum value for the mass fraction of one or a combination of components (e.g., noble metals: $\mathrm{Rh}_{2} \mathrm{O}_{3}+\mathrm{PdO}+\mathrm{Ru}_{2} \mathrm{O}_{3}$ ). These limits cover species not included among the nine species covered in the CVS studies. (They are, of course, included within the tenth component "Other.") These limits are of the form

$$
F G_{k} \leq F G_{k, U L}
$$

Their limits are given in Table G2.

\subsection{GLASS PROPERTY CONSTRAINTS}

These constraints will embody the findings of the composition variability study of Hrma and Piepel (1992). The calculation of each property (viscosity, electrical conductivity, durability [actual rate of release of boron] by either the Product Consistency Test or Materials Characterization Center test) is of the form

$$
\text { MinPropVal } \leq \sum_{i=1}^{10} b_{i} F G_{i}+\sum_{i=1}^{10} \sum_{j>i} b_{i j} F G_{i} F G_{j} \leq \text { MaxPropVal }
$$

where $b_{i}$ and $b_{i j}$ are the coefficients of the first and second order terms respectively and 10 is the number of components considered in the study. For each of the nonlinear property models, the coefficients of Model No. 3 from Hrma and Piepel (1992) were used. The limits on each of the constraints are given in Table G3.

\subsection{GLASS OPTIMIZATION RESULTS}

The current glass optimization models are based on analyses and experimental glass formulation data relative to expected high-level waste (HLW) stream compositions (Hrma and Piepel 1992 and Hoza 1993). Recent Tri-Party Agreement (TPA) (Ecology 1994) changes have also designated glass as the final waste product form for the low-level waste (LLW) stream. However, no exte ive analyses or experimental glass formulation data relative to expected LLW stream compositions have been done. Therefore, the HLW glass composition specifications and associated optimization models will be used for the LLW glass streams also. It is expected that the LLW glass will have different glass composition and property limits that will be defined in future work. 
The HLW glass will be formed in canisters that will be containerized for storage in an underground repository. The LLW glass is expected to be stored as gravel-sized glass chunks (cullet) within a sulfur cement matrix in underground vaults. Thus, the HLW glass disposal product units are shown as canisters, while the LLW glass disposal product units are shown as vaults. A calculation of the number of LLW glass canisters is included for comparison purposes only.

To obtain feasible solutions when optimizing the glass composition the $\mathrm{Fe}_{2} \mathrm{O}_{3}$ lower limit of 2 wt\% (al1 limits are weight percents) was removed in all HLW and LLW glass calculations. This was because the waste oxide streams resulting from processing all single- and double-shell tank wastes together (i.e., as a blended waste stream) contained $\mathrm{Fe}_{2} \mathrm{O}_{3}$ in quantities of small overail mass fraction, requiring unattainable high waste loadings to achieve the lower constraint imit. The $\mathrm{Fe}_{2} \mathrm{O}_{3}$ lower limit was originally based on maintaining sufficient $\mathrm{Fe}_{2} \mathrm{O}_{3}$ in the glass to act as a redox buffer to protect against uranium +5 reduction to uranium+4. This lower limit should be reexamined to determine if it is necessary.

The initial limiting constraints for the HLW and LLW glass compositions were as follows: the $P_{2} O_{5}$ upper limit of 1 wt\% for the TRUX31 (TRUEX-C HLW stream), SLUD31 (STudge Wash C HLW stream), CLELLW (CLEAN Option LLW stream), and TRULLW (TRUEX-C Option LLW stream) models; the other upper limit (OTHHI) of 10 wt\% for the CLEAN31 (CLEAN Option $\mathrm{HLW}$ stream) model; and the $\mathrm{Na}_{2} \mathrm{O}$ upper limit of 20 wt\% for the SLULLW (SIudge Wash C LLW stream) model. Another glass optimization run was made in which the initial limiting or binding constraint for each model was relaxed. The next 1 imiting constraints became the crystallinity No. 3 upper 1 imit of 22.5 wt\% for the CLEAN3I mode 1 , the OTHHI of 10 wt\% for the TRUX31 and SLUD31 models, the $\mathrm{Na}_{2} \mathrm{O}$ upper 1 imit of 20 wt\% for the CLELLW and TRULLW models, and several limits for the SLULLW model.

Relaxing the OTHHI (this limit represents everything other than the nine components considered in the CVS study) was definitely considered to be extrapolating outside the range of applicability of the glass property models and constraints (Hrma and Piepel 1992 and Hoza 1993). However, relaxing the $\mathrm{P}_{2} \mathrm{O}_{5}$ upper 7 imit was not considered to represent a large extrapolation outside the range of model applicability. Although the same reasoning could be applied to the $\mathrm{Na}_{2} \mathrm{O}$ upper limit only the $\mathrm{P}_{2} \mathrm{O}_{5}$ upper limit was relaxed for the process strategy comparisons. Thus, for the LLW glass compositions (CLELLW, TRULLW, and SLULLW models) the limiting constraint was the $\mathrm{Na}_{2} \mathrm{O}$ upper 1 imit of 20 wt\% and for the HLW glass compositions (CLEAN31, TRUX31, and SLUD31 models) the limiting constraint was the OTHHI of 10 wt\%.

Table 64 shows the glass optimization results with the initial binding constraint relaxed (e.g., Other, $\mathrm{P}_{2} \mathrm{O}_{5}$, or $\mathrm{Na}_{2} \mathrm{O}$ upper constraint limits) while Table G5 shows the glass optimization results with the initial binding constraint. The global models used the glass compositions that result with only the $\mathrm{P}_{2} \mathrm{O}_{5}$ upper constraint limit relaxed. Table 66 shows comparison data between the HLW glass canister and LLW glass-in-sulfur vault results with and without the initial binding constraints. Figures $G 2$ and G3 show the HLW and LLW glass production results, respectively, with and without the $\mathrm{P}_{2} \mathrm{O}_{5}$ upper constraint limit. Figures $G 4$ and $G 5$ show the weight fractions of components in the HLW and LLW waste oxide streams, respectively. Figures $G 6$ and $G 7$ show 
the weight fractions of components in the $\mathrm{HLW}$ and $L L W$ glass without the $P_{2} \mathrm{O}_{5}$ upper constraint 1 imit, respectively. Figures $G 8$ and $G 9$ show the weight fractions of components in the $H L W$ and $L L W$ glass with the $P_{2} \mathrm{O}_{5}$ upper constraint 1 imit, respectively.

The impact of the glass composition constraints on waste loading in the glass is shown in Table G6. Waste loadings of approximately 19 wt\%, 36 wt\%, and 26 wt\% were achieved in the CLEAN Option, TRUEX-C, and Sludge Wash C HLW glass products, respectively, without the $\mathrm{P}_{2} \mathrm{O}_{5}$ upper limit of 1 wt\% in place. Table $G 4$ and Figure $G 6$ show that the resulting $\mathrm{P}_{2} \mathrm{O}_{5}$ weight fraction in glass was 1.7 and $3.6 \%$ for the TRUEX-C and Sludge Wash $\mathrm{C}^{2} \mathrm{HLW}$ glass products, respectively. However, with the $\mathrm{P}_{2} \mathrm{O}_{5}$ upper 1 imit, the HLW waste loadings were significantly lower, approximately 21 and 7 wt\% for the TRUEX-C and Sludge Wash $C$ models, respectively. No impact on the CLEAN Option HLW glass resulted from relaxing the $\mathrm{P}_{2} \mathrm{O}_{5} 1$ imit because it was not the limiting constraint. Increasing the OTHHI of 10 wt\% will al so have a significant impact on the HLW glass production as shown by the comparison of the CLEAN Option HLW glass stream (CLEAN31 mode1) results in Table G6. When the OTHHI was relaxed, the CLEAN Option HLW glass stream showed a 39\% reduction of canisters (from 750 to 457), with a substantial increase in waste loading from 19 to 32 wt\%.

The LLW glass had waste loadings from 22 to 24 wt\% with the $\mathrm{P}_{2} \mathrm{O}_{5}$ upper limit in place. When the $\mathrm{P}_{2} \mathrm{O}_{5}$ upper limit was relaxed and the $\mathrm{Na}_{2} \mathrm{O}^{2}$ upper limit became binding, only the CLEAN Option LLW glass waste loading increased significantly from 22 to 27 wt\%. Increasing the $\mathrm{Na}_{2} \mathrm{O}$ upper limit will also have a significant impact on the LLW glass production as shown by the comparison of the Sludge Wash C LLW giass stream (SLULLW model) results with and without the $\mathrm{Na}_{2} \mathrm{O}$ upper 7 imit of 20 wt\% relaxed, where an $8 \%$ reduction of vaults (60 down to 55 ) was seen. The resulting $\mathrm{Na}_{2} \mathrm{O}$ weight fraction in glass was 21.5\% as shown in Table G4. The Tank Waste Technical Options Report (Boomer et al. 1993) suggests that a higher $\mathrm{Na}_{2} \mathrm{O}$ limit of 25 wt\% is possible because of the ability to recycle out-of-specification glass cullet. It also claims that higher $\mathrm{Na}_{2} \mathrm{O}$ limits in borosilicate glass compositions are reported in other literature. Increasing this limit will have a significant impact on the LLW glass production as shown by the comparison of the SLULLW model results.

Table G6 gives a summary of the HLW glass canister and LLW glass-insulfur vault counts achieved by the different process strategies relative to the inclusion of the $\mathrm{P}_{2} \mathrm{O}_{5}$ upper 1 imit. Note that the CLEAN Option HLW glass canister count was dramatically lower than the other process strategies, with only approximately 5 and $1 \%$ of the TRUEX-C and Sludge Wash C process strategy glass product magnitudes with the $\mathrm{P}_{2} \mathrm{O}_{5}$ upper limit, respectively, and only approximately 8 and $2 \%$ without the $\beta_{2} \delta_{5}$ upper limit, respectively. Relaxing the $\mathrm{P}_{2} \mathrm{O}_{5}$ upper 1 imit reduced the TRUEX-C and siudge Wash C HLW canister counts by $41^{2}$ and $72 \%$, respectively, and the LLW vault counts by 18 and $0.4 \%$ for the CLEAN Option and TRUEX-C process strategies, respectively.

The CLEAN Option LLW glass-in-sulfur vault total was highest for the $\mathrm{P}_{2} \mathrm{O}_{5}$ 1 imiting case but ranged between the TRUEX-C and Sludge Wash $C$ results without the $\mathrm{P}_{2} \mathrm{O}_{5}$ upper limit, showing vault counts of 86 and $109 \%$ of the TRUEX-C and Sludge Wash $C$ results, respectively. Note the large amount of glass mass inventory (kilograms) associated with the LLW glass. This is due to the large 
amount of $\mathrm{Na}_{2} \mathrm{O}$ in the LLW oxide streams as shown in Figure 65. Indeed $\mathrm{Na}_{2} \mathrm{O}$ constitutes 74,85 , and 89 wt\% of the LLW oxtde stream for the CLEAN Option, TRUEX-C, and Siudge Wash C process strategies, respectively. Removing this sodium would dramatically reduce the LLW glass mass inventory.

As discussed in the previous report on the evaluation of the TRUEX-C and Sludge Wash C process strategies (Knutson et al. 1993), over 90\% of the HLW glass oxides in those two models come from the undissolved solids stream that is sent to glass before transuranic extraction (TRUEX) processing and eventual neutralization. Therefore, significant glass oxide reduction for those two models must come in the acid dissolution process step.

Based on previous work (Knutson et al. 1993), a $5 \%$ heel left in the tanks during retrieval would result in an approximately $5 \%$ reduction in the HLW glass canister counts (Knutson et al. 1993).

The glass former compositions resulting from the glass optimization models should not be considered unique. When a particular glass property limit becomes constraining (e.g., $\mathrm{P}_{2} \mathrm{O}_{5}$ and $\mathrm{OTHHI)}$ the feasible glass former compositions can vary considerabiy.

With these aggressive pretreatment strategies it is clear that more glass property data and glass melting data in HLW and LLW waste composition ranges could possibly allow relaxing current composition constraints. If such limits were relaxed, higher waste oxide loadings in the glass could be achieved (and hence, reduced glass canister and vault counts, costs, etc.), and other pretreatment process choices will be seen. Indeed, the critical component constraints have not been explored as a function of the mass fraction of major glass components, such as $\mathrm{SiO}_{2}$ or $\mathrm{Na}_{2} \mathrm{O}$, and, therefore, should be taken as current estimates and not as scientifically established values that cannot be challenged if they represent severe constraints on the rest of a tank waste remediation system. Therefore, there should be additional focus on HLW and LLW immobilization activities with emphas is on currently constraining limits, such as $\mathrm{P}_{2} \mathrm{O}_{5}$, Other, and $\mathrm{Na}_{2} \mathrm{O}$.

\subsection{REFERENCES}

Boomer, K. D., et al., 1993, Tank Waste Technical Options Report, WHC-EP-0616, Rev. 0, Westinghouse Hanford Company, Richland, Washington.

Ecology 1994, Hanford Federal Facility Agreement and Consent Order, as amended, Washington State Department of Ecology, U.S. Environmental

Protection Agency, and U.S. Department of Energy, Olympia, Washington.

Edgar, T. F, and D. M. Himmelblau, 1988, Optimization of Chemical Processes, McGraw-Hill, New York, New York.

Hoza, M., 1993, Optimal Waste Loading Models for Vitrification of Hanford High-Level Waste, PHTD-693.03.01M, Pacific Northwest Laboratory, Richland, Washington. 
Hrma, P. R., and G. F. Plepel, 1992, Property/Composition Relationships for Hanford Waste Vitrification Plant Glasses -- Preliminary Results Through CVS-II Phase 2, PHTD-92-03.01/K897, Paclfic Northwest Laboratory, Richland, Washington.

Knutson, B. J., et al., 1993, "Advanced Chemical Separation Study: TRUEX Strategy Compared To Sludge Wash Ion Exchange," Letter report, July 1993. 
Figure G1. Simple Function With Two Local Minima.

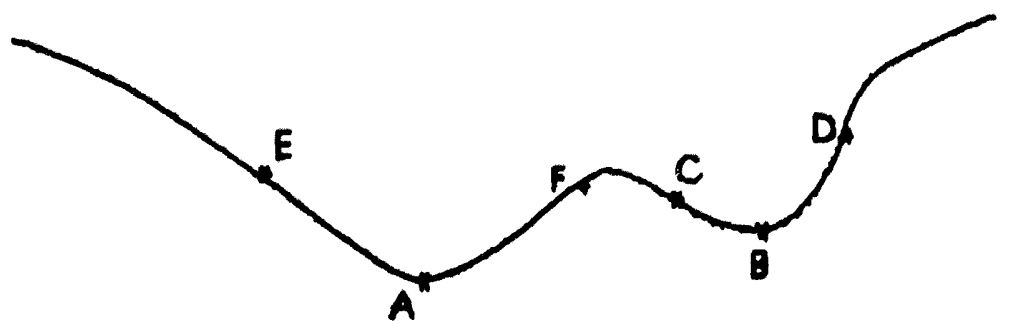

Figure G2. High-Level Waste Glass Production Results Versus $\mathrm{P}_{2} \mathrm{O}_{5}$ High-Limit Constraint.

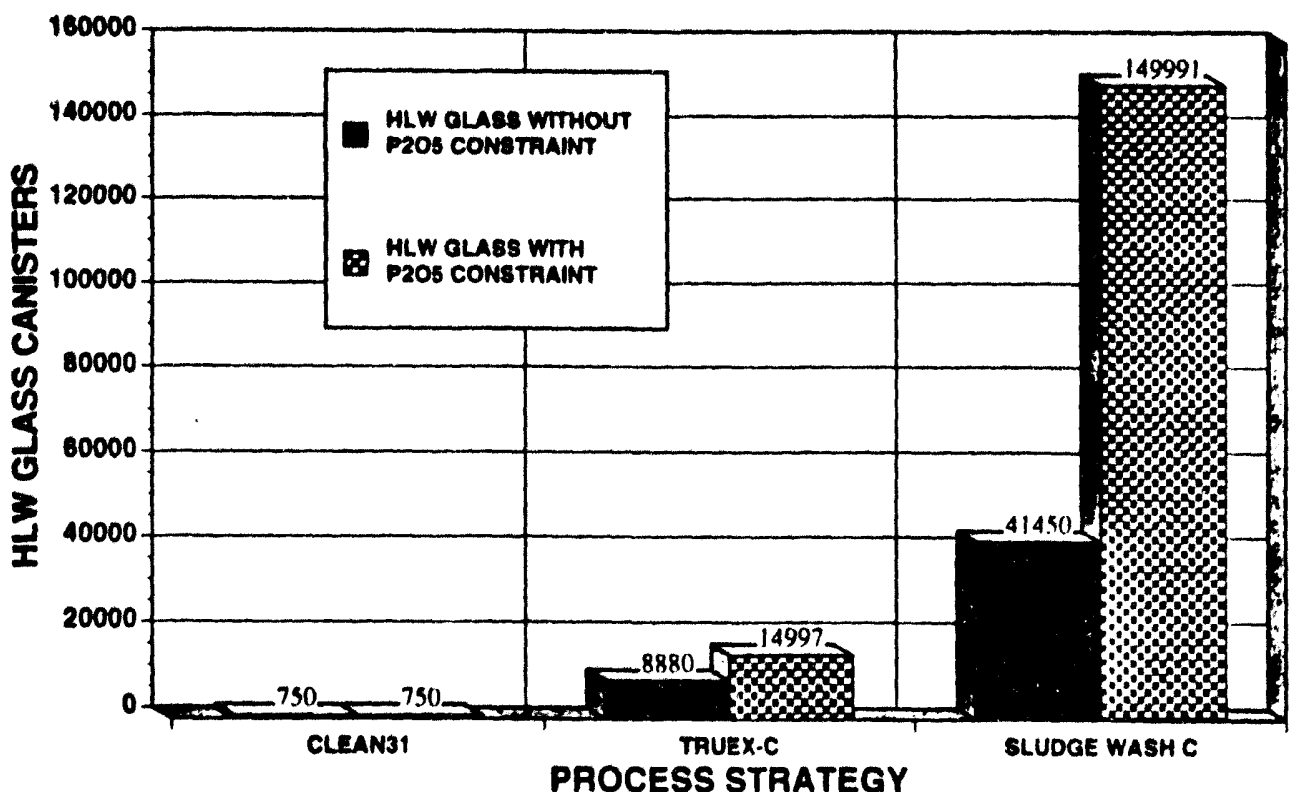

Figure G3. Low-Level Waste Glass Production Results Versus $\mathrm{P}_{2} \mathrm{O}_{5}$ High-Limit Constraint.

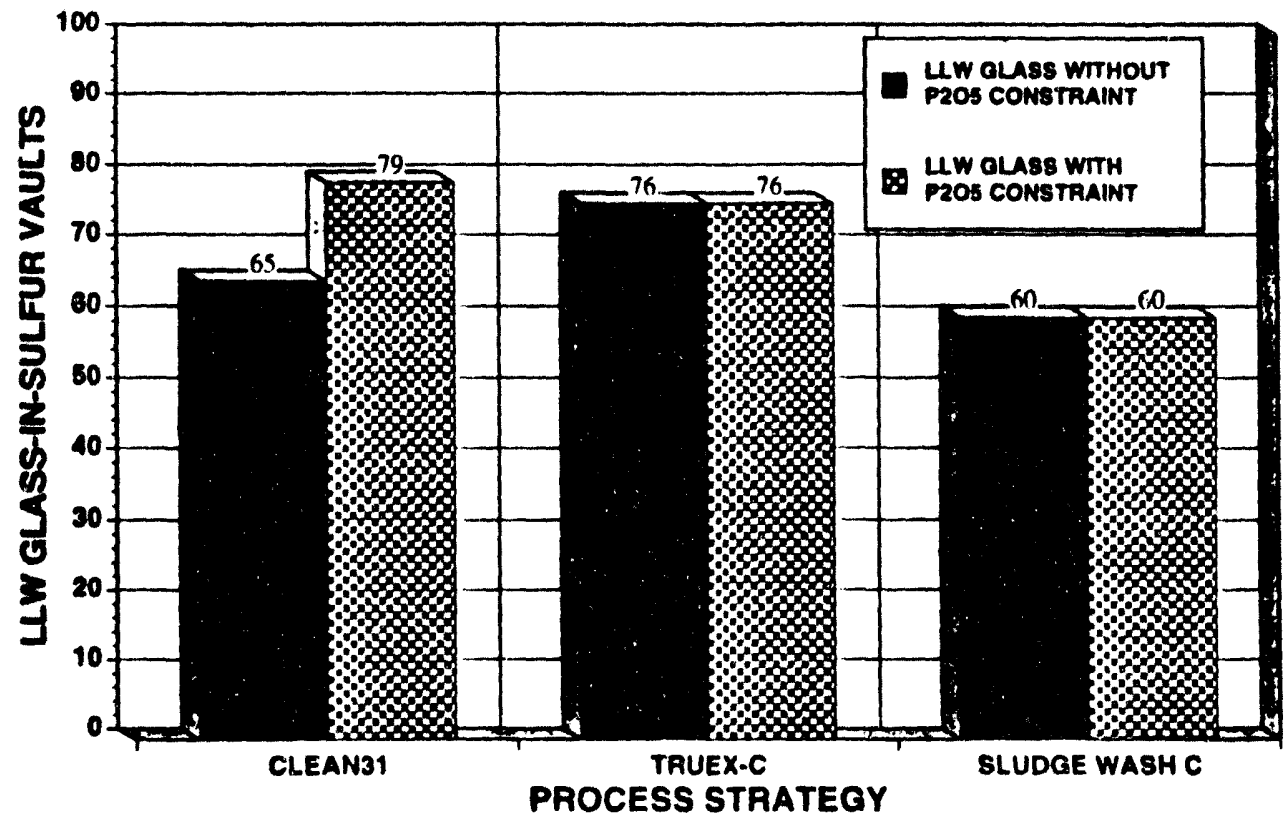


Figure G4. Weight Fractions of Components in High-Level Waste Oxide Stream.

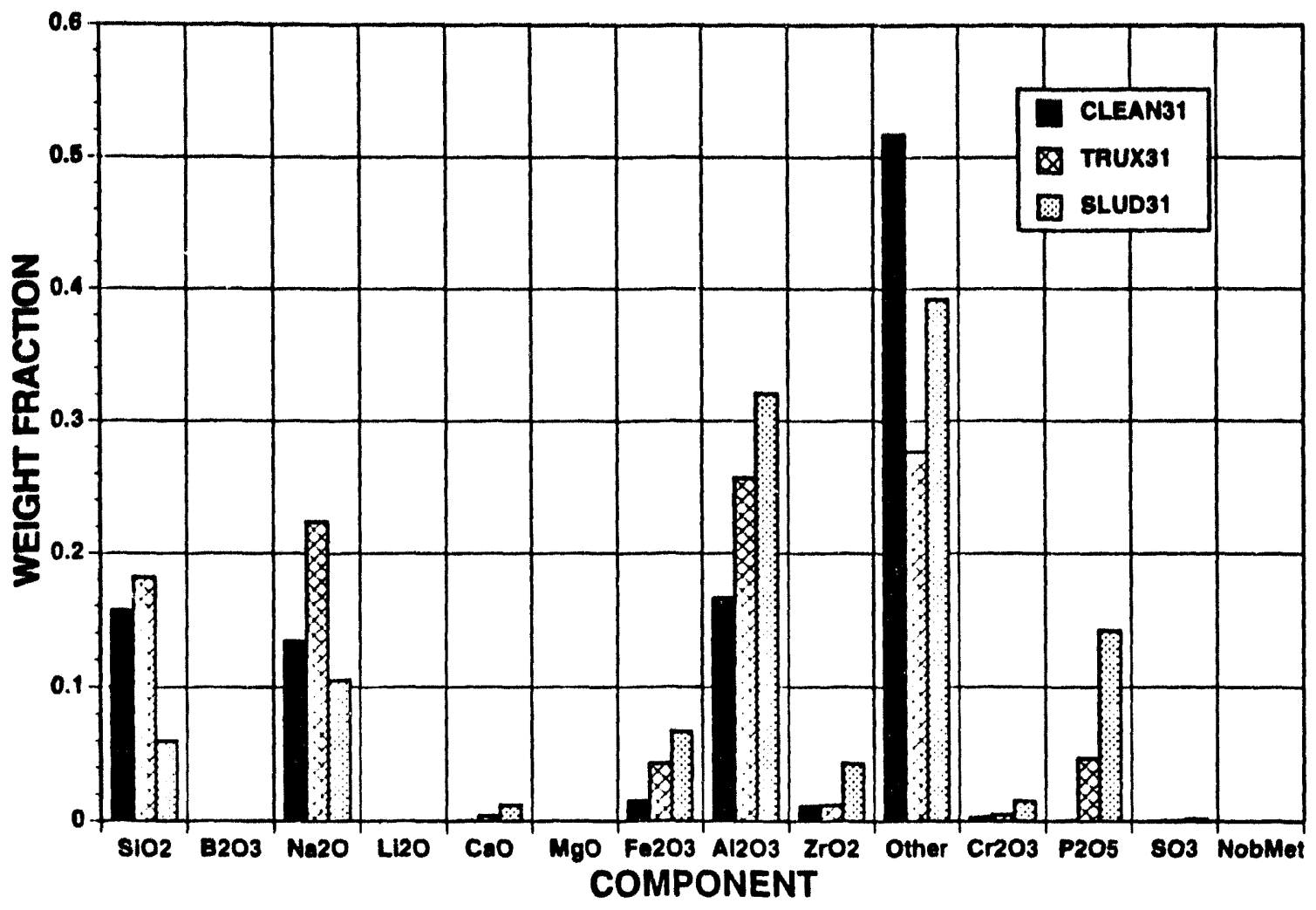

Figure G5. Weight Fractions of Components in Low-Level Waste Oxide Stream.

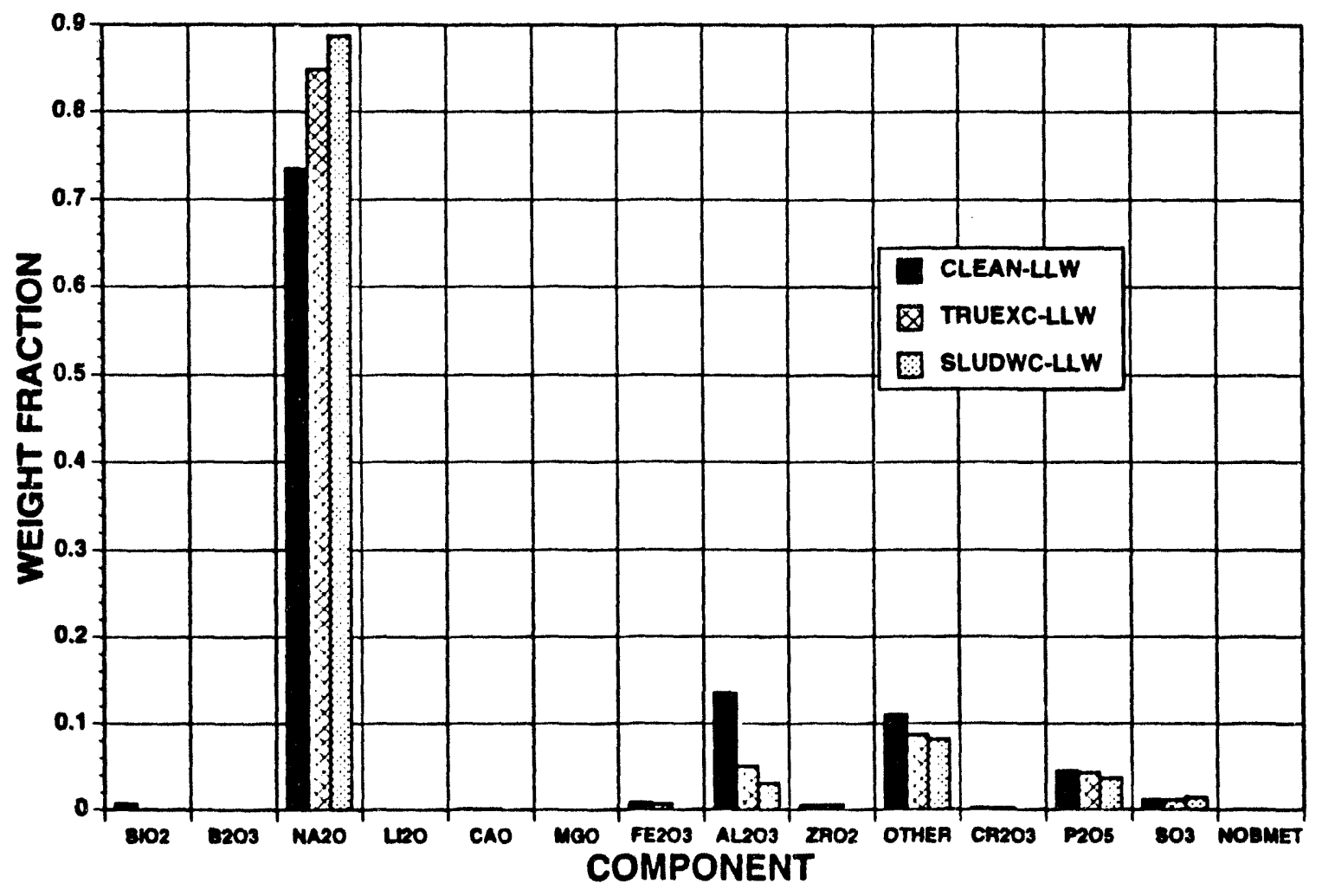

G-14 
Figure G6. Weight Fractions of Components in High-Level Waste Glass Without $\mathrm{P}_{2} \mathrm{O}_{5}$ High-Limit Constraint.

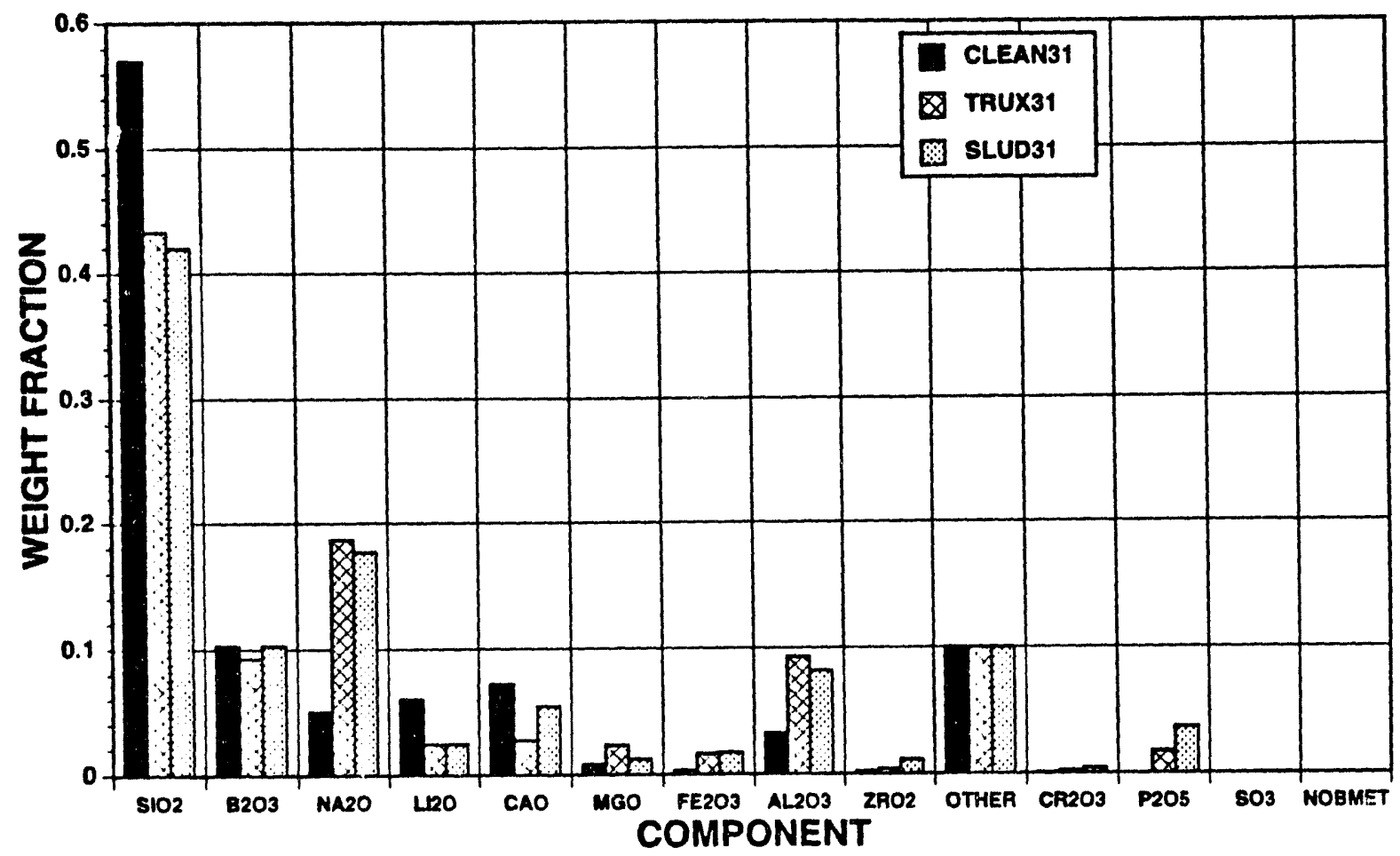

Figure 67. Weight Fractions of Components in Low-Level Waste Glass Without $\mathrm{P}_{2} \mathrm{O}_{5}$ High-Limit Constraint.

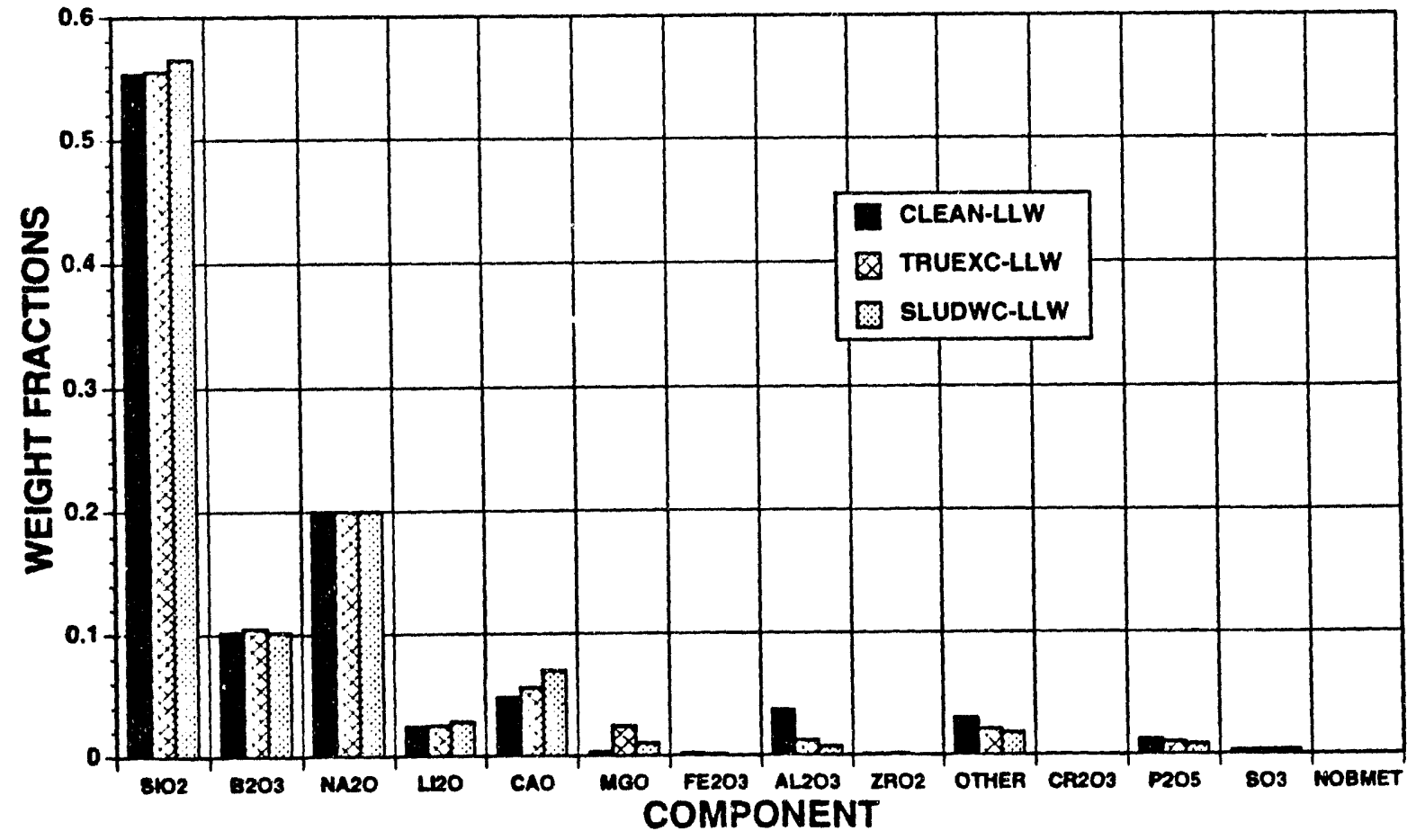


Figure G8. Weight Fractions of Components in High-Level Waste Glass With $\mathrm{P}_{2} \mathrm{O}_{5}$ High-Limit Constraint.

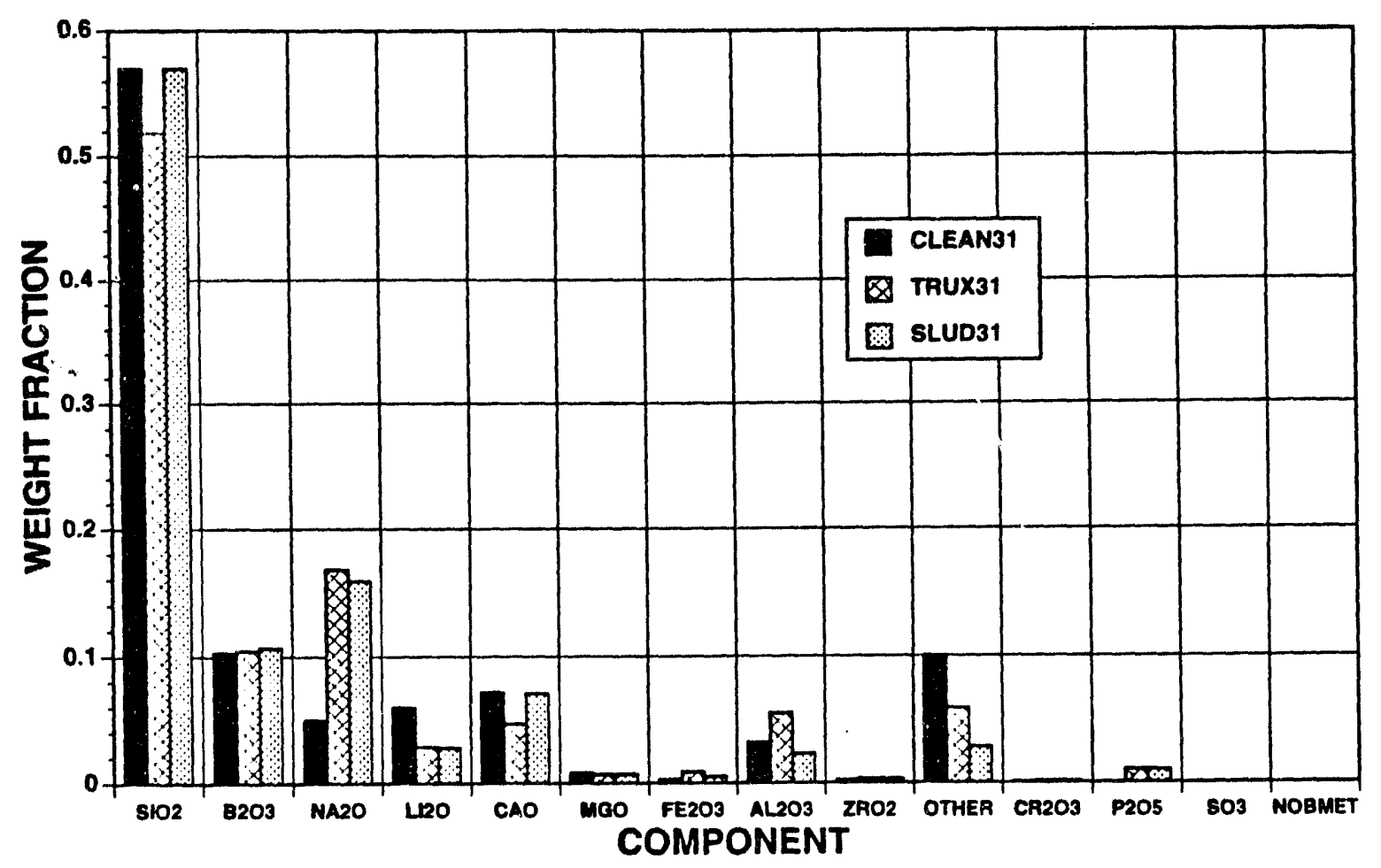

Figure 69. Weight Fractions of Components in Low-Level Waste Glass With $\mathrm{P}_{2} \mathrm{O}_{5}$ High-Limit Constraint.

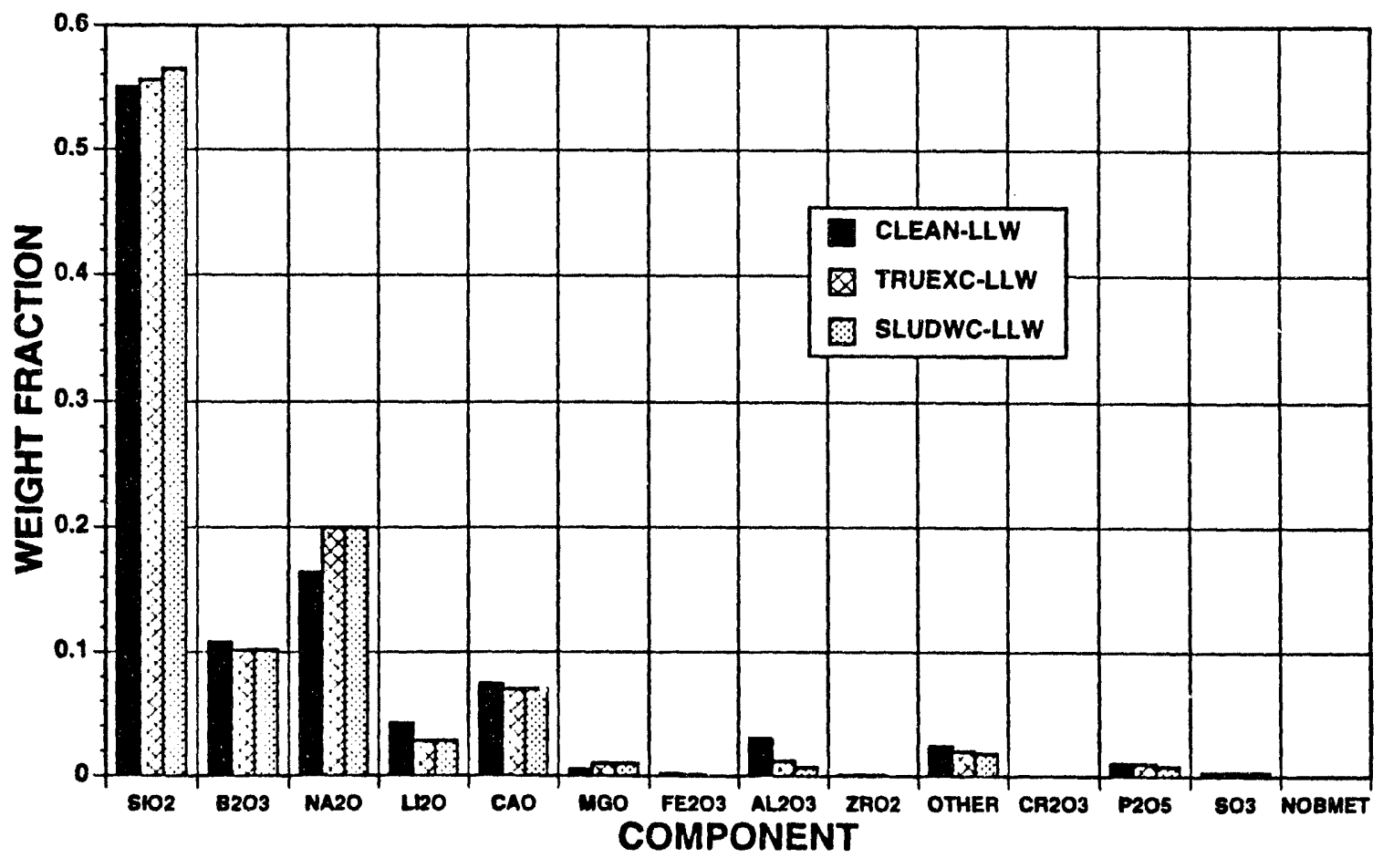


Table Gl. Upper and Lower Limits on Ten Composition Variability Study Components.

\begin{tabular}{|c|c|c|}
\hline Component, $i$ & Lower limit $-F_{i, L L}$ & Upper limit $-F G_{i, U L}$ \\
\hline sior & 0.42 & 0.57 \\
\hline${ }_{2} \mathrm{O}_{3}$ & 0.05 & 0.20 \\
\hline $\mathrm{Na}_{2} \mathrm{O}$ & 0.05 & 0.20 \\
\hline $\mathrm{Li}_{2} \mathrm{O}$ & 0.01 & 0.07 \\
\hline $\mathrm{CaO}$ & 0 & 0.10 \\
\hline MgO & 0 & 0.08 \\
\hline $\mathrm{Fe} 2 \mathrm{O}_{3}$ & 0.02 & 0.15 \\
\hline $\mathrm{A}_{12} \mathrm{O}_{3}$ & 0 & 0.15 \\
\hline $2 r 02$ & 0 & 0.13 \\
\hline Other & 0.01 & 0.10 \\
\hline
\end{tabular}

Table G2. Upper Limits on Critical Solubility Components.

\begin{tabular}{|l|c|}
\hline \multicolumn{1}{|c|}{ Solubility component, $k$} & Upper limit $-F G_{k, U L}$ \\
\hline $\mathrm{Cr}_{2} \mathrm{O}_{3}$ & 0.005 \\
\hline $\mathrm{F}$ & 0.017 \\
\hline $\mathrm{P}_{2} \mathrm{O}_{5}$ & 0.01 \\
\hline $\mathrm{SO}_{3}$ & 0.005 \\
\hline Noble metals $\left(\mathrm{Rh}_{2} \mathrm{O}_{3}+\mathrm{PdO}+\mathrm{Ru}_{2} \mathrm{O}_{3}\right)$ & 0.025 \\
\hline
\end{tabular}


Table G3. Minimum and Maximum Glass Property Values.

\begin{tabular}{|l|c|c|}
\hline \multicolumn{1}{|c|}{ Property, units } & MinPropVal & MaxPropVal \\
\hline Viscosity, PaS & 2 & 10 \\
\hline Electrical conductivity, S/m & 18 & 50 \\
\hline $\begin{array}{l}\text { Durability (release rate of } \\
\text { boron by PCT), } \mathrm{g} / \mathrm{m}^{2}\end{array}$ & $\mathrm{~N} / \mathrm{A}$ & 10 \\
\hline $\begin{array}{l}\text { Durability }(\text { release rate of } \\
\text { boron by MCC), } \mathrm{g} / \mathrm{m}^{2}\end{array}$ & $\mathrm{~N} / \mathrm{A}$ & 28 \\
\hline
\end{tabular}

MCC = Materials Characterization Center.

PCT = Product Consistency Test. 
Table G4. Glass Optimization Results With Initial Binding Constraint Relaxed.

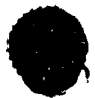

MODE:

CLEAN31
CLEAN31
HLW GLASS
(O\% HEEL)

TFUX31
TREXC
HWW GLASS
(O\% HEEL)

SLUD31
SLOWC
HWW GLASS
(O\% HEEL)

CLELW
CLEAN31
UW GLASS
(O\% HEEL)

$1.74 E+07$
$5.10 E+07$
0.255
$6.84 E+07$
41450

$1.51 E+08$

$\begin{array}{cc}2.39 E+05 & 5.28 E+06 \\ 5.15 E+05 & 8.36 E+06 \\ 0.317 & 0.361 \\ 7.55 E+05 & 1.47 E+07 \\ 457 & 8880\end{array}$

GLASS CANISTERS"

$$
\text { - } 1650 \mathrm{ko} / \mathrm{canister}
$$
ASS-IN-SURPUA VAULTS - 8.518E+6 kg/vault

PFOOUCT DrFENCES CLEAN. VS. TRUEXC
CLEAN. VS. SLUDWC

\section{COMPONENT}

FW.SIO2

FW. B2O3

FW-NA2O

FW.LI2O

FW.CAO

FWAMO

FW-FE2O3

FW-AL2O3

FW-Z7PO2

FWOTHER

FW-CR2O3

FW.P2OS

FW.SO3

FWNOEMET

CONPONENT
5.64E-01

1.61E-01

$1.76 \mathrm{E}-01$

$3.30 E-02$

$5.40 E-02$

1.30E.02

4.35E-01

1.10E-01

1.63E-01

2.20E. 02

$3.70 \mathrm{E}-02$

$9.00 \mathrm{E} .03$

5.00E-03

5.3OE-02

$4.00 \mathrm{E} .03$

1.64E.01

. $39 E .04$

$1.80 \mathrm{E} .04$

2.38E-06
.8423

$-40893$

OTH Fe2O3,P2O5
$1.51 E+08$

$4.03 E+08$
0.272

$5.53 E+08$

335251

65

Na2O

Fe203,P206

Fe2O3,P2O5

Weight Fractions in Weste Oxide Stream

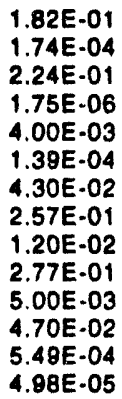

$6.00 E-02$
$4.54 E-04$
$1.05 E-01$
$2.65 E-06$
$1.20 E-02$
$4.62 E-04$
$6.70 E-02$
$3.21 E-01$
$4.30 E-02$
$3.92 E-01$
$1.50 E-02$
$1.42 E-01$
$2.00 E-03$
$6.77 E-05$

$7.00 E-03$
$5.47 E-05$
$7.35 E-01$
$5.87 E .07$
$1.00 E .03$
$5.95 E .05$
$B .00 E .03$
$1.35 E-01$
$5.00 E .03$
$1.10 E-01$
$2.00 E .03$
$4.50 E .02$
$1.10 E-02$
$8.16 E-06$

Weight Fractions In Glass Former Stream
7.57E-01

1.40E-01

3.40E-02

$6.60 E-02$

4.00E-03

1.02E-01

2.00E-01

2.40E-02

$4.80 \mathrm{E} \cdot 02$

3.00E-03

$2.00 E-03$

3.70E-02

$1.00 E-03$

3.00E-02

4.83E-04

1.20E-02

$3.00 E-03$

2.22E.06 BINONG CONSTRANT:

TRULWW
TFUEXC
LWGLASS
(OO HEEL

(O\% HEEL)

$1.52 E+08$

$4.84 E+08$

0.236

$6.46 E+08$

391704

76

.11

$\mathrm{Na2O}$

Fe2O3,P2O5

7.56E-04

4.81E.05

8.49E.01

5.20E-07

1.00E.03

5.39E-05

6. OOE.03

5.10E.02

5.00E-03

8.70E-02

2.00E -03

4.30E-02

$1.10 \mathrm{E}-02$

6.34E-06
FF.S1O2

FF. B2O3

FF-NA2O

FF-U12O

FF-CAO

FMOO

COMPONENT

FG-S1O2

FG-B2O3

FG-NAZO

FGLLI2O

FG-CAO

PGMGO

FGFE2O3

FG.AL2O3

FGZACQ

FoOThA

FG-CPROS

FG-P2OS

FG-503

FGNOENET

$5.76 E-01$
$1.46 E-01$
$1.65 E-01$
$3.80 E-02$
$4.00 E-02$
$3.60 E-02$

Welght Fractions in Glass

$4.33 E-01$
$9.30 E-02$
$1.87 E-01$
$2.40 E-02$
$2.70 E-02$
$2.30 E-02$
$1.60 E-02$
$9.30 E-02$
$4.00 E-03$
$1.00 E-01$
$2.00 E-03$
$1.70 E-02$
$1.98 E-04$
$1.80 E-05$

4.20E-01

1.03E-01

1.77E-01

2.40E-02

5.40E.02

1.20E-02

1.70E-02

B.20E.02

$1.10 E-02$

1.00E.01

$4.00 E .03$

3.60E.02

4.87E-04

1.73E-05

Class Properties

Foperity

VSCOSTr(PeS)

ELECT-CONDUCT(S/m)

DURAB.P.PCT(gm/m2/day)

DURAB.-B-MCC (gm/m2/day)

$\begin{array}{cc}2.00 & 2.81 \\ 43.22 & 49.29 \\ 2.23 & 1.10 \\ 18.08 & 15.68\end{array}$

2.00
44.44
0.01
18.08

7.25E-01

1.38E-01

3.30E-02

$7.30 \mathrm{E} .02$

3.10E-02

5.55E-01

1.05E-01

2.00E-01

2.50E-02

5.6OE-02

2.40E-02

1.00E-03

1.20E-02

1.00E-03

2.10E-02

3.72E-04

1.00E-02

3.00E-03

1. $48 E-06$ sutw

swowc

UW GLASS

1.14E+03

3.57E+0B

0.243

$4.71 E+08$

55

10

SEVERAL

$\mathrm{Fe2O3,Na2O}$
2.55E-04

2.81E-06

B.87E-01

3.68E-07

2.13E-06

$7.75 E .06$

1.43E-05

3.10E-02

1.60E-04

B.20E-02

6.69E-06

3.70E-02

1.40E.02

4.24E-07
7.53E-01

1.28E-01

1.30E-02

1.40E-02

8.20E-02

5.70E-01

9.70E-02

2.15E-01

$1.00 E-02$

1.10E-02

6.90E-02

3.48E-06

7.00E-03

3.88E-05

2.00E.02

1.62E-06

9.00E-03

$4.00 E .03$

$\begin{array}{cc}2.24 & 4.33 \\ 48.75 & 50.00 \\ 3.16 & 10.00 \\ 27.31 & 23.47\end{array}$


Table G5. Glass Optimization Results With Initial Binding Constraint.

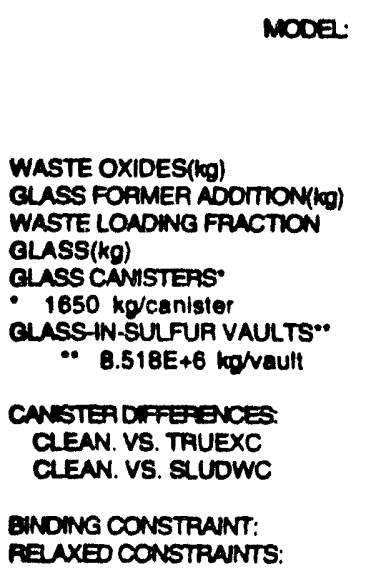

CLEAN31
CLEAN31
HLWGLASS
(O\% HEEL)

$2.39 E+05$
$9.89 E+05$
0.193
$1.24 E+06$
750

750

ortan

$\mathrm{Fa2O3}$

\section{COMPONENT}

FW.S1OR

FW-B2O3

FW-NA2O

FW.L12O

FW.CAO

FWACO

FW-FE2O3

FW.AL2O3

FW-ZRO2

FW-OTHER

FW-CR2O3

FW.P2O5

FW.SO3

FWAOEMET

\section{componert}

FF.S1O2

FF-B2O3

FFNA2O

FF.U2O

FF.CAO

FABO

\section{CONPONENT}

FQSTOR

FG-B2O3

FG.NA2O

FG-UI2O

FGCAO

FGMOO

FGFE203

FG-N2O3

FG-ZAOR

FGOTHER

FG-CF2O3

FGP2OS

Frsos

FHOENET

$1.57 E \cdot 01$
$1.83 E \cdot 05$
$1.34 E \cdot 01$
$1.07 E \cdot 07$
$4.73 E \cdot 04$
$1.86 E \cdot 05$
$1.50 E \cdot 02$
$1.66 E \cdot 01$
$1.10 E \cdot 02$
$5.17 E \cdot 01$
$3.00 E \cdot 03$
$5.68 E \cdot 04$
$7.51 E \cdot 06$
$2.73 E \cdot 06$

6.69E.01

1.28E.01

7.40 E. 02

B. 90 E.02

1.00E.02

5.70E-01

$1.03 E .01$

5.00E-02

6.00E-02

7.20E.02

8.00E.03

3.00E-03

3.20E-02

2.00E-03

1.00E-O

5.72E-04

1.10E-04

1.45E-06

TRUX31
TRUEXC
HWW GLASS
(O\% HEEL)

SLUD31
SWOWC
HWGLASS

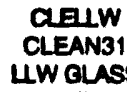

$5.29 E+0 B$

$1.94 E+07$

2.47E+07

14897

2. $30 E+0 B$

0.070

2.47E+08

149991

IWOUAS

(O\% HEEL)

$1.51 E+08$

5.24E+08

0.223

$6.74 E+08$

408378

78

$-14247$

$-149241$

$\begin{array}{lr}\mathrm{P2O5} & \mathrm{P2O5} \\ \mathrm{Fe2O3} & \mathrm{Fe2O3}\end{array}$

Fe203

Fo203

$\mathrm{F}=203$

Welght Fractions in Waste Oxide Stroam

1.82E-01

1.74E-04

2.24E-01

1.75E.06

4.00E.03

$1.39 \mathrm{E} \cdot 04$

4.30E.02

2.57E. 01

1.20E.02

2.77E-01

5.00E.03

4.70E.02

$5.49 \mathrm{E} \cdot 04$

6.00E-02
$4.54 E-04$
$1.05 E \cdot 01$
$2.65 E \cdot 06$
$1.20 E-02$
$4.62 E-04$
$6.70 E-02$
$3.21 E-01$
$4.30 E-02$
$3.92 E-01$
$1.50 E \cdot 02$
$1.42 E-01$
$2.00 E-03$
$6.77 E-05$

$7.00 E-03$

5.47E.05

$7.35 \mathrm{E}-01$

5.87E-07

$1.00 E-03$

$5.95 E-05$

$8.00 E-03$

$1.35 \mathrm{E}-01$

5.00E-03

1.10E-01

2.00E.03

$4.50 \mathrm{E}-02$

$1.10 \mathrm{E} .02$

B.16E.06

Weight Fractions in Glass Former Stream

6.09E.01
$1.15 E .01$
$1.64 E .01$
$3.00 E .02$
$7.60 E .02$
$7.00 E .03$

7.06E-01

1.39E-01

5.40E-02

$9.50 \mathrm{E} .02$

3.60E.02

5.90E.02

B.00E.03

Weight Fractions in Glass

$5.19 E-01$
$1.05 E-01$
$1.68 E-01$
$2.90 E-02$
$4.70 E-02$
$7.00 E-03$
$9.00 E \cdot 03$
$5.50 E-02$
$3.00 E-03$
$5.90 E-02$
$1.00 E-03$
$1.00 E-02$
$1.17 E-04$
$1.06 E-05$

5.70E-01
$1.07 E-01$
$1.59 E-01$
$2.80 E-02$
$7.10 E-02$
$7.00 E-03$
$5.00 E-03$
$2.30 E-02$
$3.00 E-03$
$2.80 E-02$
$1.00 E-03$
$1.00 E-02$
$1.35 E-04$
$4.77 E-06$

5.50E.01

$1.08 E .01$

1.64E.01

4.20E.02

$7.40 E \cdot 02$

$5.00 E-03$

2.00E.03

$3.00 \mathrm{E}-02$

1.00E-03

2. $40 E-02$

3.97E.04

$1.00 \mathrm{E} .02$

$3.00 E-03$

1.82E-06
3

TRULW
TRUEXC
UW GLASS
(O\% HEEL)

$1.52 E+08$

$4.97 E+0 B$

0.235

$6.49 \mathrm{E}+08$

383401

76

QLLWW
SLOWC
UWGLASS
(O\% HEEL)
$1.14 E+08$
$3.94 E+08$
0.225
$5.08 E+08$
307823

60

10

P2O5

Fo2O3

Na2O

$\mathrm{F} 92 \mathrm{O} 3$

$\begin{array}{ll}7.56 E-04 & 2.5 S E-04 \\ 4.81 E-05 & 2.81 E-06 \\ 8.49 E-01 & 8.87 E-01 \\ 5.20 E-07 & 3.6 B E-07 \\ 1.00 E-03 & 2.13 E-06 \\ 5.39 E-05 & 7.75 E-06 \\ 6.00 E-03 & 1.43 E-05 \\ 5.10 E-02 & 3.10 E-02 \\ 5.00 E-03 & 1.60 E-04 \\ \text { B.70E-02 } & 8.20 E-02 \\ 2.00 E-03 & 6.69 E-06 \\ 4.30 E-02 & 3.70 E .02 \\ 1.10 E-02 & 1.40 E-02 \\ \text { B.34E.06 } & 4.24 E-07\end{array}$

Gtass Propertles

PFoptrity

VISOOSTYY(PaS)

ELECT-CONDUCT(S/m)

DURAB.B.PCT (gm/m2/day)

DURAB.B-MCC $(g \mathrm{~m} / \mathrm{m} 2 / \mathrm{day})$

4.85
25.53
0.47

14.28
3.54

39.71

1.02
16.28

\subsection{66 \\ 1.01}

20.55
7.26E-01

1.33E-01

7.76E.04

3.60E-02

9.20E-02

1.30E.02

5.56E-01

1.02E-01

2. OOE.01

2.80E-02

$7.00 E .02$

$1.00 \mathrm{E}-02$

1.00E.03

1.20E.02

$1.00 E-03$

2.00E-02

3.70E-04

$1.00 E .02$

$3.00 E-03$
$1.40 E-06$

$3.00 E-03$
$1.40 E-06$
7.29E- 01 1.32E-01 3.00E-02

3.60E-02

9.10E.02

$1.20 E .02$

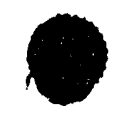

5.65E-01 1.02E.01

2.00E-01

2.80E-02

$7.00 E-02$

$1.00 \mathrm{E}-02$

3.23E-06

$7.00 \mathrm{E}-03$

3.60E-05

1.BOE-02

1.51E-06

8.00E-03

$3.00 E-03$

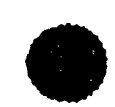


WHC-EP-0791

Table G6. Comparison of Glass Canister/Vault Results With and Without Specific Constraints.

\begin{tabular}{|c|c|c|c|c|c|c|}
\hline MODEL: & $\begin{array}{l}\text { CLEAN31 } \\
\text { CLEAN31 } \\
\text { HLW GLASS } \\
\text { (0\% HEEL) }\end{array}$ & $\begin{array}{l}\text { TRUX31 } \\
\text { TRUEX-C } \\
\text { HLW GLASS } \\
\text { (0\% HEEL) }\end{array}$ & $\begin{array}{l}\text { SLUD31 } \\
\text { SWDWC } \\
\text { HWW GLASS } \\
\text { (0\% HEEL) }\end{array}$ & $\begin{array}{l}\text { CLELLW } \\
\text { CLEAN31 } \\
\text { UW GLASS } \\
\text { (0\% HEEL) }\end{array}$ & $\begin{array}{l}\text { TRULWW } \\
\text { TREXXC } \\
\text { UW GLASS } \\
\text { (0\% HEEL) }\end{array}$ & $\begin{array}{l}\text { SLULLW } \\
\text { SUDWC } \\
\text { UW GLASS } \\
\text { (0\% HEEL) }\end{array}$ \\
\hline & \multicolumn{6}{|c|}{ With The P2O5 High Constraint } \\
\hline \multirow[t]{2}{*}{$\begin{array}{l}\text { KG GLASS } \\
\text { CANISTERS } \\
\text { VAULTS } \\
\% \text { LOAD }\end{array}$} & $\begin{array}{c}1.24 E+06 \\
750 \\
18.3\end{array}$ & $\begin{array}{c}2.47 E+07 \\
14897 \\
21.4\end{array}$ & $\begin{array}{c}2.47 E+08 \\
149991 \\
7.0\end{array}$ & $\begin{array}{c}6.74 E+08 \\
79 \\
22.3\end{array}$ & $\begin{array}{c}6.49 E+08 \\
76 \\
23.5\end{array}$ & $\begin{array}{c}5.08 E+08 \\
60 \\
22.5\end{array}$ \\
\hline & \multicolumn{6}{|c|}{ Without The P2O5 High Constraint } \\
\hline \multirow[t]{2}{*}{$\begin{array}{l}\text { KG GLASS } \\
\text { CANISTERS } \\
\text { VAULTS } \\
\% \text { LOAD }\end{array}$} & $\begin{array}{c}1.24 E+06 \\
750 \\
19.3\end{array}$ & $\begin{array}{c}1.47 E+07 \\
8880 \\
36.1\end{array}$ & $\begin{array}{c}6.84 E+07 \\
41450 \\
25.5\end{array}$ & $\begin{array}{c}5.53 E+08 \\
65 \\
27.2\end{array}$ & $\begin{array}{c}6.46 E+08 \\
76 \\
23.6\end{array}$ & $\begin{array}{c}5.08 E+08 \\
60 \\
22.5\end{array}$ \\
\hline & \multicolumn{6}{|c|}{ Without The NA2O High Constraint } \\
\hline \multirow[t]{2}{*}{$\begin{array}{l}\text { KG GLASS } \\
\text { VAULTS } \\
\% \text { LOAD }\end{array}$} & & & & & & $\begin{array}{c}4.71 E+08 \\
55 \\
24.3\end{array}$ \\
\hline & \multicolumn{6}{|c|}{ Without The OTH High Constraint } \\
\hline $\begin{array}{l}\text { KG GLASS } \\
\text { CANISTERS } \\
\% \text { LOAD }\end{array}$ & $\begin{array}{c}7.55 E+05 \\
457 \\
31.7\end{array}$ & & & & & \\
\hline \multicolumn{7}{|c|}{ Comparison of Results With And Without P2O5 Constraint } \\
\hline WITHOUT P2O5) & 1.00 & 1.69 & 3.62 & 1.22 & 1.00 & 1.00 \\
\hline $\begin{array}{r}\text { (WITHOUT P2OS/ } \\
\text { WITH P2O5) }\end{array}$ & 1.00 & 0.59 & 0.28 & 0.82 & 1.00 & 1.00 \\
\hline \% REDUCTION & 0.0 & 40.8 & 72.4 & 17.9 & 0.4 & 0.0 \\
\hline \multicolumn{7}{|l|}{ CLEAN/MODEL": } \\
\hline $\begin{array}{l}\text { WITH P2O5 } \\
\text { WTHOUT P2O5 }\end{array}$ & & $\begin{array}{l}0.05 \\
0.08\end{array}$ & $\begin{array}{l}0.01 \\
0.02\end{array}$ & & $\begin{array}{l}1.04 \\
0.86\end{array}$ & $\begin{array}{l}1.33 \\
1.09\end{array}$ \\
\hline \multicolumn{7}{|c|}{ (KG ULW GLASSKG HLW GLASS) } \\
\hline $\begin{array}{l}\text { WITH P2O5 } \\
\text { WITHOUT P2O5 }\end{array}$ & & & & $\begin{array}{l}544.39 \\
446.91\end{array}$ & $\begin{array}{l}26.23 \\
44.11\end{array}$ & $\begin{array}{l}2.05 \\
7.43\end{array}$ \\
\hline
\end{tabular}


WHC-EP-0791

This page intentionally left blank. 
WHC-EP-0791

\section{APPENDIX H}

ASPEN PLUS ${ }^{1}$ GLOBAL MODEL MASS BALANCE RESULTS

'ASPEN PLUS is a trademark of Aspen Technology, Inc.

$$
H-1
$$


WHC-EP-0791

\section{LIST OF TERMS}

CPU

HLW

LLW

TRUEX-C compact processing unit

high-level waste

low-level waste

Transuranic Extraction Option C 
WHC-EP-0791

\section{ASPEN PLUS GLOBAL MODEL MASS BALANCE RESULTS}

Table HI shows the stream mass balance and waste product summaries for each of the global model runs. Table H2 shows a comparison of the CLEAN Option. Transuranic Extraction Option C (TRUEX-C), and Sludge Wash C process strategies in terms of mass flows of the final waste product forms. Table H2 includes a breakdown of the waste products attributable to the primary waste feed stream processing and the tertiary waste processing. Also, sending the primary low-level waste (LLW) to a glass-in-sulfur product versus salt grout was considered for each process strategy (Appendix G).

As discussed in Appendix E, a large amount of tertiary waste is generated for each of the process strategies. With tertiary waste treatment, approximately $38 \%$ of the tertiary waste (S1OGRT, S3OCONC) is destined for grout, approximately $26 \%$ of the tertiary waste (S3OCOND) is destined for water treatment, and 36\% (S1OBUR, S2OASH) is destined for landfill burial. Note from Table $\mathrm{H} 2$ that the CLEAN Option process strategy produces a factor of 1.5 and 2.4 more tertiary waste than the TRUEX-C and Siudge Wash C process strategies, respectively, because of its more process-intensive strategy.

Using compact processing units (CPUS) to process the supernate and salt cake reduced the CLEAN Option tertiary waste generation by approximately $29 \%$. This reduction was estimated by processing only the sludge portion of the tank waste (i.e., approximately 36 and $4 \%$ of the single- and double-shell tank waste, respectively) through a central processing facility.

Figure HI shows the LLW vault requirements for both primary and tertiary waste. Note that the primary LLW-to-glass option required only $30 \%$ of the vaults that the LLW sait grout option required. Also, note that the CLEAN Option tertiary LLW required nearly the same number of salt grout vaults as the primary LLW. Using CPUS, the CLEAN Option tertiary LLW waste required only $73 \%$ of the sait grout vaults as the primary LLW. The TRUEX-C and Sludge Wash $C$ process strategies had tertiary grout vault requirements that were approximately 57 and $50 \%$ of those associated with primary $L L W$, respectively.

It was interesting to note that while the amount of primary LLW reporting to the salt grout facility was lower for the CLEAN Option than for the Sludge Wash $C$ process strategy, the grouting process required more chemical adds for adjustment of sodium concentration, resulting in a larger grout product volume for the CLEAN Option.

As shown in Table $\mathrm{H} 2$, in terms of kilograms of waste product produced per primary waste kilogram processed, the primary $L L W-t o-g l a s s$ option production ratios were $0.73,0.85$, and 0.67 for the CLEAN Option, TRUEX-C, and Siudge Wash C process strategies, respectively. Thus, the CLEAN Option process strategy produces $14 \%$ less primary LLW glass than the TRUEX-C process strategy and $9 \%$ more than the sludge Wash C process strategy. The primary LLW-to-sait grout option production ratios were $2.58,3.04$, and 2.17 for the CLEAN Option, TRUEX-C, and Sludge Wash $C$ process strategies, respectively. Thus, the CLEAN Option process strategy produces 15\% less primary LLW salt grout than the TRUEX-C process strategy and $19 \%$ more than the Siudge Wash C process strategy. 
For the high-level waste (HLW) stream, note the significant reduction of HLW glass canisters for the CLEAN Option. The CLEAN Option process strategy produced only 8 and $2 \%$ of the HLW glass as the TRUEX-C and sludge Wash $C$ process strategies, respectively. In terms of kilograms of waste product produced per primary waste kilograms processed, the HLW glass production ratios were $0.002,0.019$, and 0.090 for the CLEAN Option, TRUEX-C, and Siudge Wash C process strategies, respectively.

Note that the LLW and $H L W$ glass production results are based on the glass composition optimization results discussed in Appendix $G$. Relaxation of key glass component constraints in the optimization process can have a significant impact on the glass production results shown in Tables $H I$ and $H 2$.

Note that all process strategles assumed that a $0 \%$ heel (1.e., the unretrieved waste) was left in the waste tanks. Generally, If a $5 \%$ heel was assumed, the process strategy would decrease the HLW glass product by 4 to $5 \%$ as compared to the o\% heel case, while decreasing the LLW product by $2 \%$ to $3 \%$ (Knutson et al. 1993). There would be an associated major increase of the amount of waste resident in the dome-fill-tank product stream (3200HLWS) when a 5\% tank waste heel is assumed for the process strategies.

The conversion factor for $\mathrm{glass}$ canisters is based on the $60-\mathrm{cm}-(2-\mathrm{ft}-)$ diameter by $317-\mathrm{cm}-(10-\mathrm{ft}-)$ long canister ( $\mathrm{g} 1$ ass volume of $0.625 \mathrm{~m}^{3}$ )

(Boomer et al. 1993). The conversion façtor for grout vaults is based on a vault capacity of $1.4 \mathrm{E}+6 \mathrm{gal}\left(5.3 \mathrm{E}+3 \mathrm{~m}^{3}\right)$, with a grout density of $1.6 \mathrm{~g} / \mathrm{cc}$ (1.e., $8.48 \mathrm{E}+6 \mathrm{~kg}$ of salt grout/vault). The conversion factor for LLW glassin-sulfur vaults of $8.518 \mathrm{E}+6 \mathrm{~kg}$ glass/vault is based on the same vault capacity.

Table $\mathrm{H} 3$ contains the detailed ASPEN PLUS global model mass balance output results showing the model stream mass (kilograms) and curie ( $\mathrm{C}$ ) flows, separated as input, output, and all streams.

\section{REFERENCES}

Boomer, K. D., et al., 1993, Tank Waste Technical Options Report, WHC-EP-0616, Rev. 0 , Westinghouse Hanford Company, Richland, Washington.

Knutson, B. J., et al., 1993, "Advanced Chemical Separation Study: TRUEX Strategy Compared To Sludge Wash Ion Exchange," Letter report, Westinghouse Hanford Company, Richland, Washington. 


$$
\text { WHC-EP-0791 }
$$

Figure HI. Low-Level Waste Vault Requirements for Primary and Pert lacy Waste.

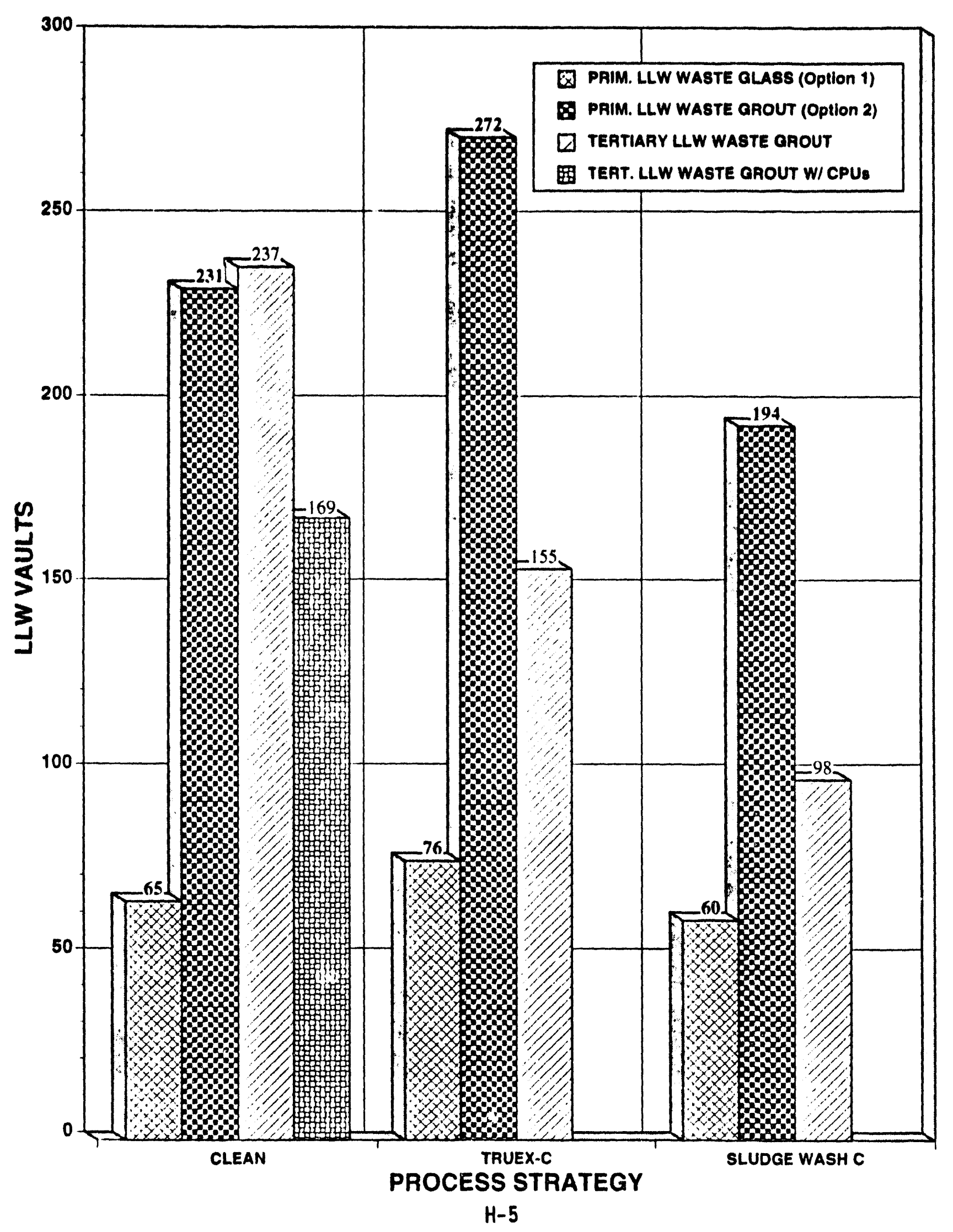


Table Hl. Stream Mass Balance and Waste Product Summaries.

SUMMARY OF PRODUCT STREAM MASS FLOWS $(\mathrm{kg})$

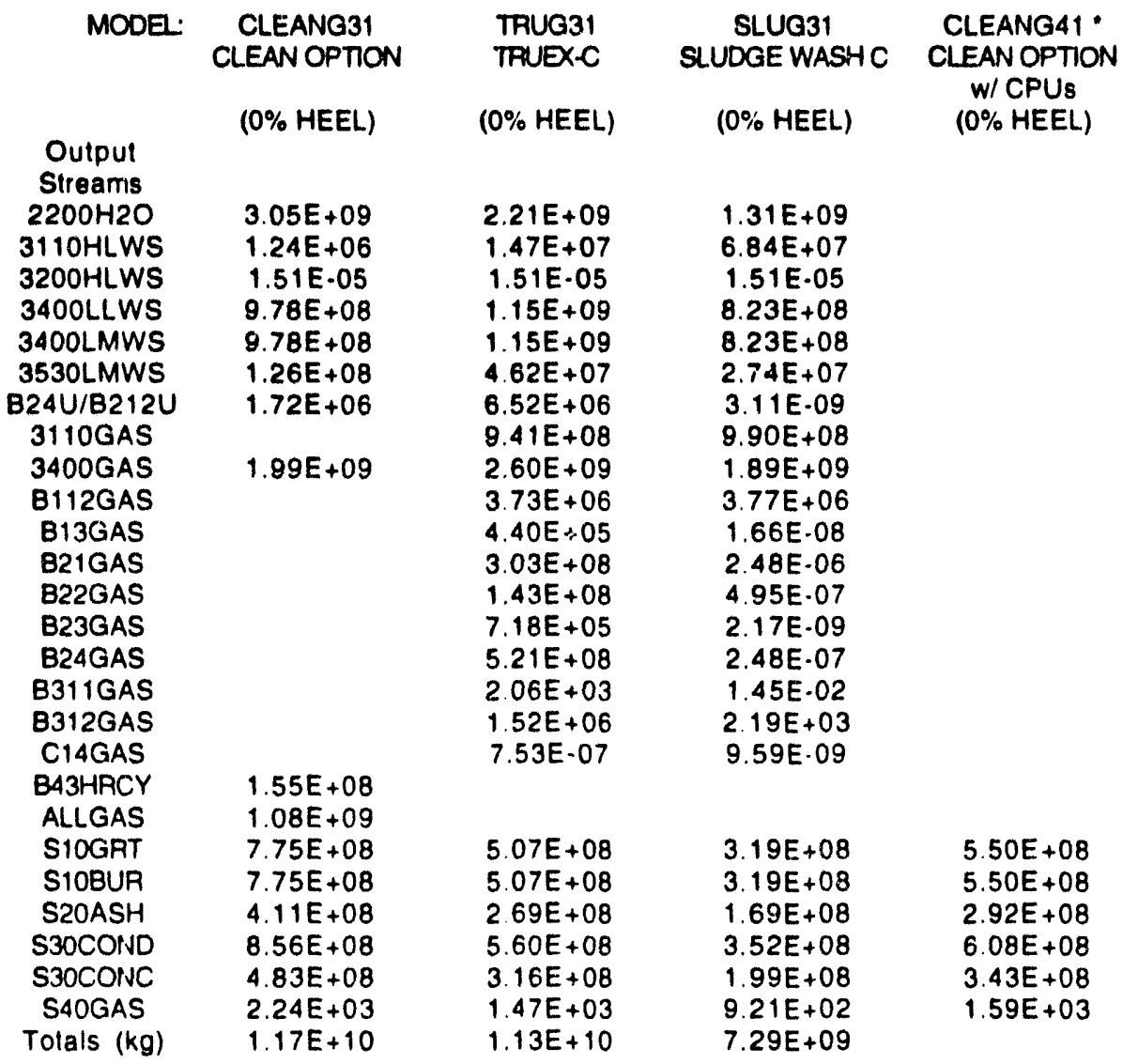

- CLEANG41 MODEL SHOWS THE EFFECT ON TERTIARY WASTE GENERATION WHEN PROCESSING ONLY TANK SLUDGE IN A CENTRAL PROCESSING FACILITY 
Table H2. Waste Product Summaries and Comparisons.

\section{WASTE FORM}

INITIAL PRIMARY WASTE FEED (kg)

HLW GLASS $(\mathrm{kg})$

LLW GLASS (kg) (Option 1)

LLW SALT-GROUT (kg) (Option 2)

WATER TREATMENT IX RESIN (kg)

DOME-FILL-TNKS $(\mathrm{kg})$

URANIUM PROD $(\mathrm{kg})$

$\mathrm{H} 2 \mathrm{O}(\mathrm{kg})$

$\mathrm{GAS}+(\mathrm{kg})$

TERTIARY WASTE TO WATER TREATMENT $(\mathrm{kg})$

TERT WASTE TO GROUT(NO CEMENT A.DD) (kg)

TERTIARY WASTE TO LANDFILL ( $\mathrm{kg}$ )

TERTIARY WASTE SALT GROUT* $(\mathrm{kg})$

('INCLUDES 1.6 FACTOR FOR CEMENT ADD)

TOTAL GENERATED TERTIARY WASTE $(\mathrm{kg})$ TERTW TO WATER TREATMENT : TOT TERTW TERTW TO LANDFILL(BUR) / TOT TERTW TERTW TO GROUT / TOT TERTW

TOT TERT WASTE / INIT PRIM WASTE

HLW GLASS / INIT PRIM FEED

LLW GLASS (Option 1) / INIT PRIM FEED

LLW SALT GROUT (Option 2) / INIT PRIM FEED

TERW GROUT / INIT PRIM FEED

ALL GROUT / INIT PRIM FEED

CLEAN/"MODEL" (TOT TERT WASTE)

CLEAN/"MODEL" (HLW GLASS)

CLEAN/"MODEL" (LLW GLASS)

CLEAN/"MODEL" (SALT-GROUT)

CLEAN"MODEL" (TERT-GROUT)

CLEAN/"MODEL" (ALL-GROUT)

HLW GLASS CANISTERS **

LLW GLASS VAULTS $\cdots$ (Option 1)

LLW GROUT VAULTS .... (Option 2)

LLW GLASS VAULTS / LLW GROUT VAULTS

TERT WASTE VAULTS ...*

GROUT VAULT RATIO: TERTIPRIM

SUMMARY OF PRODUCT MASS FLOW $(\mathrm{kg})$ BY WASTE FORM

\section{MODEL:}

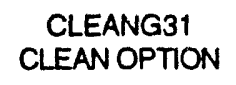

(0\% HEEL)

$7.57 E+08$
$1.24 E+06$
$5.53 E+08$
$1.96 E+09$
$1.26 E+08$
$1.51 E-05$
$1.72 E+06$
$3.05 E+09$
$3.07 E+09$

$8.56 E+08$
$1.26 E+09$
$1.19 E+09$
$2.01 E+09$

3. $30 E+09$
0.26

0.36

0.38

4.36

0.002

0.73

2.58

2.66

5.24

\section{TRUG31 \\ TRUEX-C}

(0\% HEEL)

$7.57 E+08$

$1.47 E+07$

$6.46 \mathrm{E}+08$

2.30E+09

$4.62 E+07$

$1.51 E-05$

$6.52 E+06$

2.21E+09

$4.52 E+09$

$5.60 E+08$

8. $24 E+08$

$7.76 E+08$

$1.32 E+09$

\section{2. $16 E+09$}

0.26

0.36

0.38

2.85

0.019

0.85

3.04

1.74

4.78

1.53

0.08

0.86

0.85

1.53

1.10

8880

76

272
0.28

155

0.57

\section{SLUG31 SLUDGE WASHC}

(0\% HEEL)

$7.57 E+08$

$6.84 E+07$

$5.08 E+08$

1.65E+09

2.74E+07

1.51E-05

3.11E-09

1.31E+09

2. $88 E+09$

$3.52 E+08$

$5.18 E+08$

$4.88 E+08$

8.29E+08

1.36E+09

0.26

0.36

0.38

1.79

0.090

0.67

2.17

1.09

3.27

2.43

0.02

1.09

1.19

2.43

1.60

41450

60

194

0.31

98

0.50
CLEANG41 * CLEAN OPTION W/ CPUS ( $0 \%$ HEEL)

$7.57 E+08$
$6.08 E+08$

$8.94 E+08$

$8.42 E+08$

$1.43 E+09$

2.34E+09

0.26

0.36

0.38

3.10

1.89

4.47

1.41

1.41

1.17

237

1.03

- CLEANG41 MODEL SHOWS THE EFFECT ON TERTIARY WASTE GENERATION WHEN PROCESSING ONLY TANK SLULGE IN A CENTRAL PROCESSING FACILITY

- GLASS CANISTERS (1650 KG/CANISTER)

*.. GLASS VAULTS (8.518E6 KGNAULT)

... GROUT VAULTS (8.48E6 KGNAULT) 


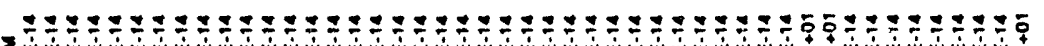

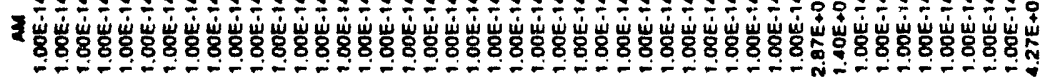

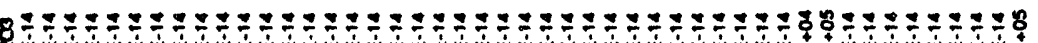

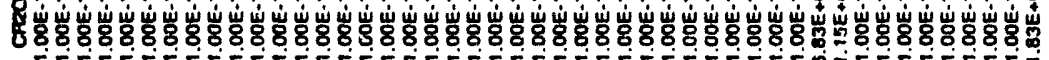

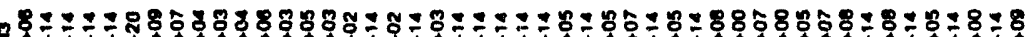

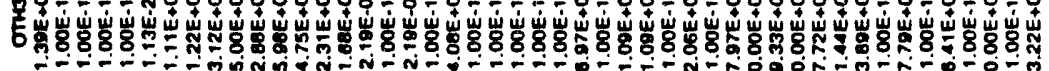

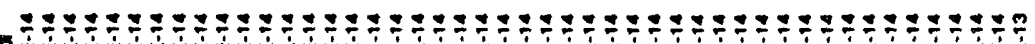

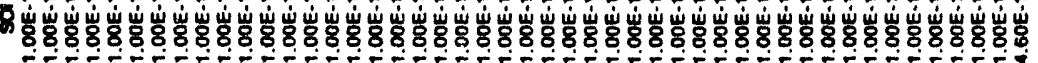

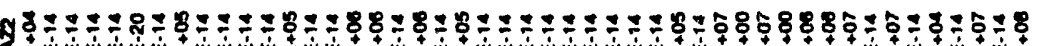

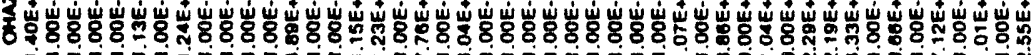

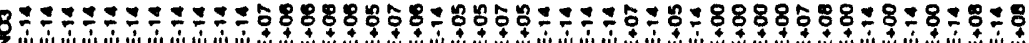

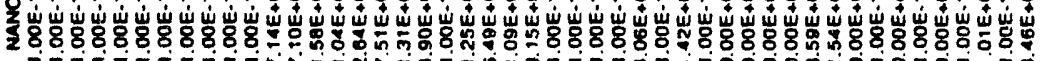

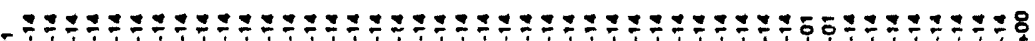

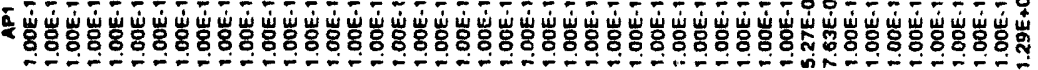

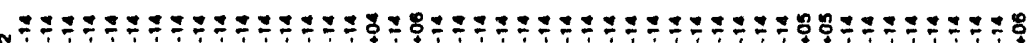

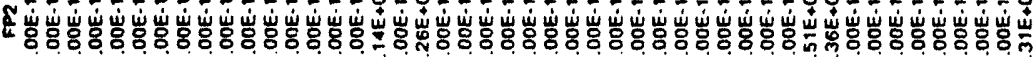

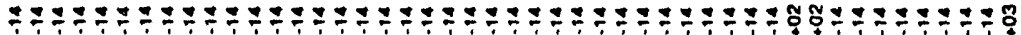

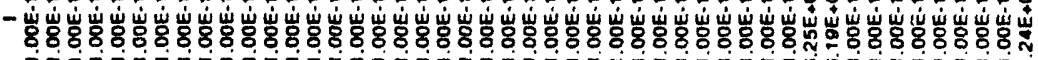

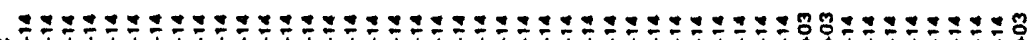

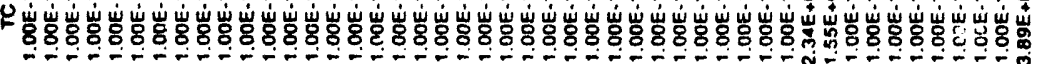

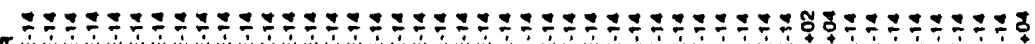

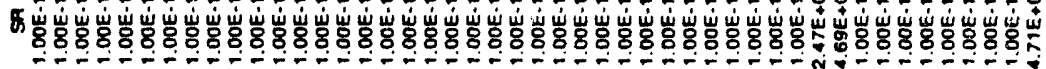

8

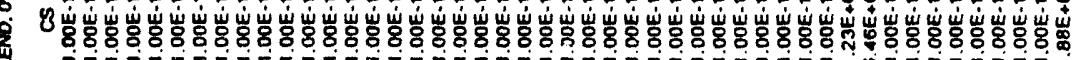

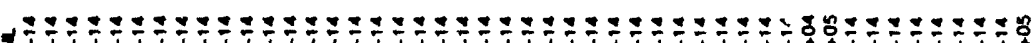

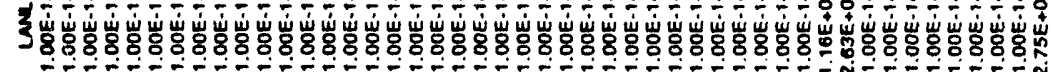

8

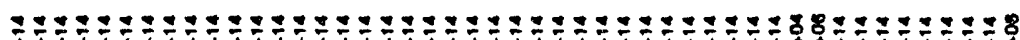

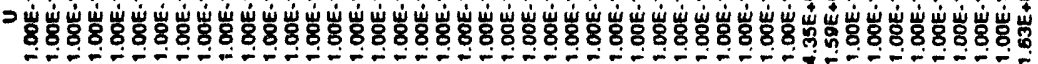

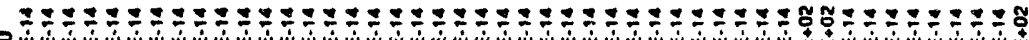

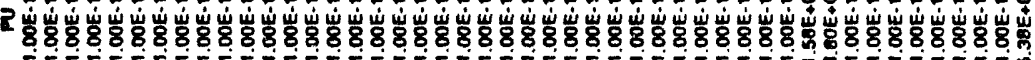

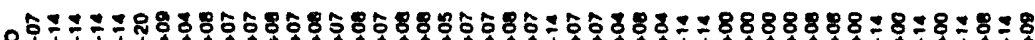

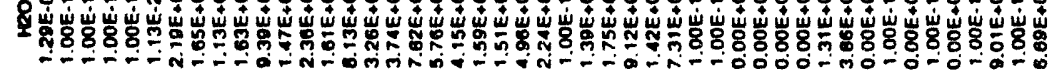

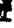

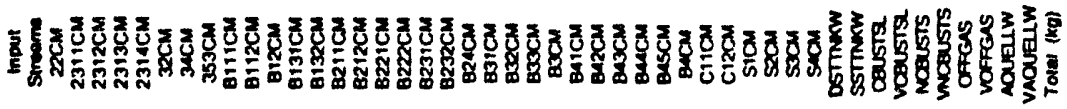

$80 \% 7 \% \div 8888888888$

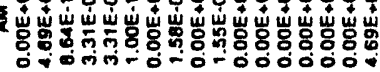

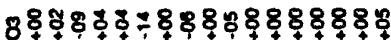

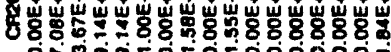

ำ

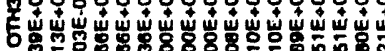

$88 \div 888898 \% 888888 \%$

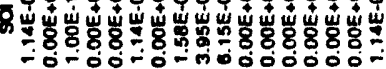

충ํำ

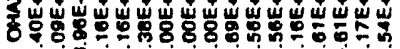

8 ช

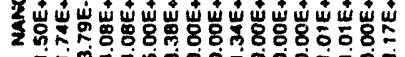

-

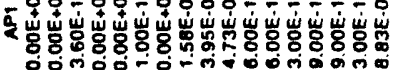

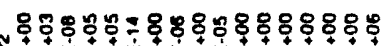

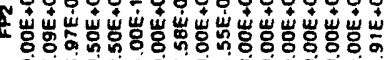
우우ำ

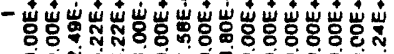

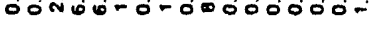

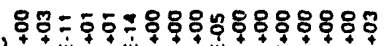

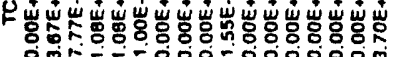
$88088 \div 888 \% 888888$

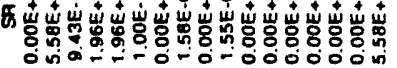
$8 \%$

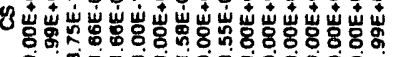

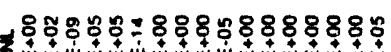

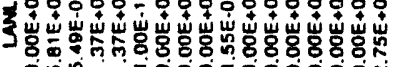

890 >

$8 \%$

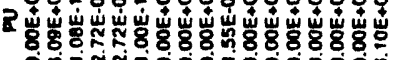

$88 \% 8055080888 \% 58$

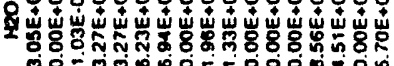

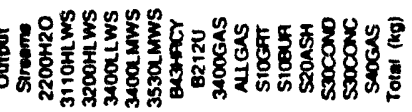

H-8 


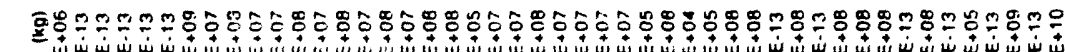

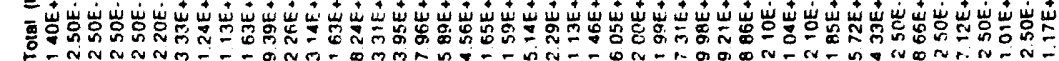

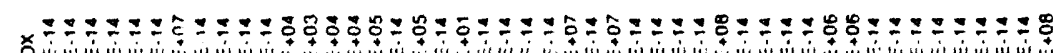

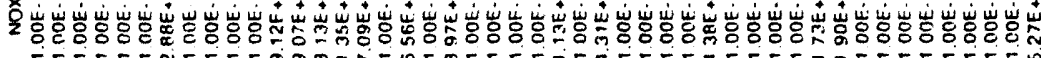

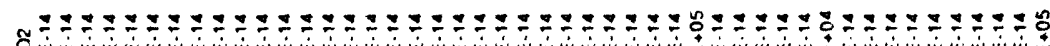

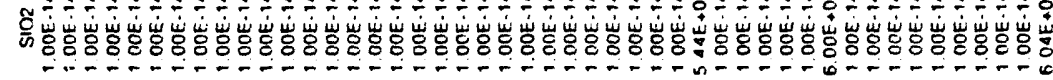

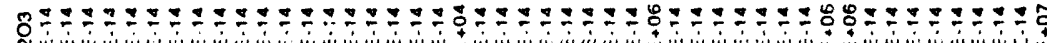

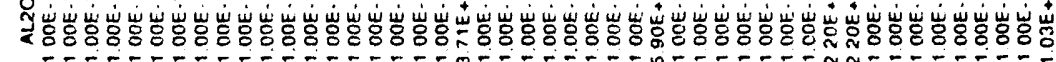
要

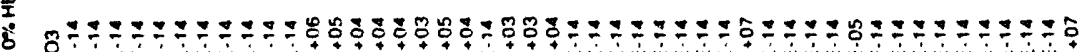

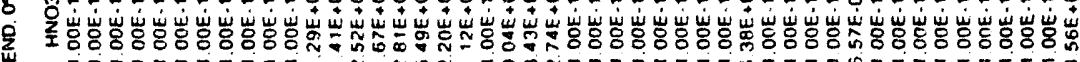
岩

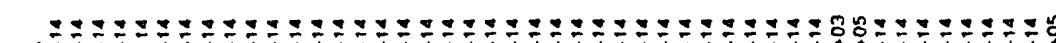
×

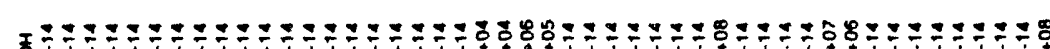

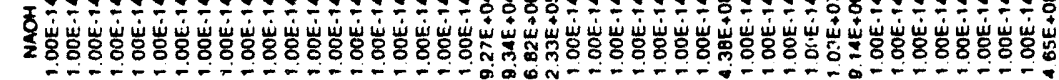
年

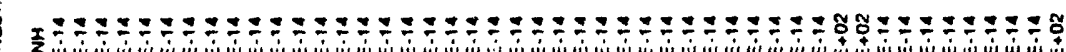

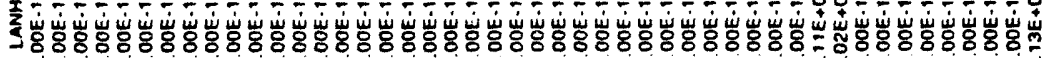

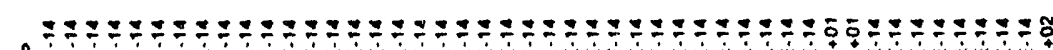

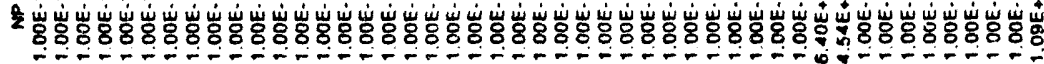

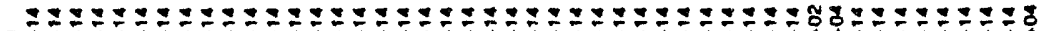

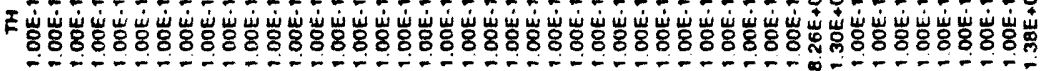

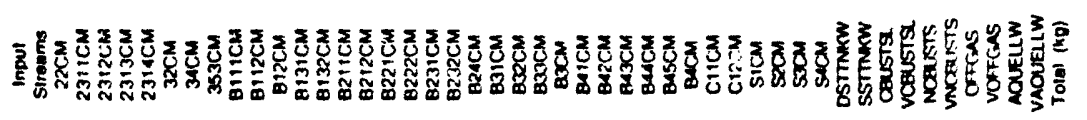

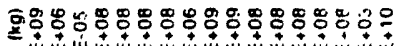

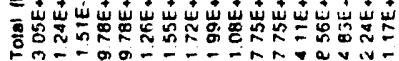

×8

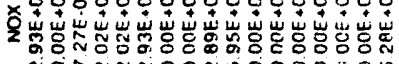

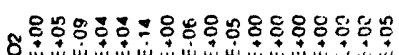

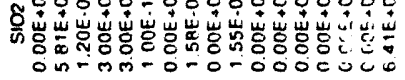

80

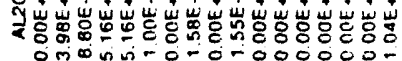

80ْ

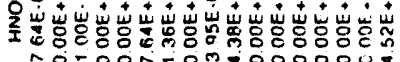

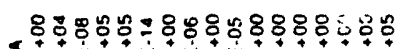
ఐ

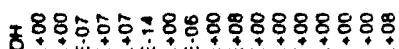

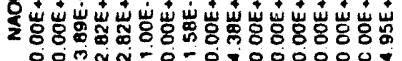

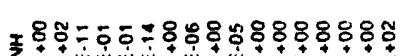

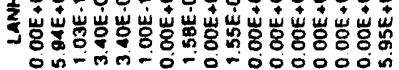
8

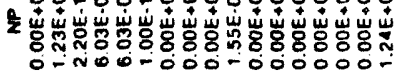

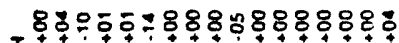

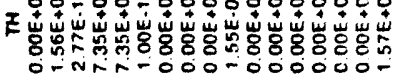

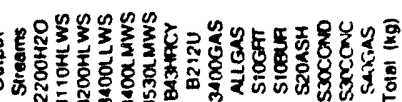


$O I-H$

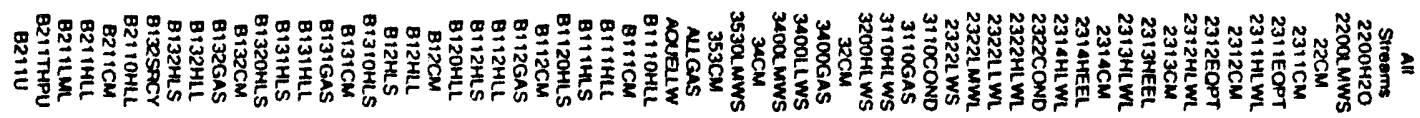

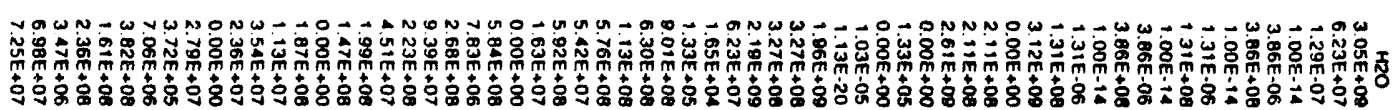

nu

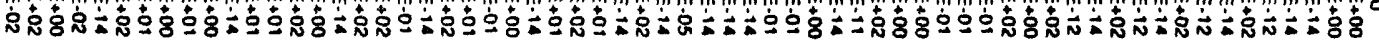

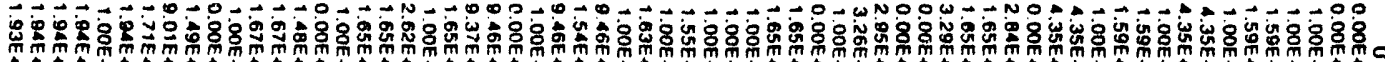

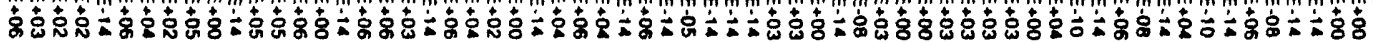

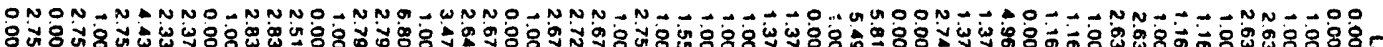

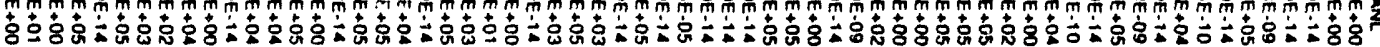

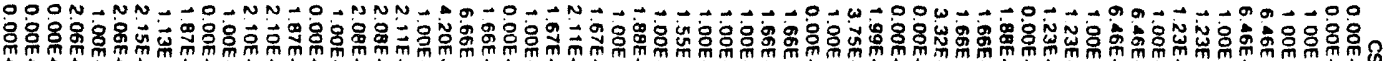

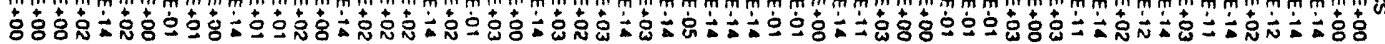

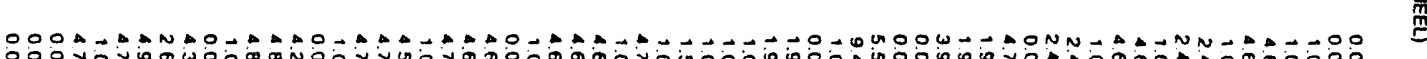

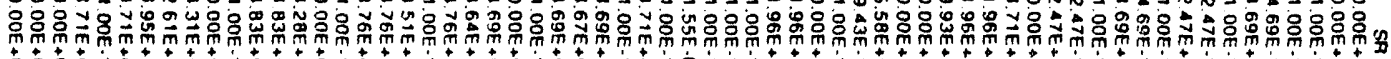

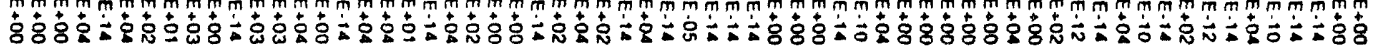

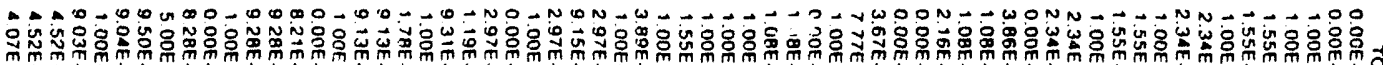

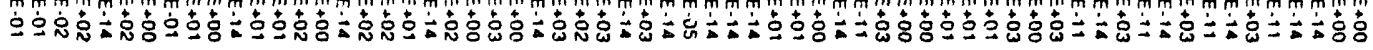

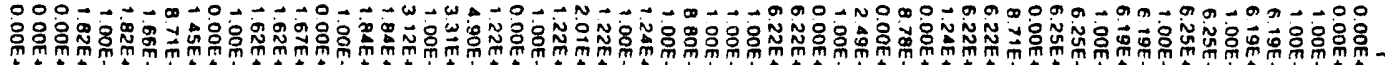

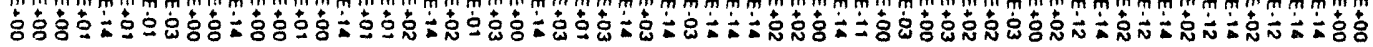

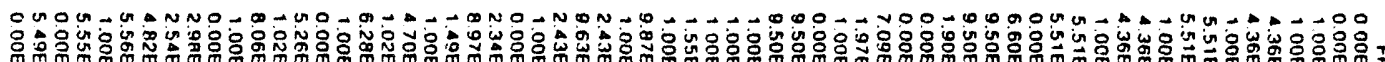

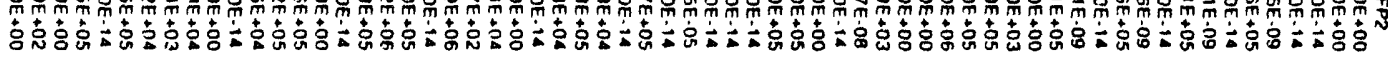

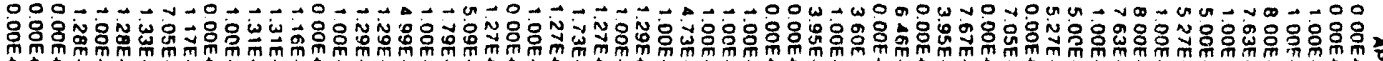

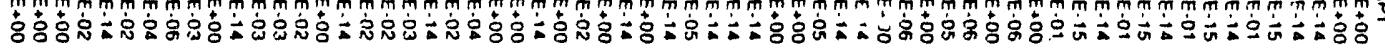

PNN -

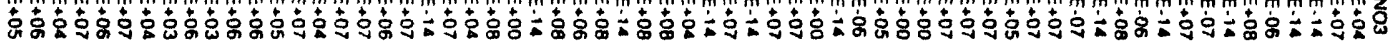

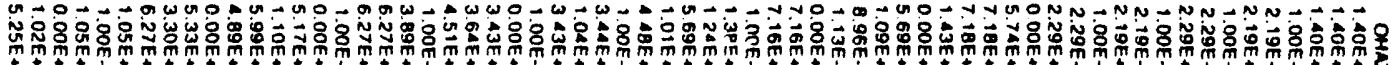

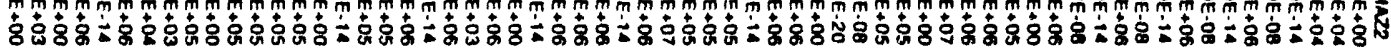

웅휴

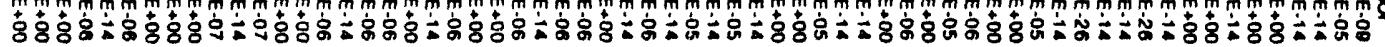

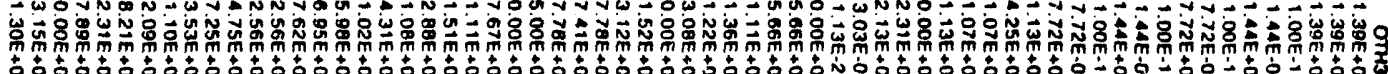

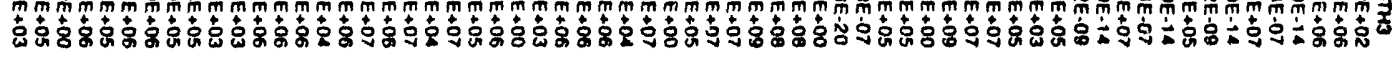

8े

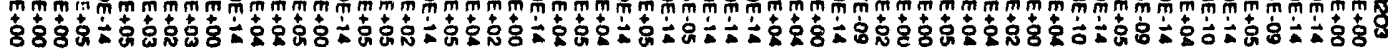

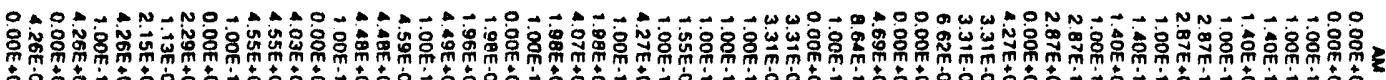

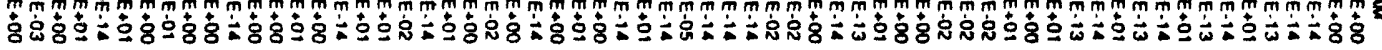


Table H3. ASPEN PLUS Global Model Mass Balance Output Results. (sheet 4 of 56)

\begin{tabular}{|c|c|c|c|c|c|c|c|c|c|c|}
\hline \\
\hline & & & & . & & 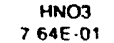 & $\begin{array}{l}A .030 \\
0.060 .00\end{array}$ & $\begin{aligned} 502 \\
0.00\end{aligned}$ & 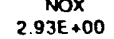 & 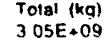 \\
\hline & 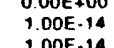 & 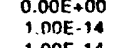 & 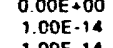 & 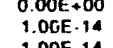 & 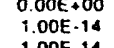 & 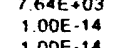 & 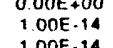 & 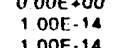 & & \\
\hline & 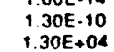 & 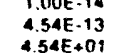 & 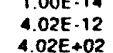 & 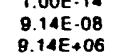 & $\begin{array}{l}5.00 \\
5.00\end{array}$ & 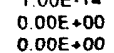 & $\begin{array}{l}2.200=08 \\
2.2060\end{array}$ & 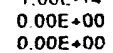 & 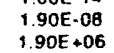 & 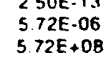 \\
\hline COAPT & 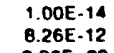 & 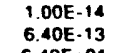 & $\begin{array}{l}1.006-14 \\
1.1 .1 E=-12\end{array}$ & $\begin{array}{l}0.060-14 \\
1.03 E-07\end{array}$ & 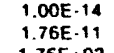 & 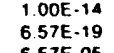 & 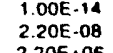 & 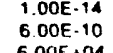 & 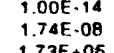 & 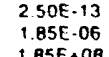 \\
\hline & & $\begin{array}{l}0.00-14 \\
4.54-14\end{array}$ & 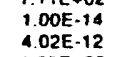 & i. $1.006-14$ & 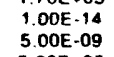 & & & & 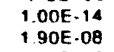 & \\
\hline & & & & & 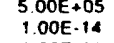 & & & & & \\
\hline & $=02$ & 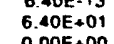 & 1 & $\begin{array}{l}0.036-0.07 \\
0.035=07\end{array}$ & $\begin{aligned} 7,6 E \\
\end{aligned}$ & 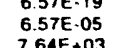 & 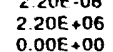 & 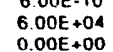 & 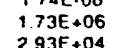 & \\
\hline & 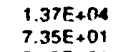 & 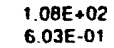 & 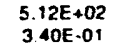 & 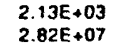 & 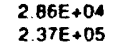 & 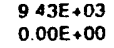 & 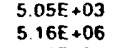 & 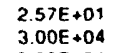 & 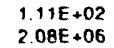 & \\
\hline 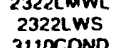 & 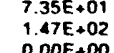 & 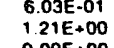 & 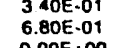 & 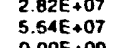 & 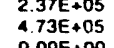 & $\begin{array}{l}7.656-05 \\
3.955\end{array}$ & 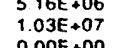 & 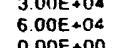 & 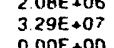 & \\
\hline 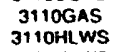 & $\begin{array}{l}0.000+00 \\
1.565=0\end{array}$ & 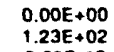 & 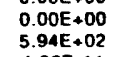 & 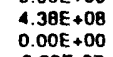 & 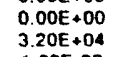 & 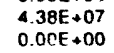 & 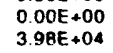 & 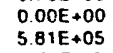 & 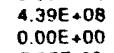 & \\
\hline$w_{4}$ & 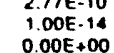 & 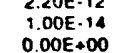 & 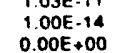 & 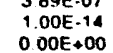 & 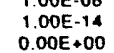 & $\begin{array}{l}1,05-14 \\
395 E-0.04\end{array}$ & 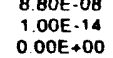 & 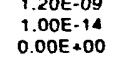 & $\begin{aligned} 7.276 \\
1.006 \\
28095\end{aligned}$ & \\
\hline ¿ws & 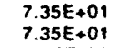 & $\begin{aligned} 6.033-01 \\
6.03 E-0.01\end{aligned}$ & $\begin{array}{l}3.400=01 \\
3.40=-01\end{array}$ & 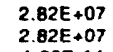 & $\begin{array}{l}2.37 F E+05 \\
2.37 t+05\end{array}$ & $\begin{array}{l}0.0 \\
0.0\end{array}$ & 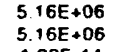 & $\begin{array}{l}3.006 \\
3.000\end{array}$ & $\begin{array}{l}2.026 \\
2.0226\end{array}$ & \\
\hline & & $\begin{array}{l}1.000 \\
1.000 \\
1.00\end{array}$ & 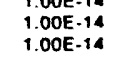 & $\begin{array}{l}.006-14 \\
1.006 E-14\end{array}$ & 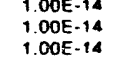 & 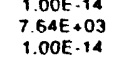 & & & & \\
\hline $\begin{array}{l}\text { tant } \\
\text { tatum }\end{array}$ & & $\begin{array}{l}1.555-05 \\
i .00=-14\end{array}$ & 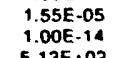 & 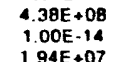 & $\begin{array}{l}1.55 E-.05 \\
1.000-14\end{array}$ & 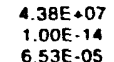 & 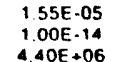 & 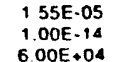 & 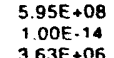 & \\
\hline & $.636+0$ & 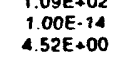 & 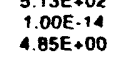 & 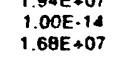 & 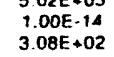 & 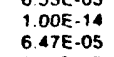 & $\begin{array}{l}\text { i. } \\
2005 \\
2.565\end{array}$ & $\begin{array}{l}.006 \\
1.0454\end{array}$ & & \\
\hline totus & 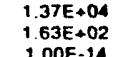 & 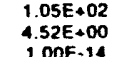 & 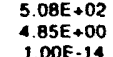 & 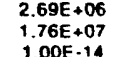 & 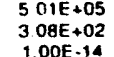 & 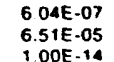 & 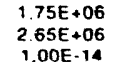 & & & \\
\hline SWS & & 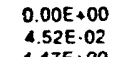 & 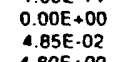 & $\begin{array}{l}0.006+00 \\
1.60=5\end{array}$ & 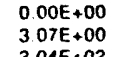 & 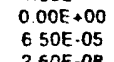 & 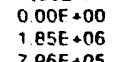 & 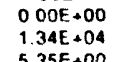 & $\begin{array}{l}3.554 \\
2.64 \\
2.64\end{array}$ & $\begin{array}{l}3.93 \\
7.99 \\
.096\end{array}$ \\
\hline thL & & 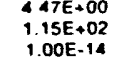 & 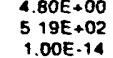 & 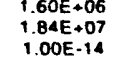 & 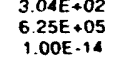 & & $\begin{array}{l}7.96 \\
5.2636 \\
1.060\end{array}$ & & & \\
\hline 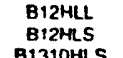 & & 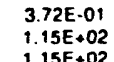 & 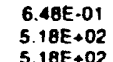 & 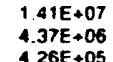 & 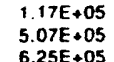 & 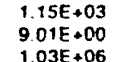 & 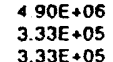 & & & \\
\hline 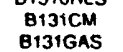 & 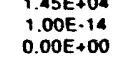 & 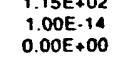 & 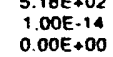 & 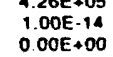 & 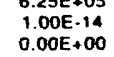 & & & $\begin{array}{l}0.065 \\
0.005\end{array}$ & & \\
\hline & & 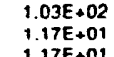 & 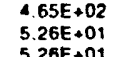 & & & & & & & \\
\hline & $\begin{array}{l}0.0 \\
0.0 \\
0.0\end{array}$ & 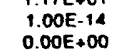 & 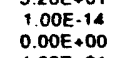 & $\begin{array}{l}0.000 .14 \\
0.006-100\end{array}$ & $\begin{array}{l}1.006-14 \\
0.006-10 \\
0.006\end{array}$ & 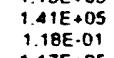 & & $\begin{array}{l}0.000 E \\
0.00 E\end{array}$ & $\begin{array}{l}90.03 \\
1.96=0\end{array}$ & \\
\hline & $\begin{array}{l}7.4 \\
3667\end{array}$ & 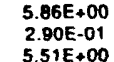 & 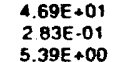 & 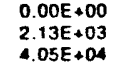 & & & & & & \\
\hline & & & & & & & & & & \\
\hline & & & 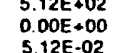 & 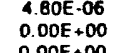 & & & & $\begin{array}{l}4.666 \\
0.000\end{array}$ & & \\
\hline & & & & & & & & & & \\
\hline
\end{tabular}




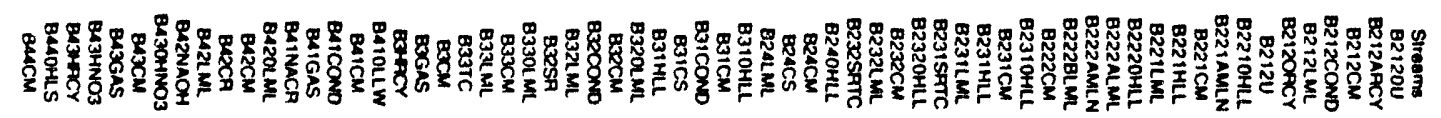

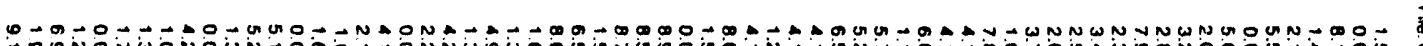

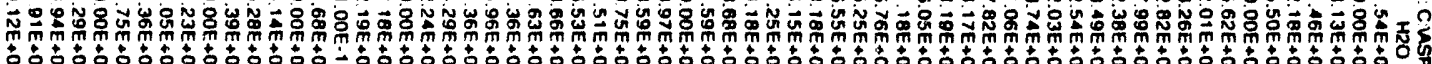

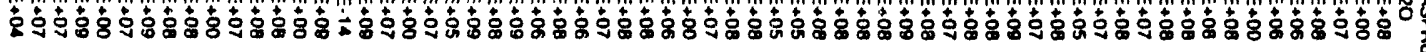

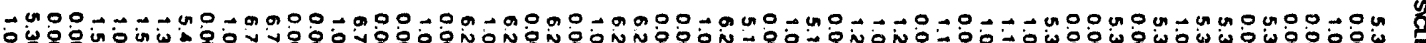

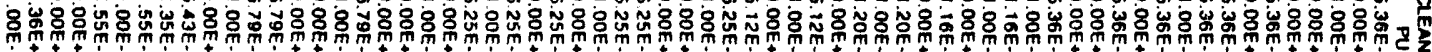

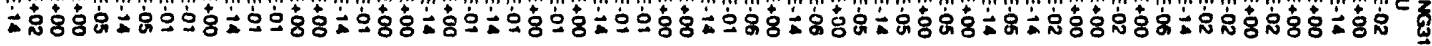

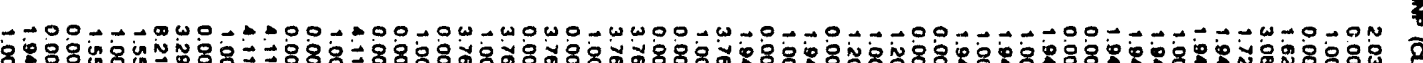

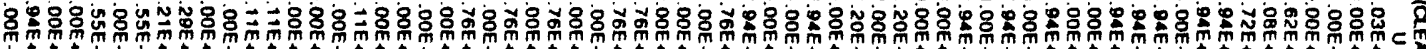

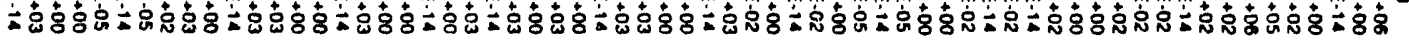

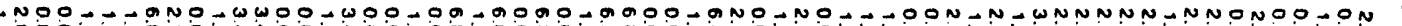

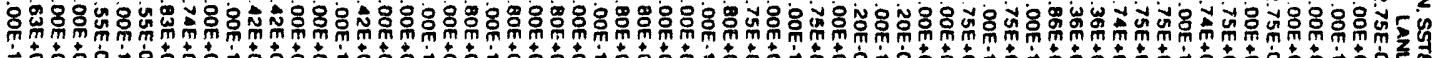

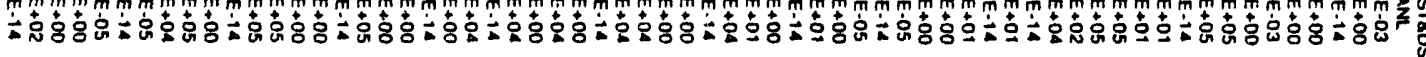

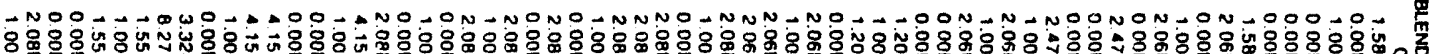

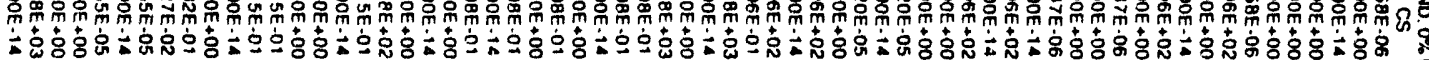

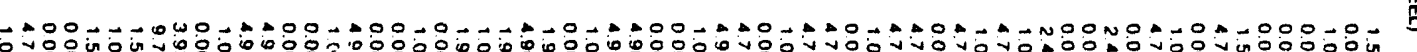

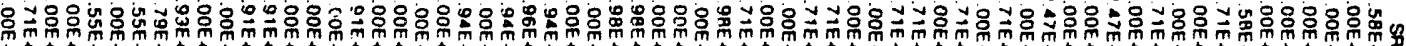

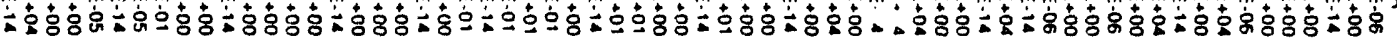

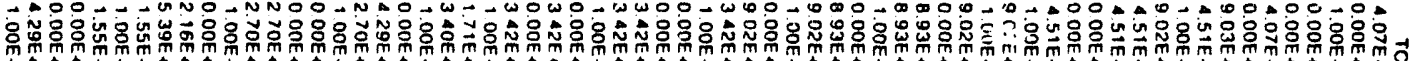

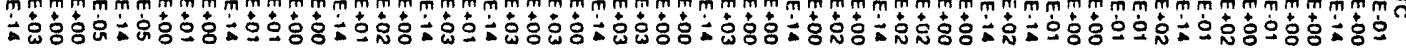

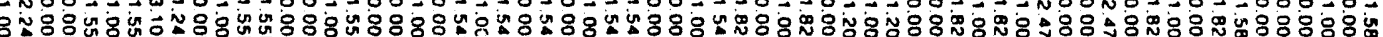

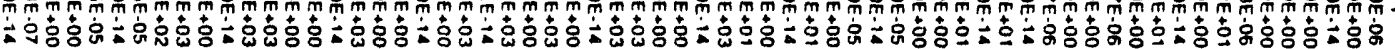

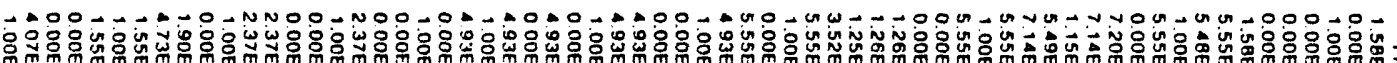

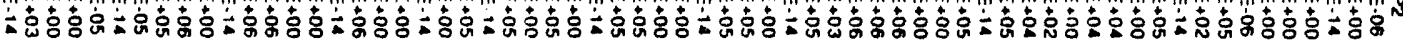

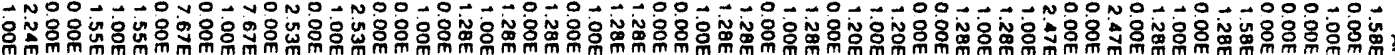

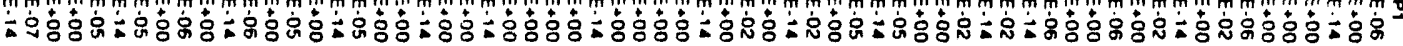

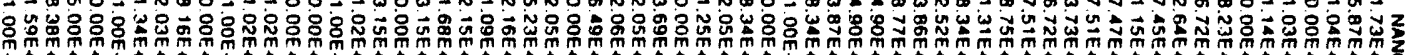

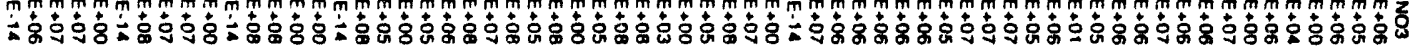

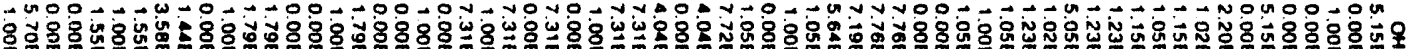

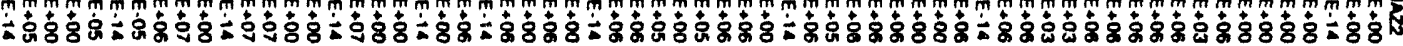

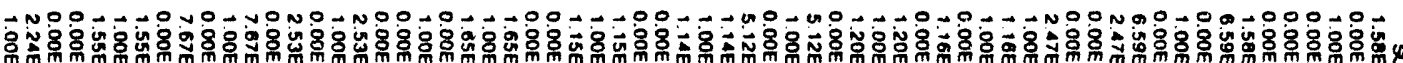

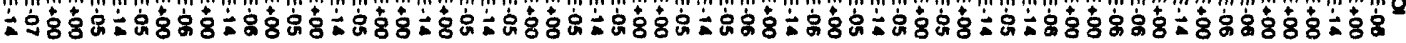

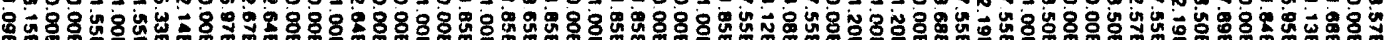

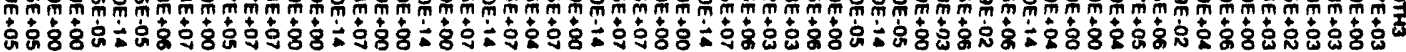

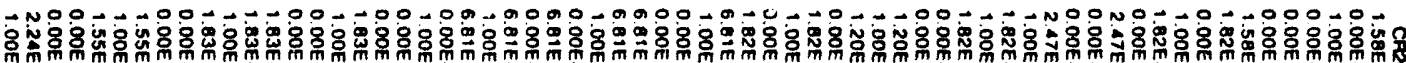

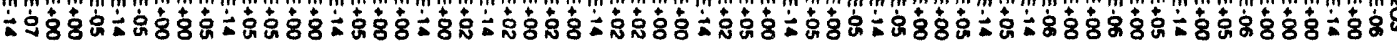

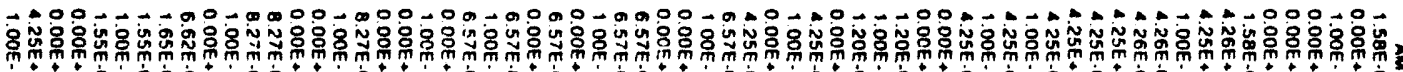

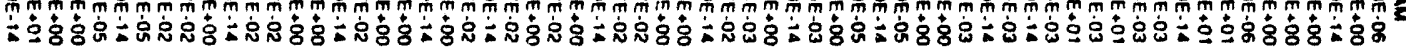


Table H3. ASPEN PLUS Global Model Mass Balance Output Results. (sheet 6 of 56)

\begin{tabular}{|c|c|c|c|c|c|c|c|c|c|c|}
\hline 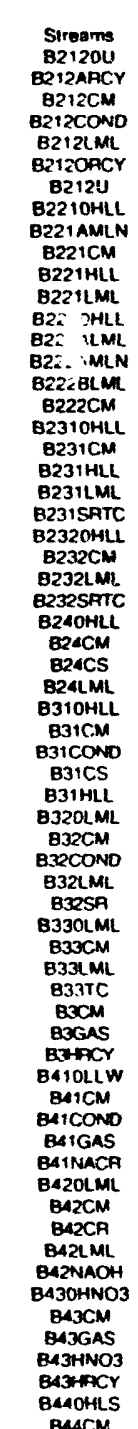 & 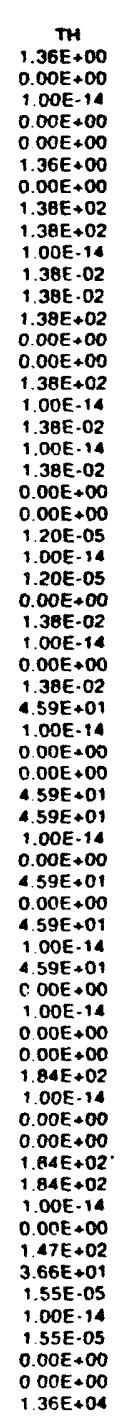 & 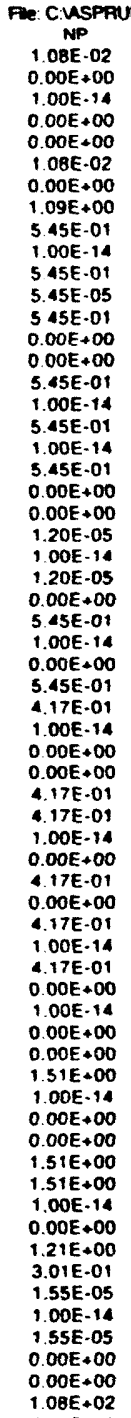 & 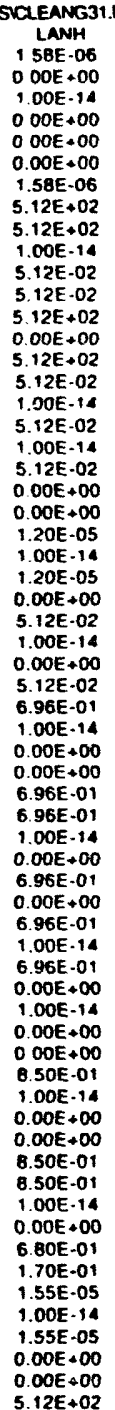 & 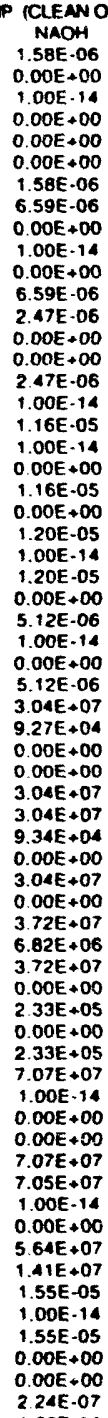 & 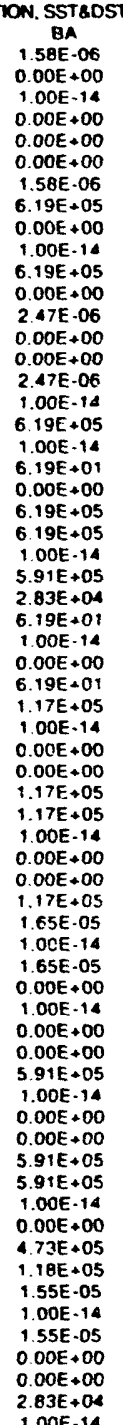 & 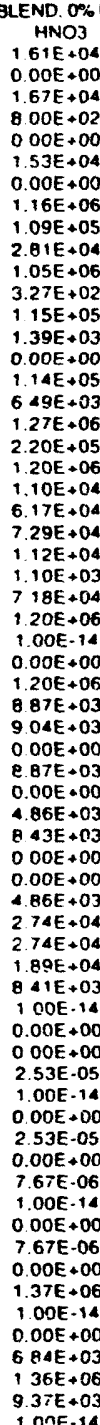 & 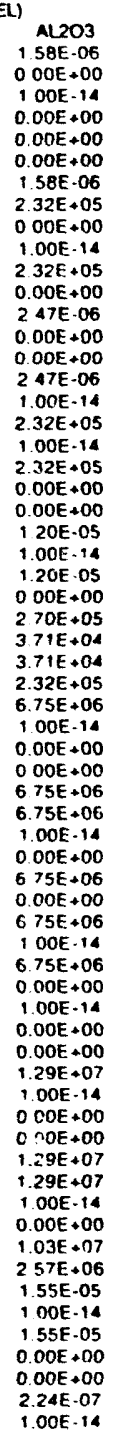 & 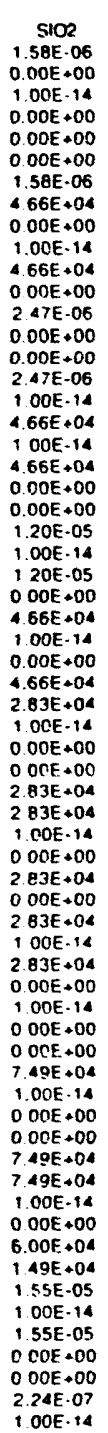 & 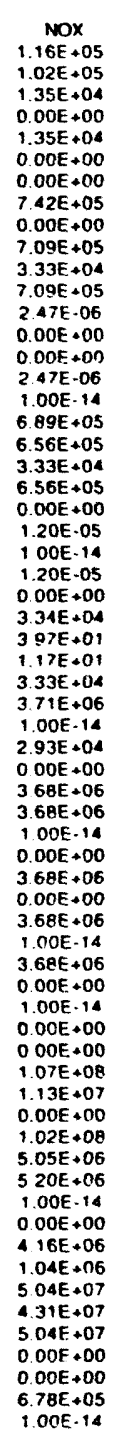 & 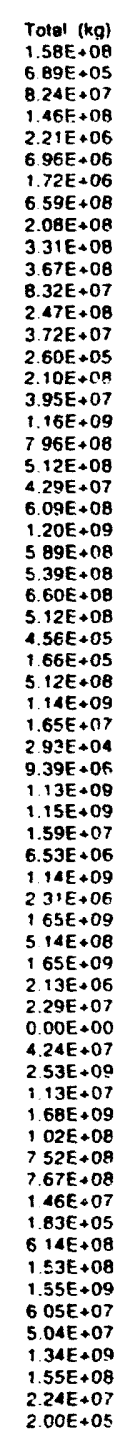 \\
\hline
\end{tabular}




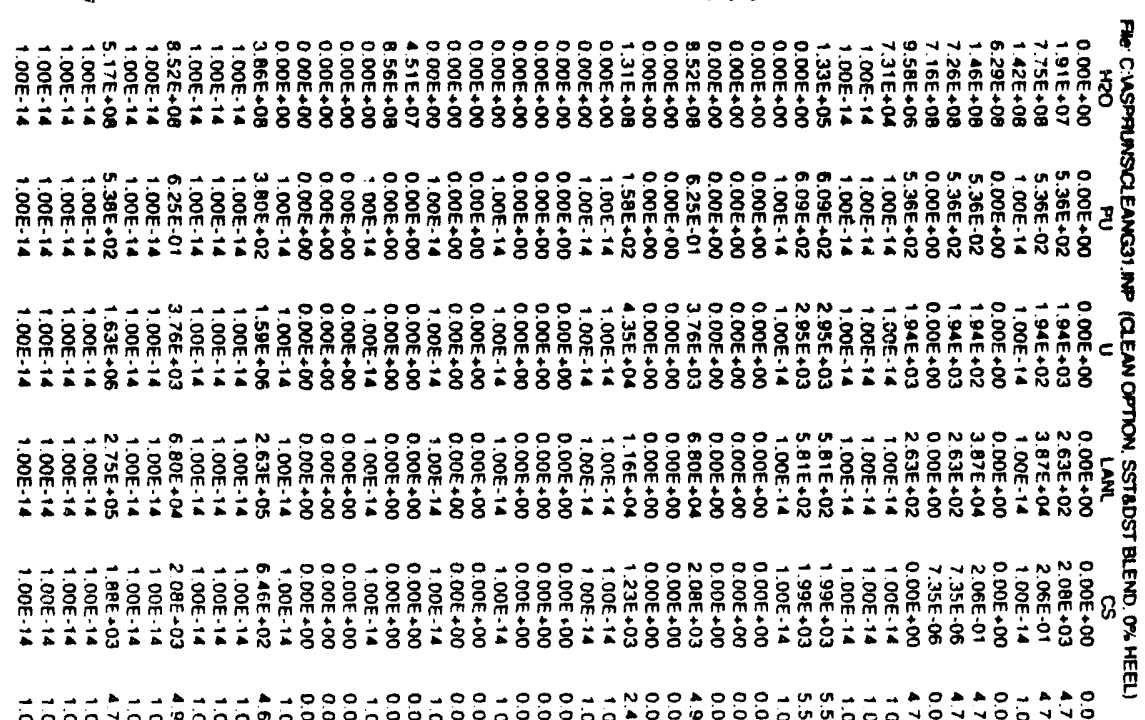

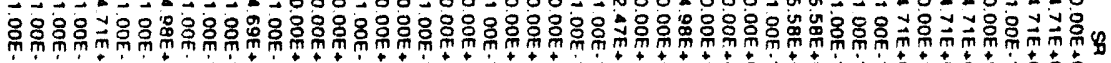

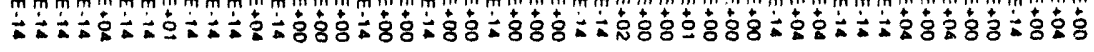

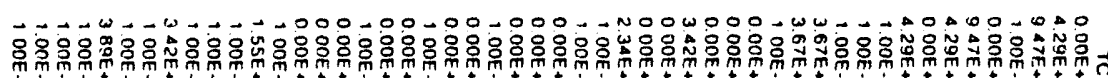

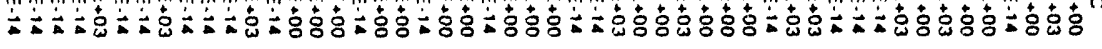

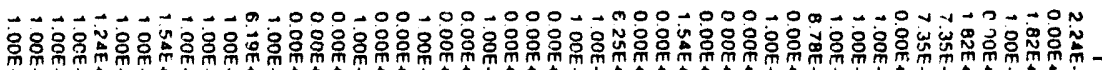

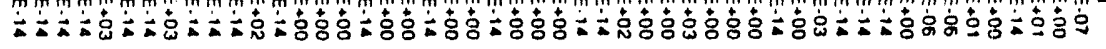

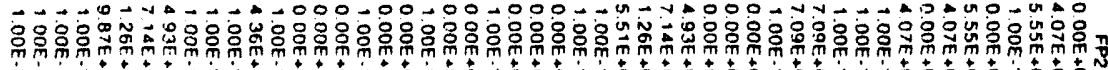

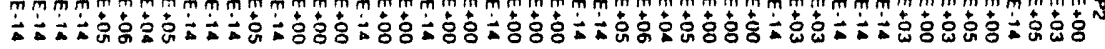

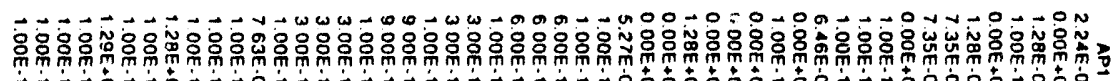

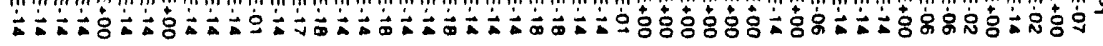

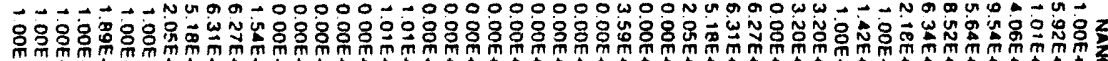

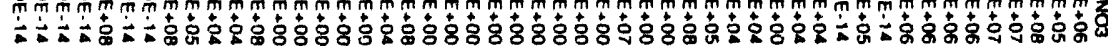

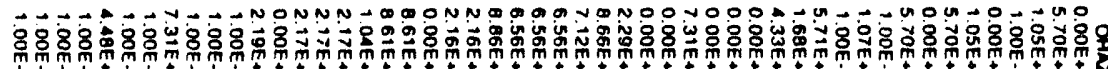

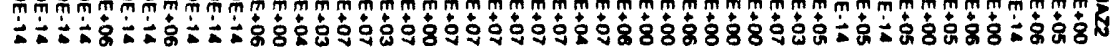

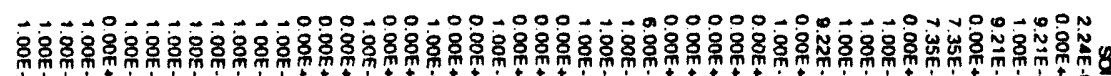

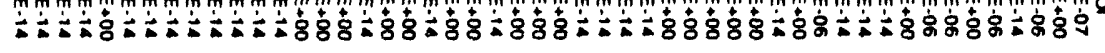

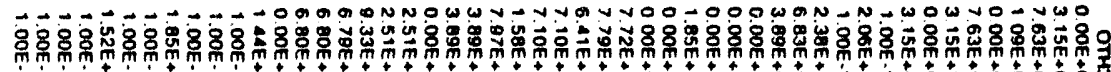

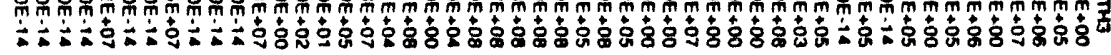

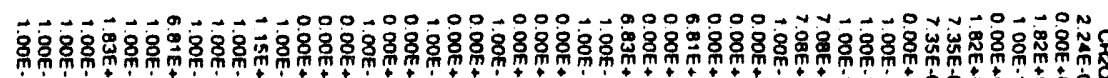

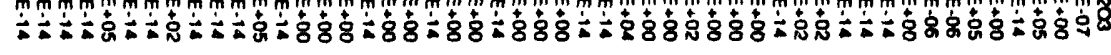

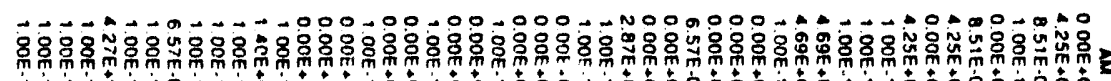

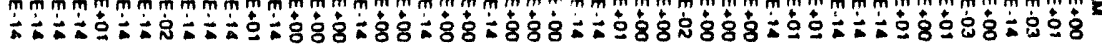


$S I-H$

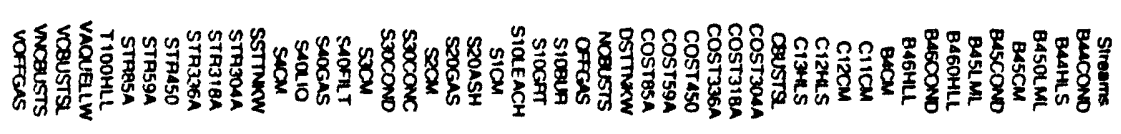

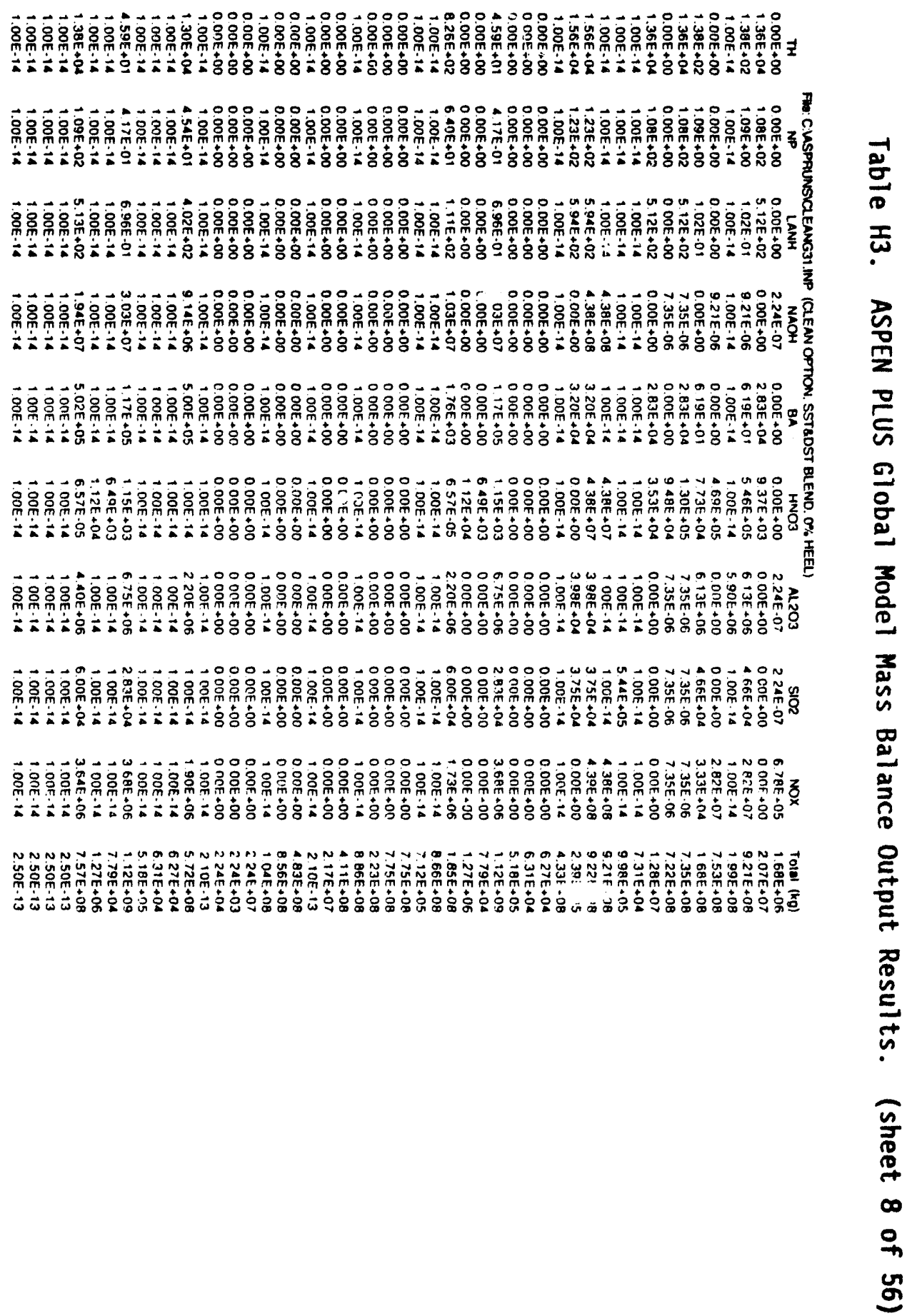




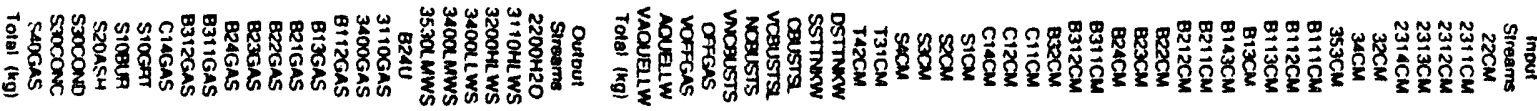

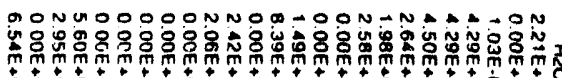

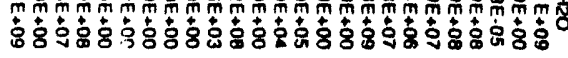

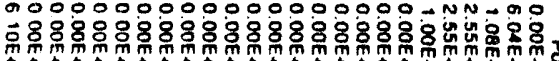

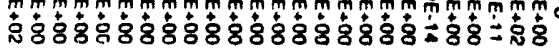

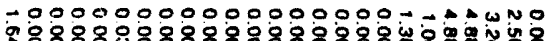

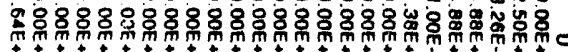

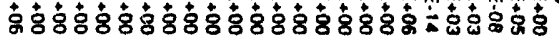

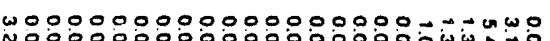

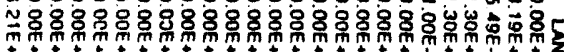

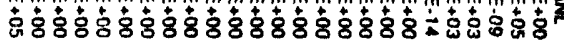

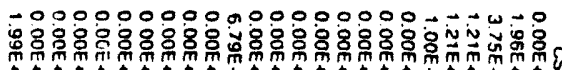

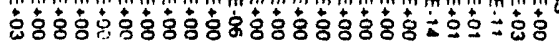

un

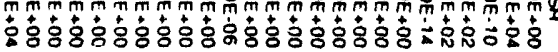

wo인

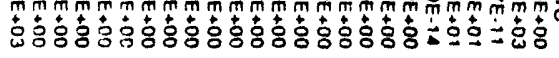

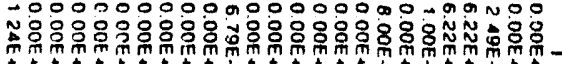

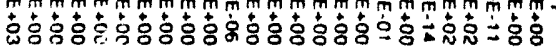
0000000000000000000 - 1 -10

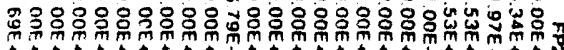

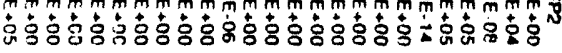

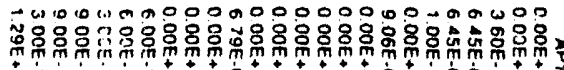

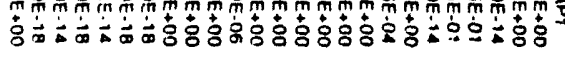

Wo9

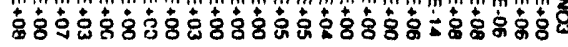

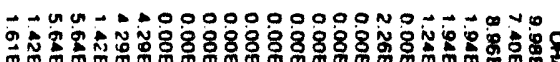

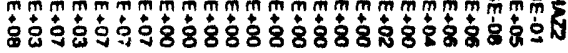

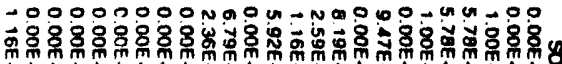

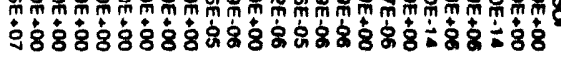

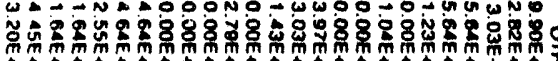

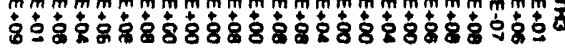

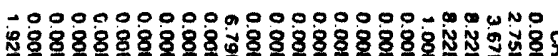

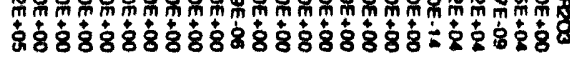

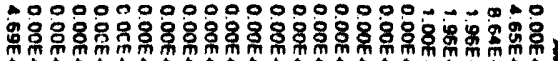

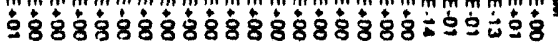

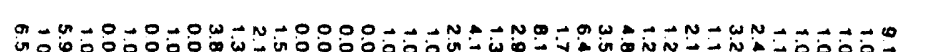

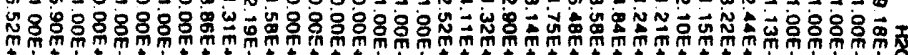

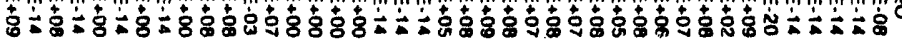

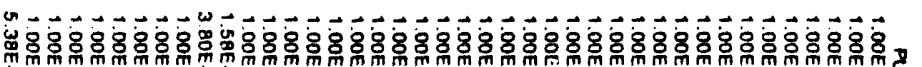
怘亡

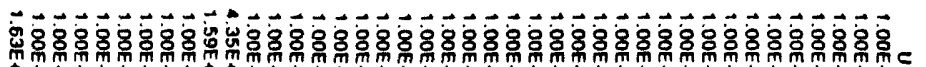

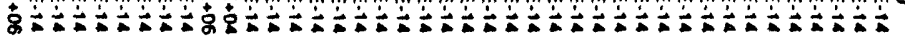

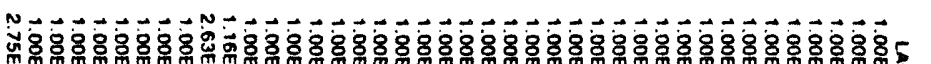

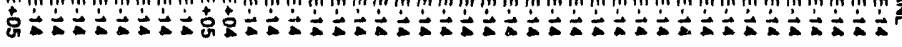

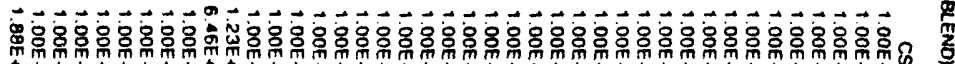

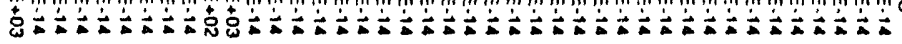

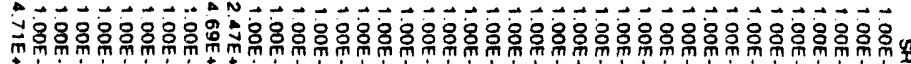

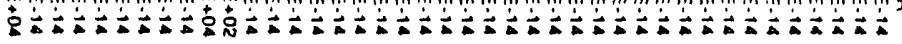

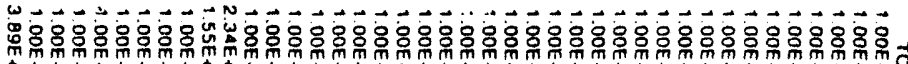

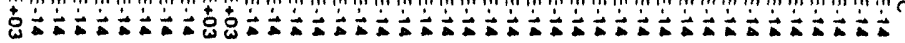

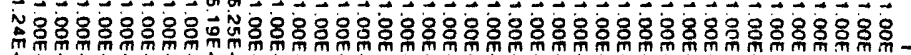

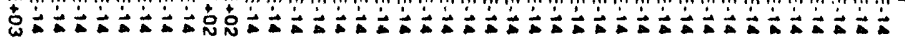

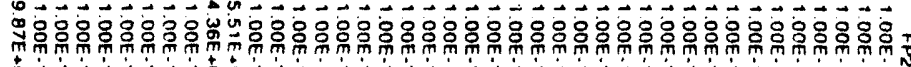

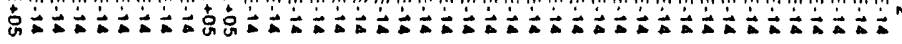

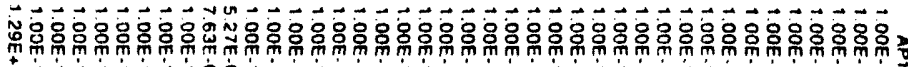

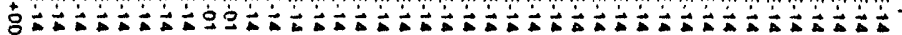

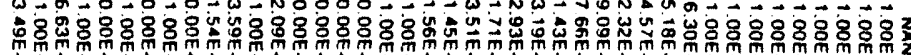

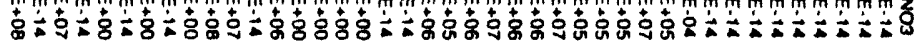

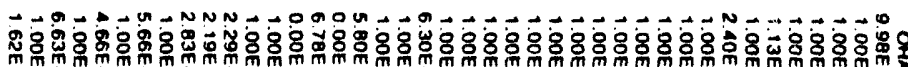

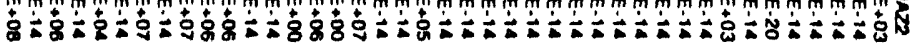

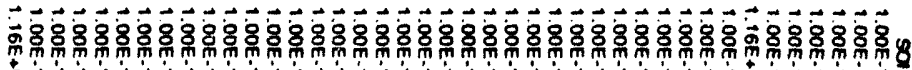

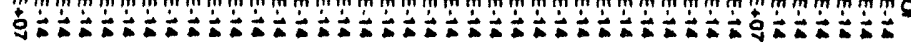

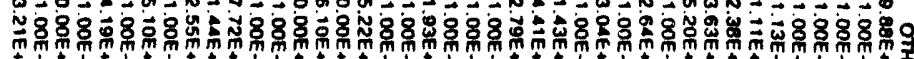

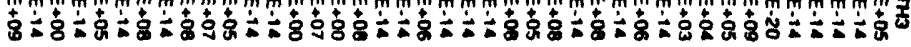

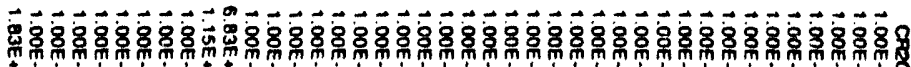

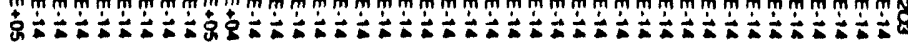

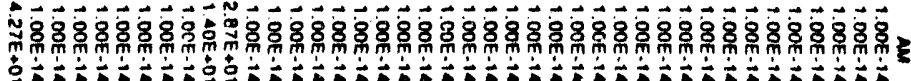




\section{$\angle I-H$}

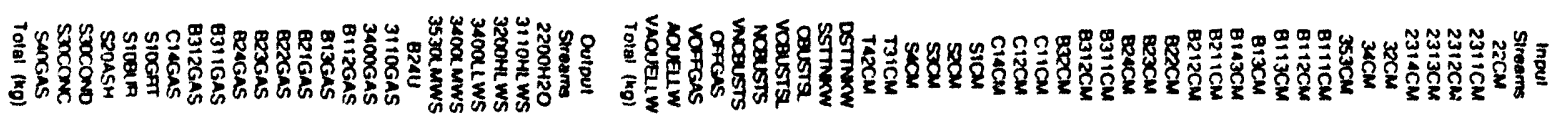

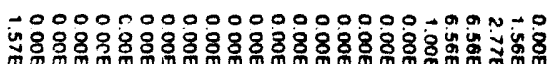

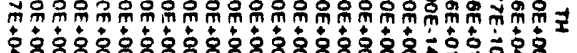

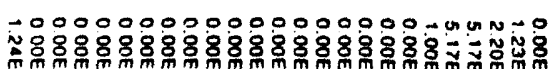

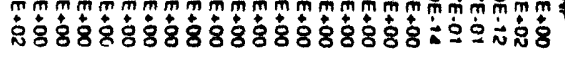

NOOOOOO0000000000000

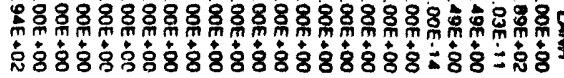

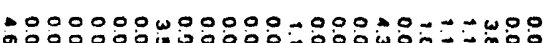
ER

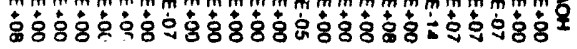

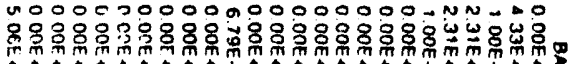
งัง

$4000000000-1000-10-000-00$

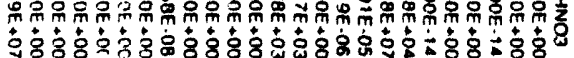
นืํำ

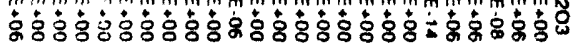

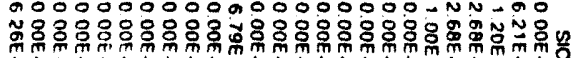

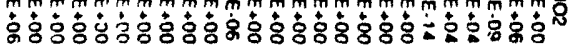

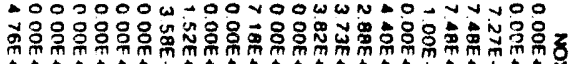

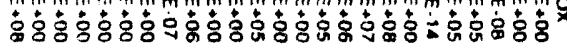

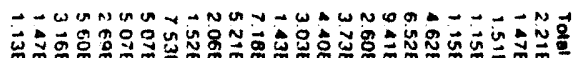

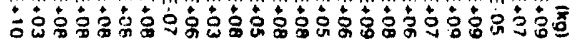

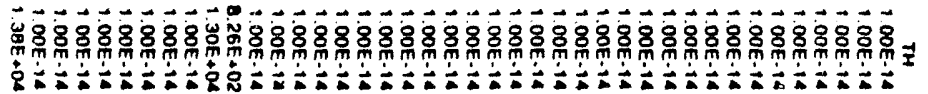

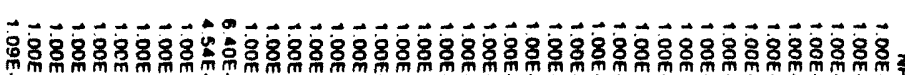

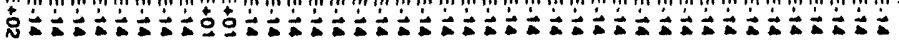

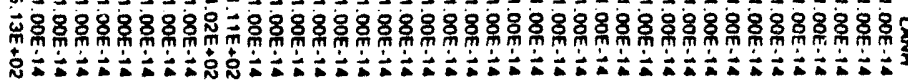

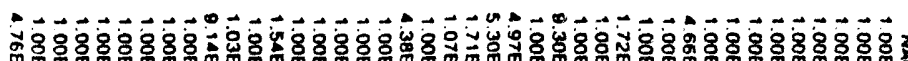

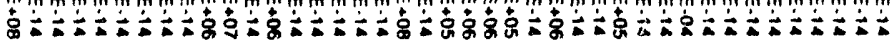

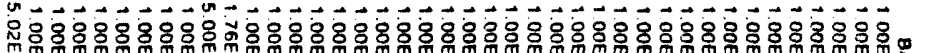

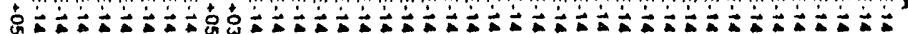

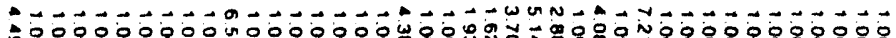

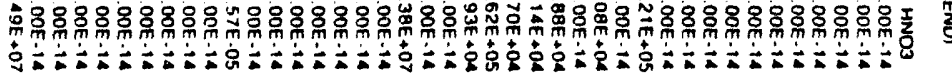

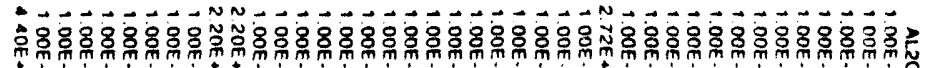

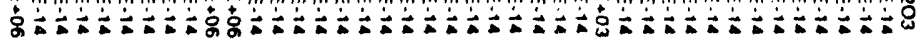

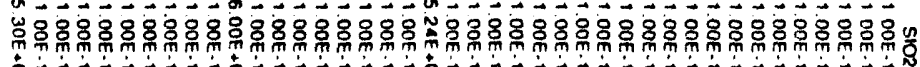

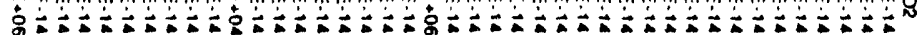

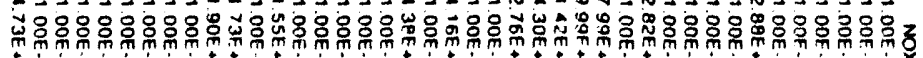

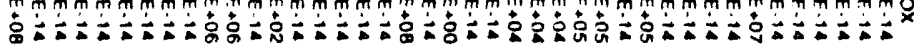

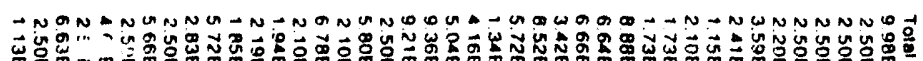

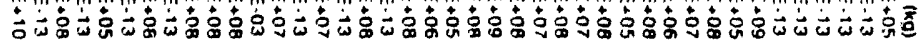

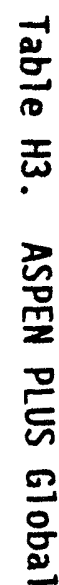

중

㐫

政

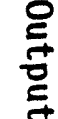

总

$\frac{1}{8}$

응

$\stackrel{\circ}{\rightarrow}$

জু 


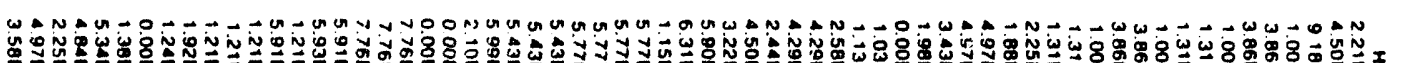

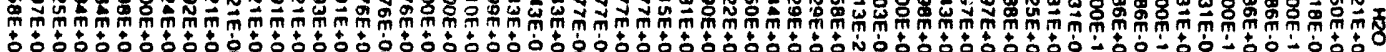

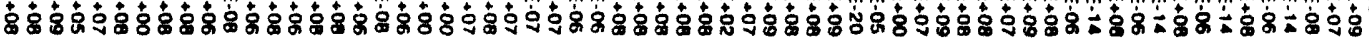

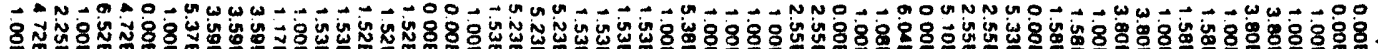

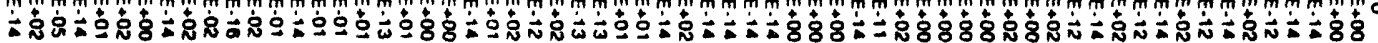

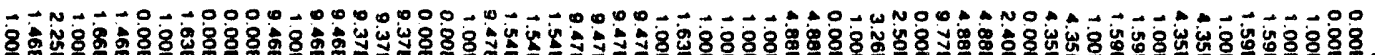
管

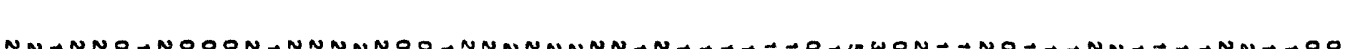

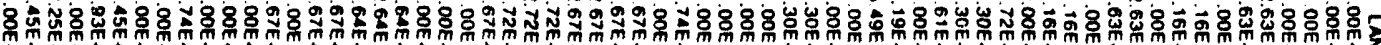

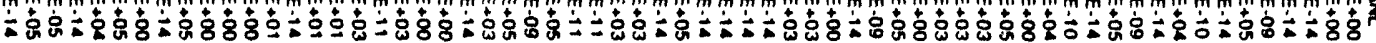

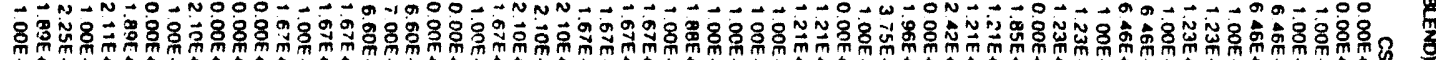

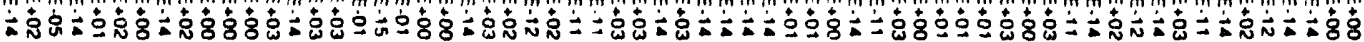

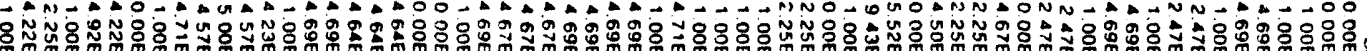

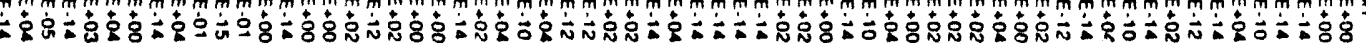

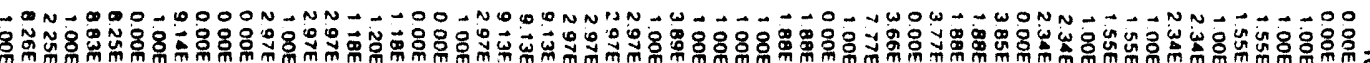

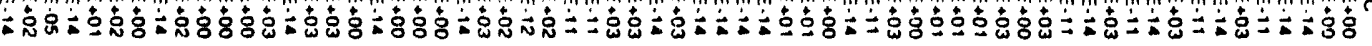

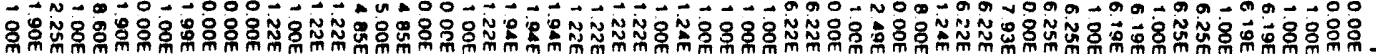

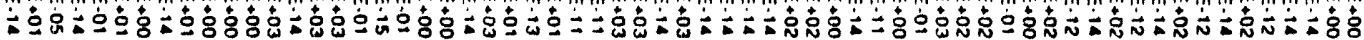

OONOW

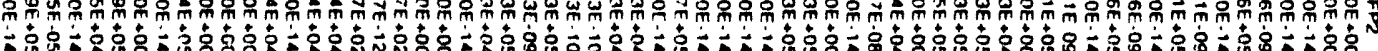

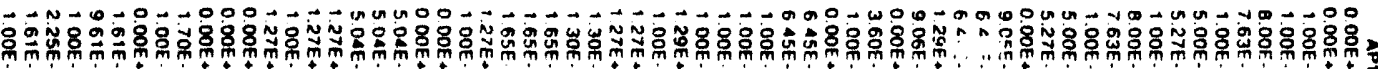

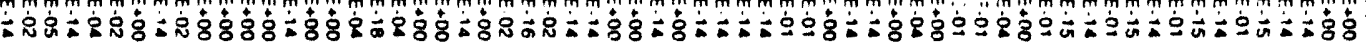

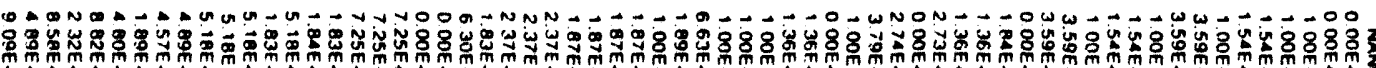

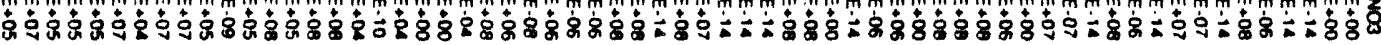

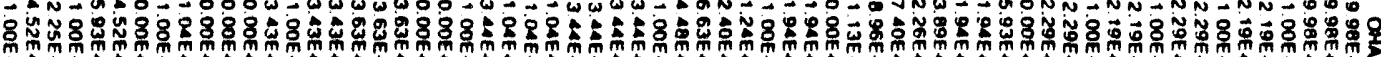

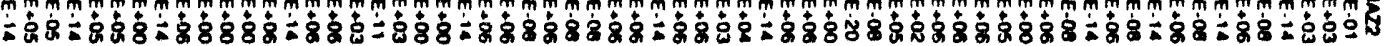

FN88 N N

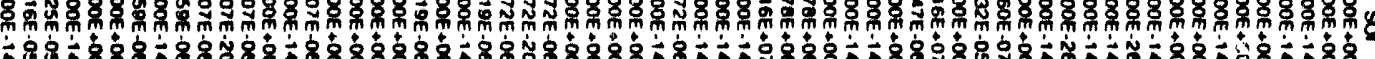

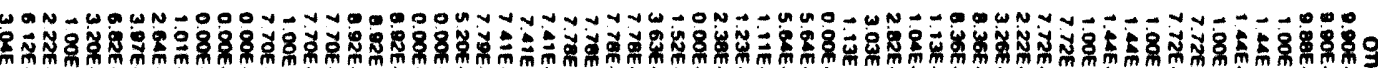

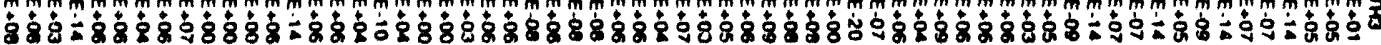

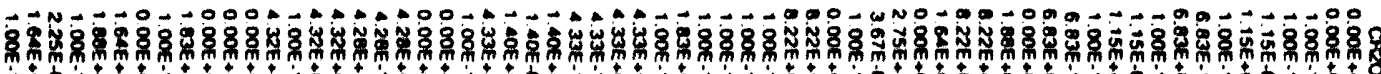

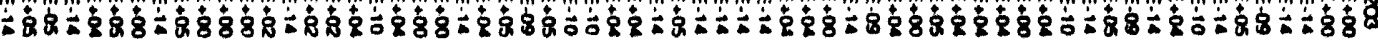

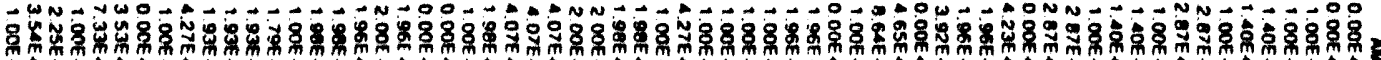

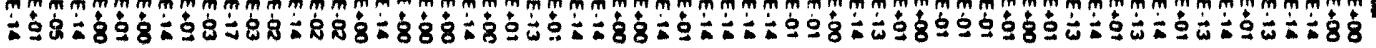




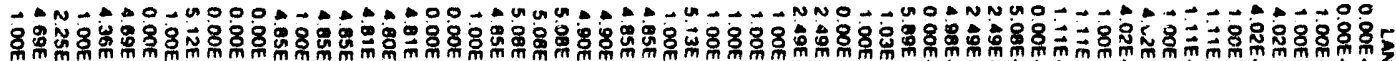

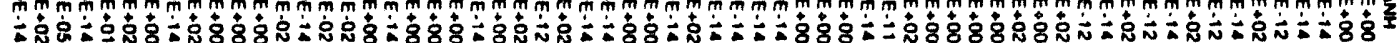

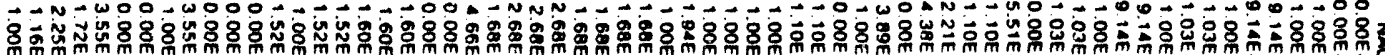

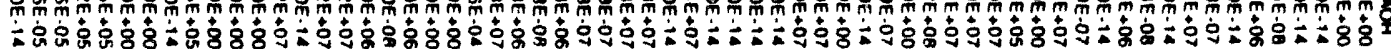

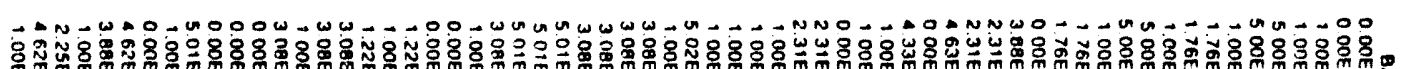

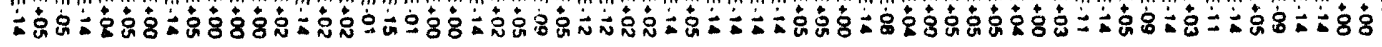

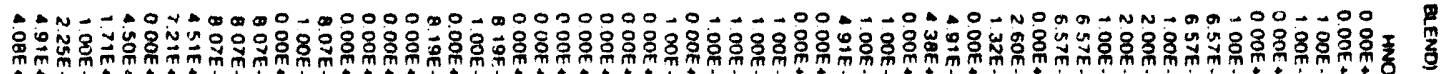

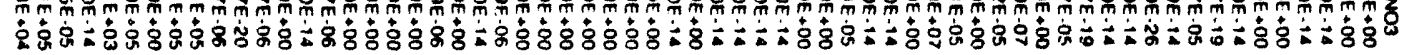

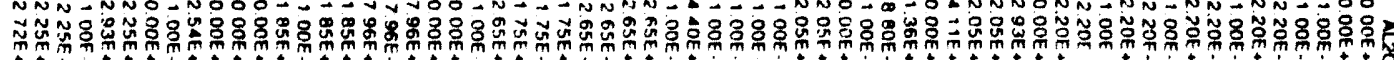

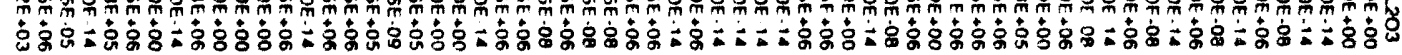

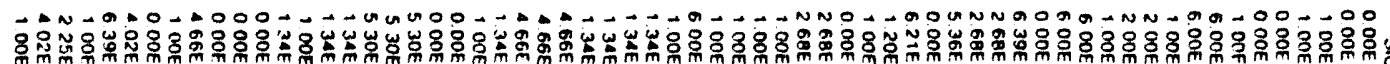

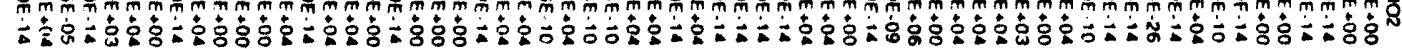

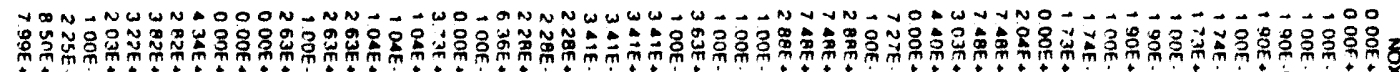

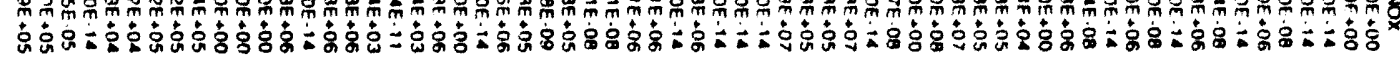

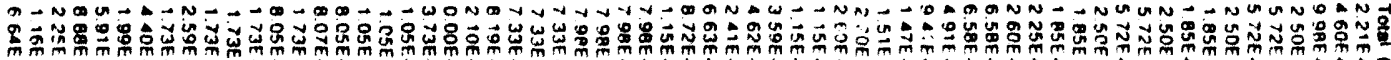

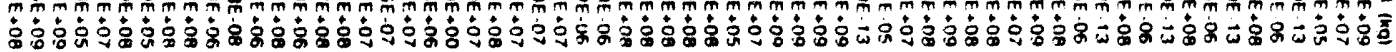




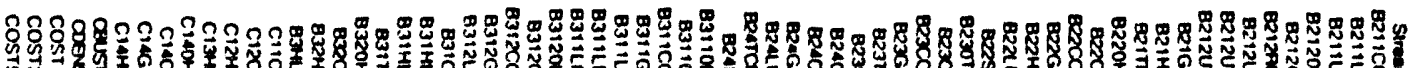

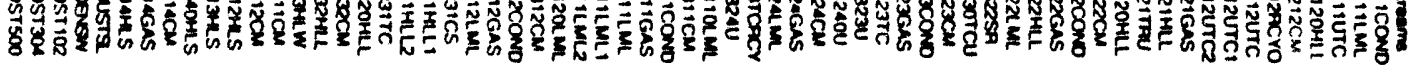

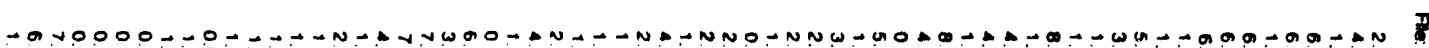

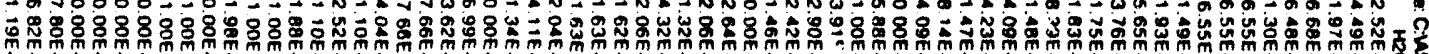

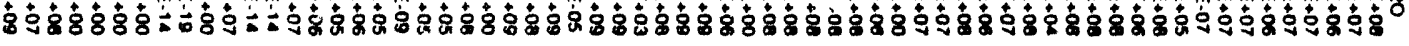

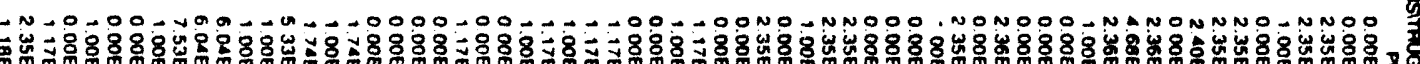

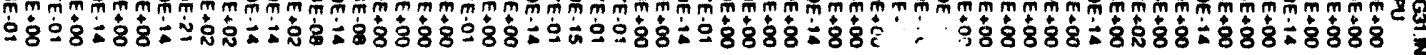

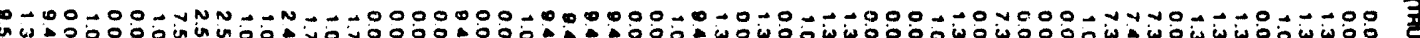

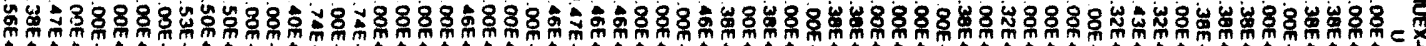

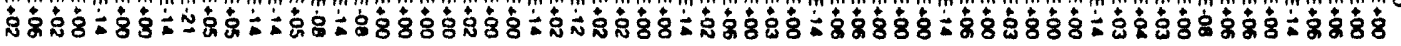

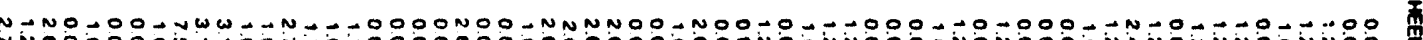

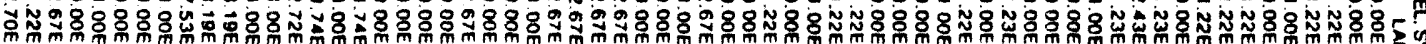

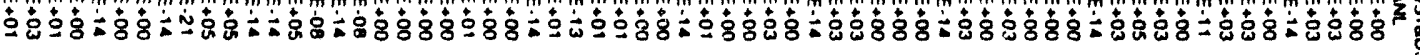

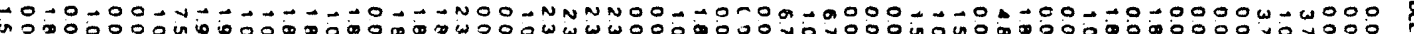

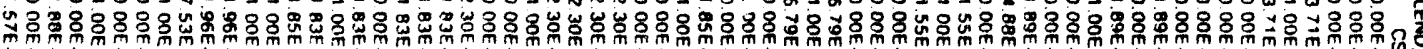

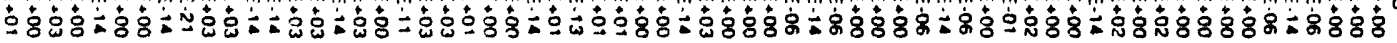

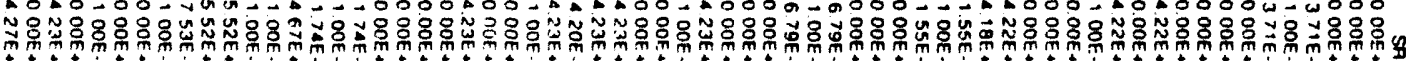

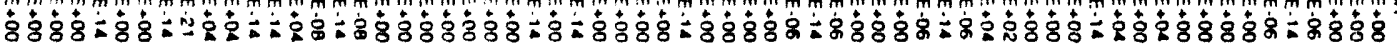

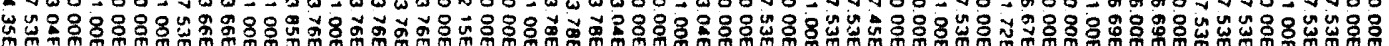

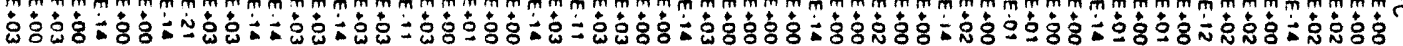

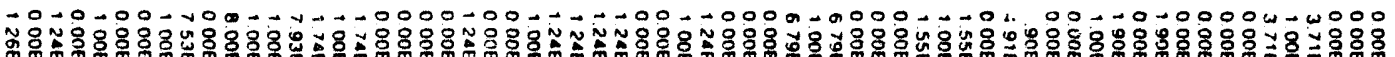

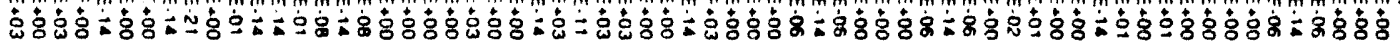

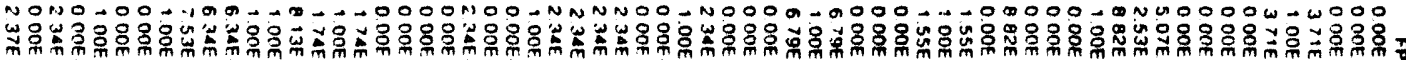

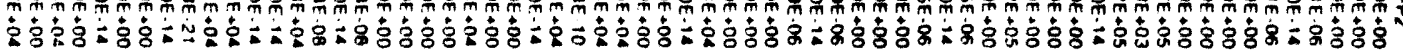

$\overrightarrow{4}$

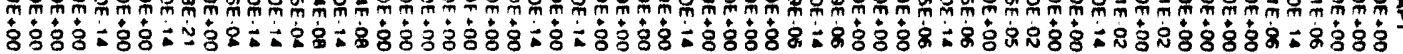

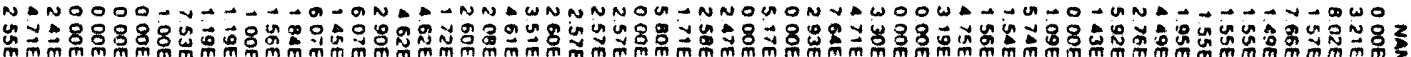

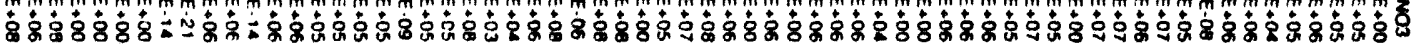

wow

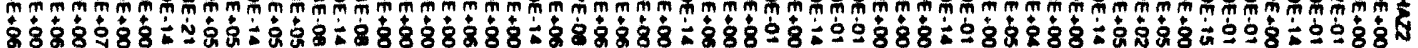

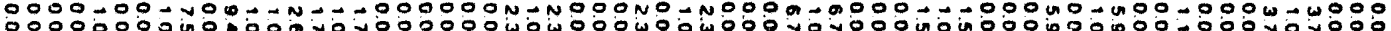

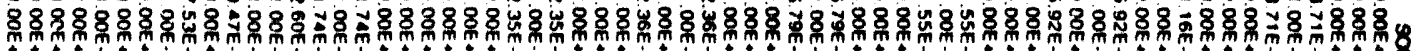

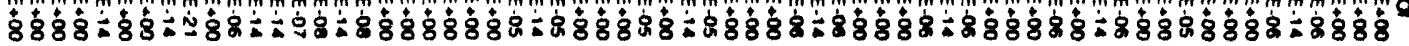

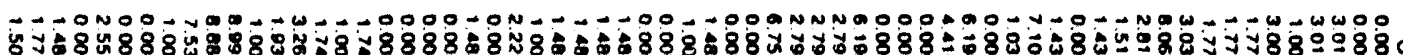

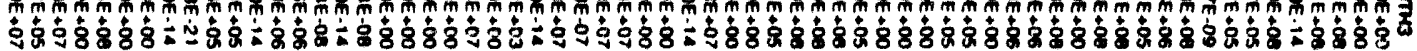

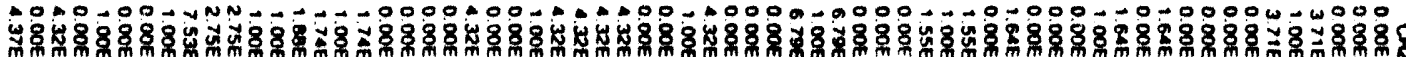

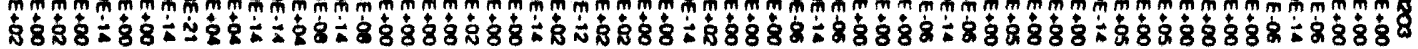

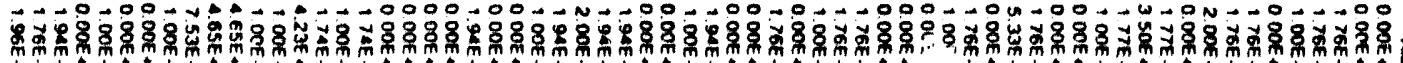

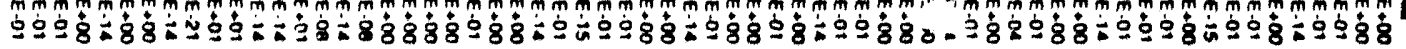



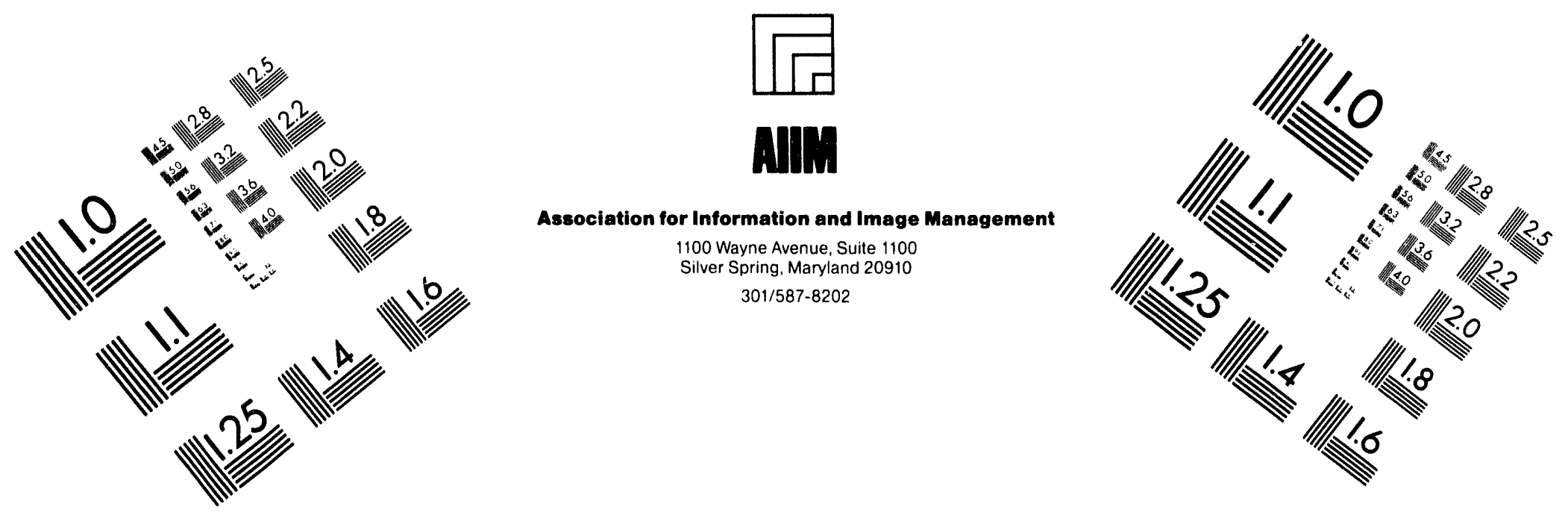

\section{Centimeter}

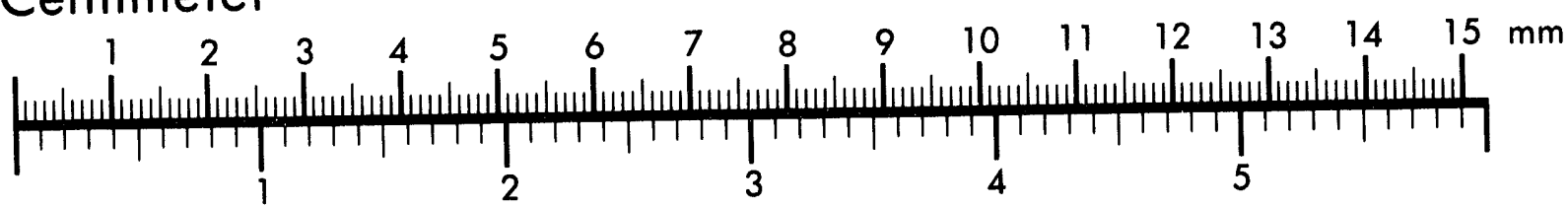
Inches

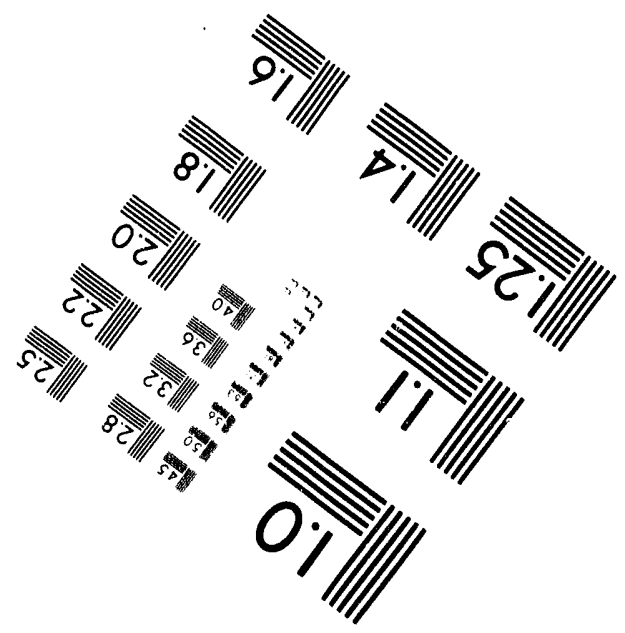

MANUFACTURED TO AIIM STANDARDS

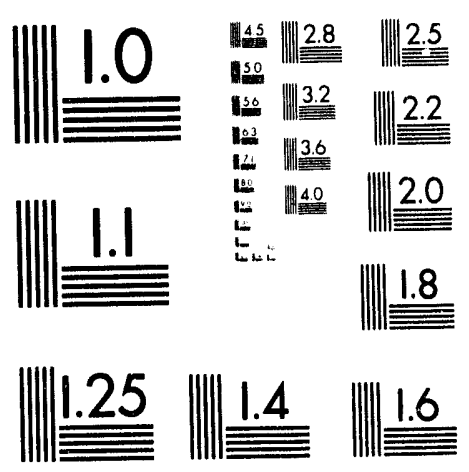

BY APPLIED IMAGE, INC.

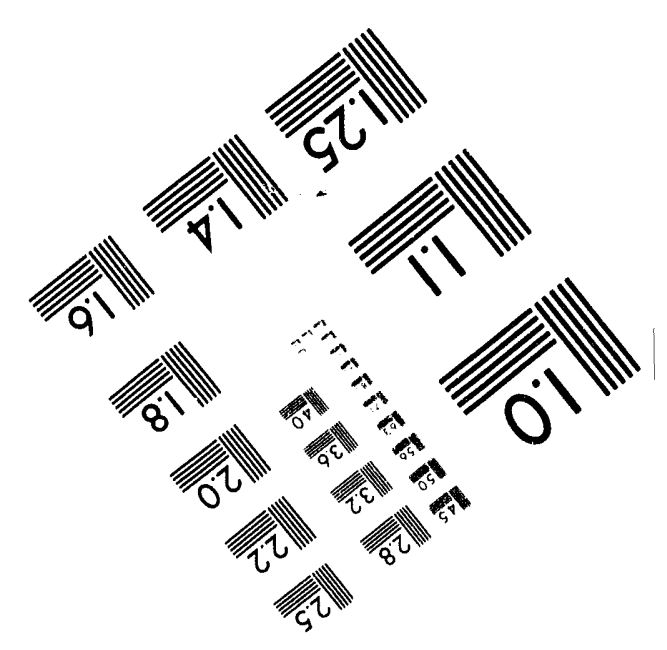



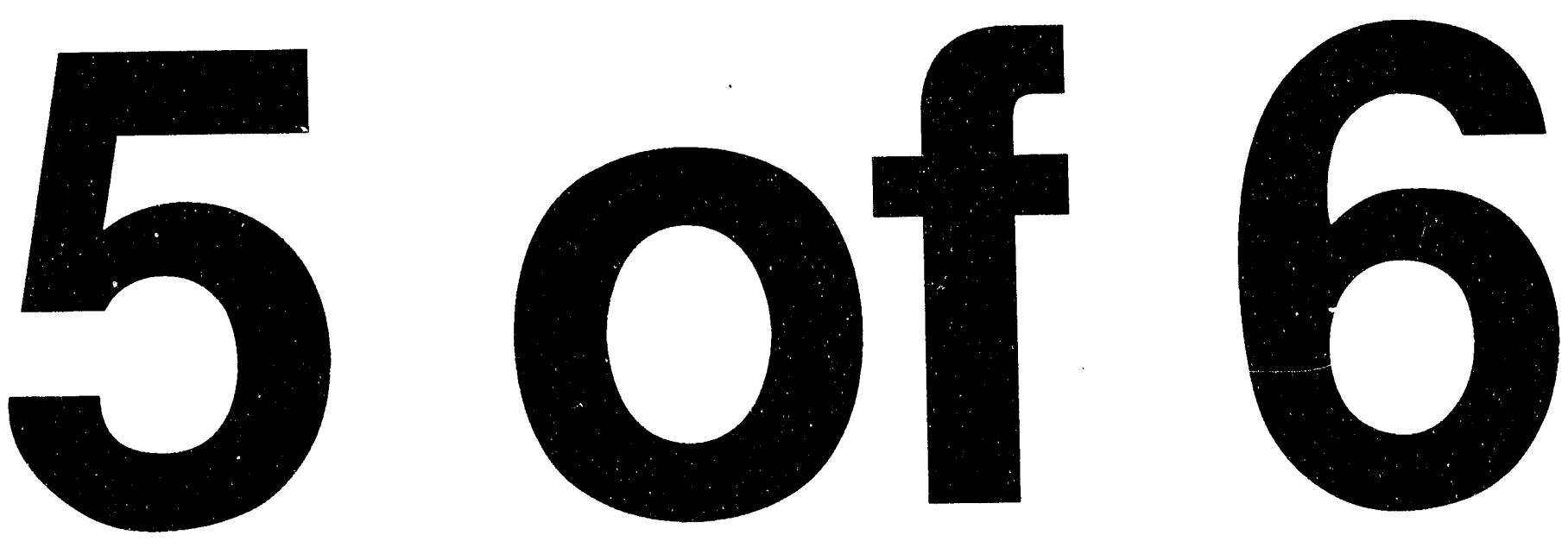
Table H3. ASPEN PLUS Global Model Mass Balance Output Results. (sheet 14 of 56)

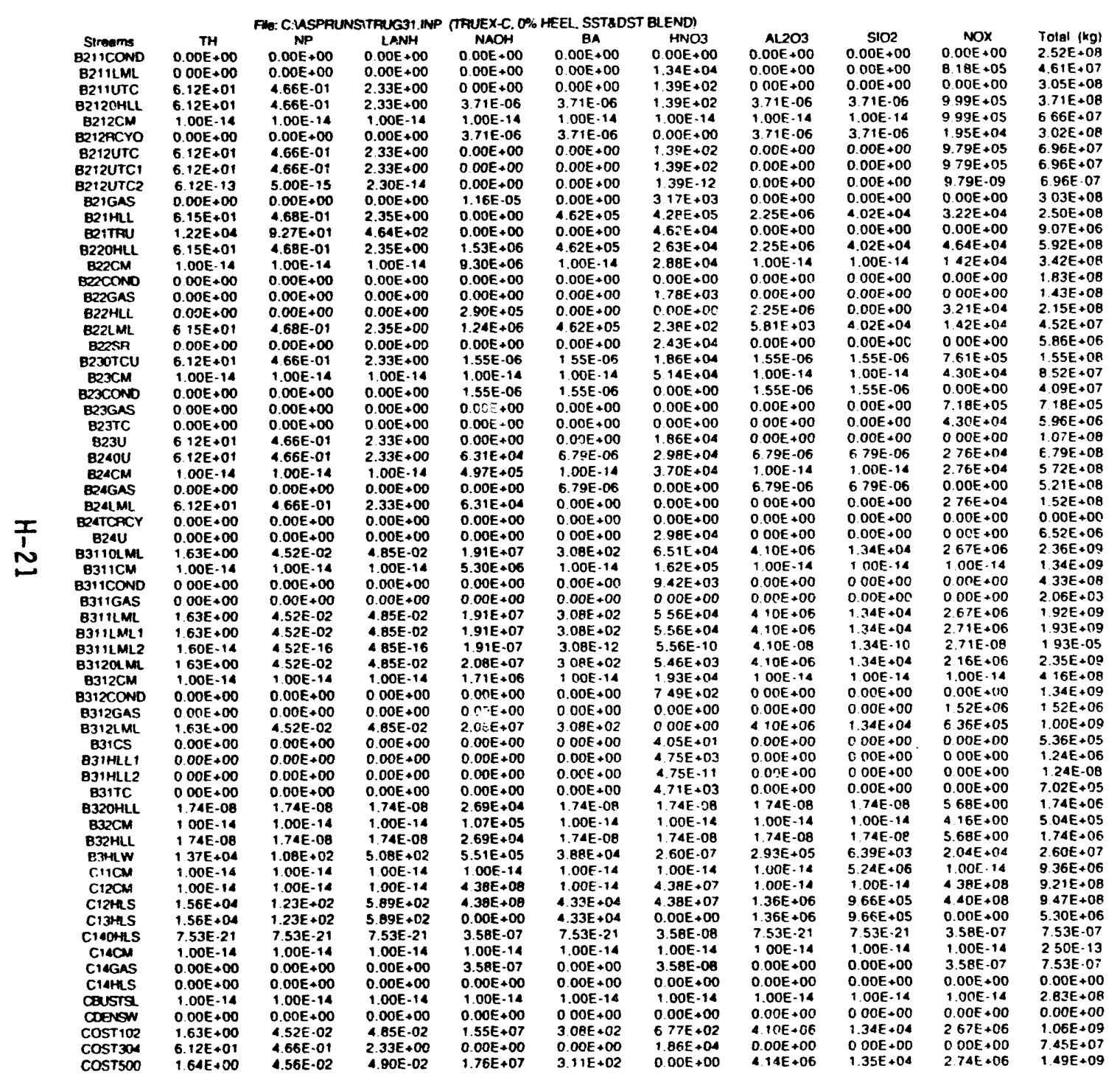


Table H3. ASPEN PLUS Global Model Mass Balance Output Results. (sheet 15 of 56)

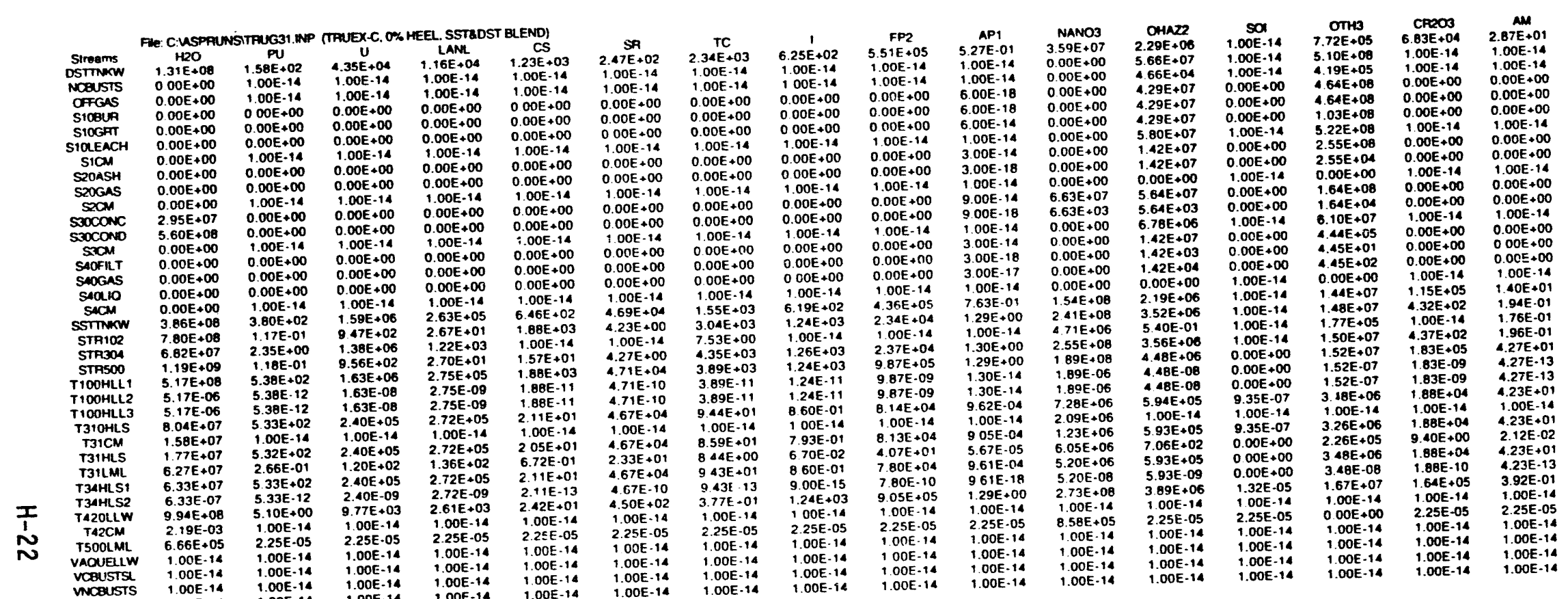


Table H3. ASPEN PLUS Global Model Mass Balance Output Results. (sheet 16 of 56)

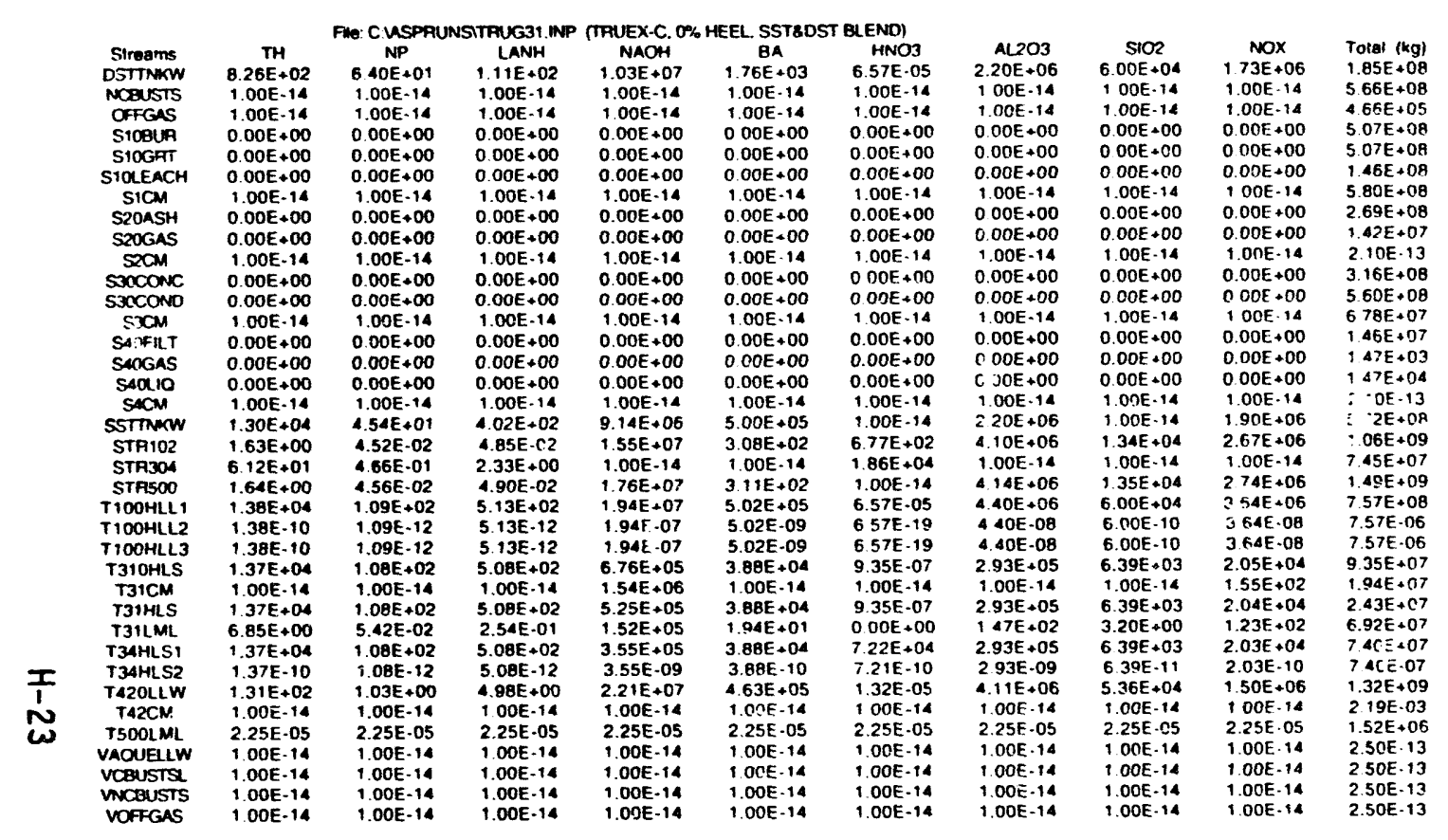




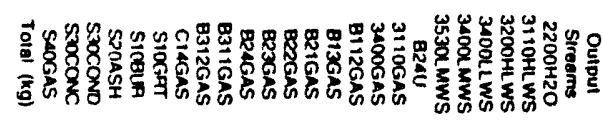

HO- $-0000000-0 n-0 N-1-N \omega \omega-0-1$

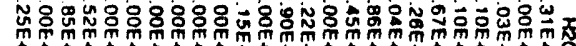

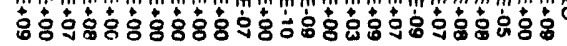

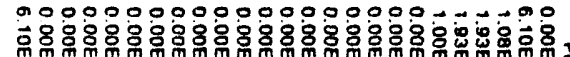

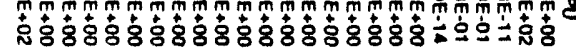

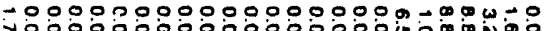

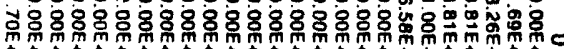

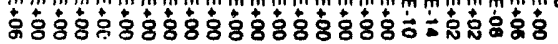

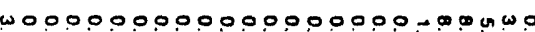

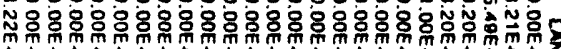

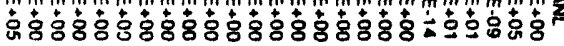

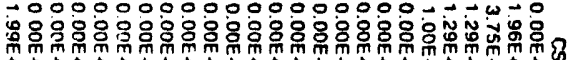

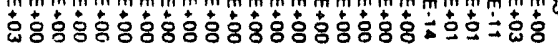

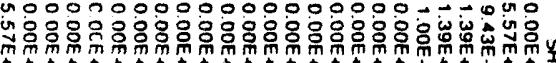

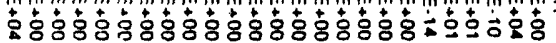

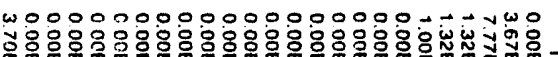

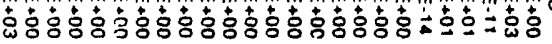

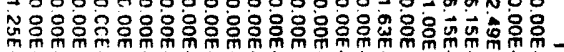

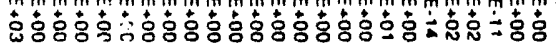

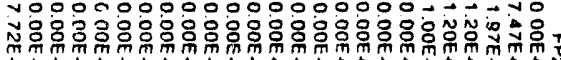

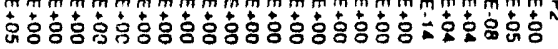

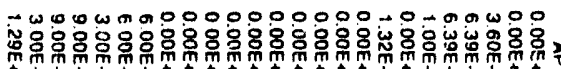

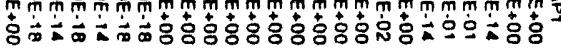

NOA

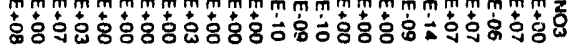

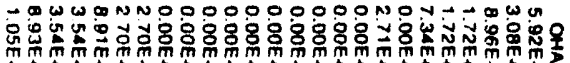

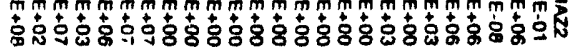

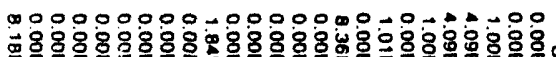

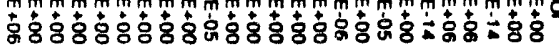

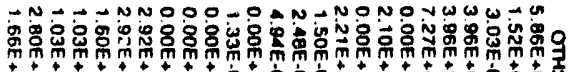

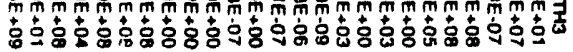

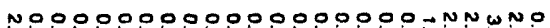

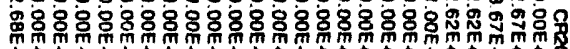

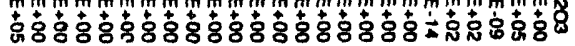

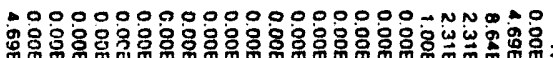

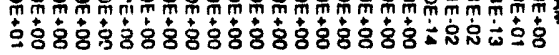

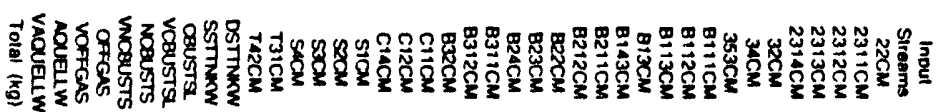

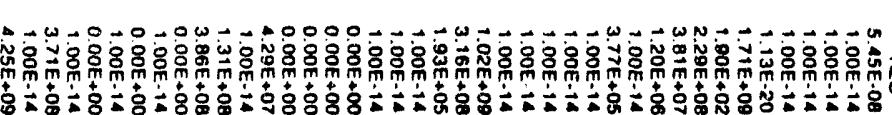

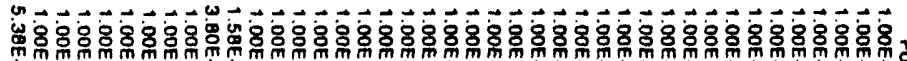

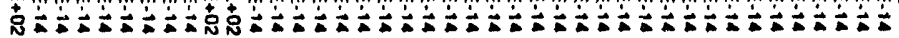

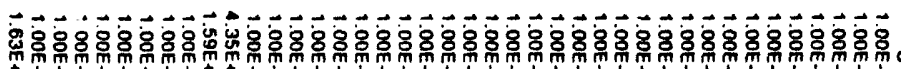

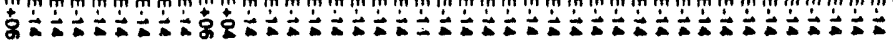

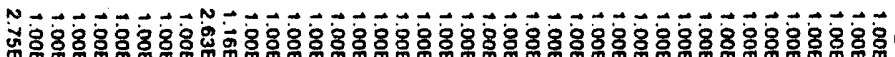

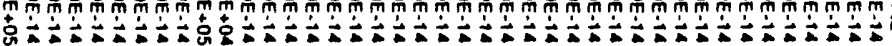

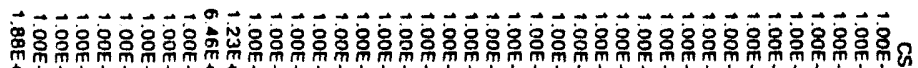

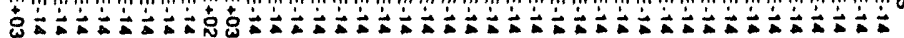

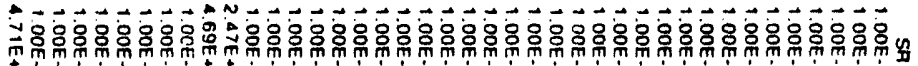

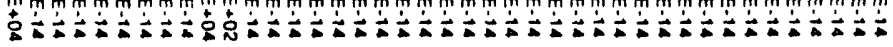

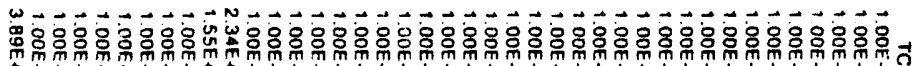

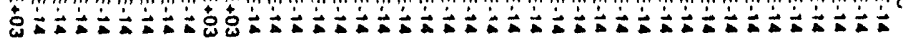

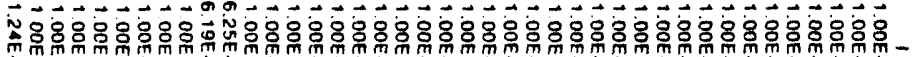

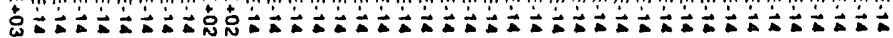

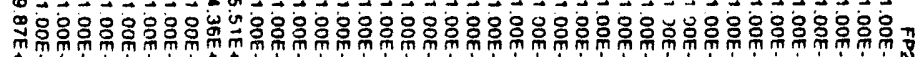

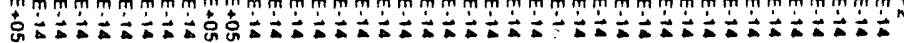

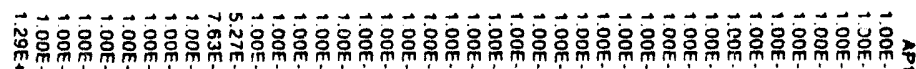

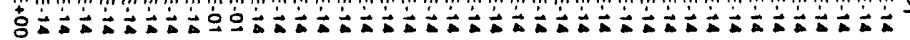

N

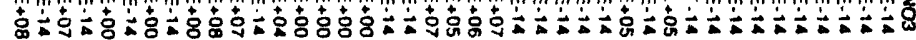

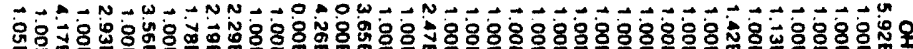

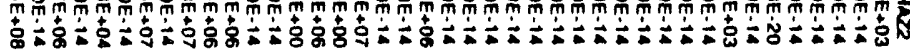

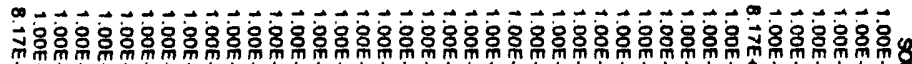

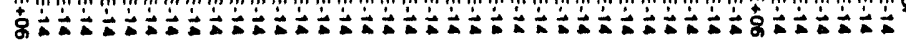

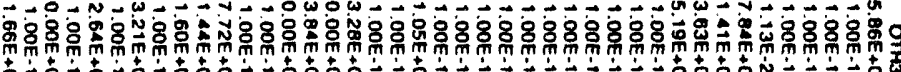

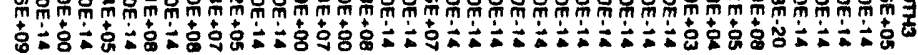

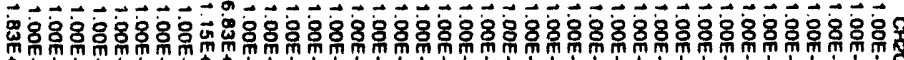

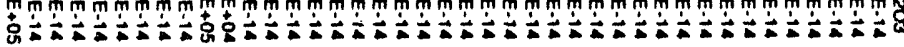

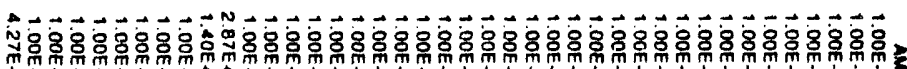

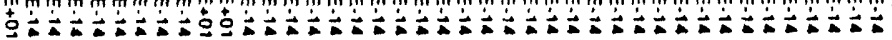




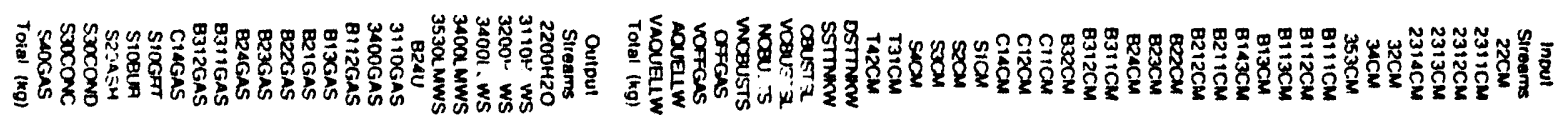

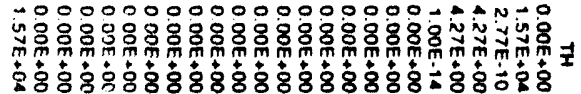

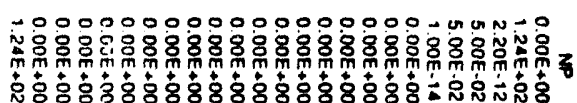

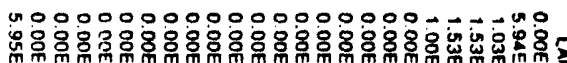

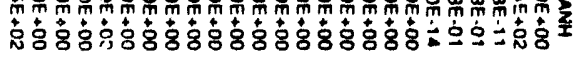

$10000001000000000+0-00000$

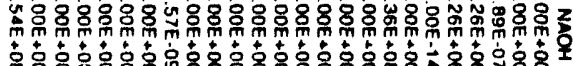

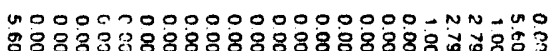

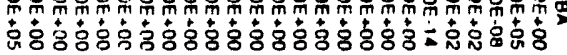

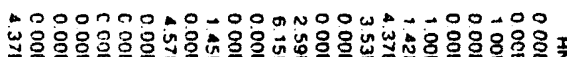

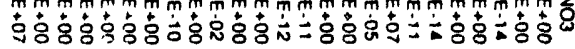

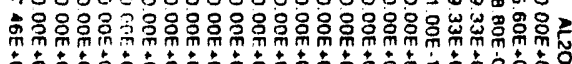

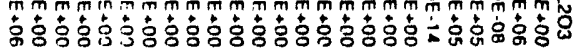

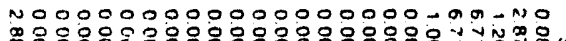

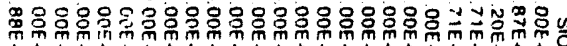

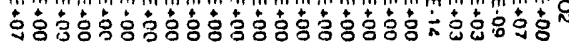

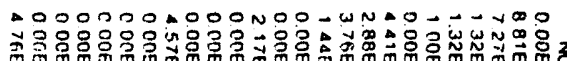

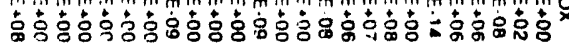

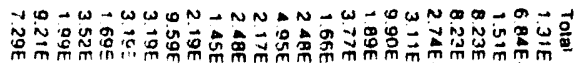

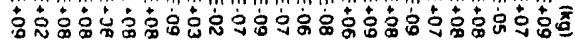

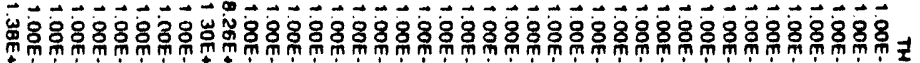

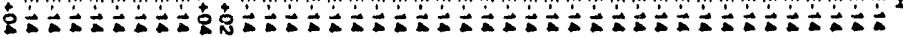

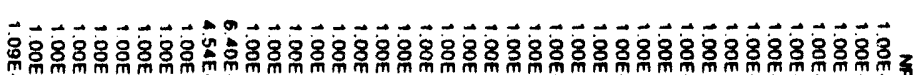

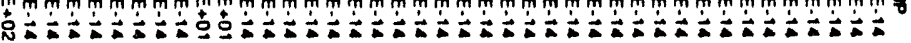

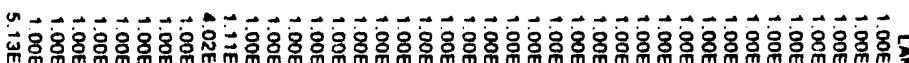

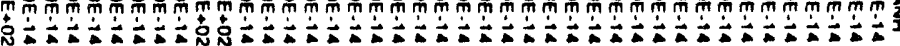

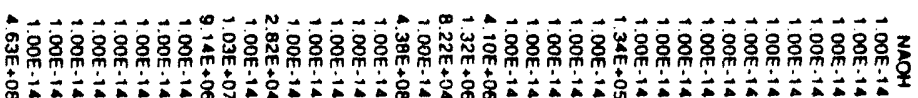

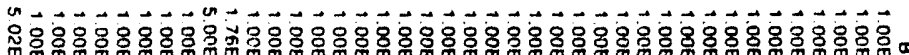

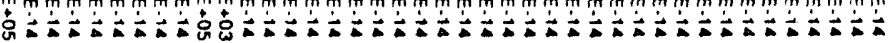

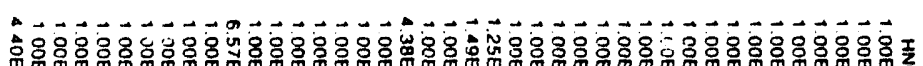

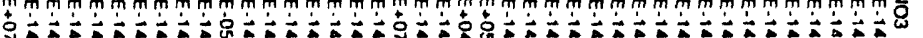

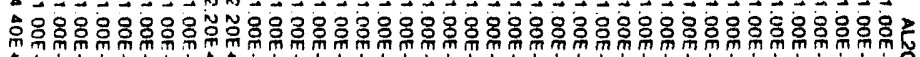

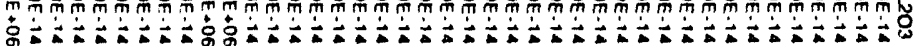

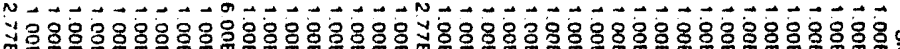
管

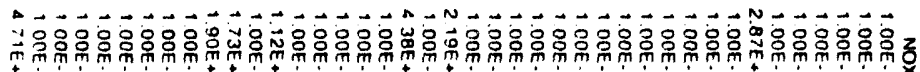

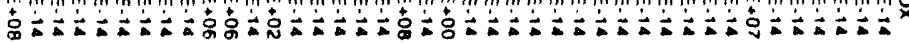

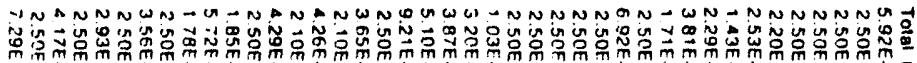

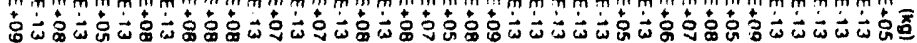




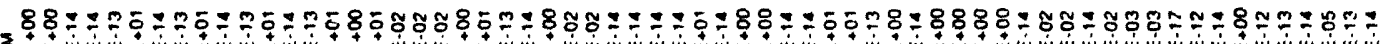

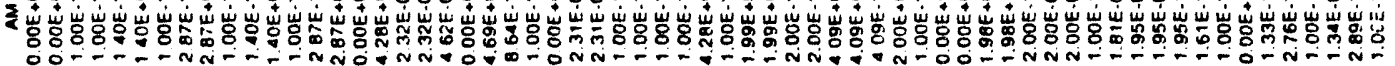

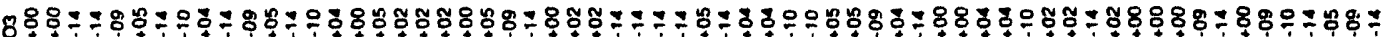

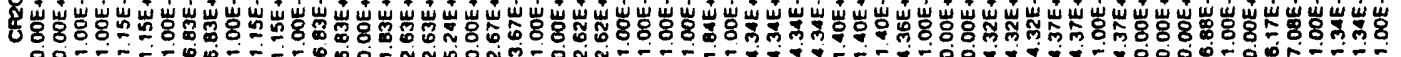

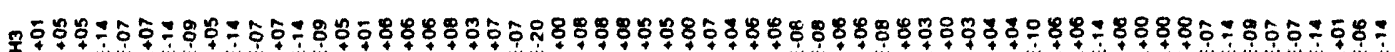

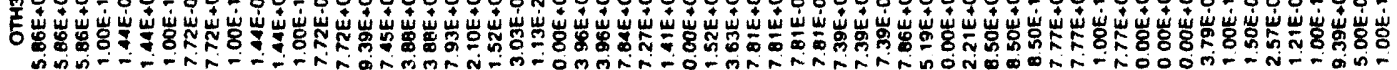

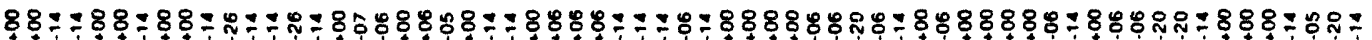

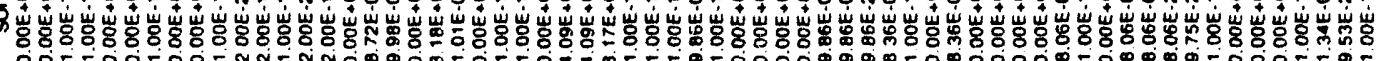

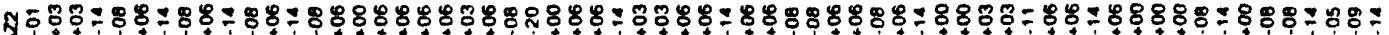

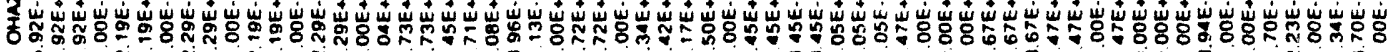

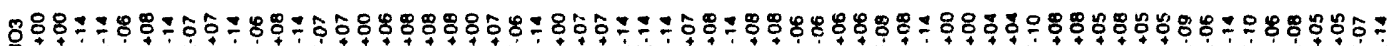

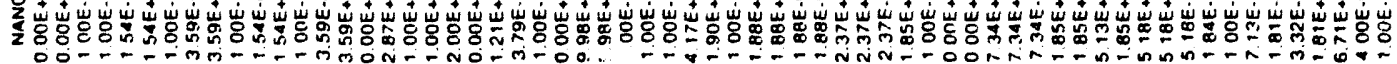

-

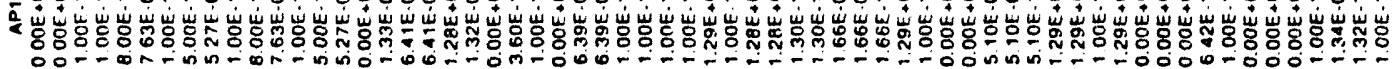

車务

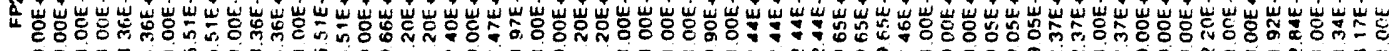

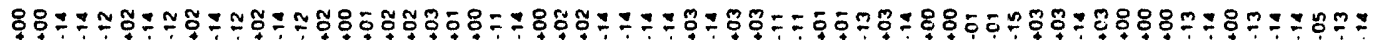

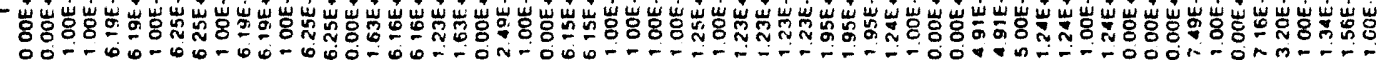

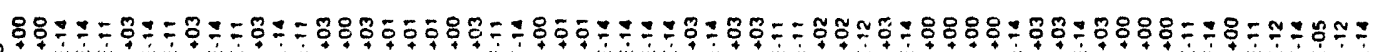

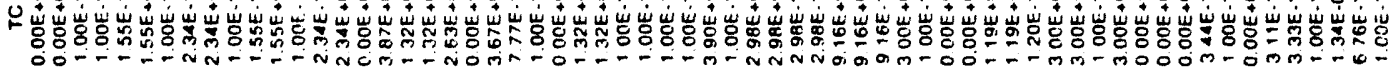

車行

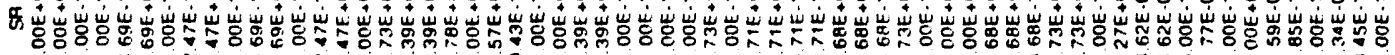

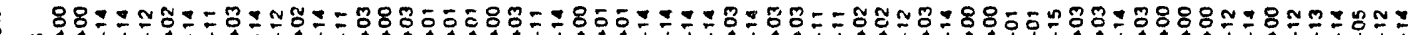

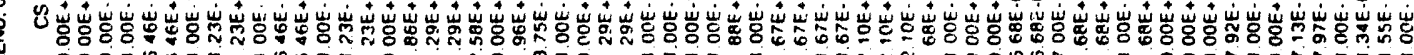

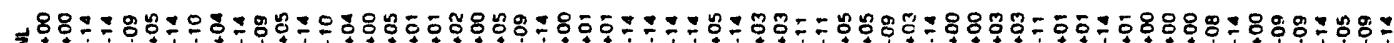

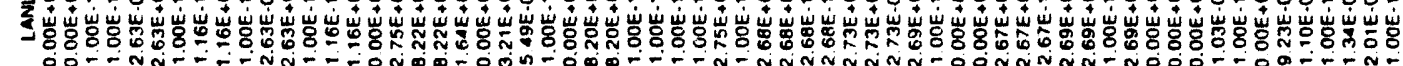
龺

88

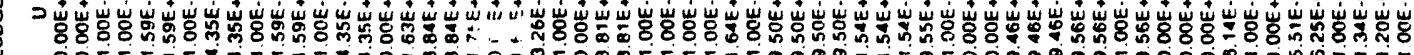

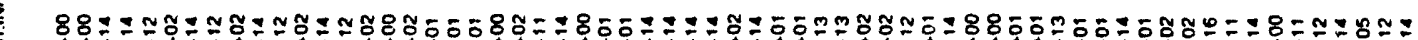

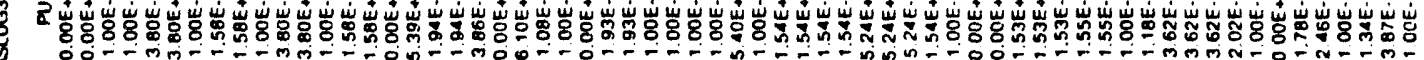

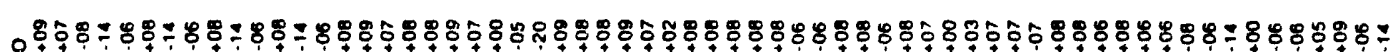

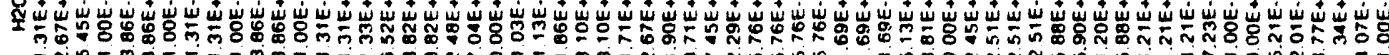

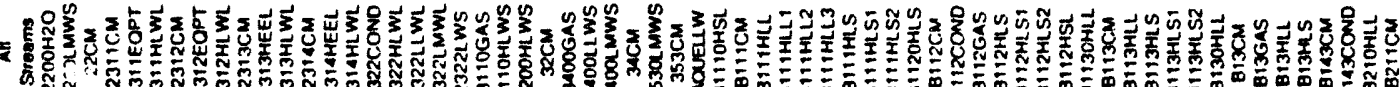

H-26 


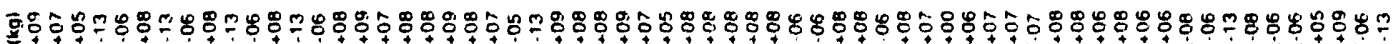

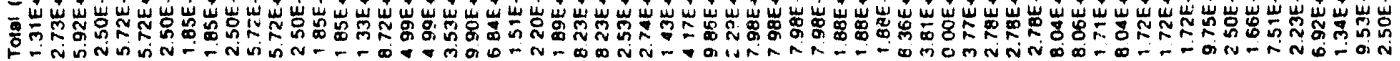

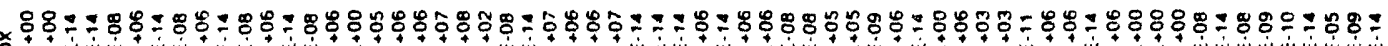

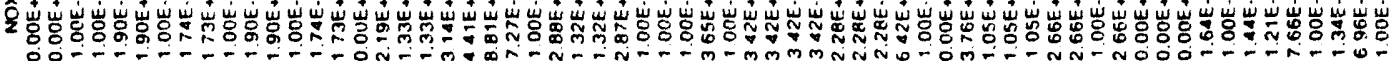

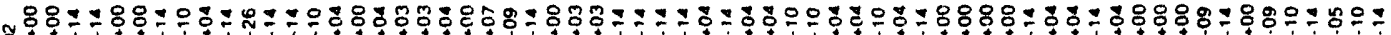

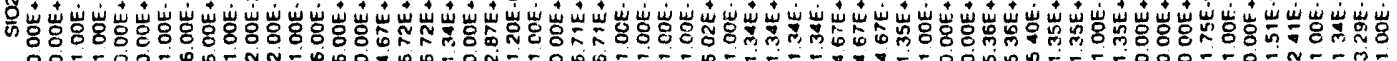

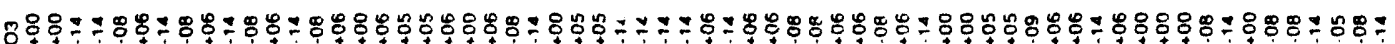

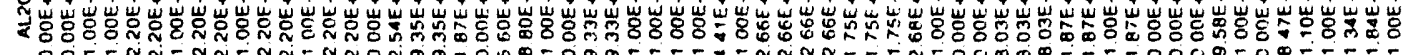

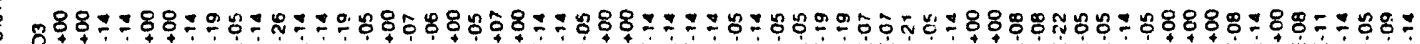

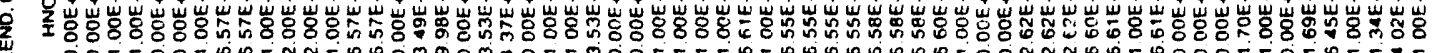

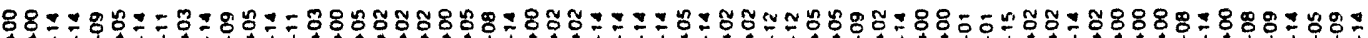

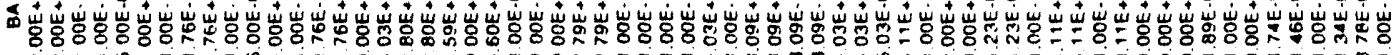

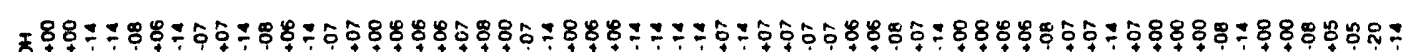

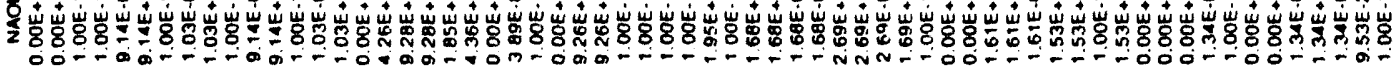

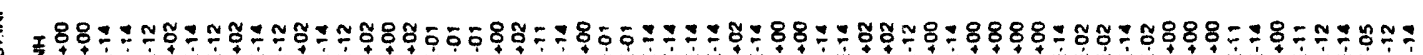

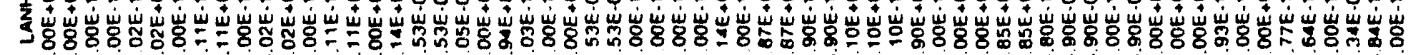

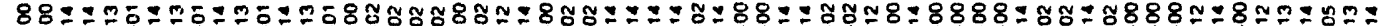

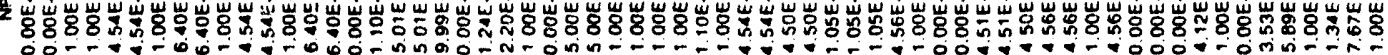

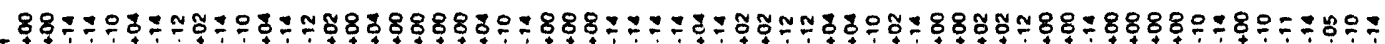

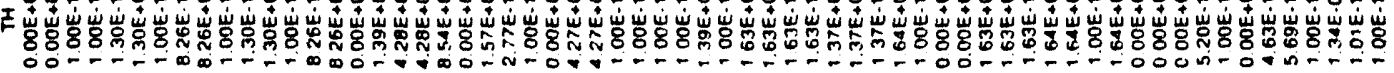

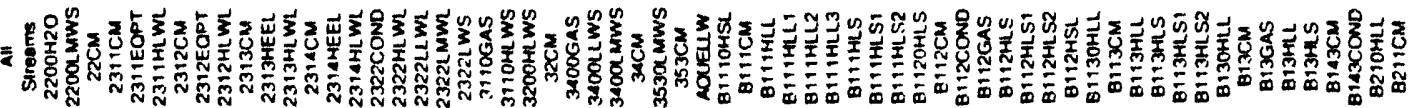




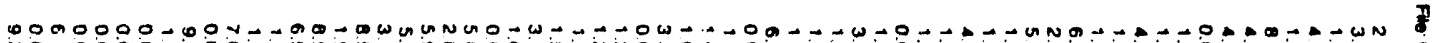

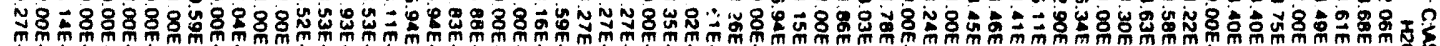

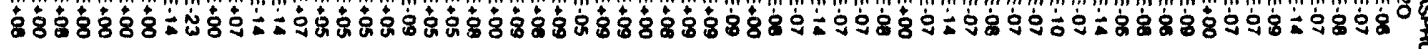

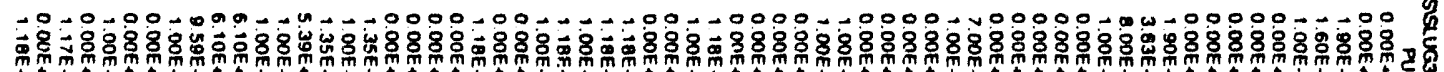

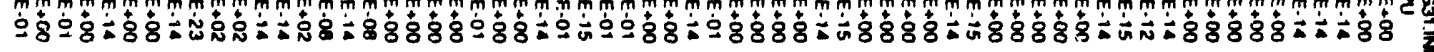

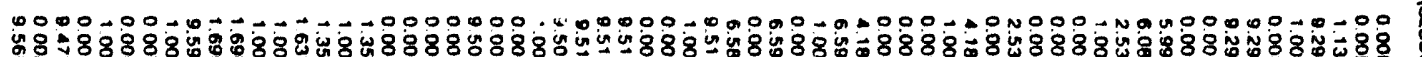

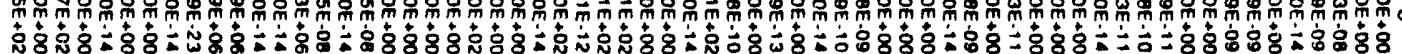

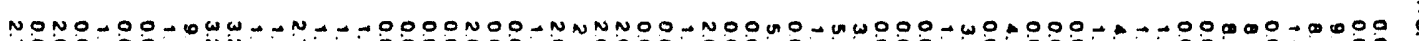

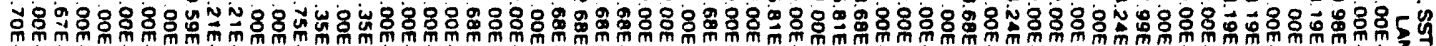

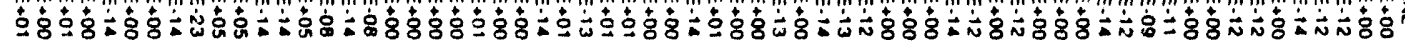

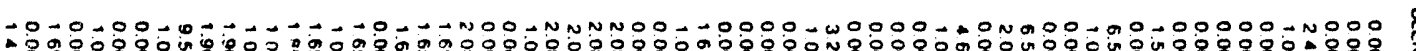

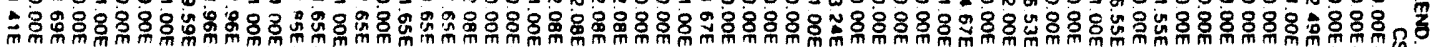

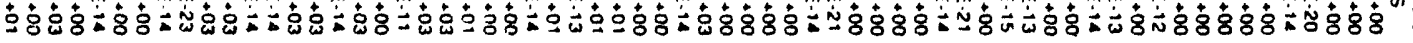

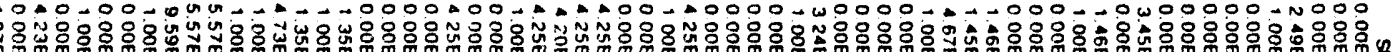

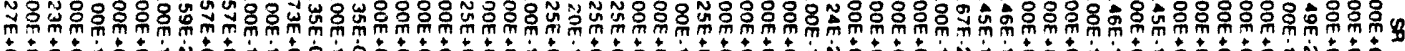

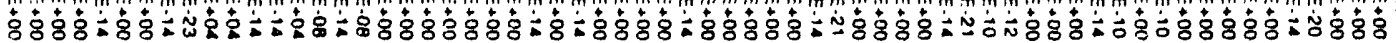

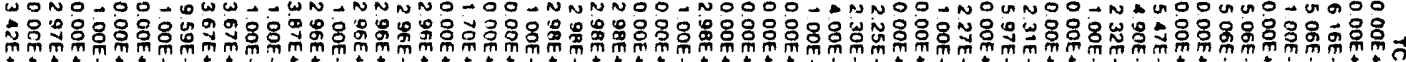

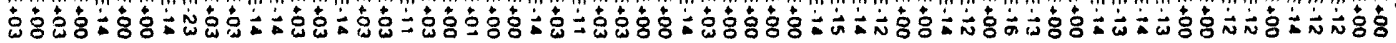

$-0-0-00-00-1-1-1-0000-00-1-1-00-10000-\omega 0000-000000-00000000-N 000$

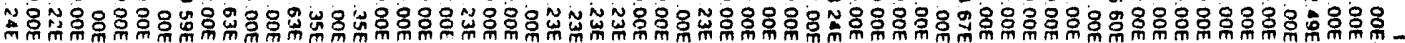

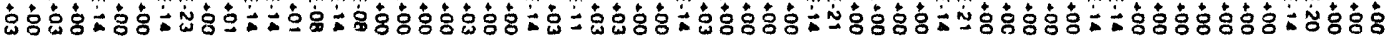

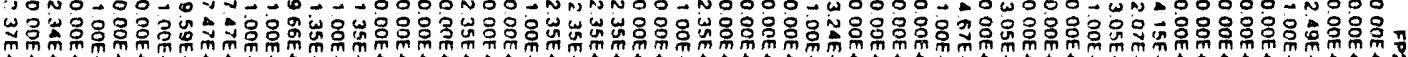

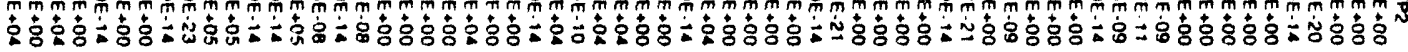

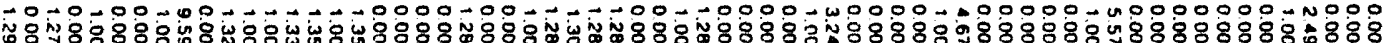

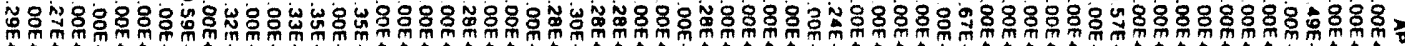

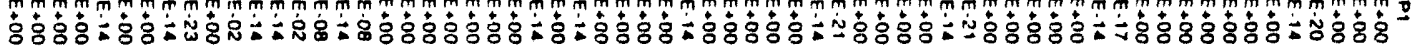

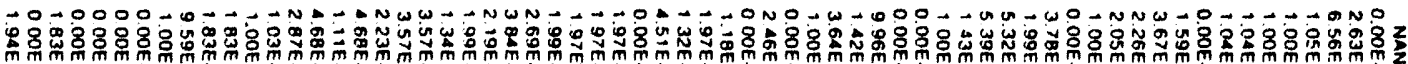

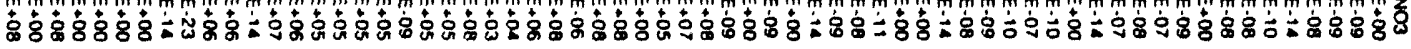

wowo-

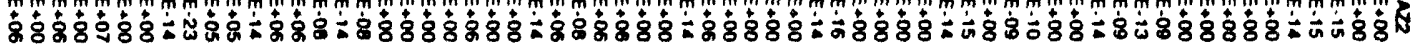

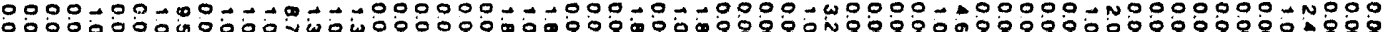

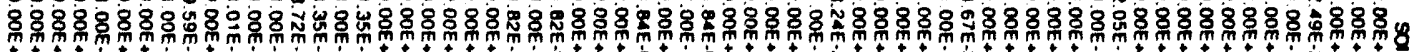

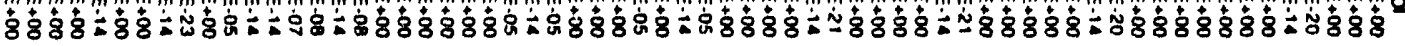

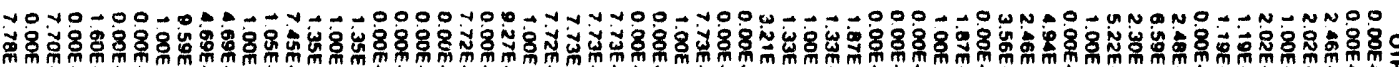

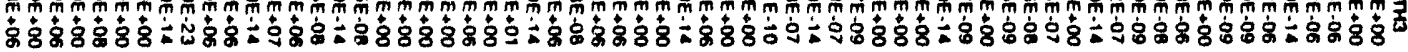

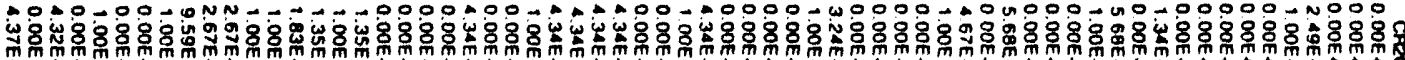

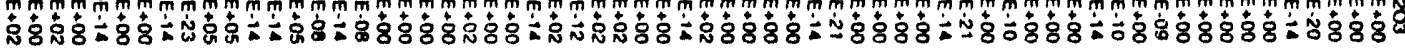

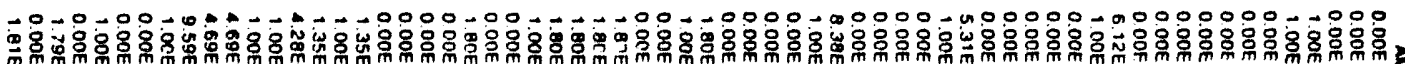

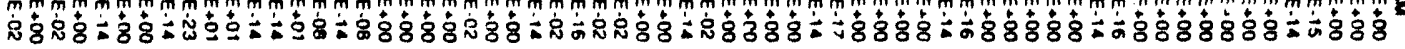


Table H3. ASPEN PLUS Global Model Mass Balance Output Results. (sheet 22 of 56)

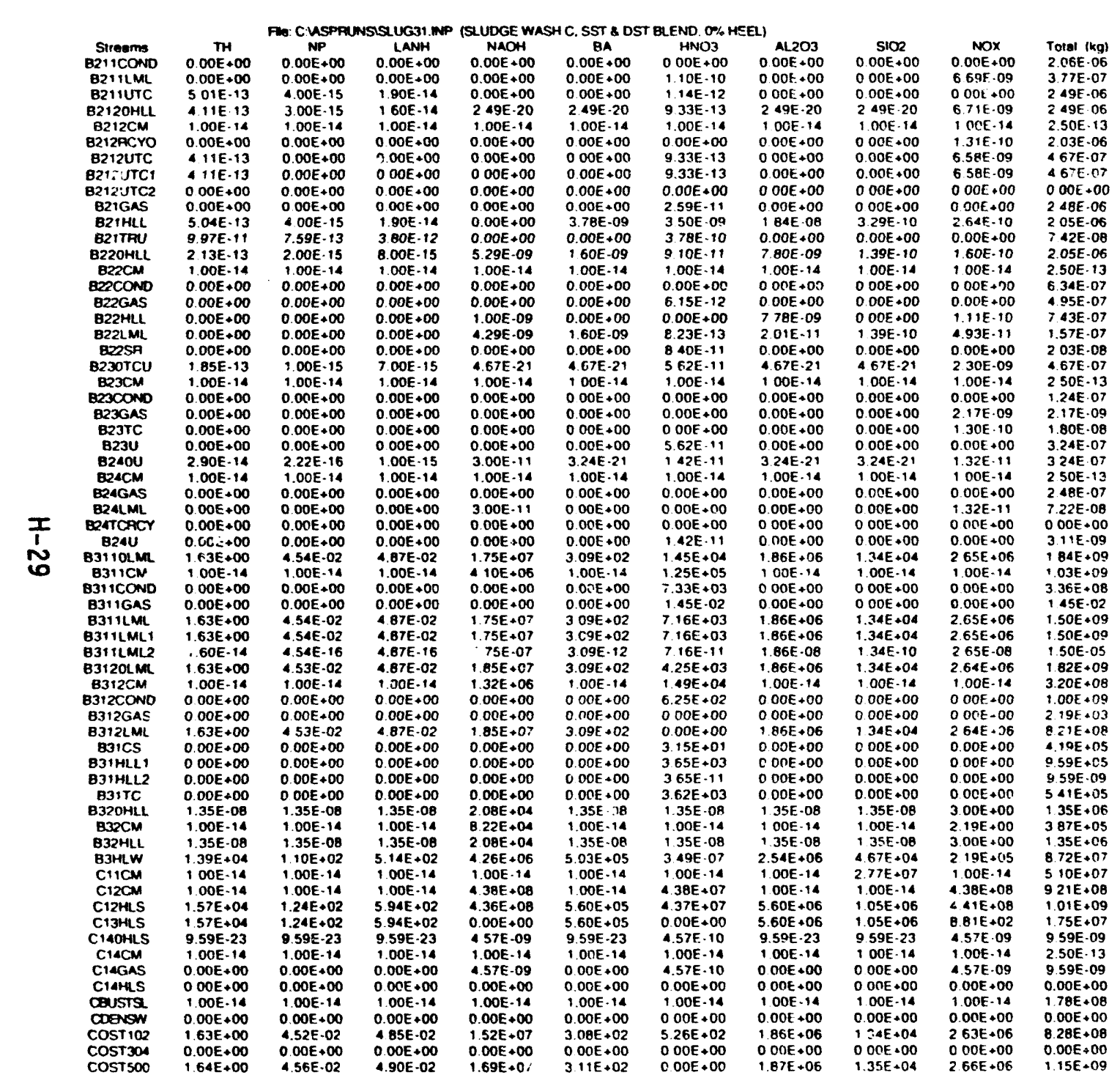




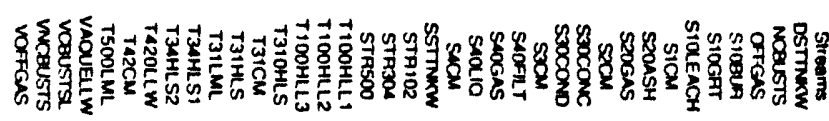

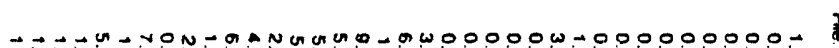

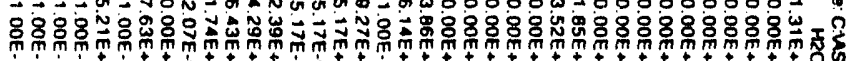

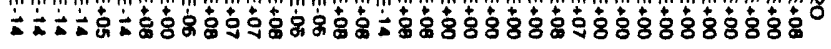

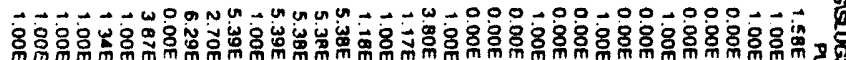

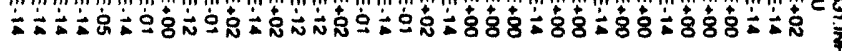

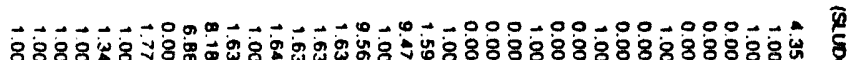

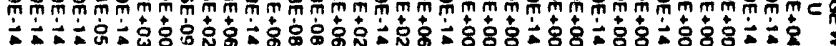

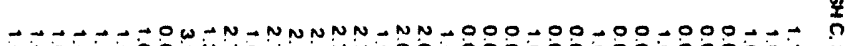

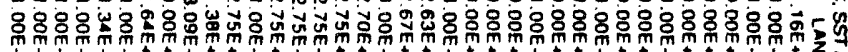

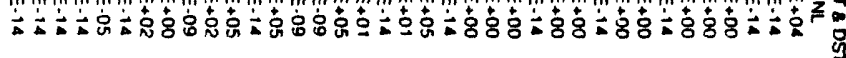

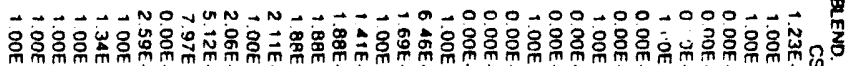

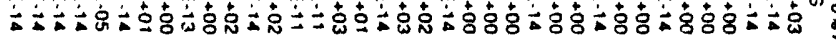

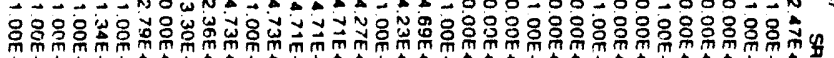

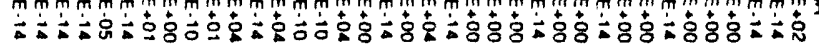

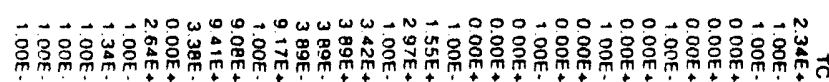

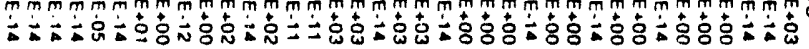

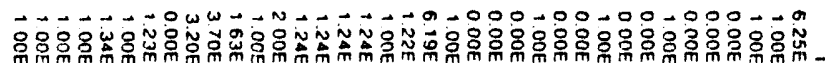

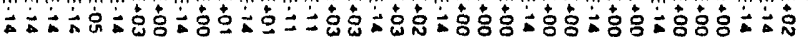

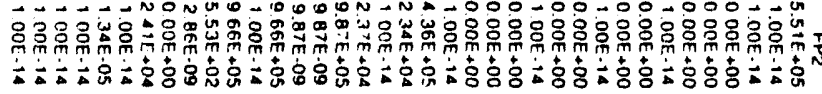

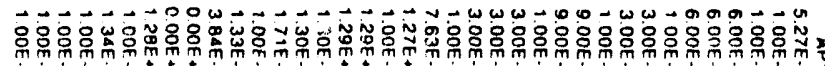

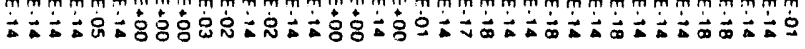

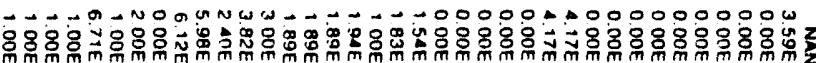

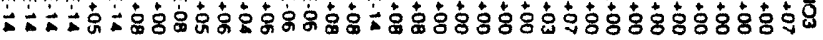

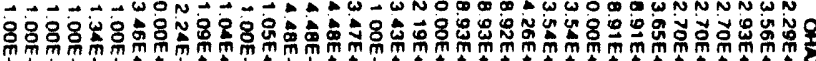

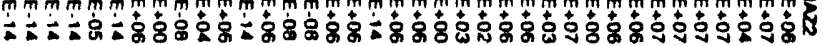

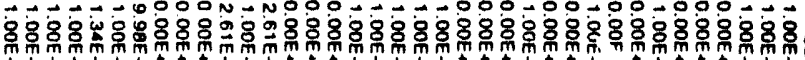

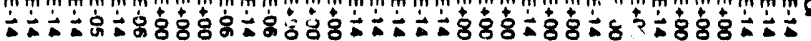

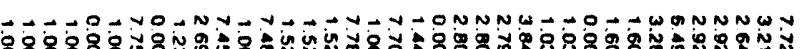

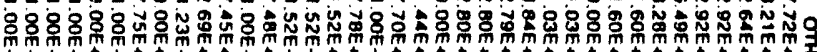

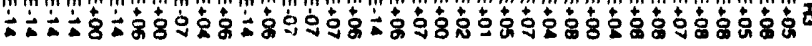

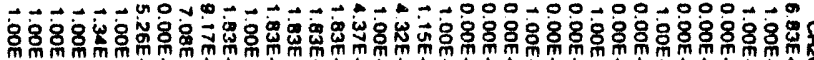

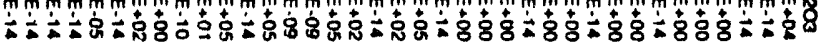

80

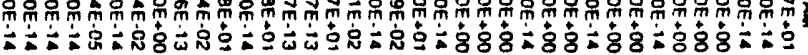




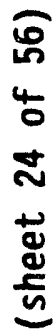

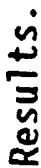

言

O

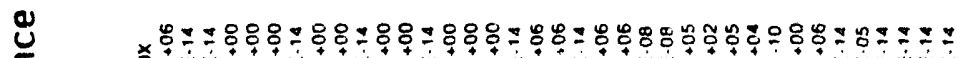

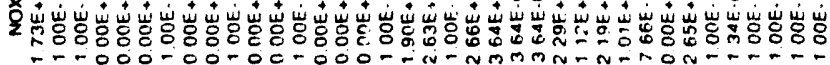

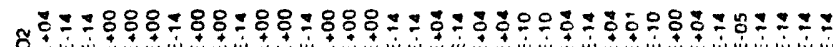

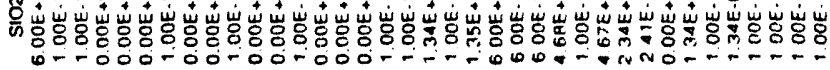

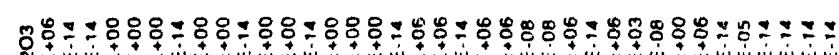

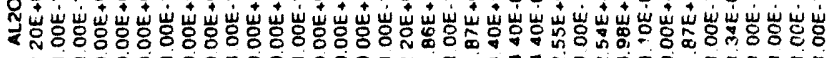
要

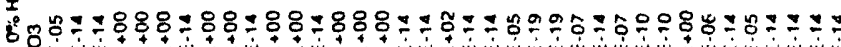

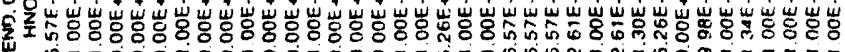

帝

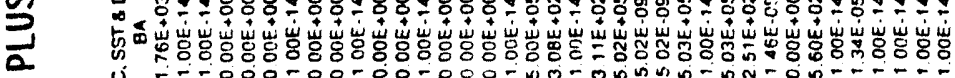

Z 意

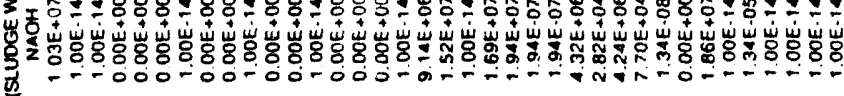

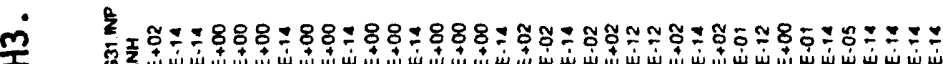

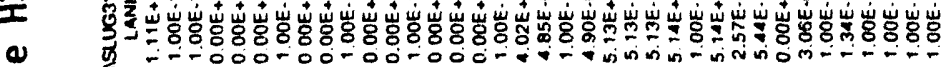
$\stackrel{\infty}{\stackrel{0}{\circ}}$

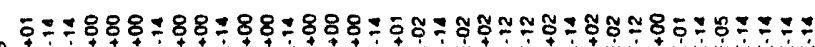

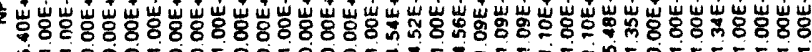

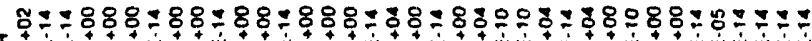

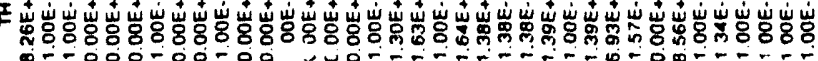

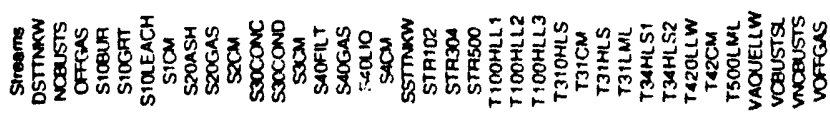


Table H3. ASPEN PLUS Global Model Mass Balance Output Results. (sheet 25 of 56)

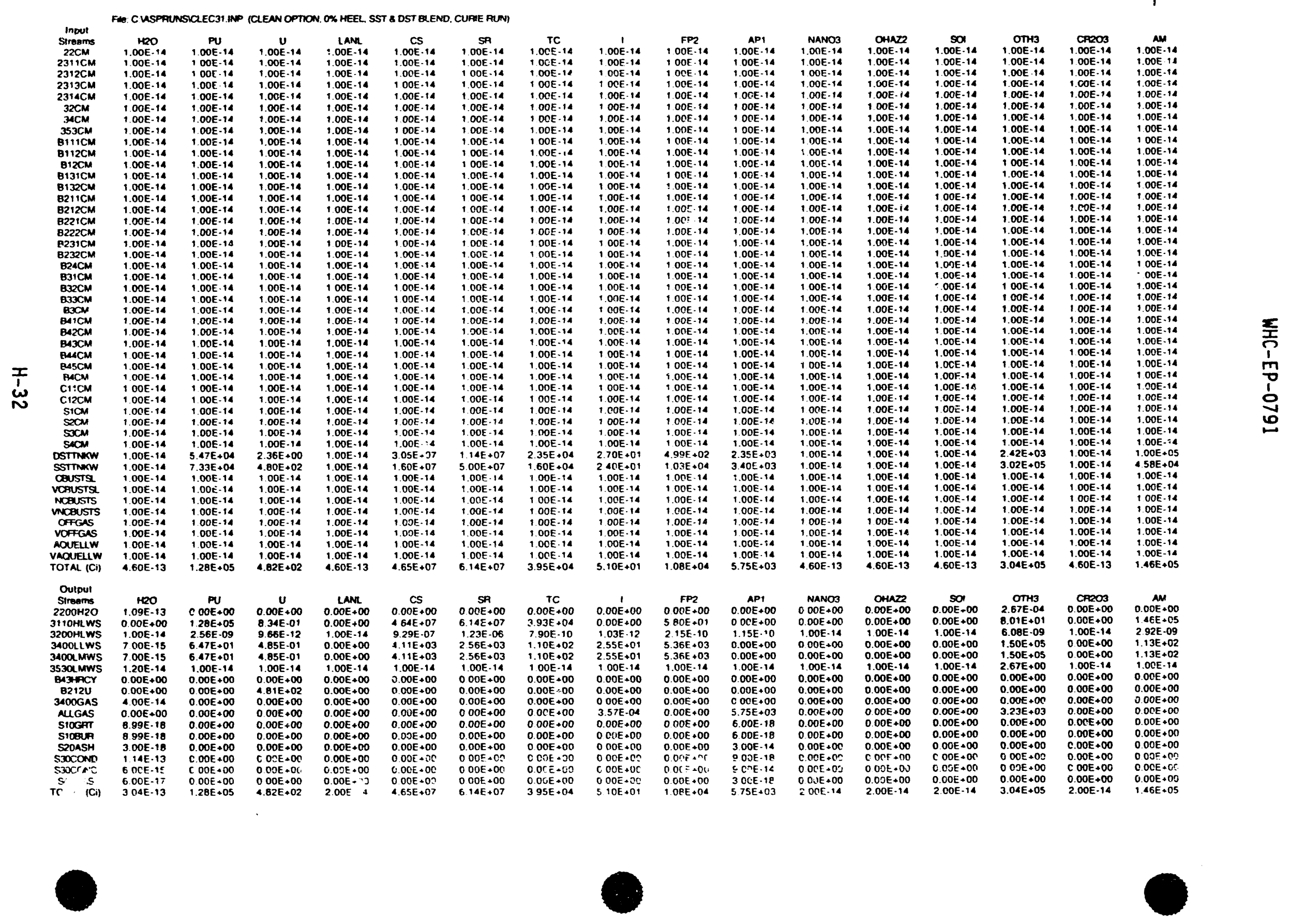


$\varepsilon \varepsilon-H$

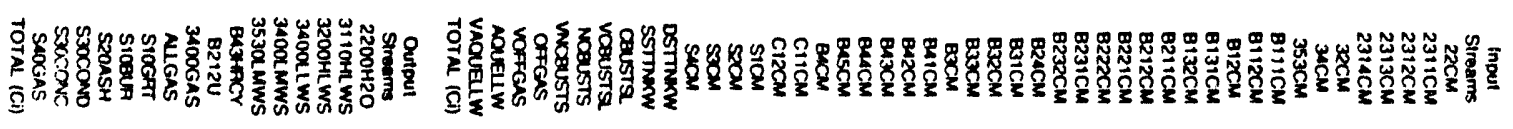

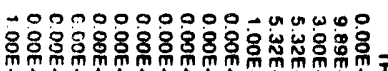

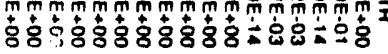

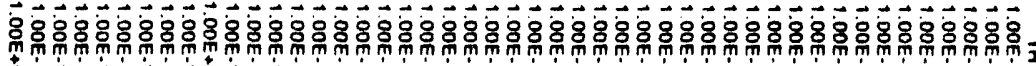

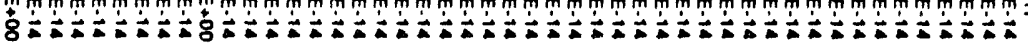

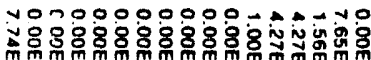

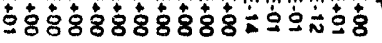

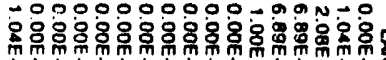

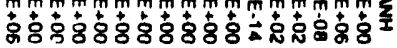

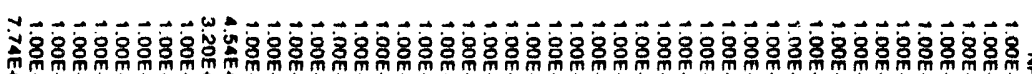

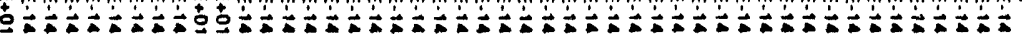

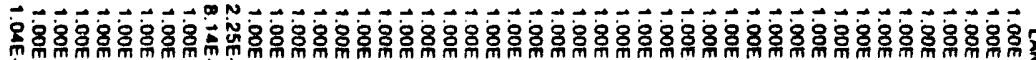

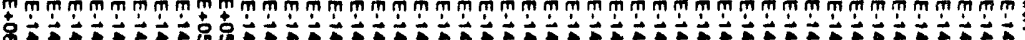

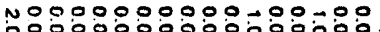

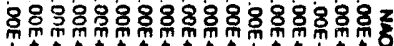

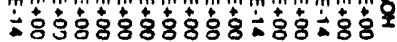

N $0000000000-00-00$

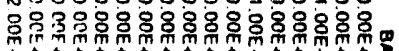

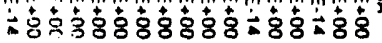

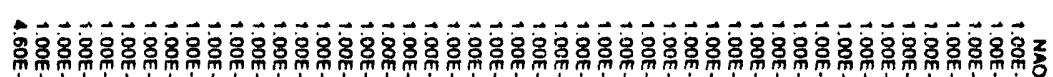

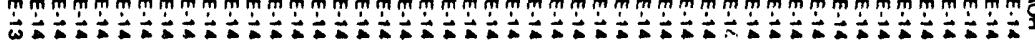

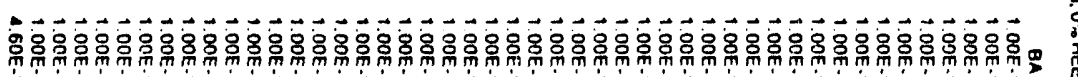

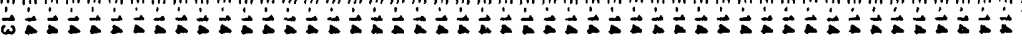
N2000000000-000 -00

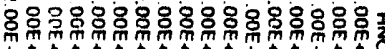

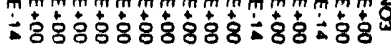

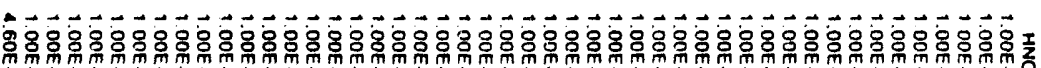

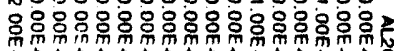

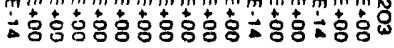

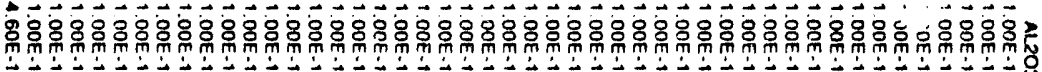

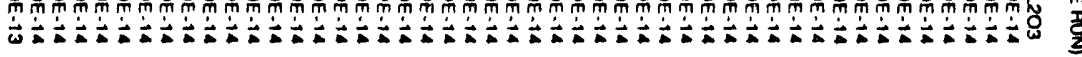

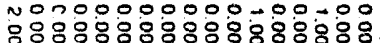

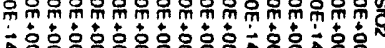

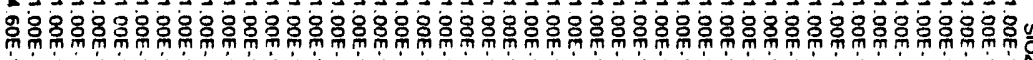

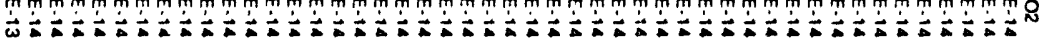

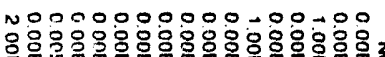

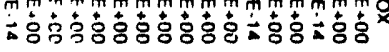

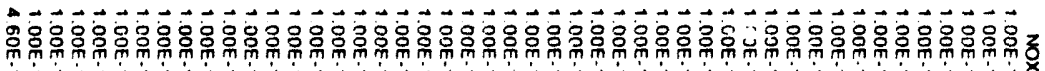

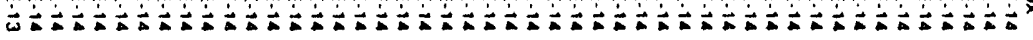

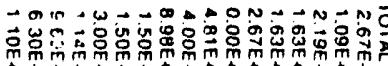

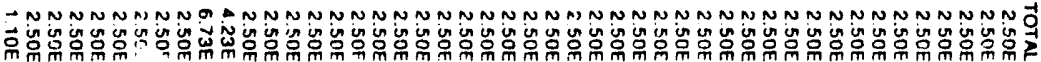

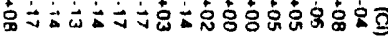

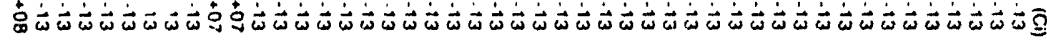




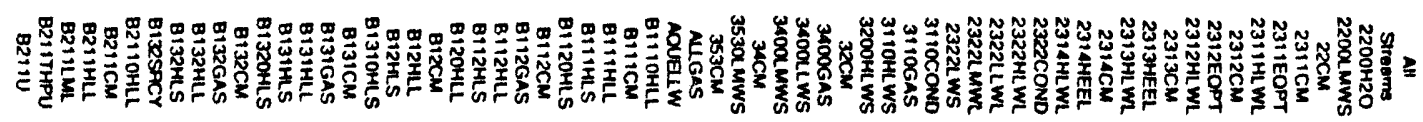

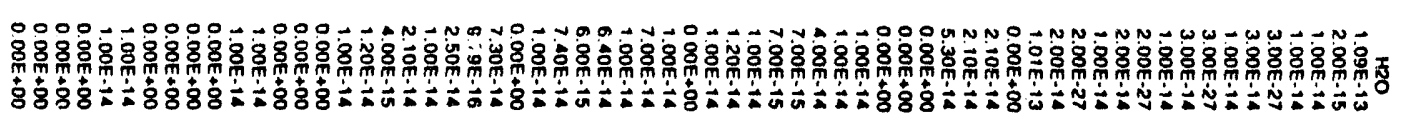

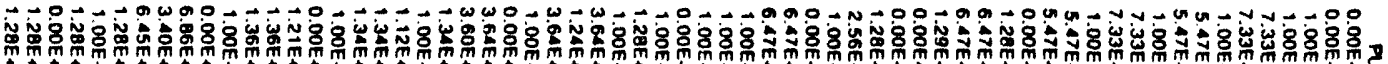

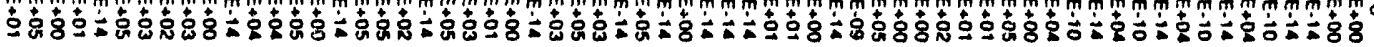

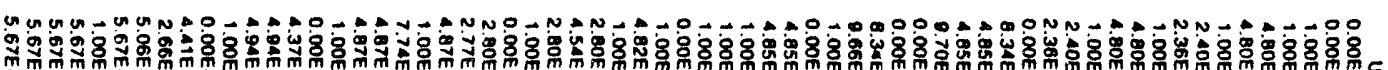

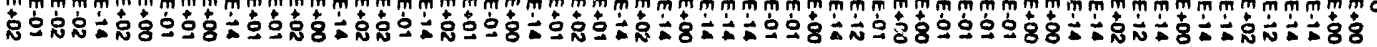

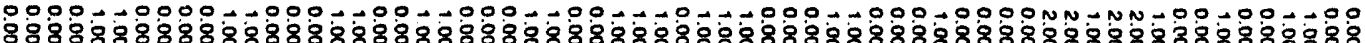

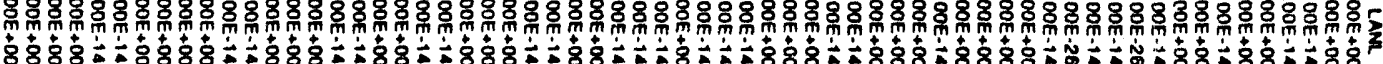

OOOH

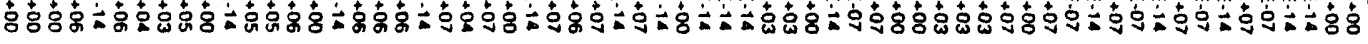

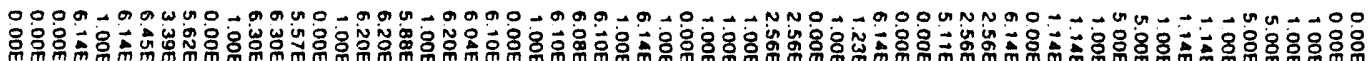

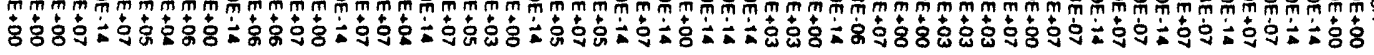

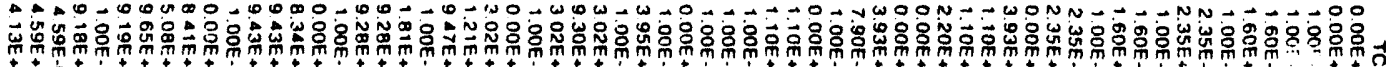

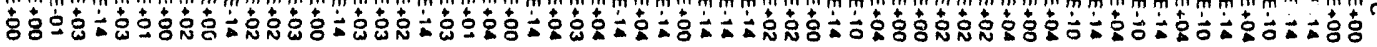

OOD N N n

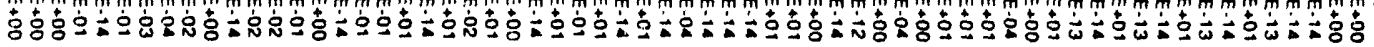

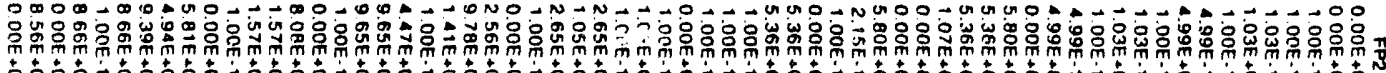

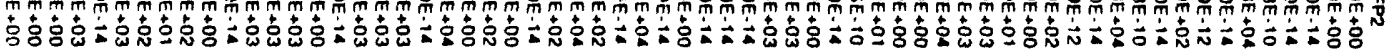

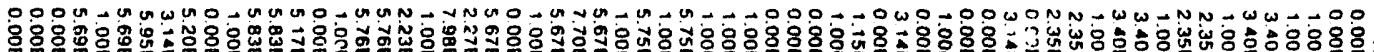

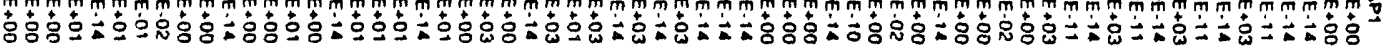

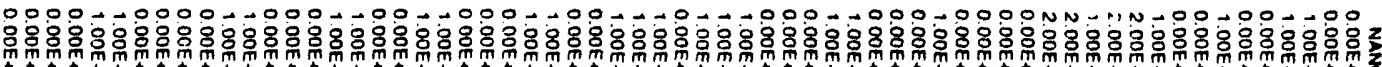

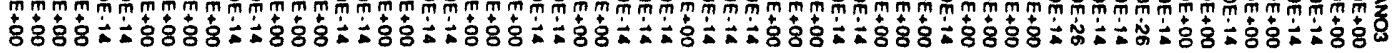

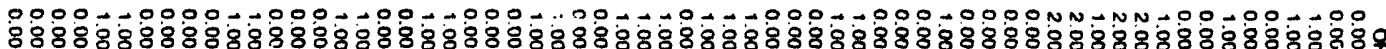

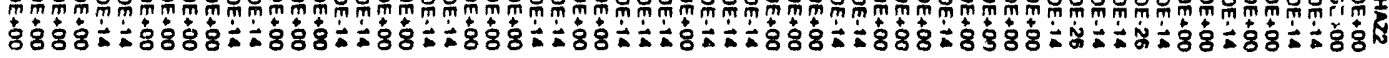

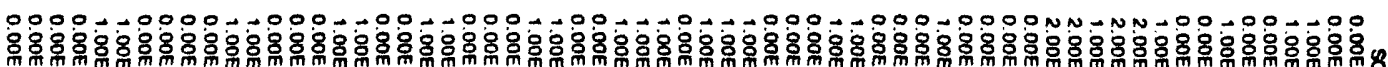

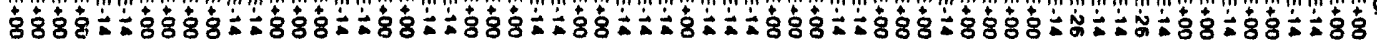

W

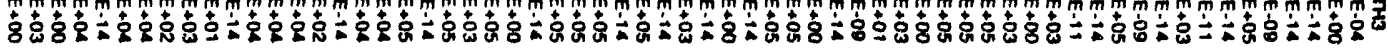

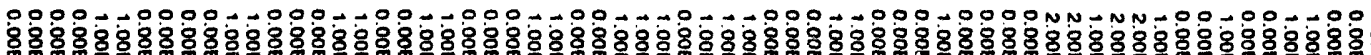
古古8

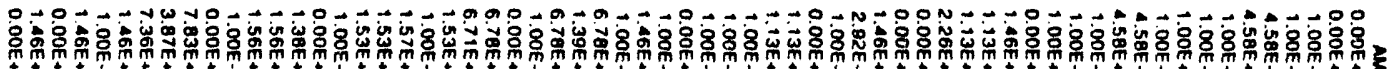

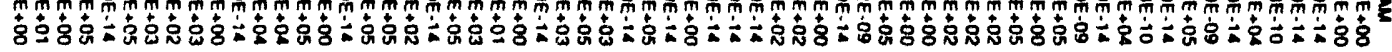




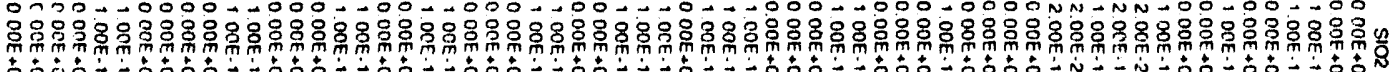

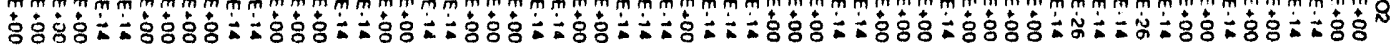

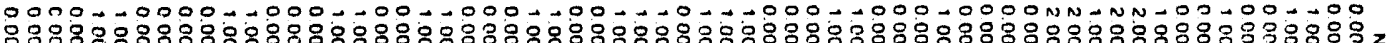

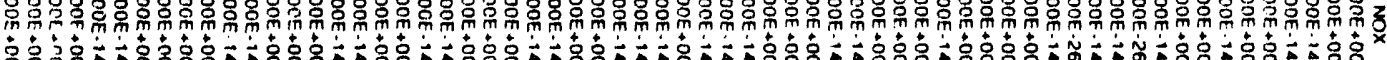

in

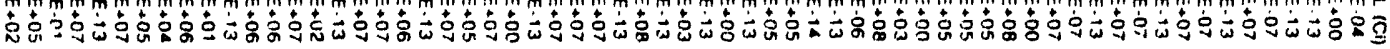




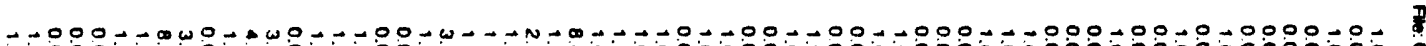

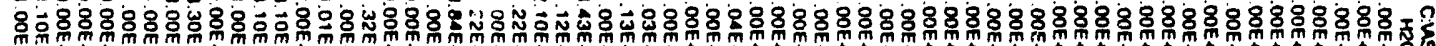

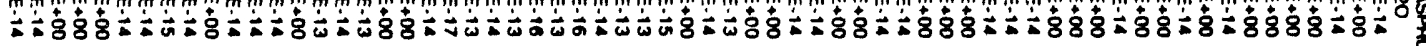

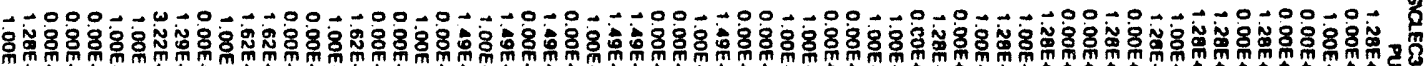

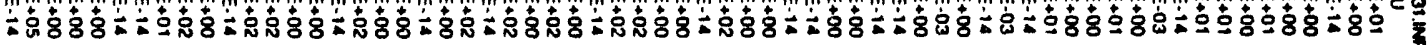

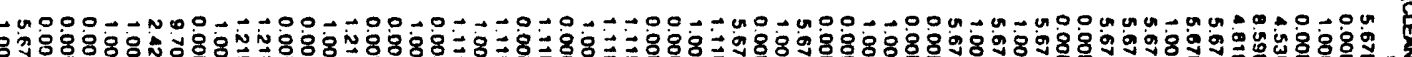

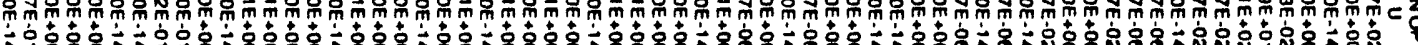
- $000-000-000-100-00-1000-1000-100-100-1000-1-000-00-0-0000-0-12$

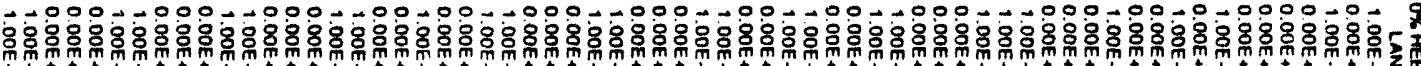

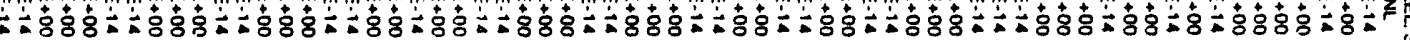

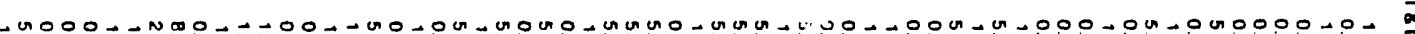

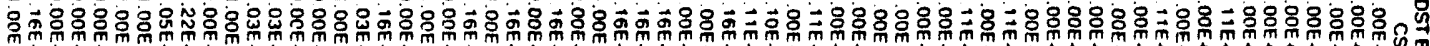

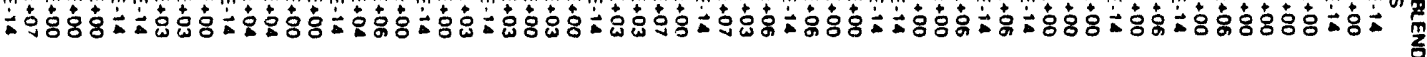

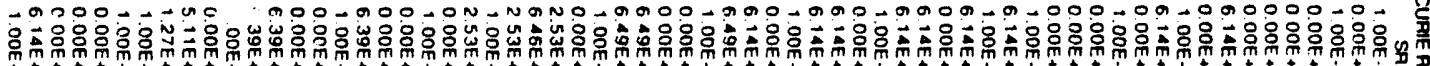

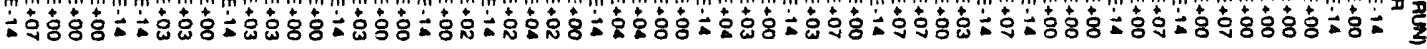

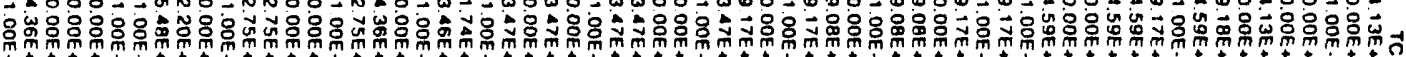

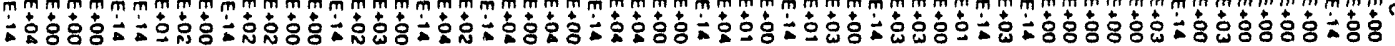

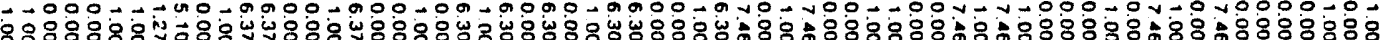

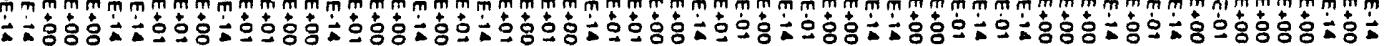

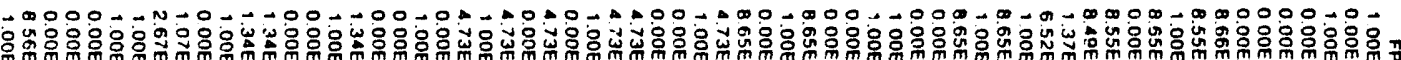

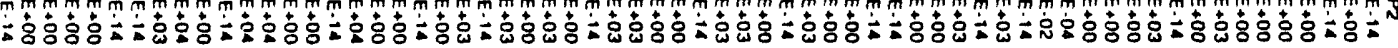

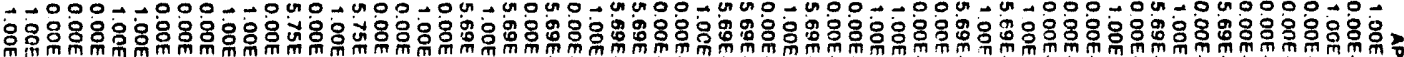

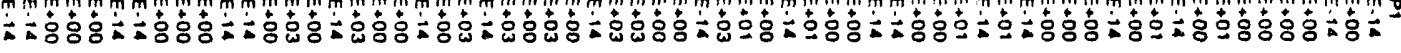

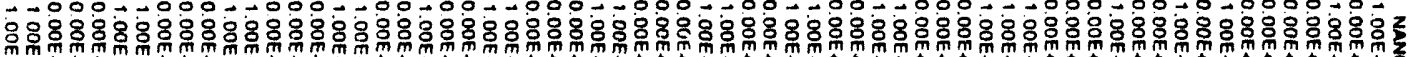

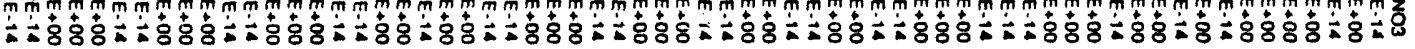

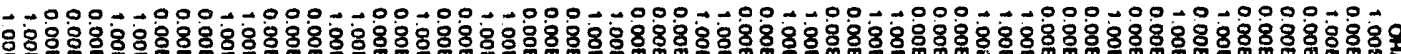

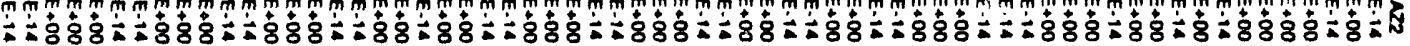

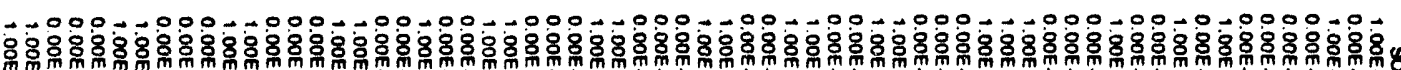

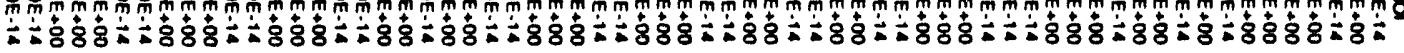

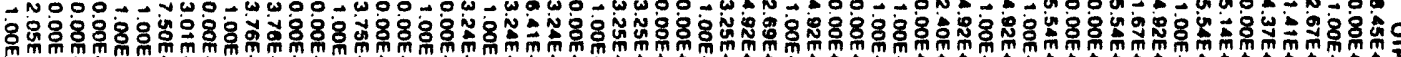

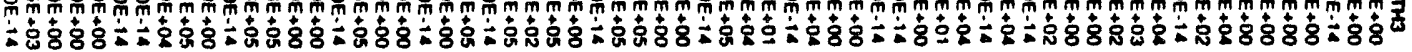

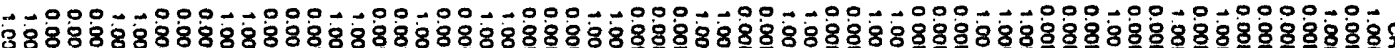

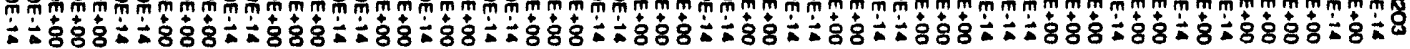

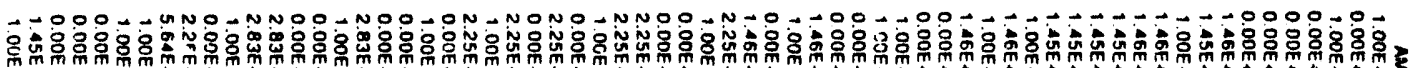

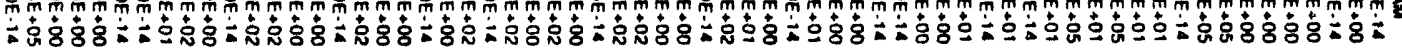


Table H3. ASPEN PLUS Global Model Mass Balance Output Results. (sheet 30 of 56)

\begin{tabular}{|c|c|c|c|c|c|c|c|c|c|c|}
\hline 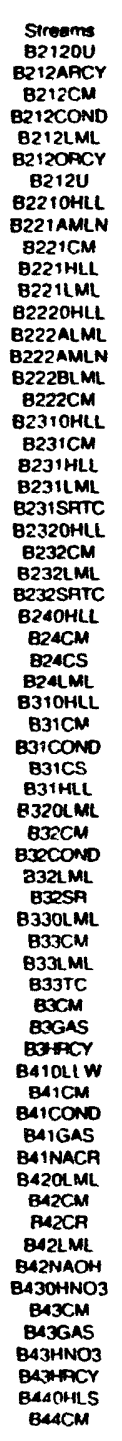 & 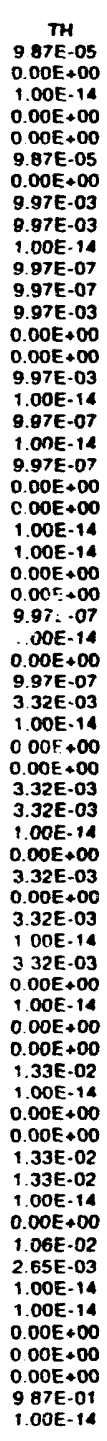 & 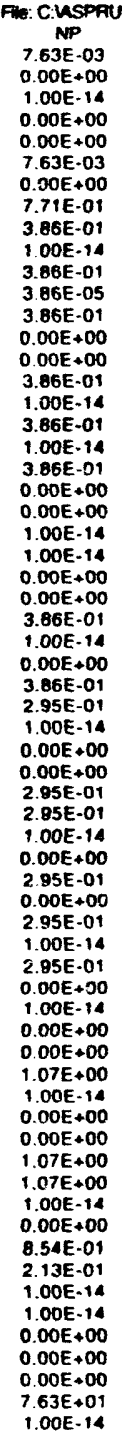 & 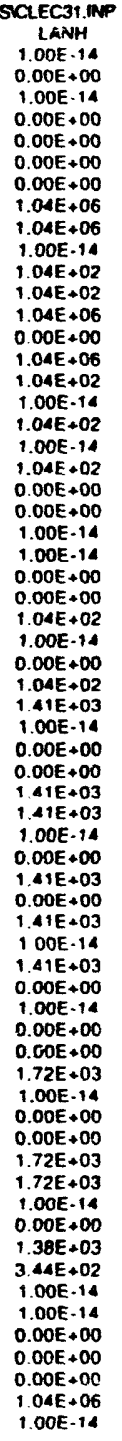 & 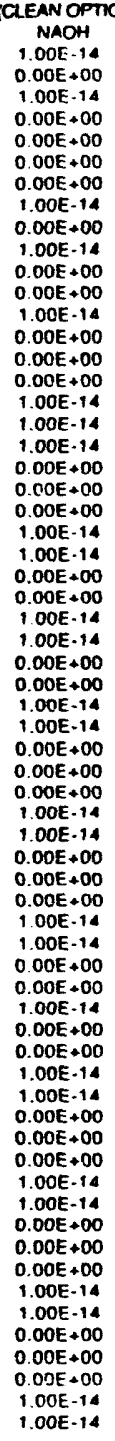 & 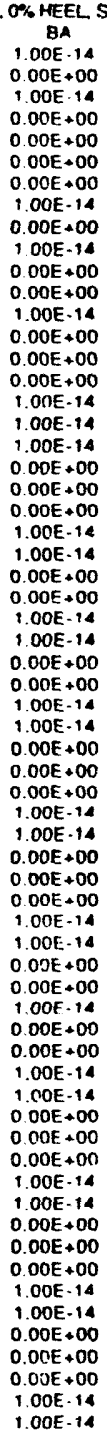 & 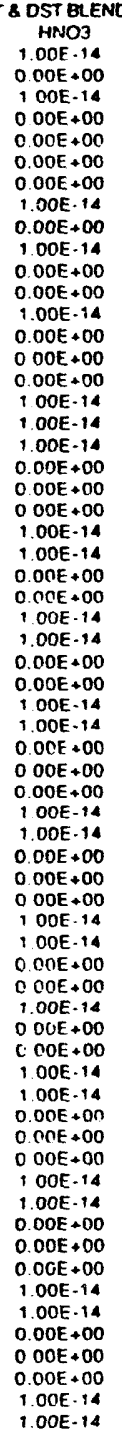 & 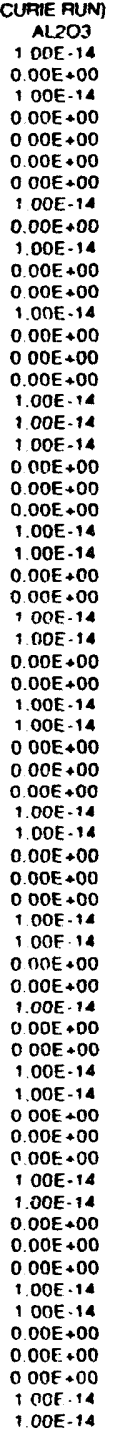 & 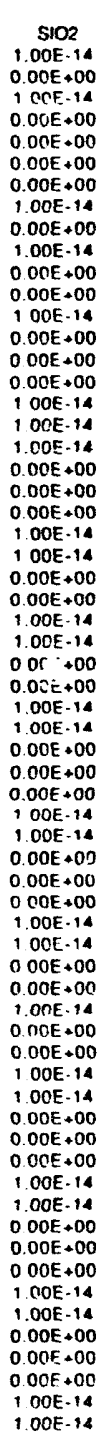 & 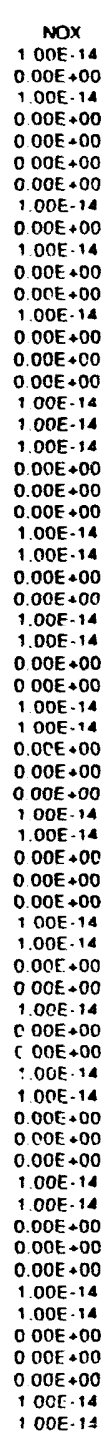 & 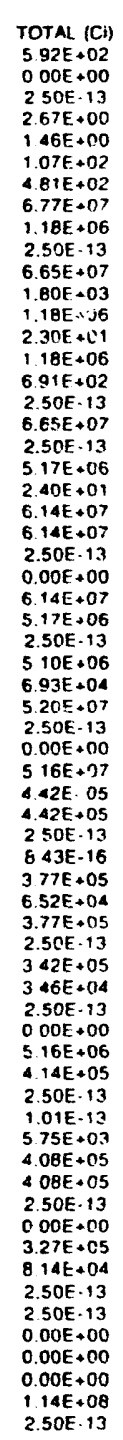 \\
\hline
\end{tabular}




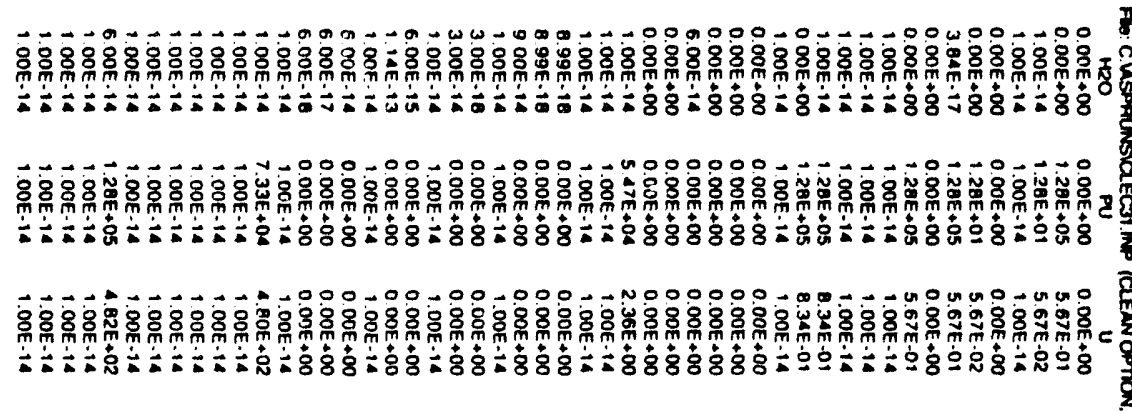

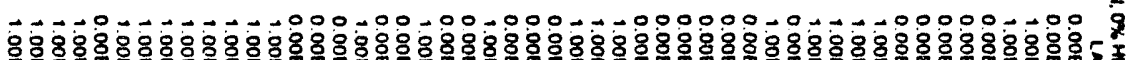

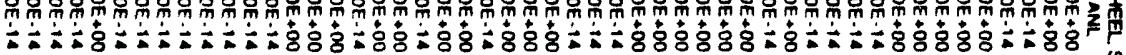

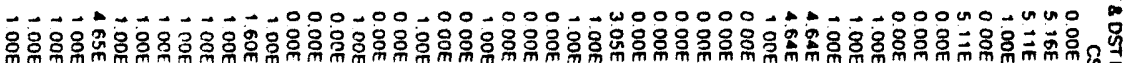

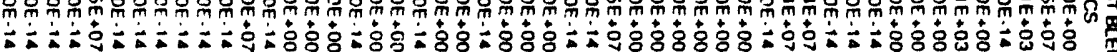

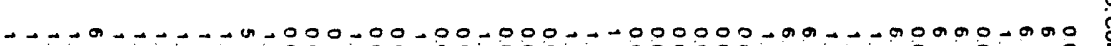

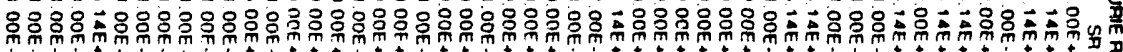

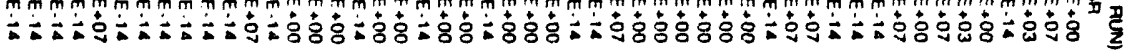

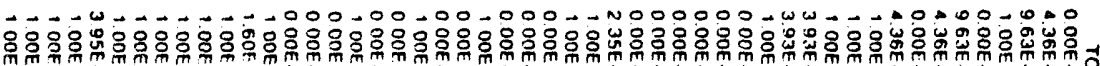

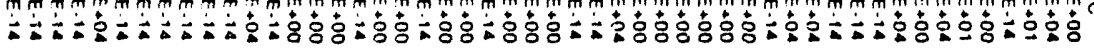

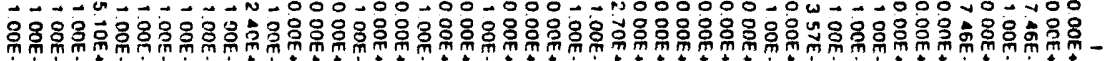

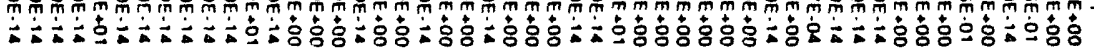

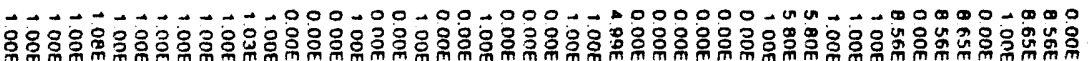

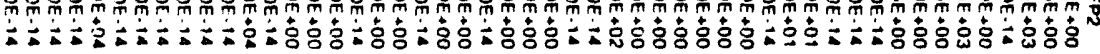

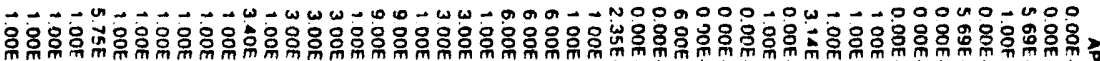

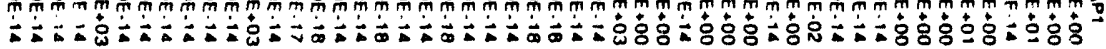

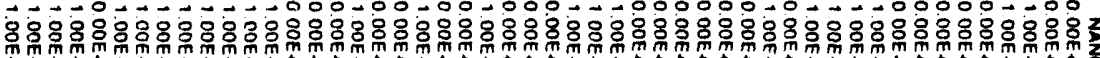

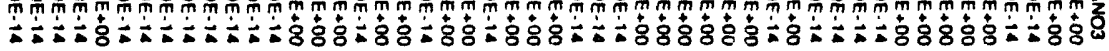

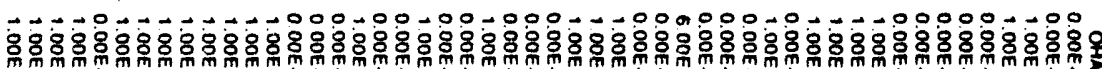

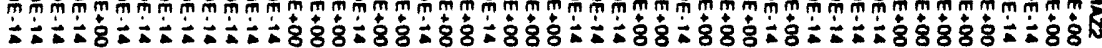

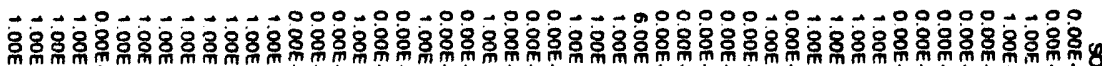

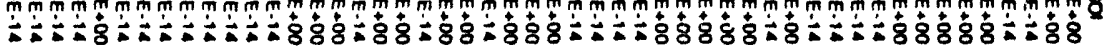

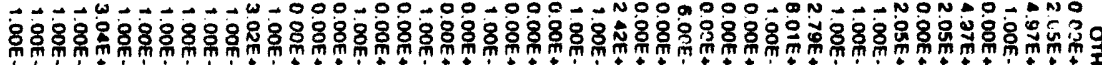

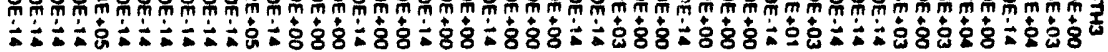

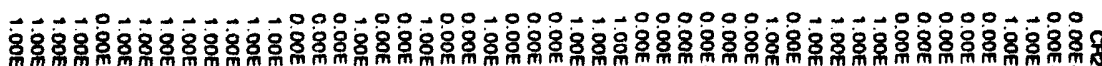

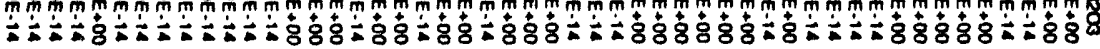

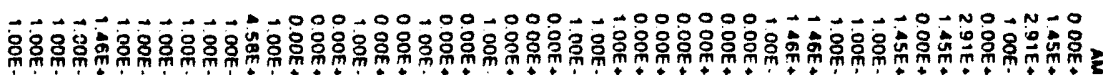

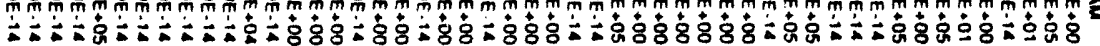




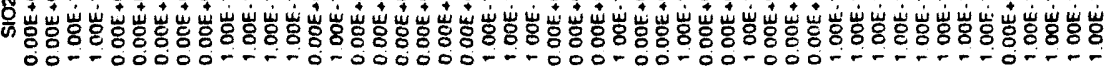
承

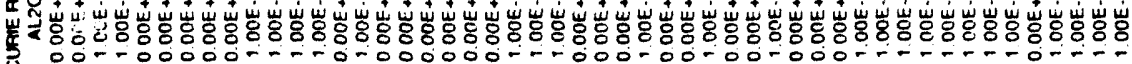
$\dot{0}$ 要

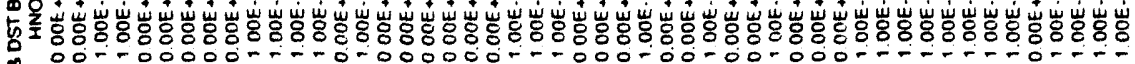
5

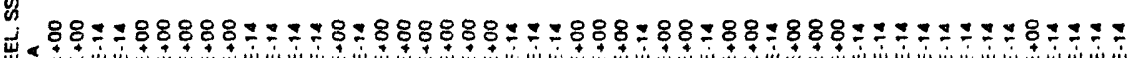

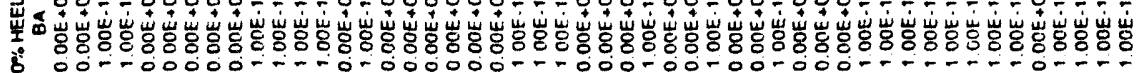
i

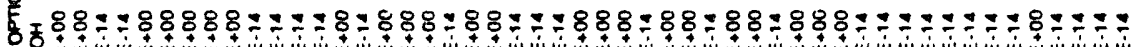

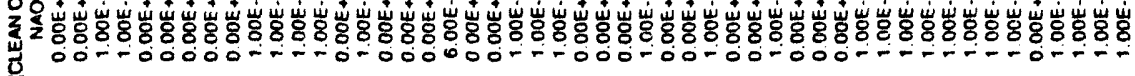

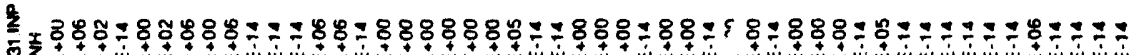

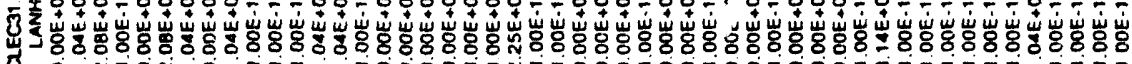

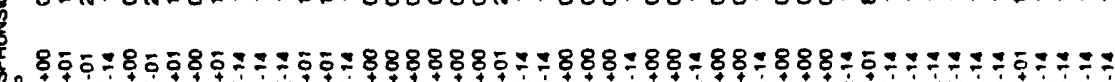

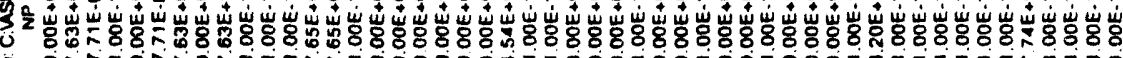

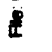

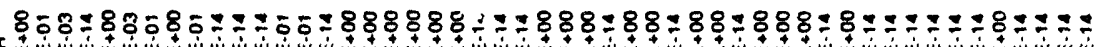

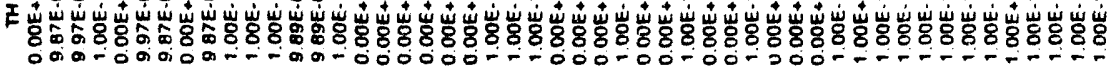

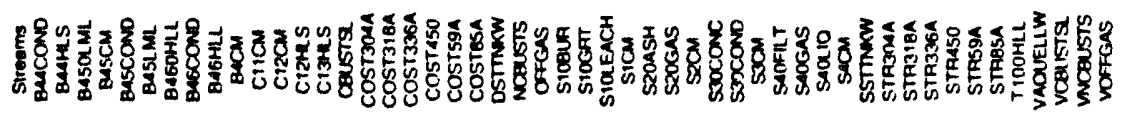


Table H3. ASPEN PLUS Global Model Mass Balance Output Results. (sheet 33 of 56)

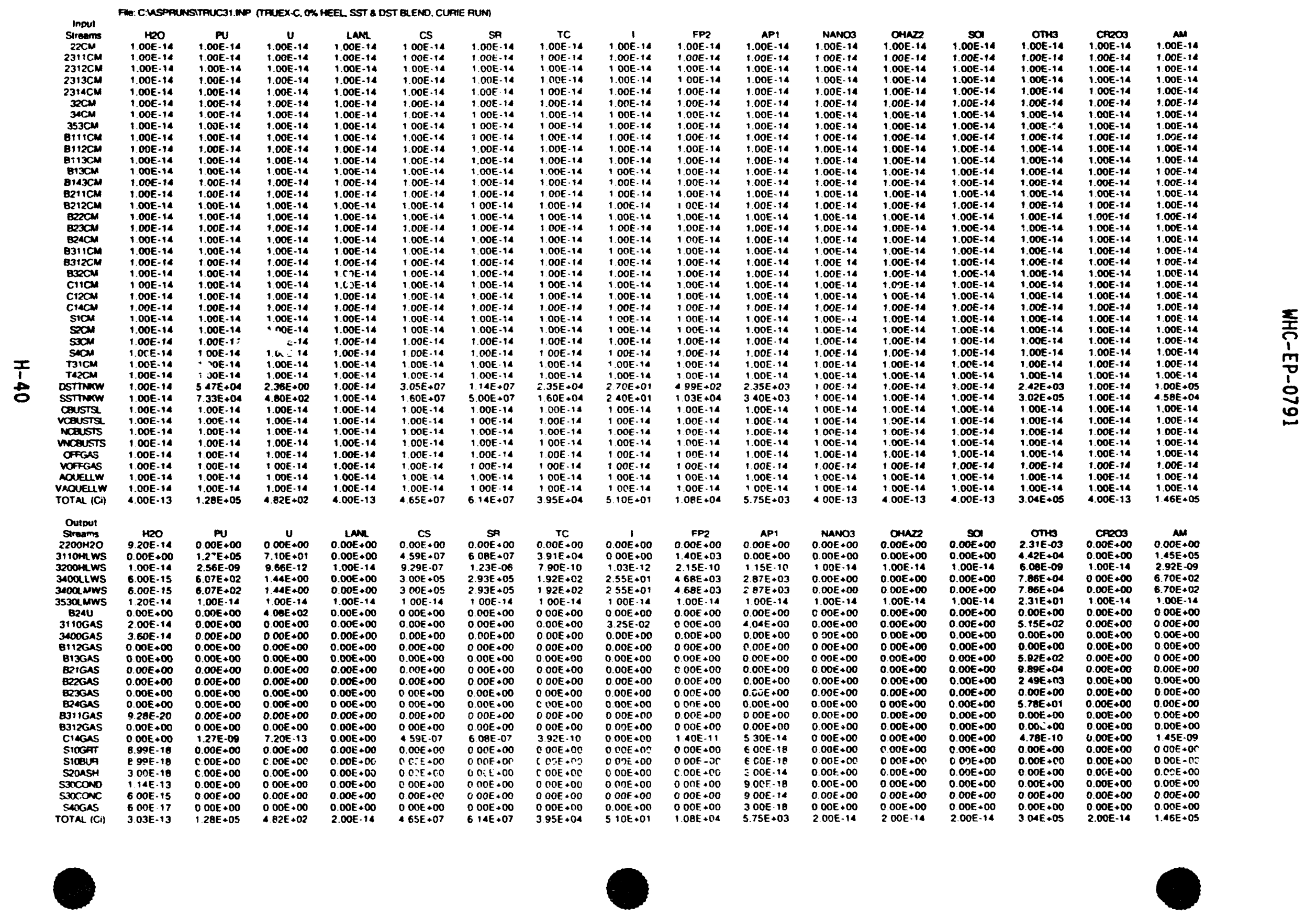




\section{Table H3. ASPEN PLUS Global Model Mass Balance Output Results. (sheet 34 of 56)}

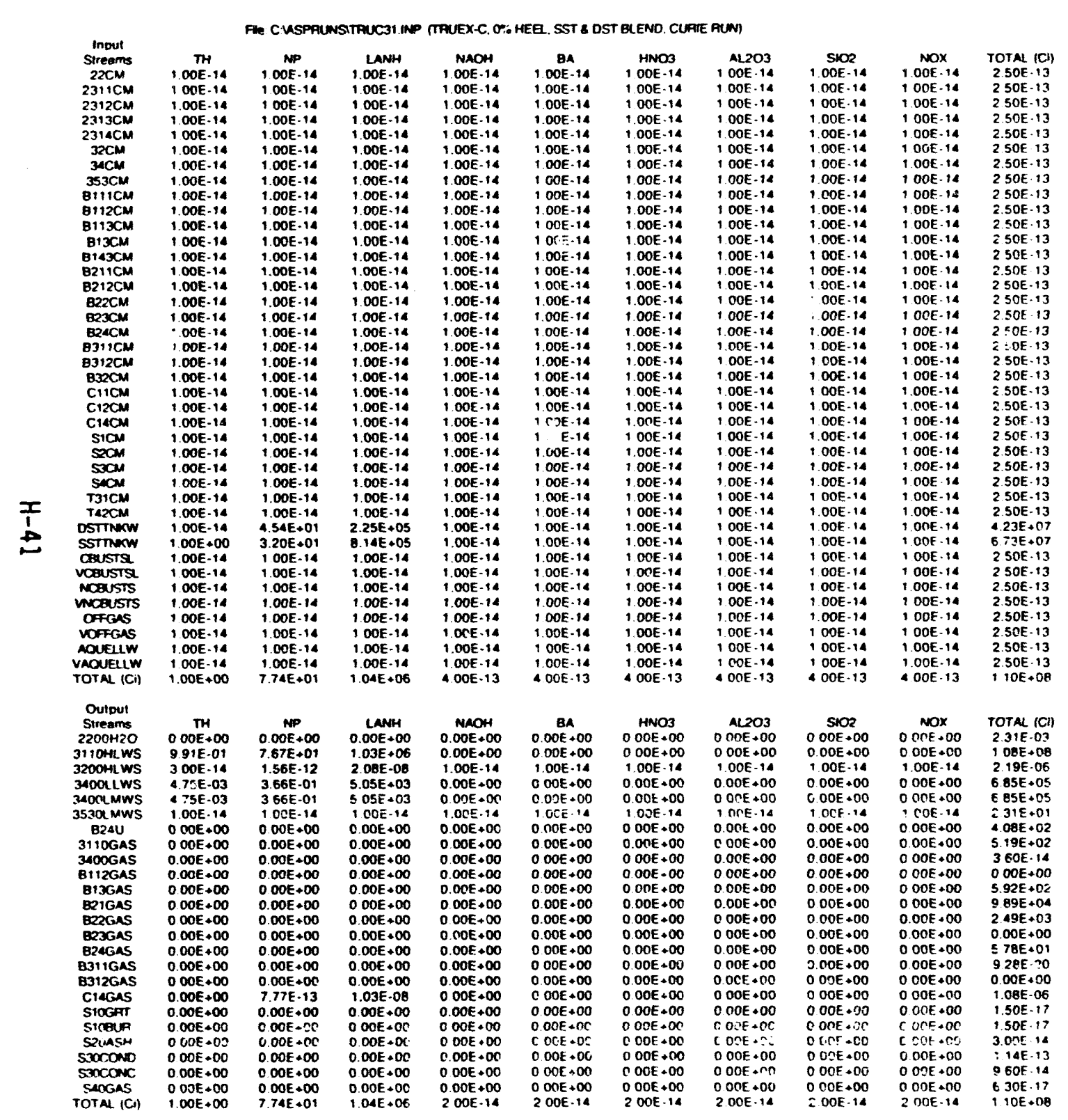




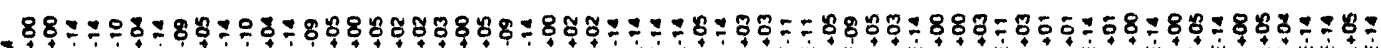

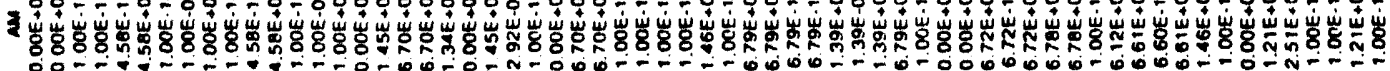

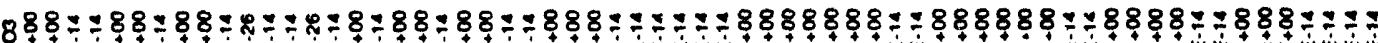

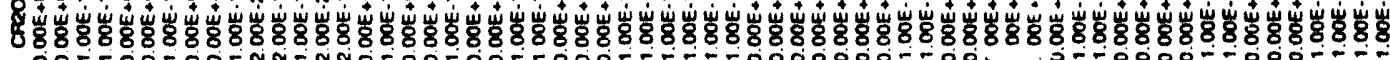

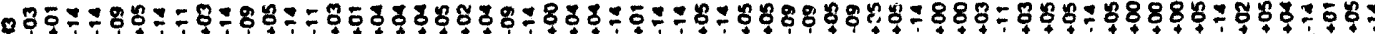

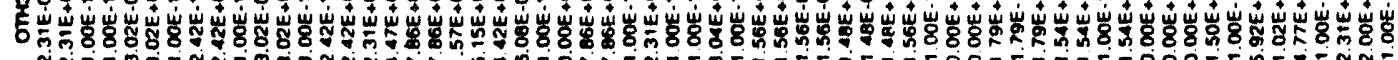

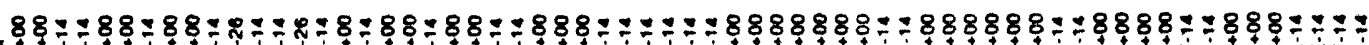

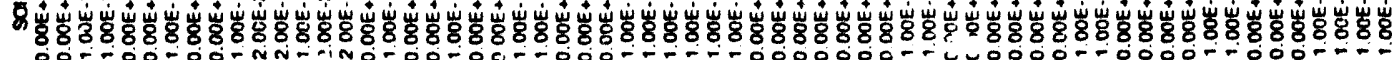

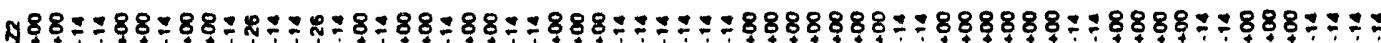

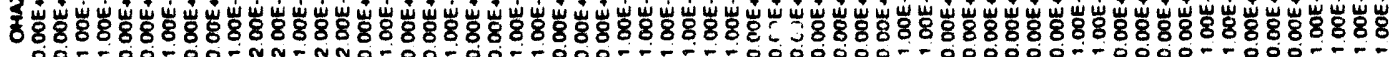

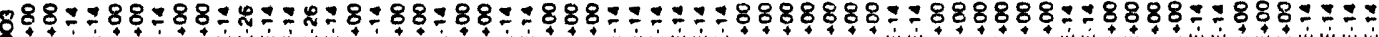

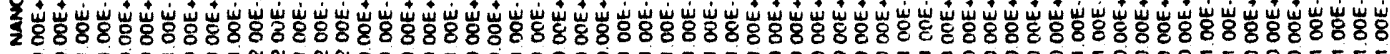

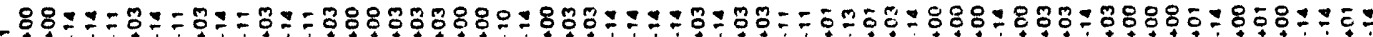

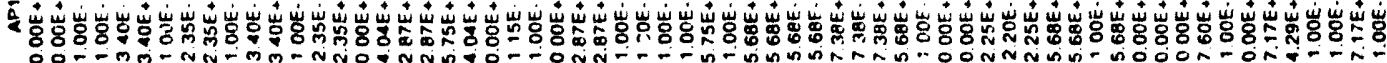
8

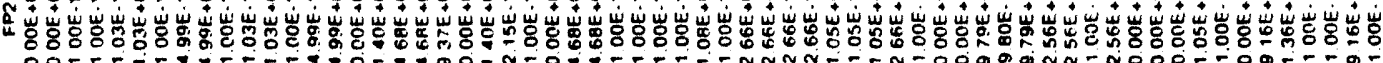

8

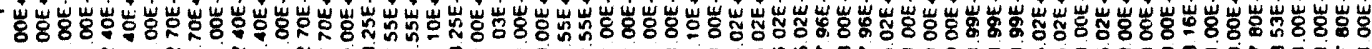

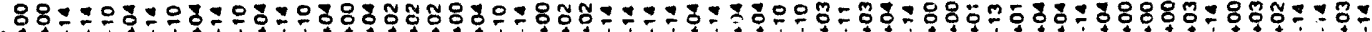

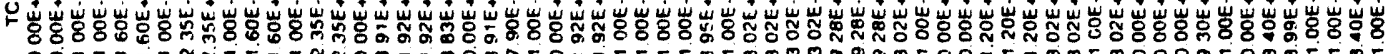

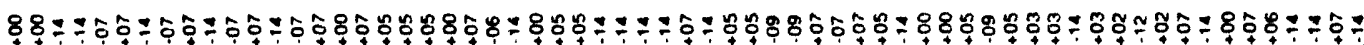
₹

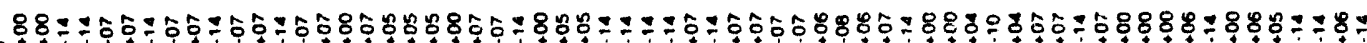

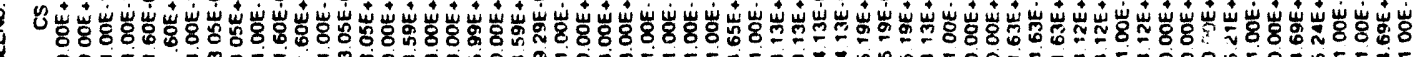

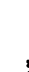

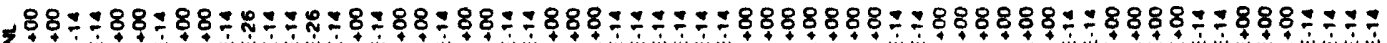

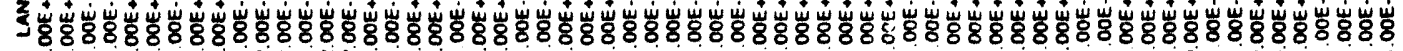

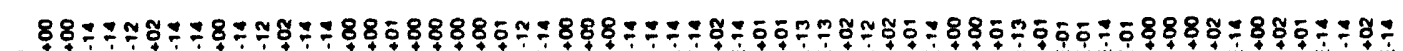

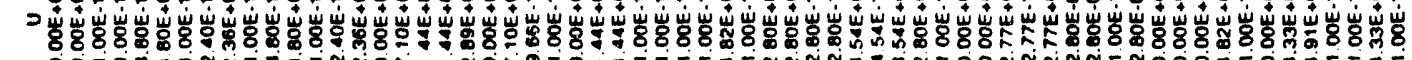

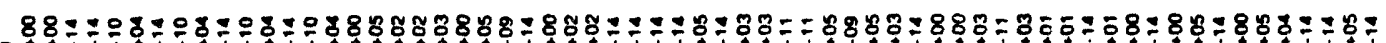

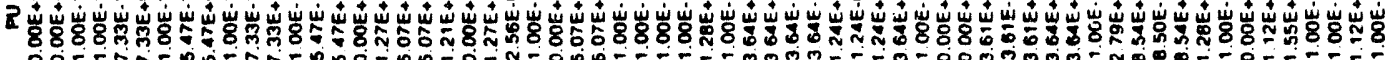

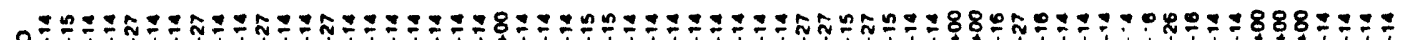
8

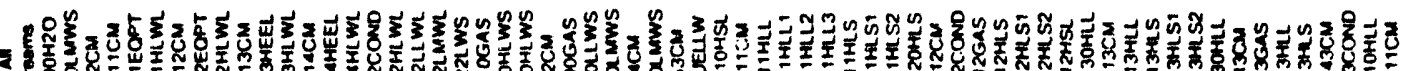

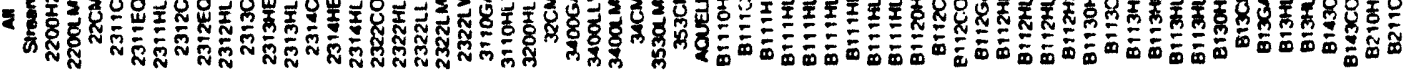




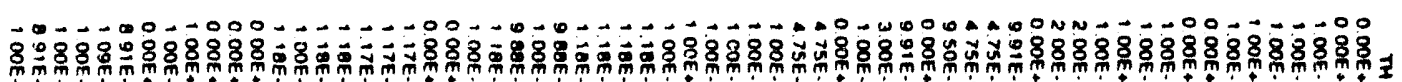

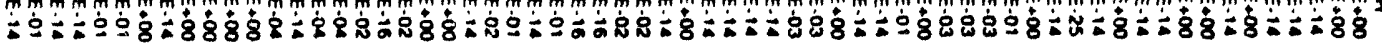

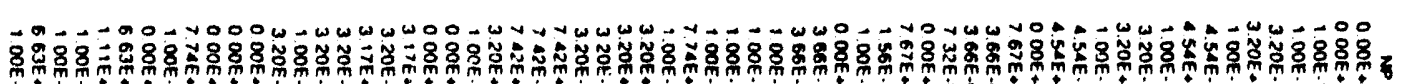

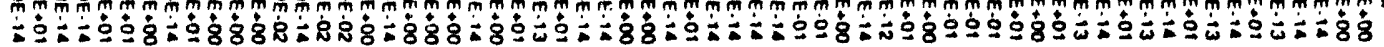

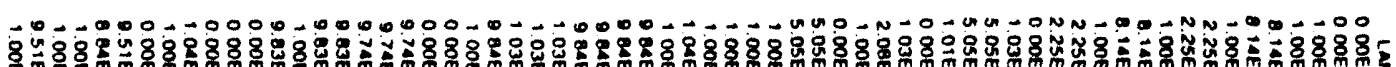

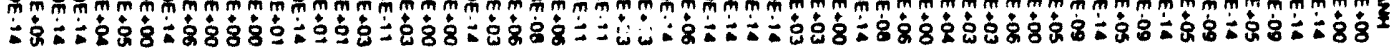

$\frac{0}{0}$

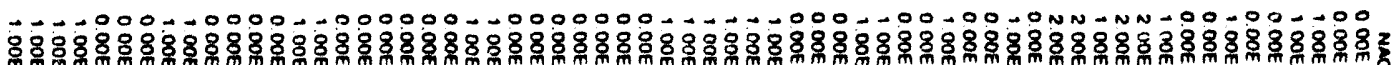

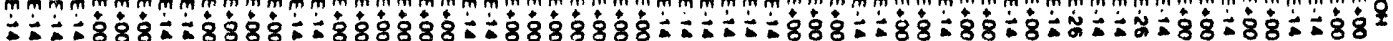

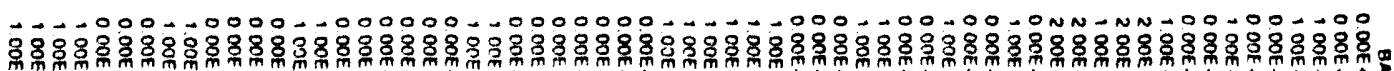

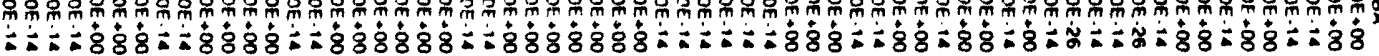

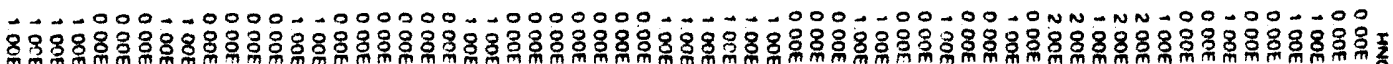

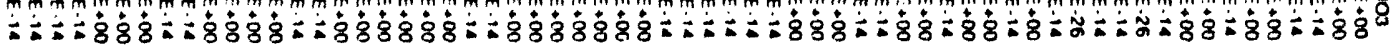

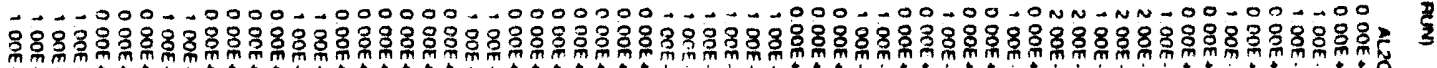

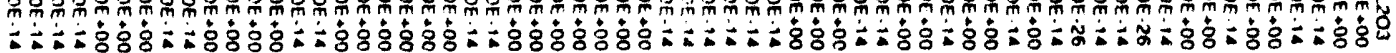

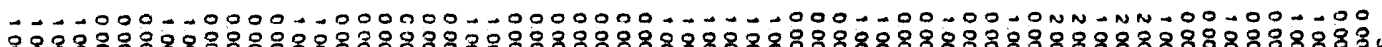

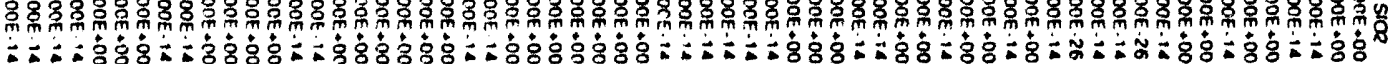

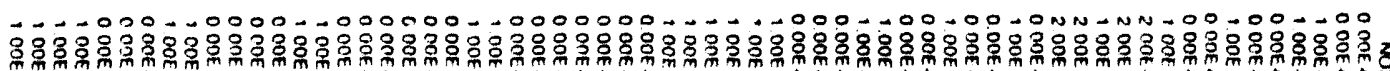

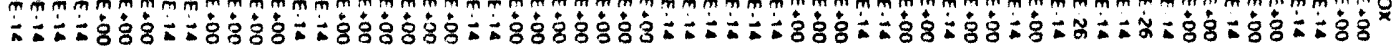

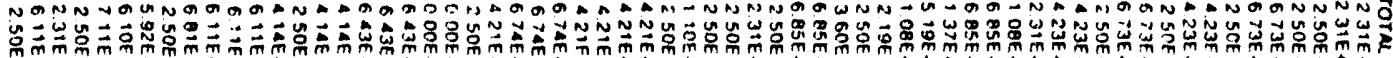

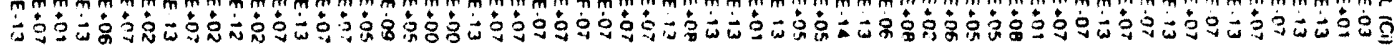


$880 \%$

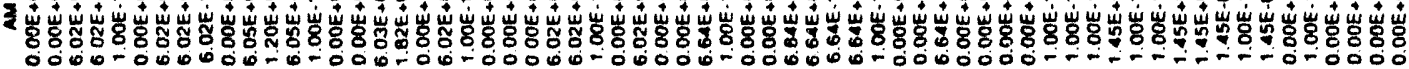

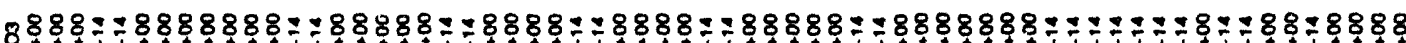

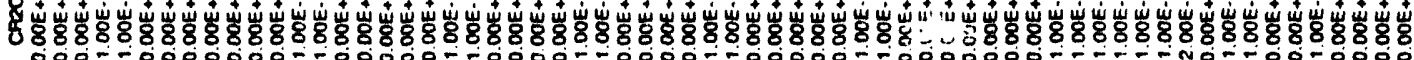

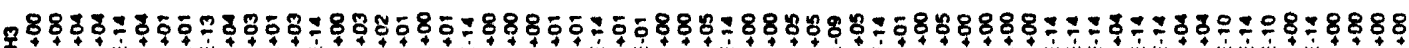

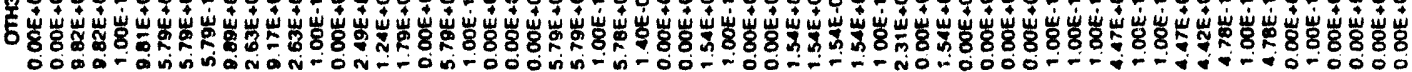

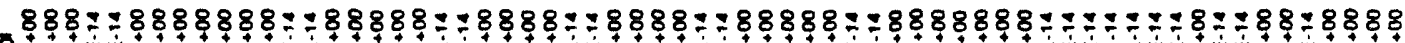

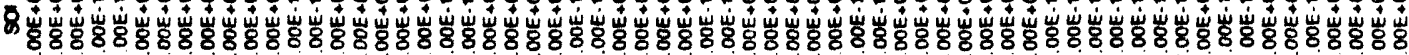

우운

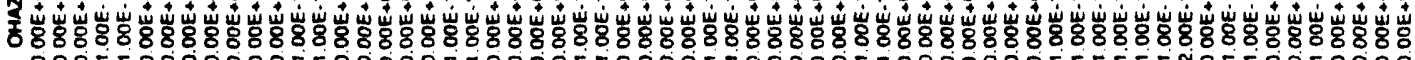

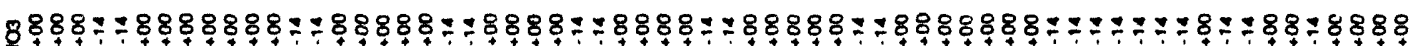

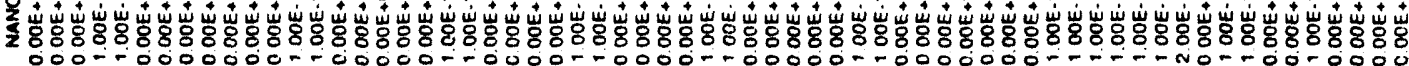

- 우운

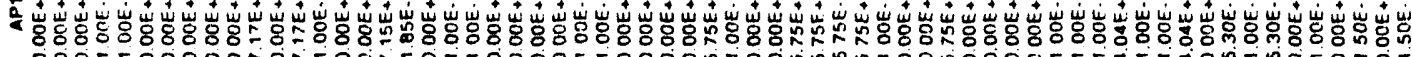

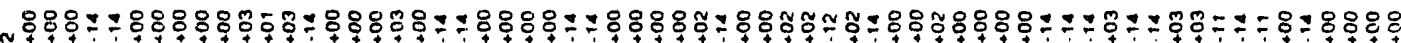

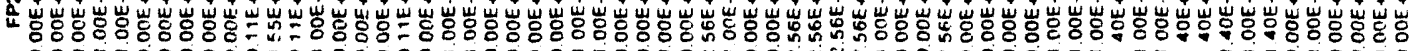

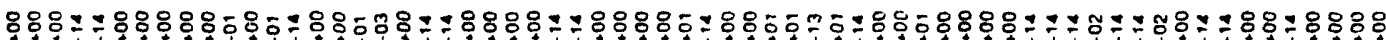

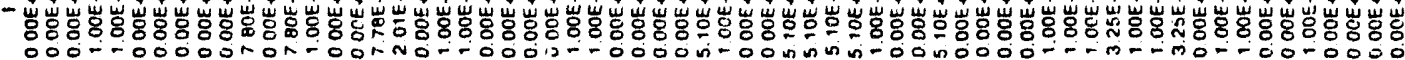

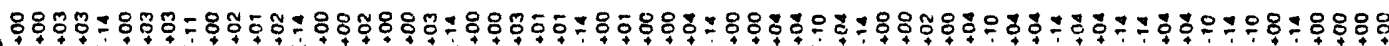

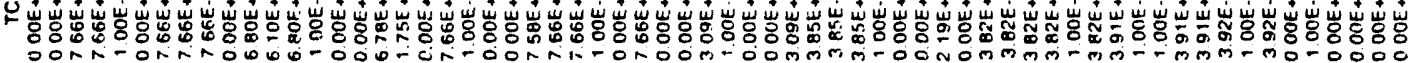

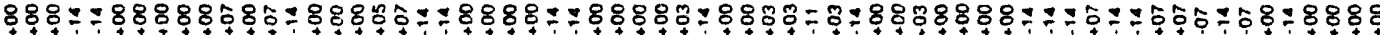

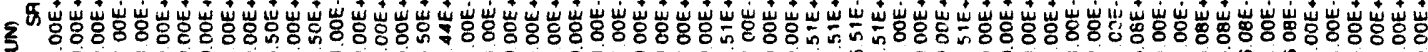

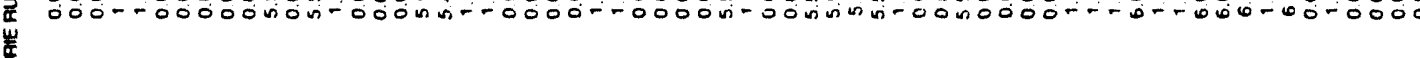

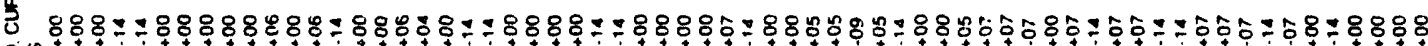

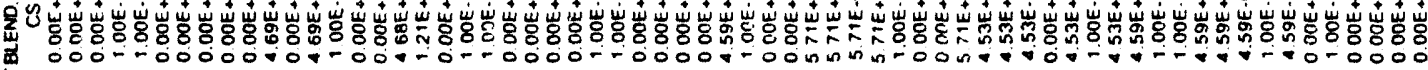
要

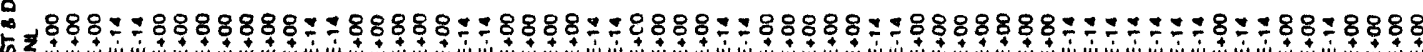

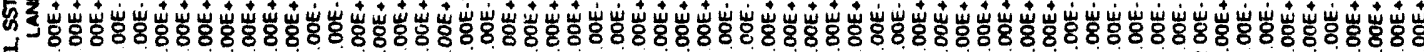

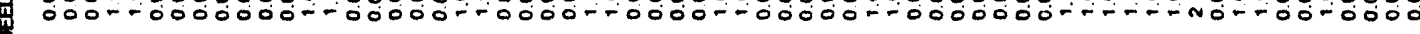

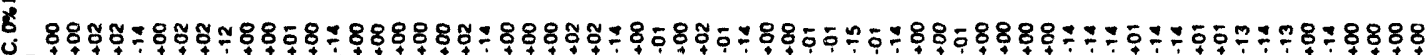

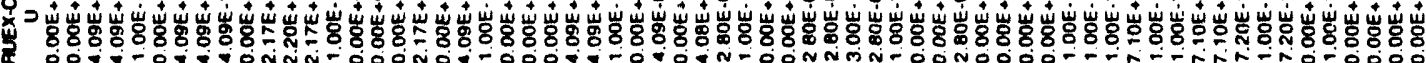

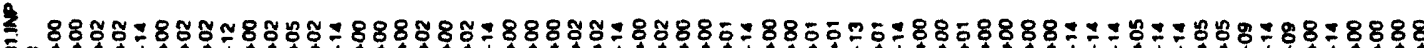

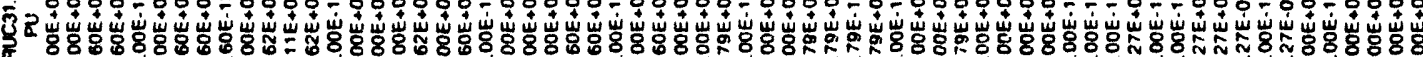

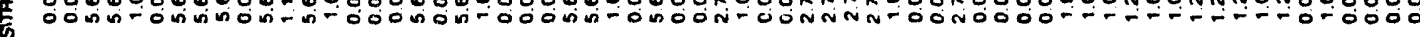

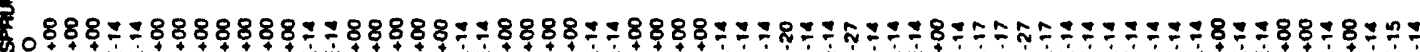

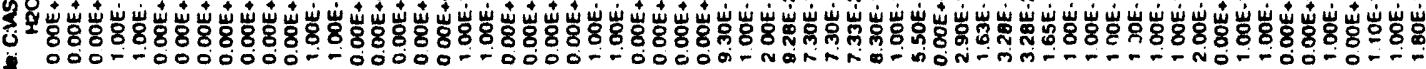

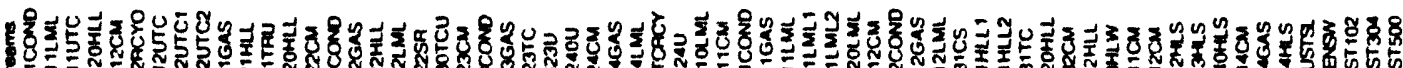

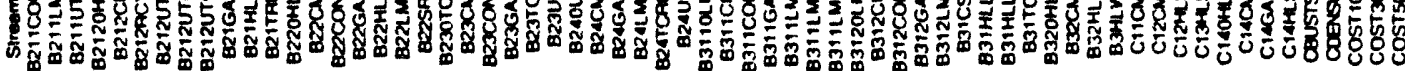




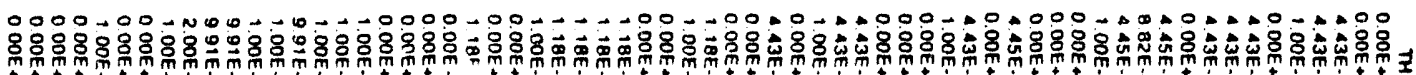

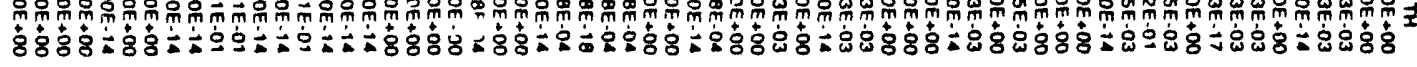

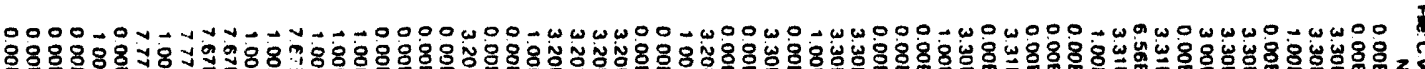

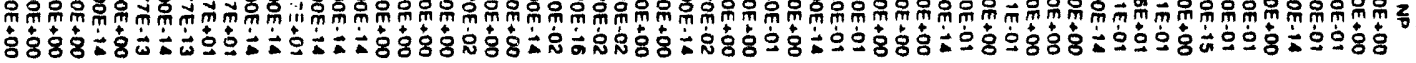

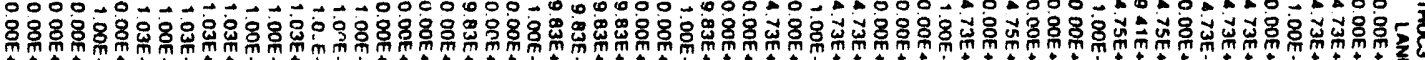

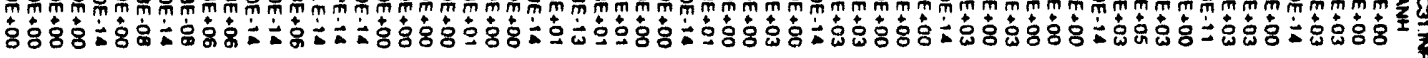

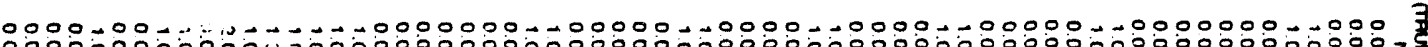

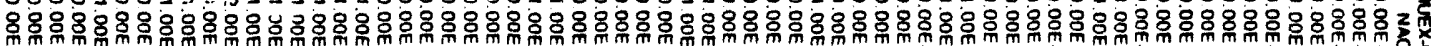

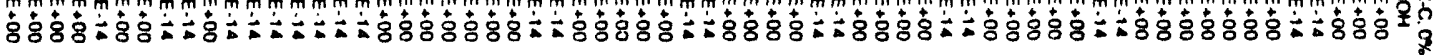

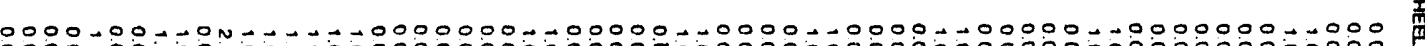

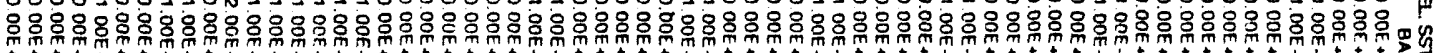

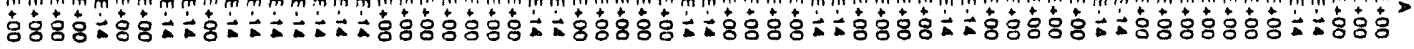

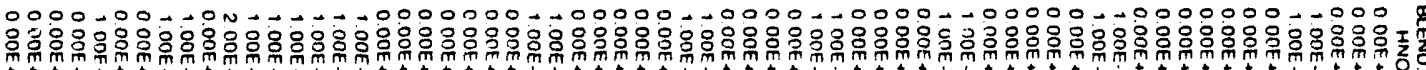

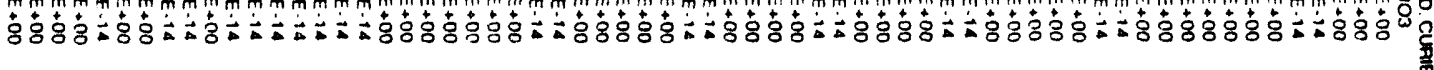

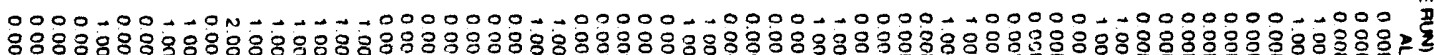

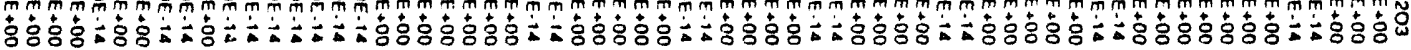

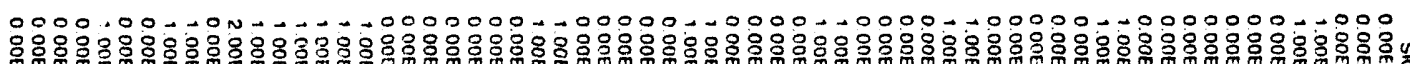

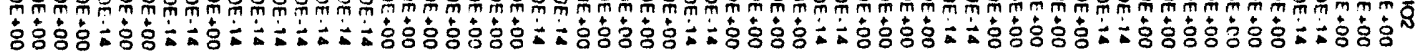

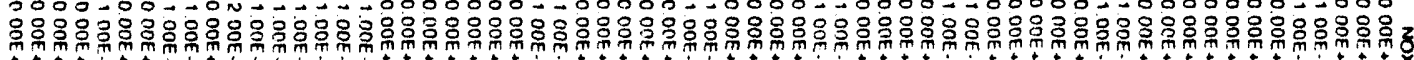

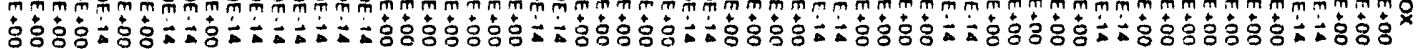

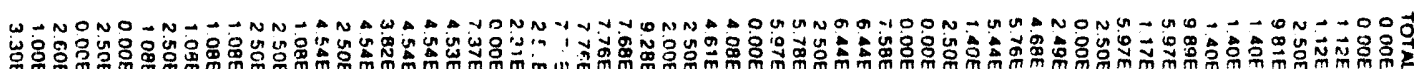

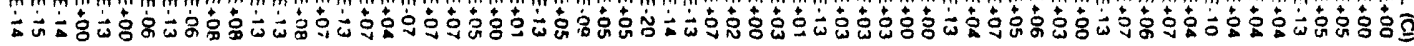


象

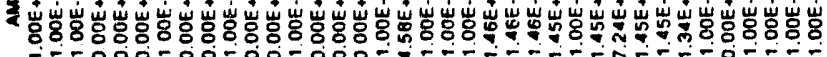

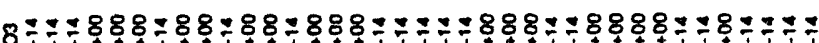

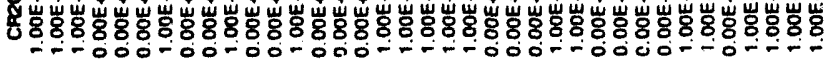

象:

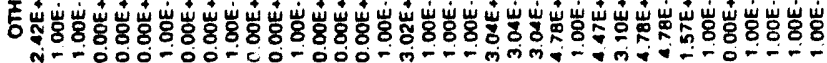

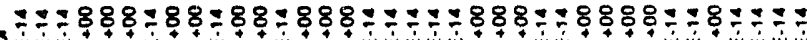
ठ

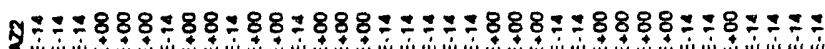

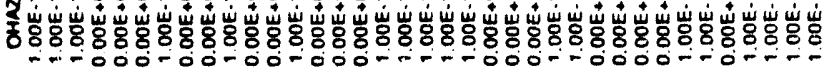

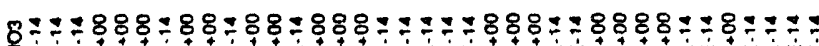

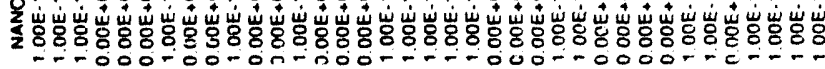

-

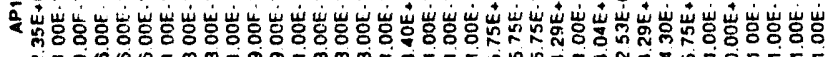

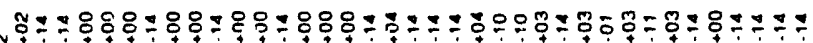

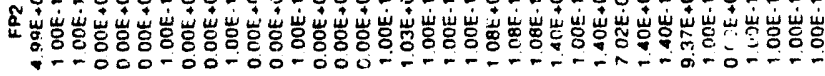

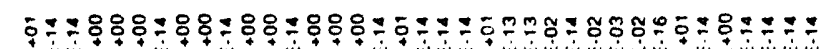

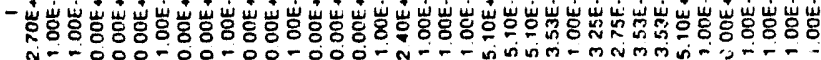

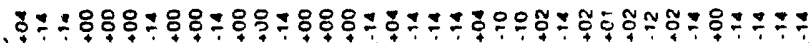

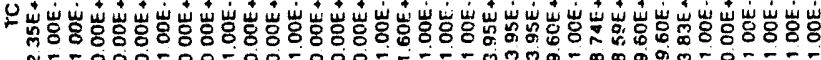

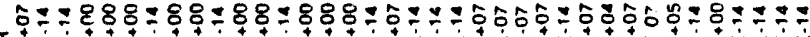

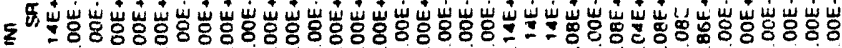

密 응

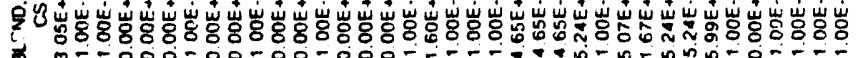
落

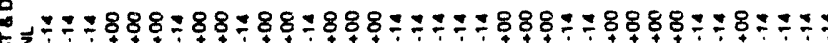

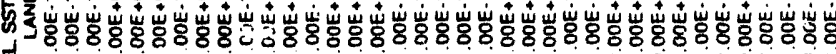
螘

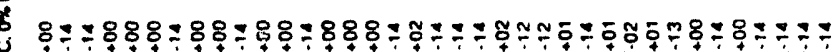
ح

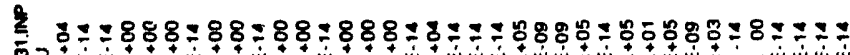

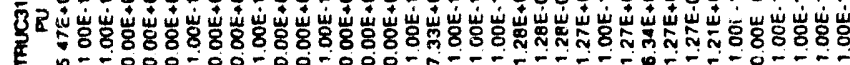
$\frac{5}{5}$

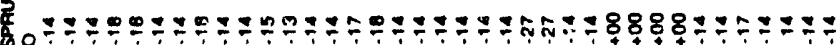

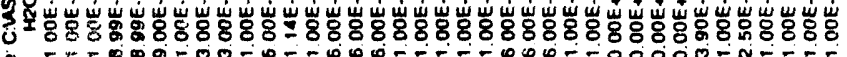
要

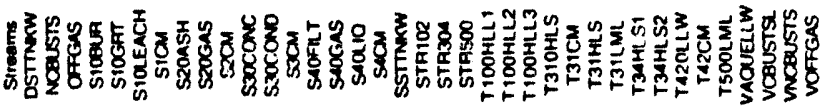


Table H3. ASPEN PLUS Global Model Mass Balance Output Results. (sheet 40 of 56)

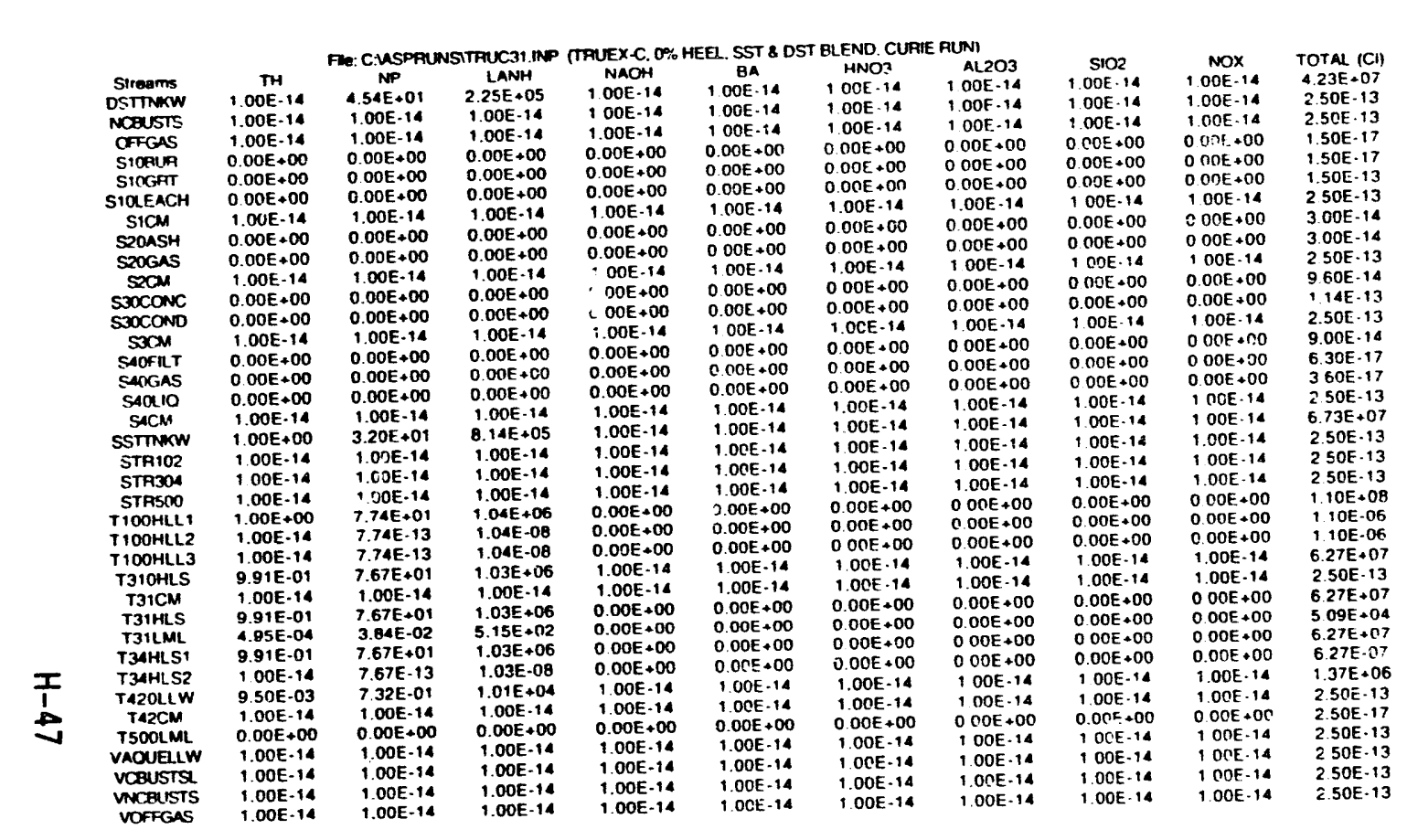




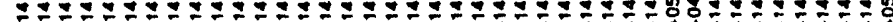

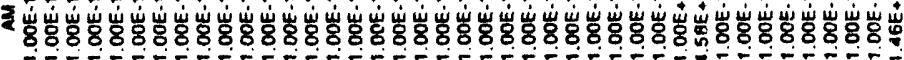

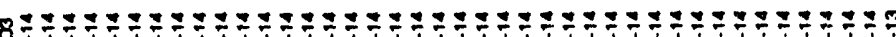

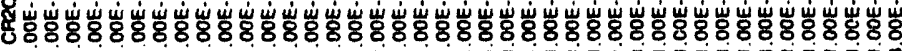

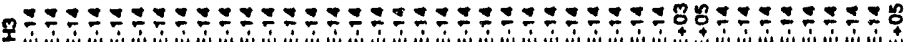

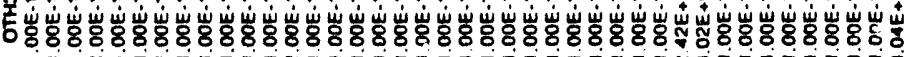

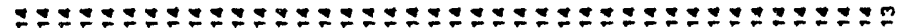

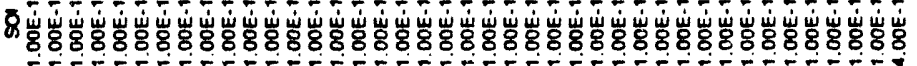

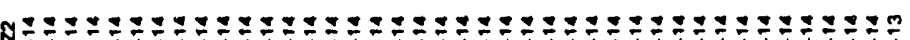

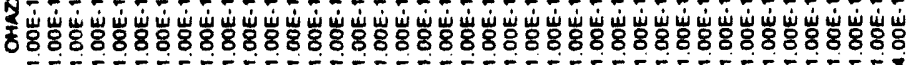

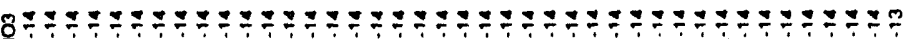

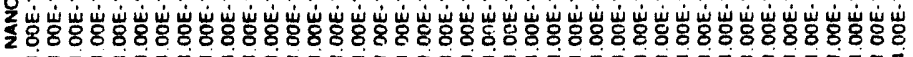

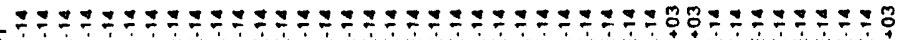

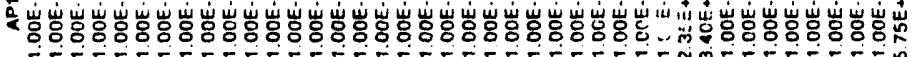

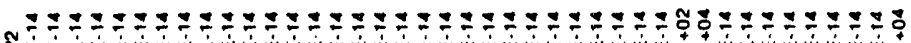

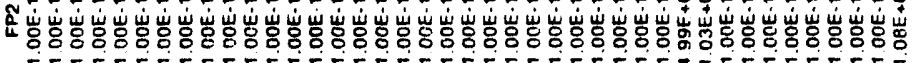

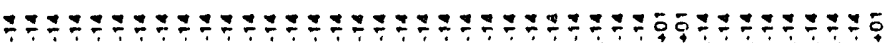

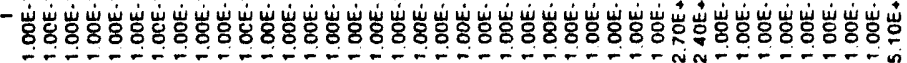

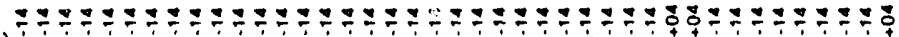

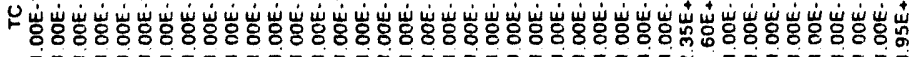
0

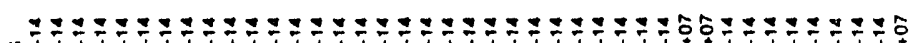

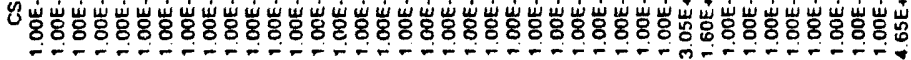

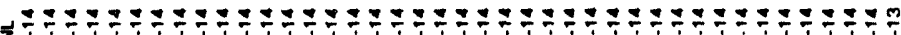

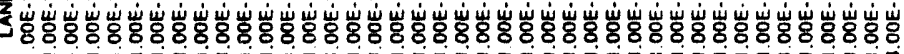

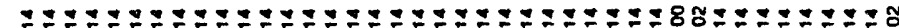

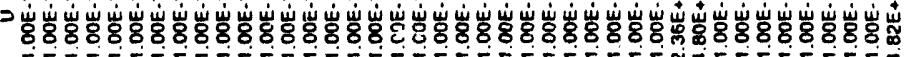

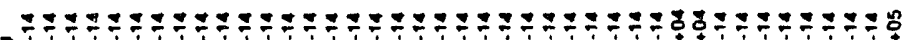

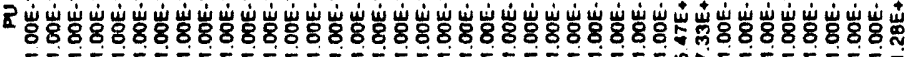

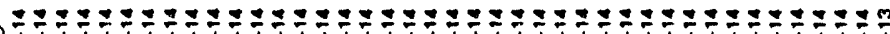

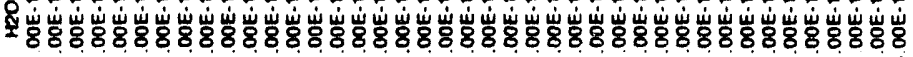

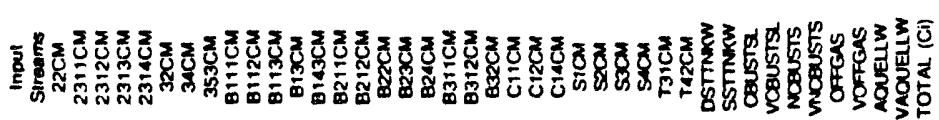

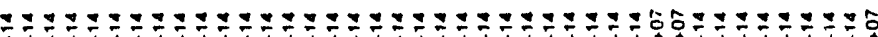

$8 \%$

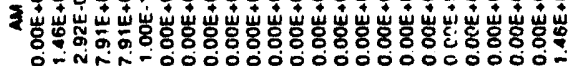

$888 \div 88 \div 888888888888888889=$

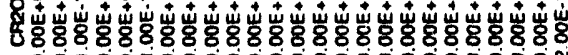

운

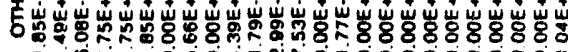

$88 \div 88 \div 888888888888888888=$

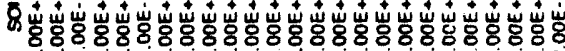
近-0000000000000000000000

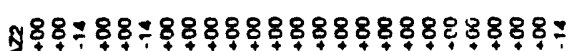

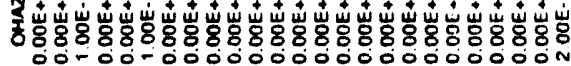

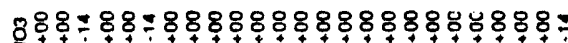

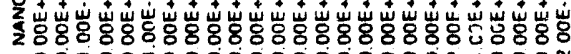

운

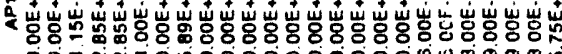

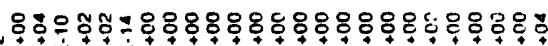

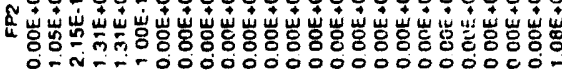
$80050 \div 8508888888888 \% 58885$

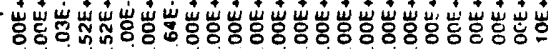

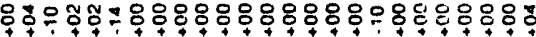

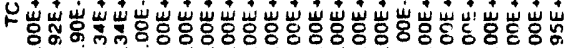

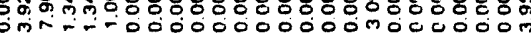

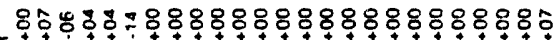

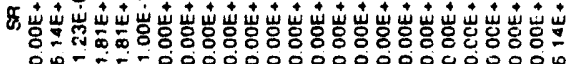

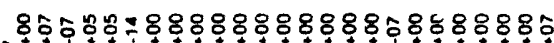

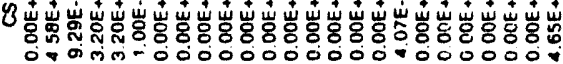

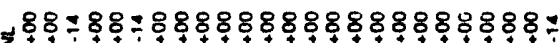

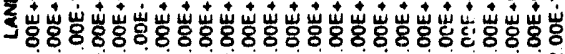

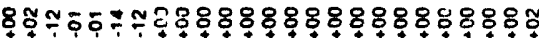
>

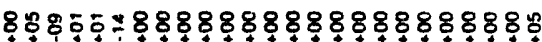

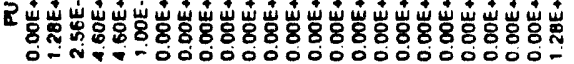

$=8 \div \div \div 8 \div=98888888000 \%=2$

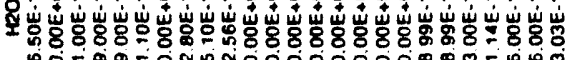

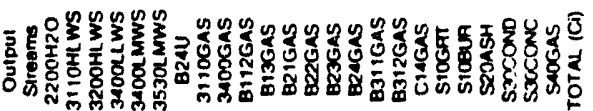




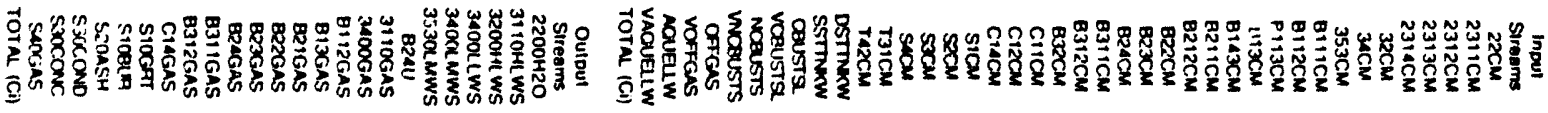

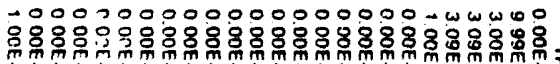

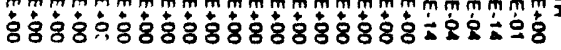

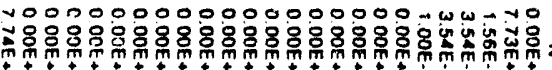

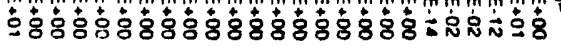

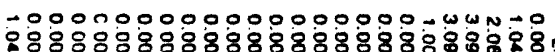

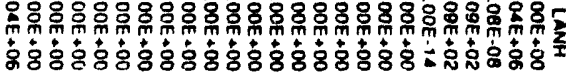

NOOOOOOOOOOOO000000-00-00

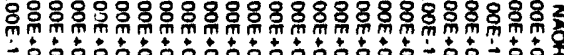

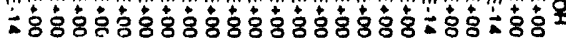

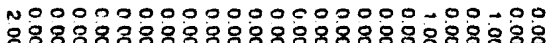

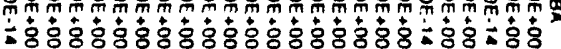

$000000000000000000-00-00$

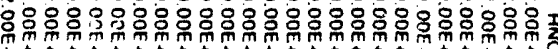

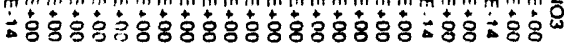

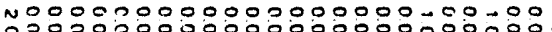

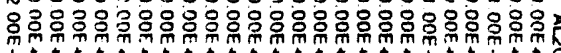

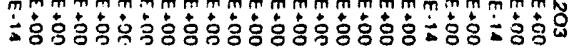

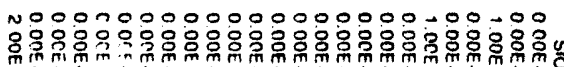

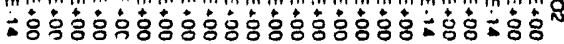

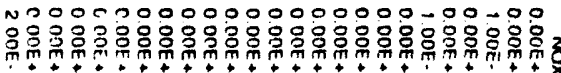

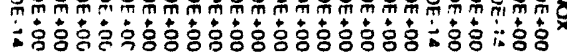

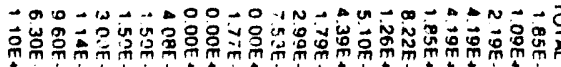

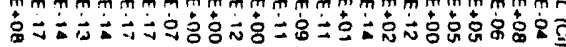

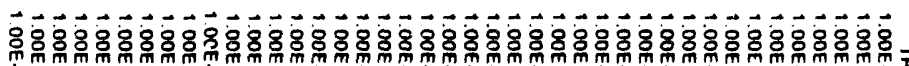

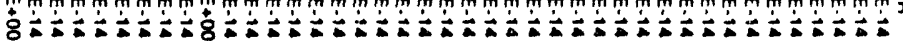

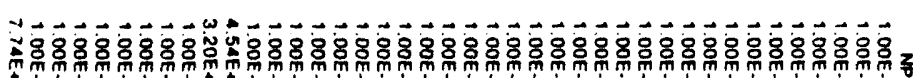

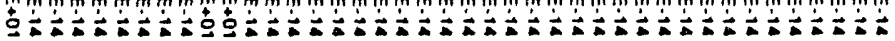

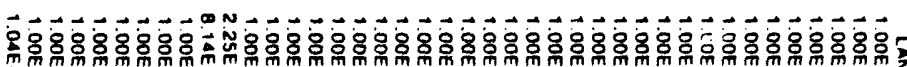

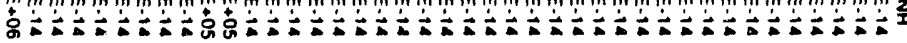

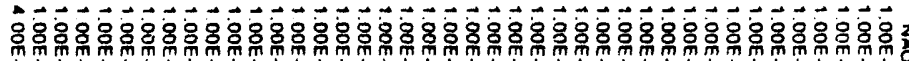

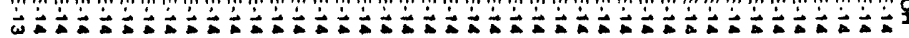

83

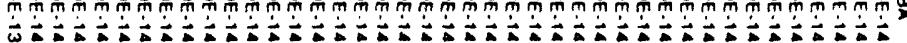

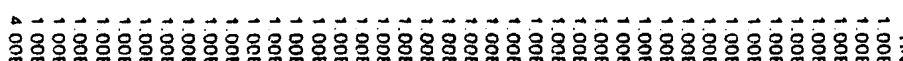

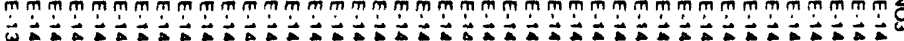

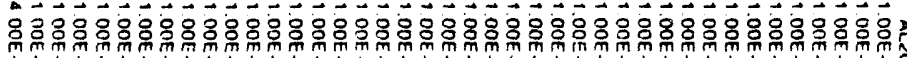

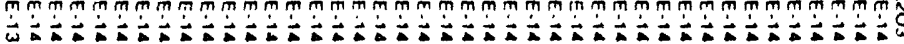

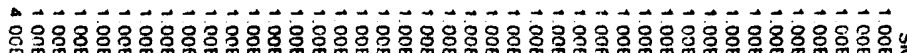

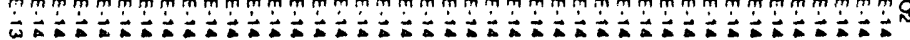

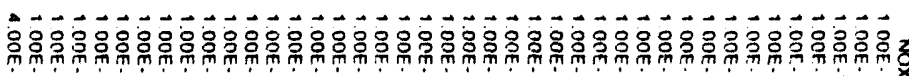

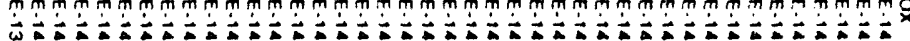

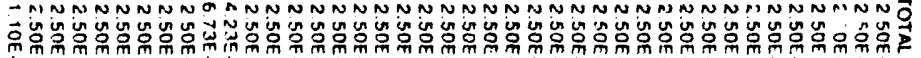

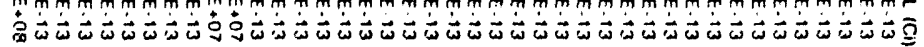




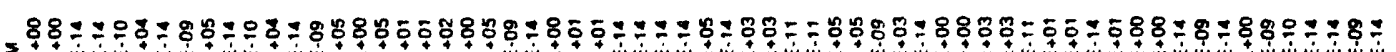

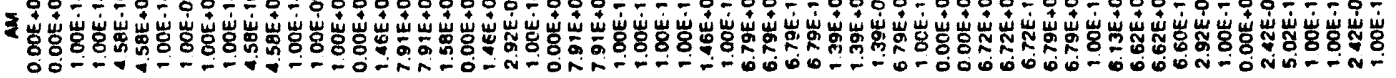

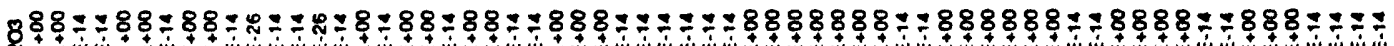

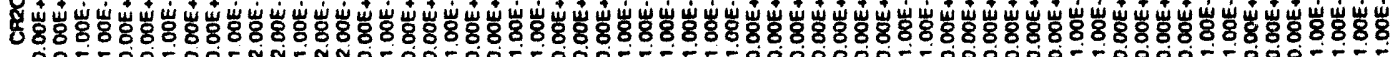

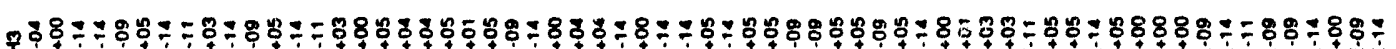

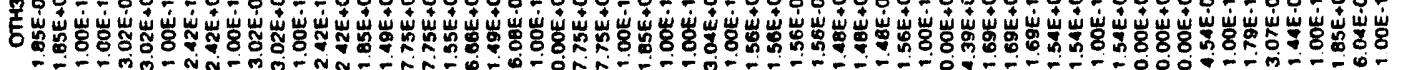

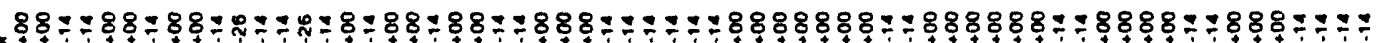

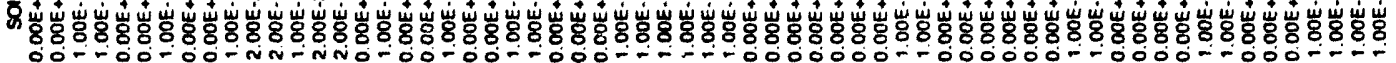

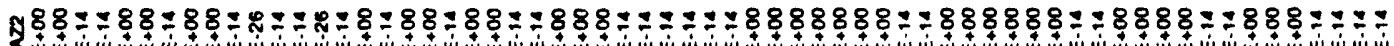

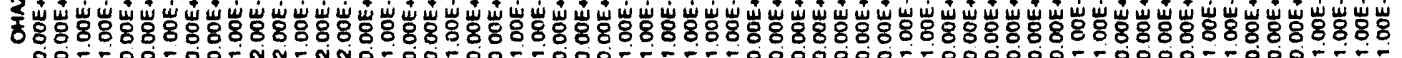

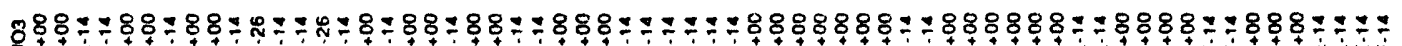

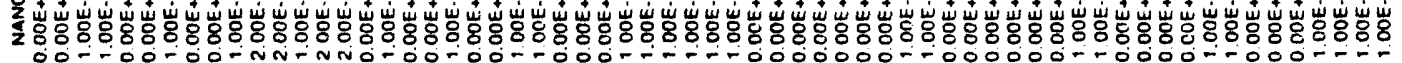

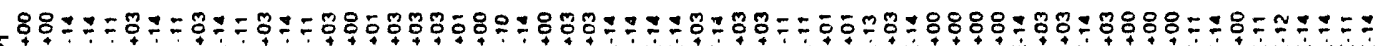

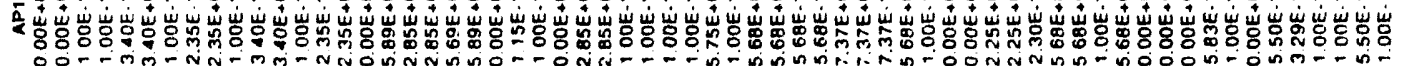

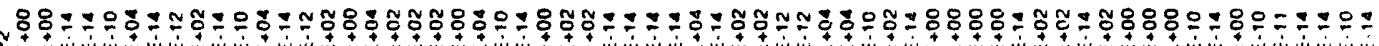

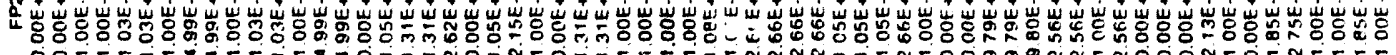

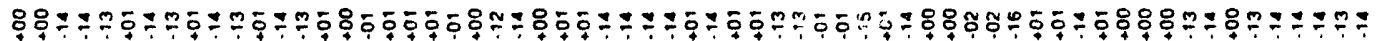

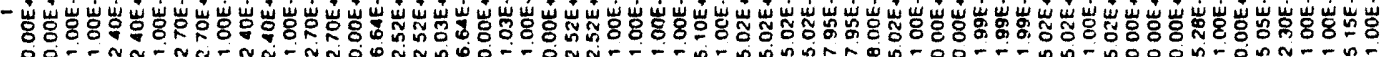

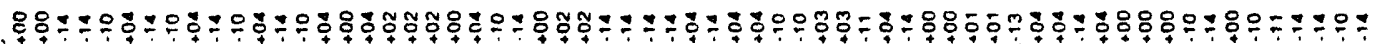

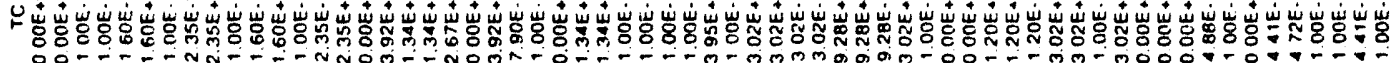

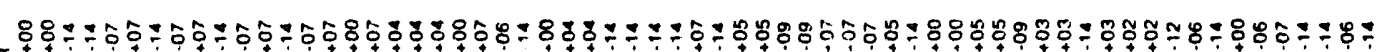

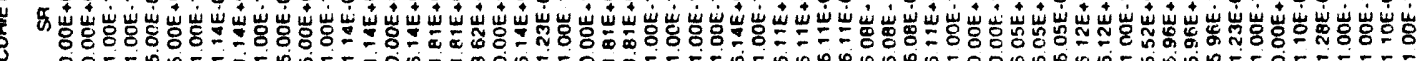

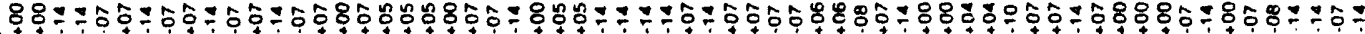

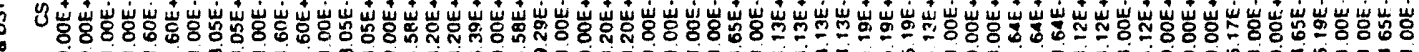
5

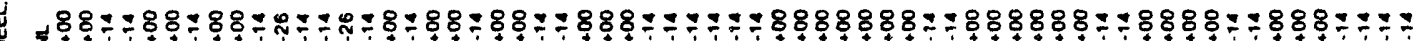

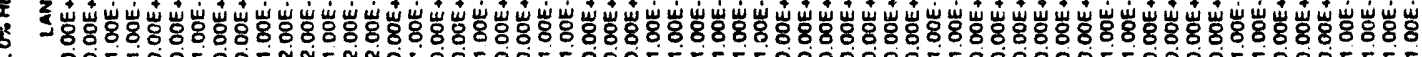

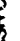

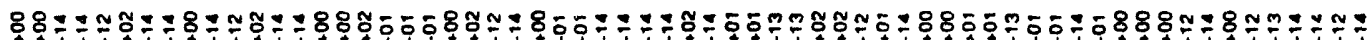

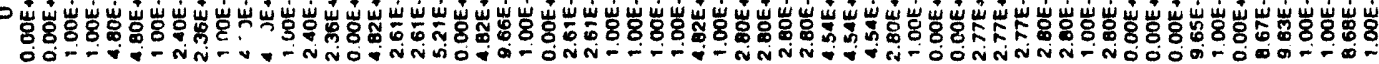

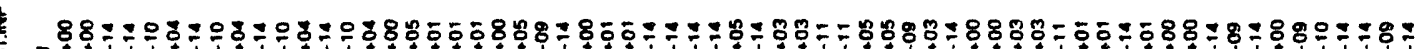

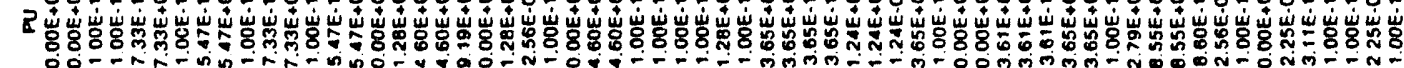

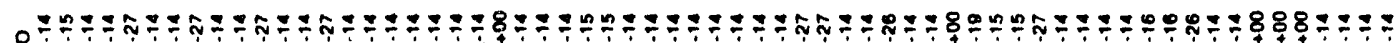

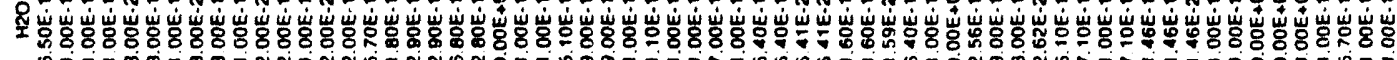

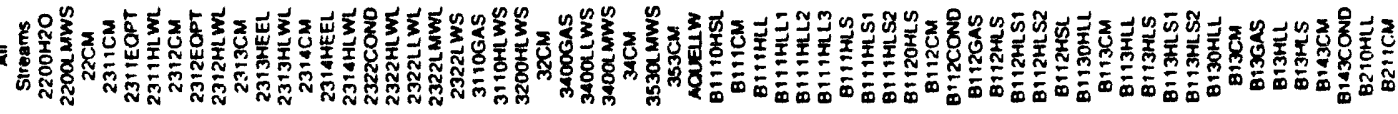


Table H3. ASPEN PLUS Global Model Mass Balance Output Results. (sheet 44 of 56)

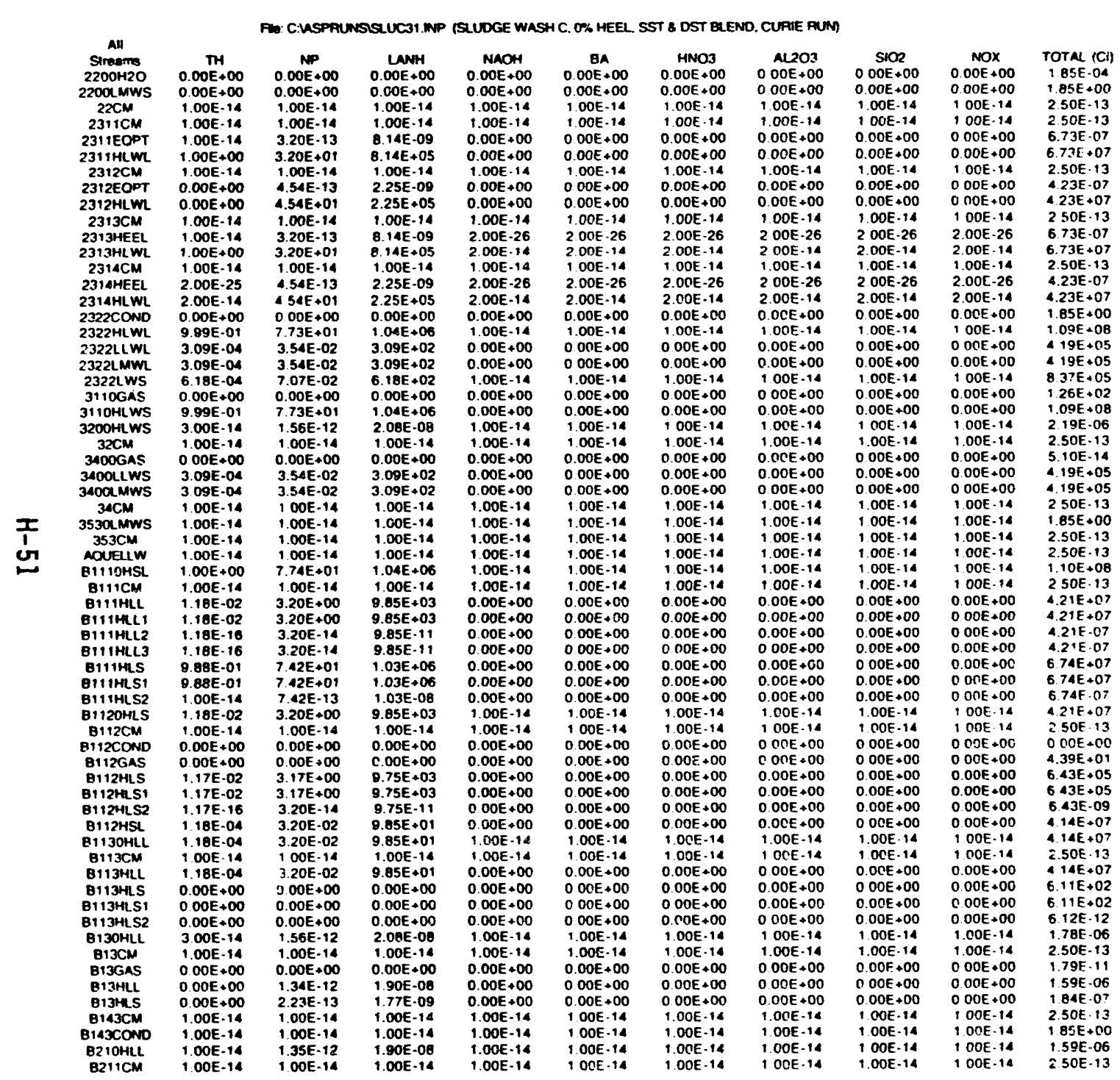




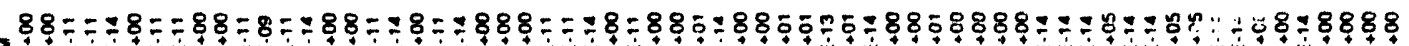

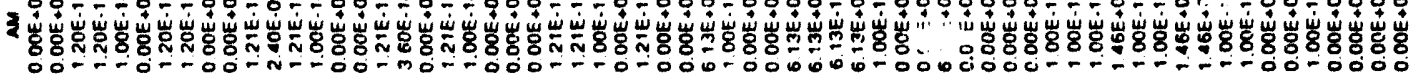

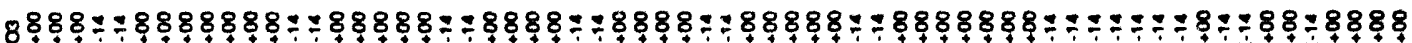

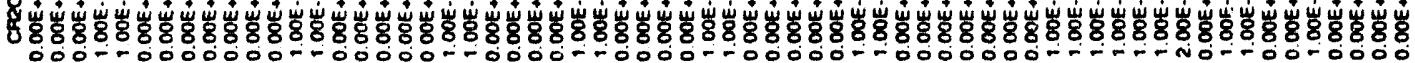

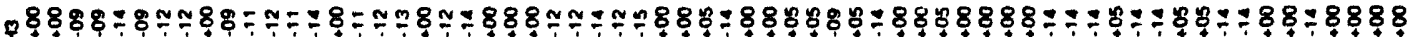

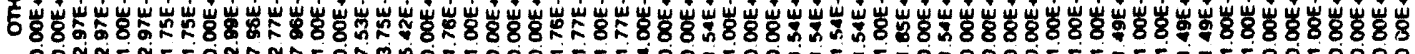

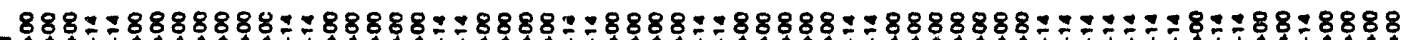

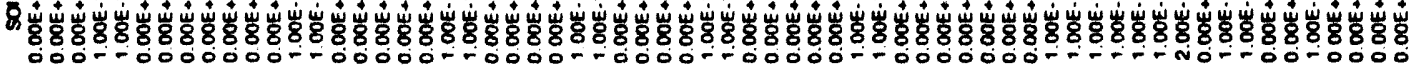

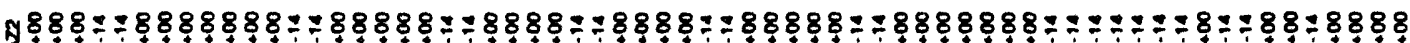

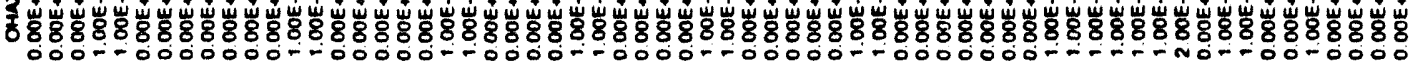

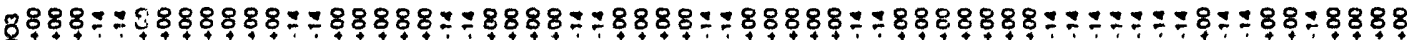

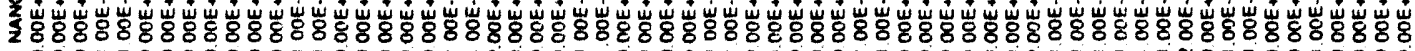

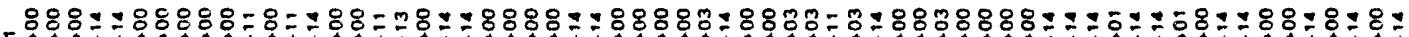

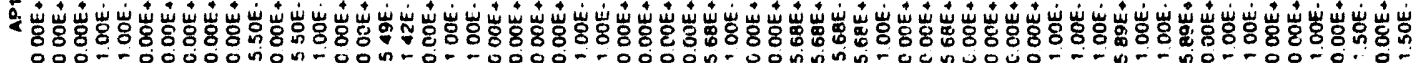

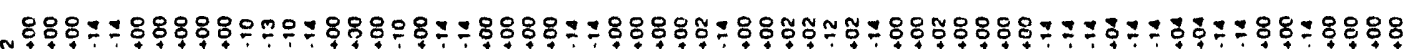

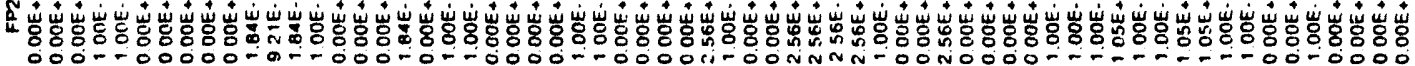

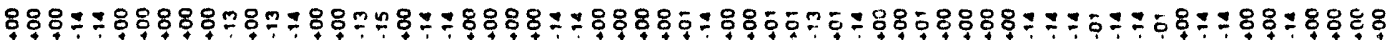

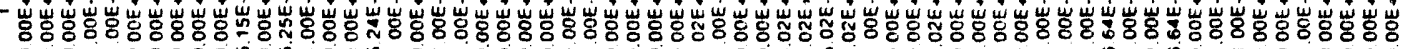

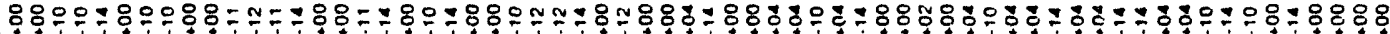

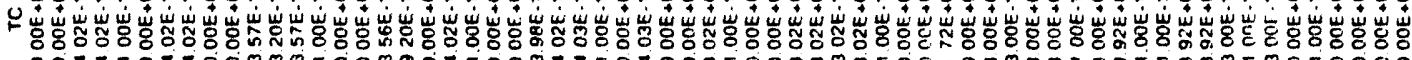

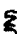

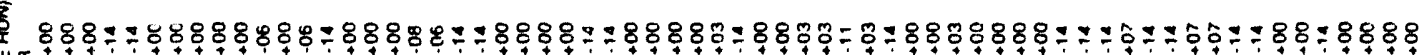

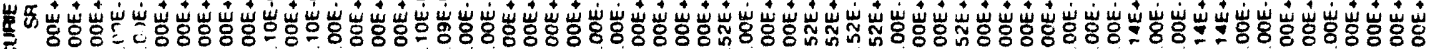

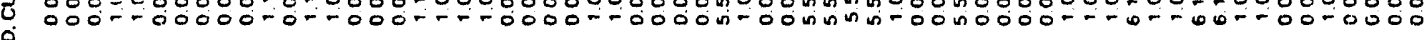
密

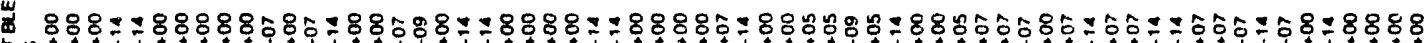

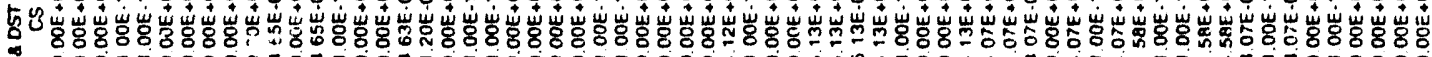
5

W

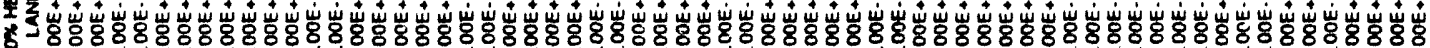

密

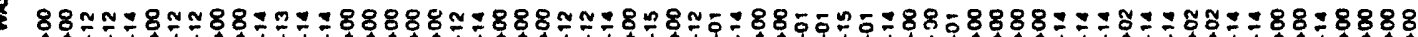

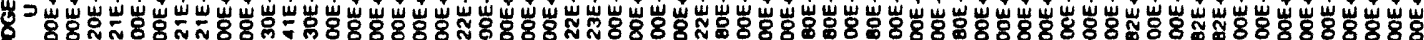

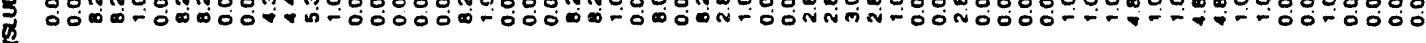

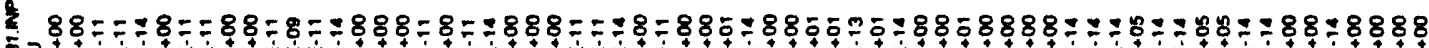

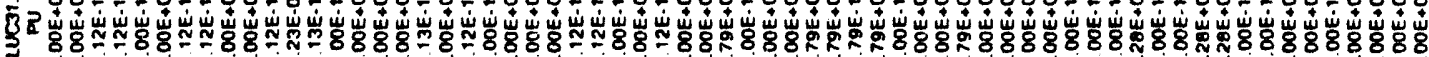

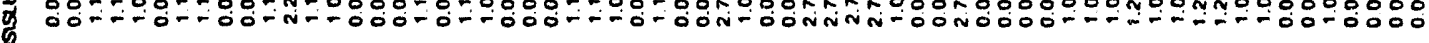

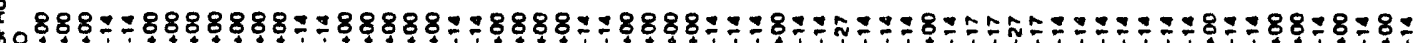

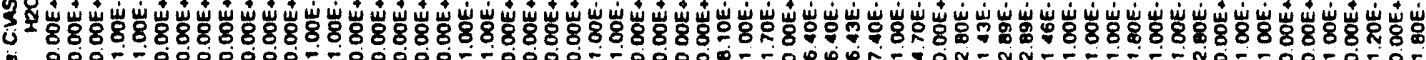

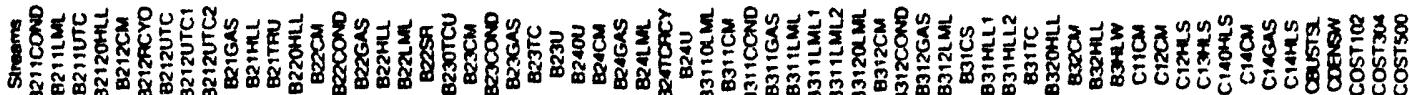




\section{-}

Table H3. ASPEN PLUS Global Model Mass Balance Output Results. (sheet 46 of 56)

\begin{tabular}{|c|c|c|c|c|c|c|c|c|c|c|}
\hline 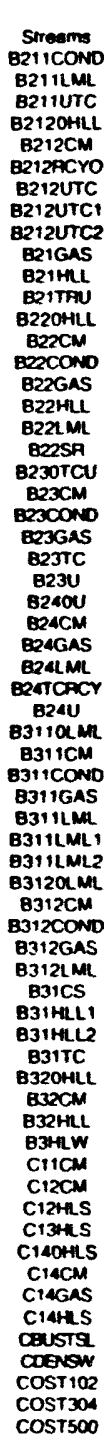 & 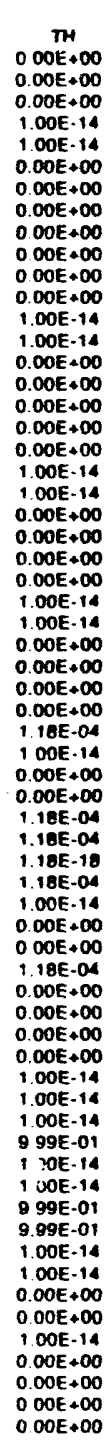 & 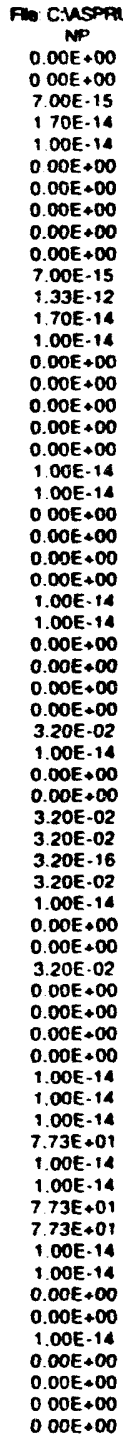 & 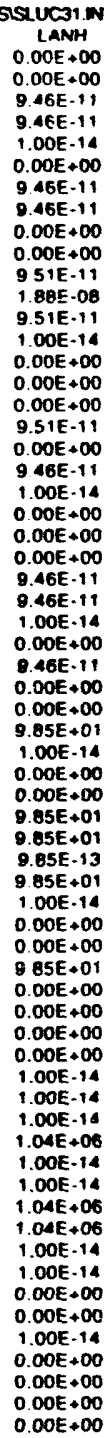 & 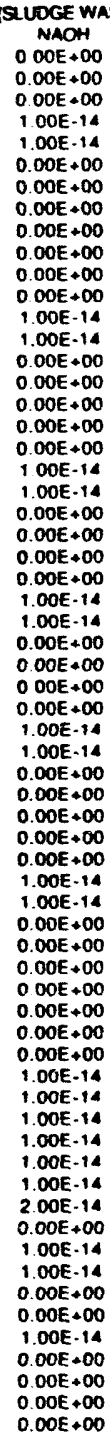 & 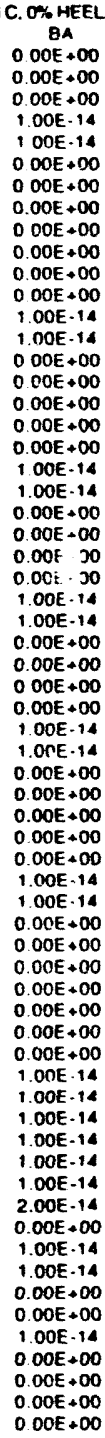 & 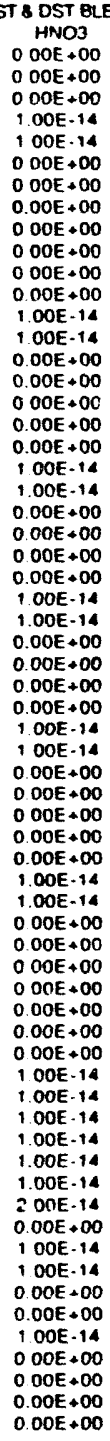 & 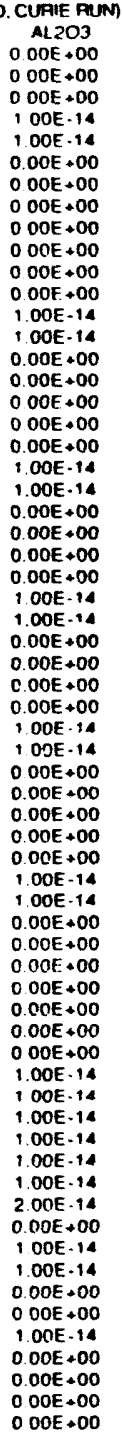 & 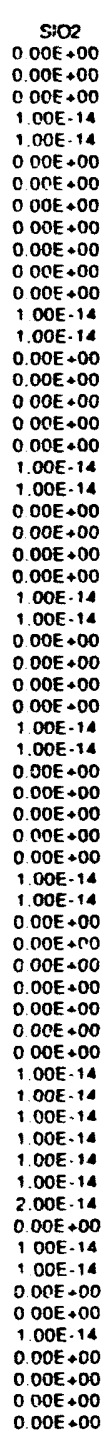 & 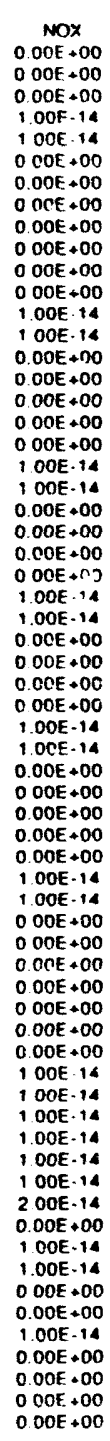 & 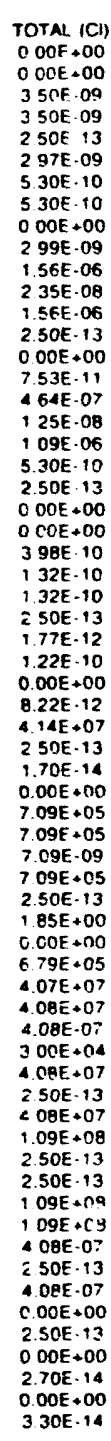 \\
\hline
\end{tabular}




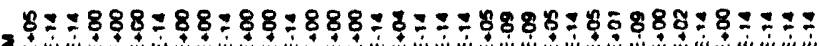

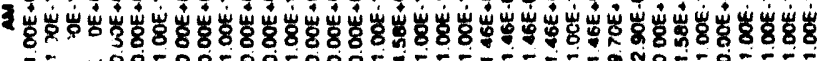

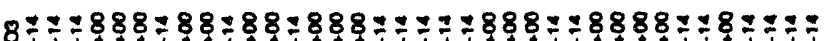

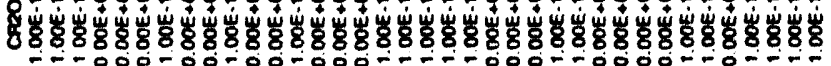

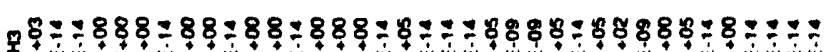

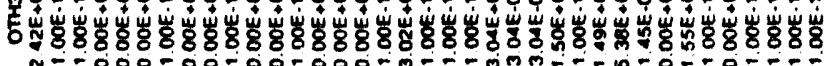
8:

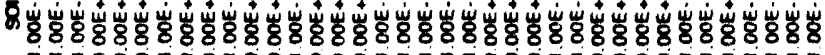

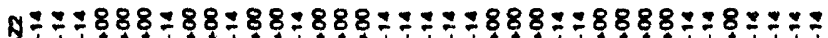

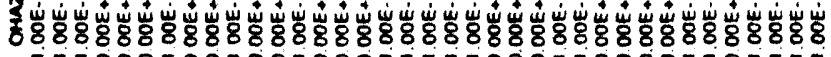

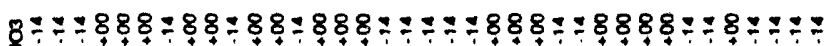

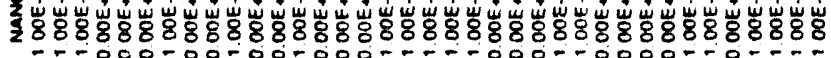

-

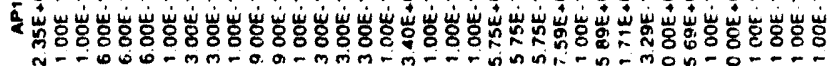

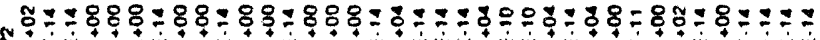
ผ

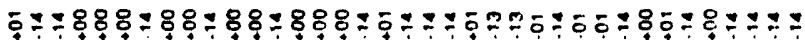

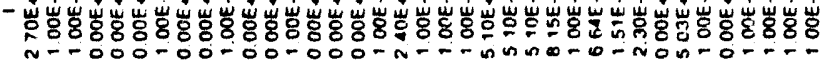

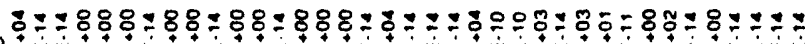

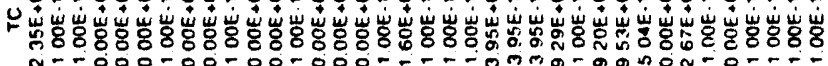
承

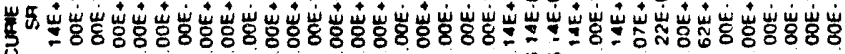

(2)

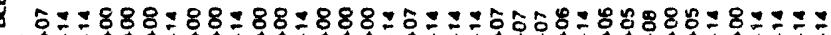

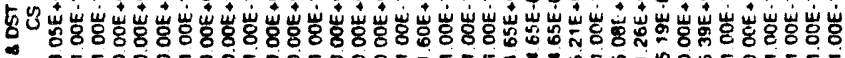
5

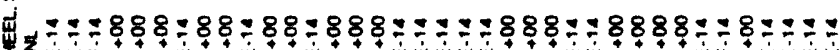

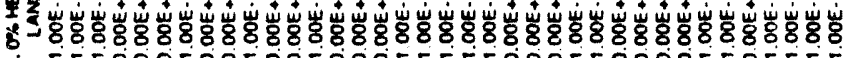
妾

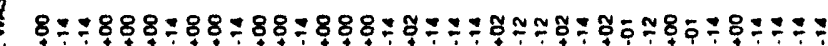

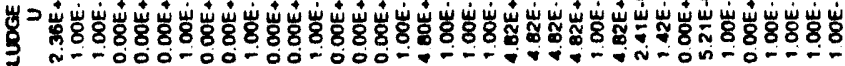

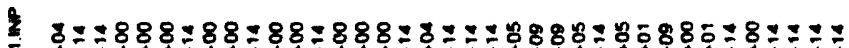

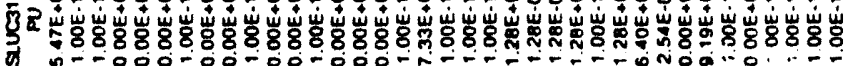
要 侟 8

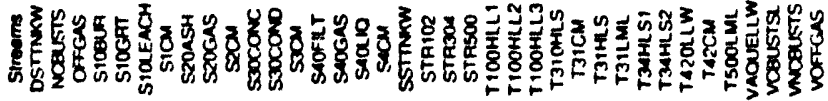


Table H3. ASPEN PLUS Global Model Mass Balance Output Results. (sheet 48 of 56)

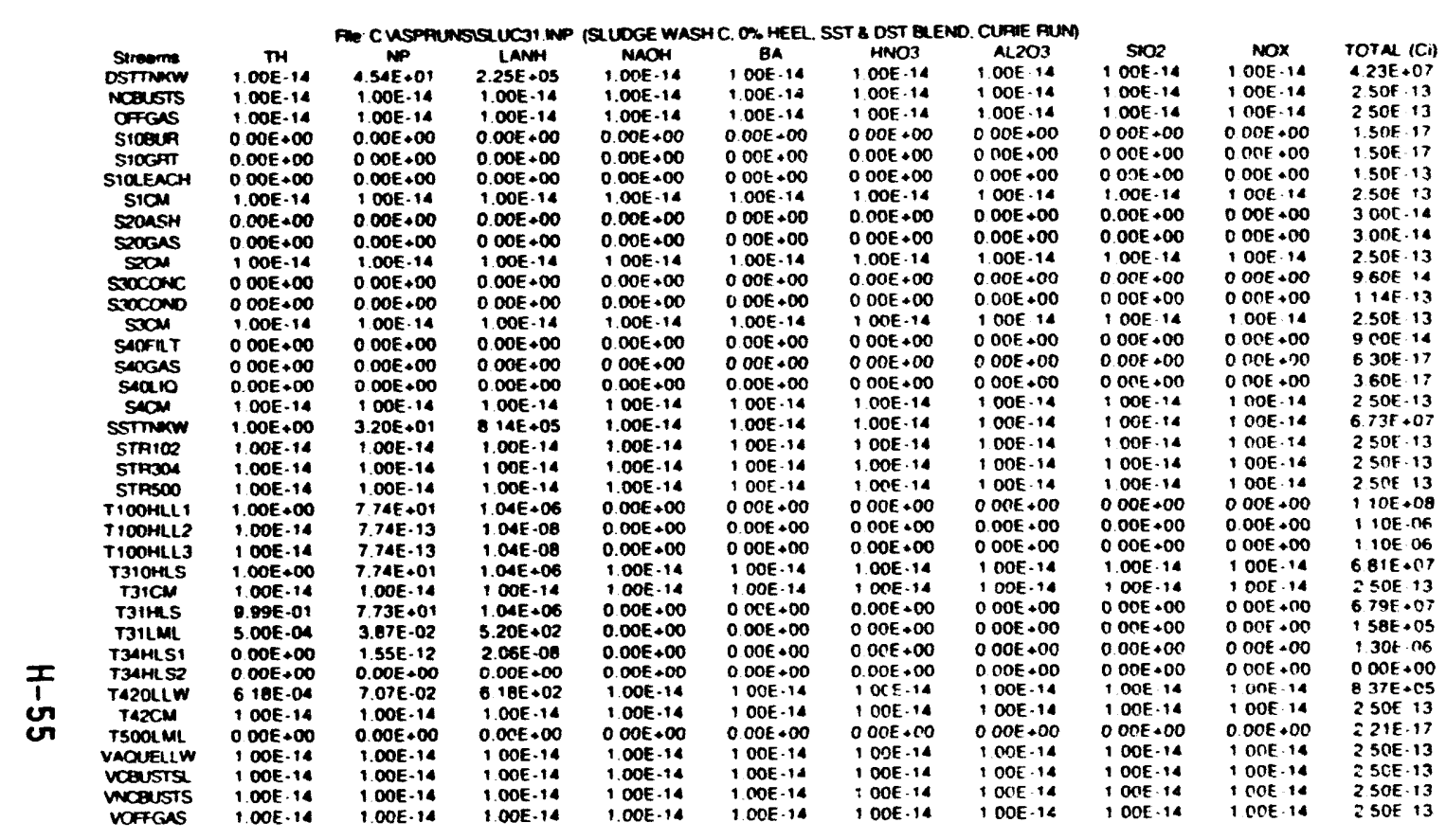




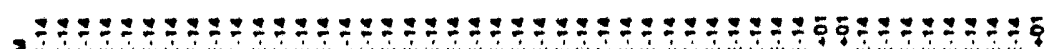

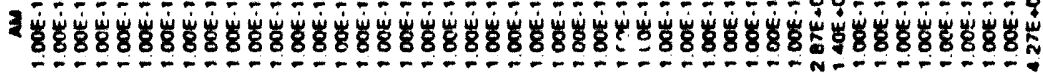

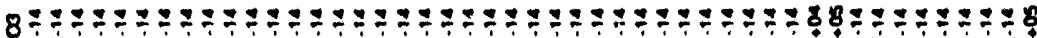

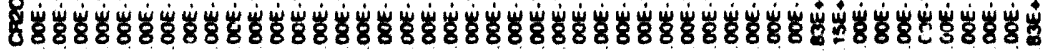

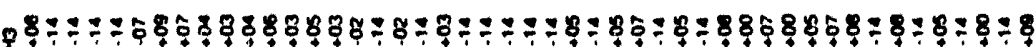

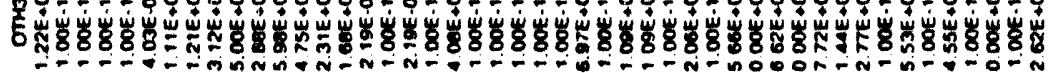

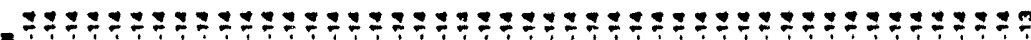
8.
\end{abstract}

ه

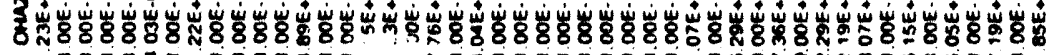

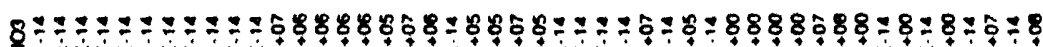

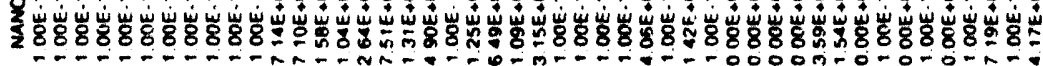

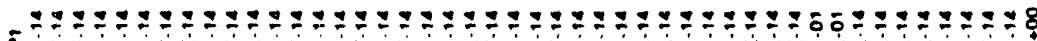

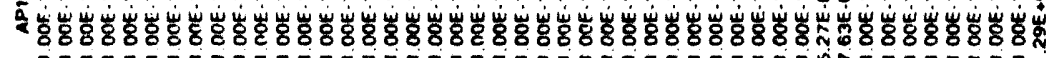

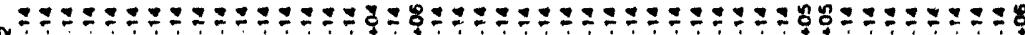

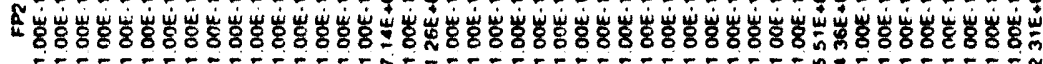
-

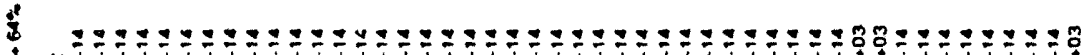

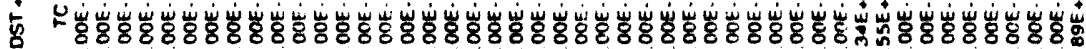

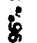

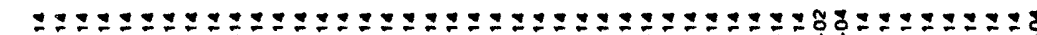

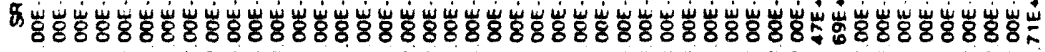

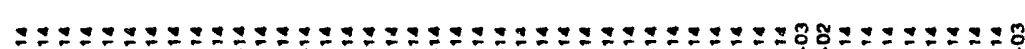

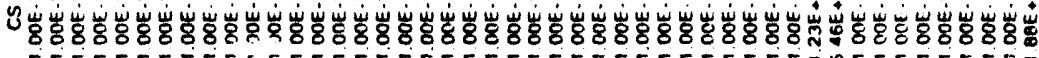

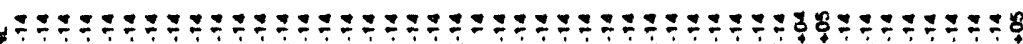

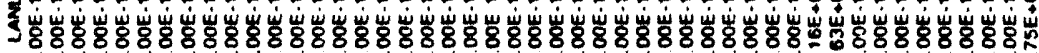

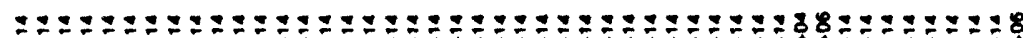
2 (1)

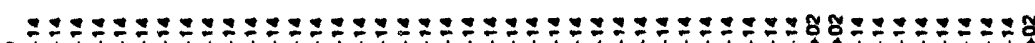

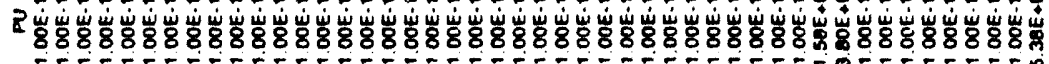

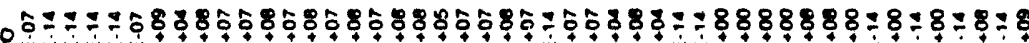

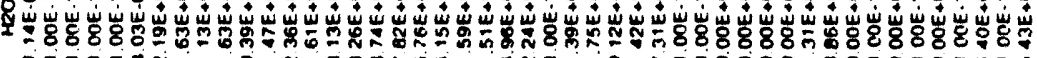
(

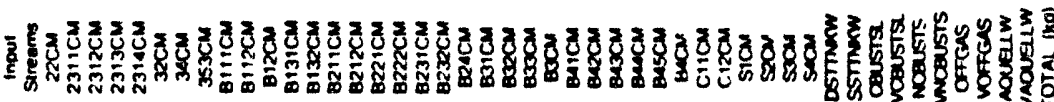

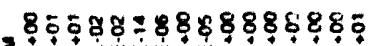

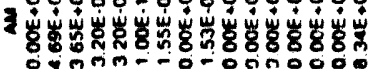

$8898 \%$ 88

8888858898888859

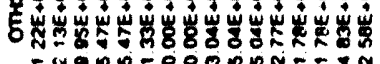

$88 \div 888888888888 \%$ 8.

ำ

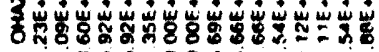

$87 \%$

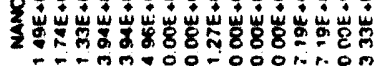

$88588 \div \div 8409=0 \div 0$

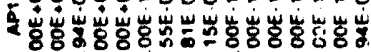

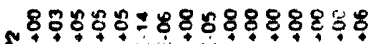

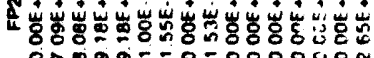

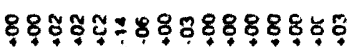

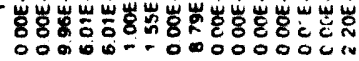

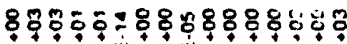

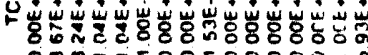

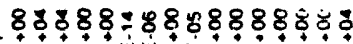

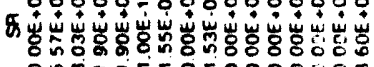

$8 \%$

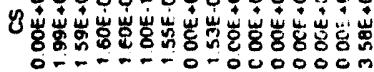

$8907 \%$

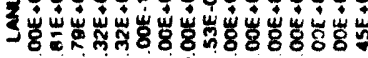

$89898 \div 8888889 \div 8$

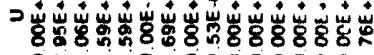

$88850=889888889$

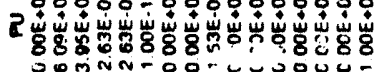
$89809098 \%$

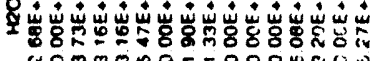

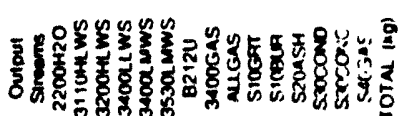


WHC-EP-0791

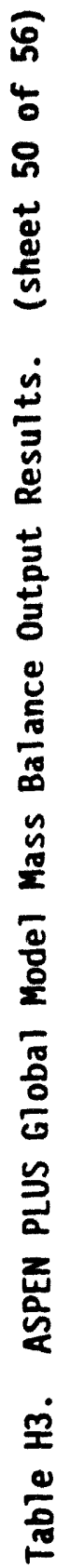

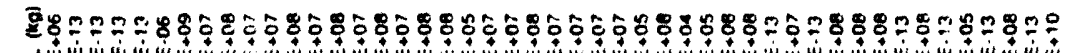

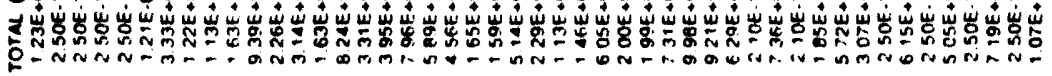

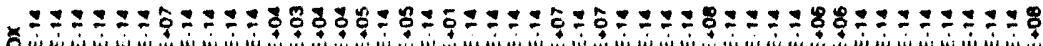
宏

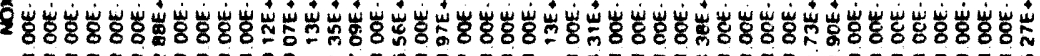

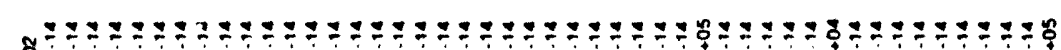

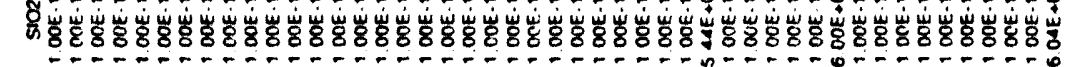

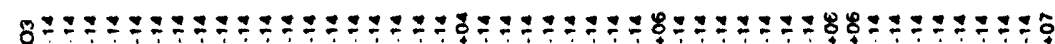

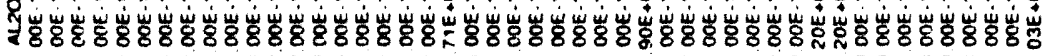

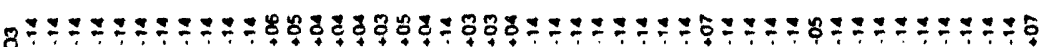

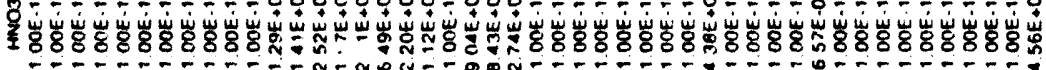

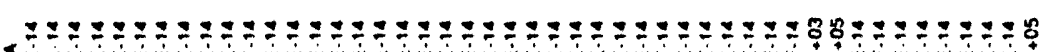

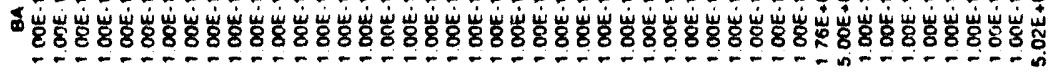

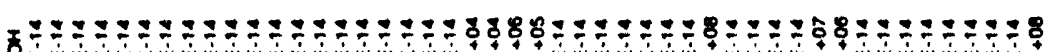

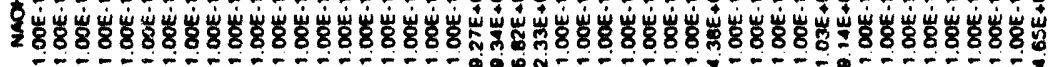

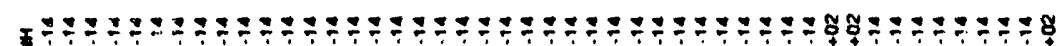

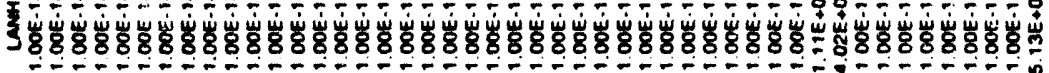

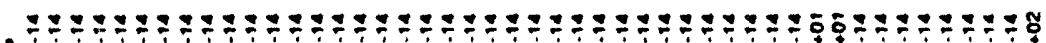

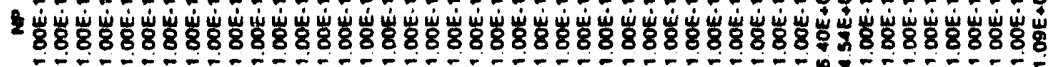

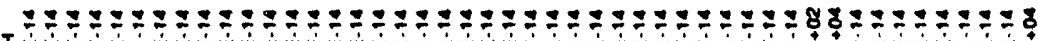

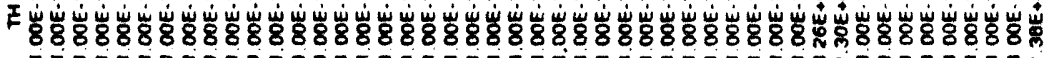

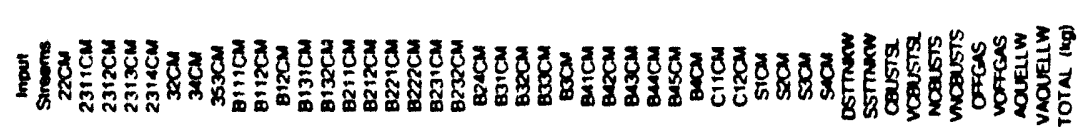

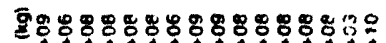

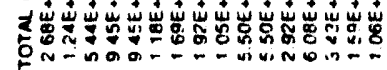

×

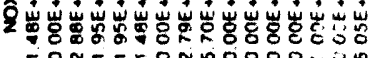

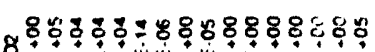

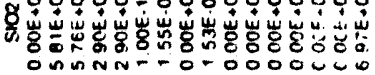
$88980 \%=8898888890$

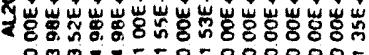
8

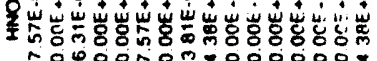

89890

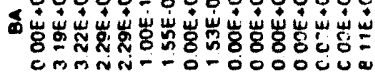

78

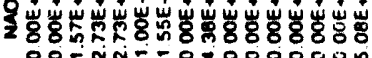

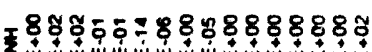

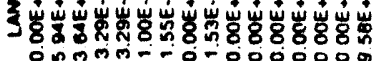

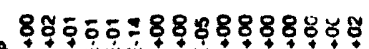

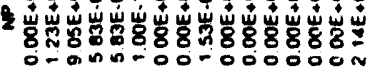
8

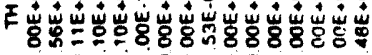

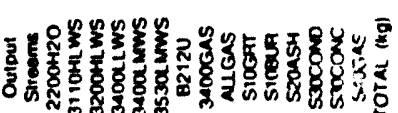




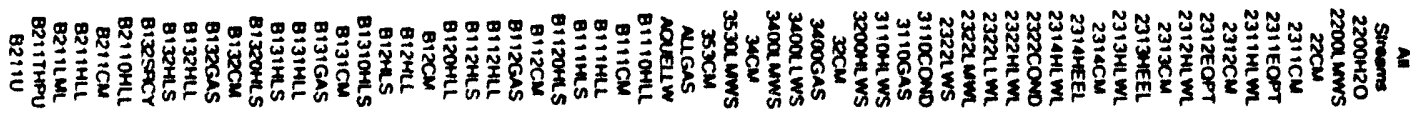

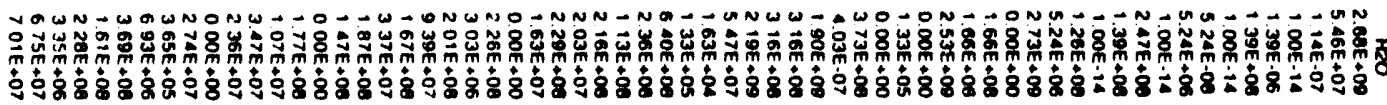

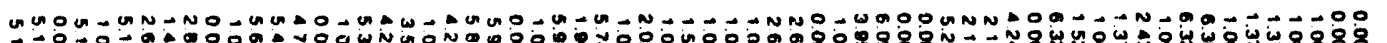

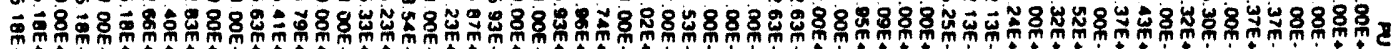

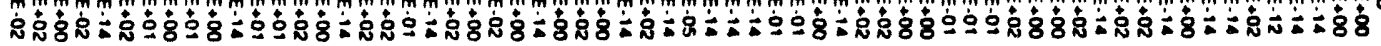

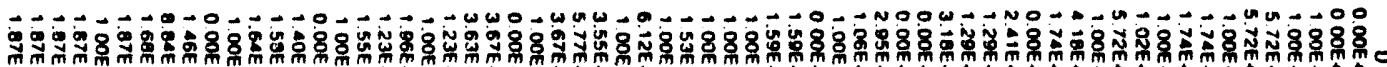

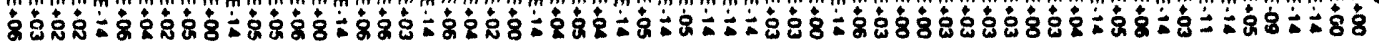

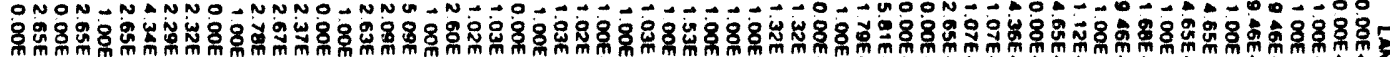

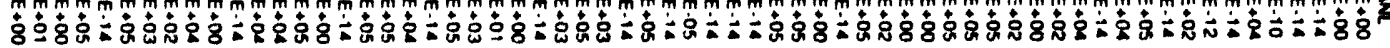

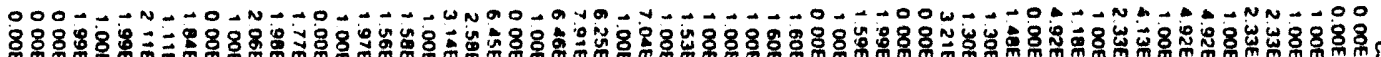

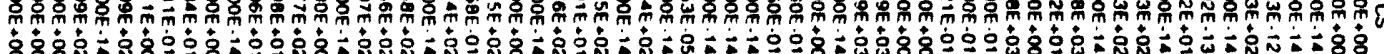

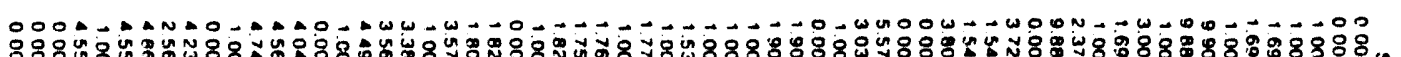

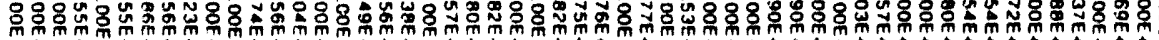

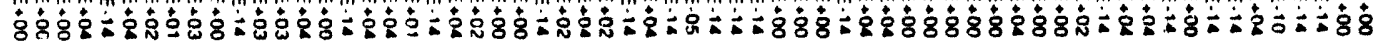

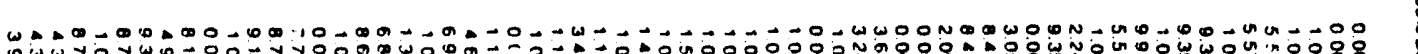

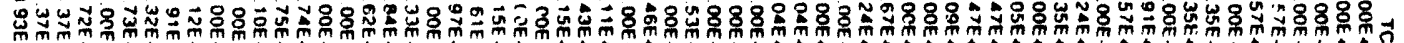

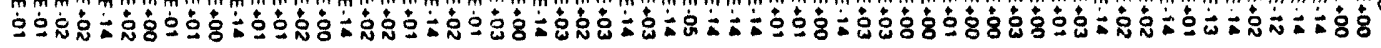

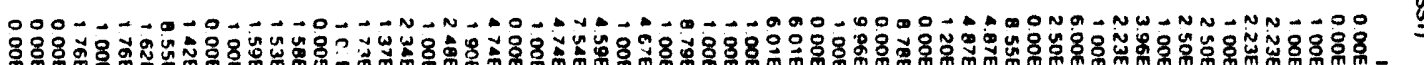

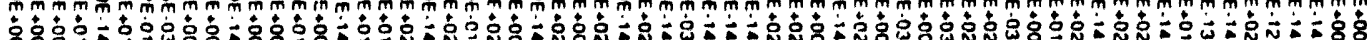

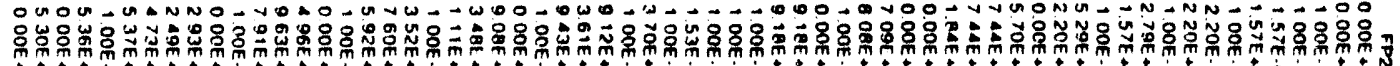

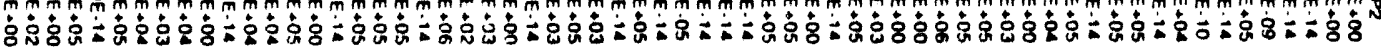

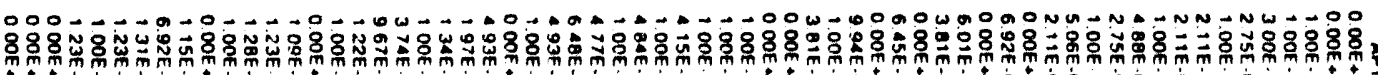

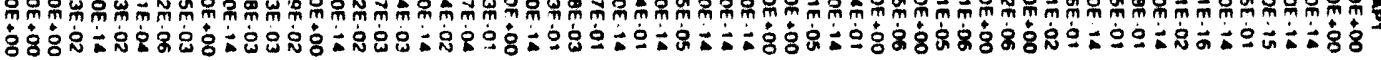

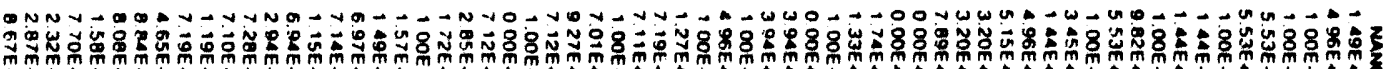

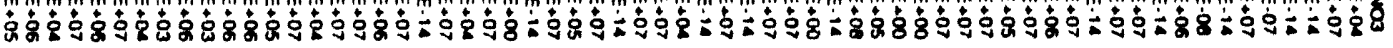

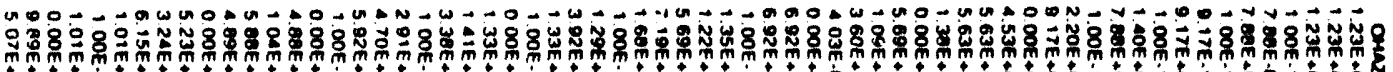

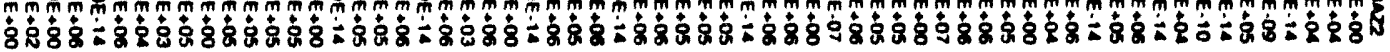

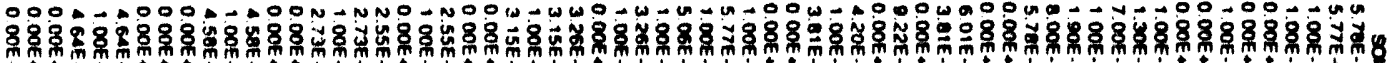

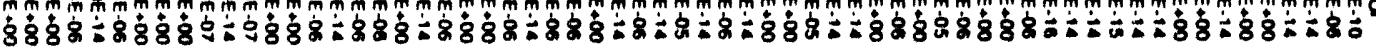

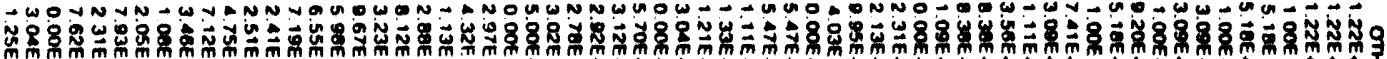

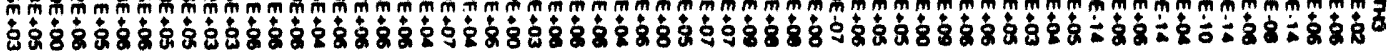

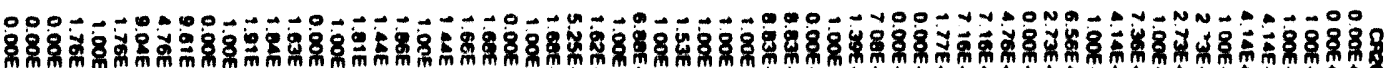

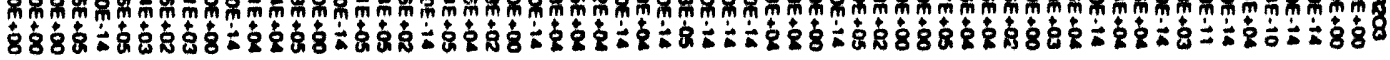

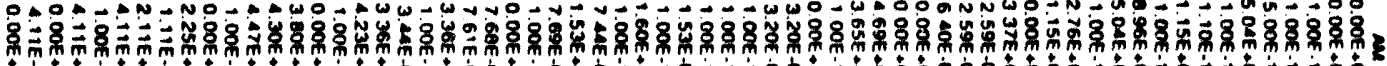

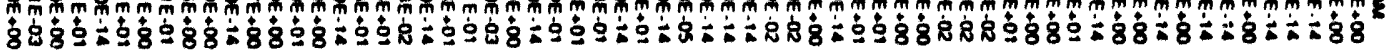


WHC-EP-0791

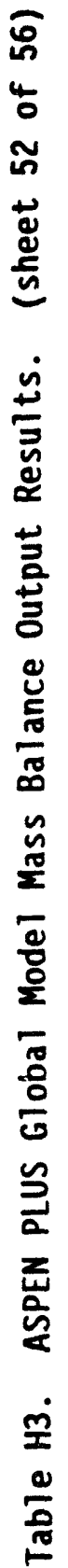

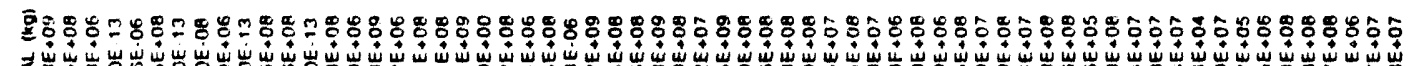

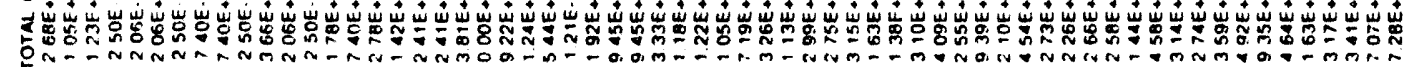

焉

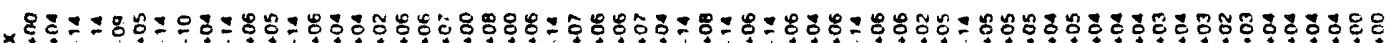

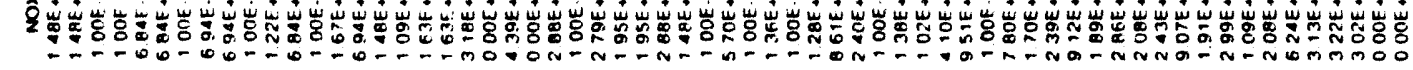

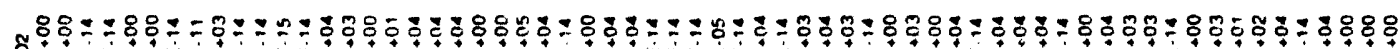

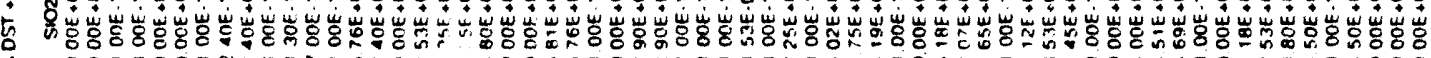

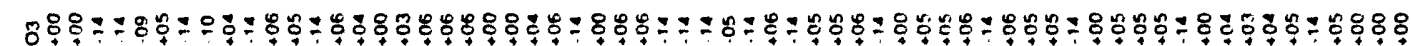

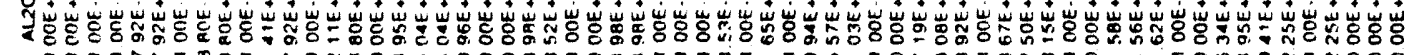
滦

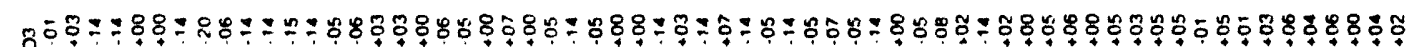

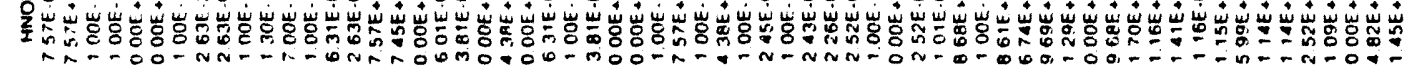

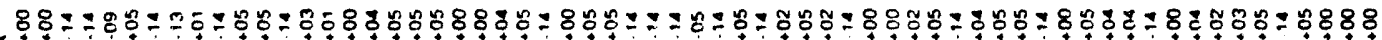

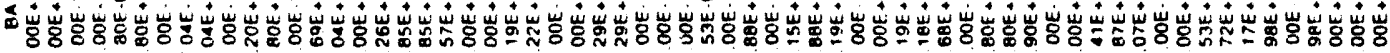

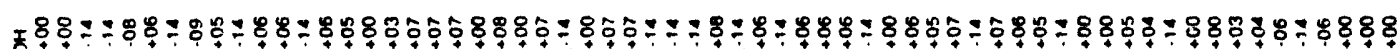

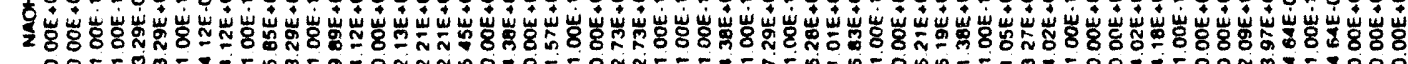
年

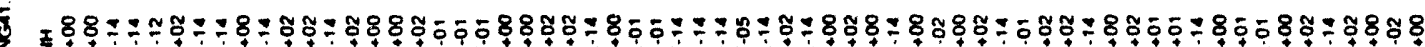

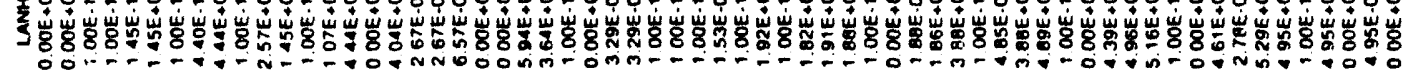

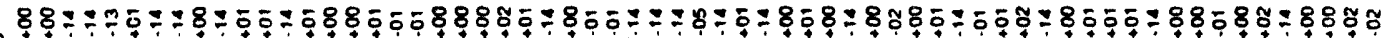

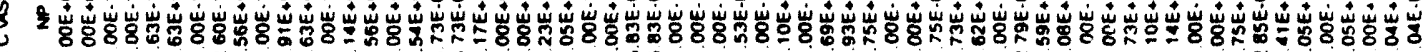

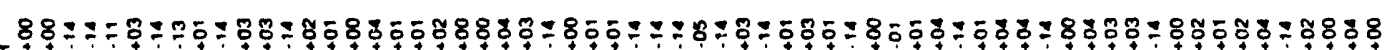

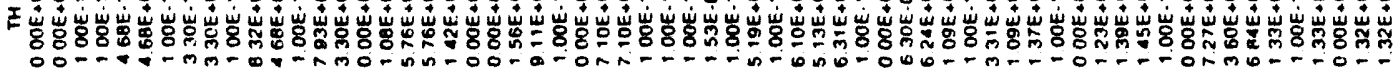

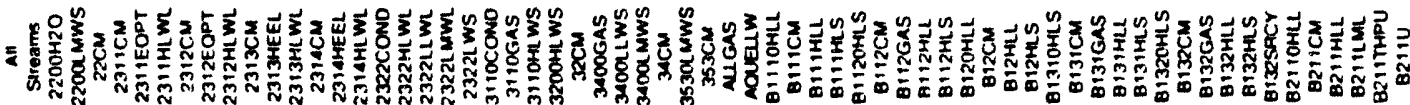




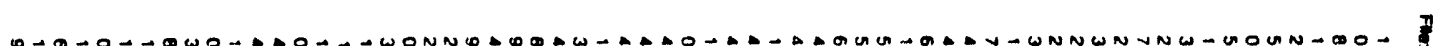

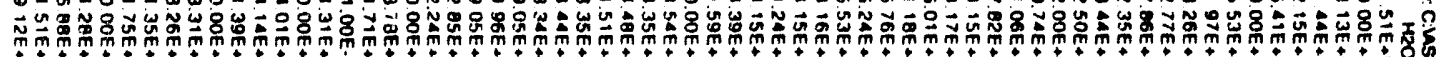

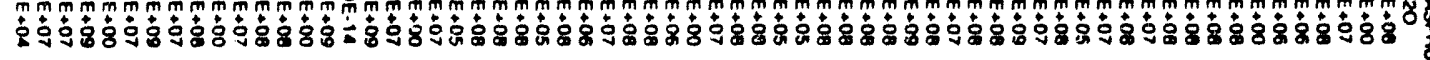

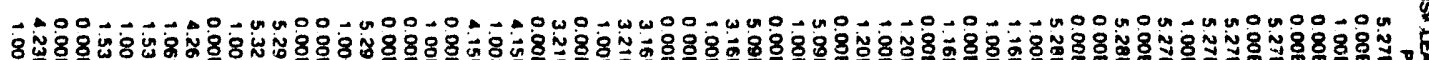

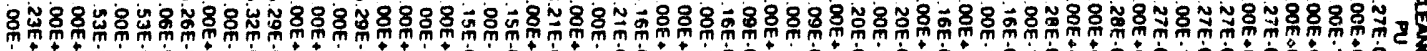

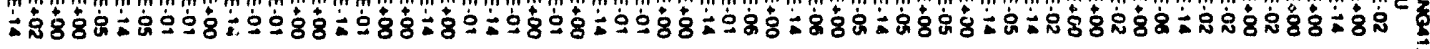

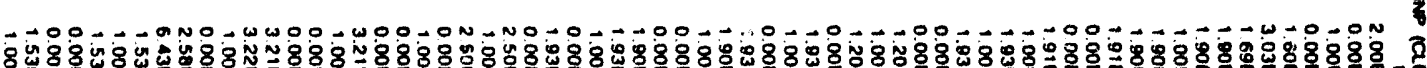

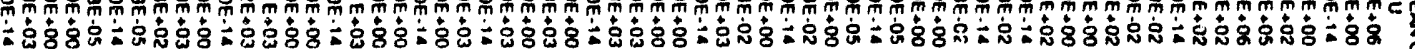

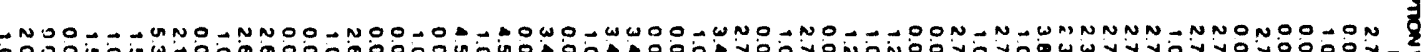

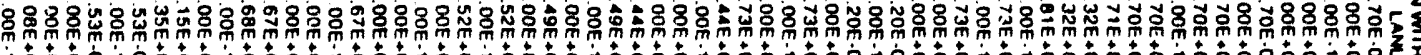

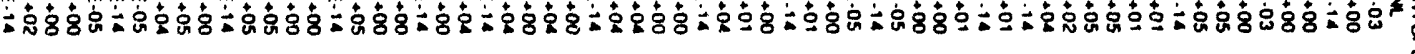

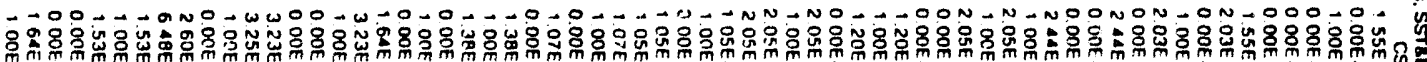

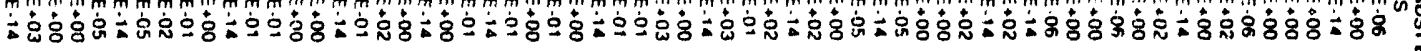

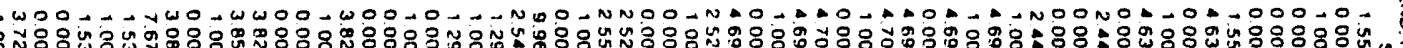

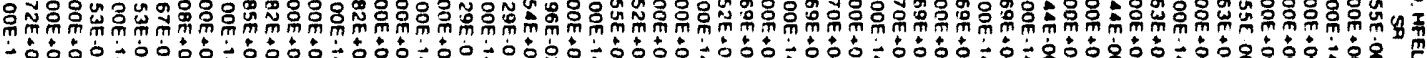

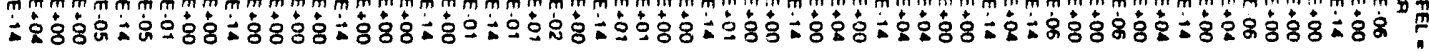

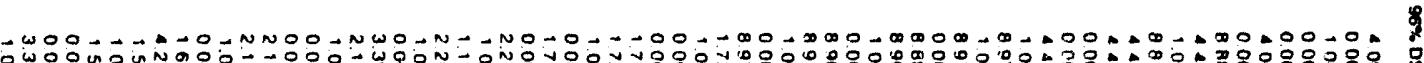

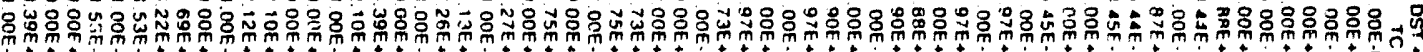

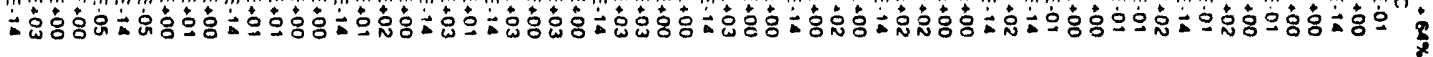

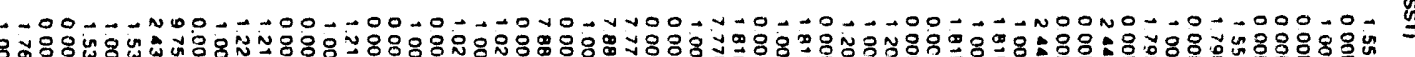

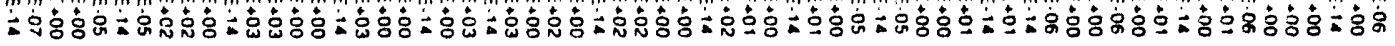

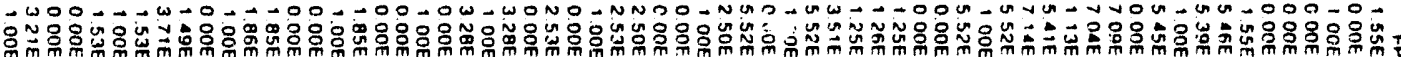

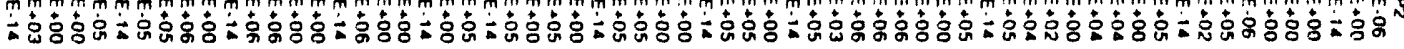

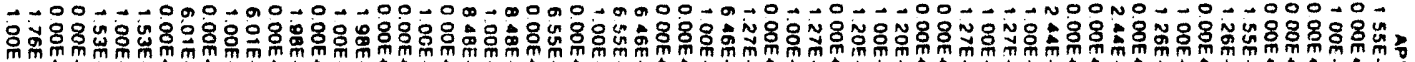

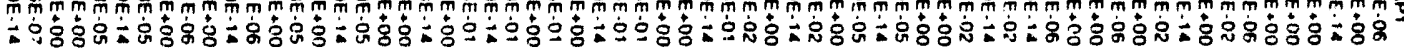

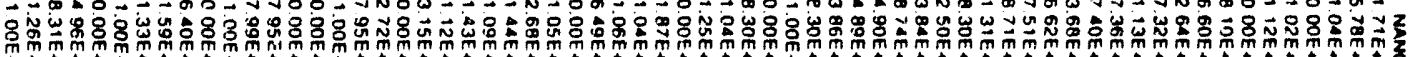

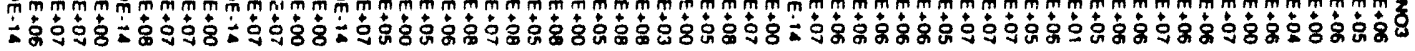

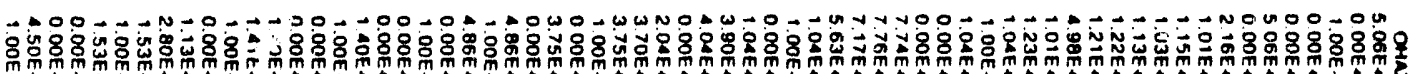

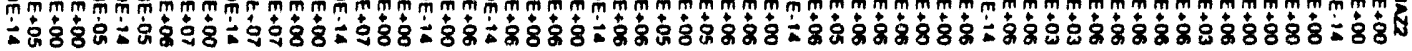

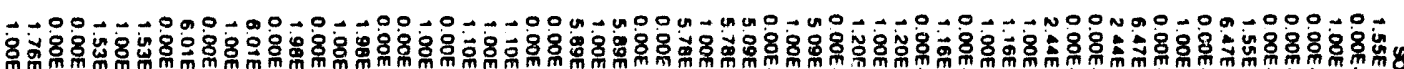

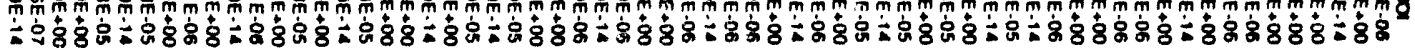

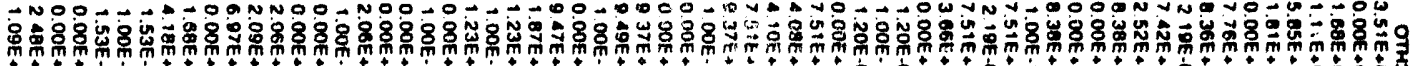

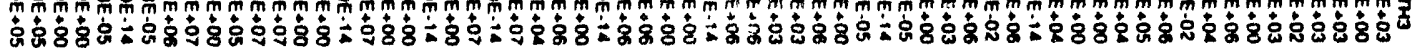

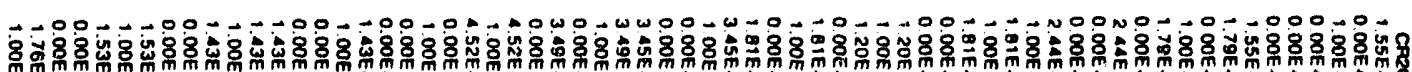

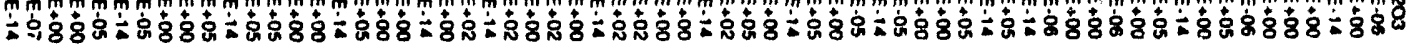

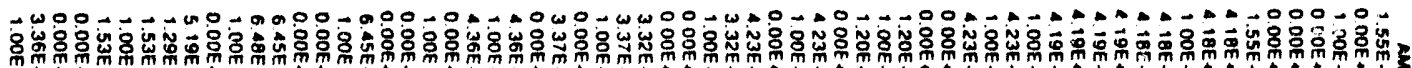

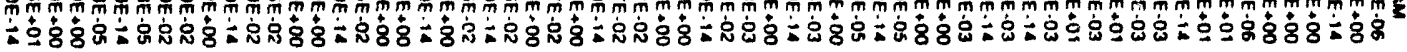


Table H3. ASPEN PLUS Global Model Mass Balance Output Results. (sheet 54 of 56)

\begin{tabular}{|c|c|c|c|c|c|c|c|c|c|c|}
\hline 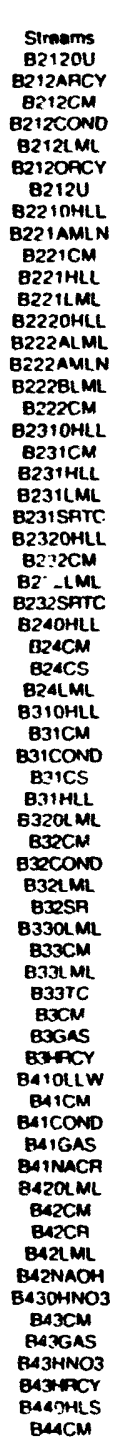 & 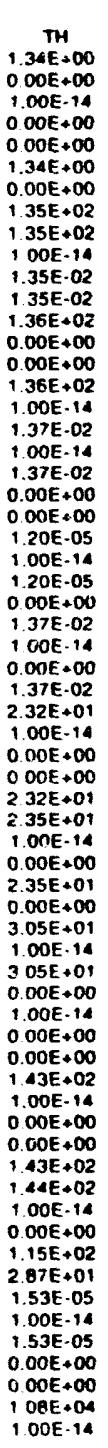 & 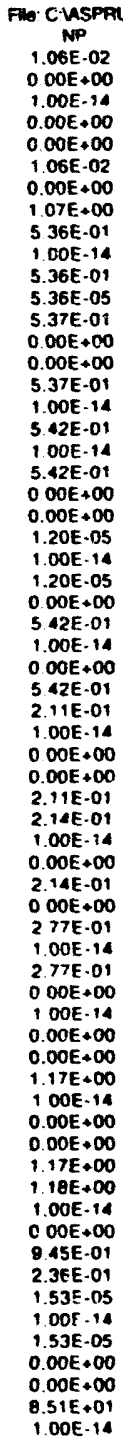 & 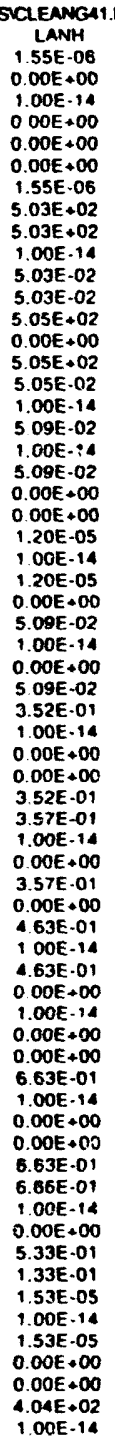 & 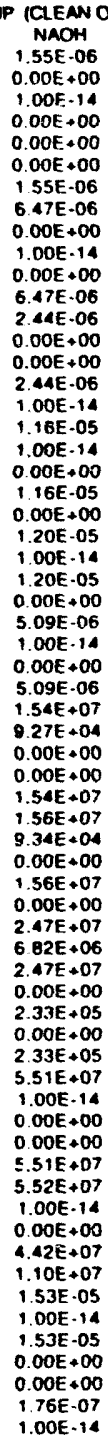 & 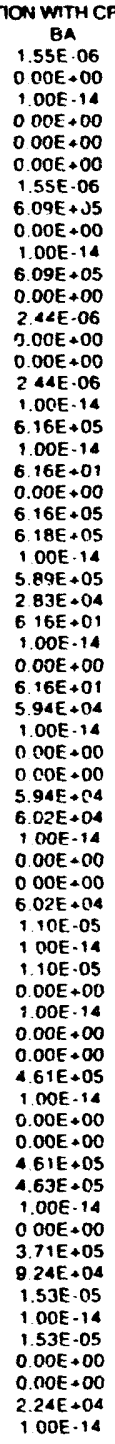 & 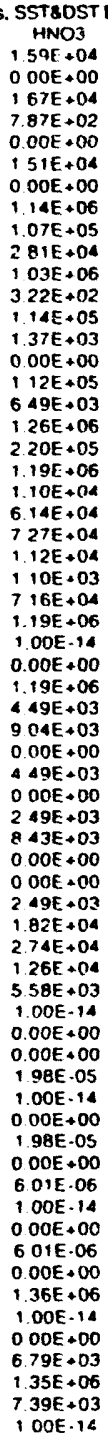 & 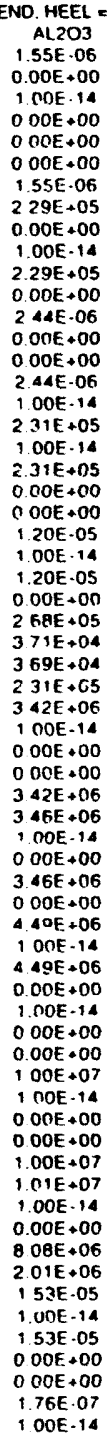 & 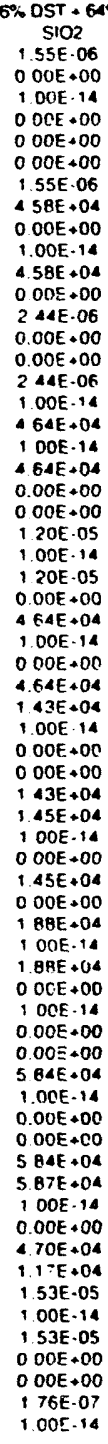 & 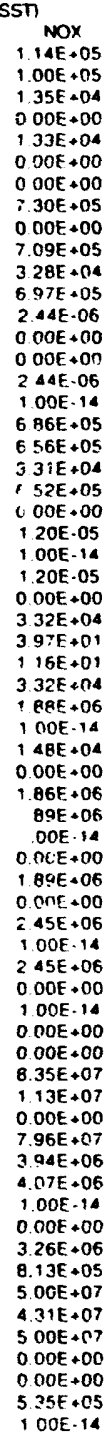 & 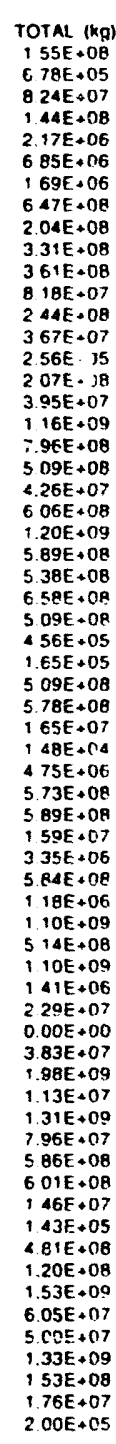 \\
\hline
\end{tabular}




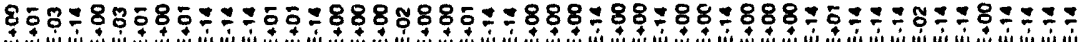

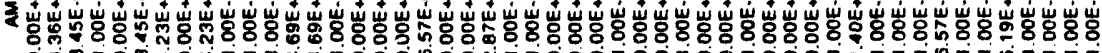

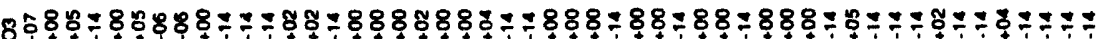

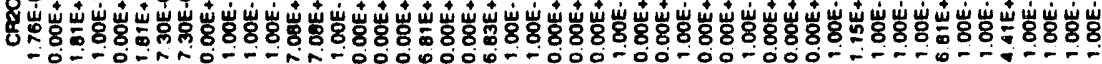

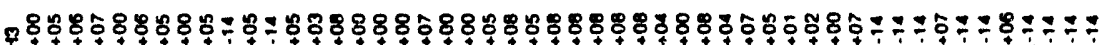

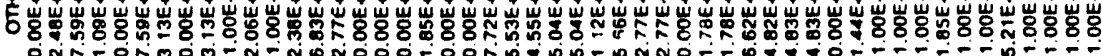

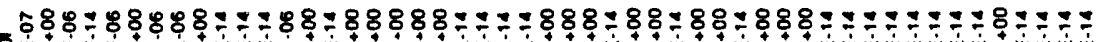

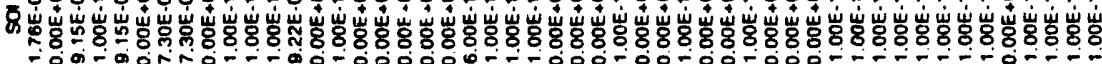

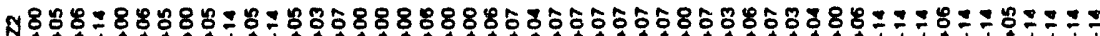

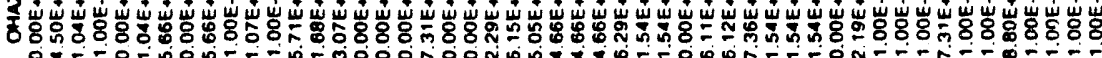

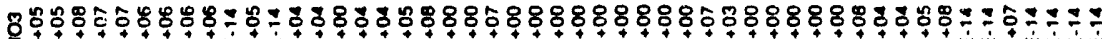

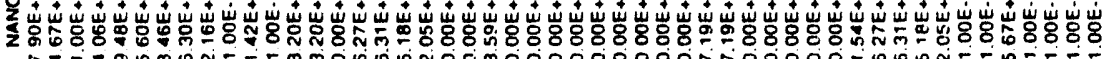

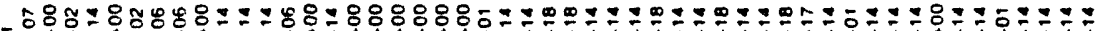

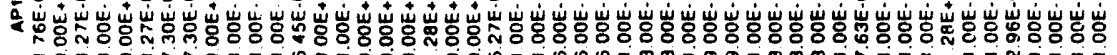

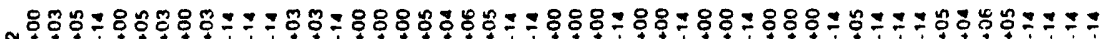

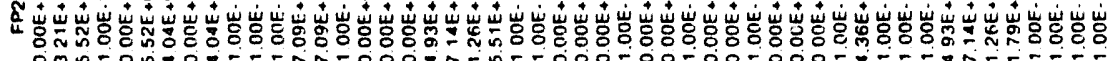

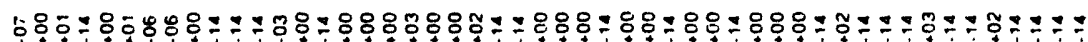

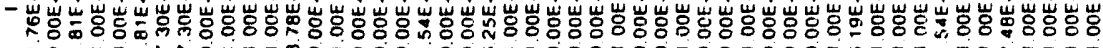

点

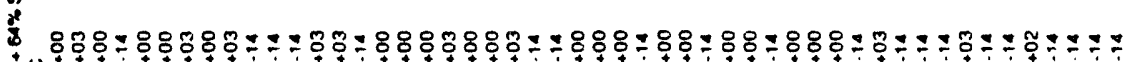

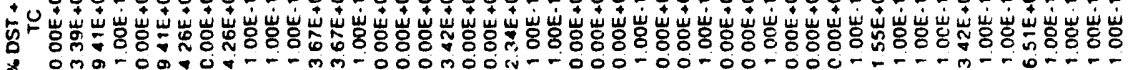

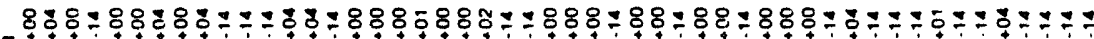

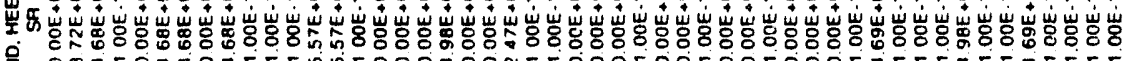

密

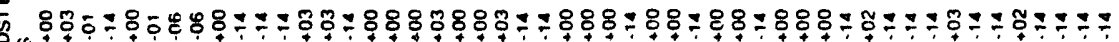

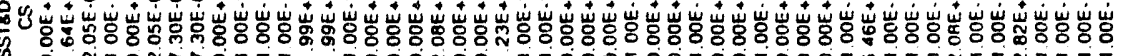
究

舟

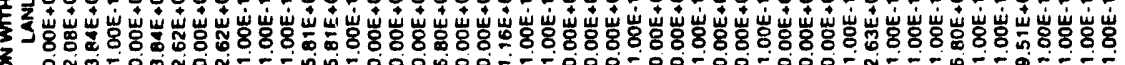

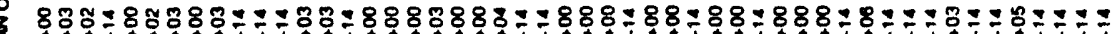

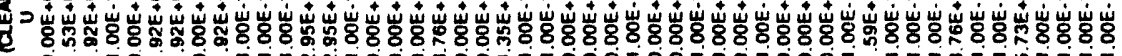
定

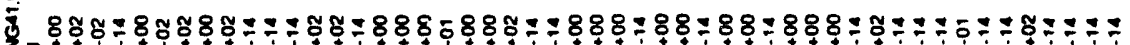

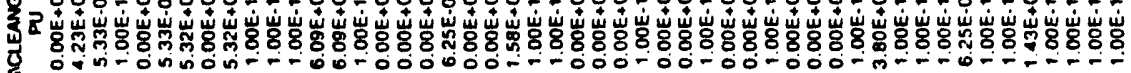
3

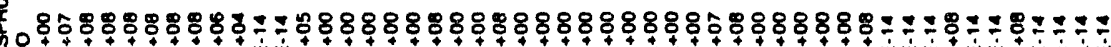

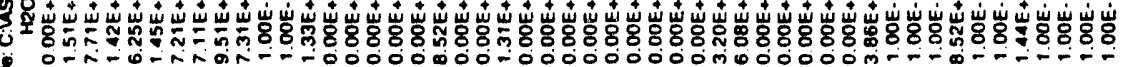

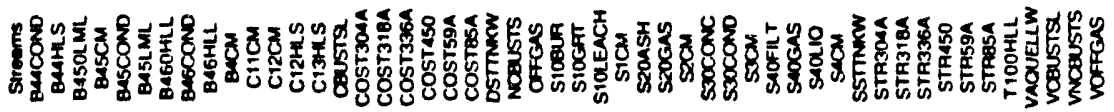


Table H3. ASPEN PLUS Global Model Mass Balance Output Results. (sheet 56 of 56)

\begin{tabular}{|c|c|c|c|c|c|c|c|c|c|c|}
\hline 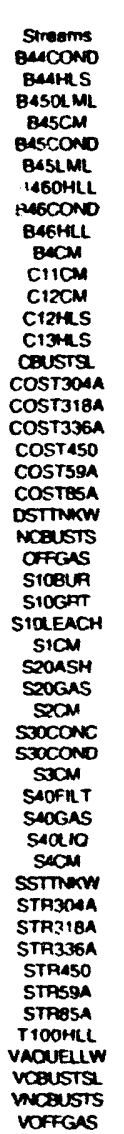 & 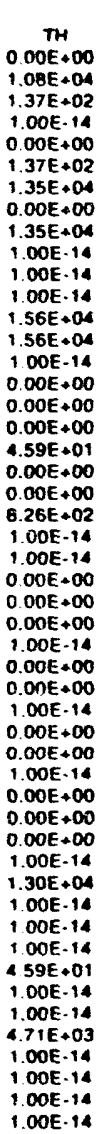 & 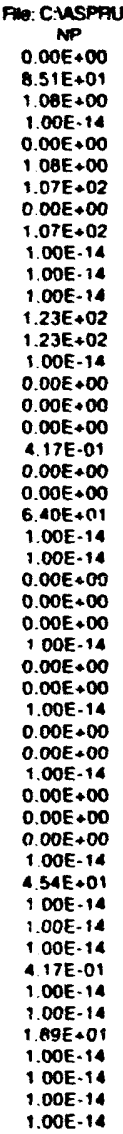 & 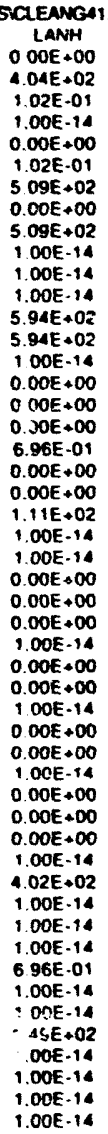 & 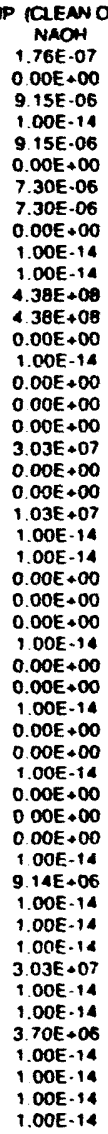 & 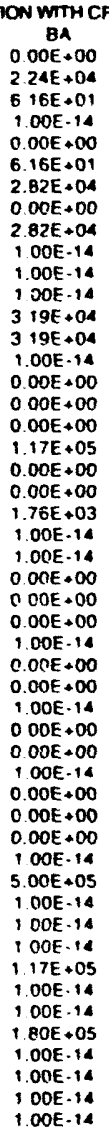 & 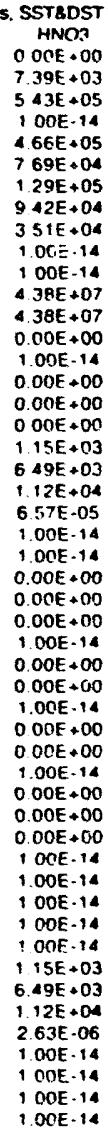 & 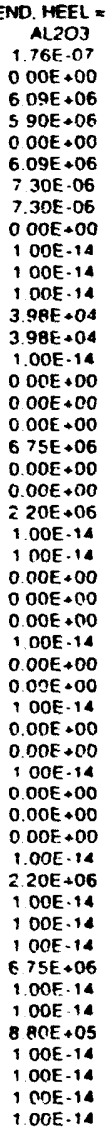 & 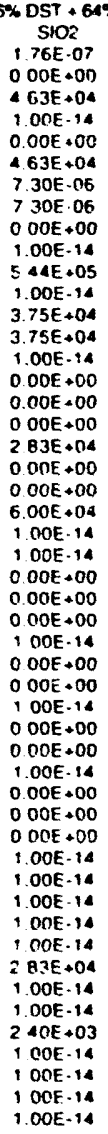 & 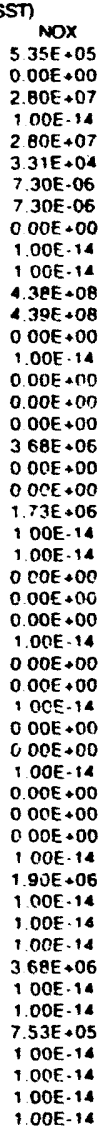 & 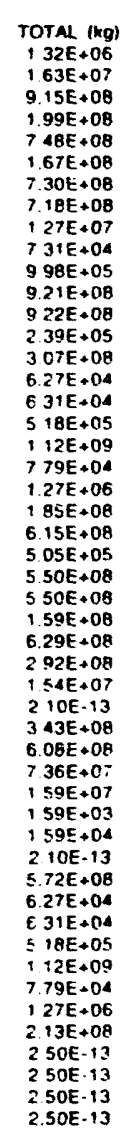 \\
\hline
\end{tabular}


WHC-EP-0791

This page intentionally left blank. 
WHC-EP-0791

APPENDIX I

\section{RELATIVE HEALTH RISK ESTIMATES}




\section{LIST OF TERMS}

$\begin{array}{ll}\text { ALARA } & \text { as low as reasonably achievable } \\ \text { DF } & \text { decontamination factor } \\ \text { DOE } & \text { U.S. Department of Energy } \\ \text { DST } & \text { double-shell tank } \\ \text { GTCC } & \text { Greater than Class C } \\ \text { HHI } & \text { Health Hazard Index } \\ \text { HLW } & \text { high-level waste } \\ \text { HSA } & \text { Hanford Strategic Analysis } \\ \text { LANH } & \text { heavy lanthanides } \\ \text { LANL } & \text { light lanthanides } \\ \text { LLW } & \text { low-level waste } \\ \text { PUREX } & \text { Plutonium-Uranium Extraction (PIant) } \\ \text { SST } & \text { single-shell tank } \\ \text { TRU } & \text { transuranic } \\ \text { TRUEX-C } & \text { Transuranic Extraction Option C }\end{array}$


WHC-EP-0791

\section{RELATIVE HEALTH RISK ESTIMATES}

\section{I1.0 RELATIVE HEALTH RISKS}

Systems analyses of radioactive waste handling systems require safety performance measures in evaluating alternative processes. Absolute health risks in terms of dose or deaths are not necessary for the purpose of this study. Oniy ratios of health risks of the same kind are calculated for the processes to be compared. Health risks of each kind can be stated as ratios to a standard or a regulation, and the values for each process can be compared with each other.

The various kinds of health risks can be subdivided into short term and long term with the time of decay of ${ }^{137} \mathrm{Cs}$ and ${ }^{90} \mathrm{Sr}$ to insignificant values as the transition point. In waste repository risk calculations, the range 300 to 1,000 years after reactor discharge was taken as a transition range, but here 500 years will be used as the dividing time between short term and iong term.

A second kind of subdivision of health risks is between onsite and offsite risks. As used here "onsite risks" refer to radionuclides or chemicals in question that remain onsite usually in solid form and undisturbed unless man intrudes and releases them into the biosphere, exposing himself or carrying them offsite to expose others. As used here, offsite risk refers to radionuclides or chemicals that migrate offsite with geochemical agents, such as water after barriers are breached. The onsite risk is directly related to the concentration in the waste at the onsite sample point, while offsite risk also depends on the solubility in water and the rate of transport to a remote sample point where the material is released to the biosphere.

A preliminary division of the safety performance measures into five categories has been made, and a relative health risk estimate for each of them has been developed. The categories are given below:

1. Long-Term Offsite Safety: This measure is confined to radionuclides that can be transported by water from the waste site and measured by an adjusted health hazard ingex (HHI). The important nuclides on our time scale are ${ }^{9} \mathrm{TC}$ and ${ }^{29} \mathrm{I}$.

2. Long-Term Onsite Safety: This measure assumes that human or animal intrusion will introduce mixed wastes directly into the biosphere. This measure is represented by the ratio of radionuclide concentration to Hanford cleanup residential sojil standards, The
important nuclides are alpha emitters, such as ${ }^{238} \mathrm{U},{ }^{230} \mathrm{Pu},{ }^{241} \mathrm{Am}$, $24 \mathrm{Am}$, and their decay chains.

3. Short-Term offsite Safety: This measure is confined to radionuclides that can be transported to the biosphere within 300 to 500 years by water or air after unusual events, or which are already present in the groundwater. It is measuren by the ratio of concentration to potabie water limits. The important radionuclides should be ${ }^{3 \mathrm{H}}$, ${ }^{139} \mathrm{Cs}$, and ${ }^{90} \mathrm{Sr}$. 
4. Short-Term Onsite Safety: This measure assumes human or animal intrusion will introduce mixed wastes directly into the biosphere within 300 to 500 years. This measure is represented by the ratio of radionuclide concentration to Hanford Site cleanup residential soll standards. The important nuclides should be ${ }^{13} \mathrm{Cs},{ }^{80} \mathrm{Sr}$, and Pu.

5. Occupational Exposure: This measure accounts for exposure to workers operating the processing plants who are taking samples and cleaning up spilils. It is directly proportional to and measured by the volume and activity level of tertiary nonprocess wastes, such as special work permit clothing, laboratory samples, gloves, removed equipment, decontamination solutions. The important radionuclides should be ${ }^{137} \mathrm{Cs},{ }^{90} \mathrm{Sr}$, and ${ }^{238} \mathrm{Pu}$. These may be classified as gamma fields, alpha emitters, and beta emitters with low energy gamma fields, and indexes can be calculated separately for each category.

\section{I2.0 PROBABILITY AND RISK}

It is certainly desirable to evaluate all five of these relative performance measures separately. However, after this step is taken, one cannot combine them into a single safety performance measure without calculating the risk from each (i.e., the product of probability of occurrence and the dose consequences).

It may be possible to come up with a useful relative measure of risk without computing the doses if the limits for delivery to man are consistent among the five performance measures. For this, one would al so need the set of probabilities for each of the five types of exposure (i.e., the probability distribution that each will occur). In the absence of much experience, this probability set might be in the form of uniform probability, log uniform probability, or a triangular distribution for each of the five consequences.

Although we have not estimated health risks here, some idea of the values of the probability sets is as follows.

1. Long-Term Offsite Safety: Barrier degradation followed by nuclide transport will have a high probability, near unity, because the process is already described by the calculation of transport through the barrier. Only the probability of water contact during a very long time period needs to be added. Additional disruptive events, such as those that shorten the flow path for water to and from the waste, will have lower probabilities; but the consequences will be greater because the barrier is less formidable.

2. Long-Term Onsite Safety: The probability of human intrusion is the subject of much debate. However, over a long period of time, it is very likely. Then, the exposure of the intruder and quantity of waste removed to the biosphere by human transport may become an important quantity. 
3. Short-Torw Offsite Safety: The very low probability of release by disruptive events during the first 300 to 500 years should be evaluated because the consequences resulting from the release of high inventories of ${ }^{137} \mathrm{Cs}$ and ${ }^{90} \mathrm{Sr}$ could be very great. Materials already released would need a transport calculation but would have a release probability of unity.

4. Short-Torm Onsite Safoty: The probability of human intrusion during the first 300 to 500 years can be made very low by administrative controls of the surrounding area. The consequences resulting from intrusion into high inventories of ${ }^{137} \mathrm{Cs}$ and ${ }^{0} \mathrm{Sr}$ may still be very large.

5. Occupational Exposure: Estimated exposures would have a probability of unity. Occupational exposure of workers resulting from accidents would have to be included, and each accident will have its own probability of a given dose exposure.

If the probability distribution part of each of the five consequences were estimated, the corresponding risks could be put on a common basis. The first four can be ratioed to concentration or inventory limits that have been derived from an allowable dose; therefore, the dose for the consequence is available by simple ratio. The occupational exposure is already ratioed to a dose limit. Proper attention should also be given to the important matter of whether each of the five risks are in terms of maximum individuals or the population as a whole. The ratio of the worker population to the total population may also be needed. Only then can we calculate and sum all five risks in dose units to estimate a single performance measure of radiological risk.

The measurement of health risk in terms of dose units would bypass the secondary concentration and inventory limits that have been derived from them to set regulatory controls on releases from waste sites. This would allow one to determine the effect on health risk of setting the concentration limits at one value or another. One could then do cost-benefit analyses on the regulations themselves and influence the setting of reasonable limits. This has not been done in this analysis, and only five separate HHIs have been est imated.

\section{I3.0 EVALUATION OF RELATIVE HEALTH MAZARDS}

\subsection{LONG-TERH OFFSITE HEALTH HAZARD}

The long-term offsite radiological safety performance measure was evaluated by combining transport through a barrier to a 100 meter distant well with the concentration limit for potable water. The resultant adjusted HHI was then used to compare the waste separation alternatives. This long-term performance measure is confined to radionuclides that can be transported by water from the waste site and measured by the adjusted HHI. The important nuclides are ${ }^{9} \mathrm{TC}$ and ${ }^{129} \mathrm{I}$. 
The relative long-term health hazard model developed below evaluates the long-term health hazard to the public. The health hazard estimates included the following assumptions.

1. All time perlods are equally important when evaluating health hazard. However, this model is directed at the time period from 500 to $10^{3}$ years or essentially long-term health hazard.

2. Waste is disposed of in dry form, with nuclide migration to groundwater and then into drinking water as the dominant hazard pathway from the waste dispo:al site to biological receptors.

3. Assessment of health hazard from benefictal use of nuclear materials is not considered.

4. The radiological health hazard to a receptor is proportional to the quantity of radioactive material disposed of. This proportionality relies on the following. First, health hazard is proportional to the dose to a receptor by a dose response constant, $d_{r}$ (endpoints/mrem). Second, dose is a linear function of disposed waste mass. To relate dose to source mass, a dose factor, $d_{f}$, and a waste disposal package/barrier attenuation factor, $a_{f}$, are used. These factors account for far - field and near - field barrier effects, respectively. The dose factor, $d$, relates liquid concentration at the biosphere (e.g., drinking water from a well) to the disposed waste mass source and al so characterizes the receptor's rate and period of ingestion. It is adjusted to support incorporation of a package/barrier attenuation factor, $a_{f}$, which is normalized to unity for unimpeded migration in soil (mrem/ $\mathrm{kg})$. The package/barrier attenuation factor, $a_{4}$, depends on diffusivity and path to soil regions without drainage barriers. This factor is normalized to unity for unimpeded migration in soil. For groundwater, it is normalized to unimpeded groundwater flow $\left(a_{f} \leq 1\right.$, dimensionless). As an example, the health hazard, $r_{k}$, from technetium mass $(\mathrm{kg})$ in output waste stream $k, T c_{k}$, would be:

$$
r_{k}=d_{r} * d_{f k} * a_{f k T c} * T c_{k}
$$

Thus, the relative long-term health hazard to the public, $r_{\text {rel }}$, may be characterized by the following equation:

$$
r_{\text {rel }}=\left(r_{11 W}+r_{1 \text { In }}+r_{\text {tru }}+r_{h(w)}\right) / r_{0}
$$

where:

$r_{11}=$ Health hazard due to low-level waste.

$r_{1}=$ Health hazard due to low-level mixed waste (hazardous and radioactive).

$r_{\text {tru }}=$ Health hazard due to transuranic waste.

$r_{\text {hiw }}=$ Health hazard due to high-level waste.

$r_{0}=$ Health hazard from current inventory in its existing configuration. 
5. Material in current inventories would in time migrate in soll without any impediment, except for materials in Facilities and Nuclear Material. For the purpose of normalizing health hazard estimates, it is assumed that the period of initially impeded inigration resulting from tanks, etc., is negligible.

6. For an initial approximation, assume all $d_{\text {fk }}$ are equal. Thus, $r_{\text {rel }}$ is only a function of afk and $T c_{k}$ (or $I_{k}$ ). "The following represent the main waste disposal product streams considered in the relative health hazard estimates.

\begin{tabular}{|c|c|}
\hline $\begin{array}{l}\text { Waste Disposal } \\
\text { Product Stream }\end{array}$ & $\begin{array}{c}99 \text { Tc Package/Barrier } \\
\text { Attenuation Factor, a } \\
\text { fkic }\end{array}$ \\
\hline $\begin{array}{l}\text { In Situ } \\
\text { Vitrification } \\
\text { Monolith: } \\
\text { 3110HLWS }\end{array}$ & $5.1 E-11$ \\
\hline $\begin{array}{l}\text { Salt Grout: } \\
3400 \text { LMWS, LLWS }\end{array}$ & $6.6 E-5$ \\
\hline $\begin{array}{l}\text { In-situ Grout: } \\
\text { 3530LMWS }\end{array}$ & $1.5 E-5$ \\
\hline $\begin{array}{l}\text { Dome Fill Tank: } \\
\text { 3200HLWS }\end{array}$ & $1.7 E-4$ \\
\hline
\end{tabular}

To estimate the iodine (I) attenuation factors, $a_{f l}$, it is was assumed that $1 \mathrm{~kg}$ of I is 1.5 times as dangerous as $1 \mathrm{~kg}$ of technetium, based on a relative dose contribution basis (e.g., iodine concentrates in the thyroid once in the human body, while technetium distributes among many organs). Thus, $a_{\text {fly }}=1.5 * a_{\mathrm{fkTc}}$. The basis for this factor is the following calculation:

$$
{ }^{129} I /\left({ }^{127} I+{ }^{129} I\right)=0.67
$$

Use 50-year committed dose equivalent factors from DOE/EH-0071(7/88):

$$
\begin{aligned}
\mathrm{CEDE}_{1} *(\mathrm{~kg} / \mathrm{Ci})_{\mathrm{TC}} /\left(\mathrm{CEDE}_{\mathrm{TC}} *(\mathrm{~kg} / \mathrm{Ci})_{1}\right) & =0.28 * 0.059 / 1.3 \mathrm{e}-3 * 5.66 / 0.67 \\
& =1.5
\end{aligned}
$$

The iodine attenuation factors were al so multiplied by a conservative 0.8 factor to account for the ratio of radiogenic iodine from nuclear reactors in the stream flows (Pajunen et al. 1992).

The above stream technetium and iodine masses along with the relative long-term health hazard estimates for the CLEAN Option, Transuranic Extraction Option C (TRUEX-C), and Sludge Wash C process strategies are shown in Table II 
for the ox heel (1.e., unretrieved waste remaining in tank) case. Figure II shows the same health hazard data. The comparison results show that the relative long-term offsite health hazard for the CLEAN Option process strategy is within $1 \%$ of the health hazard for the TRUEX-C and Sludge Wash $C$ process strategles for the 0\% tank waste heel case. Based on previous analyses of the TRUEX-C and Sludge Wash C process strategies (Knutson et al. 1993) these relative health hazards will increase by a factor of approximately 2.4 if the heel percentage increases from zero to $5 \%$. This health hazard increase is associated with more radioactive waste products left behind in the tanks that will be immobilized in place (stream 3200HLWS would reflect this health hazard increase). Thus, the data suggest that the relative long-term offsite health hazard is comparable for all of the process strategies.

These results should not be used in an absolute sense but only for comparative value in considering the various waste treatment processes.

\subsection{LONG-TERH ONSITE HEALTH HAZARD}

A preliminary set of coefficients for long-term onsite health hazard is given in Table 12 for the 25 lumped components used in the global models. These coefficients are derived from the drilling and excavation scenarios given in Dose and Risk Assessment for Intrusion Into Mixed Waste Disposal Sites (Kennedy and Aaberg 1991). For all situations, the dose from the excavation scenario predominated over the drilling scenario. Their results are presented in Table 13 in terms of rems per person per drilling or excavation event for individual radionuclides present at unit concentrations in the solid waste. They represent the worst-case scenario with pathways to the highest exposed individual. The final column in Table 13 shows the ir health hazard data in units of dose (millirem) per exposure concentration $\left(\mathrm{C} / \mathrm{m}^{3}\right)$ convenient for HHI calculations made in this report.

To calculate the long-term onsite HHI (mrem per person) associated with a particular component in a specific waste stream, the HHI coefficient (mrem/[Ci/m $]$ ) for that component was multiplied by the radioactivity leve] (or concentration) $\left(\mathrm{Ci} / \mathrm{m}^{3}\right)$ of the component in that specific waste stream. The radioactivity level calculations are discussed in Section 15.0.

The long-term onsite HHIs in mrem per person are shown in Table I4 for the Clean Option, TRUEX-C, and Sludge Wash $C$ process strategies. The data in Table I4 were normalized to the value for the total tank waste feedstreams (SSTTNKW and DSTTNKW). Cesium and strontium were excluded because of their short half-lives compared to the assumed long-term time frame of greater than 500 years. The normalized HHIs are compared in Figure 12 by waste stream for the three pretreatment process strategies with $0 \%$ tank heels.

As shown in Figure 12, the greatest long-term onsite waste health hazard would be in the high-level waste (HLW) glass stream (3110HLWS) if the environment were not protected from it by formidable barriers around the repository. The uranium product stream (B212U for Clean, B24U for TRUEX-C and Sludge Wash C) has the highest HHI for the Sludge Wash C process because it is highly concentrated. However, it is assumed that the uranium product does not remain onsite but is added to the U.S. national stockpile. Thus, the tank heel (requiring dome fill) stream (3200HLWS) dominates the onsite health 
hazard. Otherwise, the primary low-level waste (LLW) grout stream ( 3400 LLWS +3400 LMWS) contains the only significant variation between pretreatment process strategies, where the TRUEX-C process strategy value was a factor of approximately 5 and $7 \mathrm{higher}$ than for the Clean Option and Siudge Wash C process strategies, respectively. Note that while no estimate of the primary LLW glass HHI is included, it is expected to be lower than that resulting from primary LLW grout because the glass represents a more stable long-term waste form than grout.

The contributions of each component to the normalized long-term onsite HHI for the HLW glass and primary LLW grout are compared in Figures 13 and I4, respectively. Figure 13 shows that the Clean Option HLW glass has substantialiy more contribution to the long-term onsite HHI (compared to the original total feedstream value) from plutonium, technetium, americium, thorium, neptunium, and heavy lanthanides (LANH), because of the more extensive separation processing performed for these elements. This greater processing in the CLEAN Option al so produced less contribution to HLW glass from uranium, fission products (FP2), and ${ }^{63} \mathrm{Ni}$ (OTH3) compared to the other process strategies.

Overall, the Clean Option long-term onsite HHI was more than an order of magnitude greater for the HLW glass product than the TRUEX-C and Sludge Wash C process strategies because of the greater $H L W$ concentration. However, it is highly unlikely that any excavation would uncover a deep repository. The drilling scenario with different controlling components would produce more health hazard from the repository than the excavation scenario.

The contributions of each component to the normalized long-term onsite HHI for primary LLW grout show that the CLEAN Option had larger contributions from the iodine, fission products (FP2), ${ }^{63} \mathrm{Ni}$ (OTH3), thorium, and neptunium than the TRUEX-C and Sludge Wash $C$ process strategies because of the greater primary LLW concentration. However, the overall long-term onsite HHI for primary LLW grout is substantially lower when compared to the initial feedstream.

These results should not be used in an absolute sense but only for comparative value in considering the various waste treatment processes.

\subsection{SHORT-TERH ONSITE AND OFFSITE HEALTH HAZARD}

The offsite health hazard for the short term (less than 500 years) was nearly nil because the barriers to migration would be expected to be intact and unbreached for 500 years uniess man intruded. Therefore, the offsite health hazard was included in the onsite health hazard. The short-term onsite health hazard was calculated in the same way as the long-term onsite health hazard, except that the age of the waste was assumed to be only 10 years so that ${ }^{3} \mathrm{CS}$ and ${ }^{90} \mathrm{Sr}$ were still the dominant radiation dose inventories.

The HHI coefficients for short-term and long-term onsite health hazards are given in Table 12. It is apparent that the two indexes have the same values for many components. This does not mean that the two health hazards are the same because the probabilities of release are not included. While a framework has been laid for future calculations, much work needs to be done in 
estimating or finding input information to calculate these HHIs and in estimating probability sets before they can be combined to give a single index.

The short-term onsite and offsite HHIs in mrem per person are shown in Table I5 for the Clean Option, TRUEX-C, and Sludge Wash $C$ process strategies. The data in Table 15 were normalized to the value for the total tank waste feedstreams (SSTTNKW and DSTTNKW) and compared in Figure I5 by waste stream for the three pretreatment process strategies with $0 \%$ tank heels.

As shown in Figure 15, the greatest short-term onsite waste health hazard would be in the HLW glass stream (3110HLWS) if the environment were not protected from it by formidable barriers around the repository. Excluding the $\mathrm{HLW}$ glass product, the tank heel (requiring dome fill) stream (3200HLWS) dominates the short-term onsite health hazards. Otherwise, the only significant variations in the short-term onsite health hazards between the pretreatment process strategies occurs for the primary LLW grout product stream (3400LLWS+3400LMWS) and the uranium product stream (B212U for CLEAN and B24U for TRUEX-C and Sludge Wash C). Assuming that the uranium product does not remain onsite but is added to the U.S. national stockpile, only the primary LLW grout product health hazards are compared. As shown in Figure 15 the Clean Option process strategy has a primary LLW grout product health hazard value that is more than an order of magnitude less than the TRUEX-C and Sludge Wash C process strategy values. Note that while no estimate of the primary LLW glass HHI is included it is expected to be lower than that resulting from primary $L L W$ grout because the glass represents a more stable short-term (up to 500 years) waste form than grout.

The contributions of each component to the normalized short-term onsite HHI for the HLW glass and primary LLW grout are compared in Figures 16 and 17 , respectively. Figure I6 shows that the CLEAN Option HLW glass has

substantially more contribution to the short-term onsite HHI (compared to the original total feedstream value) from plutonium, cesium, strontium, technetium, americium, thorium, neptunium, and LANH, because of the more extensive separation processing performed for these elements. This greater processing in the CLEAN Option also produced less contribution to $\mathrm{HLW}$ glass from uranium, fission products (FP2), and ${ }^{63} \mathrm{Ni}$ (OTH3) compared to the other process strategies.

Overal1, the CLEAN Option short-term onsite HHI was more than an order of magnitude greater for the HLW glass product than the TRUEX-C and Sludge Wash C process strategies because of the greater HLW concentration. However, it is highly unlikely that any excavation would uncover a deep repository in the first 500 years after closure. The drilling scenario with different controlling components would probably produce more health hazard from the repository than the excavation scenario during the first 500 years.

The contributions of each component to the normalized short-term onsite HHI for primary LLW grout show that the CLEAN Option had larger contributions from the iodine, fission products (FP2), ${ }^{63} \mathrm{Ni}(0 \mathrm{TH} 3)$, thorium, and neptunium than the TRUEX-C and Sludge Wash C process strategies because of the greater primary LLW concentration. However, the CLEAN Option had substantialiy smaller contributions from cesium and strontium because of the more extensive 
WHC-EP-0791

separation processing performed for these elements. Overall, the short-term onsite HHI for primary LLW grout is substantially lower when compared to the initial feedstream.

These results should not be used in an absolute sense but only for comparative value in considering the various waste treatment processes.

\subsection{OCCUPATIONAL EXPOSURE}

The dose to workers operating a plant may be an important health hazard. In general, with similar designs, one would expect that more highly radioactive streams result in greater worker exposure, even though the processes themselves are shielded. This is because sampling and equipment repair usually have some doses associated with them. It was arbitrarily assumed that the occupational exposure was proportional to the volume of tertiary nonprocess waste from a particular process multiplied by the curie concentration of the lumped component in the feedstream to a process. This does NOT result in the curies in the tertiary waste or in an HHI in terms of dose but does leave the possibility that the index could be calibrated by data from an operating plant.

There are essentially two types of occupational exposure. They are as follows.

1. Gamma radiation (primarily ${ }^{137} \mathrm{Cs}$ ) emanating from equipment, processes, or contamination, which acts through a distance and perhaps requires shielding.

2. Contamination by alpha and beta particles where the radionuclide gets on the clothing, skin, or inside the person. The alpha contamination is quite significant in occupational exposure (e.g., uranium, plutonium, neptunium, thorium, americium). The beta contamination will be unimportant unless a low-energy gamma accompanies the beta decay.

The previous report (Knutson et al 1993) considered occupational exposure resulting from ${ }^{137} \mathrm{Cs}$ weighted by the feedstream and tertiary waste flows to particular processes, along with the relative alpha contamination from ${ }^{238} \mathrm{U}$ and ${ }^{239} \mathrm{Pu}$ and beta contamination from ${ }^{90} \mathrm{Sr}$. To increase the accuracy and detail of the occupational exposure calculations more components were evaluated in this report. Because the lumped or pseudo components of FP2, OHAZ2, and OTH3 contained a mix of isotopes, the isotope with the major exposure impact was chosen to represent the pseudo component. This impact was evaluated, based on the particular isotope radioactive inventory, half-life compared to expected processing time frames, and decay particle energies and their relative exposure importance. Isotopes with more than one decay particle emission were classified based on the decay particle energies. The following is a list of the radionuclides considered in this report and their subsequent contamination classification used in calculating the occupational exposure impact. 
WHC-EP-0791

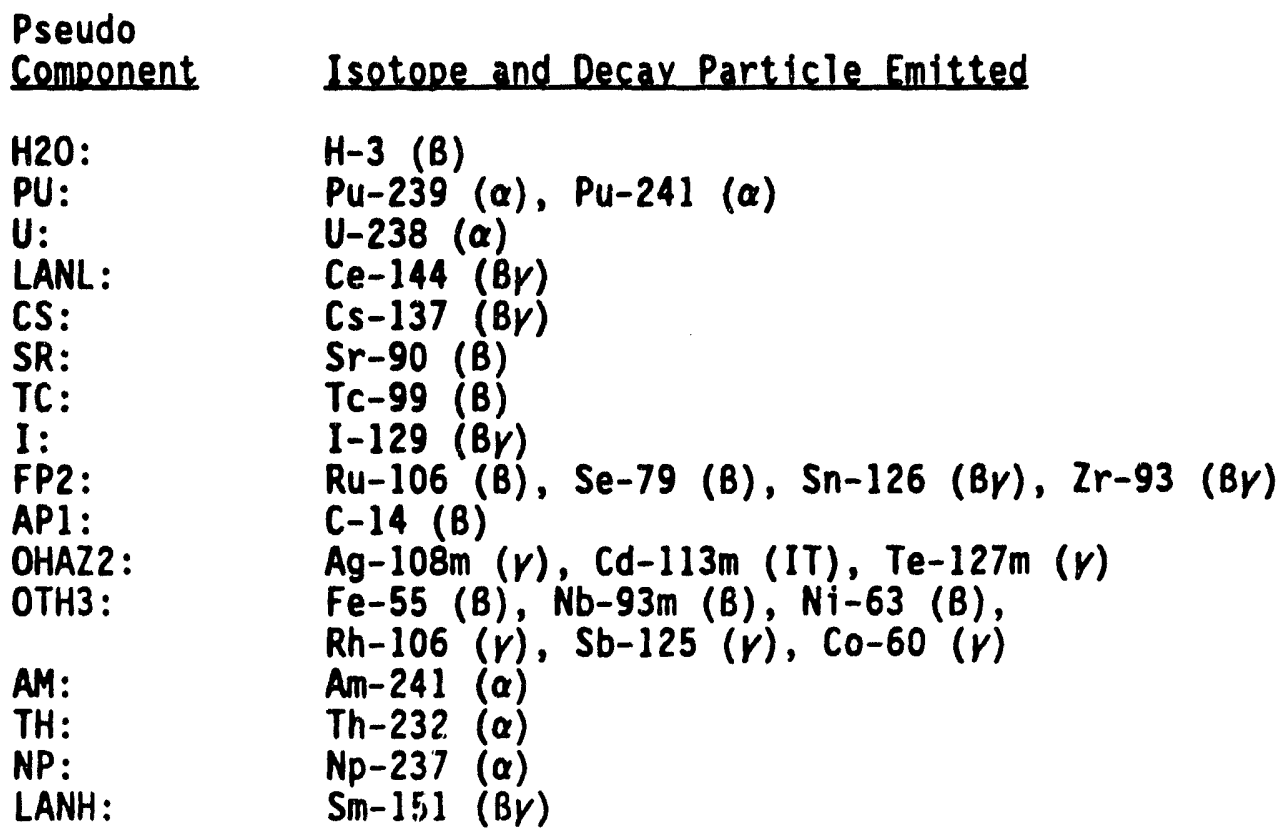

\section{I4.0 CLASSIFICATION FOR OCCUPATIONAL EXPOSURE CALCULATION}

Gamma Contamination: Cs (Cs-137)

Alpha Contamination: $\quad U(U-238)+P u(P u-239)+A m(A m-241)+T h(T h-232)$

$+N p(N p-237)$

Beta Contamination: $\operatorname{Sr}(\mathrm{Sr}-90)+\mathrm{TC}(\mathrm{TC}-99)+\mathrm{I}(\mathrm{I}-129)+\mathrm{FP2}$ (Zr-93

$+\mathrm{Sn}-126)+$ APl $(\mathrm{C}-14)+$ OTH3 $(\mathrm{Ni}-63)+$ LANH $(\mathrm{Sm}-151)$

Note that $\mathrm{H}_{2} \mathrm{O}$, light lanthanides (LANL), and OHAZ2 were not included because the radioactive inventories for their respective isotopes were ignored, based on their short half-lives compared to expected processing time frames or simply from having zero radioactive inventories. Table $\mathrm{Jl}$ of Appendix $\mathrm{J}$ shows the isotopes that were ignored in the radioactive inventory calculations.

Thus, the occupational exposure HHI was calculated for each major process within each process strategy using three separate indexes for gamma, alpha, and beta contamination. These indexes represent the process feedstream radioactive inventory concentration $(\mathrm{Ci} / \mathrm{kg})$ weighted to represent tertiary waste "curies" by multiplying by the process tertiary waste stream inventory $(\mathrm{kg})$. Assuming an arbitrary $1 \mathrm{~kg} / \mathrm{m}^{3}$ density for comparison purposes, the resulting HHIs are in units of curies times kilograms per cubic meter. Note that by calculating the HHIs for each process, due credit is given to flowsheets in which high-dose radionuclides are taken out of streams in the earlier processes, leaving lower potential for exposure in downstream processes. 
The occupational exposure HHIs are given in Tables 16 through 18 , for the CLEAN Option, TRUEX- $C$, and Sludge Wash $C$ process strategies, respectively, with a summary shown in Table 19. Figures 18 through III show the same table information for the respective process strategies. As shown in Table 19 the plant designs for the CLEAN Option flowsheets result in about 3 and $24 \%$ more occupational gamma exposure, a factor of 2.3 and 12.1 more occupational alpha exposure, and a factor of 2.2 and 19.2 more occupational beta exposure, than the TRUEX-C and Sludge Wash C plant designs, respectively. These larger indexes for the CLEAN Option result from its greater processing requirements.

Figures 16 through 18 show how the occupational exposure distributes among the various processes in each process strategy. Note that in the flowsheets the ion exchange processes are weighted more heavily in terms of exposure than the solid separation steps because they are assumed to have the same amount of tertiary waste as a single cycle of solvent extraction who was a plant engineer in the Hanford Plutonium-Uranium Extraction (PUREX) Plant for many years). The solid separation steps are only assumed to generate 0.25 as much tertiary waste as one solvent extraction cycle judged from equipment floor space in the Savannah River PUREX $F$ and $H$ canyons (see Appendix $E$ ).

Generally, more efficient removal of major exposure-causing components in the first process elements to contact the tank waste would lower the occupational exposure in all process strategies. For example, the CLEAN Option process strategy has alkal ine-side cesium removal before strontium and technetium removal, thereby reducing gamma exposure in these subsequent removal operations (Figure 18). However, cesium removal follows after other removal operations in the acid-side processing. If the acid-side cesium removal could also be placed before the other acid-side removal operations, it would reduce the gamma exposure in those subsequent removal operations.

These results should not be used in an absolute sense but only for comparative value in considering the various waste treatment processes.

\section{I5.0 MASTE CLASSIFICATION AND RADIONUCLIDE CONCENTRATIONS}

The radioactivity level (or concentration) of each component in each waste stream was determined by making a global level model run using the radioactive curie inventories (Appendices $H$ and $\mathrm{J}$ ) in $\mathrm{place}$ of the $\mathrm{kg}$ mass inventories for the two tank waste feedstreams. Multiplying the component curies $(C i)$ in a specific waste stream by the waste stream density $\left(\mathrm{kg} / \mathrm{m}^{3}\right.$ kilograms per cubic meter) and dividing by the total waste stream $\mathrm{kg}$ yields the desired component radioactivity levels $\left(\mathrm{Ci} / \mathrm{m}^{3}\right)$. These stream radioactivity levels for each process strategy are shown in Table 112.

A detailed discussion of the Hanford waste tank inventories is given in the Hanford Strategic Analysis (HSA) (Pajunen et al. 1992). In that report the TNKW inventory was divided into SSTTNKW, DSTTNKW, and UGTNKSTR and the concentrations of each of the radioactive components was shown by inventory category in bar graphs with waste classifications as shaded backgrounds. A portion of the tank heels that could not be recovered was included in UGTNKSTR. The DSTTNKW is usualiy highly concentrated, but the concentration 
in SSTTNKW varies widely from tank to tank although the total volume of SSTTNKW is much larger than DSTTNKW. The discussion of waste concentrations and classifications here is similar to that in the HSA with a breakdown between the SSTTNKW and DSTTNKW categories. However, this breakdown cannot apply to a single tank or a single tank farm. Also, because large quantities of inert strontium, LANHs, and, perhaps, lodine were added to parts of the SSTTNKW, the concentrations of radionuclides in these components vary widely between SSTTNKW and DSTTNKW.

The LLW classification criteria used for individual radionuclides are given in Table I10, expanded from those used in the HSA. Existing regulations do not explicitiy contain criteria for all of the radionuclides desired and extrapolation methods (Kocher and Croff 1988) may be in dispute. These values were converted into the component LLW classification criteria shown in Table III by using only the controliing radionuclide in each component. Only 12 out of the 25 components were actualiy evaluated because the radioactivity level of the remaining components were considered zero or no limits were established.

In the LLW classification system only the HLW glass stream (3110HLWS) for the CLEAN Option and TRUEX-C process strategies qualified as Greater than Class C (GTCC). The single-shell tank (SST) and double-shell tank (DST) tank waste feedstreams (SSTTNKW and DSTTNKW, respectively), the HLW glass stream for Sludge Wash $C$, and the tank residual waste stream (3200HLWS) qualified as LLW-C. All of these streams require deep burial by virtue of their ${ }^{137} \mathrm{CS}$ and ${ }^{9} \mathrm{Sr}$ content. As a whole the SSTTNKW has low concentrations of radionuclides, but some individual tanks contain GTCC because of their ${ }^{137} \mathrm{CS},{ }^{90} \mathrm{Sr}$, or transuranic (TRU) content. The U.S. Department of Energy (DOE) LLW classifications, such as Category 2, derived from the above system, have an upper limit identical to $C l a s s ~ C$ but Category 1 reduces the equivalent to Class $A$ to even lower values, which would place fewer SSTTNKW tanks into Category 1 than into Class $A$.

The LLW waste classifications for the process strategies are shown in Table 113 based on the radioactive concentrations shown in Table 112 . The concentrations of total radioactivity and of components plutonium, uranium, cesium, strontium, technetium, iodine, fission products (FP2), activation products (AP1), other (OTH3), americium, neptunium, and LANH in the feed and waste streams for the $0 \%$ heel option are compared with the LLW classifications in Figures 112 through 124 , respectively. The waste product streams may deviate from the target designations for some components because the LLW classifications involve setting multiple individual radionuclide (or component) limits. Modification of the process decontamination factors (DFs) to agree with all target classifications could result in minor changes in capital or operating costs. Also the flowsheet results are always subject to as low as reasonabiy achievable (ALARA) considerations so that lower LLW concentrations and classifications are preferred over higher values. Higher HLW radionuclide concentrations are usualiy preferred because they lower HLW disposal costs.

The complexity of using the $L L W$ individual radionuclide limits as multiple performance measures (applying only to the first 500 years after disposal) is evident. The method of applying these limits is that if any individual radionuclide exceeds its limit for a particular $L L W$ classification, then the waste is a higher class, and there is a specially prescribed additivity rule. 
The need for process screening methods has led us to the development of a simpler set of HHIs discussed above, which are tailored to the periods of risk. However, meeting the waste classifications are regulatory requirements and no lower bound has been set for Class A except for ALARA.

Figure 112 shows the concentration of total radioactivity. The radiological i imits applied to each feedstream or waste product stream were those for a component that controlled the final classification of that stream for all process strategies (Table I13). For example, because the HLW glass stream ( $3110 \mathrm{HLWS}$ ) classification was controlled by piutonium, cesium, strontium, and technetium limits, the plutonium ifmits were used because they were consistently controlling in all process strategies. Thus, the intent of Figure 112 is to show a comparison of the final stream classifications for each process strategy.

As shown in Figure 112, the tank waste feedstreams (SSTTNKW and DSTTNKW), the HLW glass stream (3110HLWS) destined for a HLW repository, and the tank heel (3200HLWS) stream are GTCC. The LLW grout (3400LLWS+3400LMWS) streams are LLW Class A for the CLEAN Option process strategy, while the TRUEX-C and Sludge Wash C process strategies were LLW Class B because of the strontium limits. Using uranium LLW classification limits proposed by Kocher and Croff (Kocher and Croff 1988), the uranium product stream was al so GTCC for the CLEAN and Sludge Wash C process strategies, while the TRUEX-C process strategy uranium stream was LLW Class C. Higher DFs may be needed for the atmospheric release of the vitrification offgas (VITRW-GAS) where different 1 imits apply than for solid LLW. The uranium product stream was also GTCC for the CLEAN and Sludge Wash C process strategies, while the TRUEX-C process strategy uranium stream was LLW Class C.

Figure 113 shows the concentration of the plutonium (e.g., ${ }^{239} \mathrm{Pu}$ ) component radioactivity. The HLW glass and tank heel streams were classified as TRU. The Clean Option and TRUEX-C process strategies had grout streams classified as LLW Class $A$ while the Sludge Wash C process strategy grout stream was below LLW Class A (note that the CLEAN Option was nearly below Class $A)$. The TRUEX-C vitrification offgas was LLW Class $A$ and may need further cleanup as discussed above. Summarizing, the HLW was more concentrated in the CLEAN Option process strategy because of its more extensive plutonium separation processing. The HLW was above LLW Class C, while the LLW was reduced to nearly industrial limits by the CLEAN Option and Sludge Wash C process strategies.

Figure 114 shows the concentration of the uranium (e.g., ${ }^{238} \mathrm{U}$ ) component radioactivity. The HLW glass and tank heel streams were classified as LLW Class $A$ while the grout streams were classified as less than LLW Class A. Using uranium LLW Classification limits proposed by Kocher and Croff (Kocher and Croff 1988), the uranium product stream was also GTCC for the CLEAN and Sludge Wash $C$ process strategies, while the TRUEX-C process strategy uranium stream was LLW Class C.

Figure 115 shows the concentration of the cesium (e.g., ${ }^{137} \mathrm{Cs}$ ) component radioactivity. Both the HLW glass and the tank heel are LLW-C, with the CLEAN Option process strategy HLW glass qualifying as GTCC. The grout streams for the TRUEX-C and Sludge Wash C process strategies were LLW Class A while the 
CLEAN Option grout stream qualified for industrial use. The vitrification offgas stream for the TRUEX-C and Sludge Wash C process strategies were LLW $C l$ ass $A$ and $B$, respectively.

Figure 116 shows the concentration of the strontium $\left(e . g_{.},{ }^{90} \mathrm{sr}\right)$ component radioactivity. Both the HLW glass and the tank heel are LLW-C, with the CLEAN Option process strategy HLW glass qualifying as GTCC. The grout streams for the TRUEX-C and Sludge Wash $C$ process strategies were LLW Class $B$, while the CLEAN Option grout stream qualified as LLW Class A. The vitrification offgas stream for the TRUEX-C process strategy was LLW Class $B$.

Figure 117 shows the concentration of the technetium (e.g., ${ }^{99} \mathrm{Tc}$ ) component radioactivity. The HLW glass stream for the CLEAN Option process strategy was classified as GTCC while the TRUEX-C and Sludge Wash $C$ process strategies were LLW Class C. The tank heel is below the LLW Class A limit and the grout is below the industrial soil limit. The vitrification offgas stream for the TRUEX-C and Sludge Wash $C$ process strategles qualified as residential and industrial, respectively.

Figure $I 18$ shows the concentration of the iodine (e.g., $\left.{ }^{129} \mathrm{I}\right)$ component radioactivity. All streams are well below the LLW Class A imit.

Figure 119 shows the concentration of the fission product $\left(e . g .,{ }^{93} \mathrm{Zr}\right.$, ${ }^{126} \mathrm{Sn}$ ) component radioactivity. All streams qualify as LLW Class A. The vitrification offgas stream for the TRUEX-C process strategy was below the LLW Class A limit.

Figure 120 shows the concentration of the activation product $\left(e . g .,{ }^{14} \mathrm{C}\right)$ component radioactivity. All streams qualified as LLW Class $A$. The vitrification offgas stream for the CLEAN Option and TRUEX-C process strategies were below residential limits. Note that the industrial limit exceeds the LLW class limits for ${ }^{14} \mathrm{C}$.

Figure 121 shows the concentration of the "Other" (e.g., ${ }^{63} \mathrm{Ni}$ ) component radioactivity. Most streams qualified as LLW Class $A$ or below. The in situ grout stream (3530LMWS) was below the industrial soil limit. The vitrification offgas stream for the CLEAN Option and TRUEX-C process strategies were below residential and industrial limits, respectively.

Figure 122 shows the concentration of the americium (e.g., ${ }^{241} \mathrm{Am}$ ) component radioactivity. Both the HLW glass and the tank heel are classified as TRU. The grout streams were LLW Class $A$. The vitrification offgas stream for the TRUEX-C process strategy was LLW Class $A$.

Figure 123 shows the concentration of the neptunium (e.g., ${ }^{237} \mathrm{~Np}$ ) component radioactivity. All streams were LLW Class A except for the CLEAN Option HLW glass stream, which was LLW Class C.

Figure 124 shows the concentration of the LANH (e.g., ${ }^{151} \mathrm{Sm}$ ) component radioactivity. Both the HLW glass and the tank heel streams were classified as LLW Class A except the CLEAN Option HLW glass stream, which was GTCC. The TRUEX-C process strategy grout stream was LLW Class A while the CLEAN Option and Sludge Wash C process strategy grout streams were classified as industrial-soil. The vitrification offgas stream for the TRUEX-C process strategy was LLW Class A. 


\subsection{REFERENCES}

Kennedy, Jr., W. E., and R. L. Aaberg, 1991, Dose and Risk Assessment For Intrusion Into Mixed Waste Disposal Sites, PNL-SA-20032, Pacific Northwest Laboratory, Richland, Washington.

Knutsen, B. J., et al., 1993, "Advanced Chemical Separation Study: TRUEX Strategy Compared To Sludge Wash Ion Exchange," Letter report, Westinghouse Hanford Company, Richland, Washington.

Kocher, D. C., and A. G. Croff, 1988, A Proposed Classification System For High-Level And Other Radioactive Wastes, Research Sponsored by DOE Contract DE-AC05-840R21400, Radioactive Waste Management and the Nuclear Fuel Cycle, Vol. LL(3), Oak Ridge National Laboratory, Oak Ridge, Tennessee.

Pajunen, A. L., et al., 1992, Hanford Strategic Analysis Study, WHC-EP-0549, Westinghouse Hanford Company, Richland, Washington. 
WHC-EP-0791

Figure 11. Comparison of Relative Long-Term Offsite Health Hazard.

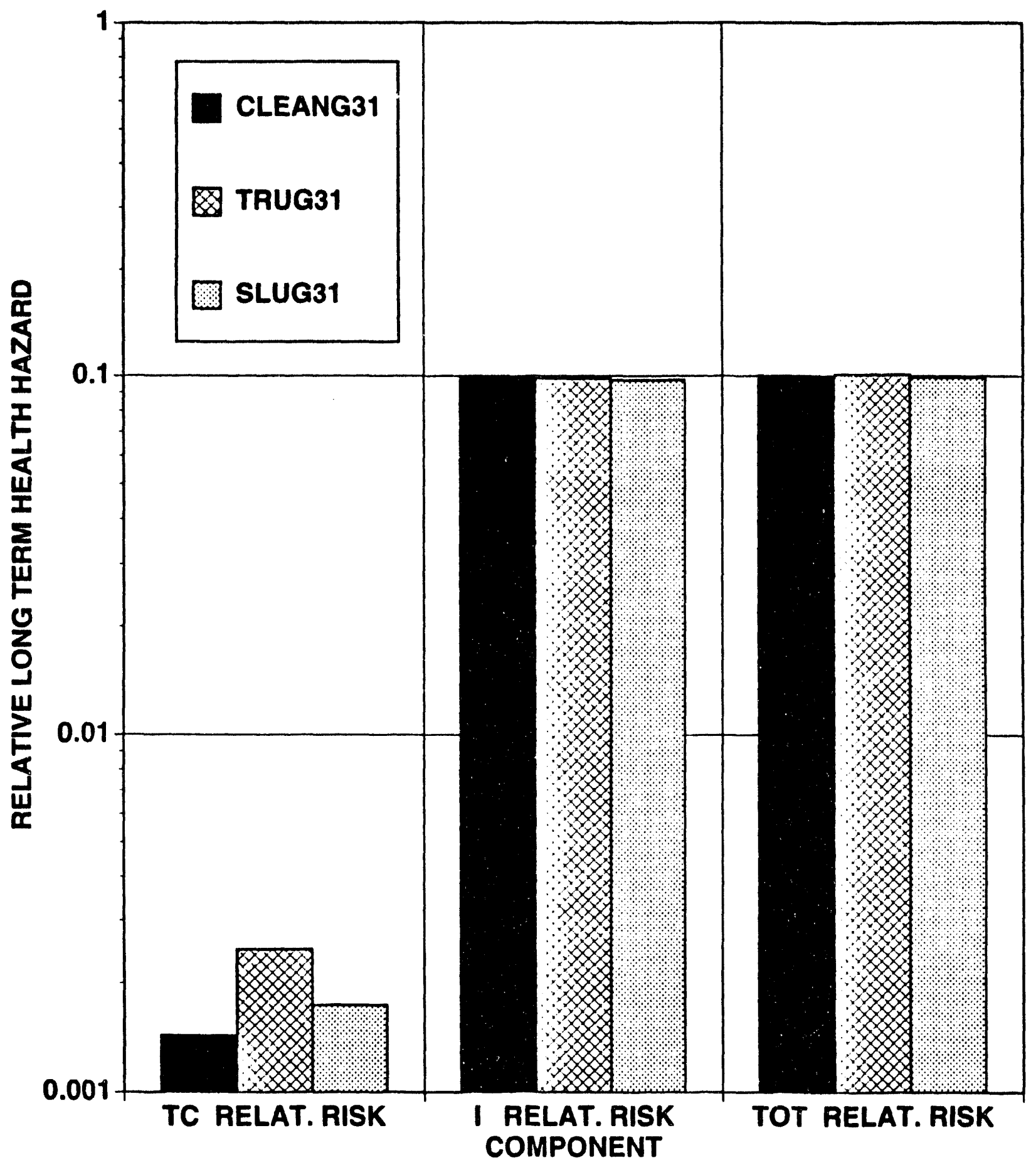


Figure 12. Long-Term Onsite Health Hazard Index.

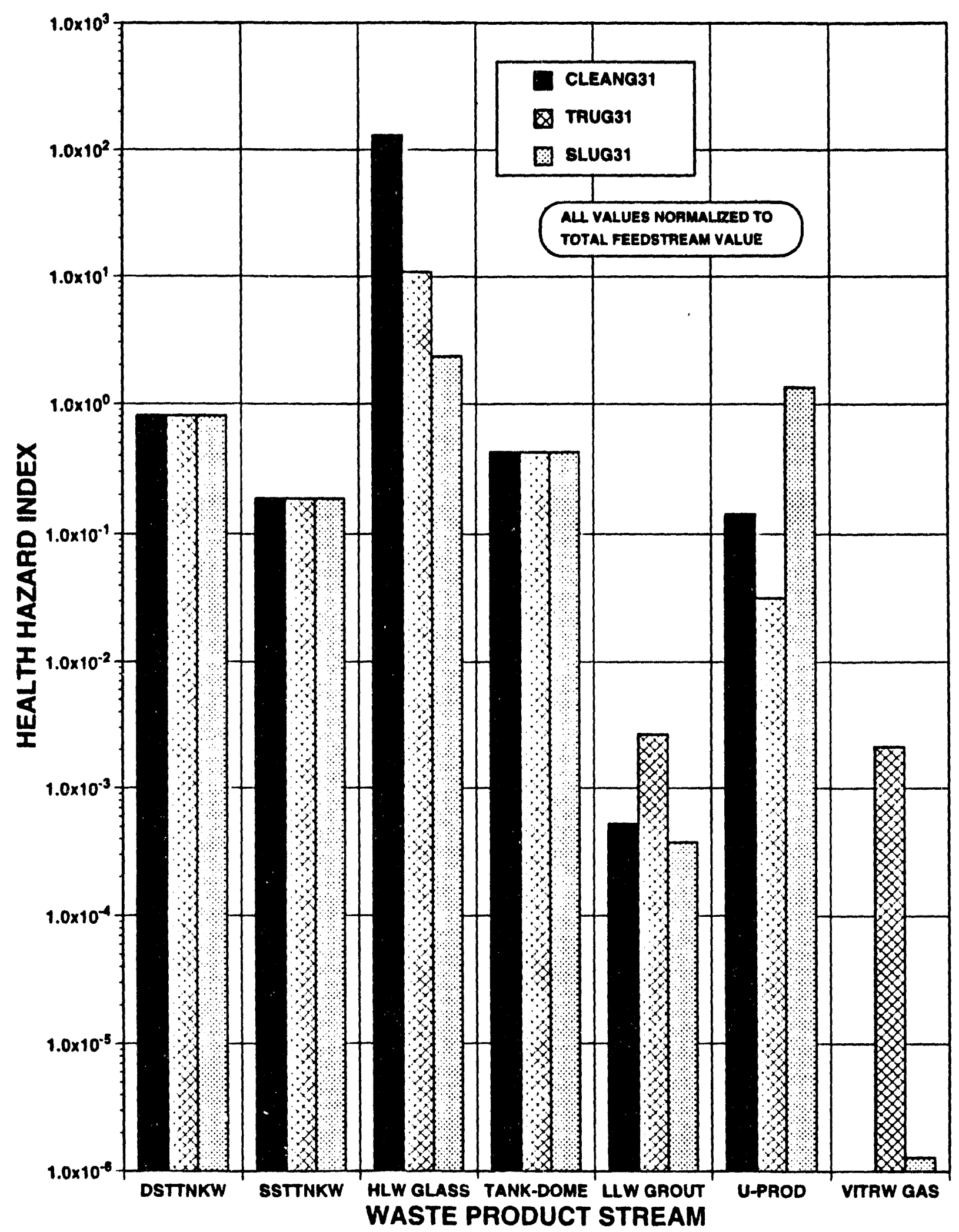


Figure 13. Long-Term Onsite Health Hazard Index for High-Level Waste Glass Stream.

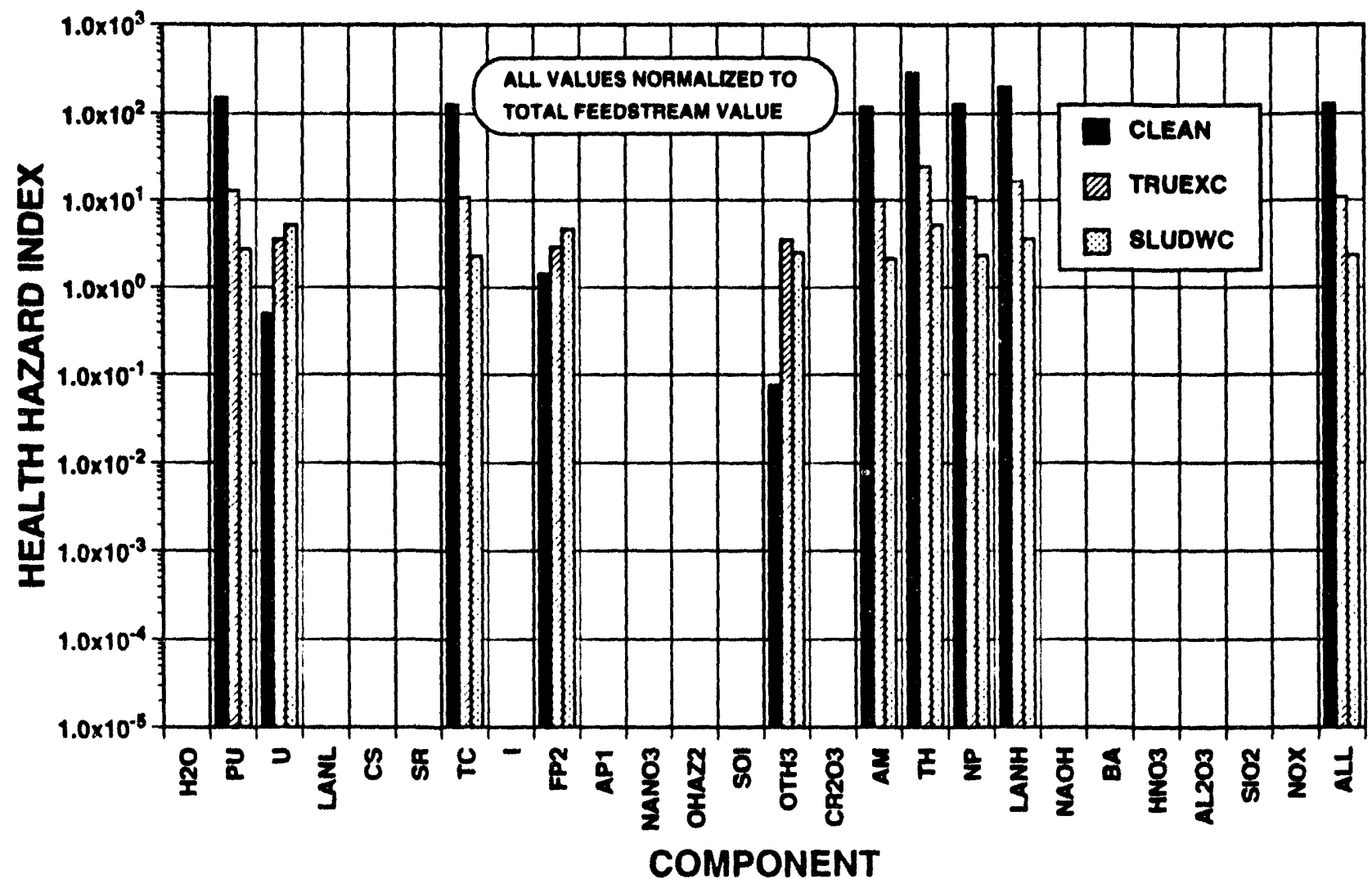

Figure 14. Long-Term Onsite Health Hazard Index for Low-Level Waste Grout Stream.

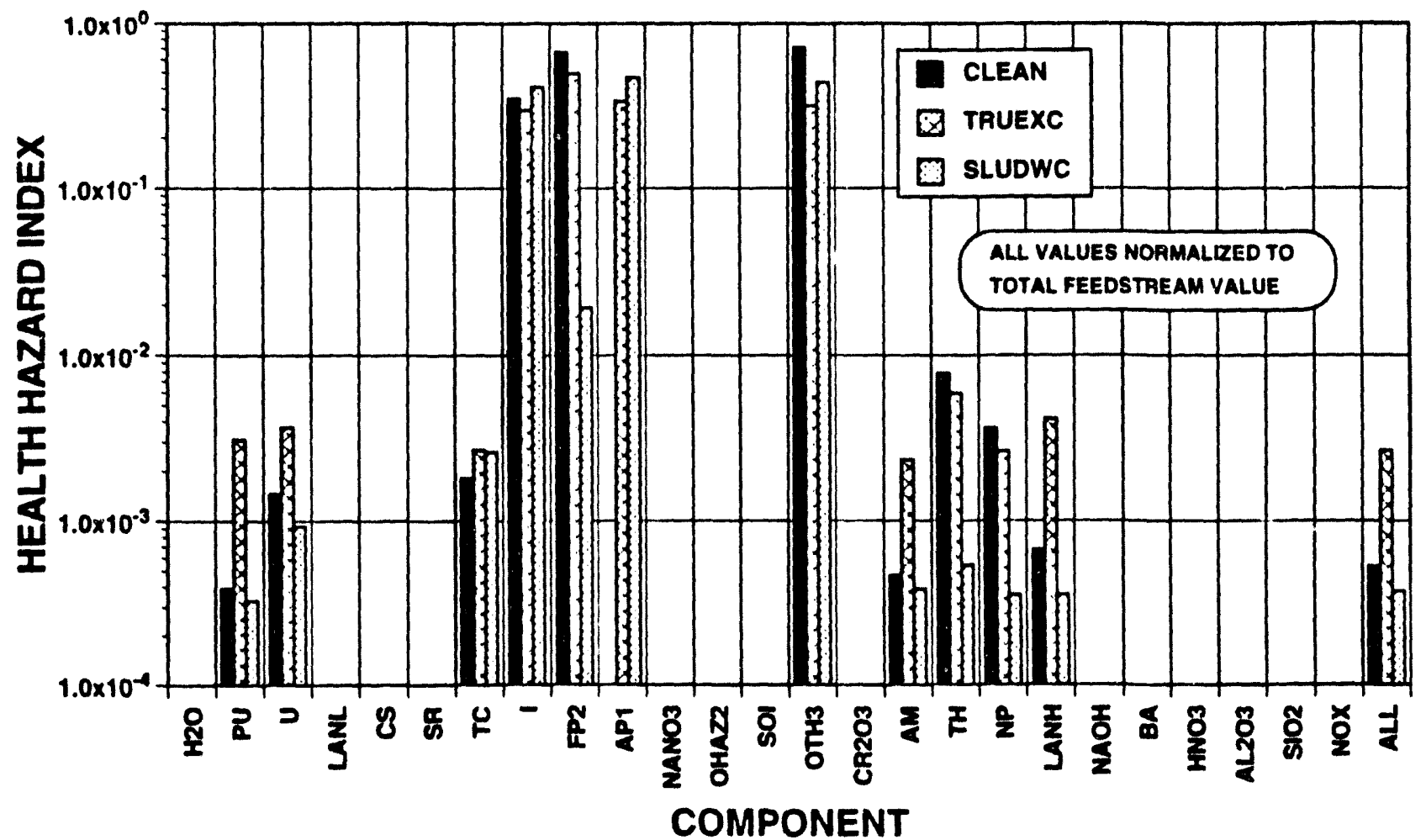


WHC-EP-0791

Figure 15. Short-Term Onsite and Offsite Health Hazard Index.

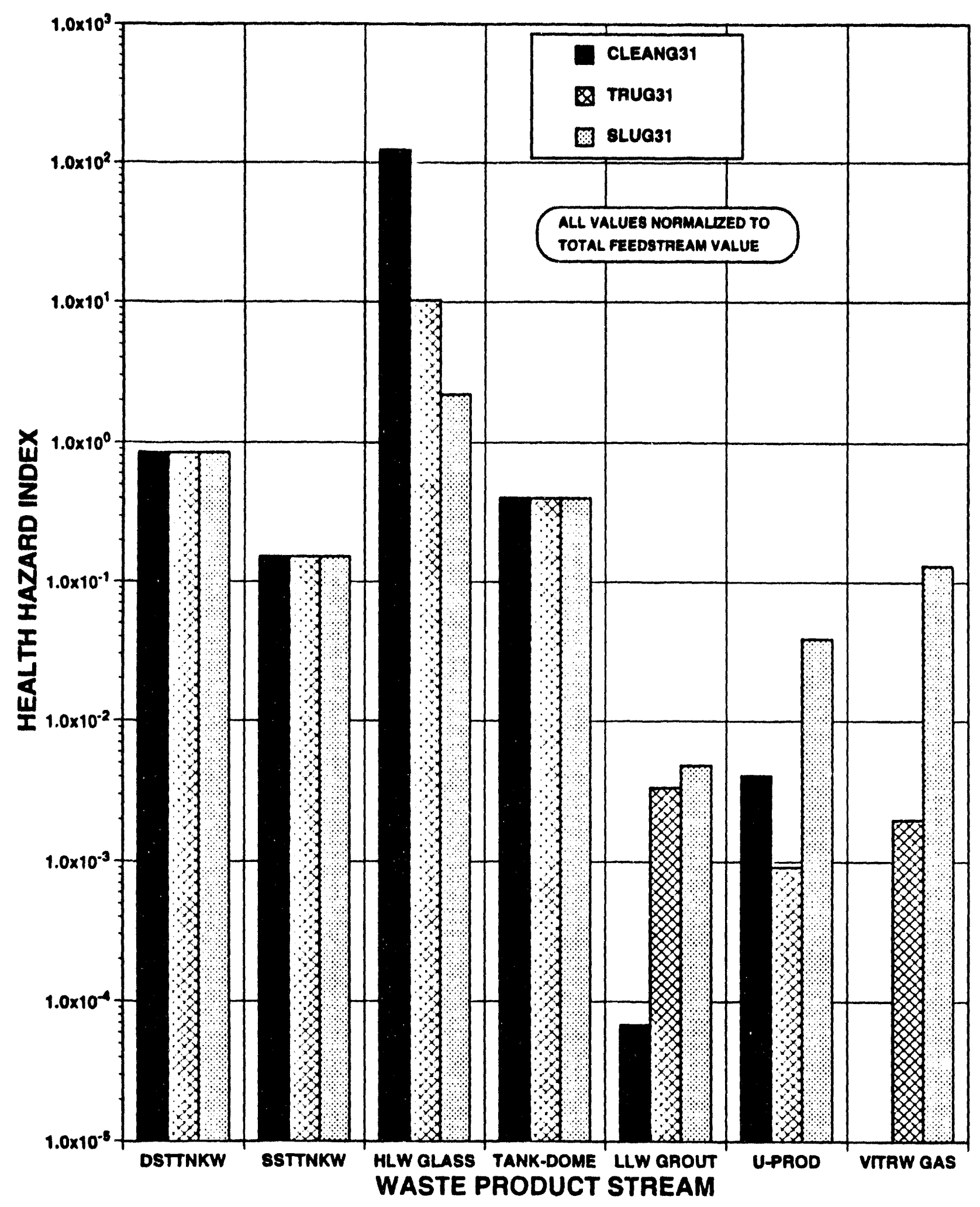


Figure 16. Short-Term Onsite and Offsite Health Hazard Index for High-Level Waste Glass Stream.

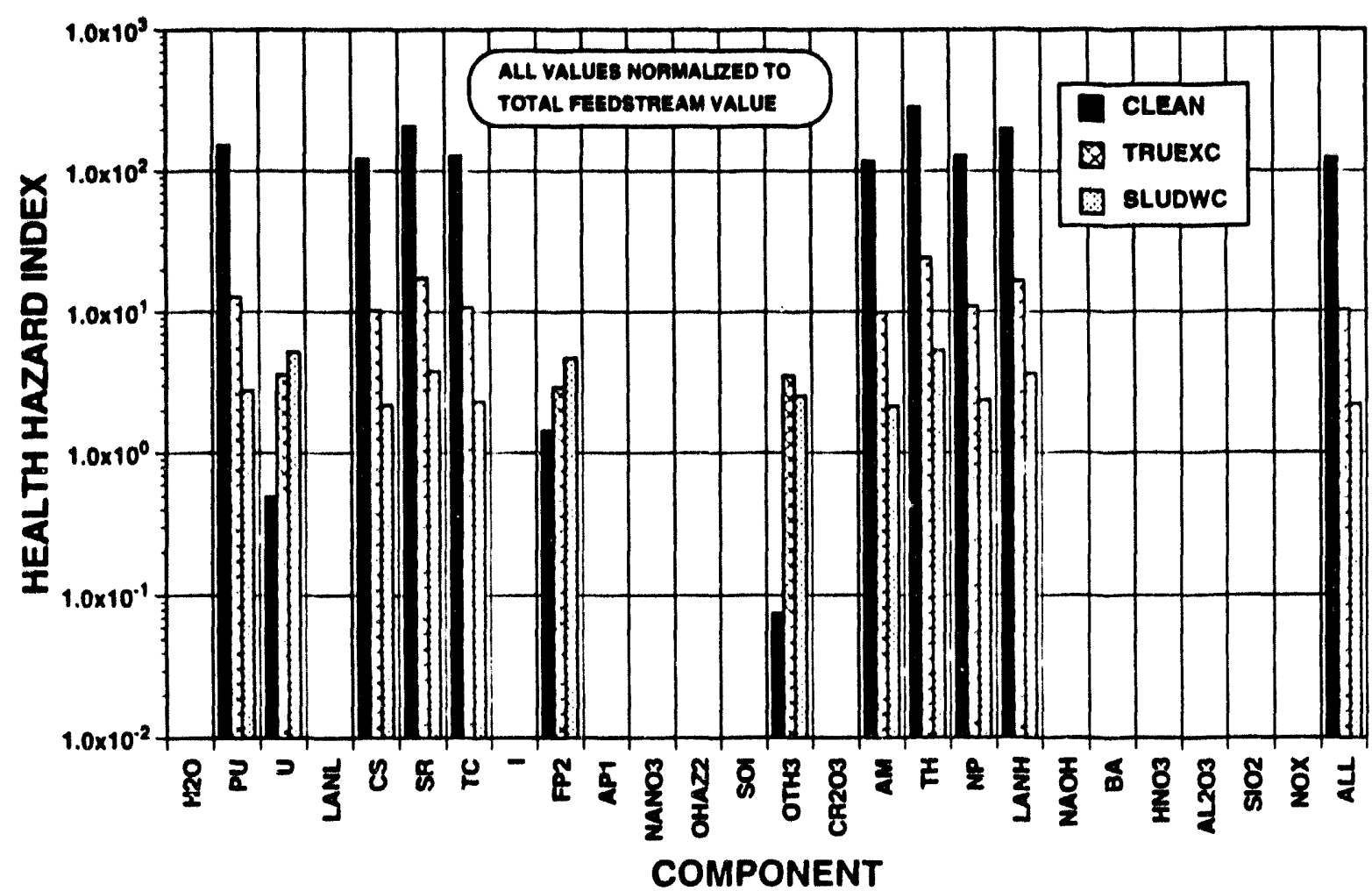

Figure 17. Short-Term Onsite and Offsite Health Hazard Index for Low-Level Waste Grout Stream.

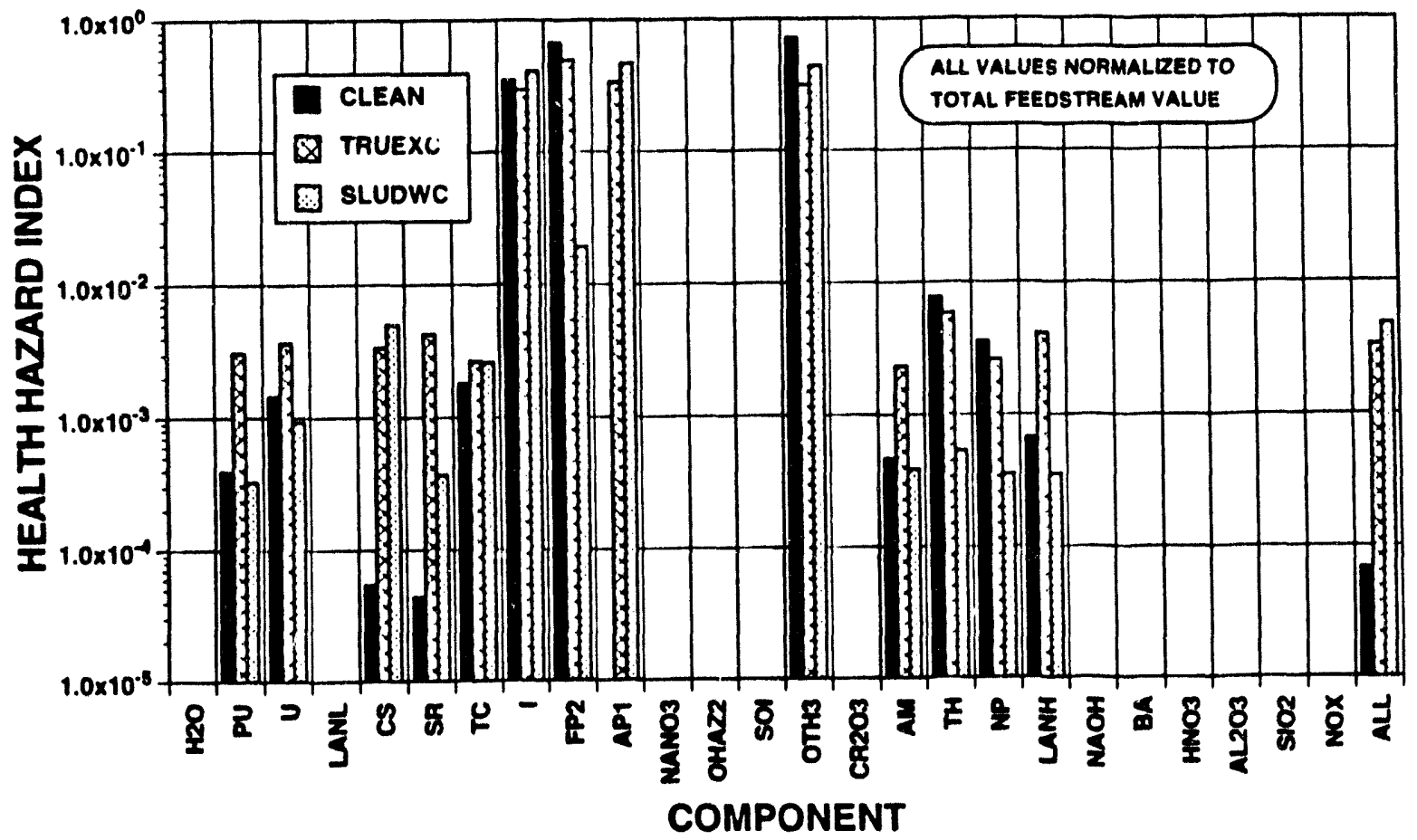




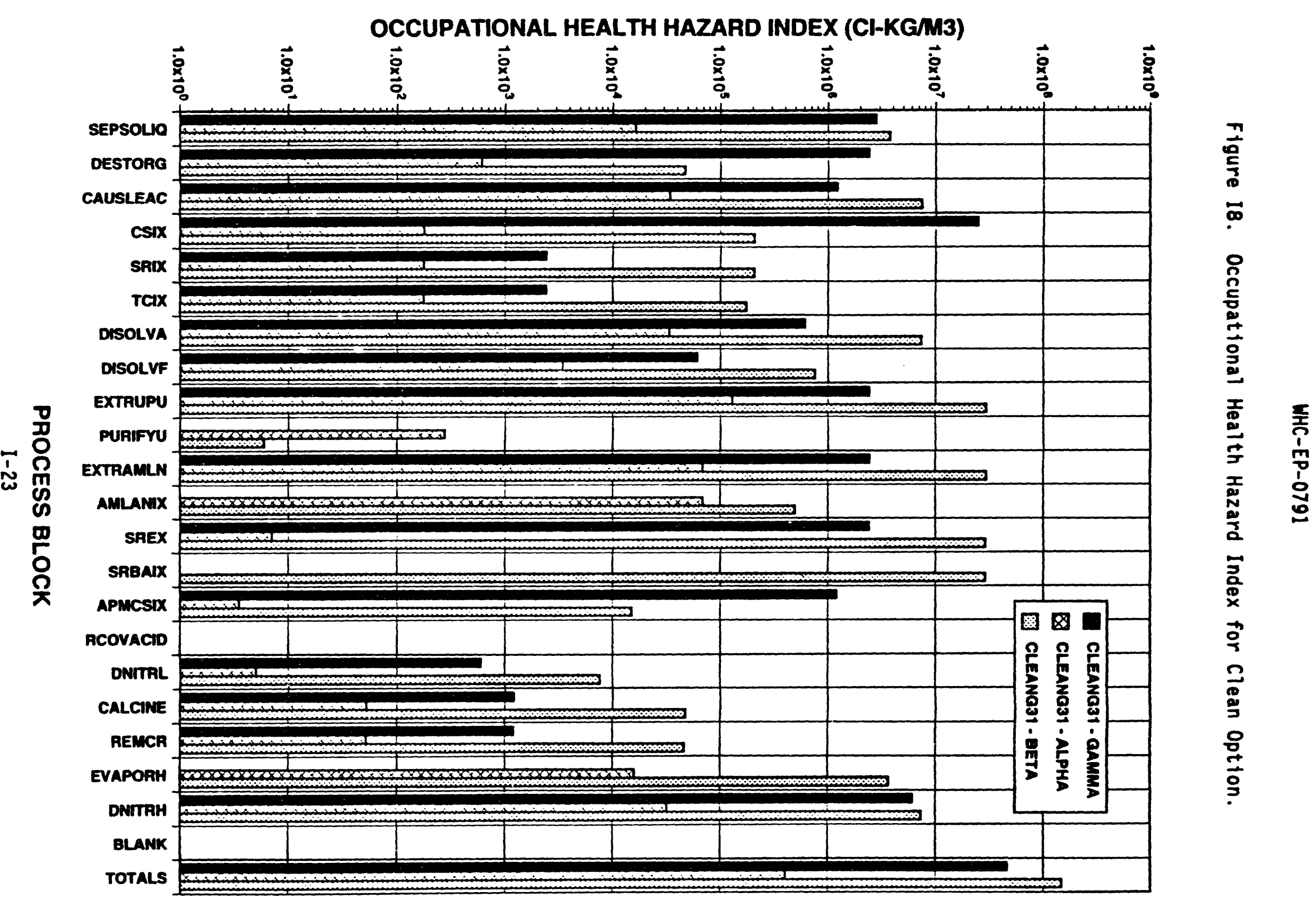


Figure 19. Occupational Health Hazard Index for Transuranic
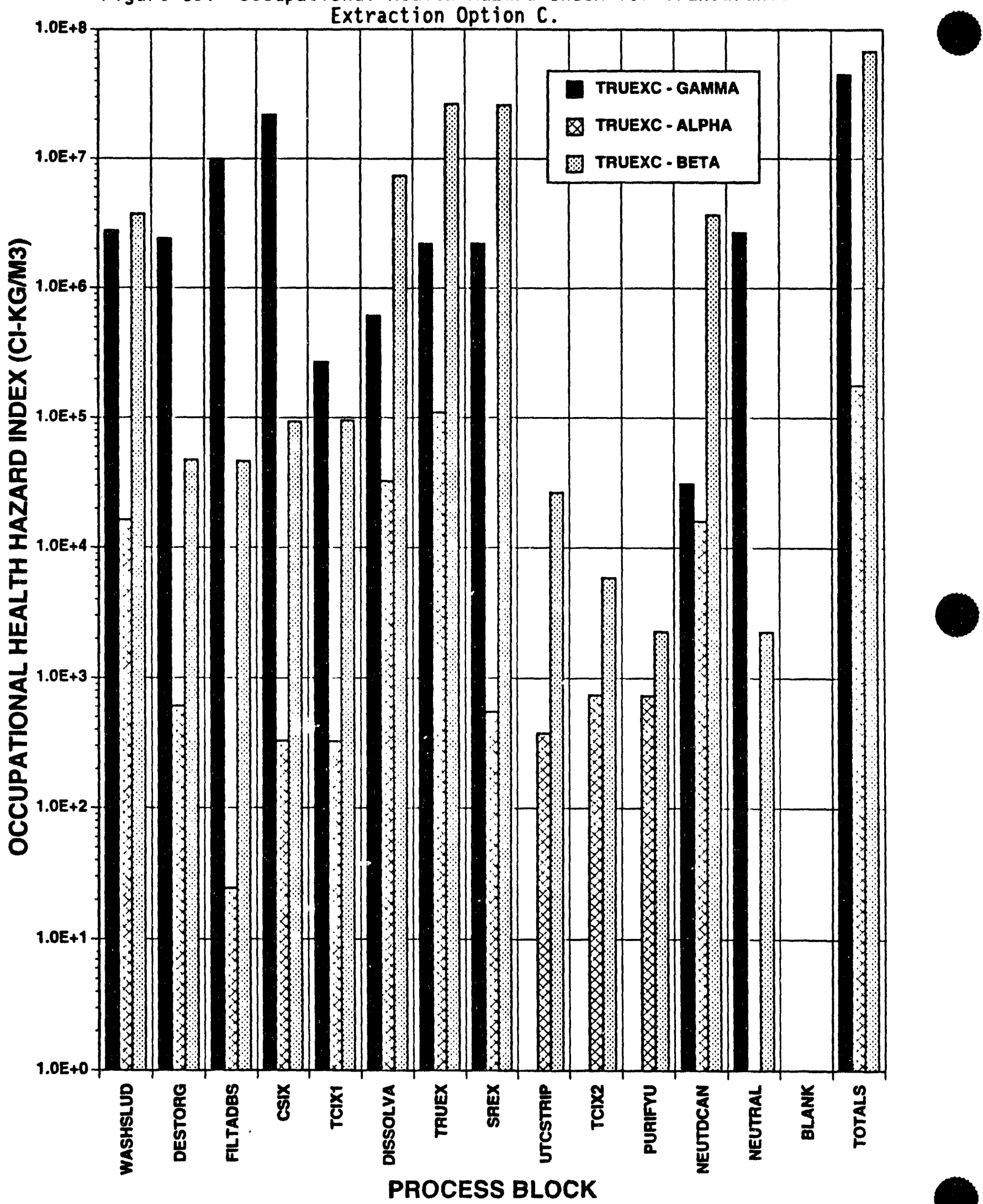


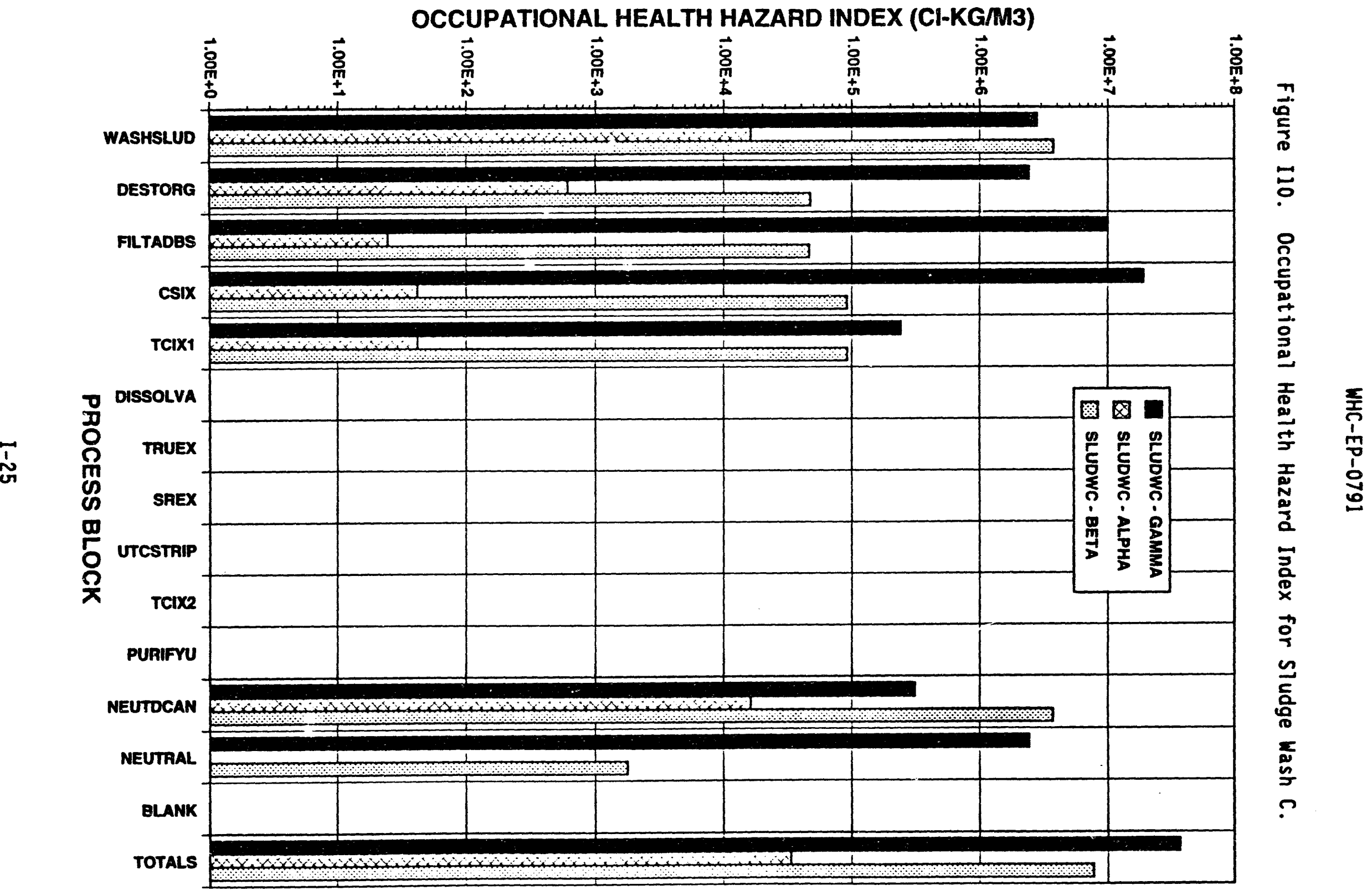


WHC-EP-0791

Figure I11. Total Occupational Health Hazard Index.

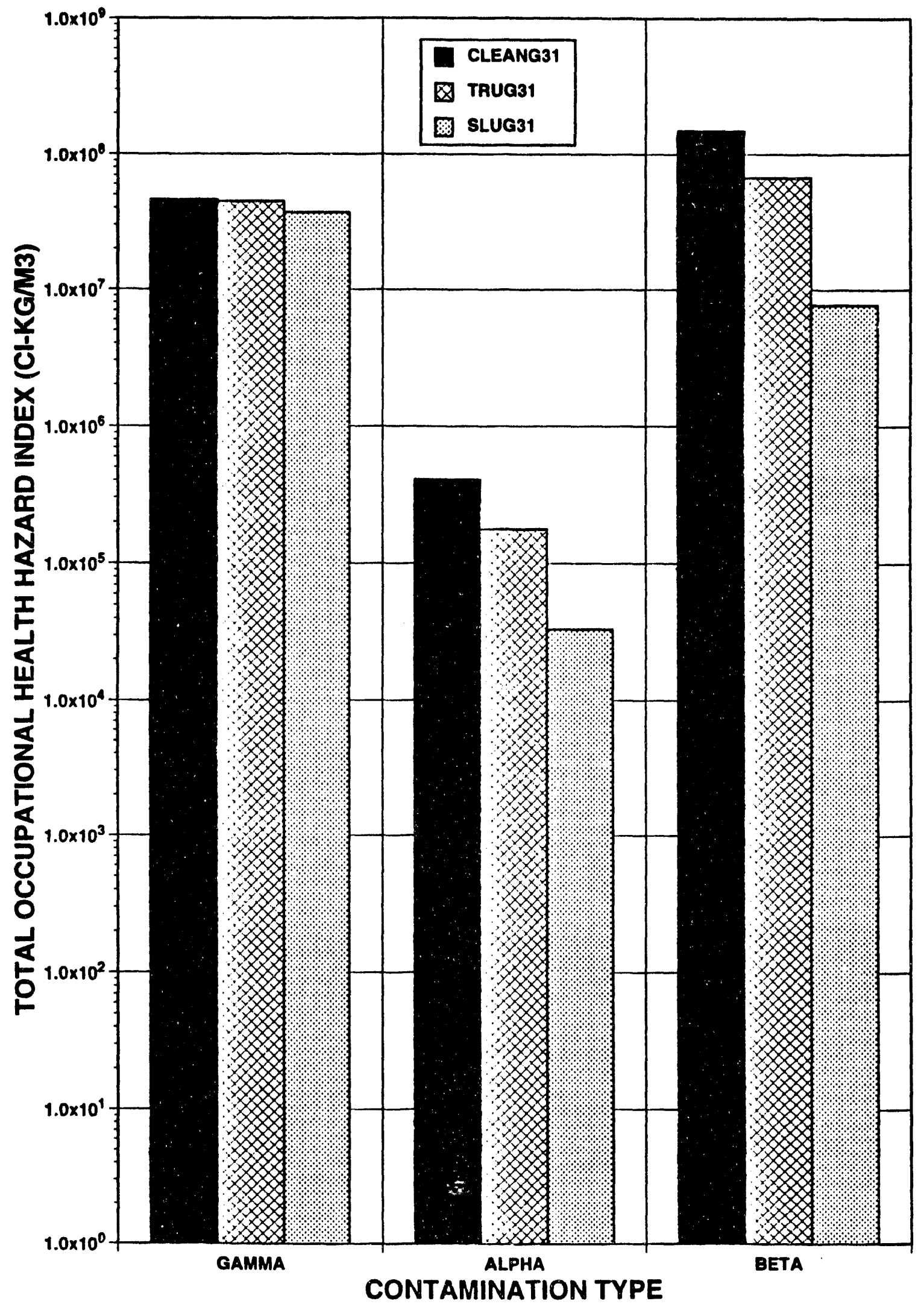


Figure 112. Total Radioactivity Level.

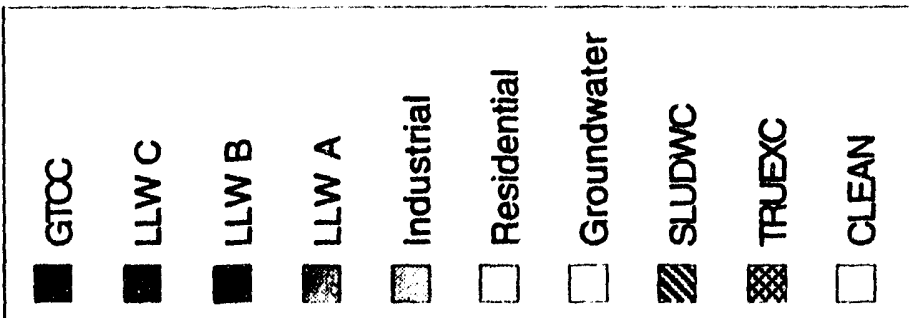

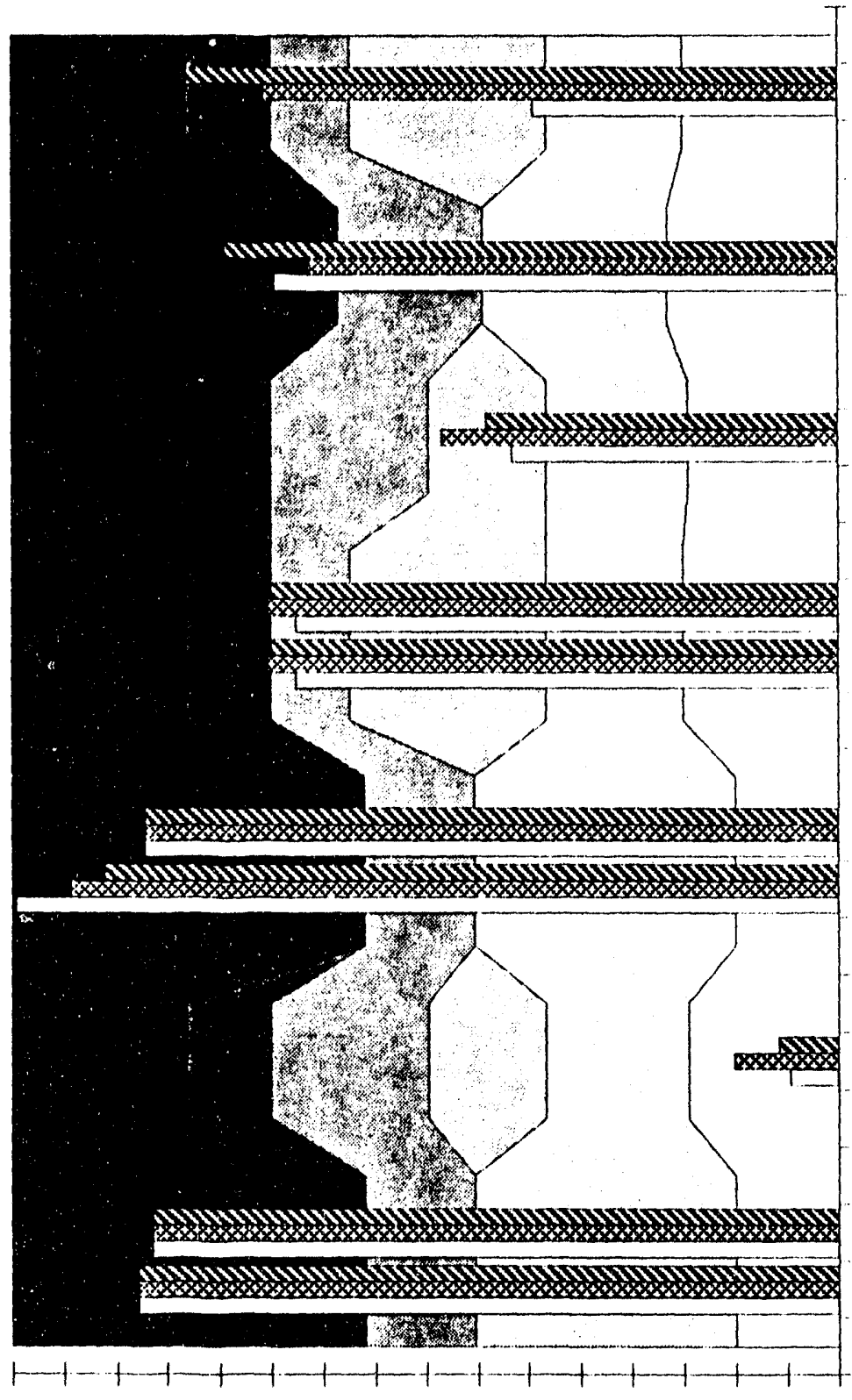

SHO-MULIN

Lonooyd-n

SMWา0๕SE

SMW700tE

SMา700†ย

E⿱

SMHOOZE

SMTHOLLE

$\mathrm{OZHOOZZ}$

MYNUSS

MYNUSO

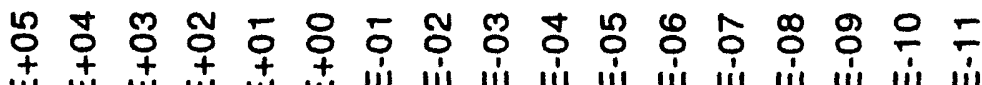

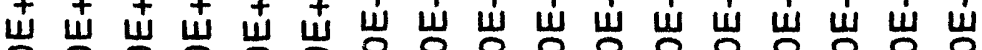

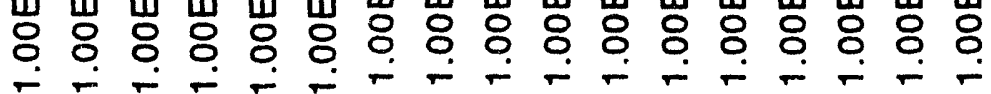

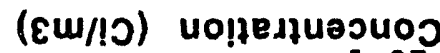


Figure 113. Plutonium Radioactivity Level.

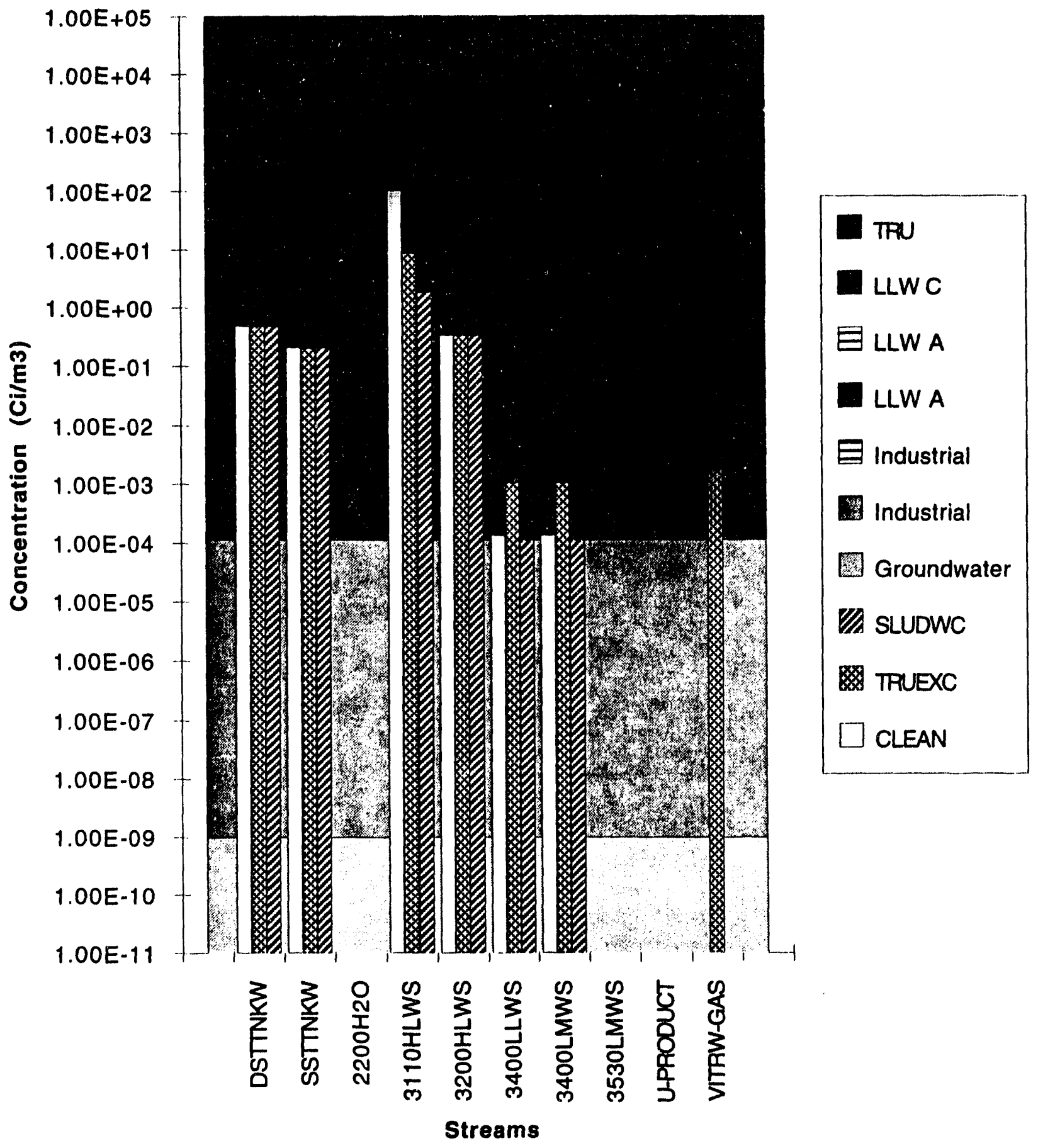


WHC-EP-0791

Figure 114. Uranium Radioactivity Level.

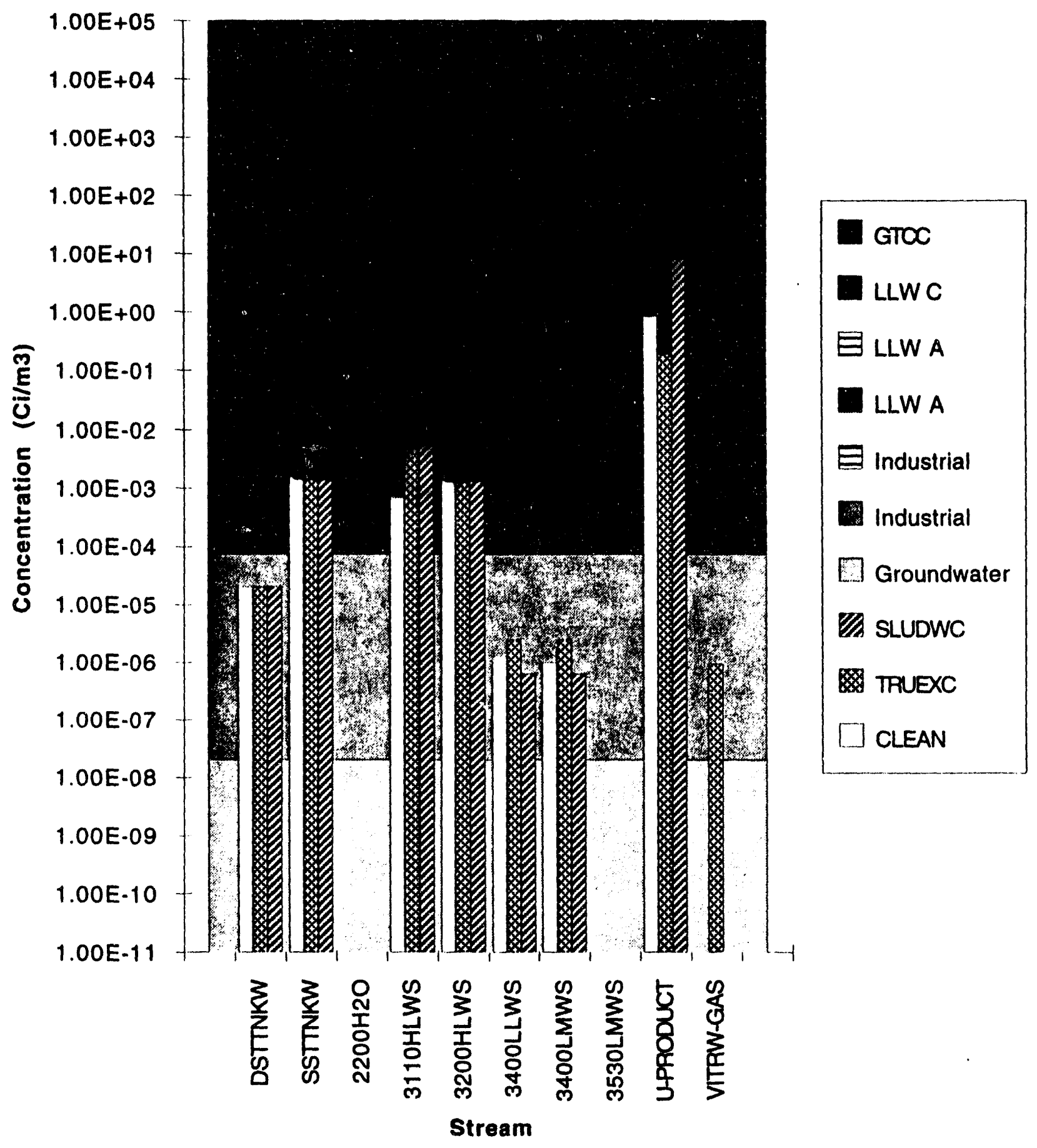


Figure I15. Cesium Radioactivity Level.

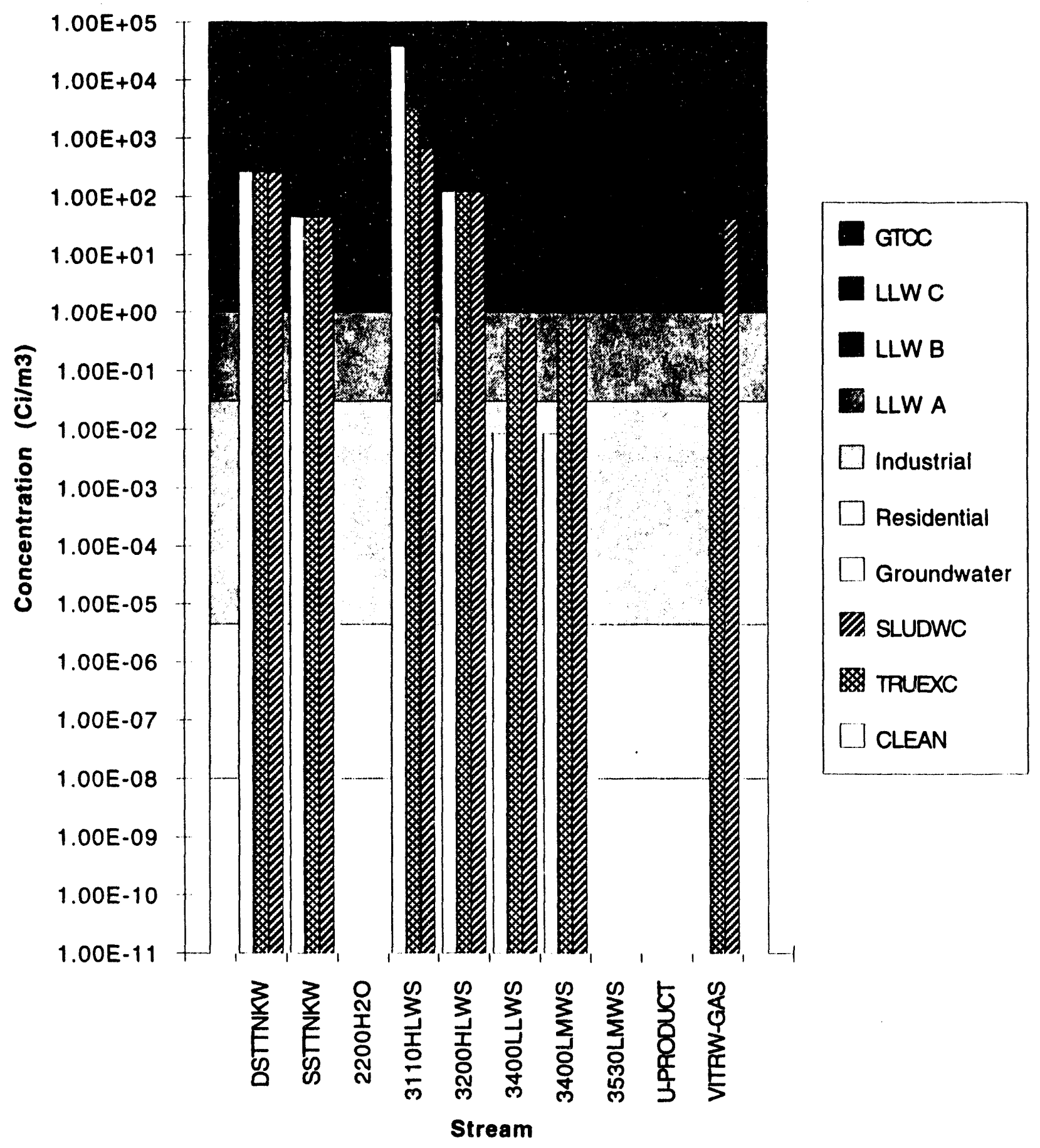


WHC-EP-0791

Figure 116. Strontium Radioactivity Level.

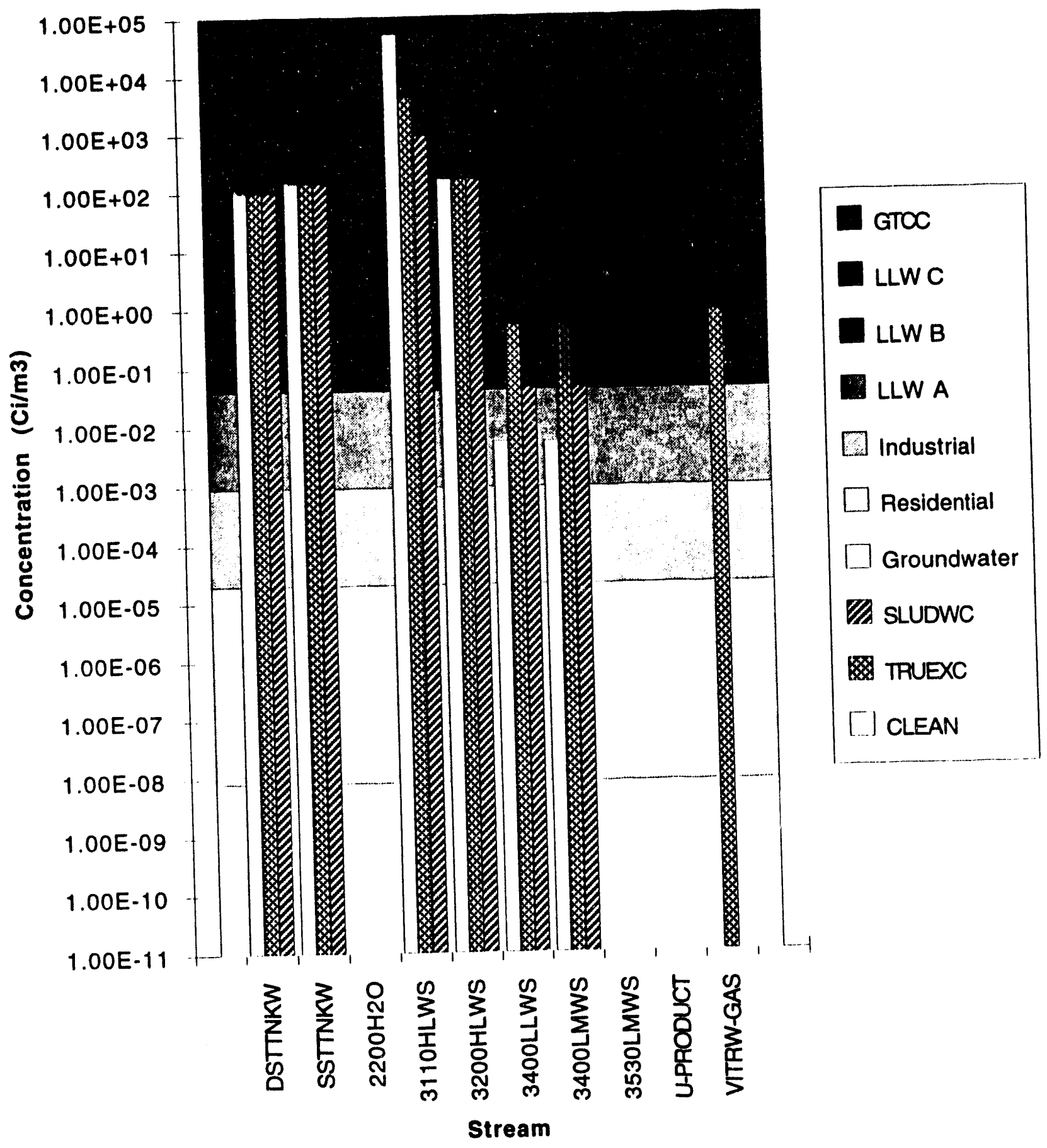


WHC-EP-0791

Figure 117. Technetium Radioactivity Level.

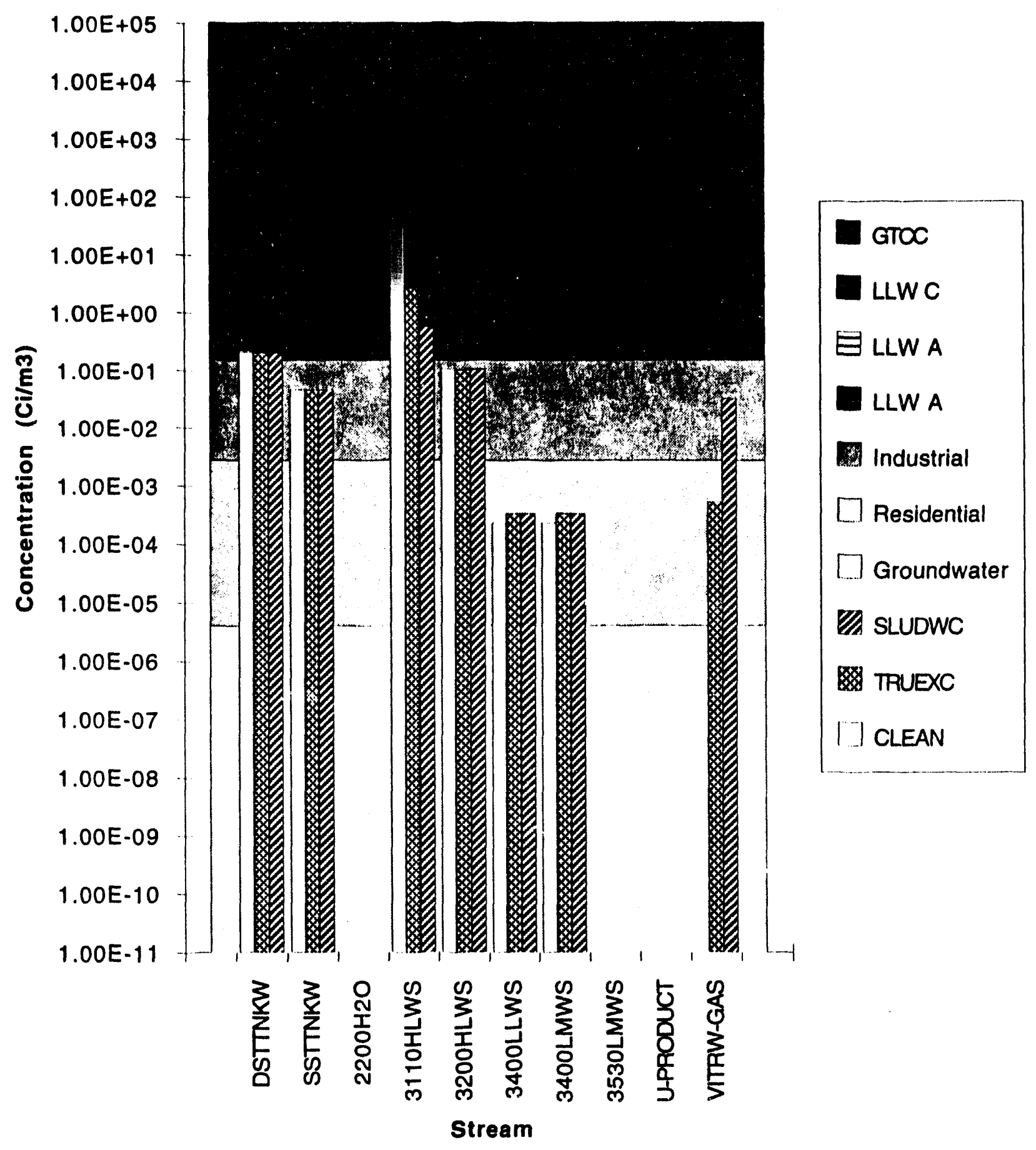


WHC-EP-0791

Figure 118. Iodine Radioactivity Level.

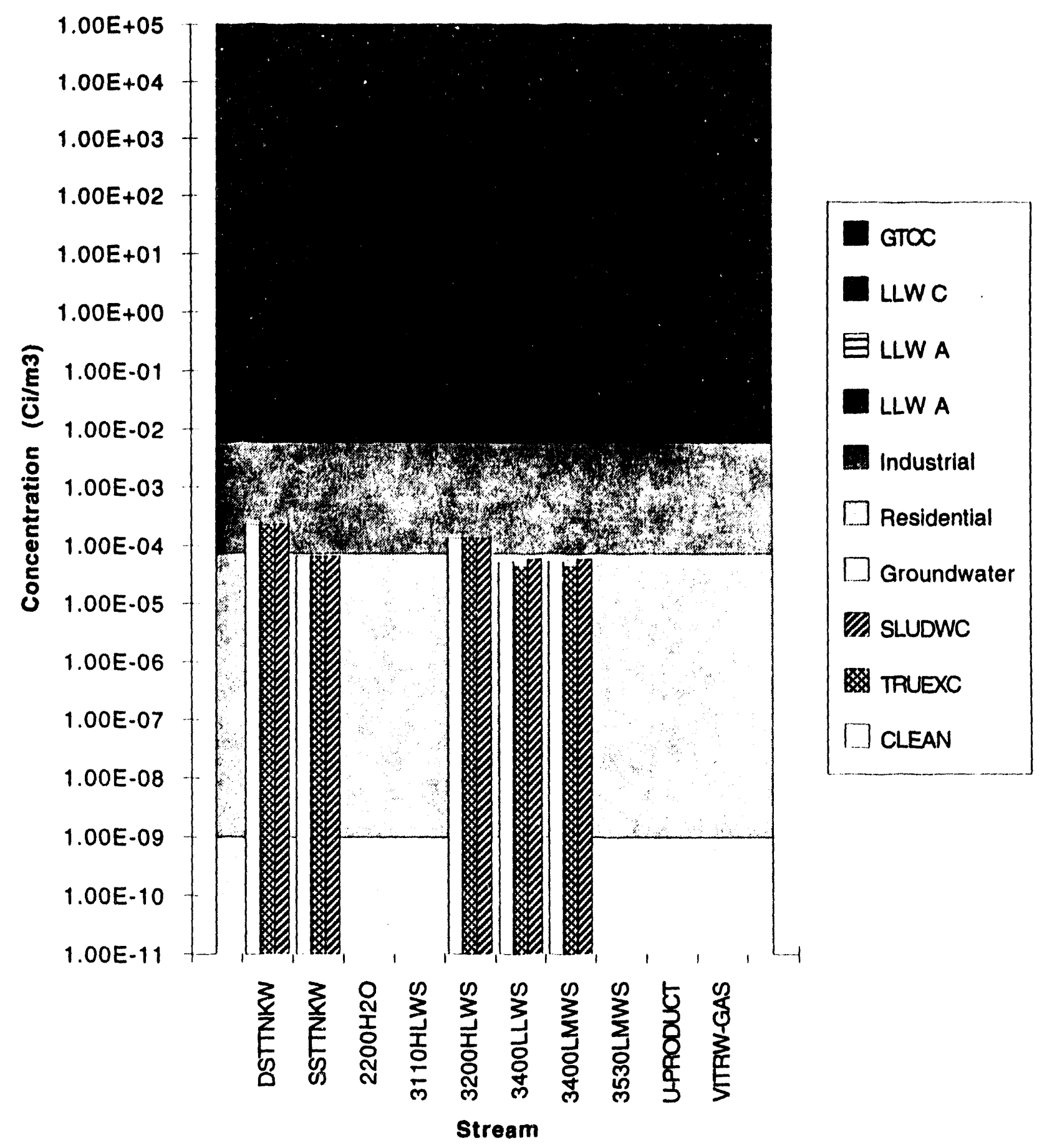


Figure 119. Fission Product Radioactivity Leve1.

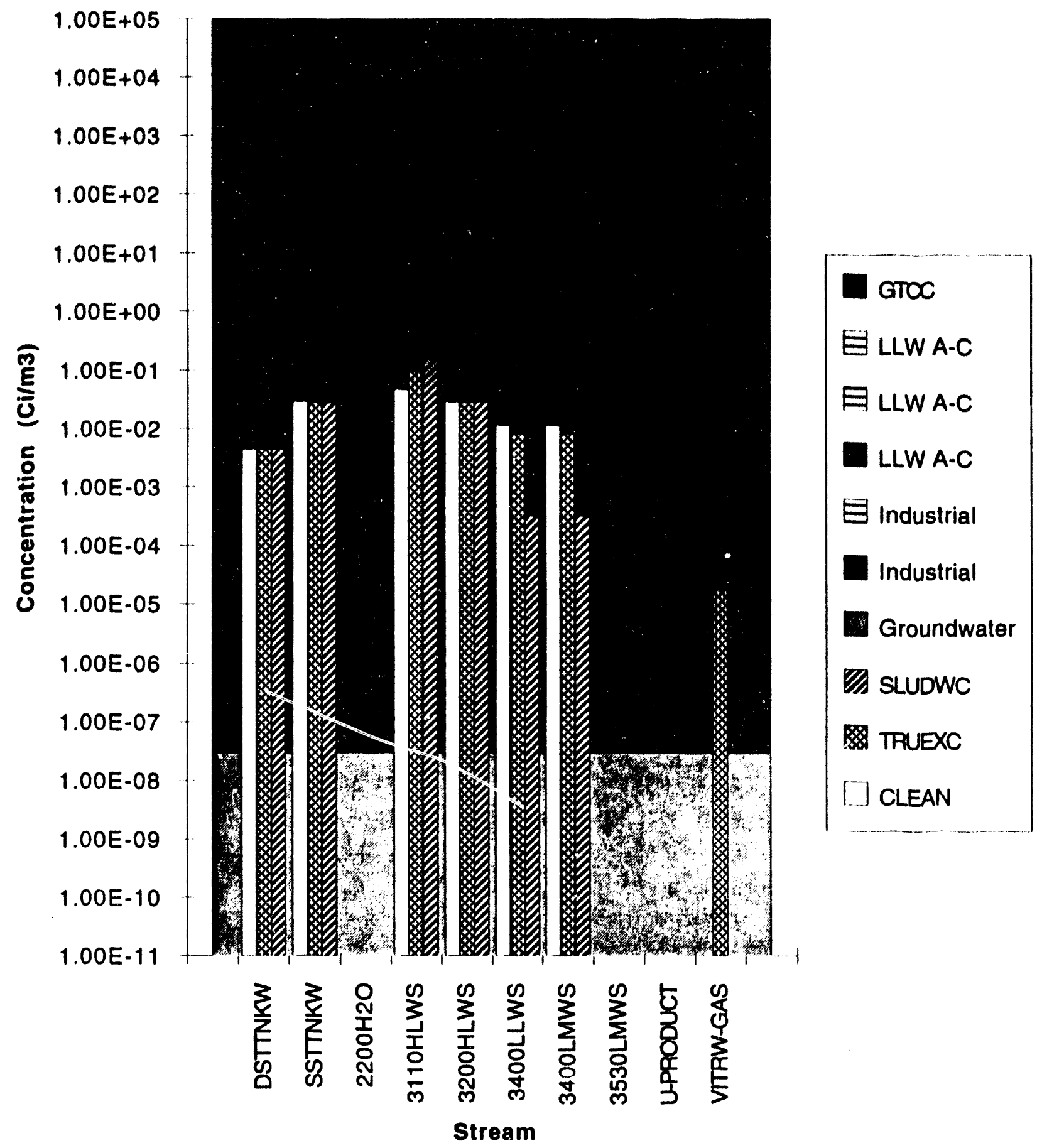


WHC-EP-0791

Figure 120. Activation Product Radioactivity Level.

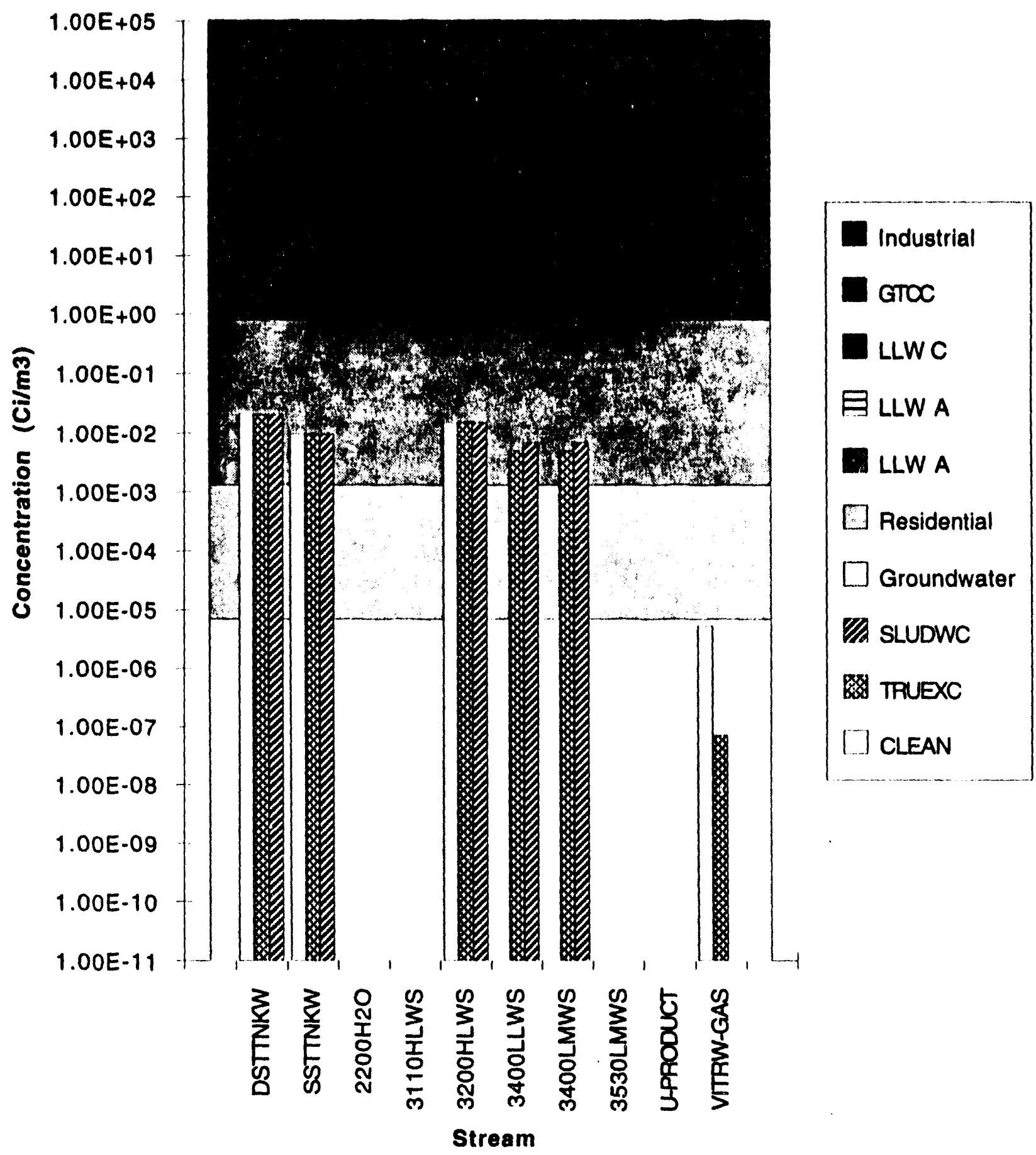


WHC-EP-0791

Figure 121. Other (e.g., Nickel) Radioactivity Level.

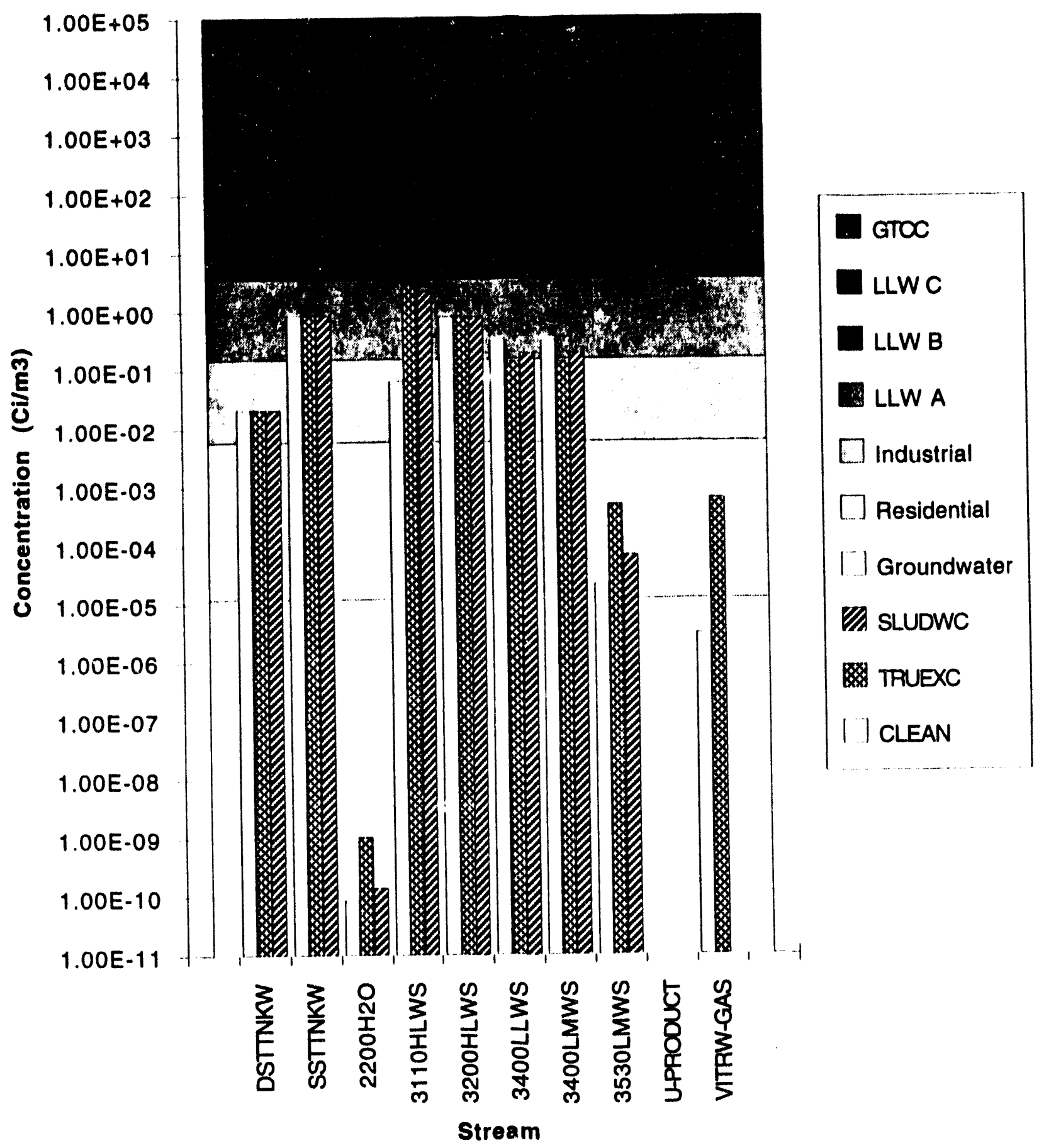


Figure 122. Americium Radioactivity Level.

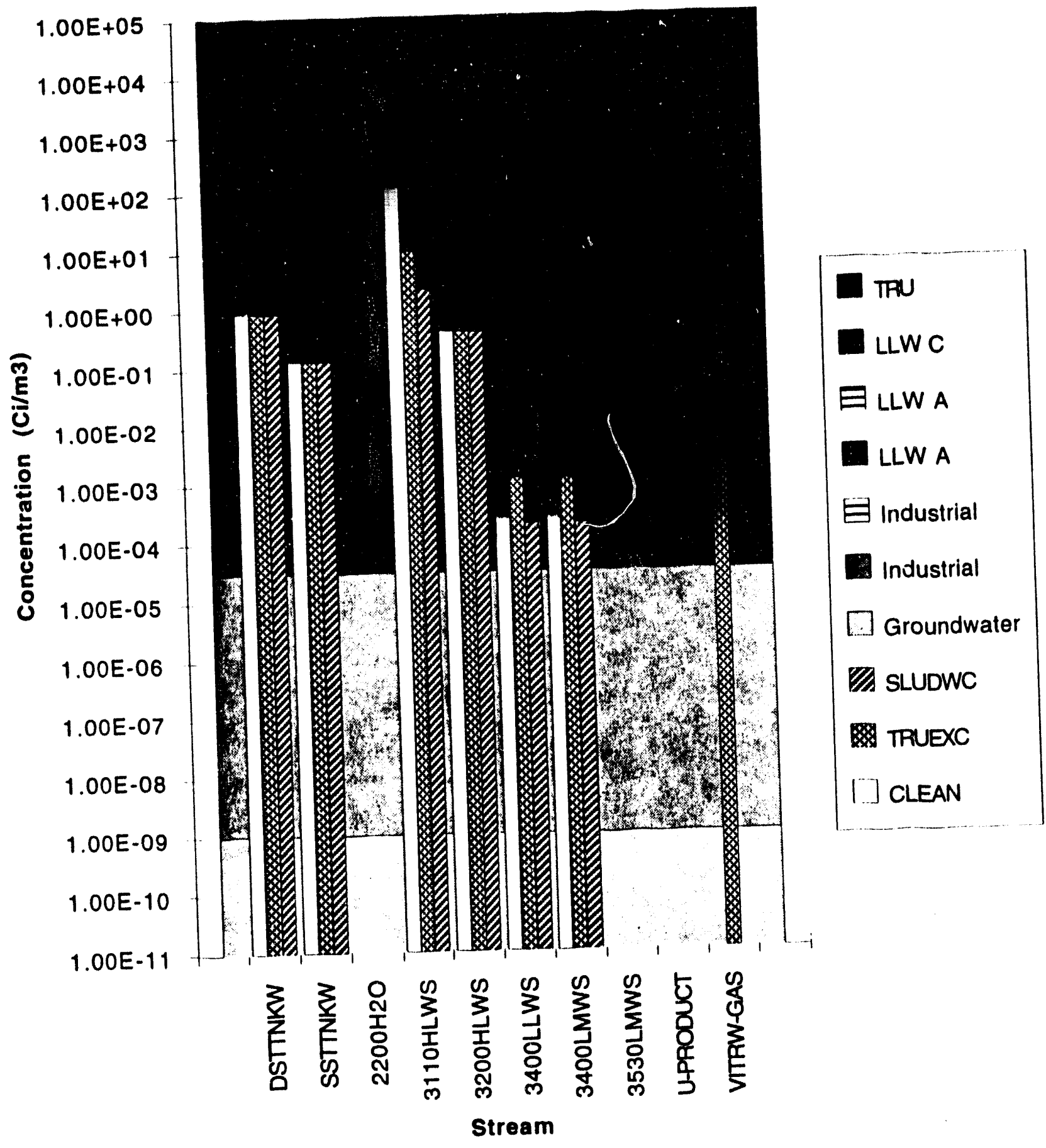




\section{WHC-EP-0791}

Figure 123. Neptunium Radioactivity Leve1.

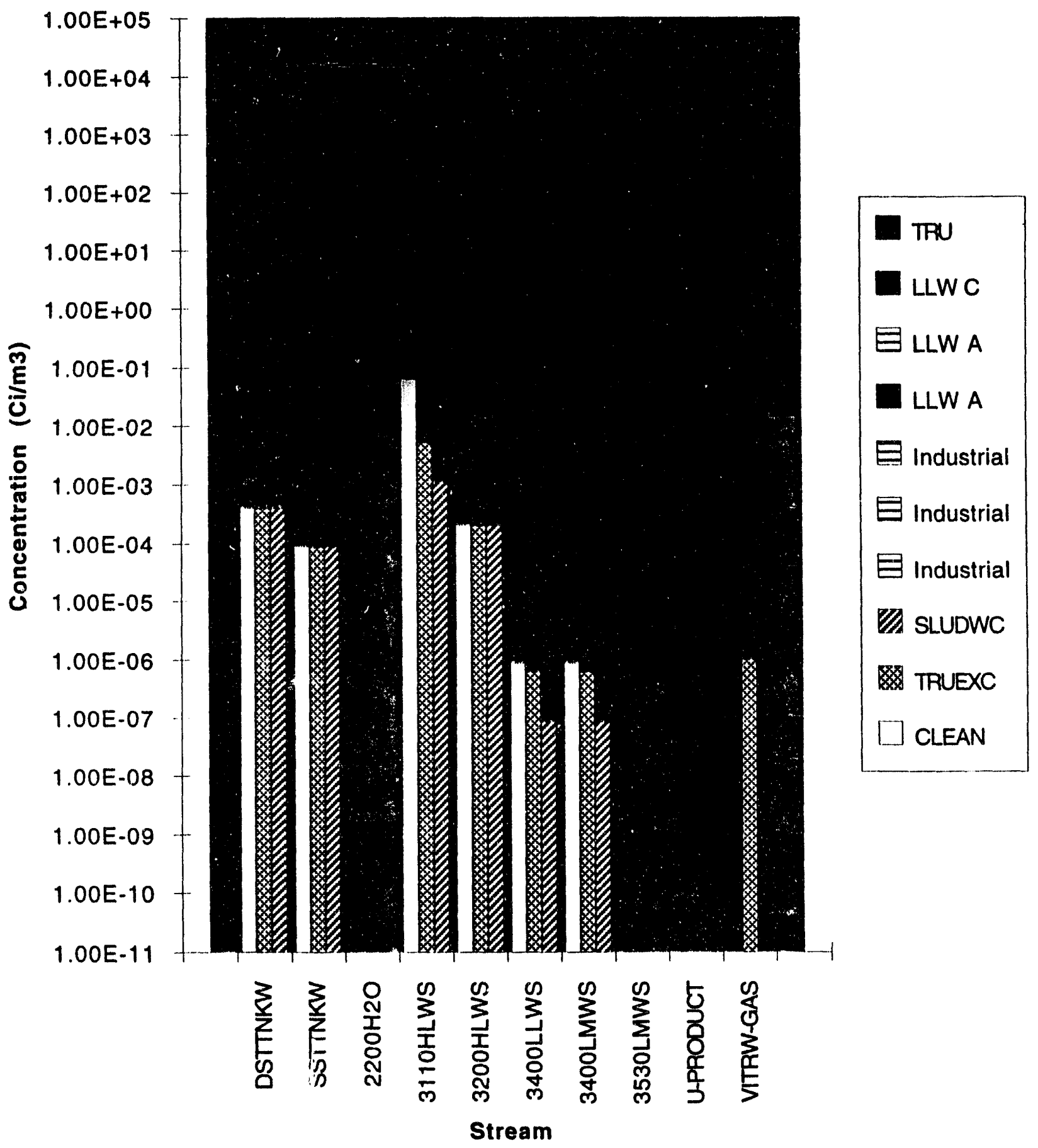


WHC-EP-0791

Figure 124. Heavy Lanthanide Radioactivity Level.

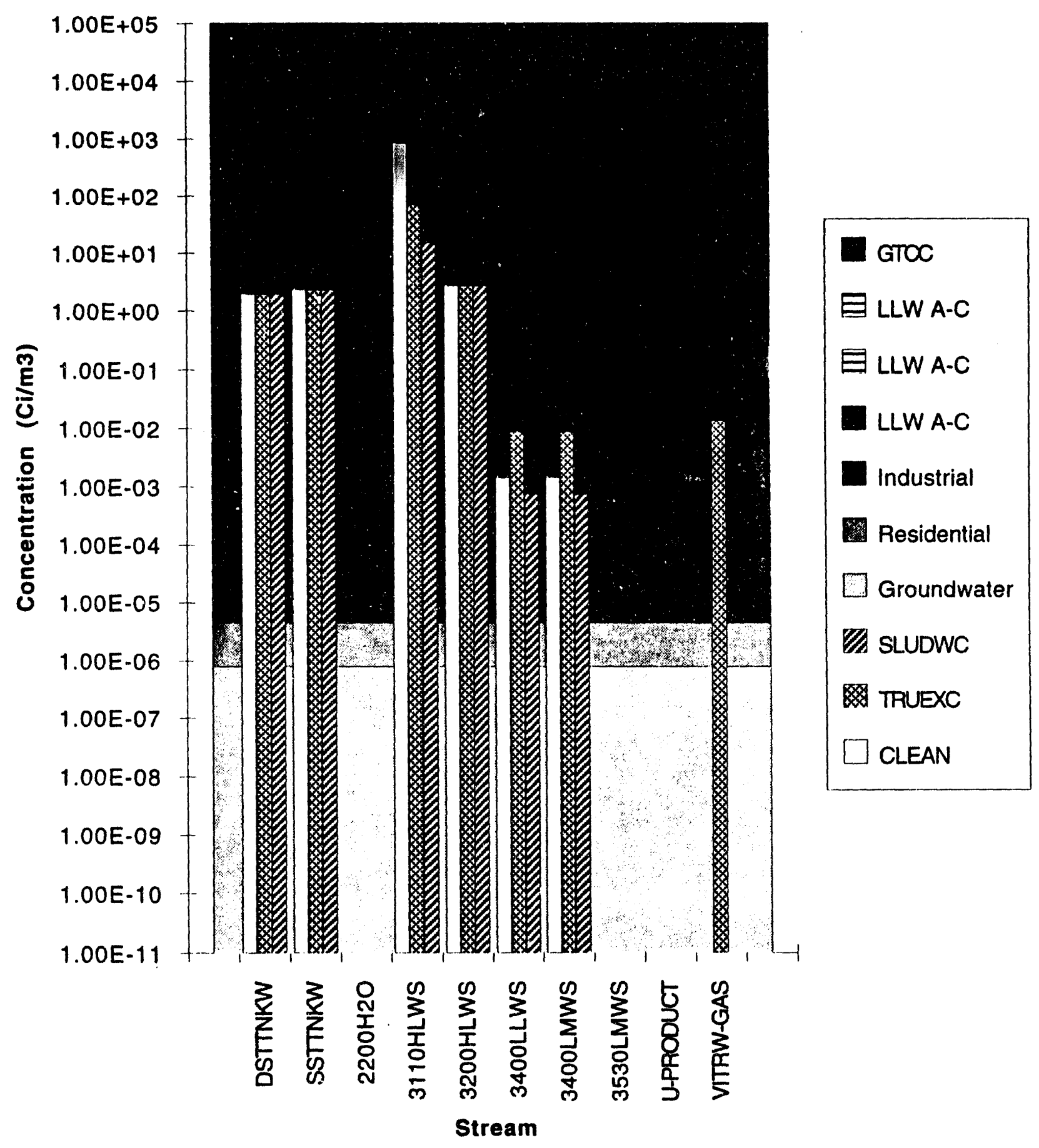


Table I1. Relative Long-Term Health Hazard Comparison Between Process Strategies.

Mass Inventories (KG)

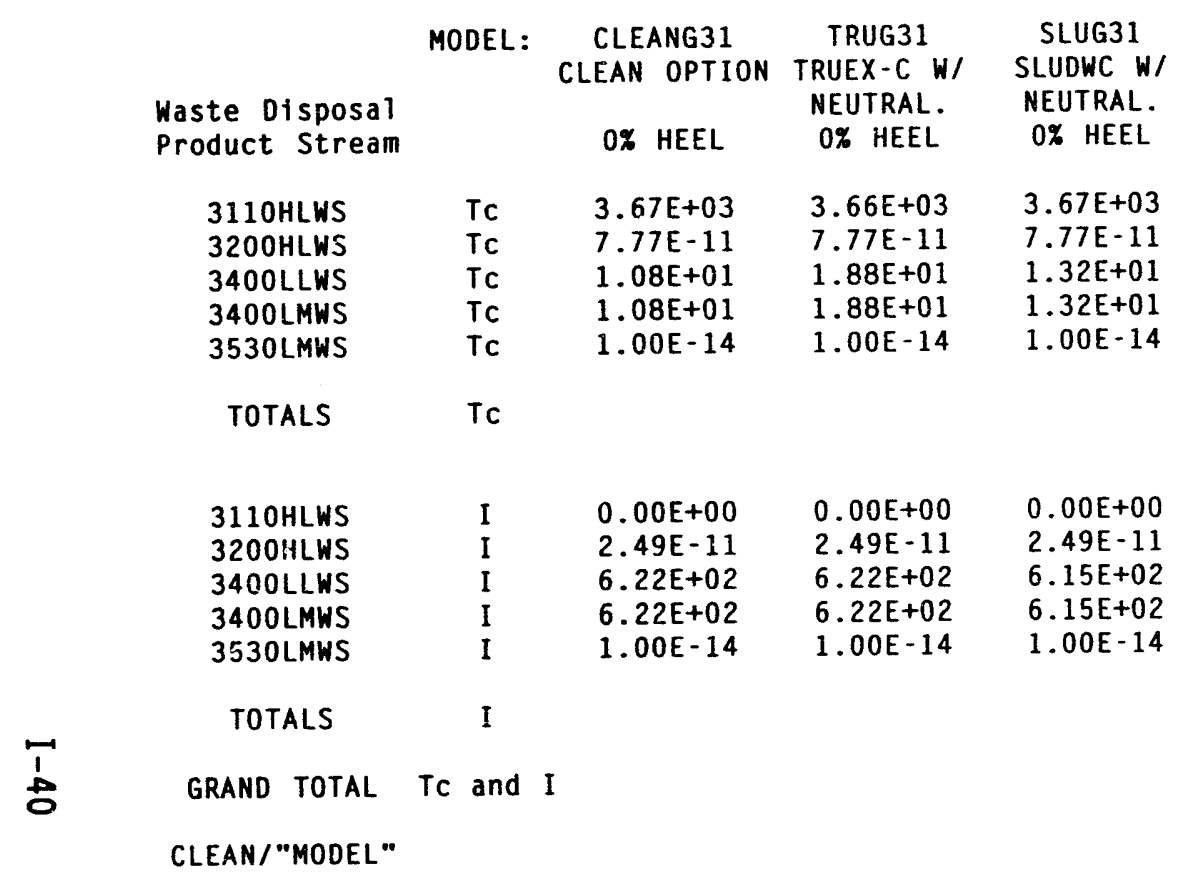

Relative Health Risk For Tc and I

CLEANG31 TRUG31 SLUG31

CLEAN OPTION TRUEX-C H/ SLUDWC W/

Pkg/barrier
NEUTRAL. NEUTRAL.

Atten fact, af $0 \%$ HEEL O\% HEEL O\% HEEL

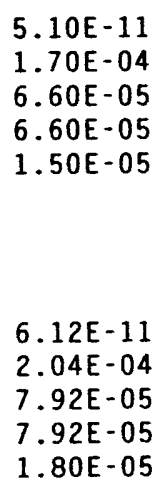

1.87E-07

$1.87 E-07$

$1.87 \mathrm{E}-07$

$1.30 E-14 \quad 1.30 E-14$

$1.30 \mathrm{E}-14$

$7.13 \mathrm{E}-04 \quad 1.24 \mathrm{E}-03 \quad 8.68 \mathrm{E}-04$

$7.13 \mathrm{E}-04 \quad 1.24 \mathrm{E}-03 \quad 8.68 \mathrm{E}-04$

$\begin{array}{lll}1.50 \mathrm{E}-19 & 1.50 \mathrm{E}-19 & 1.50 \mathrm{E}-19\end{array}$

$1.43 E-03 \quad 2.49 E-03 \quad 1.74 E-03$

$0.00 E+00 \quad 0.00 E+00 \quad 0.00 E+00$

$5.00 \mathrm{E}-15 \quad 5.00 \mathrm{E}-15 \quad 5.00 \mathrm{E}-15$

$4.93 E-02 \quad 4.92 E-02 \quad 4.87 E-02$

$4.93 \mathrm{E}-02 \quad 4.92 \mathrm{E}-02 \quad 4.87 \mathrm{E}-02$

$1.80 \mathrm{E}-19 \quad 1.80 \mathrm{E}-19 \quad 1.80 \mathrm{E}-19$

$9.85 \mathrm{E}-02 \quad 9.85 \mathrm{E}-02 \quad 9.73 \mathrm{E}-02$

$1.00 E-01 \quad 1.01 E-01 \quad 9.91 E-02$

9. $90 \mathrm{E}-01 \quad 1.01 \mathrm{E}+00$ 
Table 12. Health Hazard Index Coefficients by Component.

\section{COMPONENT}

$\mathrm{H} 2 \mathrm{O}$

PU

U

LANL

CS

P

TC

I

FP2

AP1

NANO3

OHAZ2

$\mathrm{SO}$

OTH3

CR2O3

AM

TH

NP

LANH

$\mathrm{NAOH}$

$B A$

$\mathrm{HNO} 3$

AL2O3

$\mathrm{SIO} 2$

NOX

\section{SHORT TERM \\ ONSITE}

(mrem/person)/

(Ci/m3)

$3.00 E+01$

$5.80 E+04$

2.20E+04

$0.00 E+00$

$1.40 E+04$

$2.20 E+02$

$5.30 E+00$

$4.40 E+02$

$4.40 E+02$

$5.00 \mathrm{E}+00$

$0.00 E+00$

$0.00 E+00$

$0.00 E+00$

$1.30 E+00$

$0.00 E+00$

$8.90 E+04$

$5.00 E+04$

$5.00 E+04$

4.40E+02

$0.00 E+00$

$0.00 E+00$

$0.00 E+00$

$0.00 E+00$

$0.00 E+00$

$0.00 E+00$
LONG TERM

ONSTTE

(mrem/person)/

(Ci/m3)

$0.00 E+00$

$5.80 E+04$

2.20E+04

$0.00 E+00$

$0.00 E+00$

$0.00 E+00$

5. $30 E+00$

4.40E+02

4.40E+02

$5.00 E+00$

$0.00 E+00$

$0.00 E+00$

$0.00 E+00$

1. $30 E+00$

$0.00 E+00$

$8.90 E+04$

$5.00 E+04$

$5.00 E+04$

$4.40 E+02$

$0.00 E+00$

$0.00 E+00$

$0.00 E+00$

$0.00 E+00$

$0.00 E+00$

$0.00 E+00$ 
Table 13. Calculated Radiation Dose and Cancer Risk to Individuals, Ranked by Risk, Assuming Exposure to a Buried Vlaste Concentration of $1 \mathrm{mCi} / \mathrm{m}^{3}$ (PNL-SA-20032 [Kennedy and Aaberg 1991]).

Scenario

Nuclide

$$
\begin{aligned}
& \text { Ingestion } \\
& \text { (rems) }
\end{aligned}
$$

Inhalation

(rems)

External

(rems)

Drilling:

$\begin{array}{ccccccc}\text { Co-60 } & 1.2 E-06 & 1.6 E-09 & 2.6 E-03 & 2.6 E-03 & 1 E-06 & 2.60 E+03 \\ \text { Cs-137 } & 5.7 E-06 & 2.3 E-10 & 6.2 E-04 & 6.3 E-04 & 3 E-07 & 6.30 E+02 \\ \text { Am-241 } & 4.4 E-04 & 3.5 E-06 & 4.7 E-06 & 4.5 E-04 & 2 E-07 & 4.50 E+02 \\ & & & & & & \\ 1-129 & 3.0 E-05 & 1.2 E-09 & 1.8 E-06 & 3.2 E-05 & 1 E-08 & 3.20 E+01 \\ \text { Sr-90 } & 1.5 E-05 & 1.6 E-09 & 1.2 E-07 & 1.5 E-05 & 6 E-09 & 1.50 E+01 \\ \text { Pu-239 } & 6.0 E-06 & 2.4 E-06 & 4.6 E-08 & 8.4 E-06 & 3 E-09 & 8.40 E+00 \\ \text { U-238 } & 2.8 E-06 & 9.2 E-07 & 2.8 E-08 & 3.7 E-06 & 2 E-09 & 3.70 E+00 \\ \text { Tc-99 } & 2.7 E-07 & 6.7 E-11 & 1.7 E-08 & 2.9 E-07 & 1 E-10 & 2.90 E-01 \\ \text { Ni-63 } & 6.6 E-08 & 1.7 E-11 & 6.3 E-11 & 6.6 E-08 & 3 E-11 & 6.60 E-02\end{array}$

Excavation:

$\begin{array}{ccccccc}\text { Am-241 } & 5.4 E-03 & 8.4 E-02 & 1.0 E-04 & 8.9 E-02 & 4 E-05 & 8.90 E+04 \\ \text { Co-60 } & 1.5 E-05 & 3.8 E-05 & 5.8 E-02 & 5.8 E-02 & 2 E-05 & 5.80 E+04 \\ \text { Pu-239 } & 7.5 E-05 & 5.8 E-02 & 1.0 E-06 & 5.8 E-02 & 2 E-05 & 5.80 E+04 \\ & & & & & & \\ \text { U-238 } & 3.5 E-05 & 2.2 E-02 & 6.3 E-07 & 2.2 E-02 & 9 E-06 & 2.20 E+04 \\ \text { Cs-137 } & 7.1 E-05 & 5.7 E-06 & 1.4 E-02 & 1.4 E-02 & 6 E-06 & 1.40 E+04 \\ 1-129 & 3.7 E-04 & 2.9 E-05 & 4.1 E-05 & 4.4 E-04 & 2 E-07 & 4.40 E+02 \\ & & & & & & \\ \text { Sr-90 } & 1.8 E-04 & 3.9 E-05 & 2.6 E-06 & 2.2 E-04 & 9 E-08 & 2.20 E+02 \\ \text { Tc-99 } & 3.3 E-06 & 1.6 E-06 & 3.7 E-07 & 5.3 E-06 & 2 E-09 & 5.30 E+00 \\ \text { Ni-63 } & 8.3 E-07 & 4.2 E-07 & 1.4 E-09 & 1.3 E-06 & 5 E-10 & 1.30 E+00\end{array}$

(a) Risk for workers, as given in ICRP Publication 60, is based on the conversion factor 4 E-04 fatal cancers per rem.

mrem/Ci/m3
Risk of

Fatal

New Units

(rems) 


\section{$\varepsilon b-I$}

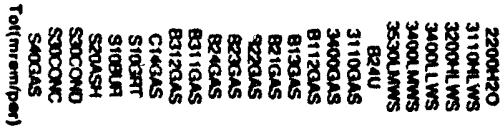

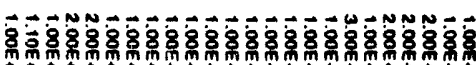

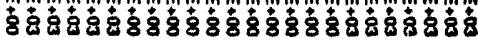

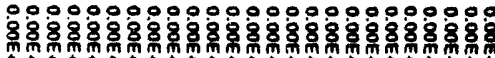

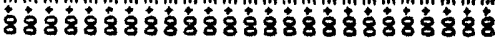

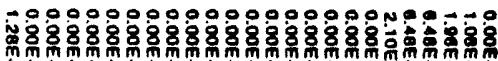

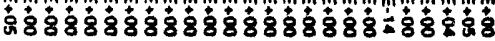

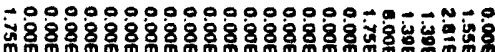
ผั่

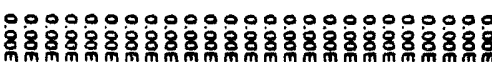

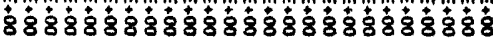

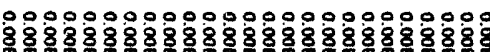

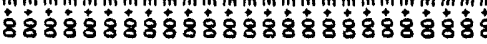

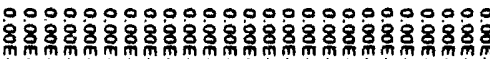

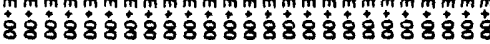

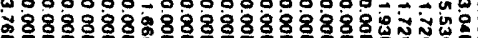

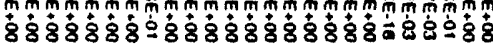

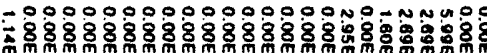

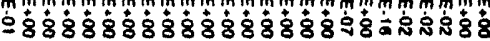

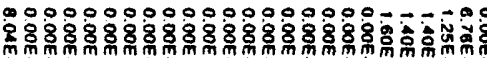
ڤัง

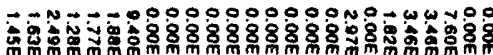
ค่

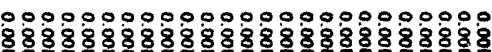
ช்

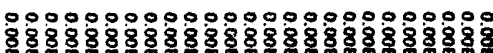

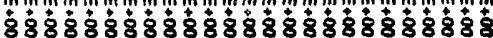

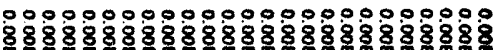

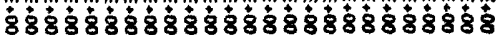

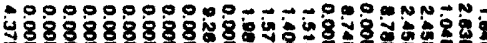

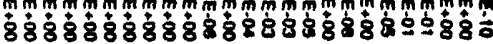

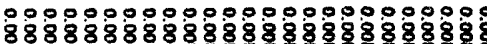
ถ்

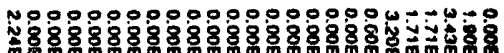

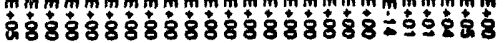

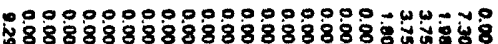
仓ั่

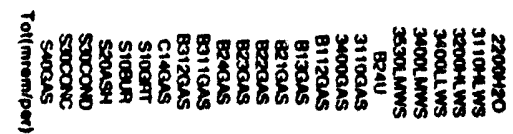

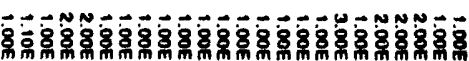

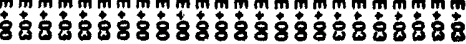

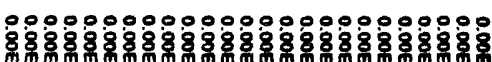
ริ8388

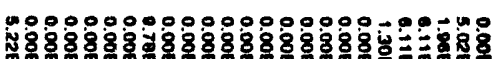
งัง

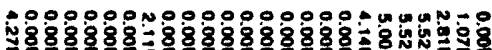

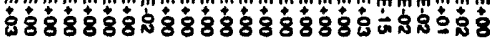

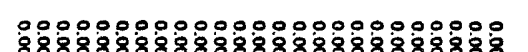
s. ช̇

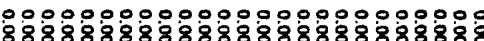
ช́ร் 880800000000000

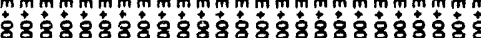

60000000000000000000-1-n -

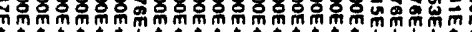

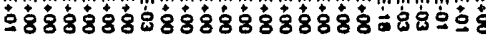

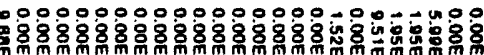

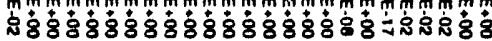

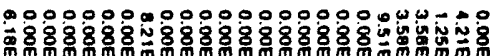

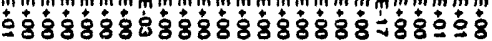

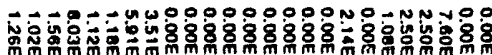

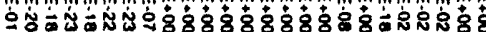

00000000000000000000000

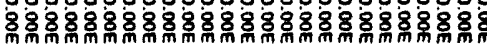
ธ்่่

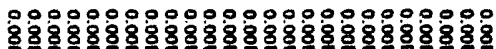

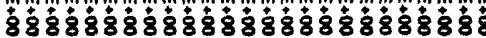

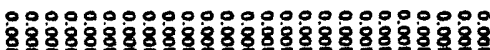

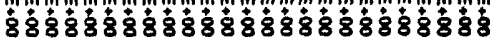

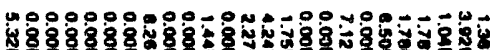

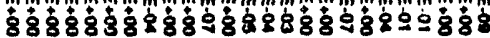

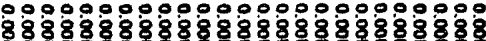

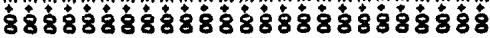

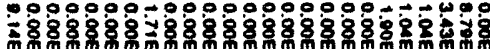

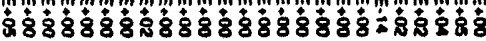

แับำ

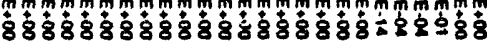

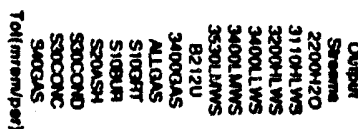

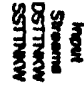

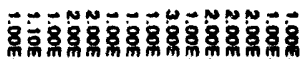

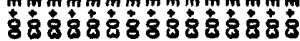

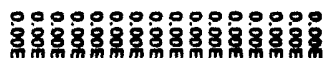

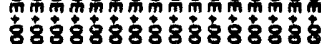

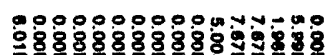
\$8

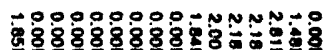

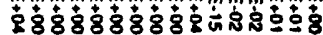

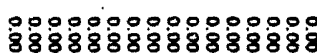

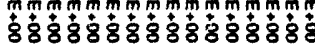

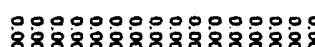

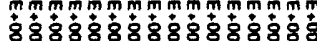

000000000000000

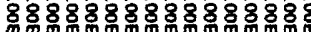

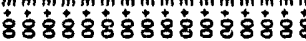

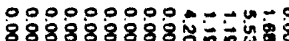
等

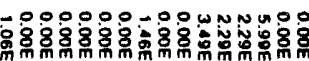

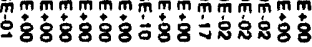

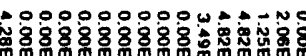

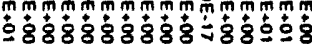

ดิ

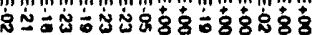

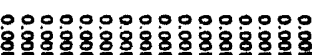

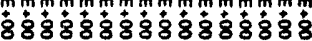

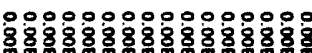

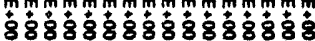

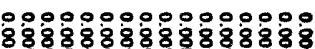

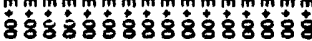

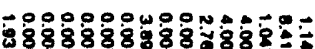

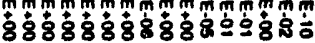

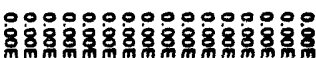

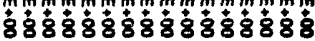

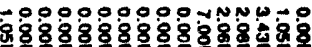
ง่ร8

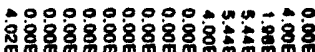
ڤ்

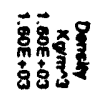

然

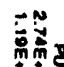

\&ें

落

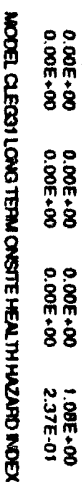

㻤宫-

这

离票

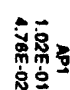

8유.

$8 \%$

8융ำ

索势

串客

88

章繁

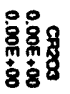

咅蒡

部客玨 
Table I4. Long-Term Onsite Health Hazard Index (mrem/person) by Component. (sheet 2 of 2)

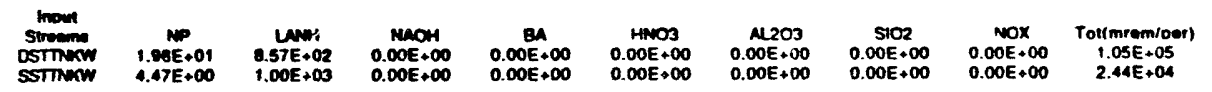

Outout

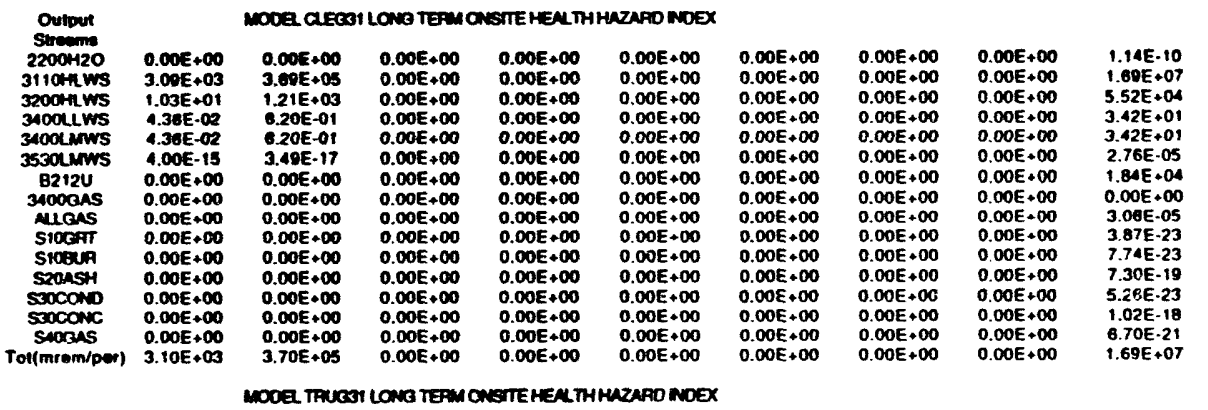

MOCE TRUCBI LONG TEPM ONSTE HENTHHUZARO MOEX

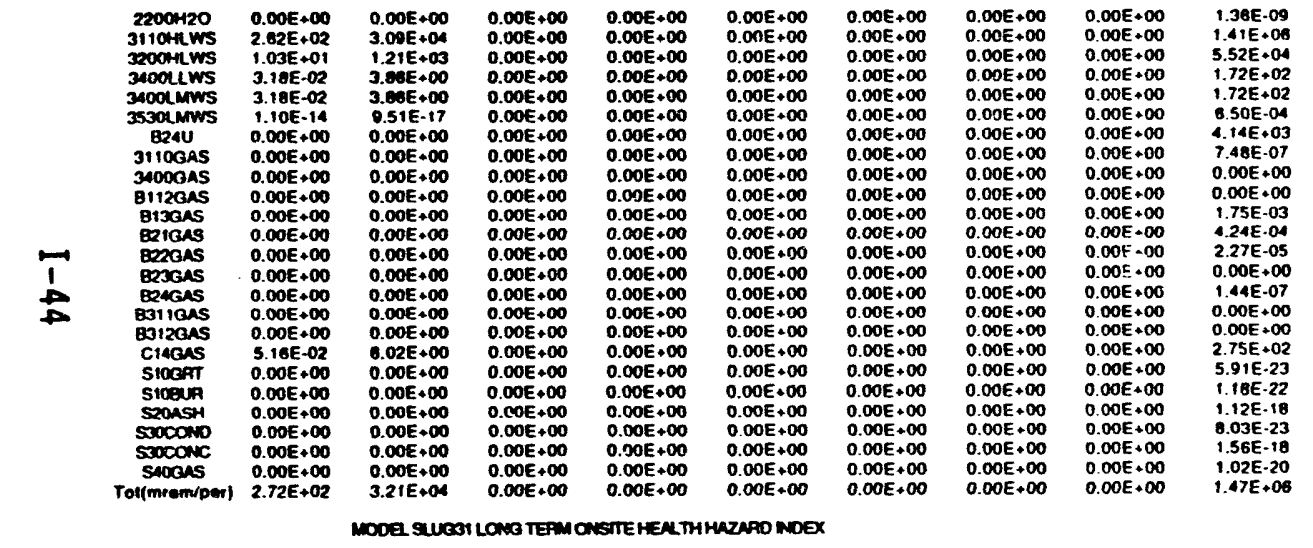

\begin{tabular}{|c|c|c|c|c|c|c|c|c|c|}
\hline 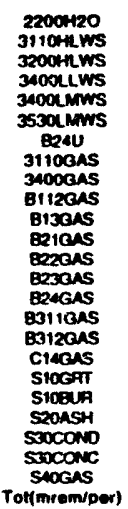 & 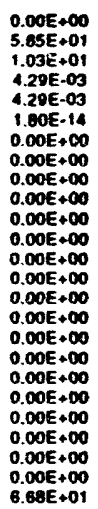 & 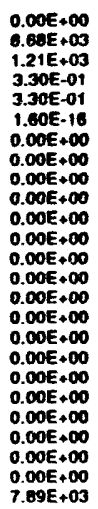 & 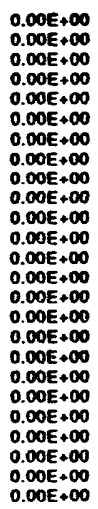 & 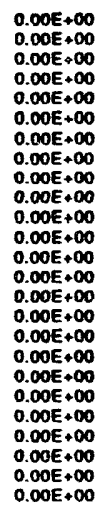 & 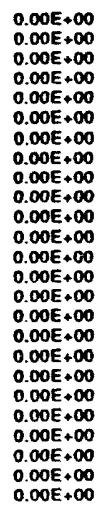 & 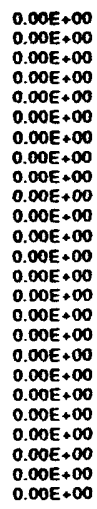 & $\begin{array}{l}0.00 E+00 \\
0.00 E+00 \\
0.00 E+00 \\
0.00 E+00 \\
0.00 E+00 \\
0.00 E+00 \\
0.00 E+00 \\
0.00 E+00 \\
0.00 E+00 \\
0.00 E+00 \\
0.00 E+00 \\
0.00 E+00 \\
0.00 E+00 \\
0.00 E+00 \\
0.00 E+00 \\
0.00 E+00 \\
0.00 E+00 \\
0.00 E+00 \\
0.00 E+00 \\
0.00 E+00 \\
0.00 E+00 \\
0.00 E+00 \\
0.00 E+00 \\
0.00 E \\
0.00 E+00 \\
0.000\end{array}$ & 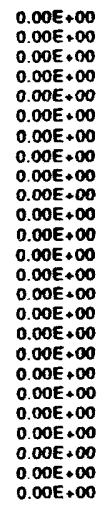 & 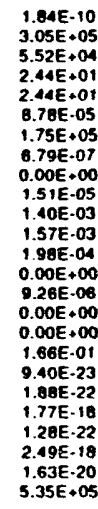 \\
\hline
\end{tabular}




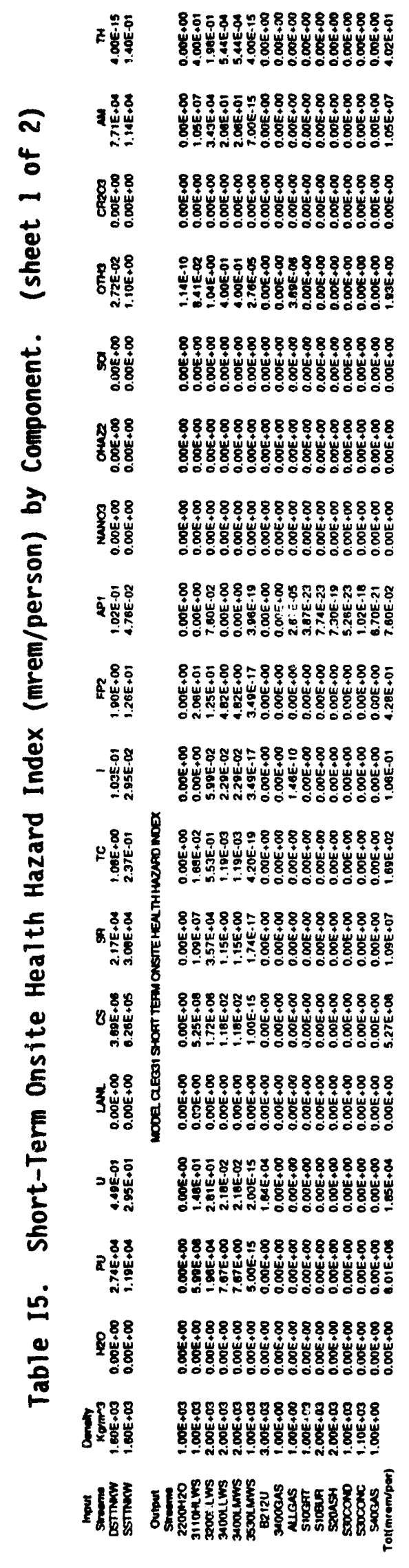

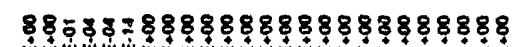

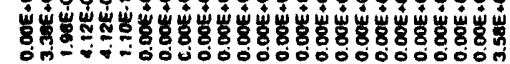

$887 \%$

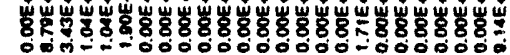

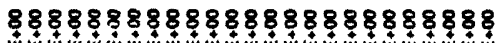

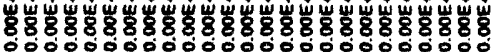

8885588588878898888888888

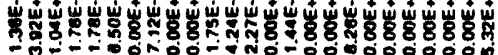

8888888888888888888888888

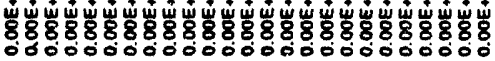

8888888888888888888888888

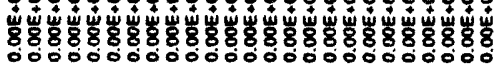

89888888888888888898888888888

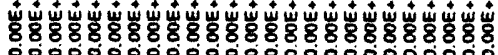

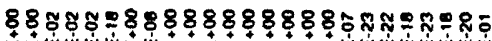

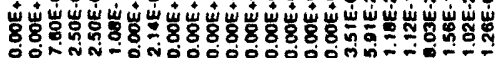

$8 \overline{8}=88=88888888888888888885$

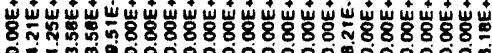

$88 \% \approx ్ 888888888888888888 \%$

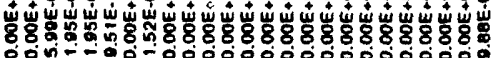

8

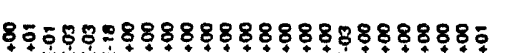

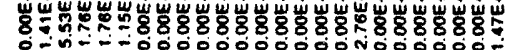

$807 \%$

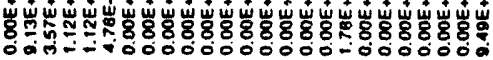

$858 \% 8=8888888888888888880$

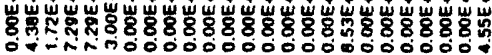

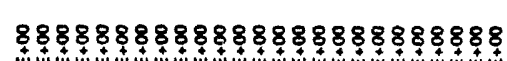

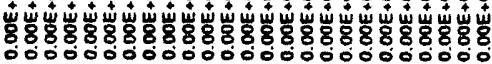

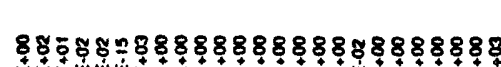

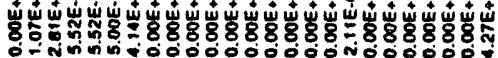

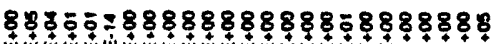

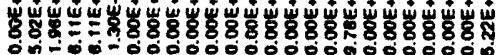

88888888888888888888888888

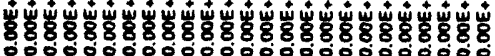

888888888888888888888888

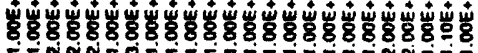

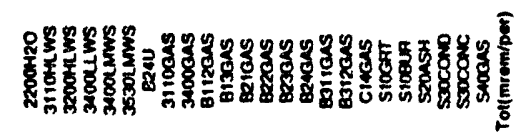
$I-45$
$85 \overline{5} 8 \div 88888888888888888885$

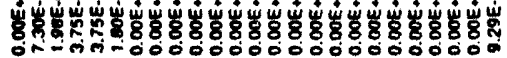

$8885 \div \div 88888888888888888 \%$ 8.

888888888888888888888898888

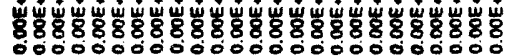

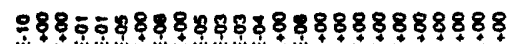

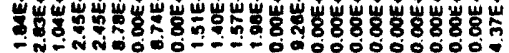

8888888888888888888888888

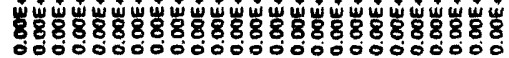

88888888888888888888888888

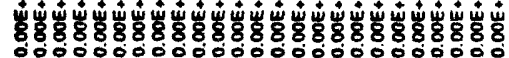

8888888888888888888888888

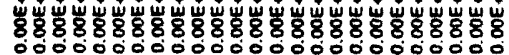

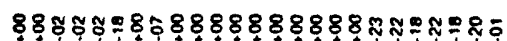

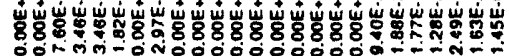

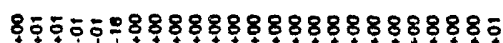

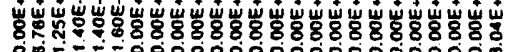

$88 \% 8 \% 8588888888888888885$

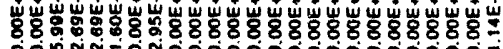

8

8858898888888888858888888

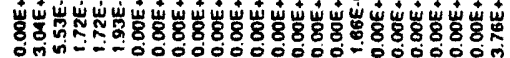

$88388=888888888888888888 \%$

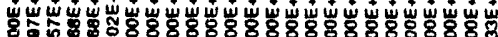

(1)

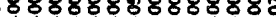

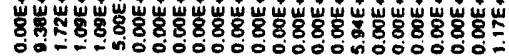
88888888888888888888888888

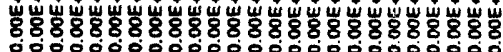

8 8ซ

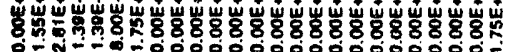

$88888 \div 888888888888888888 \%$

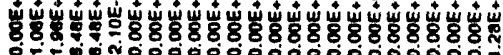

8888888888888888888888888 8.

$8888888888888888888 \% 898888$

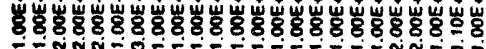

atthy 
iable 15. Short-Term Onsite Health Hazard Index (mrem/person) by Component. (sheet 2 of 2)

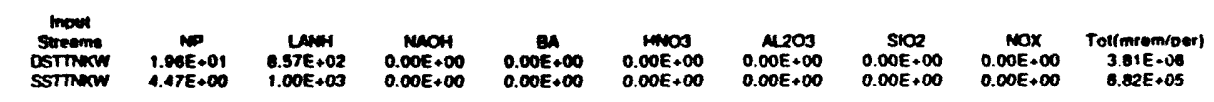

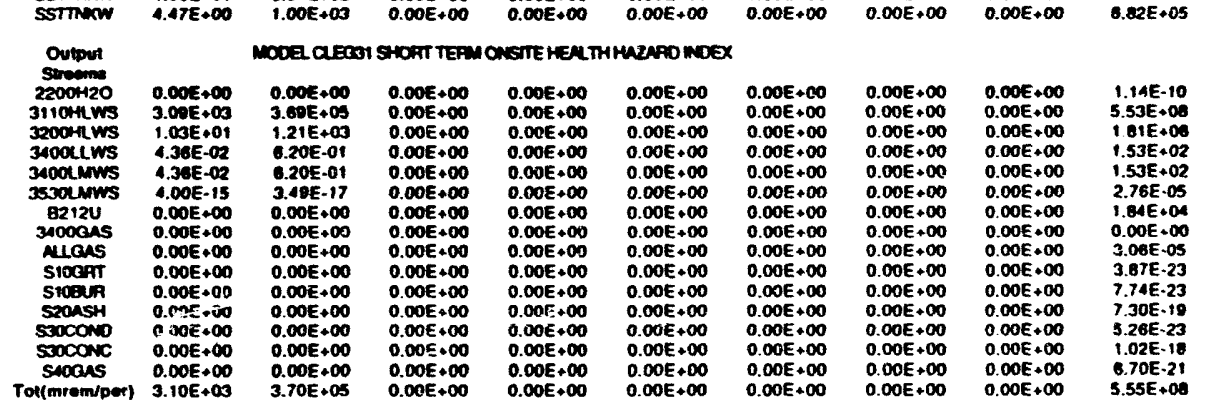

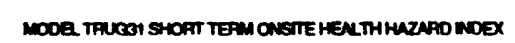

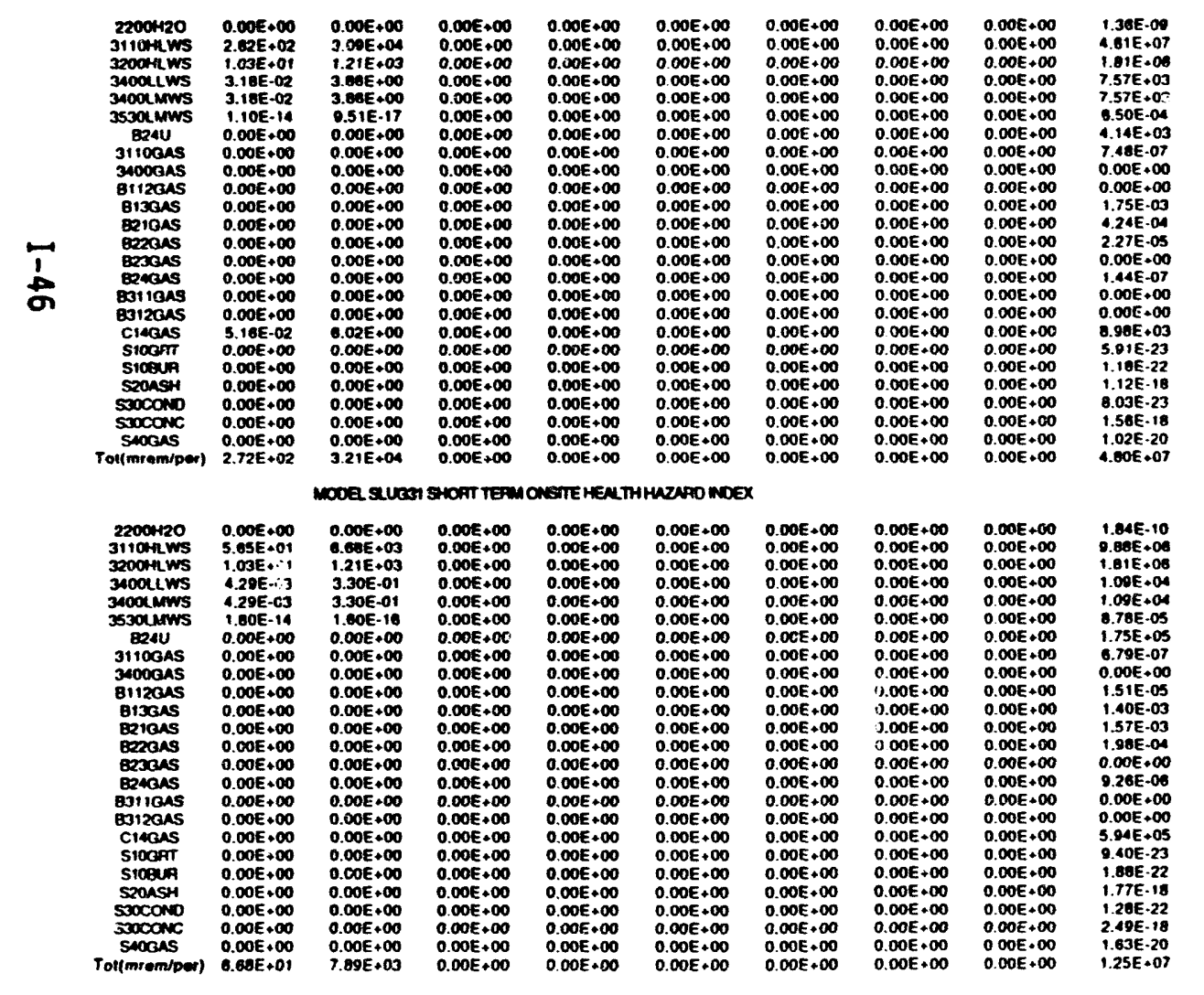


WHC-EP-0791

Table 16. Occupational Health Hazard Index for Model CLEANG31. gaMma CONTAMINATON

\begin{tabular}{|c|c|c|c|c|c|}
\hline BLoCK & CAMMA FLOW (Cl) & TOTAL FLOW (KG) & $\begin{array}{l}\text { CONCENTRATION } \\
\text { (CUKG) }\end{array}$ & $\begin{array}{l}\text { TERTARY STREA } \\
\text { FLOWS (KO) }\end{array}$ & $\begin{array}{l}\text { OCCUP. HENTH HA } \\
\text { WDEX (CHKCMM3) }\end{array}$ \\
\hline 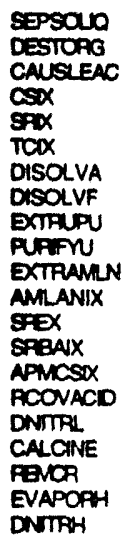 & $\begin{array}{l}4.65 E+07 \\
4.12 E+07 \\
1.04 E+07 \\
5.16 E+07 \\
5.16 E+03 \\
5.16 E+03 \\
5.16 E+06 \\
5.20 E+05 \\
5.11 E+06 \\
0.00 E+00 \\
5.11 E+06 \\
0.00 E+00 \\
5.11 E+06 \\
0.00 E+00 \\
5.11 E+06 \\
0.00 E+00 \\
5.11 E+03 \\
1.03 E+04 \\
1.03 E+04 \\
0.00 E+00 \\
5.16 E+07\end{array}$ & $\begin{array}{l}7.57 E+08 \\
7.97 E+08 \\
2.47 E+08 \\
1.12 E+09 \\
1.13 E+09 \\
1.14 E+08 \\
6.30 E+07 \\
1.53 E+07 \\
3.17 E+08 \\
7.53 E+07 \\
3.28 E+08 \\
2.08 E+08 \\
3.67 E+08 \\
6.09 E+08 \\
5.12 E+08 \\
7.62 E+08 \\
7.22 E+08 \\
2.52 E+09 \\
7.52 E+08 \\
7.35 E+08 \\
2.22 E+07\end{array}$ & $\begin{array}{l}6.13 E \cdot 02 \\
5.17 E \cdot 02 \\
4.21 E \cdot 02 \\
4.59 E \cdot 02 \\
4.56 E \cdot 06 \\
4.53 E \cdot 06 \\
8.20 E \cdot 02 \\
3.40 E \cdot 02 \\
1.61 E-02 \\
0.00 E+00 \\
1.56 E \cdot 02 \\
0.00 E+00 \\
1.30 E-02 \\
0.00 E+00 \\
9.08 E-03 \\
0.00 E+00 \\
7.07 E \cdot 06 \\
4.07 E \cdot 06 \\
1.37 E \cdot 05 \\
0.00 E+00 \\
2.33 E+00\end{array}$ & $\begin{array}{l}4.49 E+07 \\
4.63 E+07 \\
2.01 E+07 \\
5.33 E+08 \\
5.34 E+08 \\
5.35 E+08 \\
7.41 E+06 \\
1.80 E+06 \\
1.49 E+08 \\
3.59 E+07 \\
1.55 E+08 \\
9.86 E+07 \\
1.73 E+08 \\
2.88 E+08 \\
1.21 E+08 \\
9.03 E+07 \\
8.50 E+07 \\
2.07 E+08 \\
8.82 E+07 \\
1.26 E+07 \\
2.61 E+06\end{array}$ & $\begin{array}{l}2.76 E+06 \\
2.39 E+06 \\
1.22 E+06 \\
2.44 E+07 \\
2.43 E+03 \\
2.42 E+03 \\
6.08 E+05 \\
6.11 E+04 \\
2.40 E+06 \\
0.00 E+00 \\
2.42 E+06 \\
0.00 E+00 \\
2.40 E+06 \\
0.00 E+00 \\
1.20 E+06 \\
0.00 E+00 \\
6.01 E+02 \\
1.21 E+03 \\
1.20 E+03 \\
0.00 E+00 \\
6.08 E+00\end{array}$ \\
\hline TOTALS & $2.27 E+08$ & $1.32 E+10$ & $2.70 E+00$ & $3.36 E+09$ & $4.60 E+07$ \\
\hline
\end{tabular}

ALPHA CONTAMINATION

Beck

SEPSODO

DESTOAC

CALSLEAC

$\cos x$

$\operatorname{six}$

$\operatorname{TCIX}$

DISOLVA

DISOLVF

Extrify

PXTPU

EXTRAMLN

AMLANIX

SEX

SPANAX

RCOVACID

DNITIL

CALCINE

DAPOPH

DNTRH

TOTALS

Bock

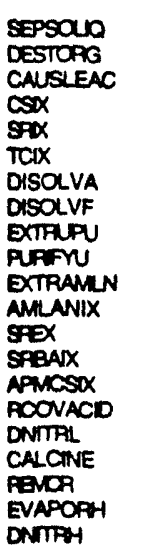

TOTALS
ALPHA FLOW (CI) TOTAL FLOW (KG)

CONCENTRATION TERTLAY STREAM OCCUP. HEALTHHAZ

$2.75 E+05$
$1.05 E+04$
$2.89 E+05$
$3.75 E+02$
$3.75 E+02$
$3.75 E+02$
$2.88 E+05$
$2.93 E+04$
$2.74 E+05$
$5.79 E+02$
$1.46 E+05$
$1.46 E+05$
$1.49 E+01$
$0.00 E+00$
$1.49 E+01$
$0.00 E+00$
$4.27 E+01$
$4.47 E+02$
$4.47 E+02$
$2.73 E+05$
$2.73 E+05$

$7.57 E+08$
$7.97 E+08$
$2.47 E+08$
$1.12 E+09$
$1.13 E+09$
$1.14 E+09$
$6.30 E+07$
$1.53 E+07$
$3.17 E+08$
$7.53 E+07$
$3.28 E+08$
$2.08 E+08$
$3.67 E+08$
$6.09 E+08$
$5.12 E+08$
$7.62 E+08$
$7.22 E+08$
$2.52 E+09$
$7.52 E+08$
$7.35 E+08$
$2.22 E+07$

$2.00 E+06$

$1.325+10$

BETA CONTAMINATION

BETAFLOW (CI) TOTAL FLOW (KG)

CONCENIRATION TERTAFY STREAM OCCUP. HEALTHHAZ (CUKG) FLOWS (KG) INDEX (CI-KGM3)

$\begin{array}{ll}6.28 E+07 & 7.57 E+08 \\ 8.12 E+05 & 7.97 E+08 \\ 6.34 E+07 & 2.47 E+08 \\ 4.36 E+05 & 1.12 E+09 \\ 4.36 E+05 & 1.13 E+09 \\ 3.71 E+05 & 1.14 E+09 \\ 6.32 E+07 & 6.30 E+07 \\ 6.42 E+06 & 1.53 E+07 \\ 6.25 E+07 & 3.17 E+08 \\ 1.26 E+01 & 7.53 E+07 \\ 6.25 E+07 & 3.28 E+08 \\ 1.04 E+06 & 2.08 E+08 \\ 6.14 E+07 & 3.67 E+08 \\ 6.14 E+07 & 6.09 E+08 \\ 6.42 E+04 & 5.12 E+08 \\ 0.00 E+00 & 7.62 E+08 \\ 6.49 E+04 & 7.22 E+08 \\ 4.03 E+05 & 2.52 E+09 \\ 3.98 E+05 & 7.52 E+08 \\ 6.24 E+07 & 7.35 E+08 \\ 6.24 E+07 & 2.22 E+07 \\ 5.72 E+08 & 1.32 E+10\end{array}$

8.29E-02

$4.49 E+07$ $1.02 E-03$
$2.57 E .01$

3.88E.04

3.86E-04

3.26E.04

$1.00 E+00$

4.20E-01

1.97E.01

1.67E-07

$1.00 E-01$
$4.99 E-03$

1.67E-01

1.01E.01

1.26E-04

$0.00 E+00$

8.89E-05

$1.60 \mathrm{E} \cdot 04$

5.28E.04

6.49E-02

$2.82 E+00$

2.91E+07

5. $33 E+08$

5. $35 E+08$

$7.41 E+06$

7. $11 E+06$

$1.80 E+06$

$1.49 E+08$

$3.59 E+07$
$1.55 E+08$

$0.86 \mathrm{E}+07$

1.73E+08

$2.8 B E+08$

$1.21 E+08$

.03E+07

B. $50 E+07$

2.87E+08

6.82E+07

$4.26 E+07$

$2.61 E+06$

$4.71 E+04$

7.47E+06

2.07E+05

2.06E+05

1.74E+05

$7.44 E+06$

7.54E+05

2.94E+07

$6.00 E+00$

$2.96 E+07$

4.03E+05

$2.89 \mathrm{E}+07$

$2.90 E+07$

$1.51 E+04$

$0.00 E+00$

$7.64 E+03$

$4.75 E+04$
$4.66 E+04$

$4.66 E+04$
$3.62 E+08$

$7.36 E+06$

5. $33 \mathrm{E}+00$

3.36E+09

$1.48 E+08$ 


\section{Table 17. Occupational Health Hazard Index for Model TRUG3l.}

GAMMA CONTAMINATION

BLOK

WASHSLUD

DESTORG

FILTADBS

$\operatorname{cox}$

TCIX1

DISSOLVA

TRUEX

SFEX

UTCSTRIP

TCIX2

PUAFY

NEUTDCAN

NEUTRAL

TOTALS

BLOCK

WASHSLUD

DESTORG

FILTADBS

$\operatorname{CSX}$

TCIX1

DISSOLVA

TRUEX

SFEX

UTCSTRIP

TCIX2

PURIFY

NEUTDCAN

NEUTRAL.

TOTALS

BLOCK
WASHSLUD
DESTOAG
FILTADBS
CSX
TCIX1
DISSOLVA
TRUEX
STEX
UTCSTRIP
TCIX2
PUPIFY
NEUTDCAN
NEUTRAL
TOTALS

TOTALS
GAMMA FLOW (CI) TOTAL FLOW (KG)

$4.65 E+07$
$4.13 E+07$
$4.12 E+07$
$4.59 E+07$
$5.71 E+05$
$5.21 E+06$
$4.69 E+06$
$4.69 E+06$
$0.00 E+00$
$0.00 E+00$
$0.00 E+00$
$5.24 E+05$
$4.53 E+07$
$2.36 E+08$

$7.98 E+08$

$8.05 E+U 8$

$1.02 E+09$

$1.93 E+09$

6.55E+07

$5.01 E+08$

$2.50 E+08$

$3.05 E+08$

$6.96 \mathrm{E}+07$

$1.07 E+08$

$7.40 E+07$

1.24E+06

$6.70 E+09$

\section{ALPHA CONTAMINATION}

ALPHA FLOW (Cl) TOTAL FLOW (KG)

$2.75 E+05$
$1.05 E+04$
$1.05 E+02$
$6.92 E+02$
$6.92 E+02$
$2.75 E+05$
$2.34 E+05$
$1.17 E+03$
$1.57 E+03$
$1.57 E+03$
$1.57 E+03$
$2.72 E+05$
$0.00 E+00$
$1.07 E+06$

$7.57 E+08$

$7.98 E+08$

8.05E+08

$1.02 E+09$

$1.93 E+09$

8. $55 \mathrm{E}+07$

$5.01 E+08$

$2.50 E+08$

$3.05 E+08$

$6.96 \mathrm{E}+07$

$1.07 E+08$

$7.40 E+07$

$1.24 E+06$

$6.70 E+09$

\section{BETA CONTAMINATION}

BETA FLOW (CI) TOTAL FLOW (KG)

$\begin{array}{ll}6.28 E+07 & 7.57 E+08 \\ 8.13 E+05 & 7.98 E+08 \\ 1.96 E+05 & 8.05 E+08 \\ 1.97 E+05 & 1.02 E+09 \\ 2.04 E+05 & 1.93 E+09 \\ 6.26 E+07 & 8.55 E+07 \\ 5.62 E+07 & 5.01 E+08 \\ 5.50 E+07 & 2.50 E+08 \\ 1.11 E+05 & 3.05 E+08 \\ 1.24 E+04 & 6.96 E+07 \\ 4.87 E+03 & 1.07 E+08 \\ 6.19 E+07 & 7.40 E+07 \\ 3.82 E+04 & 1.24 E+06 \\ 3.00 E+08 & 6.70 E+09\end{array}$

CONCENTRATION (CI/KG)

6.13E-02

5.17E-02

5.12E.02

4.50E-02

2.96E-04

6.09E-02

9.36E-03

1.87E.02

$0.00 E+00$

$0.00 E+00$

$0.00 E+00$

7.08E-03

$3.66 \mathrm{E}+01$

$3.69 E+01$
CONCENTRATION (CI/KG)

3.63E-04

1.31E-05

$1.30 E .07$

6.79F.07

3.59E-07

3.21E.03

4.67E.04

4.67E.06

5.15E-06

2.26E-05

1.47E-05

3.67E-03

$0.00 E+00$

7.77E-03 FLOWS (KG) INDEX (Cl-KG/M3)

$\begin{array}{ll}4.49 E+07 & 2.76 E+06 \\ 4.64 E+07 & 2.40 E+06 \\ 1.89 E+08 & 9.68 E+06 \\ 4.85 E+08 & 2.18 E+07 \\ 9.03 E+08 & 2.67 E+05 \\ 1.01 E+07 & 6.14 E+05 \\ 2.35 E+08 & 2.20 E+06 \\ 1.18 E+08 & 2.20 E+06 \\ 7.27 E+07 & 0.00 E+00 \\ 3.28 E+07 & 0.00 E+00 \\ 4.99 E+07 & 0.00 E+00 \\ 4.36 E+06 & 3.09 E+04 \\ 7.29 E+04 & 2.67 E+06 \\ 2.19 E+09 & 4.46 E+07\end{array}$

TERTIAPY STREAM OCCUP. HEALTHHAZ
TERTIARY STREAM OCCUP. HEALTHHAZ FLOWS (KG)

$$
\begin{aligned}
& 4.49 E+07 \\
& 4.64 E+07 \\
& 1.89 E+08 \\
& 4.85 E+08 \\
& 9.03 E+08 \\
& 1.01 E+07 \\
& 2.35 E+08 \\
& 1.18 E+08 \\
& 7.27 E+07 \\
& 3.28 E+07 \\
& 4.99 E+07 \\
& 4.36 E+06 \\
& 7.29 E+04 \\
& 2.19 E+09
\end{aligned}
$$

$$
\begin{aligned}
& 1.63 E+04 \\
& 6.07 E+02 \\
& 2.45 E+01 \\
& 3.29 E+02 \\
& 3.24 E+02 \\
& 3.24 E+04 \\
& 1.10 E+05 \\
& 5.49 E+02 \\
& 3.75 E+02 \\
& 7.41 E+02 \\
& 7.32 E+02 \\
& 1.60 E+04 \\
& 0.00 E+00 \\
& 1.78 E+05
\end{aligned}
$$

\section{CONCENTRATION (Cl/KG)}

8.29E-02

$1.02 \mathrm{E}-03$

2.44E-04

$1.93 E-04$

1.06E-04

7.32E.01

$1.12 E-01$

2.20E-01

3.63E-04

1.79E-04

4.54E-05

8.36E-01

3.09E-02

$2.02 E+00$
TERTIARY STREAM

FLOWS (KG)

$$
\begin{aligned}
& 4.49 E+07 \\
& 4.64 E+07 \\
& 1.89 E+08 \\
& 4.85 E+08 \\
& 9.03 E+08 \\
& 1.01 E+07 \\
& 2.35 E+08 \\
& 1.18 E+08 \\
& 7.27 E+07 \\
& 3.28 E+07 \\
& 4.99 E+07 \\
& 4.36 E+06 \\
& 7.29 E+04 \\
& 2.19 E+09
\end{aligned}
$$

OCCUP. HEALTHHAZ INDEX (Cl-KG/M3)
$3.73 E+06$
$4.72 E+04$
$4.61 E+04$
9.35E+04
9.55E+04
7.38E+06
2.64E+07
2.58E+07
2.64E+04
5.87E+03
2.27E+03
$3.65 E+06$
2.25E+03
$6.72 E+07$ 
Table 18. Occupational Health Hazard Index for Model SLUG31.

GAMMA CONTAMINATION

BLOK

WASHSLUD

DESTOPG

FILTADBS

CSX

$\mathrm{TCIX} 1$

DISSOLVA

TRUEX

STEX

UTCSTRIP

TCIX2

PUAFY

NEUTDCAN

NEUTRAL

TOTALS

BLOCK

WASHSLUD

DESTORG

FILTADBS

$\operatorname{cs} \alpha$

TCIX1

OISSOLVA

TAUEX

FEX

UTCSTRIP

TCIX2

PUFAFY

NEUTDCAN

NEUTRAL

TOTALS

BLOCK

WASHSLUD

DESTORG

FILTADBS

$\operatorname{cs} x$

TCIX1

DISSOLVA

TRUEX

FEX

UTCSTRIP

TCIX2

Purafr

NEUTDCAN

NEUTTAL

TOTALS
GAMMA FLOW (CI) TOTAL FLOW (KG)

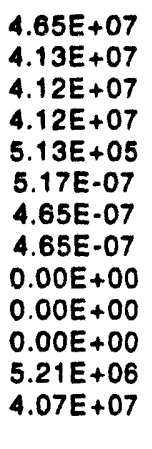

2.17E+08

$7.57 E+08$
$7.98 E+08$
$8.04 E+08$
$8.04 E+08$
$1.50 E+09$
$9.75 E-06$
$9.53 E-06$
$2.05 E-06$
$2.49 E-06$
$4.67 E-07$
$3.24 E-07$
$2.18 E+08$
$9.59 E+05$

$4.88 E+09$

ALPHA CONTAMINATION

$\begin{array}{cc}\text { ALPHA FLOW }(C) & \text { TOTAL FLOW (KG) } \\ & \\ 2.75 E+05 & 7.57 E+08 \\ 1.05 E+04 & 7.98 E+08 \\ 1.05 E+02 & 8.04 E+08 \\ 8.95 E+01 & 8.04 E+08 \\ 8.95 E+01 & 1.50 E+09 \\ 5.49 E-09 & 9.75 E-06 \\ 4.68 E-09 & 9.53 E-06 \\ 2.34 E-11 & 2.05 E-06 \\ 3.14 E-11 & 2.49 E-06 \\ 3.15 E-11 & 4.67 E-07 \\ 3.15 E-11 & 3.24 E-07 \\ 2.75 E+05 & 2.18 E+08 \\ 0.00 E+00 & 9.59 E+05 \\ 5.60 E+05 & 4.88 E+09\end{array}$

BETA CONTAMINATION

$\begin{array}{cc}\text { BETA FLOW (CI) } & \text { TOTAL FLOW (KG) } \\ 6.28 E+07 & 7.57 E+08 \\ 8.14 E+05 & 7.98 E+08 \\ 1.97 E+05 & 8.04 E+08 \\ 1.96 E+05 & 8.04 E+08 \\ 1.96 E+05 & 1.50 E+09 \\ 1.25 E-06 & 9.75 E-06 \\ 1.13 E-06 & 9.53 E-06 \\ 1.10 E-06 & 2.05 E-06 \\ 3.47 E-09 & 2.49 E-06 \\ 4.98 E-10 & 4.67 E-07 \\ 1.00 E-10 & 3.24 E-07 \\ 6.26 E+07 & 2.18 E+08 \\ 3.00 E+04 & 9.59 E+05 \\ 1.27 E+08 & 4.88 E+09\end{array}$

concentration (CI/KG)

6.13E-02

5.17E.02

5.13E.02

5.13E-02

3.41E.04

5.30E.02

4.87E-02

2.27E-01

$0.00 E+00$

$0.00 E+00$

$0.00 E+00$

2.39E-02

$4.25 E+01$

4.30E+01

CONCENTRATION (CI/KG)

3.63E-04

1.31E-05

1.30E-07

1.11E.07

5.96E.08

5.63E.04

4.91E.04

$1.14 E .05$

1.26E-05

6.73E-05

9.73E-05

$1.26 \mathrm{E}-03$

$0.00 E+00$

2.88E-03

CONCENTRATION (CI/KG)

8.29E-02

$1.02 \mathrm{E} \cdot 03$

2.45E-04

2.44E-04

1.31E-04

1.29E-01

1.18E-01

5.37E-01

1.39E-03

1.07E-03

3.10E-04

2.87E-01

3.13E-02

1.19E+00
TERTAPY STREAM OCCUP. HEALTHHAZ
FLOWS (KG)

$4.49 E+07$
$4.64 E+07$
$1.89 E+08$
$3.75 E+08$
$7.05 E+08$
$1.15 E-06$
$4.59 E-06$
$9.56 E-07$
$5.94 E-07$
$2.19 E-07$
$1.52 E-07$
$1.29 E+07$
$5.69 E+04$

$2.76 E+06$

$2.40 E+06$

$9.69 E+06$

$1.02 E+07$

2. $41 E+05$

6.11E.08

2.24E-07

2.17E-07

$0.00 E+00$

$0.00 E+00$

$0.00 E+00$

$3.08 E+05$

$2.42 E+06$

1.37E+09

3.71E+07
TERTIARY STREAM OCCUP. HEALTHHAZ FLOWS (KG) INDEX (CI-KG/M3)

$4.49 E+07$
$4.64 E+07$
$1.89 E+08$
$3.75 E+08$
$7.05 E+08$
$1.15 E-06$
$4.59 E-06$
$9.56 E-07$
$5.94 E-07$
$2.19 E-07$
$1.52 E-07$
$1.29 E+07$
$5.69 E+04$
$1.37 E+09$

$1.63 E+04$

$6.09 E+02$

2. $46 E+01$

$4.18 E+01$

4.20E+01

6.50E-10

2.25E-09

$1.09 \mathrm{E}-11$

$7.48 \mathrm{E}-12$

$1.48 E-11$

$1.48 E-11$

$1.63 E+04$

$0.00 E+00$

$3.33 E+04$
TERTLAPY STREAM OCCUP. HEALTHHAZ FLOWS (KG) INDEX (CI-KG/M3)

$\begin{array}{ll}4.49 E+07 & 3.73 E+06 \\ 4.64 E+07 & 4.73 E+04 \\ 1.89 E+08 & 4.63 E+04 \\ 3.75 E+08 & 9.16 E+04 \\ 7.05 E+08 & 9.21 E+04 \\ 1.15 E-06 & 1.48 E-07 \\ 4.59 E-06 & 5.42 E-07 \\ 9.56 E-07 & 5.13 E-07 \\ 5.94 E-07 & 8.25 E-10 \\ 2.19 E-07 & 2.34 E-10 \\ 1.52 E-07 & 4.72 E-11 \\ 1.29 E+07 & 3.71 E+06 \\ 5.69 E+04 & 1.78 E+03 \\ 1.37 E+09 & 7.71 E+06\end{array}$


Table 19. Summary of Occupational Health Hazard Index $\left(\mathrm{C} i-\mathrm{kg} / \mathrm{m}^{3}\right)$ Results.

$\begin{array}{ccc}\text { MODEL: } & \begin{array}{c}\text { CLEANG31 } \\ \text { CLEAN }\end{array} & \text { TRUG31 } \\ \text { TRUEX-C } & \text { SLUUG31 } \\ & \text { SLUDE WASHC }\end{array}$

TYPE OF

CONTAMINATION

$\begin{array}{llll}\text { GAMMA } & 4.60 E+07 & 4.46 E+07 & 3.71 E+07 \\ \text { ALPHA } & 4.04 E+05 & 1.78 E+05 & 3.33 E+04 \\ \text { BETA } & 1.48 E+08 & 6.72 E+07 & 7.71 E+06\end{array}$

CLEAN/"MODEL"

GAMMA

1.03

1.24

ALPHA

2.27

12.14

BETA

2.21

19.25 
Table 110. Waste Concentration Upper Limits.

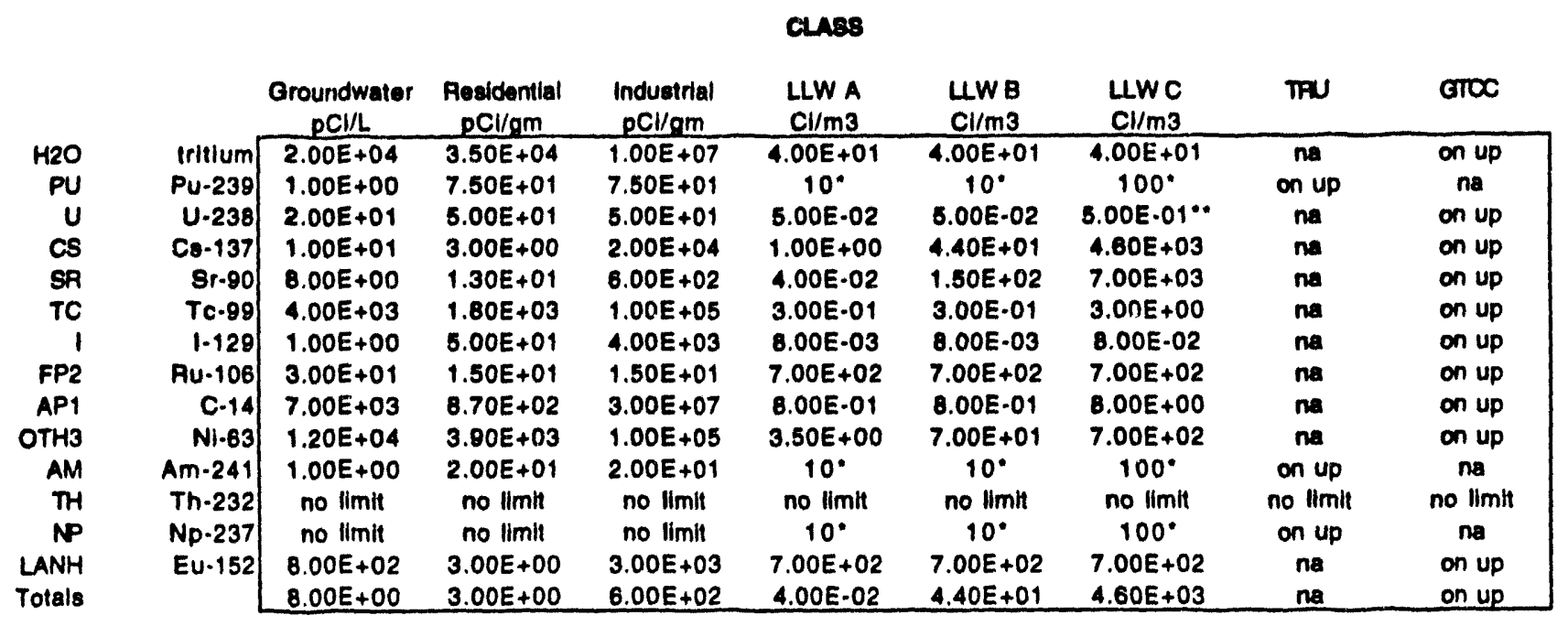

- $\mathrm{nCl} / \mathrm{am}$

-* From reference Kocher and Croff, 1988.

Table I11. Waste Concentration Upper Limits $\left(\mathrm{Ci} / \mathrm{m}^{3}\right)$.

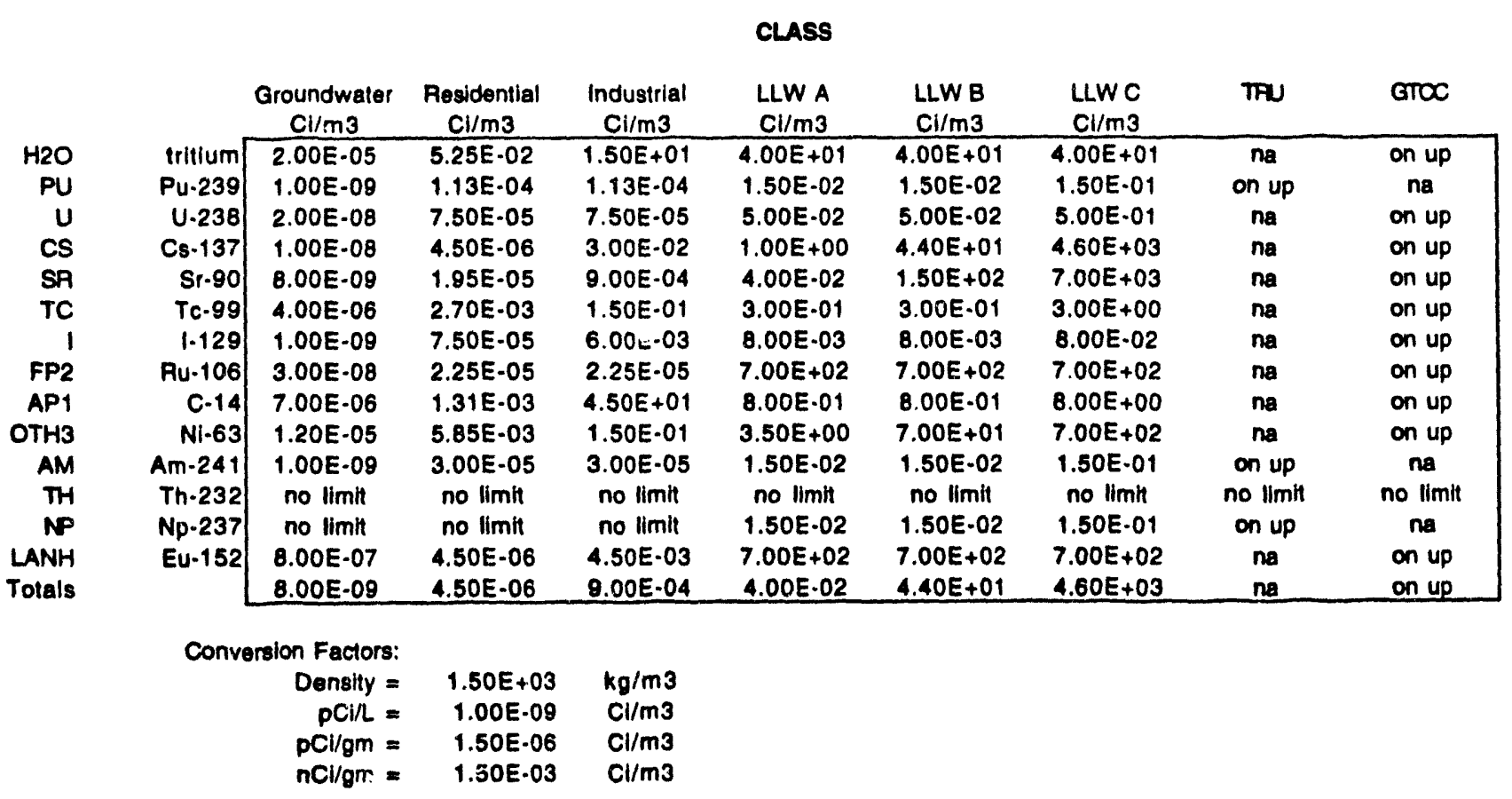



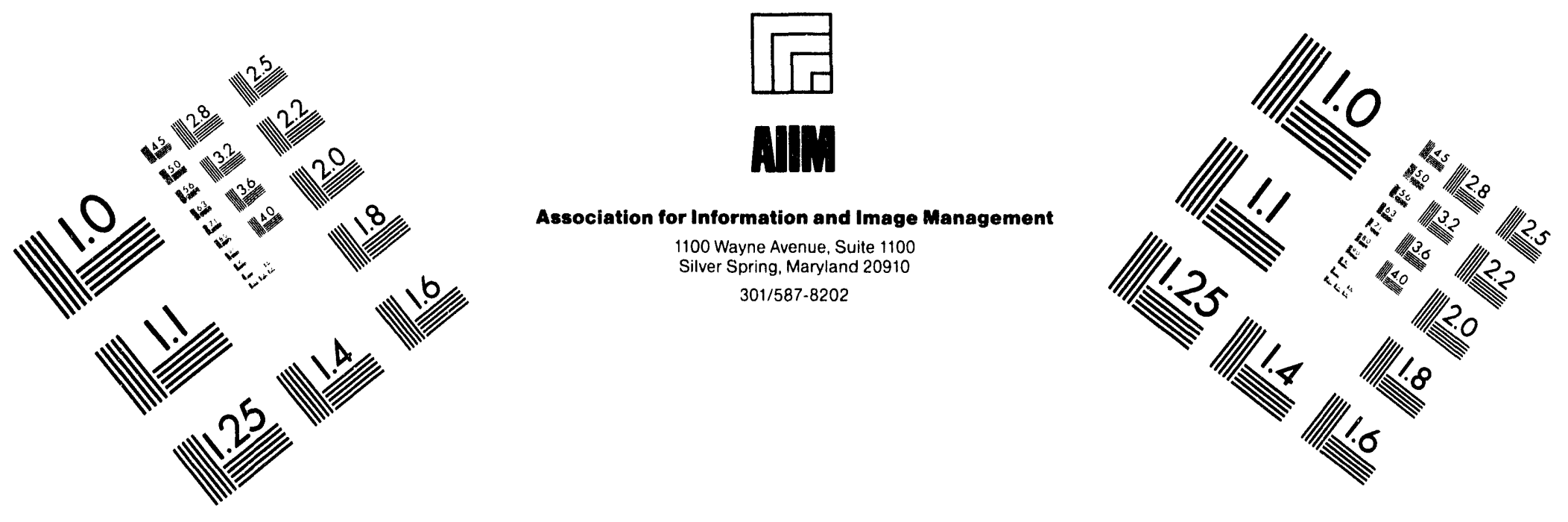

Centimeter

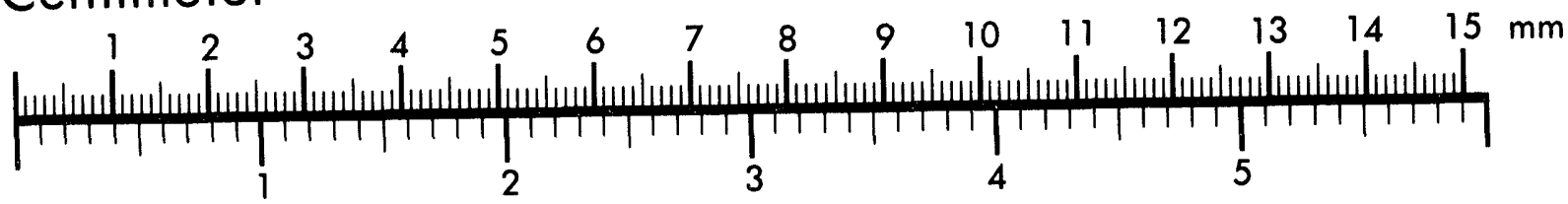
Inches
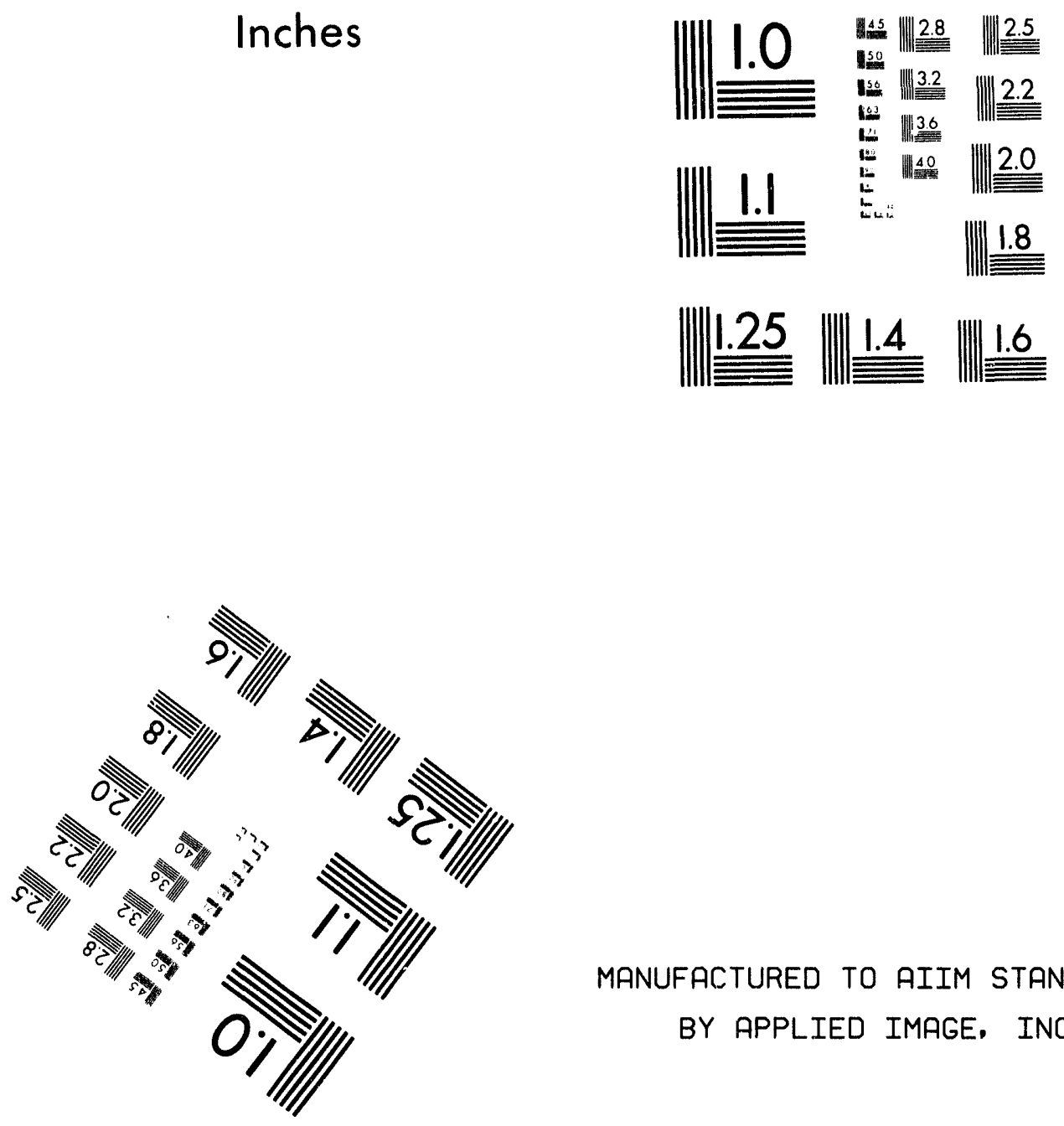

MANUFACTURED TO AIIM STANDARDS BY APPLIED IMAGE. INC.

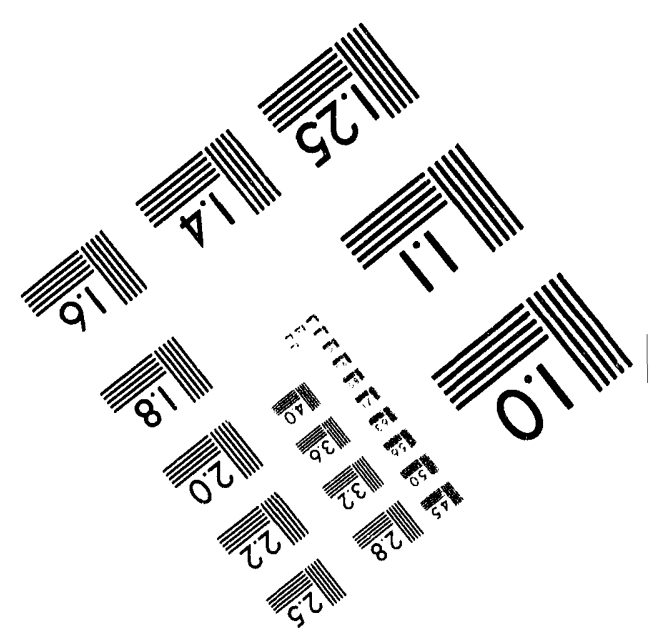



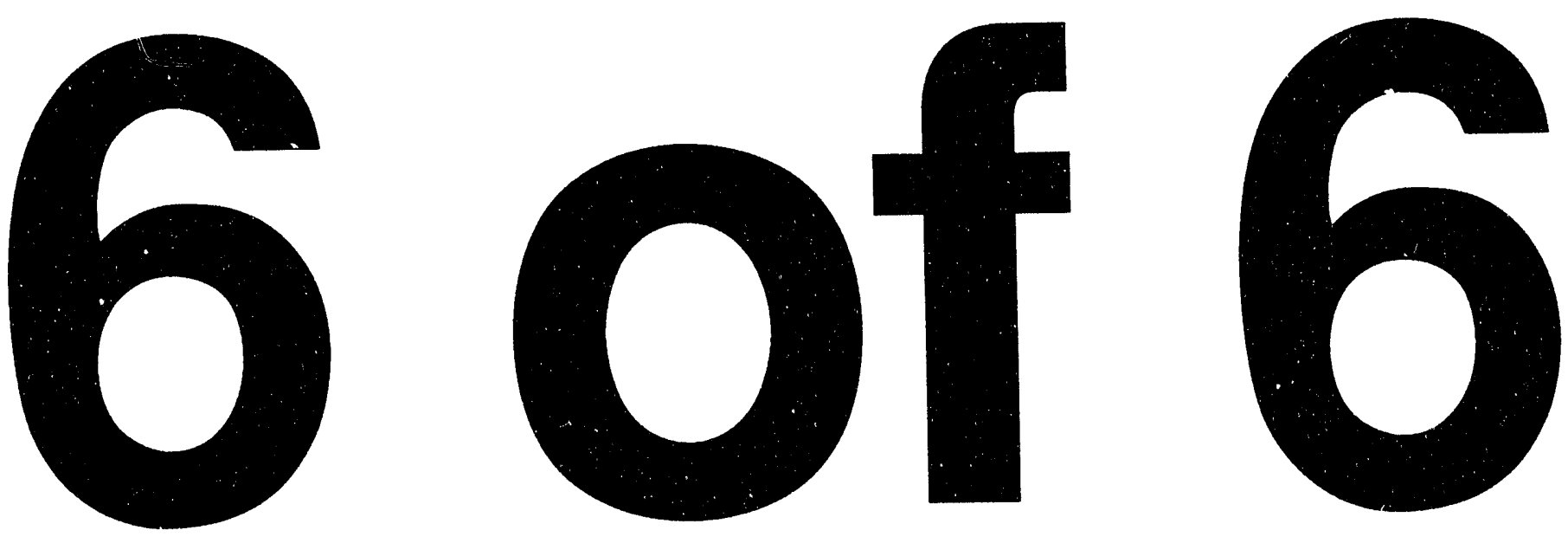


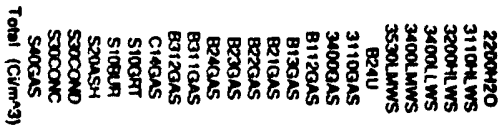

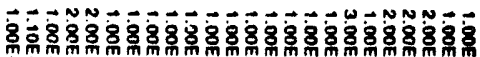

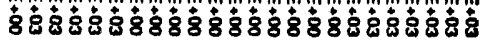

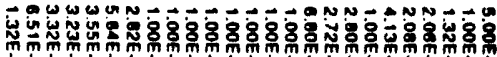

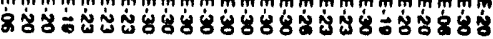

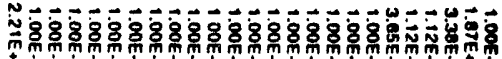

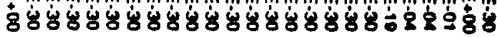

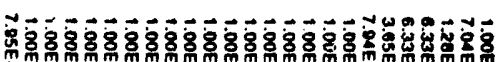

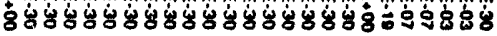

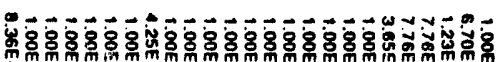

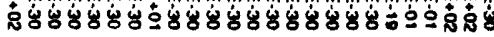

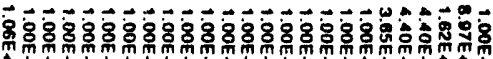

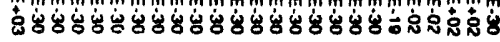

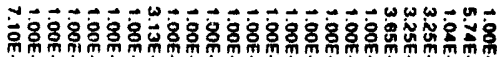

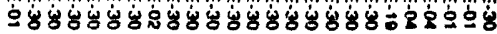

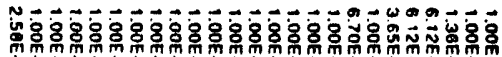

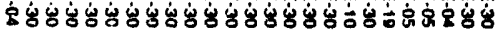

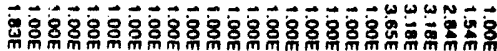

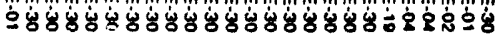

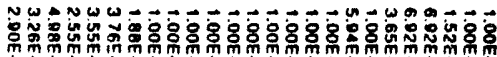

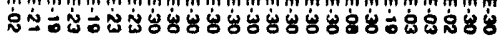

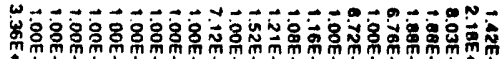

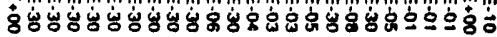

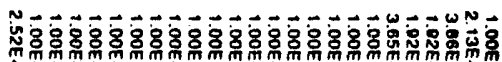

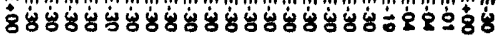

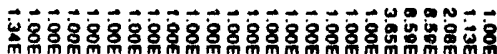

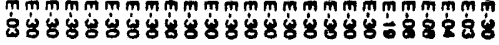

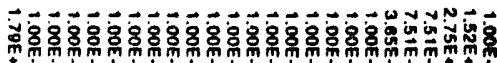

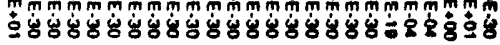

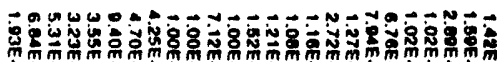

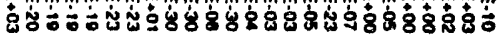

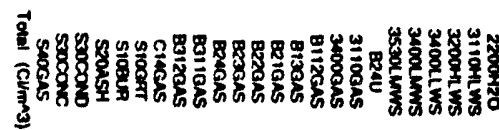

8.

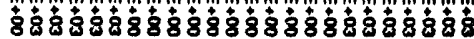

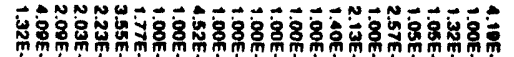

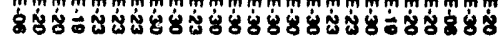

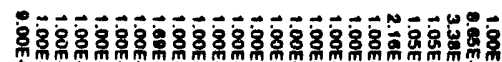

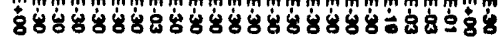

8

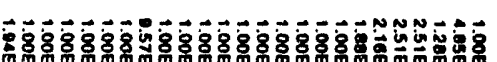

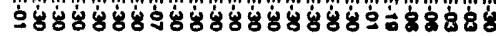

w

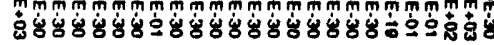

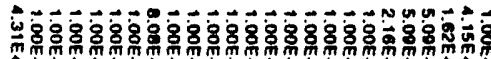

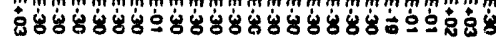

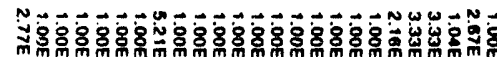

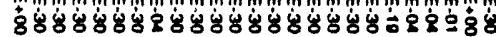

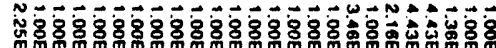

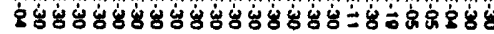

40

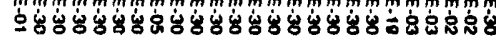

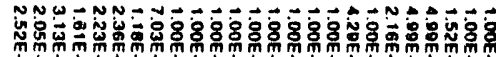

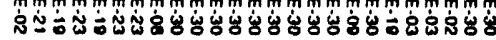

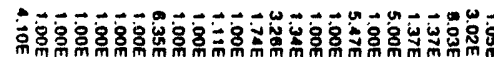

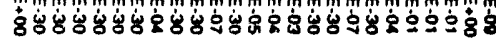

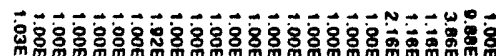

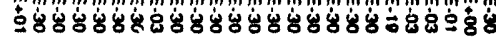

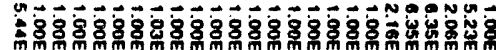

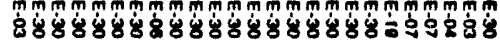

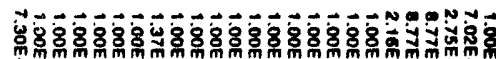

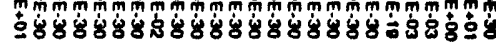

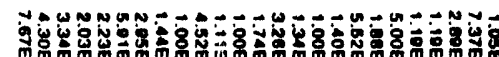

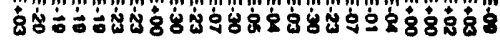

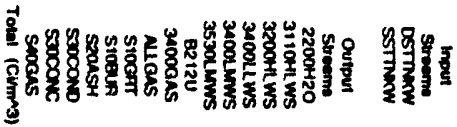

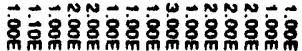
ริ

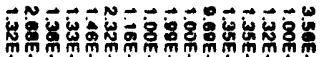

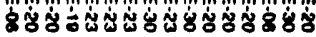

审察不

然

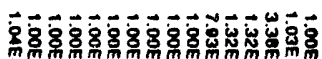

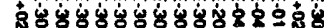

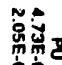

(1)

40

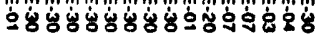

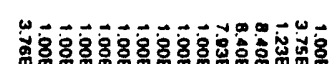

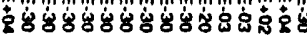

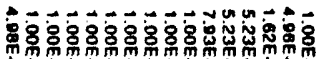

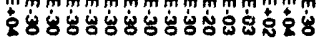

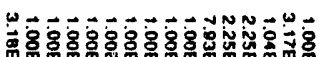

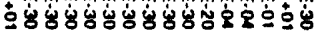

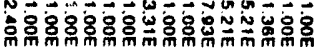

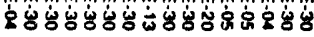

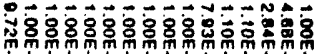

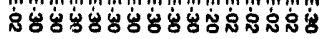

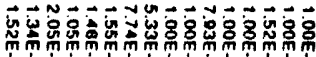

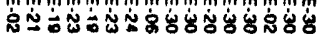

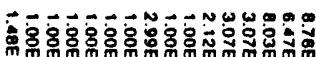

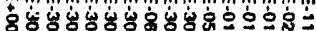

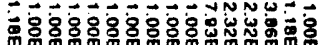

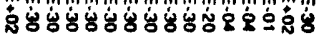

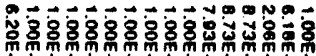

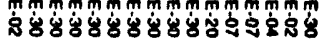

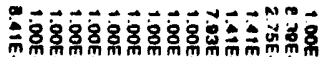

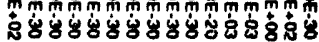

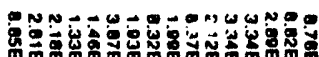

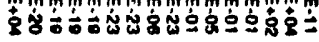

.

就

केष

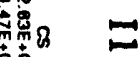

年

苯

sio

จ

․

然

ล

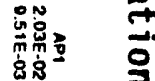

ลัก

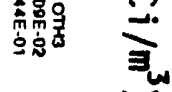

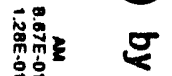

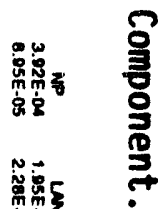

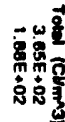


WHC-EP-0791

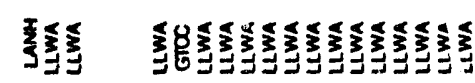

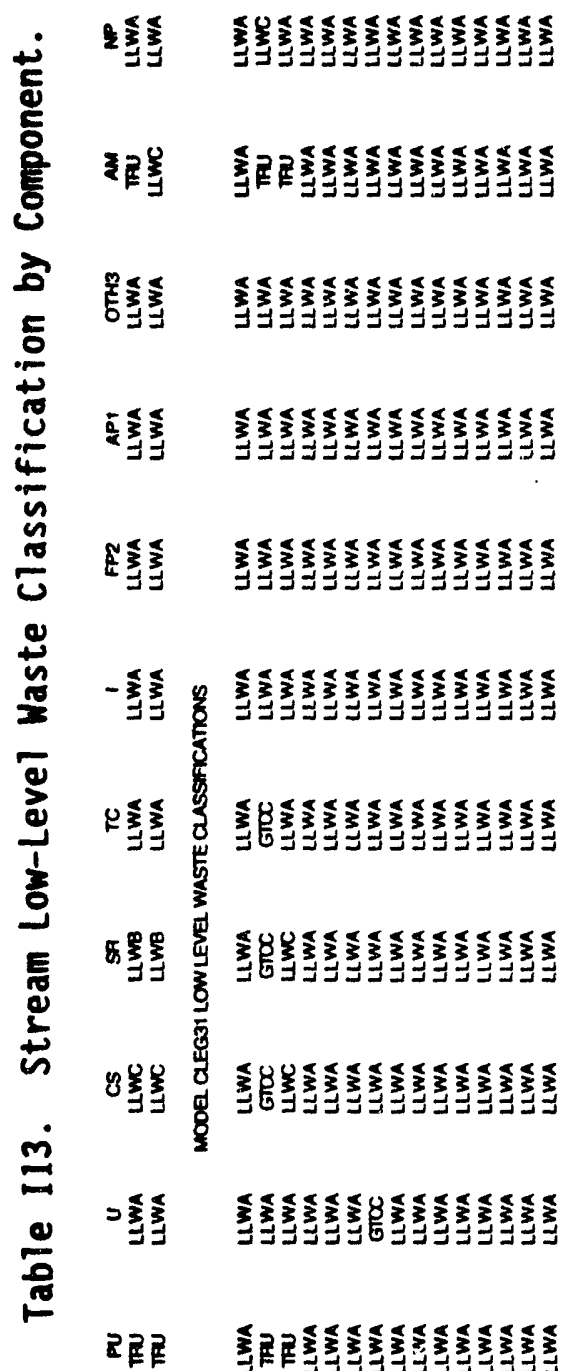

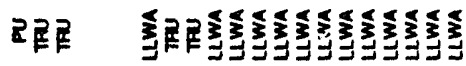

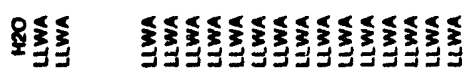

ราำ

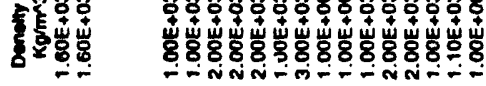

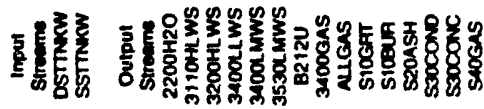

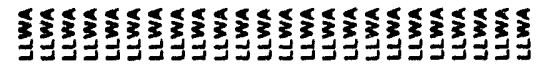

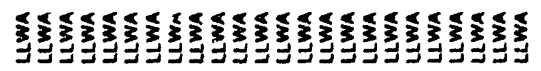

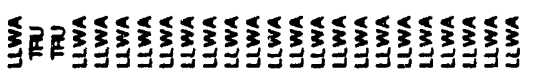

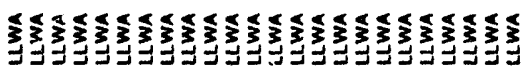

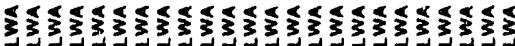

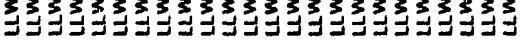

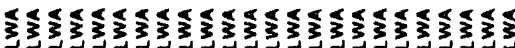

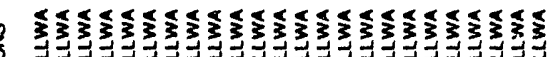

要

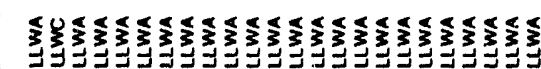

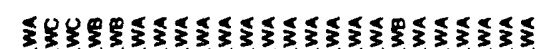

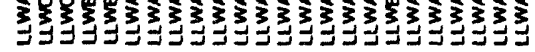

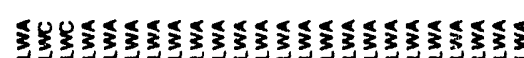

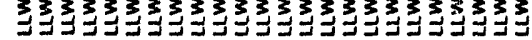

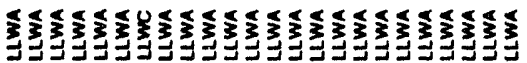

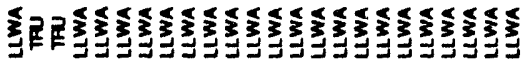

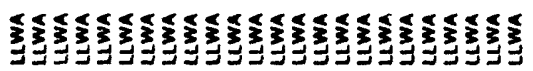

8989898988888888888989898 s8

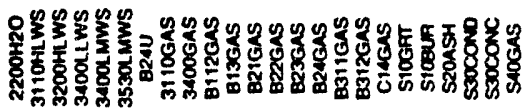

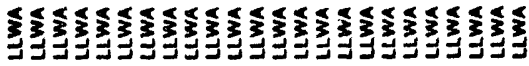

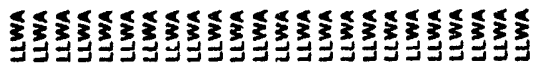

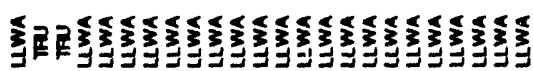

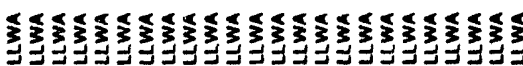

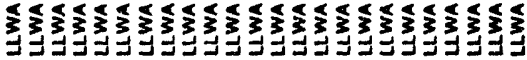

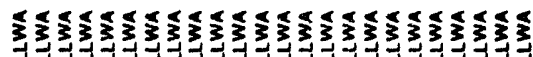

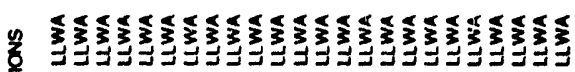

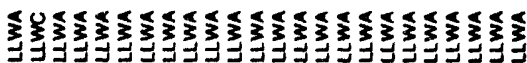
空

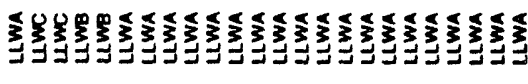

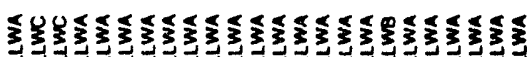

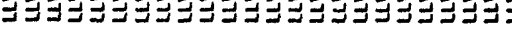

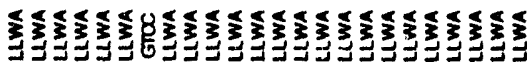

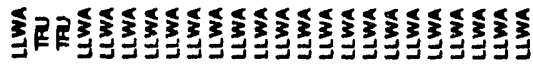

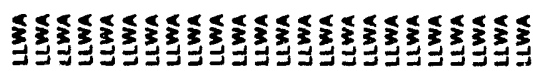

\$8马8\% 政8

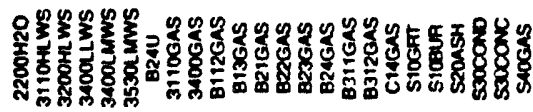


WHC-EP-0791

This page intentionally left blank. 
WHC-EP-0791

APPENDIX $\mathbf{J}$

TANK MASTE INVENTORIES 
WHC-EP-0791

\section{LIST OF TERMS}

DST

dcuble-shell tank

single-shell tank 
TANK WASTE INVENTORIES

As part of this study a review of the Hanford tank waste inventories (mass and radiological) was made based on several published documents to ensure that the most up-to-date data was used. Based on the data provided in the references listed below, tank waste inventories were determined as given in Table J1. Table J2 shows a summary of the actual radiological (i.e., curie) data provided in the following references. Actual $\mathrm{N}$ Reactor fission yield distribution data shown in Tabies $\mathrm{J} 2$ and $\mathrm{J3}$ were used to supplement the existing radiological information to provide a better estimate of existing element inventories. Note that in establishing radiological (curie) inventories several elements were ignored because of their short half-lives relative to expected processing times as noted on Table $\mathrm{Jl}$.

Table $\mathrm{J} 4$ shows the tank waste feedstream curie inventory data by radionuclide. Table J5 shows the tank waste feedstream mass inventory calculated using the curie data only.

Note that the representation of all Hanford tank waste in two feedstreams, single-shell tanks (SST) and double-she 11 tanks (DST), will not apply to a single tank or a single tank farm. Also, because large quantities of inert strontium, lanthanides, and perhaps iodine were added to parts of the SST tanks, the concentrations of radionuclides in these components vary widely between SST and DST.

\section{REFERENCES}

Designated "O'Toole" or "O'Toole-C $\mathrm{j}$ " in Table Jl:

O'Toole, S. M., 1993, "Tank Waste Inventory Revision," WHC Internal Memo 01140-93-005, from Integrated Planning Function, Westinghouse Hanford Company, Richland, Washington.

Designated "TRAC" in Table J1:

Garfield, J. S., 1993, "TWRS Systems Engineering Study Double Shell Tank Waste Inventories, Revision 1," WHC Internal Memo 25900-92-29, Westinghouse Hanford Company, Richland, Washington.

Golberg, C. E., 1993, "Technical Options Report Bases And Assumptions," WHC Internal Memo 22170-93-021, Waste Management Engineering, Westinghouse Hanford Company, Richland, Washington.

Designated "Swanson" in Table J1:

Swanson, J. L., 1993, Clean Option: An Alternative Strategy For Hanford Tank Waste Remediation, PNL-8388, V01. 2, Pacific Northwest Laboratory, Richlar 1, Washington. 
Designated "IDB93-96" or "IDB-96 Ci" in Table J1:

Integrated Data Base for 1989: Spent Fuel and Radioactive Waste Inventories, Projections, and Characteristics, November 1989, DOE/RW-0006, Rev. 5, Updated for 1993, Oak Ridge National Laboratory, Oak Ridge, Tennessee.

Designated "NEW ASPEN" in Table Jl:

Boomer, K. D., et al., 1993, Tank Waste Technical Options Report, WHC-EP-0616, Rev. 0, Westinghouse Hanford Company, Richland, Washington. 
WHC-EP-0791

Table J1. Study Tank Waste Inventory Summary.

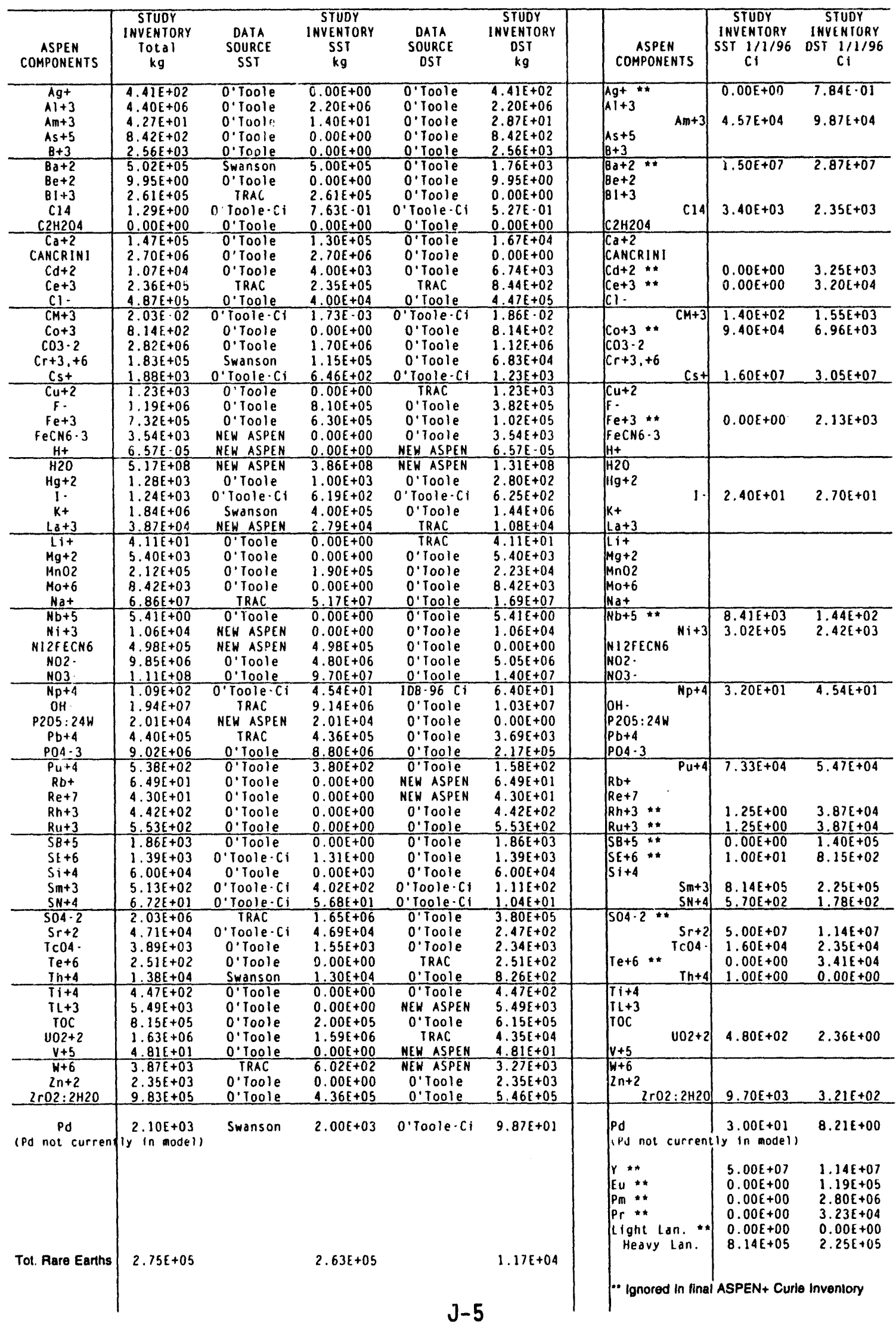




\section{Table J2. Study of Curie Inventory Data and Mass Inventory Estimates Based on the Curie Data.}

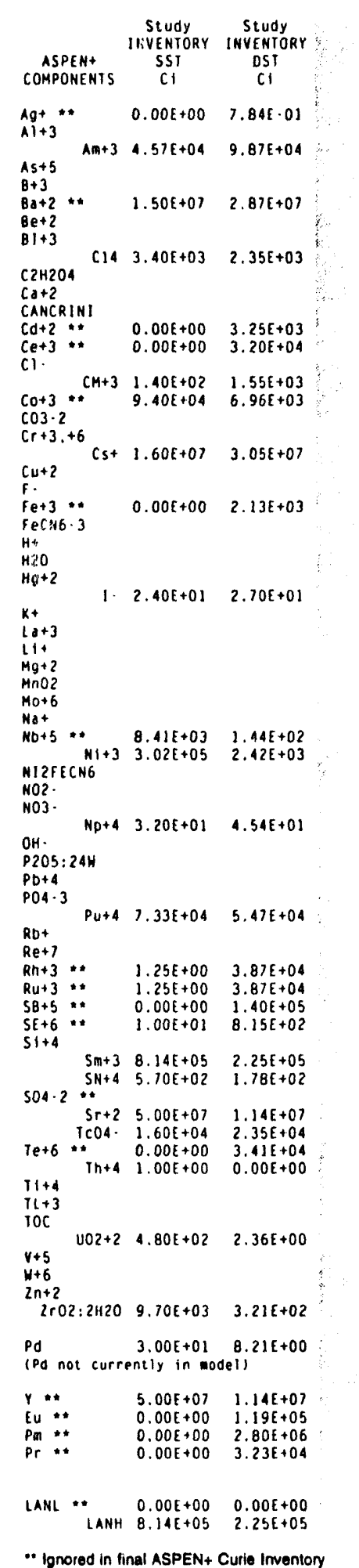

\begin{tabular}{|c|c|c|c|c|}
\hline $\begin{array}{l}\text { Study } \\
\text { INVENTORY } \\
\text { SST } \\
\text { Kg }\end{array}$ & $\begin{array}{c}\text { St UdY } \\
\text { INVENTORY } \\
\text { DST } \\
\mathrm{kg}\end{array}$ & $\begin{array}{c}\text { Study } \\
\text { INYENTORY } \\
\text { TOtal } \\
\mathrm{kg}\end{array}$ & $\begin{array}{c}\text { Estrmates } \\
\text { Fuel } 30 y \\
\text { SST } \\
\text { kg }\end{array}$ & $\begin{array}{l}\text { Sing N-Redcto } \\
\text { Decay Dota } \\
\text { OSI } \\
\mathrm{kg}\end{array}$ \\
\hline $0.00 E+00$ & $1.65 E-07$ & $1.65 \mathrm{E} \cdot 07$ & & \\
\hline $1.34 E+01$ & $2.88 \varepsilon+01$ & $4.22 \varepsilon+01$ & & \\
\hline $2.79 \mathrm{E} \cdot 05$ & $5.34 k .05$ & $8.12 t .05$ & $5.60 E+02$ & $1.07 \varepsilon+03$ \\
\hline $7.63 \mathrm{~F}-01$ & $5.27 \varepsilon \cdot 01$ & 1. $29 \varepsilon+00$ & & \\
\hline $\begin{array}{l}0.00 \varepsilon+00 \\
0.00 E+00\end{array}$ & $\begin{array}{l}1.45 E .02 \\
1.00 E-02\end{array}$ & $\begin{array}{l}1.45 E .02 \\
1.006 .02\end{array}$ & & \\
\hline $\begin{array}{l}1.736 .03 \\
3.31 E .02\end{array}$ & $\begin{array}{l}3.86 \mathrm{E} \cdot 02 \\
6.15 \mathrm{E} \cdot 03\end{array}$ & $\begin{array}{l}2.04 \mathrm{E} .02 \\
8.92 \mathrm{E}-02\end{array}$ & & \\
\hline $2.48 E+02$ & $4.03 E+02$ & $6.51 E+02$ & $6.46 E+02$ & $1.23 E+03$ \\
\hline $0.00 \varepsilon+00$ & $8.95 \mathrm{E}-04$ & $8.95 E \cdot 04$ & & \\
\hline
\end{tabular}

$\begin{array}{lllll}1.36 E+02 & 1.53 E+02 & 2.89 E+02 & 6.19 E+02 & 6.25 E+02\end{array}$

$\begin{array}{lll}3.52 E-02 & 6.02 E \cdot 04 & 3.58 E \cdot 02 \\ 5.31 E+00 & 1.56 E \cdot 01 & 5.47 E+00\end{array}$

$4.54 £+01 \quad 6.40 \varepsilon+01 \quad 1.09 \varepsilon+02$

$3.78 E+02 \quad 1.59 \varepsilon+02 \quad 5.37 E+02$

$\begin{array}{lll}3.51 E \cdot 13 & 1.09 E \cdot 08 & 1.09 E \cdot 08\end{array}$

$3.78 \mathrm{E} \cdot 07 \quad 1.17 \mathrm{E} \cdot 02 \quad 1.02 \mathrm{.3E} .02$

$\begin{array}{lll}0.00 E+00 & 1.35 E \cdot 01 & 1.35 E \cdot 01\end{array}$

$3.09 \varepsilon+01 \quad 8.55 \varepsilon+00 \quad 3.95 \varepsilon+01$

$2.01 E+01 \quad 3.66 E+00 \quad 2.37 E+01$

$3.66 \mathrm{E}+02$ \& $34 \mathrm{E}+01$

$9.435+02 \quad 1.385+03 \quad 4.495+02$

$\begin{array}{lll}9.43 E+02 & 1.38 \varepsilon+03 & 2.33 E+03\end{array}$

$\begin{array}{lll}9.00 \varepsilon+00 & 1.89 \varepsilon \cdot 03 & 1.89 \varepsilon-03 \\ 9.08 E+03 & 0.00 \varepsilon+00 & 9.08 E+03\end{array}$

$1.38 \varepsilon+06 \quad 2.85 E+03 \quad 1.38 E+06$

$3.78 E+03 \quad 3.25 E+02 \quad 3.91 E+03$

$3.09 E+01 \quad 1.19 E+01$

$5.83 E+01 \quad 1.60 E+01$

$9.20 E .02 \quad 2.10 E-02$

$0.00 E+00 \quad 3.31 E \cdot 01 \quad 3.13 E \cdot 01$
0.015 .01

$0.005+003.016+00 \quad 3.015+00$

$\begin{array}{lll}0.00 E+00 & 3.01 E+00 & 3.01 E+00 \\ 0.00 E+00 & 4.24 E .07 & 4.24 E .07\end{array}$

$\begin{array}{lllll}0.00 E+00 & 3.02 E+00 & 3.02 E+00 & 3.77 E+03 & 1.04 E+03 \\ 3.09 E+01 & 8.89 E+00 & 3.98 E+01 & 4.48 E+02 & 1.24 E+02\end{array}$

$1.31 E+00 \quad 1.07 E+02$

$4.02 E+02 \quad 1.11 E+02$ $5.48 E+01 \quad 1.04 E+01$

$4.69 E+04 \quad 2.12 E+02$
$4.22 E+03 \quad 1.17 E+03$

$3.61 E+02 \quad 9.87 E+01$

$17 E+03 \quad 1.04 E+03$
$.48 E+02 \quad 1.24 E+02$

\author{
Reactor Ylekc Distribution Date
}

N.Peactor Fuel Based on BNWL-8.350 App74 Origen Resuls

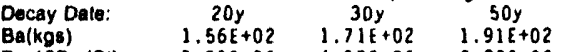

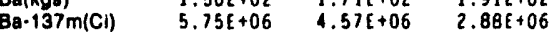
$\begin{array}{lllll}\mathrm{kg} / \mathrm{Cl} & 2.71 \mathrm{E} .05 & 3.7 \mathrm{JE} .05 & 6.64 \mathrm{E} .05\end{array}$ $\begin{array}{llll}8 \mathrm{k}+2 \text { (kg } 887) & 4.07 \mathrm{E}+02 & 5.60 \mathrm{E}+02 & 9.96 \mathrm{E}+02\end{array}$ Bat2 (kg DST) T.1.96 Wodrich Report Data: Radlogenic/NonRadiogenc lodine

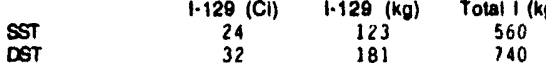
Padiogenic Ratio (1-128/1-127): SST $\quad 0.277$

PWA React. Fual Based on BMVONWI-655 Origen Fesutse

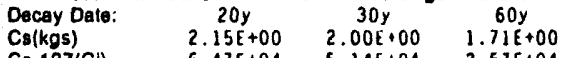
$\begin{array}{llll}\text { Cs. } & \\ \text { Cs.137(Ci) } & 6.47 E+04 & 5.14 E+04 & 2.57 E+04\end{array}$

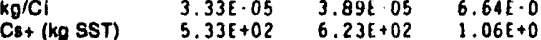
$\begin{array}{llll}\mathrm{Cs}+(\mathrm{kg} \text { SST) } & 5.33 \mathrm{E}+02 & 6.23 \mathrm{E}+02 & 1.06 \mathrm{E} \\ \mathrm{C} s+(\mathrm{kg} \text { DST) } & 1.01 \mathrm{E}+03 & 1.18 \mathrm{E}+03 & 2.02 \mathrm{E}+03\end{array}$ N-Aeactor Fuel Based on BNWL-B-350 Apr74 Oriben Results $\begin{array}{lccc}\text { Decay Dale: } & 20 y & 30 y & 50 y \\ \text { Cs(kgs) } & 2.12 \mathrm{E}+02 & 1.97 \mathrm{E}+02 & 1.76 \varepsilon+02\end{array}$

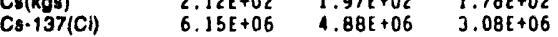
$\begin{array}{llll}\mathrm{kg} / \mathrm{Cl} & 3.44 \mathrm{E} .05 & \mathbf{1 . 0 3 6 . 0 5} & \mathbf{5 . 7 2 k . 0 5}\end{array}$

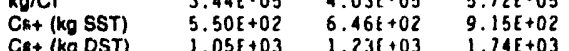
Cs+ (kgDST) 1.05k+03 $1.23 t+03 \quad 1.74 k+03$

N-Reactor Fuel Based on BNWL-B-350 Apr74 Origen Results Decay Date: $\quad 20 y \quad 30 y \quad 50 y$ Sn(kgs) $\quad 3.15 \mathrm{E}+00 \quad 3.15 \mathrm{FruN} \quad 3.15 \mathrm{E}+00$ $\begin{array}{llll}\mathrm{Sn}-128(C 1) & 3.16 \mathrm{E}+01 & 3.16 \mathrm{E}+01 & 3.16 \mathrm{t}+01\end{array}$ $\begin{array}{llll}\mathrm{kg} / \mathrm{Cl} & 9.97 \mathrm{E} \cdot 02 & 9.97 \mathrm{E} \cdot 02 & 9.97 \mathrm{E} \cdot 02 \\ \mathrm{Sn+4}(\mathrm{kg} \mathrm{SST}) & 5.68 \mathrm{E}+01 & 5.68 \mathrm{E}+01 & 5.6 \mathrm{EE}+0\end{array}$ $\begin{array}{llll}S n+4(k g D S T) & 1.04 E+01 & 1.04 E+01 & 1.04 E+01\end{array}$

N-Resclor Fuel Based on BNWL-B-350 Apr74 Origen Resulls Decay Dale: $\quad 20 y \quad 30 y \quad 50 y$ Se(kgs) $\quad 5.12 E+00 \quad 5.121+00 \quad 5.12 E+00$ Se.79(Ci) $\quad 3.91 E+01 \quad 3.91 E+01 \quad 3.91 \varepsilon+01$ $\mathrm{kg} / \mathrm{Cl} \quad 1.31 \mathrm{f} \cdot 01 \quad 1.31 \mathrm{E} \cdot 01 \quad 1.31 \mathrm{E} .01$ $\begin{array}{llll}\text { Se+6 (kg DST } & 1.31 E+00 & 1.31 E+00 & 1.31 E+00 \\ & 1.07 E+02 & 1.07 E+02 & 1.07 E+02\end{array}$

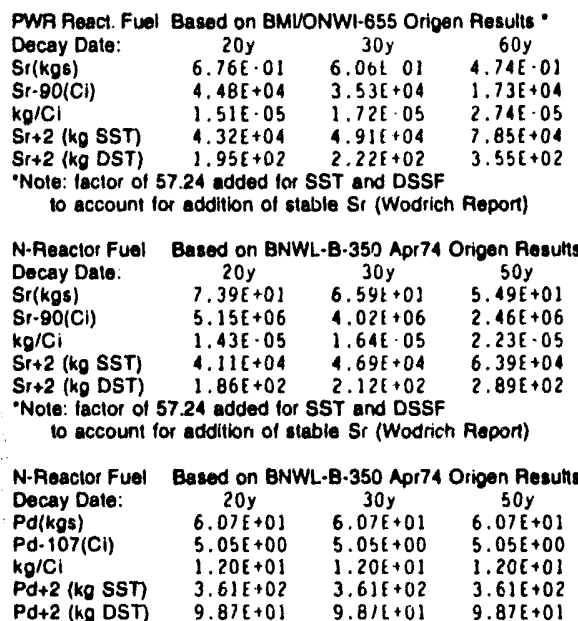




\section{Table J3. N Reactor Yield Distribution Data for Rare Earths Based on BNWL-B-350 Apr74 ORIGEN Results.}

Assume 1966 As Avg Year for Decay

$\begin{array}{cc}\text { Decayed 30y } & \text { kg-speciel } \\ \text { grams } & \text { kg-Sm151 } \\ 1.21 E+05 & 1.71 E+01 \\ 1.23 E+05 & 1.74 E+01 \\ 1.17 E+05 & 1.65 E+01 \\ 1.12 E+05 & 1.58 E+01 \\ 1.15 E+02 & 1.62 E-02 \\ 1.02 E+05 & 1.44 E+01 \\ 7.94 E-08 & 1.12 E-11 \\ 3.36 E-12 & 4.75 E-16 \\ 1.07 E+05 & 1.51 E+01 \\ 7.45 E+04 & 1.05 E+01 \\ 6.05 E+04 & 8.55 E+00 \\ 1.13 E+01 & 1.60 E-03 \\ 4.43 E+04 & 6.26 E+00 \\ 3.48 E+04 & 4.92 E+00 \\ 2.22 E+03 & 3.14 E-01 \\ 3.25 E+03 & 4.59 E-01 \\ 1.49 E+04 & 2.10 E+00 \\ 2.38 E+04 & 3.36 E+00 \\ 7.08 E+03 & 1.00 E+00 \\ 2.00 E+03 & 2.82 E-01 \\ 9.14 E+03 & 1.29 E+00 \\ 5.06 E-01 & 7.15 E-05 \\ 2.48 E+00 & 3.50 E-04 \\ 5.12 E+03 & 7.23 E-01 \\ 1.53 E-16 & 2.16 E-20 \\ 2.53 E+03 & 3.57 E-01 \\ 7.11 E+01 & 1.00 E-02 \\ 2.05 E+02 & 2.90 E-02 \\ 3.15 E-03 & 4.45 E-07 \\ 4.79 E+02 & 6.77 E-02 \\ 1.74 E+03 & 2.46 E-01 \\ 1.33 E+01 & 1.88 E-03\end{array}$

Isotope

Lal39

Ce140

Prl41

Ce142

Nd1 42

$\mathrm{Nd} 143$

Ce144

Nd144

Nd145

Nd146

Pm147

Sm14

Nd148

$\operatorname{Sm} 148$

$\operatorname{Sm} 14$

C Sm150

EuI 5

Sm 152

Eu152

Gd152

Eu153

Sm154

Eu154

Gd154

Gd157

1. $33 E+01$

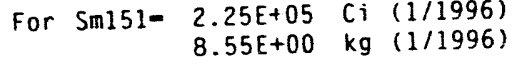

For $\begin{array}{rlll}\operatorname{Sm} 151- & 2.25 \mathrm{E}+05 & \mathrm{Ci} & (1 / 1996) \\ 8.55 \mathrm{E}+00 & \mathrm{~kg} & (1 / 1996)\end{array}$

AW
139
140
141
142
142
143
144
144
144
145
146
147
147
148
148
149
150
150
151
151
152
152
152
153
153
154
154
154
155
155
156
157

\section{Element}

$1.46 \mathrm{E}+02$

$1.49 \mathrm{E}+02$

1. $41 \mathrm{E}+02$

1. $35 E+02$

1. $39 \mathrm{E}-01$

1. $23 \mathrm{E}+02$

$9.59 \mathrm{E}-11$

$4.00 E-15$

1. $29 \mathrm{E}+02$

$9.00 E+01$

$7.31 E+01$

1. $37 \mathrm{E}-02$

5. $35 E+01$

4. $20 E+01$

2. $68 \mathrm{E}+00$

1. $80 E+01$

2. $88 \mathrm{E}+01$

$8.55 \mathrm{E}+00$

$2.42 E+00$

1. $10 E+01$

1. $6.11 E-04$

3. $00 \mathrm{E}-\mathrm{O} 3$

3.

1. $85 \mathrm{E}-19$

$1.85 \mathrm{E}-19$

$3.06 E+00$

8.5 -02

$2.48 E-01$

$3.81 \mathrm{E}-06$
$5.79 \mathrm{E}-01$

$5.79 E-01$
$2.10 E+00$

$1.61 \mathrm{E}-02$

3. $76 \mathrm{E}-17$

$\begin{array}{ll}3.76 \mathrm{E}-17 & \mathrm{La} \\ 3.80 \mathrm{E}-17 & \mathrm{Ce}\end{array}$

$3.59 \mathrm{E}-17 \mathrm{Pr}$

$3.41 \mathrm{E}-17 \mathrm{Nd}$

$3.50 \mathrm{E}-20$
$3.08 \mathrm{E}-17$

$3.06 E-04$ EU

$3.07 E-04$ GO

$1.53 \mathrm{E}-07$ TD

$2.22 \mathrm{E}-17$ Dy

$1.79 \mathrm{E}-17 \mathrm{HO}$

$1.27 \mathrm{E}+04 \mathrm{Er}$

$1.23 \mathrm{E}-03 \mathrm{Tm}$

$1.02 E-17$ YD

$4.29 \mathrm{E}-18$

$6.86 \mathrm{E}-18$

.25E+05

$5.73 \mathrm{E}-19$

$6.41 \mathrm{E}-11 \mathrm{Sm} / \mathrm{Sm} 151=$

$1.45 \mathrm{E}-18$

$6.53 E-13$

$7.10 E-19$
$2.32 E+04$

$2.32 \mathrm{E}+04$
$5.75 \mathrm{E}-20$

$5.75 \mathrm{E}-20$
$1.87 \mathrm{E}+00$

$1.87 E+00$
$1.34 E-19$

$4.82 \mathrm{E}-19$

$4.82 \mathrm{E}-19$
$3.66 \mathrm{E}-21$

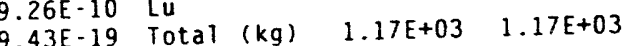

$\mathrm{kgS}$

Ci Ci fractions

$2.84 E+02$ (light) $3.06 E-04$

$3.07 E-04$

$1.53 \mathrm{E}-07$

1. $37 \mathrm{E}-02$

$1.12 \mathrm{E}+02$

$1.24 E+02 \quad 0.1060$

$2.25 \mathrm{E}+05$

$2.49 E+05 \quad 0.9515$

$3.74 \mathrm{E}+\mathrm{C} n$

$3.39 \mathrm{E}-02$

$7.12 \mathrm{E} \cdot 04$

$7.12 E \cdot 04$

$2.33 E+04$
$6.48 E-11$

$2.60 \mathrm{E}-20$

$7.51 \mathrm{E}-21$

$3.15 \mathrm{E}-04$

$5.61 E-23$

Rare Earths Based On Smi51 Data

2.60E-18 LANL(light)/Smij1=

$2.61 E+05 \quad 2.61 E+05$ 
Table J4. Tank Waste Feedstream Curie Inventory.

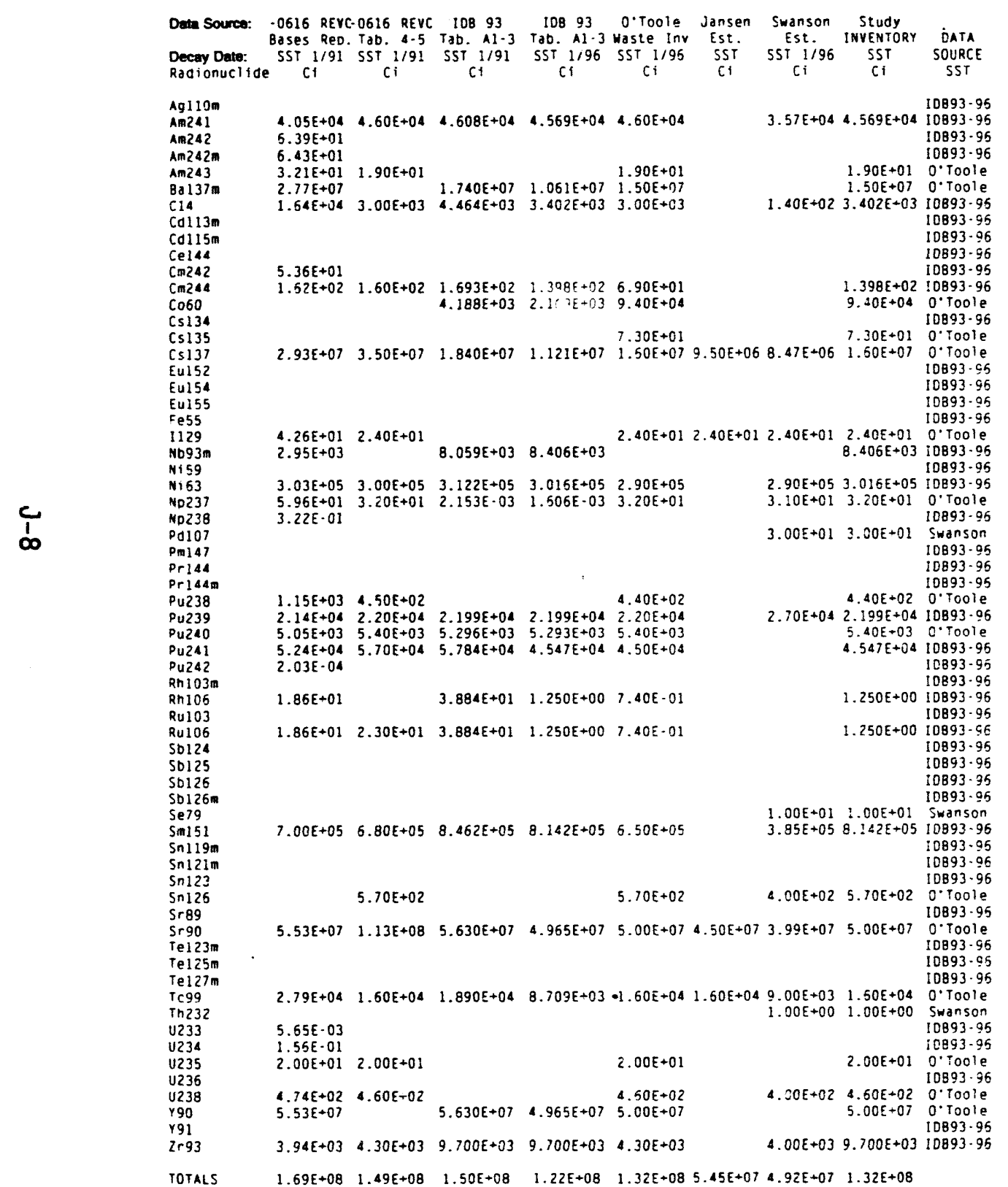

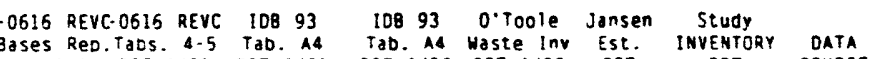

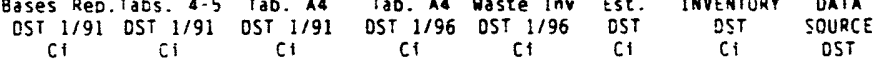

\begin{tabular}{|c|c|c|c|c|c|c|}
\hline \multirow{5}{*}{$9.86 E+04$} & \multirow{5}{*}{$9.90 E+04$} & & & & \\
\hline & & $\begin{array}{l}5.243 \mathrm{E}+04 \\
4.350 \mathrm{E}+0 \mathrm{I}\end{array}$ & $\begin{array}{l}5.856 E+04 \\
4.252 E+01\end{array}$ & $9.86 E+04$ & $\begin{array}{l}9.86 E+04 \\
4.252 E+01\end{array}$ & $\begin{array}{l}0 . \text { Toole } \\
\text { iDB93-95 }\end{array}$ \\
\hline & & & & & & \\
\hline & & $7.165 E+00$ & $7.162 E+00$ & $0.00 E+00$ & $7.16 ? E+00$ & 10893.95 \\
\hline & & $1.550 \mathrm{E}+07$ & $1.871 E+07$ & $2.87 E+07$ & $2.87 E+07$ & O. Toole \\
\hline $2.35 E+03$ & $2.30 E+03$ & $\begin{array}{l}5.693 E+02 \\
4.115 E+03\end{array}$ & $\begin{array}{l}2.629 E+03 \\
3.245+03\end{array}$ & $2.35 E+03$ & $\begin{array}{l}2.35 E+03 \\
3.246 E+03\end{array}$ & $\begin{array}{l}\text { 0. Toole } \\
13893-96\end{array}$ \\
\hline & & $1.73<\mathrm{E}-05$ & $0.000 \varepsilon+00$ & & & $10893-96$ \\
\hline & & $2.746 E+06$ & $3.198 E+04$ & $2.11 \varepsilon+03$ & $3.198 E+04$ & $10893-95$ \\
\hline & & & $3.567 E+01$ & & $3.567 E+01$ & :0893-96 \\
\hline & & $1.397 \varepsilon+03$ & $1.145 E+03$ & $1.51 E+03$ & $1.51 E+03$ & D. Tocie \\
\hline & & $1.343 E+04$ & $6.958 \mathrm{~F}+03$ & $4.65 E+03$ & $6.958 \mathrm{E}+03$ & $10893-95$ \\
\hline & & & $5.121 E+04$ & & $5.12: E+04$ & 10893.95 \\
\hline \multirow{5}{*}{$5.63 E+07$} & & $5.915 E+01$ & $5.915 E+01$ & $0.00 E+00$, & $5.5 i 5 z+0 i$ & $10893-95$ \\
\hline & $5.50 E+07$ & $\begin{array}{l}1.939 \mathrm{E}+07 \\
5.994 \mathrm{E}+02\end{array}$ & $\begin{array}{l}1.97 E+01 \\
4.546 E+02\end{array}$ & $3.04 t+0 / 1.80 t+$ & $4.645 E+02$ & $\begin{array}{l}\text { :0893-95 } \\
: 080\end{array}$ \\
\hline & & $7.934 E+04$ & $5.302 E+04$ & $5.27 E+04$ & $5.302 E+04$ & $10803-95$ \\
\hline & & 1. $300 \mathrm{E}+05$ & 5. $509 \varepsilon+04$ & $4.32 E+0.4$ & $6.509 E+04$ & $10843 \div 5$ \\
\hline & & $8.089 E+03$ & $2.133 E+03$ & & $2.133 E+03$ & I0B93-95 \\
\hline \multirow[t]{8}{*}{$2.70 E+01$} & $2.70 E+01$ & $\begin{array}{l}2.652 E-01 \\
9.783 E+01\end{array}$ & $\begin{array}{l}2.652 E-01 \\
1.438 E+02\end{array}$ & $2.7 G E+012.50 E+0 \mathrm{i}$ & $\begin{array}{l}2.70 E=1 \\
1.43 B E+02\end{array}$ & $\begin{array}{l}0.10010 \\
\text { I0893-96 }\end{array}$ \\
\hline & & $9.057 \mathrm{E}+30$ & $9.057 E+00$ & & $9.057 E+00$ & $10893-95$ \\
\hline & & $1.064 E+03$ & $1.025 E+03$ & $2.41 \mathrm{E}$ & $\begin{array}{l}2.41 E+03 \\
1\end{array}$ & $\begin{array}{l}0 . \text { Toole } \\
\text { :0893.96 }\end{array}$ \\
\hline & & $2.188 \varepsilon-01$ & $\begin{array}{l}4.5: E+01 \\
2.12=E \cdot 01\end{array}$ & $2.98 t+01$ & $2.338 \varepsilon-01$ & $10893-95$ \\
\hline & & $8.2 \mathrm{i} 0 \mathrm{E}+00$ & $9.210 E+00$ & & $8.213 E+00$ & $10893 \cdot 96$ \\
\hline & & $1.048 E+07$ & $2.797 E+06$ & $1.13 E+06$ & 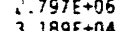 & io893-96 \\
\hline & & $\begin{array}{l}2.739 E+06 \\
288 E+04\end{array}$ & $3.828 E+02$ & & $\begin{array}{l}3.896 \\
3.328 E\end{array}$ & 10893.96 \\
\hline & & & $\begin{array}{l}4.249 E \\
4.262\end{array}$ & 4.178 & $4.44 y E+02$ & 10893.05 \\
\hline \multirow[t]{15}{*}{$2.32 E+04$} & $2.30 E+04$ & $3.277 E+03$ & $4.412 E+03$ & $9.12 E+03$ & $9.12 \mathrm{E}+03$ & 0 Toole \\
\hline & & $\begin{array}{l}8.850 E+02 \\
3.589 E+04\end{array}$ & $\begin{array}{l}1.173 E+03 \\
4.259 E+04\end{array}$ & $\begin{array}{l}2.58 E+03 \\
5.02 E+03\end{array}$ & $\begin{array}{l}2.58 E+03 \\
4.259 E+04\end{array}$ & $\begin{array}{l}0 . \text { Tole } \\
10893.96\end{array}$ \\
\hline & & $8.677 \mathrm{E}-02$ & $1.058 \mathrm{E}-01$ & & $1.058 E-01$ & $10893-95$ \\
\hline & & $5.831 E-04$ & $0.000 \mathrm{E}+$ & $1.89 \mathrm{E}+04$ & $3.965 \varepsilon+04$ & $\begin{array}{l}10893.95 \\
10893.95\end{array}$ \\
\hline & & $6.470 \varepsilon-04$ & $3.005 e^{-404}$ & .0.0 & & 10893.95 \\
\hline & & $1.203 E+06$ & $3.865 E+04$ & $1.89 \varepsilon+04$ & $3.865 E+04$ & ICB93-95 \\
\hline & & $2.012 E-04$ & & & & \\
\hline & & $\begin{array}{l}4.881 E+ \\
1.458 E+\end{array}$ & $\begin{array}{l}1.3976 \\
1.458 \mathrm{E}\end{array}$ & $6.94 \mathrm{E}+04$ & & $\begin{array}{l}10893-9.9 \\
1089.9\end{array}$ \\
\hline & & & 1.1516 & & & \\
\hline & & $6.582 \mathrm{E}+01$ & $6.582 E+01$ & $8.15 \mathrm{E}$ & B. $15 E+02$ & 0. Toole \\
\hline & & $\begin{array}{l}2.340 \mathrm{E}+05 \\
2.306 \mathrm{E}+03\end{array}$ & $\begin{array}{l}2.252 E+05 \\
1.316 E+C !\end{array}$ & $1.45 \mathrm{E}+05$ & $\begin{array}{l}2.252 E+05 \\
1.315 E+01\end{array}$ & $\begin{array}{l}10893-95 \\
10893-95\end{array}$ \\
\hline & & $\begin{array}{l}2.5706+03 \\
6.572 E+01\end{array}$ & $\begin{array}{l}1.13 E+C ! \\
6.131 E+01\end{array}$ & & 5.231 & 1089 \\
\hline & & $8.380 \mathrm{E}$ & $4.922 \mathrm{E}$ & & 4.922 & $10893-95$ \\
\hline & & & 1.0398 & $0.00 \varepsilon+00$ & & \\
\hline & & & & & 2.658 & \\
\hline \multirow{3}{*}{$2.58 E+07$} & $2.60 E+07$ & $1.144 E+07$ & $1.037 E+07$ & $1.14 E+07 \quad 4.70 E+07$ & $71.14 E+07$ & $\begin{array}{l}0 . \text { Toole } \\
0\end{array}$ \\
\hline & & & $\begin{array}{l}1.051 E-08 \\
3.40 F+04\end{array}$ & & & \\
\hline & & & $\begin{array}{l}3.409 \mathrm{E} \\
5.2 \mathrm{E}\end{array}$ & & & \\
\hline \multirow[t]{8}{*}{$2.40 E+04$} & $2.50 E+04$ & $1.3325+04$ & $2.350 E+04$ & $2.40 E+042.20 E+$ & $2.350 E+04$ & \\
\hline & & & & & & \\
\hline & & $1.232 E+00$ & $1.237 E+00$ & & $1.237 E+00$ & 10893.96 \\
\hline & & $5.177 E-02$ & $5.177 \mathrm{E}-$ & $7.04 E-02$ & $7.04 \mathrm{E}-02$ & 0.10 \\
\hline & & $1.081 E-01$ & $1.081 \mathrm{E}$ & & $181 E-01$ & $10893-95$ \\
\hline & & $9.456 E-01$ & 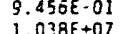 & & & $10893-96$ \\
\hline & & $3.828 \mathrm{E}+60$ & $\begin{array}{r}1.038 E+07 \\
1.542 E-09\end{array}$ & & $\begin{array}{l}1.114 \\
1.542 E\end{array}$ & \\
\hline & & $3.205 E+02$ & $3.206 \mathrm{E}+02$ & $0.00 E+00$ & $3.205 E+02$ & 10393.96 \\
\hline
\end{tabular}

$9.22 E+07 \quad 9.21 E-0: 7.46-Z+0 ; \quad 6.291 E+0 ; \quad 8.35 E+07 \quad 2.27 E+08 \quad 8.55 E+07$ 


\section{$\bullet$}

Table J5. Tank Waste Feedstream Mass Inventory Based Only on Curie Data.

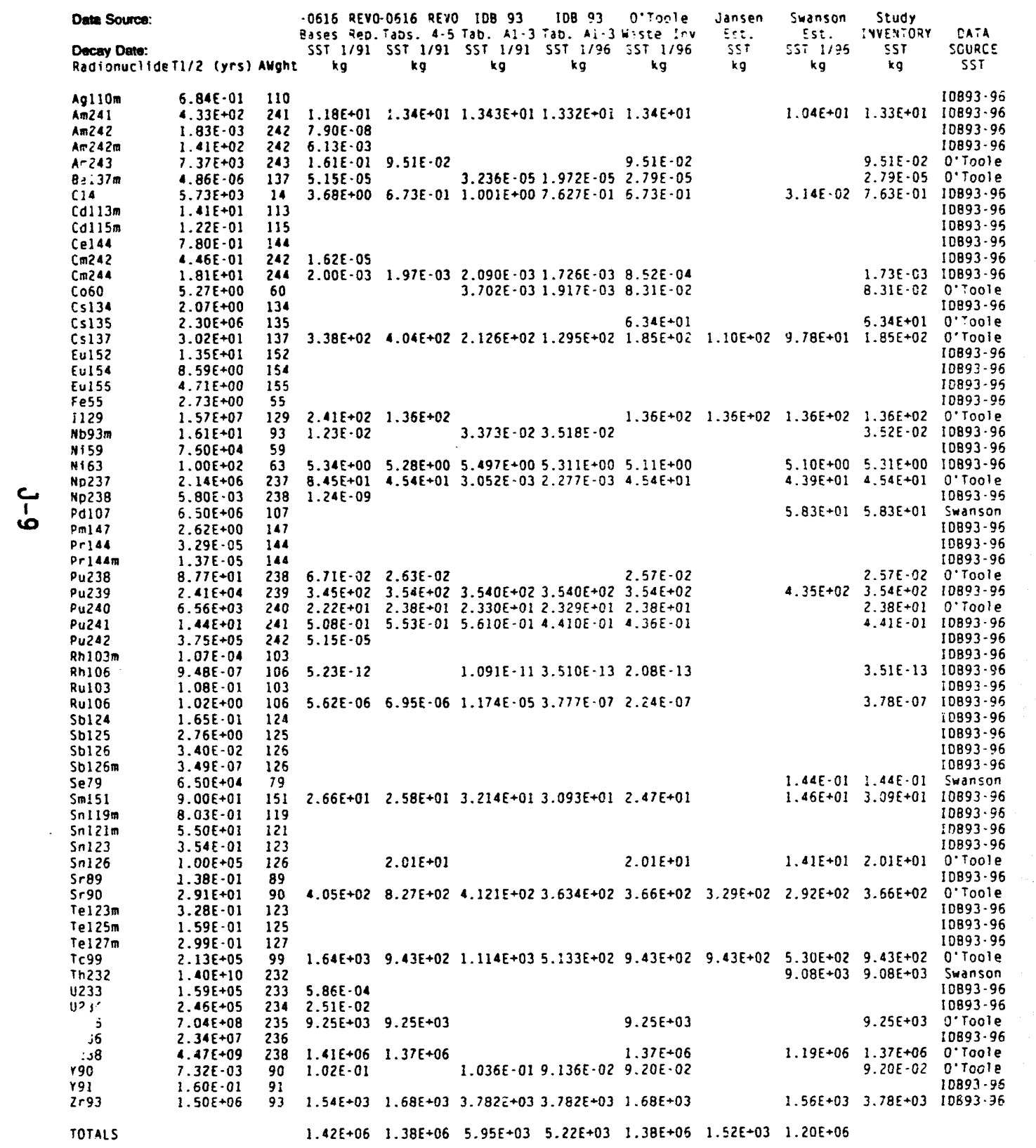

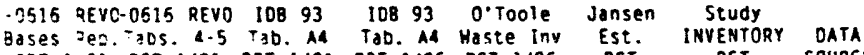
OST 1,91 DST $: / 91$ OST $1 / 91$ DST $1 / 96$ OST $1 / 96$ OST OST SOURC

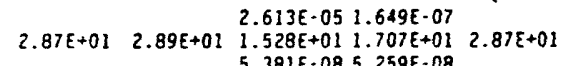
5.381E-08 5.259E-08 $4.172 \varepsilon-034.078 \varepsilon-03$
$3.5865-023.5455-02$ $\begin{array}{rl}5.26 E .08 & 10893-96 \\ 4.09 \varepsilon .03 & 10893.96\end{array}$

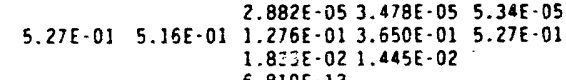
a. $617 E-01 \quad 1.004 E-02 \quad 6.62 E-04$ $1.3122-051.075 E-05$

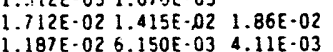
1.187E-02

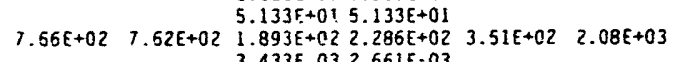
$3.433 E 032.661 E-03$

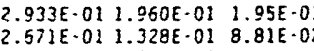

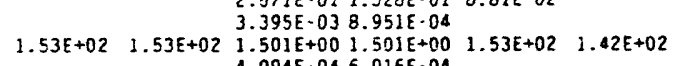
1. $135 \mathrm{E}-01 \mathrm{0l} 135 \mathrm{E}-04$ $1.873 E-021.805 E-02$ 4.24E-02 $6.39 c ;+01 \quad 6.404 E+01 \quad 4.22 E+01$ $8.441 E-103.249 \varepsilon-10$
$1.596 E+011.595 E+01$ $1.130 \mathrm{E}+013.015 \mathrm{E}+00 \quad 1.22 \mathrm{E}+00$ 3.624E-05 4.220E-07

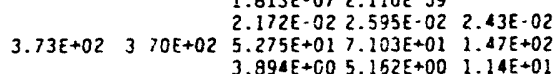

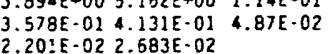

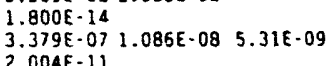
$\begin{array}{lll}2.004 E-11 & 1635-02 & 5.71 E-03\end{array}$

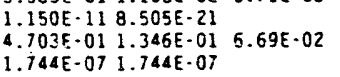
$\begin{array}{lll}9.446 E-01 & 9.445 E-01 & 1.17 E+01 \\ 8.888 E+00 & 8.554 E+00 & 5.51 E+00\end{array}$ $6.157 E-043.514 E-05$
$1.222 E-03$
$3.065 E$ $1.222 E-03$
$1.081 .140 E-05$
$3.989 E-10$
$1.051 E$ $3.659 \mathrm{E}+003.659 \mathrm{E}+00$

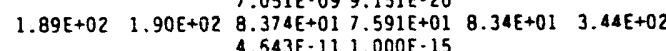
$4.543 \mathrm{E}-111.000 \mathrm{E}-15$

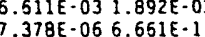
$5.34 \mathrm{E} \cdot 05$ O. Toole $5.27 \mathrm{E} \cdot 01$
$1.45 \mathrm{E} \cdot 02 \quad \mathrm{O}$. Toole
10893.96 . $00 \mathrm{E}-02 \quad 10893-96$ $1.08 \mathrm{E}-05$ 10893-96 6.85E.03 10893.06 $.96 E-02 \quad 10893-95$
$13 E+01$
10893.05 $\begin{array}{ll}3.51 E+02 & 0.1001 e \\ 2.66 E-03 & 10893-96\end{array}$ \begin{tabular}{lll}
$.96 \mathrm{E}-01$ & 108933.95 \\
$.33 \mathrm{E}-01$ & 10893.95 \\
\hline
\end{tabular} 8.95E-04 $50893-96$ $\begin{array}{ll}1.53 \mathrm{E}+02 & 0 . \mathrm{T} 001 \mathrm{e} \\ 6.02 \mathrm{E}-04 & 10893-95\end{array}$ $\begin{array}{rl}6.14 E-01 & 1083-96 \\ 4.24 \mathrm{E}-02 & 0 . T 001\end{array}$ . $40 \mathrm{E}+01 \quad \mathrm{I0893} .96$ $1.50 E+01$ 10893.96 $\begin{array}{ll}3.01 E+00 & 10893-36 \\ 4.22 E-07 & 10893-96\end{array}$ 2. 11 E- $0910893-96$ $1.47 E+02$ O. Toole $\begin{array}{ll}1.24 E+01 & 0 . T 001 e \\ 13 E-01 & 10893-96\end{array}$ 2. $58 \mathrm{BE}-02 \quad 10893.96$ $1.09 \mathrm{E}-08 \quad \begin{aligned} & \mathrm{I} \\ & 10893.95\end{aligned}$ $1.17 \mathrm{E}-02$ iDB93-96 $1.35 E-01 \quad 10893.96$

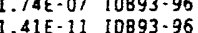
i. $17 \varepsilon+01$ 0.Toole

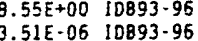
$.51 E-063$
$1.14 E-03 \quad 10893-96$ $5.99 E-10 \quad 10893-96$

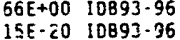
$8.34 \mathrm{E}+01 \quad 0^{\circ}$. Toole $\begin{array}{rl}1.89 E-03 & 10893-96 \\ .56 E-11 & 10893-96\end{array}$

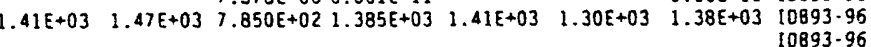

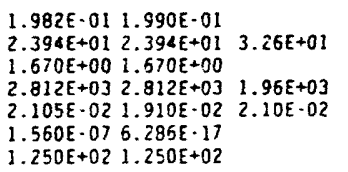
10893.96
$10893-95$

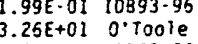
$\begin{array}{ll}1.67 E+00 & 10893.96 \\ 2.81 E+03 & 1093-96\end{array}$

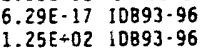

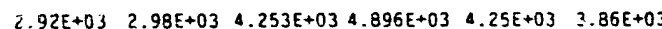


WHC-EP-0791

This page intentionally left blank.

$$
\mathrm{J}-10
$$


WHC-EP-0791

\section{APPENDIX K}

\section{PROCESS STRATEGY TECHNICAL RISK}

K-1 
WHC-EP-0791

\section{LIST OF TERMS}

CPU

compact processing unit

DOE

HLW

LLW

SREX

TCIX

TRUEX-C

U.S. Department of Energy

high-level wasto

low-level waste

strontium extraction

Technetium Ion Exchange

Transuranic Extraction Option C 
This appendix discusses the development of a process strategy technical risk estimation method based on data contained in Pretreatment status Report on the Identification and Evaluation of Alternative Processes (Sutherland et a1. 1993). The reference document presents a status report on the identification and evaluation of alternative processes. It contains an appendix showing various alternative process technology development levels and extents. This data is tabulated using the following categories:

- Maturity of particular process technologies - extent and scale of testing with simple or realistic simulants (e.g., laboratory, bench, pilot plant, full-scale, operating full-scale facility)

- Potential benefit of particular process technologies - activity level and timeliness of testing and development

- Current U.S. Department of Energy (DOE) funding level of particular process technologies - extent of funding and the associated advance of maturity.

To allow an assessment of the technical risk associated with each of the process strategies, weighting factors were assigned to the above process technology development levels and extents as shown below. The weighting factor assignments are considered first approximation using reasonable engineering judgment. Using these weights, numerical estimates of technical risk factor indexes were calculated for particular process technologies using the following formulas:

$$
\begin{gathered}
\text { Technical Risk Index }=1 /(\text { Level Weight * Extent Weight) } \quad \text { (R\&D and DT\&E) } \\
\text { Technical Risk Index }=1 /(\text { Level Weight) (Proven Levels) }
\end{gathered}
$$

where:

R\&D = Research and Develorment.

DT\&E $=$ Demonstration Testillg and Experimentation.

\begin{tabular}{|l|c|}
\hline \multicolumn{1}{|c|}{ Process Development Level } & Weight \\
\hline Research and development & 1 \\
Demonstration testing and experimentation & 10 \\
Proven in an operatirg full-scale facility \\
for processing a local high-level waste \\
$\begin{array}{l}\text { Proven in an operating full-scale facility } \\
\text { for processing Hanford high-level waste }\end{array}$ & 100 \\
\hline
\end{tabular}




\begin{tabular}{|c|c|}
\hline Process Development Extent & Weight \\
\hline \multicolumn{2}{|c|}{ Maturity of particular process technologies } \\
\hline \multicolumn{2}{|l|}{ Research and Development: } \\
\hline $\begin{array}{l}\text { High }=\text { Bench scale with realistic simulants } \\
\text { Medium }=\text { Laboratory scale with simple simulants } \\
\text { Low }=\text { Little or no tesiing }\end{array}$ & $\begin{array}{l}1.0 \\
0.3 \\
0.1\end{array}$ \\
\hline \multicolumn{2}{|l|}{ Demonstration Testing and Experimentation: } \\
\hline $\begin{array}{l}\text { High }=\text { Full scale with realistic simulants } \\
\text { Medium }=\text { Pilot plant with realistic simulants } \\
\text { Low }=\text { Pilot plant with simple simulants }\end{array}$ & $\begin{array}{l}1.0 \\
0.3 \\
0.1\end{array}$ \\
\hline \multicolumn{2}{|c|}{ Current DOE funding level of particular process technologies } \\
\hline \multicolumn{2}{|c|}{ Either R\&D or DT\&E: $\quad$ : } \\
\hline $\begin{array}{l}\text { High }=\text { Significantly advanced maturity } \\
\text { Medium }=\text { Moderately advanced maturity } \\
\text { Low }=\text { Negligible advance of maturity }\end{array}$ & $\begin{array}{l}1.0 \\
0.3 \\
0.1\end{array}$ \\
\hline \multicolumn{2}{|c|}{ Pctential benefit of particular process technologies } \\
\hline Either R\&D or DT\&E: & \\
\hline $\begin{array}{l}\text { High }=\text { High timely } \\
\text { Medium }=\text { Moderate timely } \\
\text { Low }=\text { Low, untimely or inactive concept }\end{array}$ & $\begin{array}{l}1.0 \\
0.3 \\
0.1\end{array}$ \\
\hline
\end{tabular}

Table Kl shows the technical risk factor data for various process technologies contained in the reference (Sutherland et al. 1993). These process technologies were related to the various processes represented in each process strategy as shown in Table K2. Table K2 presents the technical risk indexes calculated using the process development level and extent weights applied to the particular process technologies. Note that a low technical risk index value is desirable. The individual process technical risk indexes were summed over each risk category (i.e., maturity, timeliness, and DOE current level of effort) and averaged over each category for each process strategy. The technical risk indexes for the process technologies used in the various process strategies is shown in Figure K1. Figures K2 and K3 show the total and process average technical risk results for each process strategy, respectively.

As shown in Figure $K 1$ the technical risk for the cesium ion exchange for alkaline liquids, the low-level waste (LLW) salt grouting process, and the high-level waste (HLW) or LLW glass vitrification process had the lowest technical risk indexes, because of their essentially fully demonstrated technologies. Note that the DOE current level of effort contributes considerably to the technical risk of many process technologies, representing the slow advance of the process maturity. These processes (e.g., Solids Dissolution, Strontium Extraction [SREX], $\mathrm{NaOH} / \mathrm{HNO}_{3}$ Recycle, Technetium Ion 
Exchange [TCIX], and Uranium Separation associated with the TRUEX process) may require renewed or increased levels of effort to provide successful application of a tank waste pretreatment process strategy.

Figure K2 shows that the CLEAN Option process strategy without compact processing units (CPUS) (i.e., Model CLEANG31) had a total combined category technical risk index that was a factor of approximately 2 and 10 times higher than the Transuranic Extraction Option C (TRUEX-C) and STudge Wash C process strategies, respectively. The contributions of the individual category technical risk indexes to this total combined category technical risk index reflected essentially the same ratio factors, respectively. The exception was for the timeliness category where the CLEAN Option process strategy without CPUs had a total timeliness category technical risk index that was a factor of approximately 14 times higher than the Sludge Wash C process strategy.

The process average combined category technical risk index for the CLEAN Option process strategy without CPUs was nearly the same as the TRUEX-C process strategy and a factor of approximately 3 higher than for the sludge Wash C process strategy. Again, the contributions of the individual category technical risk indexes to this average combined category techniral risk index reflected essentially the same ratio factors, respectively. $T_{i}$ exception was for the timeliness category where the CLEAN Option process stracegy without CPUs had a total timeliness category technical risk index that was a factor of approximately 4 times higher than the Sludge Wash C process strategy.

The use of CPUs in the CLEAN Option process strategy to provide supernate and salt cake processing outside of a central processing facility will necessarily have significant technical risk because it is a new and developing concept. Thus, the CLEAN Option with CPUs should be considered to have more technical risk than the CLEAN Option based only on a central processing facility concept.

In summary, DOE current level of effort contributes considerably to the technical risk of many process technologies, representing the slow advance of the process maturity. These processes (e.g., Solids Dissolution, SREX, $\mathrm{NaOH} / \mathrm{HNO}_{3}$ Recycle, TCIX, and Uranium Separation associated with the TRUEX process) may require renewed or increased levels of effort to provide successful application of a tank waste pretreatment process strategy. The CLEAN Option process strategy without CPUs had a total combined category technical risk index that was a factor of approximately 2 and 10 times higher than the TRUEX-C and Sludge Wash $C$ process strategies, respectively. The process average combined category technical risk index for the CLEAN Option process strategy without CPUs was nearly the same as the TRUEX-C process strategy and a factor of approximately 3 higher than for the Sludge Wash $C$ process strategy. The CLEAN Option using the new and developing CPU concept should be considered to have more technical risk than the CLEAN Option based only on a central processing facility concept.

\section{REFERENCES}

Sutherland, D. G., et al., 1993, Pretreatment Status Report On. The Identification And Evaluation of Alternative Processes, WHC-SD-WM-PRS-012, Rev. O, Westinghouse Hanford Company, Richland, Washington. 
Figure Kl. Technical Risk Index.

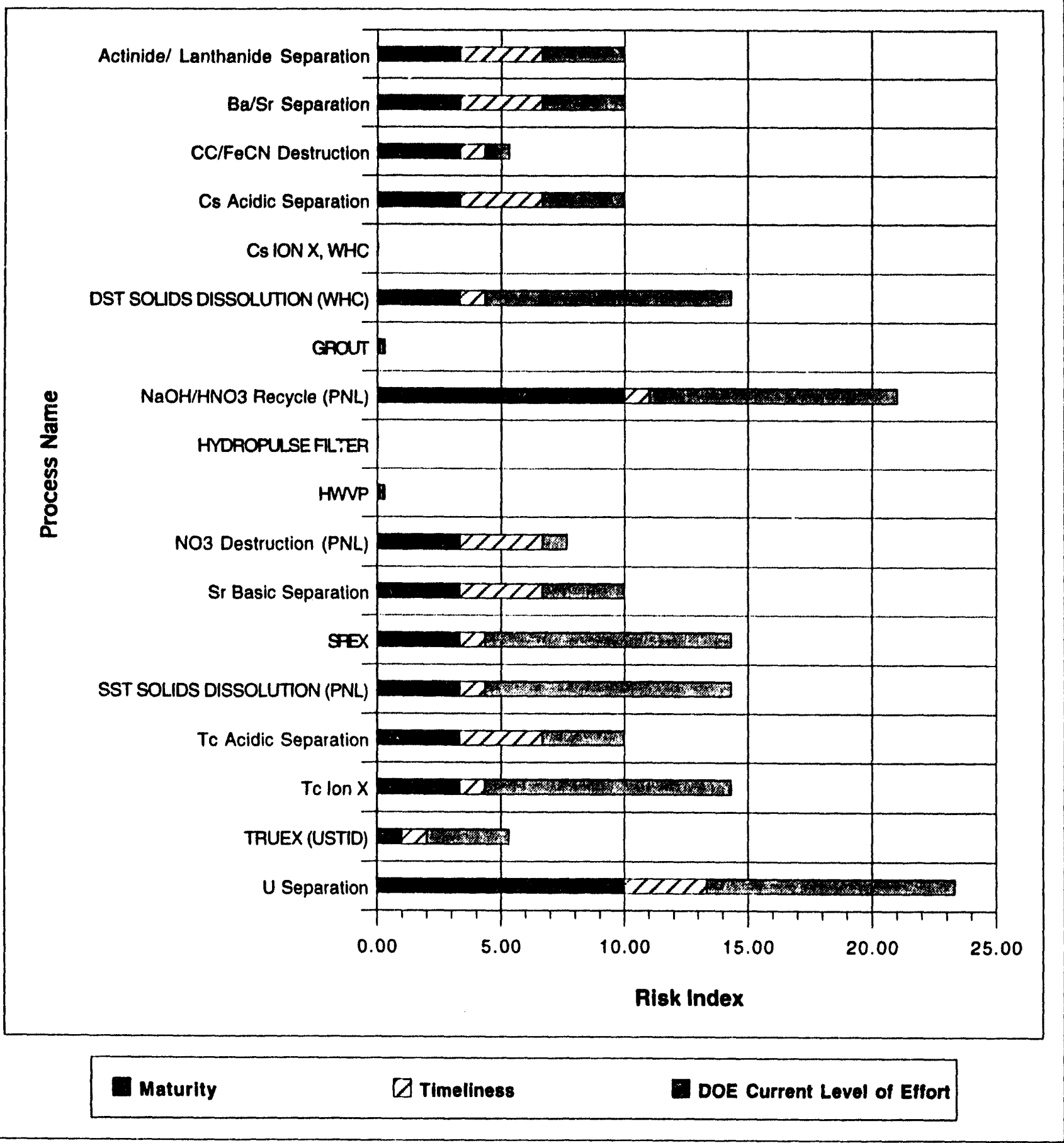


Figure K2. Total Technical Risk Index by Process Strategy.

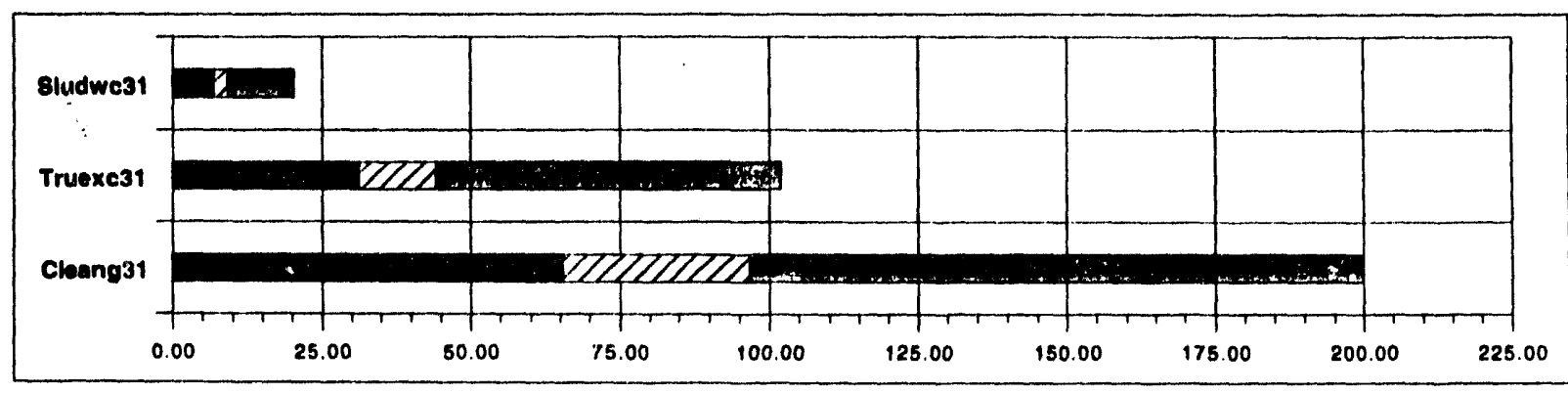

\begin{tabular}{|l|l}
\hline Maturity $\square$ Timeliness & $D$ De Current Level of Effort
\end{tabular}

Figure K3. Average Technical Risk Index by Process Strategy.

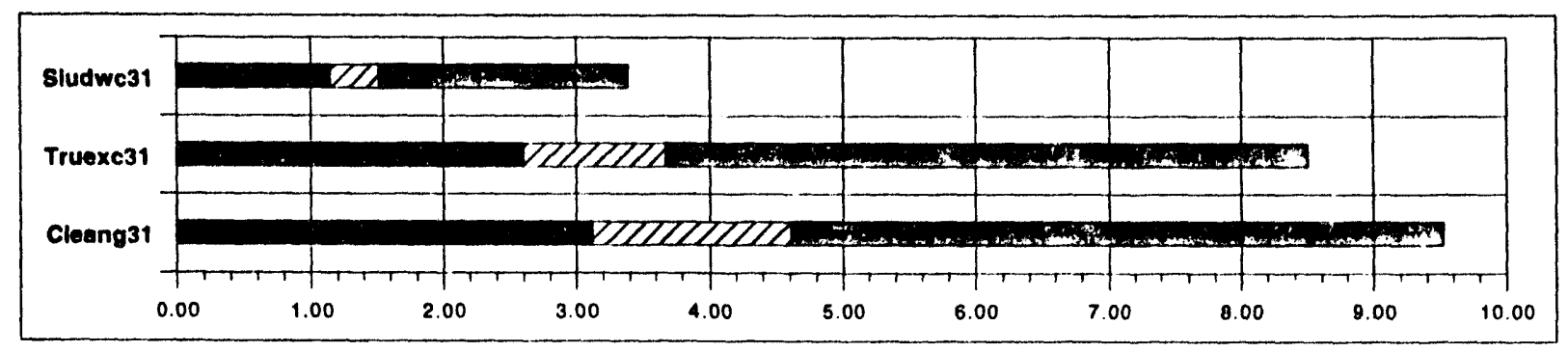

Maturity D Timeliness DOE Current Level of Effort 
Table K1. Technical Risk Factor Data for Various Process Technologies. (sheet 1 of 3)

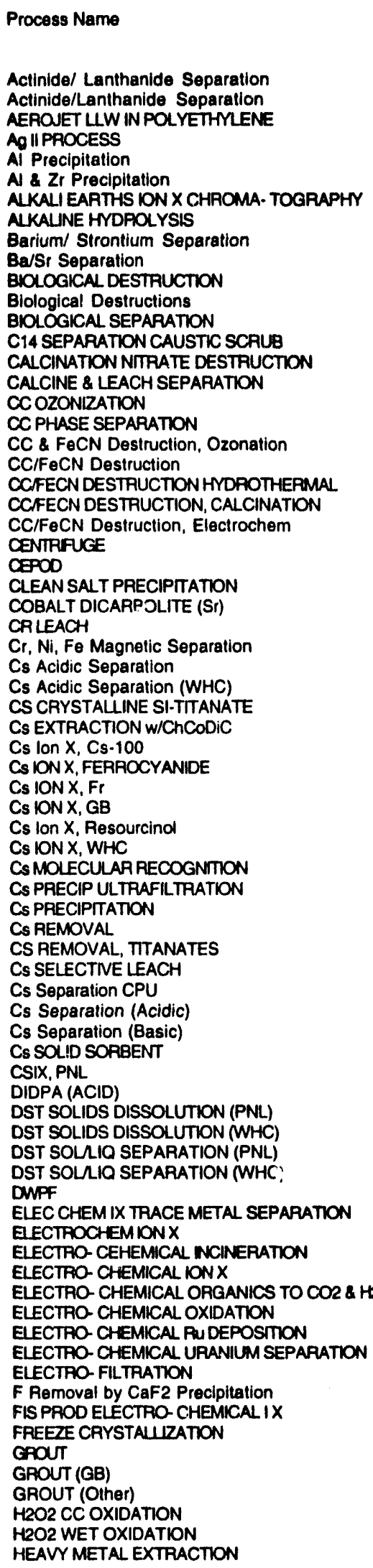

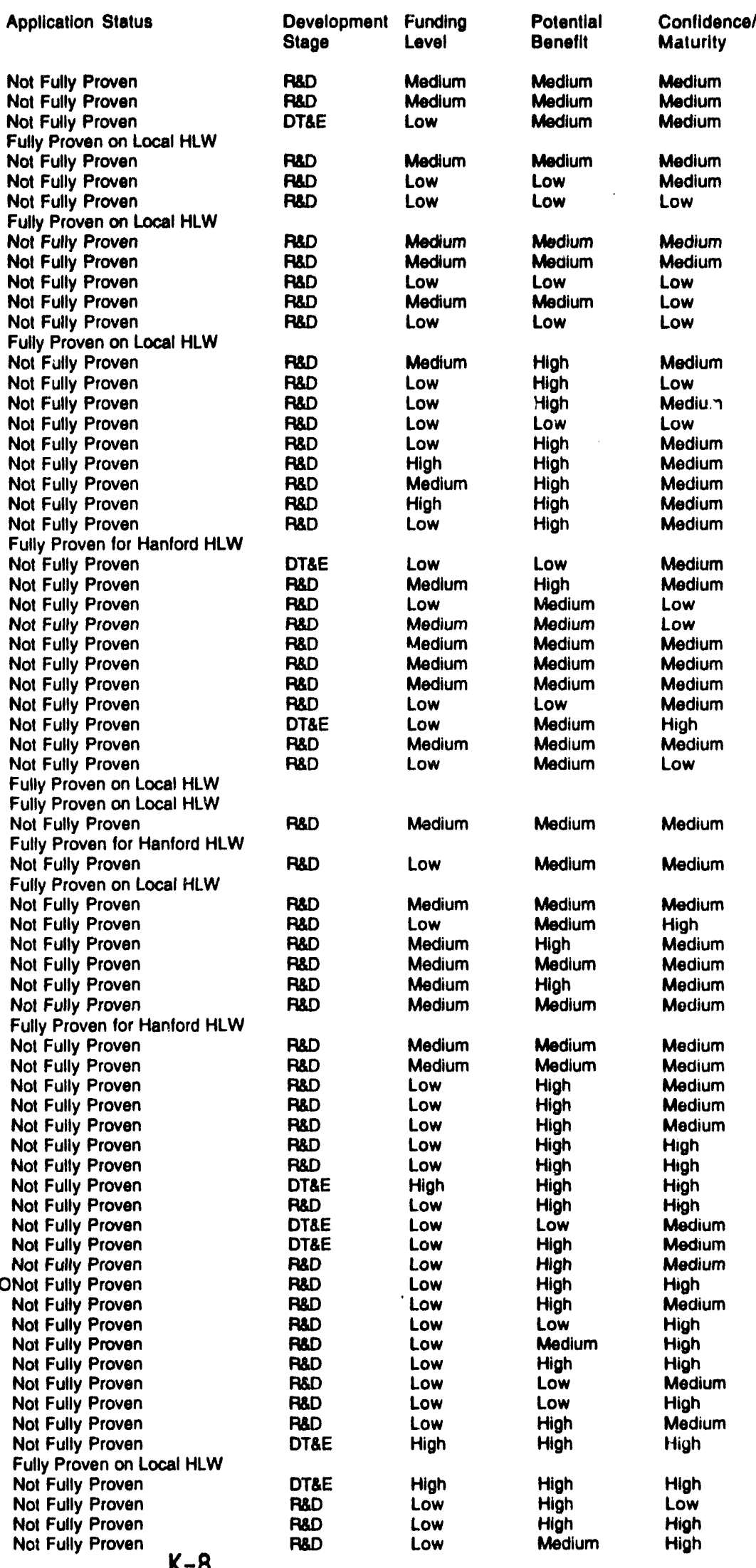

\author{
Application Status
}

Fully Proven on Local HLW

Not Fully Proven

Fully Proven on Local HLW

Not Fully Proven

Not Fully Proven

Fully Proven

Not Fully Proven

Not Fully Proven

Not Fully Proven

Not Fully Proven

Not Fully Proven

Not Fully Proven

Not Fully Proven

Not Fully Proven

Fully proven

Not Fully Proven

Not Fully Proven

Not Fully Proven

Fully Proven on Local

Fully Proven for Hanford HLW

Fully Proven on Local HLW

Not Fully Proven

Not Fully Proven

Not Fully Proven

Not Fully Proven

Fully Proven for Hanford HLW

Not Fully Proven

Nol Fully Proven

Not Fully Proven

Not Fully Proven

Not Fully Proven

Not Fully Proven

Not Fully Proven

Not Fully Proven

Not Fully Proven

Fully Proven on Local HLW

Not Fully Proven

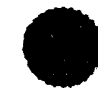


Table K1. Technical Risk Factor Data for Various Process Technologies. (sheet 3 of 3 )

Process Name

SST SOLIDS DISSOLUTION (PNL)

SST SOLIDS DISSOLUTION WHC)

SST SOLLIO SEPARATION (PNL)

SST SOLLIO SEPARATION (WHC)

SUBSTITUTED HALONAMIDES

SUPER. CRITKCAL WATEROXIUATION

Tc Acidic Separation

Tc Acidic Separation (WHC)

TC ADSORPTION (PNL)

TC ADSORPTION (WHC)

Tc Crystalline SI-Titanate

TCEXTRACTION

Tc $\operatorname{lon} X$

TC MOLECULAR RECOGNTION (TC)

TC MOLECULAR RECOGNITION (TCSU)

TCPRECIPITATYON

Tc Pre-Separation (Acidic)

TO SELECTIVE LEACH

Tc Separation (Acidic)

Tc Separation (Basic)

TC SEPARATION (Fr)

Tc SEPARATION (Japan)

Tc Solid Sorbent

TITANATE Sr REMOVAL

TITANATE TFU SEPARATION

Ti/Zeolite Separations

TRANS PU EXTRACTION W/ChCODIC

TRANS PUION $X$

TRANS PUION $X$ CHROMA- TOGRAPHY

TRANSMUTATION (Japan)

TAANSMUTATION (OTher)

TRANSMUTATION (PNL)

TRANSMUTATION (WHC)

TRU Basic Separation

TRU BY EIX + ACT-DE-CON

TRU FLOC ULTRA. FILTRATION

TRU MOLECULAR RECOGNITION

TRU PRECIPITATION

TRU SELECTIVE LEACH

TRU Separation (Acidic)

TRU Separation (Basic)

TRU SOLID SORBENT

TRU TI(OH)A PAECIPITATION

TRUEX (Japan)

TRUEX (USTID)

TRUEX (WHC)

$U$ Magnetic Separation

UMOLECULAR RECOGNITON

U Separation

ULTRAFILT. CENTRIFUGE HM FLOCCU- LATION

Uranium Separation

Vitrification to Glass

VITRIFICATION (GB)

VITAIFICATION (Japan)

Water Wash

WATER WASH SETTE-DECANT

WATER WASH $(\mathrm{Br})$

WATER WASH (SRS)

WET OXIDATION
Appilcation Status

Not Fully Proven

Not Fully Proven

Not Fully Proven

Not Fully Proven

Not Fully Proven

Not Fully Proven

Nol Fully Proven

Not Fully Proven

Not Fully Proven

Not Fully Proven

Not Fully Proven

Fully Proven on Local HLW

Not Fully Proven

Not Fully Proven

Not Fully Proven

Not Fully Proven

Not Fully Proven

Not Fully Proven

Nol Fully Proven

Not Fully Proven

Not Fully Proven

Not Fully Proven

Not Fully Proven

Not Fully Proven

Not Fully Proven

Fully Proven on Local HLW

Nol Fully Proven

Not Fully Proven

Not Fully Proven

Not Fully Proven

Not Fully Proven

Not Fully Proven

Not Fully Proven

Not Fully Proven

Not Fully Proven

Fully Proven on Local HLW

Nol Fully Proven

Not Fully Proven

Not Fully Proven

Not Fully Proven

Not Fully Proven

Not Fully Proven

Fully Proven on Local HLW

Not Fully Proven

Not Fully Proven

Not Fully Proven

Not Fully Proven

Not Fully Proven

Not Fully Proven

Fully Proven on Local HLW

Not Fully Proven

Not Fully Proven

Fully Proven on Local HLW

Fully Proven on Local HLW

Fully Proven for Hanford HLW

Fully Proven for Hanford HLW

Fully Proven on Local HLW

Fully Proven on Local HLW

Not Fully Proven

\begin{tabular}{|c|c|c|c|}
\hline $\begin{array}{l}\text { Development } \\
\text { Stage }\end{array}$ & $\begin{array}{l}\text { Funding } \\
\text { Level }\end{array}$ & $\begin{array}{l}\text { Potential } \\
\text { Benefll }\end{array}$ & $\begin{array}{l}\text { Confidenrel } \\
\text { Maturity }\end{array}$ \\
\hline $\begin{array}{l}\text { RED } \\
\text { RED } \\
\text { R\&D } \\
\text { R\&D } \\
\text { R\&D } \\
\text { R\&D } \\
\text { R\&D } \\
\text { RED } \\
\text { RED } \\
\text { RED } \\
\text { RED }\end{array}$ & $\begin{array}{l}\text { Low } \\
\text { Low } \\
\text { Low } \\
\text { Low } \\
\text { Low } \\
\text { Medium } \\
\text { Modium } \\
\text { Modium } \\
\text { Low } \\
\text { Low } \\
\text { Low }\end{array}$ & $\begin{array}{l}\text { High } \\
\text { High } \\
\text { High } \\
\text { High } \\
\text { Low } \\
\text { High } \\
\text { Modium } \\
\text { Modium } \\
\text { Modium } \\
\text { Modium } \\
\text { Low }\end{array}$ & $\begin{array}{l}\text { Medium } \\
\text { High } \\
\text { High } \\
\text { High } \\
\text { Low } \\
\text { Medium } \\
\text { Merllum } \\
\text { Medium } \\
\text { Medium } \\
\text { Medium } \\
\text { Medium }\end{array}$ \\
\hline 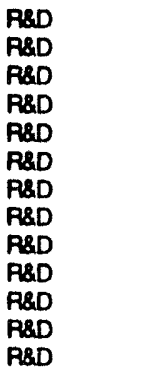 & $\begin{array}{l}\text { Low } \\
\text { Low } \\
\text { Low } \\
\text { Medium } \\
\text { Low } \\
\text { Medium } \\
\text { Medium } \\
\text { Low } \\
\text { Low } \\
\text { Low } \\
\text { Medium } \\
\text { Low } \\
\text { Low }\end{array}$ & $\begin{array}{l}\text { High } \\
\text { High } \\
\text { Modium } \\
\text { High } \\
\text { Modium } \\
\text { High } \\
\text { Modium } \\
\text { High } \\
\text { High } \\
\text { High } \\
\text { High } \\
\text { High } \\
\text { High }\end{array}$ & $\begin{array}{l}\text { Medium } \\
\text { Medium } \\
\text { Medium } \\
\text { Medium } \\
\text { Medium } \\
\text { Medium } \\
\text { Medium } \\
\text { Medium } \\
\text { Low } \\
\text { High } \\
\text { Medium } \\
\text { Medium } \\
\text { Medium }\end{array}$ \\
\hline $\begin{array}{l}\text { DT\&E } \\
\text { P\&D } \\
\text { R\&D } \\
\text { R\&D } \\
\text { R\&D } \\
\text { R\&D } \\
\text { R\&D } \\
\text { R\&D } \\
\text { R\&D }\end{array}$ & $\begin{array}{l}\text { Low } \\
\text { Low } \\
\text { Low } \\
\text { Low } \\
\text { Low } \\
\text { Low } \\
\text { Low } \\
\text { Modium } \\
\text { Low }\end{array}$ & $\begin{array}{l}\text { Medium } \\
\text { High } \\
\text { Medium } \\
\text { Low } \\
\text { Low } \\
\text { Low } \\
\text { Low } \\
\text { Modium } \\
\text { High }\end{array}$ & $\begin{array}{l}\text { High } \\
\text { Medium } \\
\text { Low } \\
\text { Medium } \\
\text { Medium } \\
\text { Medium } \\
\text { Medium } \\
\text { Medium } \\
\text { High }\end{array}$ \\
\hline $\begin{array}{l}\text { R\&D } \\
\text { R\&D } \\
\text { R\&D } \\
\text { P\&D } \\
\text { R\&D } \\
\text { P\&D }\end{array}$ & $\begin{array}{l}\text { Low } \\
\text { Medium } \\
\text { Medium } \\
\text { Medium } \\
\text { Medium } \\
\text { Medium }\end{array}$ & $\begin{array}{l}\text { High } \\
\text { High } \\
\text { High } \\
\text { High } \\
\text { Modium } \\
\text { High }\end{array}$ & $\begin{array}{l}\text { Medium } \\
\text { Medium } \\
\text { Medium } \\
\text { High } \\
\text { Medium } \\
\text { Medium }\end{array}$ \\
\hline $\begin{array}{l}\text { R\&D } \\
\text { R\&D } \\
\text { P\&D } \\
\text { P\&D } \\
\text { P\&D } \\
\text { R\&D }\end{array}$ & $\begin{array}{l}\text { Medium } \\
\text { Medium } \\
\text { Medium } \\
\text { Medium } \\
\text { Low } \\
\text { Low }\end{array}$ & $\begin{array}{l}\text { High } \\
\text { High } \\
\text { High } \\
\text { Medium } \\
\text { Medium } \\
\text { Medium }\end{array}$ & $\begin{array}{l}\text { High } \\
\text { High } \\
\text { High } \\
\text { Medium } \\
\text { Medium } \\
\text { Low }\end{array}$ \\
\hline $\begin{array}{l}\text { R\&D } \\
\text { DT\&E }\end{array}$ & $\begin{array}{l}\text { Low } \\
\text { High }\end{array}$ & $\begin{array}{l}\text { Medium } \\
\text { High }\end{array}$ & $\begin{array}{l}\text { Low } \\
\text { High }\end{array}$ \\
\hline
\end{tabular}

R\&D

Low

High

High 
WHC-EP-0791

Table K2. Technical Risk Indexes by Process Name and Overall Strategy. **** Technical Risk Category *k**

Procese Name

ATER WASH SETTLE-DECANT

U Separation

TRUEX (USTID)

Tc $\operatorname{lon} X$

HWP

SPe

SST SOLIDS DISSOLUTION (PNL)

Tc Acidic Separation GROUT

DST SOLIDS DISSOLUTION (WHC) Cs ION X, WHC

CCFFEN DESTRUCTION, CALCINATION

TOTAL, (CLEANG31/TRUEXC31) AVERAGE, (CLEANG31/TRUEXC31)

WATER WASH SETTLE-DECANT
TC ION X
HWWP
GROUT
CS ION $X$, WHC

CCFECN DESTRUCTION, CALCINATION

TOTAL, (CLEANG31/SLUDWC31) AVERAGE, (CLEANG31/SLUDWC31)

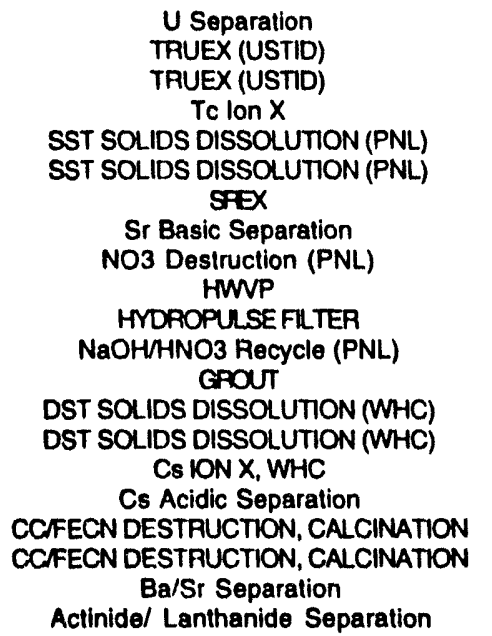

TOTAL:

Model Name
WASHSLUD
UTCSTRAP
TFUEX
TCIX1
VITHW
SFEX
DISSOLVA
TCIX2
GROUTW
DISSOLVA
CSA
DESTOPG

Maturity

Timoliness

DOE Current

Level of Effort

Combined

Categorles

TAUEXC31

\begin{tabular}{|c|c|c|c|c|}
\hline $\begin{array}{r}0.01 \\
10.00 \\
1.00 \\
3.33 \\
0.10 \\
3.33 \\
3.33 \\
3.33 \\
0.10 \\
3.33 \\
0.01 \\
3.33\end{array}$ & $\begin{array}{l}0.01 \\
3.33 \\
1.00 \\
1.00 \\
0.10 \\
1.00 \\
1.00 \\
3.33 \\
0.10 \\
1.00 \\
0.01 \\
1.00\end{array}$ & & $\begin{array}{r}0.01 \\
10.00 \\
3.33 \\
10.00 \\
0.10 \\
10.00 \\
10.00 \\
3.33 \\
0.10 \\
10.00 \\
0.01 \\
1.00\end{array}$ & \\
\hline 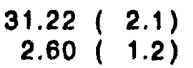 & $\begin{array}{r}12.891 \\
1.071\end{array}$ & $\begin{array}{l}2.4) \\
1.4)\end{array}$ & $\begin{array}{r}57.89(1.8) \\
4.82(1.0)\end{array}$ & 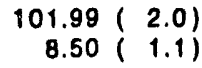 \\
\hline
\end{tabular}

SLUDWC31

WASHSLUD
TCIXI
VITRW
GROUTW
CSX
DESTORG

$\begin{array}{rrr}0.01 & 0.01 & 0.01 \\ 3.33 & 1.00 & 10.00 \\ 0.10 & 0.10 & 0.10 \\ 0.10 & 0.10 & 0.10 \\ 0.01 & 0.01 & 0.01\end{array}$

$\begin{array}{lll}3.33 & 1.00 & 1.00\end{array}$

$\begin{array}{rrrrr}6.89(9.5) & 2.22(14.1) & 11.22(9.2) & 20.33(9.8) \\ 1.15(2.7) & 0.37(4.0) & 1.87(2.6) & 3.39(2.8)\end{array}$

\section{CLEANG31}

PUPWYN
EXTRUPU
EXTRAMLN
TCIX
DISOLVA
DISOLVF
SFEX
SFIX
DNITHH
VITRW
SEPSOUQ
PCOVACID
GROUTW
DISOLVA
DISOLVF
CSO
APMOSIX
DESTOFG
CALCINE
SFBAIX
AMLANIX

10.00
1.00
1.00
3.33
3.33
3.33
3.33
3.33
3.33
0.10
0.01
10.00
0.10
3.33
3.33
0.01
3.33
3.33
3.33
3.33
3.33

65.55
3.12

$$
\begin{aligned}
& 3.33 \\
& 1.00 \\
& 1.00 \\
& 1.00 \\
& 1.00 \\
& 1.00 \\
& 1.00 \\
& 3.33 \\
& 3.33 \\
& 0.10 \\
& 0.01 \\
& 1.00 \\
& 0.10 \\
& 1.00 \\
& 1.00 \\
& 0.01 \\
& 3.33 \\
& 1.00 \\
& 1.00 \\
& 3.33 \\
& 3.33 \\
& 31.22 \\
& 1.49
\end{aligned}
$$$$
\begin{array}{r}
10.00 \\
3.33 \\
3.33 \\
10.00 \\
10.00 \\
10.00 \\
10.00 \\
3.33 \\
1.00 \\
0.10 \\
0.01 \\
10.00 \\
0.10 \\
10.00 \\
10.00 \\
0.01 \\
3.33 \\
1.00 \\
1.00 \\
3.33 \\
3.33 \\
103.22 \\
4.92
\end{array}
$$ 
WHC-EP-0791

This page intentionally left blank. 


\section{DISTRIBUTION}

\section{Number of Copies}

OEFSITE

2

Los Al amos National Laboratory

P.0. Box 1663

Los Alamos, New Mexico 87545

Dr. S. F. Agnew

Dr. M. Attrep, Jr.

1

Martin Marietta Energy Systems

P.0. Box 2008

Oak Ridge, Tennessee 37831-6149

Dr. J. Watson

2

U.S. Department of Energy. Headquarters Office of Technology Development Trevion II Building, EM-545 12800 Middlebrook Road Germantown, Maryland 20874

Dr. T. Fryberger, Program Manager

1

U.S. Department of Energy. Headquarters \% Dr. T. Fryberger

Trevion II Building, EM-545

12800 Middlebrook Road

Germantown, Maryland 20874

Dr. D. Oakley

1

Waste Policy Institute

Quince Diamond Executive Center

555 Quince Orchard Road

Gaithersburg, Maryland 20878-1437

Dr. I. Tasker

1

Westinghouse Savannah River Company

U.S. Highway 19

Aiken, South Carolina 29802

Dr. M. Thompsoil

1

Dr. R. Wymer

188A Outer Drive

Oak Ridge, Tennessee 37831 
WHC-EP-0791

\section{DISTRIBUTION (cont.)}

\section{ONSITE}

3

U.S. Department of Energy.

Richland Operations office

D. E. Trader (3) K8-50

17

Pacific Northwest Laboratory

L. K. Holton, Jr.

P7-43

M. Hoza

W. L. Kuhn (4)

K7-22

D. E. Kurath

$\mathrm{K} 2-21$

L. Lauerhass (6)

L. J. Sealock, Jr.

J. I. Straalsund

(2)

P7 -43

P8-38

J. L. Swanson

K2-10

B1 -40

P7 -25

24

Westinghouse Hanford Company

J. N. Appel

G3-21

S. A. Barker

G3-20

W. B. Barton

L4-75

J. D. Berger

LO-18

A. L. Boldt

H5- 49

G. W. Jackson

H6-33

G. Jansen, Jr.

B. J. Knutson

(6)

$\mathrm{LO}-14$

G. T. Maclean

LO-14

R. M. Orme

H5- 49

L4-75

S. E. Seeman

LO-06

E. J. Slaathaug

H5-49

D. G. Sutherland

L4-72

D. J. Washenfelder

L4-75

B. D. Zimmerman

LO-06

Information Release

Administration (3)

L8-07 

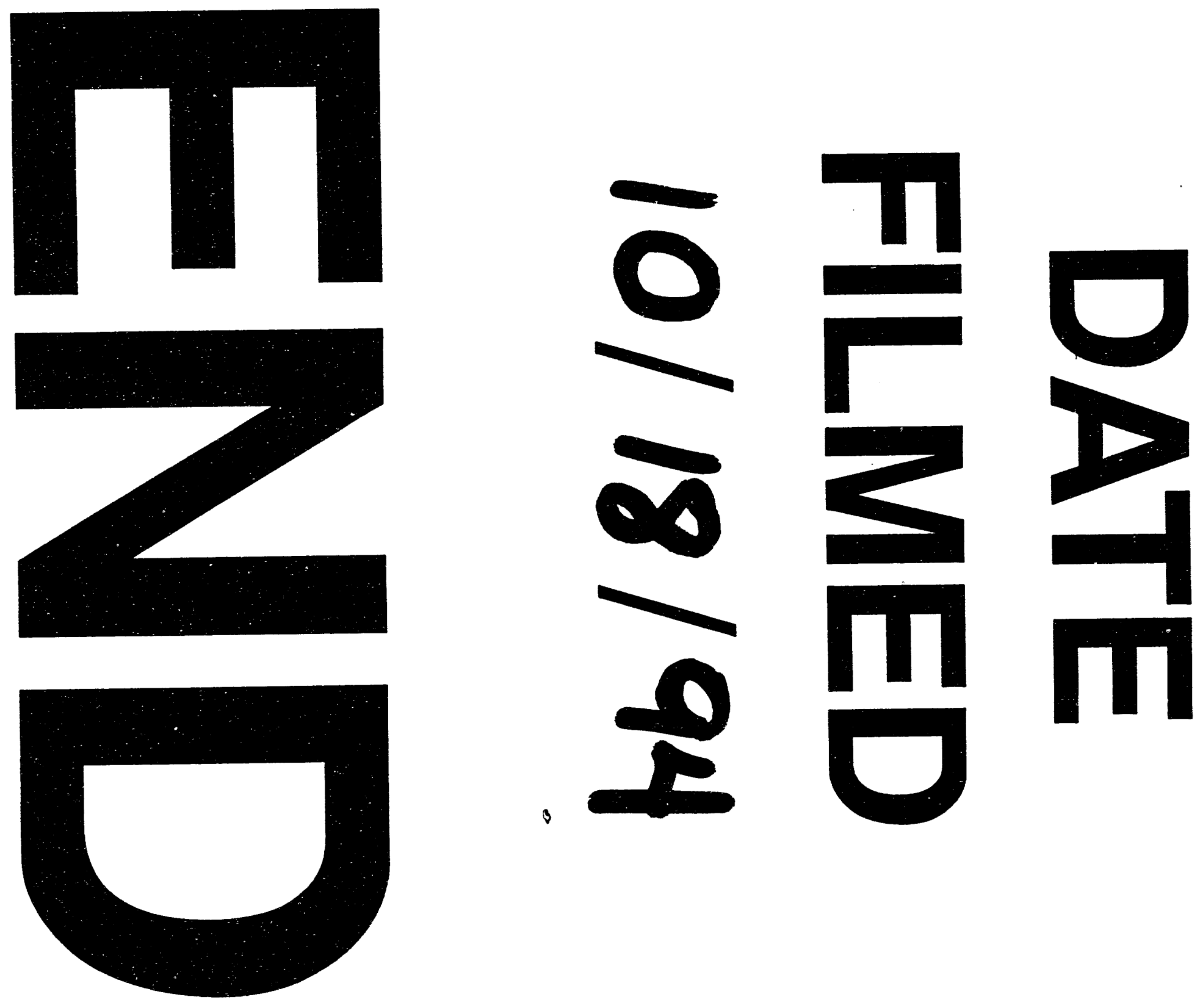
